\title{
Finite Element Modelling of Anchorage to Concrete Systems at Different Strain Rates
}

by

Lenda T. Ahmed Al Saeab

A thesis submitted to the Faculty of Graduate and Postdoctoral Affairs in partial fulfillment of the requirements for the degree of

Doctor of Philosophy

in

Civil Engineering

Carleton University

Ottawa, Ontario

(C) 2019

Lenda T. Ahmed Al Saeab 


\begin{abstract}
Demand for flexibility in design and faster construction times has resulted in the increasing use of fasteners in a variety of concrete structures. These structures are exposed to static and dynamic loading conditions. Furthermore, these structures can be exposed to high strain rate loading such as encountered in impact and blast loads. Thus, anchorage systems used to fasten elements to concrete structures are also exposed to the high strain rates of loading which can be tensile and shear loads. If not adequately designed and constructed, anchorages can fail in a catastrophic manner and pose significant threat to building safety and the life of building occupants.
\end{abstract}

Behaviour of anchors embedded into concrete and subjected to static load has been widely investigated experimentally. However, despite the fact that many structures that contain anchorage systems are exposed to dynamic loads, the research in this vital area is limited. Currently, no guidance is available in design codes for the anchorage response under high strain rate loading. The American Concrete Institute and Concrete Capacity Design methods are recommended for anchorage system subjected to static and low cycle dynamic loading only. Hence, there is a need to develop a design method to predict the anchorage response and capacity under impact and blast loading.

The project presented in this thesis aims to investigate the tensile and shear behaviour of cast-in-place, adhesive and undercut anchors subjected to different strain rates using LSDYNA software. Numerical models of the anchorage systems with different design parameters were developed and mesh sensitivity analyses were carried out to determine mesh sizes that best simulated the experimental results obtained from the literature. The 
ultimate static capacity results were verified with the design methods. Effect of strain rate, embedment depth, and anchor diameter on the tensile and shear failure loads was investigated. Failure modes for the anchorage systems were also examined at different strain rates. Concrete cone breakout diameter and failure cone angles were investigated. A relation between the ultimate loads and the strain rates was investigated and dynamic increase factors (DIF) for design were determined. Regression analysis was performed to predict a relation that accurately represents the finite element results. Results of the tensile and shear loading of the anchorage to concrete systems show that anchorage to concrete system capacity increases with an increase in the strain rates. The failure mode of the anchorage systems is influenced by the strain rate. Maximum DIFs of 1.74, 1.13 and 1.58 were obtained for the cast-in-place, adhesive and undercut anchors under tensile load respectively where concrete cone breakout failure mode was observed. Maximum DIFs of $1.17,1.13$ and 1.44 respectively were obtained for the cast-in-place, adhesive and undercut anchors exhibited steel failure mode. The maximum DIFs were $1.15,1.18$ and 1.45 respectively for the anchors subjected to shear load where steel failure was observed. 


\section{Acknowledgements}

I would like to express my sincere thanks and gratitude to my supervisor Professor Braimah for his continuous support, suggestions and valid advice throughout this research and

during the preparation of this thesis. I greatly thank Prof. Braimah for his constructive and invaluable recommendations in all phases of the research.

I would like to thank Carleton University, Department of Civil and Environmental Engineering for giving me the support during my study to accomplish my research. Also, I would like to thank Mr. Kenneth Akhiwu for his kind assistance.

Finally, I would like to extend my sincere thanks to my parents, my husband, my brothers and sisters for their continuous encouragement and support. 


\section{Dedication}

To my father and mother,

To my husband,

To the memory of my brother. 


\section{Table of contents}

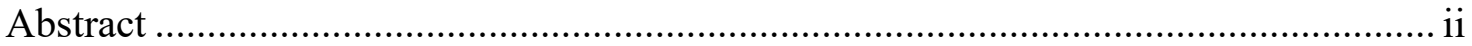

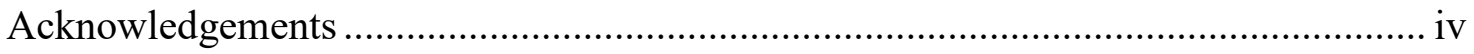

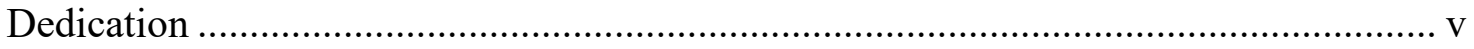

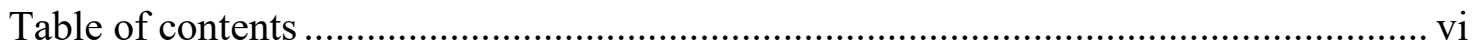

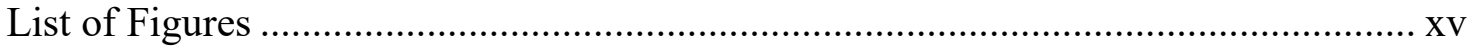

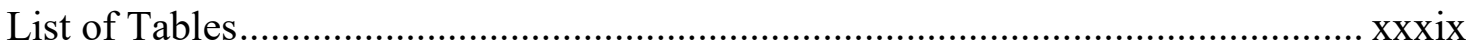

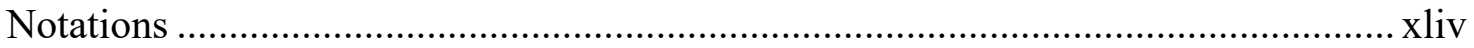

Chapter 1 : Introduction ............................................................................................................... 1

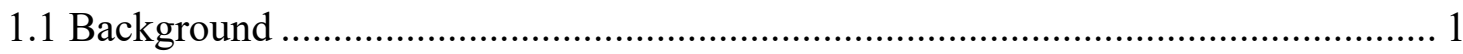

1.2 Objectives and significance of research program ........................................ 4

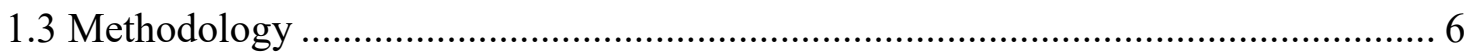

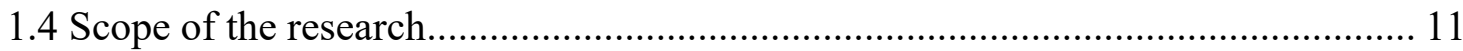

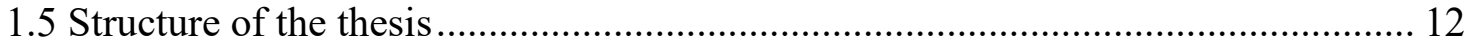

Chapter 2 : Literature review ............................................................................... 15

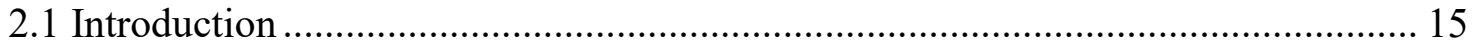

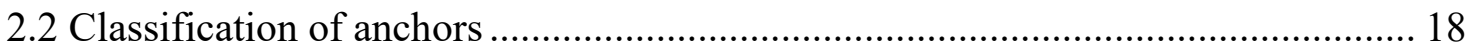

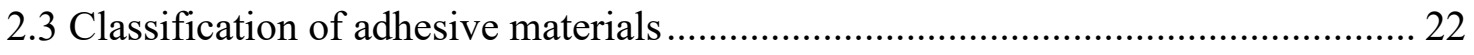

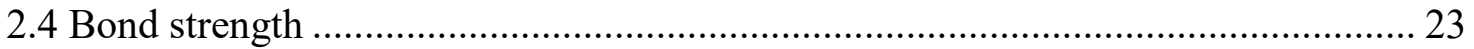

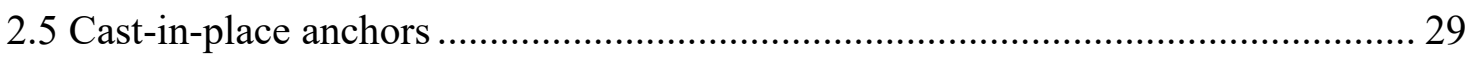

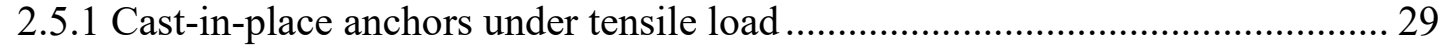

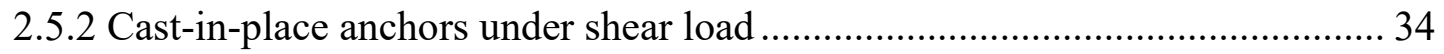


2.6.1 Adhesive anchors under tensile load .............................................................. 39

2.6.2 Adhesive anchors under shear load ............................................................. 47

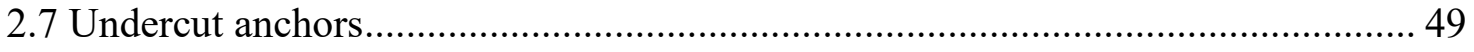

2.7.1 Undercut anchors under tensile load ............................................................ 49

2.7.2 Undercut anchors under shear load ....................................................... 51

2.8 Strain rate effect on material properties ............................................................ 52

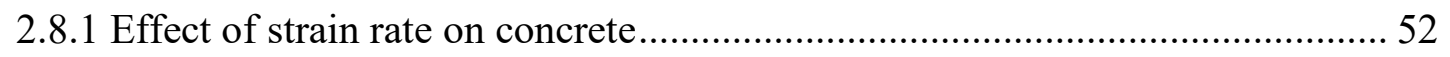

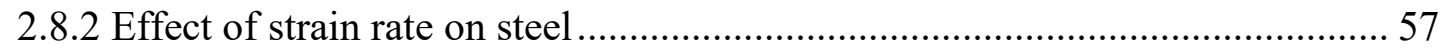

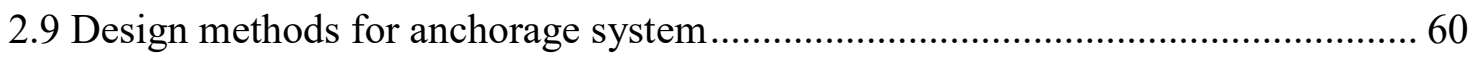

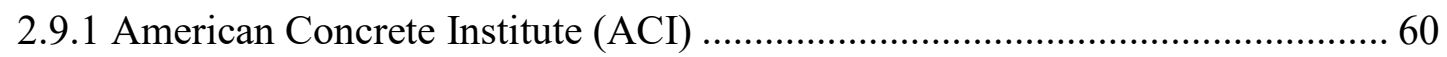

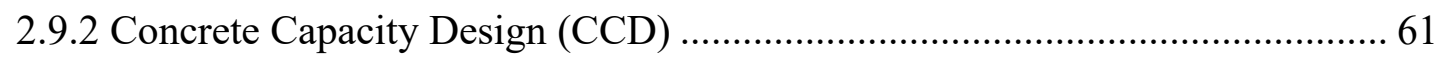

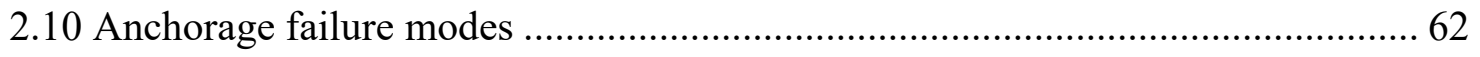

2.10.1 Failure modes of cast-in-place anchors under tensile load.............................. 62

2.10.2 Failure modes of cast-in-place anchors under shear load ................................ 63

2.10.3 Failure modes of adhesive anchors under tensile load .................................... 64

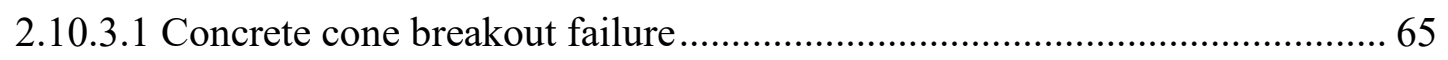

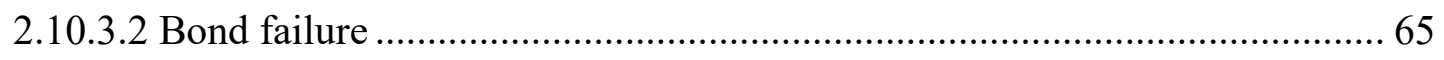

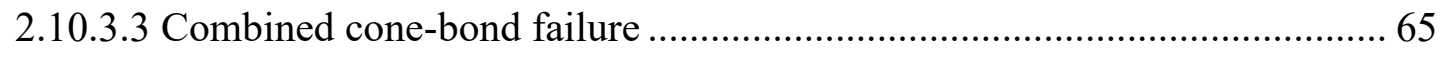

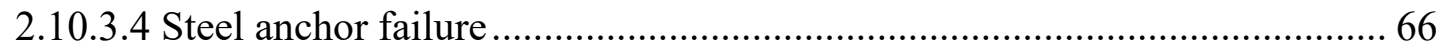

2.10.4 Failure modes of adhesive anchors under shear load ..................................... 66

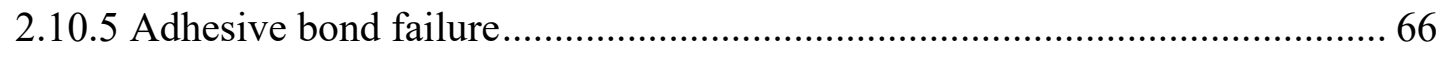

2.10.6 Failure modes of undercut anchors under tensile load ................................... 67 
Chapter 3 : Finite element modelling of anchorage systems........................................... 70

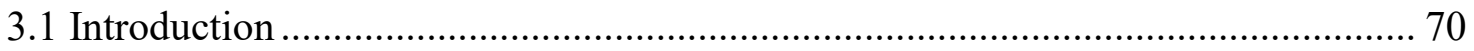

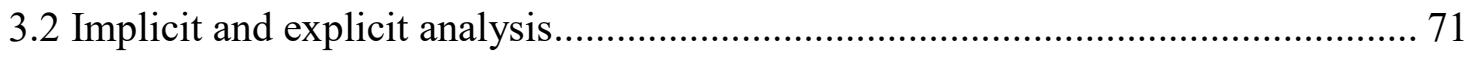

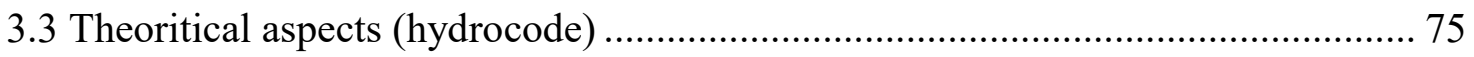

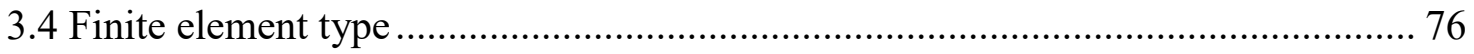

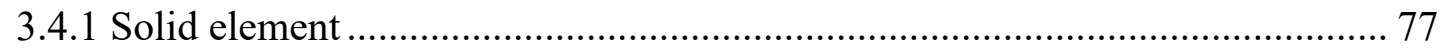

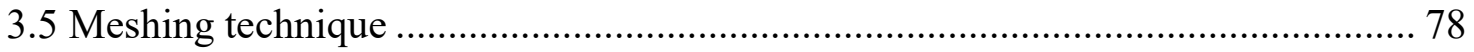

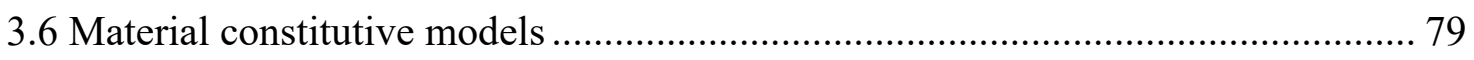

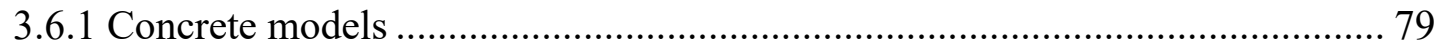

3.6.1.1 Concrete Model (MAT_CSCM_CONCRETE) .............................................. 81

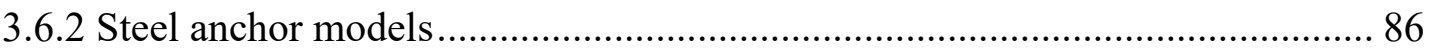

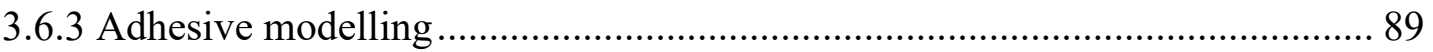

3.6.3.1 Adhesive model (MAT_ARUP_ADHESIVE).............................................. 90

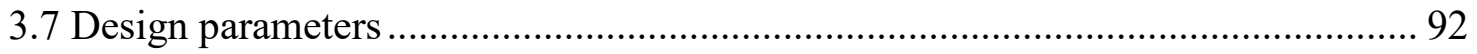

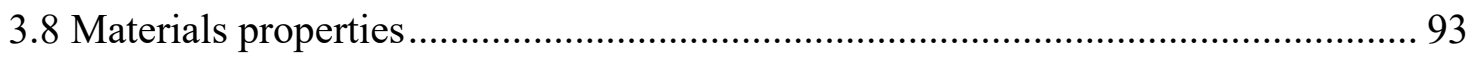

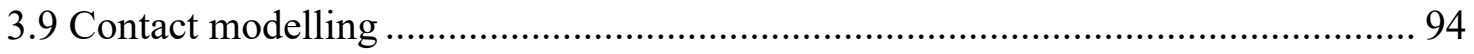

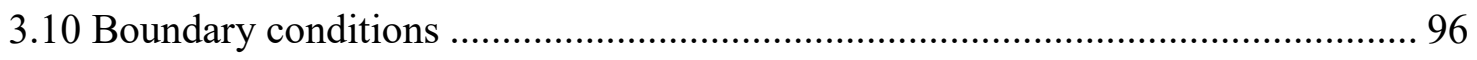

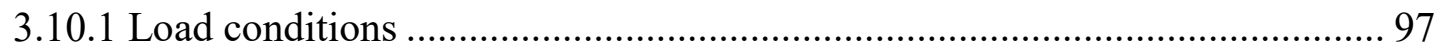

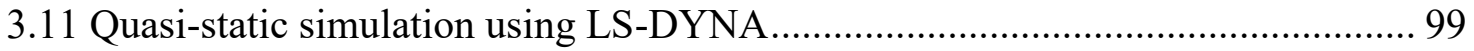

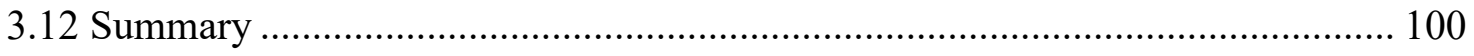


4.1 Cast-in-place anchors under tensile load 101

4.1.1 Finite element modelling of cast-in-place anchors under tensile load 101

4.1.2 Validation of cast-in-place anchor model under tensile load 102

4.1.3 Comparison of finite element results with the ACI and CCD design methods 105

4.1.4 Effect of strain rate on the level of damage and failure mode of cast-in-place anchors 108

4.1.5 Effect of design parameters on failure mode and ultimate tensile load 118

4.1.6 Effect of anchor embedment depth on the concrete cone depth

4.1.7 Effect of strain rate on concrete cone breakout diameter

4.1.8 Effect of strain rate on the tensile behaviour of cast-in-place anchors 125

4.1.9 Effect of strain rate on the ultimate tensile load and dynamic increase factor of cast-in-place anchors

4.1.10 Regression Analysis for cast-in-place anchors under tensile load

4.2 Cast-in-place anchors under shear load

4.2.1 Finite element modelling for cast-in-place anchors under shear load

4.2.2 Validation of cast-in-place anchor model under shear load

4.2.3 Comparison of finite element results with design method

4.2.4 Crack pattern for cast-in-place anchors under shear load 156

4.2.5 Effect of strain rate on the level of damage and failure mode. 158

4.2.6 Effect of design parameters on failure mode and ultimate shear load 164

4.2.7 Effect of strain rate on the shear behaviour of cast-in-place anchors 166 
4.2.8 Effect of strain rate on the ultimate shear load and DIF of cast-in-place anchors 172

4.2.9 Regression analysis for cast-in-place anchors under shear load.... 177

4.2.10 Case study: effect of concrete compressive strength on the shear behaviour of cast-in-place anchors 182

4.2.10.1 Level of damage and failure mode 182

4.2.10.2 Effect of strain rate and concrete compressive strength on shear behaviour 185

4.3 Summary 191

Chapter 5 : Strain rate effect on adhesive anchors. 193

5.1 Finite element modelling for adhesive anchors under tensile load 193

5.1.1 Validation of adhesive anchor model under tensile load. 194

5.1.2 Comparison of finite element results with ACI and CCD design methods.... 199

5.1.3 Effect of strain rate on the level of damage and failure mode of adhesive anchors 200

5.1.4 Effect of design parameters on failure mode and ultimate tensile load. 209

5.1.5 Effect of anchor embedment depth on concrete cone depth 212

5.1.6 Concrete cone breakout diameter for adhesive anchors 215

5.1.7 Effect of strain rate on the tensile behaviour of adhesive anchors 216

5.1.8 Effect of strain rate on the ultimate tensile load and DIF of adhesive anchors 225

5.1.9 Regression Analysis for adhesive anchors under tensile load.... 232

5.2 Adhesive anchors under shear load 242 
5.2.1 Finite element modelling for adhesive anchors under shear load 242

5.2.2 Validation of adhesive anchor model under shear load............................. 242

5.2.3 Comparison of finite element results with design methods........................ 245

5.2.4 Crack pattern for adhesive anchors under shear load ................................ 246

5.2.5 Effect of strain rate on the level of damage and failure mode...................... 248

5.2.6 Effect of design parameters on failure mode and ultimate shear load........... 252

5.2.7 Effect of strain rate on the shear behaviour of adhesive anchors .................. 256

5.2.8 Effect of strain rate on the ultimate shear load and DIF of adhesive anchors 262

5.2.9 Regression analysis for adhesive anchor under shear load......................... 268

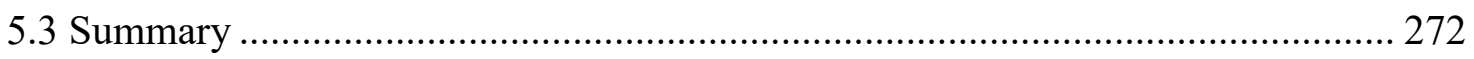

Chapter 6 : Strain rate effect on the undercut anchors ....................................... 274

6.1 Finite element modelling for undercut anchors under tensile load .................... 274

6.1.1 Validation of undercut anchor model under tensile load............................ 275

6.1.2 Comparison of FEA results with the ACI and CCD design methods ........... 278

6.1.3 Effect of strain rate on the level of damage and failure mode..................... 279

6.1.4 Effect of design parameters on failure mode and ultimate tensile load.......... 283

6.1.5 Effect of anchor embedment depth on the ultimate tensile load .................. 286

6.1.6 Effect of strain rate on concrete cone breakout diameter and cone propagation

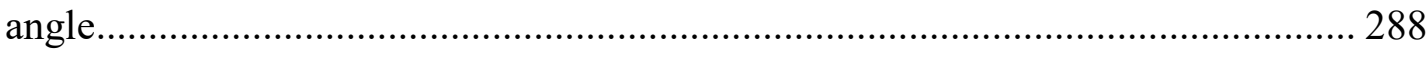

6.1.7 Effect of strain rate on the tensile behaviour of the undercut anchors .......... 290

6.1.8 Effect of strain rate on the ultimate tensile load and DIF of undercut anchors 298

6.1.9 Regression analysis for the undercut anchors under tensile load 303 
6.2 Finite element modeling for undercut anchors under shear load 309

6.2.1 Validation of undercut anchor model under shear load.................................. 309

6.2.2 Comparison of finite element results with design methods............................ 311

6.2.3 Crack pattern for the undercut anchors under shear load ............................... 313

6.2.4 Effect of strain rate on the level of damage and failure mode........................ 314

6.2.5 Effect of design parameters on failure mode and ultimate shear load............ 321

6.2.6 Effect of strain rate on the shear behaviour of undercut anchors ................... 323

6.2.7 Effect of strain rate on the ultimate shear load and DIF of undercut anchors 329

6.2.8 Regression analysis for undercut anchors under shear load .......................... 334

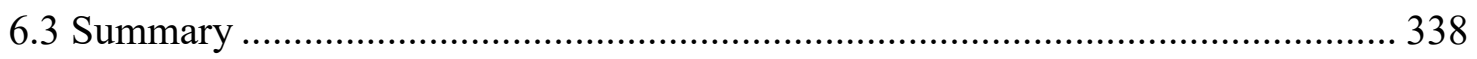

Chapter 7 :Conclusions and recommendations for future research ............................ 340

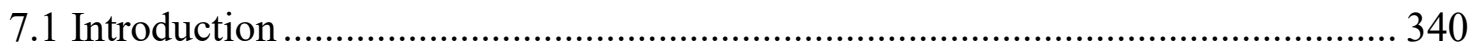

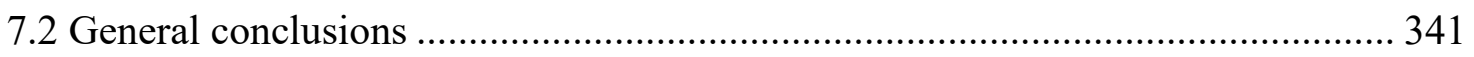

7.3 Behaviour of cast-in-place anchorage system under different strain rates............ 342

7.4 Behaviour of adhesive anchorage system under different strain rates .................. 344

7.5 Behaviour of undercut anchorage system under different strain rates ................. 345

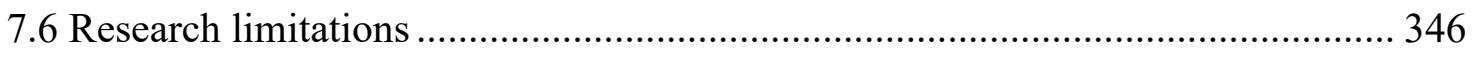

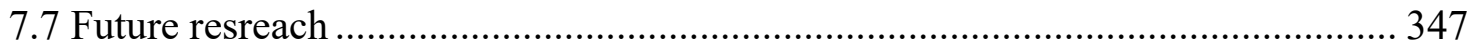

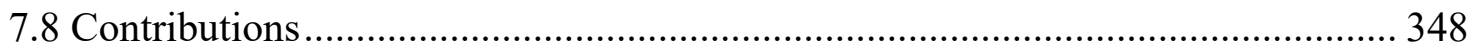

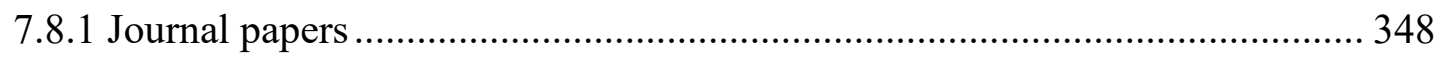

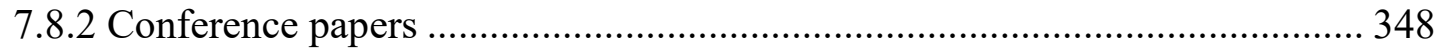

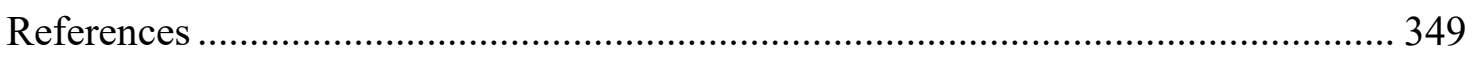


A.1 Design methods for cast-in-place anchors under tensile load ......................... 370

A.1.1 American Concrete Institute (ACI) .................................................. 370

A.1.2 Concrete Capacity Design (CCD) ........................................................... 375

A.2 Design methods for cast-in-place anchors under shear load ............................ 378

A.2.1 American concrete institute (ACI) ................................................. 378

A.2.2 Concrete Capacity Design (CCD) …................................................... 383

Appendix B: Design methods for adhesive anchors ......................................... 385

B.1 Design methods for adhesive anchors under tensile load............................... 385

B.1.1 Concrete cone breakout failure .......................................................... 385

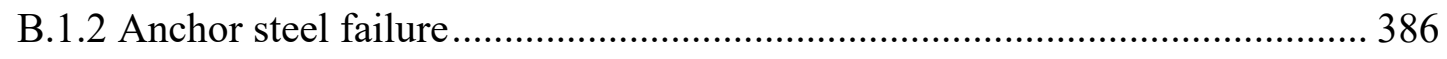

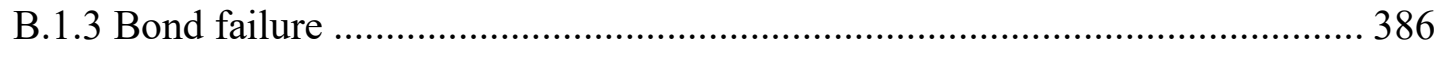

B.1.4 Combined cone-bond failure.............................................................. 388

B.2 Design methods for adhesive anchors under shear load ................................ 390

Appendix C:Tensile load-displacement relation for cast-in place anchors ............. 393

Appendix D: Shear load-displacement relation for cast-in-place anchors .............. 399

Appendix E: Tensile load-displacement relation for adhesive anchors.................... 405

Appendix F: Shear load-displacement relation for adhesive anchors .................... 411

Appendix G: Tensile load-displacement relation for undercut anchors.................. 417

Appendix H: Shear load-displacement relation for undercut anchors..................... 423

Appendix I: LS-DYNA keyword files for anchorage to concrete systems............... 429 
Cast-in-place anchor under tensile load

Cast-in-place anchor under shear load

Adhesive anchor under tensile load

Adhesive anchor under shear load 445

Undercut anchor under tensile load............................................................. 453

Undercut anchor under shear load 457 


\section{List of Figures}

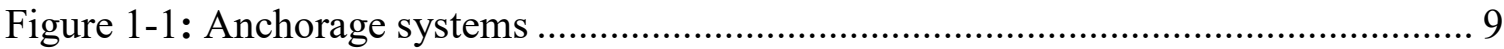

Figure 1-2: Flow chart for the methodology of the project ………................................. 10

Figure 2-1: Applications of anchorage system in rock burst (Cai et al., 2010) ................ 16

Figure 2-2: Applications of anchorage system in window; (a) glass window under blast load (Madico Safety Shield Premier Partener, 2012), (b) anchorage to base-plate (Johnson

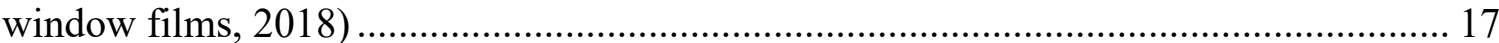

Figure 2-3: Types of anchors: (a) cast-in-place anchors, (b) post installed anchors (ACI

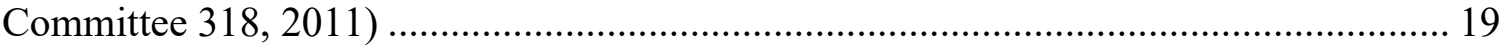

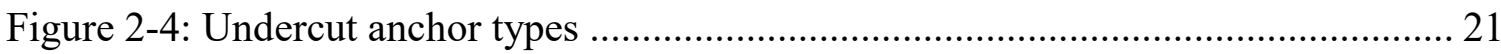

Figure 2-5: Failure modes under tensile loading: (a) steel anchor failure, (b) concrete cone breakout, (c) side face blowout, (d) concrete splitting (Cement Association of Canada 2010; ACI Committee 318, 2011) 63

Figure 2-6: Failure modes for anchors under shear load (a) steel anchor failure preceded by concrete spall; (b) concrete pryout failure; (c) concrete breakout failure; (d) thin concrete breakout; (e) edge breakout, corner breakout (f) narrow concrete edge breakout (Cement Association of Canada 2010; ACI Committee 318, 2011)

Figure 2-7: Failure modes for adhesive anchors, (a) concrete cone breakout failure; (b) combined cone-bond failure (bond failure at adhesive/concrete interface; (c) combined cone-bond failure (bond failure at steel/adhesive interface); (d) combined cone-bond failure (bond failure at combination of adhesive/concrete and steel/adhesive interface); (e)

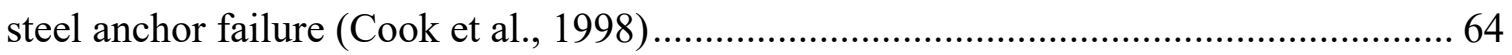


Figure 3-1: Eight node hexahedron solid element (Livermore Software Technology Corporation, 2015) 77

Figure 3-2: Four node tetrahedron solid element (Livermore Software Technology

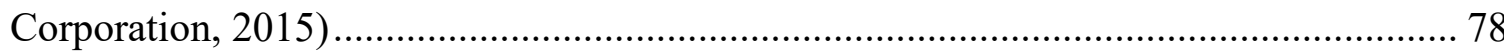

Figure 3-3: General shape of the concrete model yield surface (Murray, 2007) ............. 82

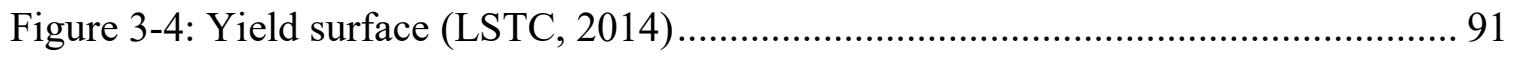

Figure 3-5: Stress-displacement relation of MAT_169 for (a) tension and (b) shear (LSTC,

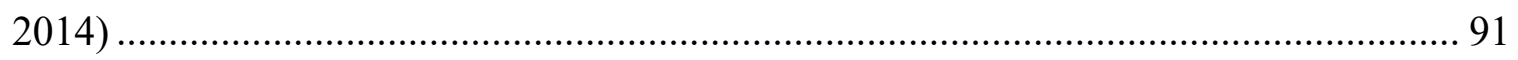

Figure 3-6: Boundary conditions for the anchorage models under (a) tensile load and (b)

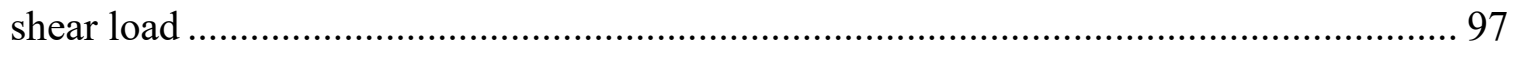

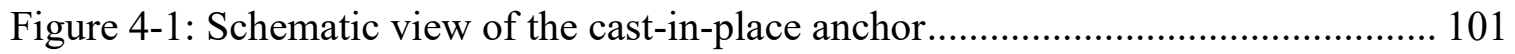

Figure 4-2: Geometric configuration with boundary condition of cast-in-place anchor

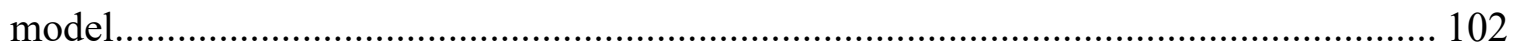

Figure 4-3: Comparison of experimental and numerical tensile load-displacement behaviour of 8-mm diameter cast-in-place anchor with $50 \mathrm{~mm}$ embedment depth ....... 104 Figure 4-4: Comparison of experimental and numerical tensile load-displacement behaviour of 24-mm diameter cast-in-place anchor with $150 \mathrm{~mm}$ embedment depth ... 104 Figure 4-5: Plastic strain contours for cast-in-place anchor at strain rate of $10^{-5} \mathrm{~s}^{-1} \ldots \ldots 110$ Figure 4-6: Effect of anchor embedment depth on the concrete cone diameter 111 Figure 4-7: Cone breakout and crack propagation angles on the cast-in-place anchorage to concrete system 112 Figure 4-8: Failure mode of $12.7 \mathrm{~mm}$ diameter cast-in-place anchor at different strain rates 
Figure 4-9: Failure mode of $15.9 \mathrm{~mm}$ diameter cast-in-place anchor at different strain rates 115

Figure 4-10: Failure mode of $19.1 \mathrm{~mm}$ diameter cast-in-place anchor at different strain rates 116

Figure 4-11: Strain rate effect on the failure mode and ultimate tensile load for cast-inplace anchor diameter of $12.7 \mathrm{~mm}$. 119 Figure 4-12: Strain rate effect on the failure mode and ultimate tensile load for cast-inplace anchor diameter of $15.9 \mathrm{~mm}$ 120 Figure 4-13: Strain rate effect on the failure mode and ultimate tensile load for cast-inplace anchor diameter of $19.1 \mathrm{~mm}$...... 121

Figure 4-14: Effect of anchor embedment depth on the concrete cone depth and ultimate tensile load for $12.7 \mathrm{~mm}$ diameter cast-in-place anchor..... 122 Figure 4-15: Effect of anchor embedment depth on the concrete cone depth and ultimate tensile load for $15.9 \mathrm{~mm}$ diameter cast-in-place anchor. 123 Figure 4-16: Effect of anchor embedment depth on the concrete cone depth and ultimate tensile load for $19.1 \mathrm{~mm}$ diameter cast-in-place anchor..... 123 Figure 4-17: Displacement contours of $15.9 \mathrm{~mm}$ diameter cast-in-place anchor at strain rates ranging from $10^{-5} \mathrm{~s}^{-1}$ to $10^{3} \mathrm{~s}^{-1}$ 124 Figure 4-18: Tensile load-displacement graph for $12.7 \mathrm{~mm}$ diameter cast-in-place anchor at strain rate of $10^{-5} \mathrm{~s}^{-1}$ 125 Figure 4-19: Tensile load-displacement graph for $12.7 \mathrm{~mm}$ diameter cast-in-place anchor at strain rate of $10^{-3} \mathrm{~s}^{-1}$ 126 
Figure 4-20: Tensile load-displacement graph for $12.7 \mathrm{~mm}$ diameter cast-in-place anchor at strain rate of $10^{-1} \mathrm{~s}^{-1}$ 126

Figure 4-21: Tensile load-displacement graph for $12.7 \mathrm{~mm}$ diameter cast-in-place anchor at strain rate of $10 \mathrm{~s}^{-1}$ 127

Figure 4-22: Tensile load-displacement graph for $12.7 \mathrm{~mm}$ diameter cast-in-place anchor at strain rate of $10^{2} \mathrm{~s}^{-1}$ 127

Figure 4-23: Tensile load-displacement graph for $12.7 \mathrm{~mm}$ diameter cast-in-place anchor at strain rate of $10^{3} \mathrm{~s}^{-1}$ 128

Figure 4-24: Ultimate tensile load and DIF versus strain rate for the cast-in-place anchor of $76.2 \mathrm{~mm}$ embedment depth 136

Figure 4-25: Ultimate tensile load and DIF versus strain rate for the cast-in-place anchor of $101.6 \mathrm{~mm}$ embedment depth 136

Figure 4-26: Ultimate tensile load and DIF versus strain rate for the cast-in-place anchor of $127 \mathrm{~mm}$ embedment depth 137

Figure 4-27: Ultimate tensile load and DIF versus strain rate for the cast-in-place anchor of $152.4 \mathrm{~mm}$ embedment depth 138

Figure 4-28: Effect of strain rate ratio on the DIF for cast-in-place anchor exhibiting concrete cone breakout failure 142 Figure 4-29: Effect of strain rate ratio on the DIF for cast-in-place anchor exhibiting steel failure. 142

Figure 4-30: Residual versus strain rate ratio for the cast-in-place anchors exhibited concrete cone breakout failure 145 
Figure 4-31: Residual versus strain rate ratio for the cast-in-place anchors exhibited steel failure. 146

Figure 4-32: DIF obtained from the finite element analysis versus the predicted DIF for the cast-in-place anchor exhibited concrete cone breakout failure. 147

Figure 4-33: DIF obtained from the finite element analysis versus the predicted DIF for the cast-in-place anchor exhibited steel failure. 148

Figure 4-34: Geometric configuration of cast-in-place anchor model under shear load 151 Figure 4-35: Applied shear load in the direction parallel to the edge distance $c_{1}$ and perpendicular to the edge distance $c_{2}$ 152

Figure 4-36: Plastic strain contours for cast-in-place anchor under shear load at strain rate of $10^{-5} \mathrm{~s}^{-1}$ 157

Figure 4-37: Pryout failure mechanism of the cast-in-place anchor 158

Figure 4-38: Failure mode for $12.7 \mathrm{~mm}$ diameter cast-in-place anchor at different strain rates 160

Figure 4-39: Failure mode for $15.9 \mathrm{~mm}$ diameter cast-in-place anchor at different strain rates 160

Figure 4-40: Failure mode for $19.1 \mathrm{~mm}$ diameter cast-in-place anchor at different strain rates 161

Figure 4-41: Steel failure process of $19.1 \mathrm{~mm}$ diameter cast-in-place anchor with $101.6 \mathrm{~mm}$ embedment depth; (a) stress concentration around the anchor, (b) anchor bending, (c) initiation of anchor fracture and (d) complete anchor fracture 163

Figure 4-42: Effect of strain rate on the failure mode and ultimate shear load for the castin-place anchor diameter of $12.7 \mathrm{~mm}$ 165 
Figure 4-43: Effect of strain rate on the failure mode and ultimate shear load for the castin-place anchor diameter of $15.9 \mathrm{~mm}$ 165

Figure 4-44: Effect of strain rate on the failure mode and ultimate shear load for the castin-place anchor diameter of $19.1 \mathrm{~mm}$ 166

Figure 4-45: Shear load-displacement graph for $12.7 \mathrm{~mm}$ diameter cast-in-place anchor at strain rate of $10^{-5} \mathrm{~s}^{-1}$ 167

Figure 4-46: Shear load-displacement graph for $12.7 \mathrm{~mm}$ diameter cast-in-place anchor at strain rate of $10^{-3} \mathrm{~s}^{-1}$ 167

Figure 4-47: Shear load-displacement graph for $12.7 \mathrm{~mm}$ diameter cast-in-place anchor at strain rate of $10^{-1} \mathrm{~s}^{-1}$ 168

Figure 4-48: Shear load-displacement graph for $12.7 \mathrm{~mm}$ diameter cast-in-place anchor at strain rate of $10 \mathrm{~s}^{-1}$ 168

Figure 4-49: Shear load-displacement graph for $12.7 \mathrm{~mm}$ diameter cast-in-place anchor at strain rate of $10^{2} \mathrm{~s}^{-1}$

Figure 4-50: Shear load-displacement graph for $12.7 \mathrm{~mm}$ diameter cast-in-place anchor at strain rate of $10^{3} \mathrm{~s}^{-1}$ 169

Figure 4-51: Ultimate shear load and DIF versus strain rate for cast-in-place anchor with $76.2 \mathrm{~mm}$ embedment depth. 174

Figure 4-52: Ultimate shear load and DIF versus strain rate for cast-in-place anchor with $101.6 \mathrm{~mm}$ embedment depth. 174

Figure 4-53: Ultimate shear load and DIF versus strain rate for cast-in-place anchor with $152.4 \mathrm{~mm}$ embedment depth. 175 
Figure 4-54: Effect of strain rate ratio on the DIF for cast-in-place anchors exhibit steel failure under shear load. 178

Figure 4-55: Residual versus strain rate ratio for the cast-in-place anchor subjected to shear load and exhibited steel failure 180

Figure 4-56: DIF obtained from the finite element analysis versus the predicted DIF for the cast-in-place anchor exhibited steel failure under shear load 181 Figure 4-57: Effect of strain rate and concrete compressive strength on the failure mode for the $12.7 \mathrm{~mm}$ diameter cast-in-place anchors. 183 Figure 4-58: Effect of strain rate and concrete compressive strength on the failure mode for the $19.1 \mathrm{~mm}$ diameter cast-in-place anchors. 184 Figure 4-59: Shear load-displacement response of $12.7 \mathrm{~mm}$ cast-in-place anchor diameter with $76.2 \mathrm{~mm}$ embedment depth at strain rate of $10^{-5} \mathrm{~s}^{-1}$ 186 Figure 4-60: Shear load-displacement response of $12.7 \mathrm{~mm}$ cast-in-place anchor diameter with $152.4 \mathrm{~mm}$ embedment depth at strain rate of $10^{-5} \mathrm{~s}^{-1}$ 187 Figure 4-61: Shear load-displacement response of $19.1 \mathrm{~mm}$ cast-in-place anchor diameter with $76.2 \mathrm{~mm}$ embedment depth at strain rate of $10^{-5} \mathrm{~s}^{-1}$ 187 Figure 4-62: Shear load-displacement response of $19.1 \mathrm{~mm}$ cast-in-place anchor diameter with $152.4 \mathrm{~mm}$ embedment depth at strain rate of $10^{-5} \mathrm{~s}^{-1}$ 188 Figure 4-63: Shear load-displacement response of $12.7 \mathrm{~mm}$ cast-in-place anchor diameter with $76.2 \mathrm{~mm}$ embedment depth at strain rate of $10^{3} \mathrm{~s}^{-1}$ 189 Figure 4-64: Shear load-displacement response of $12.7 \mathrm{~mm}$ cast-in-place anchor diameter with $152.4 \mathrm{~mm}$ embedment depth at strain rate of $10^{3} \mathrm{~s}^{-1}$ 189 
Figure 4-65: Shear load-displacement response of $19.1 \mathrm{~mm}$ diameter cast-in-place anchor with $76.2 \mathrm{~mm}$ embedment depth at strain rate of $10^{3} \mathrm{~s}^{-1}$ 190

Figure 4-66: Shear load-displacement response of $19.1 \mathrm{~mm}$ cast-in-place anchor diameter with $152.4 \mathrm{~mm}$ embedment depth at strain rate of $10^{3} \mathrm{~s}^{-1}$ 190

Figure 5-1: A schematic view of the adhesive anchorage to concrete system. 193

Figure 5-2: Geometric configuration with boundary condition for the adhesive anchor model 194

Figure 5-3: Comparison of tensile load-displacement response between FEA and experimental results obtained by Braimah et al. (Braimah et al., 2004) for anchor diameter of $6.4 \mathrm{~mm}$. 197

Figure 5-4: Failure mode obtained from the finite element analysis and the experimental results obtained by Braimah et al. (Braimah et al., 2004). 197

Figure 5-5: Comparison of tensile load-displacement response between FEA and experimental results obtained by Braimah et al. (Braimah et al., 2004) for anchor diameter of $9.5 \mathrm{~mm}$. 198

Figure 5-6: Plastic strain contours for adhesive anchor with different anchor diameters and embedment depths at strain rate of $10^{-5} \mathrm{~s}^{-1}$ 202

Figure 5-7: Cone breakout angle on the adhesive anchorage to concrete system. 203 Figure 5-8: Failure mode of 12.7-mm diameter adhesive anchor at different strain rates 206 Figure 5-9: Failure mode of 15.9-mm diameter adhesive anchor at different strain rates 207 
Figure 5-10: Failure mode of 19.1-mm diameter adhesive anchor at different strain rates 208

Figure 5-11: Strain rate effect on the failure mode and ultimate tensile load for adhesive anchor diameter of $12.7 \mathrm{~mm}$ 209

Figure 5-12: Strain rate effect on the failure mode and ultimate tensile load for adhesive anchor diameter of $15.9 \mathrm{~mm}$ 210 Figure 5-13: Strain rate effect on the failure mode and ultimate tensile load for adhesive anchor diameter of $19.1 \mathrm{~mm}$ 211

Figure 5-14: Effect of anchor embedment depth on the concrete cone depth and ultimate tensile load for $12.7 \mathrm{~mm}$ diameter adhesive anchor 214 Figure 5-15: Effect of anchor embedment depth on the concrete cone depth and ultimate tensile load for $15.9 \mathrm{~mm}$ diameter adhesive anchor 214

Figure 5-16: Effect of anchor embedment depth on the concrete cone depth and ultimate tensile load for $19.1 \mathrm{~mm}$ diameter adhesive anchor 215

Figure 5-17: Displacement contours for adhesive anchors at strain rate of $10^{-5}$ with

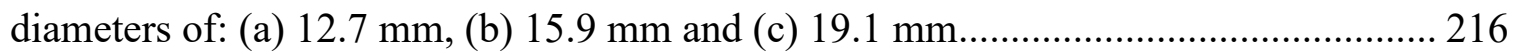
Figure 5-18: Tensile load-displacement graph for $12.7 \mathrm{~mm}$ diameter adhesive anchor at strain rate of $10^{-5} \mathrm{~s}^{-1}$ 217 Figure 5-19: Tensile load-displacement graph for $12.7 \mathrm{~mm}$ diameter adhesive anchor at strain rate of $10^{-3} \mathrm{~s}^{-1}$ 217 Figure 5-20: Tensile load-displacement graph for $12.7 \mathrm{~mm}$ diameter adhesive anchor at strain rate of $10^{-1} \mathrm{~s}^{-1}$ 218 
Figure 5-21: Tensile load-displacement graph for $12.7 \mathrm{~mm}$ diameter adhesive anchor at strain rate of $10 \mathrm{~s}^{-1}$ 218

Figure 5-22: Tensile load-displacement graph for $12.7 \mathrm{~mm}$ diameter adhesive anchor at strain rate of $10^{2} \mathrm{~s}^{-1}$ 219

Figure 5-23: Tensile load-displacement graph for $12.7 \mathrm{~mm}$ diameter adhesive anchor at strain rate of $10^{3} \mathrm{~s}^{-1}$ 219

Figure 5-24: Ultimate tensile load and DIF versus strain rate for the adhesive anchor at $76.2 \mathrm{~mm}$ embedment depth. 228

Figure 5-25: Ultimate tensile load and DIF versus strain rate for the adhesive anchor at $101.6 \mathrm{~mm}$ embedment depth.

Figure 5-26: Ultimate tensile load and DIF versus strain rate for the adhesive anchor at 127 mm embedment depth. 229

Figure 5-27: Ultimate tensile load and DIF versus strain rate for the adhesive anchor at $152.4 \mathrm{~mm}$ embedment depth 230 Figure 5-28: Effect of strain rate ratio on DIF for adhesive anchor exhibited combined cone bond failure under tensile load. 232 Figure 5-29: Effect of strain rate ratio on DIF for adhesive anchor exhibited steel failure under tensile load 233 Figure 5-30: Residual versus strain rate ratio for the adhesive anchor exhibited combined cone bond failure. 235 Figure 5-31: Residual versus strain rate ratio for the adhesive anchor exhibited steel failure 236 
Figure 5-32: DIF obtained from the finite element analysis versus the predicted DIF for the adhesive anchors exhibited combined cone bond failure 237

Figure 5-33: DIF obtained from the finite element analysis versus the predicted DIF for the adhesive anchors exhibited steel failure 237

Figure 5-34: Geometric configuration and boundary conditions of adhesive anchor model 242

Figure 5-35: Comparison of shear load-displacement relation between FEA and experimental results obtained by Cattaneo et al. (Cattaneo \& Muciaccia, 2015). 244 Figure 5-36: Failure mode obtained from:(a) finite element analysis and (b) experimental results obtained by Cattaneo et al. (Cattaneo \& Muciaccia, 2015)..... 244

Figure 5-37: Plastic strain contours for adhesive anchor under shear load at strain rate of $10^{-5} \mathrm{~s}^{-1}$ 247

Figure 5-38: Failure mode for $12.7 \mathrm{~mm}$ diameter adhesive anchor at different strain rates 249

Figure 5-39: Failure mode for $15.9 \mathrm{~mm}$ diameter adhesive anchor at different strain rates 249

Figure 5-40: Failure mode for $19.1 \mathrm{~mm}$ diameter adhesive anchor at different strain rates 250

Figure 5-41: Failure mechanism of $19.1 \mathrm{~mm}$ diameter adhesive anchor with $152.4 \mathrm{~mm}$ embedment depth; (a) stress concentration around the anchor, (b) anchor bending with bond failure, (c) initiation of anchor fracture, (d) complete anchor failure. 251 Figure 5-42: Effect of strain rate on the failure mode and ultimate shear load for the adhesive anchor diameter of $12.7 \mathrm{~mm}$...... 254 
Figure 5-43: Effect of strain rate on the failure mode and ultimate shear load for the adhesive anchor diameter of $15.9 \mathrm{~mm}$ 255

Figure 5-44: Effect of strain rate on the failure mode and ultimate shear load for the adhesive anchor diameter of $19.1 \mathrm{~mm}$ 255

Figure 5-45: Shear load-displacement graph for 12.7- $\mathrm{mm}$ diameter adhesive anchor at strain rate of $10^{-5} \mathrm{~s}^{-1}$ 256 Figure 5-46: Shear load-displacement graph for 12.7- $\mathrm{mm}$ diameter adhesive anchor at strain rate of $10^{-3} \mathrm{~s}^{-1}$ 257 Figure 5-47: Shear load-displacement graph for 12.7-mm diameter adhesive anchor at strain rate of $10^{-1} \mathrm{~s}^{-1}$ 257

Figure 5-48: Shear load-displacement graph for 12.7-mm diameter adhesive anchor at strain rate of $10 \mathrm{~s}^{-1}$ 258

Figure 5-49: Shear load-displacement graph for 12.7-mm diameter adhesive anchor at strain rate of $10^{2} \mathrm{~s}^{-1}$ 258

Figure 5-50: Shear load-displacement graph for 12.7- $\mathrm{mm}$ diameter adhesive anchor at strain rate of $10^{3} \mathrm{~s}^{-1}$ 259

Figure 5-51: Ultimate shear load and DIF versus strain rate for adhesive anchors at 76.2 mm embedment depth. 265 Figure 5-52: Ultimate shear load and DIF versus strain rate for adhesive anchors at 101.6 mm embedment depth. 266 Figure 5-53: Ultimate shear load and DIF versus strain rate for adhesive anchors at 152.4 mm embedment depth. 266 
Figure 5-54: Effect of strain rate ratio on the DIF for adhesive anchor exhibiting steel failure under shear load. 269

Figure 5-55: Residual versus strain rate ratio for the adhesive anchor subjected to shear load and exhibited steel failure 271

Figure 5-56: DIF obtained from the finite element analysis versus the predicted DIF for the adhesive anchor exhibited steel failure under shear load 272 Figure 6-1: A schematic view for the undercut anchor model. 274 Figure 6-2: Geometric configuration and boundary conditions for the undercut anchor model. 275

Figure 6-3: Tensile load-displacement response of the FEA and experimental results obtained by Mahadik et al. (Mahadik et al., 2016) 277 Figure 6-4: Failure mode of the undercut anchors under tensile load observed from the finite element analysis and the experimental results by (Mahadik et al., 2016) 277 Figure 6-5: Plastic strain contours for the undercut anchors at strain rate of $10^{-5} \mathrm{~s}^{-1} \ldots . .280$ Figure 6-6: Failure mode of $12 \mathrm{~mm}$ diameter undercut anchor at different strain rates . 281 Figure 6-7: Failure mode of $16 \mathrm{~mm}$ diameter undercut anchor at different strain rates . 281 Figure 6-8: Failure mode of $20 \mathrm{~mm}$ diameter undercut anchor at different strain rates . 282 Figure 6-9: Strain rate effect on the failure mode and ultimate tensile load for undercut anchor diameter of $12 \mathrm{~mm}$ 283 Figure 6-10: Strain rate effect on the failure mode and ultimate tensile load for undercut anchor diameter of $16 \mathrm{~mm}$ 284 Figure 6-11: Strain rate effect on the failure mode and ultimate tensile load for undercut anchor diameter of $20 \mathrm{~mm}$ 284 
Figure 6-12: Effect of anchor embedment depth on the concrete cone depth and ultimate tensile load for $12 \mathrm{~mm}$ diameter undercut anchor 287

Figure 6-13: Effect of anchor embedment depth on the concrete cone depth and ultimate tensile load for $16 \mathrm{~mm}$ diameter undercut anchor 287

Figure 6-14: Effect of anchor embedment depth on the concrete cone depth and ultimate tensile load for $20 \mathrm{~mm}$ diameter undercut anchor 288 Figure 6-15: Displacement contours for $20 \mathrm{~mm}$ undercut anchor diameter at strain rates ranging from $10^{-5} \mathrm{~s}^{-1}$ to $10^{3} \mathrm{~s}^{-1}$ 289

Figure 6-16: Tensile load-displacement graph for the 12-mm diameter undercut anchor at strain rate of $10^{-5} \mathrm{~s}^{-1}$

Figure 6-17: Tensile load-displacement graph for the 12-mm diameter undercut anchor at strain rate of $10^{-3} \mathrm{~s}^{-1}$ 291

Figure 6-18: Tensile load-displacement graph for the 12-mm diameter undercut anchor at strain rate of $10^{-1} \mathrm{~s}^{-1}$ 292

Figure 6-19: Tensile load-displacement graph for the 12-mm diameter undercut anchor at strain rate of $10 \mathrm{~s}^{-1}$ 292

Figure 6-20: Tensile load-displacement graph for the 12-mm diameter undercut anchor at strain rate of $10^{2} \mathrm{~s}^{-1}$ 293

Figure 6-21: Tensile load-displacement graph for the 12-mm diameter undercut anchor at strain rate of $10^{3} \mathrm{~s}^{-1}$ 293

Figure 6-22: Tensile load-displacement response of $100 \mathrm{~mm}$ embedment depth undercut anchors at strain rate of $10^{-5} \mathrm{~s}^{-1}$ 295 
Figure 6-23: Tensile load-displacement response of $190 \mathrm{~mm}$ embedment depth undercut anchors at strain rate of $10^{-5} \mathrm{~s}^{-1}$ 295

Figure 6-24: Ultimate tensile load and DIF versus strain rate for the undercut anchor at 100 mm embedment depth. 300

Figure 6-25: Ultimate tensile load and DIF versus strain rate for the undercut anchor at 125 mm embedment depth. 301

Figure 6-26: Ultimate tensile load and DIF versus strain rate for the undercut anchor at 190 mm embedment depth. 301

Figure 6-27: Ultimate tensile load and DIF versus strain rate for the undercut anchor at 250 mm embedment depth. 302

Figure 6-28: Effect of strain rate ratio on the DIF for the undercut anchors exhibited steel failure under tensile load. 304

Figure 6-29: Residual versus strain rate ratio for the undercut anchors exhibited steel failure 306

Figure 6-30: DIF obtained from the finite element analysis versus the predicted DIF for the undercut anchors exhibited steel failure 307 Figure 6-31: Geometric configuration and boundary conditions for the undercut anchor model... 309

Figure 6-32: Comparison of shear load-displacement relation between FEA and experimental results obtained by Mahadik et al. (Mahadik et al., 2016)..... 311 Figure 6-33: Failure mode obtained from:(a) finite element analysis and (b) experimental results obtained by Mahadik et al. (Mahadik et al., 2016) 311 
Figure 6-34: Plastic strain contours for undercut anchors under shear load at strain rate of $10^{-5} \mathrm{~s}^{-1}$ 314

Figure 6-35: Failure mode of 12-mm diameter undercut anchor at different strain rates 316 Figure 6-36: Failure mode of 16-mm diameter undercut anchor at different strain rates 317 Figure 6-37: Failure mode of 20-mm diameter undercut anchor at different strain rates318 Figure 6-38: Failure process of 12-mm diameter undercut anchor with $190 \mathrm{~mm}$ embedment depth; (a) stress concentration around the undercut anchor, (b) anchor bending, (c) crack initiation and (d) anchor fracture 320 Figure 6-39: Effect of strain rate on the failure mode and ultimate shear load for the undercut anchor diameter of $12 \mathrm{~mm}$ 321

Figure 6-40: Effect of strain rate on the failure mode and ultimate shear load for the undercut anchor diameter of $16 \mathrm{~mm}$ 322

Figure 6-41: Effect of strain rate on the failure mode and ultimate shear load for the undercut anchor diameter of $20 \mathrm{~mm}$ 322 Figure 6-42: Shear load-displacement graph for 12-mm diameter undercut anchor at strain rate of $10^{-5} \mathrm{~s}^{-1}$ 324 Figure 6-43: Shear load-displacement graph for 12-mm diameter undercut anchor at strain rate of $10^{-3} \mathrm{~s}^{-1}$ 324 Figure 6-44: Shear load-displacement graph for 12-mm diameter undercut anchor at strain rate of $10^{-1} \mathrm{~s}^{-1}$ 325 Figure 6-45: Shear load-displacement graph for 12-mm diameter undercut anchor at strain rate of $10 \mathrm{~s}^{-1}$ 325 
Figure 6-46: Shear load-displacement graph for 12-mm diameter undercut anchor at strain rate of $10^{2} \mathrm{~s}^{-1}$ 326

Figure 6-47: Shear load-displacement graph for 12-mm diameter undercut anchor at strain rate of $10^{3} \mathrm{~s}^{-1}$ 326

Figure 6-48: Ultimate shear load and DIF versus strain rate for undercut anchors at 100 mm embedment depth.

Figure 6-49: Ultimate shear load and DIF versus strain rate for undercut anchors at 125

mm embedment depth.

Figure 6-50: Ultimate shear load and DIF versus strain rate for undercut anchors at 190 mm embedment depth 332

Figure 6-51: Ultimate shear load and DIF versus strain rate for undercut anchors at 250 mm embedment depth 332

Figure 6-52: Effect of strain rate ratio on the DIF for undercut anchors exhibited steel failure under shear load. 335

Figure 6-53: Residual versus strain rate ratio for the undercut anchors under shear load exhibited steel failure 337

Figure 6-54: DIF obtained from the finite element analysis versus the predicted DIF for the undercut anchors exhibited steel failure under shear load 338 Figure A-1: Calculation of Projected area $A_{N o}$ and actual area $A_{N}$ for single anchor and double anchors (Cement Association of Canada 2010; ACI Committee 318 2011) ...... 374 Figure A-2: Calculation of projected area $A_{v c o}$ for single anchor (Cement Association of Canada, 2010; ACI Committee 318, 2011) 381 
Figure C-1: Tensile load-displacement graph for $15.9 \mathrm{~mm}$ diameter cast-in-place anchor at strain rate of $10^{-5} \mathrm{~s}^{-1}$ 393

Figure C-2: Tensile load-displacement graph for $15.9 \mathrm{~mm}$ diameter cast-in-place anchor at strain rate of $10^{-3} \mathrm{~s}^{-1}$ 393

Figure C-3: Tensile load-displacement graph for $15.9 \mathrm{~mm}$ diameter cast-in-place anchor at strain rate of $10^{-1} \mathrm{~s}^{-1}$ 394

Figure C-4: Tensile load-displacement graph for $15.9 \mathrm{~mm}$ diameter cast-in-place anchor at strain rate of $10 \mathrm{~s}^{-1}$ 394

Figure C-5: Tensile load-displacement graph for $15.9 \mathrm{~mm}$ diameter cast-in-place anchor at strain rate of $10^{2} \mathrm{~s}^{-1}$ 395

Figure C-6: Tensile load-displacement graph for $15.9 \mathrm{~mm}$ diameter cast-in-place anchor at strain rate of $10^{3} \mathrm{~s}^{-1}$ 395

Figure C-7: Tensile load-displacement graph for $19.1 \mathrm{~mm}$ diameter cast-in-place anchor at strain rate of $10^{-5} \mathrm{~s}^{-1}$ 396

Figure C-8: Tensile load-displacement graph for $19.1 \mathrm{~mm}$ diameter cast-in-place anchor at strain rate of $10^{-3} \mathrm{~s}^{-1}$ 396

Figure C-9: Tensile load-displacement graph for $19.1 \mathrm{~mm}$ diameter cast-in-place anchor at strain rate of $10^{-1} \mathrm{~s}^{-1}$ 397 Figure C-10: Tensile load-displacement graph for $19.1 \mathrm{~mm}$ diameter cast-in-place anchor at strain rate of $10 \mathrm{~s}^{-1}$ 397 Figure C-11: Tensile load-displacement graph for $19.1 \mathrm{~mm}$ diameter cast-in-place anchor at strain rate of $10^{2} \mathrm{~s}^{-1}$ 398 
Figure C-12: Tensile load-displacement graph for $19.1 \mathrm{~mm}$ diameter cast-in-place anchor at strain rate of $10^{3} \mathrm{~s}^{-1}$ 398

Figure D-1: Shear load-displacement graph for $15.9 \mathrm{~mm}$ diameter cast-in-place anchor at strain rate of $10^{-5} \mathrm{~s}^{-1}$ 399

Figure D-2: Shear load-displacement graph for $15.9 \mathrm{~mm}$ diameter cast-in-place anchor at strain rate of $10^{-3} \mathrm{~s}^{-1}$ 399

Figure D-3: Shear load-displacement graph for $15.9 \mathrm{~mm}$ diameter cast-in-place anchor at strain rate of $10^{-1} \mathrm{~s}^{-1}$ 400

Figure D-4: Shear load-displacement graph for $15.9 \mathrm{~mm}$ diameter cast-in-place anchor at strain rate of $10 \mathrm{~s}^{-1}$ 400

Figure D-5: Shear load-displacement graph for $15.9 \mathrm{~mm}$ diameter cast-in-place anchor at strain rate of $10^{2} \mathrm{~s}^{-1}$ 401

Figure D-6: Shear load-displacement graph for $15.9 \mathrm{~mm}$ diameter cast-in-place anchor at strain rate of $10^{3} \mathrm{~s}^{-1}$ 401

Figure D-7: Shear load-displacement graph for $19.1 \mathrm{~mm}$ diameter cast-in-place anchor at strain rate of $10^{-5} \mathrm{~s}^{-1}$ 402

Figure D-8: Shear load-displacement graph for $19.1 \mathrm{~mm}$ diameter cast-in-place anchor at strain rate of $10^{-3} \mathrm{~s}^{-1}$ 402

Figure D-9: Shear load-displacement graph for $19.1 \mathrm{~mm}$ diameter cast-in-place anchor mm at strain rate of $10^{-1} \mathrm{~s}^{-1}$ 403 Figure D-10: Shear load-displacement graph for $19.1 \mathrm{~mm}$ diameter cast-in-place anchor at strain rate of $10 \mathrm{~s}^{-1}$ 403 
Figure D-11: Shear load-displacement graph for $19.1 \mathrm{~mm}$ diameter cast-in-place anchor at strain rate of $10^{2} \mathrm{~s}^{-1}$ 404

Figure D-12: Shear load-displacement graph for $19.1 \mathrm{~mm}$ diameter cast-in-place anchor at strain rate of $10^{3} \mathrm{~s}^{-1}$ 404

Figure E-1: Tensile load-displacement graph for $15.9 \mathrm{~mm}$ diameter adhesive anchor at strain rate of $10^{-5} \mathrm{~s}^{-1}$ 405

Figure E-2: Tensile load-displacement graph for $15.9 \mathrm{~mm}$ diameter adhesive anchor at strain rate of $10^{-3} \mathrm{~s}^{-1}$ 405

Figure E-3: Tensile load-displacement graph for $15.9 \mathrm{~mm}$ diameter adhesive anchor at strain rate of $10^{-1} \mathrm{~s}^{-1}$ 406

Figure E-4: Tensile load-displacement graph for $15.9 \mathrm{~mm}$ diameter adhesive anchor at strain rate of $10 \mathrm{~s}^{-1}$ 406

Figure E-5: Tensile load-displacement graph for $15.9 \mathrm{~mm}$ diameter adhesive anchor at strain rate of $10^{2} \mathrm{~s}^{-1}$ 407

Figure E-6: Tensile load-displacement graph for $15.9 \mathrm{~mm}$ diameter adhesive anchor at strain rate of $10^{3} \mathrm{~s}^{-1}$ 407

Figure E-7: Tensile load-displacement graph for $19.1 \mathrm{~mm}$ diameter adhesive anchor at strain rate of $10^{-5} \mathrm{~s}^{-1}$ 408

Figure E-8: Tensile load-displacement graph for $19.1 \mathrm{~mm}$ diameter adhesive anchor at strain rate of $10^{-3} \mathrm{~s}^{-1}$ 408

Figure E-9: Tensile load-displacement graph for $19.1 \mathrm{~mm}$ diameter adhesive anchor at strain rate of $10^{-1} \mathrm{~s}^{-1}$ 409 
Figure E-10: Tensile load-displacement graph for $19.1 \mathrm{~mm}$ diameter adhesive anchor at strain rate of $10 \mathrm{~s}^{-1}$ 409

Figure E-11: Tensile load-displacement graph for $19.1 \mathrm{~mm}$ diameter adhesive anchor at strain rate of $10^{2} \mathrm{~s}^{-1}$ 410

Figure E-12: Tensile load-displacement graph for $19.1 \mathrm{~mm}$ diameter adhesive anchor at strain rate of $10^{3} \mathrm{~s}^{-1}$ 410

Figure F-1: Shear load-displacement graph for $15.9 \mathrm{~mm}$ diameter adhesive anchor at strain rate of $10^{-5} \mathrm{~s}^{-1}$ 411

Figure F-2: Shear load-displacement graph for $15.9 \mathrm{~mm}$ diameter adhesive anchor at strain rate of $10^{-3} \mathrm{~s}^{-1}$ 411

Figure F-3: Shear load-displacement graph for $15.9 \mathrm{~mm}$ diameter adhesive anchor at strain rate of $10^{-1} \mathrm{~s}^{-1}$ 412

Figure F-4: Shear load-displacement graph for $15.9 \mathrm{~mm}$ diameter adhesive anchor at strain rate of $10 \mathrm{~s}^{-1}$ 412

Figure F-5: Shear load-displacement graph for $15.9 \mathrm{~mm}$ diameter adhesive anchor at strain rate of $10^{2} \mathrm{~s}^{-1}$ 413 Figure F-6: Shear load-displacement graph for $15.9 \mathrm{~mm}$ diameter adhesive anchor at strain rate of $10^{3} \mathrm{~s}^{-1}$ 413 Figure F-7: Shear load-displacement graph for $19.1 \mathrm{~mm}$ diameter adhesive anchor at strain rate of $10^{-5} \mathrm{~s}^{-1}$ 414 Figure F-8: Shear load-displacement graph for $19.1 \mathrm{~mm}$ diameter adhesive anchor at strain rate of $10^{-3} \mathrm{~s}^{-1}$ 414 
Figure F-9: Shear load-displacement graph for $19.1 \mathrm{~mm}$ diameter adhesive anchor at strain rate of $10^{-1} \mathrm{~s}^{-1}$ 415

Figure F-10: Shear load-displacement graph for $19.1 \mathrm{~mm}$ diameter adhesive anchor at strain rate of $10 \mathrm{~s}^{-1}$ 415

Figure F-11: Shear load-displacement graph for $19.1 \mathrm{~mm}$ diameter adhesive anchor at strain rate of $10^{2} \mathrm{~s}^{-1}$ 416 Figure F-12: Shear load-displacement graph for $19.1 \mathrm{~mm}$ diameter adhesive anchor at strain rate of $10^{3} \mathrm{~s}^{-1}$ 416

Figure G-1: Tensile load-displacement graph for the $16 \mathrm{~mm}$ diameter undercut anchor at strain rate of $10^{-5} \mathrm{~s}^{-1}$

Figure G-2: Tensile load-displacement graph for the $16 \mathrm{~mm}$ diameter undercut anchor at strain rate of $10^{-3} \mathrm{~s}^{-1}$ 417

Figure G-3: Tensile load-displacement graph for the $16 \mathrm{~mm}$ diameter undercut anchor at strain rate of $10^{-1} \mathrm{~s}^{-1}$ 418

Figure G-4: Tensile load-displacement graph for the $16 \mathrm{~mm}$ diameter undercut anchor at strain rate of $10 \mathrm{~s}^{-1}$ 418

Figure G-5: Tensile load-displacement graph for the $16 \mathrm{~mm}$ diameter undercut anchor at strain rate of $10^{2} \mathrm{~s}^{-1}$ 419

Figure G-6: Tensile load-displacement graph for the $16 \mathrm{~mm}$ diameter undercut anchor at strain rate of $10^{3} \mathrm{~s}^{-1}$ 419 Figure G-7: Tensile load-displacement graph for the $20 \mathrm{~mm}$ diameter undercut anchor at strain rate of $10^{-5} \mathrm{~s}^{-1}$ 420 
Figure G-8: Tensile load-displacement graph for the $20 \mathrm{~mm}$ diameter undercut anchor at strain rate of $10^{-3} \mathrm{~s}^{-1}$ 420

Figure G-9: Tensile load-displacement graph for the $20 \mathrm{~mm}$ diameter undercut anchor at strain rate of $10^{-1} \mathrm{~s}^{-1}$ 421

Figure G-10: Tensile load-displacement graph for the $20 \mathrm{~mm}$ diameter undercut anchor at strain rate of $10 \mathrm{~s}^{-1}$ 421

Figure G-11: Tensile load-displacement graph for the $20 \mathrm{~mm}$ diameter undercut anchor at strain rate of $10^{2} \mathrm{~s}^{-1}$ 422

Figure G-12: Tensile load-displacement graph for the $20 \mathrm{~mm}$ diameter undercut anchor at strain rate of $10^{3} \mathrm{~s}^{-1}$ 422

Figure H-1: Shear load-displacement graph for $16 \mathrm{~mm}$ diameter undercut anchor at strain rate of $10^{-5} \mathrm{~s}^{-1}$ 423

Figure H-2: Shear load-displacement graph for $16 \mathrm{~mm}$ diameter undercut anchor at strain rate of $10^{-3} \mathrm{~s}^{-1}$ 423

Figure H-3: Shear load-displacement graph for $16 \mathrm{~mm}$ diameter undercut anchor at strain rate of $10^{-1} \mathrm{~s}^{-1}$ 424

Figure H-4: Shear load-displacement graph for $16 \mathrm{~mm}$ diameter undercut anchor at strain rate of $10 \mathrm{~s}^{-1}$ 424

Figure H-5: Shear load-displacement graph for $16 \mathrm{~mm}$ diameter undercut anchor at strain rate of $10^{2} \mathrm{~s}^{-1}$ 425

Figure H-6: Shear load-displacement graph for $16 \mathrm{~mm}$ diameter undercut anchor at strain rate of $10^{3} \mathrm{~s}^{-1}$ 425 
Figure H-7: Shear load-displacement graph for $20 \mathrm{~mm}$ diameter undercut anchor at strain rate of $10^{-5} \mathrm{~s}^{-1}$ 426

Figure H-8: Shear load-displacement graph for $20 \mathrm{~mm}$ diameter undercut anchor at strain rate of $10^{-3} \mathrm{~s}^{-1}$ 426

Figure H-9: Shear load-displacement graph for $20 \mathrm{~mm}$ diameter undercut anchor at strain rate of $10^{-1} \mathrm{~s}^{-1}$ 427

Figure H-10: Shear load-displacement graph for $20 \mathrm{~mm}$ diameter undercut anchor at strain rate of $10 \mathrm{~s}^{-1}$ 427

Figure H-11: Shear load-displacement graph for $20 \mathrm{~mm}$ diameter undercut anchor at strain rate of $10^{2} \mathrm{~s}^{-1}$ 428

Figure H-12: Shear load-displacement graph for $20 \mathrm{~mm}$ diameter undercut anchor at strain rate of $10^{3}$ 428 


\section{List of Tables}

Table 3-1: Material specifications for concrete models (LSTC, 2014) ........................... 80

Table 3-2: Boundary conditions of the anchorage to concrete systems............................ 97

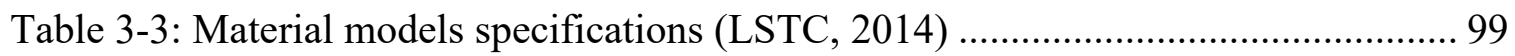

Table 4-1: Effect of mesh size refinement on the convergence of ultimate tensile load for

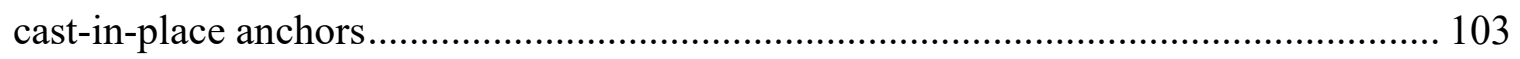

Table 4-2: Comparison of ultimate tensile loads obtained from FEA with ACI and CCD

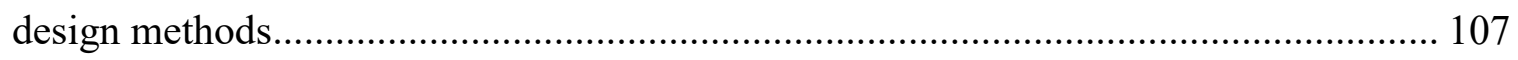

Table 4-3: Concrete cone diameter and cone breakout angle for the cast-in-place anchor at

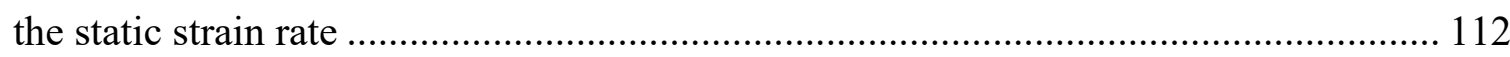

Table 4-4: Failure mode for cast-in-place anchors under tensile load at different strain rates

Table 4-5: Crack propagation angle for the cast-in-place anchors at different strain rates

Table 4-6: Ultimate tensile load and displacement results for the $12.7 \mathrm{~mm}$ diameter castin-place anchor. 130

Table 4-7: Ultimate tensile load and displacement results for the $15.9 \mathrm{~mm}$ diameter castin-place anchor. 130 Table 4-8: Ultimate tensile load and displacement results for the $19.1 \mathrm{~mm}$ diameter castin-place anchor. 131

Table 4-9: Maximum dynamic increase factor for the cast-in-place anchors under tensile load. 140 
Table 4-10: Statistical models and coefficient of determination to predict the DIF for castin-place anchors exhibited concrete cone breakout failure under tensile load 143

Table 4-11: Statistical models and coefficient of determination to predict the DIF for castin-place anchors exhibited steel failure under tensile load..... 143

Table 4-12: Comparison between ultimate dynamic load obtained from the FEA and proposed equations by Fujikake et al. (2003) for the cast-in-place anchor exhibited concrete cone breakout failure. 150

Table 4-13: Effect of mesh size refinement on the convergence of ultimate shear load for cast-in-place anchor 153

Table 4-14: Comparison between FEA and ACI 318 method. 155

Table 4-15: Failure mode for cast-in-place anchors under shear load at different strain rates 164

Table 4-16: Ultimate shear load and displacement results for the $12.7 \mathrm{~mm}$ diameter castin-place anchor 170

Table 4-17: Ultimate shear load and displacement results for the $15.9 \mathrm{~mm}$ diameter castin-place anchor. 171

Table 4-18: Ultimate shear load and displacement results for the $19.1 \mathrm{~mm}$ diameter castin-place anchor. 172

Table 4-19: Maximum DIF for the cast-in-place anchors under shear load 176 Table 4-20: Statistical models and coefficient of determination to predict the DIF for castin-place anchor exhibited steel failure under shear load. 178 Table 5-1: Effect of mesh size refinement on the convergence of ultimate tensile load for adhesive anchor. 195 
Table 5-2: Comparison of ultimate tensile loads obtained from FEA with ACI and CCD methods 199

Table 5-3: Concrete cone diameter and cone breakout angle for the adhesive anchor... 204 Table 5-4: Failure mode for adhesive anchors under tensile load at different strain rates

Table 5-5: Ultimate tensile load and displacement results for the $12.7 \mathrm{~mm}$ diameter adhesive anchor. 221

Table 5-6: Ultimate tensile load and displacement results for the $15.9 \mathrm{~mm}$ diameter adhesive anchor. 222

Table 5-7: Ultimate tensile load and displacement results for the $19.1 \mathrm{~mm}$ diameter adhesive anchor. 223

Table 5-8: Maximum dynamic increase factor for the adhesive anchors under tensile load 231

Table 5-9: Statistical models and coefficient of determination to predict the DIF for adhesive anchors exhibited combined cone bond failure under tensile load. 233 Table 5-10: Statistical models and coefficient of determination to predict the DIF for adhesive anchors exhibited steel failure under tensile load. 234

Table 5-11: Comparison between ultimate load obtained from the FEA and the proposed equations by Fujikake et al. (2003) for concrete cone breakout failure mode..... 239 Table 5-12: Comparison between ultimate dynamic load obtained from the FEA and the proposed equations by Fujikake et al. (2003) for combined cone bond failure mode.... 240 Table 5-13: Effect of mesh size refinement on the convergence of ultimate shear load for adhesive anchor. 243 
Table 5-14: Comparison between FEA and ACI 318 method. 245

Table 5-15: Failure mode for adhesive anchors under shear load at different strain rates 251

Table 5-16: Ultimate shear load and displacement results for the 12.7-mm diameter adhesive anchor. 261

Table 5-17: Ultimate shear load and displacement results for the 15.9-mm diameter adhesive anchor. 261

Table 5-18: Ultimate shear load and displacement results for the 19.1-mm diameter adhesive anchor. 262

Table 5-19: Maximum dynamic increase factor for the adhesive anchors under shear load 268

Table 5-20: Statistical models and coefficient of determination to predict the DIF for adhesive anchor exhibited steel failure under shear load 270 Table 6-1: Effect of mesh size refinement on the convergence of ultimate tensile load for undercut anchor 276 Table 6-2: Comparison of ultimate tensile loads obtained from FEA with ACI and CCD methods 279

Table 6-3: Failure mode for undercut anchors under tensile load at different strain rates 285

Table 6-4: Crack propagation angle for the undercut anchors at different strain rates... 290 Table 6-5: Ultimate tensile load and displacement for the $12 \mathrm{~mm}$ diameter undercut anchor 296 
Table 6-6: Ultimate tensile load and displacement for the $16 \mathrm{~mm}$ diameter undercut anchor 297

Table 6-7: Ultimate tensile load and displacement for the $20 \mathrm{~mm}$ diameter undercut anchor 298

Table 6-8: Maximum Dynamic increase factor for the undercut anchors under tensile load 303

Table 6-9: Statistical models and coefficient of determination to predict the DIF for the undercut anchors exhibited steel failure under tensile load. 304

Table 6-10: Comparison between ultimate dynamic load for the undercut anchor obtained from the FEA and proposed equation by Fujikake et al. (2003). 308

Table 6-11: Effect of mesh size refinement on the convergence of ultimate shear load for undercut anchor. 310

Table 6-12: Comparison of ultimate shear load obtained from FEA and ACI 318 method 312

Table 6-13: Failure mode for the undercut anchors under shear load at different strain rates 323

Table 6-14: Ultimate shear load and displacement results for the 12-mm diameter undercut anchor. 328

Table 6-15: Ultimate shear load and displacement results for the 16-mm diameter undercut anchor. 328

Table 6-16: Ultimate shear load and displacement results for the 20-mm diameter undercut anchor. 329

Table 6-17: Maximum dynamic increase factor for the undercut anchors under shear load 334

Table 6-18: Statistical models and coefficient of determination to predict the DIF for the undercut anchors exhibited steel failure under shear load 335 


\section{Notations}

$a \quad$ Acceleration

$A_{\text {brg }} \quad$ Bearing area

$A_{e} \quad$ Projected area of concrete cone failure

$A_{N} \quad$ Actual projected area of stress cone of a single anchor in tension

$A_{N o} \quad$ Projected area of stress cone of a single anchor unlimited by edge effect

or spacing between anchors in tension

$A_{S} \quad$ Effective cross-sectional area of the anchor

$A_{v} \quad$ Actual projected area considering edge effects and overlap with adjacent

anchors in shear

$A_{v o} \quad$ Projected area for one anchor unlimited by edge effects, concrete depth or cone overlapping in shear

$a^{n} \quad$ Nodal acceleration at time step (n)

$a^{n+1} \quad$ Nodal acceleration at time step $(\mathrm{n}+1)$

$c \quad$ Distance from center of anchor to edge of concrete

$c_{1} \quad$ Edge distance parallel to the direction of the applied load

$c_{2} \quad$ Edge distance perpendicular to the direction of the applied load

$c_{c} \quad$ Critical distance

$C, p \quad$ Cowper-Symonds constants

$d \quad$ Diameter of the anchor

$d_{\text {cone }} \quad$ Cone diameter

$d_{h} \quad$ Anchor head diameter

$d_{0} \quad$ Diameter of the hole 


\begin{tabular}{ll}
$d^{n}$ & Nodal displacement at time step (n) \\
$e_{N}^{\prime}$ & Distance between the resultant tensile force of the group anchors and the \\
& centroid of the group anchors \\
$e_{v}^{\prime}$ & Distance between resultant shear force of the anchor group and the centroid \\
& of the anchors \\
$E_{p}$ & Plastic hardening modulus \\
$E_{t}$ & Tangent modulus \\
$f_{c}^{\prime}$ & Compressive strength of concrete measured on cylinder \\
$f_{c c}^{\prime}$ & Compressive strength of concrete measured on cube \\
$F_{c d}$ & Ultimate dynamic concrete cone breakout \\
$f_{c t}$ & Concrete capacity \\
$f_{c, l o w}^{\prime}$ & Low strength concrete \\
$F_{s}$ & Tensile static load \\
$F_{t d}$ & Tensile dynamic load \\
$F_{u}$ & Ultimate tensile load for anchors with eccentricity effect and/or edge effect \\
$F_{u o}$ & Ultimate tensile load without eccentricity effect and/or edge effect \\
$f_{u t}$ & Ultimate tensile strength of the steel \\
$f_{i n}$ & Internal force \\
$F_{c}(t)$ & External force \\
$F_{f}$ & Shear (failure) surface \\
$F^{\prime}$ & Hardening cap surface \\
& Shear modulus \\
\hline
\end{tabular}




\begin{tabular}{ll}
$h_{c o n e}$ & Concrete cone depth \\
$h_{e f}$ & Effective embedment depth \\
$J_{1}$ & First invariant of the stress tensor \\
$J_{2}^{\prime}$ & Second invariant of the deviatoric stress tensor \\
$J_{3}^{\prime}$ & Third invariant of the deviatoric stress tensor \\
$k, k_{1}, k_{2}, k_{3}$ & Calibrated factors \\
$k_{c p}$ & Calibrated factor \\
$m$ & Mass \\
$n$ & Number of anchors \\
$N_{a}$ & Nominal bond strength for single adhesive anchor \\
$N_{b}$ & Basic concrete breakout strength in tension of a single anchor \\
$N_{c b}$ & Nominal concrete breakout strength in tension for a single anchor \\
$N_{c b g}$ & Concrete breakout strength in tension for a group of anchors \\
$N_{u}$ & Ultimate tensile load with edge effect and spacing \\
$N_{u o}$ & Ultimate tensile load \\
$N_{s b}$ & Side face blowout strength for single anchor \\
$N_{s b g}$ & Side face blowout strength for a group of anchors \\
$N_{p n}$ & Pullout load of single anchor \\
$N_{p}$ & Nominal pullout stress \\
$N_{c o n e}$ & Concrete cone failure load \\
$N_{b o n d}$ & Bond failure load \\
$P$ & Pressure \\
\hline
\end{tabular}




$$
\begin{array}{ll}
\text { PWRS } & \text { Power law term for shear } \\
s & \text { Distance between anchors (spacing) } \\
S_{i j}^{*} & \text { Trial deviatoric stress }
\end{array}
$$

$S_{i j}, S_{j k}, S_{k i}$ Deviatoric stress tensors

SHT_SL Slope of the yield surface at zero tension

$\begin{array}{ll}v & \text { Velocity } \\ V_{c b} & \text { Nominal concrete breakout strength for a single anchor in shear } \\ V_{b} & \text { Basic concrete breakout strength of a single anchor in shear in cracked } \\ & \text { concrete }\end{array}$

$V_{c b g} \quad$ Concrete breakout capacity for a group of anchors in shear

$V_{c p} \quad$ Pryout capacity of single cast-in anchor

$V_{c p g} \quad$ Pryout capacity of a group of cast-in anchors

$V_{n} \quad$ Ultimate shear load for anchors considering the effect of edge distance, concrete depth, and spacing

$V_{n o} \quad$ Ultimate shear load

$v^{n} \quad$ Nodal velocity at time step (n)

$v^{n+1} \quad$ Nodal velocity at time step $(\mathrm{n}+1)$

$Y \quad$ Yield function of the concrete material

$\rho \quad$ Density

$p \quad$ Hydrostatic pressure

$\psi_{1} \quad$ Factor taking into account the eccentricity of the resultant tensile force on the anchors

$\psi_{2} \quad$ Modification factor considers disorder of the radial symmetric stress 
distribution due to the edge effect for the anchors far from the edge

$\psi_{4} \quad$ Eccentricity effect of shear load on anchor groups

$\psi_{5} \quad$ Modification factor considers disorder of symmetric stress distribution in shear

$\psi_{e c, N} \quad$ Modification factor account for eccentricity effect for group anchors in tension

$\psi_{e d, N} \quad$ Modification factor account for edge effect in tension

$\psi_{c, N} \quad$ Modification factor account for cracking under tensile load

$\psi_{e c, v} \quad$ Modification factor accounts for eccentricity effect for group anchors subjected to eccentric shear load

$\psi_{e d, v} \quad$ Modification factor accounts for edge effect in shear

$\psi_{c, v} \quad$ Modification factor accounts for cracking under shear load

$\psi_{c p, N} \quad$ Modification factor account for post installed anchors embedded in uncracked concrete

$\psi_{h, v} \quad$ Modification factor account of the thickness of concrete

$\sum_{0} \quad$ Nominal perimeter of the reinforcing

$\sigma_{u} \quad$ Ultimate strength of the anchor

$\sigma_{0} \quad$ Initial yield stress

$\sigma_{y} \quad$ Yield stress

$\sigma_{s} \quad$ Tensile stress of the steel bar

$\tau \quad$ Shear stress

$\tau_{o} \quad$ Uniform bond stress in the adhesive layer

$\tau_{\max } \quad$ Maximum bond stress in the adhesive layer 


$\begin{array}{ll}\lambda^{\prime} & \text { Elastic constant } \\ \Re & \text { Rubin three invariant reduction factor } \\ \propto, \lambda, \beta, \theta & \text { Constants } \\ \theta_{a} & \text { Cone breakout angle } \\ \theta_{b} & \text { Crack propagation angle } \\ \Delta t & \text { Time step } \\ \dot{\varepsilon} & \text { Effective strain rate } \\ \varepsilon_{e f f}^{p} & \text { Effective plastic strain } \\ \dot{\varepsilon}_{i j}^{e} & \text { Elastic strain rate } \\ \dot{\varepsilon}_{i j}^{p} & \text { Plastic strain rate } \\ \dot{\varepsilon}_{i j} & \text { Total strain rate } \\ \varepsilon_{i j} & \text { Strain } \\ \Delta \varepsilon_{e f f}^{p} & \text { Effective plastic strain increment } \\ f_{h}\left(\varepsilon_{e f f}^{p}\right) & \text { Hardening function }\end{array}$




\section{Chapter 1 : Introduction}

\subsection{Background}

Modern construction techniques depend on the ability to connect or join building components. There are different joining techniques depending on the construction materials, structural system, and building design requirements (Eligehausen et al., 2006). Steel anchorage techniques are widely used for joining steel structural components to concrete structures (Eligehausen et al., 2006; Fuchs, 2001; Zamora et al., 2003; Eligehausen et al., 2001; Çalışkan et al., 2013).

There are two broad anchorage techniques for fastening structural components to concrete structure: cast-in-place and post-installed anchorage systems. Cast-in-place anchors are installed before casting the concrete, while post-installed anchors are installed in hardened concrete by drilling holes and inserting the steel anchors. The connections between parts in a structure are important to transfer the load between these parts (Primavera et al., 1997). Load transfer mechanism for cast-in-place anchors is mainly by mechanical interlocking between anchor and the concrete. The load transfer mechanism in post-installed anchorage systems is by mechanical interlocking, friction and bonding (adhesion) (Çalışkan et al., 2013; Eligehausen et al., 2001; Eligehausen et al., 2006; Fuchs, 2001; Zamora et al., 2003).

Many factors influence the strength of the anchorage system to concrete. These are: 
a) factors related to the anchor such as anchor type (cast-in-place or post installed), anchor strength, embedment depth, edge distance and spacing (in multi-anchor applications);

b) factors related to the base material such as type of the base material (concrete or masonry), strength and condition of the base material (cracked or uncracked), and reinforcement detailing of the base material;

c) factors related to the applied load such as direction of the applied load (shear, tension, combined shear-tension load), and type of load (static or dynamic); and

d) factors related to the environmental conditions such as corrosion and temperature (Committee Euro-International du Beton (CEB), 1994).

Failure load of the anchorage system can be determined either by experimental testing, numerical modelling or by predictive methods. The predictive methods are such as American Concrete Institute (ACI) (ACI Committee (349), 1990; Fuchs, 2001; Zamora et al., 2003) and Concrete Capacity Design (CCD) (Committee Euro-International du Beton (CEB) 1994; Fuchs et al., 1995; Subramanian, 2000; Fuchs, 2001; Ashour and Alqedra, 2005). Behaviour of anchors embedded into concrete and subjected to static load has been widely investigated experimentally (Cook et al., 1992; Fuchs, 2001; Hashimoto and Takiguchi, 2004; Yilmaz et al., 2013; Zamora et al., 2003; Çalışkan et al., 2013; Eligehausen et al., 2001; Eligehausen et al., 2006). Although many structures that contain anchorage systems are exposed to dynamic loads, most of the research is under static loading conditions. Research focusing on the effects of dynamic loads from structural 
vibration, earthquakes, impacts and blasts is limited. Investigating the dynamic response of the anchorage systems at high strain rates is crucial for the following reasons:

- Complex concrete response at high dynamic loading (Tu and Lu, 2009).

- Significant increase in the strength of concrete and steel due to high strain rate (Malvar \& Crawford, 1998; Shkolnik, 2008; Min et al., 2014).

- The high strain rate has an influence on the properties and deformation of concrete (Park et al., 2001) and steel (Lee et al., 2007) materials.

- Exposure to high strain rate can result in a catastrophic anchorage failure such as in the structures under the earthquake, impact or blast events.

- Lack of a comprehensive and validated database on the behaviour of anchorage to concrete systems in structures subjected to different strain rates is considered a critical barrier to the designers to estimate the performance of these systems under different strain rate loading conditions.

- Current codes and guidelines for the design of anchorage systems such as ACI and CCD methods address anchorage designs under static and low cycle dynamic loading. Hence, there is a need to develop a design method to predict the anchorage response under high dynamic loading to ensure structural safety and to minimize or prevent anchorage failure. 


\subsection{Objectives and significance of research program}

A review of the literature shows little information on the response of anchorage systems under high strain rates arising from blast and impact loading. Moreover, there is no accepted method for the design of anchorages subjected to high strain rates. Experimental research involving high strain rate loading can be complex, time consuming and expensive. Consequently, numerical analysis using a commercially available finite element package, LS-DYNA, was used to investigate the response of steel anchorage systems under high strain rates. The results of the numerical analyses will enable the prediction of the structural response of the anchorage systems under different strain rates.

The main objective of this research project is to investigate the effect of strain rate on the tensile and shear behaviour of cast-in-place, adhesive and undercut anchors. The main objectives of the research were achieved through the following research tasks:

- Development of finite element models for the cast-in-place, adhesive and undercut anchors to investigate the effect of strain rate on the tensile and shear behaviour of the anchorage to concrete systems.

- Investigation of the effect of strain rate on the load-displacement relation for the anchorage system.

- Examination of the effect of anchor diameter and embedment depth on the capacity of anchorage to concrete systems at different strain rates.

- Determination of the concrete cone breakout angle and cone breakout diameter for the anchorage systems. 
- Establishment of dynamic increase factors (DIF) to relate the static and dynamic capacity of anchorage systems at high strain rates.

- Prediction and discussion of the failure mode of the cast-in-place, adhesive and undercut anchorage to concrete at different strain rates.

- Examination of the level of damage of the anchorage to concrete under different design parameters and strain rates.

- Development of analytical equations to relate DIF and strain rate for the anchorage to concrete systems.

It has been experimentally established that both concrete and steel experience an increase in strength under high strain rate loading. The combined effect of strength increase of concrete and steel has not been adequately investigated under strain rates experienced under blast and impact. This research presents a numerical investigation carried out to study the tensile and shear behaviour of cast-in-place, adhesive and undercut anchorage to concrete systems under strain rates ranging from $10^{-5} \mathrm{~s}^{-1}$ to $10^{3} \mathrm{~s}^{-1}$. Predicting the failure mode of anchorage to concrete system and the relationship between the anchorage system strength increase and strain rate will provide designers with the requisite information to design safe and cost-effective anchorage systems. Also, the effect of anchor diameter and embedment depth on the anchorage system capacity at different strain rates will be invaluable to designers. In addition, the research aims to provide a proven design methodology for the cast-in-place, adhesive and undercut anchorage to concrete systems under different strain rates. The findings of this research will contribute to the design of 
anchorage systems capable of resisting high strain rate loading and predicting the failure load and failure mode of the anchorage systems under these loading conditions.

\subsection{Methodology}

In this project, behaviour of anchorage to plain concrete at strain rates in the range of $10^{-5}$ $\mathrm{s}^{-1}$ to $10^{3} \mathrm{~s}^{-1}$ was investigated using finite element analysis package - LS-DYNA software. Single cast-in-place, adhesive and undercut anchors were selected for the analysis. Effect of group anchors on the tensile and shear behaviour of anchorage to concrete system was not considered for the analysis in this thesis. Standard anchor diameters of 12.7-mm, 15.9 $\mathrm{mm}$ and 19.1-mm with embedment depths $\left(h_{e f}\right)$ of $76.2 \mathrm{~mm}, 101.6 \mathrm{~mm}, 127 \mathrm{~mm}$ and 152.4 $\mathrm{mm}$ for the cast-in-place and adhesive anchors were selected for the analysis. Three undercut anchors with nominal diameters of 12-mm, 16-mm and 20-mm and recommended embedment depths of $125 \mathrm{~mm}, 190 \mathrm{~mm}$ and $250 \mathrm{~mm}$ were considered for this investigation according to Hilti Inc. (Hilti, 2011). In addition, embedment depth of $100 \mathrm{~mm}$ was investigated. Effect of edge distance was not considered in the analyses. To preclude edge effects on the behaviour of the anchors, concrete block size of $\left(4 \mathrm{~h}_{\mathrm{ef}}+125\right) \mathrm{mm} \times\left(4 \mathrm{~h}_{\mathrm{ef}}+\right.$ 125) $\mathrm{mm} \times\left(2 \mathrm{~h}_{\mathrm{ef}}\right) \mathrm{mm}$ was selected to model the tensile behaviour of the steel anchorage systems. For modelling the shear behaviour of the steel anchorage system, concrete block size of $\left(2 h_{e f}+150\right) \mathrm{mm} \times\left(2 h_{e f}+150\right) \mathrm{mm} \times\left(1.5 h_{e f}+50\right) \mathrm{mm}$ was selected to minimize the effect of edge distance on the behaviour of the anchors.

Solid elements were used to model the steel anchor, sleeve, adhesive, plate, nut, washer and the concrete. The solid elements for the anchor, sleeve, plate, nut and washer were 
formulated using constant stress while the solid elements for the concrete were formulated using one point integrated tetrahedron. Details of the solid element types used for the analyses are presented in Chapter 3. Several material constitutive models are available in LS-DYNA to model concrete, steel and adhesive materials. Some of the commonly used material constitutive models were evaluated to select the most suitable material models that can best represent the behaviour of concrete, steel and adhesive under the strain rates investigated. Continuous Surface Cap Model (MAT_CSCM_CONCRETE) was selected to model the concrete, Piecewise Linear Plasticity Model (MAT_024) was used to model the steel anchor, and Arup Adhesive Model (MAT_169) was used to model the adhesive material.

Three contact definitions were used to model the interaction between the components of the anchorage system: CONTACT_AUTOMATIC_SURFACE_TO_SURFACE, CONTACT_TIED_SURFACE_TO_SURFACE and CONTACT_AUTOMATIC_ONE_ WAY_SURFACE_TO_SURFACE_TIEBREAK. Bonding between the concrete and the anchor for the cast-in-place and undercut anchors was performed using CONTACT_AUTOMATIC_SURFACE_TO_SURFACE. This contact keycard simulates a two-way contact where the penetration of the slave nodes (the anchor) are checked against master segment (the concrete) and master nodes are checked against slave segment. For the adhesive anchor, CONTACT_AUTOMATIC_ONE_WAY_SURFACE_TO_ SURFACE_TIEBREAK was used to model the steel to adhesive bond, CONTACT_TIED_SURFACE_TO_SURFACE was used for bonding between the adhesive and the concrete. CONTACT_ERODING_SINGLE_SURFACE was implemented to remove any distorted elements of the adhesive material. All the nodes at 
the bottom surface of the concrete block were fixed to prevent displacement and rotation during the loading regime. Using symmetry, a quarter of the anchorage system with two symmetry planes boundary condition was used to model the tensile behaviour. Half of the anchorage system with one symmetry plane boundary condition was used to model the shear behaviour.

The strain rate was imposed on the steel anchor by imposing BOUNDARY_PRESCRIBED_MOTION_SET keycard, in z-direction and y-direction, to model the tensile and shear loads respectively. After completing the finite element models, finite element analysis was carried out using explicit solver of LS-DYNA.

The cast-in-place, adhesive and undercut anchor models were validated with experimental test data and compared with results from the analytical prediction methods (ACI and CCD). Effect of strain rate on the tensile and shear capacity of the cast-in-place, adhesive and undercut anchors was investigated for the different anchor diameters and embedment depths. Ultimate load values for the anchors were determined for the investigated strain rates. The DIF, defined as the ratio of dynamic to static capacity was determined at each strain rate. The relation between the DIF and strain rate was established and an equation relating the DIF and strain rate was proposed. The adequacy of the proposed equation was verified by comparing the DIF obtained from the predicted equation with the DIF obtained from new developed numerical models of the anchorage systems with different design parameters. Figure 1-1 shows the anchorage to concrete systems used in this research. The methodology of the project can be summarized as in the flow chart shown in Figure 1-2. 


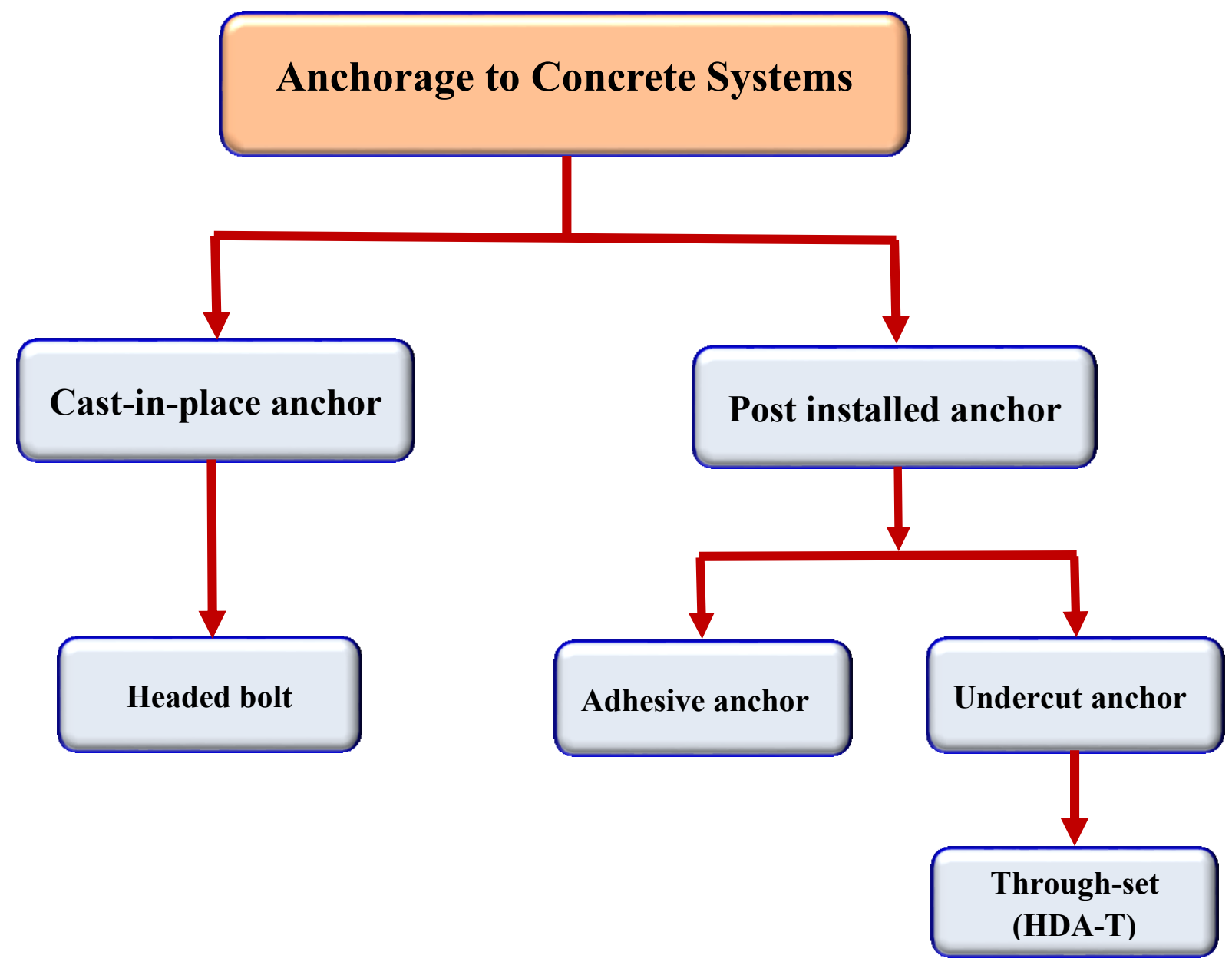

Figure 1-1: Anchorage systems 


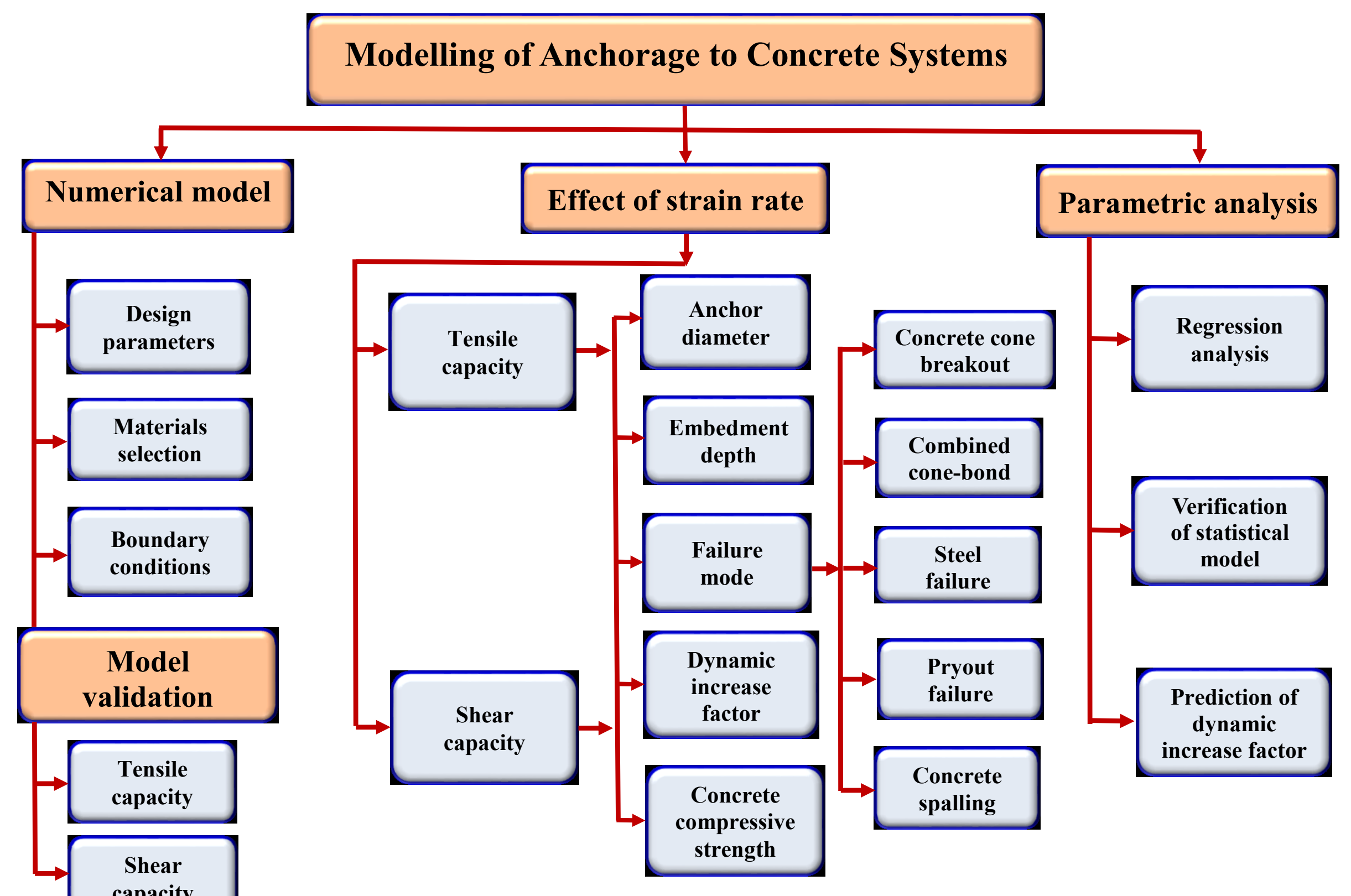

Figure 1-2: Flow chart for the methodology of the project 


\subsection{Scope of the research}

In this research, anchorage to concrete system models of different design parameters were developed using LS-DYNA software to investigate the tensile and shear behaviour of the anchorage systems embedded in plain concrete. Six strain rates of $10^{-5} \mathrm{~s}^{-1}, 10^{-3} \mathrm{~s}^{-1}, 10^{-1} \mathrm{~s}^{-1}$, $10 \mathrm{~s}^{-1}, 10^{2} \mathrm{~s}^{-1}$ and $10^{3} \mathrm{~s}^{-1}$ that represent various loading conditions were considered in the analysis. Low strain rate of $10^{-5} \mathrm{~s}^{-1}$ is considered to represent the static loading, moderate strain rates of $10^{-3} \mathrm{~s}^{-1}$ and $10^{-1} \mathrm{~s}^{-1}$ are considered to represent the earthquake, strain rate of $10 \mathrm{~s}^{-1}$ is considered for impact and high strain rates of $10^{2} \mathrm{~s}^{-1}$ and $10^{3} \mathrm{~s}^{-1}$ are associated with blast loading. The scope of this research can be summarized as follows:

1. Analyses on the behaviour of cast-in-place anchorage to concrete systems subjected to tensile and shear loads at strain rates ranging from $10^{-5} \mathrm{~s}^{-1}$ to $10^{3} \mathrm{~s}^{-1}$.

2. Analyses on the behaviour of post-installed adhesive anchorage to concrete systems subjected to tensile and shear loads at strain rates ranging from $10^{-5} \mathrm{~s}^{-1}$ to $10^{3} \mathrm{~s}^{-1}$

3. Analyses on the behaviour of post-installed undercut anchorage to concrete systems subjected to tensile and shear loads at strain rates ranging from $10^{-5} \mathrm{~s}^{-1}$ to $10^{3} \mathrm{~s}^{-1}$. 


\subsection{Structure of the thesis}

This thesis has been organized into seven chapters. The contents in each chapter are summarized as follows:

Chapter one outlines the background of anchorage to concrete systems, followed by the objectives of the research. The methodology of the research is also presented in this chapter with a flow chart that shows the process of developing the anchorage to concrete models and the factors that are investigated. Chapter one ends with the structure of the thesis that outlines all the chapters of the thesis.

Chapter two presents a comprehensive literature review on steel to concrete anchorage systems. Chapter two addresses the classifications of anchors and adhesive materials. The chapter includes a literature review on the behaviour of cast-in-place and post-installed anchors under static and dynamic tensile and shear loading. A literature survey on the effect of bond strength, embedment depth, anchor diameter, edge distance, anchor spacing, and failure mode is presented. Effect of strain rate on the concrete and steel materials is also presented. This is followed by introduction of the design methods for cast-in-place and post-installed anchors under tensile and shear loads. Failure modes of the cast-in-place and post-installed anchors and summary of the literature review are presented at the end of the chapter.

Chapter three presents the modelling and finite element analyses of the cast-in-place, adhesive and undercut anchors using LS-DYNA software. The chapter begins with introduction and presentation of the most commonly used finite element software packages in engineering applications and the main features of LS-DYNA. Implicit and explicit 
analyses methods implemented in LS-DYNA are introduced, followed by the theoretical aspects of LS-DYNA. The considerations used to select constitutive materials models, element type, and bonding type for the concrete, steel and adhesive are presented. Meshing technique, boundary conditions, contact modelling and loading conditions that accurately represents the behaviour of anchorage to concrete system are introduced. Procedure to perform quasi static analysis using LS-DYNA software is presented. Finally, a summary of chapter three is presented.

Chapter four begins with modelling the cast-in-place anchorage system using LS-DYNA software. Results and discussion of the effect of strain rate on the behaviour of cast-inplace anchors subjected to tensile and shear loads are presented. Static and dynamic loading conditions using LS-DYNA software are carried out in this chapter. Mesh sensitivity analysis is carried out to improve the accuracy of the analysis. Model validation with experimental test data in the literature as well as comparison with analytical prediction methods (ACI and CCD methods) is presented. The effect of strain rate on the ultimate tensile and shear loads, the effect of strain rate on the level of damage and failure mode as well as the DIF, the effect of anchor embedment depth on concrete cone depth, the effect of strain rate on concrete breakout diameter and crack propagation angle, and effect of concrete compressive strength on the level of damage and failure mode are presented in Chapter four. Regression analysis is performed for the cast-in-place anchors under tensile and shear loads to develop a relationship between DIF and strain rate.

Chapter five begins with modelling the adhesive anchorage to concrete system using LSDYNA software. Mesh sensitivity analysis is carried out to establish the element size and 
mesh density to maximize accuracy at least resource cost. Results and discussion of the effect of strain rate on the behaviour of adhesive anchors subjected to tensile and shear loads are presented. The adhesive anchor model is validated with experimental test data in the literature and presented in this chapter. Comparison with the ACI and CCD analytical prediction methods is presented. Level of damage and failure mode at different strain rates, the effect of strain rate on the ultimate loads and DIF, the effect of anchor embedment depth on concrete cone depth, the effect of strain rate on concrete cone breakout diameter are presented in chapter 5. Regression analysis is performed to develop a relationship between DIF and strain rate for the adhesive anchors under tensile and shear loads.

Chapter six presents modelling of the undercut anchorage to concrete system under tensile and shear loads using LS-DYNA software. Model validation with the experimental data is performed. Comparison of the finite element results with the ACI and CCD design methods is presented. Results and discussion of the effect of strain rate on the tensile and shear behaviour of undercut anchors are presented. Effect of strain rate on the ultimate loads and DIF, failure mode and crack propagation angle at different strain rates, the effect of anchor embedment depth on concrete cone depth and cone diameter are presented. Regression analysis is performed for the undercut anchors under tensile and shear loads to develop relationship between DIF and strain rate.

Chapter seven presents the main conclusions of the analyses and introduces recommendations for future work. 


\section{Chapter 2 : Literature review}

\subsection{Introduction}

Construction of buildings and building components requires joining or connecting parts together. Different anchorage techniques are widely used for joining concrete and steel structures and components. Anchorage to concrete systems can be exposed to static or dynamic loading conditions resulting from use and occupancy or from the environment or climate. Static load results from self-weight of the structure and when the live load is applied slowly. Dynamic loads result from structural vibration, earthquake, impact and blast events.

Structures can be exposed to low and high strain rate loading conditions such as encountered with creep, quasi-static, earthquake, impact and blast loads. It is essential for the structures to retain suitable structural safety with durable anchorage system. The damage that occurs due to high loading rate such as under earthquake loading, motivate researchers to study the behaviour of anchor connections at these rates. In the 1994 Northridge earthquake in the USA, anchor failures caused damage of transformers and resulted in electrical power outage to the Veteran's hospital (Zhao, 2014). In 1995 an earthquake in Osaka, Japan resulted in anchorage failure and caused transformers to slip off their foundations, resulting in power outage and severe damage to power transmission lines (Zhao, 2014). Low loading rates such as creep, on the other hand, may result in degradation of the adhesive materials and lead to failure of anchorage systems. An example of adhesive anchor failure is the collapse of part of concrete ceiling in Boston tunnel where 
adhesive anchors were used to secure the ceiling to the tunnel walls. The National Transportation Safety Board found that the collapse resulted from creep of the adhesive due to long term loading (Vuletic and Pearson, 2008).

Investigating the behaviour of anchors under high loading rates is important in roadway support and design. Anchors can be used in applications that require high loading rates such as rock burst where the anchors can be used to reduce the damage induced by rock burst (Zhao et al., 2015). For example, in mining, anchors used to secure steel mesh against rock bursts are subjected to very high impact loading (Ansell, 2006). Figure 2-1 shows applications of anchorage system in rock burst.

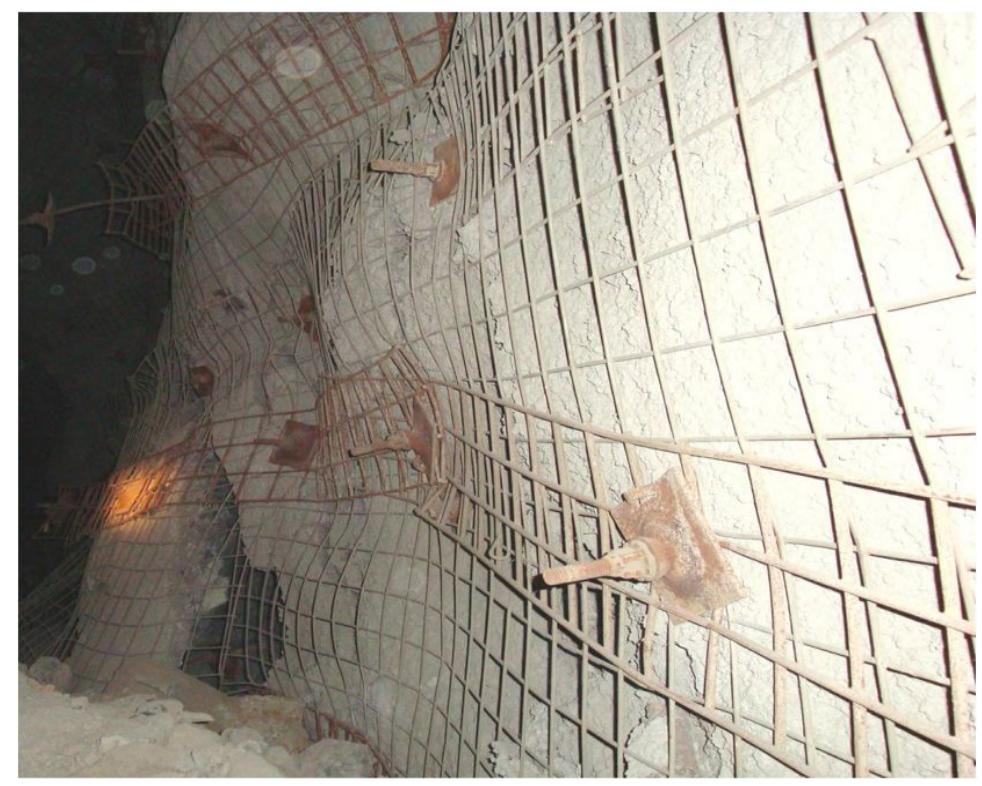

Figure 2-1: Applications of anchorage system in rock burst (Cai et al., 2010)

In addition, bolts can be used to attach blast protection appliques (such as armor) to combat vehicles, where the appliques are used to mitigate the effect of explosions. However, these 
bolts shear off under blast loads resulting in secondary fragments that may damage the vehicle and/or cause injury to people (Lou and Perciballi, 2008).

When a structure subjected to blast loading, the fractured glass windows can cause injury and fatality to the occupants of the building and the people around it. According to the Oklahoma State Department of Health, most of the injuries in the Oklahoma City bombing were caused by window glass shards (Mallonee et al., 1996; Norville \& Conrath, 2001, 2006; Shariat et al., 1998). People within a distance of $3 \mathrm{~m}$ from the windows reporting injuries due to the window glass shards represented over $40 \%$ of the total injured (Norville et al., 1999). Upgrading window glazing and using laminated glass can mitigate the injuries and fatalities. However, the load are transferred from the window glazing to the window frame and then from the window frame to the structure through the bolts (Braimah et al., 2014). Figure 2-2 shows the applications of anchors in the window to resist blast load.

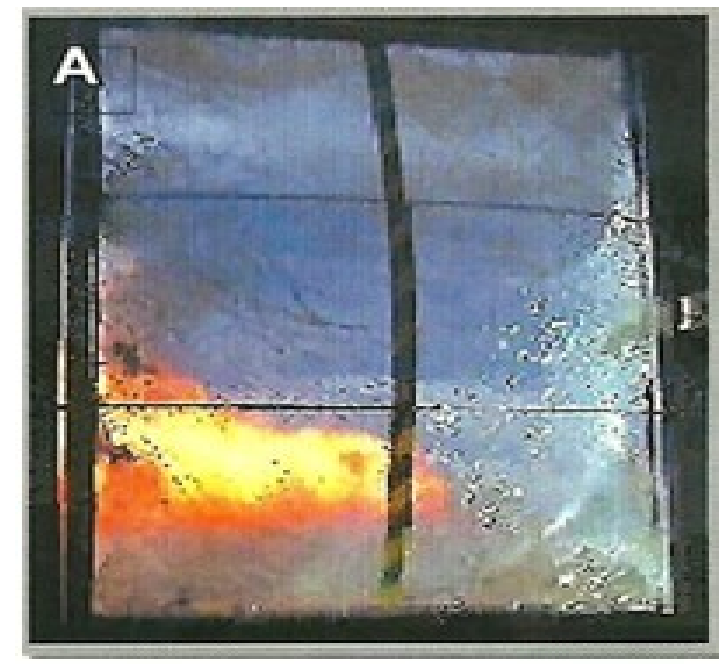

(a)

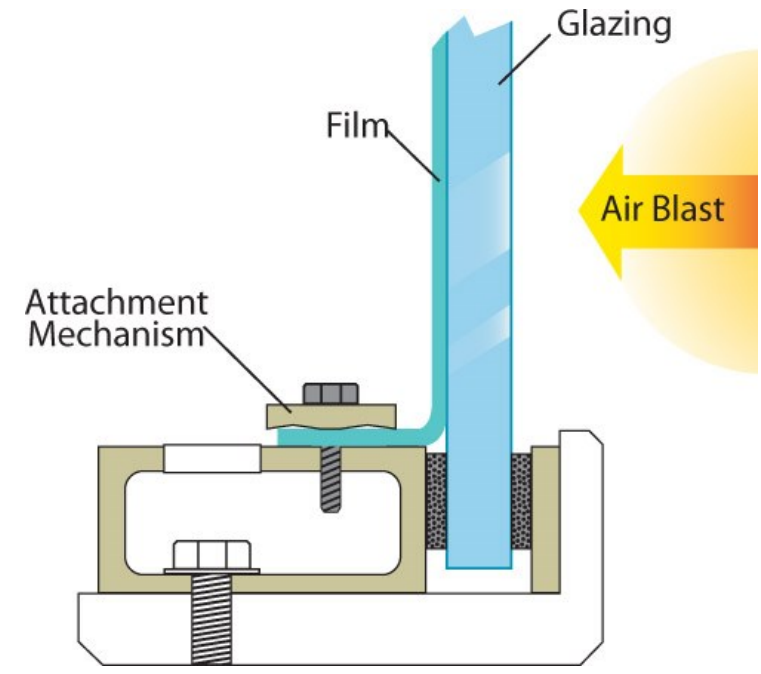

(b)

Figure 2-2: Applications of anchorage system in window; (a) glass window under blast load (Madico Safety Shield Premier Partener, 2012), (b) anchorage to base-plate (Johnson window films, 2018) 


\subsection{Classification of anchors}

The main function of the anchors is to connect structural components. As mentioned in chapter one, there are two categories of anchors commonly used for joining concrete to steel structures: cast-in-place and post-installed anchors. Cast-in-place anchors are cast into fresh concrete during forming and have been used in a wide range of structural applications for decades (Hawkins, 1987). Cast-in-place anchors are used to fasten equipment to concrete in construction in order to avoid drilling in hardened concrete, a process that creates microcracking in the concrete member as well as requiring additional finishing work (Delhomme et al., 2015a). The cast-in-place anchors can be classified according to their shape as hexagonal headed bolt, hooked L-bolt, hooked J-bolt and welded headed stud (Figure 2-3 a). Some errors may occur due to improper installation of the cast-in-place anchor and affect its performance. These errors include: inappropriate location of the anchor installation, incorrect implementation of the detailing drawing, neglect of changes that may occur in the concrete geometry during the casting, curing and under sustained loading; e.g. creep and shrinkage (Spyridis and Bergmeister, 2014).

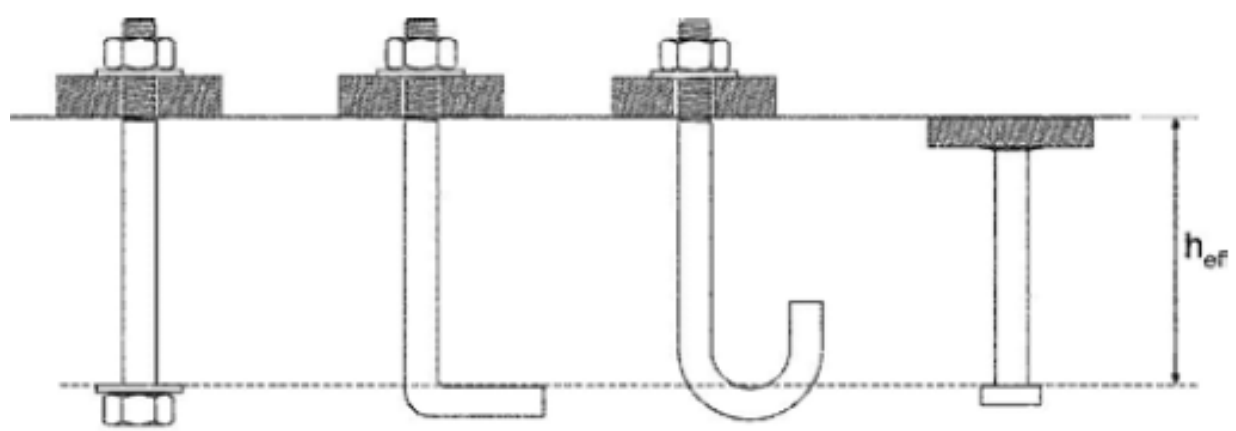

(i) hexagonal headed bolt (ii) hooked L-bolt (iii) hooked J-bolt (iv) welded headed stud (a) 


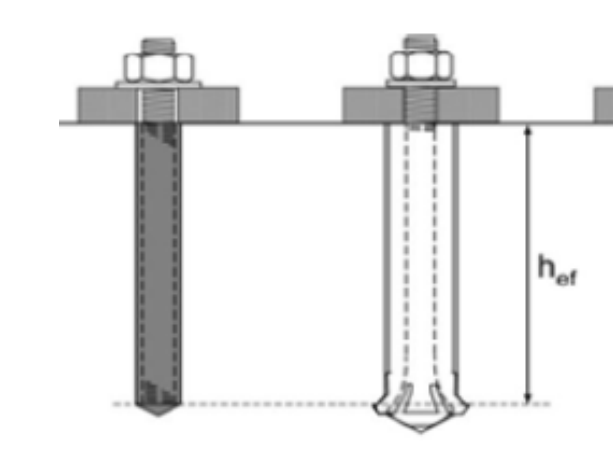

(i) Adhesive anchor

(ii) undercut anchor

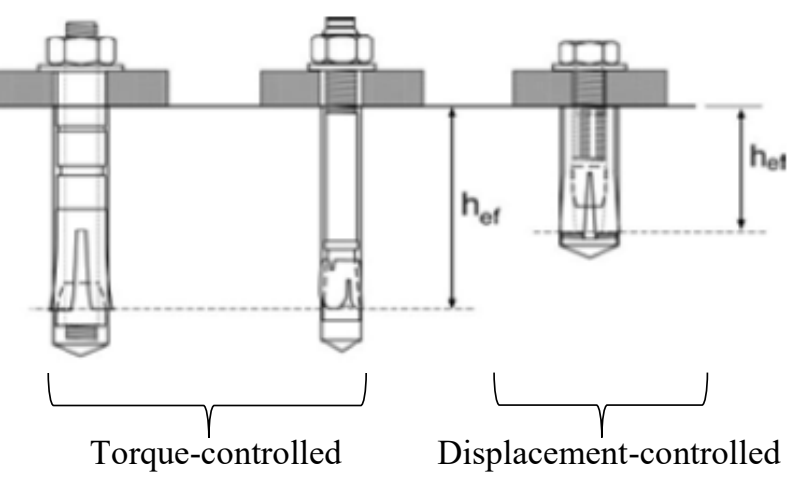

(iii) expansion anchor

(b)

Figure 2-3: Types of anchors: (a) cast-in-place anchors, (b) post installed anchors (ACI Committee 318, 2011)

Post-installed anchors are increasingly used in construction due to their flexibility of installation in any position in a substrate material and the increasing demand for shorter construction times. Post-installed anchors have found use in a variety of applications including: new construction, retrofit and rehabilitation, and repair of concrete and masonry structures (Cook, 1993). Post-installed anchors have also found use in blast retrofit applications to resist, minimize or mitigate the influence of blast load where they are subjected to high strain rate loading. Post-installed anchors are classified according to the load transfer mechanism into mechanical and bonded anchors (McMullin et al., 2016). Mechanical anchors are further classified as undercut and expansion anchors, while bonded anchors can be either adhesive anchors or grouted anchors (Figure 2-3 b). The load transfer mechanism for the undercut anchors to the substrate material is by mechanical interlocking which is similar to the cast-in-place anchors. The applied tensile load is transferred from the anchor stud to the sleeve by bearing which in turns bears on the concrete (A Mitek' Company, 1997). For expansion anchors the load transfer mechanism is by friction. On the 
other hand, the load transfer mechanism for the bonded anchors to the substrate material is by chemical bonding (Fuchs et al., 1995).

Undercut anchors are mechanical anchors. The undercutting is made either by a special drill bit or self-drilling anchor with carbide tipped segments used to undercut concrete during the installation process (ACI Committee 318, 2011). The undercut anchor is characterized by its performance that is comparable to the cast-in-place anchor with more flexibility of installation (Mackay-Sim, 1990; Hilti, 2011). In addition, undercut anchors are shown to safely and reliably resist dynamic loads and are thus suitable for applications that require high level of safety such as in nuclear power plants (Trautwein, 2017). The anchor installation can be accomplished in one of three techniques: load-controlled, displacement-controlled or torque-controlled (ACI Committee 355, 2000; Eligehausen et al., 1998). The installation by load controlled is performed by applying tensile load on the undercut anchor that expands the sleeve into a drilled hole. The displacement-controlled installation method can be performed either by applying compression force on the sleeve of the undercut anchor or by pulling out the cone and expanding the sleeve. The installation by the torque-controlled method is performed by applying a torque and resulting in expansion of the sleeve. The displacement-controlled undercut anchor is used for low strength concrete while the load-controlled and torque-controlled undercut anchors are used for low and high strength concrete.

Hilti Inc. produces two types of undercut anchors: Hilti design anchor pre-set (HDA-P) and Hilti design anchor through-set (HDA-T). In the through-set anchor type the sleeve continues through the base plate while the sleeve ends before the base plate for the pre-set 
anchor type. Through-set undercut anchor is capable of withstanding higher shear strength than the pre-set undercut anchor. This is attributed to the contribution of the sleeve to the anchor shear resistance. For the pre-set anchor type on the other hand, the shear force is transmitted form the anchor to the sleeve and then to the concrete by bearing (Hilti, 2011). Hence, the through-set undercut anchors are selected for the analysis presented in this thesis. Figure 2-4 presents the pre-set and through-set types undercut anchors.

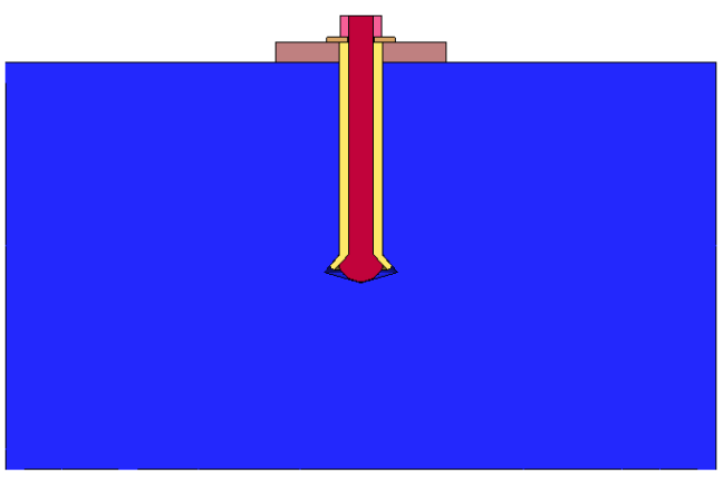

(a) Through set undercut anchor (Sleeve continues through base plate)

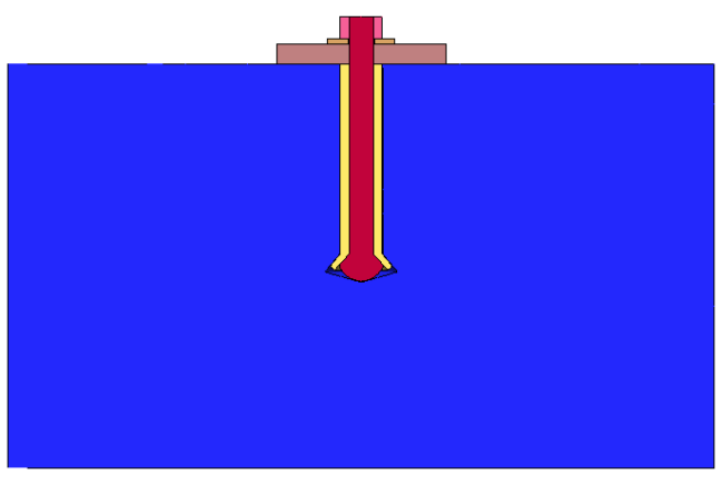

(b) Pre-set undercut anchor (Sleeve ends before base plate)

Figure 2-4: Undercut anchor types

The expansion anchors are post-installed mechanical anchors inserted in a drilled hole in hardened concrete. When torque is applied on the expansion anchor, the lower part of the expansion anchor expands resulting in increased friction and bearing against the sides of the hole. Two techniques are used to achieve the expansion of the anchor; torque-controlled and displacement-controlled techniques. Torque-controlled technique is achieved by applying a torque on the anchor, while the displacement controlled technique is achieved by applying impact force on the sleeve of anchor, the expansion is controlled by distance that the sleeve travels (ACI Committee 318, 2011). 
Adhesive anchor systems involve drilling a hole in hardened concrete and then filling the hole with an adhesive material. The anchor is then inserted in the hole, where the adhesive material serves as a bonding agent between the anchor and the concrete (Cook et al., 2007). In grouted anchors, the drilled hole in hardened concrete is grouted after placement of the steel anchor. Different types of bonding agents can be used for bonded anchors. Epoxies, polyesters and vinylesters are used as bonding agents for the adhesive anchors, while cementitious bonding agents are used for the grouted anchors.

Selection of a suitable type of anchor in construction, whether cast-in-place or post installed, can be decided based on the application, cost, and performance of the anchor.

\subsection{Classification of adhesive materials}

Adhesive materials are used to bond two or more materials together. Adhesive materials can be classified into six categories based on:

- $\quad$ origin e.g. natural and synthetic;

- functionality e.g. thermosetting and thermoplastic;

- chemical composition e.g. epoxy, silicon and polyurethane;

- $\quad$ physical form e.g. paste and liquid;

- type e.g. hot melt; and

- load carrying capacity e.g. structural, semi structural and non-structural (da Silva et al. 2011). 
Structural adhesive is used in the structural applications to sustain a long term applied load on a structure. There are several types of structural adhesives such as: epoxy and polyurethane (Goncalves and Margarido, 2015). Epoxy can be formed as two components or one component cartridges. The two component epoxies consists of resin and hardener which can be mixed together and cured at room temperature (Kinsho et al., 2000). One component epoxy can be found in liquid or paste form. Heat is required for curing the one component epoxy (Kinsho et al., 2000; da Silva et al., 2011).

\subsection{Bond strength}

The strength of anchorage system whether cast-in-place or post installed is influenced by the bond strength. In steel reinforced concrete, force is transmitted from steel to concrete through interface bond (Nammur and Naaman, 1989). The concrete composition and the surface condition of the steel influence the interface bond between concrete and steel, which in turns influences the load resistance of the reinforced concrete element (Fu and Chung, 1999). There are two types of bond models used in analysis and design: elastic and inelastic. Elastic bond is exhibited when the bond at the interface is less than the bond strength and the shear stresses depend on the relative displacement between the steel and the concrete (Nammur and Naaman, 1989).

For the adhesive anchors, the force from the anchor is transmitted to the concrete through the adhesive material. The bond strength of adhesive anchorage system is affected by two main factors: internal and external. Internal factors such as adhesive formulations, processing and packaging are under the control of the manufacturer, while external factors 
such as installation and in-service factors are under the control of the designer and installer. The installation factors include the hole condition and the strength of the substrate material while in-service factors include curing time of adhesive, thickness of adhesive layer, type of loading and environmental conditions such as temperature (high and low), moisture, and freeze-thaw cycles (Cook and Konz, 2001; Cook et al., 2007).

There are two models available to predict the bond strength of the adhesive anchors: uniform bond stress and elastic bond stress models (Cook, 1993). Bond failure occurs at the steel-adhesive interface or at the adhesive-concrete interface. The pullout force for the steel-adhesive and concrete-adhesive interfaces can be predicted from the uniform bond stress model and the elastic bond stress model. For the uniform bond stress model the pull out force can be predicted according to (Equations B.7 and B.8 in Appendix B) (McVay et al., 1996; Cook et al., 1998; Cook, 1993; Eligehausen et al., 2007). The elastic bond stress model considers the compatibility between the concrete, bonding agent and the steel anchor, while the uniform bond stress model does not (Cook et al., 1993). For the elastic bond stress model, the pullout force can be predicted according to Equation (B.9 in Appendix B) (McVay et al., 1996; Cook et al., 1998; Cook et al., 1993; Cook, 1993).

Chapman and Shah (1987) conducted pullout test to investigate bond strength between concrete and reinforcing bars at different curing ages from 1 to 28 days. They tested both smooth bars and deformed bars for different embedment lengths. They stated that bond strength of smooth bar is not affected by curing age while, bond strength of deformed bar is significantly affected by curing age. The researchers observed three types of failure: steel 
yield and fracture failure, anchor pullout (or bond) failure, and concrete splitting failure. Pullout failure was observed at early concrete ages, while yield failure was observed at later concrete ages. Splitting failure was observed at early age for embedment depth of 175 $\mathrm{mm}$ and at later ages for embedment depth of $76.2 \mathrm{~mm}$. The authors concluded that as the embedment depth increases the pullout load increases while the average bond strength decreases. The increase in the pullout load is not proportional to that of the embedment depth. The failure mode of the bond for deformed bars is influenced by the embedment depth and compressive strength. Pullout failure occurs for shallow embedment depths and low concrete compressive strengths. Splitting failure is observed for short embedment depth with high concrete compressive strength and for deep embedment depth with low concrete compressive strength. Yield failure occurs for deep embedment depths with high concrete compressive strength (Chapman and Shah, 1987). By assuming a uniform stress distribution in the pullout test, the bond stress $(u)$ is determined from Equation (2.1) (Chapman and Shah, 1987; Chan et al., 2003; Xing et al., 2015; Benmokrane et al., 1996; Larrard et al., 1993; Fabbrocino et al., 2005):

$u=\frac{A_{s} \sigma_{s}}{\sum_{0} \cdot l_{d}}=\frac{F}{\pi d_{b} l_{d}}$

Where $A_{s}$ is the cross-sectional area of the bar, $\sigma_{s}$ is the tensile stress of the steel bar, $F$ is the applied tensile load, $\sum_{0}=\pi d_{b}$ is the nominal perimeter of the reinforcing bar, $d_{b}$ is the bar diameter, $l_{d}$ is the embedment length of the bar. 
Chan et al. (2003) conducted pullout tests to investigate the bond strength of steel reinforcement in self-consolidating concrete (SCC). The authors used ordinary Portland cement concrete (OPC) for comparison purpose. Effect of age, level of reinforcing bars, and concrete type on bond strength were investigated. The authors concluded that the bond strength of reinforcement in SCC is higher than that for the OPC. Maximum bond strength of 9.51 MPa and 6.31 MPa were obtained for the SCC and OPC respectively. The decrease in the bond strength of the OPC is attributed to the bleeding and inhomogeneity of the concrete. The bond strength is very low at the early age; up to three days. The bond strength is fully developed after seven days for the OPC and after 15 days for SCC. Chan et al. (2003) stated that the bond strength varies linearly with the square root of the compressive strength. The bond strength of top reinforcement is lower than that of bottom reinforcement in an OPC and SCC member. However, the difference in bond strength for the top and bottom reinforcements in SCC members was observed to be less than that in OPC members (Chan et al., 2003).

Fu and Chung (1999) conducted electromechanical pullout tests to investigate the effect of steel rebar surface treatment and concrete composition on the bond strength. The electromechanical pullout is conducted by measuring the contact electrical resistivity and bond strength of the specimen. They concluded that the rebar surface treatment such as adding polymer (latex and Methelcellelose) to the concrete and increased water-cement ratio increases the bond strength. Surface treatment involving use of ozone is more effective than use of sand blasting and acetone. The authors stated that the effectiveness of 
adding latex to the concrete is the same as using ozone surface treatment to the rebar (Fu and Chung, 1999).

Toikka et al. (2015) conducted experimental work to investigate the effect of strain rate on the bond strength and development length of steel reinforcement in concrete beams under blast loading. The authors found that the bond strength increased while the required development length decreased at high strain rate associated with blast loading. The authors concluded that dynamic increase factor for bond stress increased with the increase in the reinforcement bar diameter (Toikka et al., 2015).

Xing et al. (2015) investigated the bond strength between the reinforcing bar and concrete. They studied the effect of bar surface type, embedment length, bar type and diameter. The authors concluded that the bond strength of plain bar is less than that of the deformed bar. The bond strength of plain bars increases significantly as the embedment depth increases from $80 \mathrm{~mm}$ to $120 \mathrm{~mm}$. As the embedment depth increases, the bond stress distribution becomes uniform. The bond strength of deformed bars is composed of mechanical interlock, friction and adhesion. It is mainly governed by the mechanical interlock. Hence, the bond strength of the deformed bars is influenced by the concrete strength. The bond strength of plain bars is composed of friction and adhesion. It mainly depends on the friction. Thus, its bond strength is not influenced by the concrete strength (Xing et al., 2015). Increasing the bar diameter decreases the bond strength (Xing et al., 2015; Larrard et al., 1993). 
Shima et al. (1987) investigated the bond mechanism in reinforced concrete using microscopic and macroscopic models. In the microscopic model, they represented the relationship between the local bond stress and local slip. In the macroscopic model, they represented the relationship between the pullout load and the displacement. Shima et al. (1987) determined the tensile stiffness in reinforced concrete. The pullout of the anchored bars resulted in large cracks at the connection area. The authors represent two types of the deformational behaviour of bond: relative displacement of anchored bars in concrete, and tension stiffness of cracked concrete (Shima et al., 1987).

Larrard et al. (1993) studied the bond strength of reinforcing bars embedded in high performance concrete (HPC) of $95 \mathrm{MPa}$ compressive strength, and ordinary concrete of 42 MPa compressive strength. They used smooth and deformed bars with different diameters. They stated that the increase in the tensile strength of the concrete increases the bond strength. The increase in the bond strength of HPC was of $80 \%$ and $30 \%$ for bar diameters of $10 \mathrm{~mm}$ and $25 \mathrm{~mm}$ respectively. They observed that, slip of the reinforcement starts at the beginning of the loading for ordinary concrete, while the slip didn't start at the beginning of the loading due to the high rigidity of the bond in the high performance concrete (Larrard et al., 1993).

Barnat et al. (2012) studied the bond strength of chemical anchors embedded in high strength concrete in the range between $71.8 \mathrm{MPa}$ to $80.7 \mathrm{MPa}$ under pullout load. The authors concluded that the performance of chemical anchorage in high strength concrete depends on the properties of the adhesive material (Barnat et al., 2012). Also, the authors 
concluded that the bond strength of the adhesive is not influenced by the concrete strength (Barnat et al., 2012). However, Cook and Konz (2001) stated that bond strength increased slightly with the increase in the concrete strength (Cook and Konz, 2001). Cook and Konz (2001) conducted tensile tests to investigate factors affecting the bond strength of adhesive anchors. The authors investigated the effect of installation condition, concrete strength, concrete aggregate types, adhesive curing time and effect of loading at high temperature. The authors found that the hole condition can have a detrimental effect on bond strength, where the bond strength decrease for anchors embedded into wet, damp and uncleaned holes. Unsuitable curing and hole preparation lead to bond failure where bond strength is insufficient. The authors used two types of aggregates: river gravel and limestone. The authors stated that the effect of using river gravel aggregates on increasing the bond strength is more than the effect of using limestone. The authors concluded that the increase in the porosity of the aggregates decrease the bond strength. Also, they concluded that the increase in the temperature to $43^{\circ} \mathrm{C}$ affects the bond strength (Cook and Konz, 2001).

\subsection{Cast-in-place anchors}

\subsubsection{Cast-in-place anchors under tensile load}

Hawkins (1987) and Delhomme et al. (2015) investigated the strength of cast-in-place headed anchors embedded in reinforced concrete and subjected to static tensile loading. Hawkins (1987) observed two types of concrete failure modes: concrete cone breakout and concrete splitting failure. Concrete cone breakout failure occurred for shallow embedment depth of $76 \mathrm{~mm}$, while concrete splitting occurred for deeper embedment depths of 127 $\mathrm{mm}$ and $187 \mathrm{~mm}$ (Hawkins, 1987). Similar observation was reported by Delhomme et al. 
(2015) where concrete cone breakout failure is observed at embedment depth of $80 \mathrm{~mm}$. In addition, Delhomme et al. (2015) observed steel failure at embedment depths of $100 \mathrm{~mm}$, $120 \mathrm{~mm}$ and $130 \mathrm{~mm}$. Furthermore, Delhomme et al. (2015) studied effect of embedment depth for quadruple anchors. The authors observed concrete cone breakout failure at embedment depths of $80 \mathrm{~mm}, 100 \mathrm{~mm}, 120 \mathrm{~mm}$, and $130 \mathrm{~mm}$ and steel failure at embedment depth of $310 \mathrm{~mm}$. The authors concluded that the failure mode changed from concrete cone breakout to steel failure at embedment depth of $130 \mathrm{~mm}$ for the quadruple anchors (Delhomme et al., 2015b).

Solomos and Berra (2006) performed experimental test on the pullout strength of anchors under static and dynamic loads. The authors used Hopkinson bar technique to apply dynamic pullout loads on post-installed and cast-in-place anchors. The authors stated that the stresses and damage generated in the concrete due to the applied load and method of installation, whether cast-in-place or post-installed, affected the failure load of anchors. Moreover, same strength for post-installed and cast-in-place anchors can be obtained when adequate installation for post-installed anchors is achieved. The authors concluded that the failure load in the dynamic test is higher than that in the static test (Solomos and Berra, 2006).

Fujikake et al. (2003), Sato et al. (2004) and Ozbolt et al. (2006) investigated the effect of loading rate on the cone resistance for cast-in-place headed anchors. Fujikake et al. (2003) and Sato et al. (2004) studied loading rates of $1 \times 10^{-1} \mathrm{kN} / \mathrm{s}, 4 \times 10^{2} \mathrm{kN} / \mathrm{s}, 4 \times 10^{3} \mathrm{kN} / \mathrm{s}$, and $4 \times 10^{4} \mathrm{kN} / \mathrm{s}$ and found that the increase in the loading rate from $1 \times 10^{-1} \mathrm{kN} / \mathrm{s}$ to $4 \times 10^{4} \mathrm{kN} / \mathrm{s}$ 
resulted in increase in the ultimate cone resistance (Fujikake et al., 2003; Sato et al., 2004). Ozbolt et al. (2006) studied loading rates ranging from $5 \mathrm{~mm} / \mathrm{s}$ to $2 \times 10^{4} \mathrm{~mm} / \mathrm{s}$ and found similar results for the static and dynamic analysis for intermediate loading rate where the microcracks are dominant. For very high loading rates, the structural behaviour is controlled by the structural inertia. The authors concluded that when the loading rate increases, the size effect on the nominal pullout strength increases significantly for the moderate high loading rates. Size effect is neglected for very high loading rates and as the embedment depth increases the nominal strength increases (Ožbolt et al., 2006).

Choi et al. (2015) studied the behaviour of cast-in-place anchors in ultra-high performance fibre-reinforced concrete (UHPFRC) under monotonic shear and tensile loads. The authors observed that the tensile and shear load capacities for cast-in-place anchors in UHPFRC are higher than that for anchors in normal concrete. The authors related this increase in the capacities to the increase in the tensile and strain capacity in UHPFRC. The authors studied the effect of embedment depth and edge distance in UHPFRC. They observed that the concrete breakout capacity increased as the embedment depth increased. They also stated that the increase in the edge distance resulted in increase in the concrete breakout capacity. The authors observed shallower angle of the failure cone for the anchors embedded in UHPFRC compared to the $35^{\circ}$ proposed in the CCD method. The authors proposed three equations to determine the tensile and shear concrete breakout capacity and anchor pryout capacity in UHPFRC (Choi et al., 2015). 
Ozbolt and Eligehausen (1990) conducted numerical analysis to investigate the behaviour of headed studs embedded in large plain concrete and subjected to tensile load. They studied the effect of concrete properties and head size on the anchor behaviour. They stated that the failure of the headed stud is induced by circumferential concrete cracking, while the displacement at failure load is induced by the concrete compression behaviour under the head. As the anchor head increased from $35 \mathrm{~mm}$ to $52 \mathrm{~mm}$, the average compression stress under the anchor head decreased and this resulted in small displacement under the anchor head. Hence, the smaller the head diameter the larger displacement at failure (Ozbolt and Eligehausen, 1990).

Jang and Suh (2006) investigated the effect of cracks on the anchorage capacity for castin-place anchors subjected to tensile load. Influence of crack depth, crack width, and distance between the cracks and the anchor was studied. The authors found that brittle failure of cracked concrete propagated the cracks faster than for anchors in uncracked concrete. The authors concluded that anchorage in uncracked concrete has higher capacity than that obtained from anchorage in cracked concrete. The authors found that, a side crack (far from the anchor) has more effect on anchorage capacity than central cracks (passing through the anchor). They stated that the crack depth and the distance between the anchor and the cracks has more influence on the anchorage capacity compared to the crack width (Jang and Suh, 2006).

Hashimoto and Takiguchi (2004) investigated the strength of cast-in-place anchor embedded $30 \mathrm{~mm}$ in concrete and subjected to tensile load under high temperature. Results 
of their study show that, the increase in the temperature decreases tensile strength (Hashimoto and Takiguchi, 2004).

Fuchs et al. (1995) proposed Concrete Capacity Design (CCD) Method to predict failure loads of cast-in-place and post-installed anchors subjected to static tensile and shear loads. Fuchs et al. compared the results of the proposed CCD method with ACI 349-85. The authors investigated the effect of edge distance, anchor spacing under tensile and shear loads. They concluded that the CCD method gives accurate results of the failure load, while the ACI method can give conservative results for the failure load when the anchors are embedded into concrete with small edge distance or unconservative results for the failure load when the edge distance is large and for deep embedment depth anchors. The size of the concrete cone breakout for the shear loading is calculated based on the edge distance, while for the tensile loading it is calculated based on the anchor depth. The anchor diameter and stiffness were found to have an effect on the failure load of the anchors (Fuchs et al., 1995).

Nilforoush et al. (2017 and 2018) investigated the tensile behaviour of cast-in-place anchors embedded into plain and fibre reinforced concrete. The authors investigated the effect of concrete thickness and adding steel fibres on the anchor capacity and failure mode. They found that the increase in the concrete thickness exhibited a slight increase in the anchor capacity. Also, the authors observed that adding steel fibres to the concrete increased the tensile capacity (Nilforoush et al., 2017; Nilforoush et al., 2018). Nilforoush et al. (2017) found that the anchorage tensile capacity and stiffness increased with the 
increase in the concrete compressive strength. Concrete cone breakout failure was observed for the anchors embedded in plain concrete while concrete splitting was observed for the anchors embedded in thin plain concrete members (Nilforoush et al., 2017). In addition, Nilforoush et al. (2018) investigated the effect of anchor head size on the tensile capacity of the cast-in-place anchors. The authors found that the increase in anchor head size resulted in a significant increase in the tensile capacity and anchor stiffness (Nilforoush et al., 2018).

Hariyadi et al. (2017) investigated the pullout capacity of anchors embedded in concrete and subjected to static pullout loads. The authors conducted experimental work on anchors with shallow embedment depth (embedment depth-to-diameter ratio is equal to 3.5) and observed combination of cone breakout and bond failure mode. The authors obtained lower tensile capacity from the experimental results in comparison with the concrete cone method (CCM) and concrete capacity design method (CCD). The authors obtained cone breakout angle in the range of $15^{\circ}$ to $23^{\circ}$ (Hariyadi et al., 2017).

\subsubsection{Cast-in-place anchors under shear load}

Shear strength of anchors embedded in concrete has been investigated experimentally by Ueda et al. (1990). The authors observed concrete failure with wedge cone for most of the tested specimens. The authors concluded that an increase in edge distance increases the shear strength of single and double anchors embedded in concrete. They also found that the shear strength for double anchors increased with the increase in anchor spacing (Ueda et al., 1990). 
In another study, Ueda et al. (1991) investigated the shear strength of steel anchor groups. The authors applied shear loads parallel and perpendicular to the edge and observed that the increase in the spacing for double anchors increased the shear capacity in the parallel and perpendicular directions to the edge. Similar observation was obtained for the adhesive anchors by Eligehausen et al. (2006) when the load was applied perpendicular to the edge (Eligehausen et al., 2006). Ueda et al. (1991) and Eligehausen et al. (2006) stated that there is a critical edge distance when the load is applied perpendicular to the edge after which the value of failure load remain constant (Eligehausen et al., 2006, Ueda et al., 1991). Ueda et al. suggested empirical equations to determine the ultimate shear strength for single and grouped anchors involving two or four anchors (Ueda et al., 1991).

Performance of cast-in-place anchors with large diameters (more than $50 \mathrm{~mm}$ ), large edge distances and deep embedment depths (more than $635 \mathrm{~mm}$ ) subjected to shear load was investigated by Lee et al. (2011). The authors made an assessment of the methods available for predicting the capacity of anchorage systems and applied them to large anchor diameters and deep embedment depths. Based on the results, the authors concluded that current prediction methods are unsuitable for large anchors with large edge distance and deep embedment depths. The authors reported that the capacity of large anchor diameters and deep embedment depths, was overestimated by the CCD method (Lee et al., 2011).

Hawkins (1987) investigated the strength of cast-in-place headed anchors embedded in reinforced concrete and subjected to static shear loading. The author stated that the increase 
in concrete compressive strength increased the ultimate shear capacity significantly (Hawkins, 1987).

Gross et al. (2001) investigated the behaviour of single and double cast-in-place, undercut and expansion anchors under static and dynamic shear loading. The anchors were installed with limited edge distance. The authors reported that the shear capacity under dynamic loading is higher than that under static loading for the anchors they tested. They also observed that the shear capacity in cracked concrete increased under dynamic loading and decreased under static loading. The authors concluded that when anchor spacing for double anchors is equal to or more than two times the embedment depth, the shear capacity of the two-anchor group is equal to the sum of the individual anchor capacities (Gross et al., 2001).

Statistical analysis on concrete breakout capacity for anchors subjected to static and dynamic shear loading has been conducted by Muratli et al. (2001). The authors compared the results of the ACI method, $\mathrm{CCD}$ method, and a regression analysis on the variation of CCD method. The authors used Monte Carlo analysis to predict the probability of failure of steel anchor systems including cast-in-place, undercut, expansion, and sleeve anchors. An increase of $20 \%$ in anchor capacity under dynamic loading in comparison with static capacity was reported by the authors. The shear breakout capacity of cast-in-place anchors was observed to be $10 \%$ higher than that for the post-installed anchors. The authors assessed the accuracy of ACI and CCD method using probability of failure and found that steel anchorage systems designed in accordance with CCD method has lower probability 
of failure than that designed in accordance with ACI method (Muratli et al., 2001). The authors also concluded that the CCD method is more accurate and thus more suitable for predicting the shear breakout capacity than the ACI method (Muratli et al., 2001; Muratli et al., 2004).

Petersen et al. (2013) investigated the shear behaviour of cast-in-place anchors subjected to seismic loading experimentally and numerically using ABAQUS finite element software. The authors investigated the exposed anchor length for the anchors of fixed ends and for the anchors of limited end rotations of $7^{\circ}$ and $14^{\circ}$. Where the exposed length is the distance between the surface of the concrete and bottom edge of loading plate. The anchors were placed in oversized hole of $3 \mathrm{~mm}$ larger than the anchor diameter in a thick load plate and fixed plate. The thick load plate is the plate where the load is applied, while the fixed plate is the plate where the anchor is fixed. The oversized holes of the thick fixed plate and load plate allow $7^{\circ}$ and $14^{\circ}$ anchor end rotations respectively. The authors stated that the exposed length has an influence on the failure mode. Shear failure mode was observed for the specimens with exposed length of $0.2 \mathrm{~d}$, while flexural dominant deformations were observed for the exposed length of $2 \mathrm{~d}$, where $\mathrm{d}$ is the anchor diameter. Strain hardening was observed for the exposed length of $4 \mathrm{~d}$, where the stiffness was increased at larger displacement. Also, the authors reported that the limited end rotation increases the ultimate shear strength and stiffness of the specimens and that the increase in the exposed length up to $4 \mathrm{~d}$ resulted in decrease of the ultimate shear load. No further decrease in the shear load was observed for anchors with larger exposed lengths than $4 d$ (Petersen et al., 2013). 
Zhao (2014) studied the behaviour of single cast-in place anchors in tension and shear installed in plastic hinge zone of concrete column. The author proposed anchor reinforcement in seismic zone to enhance the behaviour of anchors by providing protection to the concrete around the anchors. The author observed concrete spalling and cracking in the seismic zone, however, the anchor failure was due to ductile steel failure. The author concluded that the confined concrete in the plastic hinge zones can improve the performance of the anchorage system. The author stated that the anchor reinforcement play a role in carrying the load from the anchor and protecting the core concrete around the anchor from crushing and spalling (Zhao, 2014).

Jebara et al. (2016) conducted experimental work to investigate the pryout mechanism for cast-in-place welded stud subjected to shear load. They assumed pryout mechanism for cast-in-place welded studs as a pseudo-tension breakout induced by two forces: the tensile force in the stud and compression force in the plate. The authors assumed triangular compression stress distribution along the embedment depth in front of the stud. They found that the ultimate shear load increases with the increase in the anchor diameter. The increase in the anchor diameter increases the stiffness and resistance. Furthermore, the authors found that the increase in the concrete pryout capacity is proportional to the square root of the stud diameter. The authors proposed equations to calculate the pryout capacity for welded stud embedded in normal concrete and subjected to shear load (Jebara et al., 2016). 


\subsection{Adhesive anchors}

The use of adhesive anchors has gained popularity in the past decades due to the fast curing time in comparison with grouted anchors and their associated superior cost-effectiveness (Upadhyaya \& Kumar, 2015). Furthermore, the use of adhesive anchors is very common compared to other types of post installed anchors due to the flexibility of adhesive anchors in installation (Cattaneo and Muciaccia, 2015; Cook, 1993). Experimental and numerical work has been conducted by several researchers to investigate the behaviour of adhesive anchors under different loading conditions and the results of these tests are summarized in the following sections.

\subsubsection{Adhesive anchors under tensile load}

Epackachi et al. (2015) and Eligehausen et al. (2006) investigated the pullout behaviour of single and group adhesive anchors. The authors studied the influence of anchor spacing on the failure mode of adhesive anchors. Epackachi et al. (2015) used single anchors and groups of 4, 6, and 9 anchors. Anchor diameter of $20 \mathrm{~mm}$ with embedment depth of 200 $\mathrm{mm}$ and anchor spacing of $150 \mathrm{~mm}$ and $200 \mathrm{~mm}$ were investigated. Epackachi et al. (2015) observed combined cone-bond failure and steel fracture for the single anchor and concrete cone breakout failure for the groups of 4 anchors while combined cone-splitting failure was observed for the groups of 6 and 9 anchors. In the combined cone-splitting failure, splitting cracks formed in the concrete after formation of the concrete cone breakout. The authors found that the decrease in anchor spacing decreased the tensile strength of group anchors (Epackachi et al., 2015). Similar observation was reported by Li et al. (2002) for the quadruple anchor fastening. Eligehausen et al. (2006) observed that concrete breakout cone 
is initiated at the base of the anchor and propagated along the embedment depth for the anchor spacing $s=4 d$, while concrete breakout cone is initiated near the surface of the concrete for the anchor spacing $s=8 d$, and that the failure of anchor groups is similar to that of single anchor for the large spacing distance of $s=16 d$ where combined cone bond failure is observed for the deep embedment depths. Eligehausen et al. (2006) obtained concrete cone breakout failure for the high bond strength, when the mean bond strength $(\tau)$ was equal to maximum mean bond strength $\left(\tau_{\max }\right)$, while pullout failure is obtained for the very low bond strength $\left(\tau \leq 0.3 \tau_{\max }\right)$. The authors reported that the critical spacing and critical edge distance were influenced by the bond strength and anchor diameter (Eligehausen et al., 2006).

Cook et al. (1998) and McVay et al. (1996) studied the behaviour of single adhesive anchor embedded in uncracked concrete subjected to tensile load. The authors designed models for single adhesive anchor and compared it with the worldwide database of anchor test results (Cook et al., 1998; McVay et al., 1996). Cook et al. (1998) stated that the design model based on a uniform bond stress showed good agreement with the non-linear analytical studies and the results from the worldwide database with an error of about $3 \%$ (Cook et al., 1998). McVay et al. (1996) stated that the use of the uniform bond stress model will reduce the error to less than 4\% (McVay et al., 1996).

Cook et al. (1998), McVay et al. (1996) and Cattaneo and Muciaccia (2015) observed three types of failure for the adhesive anchors: concrete cone breakout failure, steel failure and combined cone-bond failure (Cook et al., 1998; McVay et al., 1996; Cattaneo and 
Muciaccia, 2015). Concrete cone breakout failure occurred for shallow embedment depth anchors (Cook et al., 1998). Steel failure induced by yielding and fracture of the steel anchor occurred for deep embedment depths where the tensile strength of the steel anchor is less than the strength of the embedded part of the anchor in the substrate (Cook et al., 1998; Cook, 1993). Combined cone-bond failure characterized by shallow concrete cone breakout at the upper embedded part of the anchor with bond failure at the lower embedded part of the anchor. In addition, Cook et al. (1998) and McVay et al. (1996) observed bond failure when the strength of the bond surface was small, as in the case of inadequate curing or improper hole preparation. The bond failure may occur either at steel-adhesive interface or at concrete-adhesive interface or at both interfaces (Cook et al., 1998).

Li et al. (2002) studied the failure mode for the adhesive anchors subjected to tensile loading. The authors observed concrete cone breakout failure for quadruple adhesive anchors with embedment depth less than or equal to $96 \mathrm{~mm}$ and small spacing, while combined cone-bond failure was observed for the large embedment depth. Pullout failure was observed for the quadruple adhesive anchors with large spacing (Li et al., 2002).

Cattaneo and Muciaccia (2015) investigated the behaviour of adhesive anchors in normal and two high performance concretes (concrete compressive strength of $75 \mathrm{MPa}$ and 90 $\mathrm{MPa}$ ) under tensile load. The authors studied the effect of embedment depth and steel fibres on the behaviour of adhesive anchors. Anchor diameter of $12 \mathrm{~mm}$ with embedment depths of $50 \mathrm{~mm}, 75 \mathrm{~mm}$ and $110 \mathrm{~mm}$ were investigated. The authors found that the ultimate tensile load was increased by adding steel fibres to the concrete. Adding steel fibre to the 
concrete resulted in combined concrete cone-bond failure mode for the embedment depth of $50 \mathrm{~mm}$. They obtained brittle/ductile failure mode for the adhesive anchors in high performance concrete and brittle failure mode for the adhesive anchors in normal concrete. They concluded that the fibre reinforced concrete is a viable alternative when there is a need to use short anchor spacing, edge distance and thinner member (Cattaneo and Muciaccia, 2015).

Yilmaz et al. (2012) investigated the behaviour of post-installed anchors embedded in low strength concrete under tensile load. The authors compared the results of the failure loads with the ACI 318 code and reported that the ACI 318 code overestimates the anchor strength in comparison with experimental data for low strength concrete. They observed that the failure mechanism depends on the concrete strength and edge distance for the anchors having an edge distance $\geq 15$ times the anchor diameter (Yilmaz et al., 2013).

Upadhyaya and Kumar (2015) introduced analytical model to predict the pullout capacity of adhesive anchors. The authors investigated the effect of material properties and design parameters (embedment depth, adhesive thickness and the relative stiffness between the steel anchor and the adhesive) on the pullout capacity of adhesive anchors. The authors stated that, using stiff anchor, the anchor can sustain higher loads before bond failure, where the adhesive layer is subjected to lower stresses. The authors investigated embedment depths in the range from $40 \mathrm{~mm}$ to $1000 \mathrm{~mm}$ and adhesive thickness in the range from $0.25 \mathrm{~mm}$ to $1.25 \mathrm{~mm}$. The authors concluded that the adhesive anchor load capacity increased with the increase in the embedment depth until a critical embedment 
depth was reached where no further increase in the anchor capacity was observed as the failure was governed by steel fracture. Also, they concluded that increase of the thickness of the adhesive layer from $0.25 \mathrm{~mm}$ to $1.25 \mathrm{~mm}$ decreases the peak stresses in the adhesive layer. The authors compared the analytical results with finite element analyses results using ABAQUS finite element analysis (FEA) package and reported good agreement (Upadhyaya and Kumar, 2015).

Cook et al. (1993) investigated the behaviour of adhesive anchors with embedment depths of $100 \mathrm{~mm}, 150 \mathrm{~mm}$ and $200 \mathrm{~mm}$ under tensile loading. The authors used fully bonded and partially bonded adhesive anchors. A debonding agent was placed at the top $50 \mathrm{~mm}$ of the embedment depth for the partially bonded adhesive anchors. The authors found that the fully bonded anchors fail either by steel anchor failure or by concrete cone breakout with the adhesive pullout, while partially bonded anchors failed by steel anchor failure or adhesive bond failure. They obtained similar failure behaviour for the fully bonded double anchors as single anchors. They found that the strength of fully bonded anchors and partially bonded anchors is nearly equal at the same embedment depth (Cook et al., 1993).

Sato et al. (2004) and Fujikake et al. (2003) investigated the pullout behaviour of adhesive anchors subjected to loading rates ranging from $1 \times 10^{-1} \mathrm{kN} / \mathrm{s}$ to $4 \times 10^{4} \mathrm{kN} / \mathrm{s}$. The authors found that the increase in the loading rate increases the failure load for bond failure of the adhesive anchors (Sato et al., 2004; Fujikake et al., 2003). Sato et al. concluded that the embedment depth has no influence on the average dynamic bond strength for the same loading rate. The authors observed bond failure at the concrete/adhesive interface at all 
loading rates tested (Sato et al., 2004). Fujikake et al. (2003) reported that bonding agent has significant effect on the performance of adhesive anchors subjected to static loading. This effect of bonding agent is probably applicable for dynamic loading condition (Fujikake et al., 2003).

Zamora et al. (2003) investigated the behaviour of single, headed and unheaded, grouted anchors subjected to static tensile load. Results of their study show identical behaviour for the adhesive and grouted unheaded anchors. Also, identical tensile behaviour was found for the headed grouted and cast-in-place anchors. The authors concluded that bond failure at the steel-grout interface is the predominant failure for the unheaded grouted anchors. However, bond failure may also occur at the grout-concrete interface. Concrete breakout cone failure or bond failure at the grout/concrete interface was observed for the headed grouted anchors. They found that the nominal bond stress at the steel-grout and concretegrout interfaces are important to identify the interface at which the bond failure occurred. The embedment strength is thus controlled by the lowest bond strength of the two interfaces (Zamora et al., 2003).

Braimah et al. (2009) investigated the behaviour of adhesive steel anchors embedded into concrete and limestone substrates and subjected to impulsive loading. The authors found that the substrate material has an influence on the failure mode. Steel failure was observed for the concrete substrate, while steel failure and limestone failure were observed for the anchors embedded into limestone substrate at similar embedment depths. The authors investigated the effect of penetration angle on the dynamic increase factor (DIF). The 
authors found that the DIF for the substrate penetration angle of $45^{\circ}$ is higher than that of $90^{\circ}$ penetration angle. The authors recommended DIF of 1.2 for the adhesive anchor of $90^{\circ}$ penetration angle for both concrete and limestone substrates, while they recommended DIF of 2.5 and 3.2 for the adhesive anchor of $45^{\circ}$ penetration angle for the limestone and concrete substrates, respectively (Braimah et al., 2009). In another study, Braimah et al. (2014) carried out impact test on the adhesive anchors embedded into concrete masonry and clay brick substrate. The authors recommended DIF of less than one for the adhesive anchor subjected to high strain rate and embedded into clay brick, and DIF greater than one for the adhesive anchor embedded into concrete masonry (Braimah et al., 2014).

Zhao et al. (2015) investigated the pullout behaviour of anchorage body under different loading rates using practical flow code (PFC) numerical software, where the anchorage body consists of the anchor, bonding materials and the concrete matrix. Loading rates in the range between $0.5 \mathrm{~mm} / \mathrm{s}$ to $1000 \mathrm{~mm} / \mathrm{s}$ corresponding to strain rates in the range from $10^{-5} \mathrm{~s}^{-1}$ to $10 \mathrm{~s}^{-1}$ were investigated. The authors studied the interfacial shear stress distribution along the anchorage embedment depth and the failure mode of the anchors. They observed irregular distribution of the axial force and interfacial shear stress. As the loading rate increases, the axial force and interfacial shear stress increase in the upper anchorage section. No clear increase of the axial force and interfacial shear stresses in the lower anchorage section was observed. The authors proposed a linear relationship between the pullout failure load and the loading rate. The authors obtained three failure modes for the anchorage substrate matrix: matrix cracking in addition to a main crack along the bottom matrix at loading rates less than $10 \mathrm{~mm} / \mathrm{s}$, crack propagation in the upper portion 
of the matrix with the main crack along the bottom matrix for the loading rates greater than $10 \mathrm{~mm} / \mathrm{s}$, pullout failure accompanied by large fracture area at loading rates greater than $100 \mathrm{~mm} / \mathrm{s}$ where the cracks propagated in the middle and top part of the bolt resulted in large fracture area. The authors attributed this failure mode at high strain rates to the breakage of the bond between the matrix and the anchors resulting from high stress concentration along the top part of the anchorage section (Zhao et al., 2015).

Kim et al. (2013) performed experimental tests and finite element analysis on the tensile behaviour of post-installed anchors. They studied the effect of torque ratio, embedment depth and anchor diameter on the tensile strength. The authors applied torque ratios of $30 \%, 50 \%$ and $70 \%$ of the total torque to tighten the nut during anchor installation. Where, the torque ratio is the ratio between the applied torque to the total torque that can be applied on the anchor. The authors used anchor diameters of $10 \mathrm{~mm}$ and $12 \mathrm{~mm}$ with embedment depths of $50 \mathrm{~mm}$ and $100 \mathrm{~mm}$. They concluded that the tensile strength is dependent on the torque ratio, embedment depth and anchor diameter. The authors stated that, the maximum load capacity for tensile tests increase with the increase in the embedment depths. The authors stated that the finite element model using ABAQUS is suitable to predicting the failure load (Kim et al., 2013).

Mahrenholtz and Eligehausen (2015) investigated the behaviour of post-installed anchors under tensile and shear loads for safety relevant applications such as nuclear power plant. The authors observed concrete breakout failure and steel anchor failure for the tensile and shear tests, respectively for the tested post-installed anchors. They also observed that the 
ultimate tensile load decreased slightly in cracked concrete. They obtained linear relationship between the displacement and cyclic loading. They found that increasing the number of load cycles increases the displacement. At the beginning of cyclic loading, large initial displacement is observed followed by a slight increase in displacement. They related the increase in the displacement to hole clearance, therefore, they suggested that a proper filler has to be used to fill the gap (Mahrenholtz and Eligehausen, 2015).

González et al. (2018) studied the tensile behaviour of post-installed (adhesive and grouted) anchors at different construction conditions. The authors used conventional (vibrated concrete) and self-compacting concrete. The authors investigated several factors such as type of hole drilling machine, type of filling material, hole condition (moisture and cleanliness) and condition of installation. The authors found that the capacity of the anchor is influenced significantly by the installation condition and the drilling machine. Also, the authors found that the hole condition has an influence on the anchor capacity (González et al., 2018).

\subsubsection{Adhesive anchors under shear load}

Caliskan et al. (2013) performed cyclic shear test on adhesive anchors embedded in low strength concrete of 5.9 MPa and 10.9 MPa. The authors used three anchor diameters of 12 $\mathrm{mm}, 16 \mathrm{~mm}$ and $20 \mathrm{~mm}$ and three embedment depths for each anchor diameter $(d)$. Embedment depths of $10 d, 15 d$ and $20 d$ were investigated. The authors found that the shear strength decreased significantly for anchor diameter more than $16 \mathrm{~mm}$ embedded into low strength concrete. Also, the authors concluded that there is no influence on the shear 
capacity of the anchors when the embedment depth was larger than 10 times the anchor diameter (Çalışkan et al., 2013).

Shear behaviour of adhesive anchors in normal and high performance concretes was investigated by Cattaneo and Muciaccia (2015). The authors studied the effect of edge distance and the effect of using steel fibre reinforced concrete on the behaviour of adhesive anchor concrete system. The authors reported increase in failure load with increase in the edge distance of adhesive anchors subjected to shear loading. Adding steel fibres to the concrete increased the ultimate shear load. The addition of steel fibres to the concrete affect the failure mode when the edge distance increased from $40 \mathrm{~mm}$ to $55 \mathrm{~mm}$, where the failure mode changed from concrete breakout to steel failure at the edge distance of $55 \mathrm{~mm}$ (Cattaneo \& Muciaccia, 2015).

Kim et al. (2013) performed experimental tests and finite element analysis on the shear behaviour of post-installed anchors. They studied the effect of torque ratio, embedment depth and anchor diameter on the shear strength. They concluded that the shear strength is dependent on the embedment depth and anchor diameter and not on the torque ratio. The authors stated that, the maximum load capacity for shear tests increase with the increase in the embedment depth (Kim et al., 2013).

Lou and Perciballi (2008) performed three point bending test on beam with bolts at the end supports. Static loads were applied on bolts with and without preloading using LS-DYNA and NEi Nastran software. The authors used preloaded bolts to ensure that the anchor will 
not fail and prevent connection separation. The authors stated that the bolts can be under tensile, shear or combined tensile and shear loads in service. The shear load on the bolts generated from joint slip or friction. Failure was analyzed using the two software programs. They observed less deformation in the beam when using preloaded anchors subjected to static loads. Higher failure loads were obtained for the preloaded bolts compared to that without preload. Quite similar results were obtained from the two software programs (Lou and Perciballi, 2008).

Epackachi et al. (2015) investigated the shear behaviour of single and group of adhesive anchors. Embedment depth of $200 \mathrm{~mm}$, anchor spacing of $150 \mathrm{~mm}$ and $200 \mathrm{~mm}$ were investigated. The authors stated that the shear strength of the adhesive anchors is not affected by the anchor spacing. The results show that, the shear strength of group of anchors can be determined by multiplying the shear strength of one anchor by the number of anchors for the spacing ranging from $150 \mathrm{~mm}$ to $200 \mathrm{~mm}$. Steel failure and concrete bearing failure were observed for the tested adhesive anchors (Epackachi et al., 2015).

\subsection{Undercut anchors}

\subsubsection{Undercut anchors under tensile load}

Primavera et al. (1997) investigated the tensile behaviour of undercut anchors in high strength concrete. Anchor embedment depth of $203 \mathrm{~mm}$ was investigated. The authors obtained shallow concrete cone breakout for the tested anchor. The authors found that the increase in the concrete compressive strength increased the pullout capacity. The authors compared the pullout capacity with the ACI and CCD methods and reported that the CCD 
method underpredicted while the ACI method overpredicted the pullout capacity of the undercut anchor (Primavera et al. 1997).

Ashour and Alqedra (2005) investigated the pullout capacity of post installed (expansion and undercut) anchors using neural network modelling under static tensile load. The authors compared the neural network results with previous experimental results and they found it to be in good agreement. The authors found that the pullout capacity for the postinstalled anchors is influenced by the anchor embedment depth (Ashour \& Alqedra, 2005).

Marcon et al. (2018) investigated the influence of the aggregate on the tensile capacity of the undercut anchors subjected to static load. The authors used three mixes of the concrete depending on the type, size and mechanical properties of the aggregate. Undercut anchors embedded in concrete at age of 28 days and 70 days were investigated. The authors stated that the size of coarse aggregate has an effect on the concrete properties and tensile capacity of the undercut anchors. However, the influence of the aggregate is within the range of the predictive equations results. Also, the authors concluded that the tensile capacity of the anchors embedded in concrete at age of 70 days is higher than that for the anchors embedded in concrete at age of 28 days (Marcon et al., 2018).

Rodriguez et al. (1997) investigated the behaviour of the post-installed (expansion and undercut) anchors in concrete subjected to static and dynamic tensile load. The authors calculated the normalized tensile capacity of the undercut and grouted anchors considering the failure mode is concrete cone breakout. They concluded that the normalized tensile 
capacity under dynamic load increased $30 \%$ compared to the normalized capacity under static load (Rodriguez et al., 1997).

\subsubsection{Undercut anchors under shear load}

Mahrenholtz and Eligehausen (2013) investigated the qualifications of the undercut anchors in nuclear power plants subjected to seismic dynamic tensile and shear loads. Two conditions, service condition and extreme cracked condition, were investigated. The authors found that the seismic dynamic load increased the cumulative displacement of the anchor causing steel failure (Mahrenholtz \& Eligehausen, 2013). In another research, Mahrenholtz and Eligehausen (2015) applied cyclic tension and shear loads on the undercut anchors used for nuclear power plants and reported that crack widths affected the strength of the anchors when subjected to tensile loading and no significant influence on the anchor strength under shear loading. The authors observed concrete failure mode under cyclic tensile loading and steel fracture failure mode under cyclic shear loading (Mahrenholtz \& Eligehausen, 2015).

Eligehausen et al. (1998) investigated the behaviour of post-installed anchors in cracked and uncracked concrete. The post installed anchors investigated were torque controlled expansion, displacement-controlled expansion (drop-in), undercut and adhesive anchors. The authors found that the capacity of the anchors decreased in the cracked concrete. The reduction in the capacity for the displacement-controlled expansion (drop-in) anchors is higher than the undercut anchors and torque controlled expansion anchors. Undercut anchors, adhesive anchors and properly designed torque controlled expansion anchors are 
suitable to install in cracked concrete where the tensile load can be transferred to the concrete (Eligehausen et al., 1998).

\subsection{Strain rate effect on material properties}

\subsubsection{Effect of strain rate on concrete}

The level of the strain rate applied on concrete structures has an effect on its capacity. Static loading is obtained at a strain rate ranging from $10^{-6} \mathrm{~s}^{-1}$ to $10^{-5} \mathrm{~s}^{-1}$. Low dynamic loading and earthquake results in strain rates ranging from $10^{-4} \mathrm{~s}^{-1}$ to $10^{-1} \mathrm{~s}^{-1}$. Impact loading results in strain rate in the range between $10^{0} \mathrm{~s}^{-1}$ to $10 \mathrm{~s}^{-1}$, while very high strain rate ranging from $10^{2} \mathrm{~s}^{-1}$ to $10^{3} \mathrm{~s}^{-1}$ can be achieved with blast loading (Bischoff and Perry, 1991).

Different testing machines can be used to apply various loading conditions on concrete structures at different levels of strain rates. Hydrulic testing machine can be used to apply static load at strain rate of $10^{-5} \mathrm{~s}^{-1}$ to $10^{-1} \mathrm{~s}^{-1}$. Charpy impact testing mahine can be used to apply dynamic load at strain rate up to $10^{0} \mathrm{~s}^{-1}$, while strain rate up to $10^{1} \mathrm{~s}^{-1}$ can be achieved by using drop weight impact. Higher strain rates up to $10^{2} \mathrm{~s}^{-1}$ can be reached by using Split Hopkinson Pressure Bar. Strain rates equal or higher than $10^{3} \mathrm{~s}^{-1}$ can be obtained using explosive charges (Bischoff and Perry, 1991; Hentz et al., 2004).

Effect of strain rate on the strength of concrete has been investigated by several researchers (Malvar and Crawford, 1998; Malvar and Ross, 1998; Bischoff and Perry, 1991; Georgin and Reynouard, 2003; Hentz et al., 2004; Ross et al., 1995; Fu et al., 1991; Shkolnik, 2008; Min et al., 2014; Park et al., 2001; Cadoni et al. 2001; Tedesco et al. 1994; Ross et al. 1996; 
Rossi et al. 1994). The authors found that the increase in the strain rate increases the tensile and compressive strength of concrete.

Ross et al. (1989) investigated the behaviour of concrete and mortar subjected to quasistatic and dynamic loads at strain rate in the range between $10 \mathrm{~s}^{-1}$ to $10^{2} \mathrm{~s}^{-1}$. The authors observed similar failure for the quasi static and dynamic tensile test. They concluded that both tensile and compressive strengths increase at high strain rate. However, the increase in the tensile strength at high strain rate is larger than that for the compressive strength. They found that the tensile strength of the mortar at high strain rate of $10^{2} \mathrm{~s}^{-1}$ is equal to three times that obtained from quasi-static load while it is equal to six times that obtained from quasi-static load when using concrete substrate (Ross et al. 1989).

Ross et al. (1995), Georgin and Reynouard (2003) and Min et al. (2014) conducted numerical analysis on the effect of strain rate on the strength of the concrete. The authors observed that the increase in the strain rate increases the concrete compressive strength. Georgin and Reynouard (2003) attributed the increase in the compression strength of concrete at high strain rates to the inertial force, the inertial confinement, structural effect and rate effect (Georgin and Reynouard, 2003). Ross et al. (1995) found that the increase in the strain rate from $10^{-7} \mathrm{~s}^{-1}$ to $300 \mathrm{~s}^{-1}$ increases the tensile and compressive strength of the concrete. However, there is a critical value of strain rate of $5 \mathrm{~s}^{-1}$ for the tensile strength and $60 \mathrm{~s}^{-1}$ for the compressive strength at which the percentage increase in the tensile strength of the concrete becomes higher than the percentage increase in the compressive strength. When the strain rate exceed the critical value, the strength of the concrete is 
significantly increased. Ross et al. (1995) also investigated the effect of strain rate on wet, partially wet and dry concrete. The authors found that the wet and partially wet concretes are more senstive to high strain rate than the dry concrete (Ross et al., 1995). Similar observation was obtained by Ross et al. (1996), Reinhardt et al. (1990) and Rossi et al. (1994). The increase in the sensisitvity in wet concerete is due to the existance of water in the wet concrete (Ross et al., 1996; Reinhardt et al., 1990; Rossi et al., 1994).

Min et al. (2014), Malvar and Crawford (1998), Hentz et al. (2004) and Shkolnik (2008) found that the strain rate sensitivity on the tensile strength of the concrete is higher than that on the compressive strength. A relationship between DIF and strain rate was introduced by Min et al. (Min et al., 2014). Malvar and Crawford (1998) stated that the DIF is very important in the design of structures subjected to high strain rates and can be expressed as a bilinear function of the strain rate. The authors obtained DIF greater than 2 and 6 for the concrete subjected to compression and tension loads, respectively (Malvar and Crawford, 1998). Moreover, Rossi et al. (1994) found that the DIF for wet concrete is higher than that obtained for the dry concrete (Rossi et al., 1994).

Hentz et al. (2004) and Shkolnik (2008) investigated the effect of strain rate on the modulus of elasticity of concrete under tensile and compressive loading. The authors found that the modulus of elasticity increased with the increase in the strain rate (Hentz et al., 2004; Shkolnik, 2008). Similar observation was obtained by Rossi et al. (1994) for the effect of strain rate on the modulus of elasticity of the wet concrete under tensile loading (Rossi et al., 1994). Shkolnik (2008) obtained linear relationship between the stress and the strain of 
concrete at high strain rate of $150 \mathrm{~s}^{-1}$ (Shkolnik, 2008). Hentz et al. (2004) observed that the strain rate has less effect on the Poison's ratio, energy absorption and strain at ultimate strength of the concrete compared to the tensile and compressive strengths (Hentz et al., 2004).

Park et al. (2001) conducted finite element analysis on the behaviour of concrete and mortar under high strain rate of $10^{4} \mathrm{~s}^{-1}$. The authors observed inelastic deformation in the mortar with less inelastic strains in the aggregates under impact loading. They stated that the increase in the aggregate volume fraction to $42 \%$ increases the strength of the concrete by $30 \%$ and increases the energy absorption 15\% (Park et al., 2001). The finite element modelling can predict the material response and provide an assessment to the strain rate effect (Ross et al., 1995; Georgin and Reynouard, 2003).

Tedesco et al. (1994) performed finite element analysis on the concrete subjected to compression load. The authors stated that the type of failure depends on the strain rate. At strain rate of $17 \mathrm{~s}^{-1}$ no cracking was observed. At strain rate of $25 \mathrm{~s}^{-1}$ cracking initiated which led to compression failure of about $35 \%$ of the concrete specimen. Strain rate of 200 $\mathrm{s}^{-1}$ resulted in about $85 \%$ concrete compression failure (Tedesco et al., 1994).

Bischoff and Perry (1991) investigated effect of concrete quality, water cement ratio, aggregate type, aging and curing time on concrete compressive strength at low and high strain rates. The authors observed that the increase in compressive strength of lower compressive strength concrete is greater than that for higher compressive strength concrete 
at high strain rate. The effect of the strain rate on the compressive strength is influenced by type of aggregate; stiffer aggregate is less sensitive to strain rate. Aggregates of good bond and smaller maximum size enhanced the concrete compressive strength at high strain rate. Also, the authors found that the compressive strength of concrete is affected by the method of curing and moisture content. Cured wet specimen exhibits higher compressive strength than the wet specimen. The increase in the age of the concrete shows less sensitivity to the increase in the strain rate (Bischoff and Perry, 1991).

Fu et al. (1991) investigated the effect of loading rate on reinforced concrete. They observed 30\% increase in the strength of the reinforced concrete at high loading rate; the yield strength of the steel is also increased. The failure mode is affected by the loading rate. At high loading rate the tensile and bond strength of concrete increase resulted in few cracks and sharp strain gradient. The increase in the bond strength at high loading rate decreased the ductility at failure (Fu et al., 1991).

Cadoni et al. (2001) investigated effect of strain rate on the tensile behaviour of concrete. They applied quasi static tensile loading at strain rate of $10^{-6} \mathrm{~s}^{-1}$ and impact loads at strain rates of $10^{0} \mathrm{~s}^{-1}$ and $10^{1} \mathrm{~s}^{-1}$ on the concrete at different humidity levels. The increase in the relative humidity of the concrete increases the tensile strength for the impact loading. The tensile strength decreases slightly for the saturated concrete subjected to quasi static load (Cadoni et al., 2001). 
Kulkarni and Shah (1998) investigated the effect of high loading rate on concrete beams. They applied static and high loading rates (piston velocity) of $0.00071 \mathrm{~cm} / \mathrm{s}$ and $38 \mathrm{~cm} / \mathrm{s}$ respectively. They found that the increase in the strain rate increases the ultimate load and the energy absorption capacity and that the failure mode is influenced by the strain rate. Shear failure mode was obtained for the concrete beam at the static test while flexural failure mode was obtained when similar beams were tested at high strain rate. The difference in the failure mode was attributed to the inertia forces. Flexural failure mode is attributed to yielding of reinforcement, beam rotation, opening up of cracks and reducing compression zone lead to concrete crushing in compression. The post peak behaviour of the load-deflection curve shows a gradual decrease in the load with increase in the deflection. Shear failure mode is attributed to the shear cracks. The post peak behaviour of the load-deflection curve shows a sharp decrease in the load (Kulkarni and Shah, 1998).

\subsubsection{Effect of strain rate on steel}

The strain rate has an effect on the mechanical properties of the steel material. The yield and tensile strength of the steel increases with the increase in the strain rate (Fu et al., 1991; Yu et al., 2009; Hopperstad et al., 2003), while the Young's modulus remain constant (Fu et al., 1991). This is due to the increase in the deformations and dislocations in the steel structure at high strain rate ( Lee et al., 2007). The deformation of the steel material at low strain rate or quasi-static loading is nearly homogeneous and controlled by slip and twin plastic deformation mechanisms. However, the deformation of the steel material at high strain rate is more complicated where the strains extremely concentrated along narrow area called adiabatic shear band. The cracks are initiated and propagated from these shear bands 
leading to fracture. At high strain rate the formation of the shear band in steel is affected by several factors such as chemical composition of the steel components, strain rate and heat treatment (Odeshi et al., 2005).

Lee et al. (2007) investigated the fracture response of stainless steel under high strain rate loading. They found that strain rate has an influence on the flow stress-strain behaviour and the microstructure of the specimen. The increase in the strain rate increased the level of deformation of the grain. The authors stated that the adiabatic shear band created plastic instability that leads to fracture (Lee et al., 2007).

Xiong et al. (2009) conducted tensile test of twin induced plasticity (TWIP) steel at strain rate ranging from $10^{-5} \mathrm{~s}^{-1}$ to $10^{3} \mathrm{~s}^{-1}$. The authors investigated two types of TWIP steel. Each steel type has different chemical composition according to the manganese (Mn) percent in the composition; steel $1^{\#}(14.3 \% \mathrm{Mn})$ and steel $3^{\#}(25.41 \% \mathrm{Mn})$. The authors stated that the tensile properties of steel exhibit strain rate sensitivity. Similar observation was obtained by Xu et al. (2013). In addition, Xiong et al. (2009) found that the yield strength and ultimate tensile strength of the steel $3^{\#}$ increased with the increase in the strain rate. The increase in ultimate tensile strength of steel $1^{\#}$ was higher than that reported for steel $3^{\#}$ (Xiong et al., 2009). On the other hand, Xu et al. (2013) observed no change in the yield strength at strain rate in the range from $10^{-3} \mathrm{~s}^{-1}$ to $10^{-2} \mathrm{~s}^{-1}$ while a significant increase in the yield strength was observed at high strain rate of $400 \mathrm{~s}^{-1}$ (Xu et al., 2013). 
Børvik et al. (2003) conducted non-linear finite element analysis using LS-DYNA on smooth and notched axisymmetric specimens of steel to investigate the tensile behaviour under quasi static and high strain rate loading. They concluded that the finite element models have the capability to represent the quasi static and dynamic behaviour of the material and give accurate results (Børvik et al., 2003).

Lee and Lam (1996) investigated the deformation behaviour of AISI 4340 alloy steel at low and high strain rates using Hopkinson pressure bar. They concluded that AISI 4340 alloy steel is sensitive to strain rate. They proposed an equation to predict the relation between the work hardening and strain rate sensitivity (Lee and Lam, 1996).

$\mathrm{Yu}$ et al. (2009) studied the effect of strain rate in the range from $10^{-4} \mathrm{~s}^{-1}$ to $10^{3} \mathrm{~s}^{-1}$ on dual phase steel (DP600 steel). The DP600 steel has tensile strength higher than $600 \mathrm{MPa}$ and composed of ferrite and martensite. They found that the mechanical behaviour of the steel is influenced by the strain rate. The authors proposed a new constitutive model to predict the mechanical behaviour of the steel (Yu et al., 2009).

Das et al. (2017) investigated the deformation of micro-mechanisms in dual phase steel at strain rates ranging from $10^{-3} \mathrm{~s}^{-1}$ to $800 \mathrm{~s}^{-1}$. The authors used two types of dual phase steel (DP600 and DP800) with different martensite percent. The authors found that the strain rate affects the steel deformation through formation of dislocation cells. The authors stated that the size and the extent of these cells depend on the strain rate and the fraction of 
martensite. The size of the dislocation cells become finer as the strain rate increase (Das et al., 2017).

Lu et al. (2013) investigated the effect of strain rate on the fracture surface and tensile behaviour of AISI304 stainless steel in the range from $10^{-4} \mathrm{~s}^{-1}$ to $10^{-1} \mathrm{~s}^{-1}$. The authors concluded that the ultimate tensile strength is affected significantly by the strain rate. The increase in the strain rate increased the tensile strength and flow stress. Also, the authors stated that the strain rate has an influence on the fracture surface. Microvoid coalescence is observed at low strain rate, while equiaxed dimple (having equal dimensions in all directions) with narrow diameter is observed at high strain rates (Lu et al., 2013).

\subsection{Design methods for anchorage system}

There are two common design methods proposed in building codes to predict the tensile and shear capacities of anchorage to concrete system. These methods are: American Concrete Institution (ACI) method (ACI Committee 349, 1990; ACI Committee 318, 2005; ACI Committee 318, 2011), and Concrete Capacity Design (CCD) method (Fuchs et al., 1995).

\subsubsection{American Concrete Institute (ACI)}

Several versions of ACI code include provision to predict the tensile and shear capacities of anchors. ACI method 349-85 was developed in 1975 (Fuchs et al., 1995) and proposed for the design of nuclear power structures or nuclear safety related concrete structures

(Fuchs et al., 1995; Subramanian, 2000). The method assumes that anchor failure is by 
steel fracture to provide a ductile failure mode and to improve the design reliability. Brittle failure is avoided by providing deep embedment $\left(h_{e f}\right)$ to prevent concrete failure. The concrete capacity is determined by assuming cone failure where the failure surface forms a $45^{\circ}$-cone. The failure load depends on the concrete tensile capacity and increases as a function of a square of $h_{e f}$ (Subramanian, 2000; Rao and Arora, 2013). The proposed equations to predict the tensile and shear capacity of anchorage system based on the ACI method are included in appendices A and B.

\subsubsection{Concrete Capacity Design (CCD)}

Concrete Capacity Design (CCD) method, which is equivalent to Kappa method in the Eurocode, was proposed in 1995 based on extensive experimental work conducted by Eligehausen (Fuchs et al, 1995). The CCD method depends on fracture mechanics (size effect) theory in predicting the failure load based on $35^{\circ}$ failure angle (or concrete cone breakout). The reliance of the CCD method on the fracture mechanics theory is attributed to existence of the high tensile stresses around the anchor head (Cement Association of Canada 2010).

The failure load according to the CCD method increases as a function of $h_{e f}^{1.5}$ (Yoon et al., 2001; Gesoglu et al., 2005; Cement Association of Canada, 2010). Furthermore, the CCD method can be used for cracked and uncracked concrete and can predict the concrete breakout capacity for different anchor types and design cases (Cement Association of Canada, 2010). The proposed equations to predict the tensile and shear capacity of anchorage system based on the CCD method are included in appendices A and B. 
Fuchs (2001) carried out extensive comparison between the ACI and CCD methods to predict the failure load of steel anchorage systems. The author investigated the ultimate tensile capacity and shear capacity of steel anchorage systems. Fuchs confirmed that the CCD method is a more accurate method for predicting failure loads of the anchorage system (Fuchs, 2001).

\subsection{Anchorage failure modes}

\subsubsection{Failure modes of cast-in-place anchors under tensile load}

When a steel anchor is subjected to tensile loading, five types of failure mechanisms are commonly observed: anchor bolt failure, concrete cone breakout failure, side face blowout failure, concrete splitting, and anchor pullout (Cement Association of Canada, 2010). Anchor bolt failure occurs when the strength of the anchor is less than the applied tensile stress. Concrete cone breakout failure occurs for shallow embedment depths where the applied tensile stress is greater than the strength of the concrete. Side face blowout failure occurs when there is insufficient edge distance. Concrete splitting occurs when the concrete tensile capacity is less than the bolt capacity with insufficient concrete member depth. Anchor pullout failure occurs when the friction between the anchor and the concrete is less

than the applied tensile load for the anchors without head (Fuchs et al., 1995; Cement Association of Canada, 2010). Figure 2-5 shows the failure modes for the cast-in-place anchors under tensile loading. 


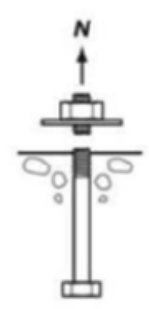

(a)

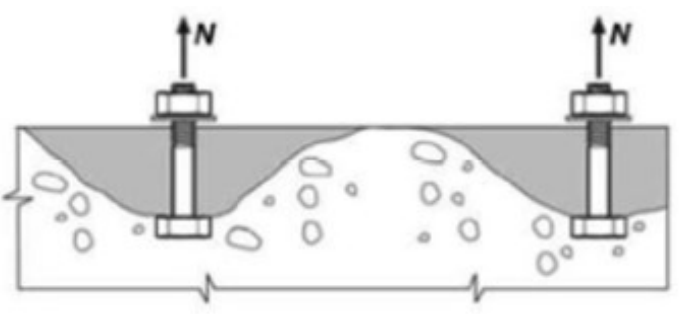

(b)

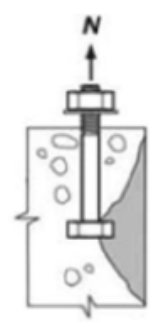

(c)

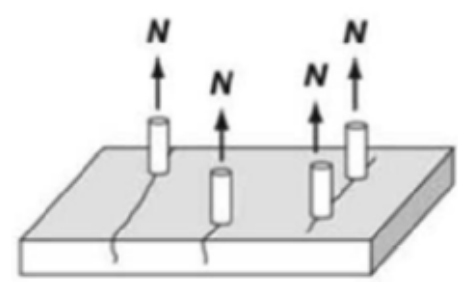

(d)

Figure 2-5: Failure modes under tensile loading: (a) steel anchor failure, (b) concrete cone breakout, (c) side face blowout, (d) concrete splitting (Cement Association of Canada 2010; ACI Committee 318, 2011)

\subsubsection{Failure modes of cast-in-place anchors under shear load}

When a steel anchorage system is subjected to shear loading, three types of failure mechanisms are commonly observed: steel anchor failure, concrete pryout, and concrete breakout (Fuchs et al., 1995; Cement Association of Canada, 2010). Steel anchor failure occurs when adequate embedment is provided without concrete edge effects, while concrete pryout is common in steel anchors without adequate embedment depth and when anchors are placed far away from the concrete free edge (Mahrenholtz \& Eligehausen, 2015). Concrete breakout failure (or side blowout) can occur for anchors close to a concrete edge or when insufficient embedment is provided. Figure 2-6 shows the failure modes for cast-in-place anchors under shear load.

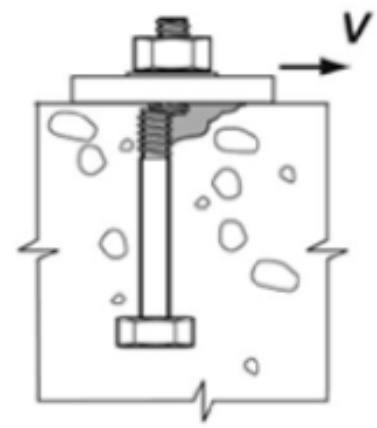

(a)

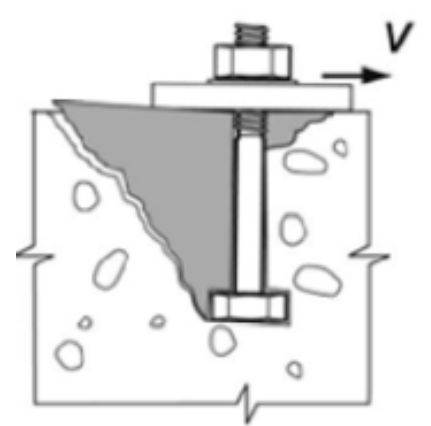

(b)

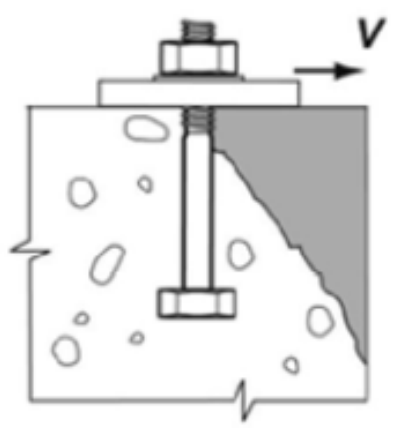

(c) 


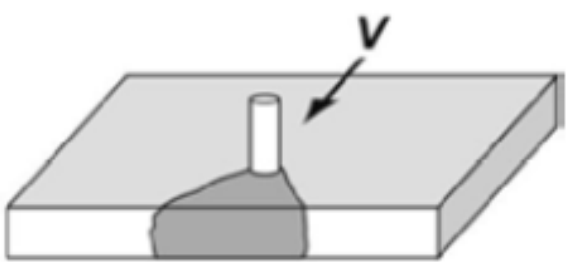

(d)

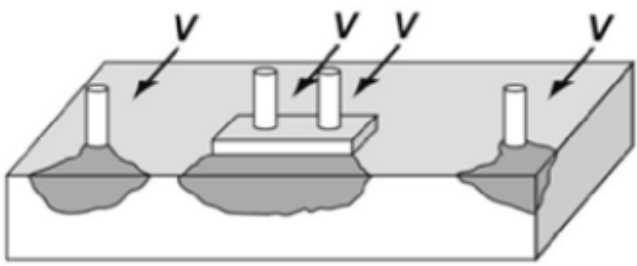

(e)

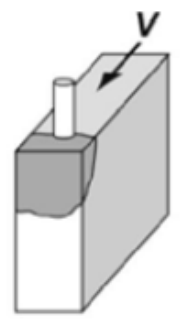

(f)

Figure 2-6: Failure modes for anchors under shear load (a) steel anchor failure preceded

by concrete spall; (b) concrete pryout failure; (c) concrete breakout failure; (d) thin concrete breakout; (e) edge breakout, corner breakout (f) narrow concrete edge breakout (Cement Association of Canada 2010; ACI Committee 318, 2011)

\subsubsection{Failure modes of adhesive anchors under tensile load}

There are four types of failure modes that can be seen in adhesive anchorage systems: concrete cone breakout failure, bond failure (including: adhesive-concrete interface, steeladhesive interface, combined adhesive-concrete and steel-adhesive interface), combined cone-bond failure, and steel anchor failure (Cook, 1993; McVay et al., 1996; Cook et al., 1998). Figure 2-7 shows the failure modes for the adhesive anchors under tensile loading (Cook et al., 1998). Each type of the failure modes is introduced in the following sections.

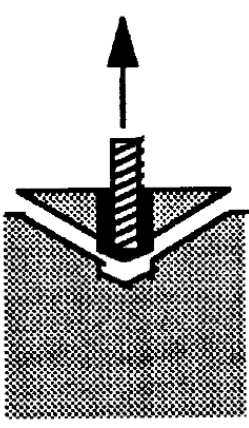

(a)

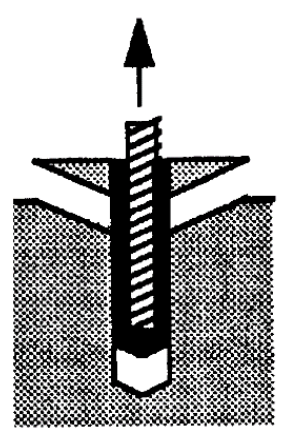

(b)

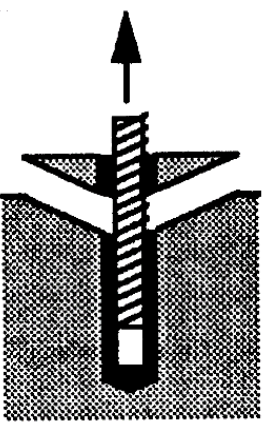

(c)

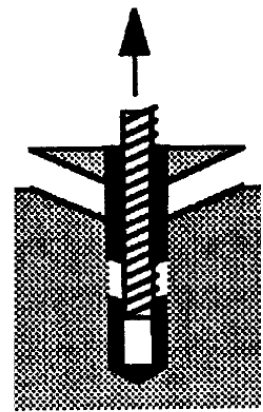

(d)

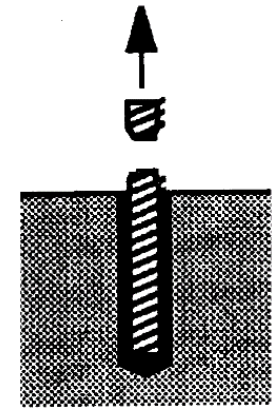

(e)

Figure 2-7: Failure modes for adhesive anchors, (a) concrete cone breakout failure; (b) combined cone-bond failure (bond failure at adhesive/concrete interface; (c) combined cone-bond failure (bond failure at steel/adhesive interface); (d) combined cone-bond failure (bond failure at combination of adhesive/concrete and steel/adhesive interface);

(e) steel anchor failure (Cook et al., 1998) 


\subsubsection{Concrete cone breakout failure}

This type of failure occurs for shallow embedment depths (Cook et al., 1993; Cook, 1993; Gesoglu et al., 2005) (Figure 2-7a). The strength of the concrete and the embedment depth of the steel anchor are the main factors that affect the ultimate failure load (Cook et al., 1998).

\subsubsection{Bond failure}

Bond failure occurs at the interface between the adhesive and the concrete, or at the interface between the steel anchor and the adhesive, or at both of the adhesive-concrete interface and steel-adhesive interface (Figure 2-7). The factors that could cause bond failure are: low bond strength of the adhesive, insufficient curing time, improper hole preparation (Cook et al., 1993).

The ultimate pullout load for bond failure mode at the steel-adhesive interface depends on the bond stress of the adhesive, anchor diameter and embedment depth. There are two models of the bond stresses: uniform bond stress model and elastic bond stress model. The equations to calculate the bond strength according to these two models are presented in Appendix B.

\subsubsection{Combined cone-bond failure}

The combined cone-bond failure is most likely to occur when the embedment depth to the anchor diameter ratio is more than 5 (McVay et al., 1996). The combined cone bond failure is characterized by a shallow cone at the top surface of the concrete accompanied by 
adhesive bond failure at the embedded part below the shallow concrete cone breakout (Figure 2-7(b),(c),(d)).

\subsubsection{Steel anchor failure}

This type of the failure is likely to occur for long embedment depth anchors, where the strength of the embedded part of the anchor into concrete is higher than the strength of the steel anchor (McVay et al., 1996; Cook, 1993). The failure occurs by steel yielding and fracture of the steel anchor (Figure 2-7e).

\subsubsection{Failure modes of adhesive anchors under shear load}

For adhesive anchors subjected to shear loading, three types of failure mechanisms similar to that obtained for the cast-in-place anchors can be observed: steel anchor failure, concrete pryout and concrete breakout (Epackachi et al., 2015). In addition, adhesive bond failure may occur in some areas along the anchor embedment depth associated with the aforementioned failure modes.

\subsubsection{Adhesive bond failure}

The exposure of adhesive material to temperature, solvents, physical stresses and environment may weaken the adhesive material and cause adhesive failure (da Silva et al., 2011). When cracks occur in the adhesive layer and lead to failure, the failure mode is called cohesive failure. When the cracks appear at the interface between the adhesive material and the adherend, the failure is called adhesive failure (Keller and Vallée, 2005). Mixed mode failure occurs when the cracks are initiated at both of the adhesive layer and the interface. 


\subsubsection{Failure modes of undercut anchors under tensile load}

Three types of failure modes are often observed for the undercut anchor-concrete systems under tensile load similar to that observed for the cast-in-place anchors: concrete cone breakout failure, anchor pullout and steel fracture failure (Cement Association of Canada, 2010; ACI Committee 355, 2000; Eligehausen et al., 1998).

\subsubsection{Failure modes of undercut anchors under shear load}

Three types of failure modes can be observed for the undercut anchors subjected to shear loads similar to that observed for the cast-in-place and adhesive anchors; pryout failure, concrete breakout and steel anchor failure (Mahrenholtz \& Eligehausen, 2015; Epackachi et al., 2015).

\subsection{Summary}

The literature review presented in this chapter has shown that the behaviour of steel anchorage systems, whether cast-in-place or post-installed, subjected to static and dynamic loads was investigated by several researchers. However, very limited attention has been allocated to these anchors at high strain rates. Hence, for the accurate and economical design of anchorages under impact and blast loading, further investigation of the effects of high strain-rates on anchorage behaviour is required. Based on the literature review presented in this chapter the main gaps in the literature can be summarized as follows:

- Several researchers investigated the tensile behaviour of cast-in-place anchors at different loading rates. Ozbolt et al. (2006) studied the size effect at different loading rate. Fujikake et al. (2003), Sato et al. (2004) and Solomos and Berra (2006) 
studied the effect of loading rate for shallow embedment depth anchors. However, effect of strain rate on the tensile behaviour of cast-in-place anchors with different design parameters has not been studied adequately.

- Most of the available studies on the effect of concrete compressive strength on the behaviour of anchorage system are focused on the anchorage behaviour under static loading. Effect of concrete compressive strength on the shear behaviour of cast-inplace anchors at low and high strain rates can help in investigating how the shear capacity and failure mode is influenced by the concrete compressive strength.

- The tensile behaviour of adhesive anchors under static load was investigated by several researches. Although loading rate effect on the tensile behaviour of adhesive anchors has been studied, effect of strain rate on the tensile behaviour of adhesive anchor has not been investigated thoroughly.

- Limited research was found on the shear behaviour of adhesive anchors under static load (Cattaneo and Muciaccia (2015), Epackachi et al. (2015)). Moreover, the shear behaviour of adhesive anchors at different strain rates has not been investigated.

- Tensile and shear behaviour of undercut anchors under static and seismic dynamic load have been studied. However, based on the literature review presented in this chapter, no studies have been found on the strain rate effect on the tensile and shear behaviour of the undercut anchors.

- Failure mode, dynamic increase factor and concrete breakout angle of the anchorage systems subjected to different strain rates have not been investigated thoroughly. 
This project aims to investigate the tensile and shear behaviour of cast-in-place, adhesive and undercut anchorage systems subjected to static and dynamic loading using LS-DYNA software. Effect of strain rate on the tensile and shear behaviour of the cast-in-place, adhesive and undercut anchors is investigated. Also, the project aims to investigate the failure mechanism and crack propagation angle of the anchorage systems at different strain rates. Additionally, this research aims to propose an equation to relate the dynamic increase factor with the strain rate for the anchorage systems. 


\section{Chapter 3 : Finite element modelling of anchorage systems}

\subsection{Introduction}

The demand to design structures to resist high dynamic loading has been increasing in recent years. When a structure is subjected to high dynamic loading, a large amount of energy is released in a very short time leading to generation of high pressure and high strain

rates in the structure. Designing such structures is a challenging task to designers due to the limited information on the response of materials to high dynamic loads (Gebbeken et al., 2001). Experimental tests are costly and require a long time to complete compared to numerical analysis which can be used to predict the structural response under different loading conditions for a wide range of structures (Davidson et al., 2005; Wu et al., 2012). In addition, numerical analysis has flexibility to change geometry, design parameters, material properties and loading condition of the structural component. Furthermore, the numerical analysis has the ability to investigate the internal damage to the structure that is difficult to be investigated by the experimental tests (Qian and Li, 2011).

LS-DYNA software was selected for the analysis in this research. LS-DYNA is a nonlinear explicit finite element code developed by Livermore Software Technology Corporation (LSTC, 2014). The original version of LS-DYNA, DYNA3D, was designed for stress analysis of structures under impact loading, and then later developed to be used for different applications (Hallquist, 2006). In early 1989 a commercial version of DYNA3D was introduced as LS-DYNA. LS-DYNA is a high fidelity software that can solve nonlinear dynamic problems and perform the analysis efficiently with less computation cost 
(Liu, 2008). It can analyze the behaviour of structures under static and dynamic loads for a wide range of variables (Hallquist, 2006). The failure mechanism can be illustrated using LS-DYNA software through its capability to calculate the strain distribution in the elements at each time step (Davidson et al., 2005). Finite element analysis using LS-DYNA software can analyze problems that involves high deformation, strain rate, high loading conditions such as impact and blast (Hallquist, 2006; Chen and Hao, 2012). Several researchers have predicted the structural response under high strain rates using LS-DYNA software (Zhao et al., 2012; Bermejo et al., 2011; Kyei and Braimah, 2013; Wu et al. 2012; Chen and Hao, 2012).

In this chapter, finite element modelling for the tensile and shear behaviour of cast-inplace, adhesive and undercut anchors subjected to static and dynamic loading using LSDYNA software is introduced. Implicit and explicit analyses for structures are presented. Material constitutive models for concrete, steel anchor and adhesive are described. Considerations to select suitable material constitutive models, boundary conditions are also presented. Finite element type, meshing and bonding techniques to model the steel anchor and concrete substrate systems are addressed.

\subsection{Implicit and explicit analysis}

Material response to applied loading can be linear or non-linear. The linear behaviour is observed in the elastic range, while the nonlinear behaviour can result from geometric effect, inelastic behaviour of materials, and large deformations. The general equations of 
motion for linear damped single-degree-of-freedom (SDOF) system can be represented by Equation (3.1) (Hallquist, 2006):

$m a+c v+k d=\mathrm{F}(t)$

and for the nonlinear damped SDOF systems by Equation (3.2):

$m a+c v+f_{\text {in }}(d)=\mathrm{F}(t)$

Where $m=$ mass, $c=$ damping coefficient, $k=$ stiffness, $a=$ acceleration, $v=$ velocity, $d$ $=$ displacement, $f_{\text {in }}=$ internal force, $\mathrm{F}(\mathrm{t})=$ external force.

Linear behaviour problems can be solved by using analytical equations, while nonlinear behaviour problems can be solved by numerical methods such as Newton-Raphson method (with using direct or iterative solvers) (Rust \& Schweizerhof, 2003) and finite difference method (Remani, 2013).

Explicit central difference method is used in LS-DYNA to solve the equations of motion and to find the parameters of acceleration, velocity and displacement (Hallquist, 2006).

For time step $t^{n+1}$ the central difference method can be represented by the following equations:

$V^{n+1 / 2}=V^{n-1 / 2}+A^{n} \Delta t^{n}$ 
$D^{n+1}=D^{n}+V^{n+1 / 2} \Delta t^{n+1 / 2}$

$\Delta t^{n+1 / 2}=\frac{\Delta t^{n}+\Delta t^{n+1}}{2}$

Where $D$ is the global nodal displacement vector, $V$ is the global nodal velocity vector.

LS-DYNA consists of implicit solver with limited capabilities and an explicit solver. For the implicit analysis, the solution is performed by integrating the nodal displacement with large time step size, the nodal displacement is determined by multiplying nodal force by the inverse of the stiffness matrix (k). The user can specify the time step size for the implicit analysis. However, the implicit analysis depends on the iterative method to get the solution and uses a small number of time steps. This requires enormous computational effort and large storage capacity (Jensen et al., 2007). The explicit solver can be used for both quasistatic and dynamic analysis (Hallquist, 2006). The solution for the explicit analysis is performed by integrating the nodal acceleration with a small time step size. Where the nodal acceleration is calculated by dividing the total of internal and external forces to the nodal mass (Jensen et al., 2007). In the explicit analysis, nodal displacement, velocity, and acceleration at time step (n) are known hence direct solution for the nodal displacement at the next time step can be obtained. Equation (3.6) expresses the explicit analysis.

$d^{n+1}=f\left(d^{n}, v^{n}, a^{n}, d^{n-1}, \ldots\right)$ 
On the other hand, implicit analysis requires to identify the nodal velocity and acceleration at time step (n+1) (Jensen et al., 2007).

$d^{n+1}=f\left(v^{n+1}, a^{n+1}, d^{n}, v^{n}, \ldots\right)$

Where $d^{n}, v^{n}, a^{n}$ are the nodal displacement, velocity and acceleration at time step (n), respectively, $v^{n+1}, a^{n+1}$ are the nodal velocity and acceleration at time step $(\mathrm{n}+1)$, respectively.

The nonlinear dynamic analysis can be used to evaluate the structural response and is considered an accurate technique (Kwasniewski, 2010). However, using non-linear analysis requires an understanding of the non-linear problem and its numerical solution. Nonlinear problems can have multiple degrees of freedom and require solution of a number of equations. In the field of structural mechanics, the nonlinear problems can be either path dependent or have multiple solutions. Therefore, it is necessary to select reliable solutions that can represent the nonlinear structural problems (Bergan et al., 1978). Unlike linear problems, in nonlinear problems, it is difficult to develop a method that is valid for all applications. Many of the solutions for nonlinear problems are either for specific types of non-linear problems or need specific requirement to be achieved to get converged solution. Hence, set of alternative algorithms to solve the non-linear problems have been implemented in the computer program. The computer program can control the solution through a number of parameters that are implemented in it. So that, the flexibility, reliability and efficiency to solve a specific problem will increase (Bergan et al., 1978). 


\subsection{Theoritical aspects (hydrocode)}

The dynamic response of a structure to high strain rate loading can be predicted using simplified method such as SDOF method of analysis. The SDOF method is not able to predict localized structural damage (Zhou et al., 2008). The development in the computer programming has produced hydrocodes such as AUTODYN and LS-DYNA to predict the dynamic response of a structure ( $\mathrm{Tu}$ and $\mathrm{Lu}, 2009)$. Hydrocode depends on three fundamentals postulations: conservation of mass, momentum and energy (Gebbeken and Ruppert, 2000). In hydrocodes there are two stress components: volumetric and deviatoric stresses to deal with stresses and strains individually. In the volumetric stress component, the relationship between hydrostatic pressure, local density, and local energy can be identified by the equation of state. The deviatoric stress component is dependent on the strength of the surface which in turns depends on the first stress invariant $J_{1}$, second invariant of deviatoric stress $J_{2}^{\prime}$ and third invariant of deviatoric stress $J_{3}^{\prime}$ (Zhou et al., 2008). Tensile and compressive strength of concrete is sensitive to strain rate effect. Dynamic increase factor is used to express the effect of strain rate on concrete strength (Zhou et al., 2008). When concrete is subjected to compression load it behaves as a nonlinear material, hence the yielding (decrease in the strength and stiffness) depends on the hydrostatic pressure. Ductile behaviour of concrete is obtained at high hydrostatic pressure, where the strain rate has an effect on the tensile and compressive strength of the concrete. On the other hand, according to plasticity theory, yielding does not rely on the hydrostatic pressure; this gives a possibility of separation of hydrostatic tensor from stress tensor. As a result, deviatoric stress tensor is generated. The deviatoric stresses $\left(s_{i j}\right)$ can be expressed as in Equation (3.8) (Gebbeken \& Ruppert, 2000; Gebbeken et al., 2001): 
$s_{i j}=f\left\{\varepsilon_{i j}, \dot{\varepsilon}_{i j}, G\left[\varepsilon_{i j}, s_{i j}(p)\right], K(p, \rho)\right\}$

Where $\varepsilon_{i j}$ and $\dot{\varepsilon}_{i j}$ are the strain and strain rate respectively, and $G$ the shear modulus, $K$ the bulk modulus, $\rho$ the density, and $p$ the hydrostatic pressure. However, $G$ and $K$ vary under high loading rates.

Concrete constitutive models can be identified depending on the volumetric and deviatoric response characteristics. The common form of yield function of the concrete material can be represented by Equation (3.9), considering the volumetric response represented by first invariant of the stress tensor $J_{l}$, and the deviatoric response is represented by second and third invariant of the deviatoric stress tensors $J_{2}^{\prime}$ and $J_{3}^{\prime}$, respectively (Wu et al., 2012).

$Y\left(J_{1}, J_{2}^{\prime}, J_{3}^{\prime}\right)=0$

\subsection{Finite element type}

LS-DYNA software provides different types of elements such as solid, beam, spring, cohesive and shell elements. For each element type there is an option for element formulation to best describe the material of the model (Hallquist, 2006). Solid elements are used for modelling the anchorage system and are described in the following subsections. 


\subsubsection{Solid element}

Eight-noded hexahedron solid elements were used to model the anchor, sleeve, steel plate, washer and nut. The solid elements for the anchor, sleeve, steel plate, washer and nut were formulated using constant stress. Four-noded tetrahedron solid elements were used to model the concrete in this research. Teterahedron element is a fast, stable and simple solid element. The tetrahedron elements permit transition from fine mesh to coarse mesh (Livermore Software Technology Corporation, 2015). Tetrahedron solid elements were used by Fang and Zhang to model the reinforced concrete under dynamic load (Fang \& Zhang, 2013). The solid elements for the concrete were formulated using one point integrated solid tetrahedron (Fang \& Zhang, 2013). Each node of the solid element has three translational degrees of freedom in ( $\mathrm{x}, \mathrm{y}$, and $\mathrm{z}$ directions). The geometry of the eightnoded hexahedron solid element is shown in Figure 3-1 while the geometry of the tetrahedron solid element is shown in Figure 3-2.

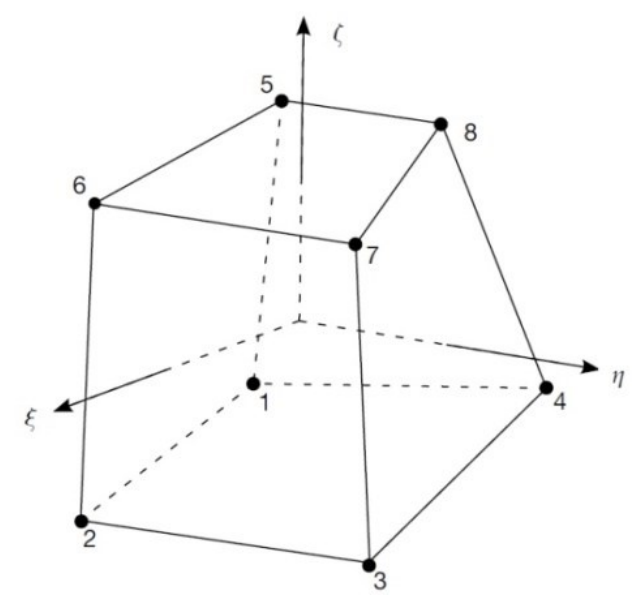

Figure 3-1: Eight node hexahedron solid element (Livermore Software Technology Corporation, 2015) 


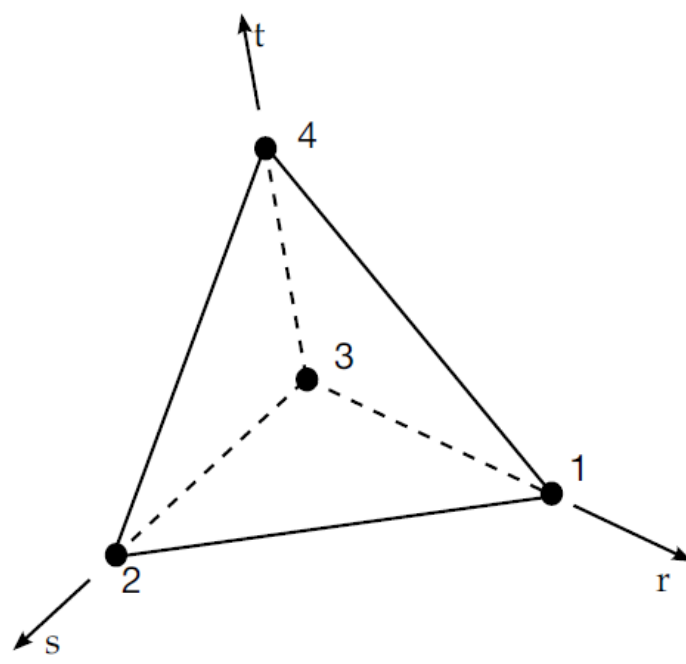

Figure 3-2: Four node tetrahedron solid element (Livermore Software Technology Corporation, 2015)

\subsection{Meshing technique}

The dynamic analysis of the structures can be performed analytically using mathematical equations or numerically using the finite element method by dividing the volume of the structure into finite elements linked at nodal points and forming a mesh. In the finite element method two types of errors may appear; numerical errors and discretizing errors that can cause the numerical model to give different answers from the analytical model. The discretizing errors can be minimized through mesh refinement (Paultre, 2010). Mesh size has an influence on the accuracy of the results and the time required to perform the analysis. Fine mesh size increases the accuracy and computation time resulting in a high overall computation cost (LSTC, 2014a). 


\subsection{Material constitutive models}

Selecting a suitable material model is important to get a reliable prediction of the structural response to applied load. A wide range of materials constitutive models have been implemented in the LS-DYNA software to represent the behaviour for different materials. In addition, the software gives the user additional options to add new materials to LSDYNA materials library using the keyword User Defined Material (Hallquist, 2006). Different options can be considered in LS-DYNA to select the appropriate material model for a specific application.

\subsubsection{Concrete models}

Concrete is a heterogeneous material consisting of aggregate and cement paste. It is ductile in compression and brittle in tension and shear (Gebbeken and Ruppert, 2000). The heterogeneity and porosity of concrete leads to complex response especially at high dynamic load where the internal energy cannot be neglected ( $\mathrm{Tu}$ and $\mathrm{Lu}, 2009)$. A variety of constitutive models implemented in LS-DYNA library can be used to model the concrete materials. These include:

- Karagozian and Case concrete damage model Release III (MAT_concrete_damage_rel3, MAT_72_R3)

- Winfrith concrete model (MAT_084)

- Johnson Holmquist concrete model (MAT_111)

- Continuous surface cap model for concrete (MAT_CSCM_CONCRETE) (MAT_159) 
Table 3-1 shows the material specifications and capabilities of the concrete models presented above.

Table 3-1: Material specifications for concrete models (LSTC, 2014)

\begin{tabular}{|c|c|c|c|c|c|c|c|c|}
\hline $\begin{array}{c}\text { Material } \\
\text { No. in } \\
\text { LS- } \\
\text { DYNA }\end{array}$ & $\begin{array}{c}\text { Material } \\
\text { model } \\
\text { title }\end{array}$ & $\begin{array}{c}\text { Element } \\
\text { formulation }\end{array}$ & $\begin{array}{c}\text { Strain } \\
\text { rate } \\
\text { effects }\end{array}$ & $\begin{array}{c}\text { Failure } \\
\text { criteria }\end{array}$ & $\begin{array}{c}\text { Equation of } \\
\text { state } \\
\text { requirement }\end{array}$ & Anisotropic & $\begin{array}{c}\text { Damage } \\
\text { effects }\end{array}$ & Applications \\
\hline 72 & $\begin{array}{c}\text { Concrete } \\
\text { damage }\end{array}$ & $\begin{array}{c}\text { Solid, thick } \\
\text { shell, SPH } \\
\text { element }\end{array}$ & Yes & Yes & Yes & No & Yes & $\begin{array}{c}\text { Soil, concrete, } \\
\text { rock }\end{array}$ \\
\hline 84 & $\begin{array}{c}\text { Winfrith } \\
\text { concrete }\end{array}$ & Solid & Yes & No & No & No & No & $\begin{array}{c}\text { Soil, concrete, } \\
\text { rock, foam }\end{array}$ \\
\hline 111 & $\begin{array}{c}\text { Johnson } \\
\text { Holmquist }\end{array}$ & $\begin{array}{c}\text { Solid, thick } \\
\text { shell, SPH } \\
\text { element }\end{array}$ & Yes & Yes & No & No & Yes & $\begin{array}{c}\text { Soil, concrete, } \\
\text { rock }\end{array}$ \\
\hline 159 & CSCM & $\begin{array}{c}\text { Solid, thick } \\
\text { shell, SPH } \\
\text { element }\end{array}$ & Yes & Yes & No & No & Yes & $\begin{array}{c}\text { Soil, concrete, } \\
\text { rock }\end{array}$ \\
\hline
\end{tabular}

In concrete material models MAT_72_R3, MAT_84 and MAT_111, the erosion criteria for deletion of highly distorted elements are not included implicitly in the formation of these models. Therefore, these material models require an additional element erosion model. This can be done by using MAT_ADD_EROSION. However, the erosion criteria for MAT_ADD_EROSION does not have a direct relation to concrete damage under impact (Sagals et al., 2011). Element erosion is necessary to simulate material damage, failure and prevent non convergence resulting from exaggerated deformation in affected area (Sagals et al., 2011). In addition, the simulation time may be increased due to the distorted elements. Material Erosion card is included in MAT_CSCM_CONCRETE material model to represent element deletion upon failure. 
MAT_CSCM_CONCRETE has been used in many applications (Wu et al., 2012) due to its simple input parameters represented by uniaxial compressive strength and maximum aggregate size. Other concrete material models on the other hand require many input parameters (Mousavi et al., 2016). In addition to the simplified input, MAT_CSCM_CONCRETE has key card to include or exclude rate effect. Furthermore, MAT_CSCM_CONCRETE model is able to represent the nonlinear behaviour of concrete material and complex concrete response in tension and compression (damage in tension and softening in compression), strain rate effect, and erosion (Bermejo et al., 2011; Wu et al., 2012). The Continuous Surface Cap Model (MAT_CSCM_CONCRETE) was thus selected to model the concrete in this research.

\subsubsection{Concrete Model (MAT_CSCM_CONCRETE)}

Continuous Surface Cap Model (MAT_CSCM_CONCRETE) (MAT_159) model was originally designed for roadside safety analysis in 1990 and then incorporated in LSDYNA in 2005 to analyze many different types of applications (Wu et al., 2012). MAT_159 can be used for solid elements to predict the elastic-plastic behaviour of concrete (Wu et al., 2012). Where concrete material is assumed to be an isotropic material and behaves as elastic material obeying Hooke's law before cracking. After cracking and as the stress increases, the concrete material yields and behaves plastically (Murray, 2007).

MAT_159 model is characterized by smooth intersection between two failure surfaces; shear failure surface $\left(F_{f}\right)$ and hardening cap $\left(F_{c}\right)$ surface. Both surfaces: $F_{f}$ and $F_{c}$ are combined through multiplication form $\left(F_{f}^{2} F_{c}\right)$ into the yield equation. This multiplication form provides the smooth and continuous intersection between the cap $\left(F_{c}\right)$ and shear $\left(F_{f}\right)$ 
surfaces. The smooth intersection makes the numerical analysis simple and removes the intricacy induced by the existence of corner region between the failure surface and the hardening cap. Three dimensional yield surface can be used to represent the yield stresses in the concrete model (LSTC, 2014). Figure 3-3 shows the general shape of the concrete model yield surface in two and three dimensions.

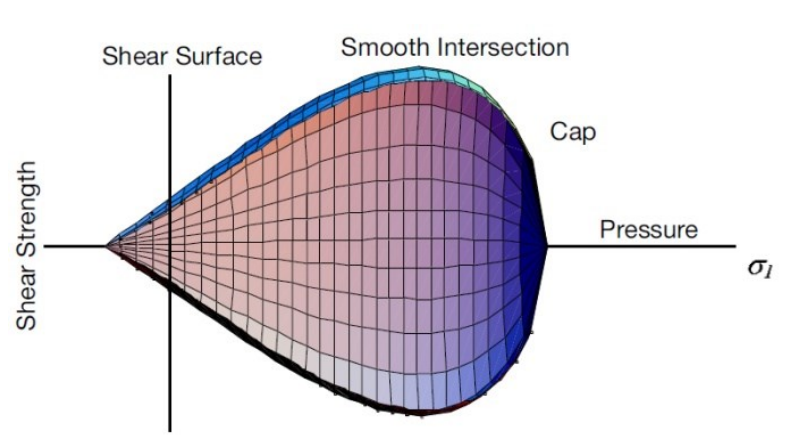

(a) In two dimensions

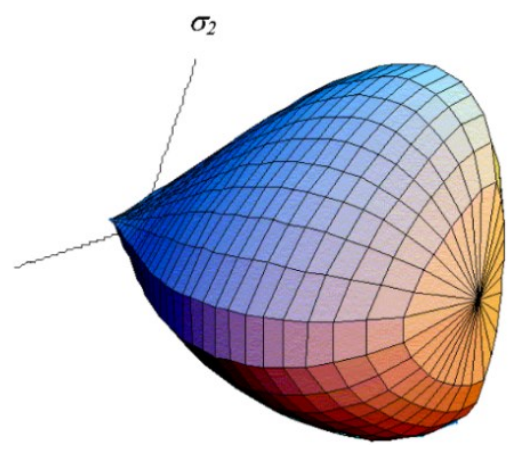

(b) In three dimensions

Figure 3-3: General shape of the concrete model yield surface (Murray, 2007)

The yield function for the MAT_159 model is represented by three stress invariants $\left(J_{1}, J_{2}^{\prime}, J_{3}^{\prime}\right)$ and cap hardening $(k)$ in Equation (3.10) (LSTC, 2014):

$f\left(J_{1}, J_{2}^{\prime}, J_{3}^{\prime}, k\right)=J_{2}^{\prime}-\Re^{2} F_{f}^{2} F_{c}$

$J_{1}=3 P$

$J_{2}^{\prime}=\frac{1}{2} S_{i j} S_{i j}$ 
$J_{3}^{\prime}=\frac{1}{3} S_{i j} S_{j k} S_{k i}$

Where $J_{1}$ is the first invariant for the stress tensor, $J_{2}^{\prime}$ and $J_{3}^{\prime}$ are the second and third invariant for the deviatoric stress tensor, respectively, $k$ is the cap hardening parameter, $\mathfrak{R}$ is the Rubin three invariant reduction factor. The concrete strength can be calculated by the Rubin scaling factor $(\mathfrak{R})$ at different stress states relative to concrete strength for triaxial compression (TXC). $S_{i j}, S_{j k}, S_{k i}$ are the deviatoric stress tensor.

LS-DYNA software provides updated values of the stress $\left(J_{1}^{T}, J_{2}^{\prime T}, J_{3}^{\prime T}\right)$ at each time step. If the updated stress value is located on or inside the yield surface, $f\left(J_{1}^{T}, J_{2}^{\prime T}, J_{3}^{\prime T}, k\right) \leq 0$ the concrete behaves as elastic material. If the updated stress value is located outside the yield surface, $f\left(J_{1}^{T}, J_{2}^{\prime T}, J_{3}^{\prime T}, k\right)>0$, the concrete behaves as elastic-plastic material, where the updated stress is returned to the yield surface, $f\left(J_{1}^{p}, J_{2}^{\prime p}, J_{3}^{\prime p}, k\right)=0$, by a plasticity algorithm (Murray, 2007).

The shear failure surface can be expressed as follows:

$F_{f}\left(J_{1}\right)=\propto-\lambda \exp ^{-\beta J_{1}}+\theta J_{1}$

Where $\propto, \lambda, \beta$ and $\theta$ are constants measured from a triaxial compression test. 
The plastic volume change (expansion and compaction) can be simulated using the cap surface, where the cap movement can represent the expansion or compaction. The hardening cap surface can be represented as follows:

$F_{c}\left(J_{1}, k\right)= \begin{cases}1-\frac{\left[J_{1-}-L(k)\right]\left[\left|J_{1}-L(k)\right|+J_{1}-L(k)\right]}{2[X(k)-L(k)]^{2}} & \text { if } J_{1}>L(k) \\ 1 & \text { if } J_{1} \leq L(k)\end{cases}$

Where

$L(k)=\left\{\begin{array}{lll}k & \text { if } & k>k_{o} \\ k_{o} & \text { if } & k \leq k_{o}\end{array}\right.$

The hardening cap function $\left(F_{c}\right)$ is either unity or an ellipse depending on the stress state. Unity function $\left(F_{c}=1\right)$ is obtained when $\left(J_{1} \leq L(k)\right)$, where the stress is located in the tensile or low confining pressure zone, consequently, the yield function depends on the shear surface only. Ellipse function is obtained when $\left(J_{1}>L(k)\right)$, where the stress located in low to high confining pressure zone and the yield function is dependent on both the cap surface and the shear surface.

The shear failure surface intersects the cap failure surface when $J_{1}=k$. At the initial intersection between shear surface and cap surface $J_{1}=k_{o}$ (Murray, 2007).

MAT_159 has built-in erosion criterion that can represent the concrete failure (Bermejo et al., 2011). When the damage $(d)$ is equal to or greater than 1 and the maximum principal strain is greater than 1-ERODE, the element is eroded. When the damage is less than 1 the 
erosion does not occur (Murray, 2007). Furthermore, MAT_159 has built-in damage criterion to represent the damage of the concrete when subjected to compression or tension load. The value of damage parameter $(d)$ is ranging from zero to one and allows for the prediction of crack initiation and propagation in concrete. The MAT_159 material model has been reported to be more suitable for modelling concrete compared to the other material models available in LS-DYNA (Jaime, 2011).

Murray (2004) conducted finite element analysis to investigate the damage on reinforced concrete beam subjected to impact load using MAT_159. Murray's analysis revealed that the damage and displacement were adequately simulated when using MAT_159. Moutossamy et al. (2011) investigated the validation of MAT_159 concrete model using Lagrange in solid to connect the reinforcement with the concrete using analytical and numerical analysis on frame structure. The authors reported that MAT_159 model can represent the real behaviour of the concrete in many conditions. The authors also reported that the cracks in the concrete structure can be modeled properly using plastic strain contours (Moutoussamy et al., 2011).

\section{Rate Effect:}

Rate effect is used to model a materials dependence on loading or strain rates. LS-DYNA has a built-in parameter (IRATE) to represent the strain rate effect on the concrete strength. By activating the IRATE formulation, increase in the strain rate increases the strength of the concrete material. The rate effect is applied through using viscoplastic parameters on 
the plasticity surface, damage surface and fracture energy. The viscoplastic parameters are used for compatibility of the uniaxial tensile and compressive strength data (Murray, 2007).

\subsubsection{Steel anchor models}

PIECEWISE_LINEAR_PLASTICITY (MAT_024) and MAT_PLASTIC_KINEMATIC (MAT_003) material models can be used to model the steel material. Rate effect is included in both material models. The two material models are cost effective and commonly used to model steel material and take into account the isotropic and kinematic hardening plasticity of steel (Bi and Hao, 2013; Livermore software technology Corporation, 2012). Analysis has been made on the anchorage to concrete system using MAT_024 and MAT_003. Results show that both material models give same load-displacement response, ultimate load and failure mode. MAT_024 is the most commonly used to model steel and hence MAT_024 is used to model steel material in this research. MAT_024 is an elasto-plastic material model that can represent failure depending on plastic strain or a minimum time step size.

The deviatoric stresses required to attain the yield function for MAT_024 can be expressed by Equation (3.17) (Livermore Software Technology Corporation, 2015):

$$
\begin{aligned}
& \varnothing=\frac{1}{2} S_{i j} S_{i j}-\frac{\sigma_{y}^{2}}{3} \leq 0 \\
& \sigma_{y}=\beta\left[\sigma_{0}+f_{h}\left(\varepsilon_{e f f}^{p}\right)\right]
\end{aligned}
$$


Where

$\beta$ is a constant representing strain rate effect, $\sigma_{0}$ is the initial yield stress, and $f_{h}\left(\varepsilon_{\text {eff }}^{p}\right)$ is the hardening function. The hardening function can be represented in linear hardening form expressed in Equation (3.19).

$f_{h}\left(\varepsilon_{e f f}^{p}\right)=E_{p}\left(\varepsilon_{e f f}^{p}\right)$

$E_{p}=\frac{E_{t} E}{E-E_{t}^{\prime}}$

$\varepsilon_{e f f}^{p}=\int_{0}^{t}\left(\frac{2}{3} \dot{\varepsilon}_{i j}^{p} \dot{\varepsilon}_{i j}^{p}\right)^{1 / 2} d t$

$\dot{\varepsilon}_{i j}^{p}=\dot{\varepsilon}_{i j}-\dot{\varepsilon}_{i j}^{e}$

Where $E_{p}$ is the plastic hardening modulus, $E_{t}$ is the tangent modulus, $\varepsilon_{e f f}^{p}$ is the effective plastic strain, $\dot{\varepsilon}_{i j}^{p}$ is the plastic strain rate, $\dot{\varepsilon}_{i j}^{e}$ is the elastic strain rate, $\dot{\varepsilon}_{i j}$ is the total strain rate.

The deviatoric stresses are calculated and updated elastically to satisfy the aforementioned yield function. If the yield function is not satisfied, then the increase in the effective plastic strain is calculated according to Equation (3.23).

$\Delta \varepsilon_{e f f}^{p}=\frac{\left(\frac{3}{2} S_{i j}^{*} S_{i j}^{*}\right)^{\frac{1}{2}}-\sigma_{y}}{3 G+E_{p}}$ 
Where

$\Delta \varepsilon_{e f f}^{p}$ is the effective plastic strain increment, $\mathrm{G}$ is the shear modulus, $S_{i j}^{*}$ is the trial deviatoric stress. If the deviatoric stresses $S_{i j}^{*}$ is higher than the yield stress $\sigma_{y}$, it is scaled back to the yield surface and the updated deviatoric stress, $S_{i j}^{n+1}$ is given by Equation (3.24).

$S_{i j}^{n+1}=\frac{\sigma_{y}}{\left(\frac{3}{2} S_{i j}^{*} S_{i j}^{*}\right)^{\frac{1}{2}}} S_{i j}^{*}$

\section{Rate effect}

The strain rate effect is considered for Piecewise Linear Plasticity model (MAT_024) as follows:

- The Cowper-Symonds model which scales the yield stress with a factor as per Equation (3.25) (Livermore Software Technology Corporation, 2015):

$$
\begin{aligned}
& \beta=1+\left(\frac{\dot{\varepsilon}}{C}\right)^{1 / p} \\
& \dot{\varepsilon}=\sqrt{\dot{\varepsilon}_{i j} \dot{\varepsilon}_{i j}}
\end{aligned}
$$

Where $\mathrm{C}$ and $\mathrm{P}$, are Cowper-Symonds constants and $\dot{\varepsilon}$ is the strain rate.

Cowper-Symonds parameters $\mathrm{C}$ of $40 \mathrm{~s}^{-1}$ and $\mathrm{P}$ of 5 are recommended for steel (Boh et al., 2004) and were used in this research to represent the strain rate effect. 
- Using a table if there are different stress-strain curves for different strain rates where a curve ID can be defined for each strain rate. If the strain rate is not in the range included in the table (higher or lower than the strain rate in the table), then effective stress-strain curve for the maximum value of strain rate in the table is used for the strain rates higher than the maximum value. Effective stress-strain curve for the minimum strain rate in the table is used for the strain rates lower than the minimum value (Livermore Software Technology Corporation, 2015).

\subsubsection{Adhesive modelling}

The adhesive layer in post-installed adhesive anchorage systems can be modeled by using either a mesh dependent or mesh independent method. The mesh dependent method can be implemented by using identical connection between the adhesive and the adherents, while the mesh independent method can be implemented by using CONTACT_TIED_NODES_TO_SURFACE keycard where the connection between the adhesive and the adherents is incompatible (Marzi et al., 2008).

There are two approaches that can be used to describe failure behaviour of the adhesive layer: cohesive zone model (CZM) and continuum damage mechanics (CDM) approach. The CZM describes the damage and failure of adhesive material efficiently by employing simple traction separation law (Marzi et al., 2008; Marzi et al., 2009; May et al., 2014). The CDM approach on the other hand describes the damage of the adhesive material by employing damage parameter to include the stiffness degradation of the adhesive material (Tserpes \& Koumpias, 2012). Both CZM and CDM approaches can represent the damage initiation until complete failure (Sugiman \& Ahmad, 2017). However, the CZM approach 
is faster and less complicated than the continuum damage mechanics approach (Marzi et al., 2008; Marzi et al., 2009; May et al., 2014). In addition, the continuum damage mechanics approach depends more on mesh size compared to the (CZM) (Tserpes \& Koumpias, 2012; Sugiman \& Ahmad, 2017).

There are several cohesive zone models that can be used to model the adhesive material implemented in LS-DYNA. These models include ARUP_ADHESIVE (MAT_169), COHESIVE_MIXED_MODE (MAT_138), COHESIVE_ELASTIC (MAT_184), COHESIVE_THERMAL (MAT_185) and COHESIVE_GENERAL (MAT_186) (LSTC, 2014). MAT_138, MAT_184, MAT_185, MAT_186 don't have a keycard to represent the rate effect. However, MAT_169 has rate dependent keycard that can represent the strain rate (LSTC, 2014). Hence, among these models MAT_ARUP_ADHESIVE (MAT_169) was selected to model the adhesive material. MAT_ARUP_ADHESIVE is structural adhesive and can represent the behaviour of the adhesive anchor (Graf et al., 2014).

\subsubsection{Adhesive model (MAT_ARUP_ADHESIVE)}

MAT_ARUP_ADHESIVE (MAT_169) was used to model the adhesive material. Solid element formulations 1,2 and 15 are suitable to model the adhesive material. The yield function and the failure surfaces for the MAT_169 model is represented by a power-law combination of direct tension and shear as in Equation (3.27) (LSTC, 2014).

$\left(\frac{\sigma}{\sigma_{\max }}\right)^{P W R T}+\left(\frac{\tau}{\tau_{\max }-S H T_{-} S L \times \sigma}\right)^{P W R S}=1$ 
Where

PWRT , PWRS are power law terms for tension and shear respectively. $\sigma$ is the tensile stress, $\tau$ is the shear stress, $S H T \_S L$ is the slope of the yield surface at zero tension.

Figure 3-4 shows the yield surface for MAT_169.

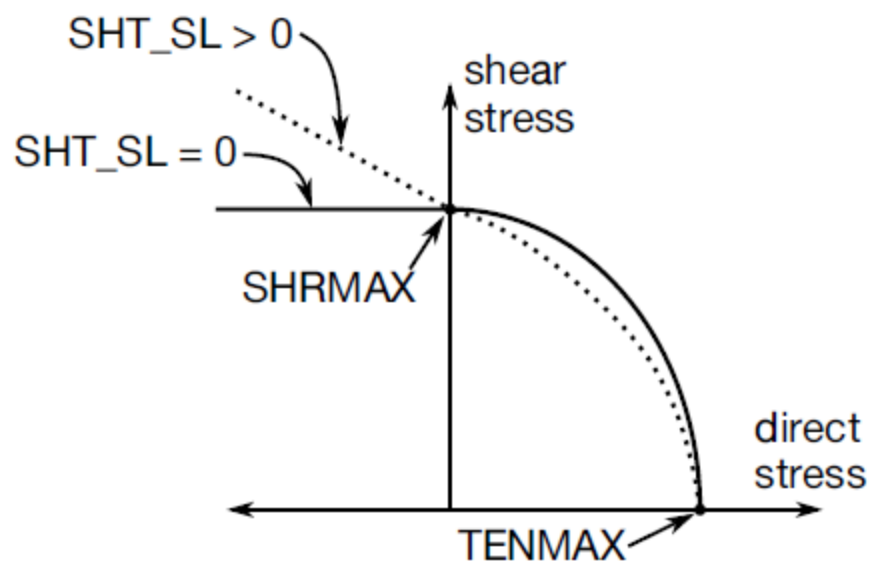

Figure 3-4: Yield surface (LSTC, 2014)

Figure 3-5 shows the stress-displacement relation for MAT_169 in tension and shear.

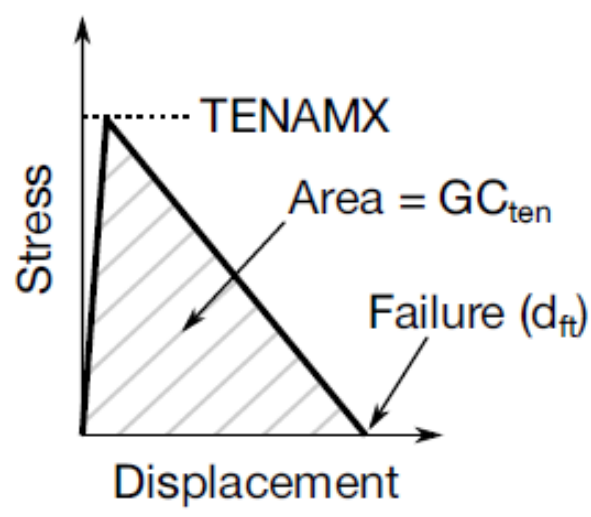

(a)

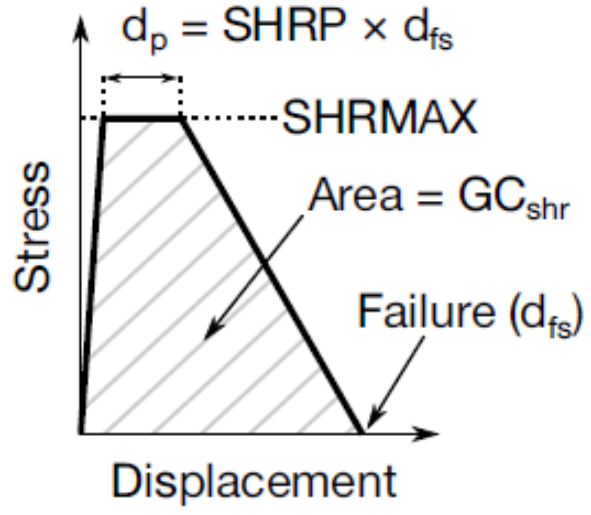

(b)

Figure 3-5: Stress-displacement relation of MAT_169 for (a) tension and (b) shear (LSTC, 2014) 


\section{Rate effect:}

The strain rate effect is considered for MAT_ARUP_ADHESIVE model (MAT_169) through parameters EDOT0 and EDOT2 for the static and dynamic strain rates respectively (LSTC, 2015).

\subsection{Design parameters}

Concrete block size of $\left(4 h_{e f}+125\right) m m \times\left(4 h_{e f}+125\right) m m \times\left(2 h_{e f}\right) m m$ was modeled and used for the tensile analysis of anchorage systems. The size of the concrete block for the tensile analyses was selected to preclude edge effect on the failure of concrete cone formation. A concrete block size of $\left(2 h_{e f}+150\right) m m \times\left(2 h_{e f}+150\right) m m \times$ $\left(1.5 h_{e f}+50\right) m m$ was used for the shear analysis to minimize concrete edge effects on the behaviour of the anchors. Single anchor was used for the analysis. The anchor placement in the concrete block was such that the edge distance (c) does not affect the concrete breakout cone formation. Anchorage failure by concrete breakout near the free edge is not considered in this research.

Cast-in-place and adhesive anchor diameters of $12.7 \mathrm{~mm}, 15.9 \mathrm{~mm}$ and $19.1 \mathrm{~mm}$ were used. According to the design guidelines of ASTM A 307, the recommended effective embedment depths are $101.6 \mathrm{~mm}, 127 \mathrm{~mm}$ and $152.4 \mathrm{~mm}$ for the $12.7-\mathrm{mm} 15.9-\mathrm{mm}$ and 19.1-mm diameters anchors, respectively were used for the analysis (Bridge Design Aids, 2012). In this research, anchor embedment depths of $76.2 \mathrm{~mm}, 101.6 \mathrm{~mm}, 127 \mathrm{~mm}$ and $152.4 \mathrm{~mm}$ were investigated for each anchor diameter to investigate the influence of various embedment depths (the recommended, less than the recommended and higher than 
the recommended embedment depths) on the behaviour of the anchorage to concrete system, ultimate load, level of damage and the failure mode.

According to Hilti Inc. the minimum embedment depths for adhesive anchor diameters of $12.7 \mathrm{~mm}, 15.9 \mathrm{~mm}$ and $19.1 \mathrm{~mm}$ are $70 \mathrm{~mm}, 79 \mathrm{~mm}$ and $89 \mathrm{~mm}$ respectively (Hilti, 2014). Cast-in-place anchors with hexagonal head were used for the analysis. The hexagonal head sizes $(\mathrm{D} \times \mathrm{H})$ of $(19 \times 8) \mathrm{mm},(24 \times 10)$ and $(28.5 \times 12)$ were used for anchor diameters of $12.7 \mathrm{~mm}, 15.9 \mathrm{~mm}$ and $19.1 \mathrm{~mm}$ respectively (Cement Association of Canada, 2010). Three nominal undercut anchor diameters of $12 \mathrm{~mm}, 16 \mathrm{~mm}$ and $20 \mathrm{~mm}$ with effective embedment depths of $125 \mathrm{~mm}, 190 \mathrm{~mm}$ and $250 \mathrm{~mm}$ recommended by Hilti (Hilti, 2011) were investigated. In this research embedment depths of $100 \mathrm{~mm}, 125 \mathrm{~mm}, 190 \mathrm{~mm}$ and $250 \mathrm{~mm}$ were investigated for all the anchor diameters to investigate the behaviour of anchorage system, ultimate load, failure mode and level of damage for various embedment depths (the recommended, less than and higher than the recommended embedment depths). The embedment depth $\left(h_{e f}\right)$ of the undercut anchor is measured from the surface of the concrete to the end of the sleeve.

\subsection{Materials properties}

The cast-in-place, adhesive and undercut anchorage systems consisted of steel anchor, steel plate, washer, nut and concrete block. In addition to the adhesive material for the adhesive anchorage system and sleeve for the undercut anchorage system. The concrete blocks were modeled using continuous surface cap model (MAT_159) while the steel anchor, steel plate, sleeve, washer and nut were modeled using piecewise linear plasticity model (MAT_024). MAT_ARUP_ADHESIVE (MAT_169) was used to model the adhesive 
material. The concrete material was modeled with a density of $0.0024 \mathrm{~g} / \mathrm{mm}^{3}$ and compressive strength $\left(f_{c}^{\prime}\right)$ of $30 \mathrm{MPa}$. The concrete material is assumed free from cracking or damage, and this is implemented in the concrete material model (MAT_159) through the pre-existing damage parameter PRED equal to zero.

For the cast-in-place and adhesive anchors the properties of the steel anchor, steel plate, washer and nut were in accordance with ASTM A354 specification with yield strength of $896 \mathrm{MPa}$, ultimate tensile strength of $1034 \mathrm{MPa}$, density of $0.00785 \mathrm{~g} / \mathrm{mm}^{3}$, Young's modulus of $200000 \mathrm{MPa}$, failure strain of $14 \%$ and Poisson's ratio of 0.3 . The adhesive material was modeled with a density of $0.0012 \mathrm{~g} / \mathrm{mm}^{3}$, tensile strength of $56 \mathrm{MPa}$, shear strength of $44 \mathrm{MPa}$ (Dogan et al., 2012), Elastic modulus of $3034 \mathrm{MPa}$ and Poisson's ratio of 0.4 .

For the undercut anchors the steel anchor, sleeve, steel plate, washer and nut were modeled in accordance with Hilti Inc. specification with yield strength of $640 \mathrm{MPa}$ and tensile strength of $800 \mathrm{MPa}$ (Hilti, 2011).

\subsection{Contact modelling}

There are different types of contacts that can be applied using LS-DYNA software. In general, the contact between two surfaces can be automatic or non-automatic. Automatic contact can be used for explicit analysis while, non-automatic contact can be used for implicit analysis (LSTC, 2014). 
In the present investigation, CONTACT_AUTOMATIC_SURFACE_TO_SURFACE was used to define the contact interfaces between the concrete, steel anchor, washer, nut and anchor plate for cast-in-place and undercut anchors. This contact bonds the steel anchor and concrete surfaces. The contact algorithm is characterized by its dual treatment, where the slave and master nodes checked against penetration in each other. Hence, the user has flexibility to switch slave part/ master part and obtain identical behaviour. This contact formulation is useful to prevent penetration between contacted surfaces (LSTC, 2014).

For the adhesive anchor CONTACT_AUTOMATIC_ONE_WAY_SURFACE_TO_ SURFACE_TIEBREAK was used to model the interaction between the adhesive and anchor. The tiebreak contact is penalty-based contact which allows transmission of the forces in normal and tangential directions and has the capability to model the failure (LSTC, 2014a). Before failure, the tiebreak contact prevents the separation between slave node (adhesive) and the master segment. After failure, the coupling in normal direction is eliminated and the contact behaves as surface to surface contact with thickness offset (LSTC, 2014). CONTACT_ERODING_SINGLE_SURFACE was used to remove the distorted elements of the adhesive material. ENMASS parameter is set equal to one in the CONTROL_CONTACT key card to retain the mass of these removed distorted elements and they remain active in contact (LSTC, 2014a).

CONTACT_TIED_SURFACE_TO_SURFACE key card was used to model the bond between adhesive and the concrete. The tied contact is a constrained-based contact used to ensure that the adhesive material is tied to the concrete material with no gap (LSTC, 2014). 
The tied contact is used when the mesh sizes of two surfaces in contact are different to ensure that the slave nodes (adhesive) located on the master segment (concrete) with no gap (LSTC, 2014; Bala, 2008). LS-DYNA updates the coordinates of the slave nodes to be identical on the master surface and neglect the slave nodes that are located far from the master surface (Bala, 2008). Moreover, the tied contact allows mesh transition (Hallquist, 2006). When the failure of the elements in contact occurs, the elements in the tied contact are automatically deleted (Chen et al., 2017). The interaction between the anchor plate, washer, nut, steel anchor and the concrete was modeled using CONTACT_AUTOMATIC_SURFACE_TO_SURFACE.

\subsection{Boundary conditions}

Bottom surface of the concrete block (XY plane) was fixed to prevent translational and rotational motion during the application of loading. Due to the symmetry of the geometric configuration of the anchorage system, a quarter of the anchorage to concrete system was modelled to investigate the tensile behaviour while half of the anchorage to concrete system was modelled to investigate the shear behaviour. Using the symmetry increases the efficiency and reduces the computational time. Symmetry planes boundary conditions were applied on the anchorage to concrete models. Two symmetry planes (XZ and YZ) boundary conditions were applied to represent the tensile behaviour. One symmetry plane (YZ) was applied to represent the shear behaviour. Figure 3-6 shows the geometric configuration and boundary conditions for the anchorage system model under tensile and shear loads. Table 3-2 summarizes the boundary conditions used for the anchorage models. 


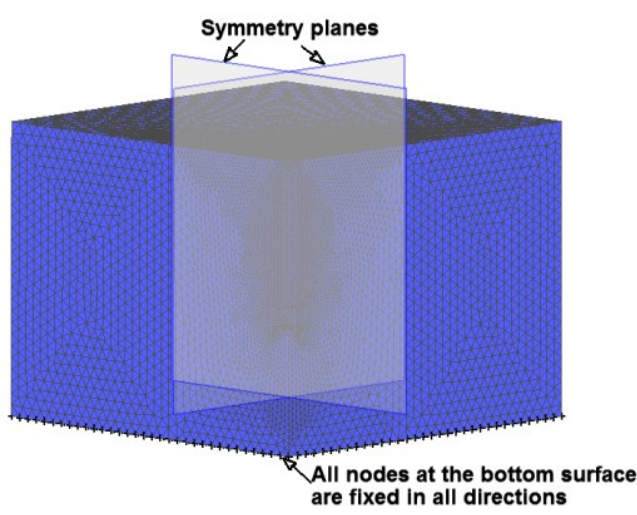

(a) Quarter of the concrete block

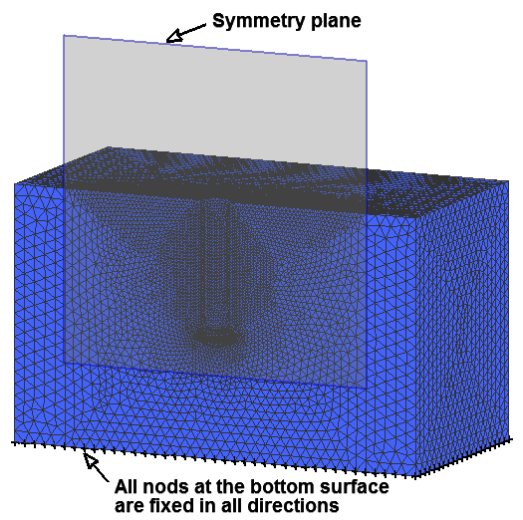

(b) Half of the concrete block

Figure 3-6: Boundary conditions for the anchorage models under (a) tensile load and (b) shear load

Table 3-2: Boundary conditions of the anchorage to concrete systems

\begin{tabular}{|c|c|c|c|c|c|c|}
\hline \multirow{2}{*}{ Boundary conditions } & \multicolumn{6}{|c|}{ *Degree of freedom } \\
\cline { 2 - 7 } & \multicolumn{3}{|c|}{ Translational } & \multicolumn{3}{c|}{ Rotational } \\
\cline { 2 - 7 } & $\mathrm{x}$ & $\mathrm{y}$ & $\mathrm{z}$ & $\mathrm{rx}$ & ry & $\mathrm{rZ}$ \\
\hline XZ symmetry plane & 0 & 1 & 0 & 1 & 0 & 1 \\
\hline YZ symmetry plane & 1 & 0 & 0 & 0 & 1 & 1 \\
\hline $\begin{array}{c}\text { XY plane (at the bottom } \\
\text { of the concrete) }\end{array}$ & 1 & 1 & 1 & 1 & 1 & 1 \\
\hline
\end{tabular}

*Degree of freedom: $1=$ fixed, $0=$ free

\subsubsection{Load conditions}

The load in LS-DYNA can be applied using five options: BOUNDARY_SPC_SET, INITIAL_VELOCITY, LOAD_SEGMENT_SET, LOAD_NODE_SET, and BOUNDARY_PRESCRIBED_MOTION_SET. The BOUNDARY_SPC_SET (single point constraint) can be applied to determine the reaction force. INITIAL_VELOCITY can be used to apply the velocity to all nodes of the object. LOAD_SEGMENT_SET can be used to apply pressure load (Chou et al, 2004). LOAD_NODE_SET can be used to apply 
static load (Abebe \& Qiu, 2016). BOUNDARY_PRESCRIBED_MOTION_SET is used to apply the quasi static loading (Kashani et al., 2013) and dynamic loading (Abebe \& Qiu, 2016) and to maintain uniform rate of velocity. DATABASE_CROSS_SECTION_PLANE command is used to specify the location of the applied load, where the load-displacement relation can be drawn. BOUNDARY_PRESCRIBED_MOTION_SET is used to apply the tensile and shear loads. In this research, BOUNDARY_PRESCRIBED_MOTION_SET was applied in z-direction at the top nodes of the anchor to simulate the tensile load on the anchorage systems. BOUNDARY_PRESCRIBED_MOTION_SET was applied in y-direction on the anchor plate to simulate the shear load on the anchorage systems. When using BOUNDARY_PRESCRIBED_MOTION_SET, velocity, or displacement can be prescribed. The displacement is used for the implicit analysis (Maker \& Zhu, 2000), whereas the velocity is recommended for explicit analysis. The strain rate is applied using the velocity-time curve in the DEFINE_CURVE command in LS-DYNA. The applied strain rate can be checked using SRate in the fringe component of the LS-DYNA post processor and history-scalar option. Also, the strain rate can be measured using DATABASE_EXTENT_BINARY, setting the strain rate flag (STRFLG) to one and the energy output (N3THDT) to one to minimize the output (LSTC, 2014b). In this research, for the anchors subjected to tensile and shear loads, the strain rate has been measured using SRate in the fringe component of the LS-DYNA post processor (Fcomp) that presents strain rate plot and then using History-Scalar-option that plots a time history of the strain rate (LSTC, 2014b). 


\subsection{Quasi-static simulation using LS-DYNA}

Explicit LS-DYNA code is designed for dynamic analysis; however quasi-static analysis is performed by decreasing the loading rate and scaling up the mass of the anchor, adhesive and concrete materials by a factor of 1000 . This procedure decreases the number of time steps required, reduces the simulation time and improve the computation efficiency. To ensure that the static analysis is achieved, the ratio of the kinetic energy to the internal energy has to be less than 5\% during the analysis (Bathe et al., 1999; El-Hage et al., 2005; Han et al., 2007). On the other hand, implicit solver can also be used to perform the static analysis. However, the analysis using the implicit solver generates few number of time steps with large disk storage requirements. This increases the computation time and requires a lot of effort and large computer capacity to complete the run and store the file. As a result, the computational cost will increase (Jensen et al., 2007). Table 3-3 shows the specifications for the material models used in the analysis.

Table 3-3: Material models specifications (LSTC, 2014)

\begin{tabular}{|c|c|c|c|c|c|c|c|c|}
\hline $\begin{array}{c}\text { Material } \\
\text { No. in } \\
\text { LS- } \\
\text { DYNA }\end{array}$ & $\begin{array}{c}\text { Material } \\
\text { title }\end{array}$ & $\begin{array}{c}\text { Element } \\
\text { formulation }\end{array}$ & $\begin{array}{l}\text { Strain } \\
\text { rate } \\
\text { effects }\end{array}$ & $\begin{array}{l}\text { Failure } \\
\text { criteria }\end{array}$ & $\begin{array}{l}\text { Equation of } \\
\text { state } \\
\text { requirement }\end{array}$ & Anisotropic & $\begin{array}{r}\text { Damage } \\
\text { effects }\end{array}$ & Applications \\
\hline 24 & $\begin{array}{l}\text { Piecewise } \\
\text { Linear } \\
\text { Plasticity }\end{array}$ & $\begin{array}{c}\text { Solid, beam, } \\
\text { shell, SPH, } \\
\text { ALE solid }\end{array}$ & Yes & Yes & No & No & No & Metal, plastic \\
\hline 159 & $\mathrm{CSCM}$ & $\begin{array}{l}\text { Solid, thick } \\
\text { shell, SPH }\end{array}$ & Yes & Yes & No & No & Yes & $\begin{array}{c}\text { Soil, } \\
\text { concrete, rock }\end{array}$ \\
\hline 169 & $\begin{array}{c}\text { Arup } \\
\text { Adhesive }\end{array}$ & Solid & Yes & Yes & No & Yes & No & Adhesive \\
\hline
\end{tabular}




\subsection{Summary}

This chapter presents finite element modelling for cast-in-place, adhesive and undercut anchorage systems. The chapter started with introduction on the development of the LSDYNA code and its capabilities to predict the structural response under low and high strain rates. The characteristics of the LS-DYNA finite element software package are introduced. Implicit and explicit solvers of LS-DYNA software and their capabilities are presented. Theoretical aspects of LS-DYNA hydrocode used to predict the dynamic response of a structure are presented. Finite element types are introduced and selection criteria for element type are presented. Solid elements that are used to model anchorage to concrete system are described.

Meshing technique to discretize the model in small elements is presented. Material constitutive models for the anchorage to concrete system are presented, followed by selecting the suitable material models to model the concrete, steel anchor and adhesive. Selection criteria are presented for each material model. Materials properties and design parameters for the anchorage systems are presented. Contact keycard to model the interaction between the materials is introduced. Boundary conditions to capture the tensile and shear behaviour of the anchorage to concrete systems are introduced. Application of tensile and shear loads is introduced. Performing quasi static analysis using LS-DYNA software is presented at the end of the chapter. 


\section{Chapter 4 : Strain rate effect on cast-in-place anchors}

\subsection{Cast-in-place anchors under tensile load}

\subsubsection{Finite element modelling of cast-in-place anchors under tensile load}

Analysis of the behaviour of cast-in-place anchors embedded into concrete under tensile loading at different strain rates was performed using the explicit commercial finite element code LS-DYNA (LSTC, 2014). Strain rates ranging from low (static) to high $\left(10^{-5} \mathrm{~s}^{-1}, 10^{-}\right.$ ${ }^{3} \mathrm{~s}^{-1}, 10^{-1} \mathrm{~s}^{-1}, 10 \mathrm{~s}^{-1}, 10^{2} \mathrm{~s}^{-1}$ and $\left.10^{3} \mathrm{~s}^{-1}\right)$ were investigated. The design parameters, materials properties, boundary conditions and contacts for the anchorage to concrete system were introduced in chapter 3 sections 3.7 to 3.11 . The friction between the cast-in-place anchor and the concrete and between the anchor and the anchor plate was neglected. For the castin-place anchor, the anchor head has a major role on resisting the applied load on the anchor. A schematic view of the cast-in-place anchor is shown in Figure 4-1. The geometric configuration and boundary condition for the cast-in-place anchor model are shown in Figure 4-2. The mesh of the concrete block was biased (refined) towards the cast-in-place anchor and along the embedment depth to improve the accuracy of the finite element analysis and to minimize discretization errors.

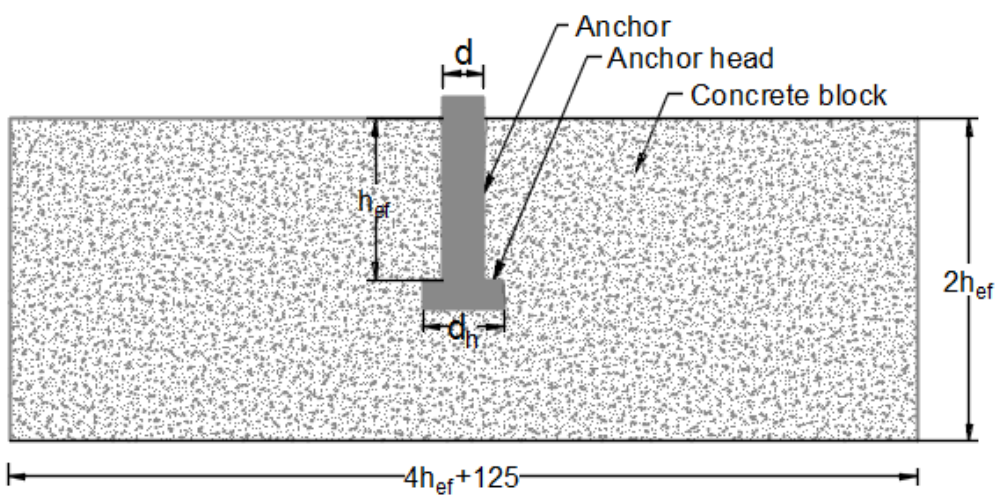

Figure 4-1: Schematic view of the cast-in-place anchor 


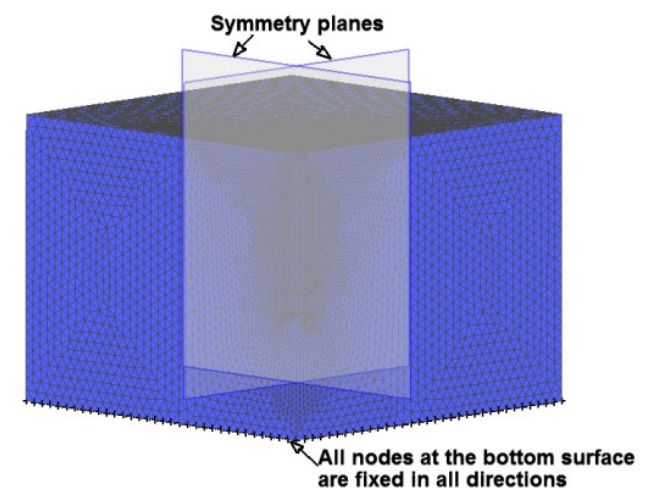

(a) Concrete mesh

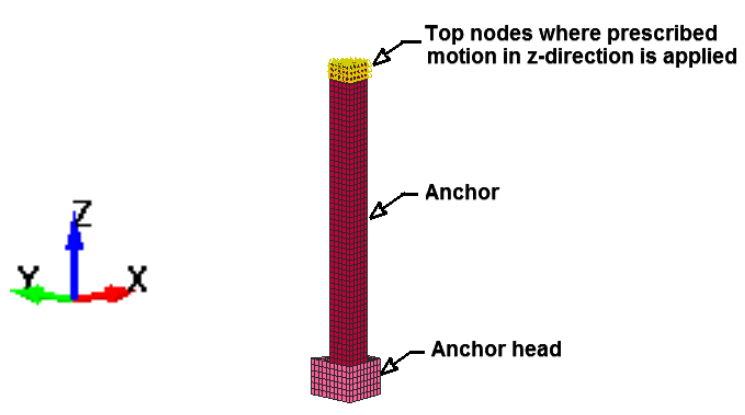

Figure 4-2: Geometric configuration with boundary condition of cast-in-place anchor model

\subsubsection{Validation of cast-in-place anchor model under tensile load}

The finite element model was validated by comparing the ultimate tensile load obtained from the finite element analysis with the experimental test results in the literature. Two finite element models, representing the test specimen used by Eligehausen et al. (Eligehausen et al., 1992) were developed and used to validate the numerical model. The concrete block size was $\left(4 h_{e f}+200\right) \mathrm{mm} \times\left(4 h_{e f}+200\right) \mathrm{mm} \times\left(2 h_{e f}\right) \mathrm{mm}$ and the steel anchor diameters were 8-mm and 24-mm with embedment depths of $50 \mathrm{~mm}$ and 150 $\mathrm{mm}$ respectively. The authors used concrete block of $31 \mathrm{MPa}$ cube compressive strength $\left(f_{c c}^{\prime}\right)$ that is approximately equivalent to $25.7 \mathrm{MPa}$ cylinder compressive strength (British Standards Institution Draft for development, 1992; Committee Euro-International du Beton (CEB), 1994). Finite element analysis (FEA) was performed under static loading (strain rate of $\left.10^{-5} \mathrm{~s}^{-1}\right)$.

Mesh sensitivity analysis was carried out to obtain the optimum mesh size that gives converged results with the experimental results. Several models with different mesh sizes 
were developed for the cast-in-place anchors. Table 4-1 presents the effect of mesh size refinement on the ultimate tensile load for the cast-in-place anchor.

Table 4-1: Effect of mesh size refinement on the convergence of ultimate tensile load for cast-in-place anchors

\begin{tabular}{|c|c|c|c|c|c|c|c|c|c|c|}
\hline \multirow[t]{3}{*}{$\begin{array}{c}\text { Model } \\
\text { No. }\end{array}$} & \multirow[t]{3}{*}{$\begin{array}{c}\mathrm{d} \\
(\mathrm{mm})\end{array}$} & \multirow[t]{3}{*}{$\begin{array}{l}\mathrm{h}_{\mathrm{ef}} \\
(\mathrm{mm})\end{array}$} & \multicolumn{3}{|c|}{$\begin{array}{l}\text { Mesh size } \\
(\mathrm{mm})\end{array}$} & \multicolumn{2}{|c|}{$\begin{array}{l}\text { Ultimate tensile } \\
\text { load }(\mathrm{kN})\end{array}$} & \multicolumn{2}{|c|}{$\begin{array}{l}\text { Displacement } \\
(\mathrm{mm})\end{array}$} & \multirow[t]{3}{*}{$\begin{array}{l}\text { Failure } \\
\text { mode }\end{array}$} \\
\hline & & & \multirow[t]{2}{*}{ Anchor } & \multicolumn{2}{|c|}{ Concrete } & \multirow[t]{2}{*}{ FEA } & \multirow[t]{2}{*}{ EXP. } & \multirow[t]{2}{*}{ FEA } & \multirow[t]{2}{*}{ EXP. } & \\
\hline & & & & Min. & Max. & & & & & \\
\hline 1 & \multirow{3}{*}{8} & \multirow{3}{*}{50} & 1 & 2 & 8 & 30.23 & \multirow{3}{*}{31.48} & 1.92 & \multirow{3}{*}{1.68} & \multirow{3}{*}{$\begin{array}{l}\text { Concrete } \\
\text { cone } \\
\text { breakout }\end{array}$} \\
\hline 2 & & & 1 & 1 & 8 & 30.45 & & 1.87 & & \\
\hline 3 & & & 0.75 & 1 & 8 & 32.15 & & 1.75 & & \\
\hline 4 & \multirow{3}{*}{24} & \multirow{3}{*}{150} & 1 & 2 & 8 & 143.98 & \multirow{3}{*}{152.66} & 4.19 & \multirow{3}{*}{4.43} & \multirow{3}{*}{$\begin{array}{c}\text { Concrete } \\
\text { cone } \\
\text { breakout }\end{array}$} \\
\hline 5 & & & 1 & 1 & 8 & 147.14 & & 4.16 & & \\
\hline 6 & & & 0.75 & 1 & 8 & 156.47 & & 4.41 & & \\
\hline
\end{tabular}

Mesh sizes of $1 \times 2 \times 8 \mathrm{~mm}, 1 \times 1 \times 8 \mathrm{~mm}$ and $0.75 \times 1 \times 8 \mathrm{~mm}$ were investigated. The mesh sizes of $1 \times 1 \times 8 \mathrm{~mm}$ and $0.75 \times 1 \times 8 \mathrm{~mm}$ give ultimate load and displacement results converged to the experimental results. The ultimate load and displacement at maximum load obtained using mesh size of $0.75 \times 1 \times 8 \mathrm{~mm}$ were found closer $1.3 \%$ and $7.1 \%$ respectively than that obtained using mesh size of $1 \times 1 \times 8 \mathrm{~mm}$ for the anchor diameter of $8 \mathrm{~mm}$. The ultimate load and displacement were found closer $1.3 \%$ and $6.1 \%$ respectively for the anchor diameter of $24 \mathrm{~mm}$. Mesh size of $(0.75 \times 1 \times 8) \mathrm{mm}$ exhibits good agreement with the experimental results obtained by Eligehausen et al. (Eligehausen et al., 1992) and was selected for the analysis.

Figures 4-3 and 4-4 present a comparison of tensile load-displacement results from experimental tests by Eligehausen et al. (Eligehausen et al., 1992) and the finite element analysis for anchor embedment depths of $50 \mathrm{~mm}$ and $150 \mathrm{~mm}$ respectively. Ultimate tensile load obtained from the FEA (mesh size of $0.75 \times 1 \times 8 \mathrm{~mm}$ ) was found to be $2.1 \%$ and $2.5 \%$ 
higher than the experimental results for the $8-\mathrm{mm}$ and $24-\mathrm{mm}$ diameter anchors respectively while the initial stiffness of the concrete anchorage system is almost the same.

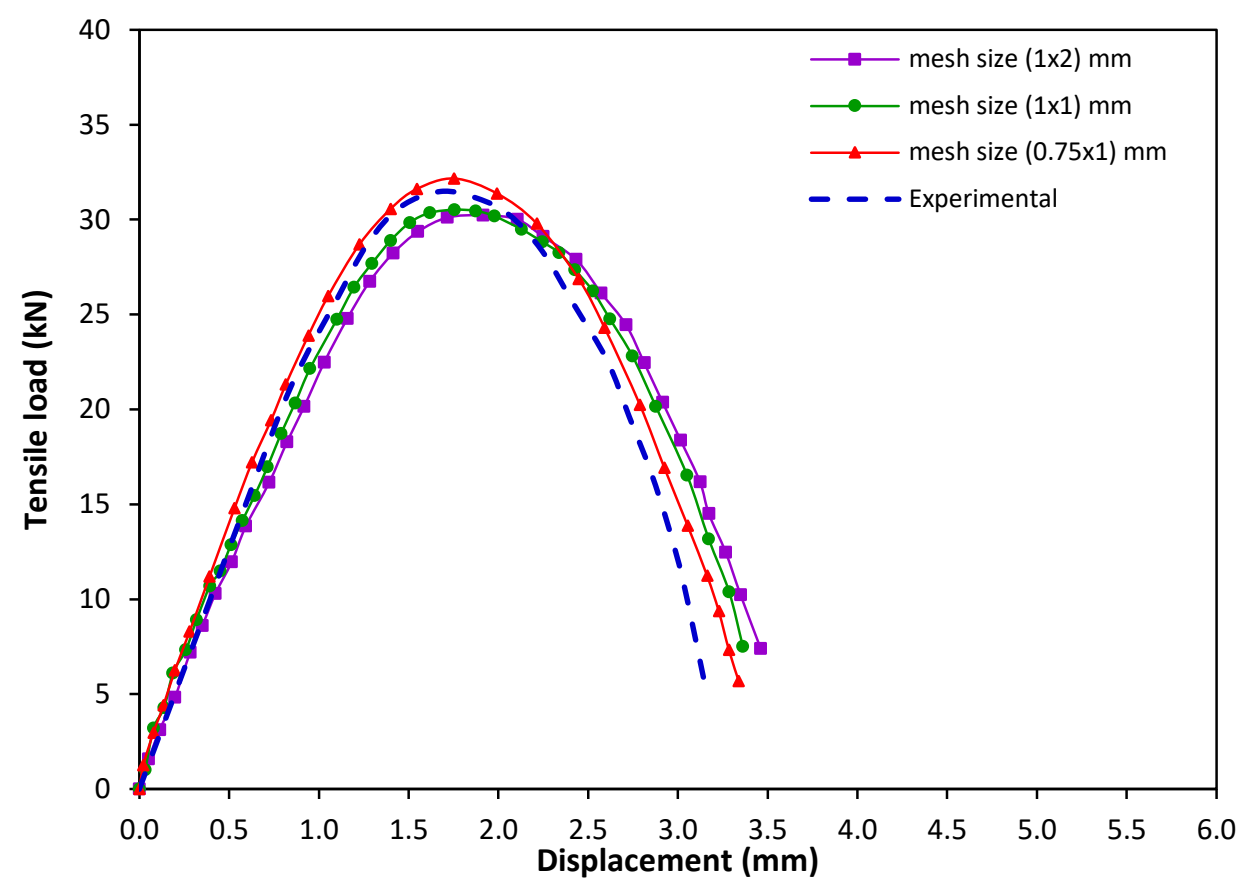

Figure 4-3: Comparison of experimental and numerical tensile load-displacement behaviour of 8-mm diameter cast-in-place anchor with $50 \mathrm{~mm}$ embedment depth

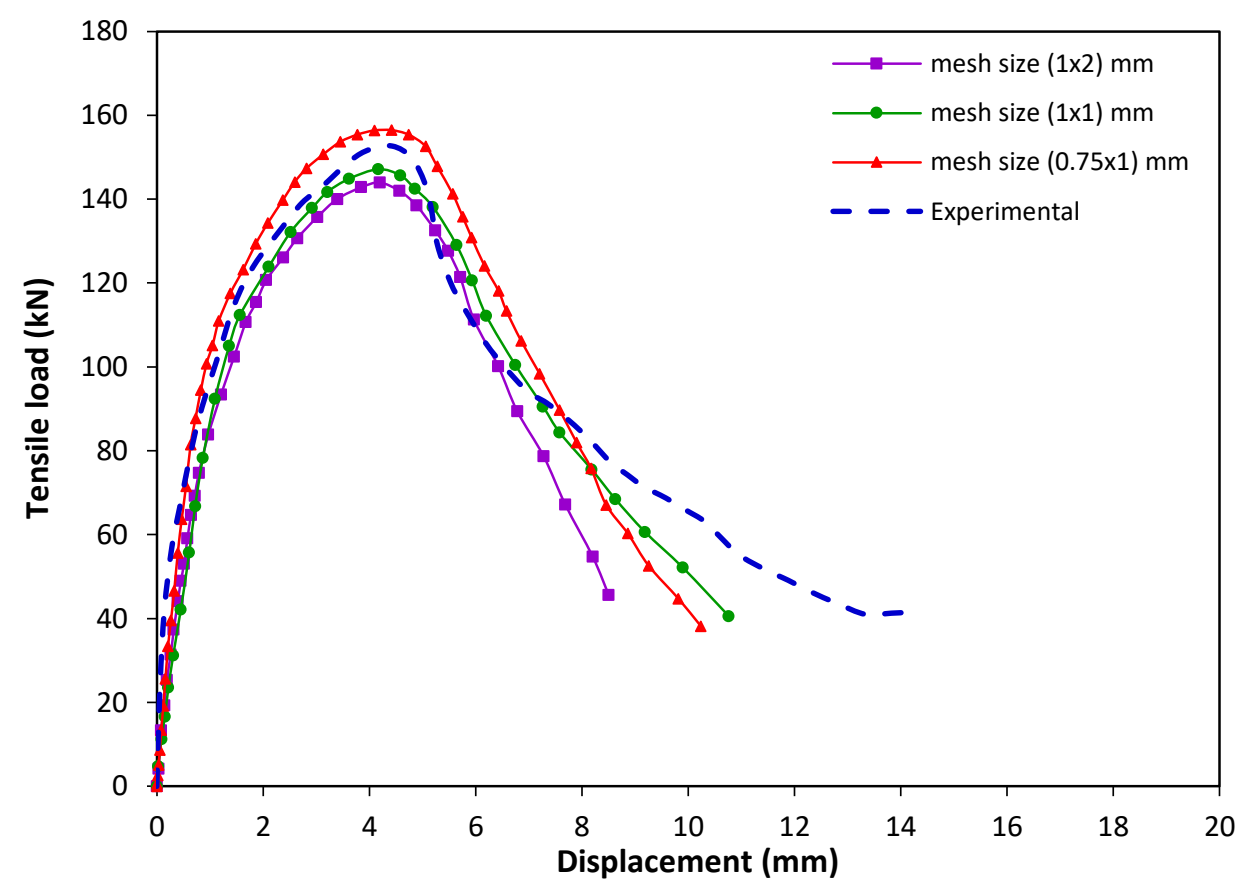

Figure 4-4: Comparison of experimental and numerical tensile load-displacement behaviour of 24-mm diameter cast-in-place anchor with $150 \mathrm{~mm}$ embedment depth 
Concrete cone breakout failure mode was observed from the finite element analysis similar to that obtained from the experimental tests conducted by Eligehausen et al. (Eligehausen et al., 1992). Concrete breakout cone diameters of $160 \mathrm{~mm}$ and $494 \mathrm{~mm}$ were obtained from the FEA analyses for 8-mm and 24-mm diameter anchors with $50 \mathrm{~mm}$ and $150 \mathrm{~mm}$ embedment depths respectively. A good agreement was obtained with the experimental results by Eligehausen et al. (1992) where the concrete cone breakout diameter was reported to be less than $4 h_{e f}(200 \mathrm{~mm}$ and $600 \mathrm{~mm}$ for the $8-\mathrm{mm}$ and $24-\mathrm{mm}$ diameter anchors respectively).

After validation of the cast-in-place anchorage to concrete system numerical model, it was used to investigate the effect of strain rate on the capacity of the anchorage system for various design parameters (anchor diameter and embedment depth). The results of the analysis are presented in the following sections.

\subsubsection{Comparison of finite element results with the ACI and CCD design methods}

Ultimate tensile loads obtained from the finite element analysis at the static strain rate of $10^{-5} \mathrm{~s}^{-1}$ were compared with the results obtained from American Concrete Institute (ACI 349-85) (Fuchs, 2001; Fuchs et al., 1995) and Concrete Capacity Design (CCD) methods. According to ACI design method, the ultimate tensile load of the cast-in-place anchors is the minimum of the concrete breakout load and the steel fracture load. The concrete cone breakout failure load $\left(N_{u o}\right)$ can be determined from Equation (4.1) (Fuchs et al., 1995).

$$
N_{u o}=0.96 \sqrt{f_{c c}^{\prime}} h_{e f}^{2}\left(1+\frac{d_{h}}{h_{e f}}\right)
$$


Where $f_{c c}^{\prime}$ is the cube compressive strength of concrete, $h_{e f}$ is the effective embedment depth, $d_{h}$ is anchor head diameter. Anchor head diameters of $19 \mathrm{~mm}, 24 \mathrm{~mm}$ and $28.5 \mathrm{~mm}$ were used for anchor diameters of 12.7-mm, 15.9-mm and 19.1-mm respectively (Cement Association of Canada, 2010).

The tensile load for steel anchor failure mode can be determined by Equation (4.2).

$N_{u o}=A_{s} f_{u t}$

Where $N_{u o}$, is the tensile load, $A_{s}$ is the effective cross-sectional area of the anchor, $f_{u t}$ is the ultimate tensile strength of the steel. The minimum value of the ultimate tensile load obtained from Equations (4.1) and (4.2) is considered for comparing the design capacity by the ACI method with the finite element results and presented in Table 4-2.

According to CCD Method (Ashour \& Alqedra, 2005; Committee Euro-International du Beton (CEB), 1994; Fuchs, 2001; Fuchs et al., 1995; Subramanian, 2000), the ultimate tensile load of the cast-in-place anchors can be determined for the concrete cone breakout from Equation 4.3.

$N_{u o}=K \sqrt{f_{c c}^{\prime}} h_{e f}^{1.5}$

Where

$K=13.5$ for post installed anchors embedded in uncracked concrete 
$=15.5$ for the cast-in-place anchors embedded in uncracked concrete (Ashour \& Alqedra, 2005; Committee Euro-International du Beton (CEB), 1994; Fuchs, 2001; Fuchs et al., 1995; Subramanian, 2000).

Concrete cube compressive strength of $37 \mathrm{MPa}$, equivalent to a concrete cylinder compressive strengths of $30 \mathrm{MPa}$, was used in Equation (4.1) and Equation (4.3) (British Standards Institution Draft for development, 1992; British Standards Institution, 2013; Committee Euro-International du Beton (CEB), 1994).

Table 4-2 presents a comparison of ultimate tensile loads obtained from the finite element analysis, ACI and CCD methods for the cast-in-place anchors. As shown in the Table 4-2, the ultimate tensile load increased with increasing anchor embedment depth for the finite element analysis and the design methods. In general the results show that the ACI method underpredicts the capacity of the anchorage system. The CCD method gives a better agreement with the finite element method. Similar observation was reported by Fuchs et al. (1995) where the ACI method was reported to underpredict the failure load for shallow embedment depths and unconservative for the deep embedment depths (Fuchs et al., 1995).

Table 4-2: Comparison of ultimate tensile loads obtained from FEA with ACI and CCD design methods

\begin{tabular}{|c|c|c|c|c|c|c|c|}
\hline \multirow{2}{*}{$\begin{array}{c}\mathrm{d} \\
(\mathrm{mm})\end{array}$} & $\begin{array}{c}\mathrm{h}_{\mathrm{ef}} \\
(\mathrm{mm})\end{array}$ & \multicolumn{2}{|c|}{ Ultimate tensile load $(\mathrm{kN})$} & FEA/ACI & FEA/CCD & *Failure mode \\
\cline { 3 - 6 } & $\mathrm{ACI}$ & $\mathrm{CCD}$ & FEA & & & $($ FEA $)$ \\
\hline \multirow{4}{*}{12.7} & 76.2 & 42.36 & 62.71 & 64.48 & 1.52 & 1.03 & $\mathrm{CC}$ \\
\cline { 2 - 8 } & 101.6 & 71.55 & 96.55 & 98.03 & 1.37 & 1.02 & $\mathrm{~S}$ \\
\cline { 2 - 8 } & 127 & 108.28 & 130.98 & 98.64 & 0.91 & 0.75 & $\mathrm{~S}$ \\
\cline { 2 - 8 } & 152.4 & 130.98 & 130.98 & 99.86 & 0.76 & 0.76 & $\mathrm{~S}$ \\
\hline
\end{tabular}




\begin{tabular}{|c|c|c|c|c|c|c|c|}
\hline \multirow{4}{*}{15.9} & 76.2 & 44.59 & 62.71 & 68.61 & 1.54 & 1.09 & CC \\
\cline { 2 - 8 } & 101.6 & 74.52 & 96.55 & 104.17 & 1.40 & 1.08 & $\mathrm{CC}$ \\
\cline { 2 - 8 } & 127 & 111.98 & 134.94 & 163.26 & 1.46 & 1.21 & $\mathrm{~S}$ \\
\cline { 2 - 8 } & 152.4 & 156.98 & 177.38 & 166.35 & 1.06 & 0.94 & $\mathrm{~S}$ \\
\hline \multirow{3}{*}{19.1} & 76.2 & 46.59 & 62.71 & 72.88 & 1.56 & 1.16 & $\mathrm{CC}$ \\
\cline { 2 - 8 } & 101.6 & 77.19 & 96.55 & 116.65 & 1.51 & 1.21 & $\mathrm{CC}$ \\
\cline { 2 - 8 } & 127 & 115.32 & 134.94 & 181.29 & 1.57 & 1.34 & $\mathrm{CC}$ \\
\cline { 2 - 8 } & 152.4 & 160.99 & 177.38 & 241.37 & 1.50 & 1.36 & $\mathrm{~S}$ \\
\hline
\end{tabular}

*Failure mode: $\mathrm{CC}=$ concrete cone breakout, $\mathrm{S}=$ steel anchor failure

\subsubsection{Effect of strain rate on the level of damage and failure mode of cast-in-place} anchors

Plastic strain contours can be used to represent level of damage for concrete (Moutoussamy et al., 2011; Wu et al., 2012). The range of the damage in the concrete is expressed by fringe levels associated with crack pattern. The fringe level ranges from no damage at a value of 0 to complete damage at a value of 1 .

Contours of effective plastic strain for cast-in-place anchor with diameters of $12.7-\mathrm{mm}$, 15.9-mm and 19.1-mm and different embedment depths are shown in Figure 4-5 at strain rate of $10^{-5} \mathrm{~s}^{-1}$. As shown in the figure, for all embedment depths investigated, two failure modes were observed: concrete cone breakout failure and steel anchor failure. Concrete cone breakout failure was observed at embedment depth of $76.2 \mathrm{~mm}$ for all the anchor diameters investigated. At embedment depth of $101.6 \mathrm{~mm}$, concrete cone breakout failure was observed for $15.9 \mathrm{~mm}$ and 19.1-mm diameter anchors while steel anchor failure was observed for the $12.7 \mathrm{~mm}$ diameter anchor. With the increase in the anchor embedment 
depth, the anchor requires higher loads to break the concrete and form concrete cone failure. When the anchor is not capable to afford the high applied tensile load, anchor fracture will occur.

At embedment depths of $127 \mathrm{~mm}$ and $152.4 \mathrm{~mm}$, steel anchor failure was observed for all anchor diameters except for anchor diameter of 19.1-mm with embedment depth of 127 $\mathrm{mm}$ where concrete cone breakout failure was observed. As shown in Figure 4-5, the damage increases with the increase in the anchor diameter from 12.7- $\mathrm{mm}$ to 19.1-mm. The increase in the anchor diameter increases the anchor head diameter and in turns the amount of cracking in the concrete is increased.

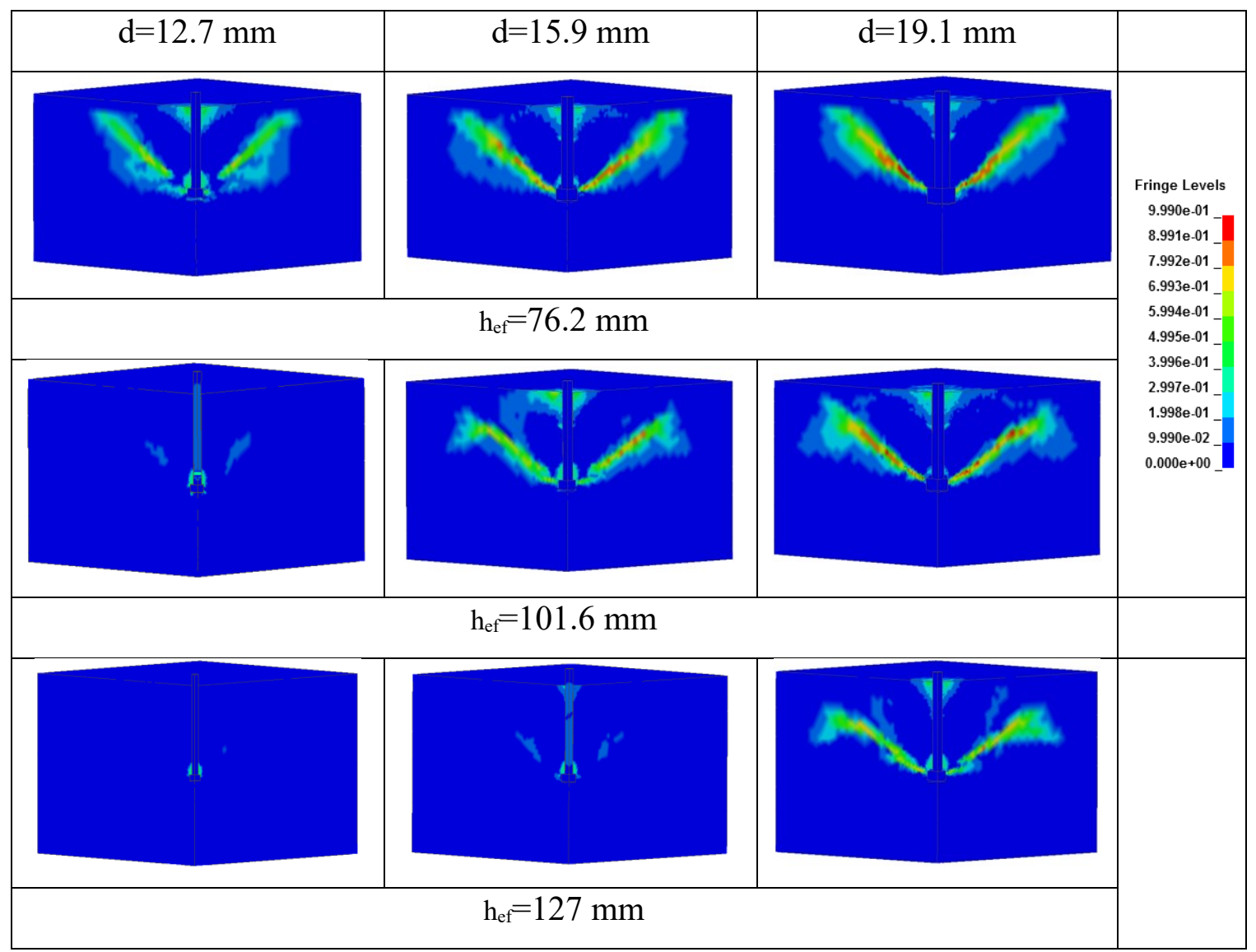




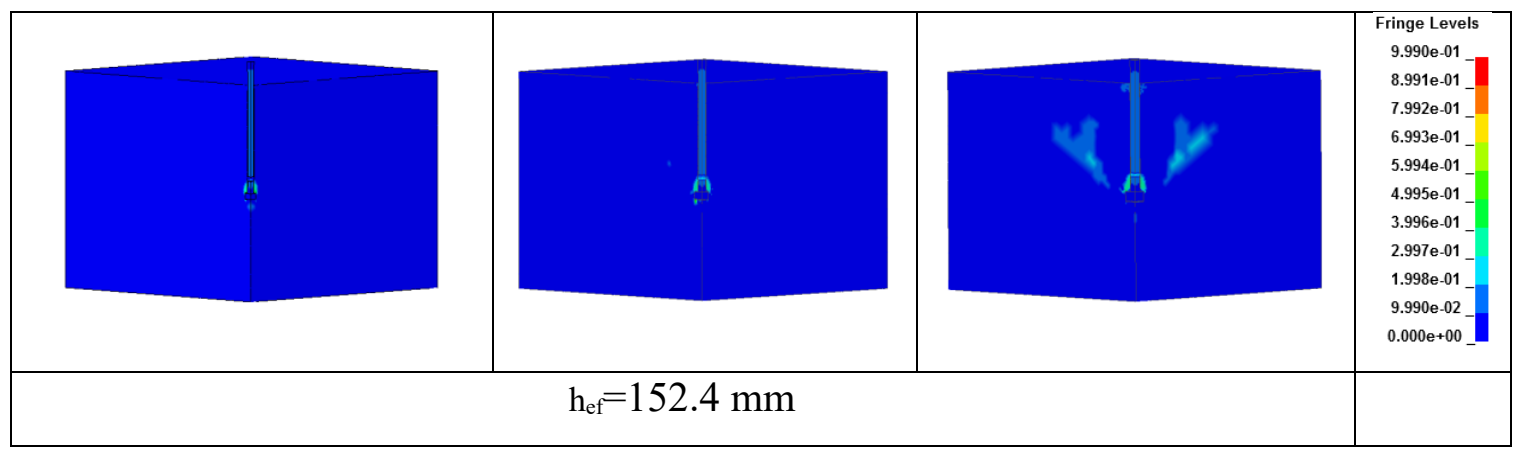

Figure 4-5: Plastic strain contours for cast-in-place anchor at strain rate of $10^{-5} \mathrm{~s}^{-1}$

Concrete cone breakout diameter $\left(d_{\text {cone }}\right)$ of $230 \mathrm{~mm}$ was obtained for the $12.7-\mathrm{mm}$ diameter anchor with embedment depth of $76.2 \mathrm{~mm}$. An increase in the concrete cone breakout diameter from $246 \mathrm{~mm}$ to $292 \mathrm{~mm}$ was obtained for the increase in the embedment depth from $76.2 \mathrm{~mm}$ to $101.6 \mathrm{~mm}$ for the $15.9-\mathrm{mm}$ diameter anchor. The increase in the concrete cone breakout diameter was from $255 \mathrm{~mm}$ to $390 \mathrm{~mm}$ for the increase in the embedment depth from $76.2 \mathrm{~mm}$ to $127 \mathrm{~mm}$ for the $19.1-\mathrm{mm}$ diameter anchor. The concrete cone breakout diameter increased with increase in the anchor embedment depth. Hence, the load required to cause failure in the concrete increased due to the increase in the size of concrete above the anchor head for the deep embedment depths. Figure 4-6 shows the relation between the concrete cone breakout diameter, embedment depth and anchor diameter. 


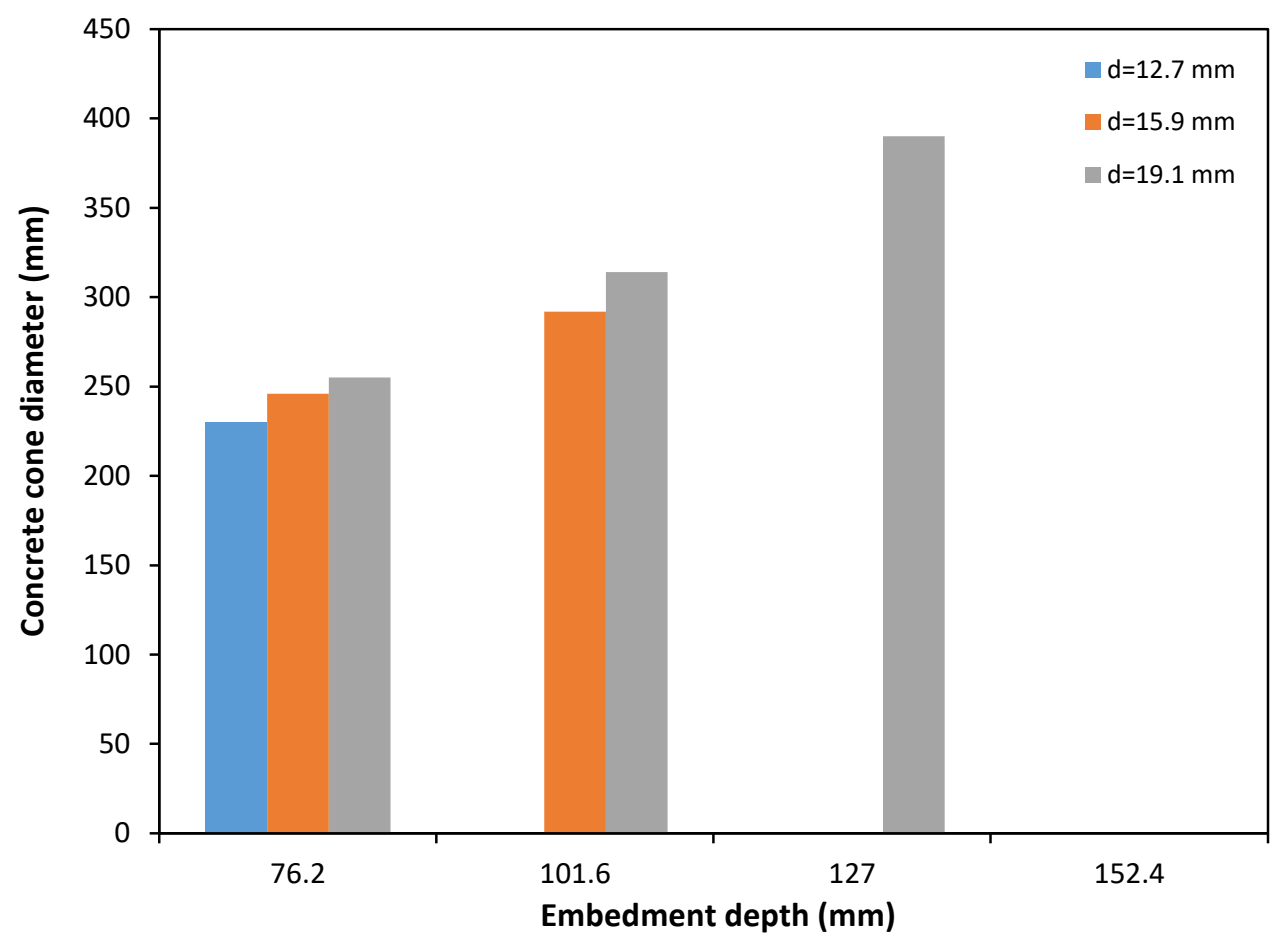

Figure 4-6: Effect of anchor embedment depth on the concrete cone diameter

The concrete cone breakout angle was determined by measuring the angle of inclination of the failure surface. Figure 4-7 shows the concrete cone breakout angle $\left(\theta_{a}\right)$ and the crack propagation angle $\left(\theta_{b}\right)$. Where, the crack propagation angle is equal to $90-\theta_{a}$. Table $4-3$ shows the concrete cone breakout angle, cone diameter and corresponding failure mode for the embedment depths and diameters of the cast-in-place anchor under tensile load investigated. As shown in Table 4-3, average concrete cone breakout angle was $31.8^{\circ}$. 


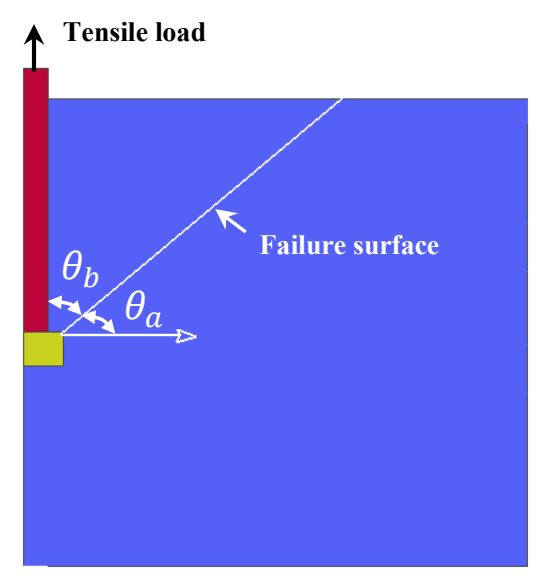

Figure 4-7: Cone breakout and crack propagation angles on the cast-in-place anchorage to concrete system

Table 4-3: Concrete cone diameter and cone breakout angle for the cast-in-place anchor at the static strain rate

\begin{tabular}{|c|c|c|c|c|}
\hline $\begin{array}{c}\mathrm{h}_{\mathrm{ef}} \\
(\mathrm{mm})\end{array}$ & $\begin{array}{c}\mathrm{d} \\
(\mathrm{mm})\end{array}$ & $\begin{array}{c}\mathrm{d}_{\text {cone }} \\
(\mathrm{mm})\end{array}$ & $\begin{array}{c}\theta_{a} \\
(\mathrm{deg})\end{array}$ & Failure mode \\
\hline \multirow{3}{*}{76.2} & 12.7 & 230 & 32 & $\mathrm{CC}$ \\
\cline { 2 - 5 } & 15.9 & 246 & 32 & $\mathrm{CC}$ \\
\cline { 2 - 5 } & 19.1 & 255 & 33 & $\mathrm{CC}$ \\
\hline \multirow{3}{*}{101.6} & 12.7 & - & - & $\mathrm{S}$ \\
\cline { 2 - 5 } & 15.9 & 292 & 31 & $\mathrm{CC}$ \\
\hline \multirow{3}{*}{127} & 19.1 & 314 & 31 & $\mathrm{CC}$ \\
\cline { 2 - 5 } & 12.7 & - & - & $\mathrm{S}$ \\
\cline { 2 - 5 } & 15.9 & - & - & $\mathrm{CC}$ \\
\hline \multirow{3}{*}{152.4} & 19.1 & 390 & 32 & $\mathrm{~S}$ \\
\cline { 2 - 5 } & 12.7 & - & - & $\mathrm{S}$ \\
\cline { 2 - 5 } & 15.9 & - & - & $\mathrm{S}$ \\
\hline
\end{tabular}

The failure mode of 12.7-mm, 15.9-mm and 19.1-mm diameter cast-in-place anchors with different embedment depths at strain rates ranging from $10^{-3} \mathrm{~s}^{-1}$ to $10^{3} \mathrm{~s}^{-1}$ is presented in Figures 4-8, 4-9 and 4-10 respectively. At early stage of loading, cracks started to appear above the anchor head, and with further increase in time the cracks appeared on the top 
surface of the concrete around the anchor circumference. As shown in Figure 4-8, for the anchor diameter of 12.7- $\mathrm{mm}$ with shallow embedment depth of $76.2 \mathrm{~mm}$, cracks grow along the embedment depth and transferred to a wider area on the concrete. These cracks then propagated diagonally forming concrete cone and leads to concrete cone breakout failure at strain rates up to $10 \mathrm{~s}^{-1}$. At high strain rates of $10^{2} \mathrm{~s}^{-1}$ and $10^{3} \mathrm{~s}^{-1}$ steel anchor failure was observed. For embedment depths of $101.6 \mathrm{~mm}, 127 \mathrm{~mm}$ and $152.4 \mathrm{~mm}$, steel anchor failure is the dominant failure mode at the strain rates ranging from $10^{-3} \mathrm{~s}^{-1}$ to $10^{3}$ $\mathrm{s}^{-1}$.

As shown in Figure 4-9, the failure mode is concrete cone breakout failure for the anchor diameter of $15.9-\mathrm{mm}$ with embedment depths of $76.2 \mathrm{~mm}$ and $101.6 \mathrm{~mm}$ at strain rates of $10^{-3} \mathrm{~s}^{-1}$ to $10 \mathrm{~s}^{-1}$. Concrete cracking was observed at strain rate of $10^{2} \mathrm{~s}^{-1}$ whereas steel anchor failure was observed at strain rate of $10^{3} \mathrm{~s}^{-1}$. For the anchor embedment depths of $127 \mathrm{~mm}$ and $152.4 \mathrm{~mm}$ steel anchor failure was observed at all the strain rates investigated.

Figure 4-10 shows concrete cone breakout failure mode for the 19.1-mm anchor diameter with embedment depths of $76.2 \mathrm{~mm}$ and $101.6 \mathrm{~mm}$ at strain rates ranging from $10^{-3} \mathrm{~s}^{-1}$ to $10 \mathrm{~s}^{-1}$. Concrete cracking was observed at strain rates of $10^{2} \mathrm{~s}^{-1}$ while steel anchor failure was observed at strain rate of $10^{3} \mathrm{~s}^{-1}$. The increase in the strain rate increased the concrete strength and thus resulting in steel anchor failure. At embedment depth of $127 \mathrm{~mm}$ concrete cone breakout failure was observed at strain rates of $10^{-3} \mathrm{~s}^{-1}$ to $10 \mathrm{~s}^{-1}$. Steel anchor failure was observed at strain rates of $10^{2} \mathrm{~s}^{-1}$ and $10^{3} \mathrm{~s}^{-1}$. Also, steel anchor failure was observed at embedment depth of $152.4 \mathrm{~mm}$ at all the strain rates investigated. 


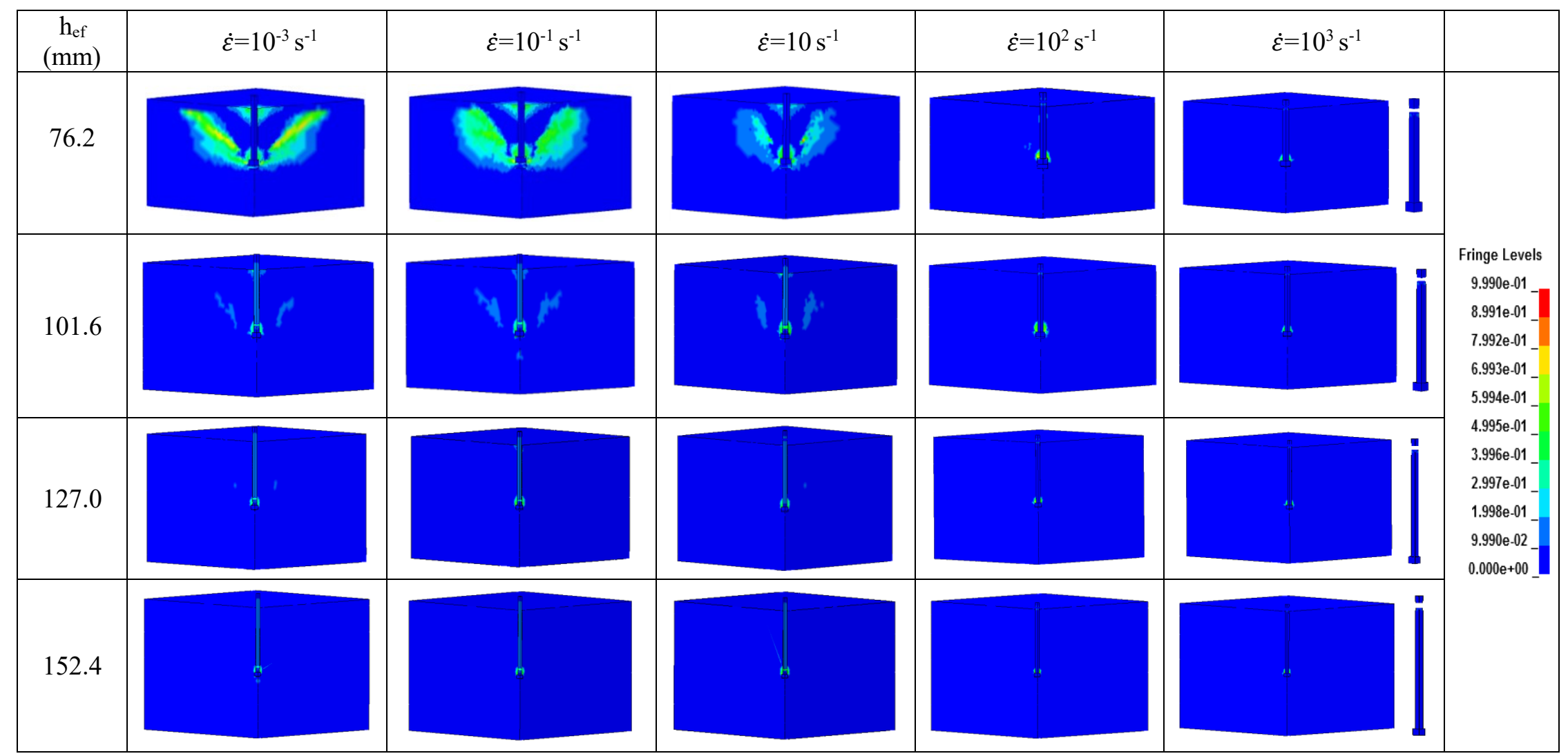

Figure 4-8: Failure mode of $12.7 \mathrm{~mm}$ diameter cast-in-place anchor at different strain rates 


\begin{tabular}{|c|c|c|c|c|c|c|}
\hline $\begin{array}{c}\mathrm{h}_{\mathrm{ef}} \\
(\mathrm{mm})\end{array}$ & $\dot{\varepsilon}=10^{-3} \mathrm{~s}^{-1}$ & $\dot{\varepsilon}=10^{-1} \mathrm{~s}^{-1}$ & $\dot{\varepsilon}=10 \mathrm{~s}^{-1}$ & $\dot{\varepsilon}=10^{2} \mathrm{~s}^{-1}$ & $\dot{\varepsilon}=10^{3} \mathrm{~s}^{-1}$ & \\
\hline 76.2 & & & & & A & \\
\hline 101.6 & & & & & 11 & $\begin{array}{l}9.990 \cdot 01 \\
8.911 .01\end{array}$ \\
\hline 127.0 & & & & Al & 4i. & $\begin{array}{l}2.997 \cdot .01 \\
1098\end{array}$ \\
\hline 152.4 & म & $\pi$ & 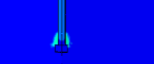 & " & , & \\
\hline
\end{tabular}

Figure 4-9: Failure mode of $15.9 \mathrm{~mm}$ diameter cast-in-place anchor at different strain rates 


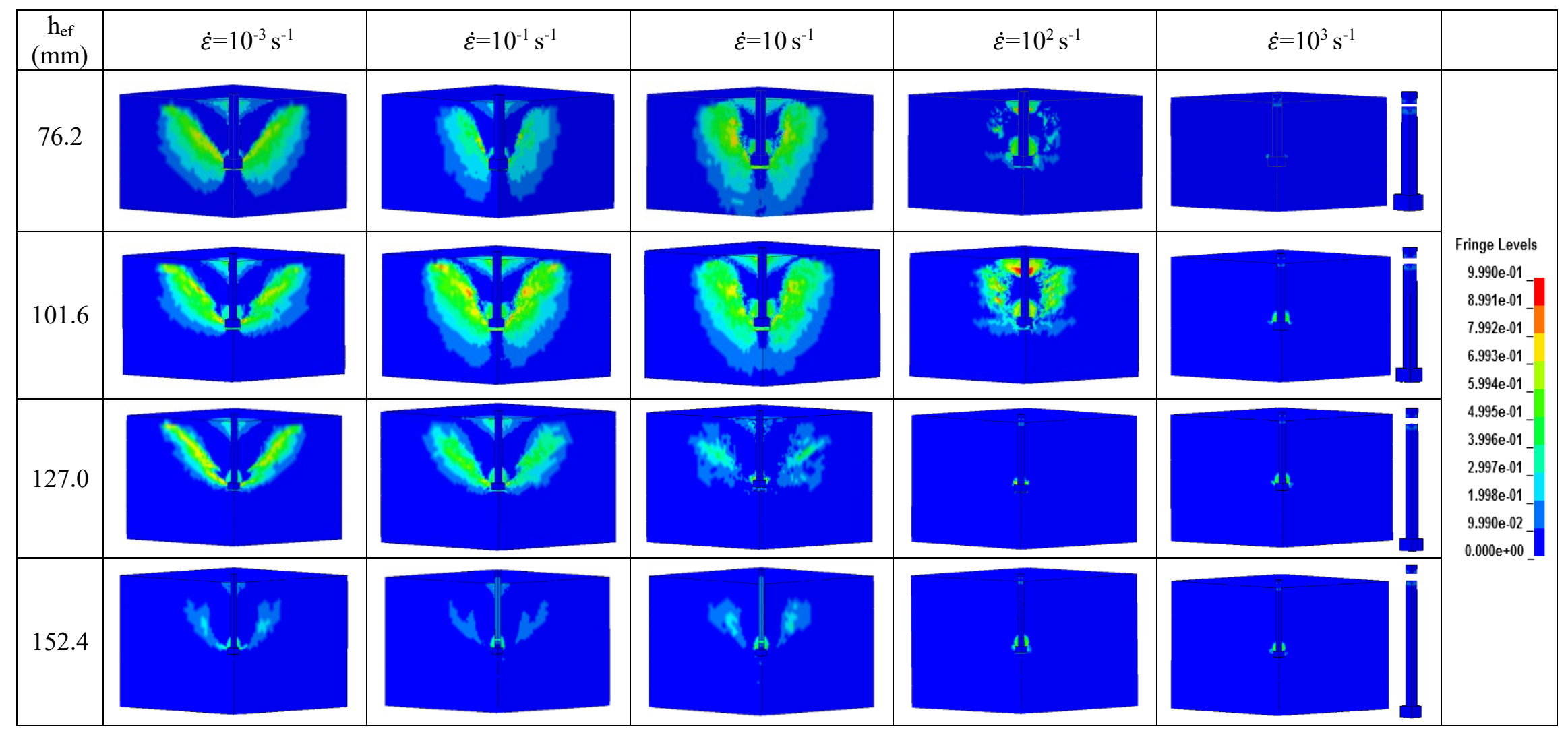

Figure 4-10: Failure mode of $19.1 \mathrm{~mm}$ diameter cast-in-place anchor at different strain rates 
From Figures 4-8, 4-9 and 4-10, it can be seen that the failure mode is affected by the strain rate. The increase in the strain rate decreased the concrete cone breakout diameter, however the increase in the strain rate increased the strength of the concrete and steel resulted in increasing the failure load. Table 4-4 shows the failure mode for cast-in-place anchors under tensile load at different strain rates.

Table 4-4: Failure mode for cast-in-place anchors under tensile load at different strain

\begin{tabular}{|c|c|c|c|c|c|c|c|c|}
\hline \multirow{3}{*}{$\begin{array}{c}\text { Model } \\
\text { No. }\end{array}$} & \multirow{3}{*}{$\begin{array}{c}d \\
(\mathrm{~mm})\end{array}$} & \multirow{3}{*}{$\begin{array}{c}\mathbf{h}_{\text {ef }} \\
(\mathbf{m m})\end{array}$} & \multicolumn{6}{|c|}{ Failure mode $^{*}$} \\
\hline & & & \multicolumn{6}{|c|}{ Strain rate $\left(\mathrm{s}^{-1}\right)$} \\
\hline & & & $\dot{\varepsilon}=10^{-5}$ & $\dot{\varepsilon}=10^{-3}$ & $\dot{\varepsilon}=10^{-1}$ & $\dot{\varepsilon}=10$ & $\dot{\varepsilon}=10^{2}$ & $\dot{\varepsilon}=10^{3}$ \\
\hline 1 & 12.7 & 76.2 & $\mathrm{CC}$ & $\mathrm{CC}$ & $\mathrm{CC}$ & $\mathrm{CC}$ & $\mathrm{S}$ & $\mathrm{S}$ \\
\hline 2 & 12.7 & 101.6 & $\mathrm{~S}$ & $\mathrm{~S}$ & $\mathrm{~S}$ & $\mathrm{~S}$ & $\mathrm{~S}$ & $\mathrm{~S}$ \\
\hline 3 & 12.7 & 127.0 & $\mathrm{~S}$ & $\mathrm{~S}$ & $\mathrm{~S}$ & $\mathrm{~S}$ & $\mathrm{~S}$ & $\mathrm{~S}$ \\
\hline 4 & 12.7 & 152.4 & $\mathrm{~S}$ & $\mathrm{~S}$ & $\mathrm{~S}$ & $\mathrm{~S}$ & $\mathrm{~S}$ & $\mathrm{~S}$ \\
\hline 5 & 15.9 & 76.2 & $\mathrm{CC}$ & $\mathrm{CC}$ & $\mathrm{CC}$ & $\mathrm{CC}$ & $\mathrm{COC}$ & $\mathrm{S}$ \\
\hline 6 & 15.9 & 101.6 & $\mathrm{CC}$ & $\mathrm{CC}$ & $\mathrm{CC}$ & $\mathrm{CC}$ & $\mathrm{COC}$ & $\mathrm{S}$ \\
\hline 7 & 15.9 & 127.0 & $\mathrm{~S}$ & $\mathrm{~S}$ & $\mathrm{~S}$ & $\mathrm{~S}$ & $\mathrm{~S}$ & $\mathrm{~S}$ \\
\hline 8 & 15.9 & 152.4 & $\mathrm{~S}$ & $\mathrm{~S}$ & $\mathrm{~S}$ & $\mathrm{~S}$ & $\mathrm{~S}$ & $\mathrm{~S}$ \\
\hline 9 & 19.1 & 76.2 & $\mathrm{CC}$ & $\mathrm{CC}$ & $\mathrm{CC}$ & $\mathrm{CC}$ & $\mathrm{COC}$ & $\mathrm{S}$ \\
\hline 10 & 19.1 & 101.6 & $\mathrm{CC}$ & $\mathrm{CC}$ & $\mathrm{CC}$ & $\mathrm{CC}$ & $\mathrm{COC}$ & $\mathrm{S}$ \\
\hline 11 & 19.1 & 127.0 & $\mathrm{CC}$ & $\mathrm{CC}$ & $\mathrm{CC}$ & $\mathrm{CC}$ & $\mathrm{S}$ & $\mathrm{S}$ \\
\hline 12 & 19.1 & 152.4 & $\mathrm{~S}$ & $\mathrm{~S}$ & $\mathrm{~S}$ & $\mathrm{~S}$ & $\mathrm{~S}$ & $\mathrm{~S}$ \\
\hline
\end{tabular}

*Failure mode: $\mathrm{CC}=$ concrete cone breakout, $\mathrm{COC}=$ concrete cracking, $\mathrm{S}=$ steel anchor failure

The crack propagation angle $\left(\theta_{b}\right)$ was measured for the cast-in-place anchors at the ultimate tensile load and presented in Table 4-5. As shown in Table 4-5 and Figures 4-8, 4-9 and 410 , the crack propagation angle decreased with increasing the strain rate for the cast-inplace anchors where concrete cone breakout failure is observed. 
Table 4-5: Crack propagation angle for the cast-in-place anchors at different strain rates

\begin{tabular}{|c|c|c|c|c|c|c|c|c|}
\hline \multirow{3}{*}{$\begin{array}{c}\text { Model } \\
\text { No. }\end{array}$} & \multirow{3}{*}{$\begin{array}{c}\mathrm{d} \\
(\mathrm{mm})\end{array}$} & \multirow{3}{*}{$\begin{array}{c}\mathbf{h}_{\text {ef }} \\
(\mathbf{m m})\end{array}$} & \multicolumn{6}{|c|}{ Crack propagation angle (deg) } \\
\hline & & & \multicolumn{6}{|c|}{ Strain rate $\left(\mathrm{s}^{-1}\right)$} \\
\hline & & & $\dot{\varepsilon}=10^{-5}$ & $\dot{\varepsilon}=10^{-3}$ & $\dot{\varepsilon}=10^{-1}$ & $\dot{\varepsilon}=10$ & $\dot{\varepsilon}=10^{2}$ & $\dot{\varepsilon}=10^{3}$ \\
\hline 1 & 12.7 & 76.2 & 58 & 56 & 53 & 50 & - & - \\
\hline 2 & 12.7 & 101.6 & - & - & - & - & - & - \\
\hline 3 & 12.7 & 127.0 & - & - & - & - & - & - \\
\hline 4 & 12.7 & 152.4 & - & - & - & - & - & - \\
\hline 5 & 15.9 & 76.2 & 58 & 56 & 53 & 48 & - & - \\
\hline 6 & 15.9 & 101.6 & 59 & 57 & 55 & 50 & - & - \\
\hline 7 & 15.9 & 127.0 & - & - & - & - & - & - \\
\hline 8 & 15.9 & 152.4 & - & - & - & - & - & - \\
\hline 9 & 19.1 & 76.2 & 57 & 55 & 50 & 46 & - & - \\
\hline 10 & 19.1 & 101.6 & 59 & 56 & 50 & 46 & - & - \\
\hline 11 & 19.1 & 127.0 & 58 & 55 & 51 & 48 & - & - \\
\hline 12 & 19.1 & 152.4 & - & - & - & - & - & - \\
\hline
\end{tabular}

\subsubsection{Effect of design parameters on failure mode and ultimate tensile load}

Figures 4-11, 4-12 and 4-13 show the effect of strain rate on the failure mode and ultimate tensile load for the $12.7-\mathrm{mm}, 15.9-\mathrm{mm}$ and $19.1-\mathrm{mm}$ diameter cast-in-place anchors respectively. As shown in Figure 4-11, the tensile load increased from $64.5 \mathrm{kN}$ to $79.4 \mathrm{kN}$ for the $76.2 \mathrm{~mm}$ embedment depth at the strain rates up to $10 \mathrm{~s}^{-1}$ where concrete cone breakout failure was observed. At strain rate of $10^{2} \mathrm{~s}^{-1}$ the ultimate tensile load increased to $105.3 \mathrm{kN}$ where steel anchor failure was observed. At high strain rate of $10^{3} \mathrm{~s}^{-1}$ insignificant increase in the ultimate tensile load was obtained where steel anchor failure was observed. Steel anchor failure was observed for $101.6 \mathrm{~mm}, 127 \mathrm{~mm}$ and $152.4 \mathrm{~mm}$ embedment depths for all the strain rates investigated. The increase in the strain rate from $10^{-5} \mathrm{~s}^{-1}$ to $10^{3} \mathrm{~s}^{-1}$ increased the ultimate tensile load $12.6 \%, 12 \%$ and $10.6 \%$ for the anchor embedment depths of $101.6 \mathrm{~mm}, 127 \mathrm{~mm}$ and $152.4 \mathrm{~mm}$ respectively. 


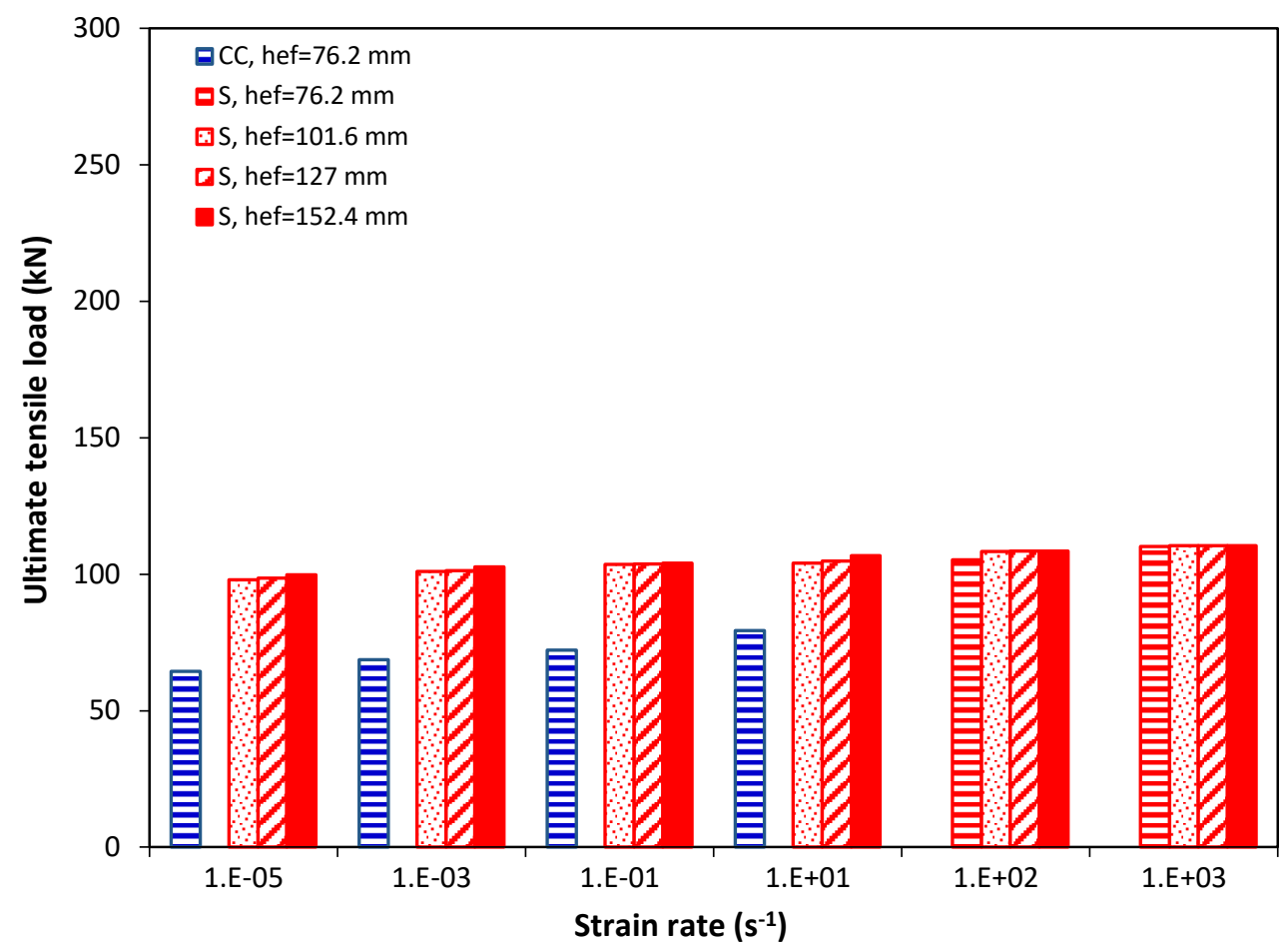

Figure 4-11: Strain rate effect on the failure mode and ultimate tensile load for cast-inplace anchor diameter of $12.7 \mathrm{~mm}$

As shown in Figure 4-12, the tensile load increased from $68.61 \mathrm{kN}$ to $98.14 \mathrm{kN}$ and from $104.17 \mathrm{kN}$ to $128.75 \mathrm{kN}$ for the $76.2 \mathrm{~mm}$ and $101.6 \mathrm{~mm}$ embedment depths as the strain rate increased from $10^{-5} \mathrm{~s}^{-1}$ to $10 \mathrm{~s}^{-1}$ where concrete cone breakout failure was observed. Further increase in the strain rate to $10^{2} \mathrm{~s}^{-1}$ and $10^{3} \mathrm{~s}^{-1}$ increased the ultimate tensile load, where concrete cracking and steel anchor failure were observed at strain rates of $10^{2} \mathrm{~s}^{-1}$ and $10^{3} \mathrm{~s}^{-1}$ respectively. Also, steel anchor failure was observed for $127 \mathrm{~mm}$ and $152.4 \mathrm{~mm}$ embedment depths for all the strain rates investigated. The increase in the strain rate from $10^{-5} \mathrm{~s}^{-1}$ to $10^{3} \mathrm{~s}^{-1}$ increased the ultimate tensile load $17.4 \%$ and $16.5 \%$ for the anchor embedment depths of $127 \mathrm{~mm}$ and $152.4 \mathrm{~mm}$ respectively. 


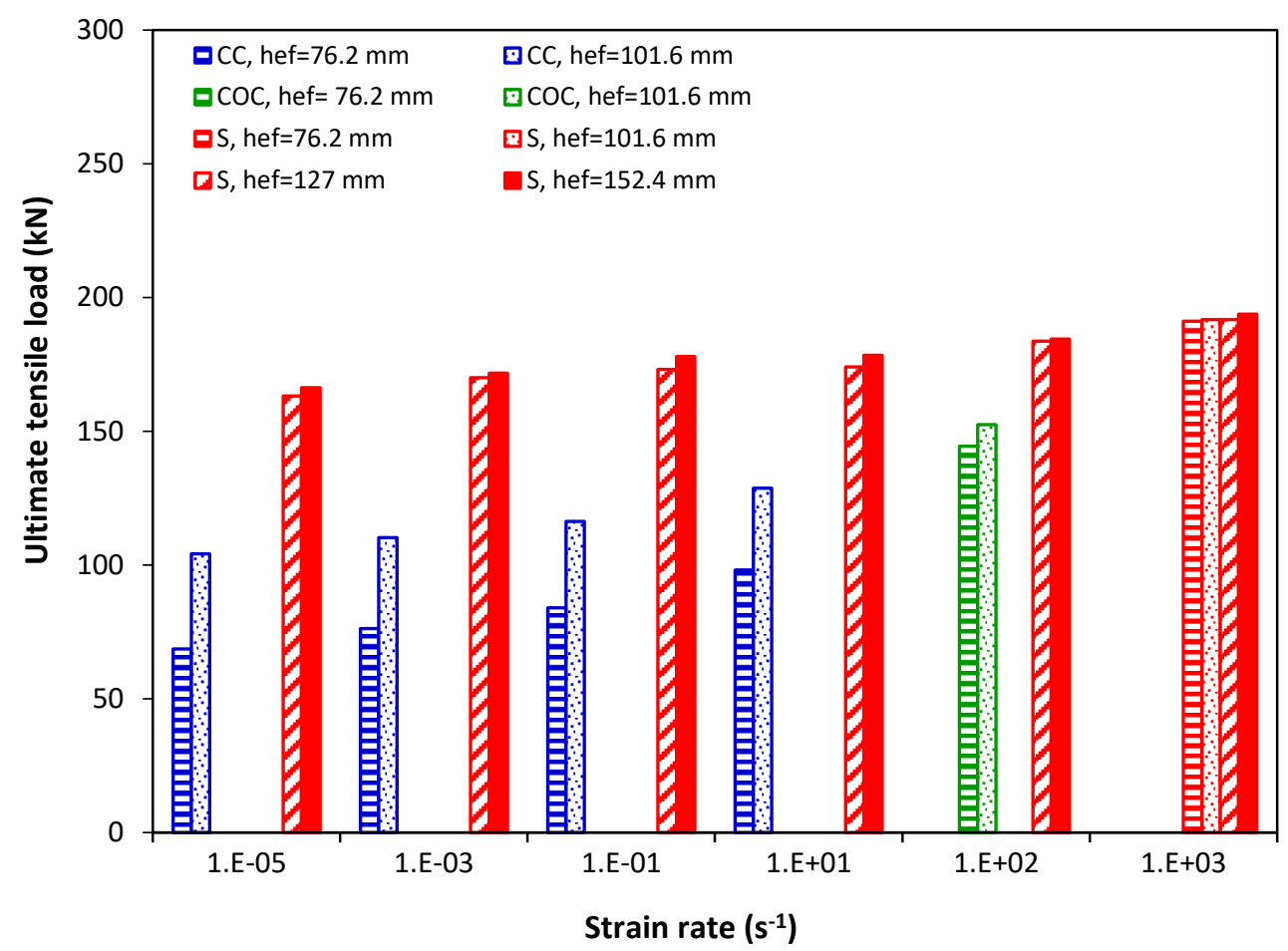

Figure 4-12: Strain rate effect on the failure mode and ultimate tensile load for cast-inplace anchor diameter of $15.9 \mathrm{~mm}$

As shown in Figure 4-13, the tensile load increased with the increase in the strain rates up to $10 \mathrm{~s}^{-1}$ for anchor embedment depths of $76.2 \mathrm{~mm}, 101.6 \mathrm{~mm}$ and $127 \mathrm{~mm}$ where concrete cone breakout failure was observed. The ultimate tensile load increased with further increase in the strain rate to $10^{2} \mathrm{~s}^{-1}$ where concrete cracking was observed for embedment depths of $76.2 \mathrm{~mm}$ and $101.6 \mathrm{~mm}$ while steel anchor failure was observed for embedment depth of $127 \mathrm{~mm}$. At high strain rate of $10^{3} \mathrm{~s}^{-1}$, the ultimate tensile load increased where steel anchor failure was observed. Also, steel anchor failure was observed for $152.4 \mathrm{~mm}$ embedment depth at all the strain rates investigated with an increase in the ultimate tensile load of $13.4 \%$. 


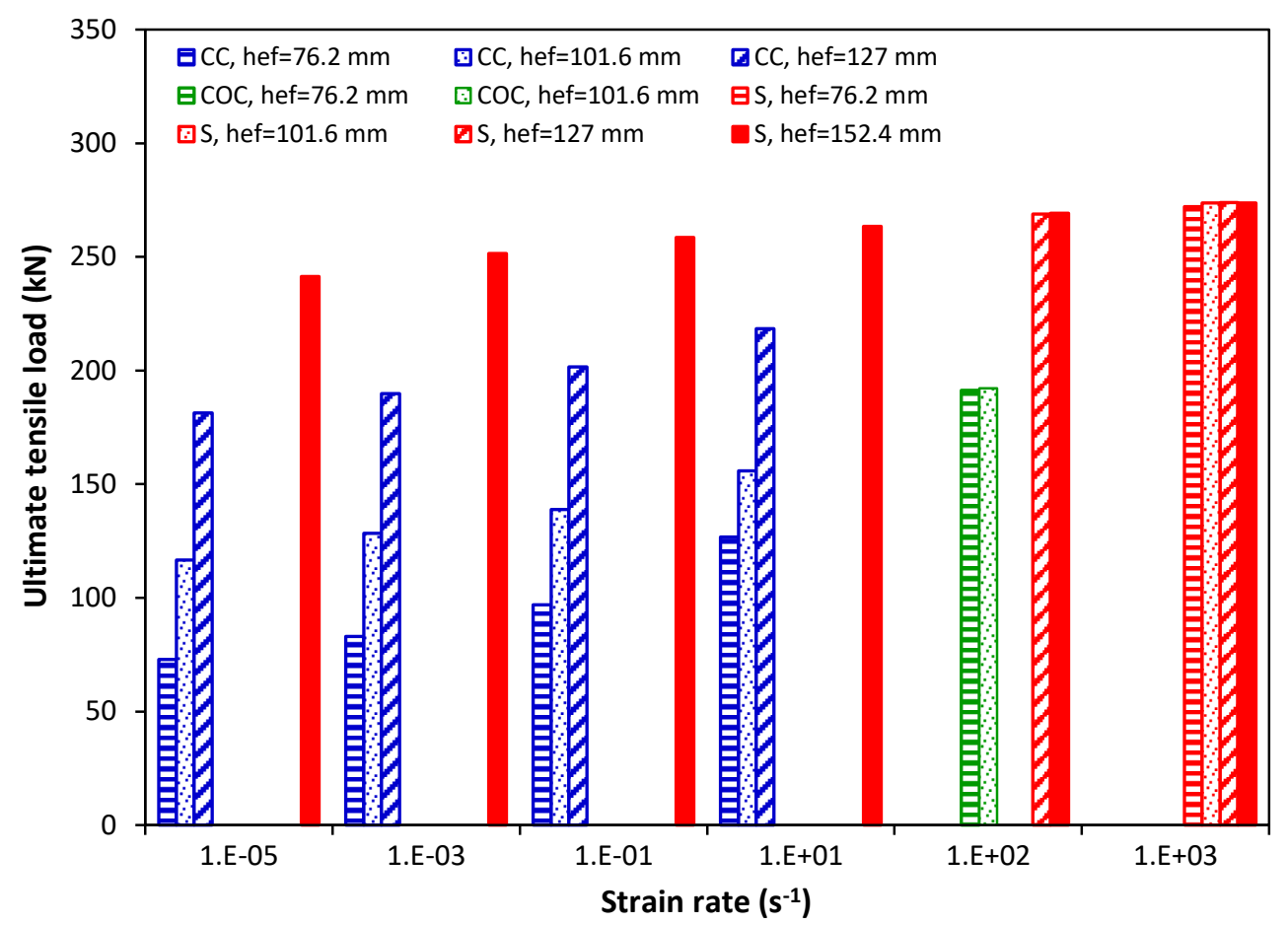

Figure 4-13: Strain rate effect on the failure mode and ultimate tensile load for cast-inplace anchor diameter of $19.1 \mathrm{~mm}$

\subsubsection{Effect of anchor embedment depth on the concrete cone depth}

Figures 4-14, 4-15 and 4-16 show the effect of anchor embedment depth on concrete cone depth and ultimate tensile load for 12.7-mm, 15.9-mm and 19.1-mm diameter cast-in-place anchors respectively. Different concrete cone depths are obtained from the models for tension response at strain rate of $10^{-5} \mathrm{~s}^{-1}$ for the embedment depths of $76.2 \mathrm{~mm}, 101.6 \mathrm{~mm}$, $127 \mathrm{~mm}$, and $152.4 \mathrm{~mm}$. As shown in Figure 4-14, for the 12.7-mm diameter cast-in-place anchor, concrete cone breakout is obtained at embedment depth of $76.2 \mathrm{~mm}$. For anchor embedment depths of $101.6 \mathrm{~mm}, 127 \mathrm{~mm}$ and $152.4 \mathrm{~mm}$ steel anchor failure mode was observed. For the anchor diameter of 15.9-mm (Figure 4-15), concrete cone breakout failure is obtained for the embedment depths of $76.2 \mathrm{~mm}$ and $101.6 \mathrm{~mm}$ whereas steel 
anchor failure is observed for the embedment depths of $127 \mathrm{~mm}$ and $152.4 \mathrm{~mm}$. For the 19.1-mm diameter cast-in-place anchor, as shown in Figure 4-16, the concrete cone depth increased with the increase in the embedment depth from $76.2 \mathrm{~mm}$ to $127 \mathrm{~mm}$ where concrete cone breakout failure was observed. Steel anchor failure was observed at embedment depth of $152.4 \mathrm{~mm}$. From Figures 4-14, 4-15 and 4-16, it can be seen that the ultimate tensile load increases with the increase in the anchor diameter. Also, the ultimate tensile load increased with the increase in the embedment depth when the concrete cone breakout failure or transition from concrete cone breakout failure to steel anchor failure is observed.

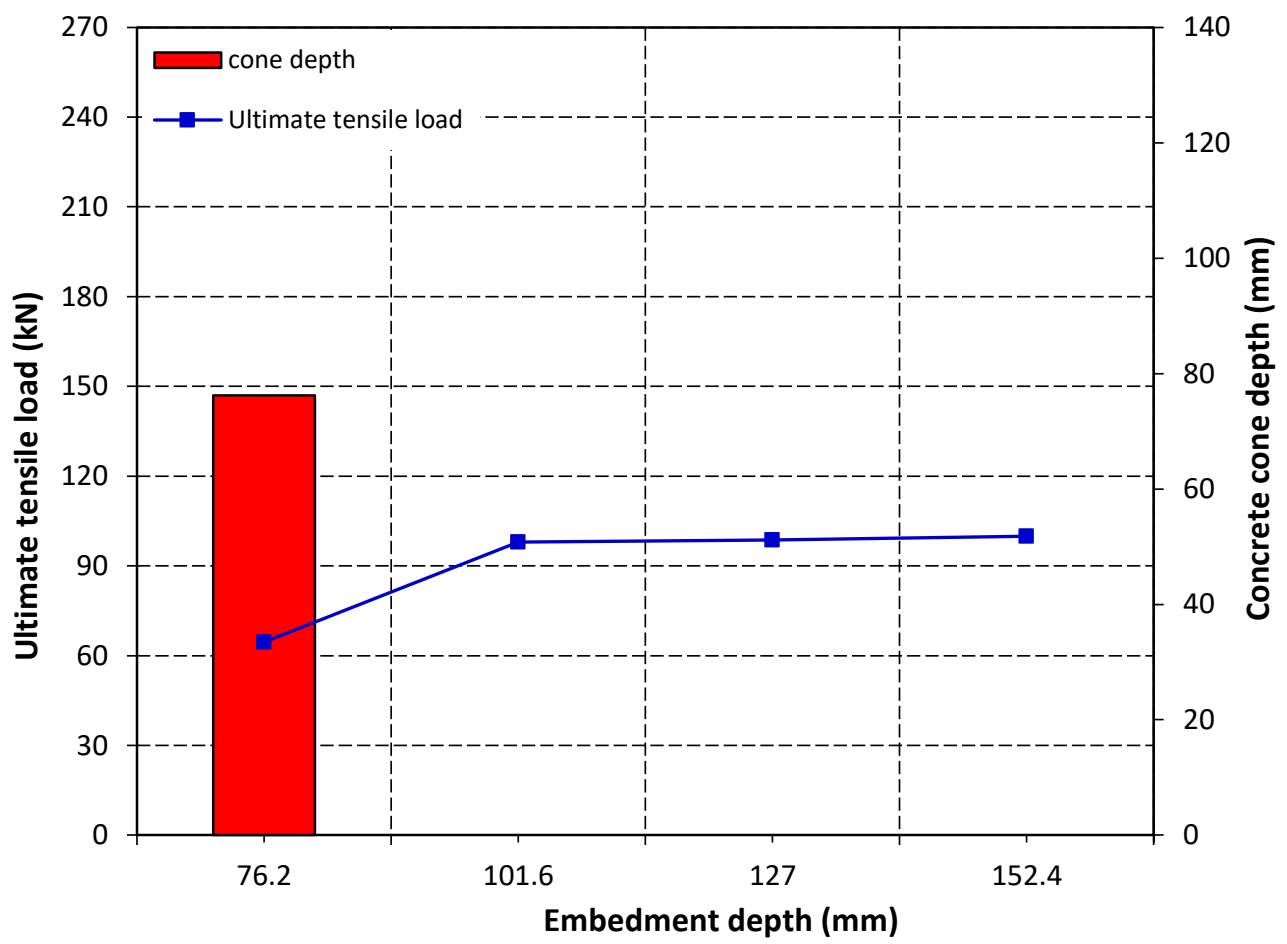

Figure 4-14: Effect of anchor embedment depth on the concrete cone depth and ultimate tensile load for $12.7 \mathrm{~mm}$ diameter cast-in-place anchor 


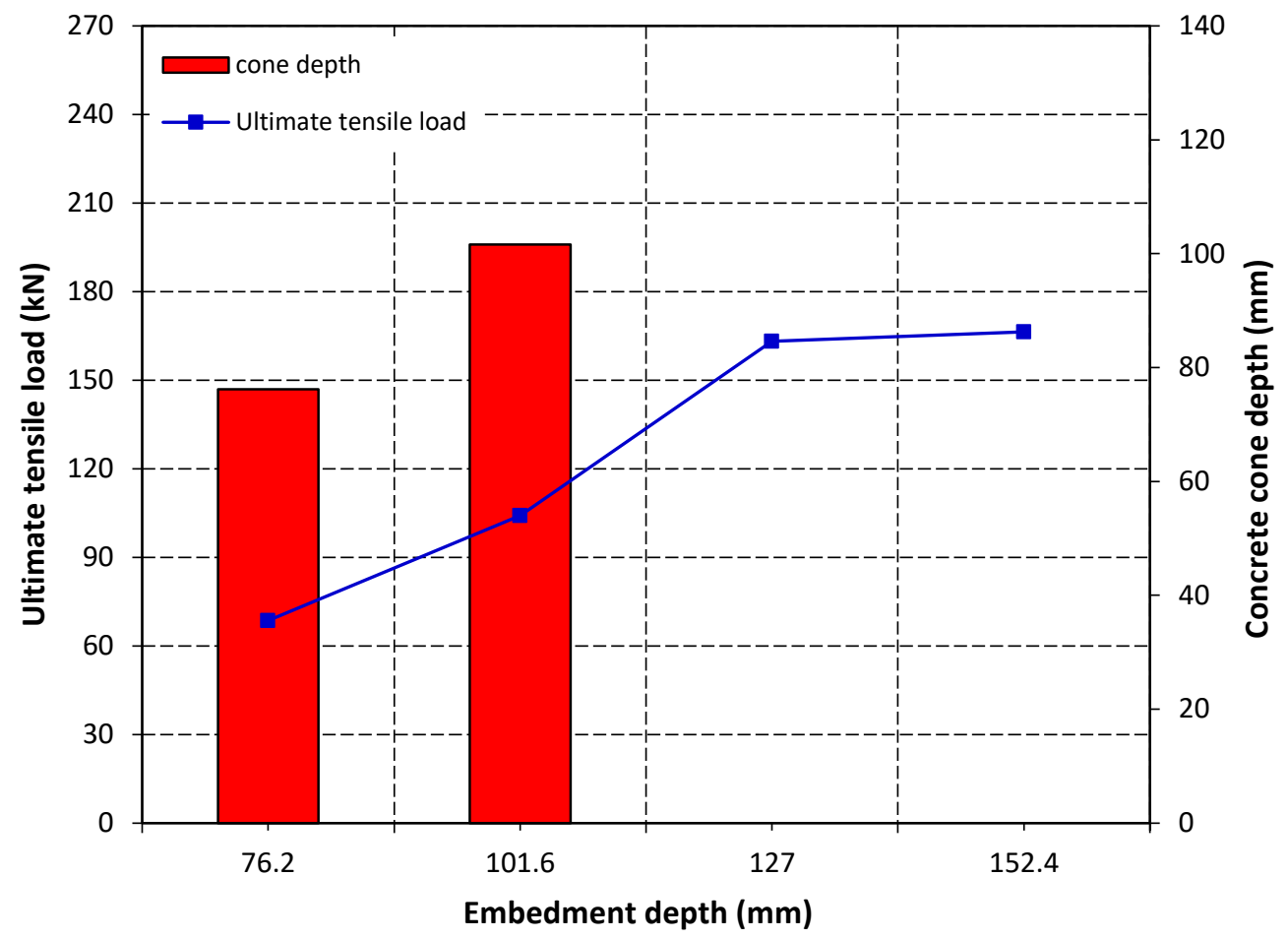

Figure 4-15: Effect of anchor embedment depth on the concrete cone depth and ultimate tensile load for $15.9 \mathrm{~mm}$ diameter cast-in-place anchor

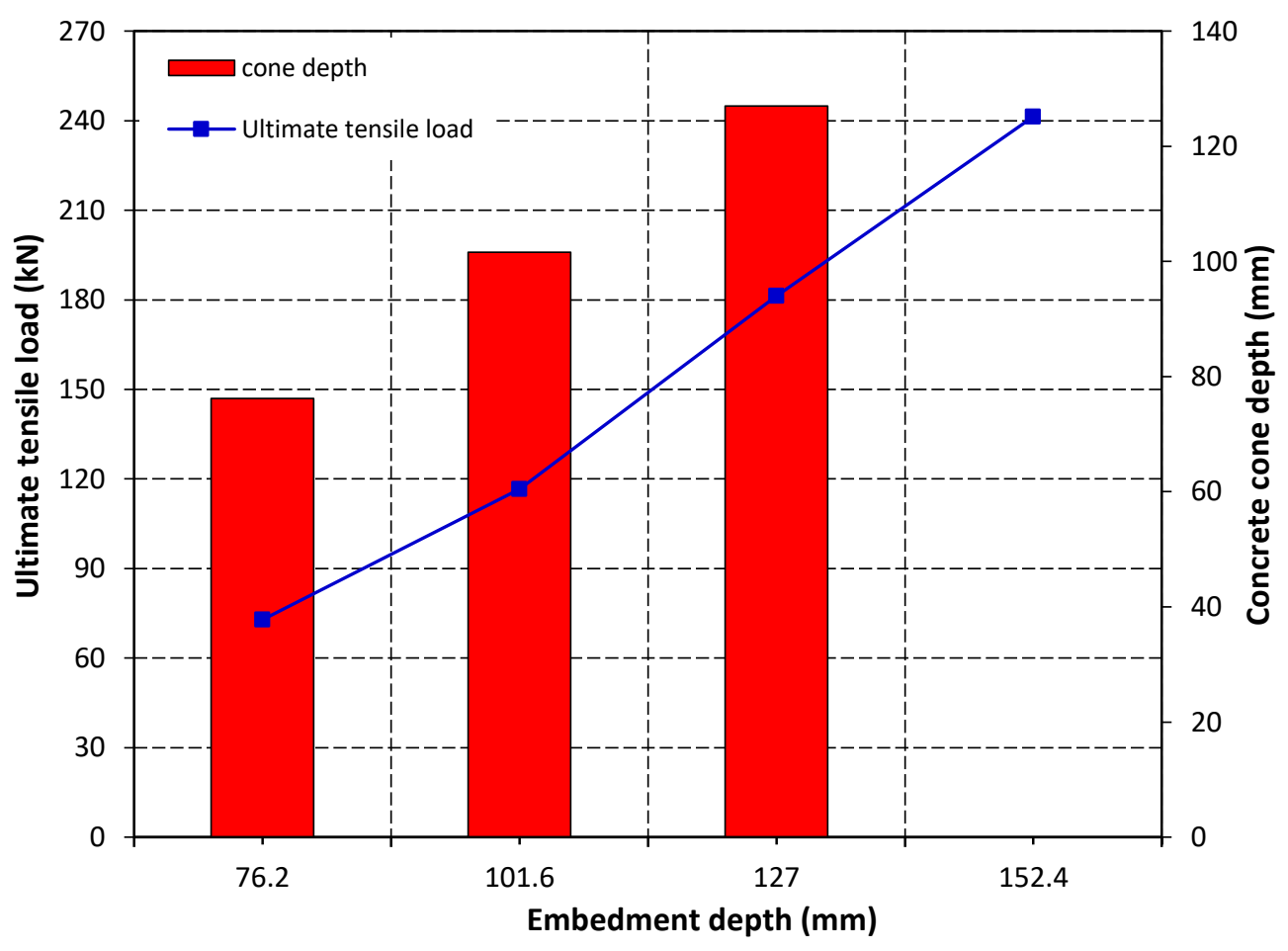

Figure 4-16: Effect of anchor embedment depth on the concrete cone depth and ultimate tensile load for $19.1 \mathrm{~mm}$ diameter cast-in-place anchor 


\subsubsection{Effect of strain rate on concrete cone breakout diameter}

Figure 4-17 shows the displacement contours for 15.9-mm diameter cast-in-place anchor with embedemnt depth of $76.2 \mathrm{~mm}$ under tensile load at strain rates of $10^{-5} \mathrm{~s}^{-1}, 10^{-3} \mathrm{~s}^{-1}, 10^{-}$ ${ }^{1} \mathrm{~s}^{-1}, 10 \mathrm{~s}^{-1}, 10^{2} \mathrm{~s}^{-1}$ and $10^{3} \mathrm{~s}^{-1}$. As shown in the figure, the increase in the strain rate decreased the concrete cone breakout diameter. The decrease in the concrete cone breakout diameter is significant at strain rate of $10^{2} \mathrm{~s}^{-1}$ while steel anchor failure is observed at highest strain rate of $10^{3} \mathrm{~s}^{-1}$.

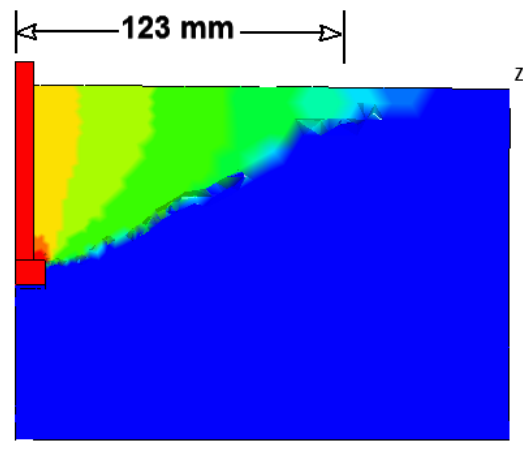

(a) $\dot{\varepsilon}=10^{-5} \mathrm{~s}^{-1}$

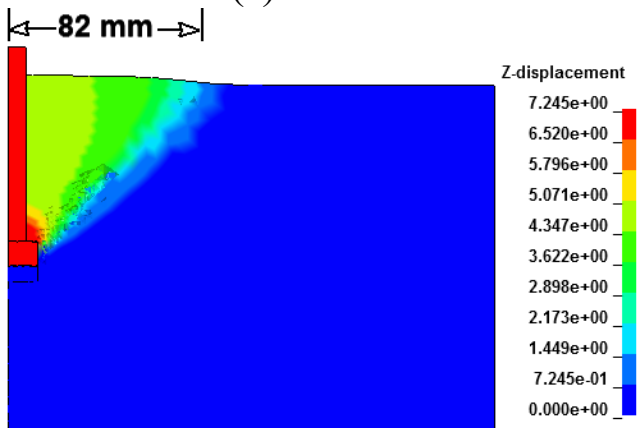

(c) $\dot{\varepsilon}=10^{-1} \mathrm{~s}^{-1}$

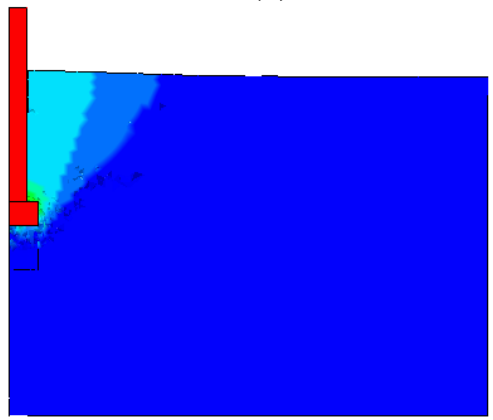

(e) $\dot{\varepsilon}=10^{2} \mathrm{~s}^{-1}$

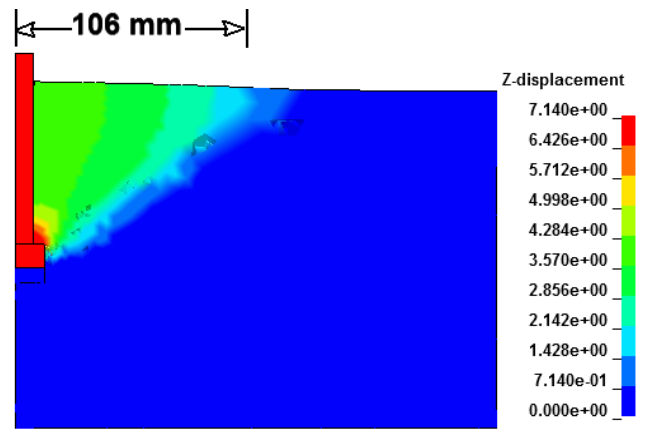

(b) $\dot{\varepsilon}=10^{-3} \mathrm{~s}^{-1}$

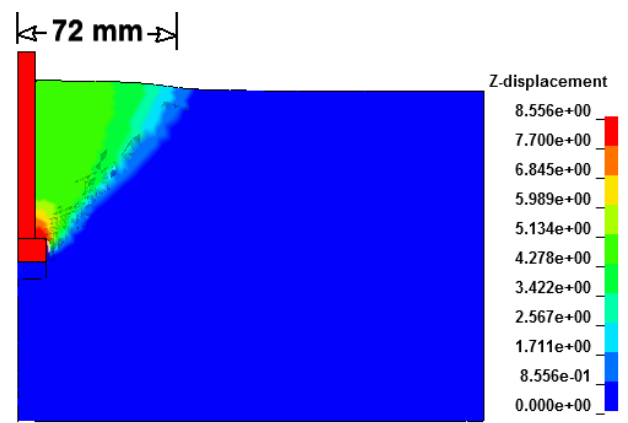

(d) $\dot{\varepsilon}=10 \mathrm{~s}^{-1}$

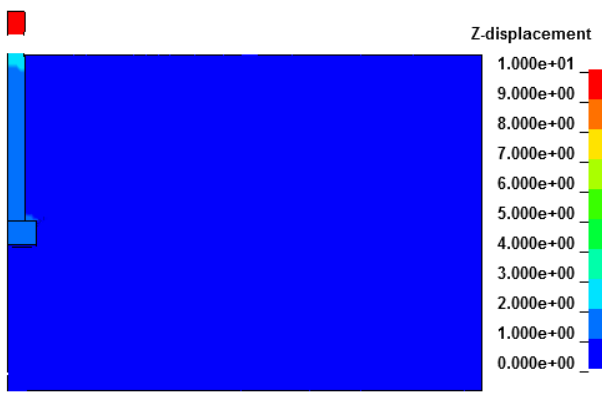

(f) $\dot{\varepsilon}=10^{3} \mathrm{~s}^{-1}$

Figure 4-17: Displacement contours of $15.9 \mathrm{~mm}$ diameter cast-in-place anchor at strain rates ranging from $10^{-5} \mathrm{~s}^{-1}$ to $10^{3} \mathrm{~s}^{-1}$ 


\subsubsection{Effect of strain rate on the tensile behaviour of cast-in-place anchors}

Tensile behaviour of cast-in-place anchor embedded into concrete was investigated at strain rates ranging from $10^{-5} \mathrm{~s}^{-1}$ to $10^{3} \mathrm{~s}^{-1}$. Figures 4-18, 4-19, 4-20, 4-21, 4-22 and 4-23 present the load-displacement response for the $12.7-\mathrm{mm}$ diameter cast-in-place anchor at strain rates of $10^{-5} \mathrm{~s}^{-1}, 10^{-3} \mathrm{~s}^{-1}, 10^{-1} \mathrm{~s}^{-1}, 10 \mathrm{~s}^{-1}, 10^{2} \mathrm{~s}^{-1}$ and $10^{3} \mathrm{~s}^{-1}$ respectively. The displacement in the z-direction (shown in Figure 4-2) was measured at the top of the anchor using History-Nodal-z-displacement in LS-DYNA post processor.

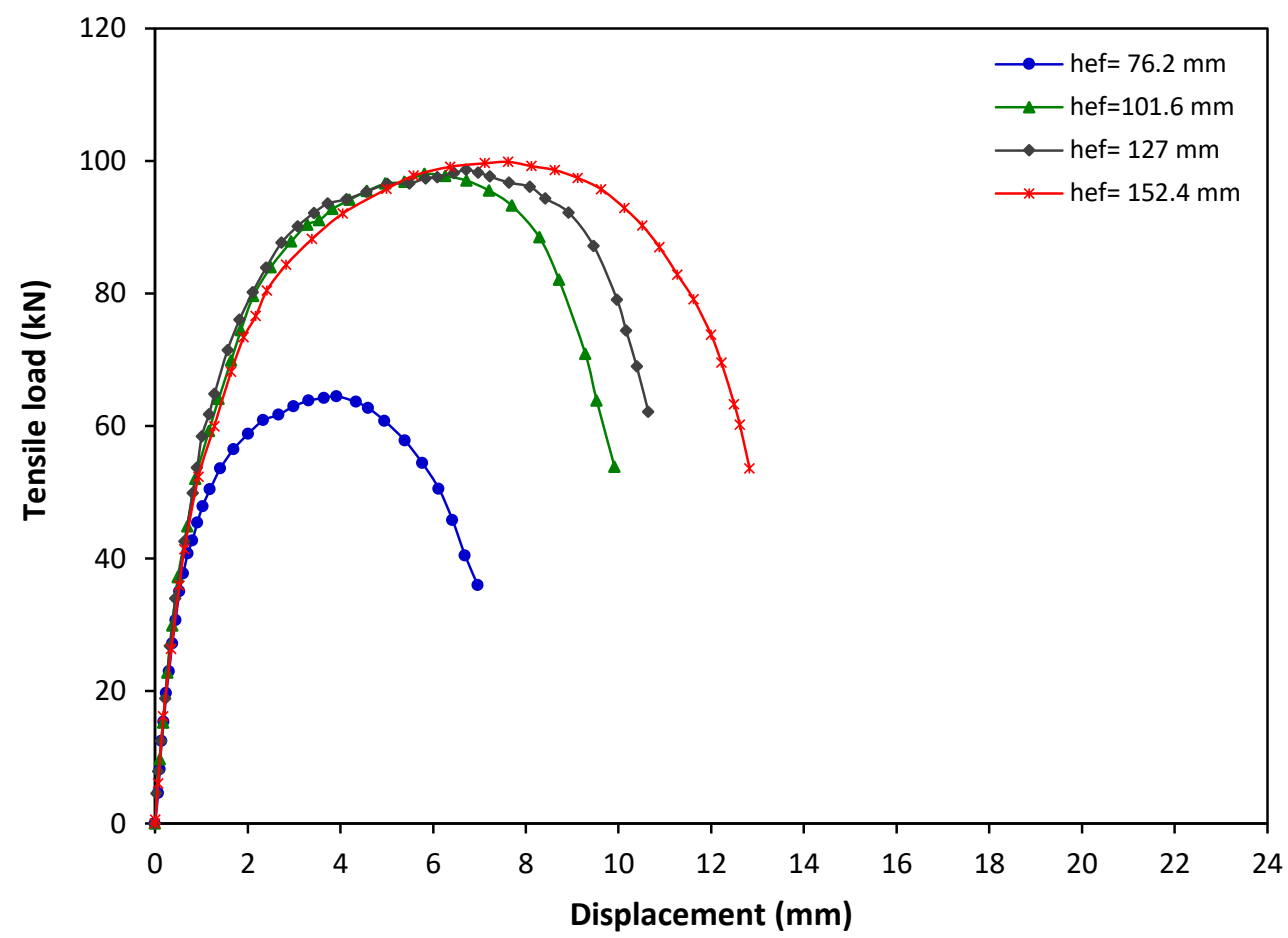

Figure 4-18: Tensile load-displacement graph for $12.7 \mathrm{~mm}$ diameter cast-in-place anchor at strain rate of $10^{-5} \mathrm{~s}^{-1}$ 


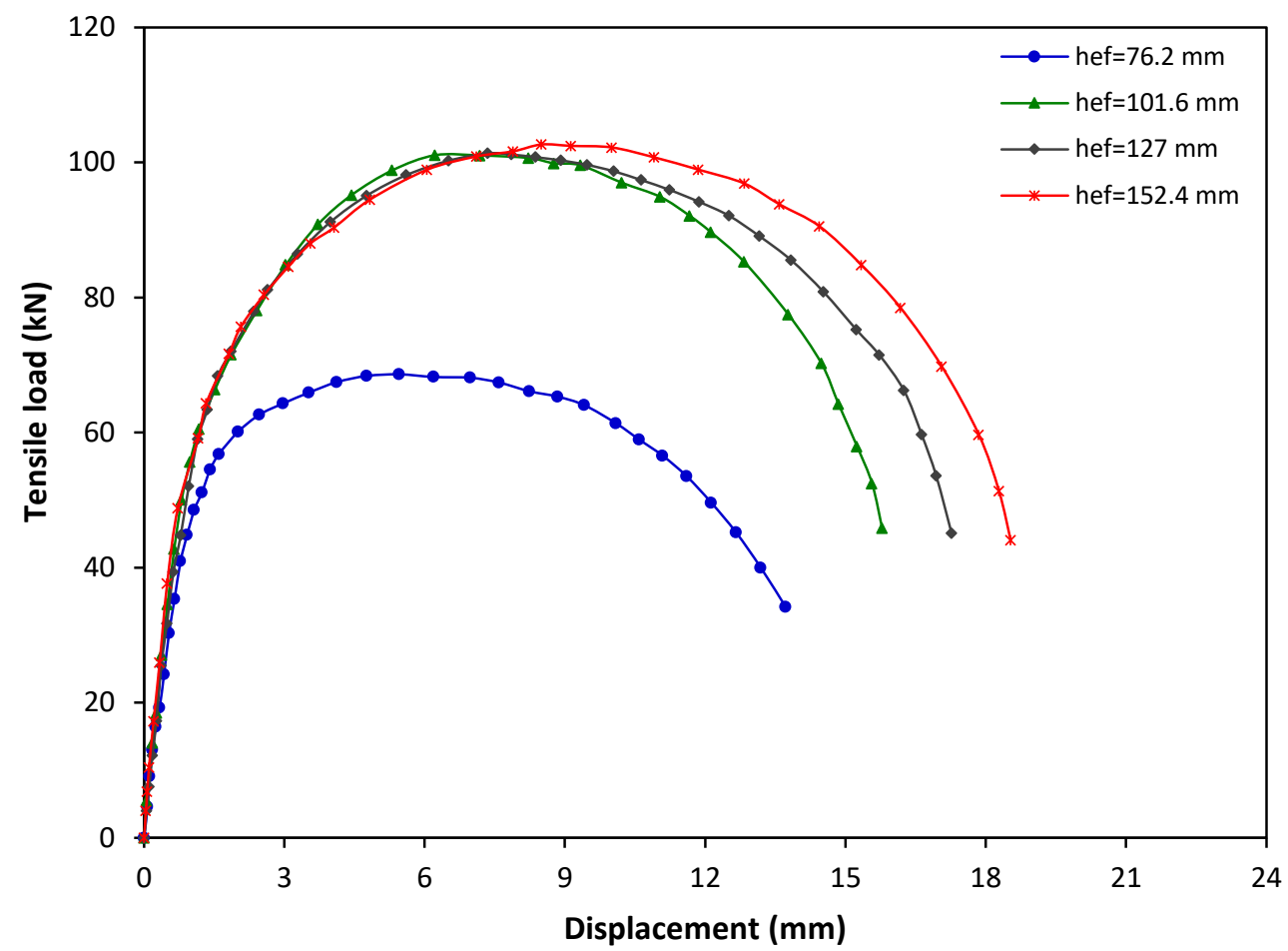

Figure 4-19: Tensile load-displacement graph for $12.7 \mathrm{~mm}$ diameter cast-in-place anchor at strain rate of $10^{-3} \mathrm{~s}^{-1}$

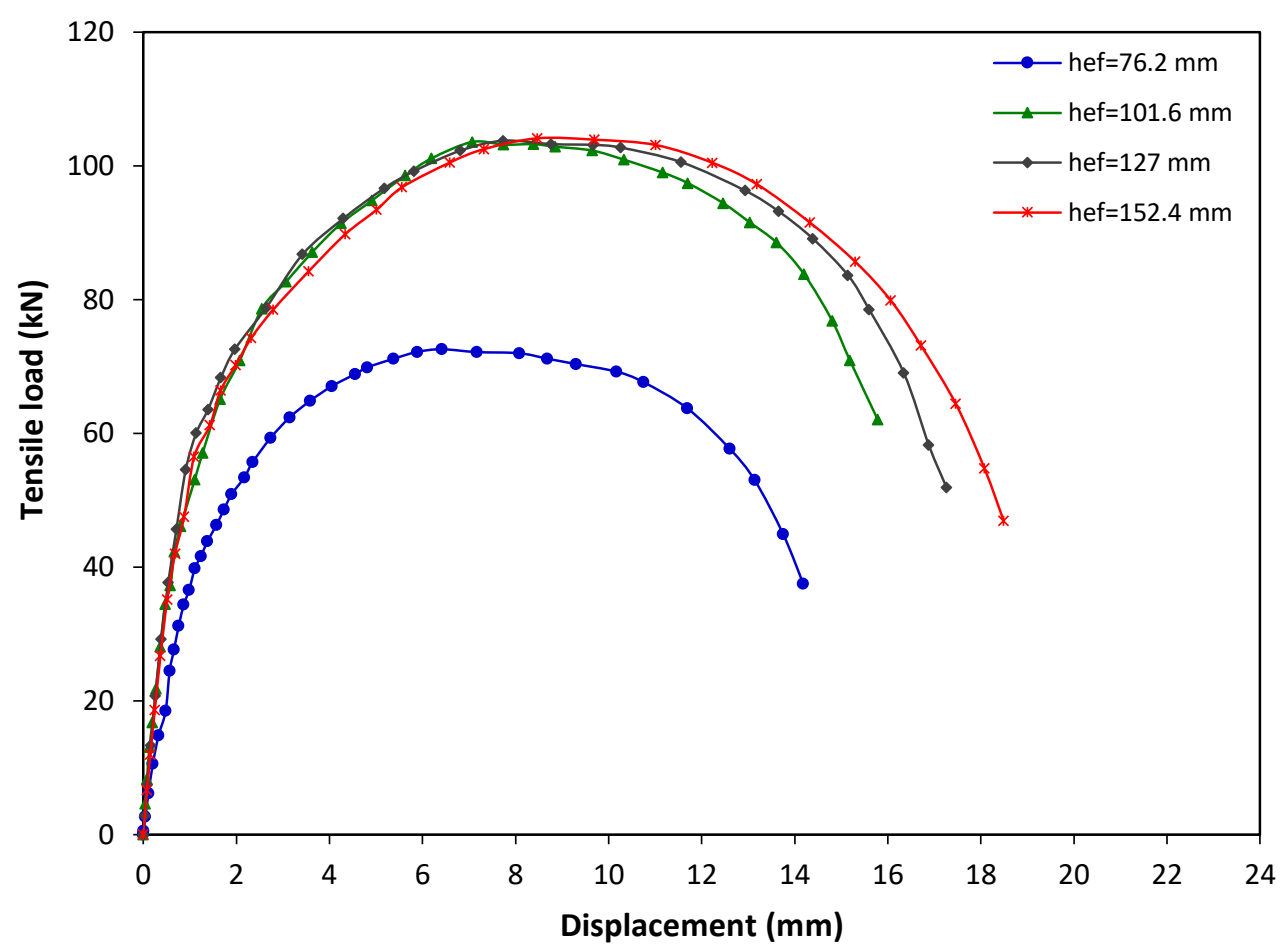

Figure 4-20: Tensile load-displacement graph for $12.7 \mathrm{~mm}$ diameter cast-in-place anchor at strain rate of $10^{-1} \mathrm{~s}^{-1}$ 


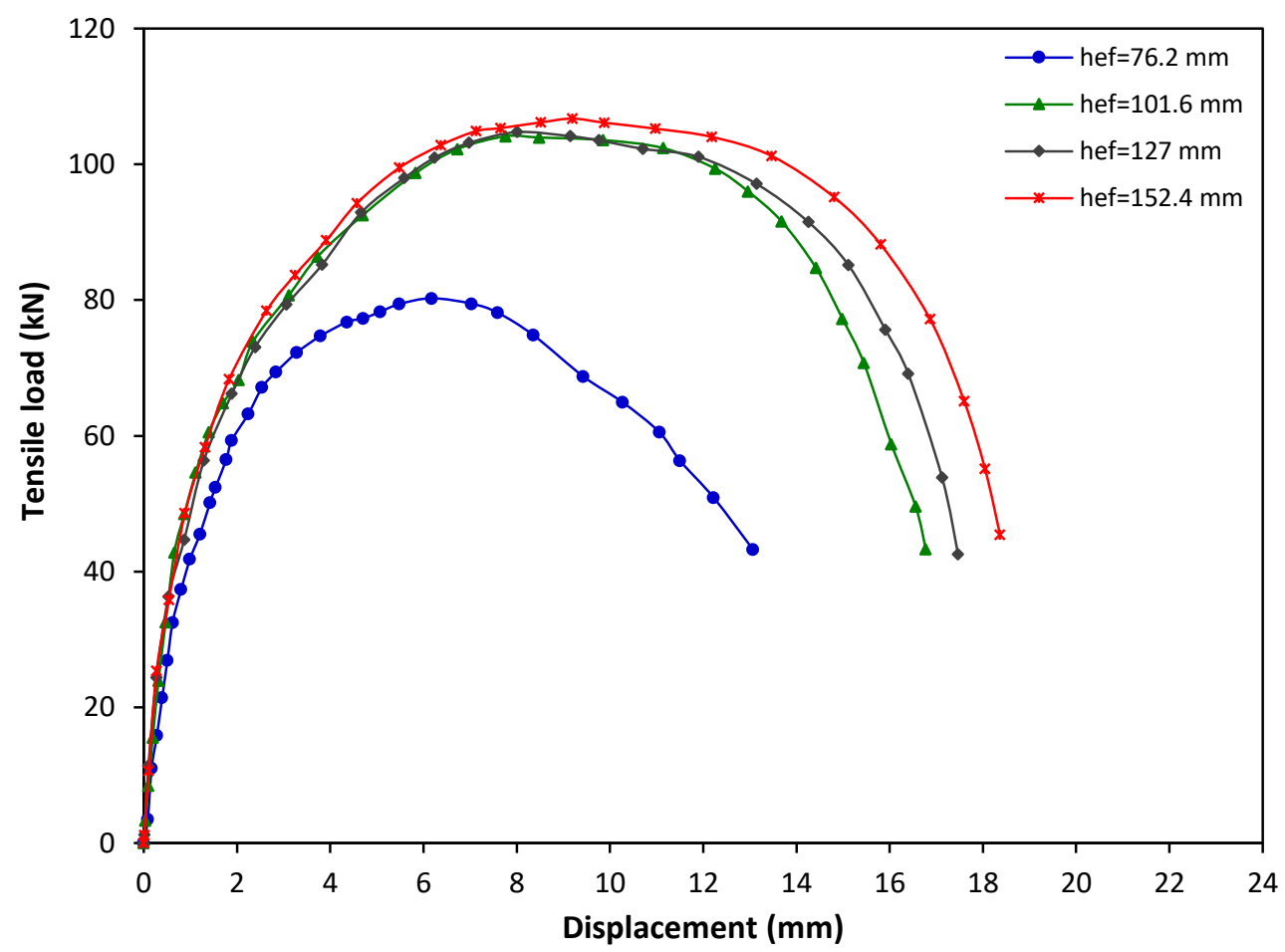

Figure 4-21: Tensile load-displacement graph for $12.7 \mathrm{~mm}$ diameter cast-in-place anchor at strain rate of $10 \mathrm{~s}^{-1}$

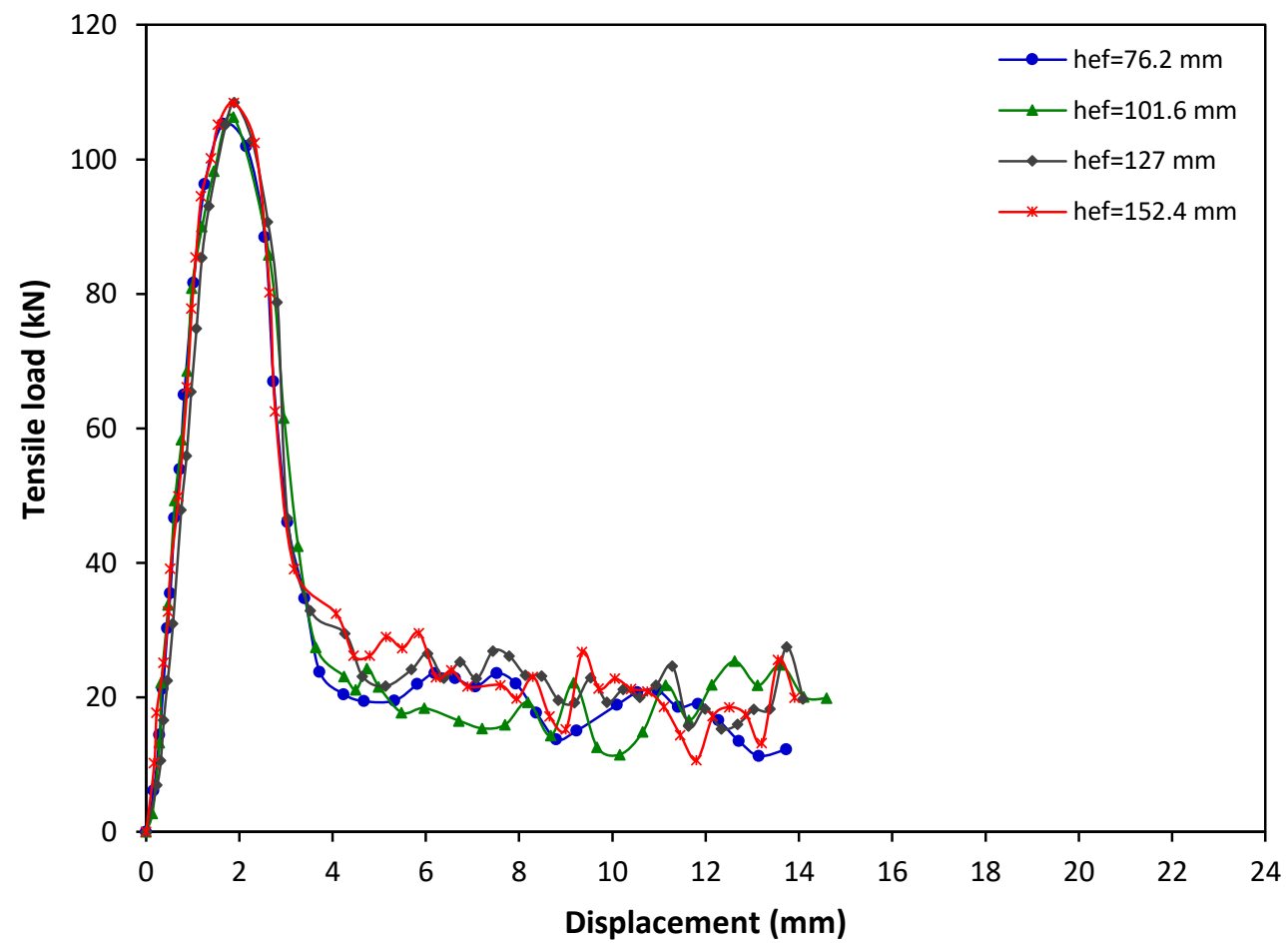

Figure 4-22: Tensile load-displacement graph for $12.7 \mathrm{~mm}$ diameter cast-in-place anchor at strain rate of $10^{2} \mathrm{~s}^{-1}$ 


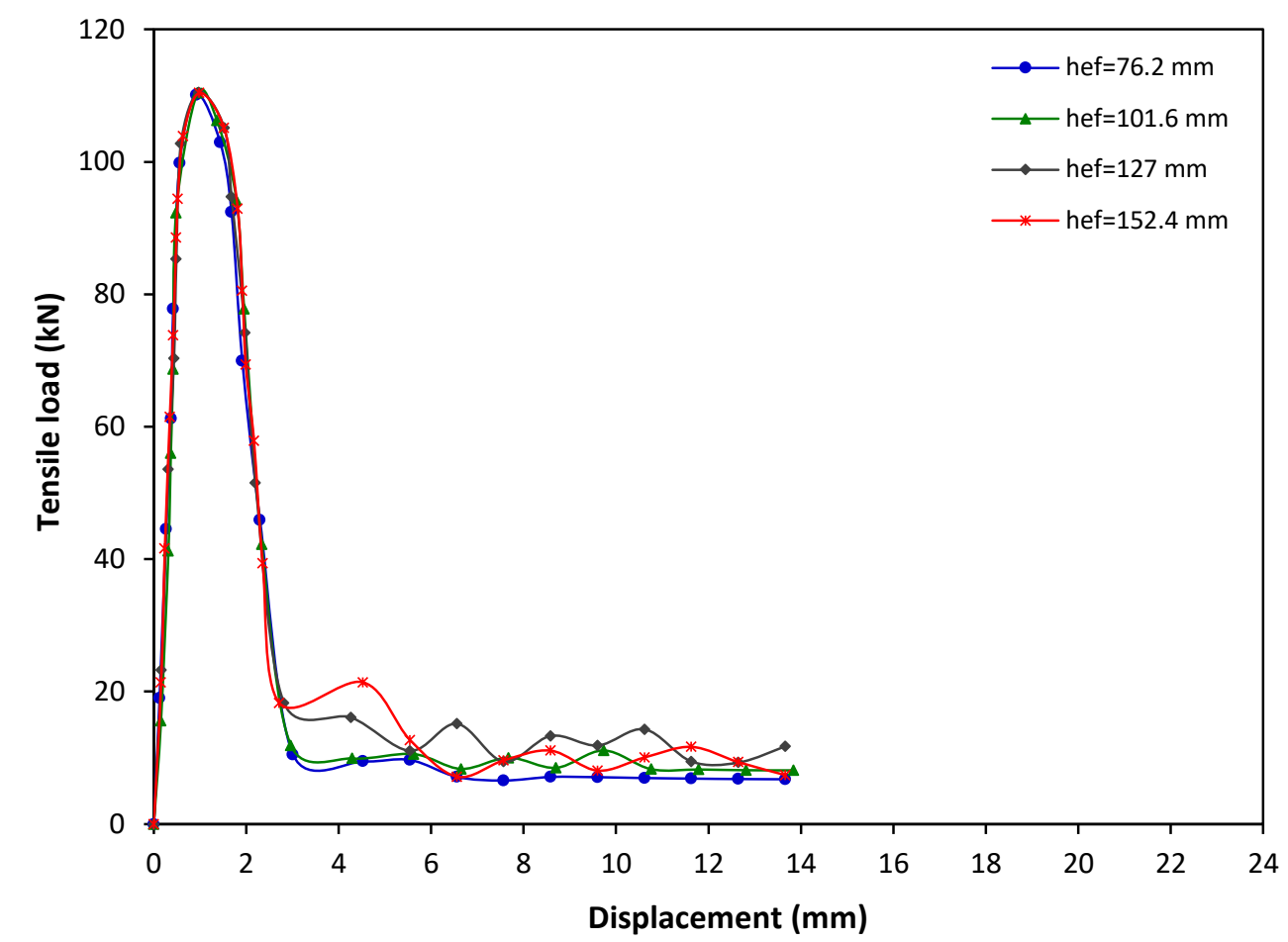

Figure 4-23: Tensile load-displacement graph for $12.7 \mathrm{~mm}$ diameter cast-in-place anchor at strain rate of $10^{3} \mathrm{~s}^{-1}$

As shown from the figures, the tensile load increased with the displacement until it reaches the ultimate load, then the load decreased with further increase in displacement until failure. The increase in the embedment depth from $76.2 \mathrm{~mm}$ to $101.6 \mathrm{~mm}$ shows an increase in the ultimate tensile load for the cast in place anchor. This is attributed to the increase in the amount of concrete above the anchor head and increase in the failure area as the embedment depth increased. The increased failure surface area results in increased loads required to breakout the concrete. Same trend for the effect of anchor embedment depth on the tensile load was reported by Eligehausen et al. (Eligehausen et al., 1992). The increase in embedment depth from $101.6 \mathrm{~mm}$ to $152.4 \mathrm{~mm}$ show very little increase in the ultimate tensile load of the cast-in-place concrete anchorage system as the failure mode was by steel anchor failure. As the strain rate increased the failure mode transitions from concrete cone 
breakout failure to steel anchor failure. At this point the ultimate failure load of the anchorage systems is the same irrespective of embedment depth. This trend is observed at strain rate of $10^{2} \mathrm{~s}^{-1}$ and higher for $12.7-\mathrm{mm}$ diameter anchor.

In general, the increase in the strain rate from $10^{-5} \mathrm{~s}^{-1}$ to $10^{3} \mathrm{~s}^{-1}$ increases the ultimate tensile load. The ultimate tensile load increased from $64.48 \mathrm{kN}$ to $110.13 \mathrm{kN}$ for the anchor embedment depth of $76.2 \mathrm{~mm}$ when the strain rate increased from $10^{-5} \mathrm{~s}^{-1}$ to $10^{3} \mathrm{~s}^{-1}$. The load-displacement response of the cast-in-place anchorage system at increased strain rate of $10^{2} \mathrm{~s}^{-1}$ and $10^{3} \mathrm{~s}^{-1}$ is characterized by a linear response to the peak load and then shows a drop in load.

Tensile load-displacement response of the 15.9-mm and 19.1-mm diameter cast-in-place anchors with embedment depths of $76.2 \mathrm{~mm}, 101.6 \mathrm{~mm}, 127 \mathrm{~mm}$ and $152.4 \mathrm{~mm}$ at strain rates of $10^{-5} \mathrm{~s}^{-1}, 10^{-3} \mathrm{~s}^{-1}, 10^{-1} \mathrm{~s}^{-1}, 10 \mathrm{~s}^{-1}, 10^{2} \mathrm{~s}^{-1}$ and $10^{3} \mathrm{~s}^{-1}$ show similar trends to the 12.7$\mathrm{mm}$ diameter anchor and are presented in Appendix C.

Tables 4-6, 4-7 and 4-8 show the ultimate tensile load $\left(F_{u}\right)$ and corresponding displacements $(\delta)$ for the 12.7-mm, 15.9-mm and 19.1-mm diameter cast-in-place anchors at strain rates ranging from $10^{-5} \mathrm{~s}^{-1}$ to $10^{3} \mathrm{~s}^{-1}$. As shown in the tables, the increase in the strain rate from $10^{-5} \mathrm{~s}^{-1}$ to $10^{3} \mathrm{~s}^{-1}$ increases the ultimate tensile load for the cast-in-place anchors. 
Table 4-6: Ultimate tensile load and displacement results for the $12.7 \mathrm{~mm}$ diameter castin-place anchor

\begin{tabular}{|c|c|c|c|c|c|c|}
\hline $\begin{array}{l}\text { Model } \\
\text { No. }\end{array}$ & $\begin{array}{c}\mathrm{d} \\
(\mathrm{mm})\end{array}$ & $\begin{array}{c}\mathrm{h}_{\mathrm{ef}} \\
(\mathrm{mm})\end{array}$ & $\begin{array}{c}\dot{\varepsilon} \\
\left(\mathrm{s}^{-1}\right)\end{array}$ & $\begin{array}{c}F_{\mathrm{u}} \\
(\mathrm{kN})\end{array}$ & $\begin{array}{c}\delta \\
(\mathrm{mm})\end{array}$ & $\begin{array}{l}{ }^{*} \text { Failure } \\
\text { mode }\end{array}$ \\
\hline 1 & \multirow{4}{*}{12.7} & 76.2 & \multirow{4}{*}{$10^{-5}$} & 64.48 & 3.91 & $\mathrm{CC}$ \\
\hline 2 & & 101.6 & & 98.03 & 5.82 & $\mathrm{~S}$ \\
\hline 3 & & 127 & & 98.64 & 6.72 & $S$ \\
\hline 4 & & 152.4 & & 99.86 & 7.62 & $S$ \\
\hline 5 & \multirow{4}{*}{12.7} & 76.2 & \multirow{4}{*}{$10^{-3}$} & 68.63 & 5.46 & $\mathrm{CC}$ \\
\hline 6 & & 101.6 & & 101.05 & 6.22 & $\mathrm{~S}$ \\
\hline 7 & & 127 & & 101.37 & 7.35 & $\mathrm{~S}$ \\
\hline 8 & & 152.4 & & 102.63 & 8.49 & $\mathrm{~S}$ \\
\hline 9 & \multirow{4}{*}{12.7} & 76.2 & \multirow{4}{*}{$10^{-1}$} & 72.16 & 5.89 & $\mathrm{CC}$ \\
\hline 10 & & 101.6 & & 103.55 & 7.07 & $\mathrm{~S}$ \\
\hline 11 & & 127 & & 103.74 & 7.74 & $\mathrm{~S}$ \\
\hline 12 & & 152.4 & & 104.10 & 8.47 & $\mathrm{~S}$ \\
\hline 13 & \multirow{4}{*}{12.7} & 76.2 & \multirow{4}{*}{10} & 79.39 & 5.49 & $\mathrm{CC}$ \\
\hline 14 & & 101.6 & & 104.13 & 7.76 & $\mathrm{~S}$ \\
\hline 15 & & 127 & & 104.76 & 8.01 & $\mathrm{~S}$ \\
\hline 16 & & 152.4 & & 106.75 & 9.19 & $\mathrm{~S}$ \\
\hline 17 & \multirow{4}{*}{12.7} & 76.2 & \multirow{4}{*}{$10^{2}$} & 105.30 & 1.65 & $\mathrm{~S}$ \\
\hline 18 & & 101.6 & & 108.28 & 1.88 & $\mathrm{~S}$ \\
\hline 19 & & 127 & & 108.48 & 1.89 & $\mathrm{~S}$ \\
\hline 20 & & 152.4 & & 108.41 & 1.88 & $\mathrm{~S}$ \\
\hline 21 & \multirow{4}{*}{12.7} & 76.2 & \multirow{4}{*}{$10^{3}$} & 110.13 & 0.93 & $\mathrm{~S}$ \\
\hline 22 & & 101.6 & & 110.40 & 0.94 & $\mathrm{~S}$ \\
\hline 23 & & 127 & & 110.49 & 0.97 & $S$ \\
\hline 24 & & 152.4 & & 110.42 & 0.98 & $\mathrm{~S}$ \\
\hline
\end{tabular}

Table 4-7: Ultimate tensile load and displacement results for the $15.9 \mathrm{~mm}$ diameter castin-place anchor

\begin{tabular}{|c|c|c|c|c|c|c|}
\hline $\begin{array}{c}\text { Model } \\
\text { No. }\end{array}$ & $\begin{array}{c}\mathrm{d} \\
(\mathrm{mm})\end{array}$ & $\begin{array}{c}\mathrm{h}_{\mathrm{ef}} \\
(\mathrm{mm})\end{array}$ & $\begin{array}{c}\dot{\varepsilon} \\
\left(\mathrm{s}^{-1}\right)\end{array}$ & $\begin{array}{c}\mathrm{F}_{\mathrm{u}} \\
(\mathrm{kN})\end{array}$ & $\begin{array}{c}\delta \\
(\mathrm{mm})\end{array}$ & $\begin{array}{c}{ }^{*} \text { Failure } \\
\text { mode }\end{array}$ \\
\hline 1 & \multirow{4}{*}{15.9} & 76.2 & \multirow{4}{*}{$10^{-5}$} & 68.61 & 1.34 & $\mathrm{CC}$ \\
\hline 2 & & 101.6 & & 104.17 & 3.77 & $\mathrm{CC}$ \\
\hline 3 & & 127 & & 163.26 & 6.23 & $\mathrm{~S}$ \\
\hline 4 & & 152.4 & & 166.35 & 7.14 & $\mathrm{~S}$ \\
\hline 5 & 15.9 & 76.2 & $10^{-3}$ & 76.32 & 3.20 & $\mathrm{CC}$ \\
\hline
\end{tabular}




\begin{tabular}{|c|c|c|c|c|c|c|}
\hline 6 & & 101.6 & & 110.25 & 3.86 & $\mathrm{CC}$ \\
\hline 7 & & 127 & & 170.01 & 7.54 & $\mathrm{~S}$ \\
\hline 8 & & 152.4 & & 171.81 & 8.17 & $\mathrm{~S}$ \\
\hline 9 & \multirow{4}{*}{15.9} & 76.2 & \multirow{4}{*}{$10^{-1}$} & 83.96 & 3.08 & $\mathrm{CC}$ \\
\hline 10 & & 101.6 & & 116.33 & 6.22 & $\mathrm{CC}$ \\
\hline 11 & & 127 & & 173.22 & 7.68 & $\mathrm{~S}$ \\
\hline 12 & & 152.4 & & 177.95 & 8.58 & $\mathrm{~S}$ \\
\hline 13 & \multirow{4}{*}{15.9} & 76.2 & \multirow{4}{*}{10} & 98.14 & 3.67 & $\mathrm{CC}$ \\
\hline 14 & & 101.6 & & 128.75 & 6.91 & $\mathrm{CC}$ \\
\hline 15 & & 127 & & 174.02 & 8.06 & $\mathrm{~S}$ \\
\hline 16 & & 152.4 & & 178.35 & 9.29 & $\mathrm{~S}$ \\
\hline 17 & \multirow{4}{*}{15.9} & 76.2 & \multirow{4}{*}{$10^{2}$} & 144.46 & 1.05 & $\mathrm{COC}$ \\
\hline 18 & & 101.6 & & 152.52 & 1.32 & $\mathrm{COC}$ \\
\hline 19 & & 127 & & 183.74 & 1.54 & S \\
\hline 20 & & 152.4 & & 184.52 & 1.30 & $\mathrm{~S}$ \\
\hline 21 & \multirow{4}{*}{15.9} & 76.2 & \multirow{4}{*}{$10^{3}$} & 191.15 & 1.24 & $\mathrm{~S}$ \\
\hline 22 & & 101.6 & & 191.71 & 1.39 & $\mathrm{~S}$ \\
\hline 23 & & 127 & & 191.73 & 1.36 & S \\
\hline 24 & & 152.4 & & 193.82 & 1.41 & $\mathrm{~S}$ \\
\hline
\end{tabular}

Table 4-8: Ultimate tensile load and displacement results for the $19.1 \mathrm{~mm}$ diameter castin-place anchor

\begin{tabular}{|c|c|c|c|c|c|c|}
\hline $\begin{array}{c}\text { Model } \\
\text { No. }\end{array}$ & $\begin{array}{c}\mathrm{d} \\
(\mathrm{mm})\end{array}$ & $\begin{array}{c}\mathrm{h}_{\mathrm{ef}} \\
(\mathrm{mm})\end{array}$ & $\begin{array}{c}\dot{\varepsilon} \\
\left(\mathrm{s}^{-1}\right)\end{array}$ & $\begin{array}{c}\mathrm{F}_{\mathrm{u}} \\
(\mathrm{kN})\end{array}$ & $\begin{array}{c}\delta \\
(\mathrm{mm})\end{array}$ & $\begin{array}{c}{ }^{*} \text { Failure } \\
\text { mode }\end{array}$ \\
\hline 1 & \multirow{4}{*}{19.1} & 76.2 & \multirow{4}{*}{$10^{-5}$} & 72.88 & 0.82 & $\mathrm{CC}$ \\
\hline 2 & & 101.6 & & 116.65 & 1.31 & $\mathrm{CC}$ \\
\hline 3 & & 127 & & 181.29 & 2.76 & $\mathrm{CC}$ \\
\hline 4 & & 152.4 & & 241.37 & 6.91 & $\mathrm{~S}$ \\
\hline 5 & \multirow{4}{*}{19.1} & 76.2 & \multirow{4}{*}{$10^{-3}$} & 83.09 & 1.76 & $\mathrm{CC}$ \\
\hline 6 & & 101.6 & & 128.44 & 3.45 & $\mathrm{CC}$ \\
\hline 7 & & 127 & & 189.80 & 4.63 & $\mathrm{CC}$ \\
\hline 8 & & 152.4 & & 251.55 & 6.88 & $\mathrm{~S}$ \\
\hline
\end{tabular}




\begin{tabular}{|c|c|c|c|c|c|c|}
\hline 9 & \multirow{4}{*}{19.1} & 76.2 & \multirow{4}{*}{$10^{-1}$} & 96.94 & 1.90 & $\mathrm{CC}$ \\
\hline 10 & & 101.6 & & 138.88 & 3.98 & $\mathrm{CC}$ \\
\hline 11 & & 127 & & 201.63 & 5.59 & $\mathrm{CC}$ \\
\hline 12 & & 152.4 & & 258.58 & 7.82 & $\mathrm{~S}$ \\
\hline 13 & \multirow{4}{*}{19.1} & 76.2 & \multirow{4}{*}{10} & 126.83 & 2.14 & $\mathrm{CC}$ \\
\hline 14 & & 101.6 & & 155.88 & 3.55 & $\mathrm{CC}$ \\
\hline 15 & & 127 & & 218.48 & 4.19 & $\mathrm{CC}$ \\
\hline 16 & & 152.4 & & 263.37 & 7.09 & $\mathrm{~S}$ \\
\hline 17 & \multirow{4}{*}{19.1} & 76.2 & \multirow{4}{*}{$10^{2}$} & 191.30 & 1.70 & $\mathrm{COC}$ \\
\hline 18 & & 101.6 & & 192.14 & 1.98 & $\mathrm{COC}$ \\
\hline 19 & & 127 & & 268.75 & 2.19 & $\mathrm{~S}$ \\
\hline 20 & & 152.4 & & 269.15 & 2.25 & $\mathrm{~S}$ \\
\hline 21 & \multirow{4}{*}{19.1} & 76.2 & \multirow{4}{*}{$10^{3}$} & 272.09 & 1.74 & $\mathrm{~S}$ \\
\hline 22 & & 101.6 & & 273.78 & 2.07 & $\mathrm{~S}$ \\
\hline 23 & & 127 & & 273.95 & 1.83 & $\mathrm{~S}$ \\
\hline 24 & & 152.4 & & 273.79 & 1.93 & $\mathrm{~S}$ \\
\hline
\end{tabular}

Tables 4-6, 4-7 and 4-8 show that the increase in the anchor diameter from $12.7 \mathrm{~mm}$ to $19.1 \mathrm{~mm}$ increased the ultimate tensile load for all the embedment depths and strain rates investigated. The increase in the anchor diameter requires larger anchor head that increase the bearing area above the anchor head and the failure surface area. Also, it can be seen that the embedment depth has a greater effect on the ultimate tensile load at the same strain rate when concrete cone breakout failure is the dominant failure mode. However, the increase in the embedment depth has no influence on the ultimate tensile load when steel anchor failure is the dominant failure mode. The increase in the ultimate tensile load with the increase in the strain rate is attributed to the increase in concrete resistance under increased strain rate (Ožbolt et al., 2006; Sato et al., 2004; Solomos and Berra, 2006). 


\subsubsection{Effect of strain rate on the ultimate tensile load and dynamic increase factor of cast-in-place anchors}

Investigating the influence of strain rate on the behaviour of anchors is necessary to predict a suitable DIF that can be used for design of anchorage systems subjected to different strain rates. The ratio of the dynamic to static strength of the cast-in-place anchor is defined as the dynamic increase factor (DIF). A safe and economical design of the anchors under dynamic loading can be achieved by applying the DIF to the ultimate load measured under static loading (Braimah et al., 2009). The DIF can be used to represent the effect of strain rate on concrete strength (compressive and tensile), yield and ultimate strength of steel (Malvar and Crawford, 1998; Murray, 2007; Solomos and Berra, 2006; Zhou et al., 2008). The common approach to model the effect of strain rate on the concrete under tension and compression loading is proposed by Committee Euro International du Beton (CEB) code that introduced equations to predict the DIF of the concrete subjected to different strain rates (Malvar \& Ross, 1998). The CEB proposed equations demonstrate that the DIF is a power function of the strain rate ratio (Malvar \& Crawford, 1998; Malvar \& Ross, 1998; Gebbeken \& Ruppert, 2000). In this research, in order to predict the increase in strength of anchorage system due to increase in the steel and concrete strength with the increase in the strain rate, DIF for the anchorage to concrete system was investigated. The lowest strain rate of $10^{-5} \mathrm{~s}^{-1}$ is representative of static loading and will be used as the baseline for comparison with cast-in-place anchor capacity at the higher strain rates for the anchors exhibiting concrete cone breakout failure. For the anchors exhibiting steel failure mode at strain rate higher than $10^{-5} \mathrm{~s}^{-1}$, the dynamic increase factor is taken as the ratio of ultimate dynamic load to the ultimate static steel failure load. 
Figures 4-24, 4-25, 4-26 and 4-27 present the ultimate tensile load and DIF plotted as a function of the strain rate for the 12.7- $\mathrm{mm}, 15.9-\mathrm{mm}$ and 19.1-mm diameter cast-in-place anchors with embedment depths of $76.2 \mathrm{~mm}, 101.6 \mathrm{~mm}, 127 \mathrm{~mm}$ and $152.4 \mathrm{~mm}$ respectively. As shown in the figures, the ultimate tensile load increases with increase in the anchor diameter from $12.7-\mathrm{mm}$ to $19.1-\mathrm{mm}$. It can be seen from Figures 4-24 and 425 , the ultimate tensile load versus strain rate relationship is bilinear with a change in slope at strain rate of $10 \mathrm{~s}^{-1}$ for anchor diameters of 15.9-mm and 19.1-mm. The ultimate tensile load increases with increase in the strain rate from $10^{-5} \mathrm{~s}^{-1}$ to $10 \mathrm{~s}^{-1}$. A significant increase in the ultimate tensile load is observed when the strain rate increased from $10 \mathrm{~s}^{-1}$ to $10^{3} \mathrm{~s}^{-1}$ for anchor diameters of 15.9-mm and 19.1-mm. The increase in the strain rate from $10^{2} \mathrm{~s}^{-1}$ to $10^{3} \mathrm{~s}^{-1}$ for the anchor diameter of $12.7-\mathrm{mm}$ with embedment depth of $76.2 \mathrm{~mm}$ has a slight influence on the ultimate tensile load where steel anchor failure is observed (Figure 4-24). For the anchor diameter of 12.7-mm with embedment depth of $101.6 \mathrm{~mm}$ (Figure 425), an increase in the ultimate tensile load from $98.03 \mathrm{kN}$ to $110.4 \mathrm{kN}(12.6 \%$ increase) was obtained where steel anchor failure is observed at all strain rates investigated.

Figure 4-24 is representative of DIF of anchorage systems failing by concrete cone breakout. It can be seen from Figure 4-24 for anchor embedment depth of $76.2 \mathrm{~mm}$ the DIF increased with the increase in the strain rate from $10^{-5} \mathrm{~s}^{-1}$ to $10^{2} \mathrm{~s}^{-1}$ for all anchor diameters, except for anchor diameter of $12.7 \mathrm{~mm}$ where the dynamic increase factor is measures as the ratio of the dynamic load to the steel failure load. The increase in the strain rate from $10^{-5} \mathrm{~s}^{-1}$ to $10^{-1} \mathrm{~s}^{-1}$ increased the DIF from 1 to $1.1,1.2$ and 1.3 for the anchor diameters of 12.7- $\mathrm{mm}, 15.9-\mathrm{mm}$ and $19.1-\mathrm{mm}$ respectively. The increase in the strain rate from $10 \mathrm{~s}^{-1}$ 
to $10^{2} \mathrm{~s}^{-1}$ increased the DIF significantly from 1.43 to 2.11 and 1.74 to 2.62 for the anchor diameters of $15.9-\mathrm{mm}$ and 19.1- $\mathrm{mm}$ respectively. For the anchor diameter of $12.7 \mathrm{~mm}$, DIF of 1.07 was obtained where steel failure was observed. At high strain rate of $10^{3} \mathrm{~s}^{-1}$, DIF of $1.12,1.17$ and 1.13 were obtained for the anchor diameters of $12.7-\mathrm{mm}, 15.9-\mathrm{mm}$ and 19.1-mm respectively where steel failure was observed.

Figure 4-25 for anchor embedment depth of $101.6 \mathrm{~mm}$ shows that the DIF increased with the increase in the strain rate from $10^{-5} \mathrm{~s}^{-1}$ to $10^{2} \mathrm{~s}^{-1}$ for the $15.9-\mathrm{mm}$ and $19.1-\mathrm{mm}$ diameter cast-in-place anchors where concrete cone failure was observed. At high strain rate of $10^{3}$ $\mathrm{s}^{-1}$, DIF of 1.17 and 1.13 were obtained for the anchor diameters of $15.9 \mathrm{~mm}$ and $19.1 \mathrm{~mm}$ where steel failure was observed. A slight increase in the DIF to 1.13 was obtained for the anchor diameter of $12.7 \mathrm{~mm}$ with the increase in the strain rate from $10^{-5} \mathrm{~s}^{-1}$ to $10^{3} \mathrm{~s}^{-1}$ where steel anchor failure was the dominant failure mode at all the strain rates investigated. This increase is attributed to the increase in the strength of steel with the increase in the strain rate. 


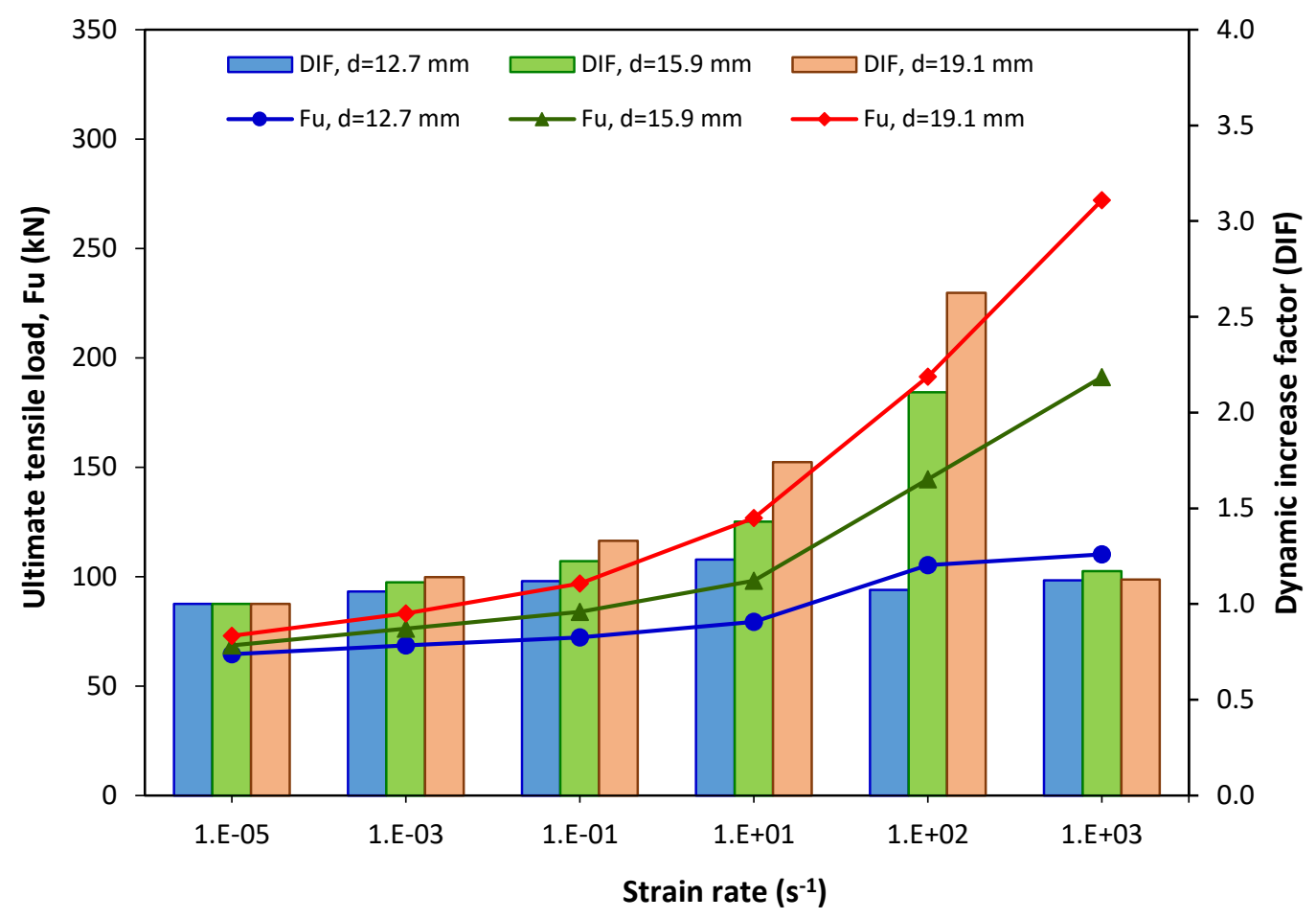

Figure 4-24: Ultimate tensile load and DIF versus strain rate for the cast-in-place anchor of $76.2 \mathrm{~mm}$ embedment depth

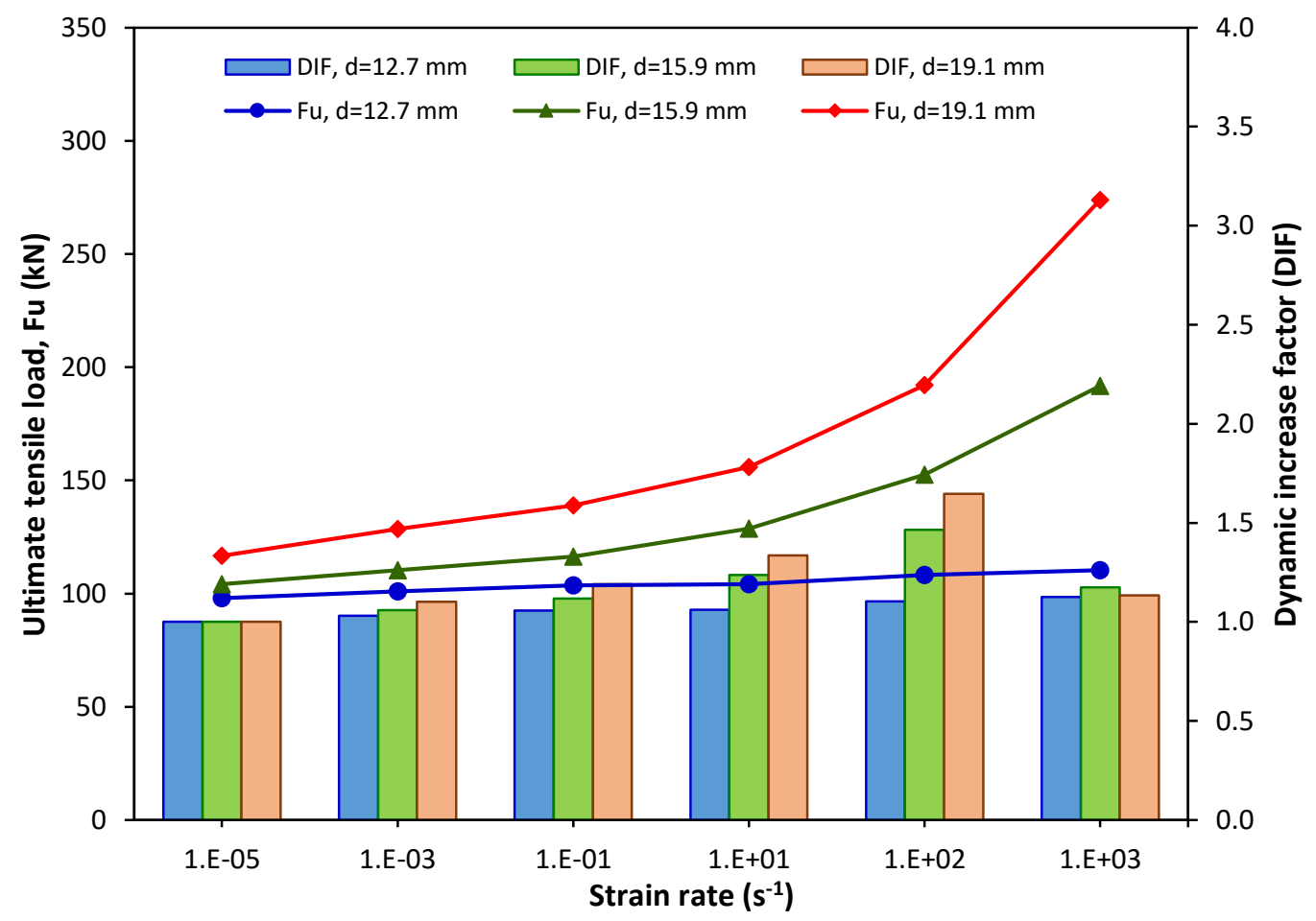

Figure 4-25: Ultimate tensile load and DIF versus strain rate for the cast-in-place anchor of $101.6 \mathrm{~mm}$ embedment depth 
As shown in Figure 4-26 for the embedment depth of $127 \mathrm{~mm}$, the ultimate tensile load increased $12 \%$ and $17.4 \%$ as the strain rate increased from $10^{-5} \mathrm{~s}^{-1}$ to $10^{3} \mathrm{~s}^{-1}$ for anchor diameters of 12.7-mm and 15.9-mm respectively. For the cast-in-place anchor diameter of 19.1-mm, the ultimate tensile load increased $20.5 \%$ when the strain rate increased from $10^{-}$ ${ }^{5} \mathrm{~s}^{-1}$ to $10 \mathrm{~s}^{-1}$ where concrete cone breakout failure was observed. The increase in the ultimate load was $25.4 \%$ when the strain rate increased from $10 \mathrm{~s}^{-1}$ to $10^{3} \mathrm{~s}^{-1}$. Transition from concrete cone breakout to steel anchor failure was observed at strain rate of $10^{2} \mathrm{~s}^{-1}$. DIF of 1.12 and 1.17 are obtained when the strain rate increased from $10^{-5} \mathrm{~s}^{-1}$ to $10^{3} \mathrm{~s}^{-1}$ for anchor diameters of 12.7-mm and 15.9-mm respectively. For anchor diameter of $19.1 \mathrm{~mm}$ the DIF increased to 1.21 at strain rate of $10 \mathrm{~s}^{-1}$ where concrete cone breakout failure is observed. The DIF of 1.11 and 1.13 were obtained at strain rates of $10^{2} \mathrm{~s}^{-1}$ and $10^{3} \mathrm{~s}^{-1}$ where steel anchor failure is observed.

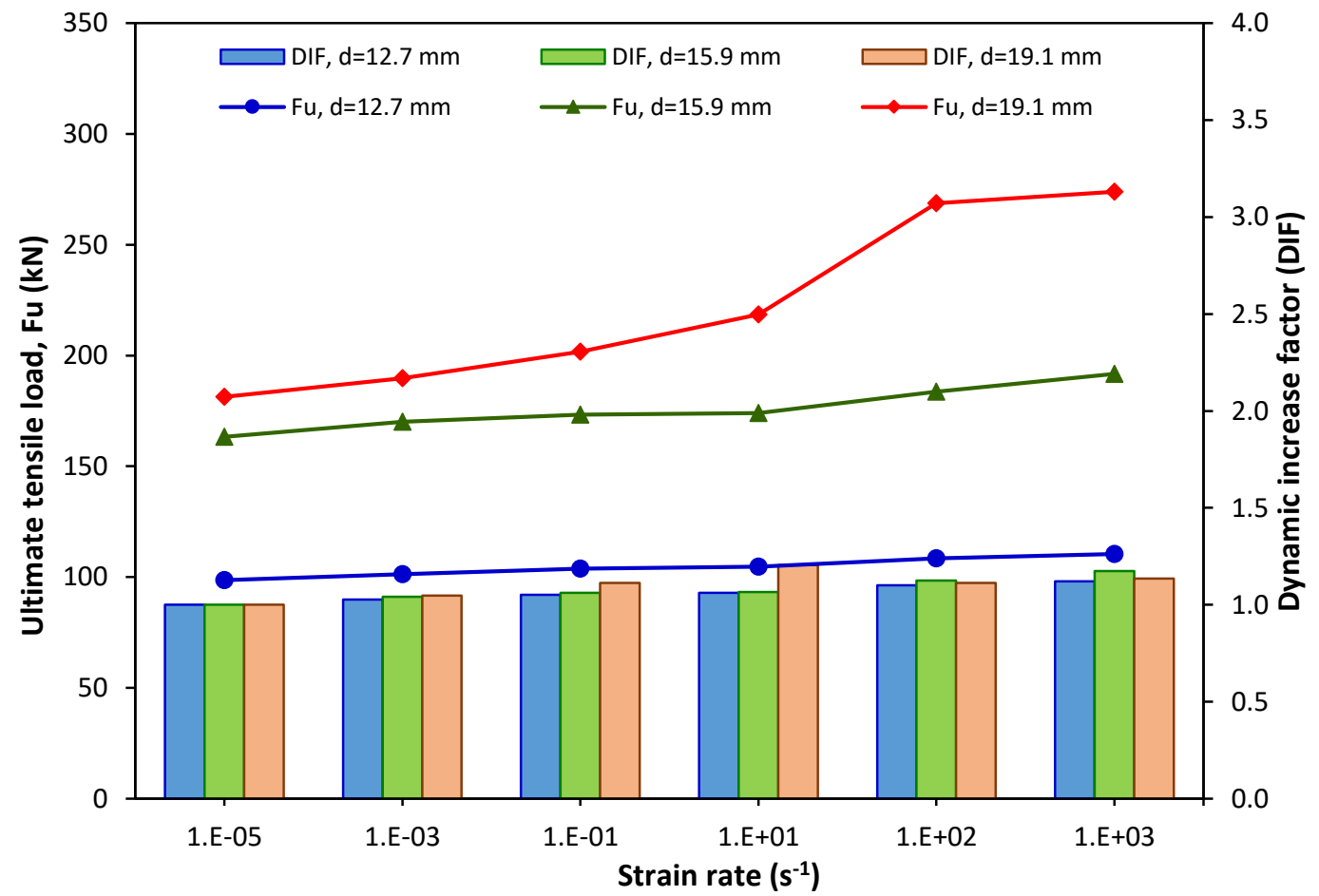

Figure 4-26: Ultimate tensile load and DIF versus strain rate for the cast-in-place anchor of $127 \mathrm{~mm}$ embedment depth 
As shown in Figure 4-27, at embedment depth of $152.4 \mathrm{~mm}$, the ultimate tensile load increased $10.6 \%, 16.5 \%$ and $13.4 \%$ for the $12.7-\mathrm{mm}, 15.9-\mathrm{mm}$ and $19.1-\mathrm{mm}$ diameter castin-place anchors when the strain rate increased from $10^{-5} \mathrm{~s}^{-1}$ to $10^{3} \mathrm{~s}^{-1}$ where the steel fracture is the dominant failure mode. It can be seen that, approximately linear relation is obtained between the ultimate tensile load and the strain rate. Figure 4-27 is representative of the DIF of cast-in-place anchorage system failing by steel anchor fracture. DIF of 1.11, 1.17 and 1.13 were obtained for anchor diameters of $12.7 \mathrm{~mm}, 15.9 \mathrm{~mm}$ and $19.1 \mathrm{~mm}$ with embedment depth of $152.4 \mathrm{~mm}$ at high strain rate of $10^{3} \mathrm{~s}^{-1}$.

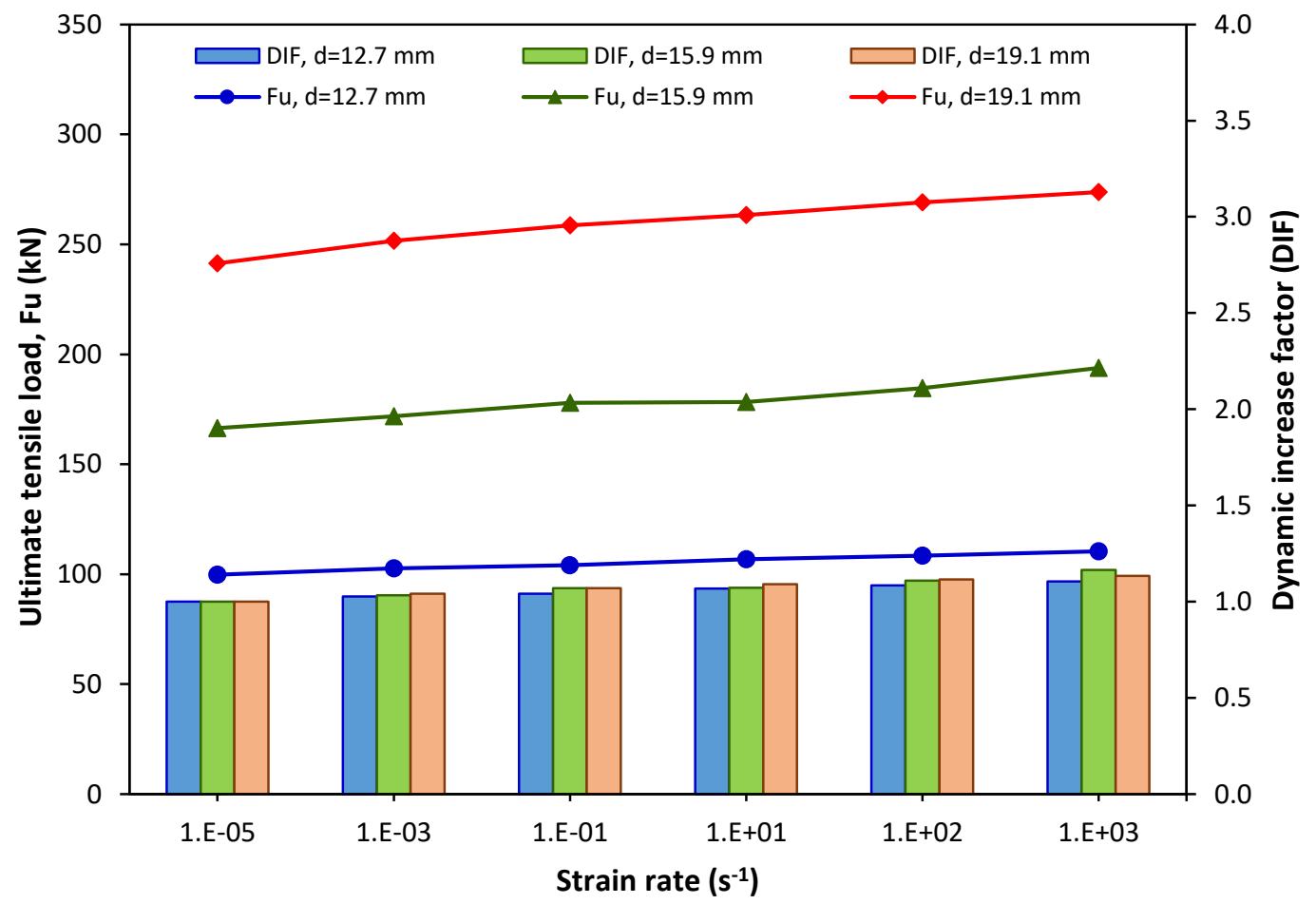

Figure 4-27: Ultimate tensile load and DIF versus strain rate for the cast-in-place anchor of $152.4 \mathrm{~mm}$ embedment depth

It can be seen that maximum tensile loads of $110.42 \mathrm{kN}, 193.82 \mathrm{kN}$ and $273.79 \mathrm{kN}$ were obtained at high strain rate of $10^{3} \mathrm{~s}^{-1}$ and embedment depth of $152.4 \mathrm{~mm}$ for the $12.7-\mathrm{mm}$, 15.9-mm and 19.1-mm diameter cast-in-place anchors respectively. 
The increase in the ultimate load with the increase in strain rate is attributed to the fact that the strain rate has an effect on the mechanical properties of the concrete and steel materials. For the concrete material, the tensile and compressive strengths increase with the increase in the strain rate (Hentz et al., 2004). Hence, the concrete resistance increase results in increase of the ultimate load capacity of the anchorage system. Also, increasing the strain rate increases the modulus of elasticity of the concrete (Shkolnik, 2008) and its energy absorption capacity (Bischoff \& Perry, 1991). Moreover, the yield and ultimate strength of the steel increase with increase in the strain rate (Fu et al., 1991). This is due to the increase in the deformations and dislocations of steel at high strain rate. The strain rate has an effect on the microstructure of the steel, increasing the strain rate resulted in increasing the deformation level (Lee et al., 2007). In general, the DIF increased with the increase in the strain rate where the concrete and steel strengths increased with the increase in strain rate. The increase in the tensile strength of concrete with the increase in the strain rate is higher than that for the steel (Malvar \& Ross, 1998; Malvar \& Crawford, 1998). Hence, maximum DIF of 1.23 was obtained for anchor diameter of $12.7 \mathrm{~mm}$ with embedment depth of 76.2 $\mathrm{mm}$ at strain rate of $10 \mathrm{~s}^{-1}$ where concrete cone breakout failure was observed. Maximum DIF of 2.11 and 2.62 were obtained for the anchor diameters of 15.9-mm and 19.1-mm respectively with anchor embedment depth of $76.2 \mathrm{~mm}$ at high strain rate of $10^{2} \mathrm{~s}^{-1}$.

The DIF of cast-in-place anchors at different strain rates can be represented as a function of the strain rate ratio in accordance with Equation (4.4).

$$
D I F=\frac{F_{u d}}{F_{u s}}=A\left(\frac{\dot{\varepsilon}_{d}}{\dot{\varepsilon}_{s}}\right)^{B}
$$


$F_{u d}=F_{u s} \cdot A\left(\frac{\varepsilon_{d}}{\dot{\varepsilon}_{s}}\right)^{B}$

Where

$F_{u s}, F_{u d}$ are the ultimate static and dynamic tensile loads of the anchorage to concrete systems respectively. A and $\mathrm{B}$ are constants to be determined from experimental or numerical parametric studies, and $\dot{\varepsilon}_{s}$ and $\dot{\varepsilon}_{d}$ are the static and dynamic strain rates respectively.

Table 4-9 shows the maximum DIF for the cast-in-place anchors under tensile load where concrete cone breakout failure and steel anchor failure modes were observed.

Table 4-9: Maximum dynamic increase factor for the cast-in-place anchors under tensile

\begin{tabular}{|c|c|c|c|c|c|c|}
\hline \multirow{2}{*}{$\begin{array}{c}\text { Model } \\
\text { No. }\end{array}$} & \multirow{2}{*}{$\begin{array}{c}\mathrm{d} \\
(\mathrm{mm})\end{array}$} & \multirow{2}{*}{$\begin{array}{c}\mathrm{h}_{\mathrm{ef}} \\
(\mathrm{mm})\end{array}$} & \multirow[t]{2}{*}{$\dot{\varepsilon}\left(\mathrm{s}^{-1}\right)$} & DIF & \multirow[t]{2}{*}{$\dot{\varepsilon}\left(\mathrm{s}^{-1}\right)$} & DIF \\
\hline & & & & $\mathrm{CC}$ & & $\mathrm{S}$ \\
\hline 1 & 12.7 & 76.2 & 10 & 1.23 & $10^{3}$ & 1.12 \\
\hline 2 & 12.7 & 101.6 & - & - & $10^{3}$ & 1.13 \\
\hline 3 & 12.7 & 127.0 & - & - & $10^{3}$ & 1.12 \\
\hline 4 & 12.7 & 152.4 & - & - & $10^{3}$ & 1.11 \\
\hline 5 & 15.9 & 76.2 & 10 & 1.43 & $10^{3}$ & 1.17 \\
\hline 6 & 15.9 & 101.6 & 10 & 1.24 & $10^{3}$ & 1.17 \\
\hline 7 & 15.9 & 127.0 & - & - & $10^{3}$ & 1.17 \\
\hline 8 & 15.9 & 152.4 & - & - & $10^{3}$ & 1.17 \\
\hline 9 & 19.1 & 76.2 & 10 & 1.74 & $10^{3}$ & 1.13 \\
\hline 10 & 19.1 & 101.6 & 10 & 1.34 & $10^{3}$ & 1.13 \\
\hline 11 & 19.1 & 127.0 & 10 & 1.21 & $10^{3}$ & 1.13 \\
\hline 12 & 19.1 & 152.4 & - & - & $10^{3}$ & 1.13 \\
\hline
\end{tabular}




\subsubsection{Regression Analysis for cast-in-place anchors under tensile load}

To develop an accurate predictive model based on the finite element results for determining the DIF of the cast-in-place anchorage to concrete systems, regression analysis was performed. The DIF is plotted as a function of the strain rate ratio $\left(\dot{\varepsilon}_{d} / \dot{\varepsilon}_{s}\right)$ based on the numerical results obtained to account for the effect of the strain rate on the ultimate tensile load. Where the strain rate ratio $\left(\dot{\varepsilon}_{d} / \dot{\varepsilon}_{s}\right)$ is the ratio of dynamic strain rate $\left(\dot{\varepsilon}_{d}\right)$ to the static strain rate $\left(\dot{\varepsilon}_{s}\right)$. Average value of the DIF for the cast-in-place anchorage systems with anchor diameters of $12.7-\mathrm{mm}, 15.9-\mathrm{mm}$ and $19.1-\mathrm{mm}$ was calculated to adjust the DIF for the effect of anchor diameter. Figure 4-28 shows the relation between the DIF and the strain rate ratio $\left(\dot{\varepsilon}_{d} / \dot{\varepsilon}_{s}\right)$ for the cast-in-place anchors exhibited concrete cone breakout failure mode. The increase in the strain rate increased the strengths of concrete and steel materials resulted in increasing the ultimate load and the DIF. Figure 4-29 shows the relation between the DIF and the strain rate ratio $\left(\dot{\varepsilon}_{d} / \dot{\varepsilon}_{S}\right)$ for the cast-in-place anchors exhibited steel anchor failure mode. 


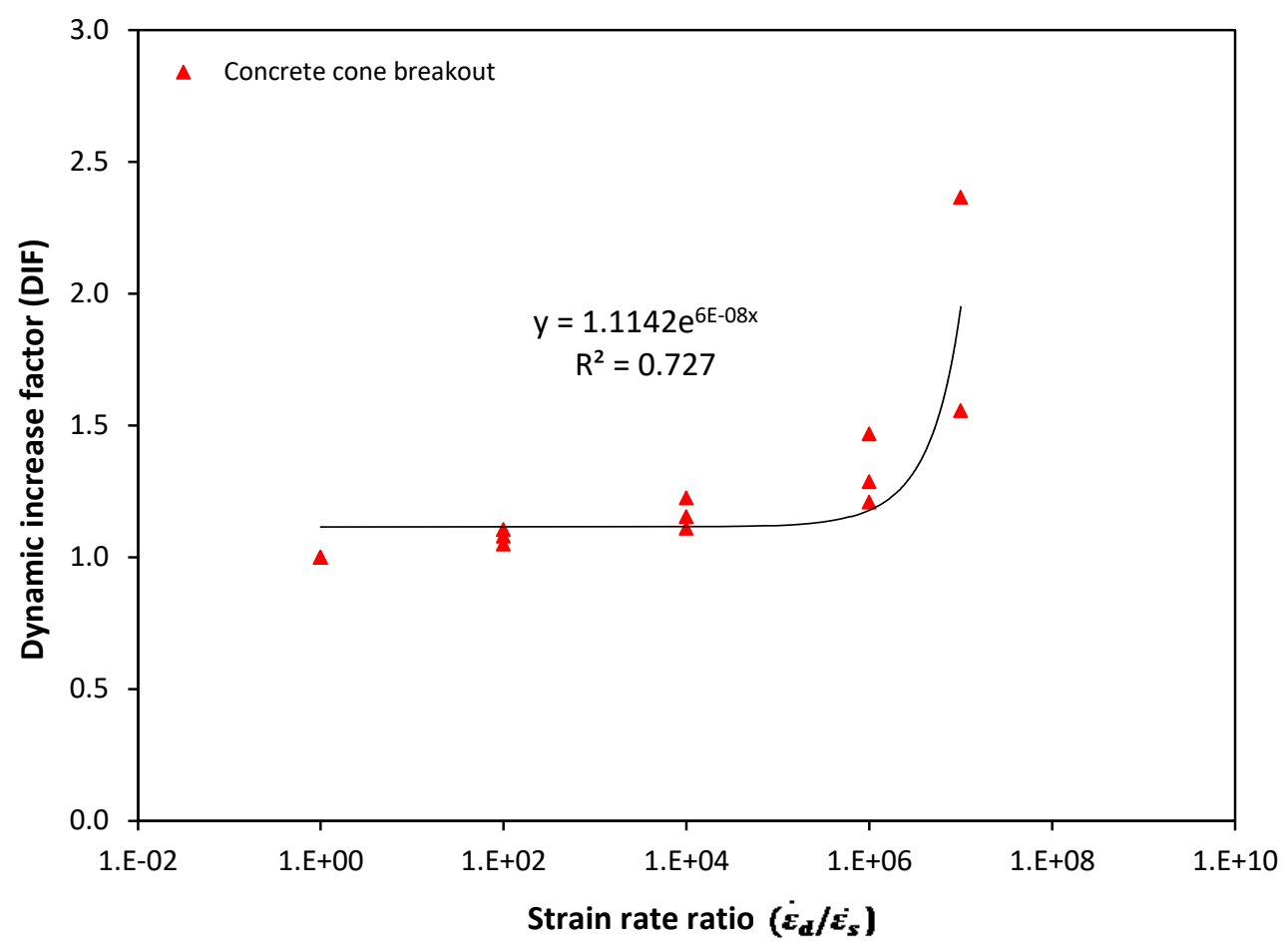

Figure 4-28: Effect of strain rate ratio on the DIF for cast-in-place anchor exhibiting concrete cone breakout failure

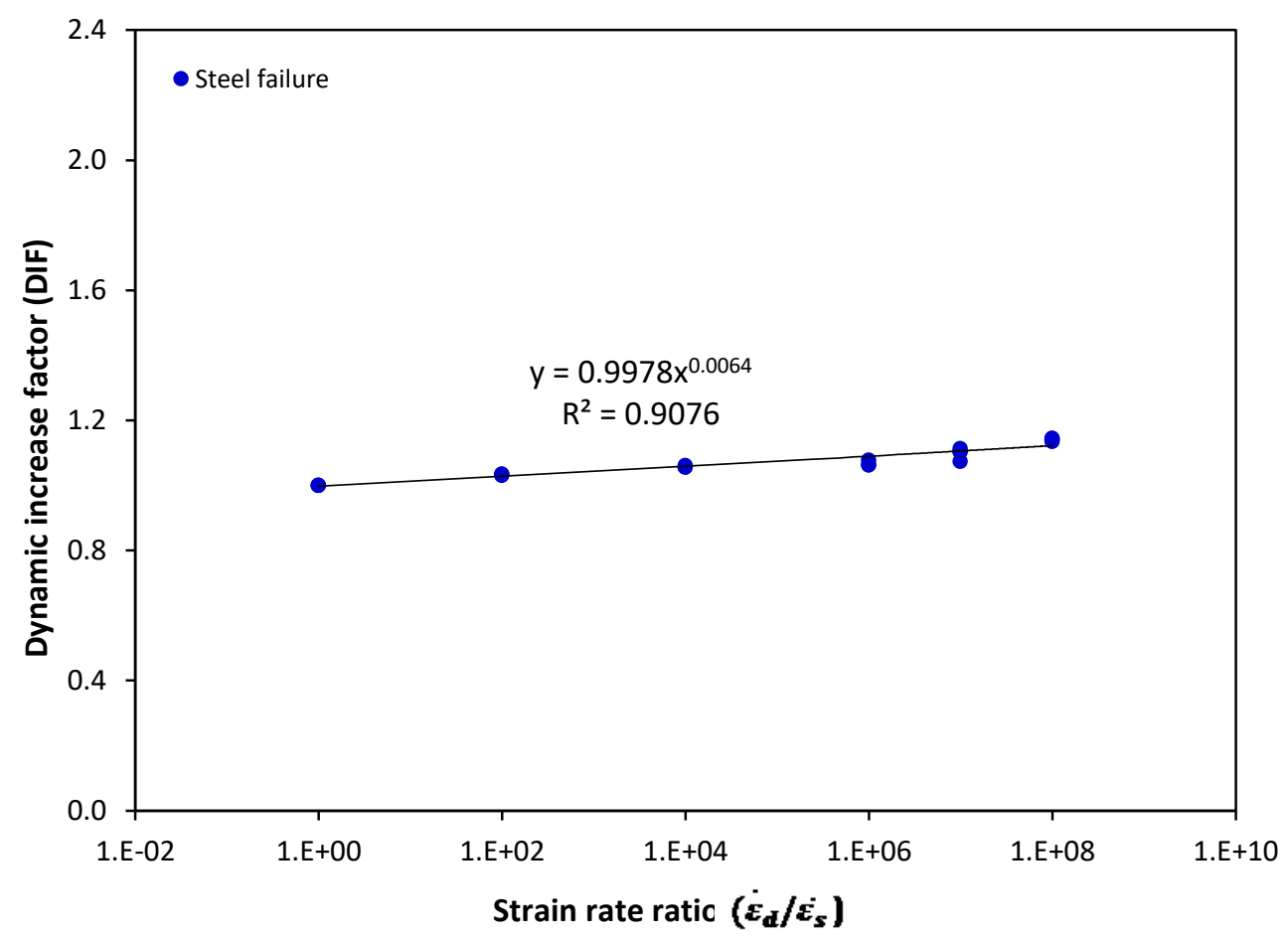

Figure 4-29: Effect of strain rate ratio on the DIF for cast-in-place anchor exhibiting steel failure 
Various regression models; exponential, linear, logarithmic and power models were used to develop a formula that relates the DIF with the strain rate as shown in Tables 4-10 and 4-11 for concrete cone breakout and steel anchor failure respectively. The adequacy of the predicted model is verified according to the following tests: calculating coefficient of determination $\mathrm{R}^{2}$ and residual analysis (Montgomery, 2013). A good probability distribution of the results is obtained when the coefficient of determination $\left(\mathrm{R}^{2}\right)$ is closer to one (Ceci et al., 2012).

Table 4-10: Statistical models and coefficient of determination to predict the DIF for cast-in-place anchors exhibited concrete cone breakout failure under tensile load

\begin{tabular}{|c|c|c|}
\hline Statistical models & Formulae & Coefficient of determination $\left(\mathrm{R}^{2}\right)$ \\
\hline Exponential & $D I F=1.1142 e^{6 \mathrm{E}-08\left(\frac{\dot{\varepsilon}_{d}}{\dot{\varepsilon}_{s}}\right)}$ & 0.727 \\
\hline Linear & $D I F=9 E-08\left(\frac{\dot{\varepsilon}_{d}}{\dot{\varepsilon}_{s}}\right)+1.1158$ & 0.726 \\
\hline Logarithmic & $D I F=0.0435 \ln \left(\frac{\dot{\varepsilon}_{d}}{\dot{\varepsilon}_{s}}\right)+0.8996$ & 0.640 \\
\hline Power & $D I F=0.9442\left(\frac{\dot{\varepsilon}_{d}}{\dot{\varepsilon}_{s}}\right)^{0.0313}$ & \\
\hline
\end{tabular}

Table 4-11: Statistical models and coefficient of determination to predict the DIF for cast-in-place anchors exhibited steel failure under tensile load

\begin{tabular}{|c|c|c|}
\hline Statistical models & Formulae & Coefficient of determination $\left(\mathrm{R}^{2}\right)$ \\
\hline Exponential & $D I F=1.0505 e^{9 \mathrm{E}-10\left(\frac{\dot{\varepsilon}_{d}}{\dot{\varepsilon}_{s}}\right)}$ & 0.600 \\
\hline Linear & $D I F=9 E-10\left(\frac{\dot{\varepsilon}_{d}}{\dot{\varepsilon}_{s}}\right)+1.051$ & 0.618 \\
\hline Logarithmic & $D I F=0.0068 \ln \left(\frac{\dot{\varepsilon}_{d}}{\dot{\varepsilon}_{s}}\right)+0.9964$ & 0.908 \\
\hline Power & $D I F=0.9978\left(\frac{\dot{\varepsilon}_{d}}{\dot{\varepsilon}_{s}}\right)^{0.0064}$ & \\
\hline
\end{tabular}


As shown in Tables 4-10 and 4-11, the highest coefficients of determination of $73 \%$ and $91 \%$ are obtained from the exponential model and power model for the concrete cone breakout and steel anchor failure modes respectively. The exponential model can represent $73 \%$ and $91 \%$ of the total variation between the DIF obtained from the finite element analysis and the predicted values for the concrete cone breakout and steel anchor failure modes respectively. The predicted formulae for the DIF can be presented in Equations (4.6) and (4.7) for the concrete cone breakout and steel anchor failure modes respectively as follows:

$D I F=1.1142 e^{6 \mathrm{E}-08\left(\frac{\dot{\varepsilon}_{d}}{\dot{\varepsilon}_{s}}\right)}$

$D I F=0.9978\left(\frac{\dot{\varepsilon}_{d}}{\dot{\varepsilon}_{s}}\right)^{0.0064}$

Where $\dot{\varepsilon}_{S}$ is the static strain rate $=10^{-5} \mathrm{~s}^{-1}, \dot{\varepsilon}_{d}$ is the dynamic strain rate ranging from $10^{-3} \mathrm{~s}^{-}$ ${ }^{1}$ to $10^{3} \mathrm{~s}^{-1}$.

The proposed Equations (4.6) and (4.7) can be used to predict the ultimate dynamic load $\left(F_{u d}\right)$ for the concrete cone breakout and steel anchor failure modes respectively as follows:

$F_{u d}=F_{u s} \times 1.1142 e^{6 \mathrm{E}-08\left(\frac{\dot{\varepsilon}_{d}}{\dot{\varepsilon}_{s}}\right)}$

$F_{u d}=F_{u s} \times 0.9978\left(\frac{\dot{\varepsilon}_{d}}{\dot{\varepsilon}_{s}}\right)^{0.0064}$ 
Where the ultimate static load $\left(F_{u s}\right)$ can be determined from Equations 4.1 and 4.3 for the concrete cone breakout and from Equation (4.2) for steel anchor failure mode.

Residual analysis has been performed to measure the difference between the results obtained from finite element analysis of the cast-in-place anchors under tensile load and fitted results of DIF obtained from Equations (4.6) and (4.7). Where the residual is the difference between the observed values (FEA results) and the predicted values (obtained from the equations). The residual plots are presented in Figures 4-30 and 4-31.

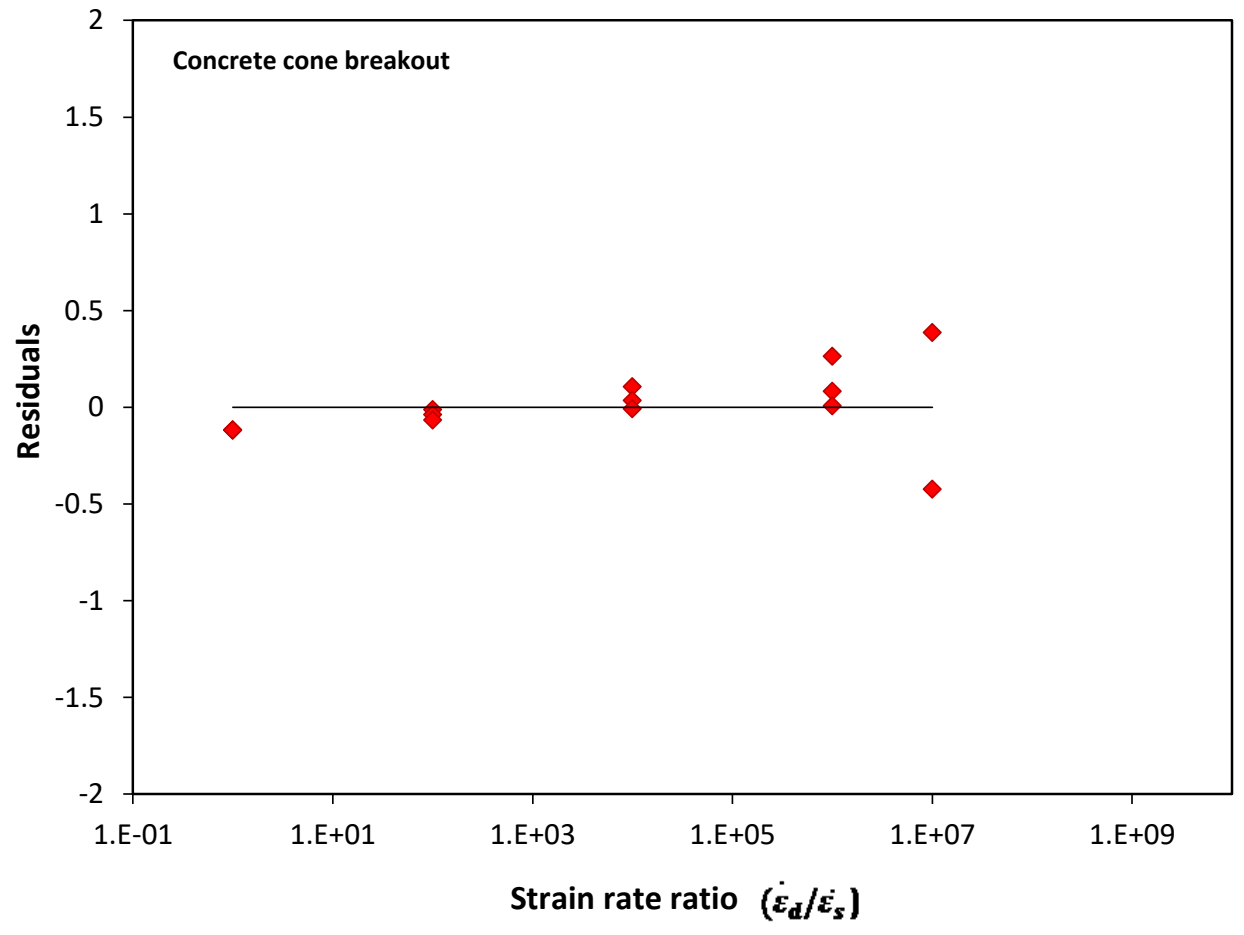

Figure 4-30: Residual versus strain rate ratio for the cast-in-place anchors exhibited concrete cone breakout failure 


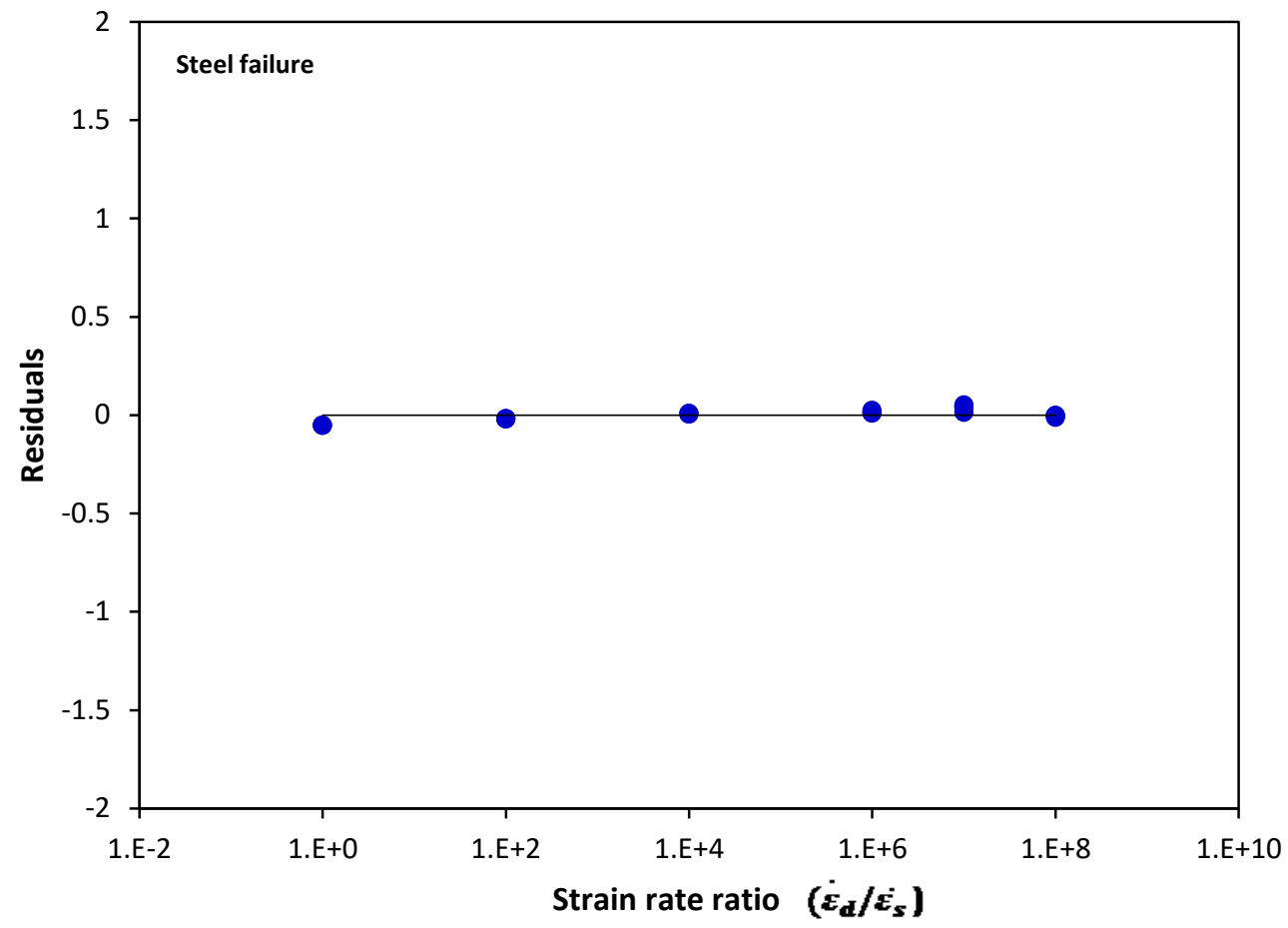

Figure 4-31: Residual versus strain rate ratio for the cast-in-place anchors exhibited steel failure

As shown in Figure 4-30, for the concrete cone breakout failure the variance in the residual increases with the increase in the strain rate ratio. For the steel anchor failure (Figure 431), the residual exhibited horizontal trend line at strain rate ratio up to $10^{8}$.

In addition, to evaluate the adequacy of the proposed equations, new cast-in-place anchor models with diameters of $9.5 \mathrm{~mm}, 12.7 \mathrm{~mm}, 15.9 \mathrm{~mm}$ and $19.1 \mathrm{~mm}$ and embedment depths of $89 \mathrm{~mm}, 114 \mathrm{~mm}$ and $140 \mathrm{~mm}$ were developed. The relation between the DIF results of the newly developed numerical models and the regression models (Equations (4.6) and (4.7)) are presented as shown in Figures 4-32 and 4-33 for the concrete cone breakout failure and steel failure modes respectively. As shown in the Figure 4-32, the DIF is observed distributed around the equality line with some divergence for the anchors 
exhibited concrete cone breakout failure for the higher values of the DIF where the residual increased at higher strain rates. For the anchors exhibited steel failure as shown in Figure $4-33$, the DIF is distributed around the equality line.

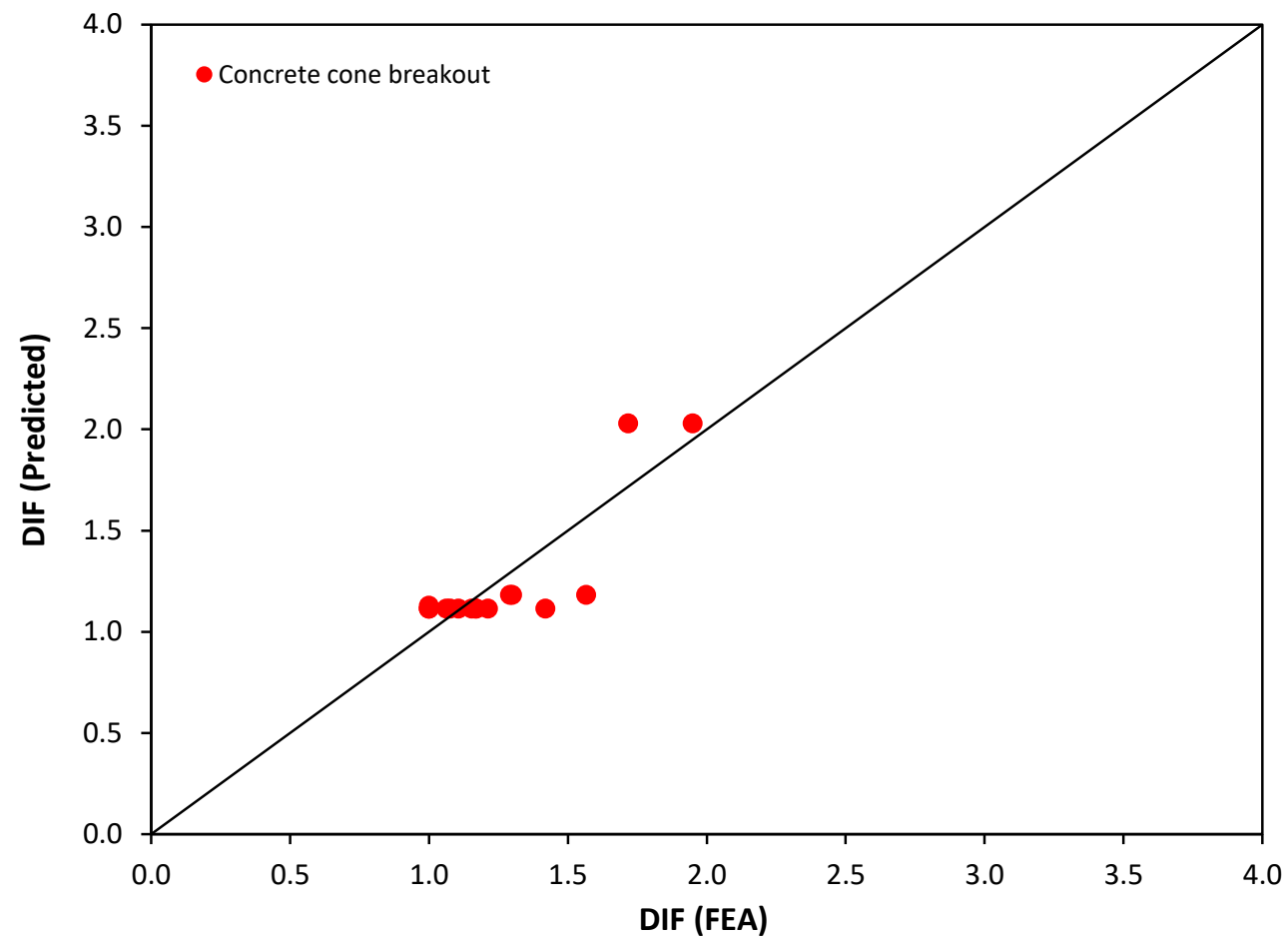

Figure 4-32: DIF obtained from the finite element analysis versus the predicted DIF for the cast-in-place anchor exhibited concrete cone breakout failure 


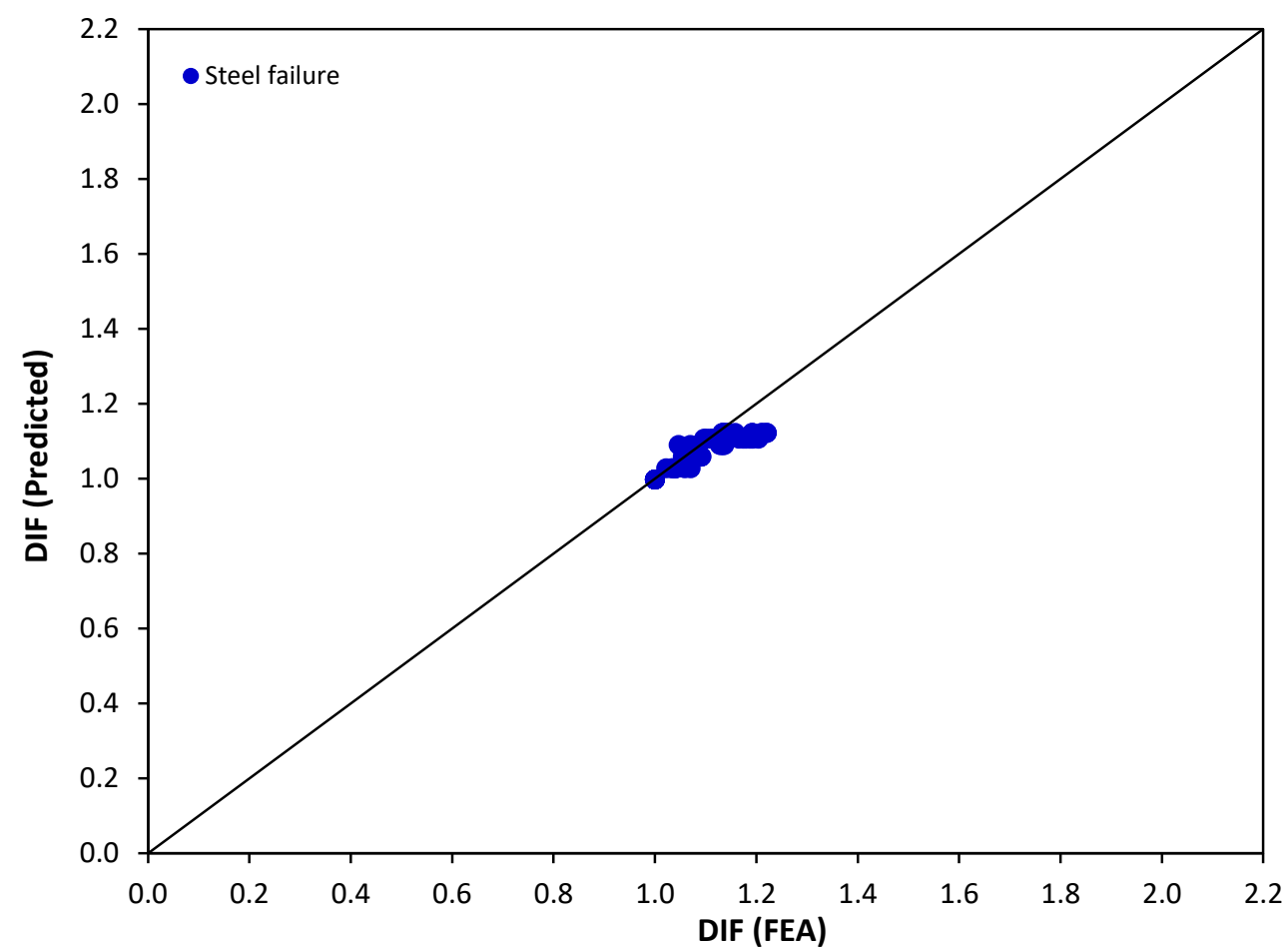

Figure 4-33: DIF obtained from the finite element analysis versus the predicted DIF for the cast-in-place anchor exhibited steel failure

In order to verify the results obtained from the finite element analysis, a comparison has been made between the ultimate dynamic load obtained from the finite element analysis for the cast-in-place anchors exhibited concrete cone breakout failure and the proposed Equation by Fujikake et al. (Equation (4.10)) (Fujikake et al., 2003). Fujikake et al. (2003) proposed an equation to determine the ultimate dynamic cone resistance for the shallow embedment depths (Fujikake et al., 2003) as follows:

$$
\begin{aligned}
& F_{c d}=A_{e} \cdot F_{t d} \cdot \frac{\alpha}{\sqrt{h_{e f}}} \\
& A_{e}=\pi \cdot h_{e f} \cdot \tan \theta\left(d+h_{e f} \cdot \tan \theta\right)
\end{aligned}
$$


Where, $F_{t d}$ is determined according to the proposed equation by Ross et al. (1989) (Ross et al., 1989) as follows:

$\frac{F_{t d}}{F_{s}}=\exp \left[0.00126\left(\log _{10} \frac{\dot{\varepsilon}_{d}}{\dot{\varepsilon}_{s}}\right)^{3.373}\right]$

$F_{S}=0.23\left(f_{c}^{\prime}\right)^{2 / 3}$

Where $F_{c d}$ is the ultimate dynamic concrete cone breakout, $A_{e}$ is the projected area of concrete cone failure, $\alpha=3.48 \times 10^{-3}, \theta$ is the crack propagation angle $\left(\theta=60^{\circ}\right)$, where the concrete cone breakout angle is equal to $30^{\circ} . F_{s}$ and $F_{t d}$ are the static and dynamic tensile strength of concrete respectively. Table 4-12 shows a comparison of the ultimate dynamic load obtained from the finite element analysis and the proposed equation by Fujikake et al. (Fujikake et al., 2003). As shown in the table, the FEA results for the anchors overpredict the ultimate load obtained by Fujikake et al. prediction equation. 
Table 4-12: Comparison between ultimate dynamic load obtained from the FEA and proposed equations by Fujikake et al. (2003) for the cast-in-place anchor exhibited concrete cone breakout failure

\begin{tabular}{|c|c|c|c|c|c|c|c|c|c|}
\hline \multirow{2}{*}{$\begin{array}{c}\mathrm{d} \\
(\mathrm{mm})\end{array}$} & \multirow{2}{*}{$\begin{array}{c}\mathbf{h}_{\mathrm{ef}} \\
(\mathrm{mm})\end{array}$} & \multirow{2}{*}{$\begin{array}{c}\dot{\varepsilon}_{s} \\
\left(\mathrm{~s}^{-1}\right)\end{array}$} & \multirow{2}{*}{$\begin{array}{c}\dot{\varepsilon}_{d} \\
\left(\mathrm{~s}^{-1}\right)\end{array}$} & \multirow[t]{2}{*}{$\dot{\varepsilon}_{d} / \dot{\varepsilon}_{s}$} & \multicolumn{2}{|c|}{ FEA } & \multirow{2}{*}{$\begin{array}{c}F_{\text {ud }} \\
\text { Regression } \\
(\mathrm{kN})\end{array}$} & \multirow{2}{*}{$\begin{array}{c}\text { Fud }_{\text {ud }} \\
\text { Fujikake } \\
(\mathbf{k N}) \\
\end{array}$} & \multirow{2}{*}{$\begin{array}{c}\text { Fud }_{\text {uEA/ }} \\
\text { Fud }_{\text {udikake }} \text { Fujikak }\end{array}$} \\
\hline & & & & & $\begin{array}{c}\mathbf{F}_{\text {us }} \\
(\mathbf{k N})\end{array}$ & $\begin{array}{c}F_{\text {ud }} \\
(\mathbf{k N})\end{array}$ & & & \\
\hline 12.7 & 76.2 & $10^{-5}$ & $10^{-3}$ & $10^{2}$ & 64.48 & 68.63 & 71.84 & 53.80 & 1.28 \\
\hline 12.7 & 76.2 & $10^{-5}$ & $10^{-1}$ & $10^{4}$ & 64.48 & 72.16 & 71.89 & 60.79 & 1.19 \\
\hline 15.9 & 76.2 & $10^{-5}$ & $10^{-3}$ & $10^{2}$ & 68.61 & 76.32 & 76.45 & 54.99 & 1.39 \\
\hline 15.9 & 76.2 & $10^{-5}$ & $10^{-1}$ & $10^{4}$ & 68.61 & 83.96 & 76.49 & 62.13 & 1.35 \\
\hline 15.9 & 101.6 & $10^{-5}$ & $10^{-3}$ & $10^{2}$ & 104.17 & 110.25 & 116.07 & 82.38 & 1.34 \\
\hline 15.9 & 101.6 & $10^{-5}$ & $10^{-1}$ & $10^{4}$ & 104.17 & 116.33 & 116.14 & 93.09 & 1.25 \\
\hline 19.1 & 76.2 & $10^{-5}$ & $10^{-3}$ & $10^{2}$ & 72.88 & 83.09 & 81.20 & 56.18 & 1.48 \\
\hline 19.1 & 76.2 & $10^{-5}$ & $10^{-1}$ & $10^{4}$ & 72.88 & 96.94 & 81.25 & 63.48 & 1.53 \\
\hline 19.1 & 101.6 & $10^{-5}$ & $10^{-3}$ & $10^{2}$ & 116.65 & 128.44 & 129.97 & 83.76 & 1.53 \\
\hline 19.1 & 101.6 & $10^{-5}$ & $10^{-1}$ & $10^{4}$ & 116.65 & 138.88 & 130.05 & 94.64 & 1.47 \\
\hline
\end{tabular}

${ }^{*} \mathrm{~F}_{\text {us }}$ FEA: ultimate static load obtained from FEA, $\mathrm{F}_{\mathrm{ud}}$ FEA: ultimate dynamic load obtained from FEA 


\subsection{Cast-in-place anchors under shear load}

\subsubsection{Finite element modelling for cast-in-place anchors under shear load}

Analysis on the behaviour of cast-in-place anchors embedded into concrete under shear load at different strain rates was performed using the explicit commercial finite element code LS-DYNA (LSTC, 2014). Figure 4-34 shows the geometric configuration and boundary conditions for the cast-in-place anchor model for investigating shear behaviour.

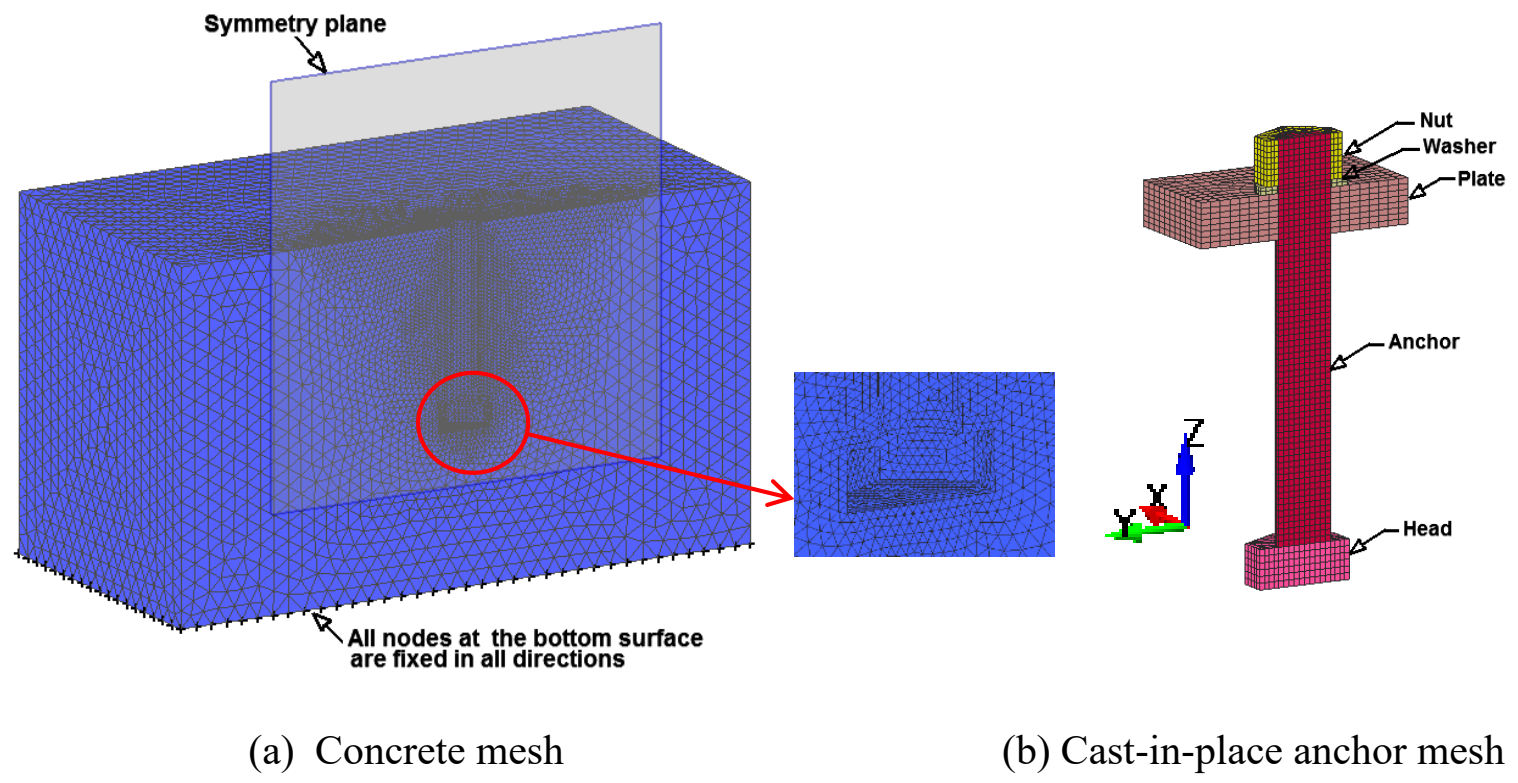

Figure 4-34: Geometric configuration of cast-in-place anchor model under shear load

\subsubsection{Validation of cast-in-place anchor model under shear load}

The finite element model was validated by modeling the specimens in an experimental investigation by Ueda et al. (1991) and comparing ultimate shear loads obtained from the analysis with experimental tests. Two finite element models, representative of the test specimens of Ueda et al. (1991) were developed. Two edge distances of $56 \mathrm{~mm}$ and 106 $\mathrm{mm}$ in the parallel direction $\left(c_{1}\right)$ to direction of the applied load and a larger edge distance 
perpendicular to the applied shear load direction $\left(c_{2}\right)$ were investigated. Figure 4-35 shows the direction of the applied load and the edge distances $c_{1}$ and $c_{2}$.

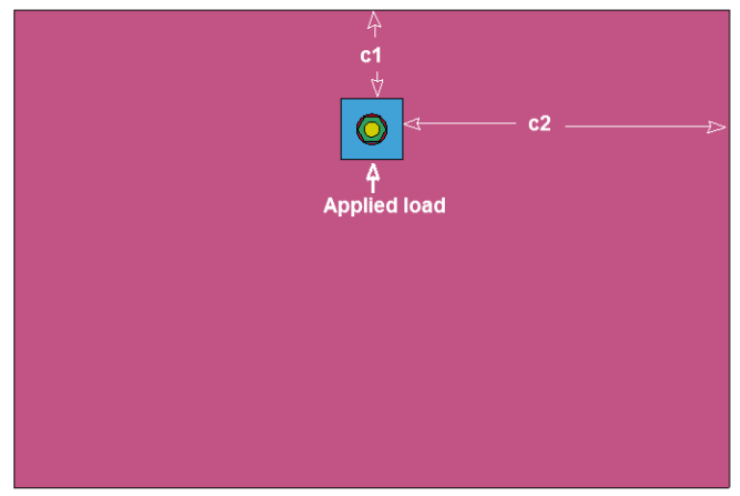

Figure 4-35: Applied shear load in the direction parallel to the edge distance $c_{l}$ and perpendicular to the edge distance $c_{2}$

The anchors had a diameter of 16- $\mathrm{mm}$ and embedment depth of $200 \mathrm{~mm}$ and were cast into concrete with compressive cylinder strength of $24 \mathrm{MPa}$. Mesh sensitivity analysis was performed to determine the suitable mesh size that can best represent the experimental results. The load-displacement relation was not presented in the experimental work conducted by Ueda et al., hence the ultimate shear loads were considered for comparison purpose. Table 4-13 presents a comparison of ultimate shear load from experimental tests by Ueda et al. (Ueda et al., 1991) and finite element analysis (FEA) results under static shear loading. Mesh sizes of $1 \times 2 \times 8 \mathrm{~mm}, 1 \times 1 \times 8 \mathrm{~mm}$ and $0.75 \times 1 \times 8 \mathrm{~mm}$ were investigated. As shown in Table 4-13 the finite element analysis results with the mesh sizes of $1 \times 1 \times 8$ $\mathrm{mm}$ and $0.75 \times 1 \times 8 \mathrm{~mm}$ give converged ultimate shear load results to the experimental results with a percentage difference of $4.5 \%$ and $2.9 \%$ respectively for the edge distance of $56 \mathrm{~mm}$ and a percentage difference of $4.1 \%$ and $3.2 \%$ for the edge distance of $106 \mathrm{~mm}$. The finite element analysis results with mesh size of $0.75 \mathrm{~mm}$ for the steel anchor and a 
biased mesh from 1 to $8 \mathrm{~mm}$ for the concrete show good agreement with the experimental results and was selected for the analysis.

Table 4-13: Effect of mesh size refinement on the convergence of ultimate shear load for cast-in-place anchor

\begin{tabular}{|c|c|c|c|c|c|c|c|}
\hline \multirow[t]{3}{*}{$\begin{array}{l}\text { Model } \\
\text { No. }\end{array}$} & \multirow[t]{3}{*}{$\begin{array}{c}\mathrm{c}_{1} \\
(\mathrm{~mm})\end{array}$} & \multicolumn{3}{|c|}{$\begin{array}{c}\text { Mesh size } \\
(\mathrm{mm})\end{array}$} & \multicolumn{2}{|c|}{$\begin{array}{l}\text { Ultimate shear load } \\
\qquad(\mathrm{kN})\end{array}$} & \multirow[t]{3}{*}{ Failure mode } \\
\hline & & \multirow[t]{2}{*}{ Anchor } & \multicolumn{2}{|c|}{ Concrete } & \multirow[t]{2}{*}{ FEA } & \multirow[t]{2}{*}{ EXP. } & \\
\hline & & & Min. & Max. & & & \\
\hline 1 & \multirow{3}{*}{56} & 1 & 2 & 8 & 12.75 & \multirow{3}{*}{13.70} & \multirow{3}{*}{$\begin{array}{c}\text { Concrete } \\
\text { cone } \\
\text { breakout }\end{array}$} \\
\hline 2 & & 1 & 1 & 8 & 13.11 & & \\
\hline 3 & & 0.75 & 1 & 8 & 14.10 & & \\
\hline 4 & \multirow{3}{*}{106} & 1 & 2 & 8 & 36.82 & \multirow{3}{*}{39.60} & \multirow{3}{*}{$\begin{array}{l}\text { Concrete } \\
\text { cone } \\
\text { breakout }\end{array}$} \\
\hline 5 & & 1 & 1 & 8 & 38.05 & & \\
\hline 6 & & 0.75 & 1 & 8 & 40.88 & & \\
\hline
\end{tabular}

\subsubsection{Comparison of finite element results with design method}

When the anchors are installed far from the concrete free edge and subjected to shear loading, pryout failure and or steel anchor failure are the dominant failure modes. The pryout failure load of the anchor can be calculated according to ACI 318 (ACI Committee $318,2011)$ as in Equation (4.14).

$V_{c p}=k_{c p} N_{c b}$

where

$k_{c p}=\left\{\begin{array}{l}1 \text { for } h_{e f}<63.5 \mathrm{~mm} \\ 2 \text { for } h_{e f} \geq 63.5 \mathrm{~mm}\end{array}\right.$

and 
$N_{c b}=\frac{A_{N}}{A_{N o}} \psi_{e d, N} \cdot \psi_{c, N} \cdot \psi_{c p, N} \cdot N_{b}$

$N_{b}=K \sqrt{f_{c c}^{\prime}} h_{e f}^{1.5}$

$N_{c b}$ is the concrete tensile breakout capacity for the anchor, $A_{N}$ is the actual projected area, $A_{N o}$ is the projected area for the failure surface of concrete, $\psi_{e d, N}$ is a modification factor accounting for edge effects, $\psi_{c, N}$ is a modification factor accounting for cracking, $\psi_{c p, N}$ is a modification factor accounting for post-installed anchors embedded in concrete without reinforcement, $N_{b}$ is the basic concrete breakout strength of a single anchor, $k_{c p}$ is a modification factor for embedment depth effects. $K=15.5$ for the cast-in-place anchors and 13.5 for post installed anchors (Fuchs et al., 1995).

The steel anchor failure for cast-in-place headed bolt can be calculated according to ACI 318 as in Equation (4.18) (ACI Committee 318, 2011).

$V_{u}=0.6 A_{s} \cdot F_{u t}$

where

$A_{s}$ is the effective cross section area of the anchor, $F_{u t}$ is ultimate tensile strength of the anchor.

Ultimate shear load obtained from the finite element analysis was compared with results obtained from ACI 318 method. The anchors were embedded in $30 \mathrm{MPa}$ compressive 
strength concrete. The ultimate shear load was observed to increase with the increase of the anchor diameter from $12.7 \mathrm{~mm}$ to $19.1 \mathrm{~mm}$.

Table 4-14 presents a comparison of ultimate shear load obtained from the finite element analysis (FEA) and ACI 318 (ACI Committee 318, 2011) for the anchor diameters and embedment depths investigated. The failure mode observed from the finite element analysis is also presented in the Table. Minimum value of the ultimate shear load obtained from Equations (4.14) and (4.18) is considered for comparison with the finite element results and included in Table 4-14. It can be seen from Table 4-14 that the ACI method underpredicts the failure load for the anchor diameter of $12.7-\mathrm{mm}$. This can be attributed to the high bending, tensile and shear stresses that are generated in anchor diameter of 12.7$\mathrm{mm}$ due to the applied shear load where the interaction of these stresses causes steel anchor failure. In general, the results show that the ultimate shear load obtained from the finite element analysis is in a good agreement with the ACI 318 method.

Table 4-14: Comparison between FEA and ACI 318 method

\begin{tabular}{|c|c|c|c|c|c|c|}
\hline \multirow{2}{*}{$\begin{array}{c}\text { Model } \\
\text { No. }\end{array}$} & \multirow{2}{*}{$\begin{array}{c}\mathrm{d} \\
(\mathrm{mm})\end{array}$} & $\begin{array}{c}\mathrm{h}_{\mathrm{ef}} \\
(\mathrm{mm})\end{array}$ & \multicolumn{2}{|c|}{$\begin{array}{c}\text { Failure Load } \\
(\mathrm{kN})\end{array}$} & FEA/ACI & $\begin{array}{c}\text { *Failure mode } \\
(\text { FEA })\end{array}$ \\
\cline { 3 - 6 } & & $\begin{array}{c}\text { FEA Static } \\
\left(\dot{\varepsilon}=10^{-5} \mathrm{~s}^{-1}\right)\end{array}$ & ACI 318 & & \\
\hline 1 & 12.7 & 76.2 & 86.64 & 78.59 & 1.10 & $\mathrm{~S}$ \\
\hline 2 & 12.7 & 101.6 & 88.81 & 78.59 & 1.13 & $\mathrm{~S}$ \\
\hline 3 & 12.7 & 152.4 & 90.16 & 78.59 & 1.15 & $\mathrm{~S}$ \\
\hline 4 & 15.9 & 76.2 & 104.37 & 123.18 & 0.85 & $\mathrm{PR}$ \\
\hline 5 & 15.9 & 101.6 & 118.79 & 123.18 & 0.96 & $\mathrm{~S}$ \\
\hline 6 & 15.9 & 152.4 & 120.67 & 123.18 & 0.98 & $\mathrm{~S}$ \\
\hline
\end{tabular}




\begin{tabular}{|c|c|c|c|c|c|c|}
\hline 7 & 19.1 & 76.2 & 124.18 & 125.43 & 0.99 & PR \\
\hline 8 & 19.1 & 101.6 & 152.14 & 177.75 & 0.86 & PR \\
\hline 9 & 19.1 & 152.4 & 174.09 & 177.75 & 0.98 & S \\
\hline
\end{tabular}

* Failure mode: $\mathrm{PR}=$ pryout, $\mathrm{S}=$ steel anchor failure

\subsubsection{Crack pattern for cast-in-place anchors under shear load}

Contours of effective plastic strain for cast-in-place anchors with different anchor diameters and embedment depths at failure are shown in Figure 4-36 at strain rate of $10^{-5}$ $\mathrm{s}^{-1}$. Fringe levels in Figure 4-36 (level of plastic straining) represent the localized damage. As shown in the figure, concrete pryout failure is observed at low strain rate of $10^{-5} \mathrm{~s}^{-1}$ for anchor diameters of $15.9 \mathrm{~mm}$ and $19.1 \mathrm{~mm}$ with embedment depth of $76.2 \mathrm{~mm}$, while steel anchor failure was observed for anchor diameter of $12.7 \mathrm{~mm}$. Steel anchor failure was also observed for all the anchor diameters at embedment depths of $101.6 \mathrm{~mm}$ and $152.4 \mathrm{~mm}$ except for anchor diameter of $19.1 \mathrm{~mm}$ with embedment depth of $101.6 \mathrm{~mm}$ which exhibited concrete pryout failure. This is attributed to the increase in the anchor diameter which increased the strength of the anchor leading to initiation of cracking in the concrete at the bottom of the anchor that propagate diagonally towards the concrete surface and resulted in pryout failure. The pryout failure is characterized by concrete half cone breakout behind the anchor associated with concrete cracking/crushing in front of the anchor. The level of damage increased with the increase in the anchor diameter from $12.7-\mathrm{mm}$ to 19.1 $\mathrm{mm}$ where more cracking was observed in the concrete. 


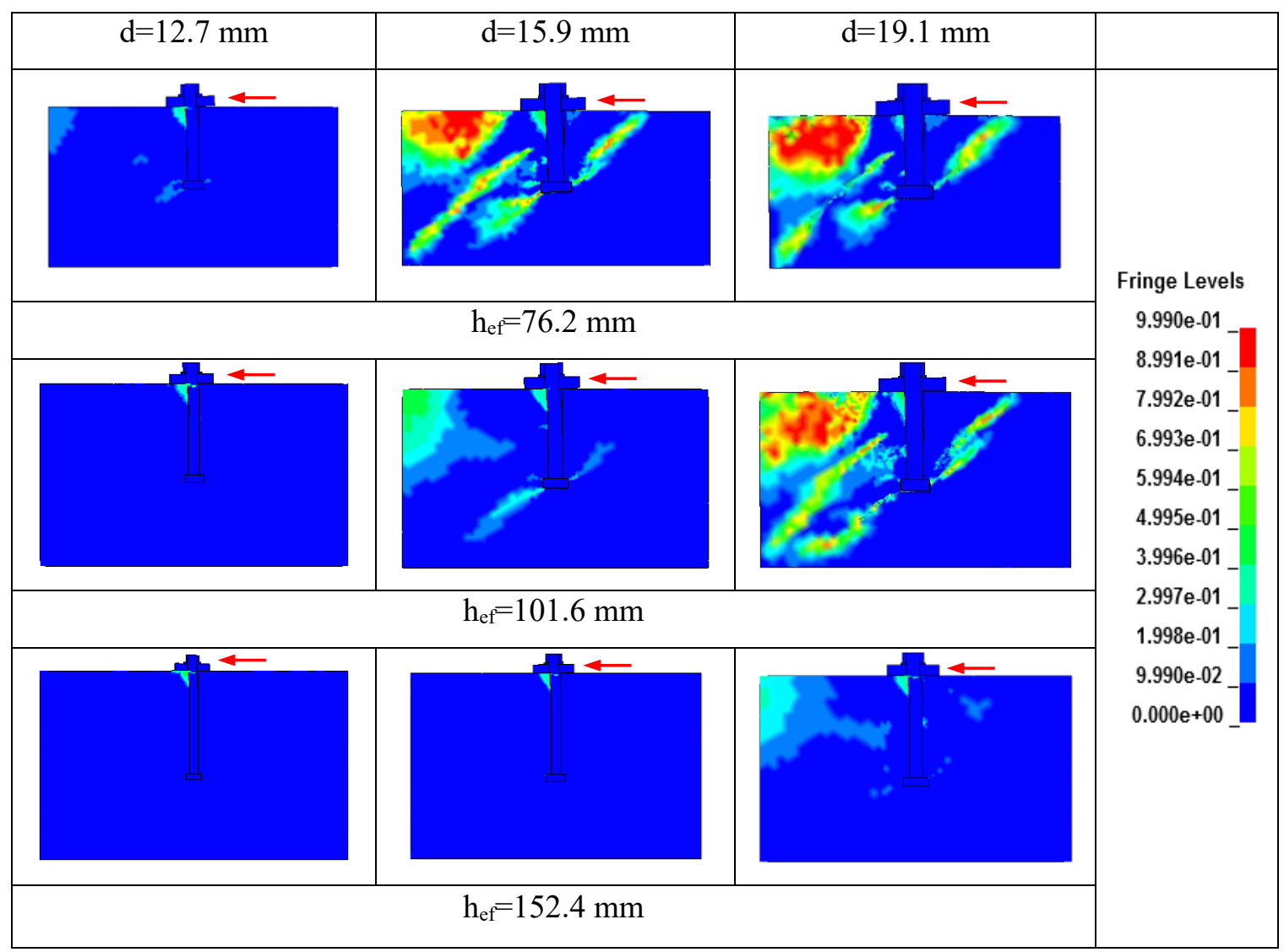

Figure 4-36: Plastic strain contours for cast-in-place anchor under shear load at strain rate of $10^{-5} \mathrm{~s}^{-1}$

According to Zhao (Zhao, 1994) the applied shear load is transferred to the concrete through four mechanisms. The first is the friction force between the concrete and the anchor plate and oriented in opposite direction to the applied load $\left(R_{1}\right)$. The second is the reaction force of the concrete towards the anchor bolt $\left(R_{2}\right)$. The third is the reaction force at the front tip of the plate $\left(R_{3}\right)$. The fourth is the tensile force of the anchor bolt $\left(R_{4}\right)$ (Zhao, 1994). In addition, there is a reaction force on the anchor head that pushes on the concrete resulting in concrete pryout failure $\left(R_{5}\right)$. Figure 4-37 presents the reaction forces on the concrete due to the applied shear load on the anchor. 


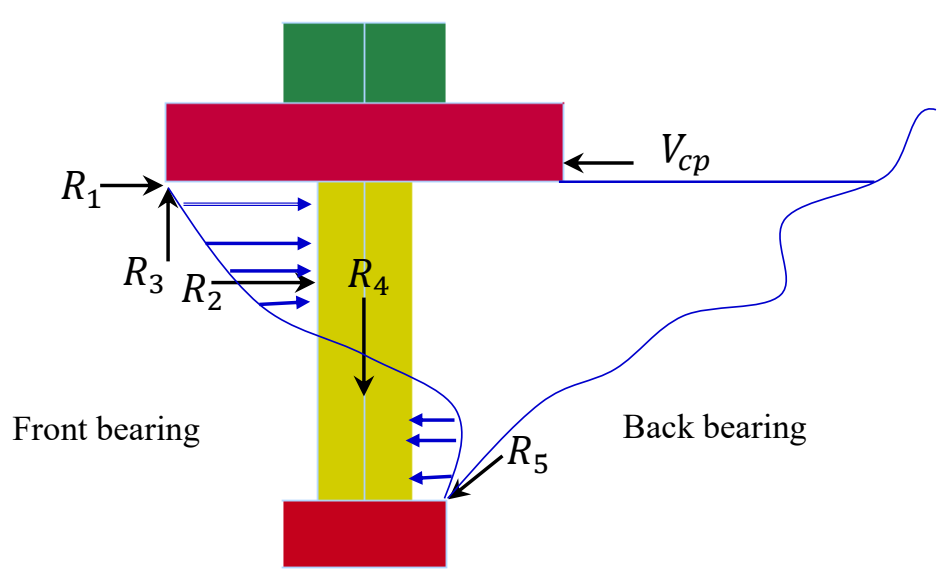

Figure 4-37: Pryout failure mechanism of the cast-in-place anchor

The shear load applied on the anchor plate develops stresses in the concrete in front of the anchor, produces a rotation moment of the anchor plate. As a result two forces are developed; compression force on the concrete under the tip of the anchor plate and tensile force in the anchor (Zhao, 1994). The stresses on the concrete in front of the anchor increase with the increase in the applied shear load, create concrete fracture under the anchor plate and increase the rotation of the anchor plate. As a result, half concrete cone breakout is formed at the back side of the anchor leads to pryout failure.

\subsubsection{Effect of strain rate on the level of damage and failure mode}

To demonstrate the effect of strain rate on the failure mechanism and level of damage, plastic strain contours for cast-in-place anchors subjected to different shear strain rates were investigated. Figures 4-38, 4-39 and 4-40 present the failure mode for the 12.7-mm, 15.9-mm and 19.1-mm diameter cast-in-place anchors respectively. As shown in Figure 438 , steel anchor failure is the dominant failure mode for the $12.7-\mathrm{mm}$ diameter cast-inplace anchor at strain rates ranging from $10^{-3} \mathrm{~s}^{-1}$ to $10^{3} \mathrm{~s}^{-1}$. Also, steel anchor failure is the 
dominant failure mode for the anchor diameter of 15.9-mm (Figure 4-39) at the strain rates of $10^{-3} \mathrm{~s}^{-1}$ to $10^{3} \mathrm{~s}^{-1}$ except for embedment depth of $76.2 \mathrm{~mm}$ at strain rate of $10^{-3} \mathrm{~s}^{-1}$ where pryout failure was observed. For the anchor diameter of $19.1 \mathrm{~mm}$ (Figure 4-40), pryout failure mode was observed at embedment depths of $76.2 \mathrm{~mm}$ and $101.6 \mathrm{~mm}$ at strain rate of $10^{-3} \mathrm{~s}^{-1}$. At early stage of loading, the cracks initiated on the top surface of the concrete around the anchor in the direction of the applied load and propagated along the anchor embedment depth. Cracking in the concrete was subsequently transferred to a wider area in the concrete, leading to pryout failure. Steel anchor failure was observed at embedment depth of $152.4 \mathrm{~mm}$. At strain rate of $10^{-1} \mathrm{~s}^{-1}$, pryout failure mode was observed with embedment depth of $76.2 \mathrm{~mm}$ while steel anchor failure was observed at embedment depths of $101.6 \mathrm{~mm}$ and $152.4 \mathrm{~mm}$. At strain rates of $10 \mathrm{~s}^{-1}, 10^{2} \mathrm{~s}^{-1}$ and $10^{3} \mathrm{~s}^{-1}$ steel anchor failure was observed for all the embedment depths investigated.

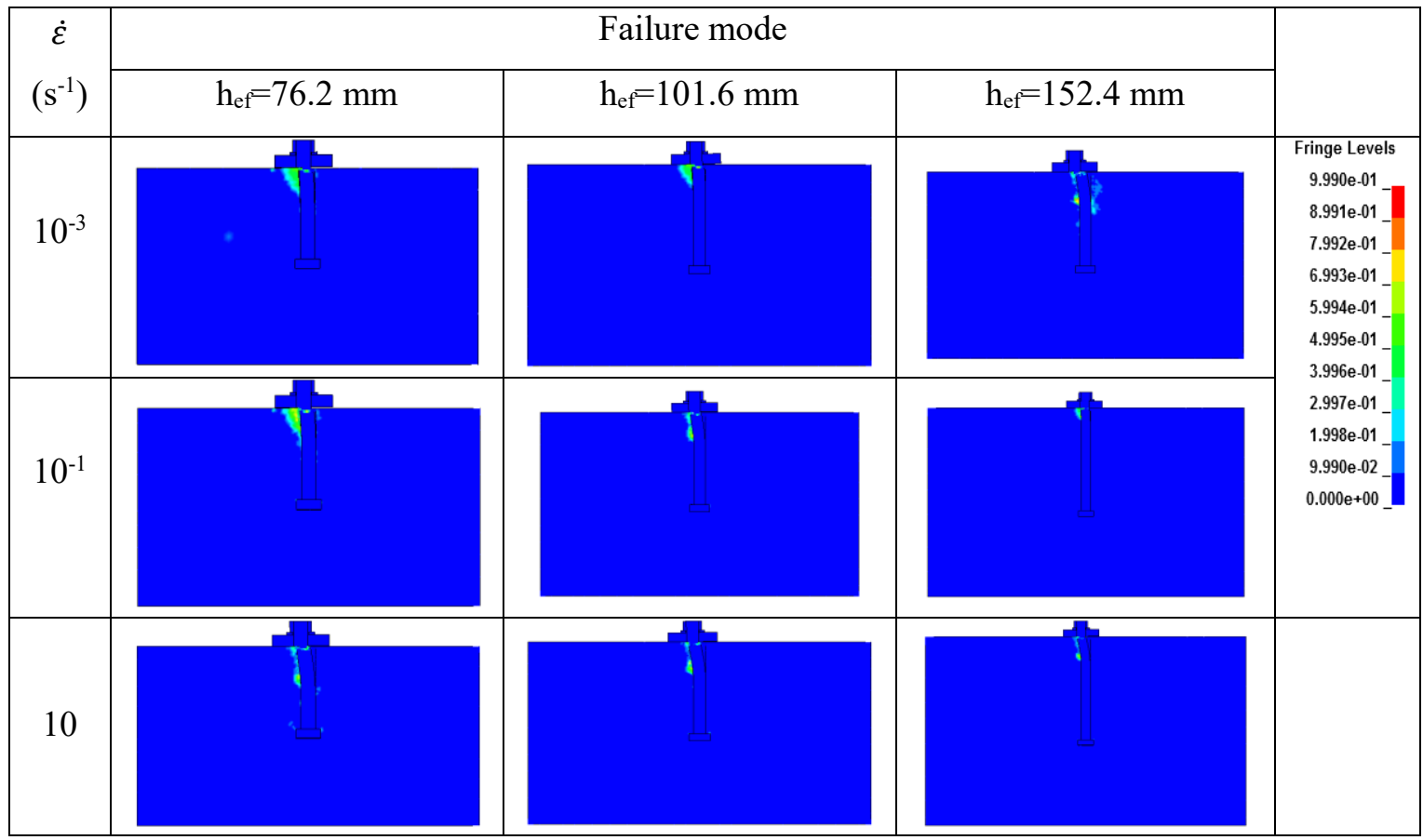




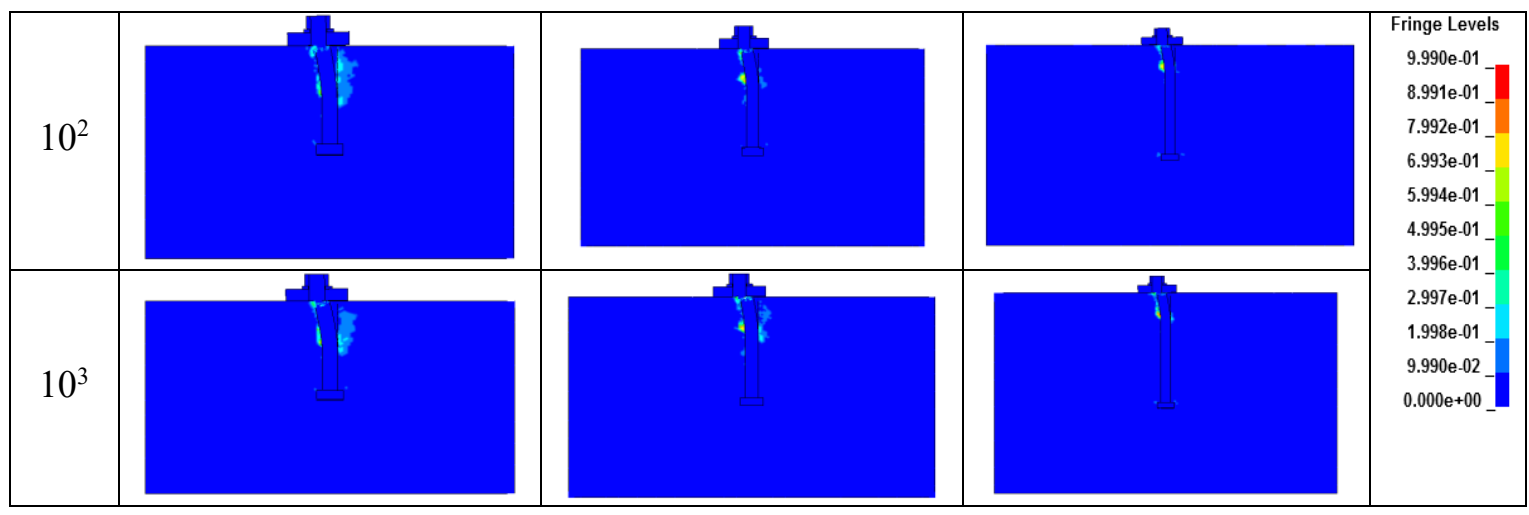

Figure 4-38: Failure mode for $12.7 \mathrm{~mm}$ diameter cast-in-place anchor at different strain rates

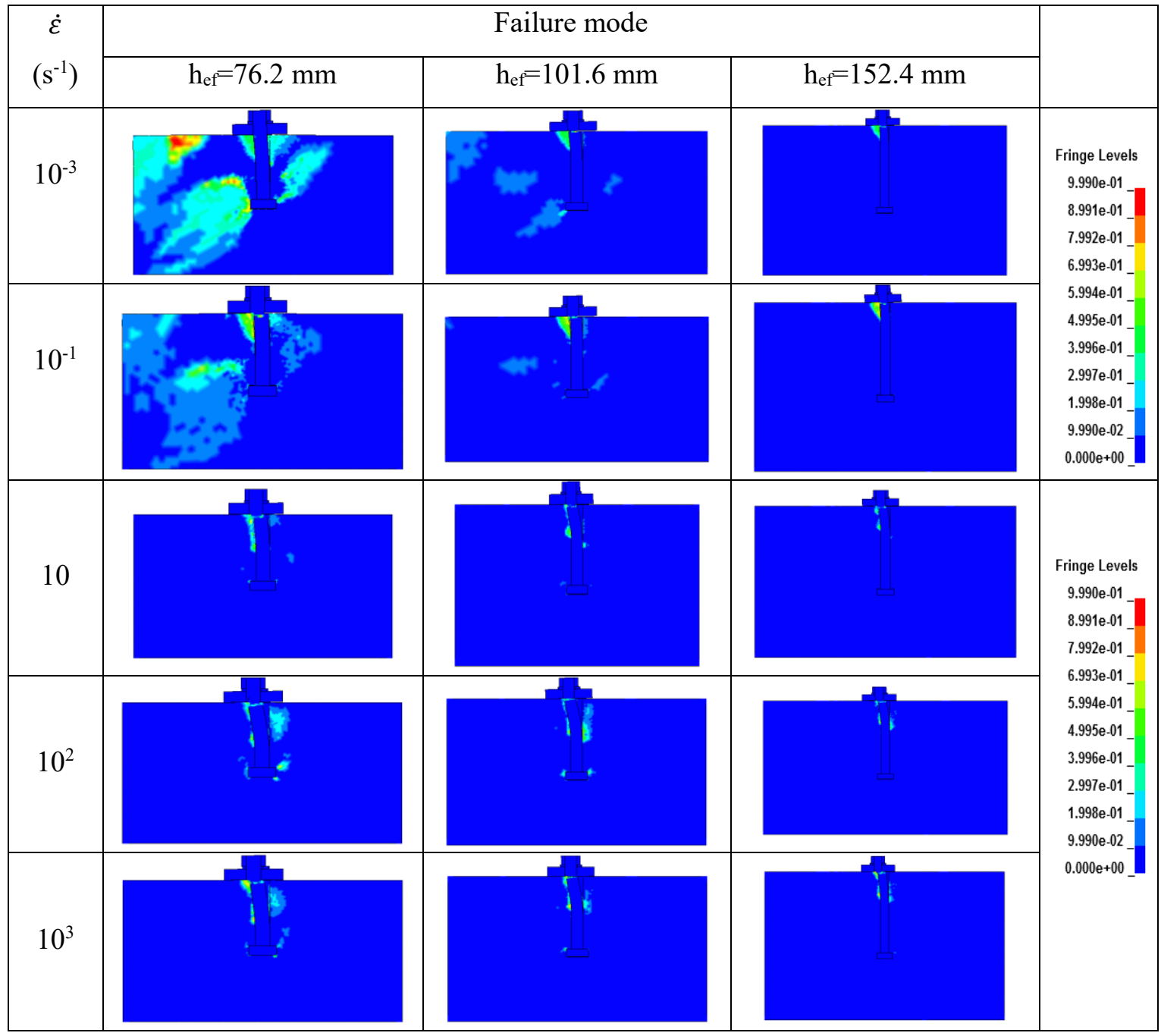

Figure 4-39: Failure mode for $15.9 \mathrm{~mm}$ diameter cast-in-place anchor at different strain rates 


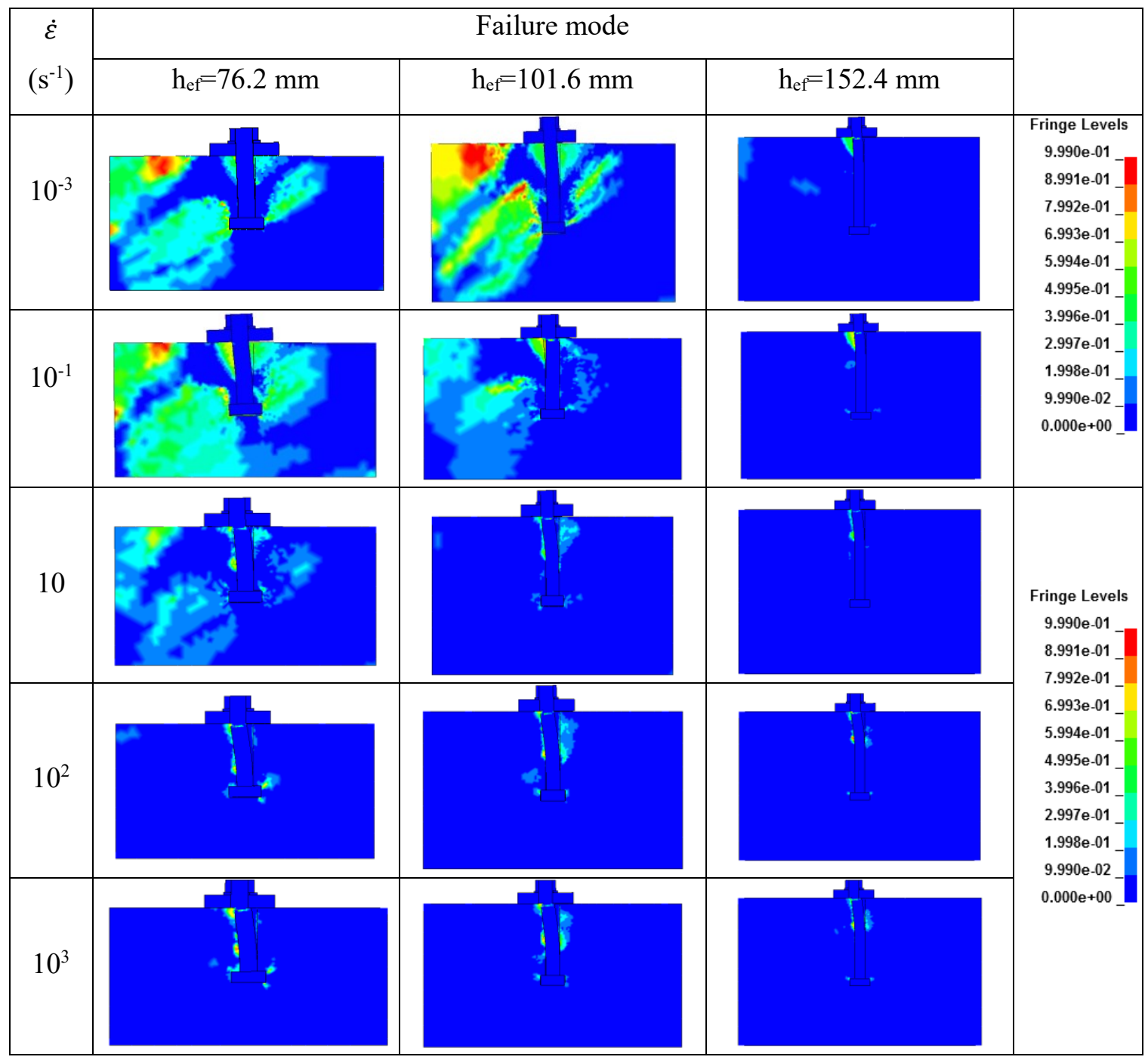

Figure 4-40: Failure mode for $19.1 \mathrm{~mm}$ diameter cast-in-place anchor at different strain rates

It can be seen from Figures 4-36, 4-38, 4-39 and 4-40 that as the anchor diameter increased from $12.7 \mathrm{~mm}$ to $19.1 \mathrm{~mm}$ cracking and level of damage in the concrete increased at all the strain rates investigated. Also, it can be seen that the increase in the strain rate has an effect on the failure mode of cast-in-place anchorage systems. Concrete pryout failure was observed at strain rates of $10^{-5} \mathrm{~s}^{-1}$ and $10^{-3} \mathrm{~s}^{-1}$ for $15.9 \mathrm{~mm}$ and $19.1 \mathrm{~mm}$ diameters with 76.2 mm embedment depth. Also, pryout failure was observed for 19.1-mm diameter with 
embedment depth of $101.6 \mathrm{~mm}$ at strain rates of $10^{-5} \mathrm{~s}^{-1}$ and $10^{-3} \mathrm{~s}^{-1}$. Concrete spalling followed by steel anchor failure was observed at higher strain rates of $10^{-1} \mathrm{~s}^{-1}$ to $10^{3} \mathrm{~s}^{-1}$. In general, the faiure mode for the cast-in-place anchors subjected to shear load depends on several factors such as edge distance, concrete compressive strength, embedment depth to diameter ratio $\left(h_{e f} / d\right)$ and strain rate. In this section, concrete compressive strength of $30 \mathrm{MPa}$ was investigated and the anchor was placed far from the free edge of the concrete. Thus, the ratio $\left(h_{e f} / d\right)$ and strain rate are the factors that affect the failure mode and considered for the analysis. At static strain rate of $10^{-5} \mathrm{~s}^{-1}$, the occurance of pryout failure mode depends mainly on the ratio of $\left(h_{e f} / d\right)$. The failure mode is goverend by pryout failure for the large anchor diameters with shallow embedment depths. On the other hand, the failure mode is goverened by steel anchor failure when the embedment depth is large enough compared to anchor diameter. Thus pryout failure mode was observed for the 15.9$\mathrm{mm}$ and 19.1-mm diameter cast-in-place anchors for the shallow embedment depth of 76.2 $\mathrm{mm}$. Also, the strain rate affect the failure mode where transition from pryout failure to steel anchor failure is observed with the increase in the strain rate.

The steel failure process of 19.1-mm diameter cast-in-place anchor with $101.6 \mathrm{~mm}$ embedment depth at strain rate of $10 \mathrm{~s}^{-1}$ is shown in Figure 4-41. Contours of Von Mises stresses (MPa) are presented in the figure. As shown, the stresses are concentrated around the top part of the anchor where the shear load is introduced followed by anchor bending and fracturing. When the shear load is applied on the anchor plate, friction forces between the anchor plate and the concrete surface are generated. When the applied shear load exceeds the friction force, the anchor plate will slip and transfer the shear load to the bolt 
(Cook et al., 2013), which in turns transfers the load to the concrete through bearing. The concrete resists the shear forces transferred from the anchor causing concrete spall and fracture. In addition to the shear stress on the anchor, bending and tensile stresses are also generated. According to Cook et al., the interaction of these three stresses can lead to anchor fracture (Cook et al., 2013).

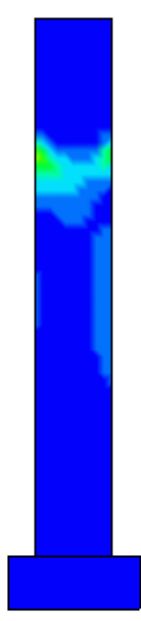

(a) $\mathrm{t}=0.13 \mathrm{~ms}$

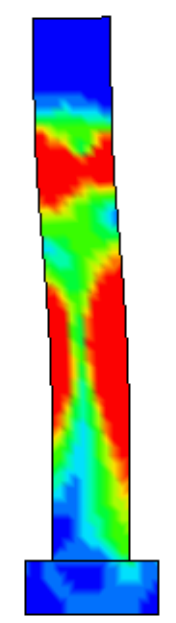

(b) $\mathrm{t}=0.66 \mathrm{~ms}$

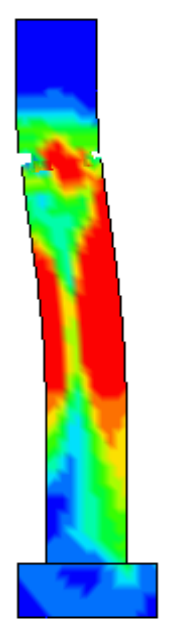

(c) $\mathrm{t}=0.84 \mathrm{~ms}$

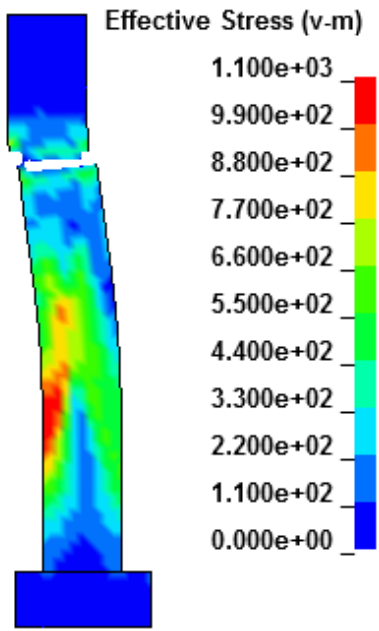

(d) $\mathrm{t}=0.91 \mathrm{~ms}$

Figure 4-41: Steel failure process of $19.1 \mathrm{~mm}$ diameter cast-in-place anchor with 101.6 mm embedment depth; (a) stress concentration around the anchor, (b) anchor bending, (c) initiation of anchor fracture and (d) complete anchor fracture

The failure modes for the cast-in-place anchors at different strain rates is shown in Table 4-15. As shown in Table 4-15, at strain rates of $10^{-5} \mathrm{~s}^{-1}$ to $10^{3} \mathrm{~s}^{-1}$, steel anchor failure was observed for 12.7-mm diameter cast-in-place anchor. Pryout failure mode was observed for anchor diameters of 15.9-mm and 19.1-mm with embedment depth of $76.2 \mathrm{~mm}$. Also, pryout failure mode is observed for anchor diameter of $19.1 \mathrm{~mm}$ with embedment depth of $101.6 \mathrm{~mm}$ at strain rates of $10^{-5} \mathrm{~s}^{-1}$ and $10^{-3} \mathrm{~s}^{-1}$. Steel anchor failure is observed at the strain 
rates of $10^{-1} \mathrm{~s}^{-1}$ up to $10^{3} \mathrm{~s}^{-1}$ for all anchor diameters and embedment depths except for anchor diameter of $19.1 \mathrm{~mm}$ with embedment depth of $76.2 \mathrm{~mm}$ at strain rate of $10^{-1} \mathrm{~s}^{-1}$ where pryout failure mode is observed.

Table 4-15: Failure mode for cast-in-place anchors under shear load at different strain

\begin{tabular}{|c|c|c|c|c|c|c|c|c|}
\hline \multirow{3}{*}{$\begin{array}{c}\text { Model } \\
\text { No. }\end{array}$} & \multirow{3}{*}{$\begin{array}{c}d \\
(\mathbf{m m})\end{array}$} & \multirow{3}{*}{$\begin{array}{c}\mathbf{h}_{\text {ef }} \\
(\mathbf{m m})\end{array}$} & \multicolumn{6}{|c|}{ "Failure mode } \\
\hline & & & \multicolumn{6}{|c|}{ Strain rate $\left(\mathrm{s}^{-1}\right)$} \\
\hline & & & $\dot{\varepsilon}=10^{-5}$ & $\dot{\varepsilon}=10^{-3}$ & $\dot{\varepsilon}=10^{-1}$ & $\dot{\varepsilon}=10$ & $\dot{\varepsilon}=10^{2}$ & $\dot{\varepsilon}=10^{3}$ \\
\hline 1 & 12.7 & 76.2 & $\mathrm{~S}$ & $\mathrm{~S}$ & S & $\mathrm{S}$ & $\mathrm{S}$ & $\mathrm{S}$ \\
\hline 2 & 12.7 & 101.6 & $\mathrm{~S}$ & $\mathrm{~S}$ & $\mathrm{~S}$ & $\mathrm{~S}$ & $\mathrm{~S}$ & $\mathrm{~S}$ \\
\hline 3 & 12.7 & 152.4 & $\mathrm{~S}$ & $\mathrm{~S}$ & $\mathrm{~S}$ & $\mathrm{~S}$ & $\mathrm{~S}$ & $\mathrm{~S}$ \\
\hline 4 & 15.9 & 76.2 & PR & PR & $\mathrm{S}$ & $\mathrm{S}$ & $\mathrm{S}$ & $\mathrm{S}$ \\
\hline 5 & 15.9 & 101.6 & $\mathrm{~S}$ & $\mathrm{~S}$ & $\mathrm{~S}$ & $\mathrm{~S}$ & $\mathrm{~S}$ & $\mathrm{~S}$ \\
\hline 6 & 15.9 & 152.4 & $\mathrm{~S}$ & $\mathrm{~S}$ & $\mathrm{~S}$ & $\mathrm{~S}$ & $\mathrm{~S}$ & $\mathrm{~S}$ \\
\hline 7 & 19.1 & 76.2 & PR & PR & PR & $\mathrm{S}$ & $\mathrm{S}$ & $\mathrm{S}$ \\
\hline 8 & 19.1 & 101.6 & PR & $\mathrm{PR}$ & $\mathrm{S}$ & $\mathrm{S}$ & $\mathrm{S}$ & $\mathrm{S}$ \\
\hline 9 & 19.1 & 152.4 & $S$ & $\mathrm{~S}$ & $\mathrm{~S}$ & $S$ & $S$ & $\mathrm{~S}$ \\
\hline
\end{tabular}

*Failure mode: $\mathrm{PR}=$ pryout failure, $\mathrm{S}=$ steel anchor failure

\subsubsection{Effect of design parameters on failure mode and ultimate shear load}

Figures 4-42, 4-43 and 4-44 show the effect of strain rate on the failure mode and ultimate shear load for the 12.7-mm, 15.9-mm and 19.1-mm diameter cast-in-place anchors respectively. The figures indicate that the strain rate has an effect on the failure mode for the anchor diameters of $15.9 \mathrm{~mm}$ and $19.1 \mathrm{~mm}$ where transition from pryout failure to steel anchor failure is observed. Furthermore, the anchor diameter and embedment depth have an effect on the failure mode. It can be seen from the figures that the ultimate shear load increased with the increase in the strain rate for the cast-in-place anchors. 


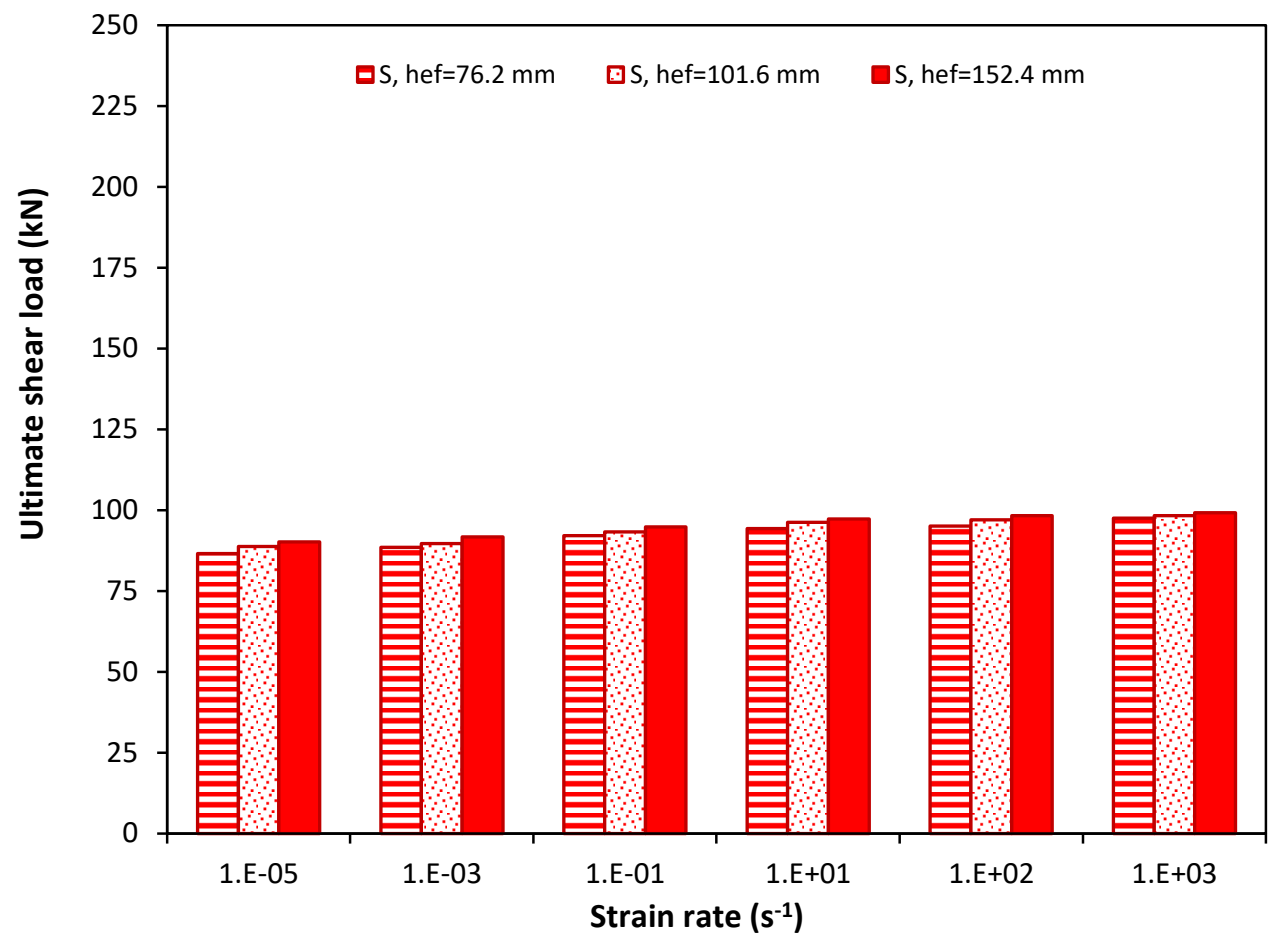

Figure 4-42: Effect of strain rate on the failure mode and ultimate shear load for the castin-place anchor diameter of $12.7 \mathrm{~mm}$

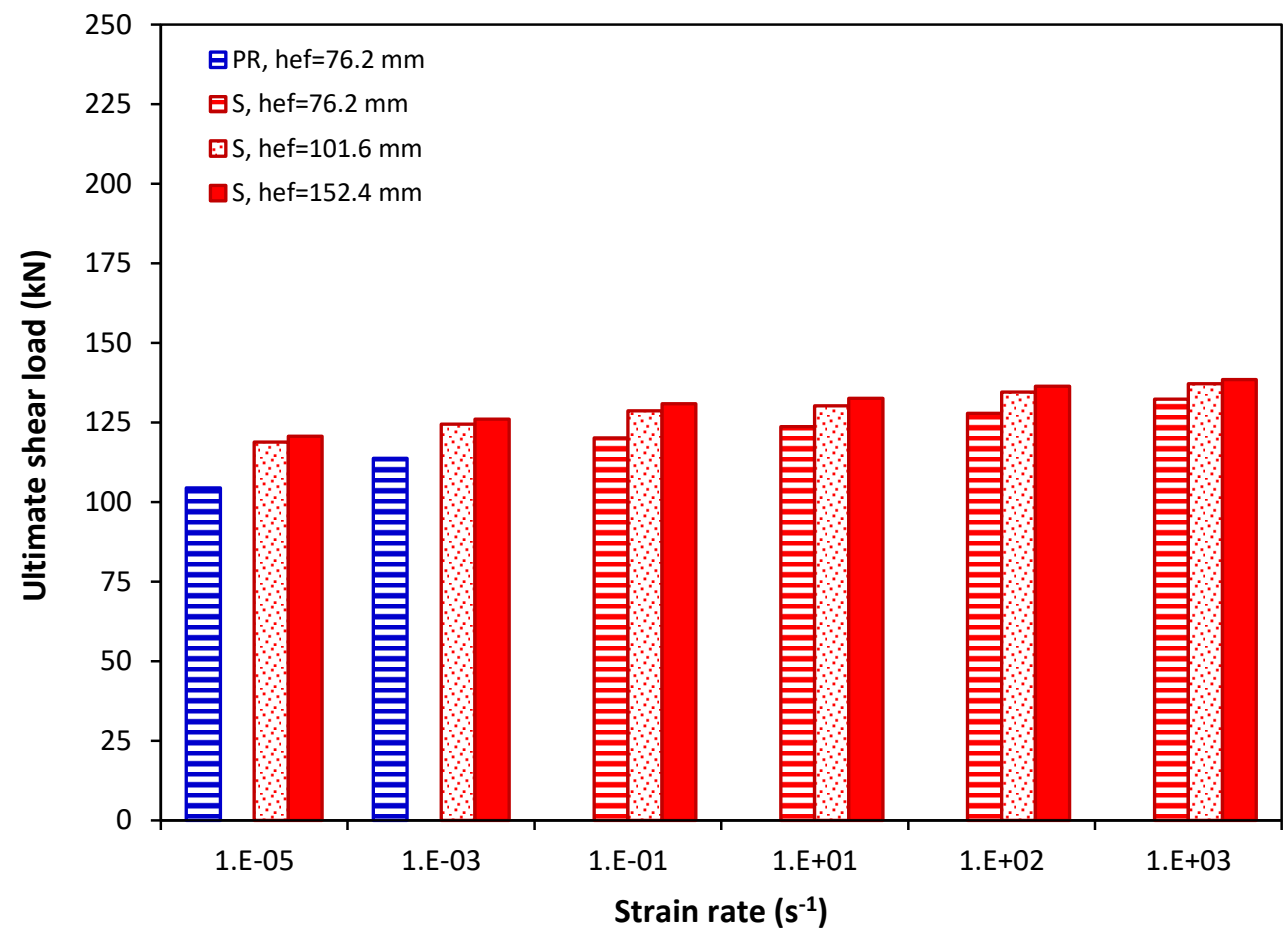

Figure 4-43: Effect of strain rate on the failure mode and ultimate shear load for the castin-place anchor diameter of $15.9 \mathrm{~mm}$ 


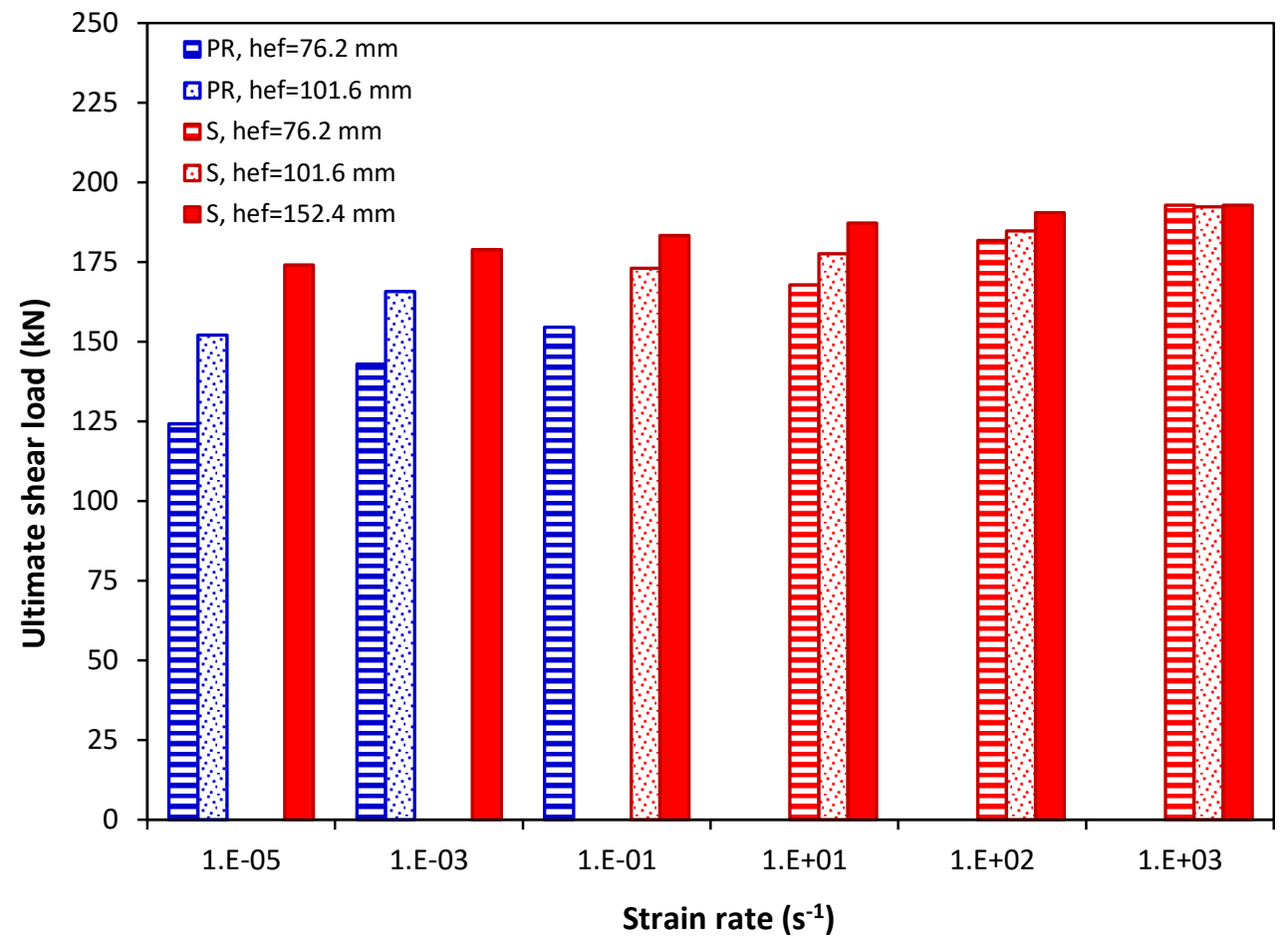

Figure 4-44: Effect of strain rate on the failure mode and ultimate shear load for the castin-place anchor diameter of $19.1 \mathrm{~mm}$

\subsubsection{Effect of strain rate on the shear behaviour of cast-in-place anchors}

Shear behaviour of cast-in-place anchors embedded into concrete was investigated using LS-DYNA finite element software. Strain rates ranging from low to high strain rates $\left(10^{-5}\right.$ $\mathrm{s}^{-1}, 10^{-3} \mathrm{~s}^{-1}, 10^{-1} \mathrm{~s}^{-1}, 10 \mathrm{~s}^{-1}, 10^{2} \mathrm{~s}^{-1}$ and $\left.10^{3} \mathrm{~s}^{-1}\right)$ were selected for the analysis. Effect of strain rate on the shear capacity for different cast-in-place anchor diameters and embedment depths was investigated. Load-displacement response for the $12.7-\mathrm{mm}$ diameter cast-inplace anchor at strain rates of $10^{-5} \mathrm{~s}^{-1}, 10^{-3} \mathrm{~s}^{-1}, 10^{-1} \mathrm{~s}^{-1}, 10 \mathrm{~s}^{-1}, 10^{2} \mathrm{~s}^{-1}$ and $10^{3} \mathrm{~s}^{-1}$ is shown in Figures 4-45, 4-46, 4-47, 4-48, 4-49 and 4-50 respectively. Embedment depths of $76.2 \mathrm{~mm}$, $101.6 \mathrm{~mm}$ and $152.4 \mathrm{~mm}$ were used for the analysis. The displacement in the y-direction (shown in Figure 4-34) was measured at the top of the anchor using History-Nodal-ydisplacement in the LS-DYNA post processor. 


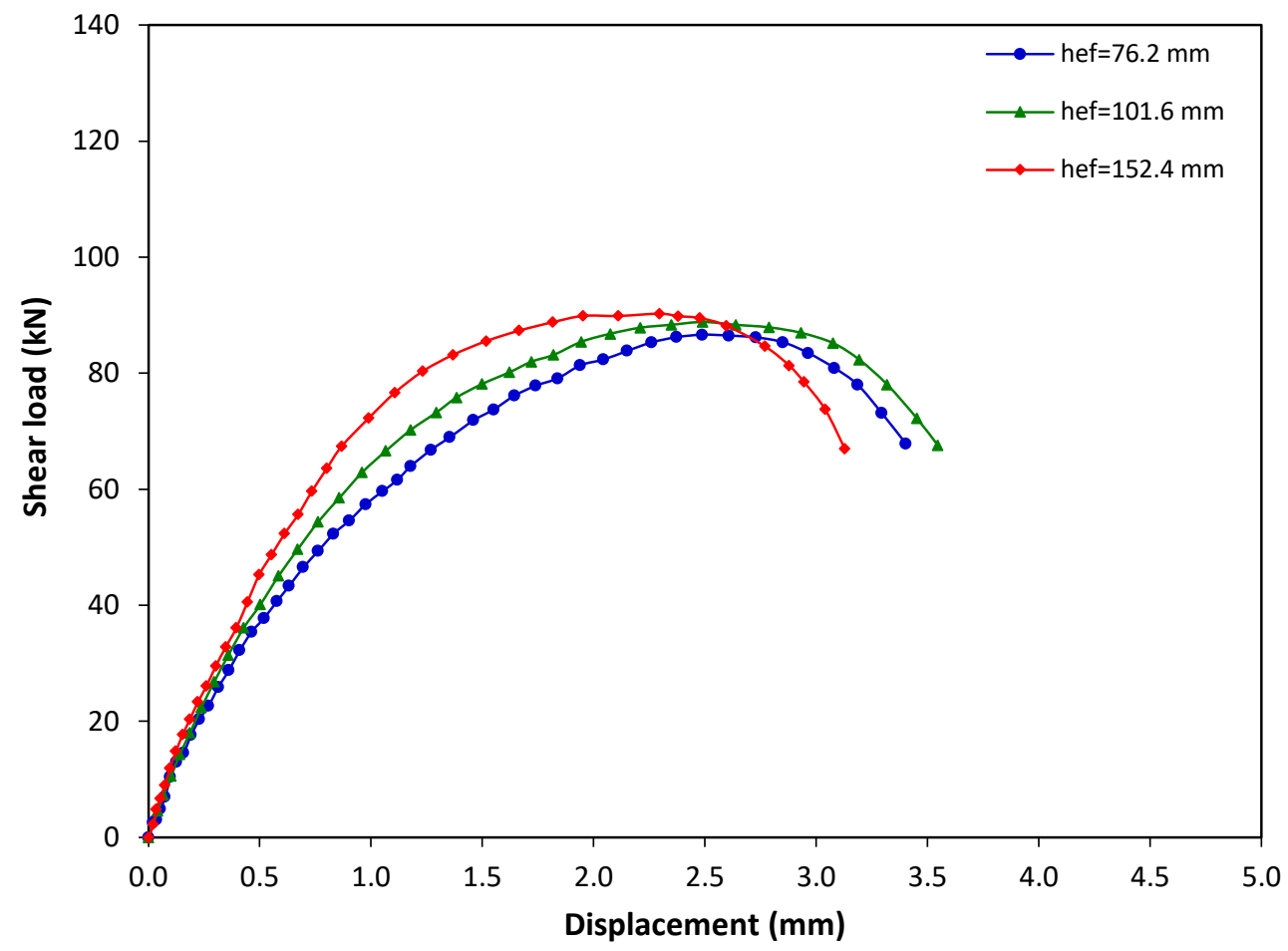

Figure 4-45: Shear load-displacement graph for $12.7 \mathrm{~mm}$ diameter cast-in-place anchor at strain rate of $10^{-5} \mathrm{~s}^{-1}$

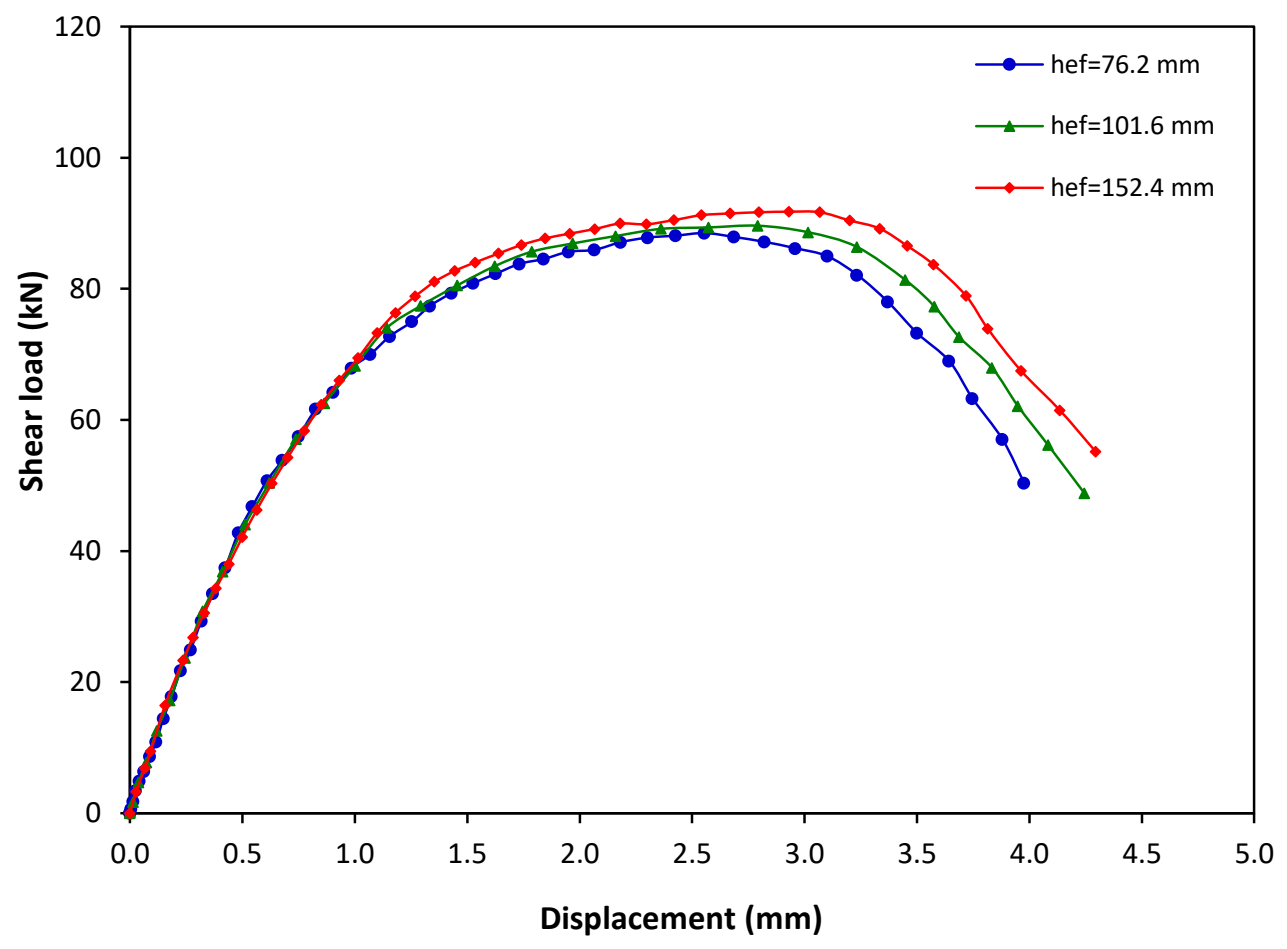

Figure 4-46: Shear load-displacement graph for $12.7 \mathrm{~mm}$ diameter cast-in-place anchor at strain rate of $10^{-3} \mathrm{~s}^{-1}$ 


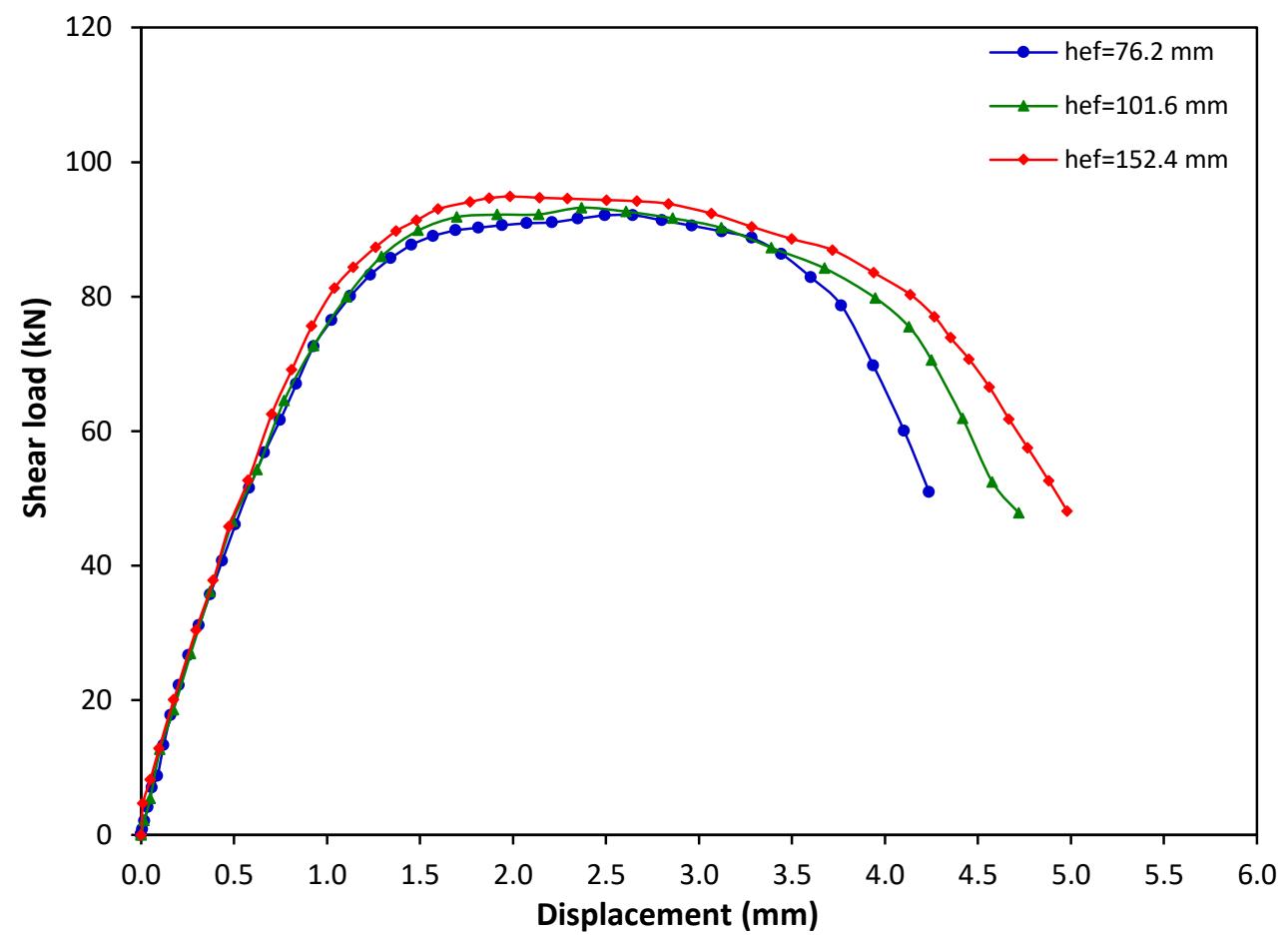

Figure 4-47: Shear load-displacement graph for $12.7 \mathrm{~mm}$ diameter cast-in-place anchor at strain rate of $10^{-1} \mathrm{~s}^{-1}$

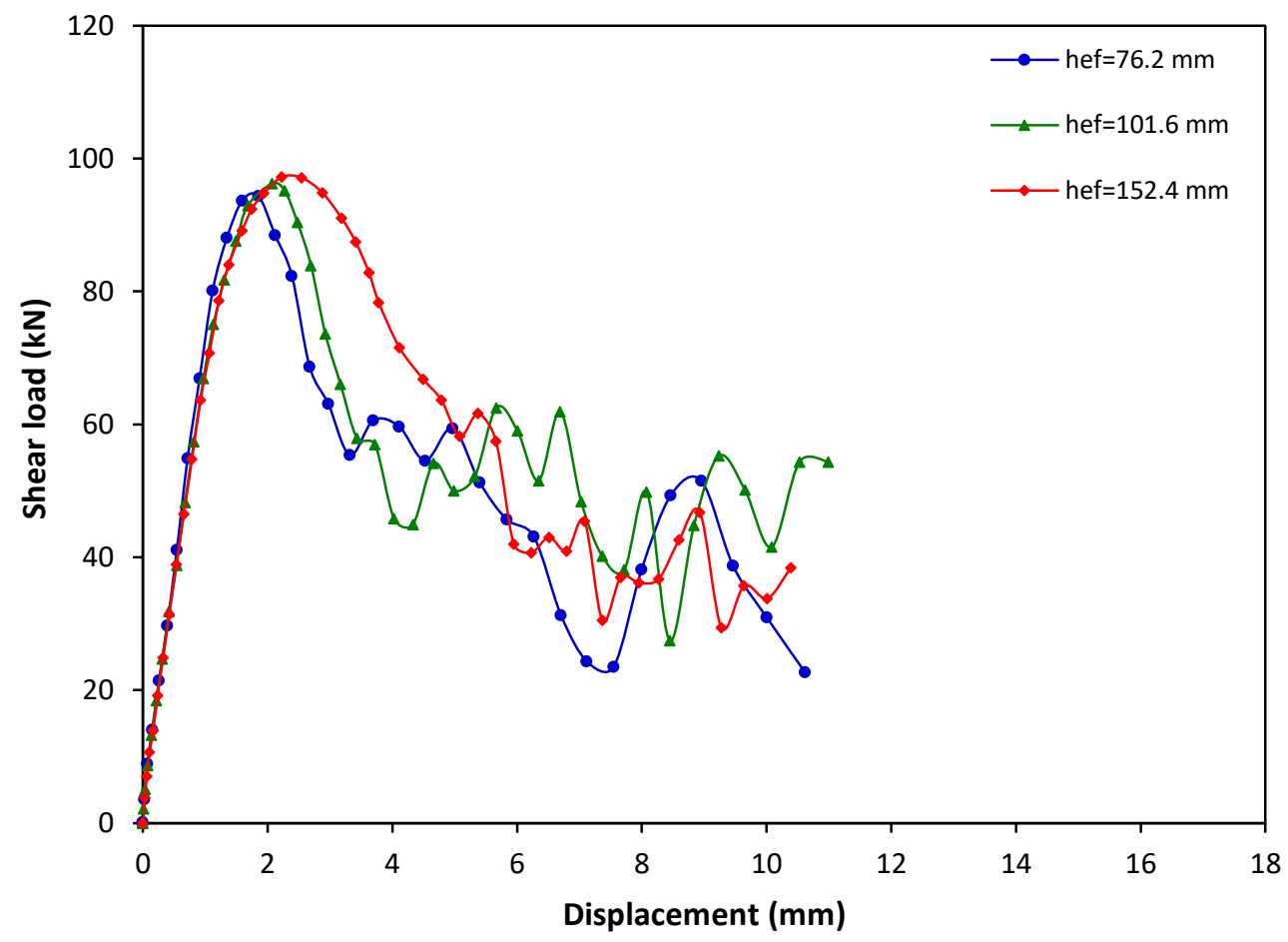

Figure 4-48: Shear load-displacement graph for $12.7 \mathrm{~mm}$ diameter cast-in-place anchor at strain rate of $10 \mathrm{~s}^{-1}$ 


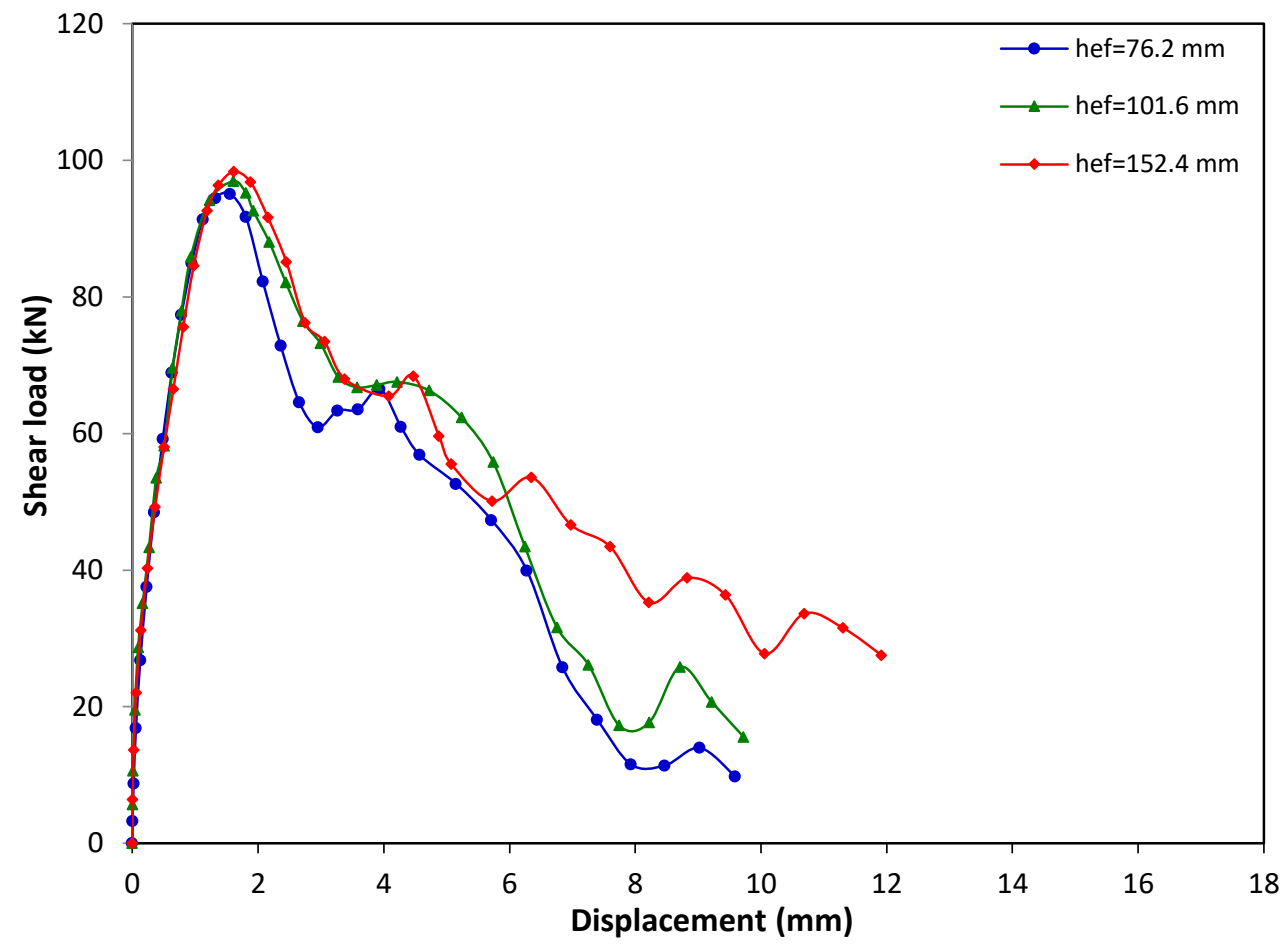

Figure 4-49: Shear load-displacement graph for $12.7 \mathrm{~mm}$ diameter cast-in-place anchor at strain rate of $10^{2} \mathrm{~s}^{-1}$

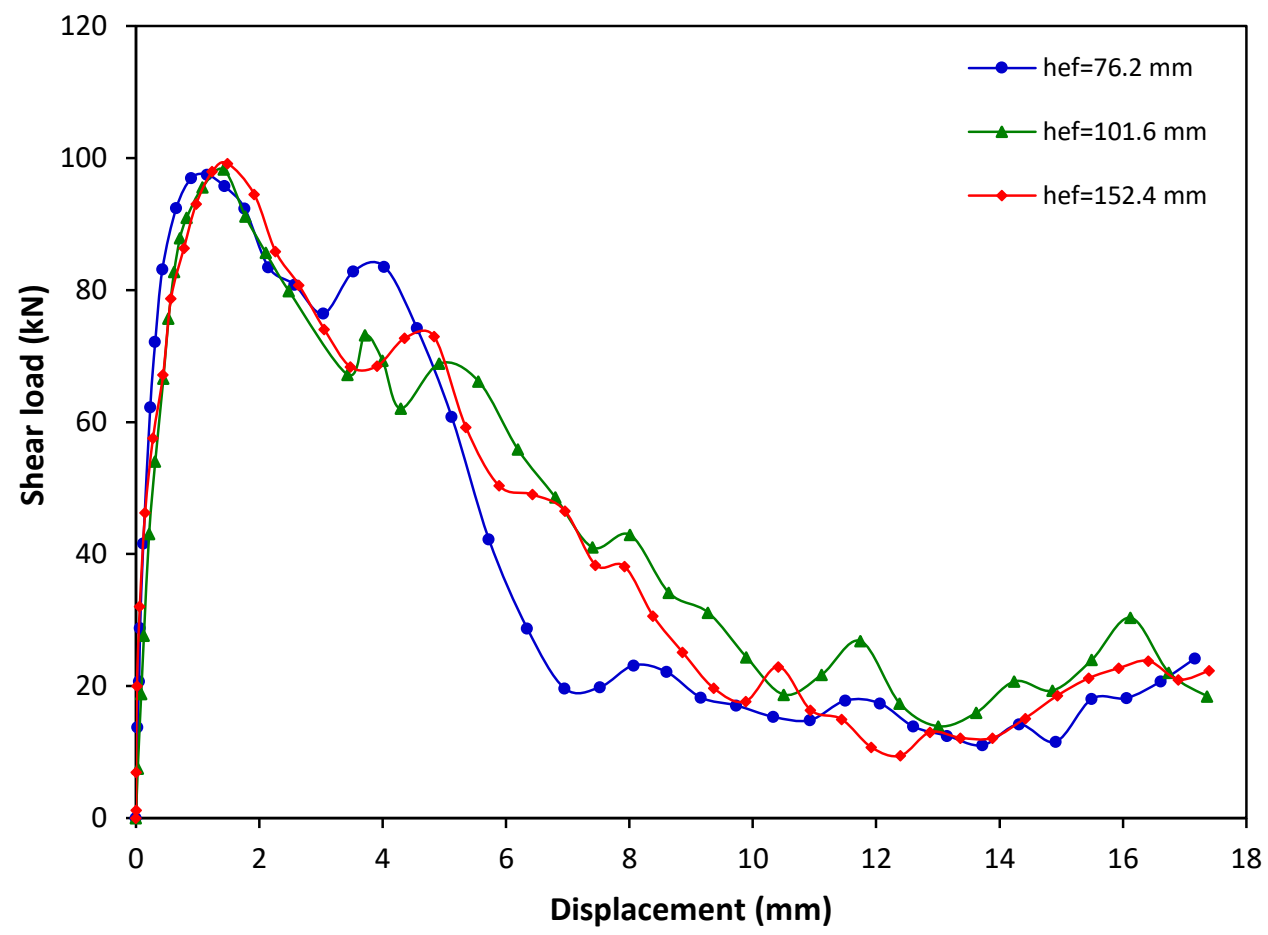

Figure 4-50: Shear load-displacement graph for $12.7 \mathrm{~mm}$ diameter cast-in-place anchor at strain rate of $10^{3} \mathrm{~s}^{-1}$ 
As shown from Figures 4-45 to 4-50, the increase in the strain rate increased the shear capacity for the $12.7-\mathrm{mm}$ diameter cast-in-place anchor. At strain rates of $10^{-5} \mathrm{~s}^{-1}$ to $10^{3} \mathrm{~s}^{-1}$, the shear load increased with the displacement until the ultimate load was reached, and then decreased until complete failure. This is attributed to the progressive crack propagation in the concrete and anchor failure.

Shear load-displacement response for 15.9-mm and 19.1-mm diameter cast-in-place anchors with embedment depths of $76.2 \mathrm{~mm}, 101.6 \mathrm{~mm}$ and $152.4 \mathrm{~mm}$ at strain rates of $10^{-}$ ${ }^{5} \mathrm{~s}^{-1}, 10^{-3} \mathrm{~s}^{-1}, 10^{-1} \mathrm{~s}^{-1}, 10 \mathrm{~s}^{-1}, 10^{2} \mathrm{~s}^{-1}$ and $10^{3} \mathrm{~s}^{-1}$ is presented in Appendix D. Tables 4-16, 417 and 4-18 present the ultimate shear load $\left(V_{u}\right)$ and corresponding displacement $(\delta)$ for the 12.7-mm, 15.9-mm and 19.1-mm diameter cast-in-place anchors respectively.

Table 4-16: Ultimate shear load and displacement results for the $12.7 \mathrm{~mm}$ diameter castin-place anchor

\begin{tabular}{|c|c|c|c|c|c|c|}
\hline $\begin{array}{c}\text { Model } \\
\text { No. }\end{array}$ & $\begin{array}{c}\mathrm{d} \\
(\mathrm{mm})\end{array}$ & $\begin{array}{c}\mathrm{h}_{\mathrm{ef}} \\
(\mathrm{mm})\end{array}$ & $\begin{array}{c}\dot{\varepsilon} \\
\left(\mathrm{s}^{-1}\right)\end{array}$ & $\begin{array}{c}\mathrm{V}_{\mathrm{u}} \\
(\mathrm{kN})\end{array}$ & $\begin{array}{c}\delta \\
(\mathrm{mm})\end{array}$ & $\begin{array}{c}\text { Failure } \\
\text { mode }\end{array}$ \\
\hline 1 & \multirow{3}{*}{12.7} & 76.2 & \multirow{3}{*}{$10^{-5}$} & 86.64 & 2.49 & $\mathrm{~S}$ \\
\hline 2 & & 101.6 & & 88.81 & 2.49 & $\mathrm{~S}$ \\
\hline 3 & & 152.4 & & 90.16 & 2.29 & $\mathrm{~S}$ \\
\hline 4 & \multirow{3}{*}{12.7} & 76.2 & \multirow{3}{*}{$10^{-3}$} & 88.49 & 2.56 & $\mathrm{~S}$ \\
\hline 5 & & 101.6 & & 89.63 & 2.79 & $\bar{S}$ \\
\hline 6 & & 152.4 & & 91.78 & 2.93 & $S$ \\
\hline 7 & \multirow{3}{*}{12.7} & 76.2 & \multirow{3}{*}{$10^{-1}$} & 92.14 & 2.65 & $\mathrm{~S}$ \\
\hline 8 & & 101.6 & & 93.25 & 2.37 & $\mathrm{~S}$ \\
\hline 9 & & 152.4 & & 94.89 & 1.99 & $\bar{S}$ \\
\hline 10 & \multirow{3}{*}{12.7} & 76.2 & \multirow{3}{*}{10} & 94.36 & 1.85 & $\mathrm{~S}$ \\
\hline 11 & & 101.6 & & 96.23 & 2.07 & $\bar{S}$ \\
\hline 12 & & 152.4 & & 97.26 & 2.23 & $\mathrm{~S}$ \\
\hline
\end{tabular}




\begin{tabular}{|c|c|c|c|c|c|c|}
\hline 13 & \multirow{3}{*}{12.7} & 76.2 & \multirow{3}{*}{$10^{2}$} & 95.08 & 1.55 & $\mathrm{~S}$ \\
\hline 14 & & 101.6 & & 96.99 & 1.61 & $\mathrm{~S}$ \\
\hline 15 & & 152.4 & & 98.35 & 1.61 & $\mathrm{~S}$ \\
\hline 16 & \multirow{3}{*}{12.7} & 76.2 & \multirow{3}{*}{$10^{3}$} & 97.48 & 1.15 & $\mathrm{~S}$ \\
\hline 17 & & 101.6 & & 98.26 & 1.42 & $\mathrm{~S}$ \\
\hline 18 & & 152.4 & & 99.15 & 1.48 & $\mathrm{~S}$ \\
\hline
\end{tabular}

Table 4-17: Ultimate shear load and displacement results for the $15.9 \mathrm{~mm}$ diameter castin-place anchor

\begin{tabular}{|c|c|c|c|c|c|c|}
\hline $\begin{array}{c}\text { Model } \\
\text { No. }\end{array}$ & $\begin{array}{c}\mathrm{d} \\
(\mathrm{mm})\end{array}$ & $\begin{array}{c}\mathrm{h}_{\mathrm{ef}} \\
(\mathrm{mm})\end{array}$ & $\begin{array}{c}\dot{\varepsilon} \\
\left(\mathrm{s}^{-1}\right)\end{array}$ & $\begin{array}{c}\mathrm{V}_{\mathrm{u}} \\
(\mathrm{kN})\end{array}$ & $\begin{array}{c}\delta \\
(\mathrm{mm})\end{array}$ & $\begin{array}{l}\text { Failure } \\
\text { mode }\end{array}$ \\
\hline 1 & \multirow{3}{*}{15.9} & 76.2 & \multirow{3}{*}{$10^{-5}$} & 104.37 & 2.05 & PR \\
\hline 2 & & 101.6 & & 118.79 & 2.42 & $S$ \\
\hline 3 & & 152.4 & & 120.67 & 2.53 & $\bar{S}$ \\
\hline 4 & \multirow{3}{*}{15.9} & 76.2 & \multirow{3}{*}{$10^{-3}$} & 113.74 & 3.67 & PR \\
\hline 5 & & 101.6 & & 124.43 & 3.49 & $\mathrm{~S}$ \\
\hline 6 & & 152.4 & & 126.01 & 3.13 & $\bar{S}$ \\
\hline 7 & \multirow{3}{*}{15.9} & 76.2 & \multirow{3}{*}{$10^{-1}$} & 120.13 & 3.58 & $S$ \\
\hline 8 & & 101.6 & & 128.58 & 3.52 & $\bar{S}$ \\
\hline 9 & & 152.4 & & 130.87 & 3.27 & $S$ \\
\hline 10 & \multirow{3}{*}{15.9} & 76.2 & \multirow{3}{*}{10} & 123.65 & 1.98 & $S$ \\
\hline 11 & & 101.6 & & 130.21 & 2.12 & $\mathrm{~S}$ \\
\hline 12 & & 152.4 & & 132.51 & 2.74 & $S$ \\
\hline 13 & \multirow{3}{*}{15.9} & 76.2 & \multirow{3}{*}{$10^{2}$} & 127.89 & 1.61 & $S$ \\
\hline 14 & & 101.6 & & 134.47 & 1.67 & $\mathrm{~S}$ \\
\hline 15 & & 152.4 & & 136.36 & 1.78 & 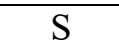 \\
\hline 16 & \multirow{3}{*}{15.9} & 76.2 & \multirow{3}{*}{$10^{3}$} & 132.30 & 1.87 & $S$ \\
\hline 17 & & 101.6 & & 137.16 & 1.85 & $\mathrm{~S}$ \\
\hline 18 & & 152.4 & & 138.42 & 1.78 & $\mathrm{~S}$ \\
\hline
\end{tabular}


Table 4-18: Ultimate shear load and displacement results for the $19.1 \mathrm{~mm}$ diameter castin-place anchor

\begin{tabular}{|c|c|c|c|c|c|c|}
\hline $\begin{array}{l}\text { Model } \\
\text { No. }\end{array}$ & $\begin{array}{c}\mathrm{d} \\
(\mathrm{mm})\end{array}$ & $\begin{array}{c}\mathrm{h}_{\mathrm{ef}} \\
(\mathrm{mm})\end{array}$ & $\begin{array}{c}\dot{\varepsilon} \\
\left(\mathrm{s}^{-1}\right)\end{array}$ & $\begin{array}{c}\mathrm{V}_{\mathrm{u}} \\
(\mathrm{kN})\end{array}$ & $\begin{array}{c}\delta \\
(\mathrm{mm})\end{array}$ & $\begin{array}{l}\text { Failure } \\
\text { mode }\end{array}$ \\
\hline 1 & \multirow{3}{*}{19.1} & 76.2 & \multirow{3}{*}{$10^{-5}$} & 124.18 & 1.73 & PR \\
\hline 2 & & 101.6 & & 152.14 & 2.12 & PR \\
\hline 3 & & 152.4 & & 174.09 & 3.08 & $\mathrm{~S}$ \\
\hline 4 & \multirow{3}{*}{19.1} & 76.2 & \multirow{3}{*}{$10^{-3}$} & 143.04 & 2.47 & PR \\
\hline 5 & & 101.6 & & 165.76 & 2.96 & PR \\
\hline 6 & & 152.4 & & 178.96 & 3.29 & $\mathrm{~S}$ \\
\hline 7 & \multirow{3}{*}{19.1} & 76.2 & \multirow{3}{*}{$10^{-1}$} & 154.56 & 3.16 & PR \\
\hline 8 & & 101.6 & & 173.03 & 3.34 & S \\
\hline 9 & & 152.4 & & 183.29 & 3.47 & S \\
\hline 10 & \multirow{3}{*}{19.1} & 76.2 & \multirow{3}{*}{10} & 167.89 & 1.92 & S \\
\hline 11 & & 101.6 & & 177.59 & 2.41 & S \\
\hline 12 & & 152.4 & & 187.20 & 2.53 & S \\
\hline 13 & \multirow{3}{*}{19.1} & 76.2 & \multirow{3}{*}{$10^{2}$} & 181.72 & 2.04 & S \\
\hline 14 & & 101.6 & & 184.83 & 2.09 & $\mathrm{~S}$ \\
\hline 15 & & 152.4 & & 190.48 & 1.98 & S \\
\hline 16 & \multirow{3}{*}{19.1} & 76.2 & \multirow{3}{*}{$10^{3}$} & 192.91 & 1.79 & $\mathrm{~S}$ \\
\hline 17 & & 101.6 & & 192.28 & 2.09 & $\mathrm{~S}$ \\
\hline 18 & & 152.4 & & 192.81 & 2.01 & $\mathrm{~S}$ \\
\hline
\end{tabular}

\subsubsection{Effect of strain rate on the ultimate shear load and DIF of cast-in-place anchors}

The relation between the ultimate shear load, DIF and the strain rate for the cast-in-place anchor of $76.2 \mathrm{~mm}, 101.6 \mathrm{~mm}$ and $152.4 \mathrm{~mm}$ embedment depths and different anchor diameters is shown in Figures 4-51, 4-52 and 4-53. It can be seen that the ultimate shear load increased with the increase in the anchor diameter from $12.7 \mathrm{~mm}$ to $19.1 \mathrm{~mm}$ for all the embedment depths and strain rates investigated. Anchor diameter of $12.7 \mathrm{~mm}$ exhibited an increase in the ultimate shear load of $12.5 \%, 10.6 \%$ and $10.0 \%$ as the strain rate 
increased from $10^{-5} \mathrm{~s}^{-1}$ to $10^{3} \mathrm{~s}^{-1}$ for the $76.2 \mathrm{~mm}, 101.6 \mathrm{~mm}$ and $152.4 \mathrm{~mm}$ embedment depths respectively. Anchor diameter of $15.9 \mathrm{~mm}$ exhibited an increase in the ultimate shear load with the increase in the strain rate at embedment depth of $76.2 \mathrm{~mm}$ where the failure mode transitioned from pryout failure to steel anchor failure. An increase of $15.5 \%$ and $14.7 \%$ in the ultimate shear load is obtained with the increase in strain rate from $10^{-5}$ $\mathrm{s}^{-1}$ to $10^{3} \mathrm{~s}^{-1}$ for anchor embedment depths of $101.6 \mathrm{~mm}$ and $152.4 \mathrm{~mm}$ respectively. For anchor diameter of $19.1 \mathrm{~mm}$, the ultimate shear load increased from $124.2 \mathrm{kN}$ to $192.9 \mathrm{kN}$ and from $152.1 \mathrm{kN}$ to $192.3 \mathrm{kN}$ as the strain rate increased from $10^{-5} \mathrm{~s}^{-1}$ to $10^{3} \mathrm{~s}^{-1}$ for anchor embedment depths of $76.2 \mathrm{~mm}$ and $101.6 \mathrm{~mm}$ respectively. An increase in the ultimate shear load of $10.75 \%$ was obtained with the increase in the strain rate from $10^{-5} \mathrm{~s}^{-1}$ to $10^{3}$ $\mathrm{s}^{-1}$ at $152.4 \mathrm{~mm}$ embedment depth. Maximum ultimate shear loads of $99.2 \mathrm{kN}, 138.4 \mathrm{kN}$ and $192.8 \mathrm{kN}$ were obtained for anchor diameters of $12.7 \mathrm{~mm}, 15.9 \mathrm{~mm}$ and $19.1 \mathrm{~mm}$ respectively with embedment depth of $152.4 \mathrm{~mm}$ at high strain rate of $10^{3} \mathrm{~s}^{-1}$. At low strain rate of $10^{-5} \mathrm{~s}^{-1}$, ultimate shear loads of $90.2 \mathrm{kN}, 120.7 \mathrm{kN}$ and $174.1 \mathrm{kN}$ were obtained for the anchor diameters of $12.7-\mathrm{mm}, 15.9-\mathrm{mm}$ and $19.1-\mathrm{mm}$ respectively with embedment depth of $152.4 \mathrm{~mm}$. It can be seen from Figures 4-51, 4-52 and 4-53, the relation between the ultimate shear load and the strain rate is almost linear for all the anchors investigated. 


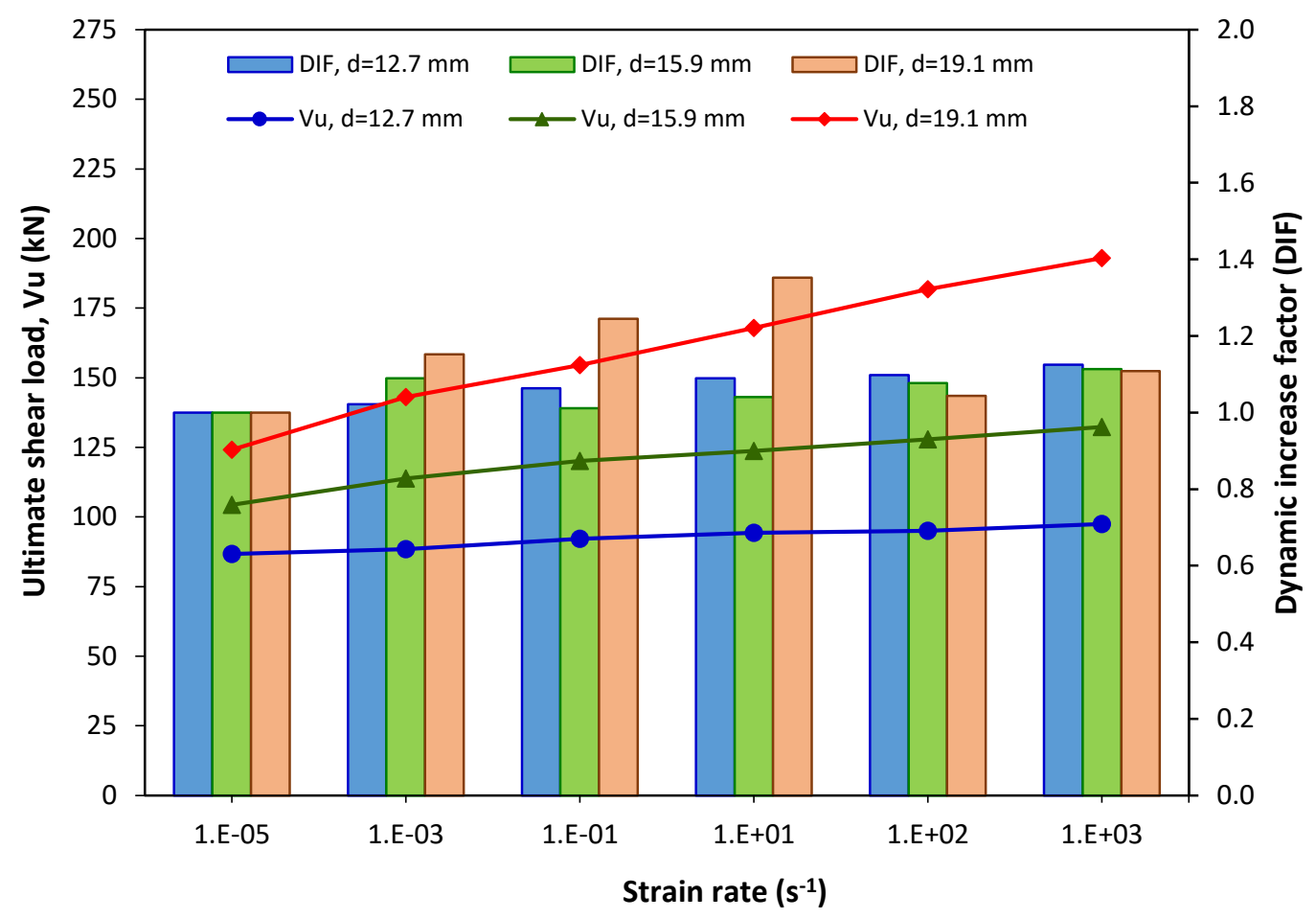

Figure 4-51: Ultimate shear load and DIF versus strain rate for cast-in-place anchor with $76.2 \mathrm{~mm}$ embedment depth

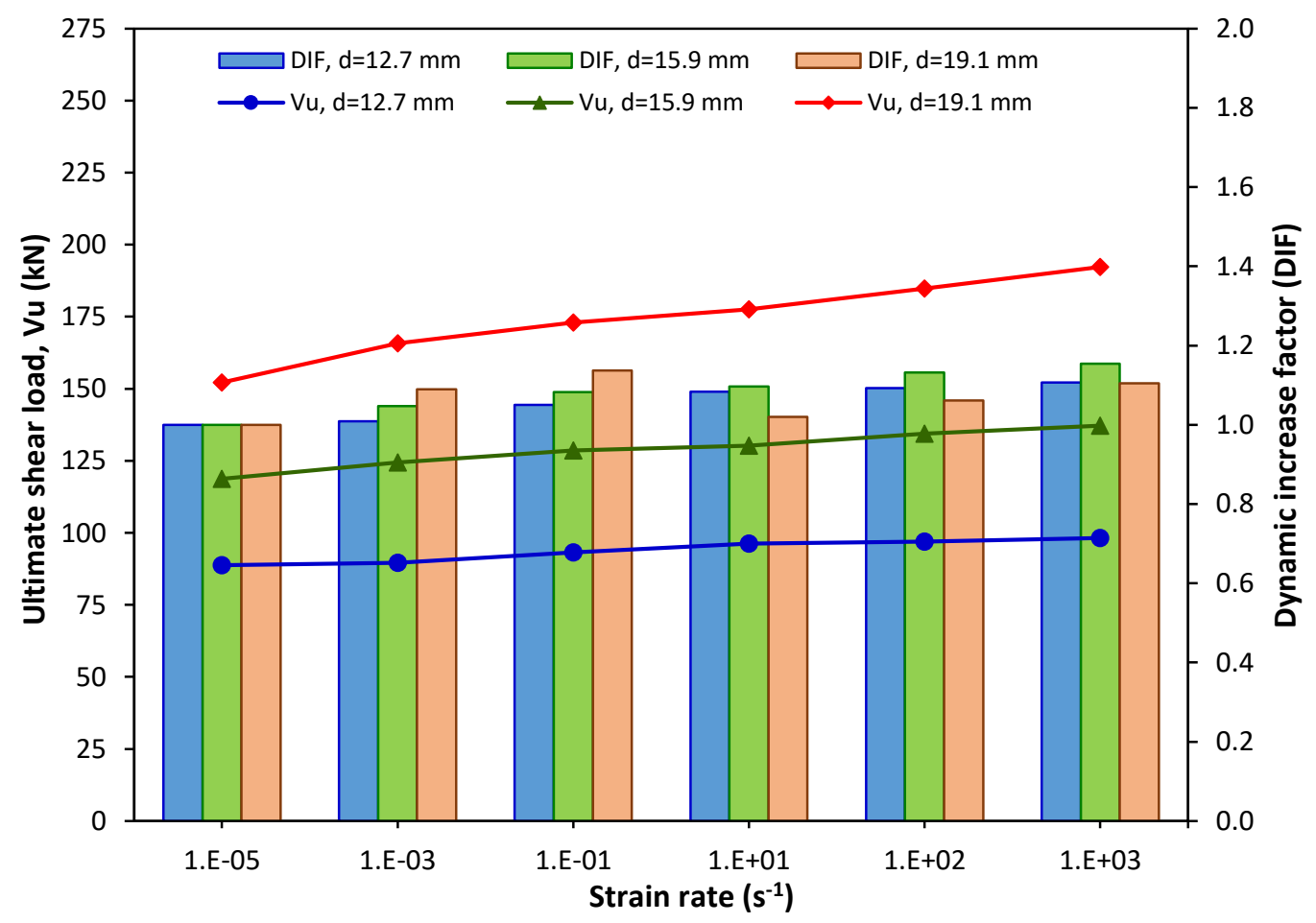

Figure 4-52: Ultimate shear load and DIF versus strain rate for cast-in-place anchor with $101.6 \mathrm{~mm}$ embedment depth 


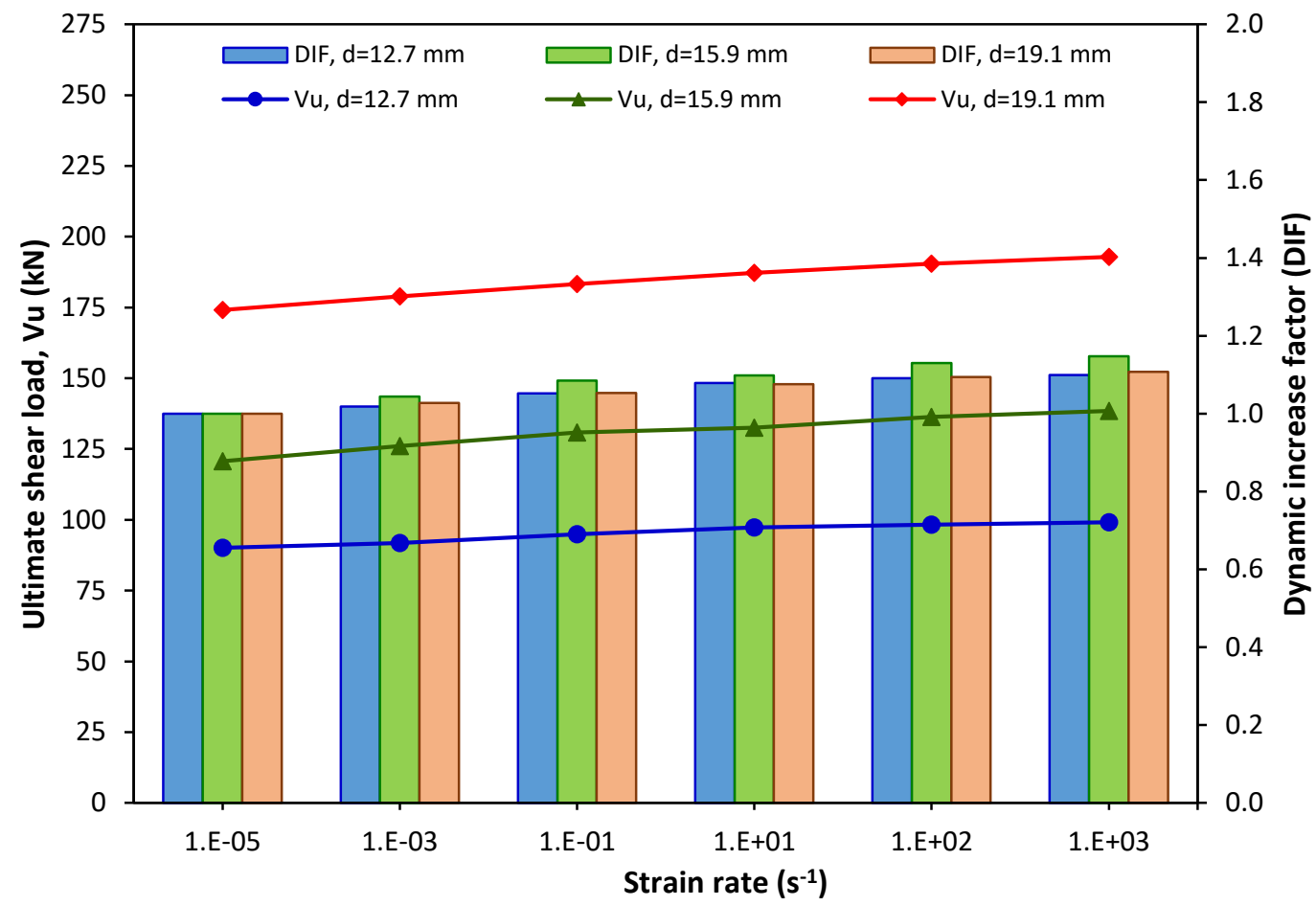

Figure 4-53: Ultimate shear load and DIF versus strain rate for cast-in-place anchor with $152.4 \mathrm{~mm}$ embedment depth

As shown in Figure 4-51, for the 12.7-mm diameter anchor the DIF increased slightly with the increase in the strain rate where steel anchor failure was observed at all the strain rates investigated. For anchor diameter of $15.9 \mathrm{~mm}$ with embedment depth of $76.2 \mathrm{~mm}$ the DIF increased from 1 to 1.09 at strain rate of $10^{-3} \mathrm{~s}^{-1}$ where pryout failure was observed. Further increase in the strain rate resulted in steel failure with DIF of 1.11 at high strain rate of $10^{3}$ $\mathrm{s}^{-1}$. The increase in the DIF is obvious for the anchor diameter of $19.1 \mathrm{~mm}$ with embedment depth of $76.2 \mathrm{~mm}$ where the failure mode transitioned from pryout failure at low strain rate of $10^{-5} \mathrm{~s}^{-1}$ to steel fracture at strain rate of $10 \mathrm{~s}^{-1}$. At high strain rates of $10^{2} \mathrm{~s}^{-1}$ and $10^{3} \mathrm{~s}^{-1}$, DIF of 1.04 and 1.11 were obtained for the $19.1 \mathrm{~mm}$ anchor diameter where steel failure was observed. From Figure 4-52, DIF of 1.14 was obtained for the $19.1 \mathrm{~mm}$ anchor diameter at strain rate of $10^{-1} \mathrm{~s}^{-1}$ where transition in the failure mode from pryout to steel 
anchor failure is observed at higher strain rates. DIF of 1.1 was obtained at high strain rate of $10^{3} \mathrm{~s}^{-1}$. Very close DIF values were obtained for the $12.7-\mathrm{mm}$ and $15.9-\mathrm{mm}$ diameter anchors that all fail by steel fracture at all strain rates investigated. From Figure 4-53, for embedment depth of $152.4 \mathrm{~mm}$, the DIF values are very close for all anchor diameters where steel failure is the dominant failure mode at all the strain rates. Maximum DIF of 1.35 is obtained for the 19.1-mm diameter cast-in-place anchor with $76.2 \mathrm{~mm}$ embedment depth at strain rate of $10 \mathrm{~s}^{-1}$. The DIF of cast-in-place anchors at different strain rates is represented as a function for the strain rate ratio as follows:

$D I F=\frac{V_{u d}}{V_{u s}}=A\left(\frac{\dot{\varepsilon}_{d}}{\dot{\varepsilon}_{s}}\right)^{B}$

$V_{u d}=V_{u s} \cdot A\left(\frac{\dot{\varepsilon}_{d}}{\dot{\varepsilon}_{s}}\right)^{B}$

Where $V_{u s}, V_{u d}$ are the ultimate static and dynamic shear loads respectively. A and $\mathrm{B}$ are constants to be determined by experimental testing or numerical parametric analysis.

Table 4-19 shows the maximum dynamic increase factor for the cast-in-place anchors under shear load where pryout failure and steel failure modes were observed.

Table 4-19: Maximum DIF for the cast-in-place anchors under shear load

\begin{tabular}{|c|c|c|c|c|c|c|}
\hline $\begin{array}{c}\text { Model } \\
\text { No. }\end{array}$ & $\begin{array}{c}\mathrm{d} \\
(\mathrm{mm})\end{array}$ & $\begin{array}{c}\mathrm{h}_{\text {ef }} \\
(\mathrm{mm})\end{array}$ & $\begin{array}{c}\dot{\varepsilon} \\
\left(\mathrm{s}^{-1}\right)\end{array}$ & $\mathrm{PR}$ & \multirow{2}{*}{$\begin{array}{c}\dot{\varepsilon} \\
\left(\mathrm{s}^{-1}\right)\end{array}$} & $\mathrm{DIF}$ \\
\hline 1 & 12.7 & 76.2 & - & - & $10^{3}$ & 1.13 \\
\hline 2 & 12.7 & 101.6 & - & - & $10^{3}$ & 1.11 \\
\hline 3 & 12.7 & 152.4 & - & - & $10^{3}$ & 1.10 \\
\hline 4 & 15.9 & 76.2 & $10^{-3}$ & 1.09 & $10^{3}$ & 1.11 \\
\hline 5 & 15.9 & 101.6 & - & - & $10^{3}$ & 1.15 \\
\hline 6 & 15.9 & 152.4 & - & - & $10^{3}$ & 1.15 \\
\hline
\end{tabular}




\begin{tabular}{|l|c|c|c|c|c|c|}
\hline 7 & 19.1 & 76.2 & $10^{-1}$ & 1.24 & $10^{3}$ & 1.11 \\
\hline 8 & 19.1 & 101.6 & $10^{-3}$ & 1.09 & $10^{3}$ & 1.10 \\
\hline 9 & 19.1 & 152.4 & - & - & $10^{3}$ & 1.11 \\
\hline
\end{tabular}

\subsubsection{Regression analysis for cast-in-place anchors under shear load}

Regression analysis has been performed to predict the relation that accurately represents the finite element results of the cast-in-place anchor subjected to shear load. As shown in Figures 4-38, 4-39 and 4-40, steel anchor failure is the dominant failure mode for most of the cast-in-place anchors subjected to shear load and subjected to strain rate in the range of $10^{-3} \mathrm{~s}^{-1}$ to $10^{3} \mathrm{~s}^{-1}$, hence regression analysis is performed for the anchors exhibiting steel failure mode. Average values of the DIF for the 12.7-mm, 15.9-mm and 19.1-mm cast-inplace diameter anchors were considered to adjust the DIF for the effect of anchor diameter. The relation between the DIF and the strain rate ratio $\left(\dot{\varepsilon}_{d} / \dot{\varepsilon}_{s}\right)$ is shown in Figure 4-54 for the cast-in-place anchors exhibited steel anchor failure mode. 


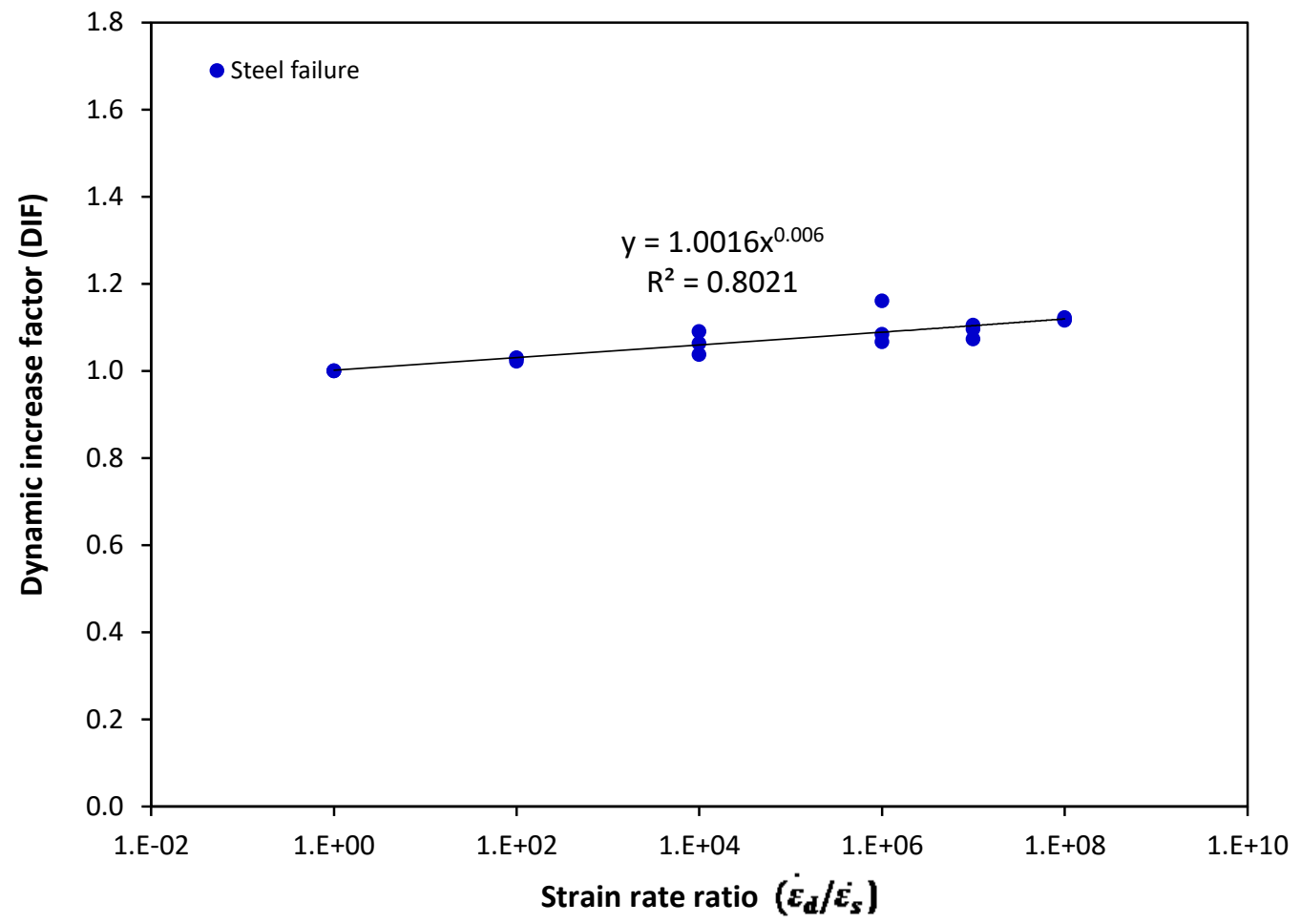

Figure 4-54: Effect of strain rate ratio on the DIF for cast-in-place anchors exhibit steel failure under shear load

Table 4-20 shows the statistical models used to predict a formula that relates the DIF with the strain rate for the cast-in-place anchors exhibited steel anchor failure.

Table 4-20: Statistical models and coefficient of determination to predict the DIF for cast-in-place anchor exhibited steel failure under shear load

\begin{tabular}{|c|c|c|}
\hline Statistical models & Formulae & $\begin{array}{c}\text { Coefficient of determination } \\
\left(\mathrm{R}^{2}\right)\end{array}$ \\
\hline Exponential & $D I F=1.0539 e^{6 E-10\left(\frac{\dot{\varepsilon}_{d}}{\dot{\varepsilon}_{s}}\right)}$ & 0.277 \\
\hline Linear & $D I F=7 E-10\left(\frac{\dot{\varepsilon}_{d}}{\dot{\varepsilon}_{s}}\right)+1.0548$ & 0.278 \\
\hline Logarithmic & $D I F=0.0064 \ln \left(\frac{\dot{\varepsilon}_{d}}{\dot{\varepsilon}_{s}}\right)+1.0009$ & 0.790 \\
\hline Power & $D I F=1.0016\left(\frac{\dot{\varepsilon}_{d}}{\dot{\varepsilon}_{s}}\right)^{0.006}$ & 0.802 \\
\hline
\end{tabular}


As shown in Table 4-20 the highest coefficients of determination of $80 \%$ is obtained from the power model for the steel anchor failure mode. The predicted formulae for the DIF for cast-in-place anchors subjected to shear load and exhibited steel failure can be presented as in Equation (4.21).

$D I F=1.0016\left(\frac{\dot{\varepsilon}_{d}}{\dot{\varepsilon}_{s}}\right)^{0.006}$

The proposed Equation (4.21) can be used to predict the ultimate dynamic shear load $\left(V_{u d}\right)$ for steel anchor failure mode as follows:

$V_{u d}=V_{u s} \times 1.0016\left(\frac{\dot{\varepsilon}_{d}}{\dot{\varepsilon}_{s}}\right)^{0.006}$

Where the ultimate static load $\left(V_{u s}\right)$ can be determined from Equation (4.18) for the castin-place anchors exhibited steel failure under shear load.

Residual analysis has been performed for the cast-in-place anchors subjected to shear load to determine the difference between the DIF results obtained from the finite element and the DIF results obtained from the proposed Equation (4.21). Figure 4-55 presents the residual plots for the cast-in-place anchors. As shown in Figure 4-55, the residual results exhibit horizontal trend line with strain rate ratio. 


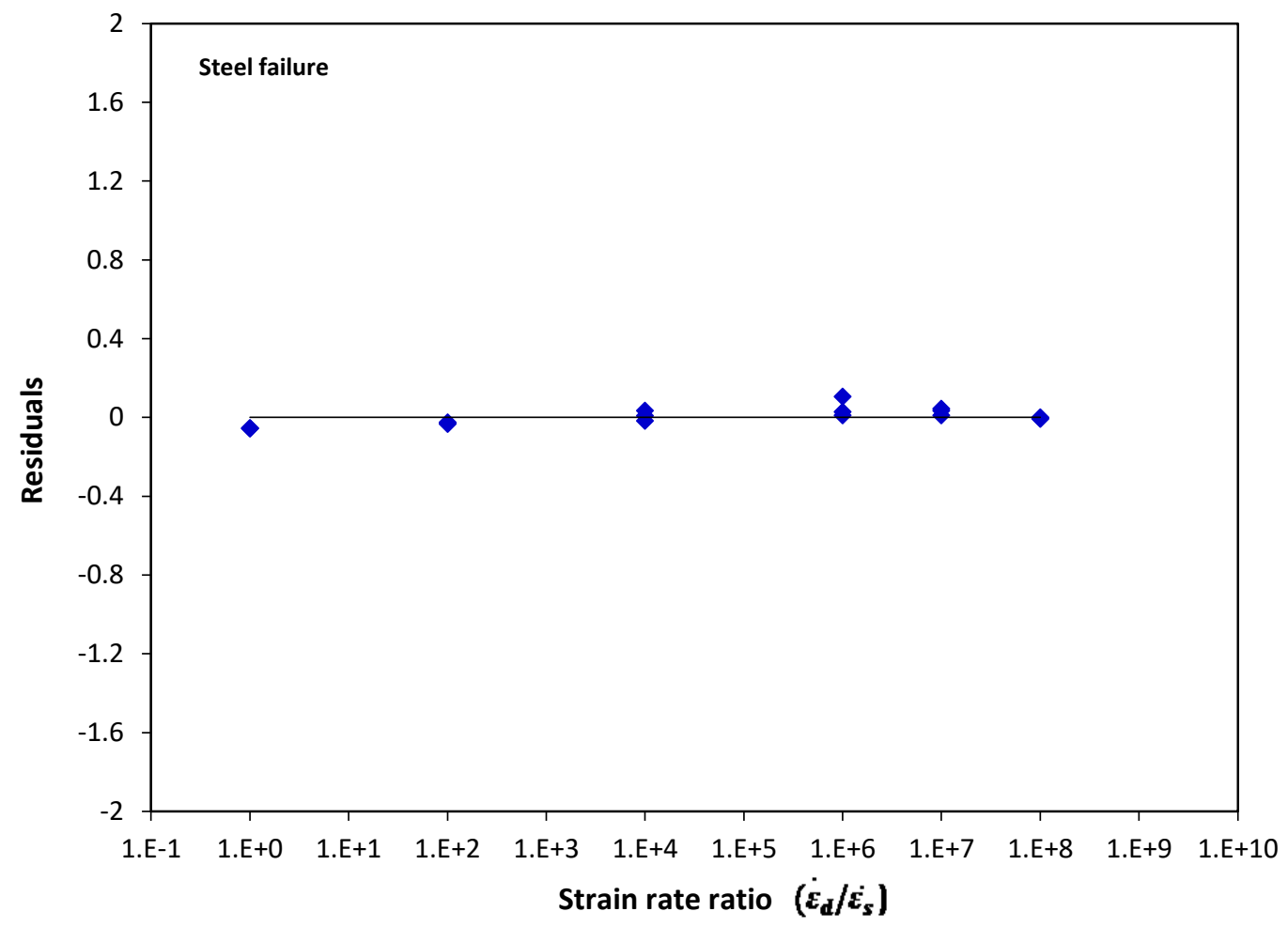

Figure 4-55: Residual versus strain rate ratio for the cast-in-place anchor subjected to shear load and exhibited steel failure

In addition, new cast-in-place anchor models were developed with diameters of $9.5 \mathrm{~mm}$, $12.7 \mathrm{~mm}, 15.9 \mathrm{~mm}$ and $19.1 \mathrm{~mm}$ and embedment depths of $89 \mathrm{~mm}, 114 \mathrm{~mm}$ and $140 \mathrm{~mm}$ to verify the proposed equation. A comparison has been made between the proposed equation and the results of the new cast-in-place anchor models. Figure 4-56 presents the relation between the DIF obtained from newly developed numerical models and the regression models (Equation (4.21)) for cast-in-place anchors exhibited steel failure. As shown in the figure, the DIF is distributed normally around the equality line, however some divergence was observed for the high DIF values. 


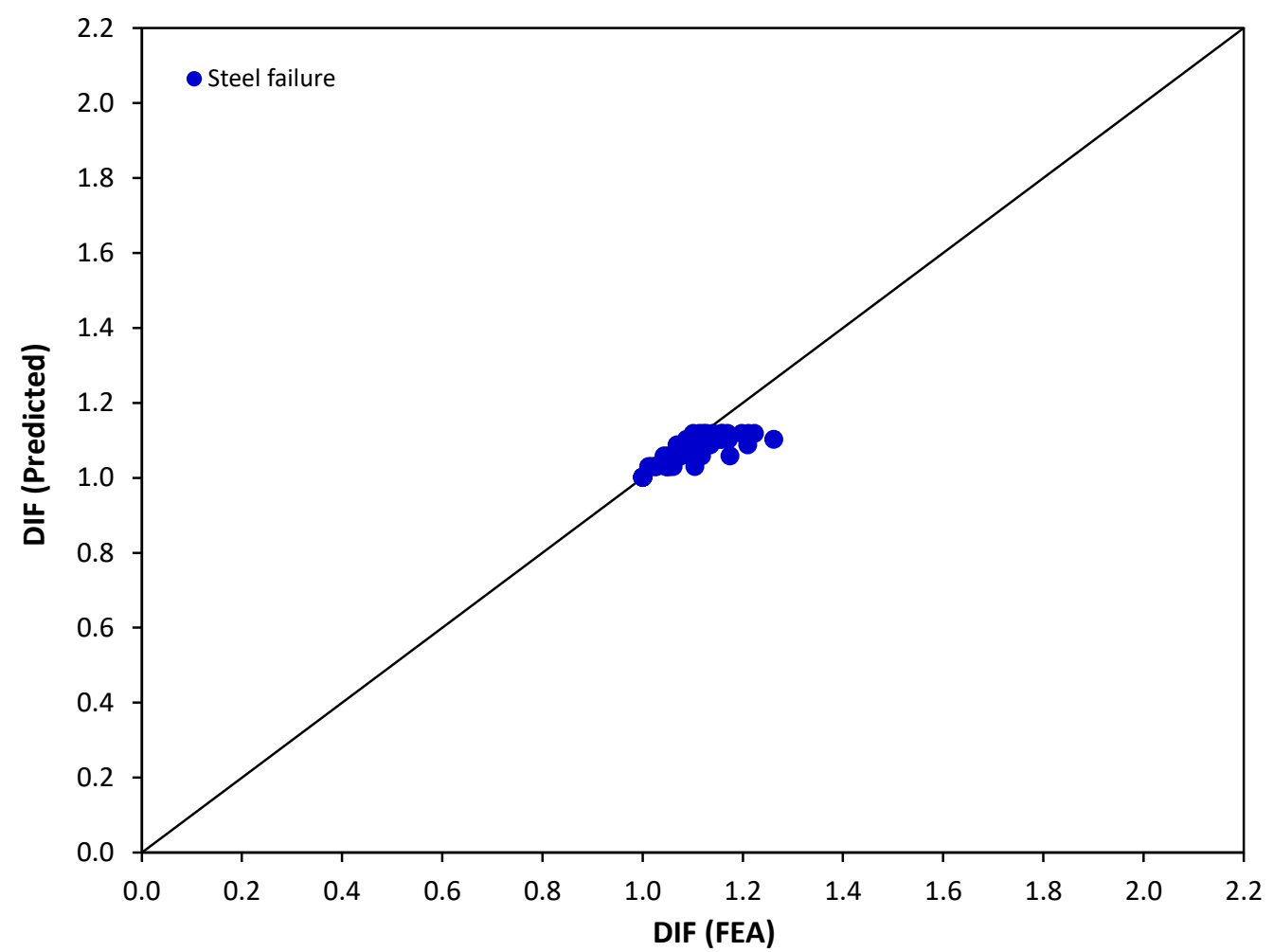

Figure 4-56: DIF obtained from the finite element analysis versus the predicted DIF for the cast-in-place anchor exhibited steel failure under shear load 


\subsubsection{Case study: effect of concrete compressive strength on the shear behaviour of cast-in-place anchors}

\subsubsection{Level of damage and failure mode}

Cast-in-place anchor diameters of $12.7 \mathrm{~mm}$ and $19.1 \mathrm{~mm}$ with embedment depths of 76.2 $\mathrm{mm}$ and $152.4 \mathrm{~mm}$ subjected to shear load were investigated at static $\left(10^{-5} \mathrm{~s}^{-1}\right)$ and high strain rate $\left(10^{3} \mathrm{~s}^{-1}\right)$. Three concrete compressive strengths of $20 \mathrm{MPa}, 30 \mathrm{MPa}$ and $40 \mathrm{MPa}$ were selected for the analysis. Figures 4-57 and 4-58 show the failure mode for the 12.7$\mathrm{mm}$ and 19.1-mm diameter cast-in-place anchors respectively embedded in concrete compressive strengths of $20 \mathrm{MPa}, 30 \mathrm{MPa}$ and $40 \mathrm{MPa}$ at low and high strain rates of $10^{-5}$ $\mathrm{s}^{-1}$ and $10^{3} \mathrm{~s}^{-1}$. As shown in Figure 4-57, at the low strain rate of $10^{-5} \mathrm{~s}^{-1}$, pryout failure mode is observed for the concrete compressive strength of $20 \mathrm{MPa}$ and embedment depth of $76.2 \mathrm{~mm}$. Steel anchor failure was observed for the concrete compressive strengths of $30 \mathrm{MPa}$ and $40 \mathrm{MPa}$. Also, steel anchor failure was observed for the embedment depth of $152.4 \mathrm{~mm}$ at all concrete compressive strengths. At strain rate of $10^{3} \mathrm{~s}^{-1}$ steel anchor failure was observed for all the concrete compressive strengths and embedment depths investigated. It can be seen from Figure 4-58, for the 19.1-mm diameter cast-in-place

anchor at low strain rate of $10^{-5} \mathrm{~s}^{-1}$, pryout failure was observed for all the concrete compressive strengths at embedment depth of $76.2 \mathrm{~mm}$. At the embedment depth of 152.4 mm pryout failure is observed for the compressive strength of $20 \mathrm{MPa}$ whereas steel anchor failure was observed for the compressive strengths of $30 \mathrm{MPa}$ and $40 \mathrm{MPa}$. At high strain rate of $10^{3} \mathrm{~s}^{-1}$, steel anchor failure was observed for all the concrete compressive strengths and embedment depths investigated. 


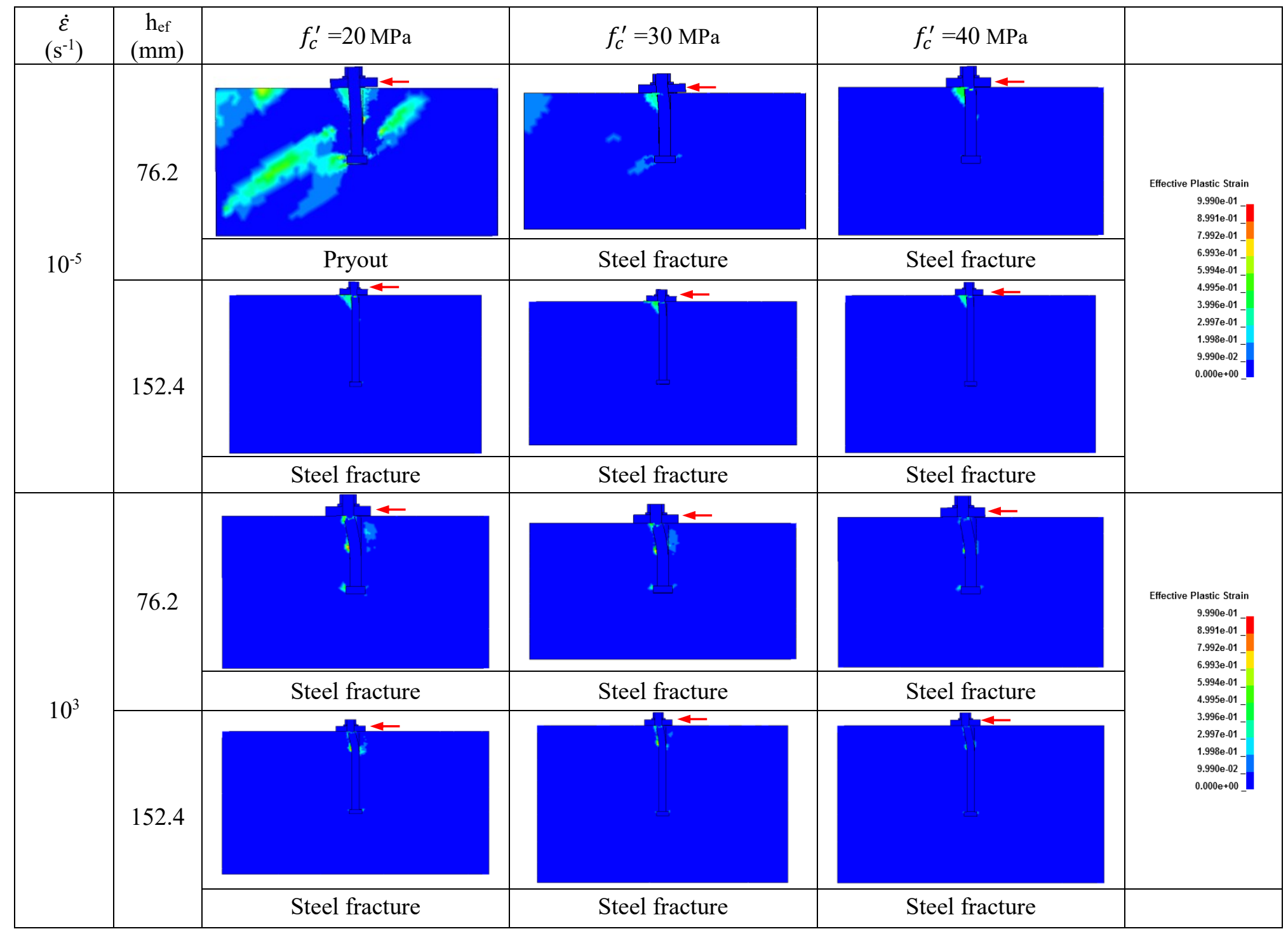

Figure 4-57: Effect of strain rate and concrete compressive strength on the failure mode for the $12.7 \mathrm{~mm}$ diameter cast-in-place anchors 


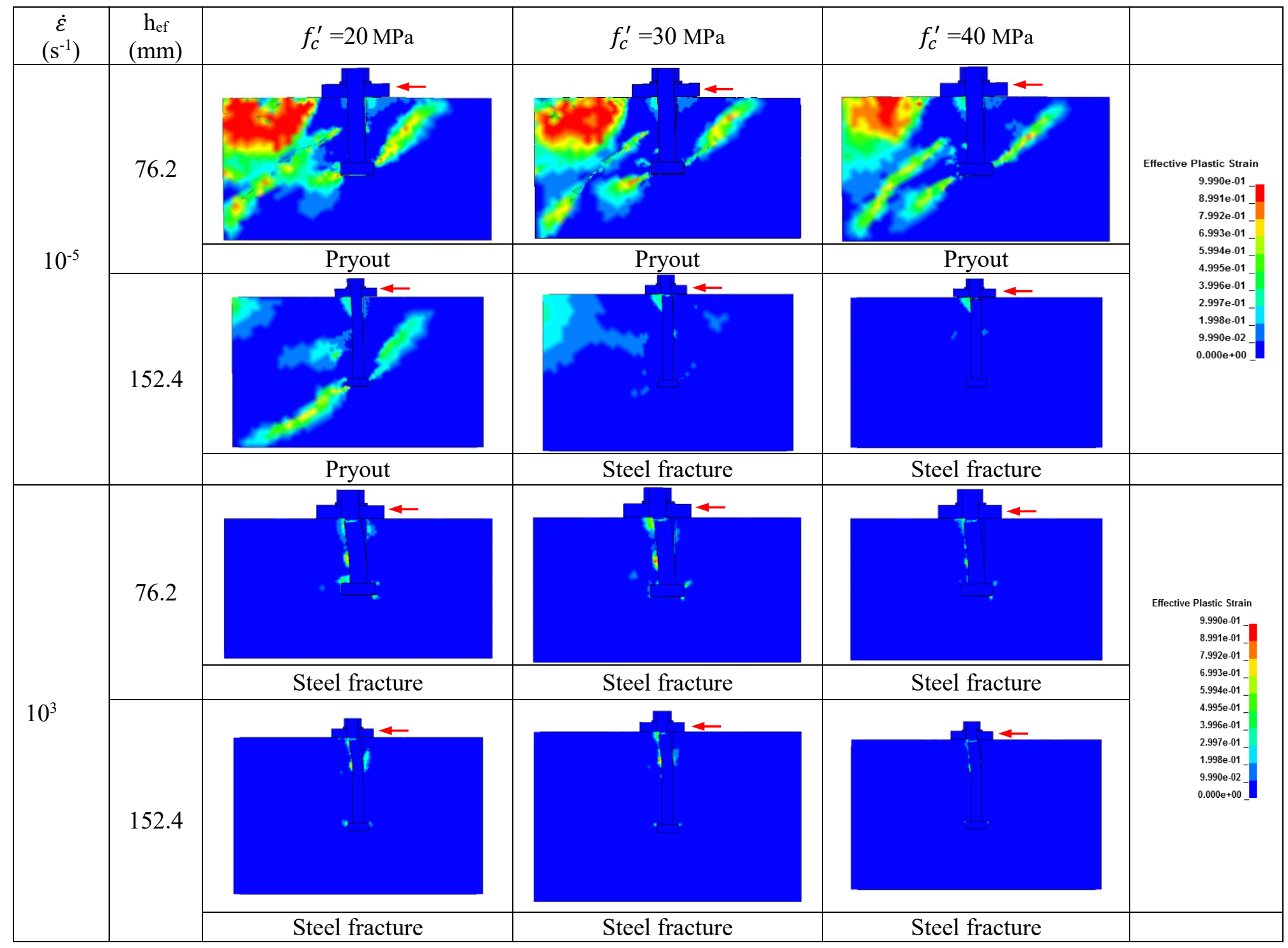

Figure 4-58: Effect of strain rate and concrete compressive strength on the failure mode for the 19.1 mm diameter cast-in-place anchors 
From Figures 4-57 and 4-58, it can be seen that for cast-in-place anchorage systems failing in concrete pryout at the static strain rate transitioned to steel fracture failure at the high strain rates. This is attributed to the higher DIF of concrete at high strain rates compared with steel (Malvar and Crawford, 1998; Malvar and Ross, 1998).

It can be stated that at low strain rate of $10^{-5} \mathrm{~s}^{-1}$, the failure mode is influenced by the concrete compressive strength where pryout failure is observed in most of the anchors at shallow embedment depth of $76.2 \mathrm{~mm}$. However, at high strain rate, the failure mode of the anchorage system transitioned to steel failure for all the concrete compressive strengths investigated. More severe concrete cracking was observed for the cast-in-place anchors embedded in $20 \mathrm{MPa}$ concrete compressive strength than that embedded in $30 \mathrm{MPa}$ and 40 MPa concrete compressive strengths at low strain rate of $10^{-5} \mathrm{~s}^{-1}$. At high strain rate, it can be seen that the damage extends to a small area in the concrete where anchor bending followed by fracturing was observed.

\subsubsection{Effect of strain rate and concrete compressive strength on shear behaviour}

Shear behaviour of cast-in-place anchors was investigated using LS-DYNA finite element software. Three concrete compressive strengths of $20 \mathrm{MPa}, 30 \mathrm{MPa}$ and $40 \mathrm{MPa}$ were selected for the analysis to investigate the effect of concrete strength on the shear response of cast-in-place anchors. Anchor diameters of $12.7 \mathrm{~mm}$ and $19.1 \mathrm{~mm}$ with embedment depths of $76.2 \mathrm{~mm}$ and $152.4 \mathrm{~mm}$ were investigated at low and high strain rates of $10^{-5} \mathrm{~s}^{-1}$ and $10^{3} \mathrm{~s}^{-1}$ respectively. Figures 4-59 and 4-60 show the effect of concrete compressive strength on the load-displacement response of the 12.7-mm diameter cast-in-place anchor at strain rate of $10^{-5} \mathrm{~s}^{-1}$ with embedment depths of $76.2 \mathrm{~mm}$ and $152.4 \mathrm{~mm}$ respectively. 
Effect of concrete compressive strength on the load displacement response of cast-in-place anchor of $19.1 \mathrm{~mm}$ diameter with $76.2 \mathrm{~mm}$ and $152.4 \mathrm{~mm}$ embedment depths at low strain rate of $10^{-5} \mathrm{~s}^{-1}$ is shown in Figures 4-61 and 4-62 respectively. As shown in the figures, the ultimate shear load of the cast-in-place anchors increased with the displacement until the maximum load then it decreased until failure. Concrete compressive strength of $40 \mathrm{MPa}$ resulted in higher ultimate shear load compared to the concrete compressive strength of 20 MPa. Similar observation was reported by Çalışkan et al. (Çalışkan et al., 2013) on the adhesive anchors embedded in low strength concrete of $5 \mathrm{MPa}$ and $10 \mathrm{MPa}$ and subjected to cyclic shear load (Çalışkan et al., 2013). The increment in the ultimate shear load is found to be higher at the shallow embedment depth of $76.2 \mathrm{~mm}$, where pryout failure is observed, compared to deeper embedment depth of $152.4 \mathrm{~mm}$ where the failure mode was by steel fracture. The increase in the embedment depth showed an increased shear capacity when the pryout failure mode is the dominant.

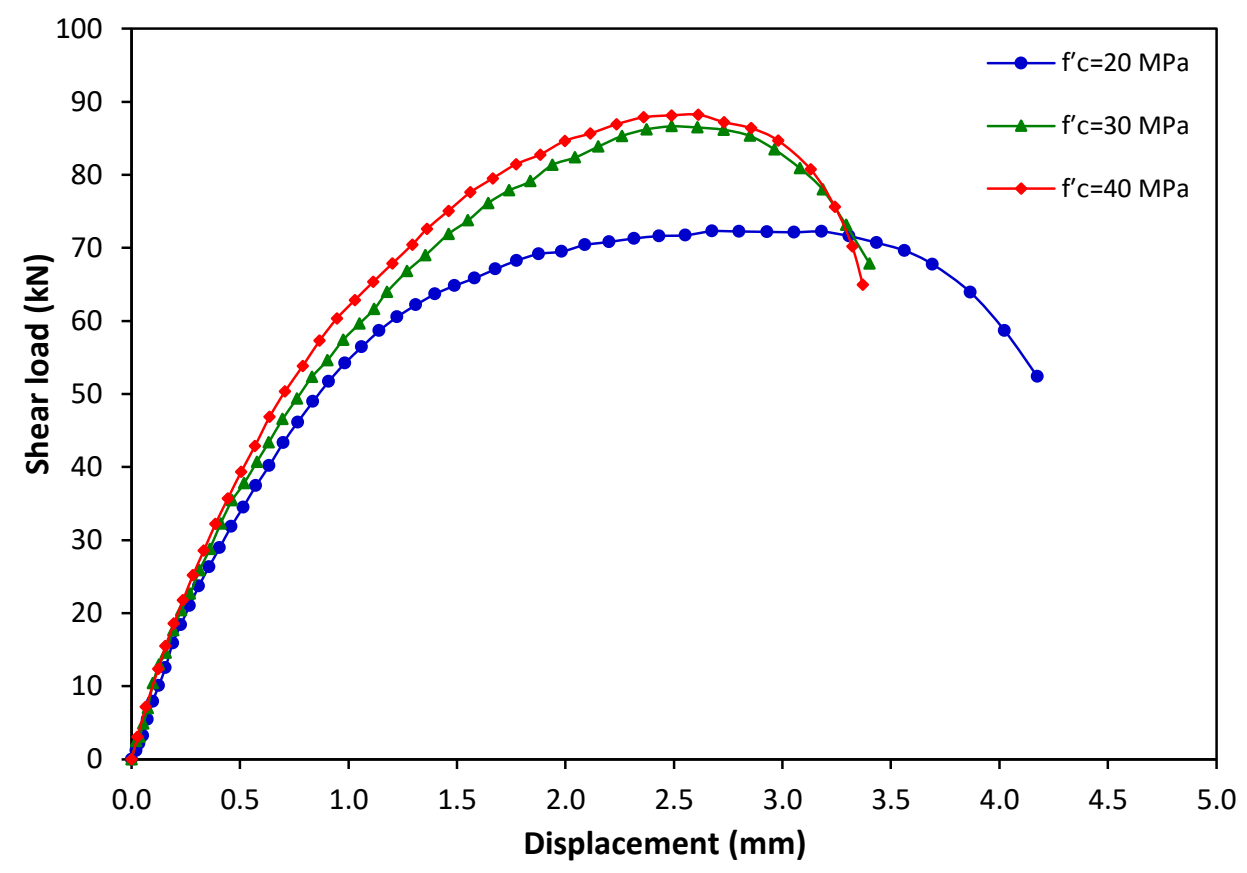

Figure 4-59: Shear load-displacement response of $12.7 \mathrm{~mm}$ cast-in-place anchor diameter with $76.2 \mathrm{~mm}$ embedment depth at strain rate of $10^{-5} \mathrm{~s}^{-1}$ 


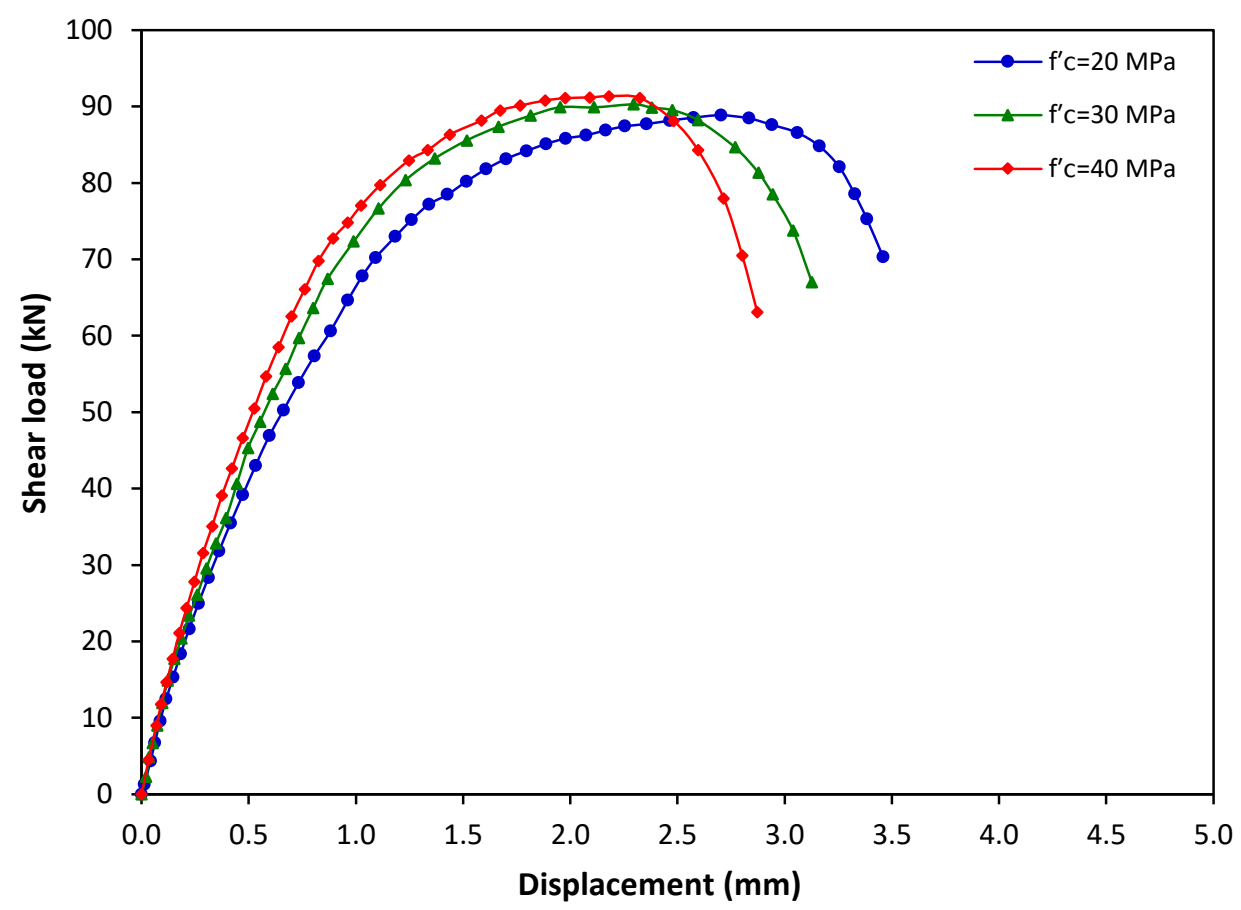

Figure 4-60: Shear load-displacement response of $12.7 \mathrm{~mm}$ cast-in-place anchor diameter with $152.4 \mathrm{~mm}$ embedment depth at strain rate of $10^{-5} \mathrm{~s}^{-1}$

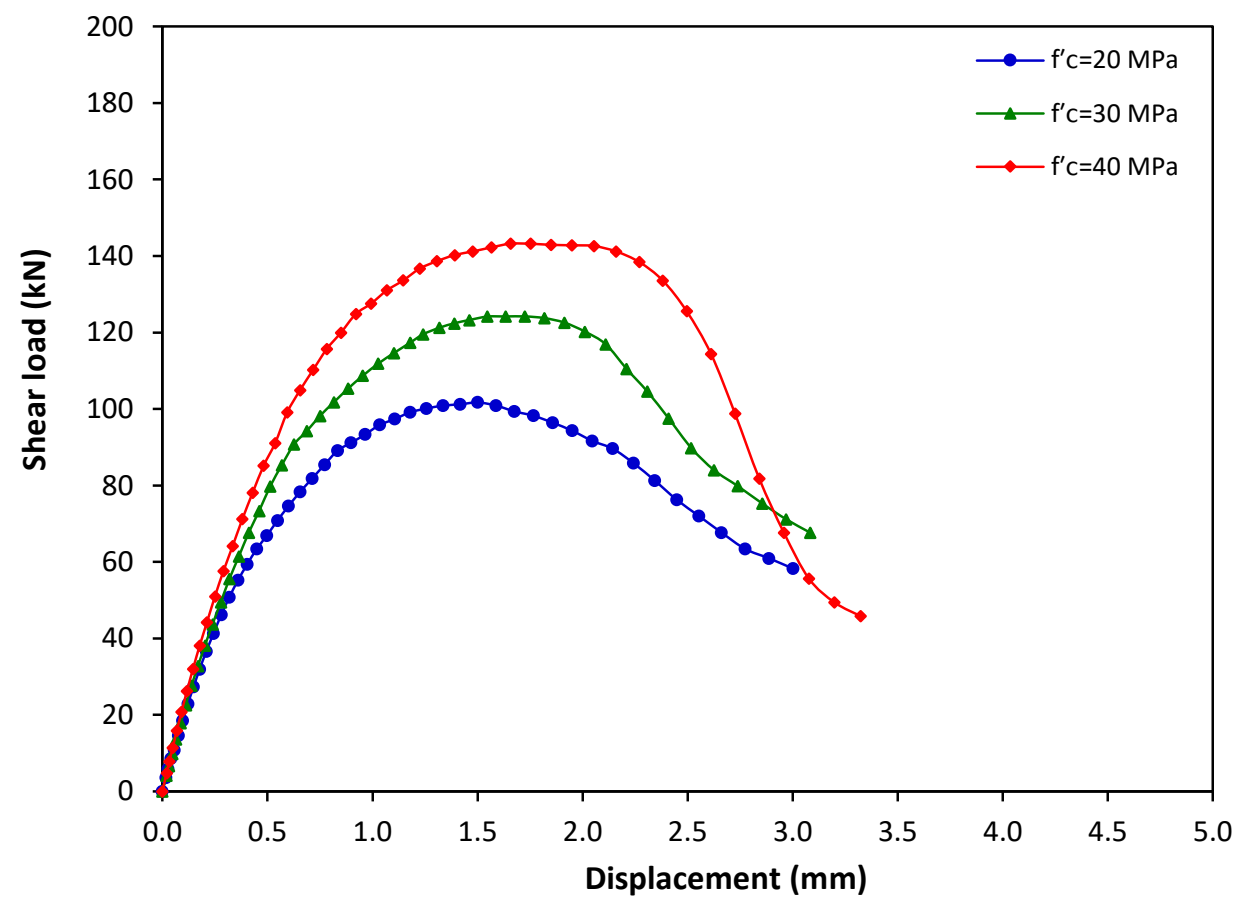

Figure 4-61: Shear load-displacement response of $19.1 \mathrm{~mm}$ cast-in-place anchor diameter with $76.2 \mathrm{~mm}$ embedment depth at strain rate of $10^{-5} \mathrm{~s}^{-1}$ 


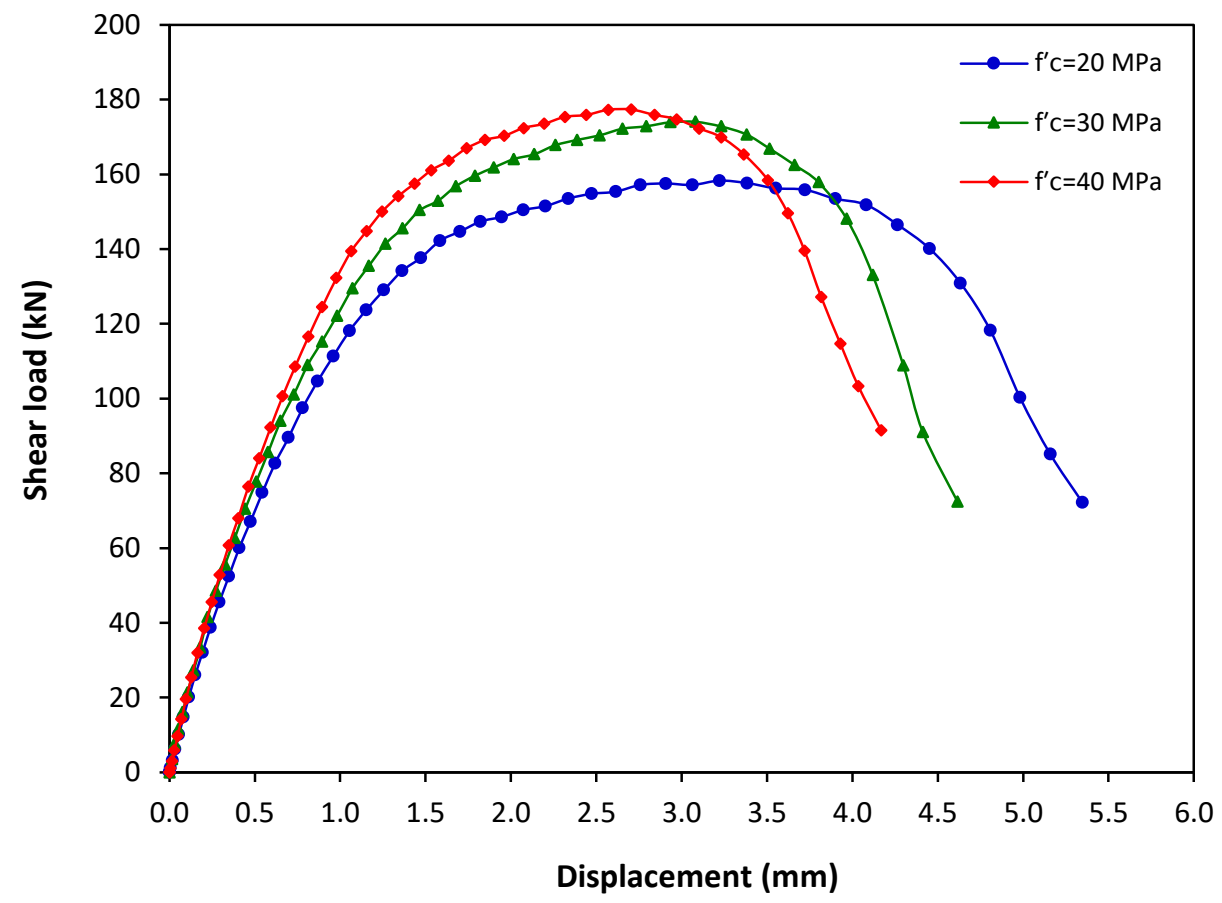

Figure 4-62: Shear load-displacement response of $19.1 \mathrm{~mm}$ cast-in-place anchor diameter with $152.4 \mathrm{~mm}$ embedment depth at strain rate of $10^{-5} \mathrm{~s}^{-1}$

At high strain rate of $10^{3} \mathrm{~s}^{-1}$, the shear load increased with the displacement until the ultimate shear load, and then decreased with increased displacement until failure (Figures $4-63,4-64,4-65$ and 4-66). The increase in the strain rate from $10^{-5} \mathrm{~s}^{-1}$ to $10^{3} \mathrm{~s}^{-1}$ resulted in increased shear capacity of the cast-in-place anchorage systems. 


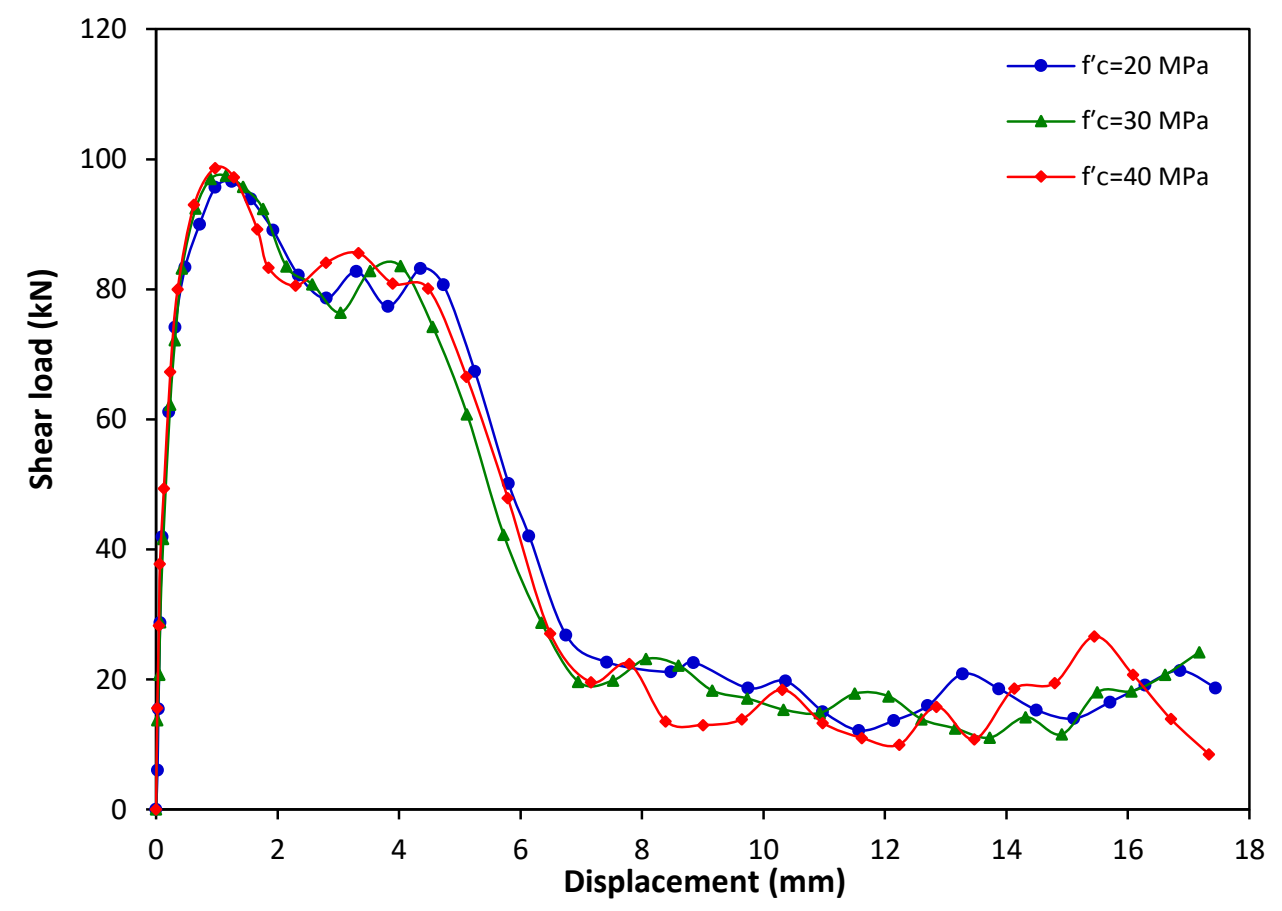

Figure 4-63: Shear load-displacement response of $12.7 \mathrm{~mm}$ cast-in-place anchor diameter with $76.2 \mathrm{~mm}$ embedment depth at strain rate of $10^{3} \mathrm{~s}^{-1}$

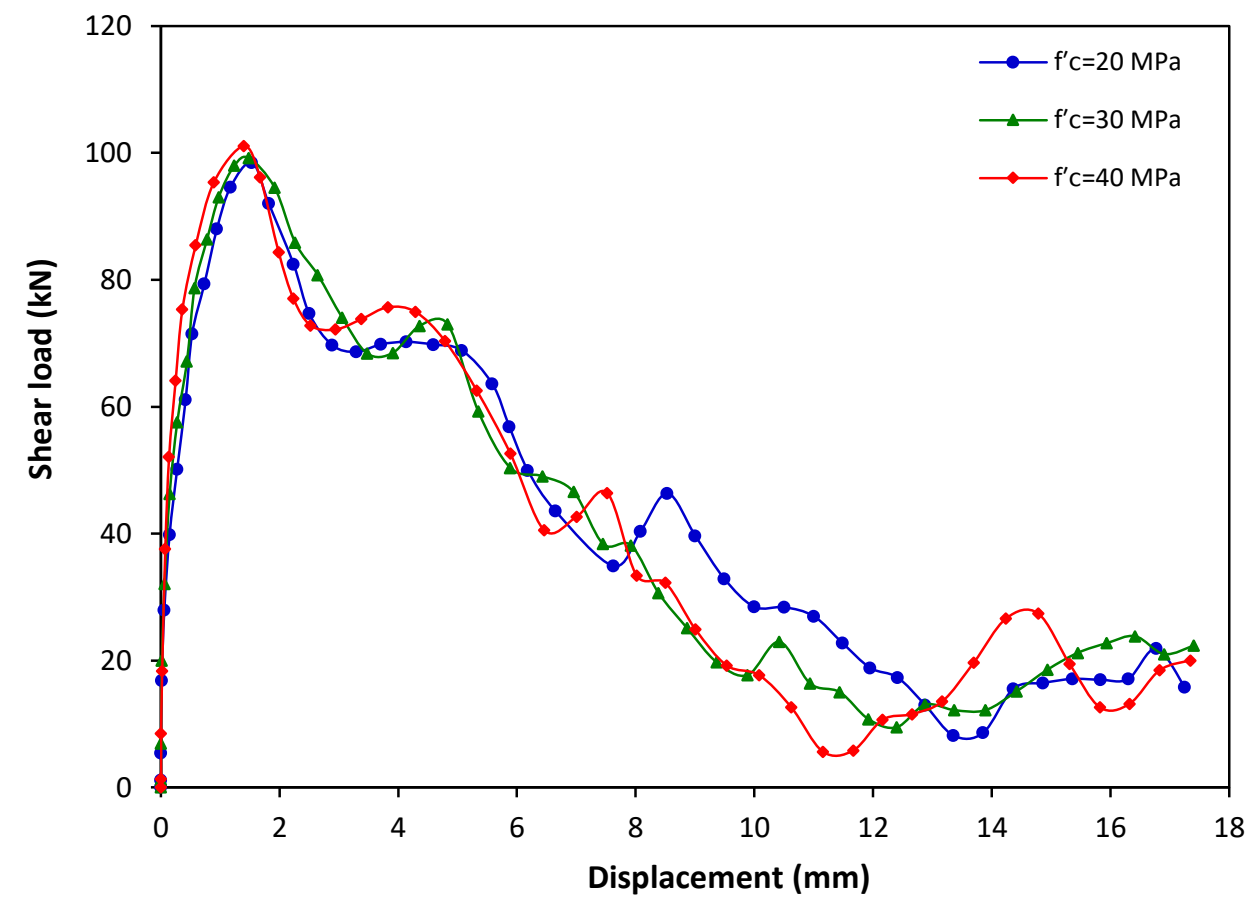

Figure 4-64: Shear load-displacement response of $12.7 \mathrm{~mm}$ cast-in-place anchor diameter with $152.4 \mathrm{~mm}$ embedment depth at strain rate of $10^{3} \mathrm{~s}^{-1}$ 


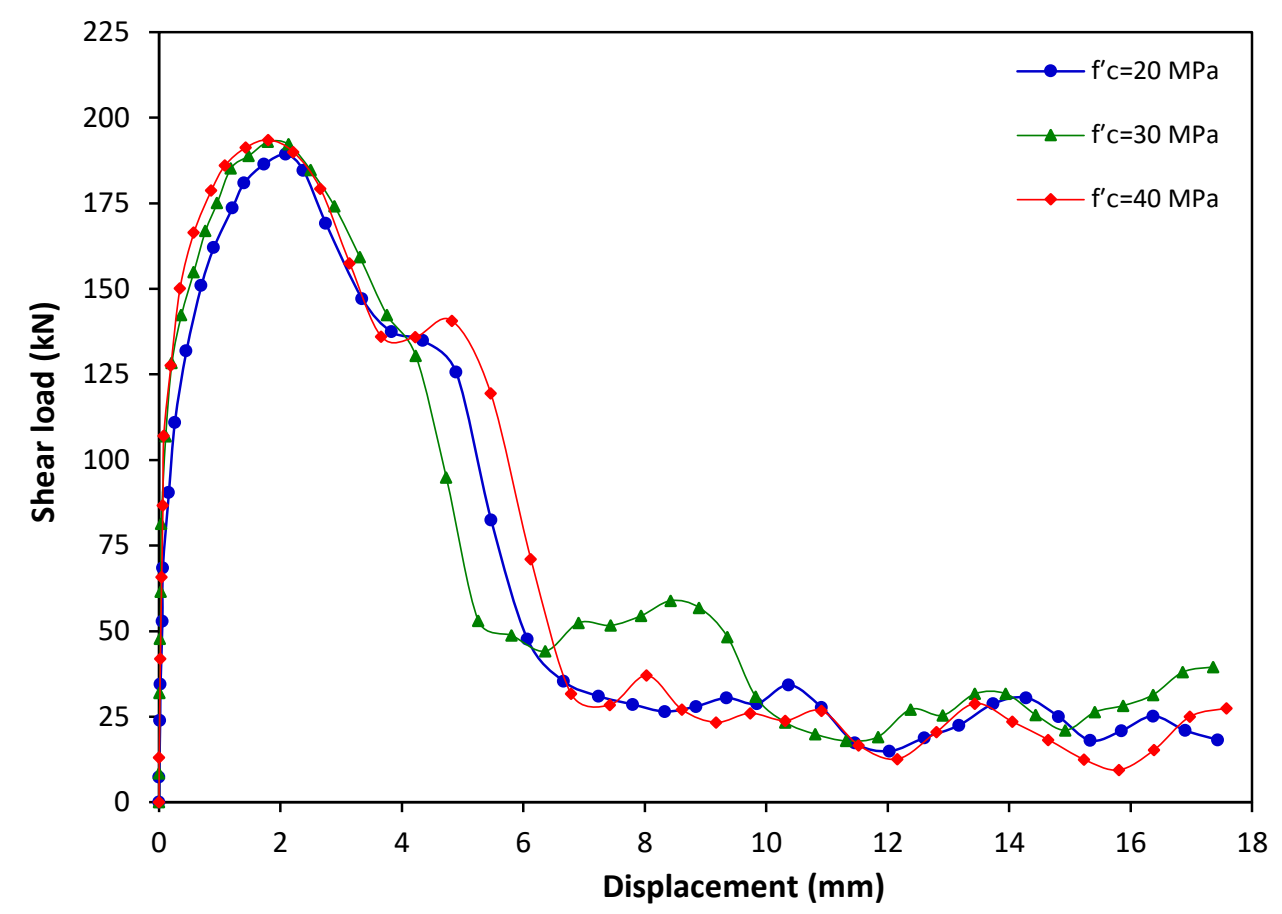

Figure 4-65: Shear load-displacement response of $19.1 \mathrm{~mm}$ diameter cast-in-place anchor with $76.2 \mathrm{~mm}$ embedment depth at strain rate of $10^{3} \mathrm{~s}^{-1}$

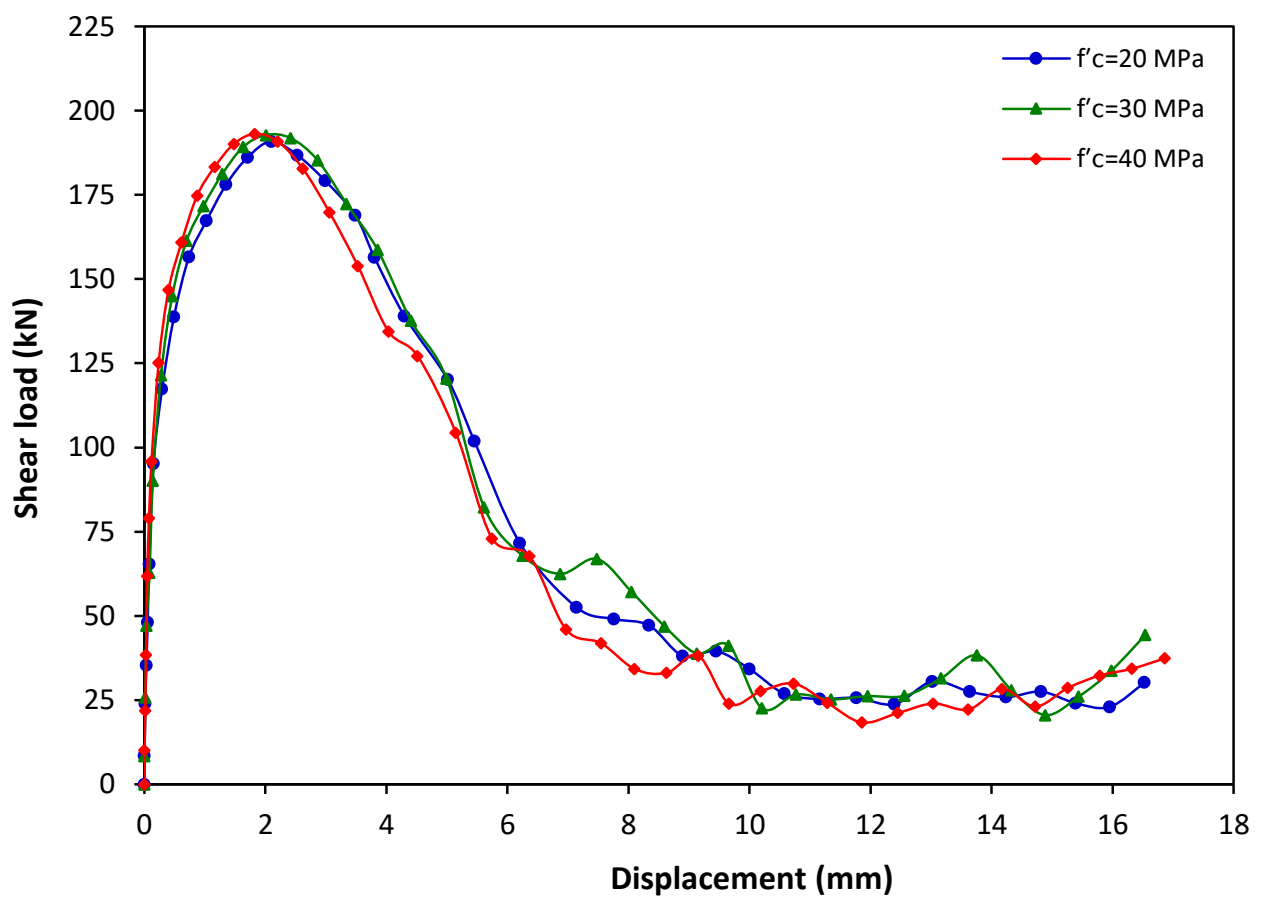

Figure 4-66: Shear load-displacement response of $19.1 \mathrm{~mm}$ cast-in-place anchor diameter with $152.4 \mathrm{~mm}$ embedment depth at strain rate of $10^{3} \mathrm{~s}^{-1}$ 
In general, it can be seen from Figures 4-59 to 4-66 that the increase in the anchor diameter from $12.7 \mathrm{~mm}$ to $19.1 \mathrm{~mm}$ increased the ultimate shear load for the cast-in-place anchors. Effect of concrete compressive strength is significant at low strain rate of $10^{-5} \mathrm{~s}^{-1}$ where pryout failure is observed while a slight (almost negligible) increase in the shear capacity is observed with the increase in the concrete compressive strength at the high strain rate of $10^{3} \mathrm{~s}^{-1}$. The increase in the shear load is attributed to increase concrete resistance to the applied shear load with the increase in the concrete compressive strength. Maximum increment in the ultimate shear load of $40.8 \%$ was obtained for the $19.1-\mathrm{mm}$ diameter castin-place anchor at strain rate of $10^{-5} \mathrm{~s}^{-1}$ when the concrete compressive strength increased from $20 \mathrm{MPa}$ to $40 \mathrm{MPa}$ where pryout failure mode is observed. Maximum increment in the ultimate shear load of $2.7 \%$ is obtained at high strain rate of $10^{3} \mathrm{~s}^{-1}$ when the concrete compressive strength increased from $20 \mathrm{MPa}$ to $40 \mathrm{MPa}$ where steel anchor failure is the dominant failure mode. At high strain rate the strength of concrete and steel materials increased. When steel failure occurs, the increment in the shear failure load is attributed to the increase in the steel strength at high strain rate rather than the increase in the concrete strength. Hence, the concrete compressive strength has a slight influence on the shear load at high strain rate.

\subsection{Summary}

This chapter introduces finite element analyses for the cast-in-place anchorage to concrete system models that were developed and presented in chapter three. Tensile and shear behaviour of the cast-in-place anchors at strain rates ranging from $10^{-5} \mathrm{~s}^{-1}$ to $10^{3} \mathrm{~s}^{-1}$ were investigated. Different design parameters (anchor diameter and embedment depth) were 
considered in the analyses. The chapter starts with validation of the cast-in-place anchor model with the experimental test data from the literature. Level of damage and failure mode for different strain rates was investigated. For the cast-in-place anchors subjected to tensile load; two types of failure modes were observed: concrete cone breakout failure and steel anchor failure. For the cast-in-place anchors subjected to shear load, two types of failure modes were observed; pryout and steel anchor failure. At high strain rate of $10^{3} \mathrm{~s}^{-1}$ steel anchor failure was the dominant failure mode for all the cast-in-place anchors investigated. Concrete cone depth, concrete cone breakout diameter and concrete cone propagation angle were determined.

Tensile and shear load-displacement relations for the cast-in-place anchors at different strain rates were drawn and analyzed. The relation between the ultimate tensile load, strain rate and failure mode for different design parameters was introduced and discussed. Also, the relation between the ultimate shear load, strain rate and failure mode for different design parameters was introduced and discussed. Comparison has been made between the results obtained from the finite element analysis and the prediction analytical methods (ACI and CCD). Dynamic increase factor was determined for the cast-in-place anchors subjected to tensile and shear loads at all the strain rates investigated. Equations were developed to predict the relation between the strain rate and the DIF for the cast-in-place anchors under tensile and shear loads. Effect of strain rate on the concrete compressive strengths of $20 \mathrm{MPa}, 30 \mathrm{MPa}$ and $40 \mathrm{MPa}$ for the cast-in-place anchors subjected to shear load was investigated. Plastic strain contours that represent the level of damage were drawn and discussed. Shear load-displacement relation for the cast-in-place anchors was drawn for the concrete compressive strengths investigated. 


\section{Chapter 5 : Strain rate effect on adhesive anchors}

\subsection{Finite element modelling for adhesive anchors under tensile load}

Finite element models were developed using LS-DYNA software (LSTC, 2014) to investigate the tensile behaviour of adhesive anchors embedded into concrete at strain rates ranging from $10^{-5} \mathrm{~s}^{-1}$ to $10^{3} \mathrm{~s}^{-1}$. A single adhesive anchor was placed in the center of each concrete block to ensure that the edge distance was sufficient for concrete cone formation without edge effects. A biased mesh refinement towards the adhesive and anchor along the embedment depth was developed to improve the accuracy of the analysis and to minimize discretization error. A schematic view of the adhesive anchorage to concrete system is shown in Figure 5-1. Figure 5-2 shows the geometric configuration and boundary condition of the adhesive anchor model under tensile load.

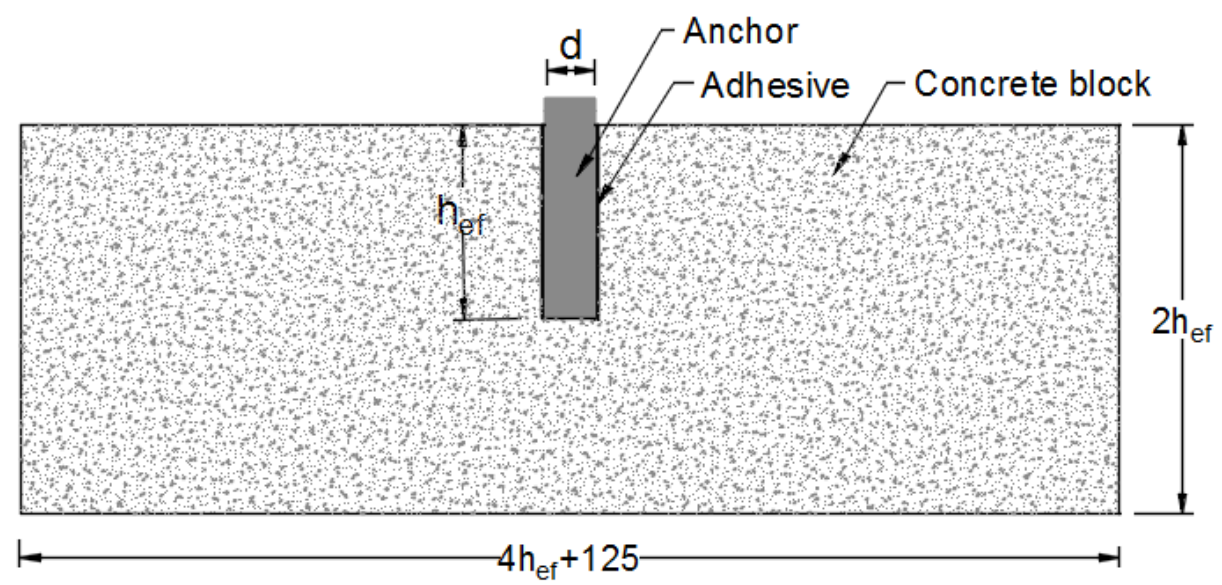

Figure 5-1: A schematic view of the adhesive anchorage to concrete system 


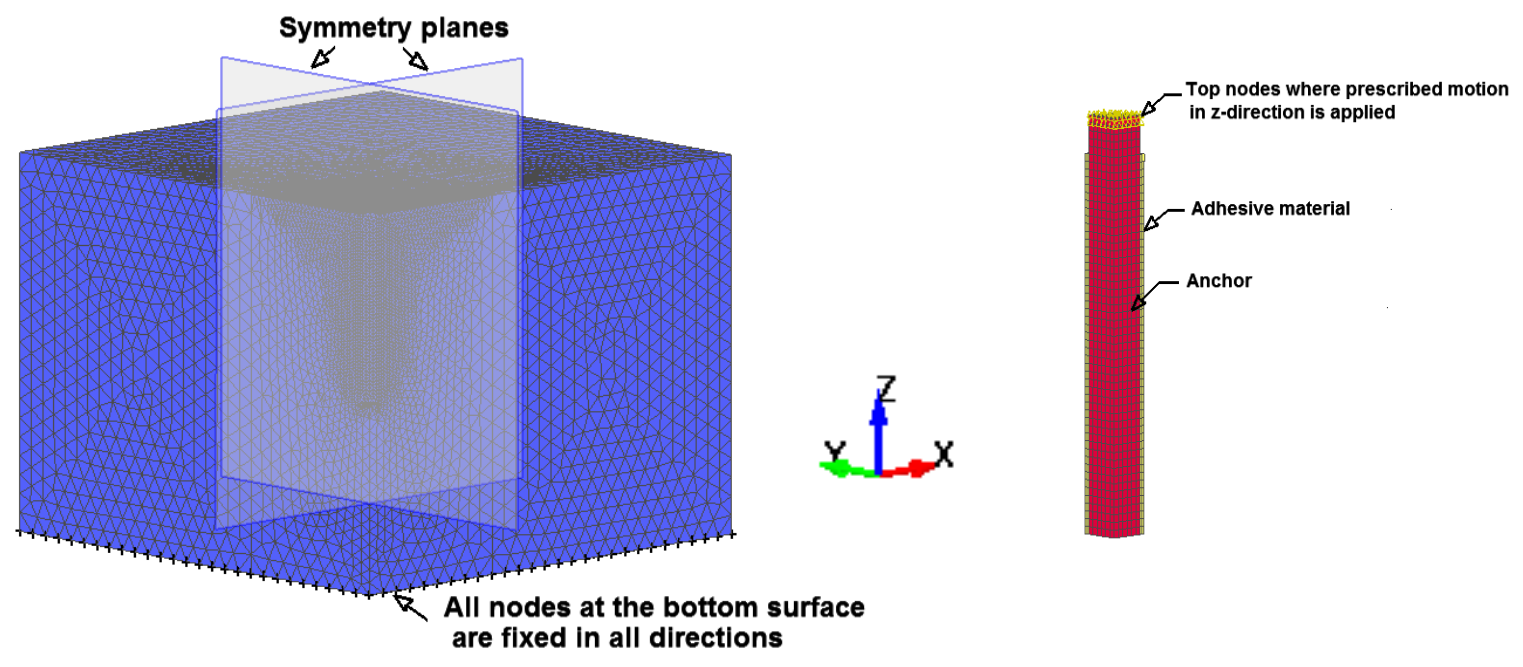

$\begin{array}{ll}\text { (a) Concrete mesh } & \text { (b) Adhesive anchor mesh }\end{array}$

Figure 5-2: Geometric configuration with boundary condition for the adhesive anchor model

\subsubsection{Validation of adhesive anchor model under tensile load}

To ensure the material models chosen for the concrete, adhesive and steel elements are adequate for the research program, two finite element models of adhesive anchorage to concrete system representing drop-mass experimental program conducted by Braimah et al. (Braimah et al., 2004) were developed in LS-DYNA to validate the numerical model. The experimental test setup consisted of a 6.4-mm diameter steel anchor with embedment depth of $114 \mathrm{~mm}$ and 9.5- $\mathrm{mm}$ diameter anchor with embedment depth of $89 \mathrm{~mm}$ embedded into a $34.5 \mathrm{MPa}$ compressive strength concrete. The 6.4- $\mathrm{mm}$ and 9.5-mm diameter adhesive anchors were modeled with yield strengths of $874 \mathrm{MPa}$ and $1030 \mathrm{MPa}$ respectively. The epoxy adhesive material was $1.5 \mathrm{~mm}$ thick with $30 \mathrm{MPa}$ tensile strength, $23 \mathrm{MPa}$ shear strength and $4900 \mathrm{MPa}$ modulus of elasticity (Braimah et al., 2004). A mesh sensitivity analysis was carried out to achieve an optimum mesh size that resulted in high accuracy in comparison with experimental results and minimized computational effort. The 
drop mass impact effect on the anchor was not modeled in the analysis. The experimental displacement profile from a drop-mass test was applied to the steel anchor in the numerical model by using BOUNDARY_PRESCRIBED_MOTION_SET in order to apply the tensile load. Mesh sizes of $0.75 \times 0.75 \times 3 \mathrm{~mm}, 0.75 \times 0.75 \times 1 \mathrm{~mm}, 0.75 \times 0.5 \times 1 \mathrm{~mm}$ and $0.5 \times 0.5 \times 1 \mathrm{~mm}$ were investigated. For the anchor diameter of $6.4 \mathrm{~mm}$, mesh sizes of $0.75 \times 0.5 \times 1 \mathrm{~mm}$ and $0.5 \times 0.5 \times 1 \mathrm{~mm}$ give a percentage difference of $3.7 \%$ and $3.3 \%$ in the ultimate tensile load respectively compared to the experimental results. Less computational time is obtained using mesh size of $0.75 \times 0.5 \times 1 \mathrm{~mm}$ than that obtained using mesh size of $0.5 \times 0.5 \times 1 \mathrm{~mm}$ and hence was selected for the analysis. Table $5-1$ shows the results of the mesh sensitivity analysis for the adhesive anchor models.

Table 5-1: Effect of mesh size refinement on the convergence of ultimate tensile load for adhesive anchor

\begin{tabular}{|c|c|c|c|c|c|c|c|c|c|c|}
\hline \multirow[t]{3}{*}{$\begin{array}{l}\text { Model } \\
\text { No. }\end{array}$} & \multirow[t]{3}{*}{$\begin{array}{c}\mathrm{d} \\
(\mathrm{mm})\end{array}$} & \multicolumn{4}{|c|}{$\begin{array}{l}\text { Mesh size } \\
\quad(\mathrm{mm})\end{array}$} & \multicolumn{2}{|c|}{$\begin{array}{c}\text { Ultimate tensile } \\
\text { load } \\
(\mathrm{kN}) \\
\end{array}$} & \multicolumn{2}{|c|}{$\begin{array}{l}\text { Displacement } \\
(\mathrm{mm})\end{array}$} & \multirow[t]{3}{*}{$\begin{array}{l}\text { Failure } \\
\text { mode }\end{array}$} \\
\hline & & \multirow{2}{*}{ Anchor } & \multirow{2}{*}{ Adhesive } & \multicolumn{2}{|c|}{ Concrete } & \multirow{2}{*}{ FEA } & \multirow{2}{*}{ EXP. } & \multirow{2}{*}{ FEA } & \multirow{2}{*}{ EXP. } & \\
\hline & & & & Min. & Max. & & & & & \\
\hline 1 & \multirow{4}{*}{6.4} & 0.75 & 0.75 & 3 & 8 & 29.50 & \multirow{4}{*}{34.37} & 0.83 & \multirow{4}{*}{0.99} & $\begin{array}{c}\text { Steel } \\
\text { failure }\end{array}$ \\
\hline 2 & & 0.75 & 0.75 & 1 & 8 & 32.11 & & 0.86 & & $\begin{array}{c}\text { Steel } \\
\text { failure }\end{array}$ \\
\hline 3 & & 0.75 & 0.50 & 1 & 8 & 33.14 & & 0.89 & & $\begin{array}{c}\text { Steel } \\
\text { failure }\end{array}$ \\
\hline 4 & & 0.50 & 0.50 & 1 & 8 & 33.28 & & 0.89 & & $\begin{array}{c}\text { Steel } \\
\text { failure }\end{array}$ \\
\hline 5 & \multirow{4}{*}{9.5} & 0.75 & 0.75 & 3 & 8 & 69.55 & \multirow{4}{*}{74.53} & 0.93 & \multirow{4}{*}{0.80} & $\begin{array}{c}\text { Concrete } \\
\text { cone }\end{array}$ \\
\hline 6 & & 0.75 & 0.75 & 1 & 8 & 72.14 & & 0.91 & & $\begin{array}{c}\text { Concrete } \\
\text { cone }\end{array}$ \\
\hline 7 & & 0.75 & 0.50 & 1 & 8 & 76.71 & & 0.88 & & $\begin{array}{l}\text { Concrete } \\
\text { cone }\end{array}$ \\
\hline 8 & & 0.50 & 0.50 & 1 & 8 & 76.71 & & 0.85 & & $\begin{array}{c}\text { Concrete } \\
\text { cone }\end{array}$ \\
\hline
\end{tabular}


For the anchor diameter of $9.5 \mathrm{~mm}$, mesh sizes of $0.75 \mathrm{~mm}$ for the steel anchor, $0.5 \mathrm{~mm}$ for the adhesive and biased 1 to $8 \mathrm{~mm}$ for the concrete yielded converged results to the experimental results obtained by Braimah et al. (Braimah et al., 2004) (Table 5-1). Further decreasing the mesh size of the steel anchor to $0.5 \mathrm{~mm}$ did not result in better results however the computational time increased significantly. Hence mesh size of $0.75 \times 0.5 \times 1$ was selected for the analysis Figure 5-3 presents a comparison of load-displacement response of the experimental tests and the finite element analysis results for the 6.4-mm diameter adhesive anchor. The figure shows that as the mesh sizes decrease the loaddisplacement response approaches the experimental. The tensile load-displacement response was almost linear up to the peak load followed by a sharp drop in the load. Load fluctuation about the residual tensile load was observed. The load fluctuation can be attributed to the pullout of the anchor to a small displacement associated with concrete cracking in a small area around the anchor followed by steel anchor failure. The results obtained from the finite element analysis were in good agreement with the experimental results with the same failure mode (steel anchor failure) as shown in Figure 5-4. 


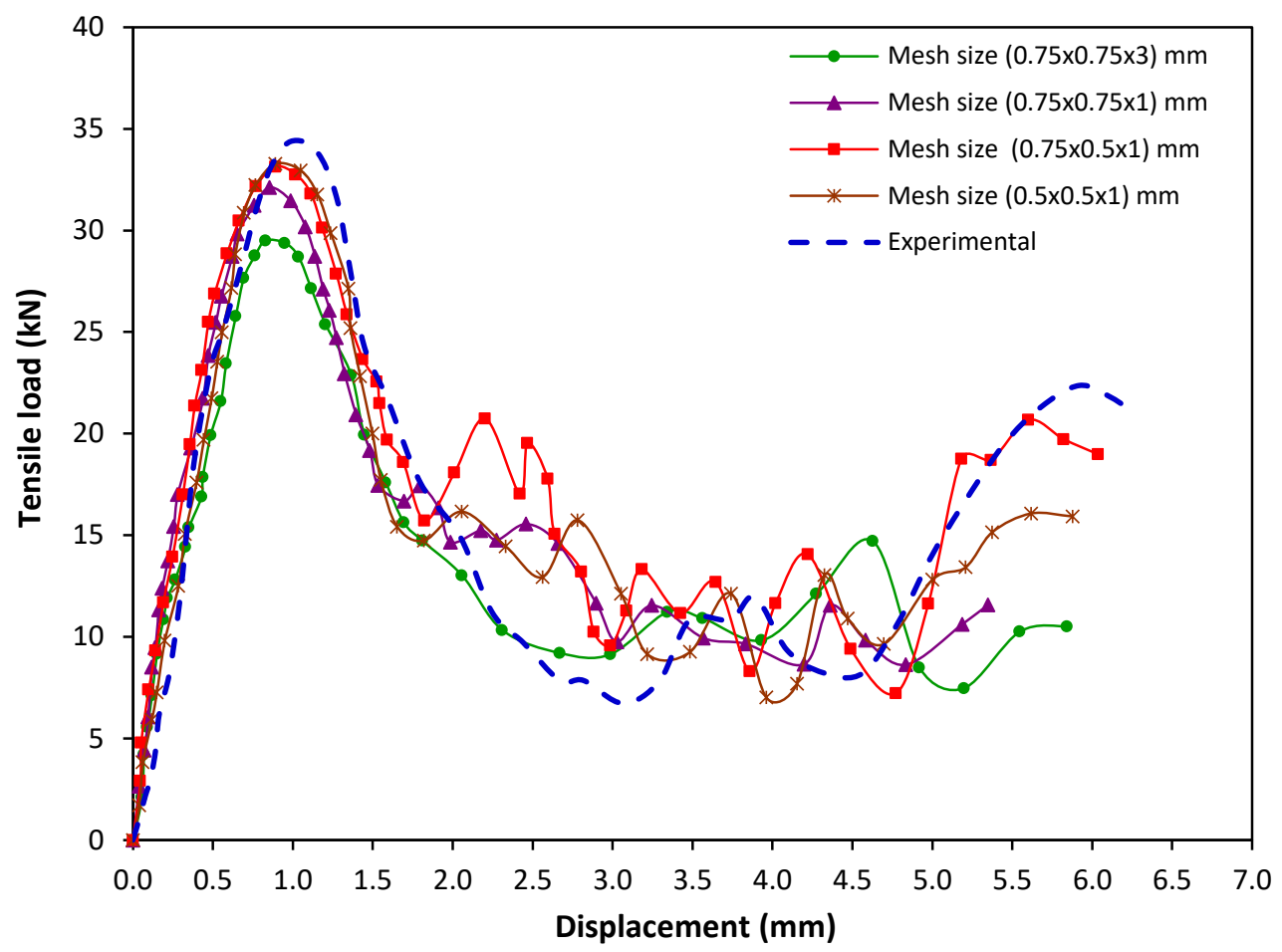

Figure 5-3: Comparison of tensile load-displacement response between FEA and experimental results obtained by Braimah et al. (Braimah et al., 2004) for anchor diameter of $6.4 \mathrm{~mm}$

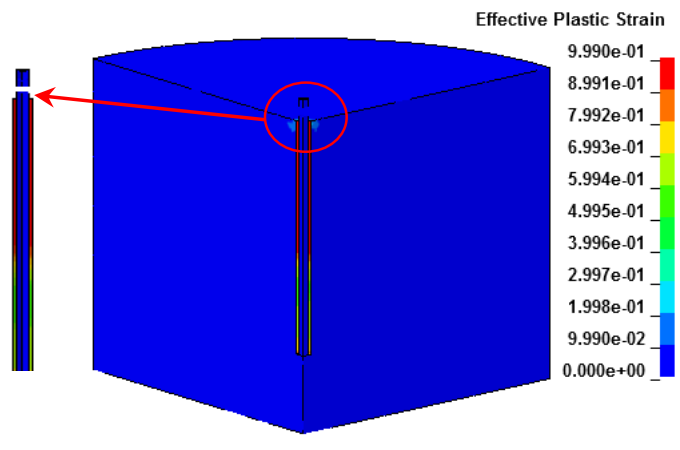

(a) FEA

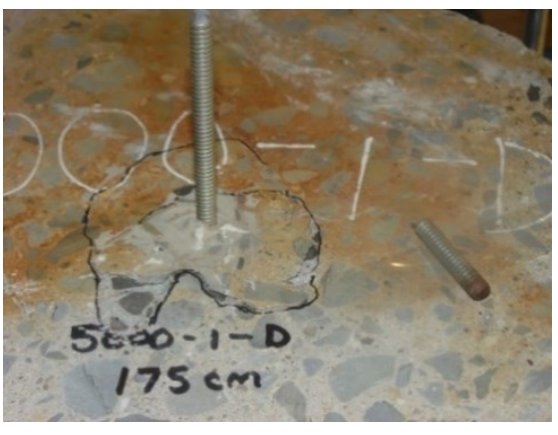

(b) Experimental ( Braimah et al., 2004)

Figure 5-4: Failure mode obtained from the finite element analysis and the experimental results obtained by Braimah et al. (Braimah et al., 2004)

Figure 5-5 presents a comparison of load-displacement response of the experimental tests and the finite element analysis results for the $9.5-\mathrm{mm}$ diameter adhesive anchor. A good agreement is obtained between the finite element analyses using mesh size of Model No. 7 
and the experimental results. Shallow concrete cone breakout failure was observed similar to that obtained from the experimental results.

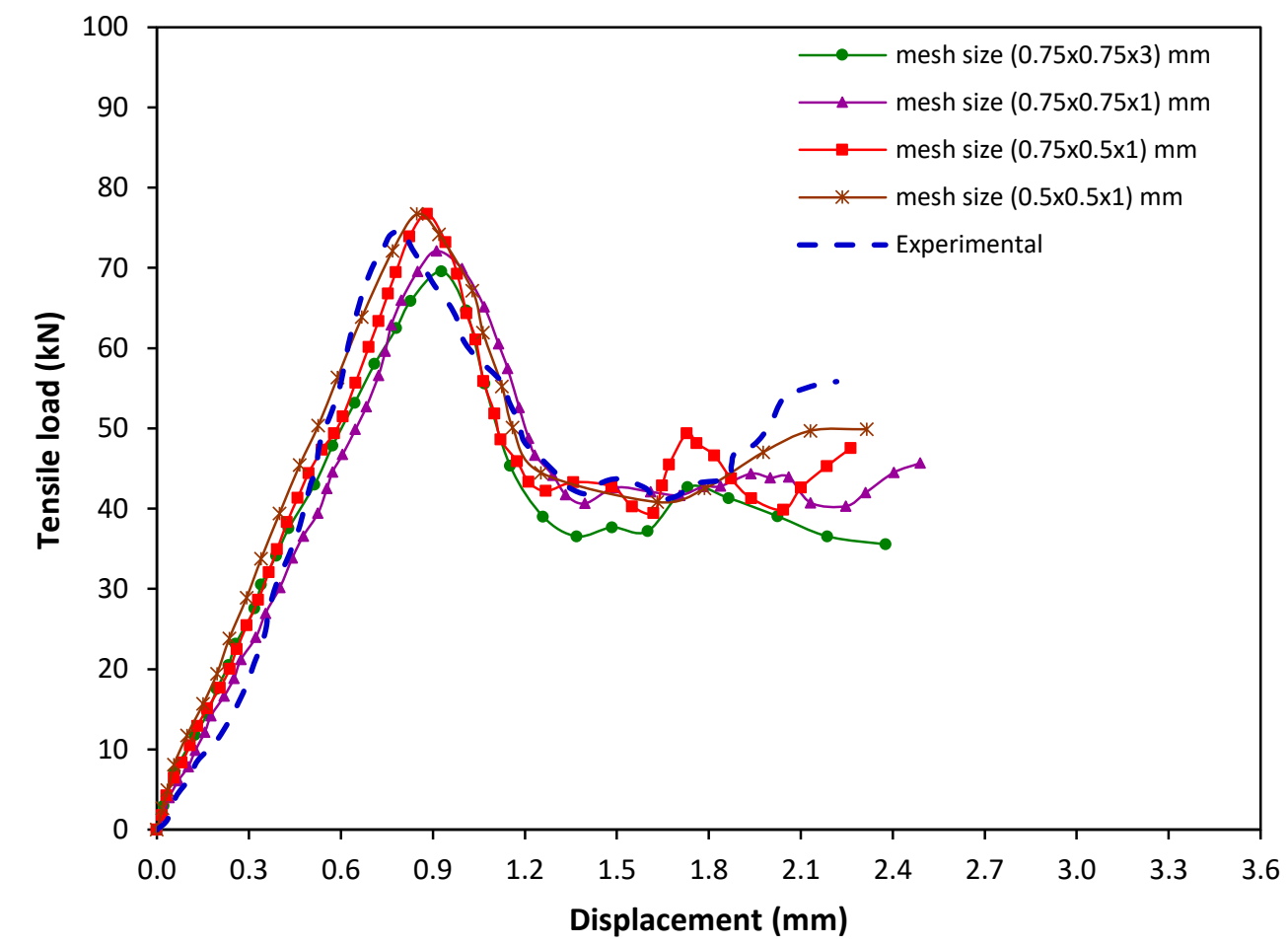

Figure 5-5: Comparison of tensile load-displacement response between FEA and experimental results obtained by Braimah et al. (Braimah et al., 2004) for anchor diameter of $9.5 \mathrm{~mm}$

After the adhesive anchor system was validated against the experimental results, the appropriate mesh size for the concrete, adhesive and steel anchor together with material models were used in a parametric analysis to investigate the effect of various design parameters (anchor diameter and embedment depth) on the capacity of the adhesive anchorage system when subjected to strain rates ranging from the static strain rate of $10^{-5}$ $\mathrm{s}^{-1}$ to higher strain rate of $10^{3} \mathrm{~s}^{-1}$. The results of the investigation are presented and discussed in the following sections. 


\subsubsection{Comparison of finite element results with $\mathrm{ACI}$ and $\mathrm{CCD}$ design methods}

Ultimate tensile loads of the adhesive anchors obtained from the finite element analysis (FEA) at the static strain rate of $10^{-5} \mathrm{~s}^{-1}$ were compared with the ACI design method (ACI 349-85) and CCD method as shown in Table 5-2. According to the ACI method (Fuchs et al., 1995), the ultimate tensile load of post-installed anchors failing by the concrete cone breakout failure mode can be determined by Equation (4.1). For the steel anchor failure, the ultimate tensile load can be determined using Equation (4.2). Hole diameters of 14.7 $\mathrm{mm}, 18 \mathrm{~mm}$, and $22 \mathrm{~mm}$ were used for the $12.7-\mathrm{mm}, 15.9-\mathrm{mm}$ and $19.1-\mathrm{mm}$ diameter adhesive anchors respectively. According to CCD design method (Committee EuroInternational du Beton (CEB), 1994; Fuchs et al., 1995), the ultimate tensile load of anchors can be determined using Equation (4.3). Minimum value of the ultimate tensile load obtained from Equations (4.1) and (4.2) is considered for comparison of ACI method with the FEA results. Also, minimum value of ultimate tensile load obtained from Equations (4.2) and (4.3) is considered for comparison of CCD method with the FEA results and included in Table 5-2.

Table 5-2: Comparison of ultimate tensile loads obtained from FEA with ACI and CCD methods

\begin{tabular}{|c|c|c|c|c|c|c|c|}
\hline $\begin{array}{c}\mathrm{d} \\
(\mathrm{mm})\end{array}$ & $\begin{array}{c}\mathrm{h}_{\mathrm{ef}} \\
(\mathrm{mm})\end{array}$ & \multicolumn{3}{|c|}{ Ultimate tensile load } & FEA/ACI & FEA/CCD & *Failure mode \\
\cline { 3 - 8 } & & ACI & CCD & FEA & & & $($ FEA $)$ \\
\hline \multirow{3}{*}{12.7} & 76.2 & 40.45 & 54.62 & 57.12 & 1.41 & 1.05 & $\mathrm{CC}$ \\
\cline { 2 - 8 } & 101.6 & 69.00 & 84.10 & 80.45 & 1.17 & 0.96 & $\mathrm{CC}$ \\
\cline { 2 - 8 } & 127.0 & 105.09 & 117.53 & 95.72 & 0.91 & 0.81 & $\mathrm{~S}$ \\
\cline { 2 - 8 } & 152.4 & 130.98 & 130.98 & 96.79 & 0.74 & 0.74 & $\mathrm{~S}$ \\
\hline 15.9 & 76.2 & 41.92 & 54.62 & 65.69 & 1.57 & 1.20 & $\mathrm{CC}$ \\
\hline
\end{tabular}




\begin{tabular}{|c|c|c|c|c|c|c|c|}
\hline \multirow{7}{*}{} & 101.6 & 70.96 & 84.10 & 87.31 & 1.23 & 1.04 & CC \\
\cline { 2 - 8 } & 127.0 & 107.53 & 117.53 & 126.18 & 1.17 & 1.07 & CCB \\
\cline { 2 - 8 } & 152.4 & 151.64 & 154.49 & 159.72 & 1.05 & 1.03 & $\mathrm{~S}$ \\
\hline \multirow{3}{*}{19.1} & 76.2 & 43.70 & 54.62 & 69.62 & 1.59 & 1.27 & $\mathrm{CC}$ \\
\cline { 2 - 8 } & 101.6 & 73.33 & 84.10 & 105.49 & 1.44 & 1.25 & $\mathrm{CC}$ \\
\cline { 2 - 8 } & 127.0 & 110.50 & 117.53 & 157.28 & 1.42 & 1.34 & $\mathrm{CC}$ \\
\cline { 2 - 8 } & 152.4 & 155.20 & 154.49 & 179.40 & 1.16 & 1.16 & $\mathrm{CC}$ \\
\hline
\end{tabular}

*Failure mode: $\mathrm{CC}=$ concrete cone breakout, $\mathrm{CCB}=$ combined cone bond, $\mathrm{S}=$ steel failure

It can be observed from Table 5-2 that the ACI and CCD methods underpredict the ultimate tensile load in comparison with the FEA results for most of the adhesive anchors as the design methods incorporate more conservatism than the FEA. However, the CCD method gives a better agreement with the finite element results. Fuchs et al. (1995) found that the CCD method agree well with the experimental results in comparison to ACI method (Fuchs et al., 1995). This is attributed to the ACI method disregard the size effect (Fuchs et al., 1995). Same material properties were used for the finite element analysis and the design codes. However, in the finite element analysis assumptions such as materials model, boundary conditions and contact formulation, were considered to develop the numerical model for the adhesive anchorage to concrete system. These assumptions may have an influence on the variation between the FEA results and the design methods results.

\subsubsection{Effect of strain rate on the level of damage and failure mode of adhesive anchors} Contours of effective plastic strain for adhesive anchor diameters of 12.7-mm, 15.9-mm and 19.1-mm with embedment depths of $76.2 \mathrm{~mm}, 101.6 \mathrm{~mm}, 127 \mathrm{~mm}$ and $152.4 \mathrm{~mm}$ at the static strain rate of $10^{-5} \mathrm{~s}^{-1}$ are shown in Figure 5-6. The crack patterns are presented 
with the plastic strain contour fringe plots in the figure. As shown in the figure, concrete cone breakout failure was observed at low strain rate of $10^{-5} \mathrm{~s}^{-1}$ for most of the adhesive anchors investigated. Cracking started to appear on the top surface of the concrete around the anchor and subsequently the cracks generated at the bottom of the anchor propagated diagonally forming concrete cone and leading to failure of the anchorage system. Also, it can be seen that the level of concrete damage increased with the increase in the anchor diameter from $12.7 \mathrm{~mm}$ to $19.1 \mathrm{~mm}$ for the same embedment depth. The increase in the anchor diameter increases the pullout load. As the anchor diameter increase the contact area between the anchor and the adhesive and between the adhesive and the concrete increases leading to increase the friction resistance (Wang et al., 2015; Wang et al., 2017).

Combined cone bond failure was observed for the 15.9-mm diameter anchor at embedment depth of $127 \mathrm{~mm}$; wherein a shallow cone was observed at the top of the concrete accompanied by adhesive bond failure at the remaining part of the embedment depth below the shallow concrete cone. Steel anchor failure was observed for the 12.7-mm diameter adhesive anchor at embedment depths of $127 \mathrm{~mm}$ and $152.4 \mathrm{~mm}$. Also, steel anchor failure was observed for the 15.9-mm diameter anchor at embedment depth of $152.4 \mathrm{~mm}$.

\begin{tabular}{|c|c|c|c|}
\hline $\mathrm{d}=12.7 \mathrm{~mm}$ & $\mathrm{~d}=15.9 \mathrm{~mm}$ & $\mathrm{~d}=19.1 \mathrm{~mm}$ & \\
\hline & & & 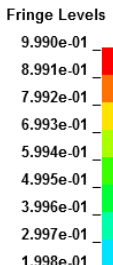 \\
\hline \multicolumn{3}{|c|}{$\mathrm{h}_{\mathrm{ef}}=76.2 \mathrm{~mm}$} & $\begin{array}{c}9.9900-02 \\
0.000 \mathrm{e}+00\end{array}$ \\
\hline
\end{tabular}




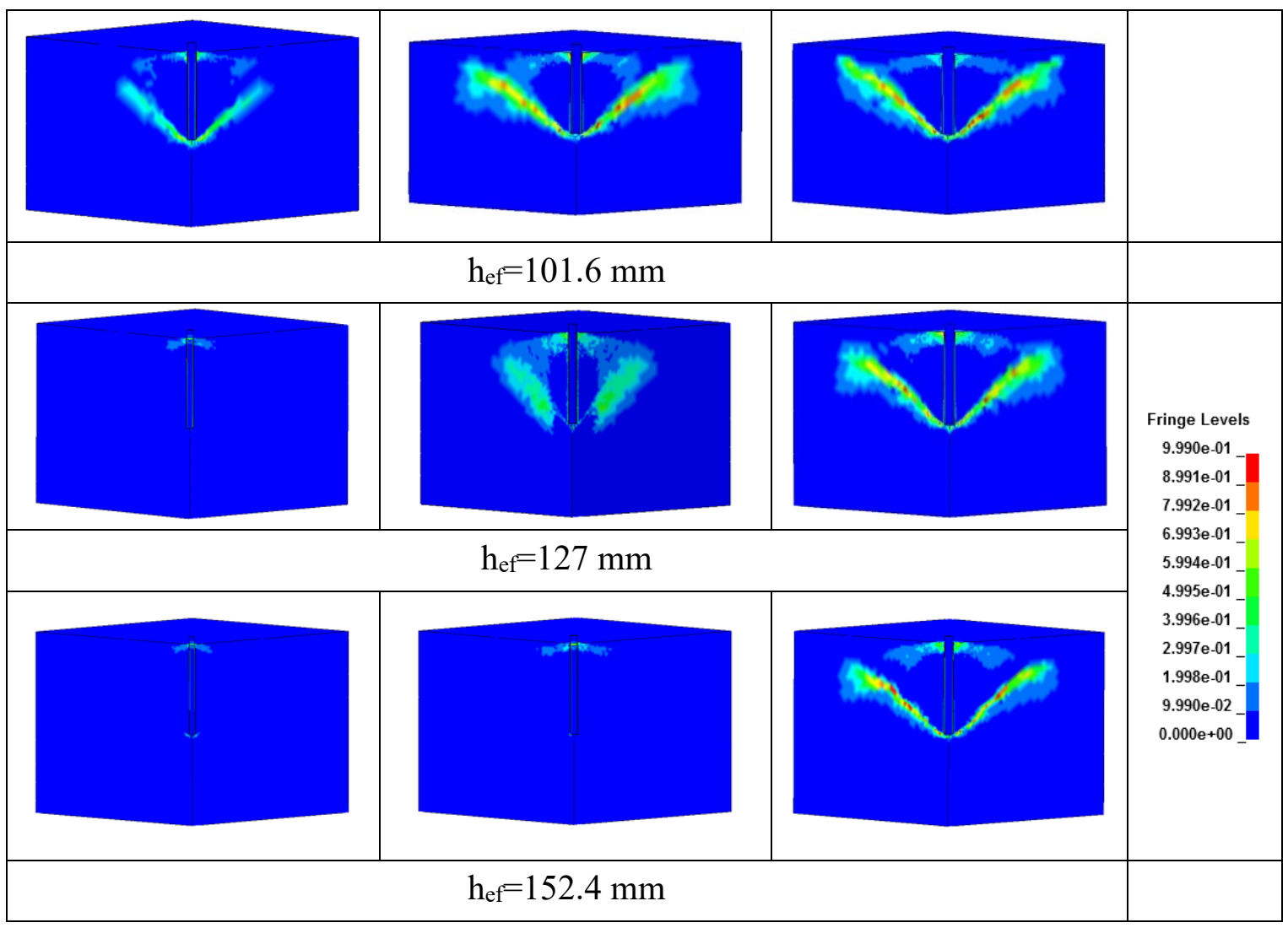

Figure 5-6: Plastic strain contours for adhesive anchor with different anchor diameters and embedment depths at strain rate of $10^{-5} \mathrm{~s}^{-1}$

Table 5-3 shows the concrete cone breakout diameter $\left(d_{\text {cone }}\right)$, cone breakout angle $\left(\theta_{a}\right)$ and corresponding failure mode of the adhesive anchor under tensile load. The increase in the embedment depth resulted in increase in the concrete cone diameter. The concrete cone breakout diameter for the 12.7- $\mathrm{mm}$ diameter adhesive anchor increases from $225 \mathrm{~mm}$ at embedment depth of $76.2 \mathrm{~mm}$ to $268 \mathrm{~mm}$ at embedment depth of $101.6 \mathrm{~mm}$. Similarly, the concrete cone breakout diameter for the 15.9-mm anchor increased from $236 \mathrm{~mm}$ at embedment depth of $76.2 \mathrm{~mm}$ to $291 \mathrm{~mm}$ at embedment depth of $101.6 \mathrm{~mm}$. Further increase in the anchor embedment depth resulted in steel anchor failure of the $12.7-\mathrm{mm}$ diameter adhesive anchor and combined concrete cone bond failure for 15.9-mm diameter adhesive anchor, as shown in Figure 5-6. The increase in the concrete cone breakout 
diameter was from $244 \mathrm{~mm}$ to $421 \mathrm{~mm}$ for the 19.1- $\mathrm{mm}$ diameter adhesive anchor for the increase in the embedment depth from $76.2 \mathrm{~mm}$ to $152.4 \mathrm{~mm}$.

Concrete cone breakout angle was determined by measuring the angle of inclination of the failure surface. Figure 5-7 shows a sketch of the concrete cone breakout angle. As shown in Table 5-3, the concrete cone breakout angle $\left(\theta_{a}\right)$ varies between $32^{\circ}$ and $35^{\circ}$ for all anchors investigated. The average concrete cone angle was $33.5^{\circ} \mathrm{mm}$. Crack propagation angle of $56.5^{\circ}$ was obtained where the crack propagation angle is equal to $\left(90-\theta_{a}\right)$. According to ACI and CCD design methods, the concrete cone angle is $45^{\circ}$ and $35^{\circ}$ respectively. The concrete cone angle obtained from the finite element analysis is approximately comparable to the concrete cone breakout angle of the CCD method. The concrete cone angle has an influence on the ultimate tensile load where the decrease in the concrete cone angle increases the concrete cone area and hence increased ultimate tensile load.

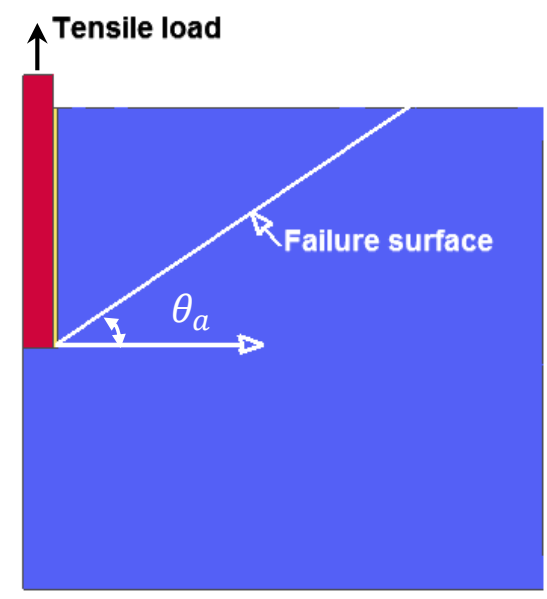

Figure 5-7: Cone breakout angle on the adhesive anchorage to concrete system. 
Table 5-3: Concrete cone diameter and cone breakout angle for the adhesive anchor

\begin{tabular}{|c|c|c|c|c|}
\hline $\begin{array}{c}\mathrm{h}_{\text {ef }} \\
(\mathrm{mm})\end{array}$ & $\begin{array}{c}\mathrm{d} \\
(\mathrm{mm})\end{array}$ & $\begin{array}{c}\mathrm{d}_{\text {cone }} \\
(\mathrm{mm})\end{array}$ & $\begin{array}{c}\theta_{a} \\
(\mathrm{deg})\end{array}$ & Failure mode \\
\hline \multirow{4}{*}{76.2} & 12.7 & 225 & 33 & $\mathrm{CC}$ \\
\cline { 2 - 5 } & 15.9 & 236 & 33 & $\mathrm{CC}$ \\
\cline { 2 - 5 } & 19.1 & 244 & 35 & $\mathrm{CC}$ \\
\hline \multirow{3}{*}{101.6} & 12.7 & 268 & 34 & $\mathrm{CC}$ \\
\cline { 2 - 5 } & 15.9 & 291 & 34 & $\mathrm{CC}$ \\
\cline { 2 - 5 } & 19.1 & 295 & 33 & $\mathrm{CC}$ \\
\hline \multirow{3}{*}{127.0} & 12.7 & - & - & $\mathrm{C}$ \\
\cline { 2 - 5 } & 15.9 & - & - & $\mathrm{CC}$ \\
\cline { 2 - 5 } & 19.1 & 370 & - & $\mathrm{S}$ \\
\hline \multirow{3}{*}{152.4} & 12.7 & - & - & $\mathrm{CC}$ \\
\cline { 2 - 5 } & 15.9 & - & 32 & \\
\cline { 2 - 5 } & 19.1 & 421 & & $\mathrm{~S}$ \\
\hline
\end{tabular}

Figures 5-8, 5-9 and 5-10 show the failure mode of 12.7-mm, 15.9-mm and 19.1-mm diameter adhesive anchors at strain rates ranging from $10^{-3} \mathrm{~s}^{-1}$ to $10^{3} \mathrm{~s}^{-1}$. As shown in Figure 5-8 for the 12.7-mm diameter adhesive anchor, combined cone bond failure was observed for the $76.2 \mathrm{~mm}$ and $101.6 \mathrm{~mm}$ embedment depths at strain rates of $10^{-3} \mathrm{~s}^{-1}$ to $10^{2} \mathrm{~s}^{-1}$. Steel anchor failure was observed for the $127 \mathrm{~mm}$ and $152.4 \mathrm{~mm}$ embedment depths at all the strain rates investigated. As shown in Figure 5-9 for the 15.9-mm diameter adhesive anchor, combined cone bond failure was observed at embedment depths of $76.2 \mathrm{~mm}, 101.6$ $\mathrm{mm}$ and $127 \mathrm{~mm}$ at strain rates of $10^{-3} \mathrm{~s}^{-1}$ to $10^{2} \mathrm{~s}^{-1}$; whereas steel anchor failure was observed at embedment depth of $152.4 \mathrm{~mm}$ at strain rates ranging from $10^{-3} \mathrm{~s}^{-1}$ to $10^{3} \mathrm{~s}^{-1}$. Also, steel anchor failure was observed at strain rate of $10^{3} \mathrm{~s}^{-1}$ for the embedment depths of $76.2 \mathrm{~mm}$ and $101.6 \mathrm{~mm}$. As shown in Figure 5-10 for the 19.1-mm diameter adhesive 
anchor at embedment depth of $76.2 \mathrm{~mm}$, concrete cone breakout failure is observed at strain rate of $10^{-3} \mathrm{~s}^{-1}$. The increase in the strain rate to $10^{2} \mathrm{~s}^{-1}$ resulted in combined cone-bond failure. For the anchor embedment depths of $101.6 \mathrm{~mm}, 127 \mathrm{~mm}$ and $152.4 \mathrm{~mm}$, combined cone-bond failure mode was observed at strain rates of $10^{-3} \mathrm{~s}^{-1}$ to $10^{2} \mathrm{~s}^{-1}$. At high strain rate of $10^{3} \mathrm{~s}^{-1}$ steel anchor failure was observed for all the anchor diameters and embedment depths investigated.

It is clear from Figures 5-8, 5-9 and 5-10 that the strain rate has an influence on the failure mode. The failure mode is observed to transition from concrete cone or combined conebond failure mode to steel anchor failure mode with increase in strain rate. This behaviour can be attributed to the increase in concrete and steel capacity with increase in strain rate. The increase in the tensile capacity of the concrete is higher than the increase in the steel capacity (Malvar \& Crawford, 1998; Malvar \& Ross, 1998). Hence, the concrete resistance to the tensile load at high strain rate increase resulted in steel anchor failure. 


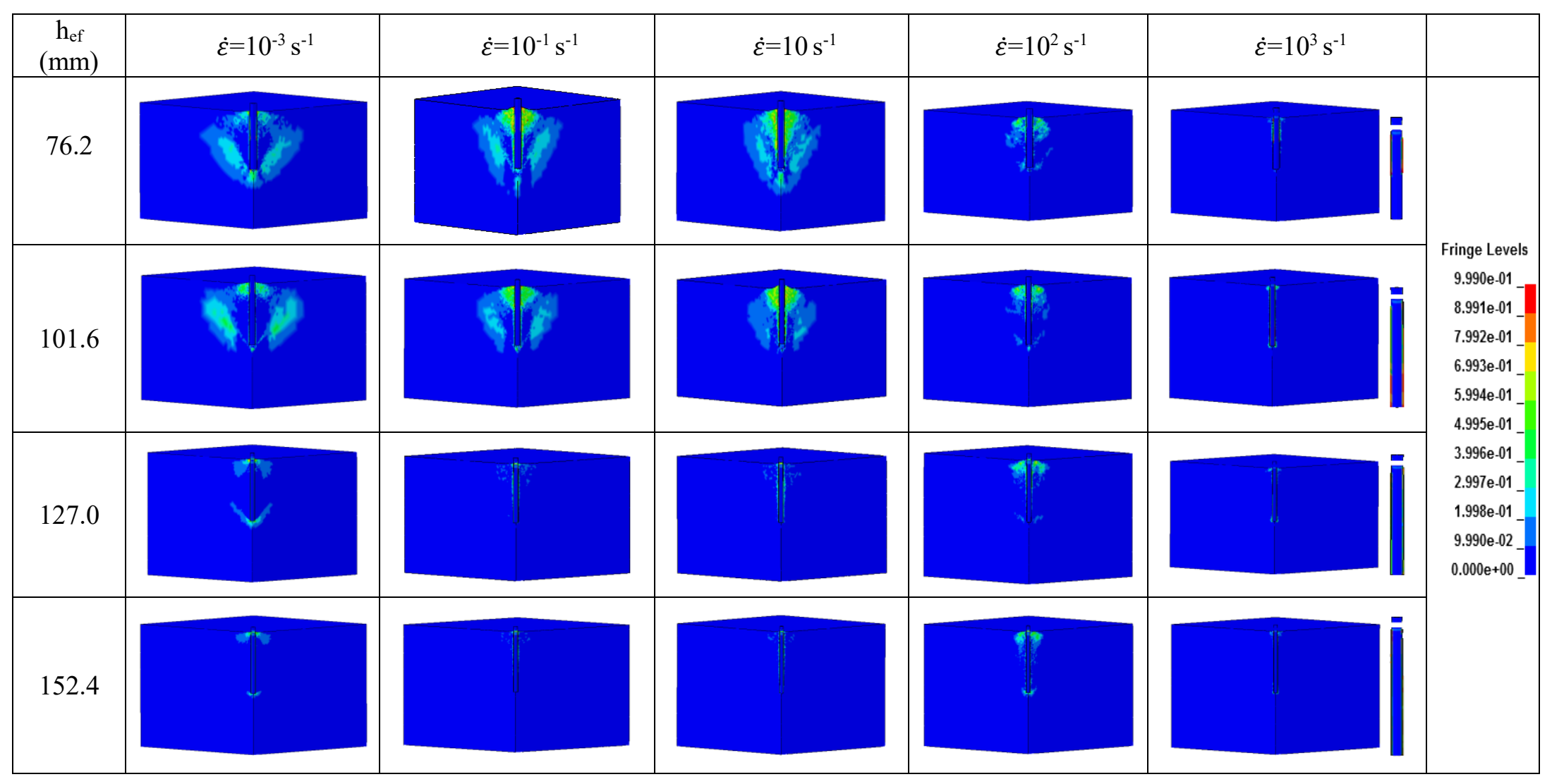

Figure 5-8: Failure mode of 12.7-mm diameter adhesive anchor at different strain rates 


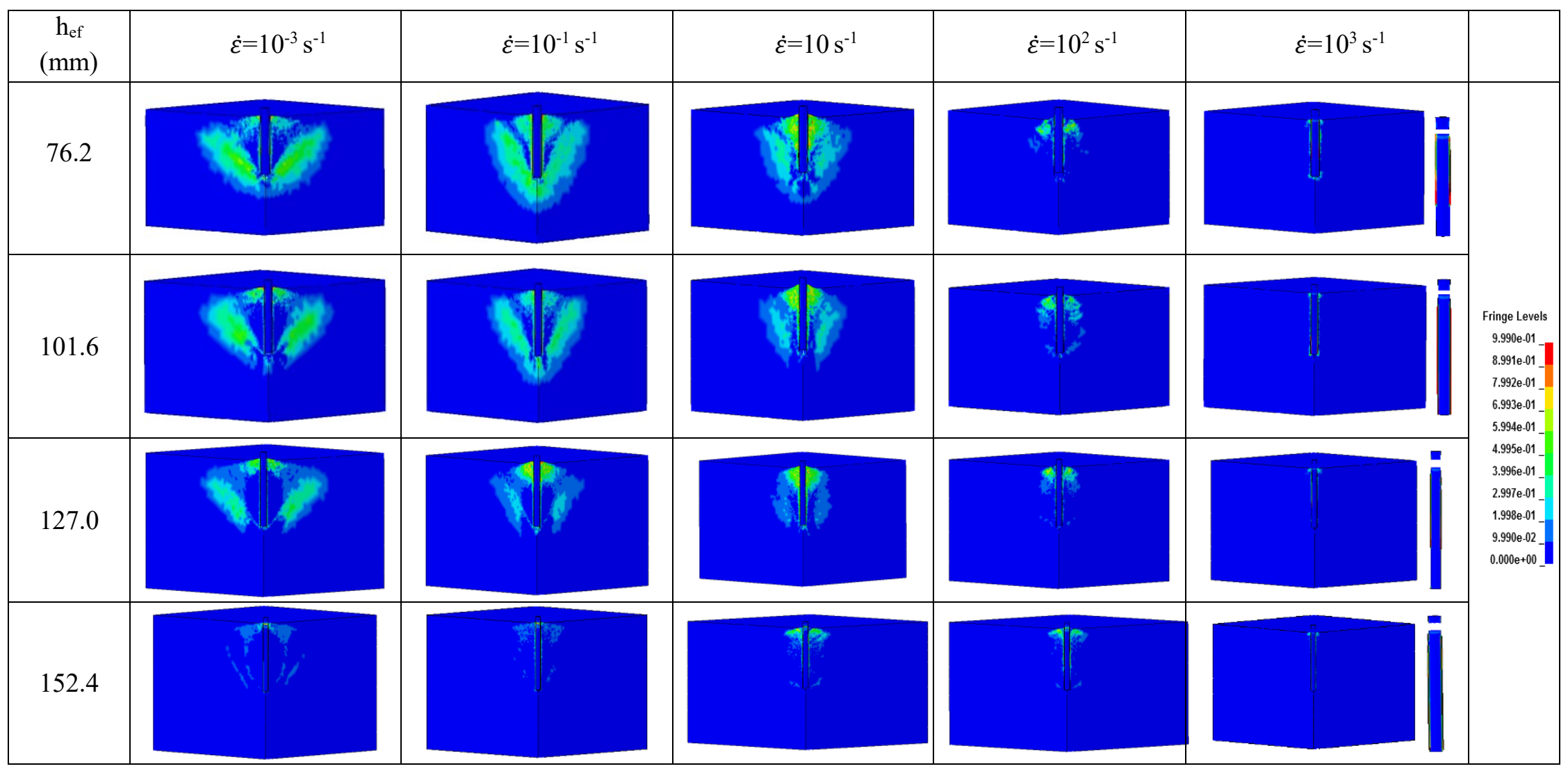

Figure 5-9: Failure mode of 15.9-mm diameter adhesive anchor at different strain rates 


\begin{tabular}{|c|c|c|c|c|c|c|}
\hline $\begin{array}{c}\mathrm{h}_{\mathrm{ef}} \\
(\mathrm{mm})\end{array}$ & $\dot{\varepsilon}=10^{-3} \mathrm{~s}^{-1}$ & $\dot{\varepsilon}=10^{-1} \mathrm{~s}^{-1}$ & $\dot{\varepsilon}=10 \mathrm{~s}^{-1}$ & $\dot{\varepsilon}=10^{2} \mathrm{~s}^{-1}$ & $\dot{\varepsilon}=10^{3} \mathrm{~s}^{-1}$ & \\
\hline 76.2 & & & & 40 & 15 & \\
\hline 101.6 & & & & - & & $\begin{array}{l}9.9900-01 \\
8.991-01\end{array}$ \\
\hline 127.0 & & & & 4 & & $\begin{array}{l}2.998 .01 \\
1.91\end{array}$ \\
\hline 152.4 & & & & & & \\
\hline
\end{tabular}

Figure 5-10: Failure mode of 19.1-mm diameter adhesive anchor at different strain rates 


\subsubsection{Effect of design parameters on failure mode and ultimate tensile load}

Figures 5-11, 5-12 and 5-13 show the effect of strain rate on the failure mode and ultimate tensile load for the adhesive anchor. As shown in Figure 5-11 the tensile load increased from $57.1 \mathrm{kN}$ to $107.2 \mathrm{kN}$ and $80.5 \mathrm{kN}$ to $107.9 \mathrm{kN}$ for the anchor embedment depths of $76.2 \mathrm{~mm}$ and $101.6 \mathrm{~mm}$ respectively, where the failure mode transitioned from concrete cone breakout at strain rate of $10^{-5} \mathrm{~s}^{-1}$ to combined cone bond failure at strain rates up to $10^{2} \mathrm{~s}^{-1}$ and steel anchor failure at strain rate of $10^{3} \mathrm{~s}^{-1}$. The ultimate tensile load increased $13.3 \%$ and $12.5 \%$ for the embedment depths of $127 \mathrm{~mm}$ and $152.4 \mathrm{~mm}$ respectively as the strain rate increased from $10^{-5} \mathrm{~s}^{-1}$ to $10^{3} \mathrm{~s}^{-1} \mathrm{~mm}$ where steel anchor failure is the dominant failure mode at all the strain rates.

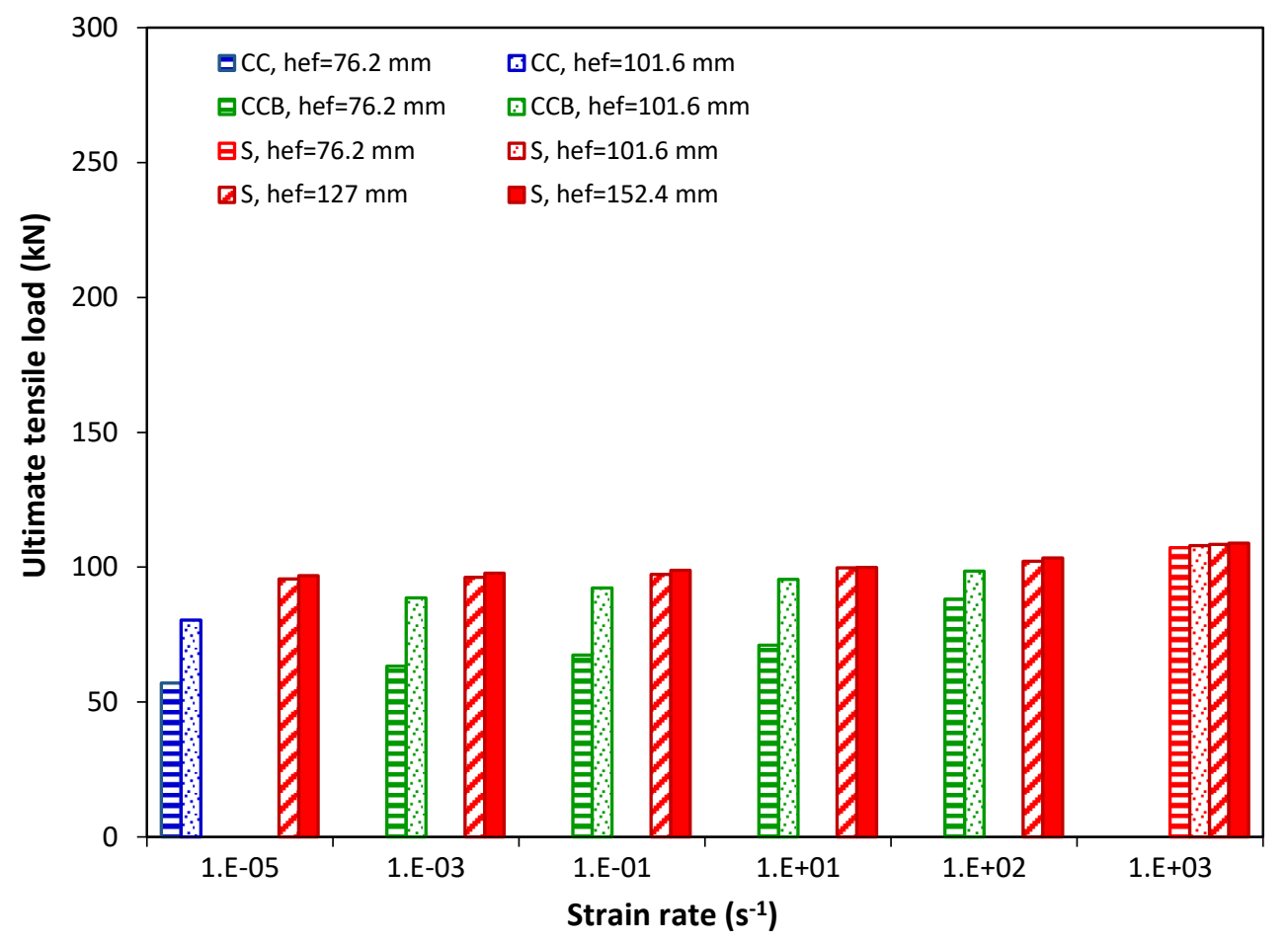

Figure 5-11: Strain rate effect on the failure mode and ultimate tensile load for adhesive anchor diameter of $12.7 \mathrm{~mm}$ 
As shown in Figure 5-12, the ultimate tensile load increased with the increase in the strain rate. For the embedment depths of $76.2 \mathrm{~mm}$ and $101.6 \mathrm{~mm}$, the failure mode transitioned from concrete cone breakout at strain rate of $10^{-5} \mathrm{~s}^{-1}$ to combined cone bond failure at strain rates up to $10^{2} \mathrm{~s}^{-1}$ and steel anchor failure at strain rate of $10^{3} \mathrm{~s}^{-1}$. For anchor embedment depth of $127 \mathrm{~mm}$, the failure mode transitioned from combined cone bond failure at strain rates up to $10^{2} \mathrm{~s}^{-1}$ to steel anchor failure at strain rate of $10^{3} \mathrm{~s}^{-1}$. For anchor embedment depth of $152.4 \mathrm{~mm}$, the ultimate tensile load increased $11.8 \%$ where steel anchor failure was observed at all strain rates investigated.

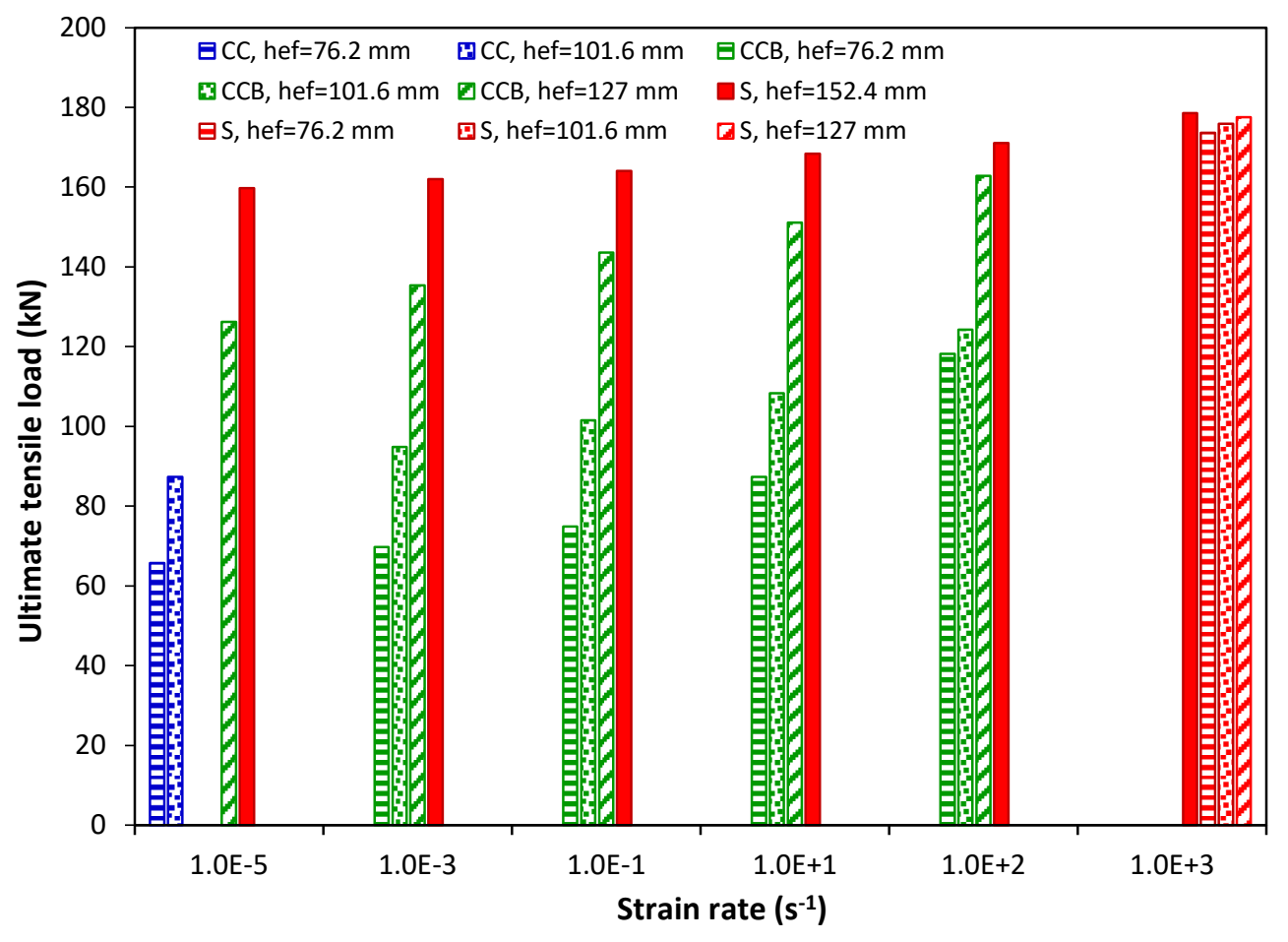

Figure 5-12: Strain rate effect on the failure mode and ultimate tensile load for adhesive anchor diameter of $15.9 \mathrm{~mm}$

As shown in Figure 5-13, the ultimate tensile load increased with the increase in the strain rates for all the embedment depths investigated. For the $76.2 \mathrm{~mm}, 101.6 \mathrm{~mm}, 127 \mathrm{~mm}$ and 
$152.4 \mathrm{~mm}$ embedment depths, the failure mode transitioned from concrete cone breakout at strain rate of $10^{-5} \mathrm{~s}^{-1}$ to combined cone bond at strain rates up to $10^{2} \mathrm{~s}^{-1}$ and then to steel anchor failure at strain rate of $10^{3} \mathrm{~s}^{-1}$. For the adhesive anchor of $76.2 \mathrm{~mm}$ embedment depth concrete cone breakout failure was observed at strain rate of $10^{-3} \mathrm{~s}^{-1}$.

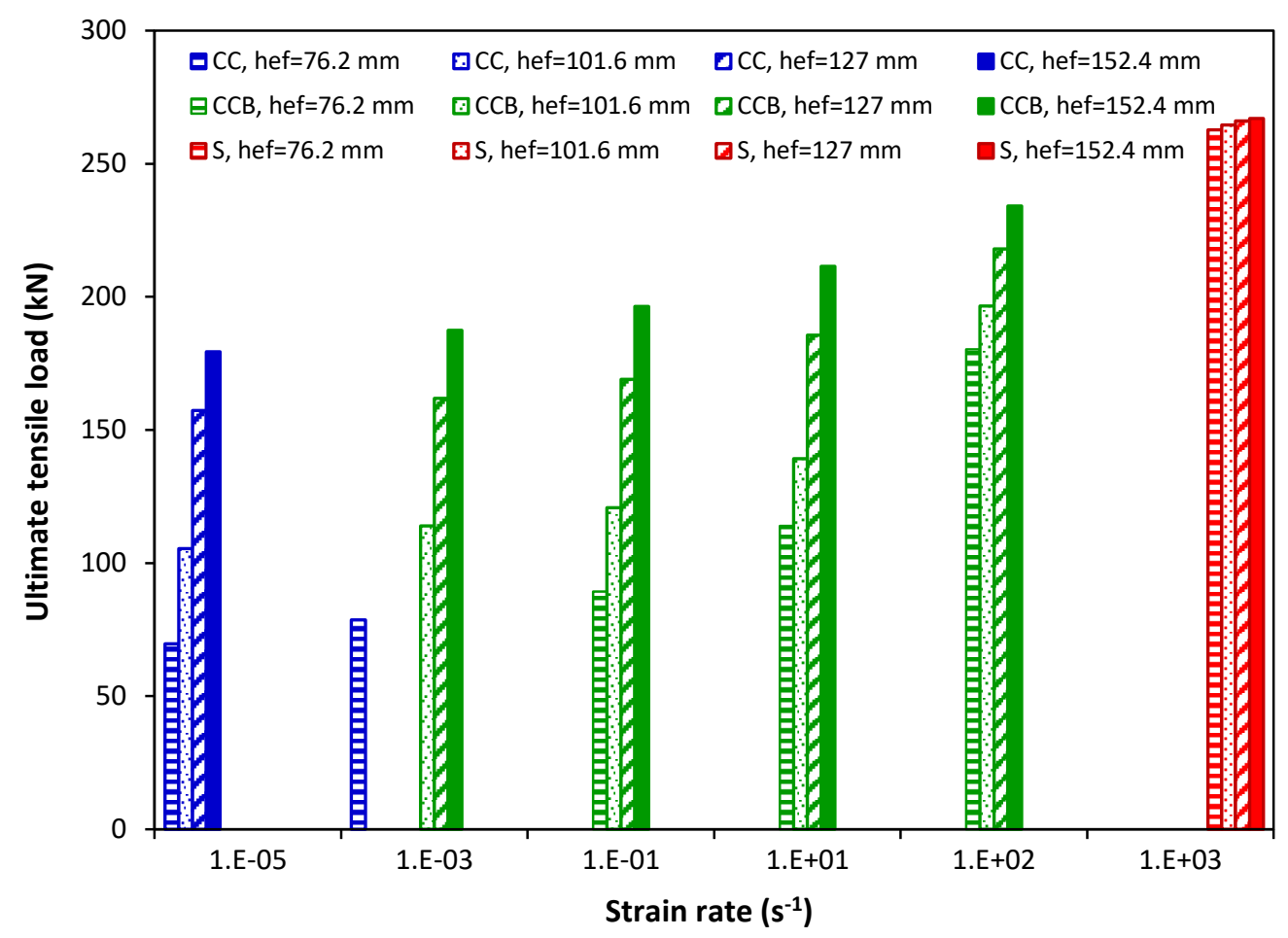

Figure 5-13: Strain rate effect on the failure mode and ultimate tensile load for adhesive anchor diameter of $19.1 \mathrm{~mm}$

It can be seen from Figures 5-11, 5-12 and 5-13, that the failure mode of the adhesive anchor is affected by the strain rate. Also, it can be seen from the figures that, the failure mode is influenced by the embedment depth and anchor diameter. The increase in the embedment depth from $76.2 \mathrm{~mm}$ to $152.4 \mathrm{~mm}$ resulted in steel anchor failure for the anchor diameter of $12.7 \mathrm{~mm}$ at low strain rate of $10^{-5} \mathrm{~s}^{-1}$. The increase in anchor diameter from $12.7 \mathrm{~mm}$ to $19.1 \mathrm{~mm}$ changed the failure mode from steel anchor failure to concrete cone breakout for the embedment depths of $127 \mathrm{~mm}$ and $152.4 \mathrm{~mm}$ at strain rate of $10^{-5} \mathrm{~s}^{-1}$ and 
from steel failure to combined cone bond failure at strain rates of $10^{-3} \mathrm{~s}^{-1}, 10^{-1} \mathrm{~s}^{-1}, 10 \mathrm{~s}^{-1}$ and $10^{2} \mathrm{~s}^{-1}$ for the same embedment depths. Also, it can be seen from the figures that the ultimate tensile load increased with the increase in the strain rate and embedment depth. The failure modes for the adhesive anchors under tensile load at different strain rates are summarized as in Table 5-4.

Table 5-4: Failure mode for adhesive anchors under tensile load at different strain rates

\begin{tabular}{|c|c|c|c|c|c|c|c|c|}
\hline Model & $\mathbf{d}$ & \multirow{2}{*}{$\begin{array}{c}\text { h } \\
\text { No. }\end{array}$} & $(\mathbf{m m})$ & $(\mathbf{m m})$ & \multicolumn{6}{|c|}{ Failure mode $^{*}$} \\
\cline { 4 - 9 } & & & $\dot{\varepsilon}=10^{-5}$ & $\dot{\varepsilon}=10^{-3}$ & $\dot{\varepsilon}=10^{-1}$ & $\dot{\varepsilon}=10$ & $\dot{\varepsilon}=10^{2}$ & $\dot{\varepsilon}=10^{3}$ \\
\hline 1 & 12.7 & 76.2 & $\mathrm{CC}$ & $\mathrm{CCB}$ & $\mathrm{CCB}$ & $\mathrm{CCB}$ & $\mathrm{CCB}$ & $\mathrm{S}$ \\
\hline 2 & 12.7 & 101.6 & $\mathrm{CC}$ & $\mathrm{CCB}$ & $\mathrm{CCB}$ & $\mathrm{CCB}$ & $\mathrm{CCB}$ & $\mathrm{S}$ \\
\hline 3 & 12.7 & 127.0 & $\mathrm{~S}$ & $\mathrm{~S}$ & $\mathrm{~S}$ & $\mathrm{~S}$ & $\mathrm{~S}$ & $\mathrm{~S}$ \\
\hline 4 & 12.7 & 152.4 & $\mathrm{~S}$ & $\mathrm{~S}$ & $\mathrm{~S}$ & $\mathrm{~S}$ & $\mathrm{~S}$ & $\mathrm{~S}$ \\
\hline 5 & 15.9 & 76.2 & $\mathrm{CC}$ & $\mathrm{CCB}$ & $\mathrm{CCB}$ & $\mathrm{CCB}$ & $\mathrm{CCB}$ & $\mathrm{S}$ \\
\hline 6 & 15.9 & 101.6 & $\mathrm{CC}$ & $\mathrm{CCB}$ & $\mathrm{CCB}$ & $\mathrm{CCB}$ & $\mathrm{CCB}$ & $\mathrm{S}$ \\
\hline 7 & 15.9 & 127.0 & $\mathrm{CCB}$ & $\mathrm{CCB}$ & $\mathrm{CCB}$ & $\mathrm{CCB}$ & $\mathrm{CCB}$ & $\mathrm{S}$ \\
\hline 8 & 15.9 & 152.4 & $\mathrm{~S}$ & $\mathrm{~S}$ & $\mathrm{~S}$ & $\mathrm{~S}$ & $\mathrm{~S}$ & $\mathrm{~S}$ \\
\hline 9 & 19.1 & 76.2 & $\mathrm{CC}$ & $\mathrm{CC}$ & $\mathrm{CCB}$ & $\mathrm{CCB}$ & $\mathrm{CCB}$ & $\mathrm{S}$ \\
\hline 10 & 19.1 & 101.6 & $\mathrm{CC}$ & $\mathrm{CCB}$ & $\mathrm{CCB}$ & $\mathrm{CCB}$ & $\mathrm{CCB}$ & $\mathrm{S}$ \\
\hline 11 & 19.1 & 127.0 & $\mathrm{CC}$ & $\mathrm{CCB}$ & $\mathrm{CCB}$ & $\mathrm{CCB}$ & $\mathrm{CCB}$ & $\mathrm{S}$ \\
\hline 12 & 19.1 & 152.4 & $\mathrm{CC}$ & $\mathrm{CCB}$ & $\mathrm{CCB}$ & $\mathrm{CCB}$ & $\mathrm{CCB}$ & $\mathrm{S}$ \\
\hline
\end{tabular}

*Failure mode: $\mathrm{CC}=$ concrete cone breakout, $\mathrm{CCB}=$ combined cone bond, $\mathrm{S}=$ steel anchor failure

\subsubsection{Effect of anchor embedment depth on concrete cone depth}

Effect of anchor embedment depth on ultimate tensile load and concrete cone depth for the 12.7-mm, 15.9-mm and 19.1-mm diameter adhesive anchors is shown in Figures 5-14, 515 and 5-16 respectively. It can be seen from Figure 5-14 the ultimate tensile load of the 12.7-mm diameter adhesive anchor increased as the anchor embedment depth increased 
from $76.2 \mathrm{~mm}$ to $127 \mathrm{~mm}$ under tensile loading at the static strain rate. The increase in the anchor embedment depth from $76.2 \mathrm{~mm}$ to $101.6 \mathrm{~mm}$ increased the concrete cone depth and hence the failure surface area. Further increase in the embedment depth to $152.4 \mathrm{~mm}$ shows no influence on the ultimate tensile load as the ultimate load was limited by the steel anchor failure.

For the 15.9-mm diameter adhesive anchor (Figure 5-15), the increase in the embedment depth from $76.2 \mathrm{~mm}$ to $152.4 \mathrm{~mm}$ increased the ultimate tensile load from $65.7 \mathrm{kN}$ to 159.7 $\mathrm{kN}$. The concrete cone depth increased with the increase in the anchor embedment depth from $76.2 \mathrm{~mm}$ to $101.6 \mathrm{~mm}$. Combined cone bond failure was observed at embedment depth of $127 \mathrm{~mm}$ with cone depth of $40 \mathrm{~mm}$ and steel anchor failure is observed at embedment depth of $152.4 \mathrm{~mm}$. For the anchor diameter of $19.1 \mathrm{~mm}$ (Figure 5-16), it can be seen that the ultimate tensile load and concrete cone depth increased with the increase in the anchor embedment depth from $76.2 \mathrm{~mm}$ to $152.4 \mathrm{~mm}$. 


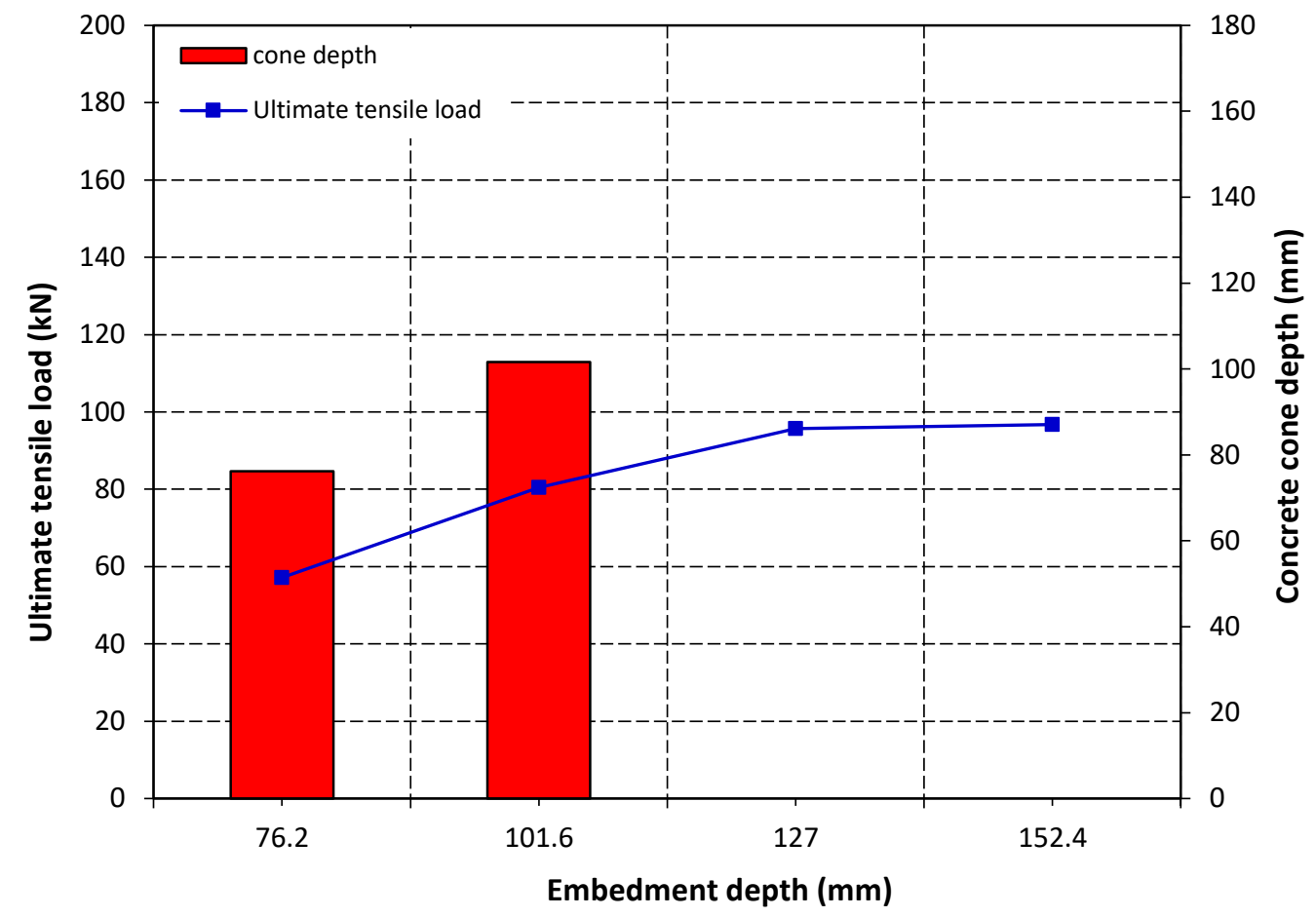

Figure 5-14: Effect of anchor embedment depth on the concrete cone depth and ultimate tensile load for $12.7 \mathrm{~mm}$ diameter adhesive anchor

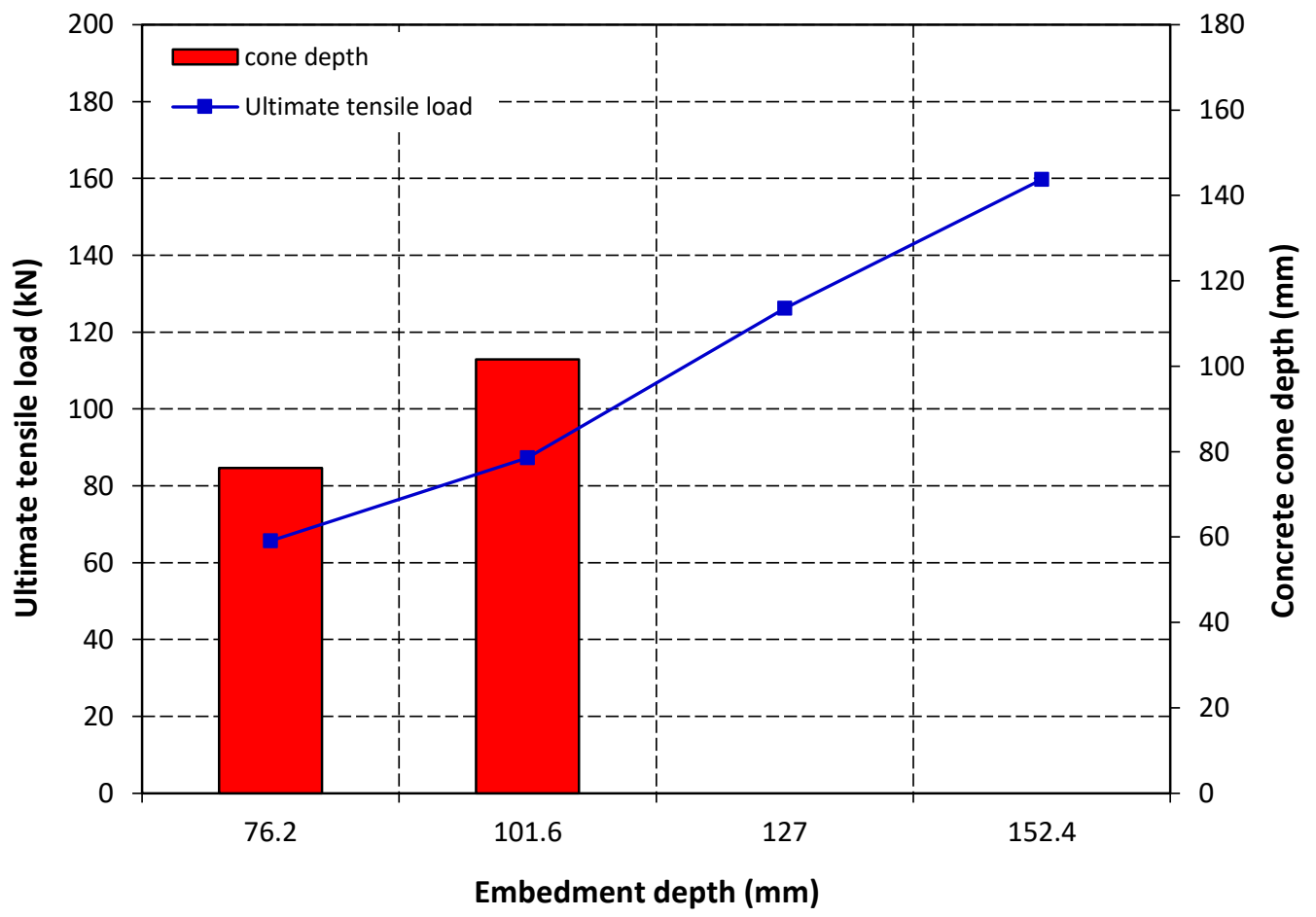

Figure 5-15: Effect of anchor embedment depth on the concrete cone depth and ultimate tensile load for $15.9 \mathrm{~mm}$ diameter adhesive anchor 


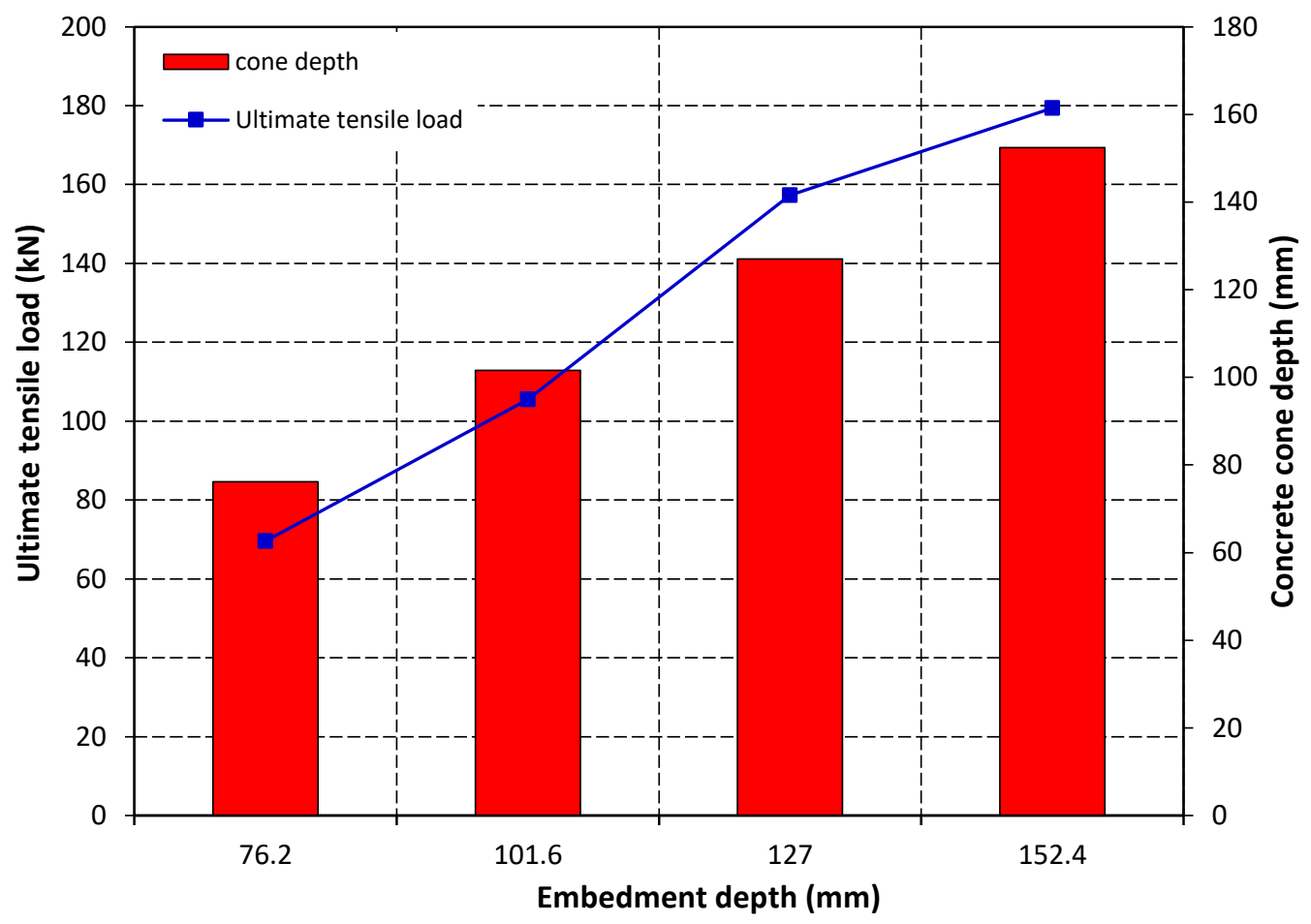

Figure 5-16: Effect of anchor embedment depth on the concrete cone depth and ultimate tensile load for $19.1 \mathrm{~mm}$ diameter adhesive anchor

\subsubsection{Concrete cone breakout diameter for adhesive anchors}

Figure 5-17 shows the displacement contours for adhesive anchors with embedment depth of $76.2 \mathrm{~mm}$ under tensile load at the static strain rate of $10^{-5} \mathrm{~s}^{-1}$. As shown in the figure, the increase in the anchor diameter from $12.7 \mathrm{~mm}$ to $19.1 \mathrm{~mm}$ increased the concrete cone breakout radius from $113 \mathrm{~mm}$ to $122 \mathrm{~mm}$.
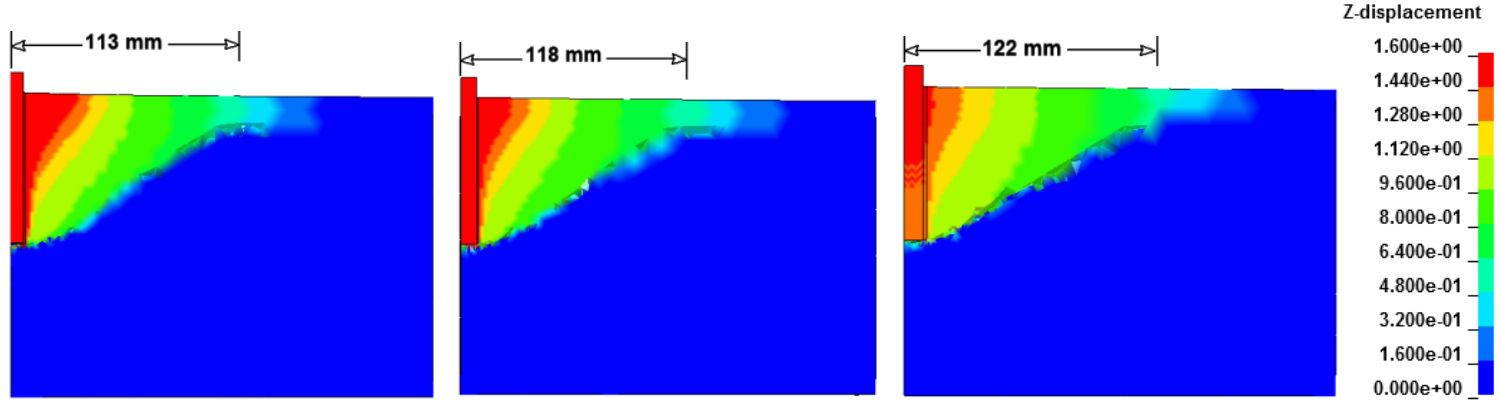

Front view 


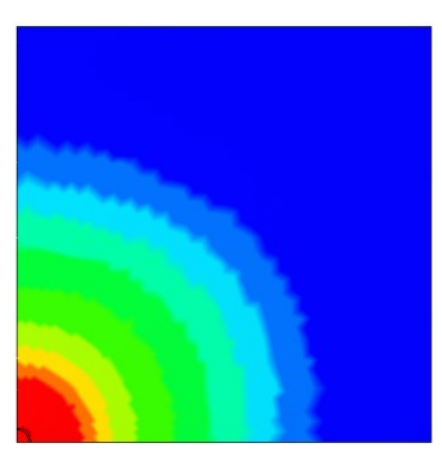

(a)

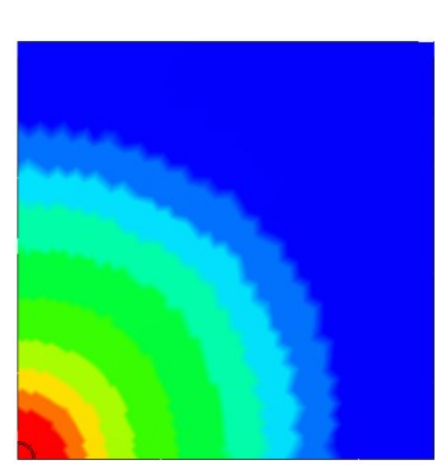

Top view

(b)

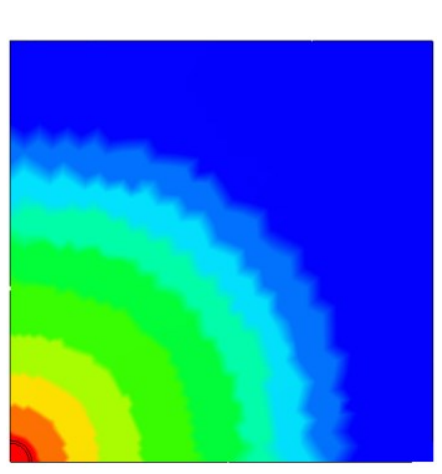

Z-displacement

$1.600 \mathrm{e}+00$

$1.440 \mathrm{e}+00$

$1.280 \mathrm{e}+00$

$1.120 \mathrm{e}+00$

$9.600 \mathrm{e}-01$

$8.000 \mathrm{e}-01$

$6.400 \mathrm{e}-01$

$4.800 \mathrm{e}-01$

$3.200 \mathrm{e}-01$

$1.600 \mathrm{e}-01$

$0.000 \mathrm{e}+00$

Figure 5-17: Displacement contours for adhesive anchors at strain rate of $10^{-5}$ with diameters of: (a) $12.7 \mathrm{~mm}$, (b) $15.9 \mathrm{~mm}$ and (c) $19.1 \mathrm{~mm}$

\subsubsection{Effect of strain rate on the tensile behaviour of adhesive anchors}

In order to investigate the tensile behaviour of the adhesive anchors, numerical models of adhesive anchorage to concrete systems were developed. Tensile capacity at different strain rates $(\dot{\varepsilon})$ was investigated for different anchor diameters and embedment depths. Figures $5-18,5-19,5-20,5-21,5-22$ and 5-23 present the load-displacement response for the 12.7$\mathrm{mm}$ diameter adhesive anchor at strain rates of $10^{-5} \mathrm{~s}^{-1}, 10^{-3} \mathrm{~s}^{-1}, 10^{-1} \mathrm{~s}^{-1}, 10 \mathrm{~s}^{-1}, 10^{2} \mathrm{~s}^{-1}$ and $10^{3} \mathrm{~s}^{-1}$ respectively. The anchor embedment depths used in the investigation were $76.2 \mathrm{~mm}$, $101.6 \mathrm{~mm}, 127 \mathrm{~mm}$ and $152.4 \mathrm{~mm}$. 


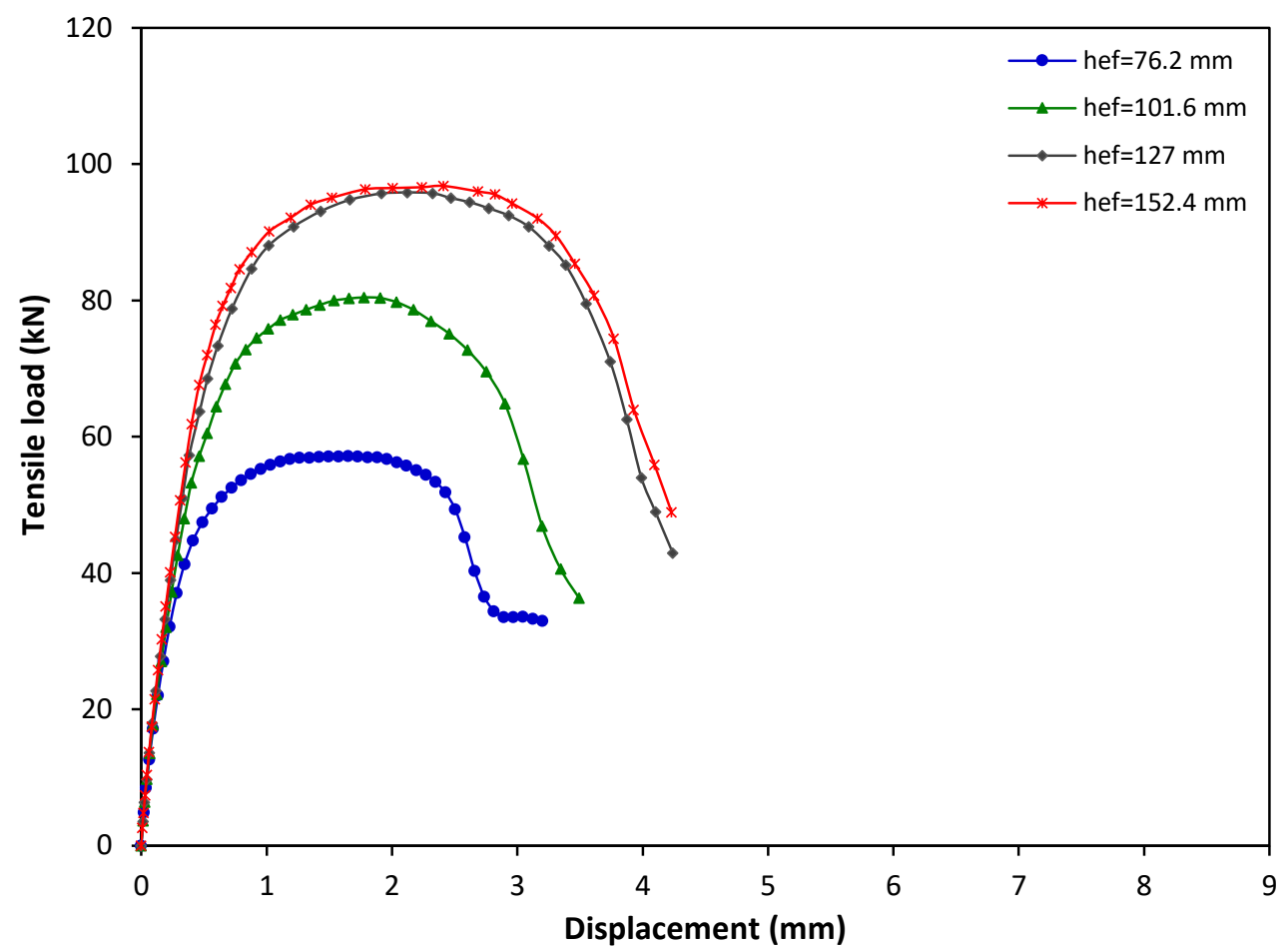

Figure 5-18: Tensile load-displacement graph for $12.7 \mathrm{~mm}$ diameter adhesive anchor at strain rate of $10^{-5} \mathrm{~s}^{-1}$

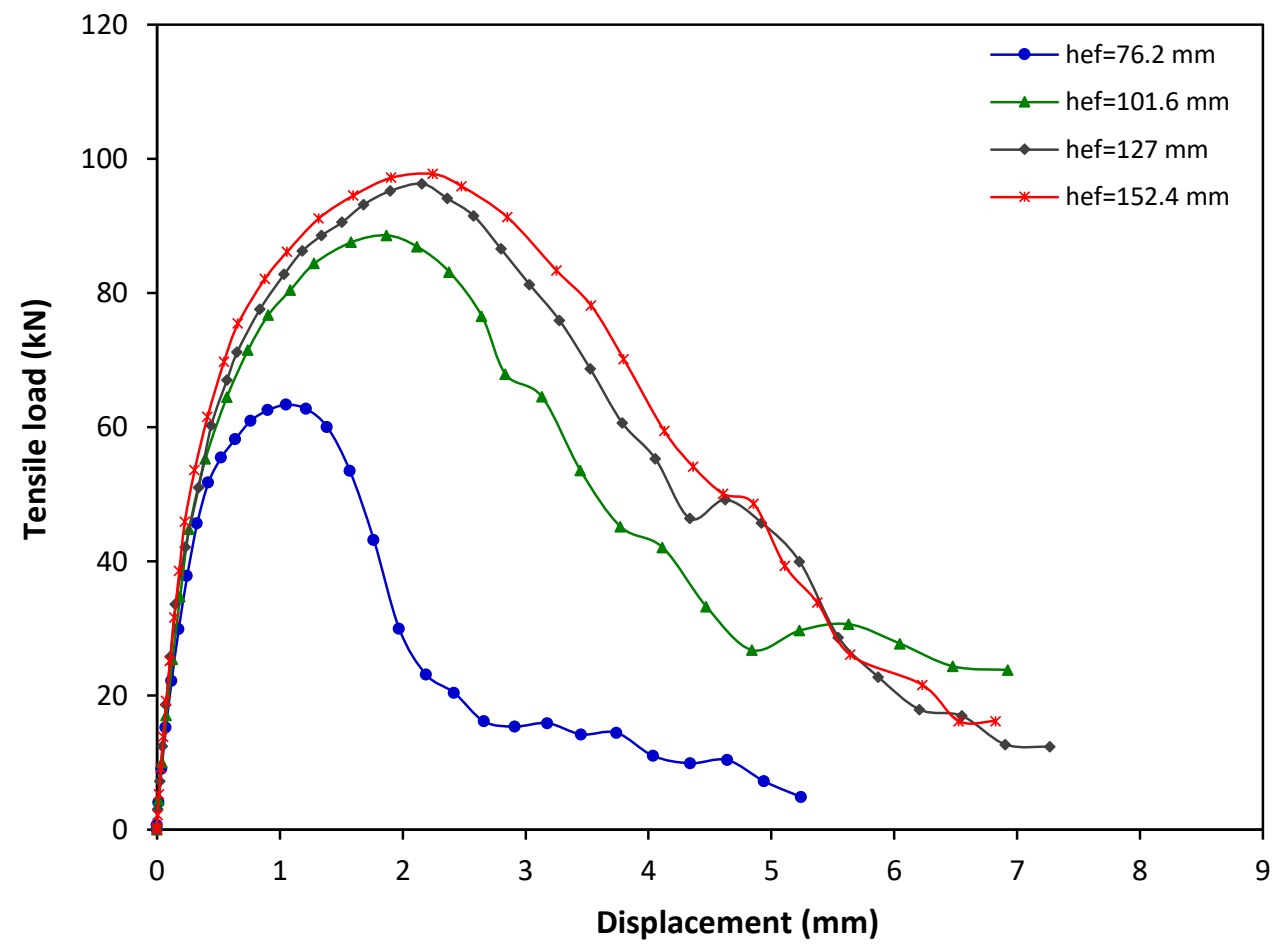

Figure 5-19: Tensile load-displacement graph for $12.7 \mathrm{~mm}$ diameter adhesive anchor at strain rate of $10^{-3} \mathrm{~s}^{-1}$ 


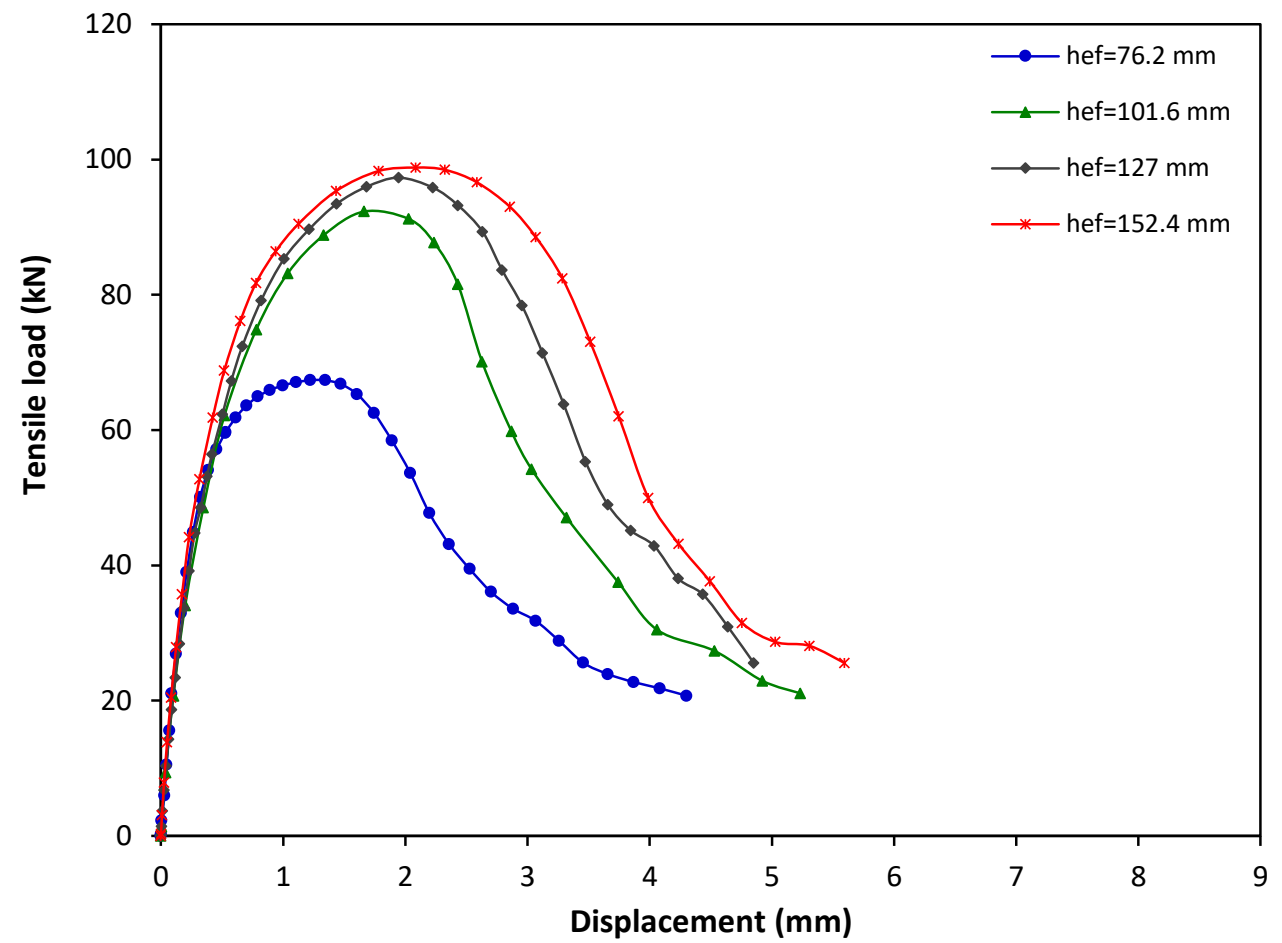

Figure 5-20: Tensile load-displacement graph for $12.7 \mathrm{~mm}$ diameter adhesive anchor at strain rate of $10^{-1} \mathrm{~s}^{-1}$

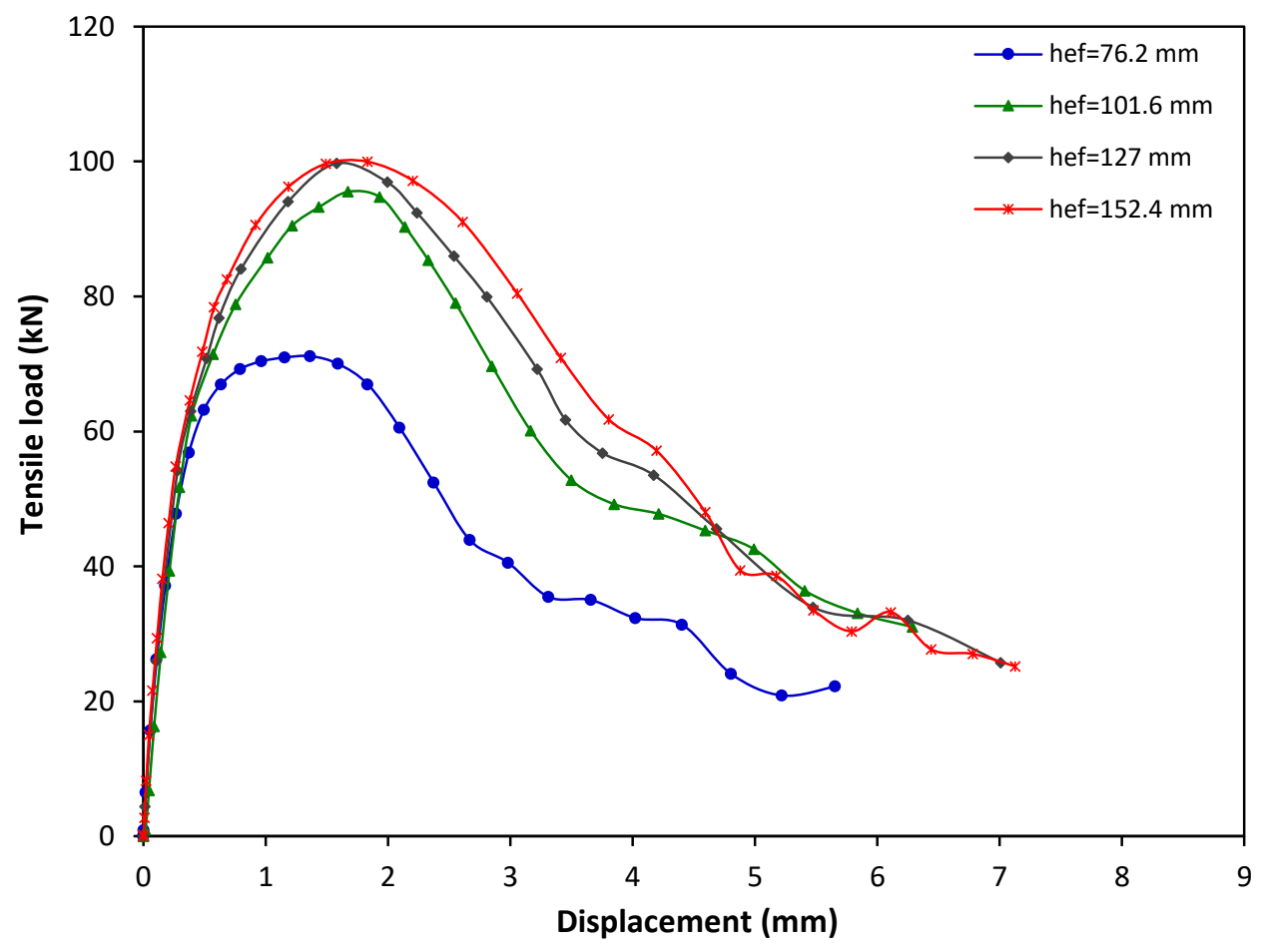

Figure 5-21: Tensile load-displacement graph for $12.7 \mathrm{~mm}$ diameter adhesive anchor at strain rate of $10 \mathrm{~s}^{-1}$ 


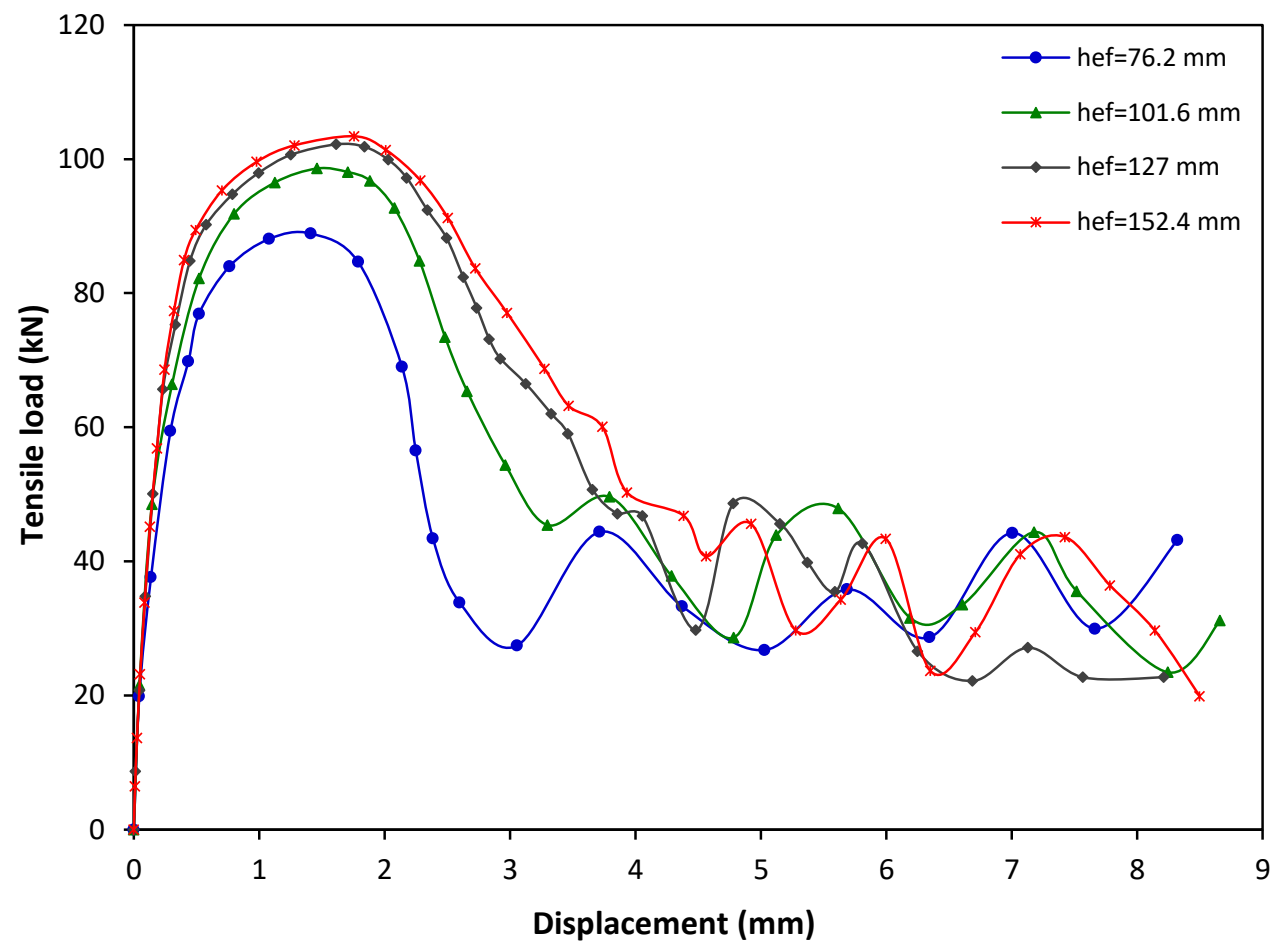

Figure 5-22: Tensile load-displacement graph for $12.7 \mathrm{~mm}$ diameter adhesive anchor at strain rate of $10^{2} \mathrm{~s}^{-1}$

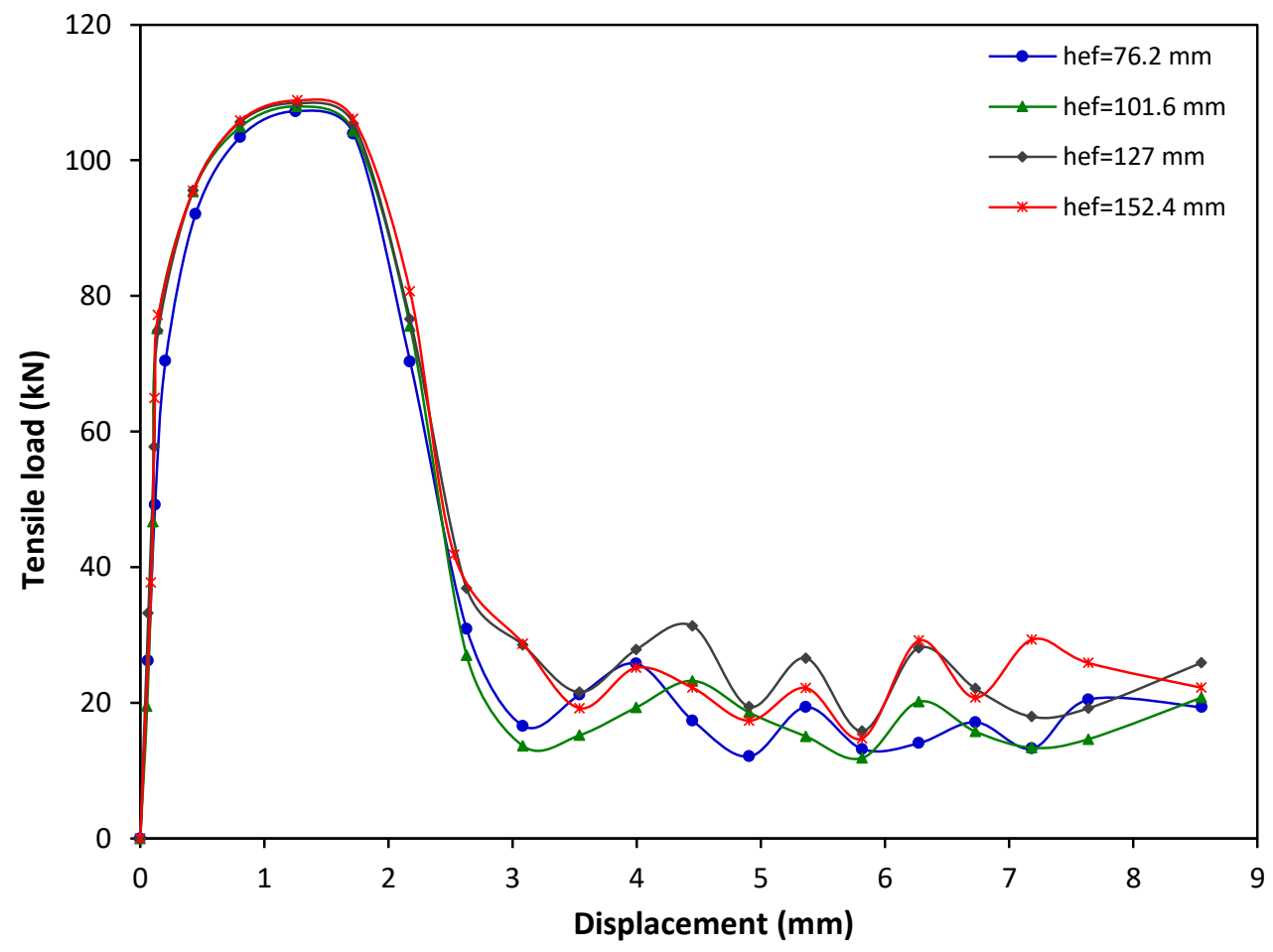

Figure 5-23: Tensile load-displacement graph for $12.7 \mathrm{~mm}$ diameter adhesive anchor at strain rate of $10^{3} \mathrm{~s}^{-1}$ 
Figures 5-18 to 5-23 show that the tensile load increased with the displacement until the ultimate load. This is attributed to the concrete resistance to the applied load where the tensile load transfers from the anchor to the concrete through the adhesive material. The post-peak response shows a reduction in the load with further increase in displacement until failure. The ultimate tensile load increased with the increase in the strain rate from $10^{-5} \mathrm{~s}^{-1}$ to $10^{3} \mathrm{~s}^{-1}$ as shown in the figures and Table 5-5. Similar observation on the effect of loading rate for the adhesive anchors was obtained by Braimah et al., where the dynamic capacity increased at high loading rate of impulse ( Braimah et al., 2009).

At intermediate strain rate of $10 \mathrm{~s}^{-1}$, the tensile load increased as the embedment depth increased from $76.2 \mathrm{~mm}$ to $127 \mathrm{~mm}$ where the failure mode changed from combined conebond failure at embedment depth of $76.2 \mathrm{~mm}$ to steel anchor failure at embedment depth of $127 \mathrm{~mm}$. Further increase in the embedment depth to $152.4 \mathrm{~mm}$ shows no increase of the tensile load as steel failure is observed. At embedment depths of $76.2 \mathrm{~mm}$ and 101.6 $\mathrm{mm}$, the post peak behaviour at strain rate of $10 \mathrm{~s}^{-1}$ shows a decrease in the tensile load due to bond failure at the lower part of the anchor accompanied by crack initiation and propagation to the top surface of the concrete. The crack initiation and propagation lead to fracturing of the concrete and results in combined cone-bond failure as shown in Figure 58.

As the strain rate increased from $10 \mathrm{~s}^{-1}$ to $10^{3} \mathrm{~s}^{-1}$, the tensile load increased with the displacement until the maximum load. The post peak behaviour shows a decrease in the tensile load due to steel anchor failure. The failure mode transitioned from combined conebond failure to steel anchor failure for the embedment depths of $76.2 \mathrm{~mm}$ and $101.6 \mathrm{~mm}$. 
At embedment depths of $127 \mathrm{~mm}$ and $152.4 \mathrm{~mm}$ steel anchor failure was the dominant failure mode (Figure 5-8). At the large embedment depths, the ultimate failure load of the anchorage systems is the same as failure is dependent on steel anchor resistance.

The increase in the ultimate load with increase in strain rate is attributed to the fact that strain rate affects the mechanical properties of the concrete and steel materials. Concrete has been reported to have increased tensile and compressive strengths with increase in the strain rate (Hentz et al., 2004). Hence, the concrete resistance increases results in increase of the ultimate load capacity of the anchorage system when failure is by either cone or combined cone-bond failure.

Tensile load-displacement relations of the 15.9-mm and 19.1-mm diameter adhesive anchors with embedment depths of $76.2 \mathrm{~mm}, 101.6 \mathrm{~mm}, 127 \mathrm{~mm}$ and $152.4 \mathrm{~mm}$ at strain rates of $10^{-5} \mathrm{~s}^{-1}, 10^{-3} \mathrm{~s}^{-1}, 10^{-1} \mathrm{~s}^{-1}, 10 \mathrm{~s}^{-1}, 10^{2} \mathrm{~s}^{-1}$ and $10^{3} \mathrm{~s}^{-1}$ are presented in Appendix E.

Tables 5-5, 5-6 and 5-7 present the results of ultimate tensile load and corresponding displacement $(\delta)$ for the 12.7-mm, 15.9-mm and 19.1-mm diameter adhesive anchors respectively.

Table 5-5: Ultimate tensile load and displacement results for the $12.7 \mathrm{~mm}$ diameter adhesive anchor

\begin{tabular}{|c|c|c|c|c|c|c|}
\hline $\begin{array}{c}\text { Model } \\
\text { No. }\end{array}$ & $\begin{array}{c}\mathrm{d} \\
(\mathrm{mm})\end{array}$ & $\begin{array}{c}\mathrm{h}_{\mathrm{ef}} \\
(\mathrm{mm})\end{array}$ & $\begin{array}{c}\dot{\varepsilon} \\
\left(\mathrm{s}^{-1}\right)\end{array}$ & $\begin{array}{c}\mathrm{F}_{\mathrm{u}} \\
(\mathrm{kN})\end{array}$ & $\begin{array}{c}\delta \\
(\mathrm{mm})\end{array}$ & $\begin{array}{l}\text { Failure } \\
\text { mode }\end{array}$ \\
\hline 1 & \multirow{4}{*}{12.7} & 76.2 & \multirow{4}{*}{$10^{-5}$} & 57.12 & 1.65 & $\mathrm{CC}$ \\
\hline 2 & & 101.6 & & 80.45 & 1.78 & $\mathrm{CC}$ \\
\hline 3 & & 127 & & 95.72 & 2.12 & $\mathrm{~S}$ \\
\hline 4 & & 152.4 & & 96.79 & 2.41 & $\mathrm{~S}$ \\
\hline
\end{tabular}




\begin{tabular}{|c|c|c|c|c|c|c|}
\hline 5 & \multirow{4}{*}{12.7} & 76.2 & \multirow{4}{*}{$10^{-3}$} & 63.33 & 1.05 & CCB \\
\hline 6 & & 101.6 & & 88.59 & 1.87 & CCB \\
\hline 7 & & 127 & & 96.27 & 2.16 & $\mathrm{~S}$ \\
\hline 8 & & 152.4 & & 97.74 & 2.23 & $\mathrm{~S}$ \\
\hline 9 & \multirow{4}{*}{12.7} & 76.2 & \multirow{4}{*}{$10^{-1}$} & 67.39 & 1.22 & CCB \\
\hline 10 & & 101.6 & & 92.31 & 1.66 & CCB \\
\hline 11 & & 127 & & 97.29 & 1.95 & $\mathrm{~S}$ \\
\hline 12 & & 152.4 & & 98.82 & 2.09 & S \\
\hline 13 & \multirow{4}{*}{12.7} & 76.2 & \multirow{4}{*}{10} & 71.14 & 1.37 & CCB \\
\hline 14 & & 101.6 & & 95.49 & 1.44 & CCB \\
\hline 15 & & 127 & & 99.73 & 1.58 & $\mathrm{~S}$ \\
\hline 16 & & 152.4 & & 99.95 & 1.83 & $\mathrm{~S}$ \\
\hline 17 & \multirow{4}{*}{12.7} & 76.2 & \multirow{4}{*}{$10^{2}$} & 88.11 & 1.08 & CCB \\
\hline 18 & & 101.6 & & 98.60 & 1.46 & CCB \\
\hline 19 & & 127 & & 102.19 & 1.62 & S \\
\hline 20 & & 152.4 & & 103.38 & 1.76 & $\mathrm{~S}$ \\
\hline 21 & \multirow{4}{*}{12.7} & 76.2 & \multirow{4}{*}{$10^{3}$} & 107.24 & 1.25 & S \\
\hline 22 & & 101.6 & & 107.93 & 1.26 & S \\
\hline 23 & & 127 & & 108.43 & 1.27 & S \\
\hline 24 & & 152.4 & & 108.85 & 1.27 & $\mathrm{~S}$ \\
\hline
\end{tabular}

Table 5-6: Ultimate tensile load and displacement results for the $15.9 \mathrm{~mm}$ diameter adhesive anchor

\begin{tabular}{|c|c|c|c|c|c|c|}
\hline $\begin{array}{c}\text { Model } \\
\text { No. }\end{array}$ & $\begin{array}{c}\mathrm{d} \\
(\mathrm{mm})\end{array}$ & $\begin{array}{c}\mathrm{h}_{\mathrm{ef}} \\
(\mathrm{mm})\end{array}$ & $\begin{array}{c}\dot{\varepsilon} \\
\left(\mathrm{s}^{-1}\right)\end{array}$ & $\begin{array}{c}\mathrm{F}_{\mathrm{u}} \\
(\mathrm{kN})\end{array}$ & $\begin{array}{c}\delta \\
(\mathrm{mm})\end{array}$ & $\begin{array}{l}\text { Failure } \\
\text { mode }\end{array}$ \\
\hline 1 & \multirow{4}{*}{15.9} & 76.2 & \multirow{4}{*}{$10^{-5}$} & 65.69 & 1.11 & $\mathrm{CC}$ \\
\hline 2 & & 101.6 & & 87.31 & 1.47 & $\mathrm{CC}$ \\
\hline 3 & & 127 & & 126.18 & 1.82 & $\mathrm{CCB}$ \\
\hline 4 & & 152.4 & & 159.72 & 2.09 & $\mathrm{~S}$ \\
\hline 5 & \multirow{3}{*}{15.9} & 76.2 & \multirow{3}{*}{$10^{-3}$} & 69.70 & 1.42 & $\mathrm{CCB}$ \\
\hline 6 & & 101.6 & & 94.88 & 1.61 & CCB \\
\hline 7 & & 127 & & 135.36 & 1.77 & CCB \\
\hline
\end{tabular}




\begin{tabular}{|c|c|c|c|c|c|c|}
\hline 8 & & 152.4 & & 162.02 & 2.24 & $\mathrm{~S}$ \\
\hline 9 & \multirow{4}{*}{15.9} & 76.2 & \multirow{4}{*}{$10^{-1}$} & 74.94 & 1.25 & CCB \\
\hline 10 & & 101.6 & & 101.48 & 1.53 & $\mathrm{CCB}$ \\
\hline 11 & & 127 & & 143.56 & 1.61 & CCB \\
\hline 12 & & 152.4 & & 164.08 & 2.36 & $\mathrm{~S}$ \\
\hline 13 & \multirow{4}{*}{15.9} & 76.2 & \multirow{4}{*}{10} & 87.36 & 1.04 & $\mathrm{CCB}$ \\
\hline 14 & & 101.6 & & 108.31 & 1.03 & $\mathrm{CCB}$ \\
\hline 15 & & 127 & & 151.14 & 1.22 & $\mathrm{CCB}$ \\
\hline 16 & & 152.4 & & 168.41 & 1.54 & $S$ \\
\hline 17 & \multirow{4}{*}{15.9} & 76.2 & \multirow{4}{*}{$10^{2}$} & 118.21 & 1.06 & CCB \\
\hline 18 & & 101.6 & & 124.28 & 1.02 & $\mathrm{CCB}$ \\
\hline 19 & & 127 & & 162.82 & 1.60 & CCB \\
\hline 20 & & 152.4 & & 171.08 & 1.76 & $S$ \\
\hline 21 & \multirow{4}{*}{15.9} & 76.2 & \multirow{4}{*}{$10^{3}$} & 173.63 & 1.26 & $\bar{S}$ \\
\hline 22 & & 101.6 & & 175.89 & 1.26 & $\mathrm{~S}$ \\
\hline 23 & & 127 & & 177.57 & 1.42 & $S$ \\
\hline 24 & & 152.4 & & 178.59 & 1.37 & $\mathrm{~S}$ \\
\hline
\end{tabular}

Table 5-7: Ultimate tensile load and displacement results for the $19.1 \mathrm{~mm}$ diameter adhesive anchor

\begin{tabular}{|c|c|c|c|c|c|c|}
\hline $\begin{array}{c}\text { Model } \\
\text { No. }\end{array}$ & $\begin{array}{c}\mathrm{d} \\
(\mathrm{mm})\end{array}$ & $\begin{array}{c}\mathrm{h}_{\mathrm{ef}} \\
(\mathrm{mm})\end{array}$ & $\begin{array}{c}\dot{\varepsilon} \\
\left(\mathrm{s}^{-1}\right)\end{array}$ & $\begin{array}{c}\mathrm{F}_{\mathrm{u}} \\
(\mathrm{kN})\end{array}$ & $\begin{array}{c}\delta \\
(\mathrm{mm})\end{array}$ & $\begin{array}{l}\text { Failure } \\
\text { mode }\end{array}$ \\
\hline 1 & \multirow{4}{*}{19.1} & 76.2 & \multirow{4}{*}{$10^{-5}$} & 69.62 & 0.80 & $\mathrm{CC}$ \\
\hline 2 & & 101.6 & & 105.49 & 1.06 & $\mathrm{CC}$ \\
\hline 3 & & 127 & & 157.28 & 1.36 & $\mathrm{CC}$ \\
\hline 4 & & 152.4 & & 179.40 & 2.04 & $\mathrm{CC}$ \\
\hline 5 & \multirow{4}{*}{19.1} & 76.2 & \multirow{4}{*}{$10^{-3}$} & 78.68 & 1.19 & $\mathrm{CC}$ \\
\hline 6 & & 101.6 & & 113.94 & 1.59 & $\mathrm{CCB}$ \\
\hline 7 & & 127 & & 161.79 & 1.77 & CCB \\
\hline 8 & & 152.4 & & 187.48 & 1.99 & $\mathrm{CCB}$ \\
\hline 9 & \multirow{2}{*}{19.1} & 76.2 & \multirow{2}{*}{$10^{-1}$} & 89.32 & 1.29 & CCB \\
\hline 10 & & 101.6 & & 120.78 & 1.32 & CCB \\
\hline
\end{tabular}




\begin{tabular}{|c|c|c|c|c|c|c|}
\hline 11 & & 127 & & 169.02 & 1.51 & $\mathrm{CCB}$ \\
\hline 12 & & 152.4 & & 196.47 & 1.47 & $\mathrm{CCB}$ \\
\hline 13 & \multirow{4}{*}{19.1} & 76.2 & \multirow{4}{*}{10} & 114.01 & 1.07 & CCB \\
\hline 14 & & 101.6 & & 139.14 & 1.02 & CCB \\
\hline 15 & & 127 & & 185.58 & 1.23 & $\mathrm{CCB}$ \\
\hline 16 & & 152.4 & & 211.45 & 1.34 & $\mathrm{CCB}$ \\
\hline 17 & \multirow{4}{*}{19.1} & 76.2 & \multirow{4}{*}{$10^{2}$} & 180.25 & 0.67 & $\mathrm{CCB}$ \\
\hline 18 & & 101.6 & & 196.53 & 0.84 & $\mathrm{CCB}$ \\
\hline 19 & & 127 & & 217.96 & 1.47 & $\mathrm{CCB}$ \\
\hline 20 & & 152.4 & & 234.18 & 1.40 & $\mathrm{CCB}$ \\
\hline 21 & \multirow{4}{*}{19.1} & 76.2 & \multirow{4}{*}{$10^{3}$} & 262.69 & 1.26 & $\mathrm{~S}$ \\
\hline 22 & & 101.6 & & 264.59 & 1.29 & $\mathrm{~S}$ \\
\hline 23 & & 127 & & 266.13 & 1.26 & $\mathrm{~S}$ \\
\hline 24 & & 152.4 & & 266.96 & 1.39 & $\mathrm{~S}$ \\
\hline
\end{tabular}

In general, it can be seen from Tables 5-5, 5-6 and 5-7 that the ultimate tensile load increased with the increase in the anchor diameter from $12.7 \mathrm{~mm}$ to $19.1 \mathrm{~mm}$ for the embedment depths and strain rates investigated. The increase in anchor diameter increases the contact area between the adhesive anchor and concrete and hence the bond capacity of the anchorage system. It can be seen from the tables that at the low strain rate of $10^{-5} \mathrm{~s}^{-1}$ for shallow embedment depths of $76.2 \mathrm{~mm}$ and $101.6 \mathrm{~mm}$ concrete cone breakout failure was observed for the 12.7- $\mathrm{mm}, 15.9-\mathrm{mm}$ and 19.1-mm diameter adhesive anchors. Also, concrete cone breakout failure was observed for the 19.1-mm diameter adhesive anchor at embedment depths of $127 \mathrm{~mm}$ and $152.4 \mathrm{~mm}$, where the tensile load was higher than the concrete strength. Steel anchor failure was observed for anchor embedment depth of 127 $\mathrm{mm}$ with anchor diameter of $12.7 \mathrm{~mm}$. Also, steel anchor failure was observed at embedment depth of $152.4 \mathrm{~mm}$ for anchor diameters of $12.7 \mathrm{~mm}$ and $15.9 \mathrm{~mm}$. For long embedment depths and smaller anchor diameters of $12.7 \mathrm{~mm}$ and $15.9 \mathrm{~mm}$, the steel anchor 
is not capable to withstand the applied load resulting in steel fracture. The tensile load is influenced by the strain rate. From Tables 5-5, 5-6 and 5-7 it can be seen that the adhesive anchors exhibited concrete cone breakout failure transitioned to steel failure at high strain rate. This can be attributed to the increased strength of concrete and steel due to increased strain rate and hence, the concrete resistance to the tensile load increased resulted in steel anchor failure.

In general, it can be seen that the displacement $(\delta)$ at the ultimate load decreased with the increase in anchor diameter from $12.7 \mathrm{~mm}$ to $19.1 \mathrm{~mm}$, at the same embedment depths. At high strain rate the effect of anchor diameter on the ultimate displacement is insignificant. The tensile load was also observed to increase with increase in the embedment depth from $76.2 \mathrm{~mm}$ to $152.4 \mathrm{~mm}$ for the anchors exhibited concrete cone breakout failure mode. The increase in the anchor embedment depth increased the displacement at the ultimate tensile load. The embedment depth was observed to have a greater effect on the ultimate tensile load at the same strain rate when concrete cone breakout failure is the dominant failure mode. However, the increase in the embedment depth has no influence on the ultimate tensile load when steel anchor failure is the dominant failure mode.

\subsubsection{Effect of strain rate on the ultimate tensile load and DIF of adhesive anchors}

In order to predict the increase in strength of anchorage system due to increase in the steel and concrete strength with the increase in the strain rate, DIF for the anchorage to concrete system was investigated. The lowest strain rate of $10^{-5} \mathrm{~s}^{-1}$ is representative of static loading rate and was used as the baseline for comparison with adhesive anchor capacity at the higher strain rates for the adhesive anchors exhibiting concrete cone breakout failure and 
combined cone bond failure. For the anchors exhibiting steel failure mode the dynamic increase factor is taken as the ratio of ultimate dynamic load to the ultimate static steel failure load. Effect of strain rate on the ultimate tensile load and DIF is shown in Figures 5-24, 5-25, 5-26 and 5-27 for the 12.7- $\mathrm{mm}, 15.9-\mathrm{mm}$ and 19.1-mm diameter adhesive anchors with embedment depths of $76.2 \mathrm{~mm}, 101.6 \mathrm{~mm}, 127 \mathrm{~mm}$ and $152.4 \mathrm{~mm}$ respectively. It can be seen from the figures that the ultimate tensile load increased with increase in strain rate from $10^{-5} \mathrm{~s}^{-1}$ to $10^{3} \mathrm{~s}^{-1}$ for all the adhesive anchors investigated. The increase in anchor embedment depth resulted in increase in ultimate tensile load at all strain rates investigated. Maximum tensile loads of $108.85 \mathrm{kN}, 178.59 \mathrm{kN}$ and $266.96 \mathrm{kN}$ were obtained at high strain rate of $10^{3} \mathrm{~s}^{-1}$ and embedment depth of $152.4 \mathrm{~mm}$ for the $12.7-\mathrm{mm}$, 15.9-mm and 19.1-mm diameter adhesive anchors, respectively. On the other hand, at low strain rate of $10^{-5} \mathrm{~s}^{-1}$ and for the same embedment depth of $152.4 \mathrm{~mm}$, the ultimate tensile loads were $96.79 \mathrm{kN}, 159.72 \mathrm{kN}$ and $179.40 \mathrm{kN}$ for the $12.7-\mathrm{mm}, 15.9-\mathrm{mm}$ and $19.1-\mathrm{mm}$ diameter adhesive anchors, respectively.

As shown from Figures 5-24 and 5-25, the relationship between the ultimate tensile load and the strain rate appears to be bilinear with a change in slope at strain rate of $10 \mathrm{~s}^{-1}$ for all anchor diameters at embedment depths of $76.2 \mathrm{~mm}$ and $101.6 \mathrm{~mm}$ where concrete cone breakout failure was observed at low strain rate of $10^{-5} \mathrm{~s}^{-1}$. Then the failure mode transitioned to combined concrete cone bond failure at strain rates up to $10^{2} \mathrm{~s}^{-1}$, and to steel anchor failure at high strain rate of $10^{3} \mathrm{~s}^{-1}$ where higher load is required to fracture the anchor. This is similar to the reported relationship between tensile concrete strength and strain rate (Malvar \& Crawford, 1998; Hentz et al., 2004). Also, Malvar \& Crawford 
reported that the relationship between compressive concrete strength and strain rate is bilinear (Malvar \& Crawford, 1998).

It can be seen from Figures 5-24 and 5-25, the 19.1-mm diameter adhesive anchor exhibited higher DIF than that obtained for anchor diameters of $12.7 \mathrm{~mm}$ and $15.9 \mathrm{~mm}$ for the strain rates ranging from $10^{-5} \mathrm{~s}^{-1}$ to $10^{2} \mathrm{~s}^{-1}$. At high strain rate of $10^{3} \mathrm{~s}^{-1}$, average DIF of 1.1 was obtained for the anchor diameters of $12.7 \mathrm{~mm}, 15.9 \mathrm{~mm}$ and $19.1 \mathrm{~mm}$ where steel failure was observed. Also, it can be seen that the DIF is higher for the shallow embedment depth of $76.2 \mathrm{~mm}$ compared to the deep embedment depth of $152.4 \mathrm{~mm}$. Maximum DIF of 1.54, 1.80 and 2.59 were obtained for the $12.7-\mathrm{mm}, 15.9-\mathrm{mm}$ and $19.1-\mathrm{mm}$ diameter adhesive anchors with embedment depth of $76.2 \mathrm{~mm}$ at the high strain rate of $10^{2} \mathrm{~s}^{-1}$ where combined cone bond failure is observed for all the adhesive anchors.

It can be seen from Figure 5-26 bilinear relationship between the ultimate tensile load and strain rate was obtained for the $15.9-\mathrm{mm}$ and $19.1-\mathrm{mm}$ anchor diameters at embedment depth of $127 \mathrm{~mm}$; whereas almost linear relation was obtained for the 12.7 -mm diameter adhesive anchor where steel anchor failure was observed at all the strain rates investigated. As shown in Figure 5-26, the DIF increased slightly from 1.01 at strain rate of $10^{-3} \mathrm{~s}^{-1}$ to 1.13 at strain rate of $10^{3} \mathrm{~s}^{-1}$ for anchor diameter of $12.7 \mathrm{~mm}$. The DIF increased from 1.07 to 1.29 and from 1.03 to 1.39 for the anchor diameters of $15.9 \mathrm{~mm}$ and $19.1 \mathrm{~mm}$ respectively when the strain rate increased from $10^{-3} \mathrm{~s}^{-1}$ to $10^{2} \mathrm{~s}^{-1}$. At high strain rate of $10^{3}$ $\mathrm{s}^{-1}$, average DIF of 1.11 was obtained for the $15.9 \mathrm{~mm}$ and $19.1 \mathrm{~mm}$ diameter adhesive anchors. Combined cone bond failure was observed for anchor diameters of $15.9 \mathrm{~mm}$ and 
$19.1 \mathrm{~mm}$ at strain rate of $10^{-3} \mathrm{~s}^{-1}$ and transitioned to steel anchor failure at high strain rate of $10^{3} \mathrm{~s}^{-1}$.

At embedment depth of $152.4 \mathrm{~mm}$ (Figure 5-27), bilinear relationship was obtained for the 19.1-mm diameter adhesive anchor where concrete cone breakout failure mode at low strain rate of $10^{-5} \mathrm{~s}^{-1}$ transitioned to combined cone bond failure at higher strain rates up to $10^{2} \mathrm{~s}^{-1}$ followed by steel anchor failure at strain rate of $10^{3} \mathrm{~s}^{-1}$. While linear relation was obtained for the $12.7-\mathrm{mm}$ and $15.9-\mathrm{mm}$ diameter adhesive anchors where steel anchor failure was the dominant failure mode at all the strain rates investigated. The DIF increased slightly for anchor diameters of $12.7 \mathrm{~mm}$ and $15.9 \mathrm{~mm}$. For the $19.1-\mathrm{mm}$ diameter adhesive anchor, the DIF increased from 1.05 at strain rate of $10^{-3} \mathrm{~s}^{-1}$ to 1.31 at strain rate of $10^{2} \mathrm{~s}^{-1}$ where combined cone bond failure was observed. At high strain rate of $10^{3} \mathrm{~s}^{-1} \mathrm{DIF}$ of 1.11 was obtained for the $19.1 \mathrm{~mm}$ diameter anchor where steel failure was observed.

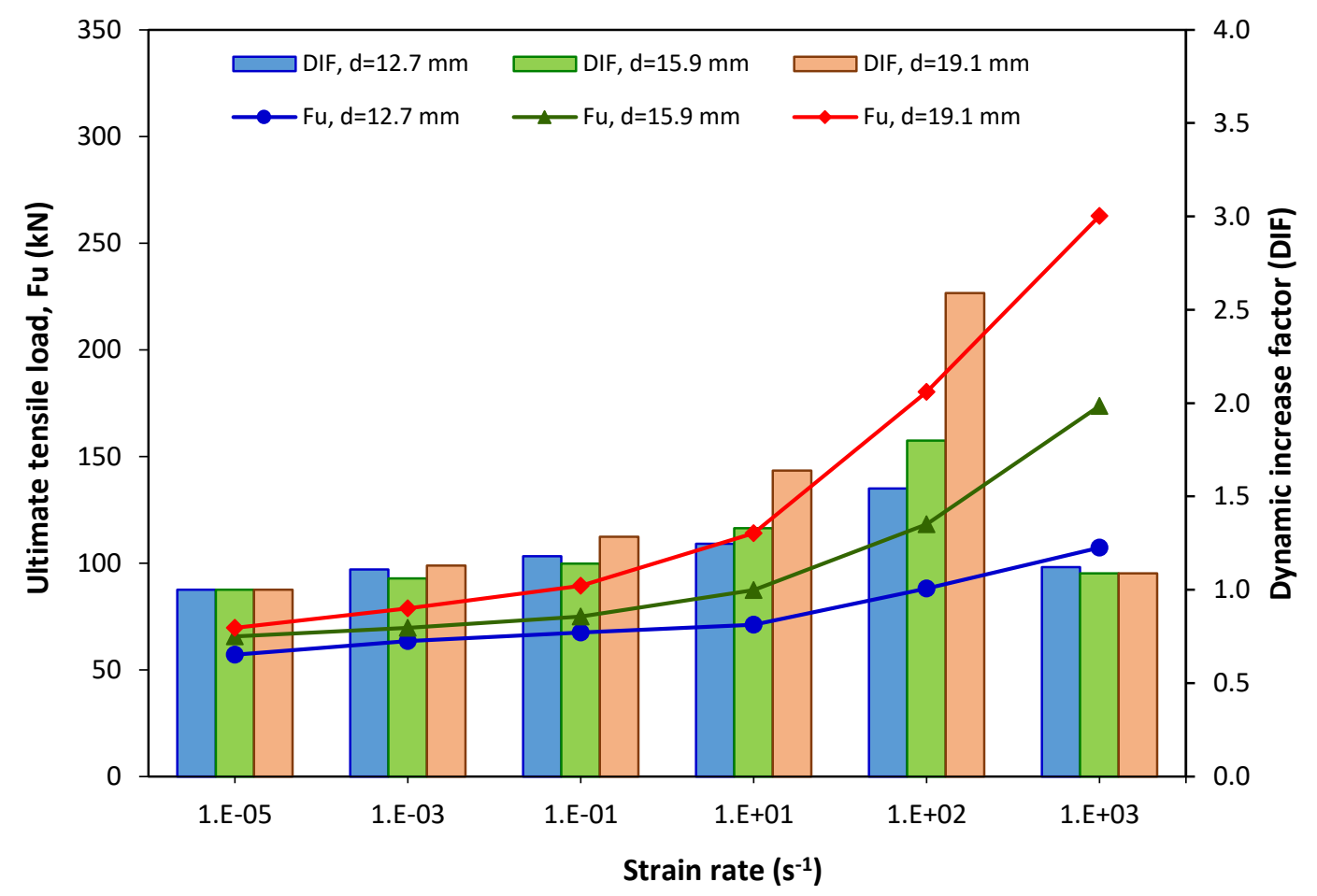

Figure 5-24: Ultimate tensile load and DIF versus strain rate for the adhesive anchor at $76.2 \mathrm{~mm}$ embedment depth 


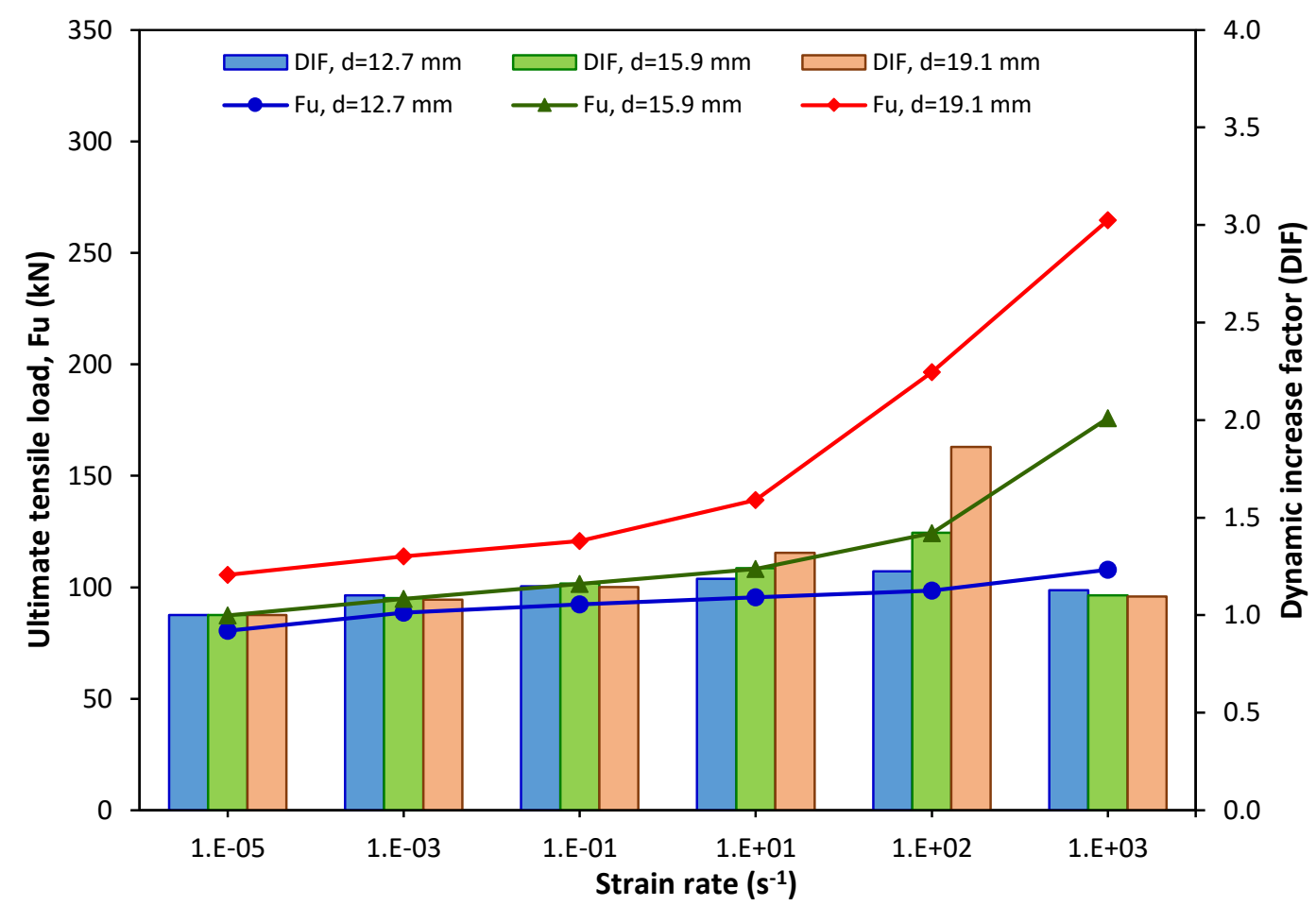

Figure 5-25: Ultimate tensile load and DIF versus strain rate for the adhesive anchor at $101.6 \mathrm{~mm}$ embedment depth

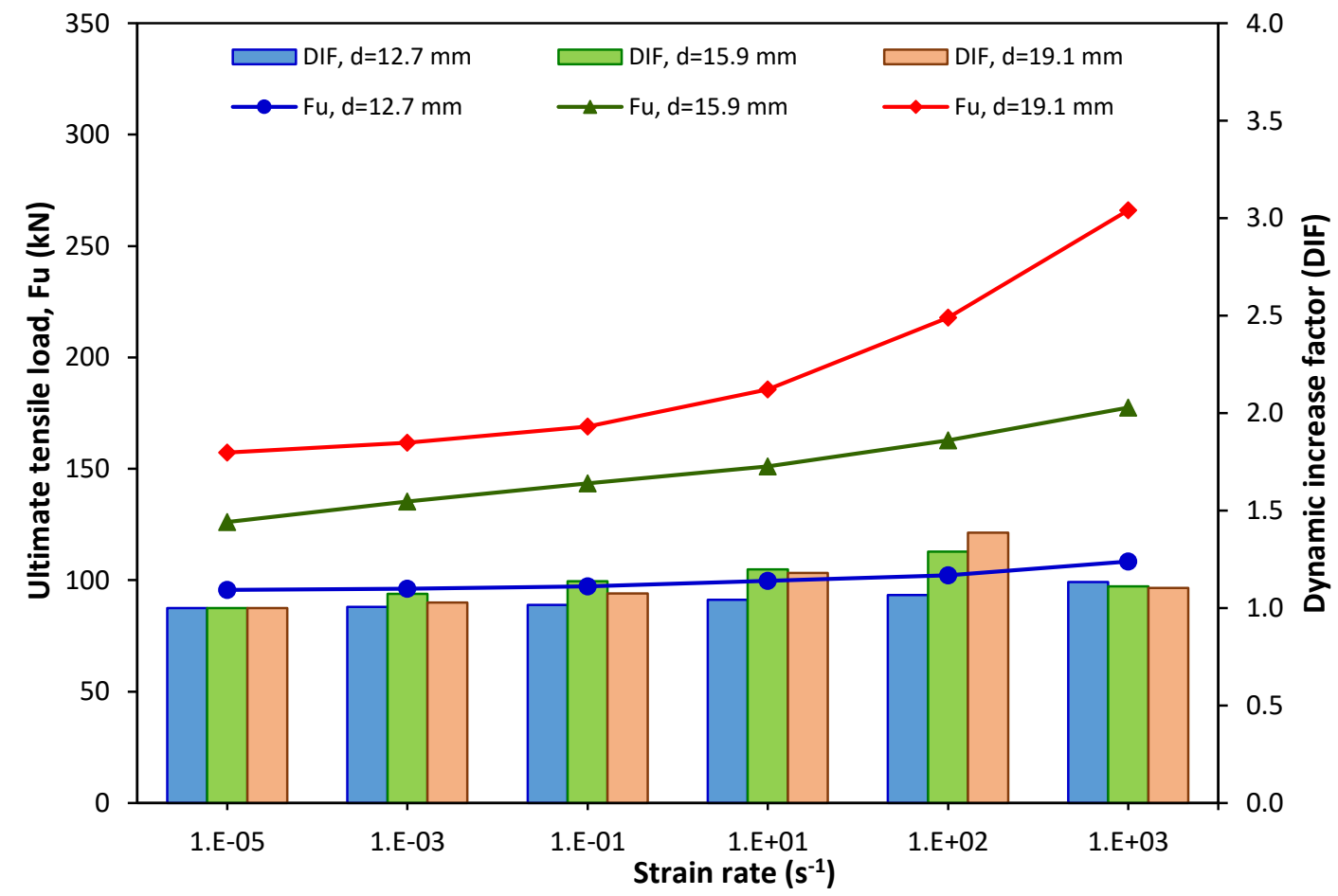

Figure 5-26: Ultimate tensile load and DIF versus strain rate for the adhesive anchor at $127 \mathrm{~mm}$ embedment depth 


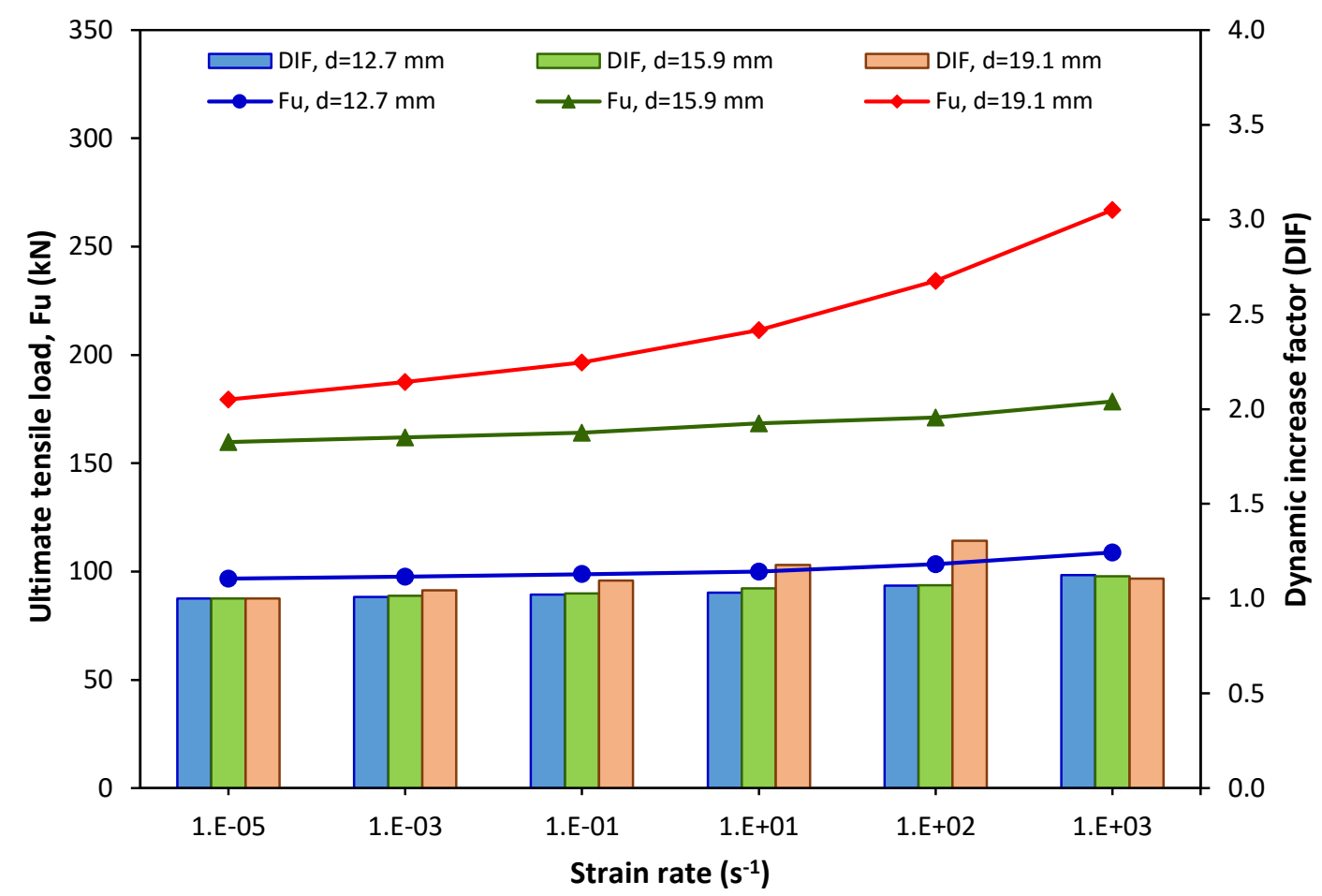

Figure 5-27: Ultimate tensile load and DIF versus strain rate for the adhesive anchor at 152.4 mm embedment depth

The increase in the strain rate increased the ultimate load and the DIF for the concrete and steel. The DIF for concrete tensile strength is much higher than that for steel (Malvar \& Crawford, 1998; Malvar \& Ross, 1998). Hence as the strain rate increases, the increase in the anchor capacity for concrete cone and combined concrete cone-bond failure exceeds the increase attributed to steel anchor failure where the concrete cone failure is observed at strain rates of $10^{-5} \mathrm{~s}^{-1}$ for most of the adhesive anchors (Figure 5-6). Combined cone-bond failure is observed at strain rates ranging from $10^{-3} \mathrm{~s}^{-1}$ to $10^{2} \mathrm{~s}^{-1}$ for most of the adhesive anchors while steel failure is the dominant failure mode for all the adhesive anchors at high strain rate of $10^{3} \mathrm{~s}^{-1}$ as shown in Figures 5-8, 5-9 and 5-10. 
Table 5-8 shows the maximum DIF for the adhesive anchors under tensile load where concrete cone breakout, combined cone bond and steel failure modes were observed. The DIF at static strain rate of $10^{-5} \mathrm{~s}^{-1}\left(\dot{\varepsilon}_{S}\right)$ is equal to one where concrete cone breakout failure was observed as shown in Table 5-8. For the anchor diameter of $12.7 \mathrm{~mm}$ with embedment depths of $127 \mathrm{~mm}$ and $152.4 \mathrm{~mm}$ and for the anchor diameter of $15.9 \mathrm{~mm}$ with embedment depth of $152.4 \mathrm{~mm}$, steel anchor failure was observed at all the strain rates investigated. For the anchor diameter of $15.9 \mathrm{~mm}$ with embedment depth of $127 \mathrm{~mm}$, combined cone bond failure was observed at strain rates of $10^{-5} \mathrm{~s}^{-1}$ and $10^{2} \mathrm{~s}^{-1}$, maximum DIF of 1.29 for the combined cone bond failure was obtained at strain rate of $10^{2} \mathrm{~s}^{-1}$. For anchor diameter of $19.1 \mathrm{~mm}$ with embedment depth of $76.2 \mathrm{~mm}$ concrete cone breakout failure was observed at strain rates of $10^{-5} \mathrm{~s}^{-1}$ and $10^{-3} \mathrm{~s}^{-1}$, maximum DIF of 1.13 for the concrete cone breakout was obtained at strain rate of $10^{-3} \mathrm{~s}^{-1}$.

Table 5-8: Maximum dynamic increase factor for the adhesive anchors under tensile load

\begin{tabular}{|c|c|c|c|c|c|c|c|c|}
\hline Model & $\mathrm{d}$ & $\mathrm{h}_{\mathrm{ef}}$ & $\dot{\varepsilon}$ & DIF & $\dot{\varepsilon}$ & DIF & $\dot{\varepsilon}$ & DIF \\
\hline No. & $(\mathrm{mm})$ & $(\mathrm{mm})$ & $\left(\mathrm{s}^{-1}\right)$ & $\mathrm{CC}$ & $\left(\mathrm{s}^{-1}\right)$ & $\mathrm{CCB}$ & $\left(\mathrm{s}^{-1}\right)$ & $\mathrm{S}$ \\
\hline 1 & 12.7 & 76.2 & $10^{-5}$ & 1.00 & $10^{2}$ & 1.54 & $10^{3}$ & 1.12 \\
\hline 2 & 12.7 & 101.6 & $10^{-5}$ & 1.00 & $10^{2}$ & 1.23 & $10^{3}$ & 1.13 \\
\hline 3 & 12.7 & 127.0 & - & - & - & - & $10^{3}$ & 1.13 \\
\hline 4 & 12.7 & 152.4 & - & - & - & - & $10^{3}$ & 1.12 \\
\hline 5 & 15.9 & 76.2 & $10^{-5}$ & 1.00 & $10^{2}$ & 1.80 & $10^{3}$ & 1.09 \\
\hline 6 & 15.9 & 101.6 & $10^{-5}$ & 1.00 & $10^{2}$ & 1.42 & $10^{3}$ & 1.10 \\
\hline 7 & 15.9 & 127.0 & - & - & $10^{2}$ & 1.29 & $10^{3}$ & 1.11 \\
\hline 8 & 15.9 & 152.4 & - & - & - & - & $10^{3}$ & 1.12 \\
\hline 9 & 19.1 & 76.2 & $10^{-3}$ & 1.13 & $10^{2}$ & 2.59 & $10^{3}$ & 1.09 \\
\hline 10 & 19.1 & 101.6 & $10^{-5}$ & 1.00 & $10^{2}$ & 1.86 & $10^{3}$ & 1.10 \\
\hline 11 & 19.1 & 127.0 & $10^{-5}$ & 1.00 & $10^{2}$ & 1.39 & $10^{3}$ & 1.10 \\
\hline 12 & 19.1 & 152.4 & $10^{-5}$ & 1.00 & $10^{2}$ & 1.31 & $10^{3}$ & 1.11 \\
\hline
\end{tabular}




\subsubsection{Regression Analysis for adhesive anchors under tensile load}

Regression analysis was carried out to develop an accurate predictive formula for determining the DIF of the adhesive anchorage to concrete systems based on the finite element results. As shown form Figures 5-8, 5-9 and 5-10, most of the adhesive anchors investigated at strain rates ranging from $10^{-3} \mathrm{~s}^{-1}$ to $10^{3} \mathrm{~s}^{-1}$ exhibited combined cone bond and steel anchor failure under tensile load. Hence regression analysis was performed for these failure modes. Average value of the DIF for the adhesive anchorage systems with anchor diameters of $12.7 \mathrm{~mm}, 15.9 \mathrm{~mm}$ and $19.1 \mathrm{~mm}$ was calculated to adjust the DIF for the effect of anchor diameter. Figures 5-28 and 5-29 present the relation between the DIF and the strain rate ratio $\left(\dot{\varepsilon}_{d} / \dot{\varepsilon}_{s}\right)$ for the combined cone bond and steel anchor failure modes respectively.

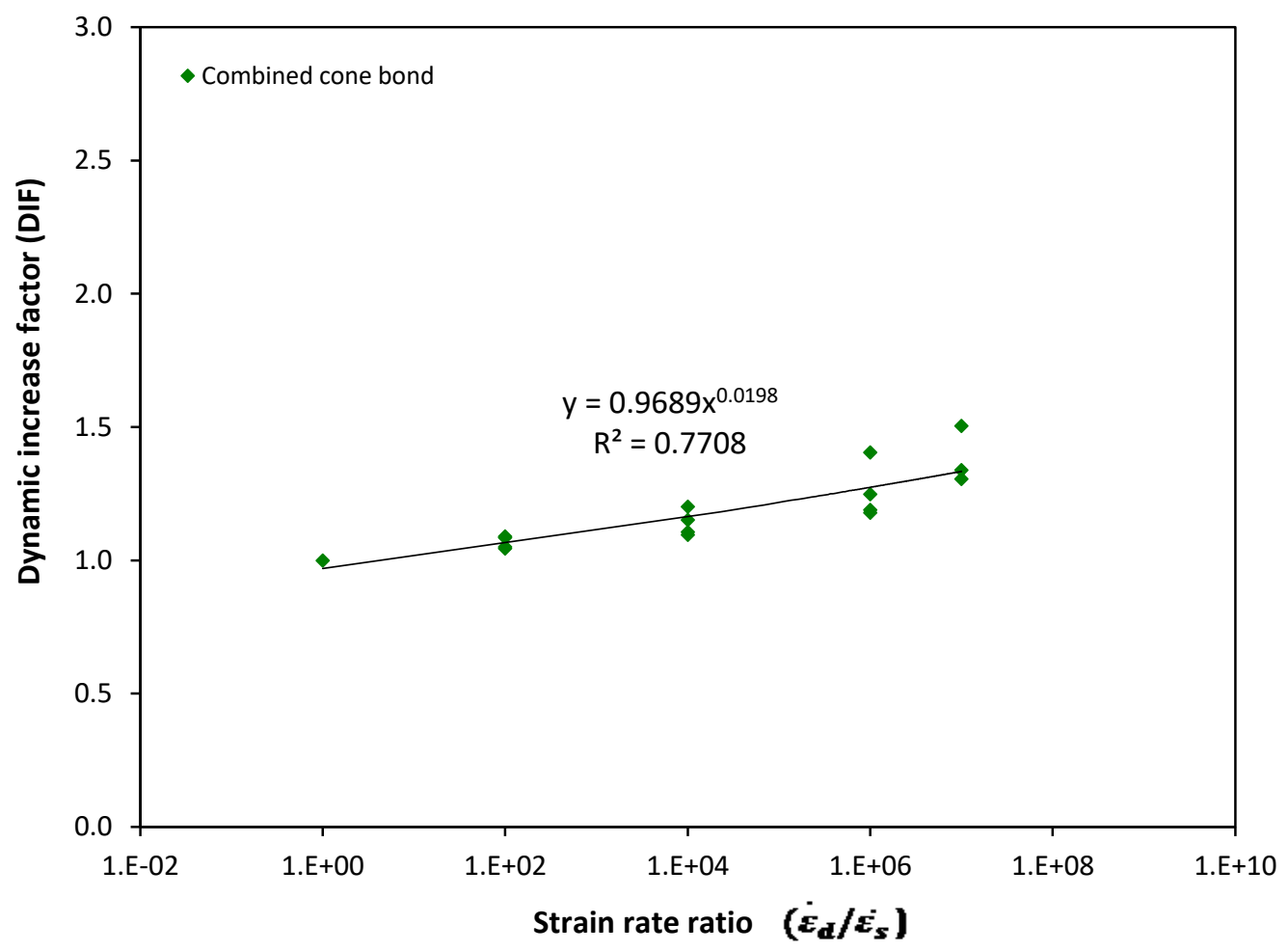

Figure 5-28: Effect of strain rate ratio on DIF for adhesive anchor exhibited combined cone bond failure under tensile load 


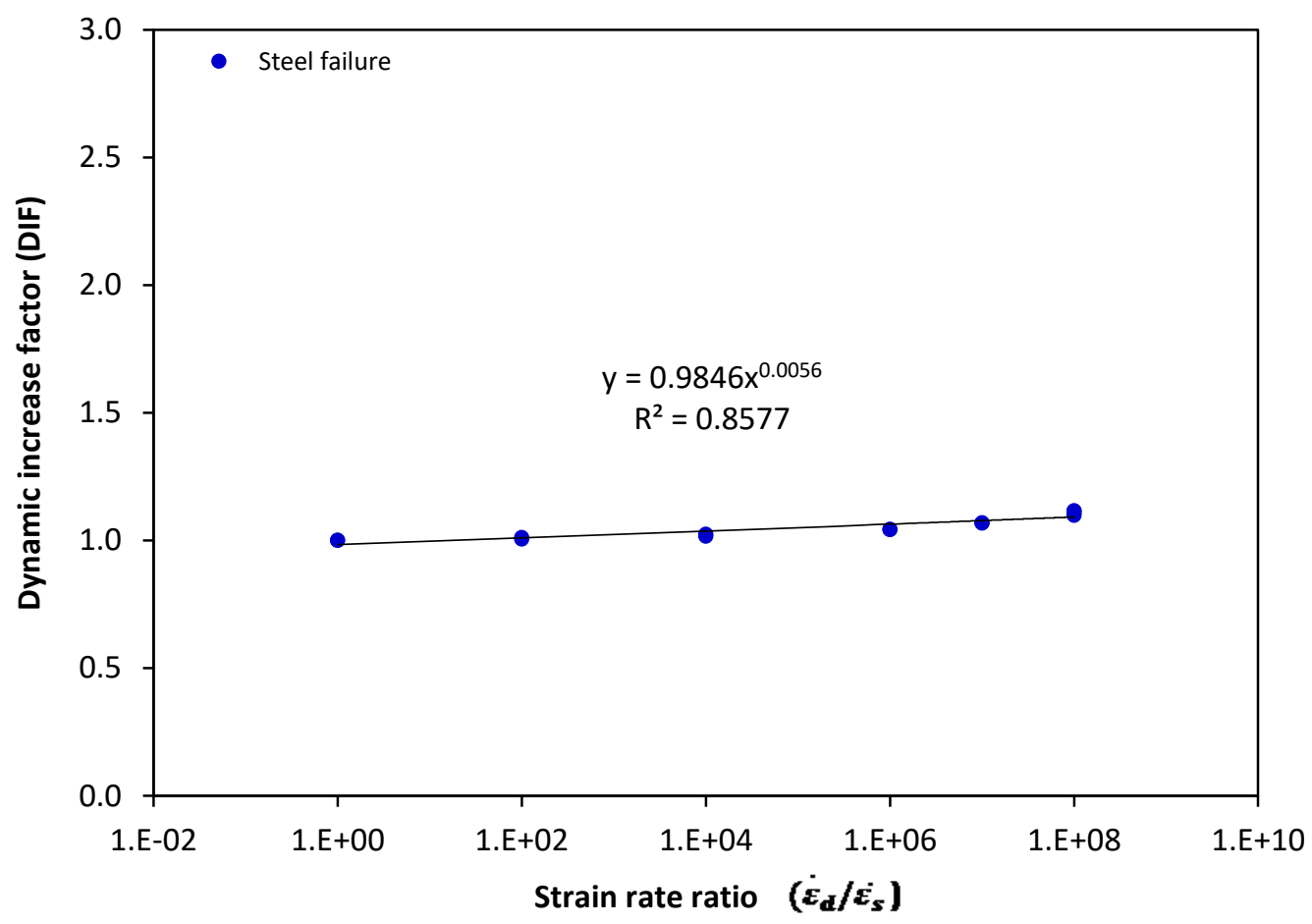

Figure 5-29: Effect of strain rate ratio on DIF for adhesive anchor exhibited steel failure under tensile load

Various statistical models are used to predict the relation between the DIF of the adhesive anchors and strain rate ratio as shown in Tables 5-9 and 5-10.

Table 5-9: Statistical models and coefficient of determination to predict the DIF for adhesive anchors exhibited combined cone bond failure under tensile load

\begin{tabular}{|c|c|c|}
\hline Statistical models & Formulae & Coefficient of determination $\left(\mathrm{R}^{2}\right)$ \\
\hline Exponential & $D I F=1.1272 e^{2 E-8\left(\frac{\dot{\varepsilon}_{d}}{\dot{\varepsilon}_{s}}\right)}$ & 0.532 \\
\hline Linear & $D I F=3 E-8\left(\frac{\dot{\varepsilon}_{d}}{\dot{\varepsilon}_{s}}\right)+1.1306$ & 0.544 \\
\hline Logarithmic & $D I F=0.0237 \ln \left(\frac{\dot{\varepsilon}_{d}}{\dot{\varepsilon}_{s}}\right)+0.9516$ & 0.733 \\
\hline Power & DIF $=0.9689\left(\frac{\dot{\varepsilon}_{d}}{\dot{\varepsilon}_{s}}\right)^{0.0198}$ & 0.771 \\
\hline
\end{tabular}


Table 5-10: Statistical models and coefficient of determination to predict the DIF for adhesive anchors exhibited steel failure under tensile load

\begin{tabular}{|c|c|c|}
\hline Statistical models & Formulae & Coefficient of determination $\left(\mathrm{R}^{2}\right)$ \\
\hline Exponential & $D I F=1.0253 e^{8 E-10\left(\frac{\dot{\varepsilon}_{d}}{\dot{\varepsilon}_{s}}\right)}$ & 0.790 \\
\hline Linear & $D I F=9 E-10\left(\frac{\dot{\varepsilon}_{d}}{\dot{\varepsilon}_{s}}\right)+1.0255$ & 0.800 \\
\hline Logarithmic & $D I F=0.0059 \ln \left(\frac{\dot{\varepsilon}_{d}}{\dot{\varepsilon}_{s}}\right)+0.9832$ & 0.849 \\
\hline Power & $D I F=0.9846\left(\frac{\dot{\varepsilon}_{d}}{\dot{\varepsilon}_{s}}\right)^{0.0056}$ & 0.858 \\
\hline
\end{tabular}

From Tables 5-9 and 5-10, it can be seen that power model exhibits the highest coefficient of determination of $77 \%$ and $86 \%$. The predicted formula for the DIF for the adhesive anchors can be presented as in Equations (5.1) and (5.2) for the combined cone bond failure and steel failure respectively.

$\mathrm{DIF}=0.9689\left(\frac{\dot{\varepsilon}_{d}}{\dot{\varepsilon}_{s}}\right)^{0.0198}$

$D I F=0.9846\left(\frac{\dot{\varepsilon}_{d}}{\dot{\varepsilon}_{s}}\right)^{0.0056}$

The ultimate dynamic load $\left(F_{u d}\right)$ for the combined cone bond and steel anchor failure modes can be determined as in Equations (5.3) and (5.4) respectively as follows:

$$
\begin{aligned}
& F_{u d}=F_{u s} \times 0.9689\left(\frac{\dot{\varepsilon}_{d}}{\dot{\varepsilon}_{s}}\right)^{0.0198} \\
& F_{u d}=F_{u s} \times 0.9846\left(\frac{\dot{\varepsilon}_{d}}{\dot{\varepsilon}_{s}}\right)^{0.0056}
\end{aligned}
$$


Where the ultimate static load $\left(F_{u s}\right)$ can be determined from Equation (B.11) for the combined cone bond and from Equation (4.2) for steel anchor failure mode.

The adequacy of the predicted model is verified by calculating coefficient of determination $\mathrm{R}^{2}$ and residual analysis (Montgomery, 2013). Residual analysis has been performed to measure the difference between the results obtained from finite element analysis and fitted results of DIF obtained from Equations (5.1) and (5.2). Figures 5-30 and 5-31 present the residual plots. As shown in Figure 5-30, for the combined cone-bond failure, the variance in the residual increases with the increase in the strain rate ratio. For the steel anchor failure (Figure 5-31), the residual exhibits horizontal trend line at strain rate ratio up to $10^{8}$.

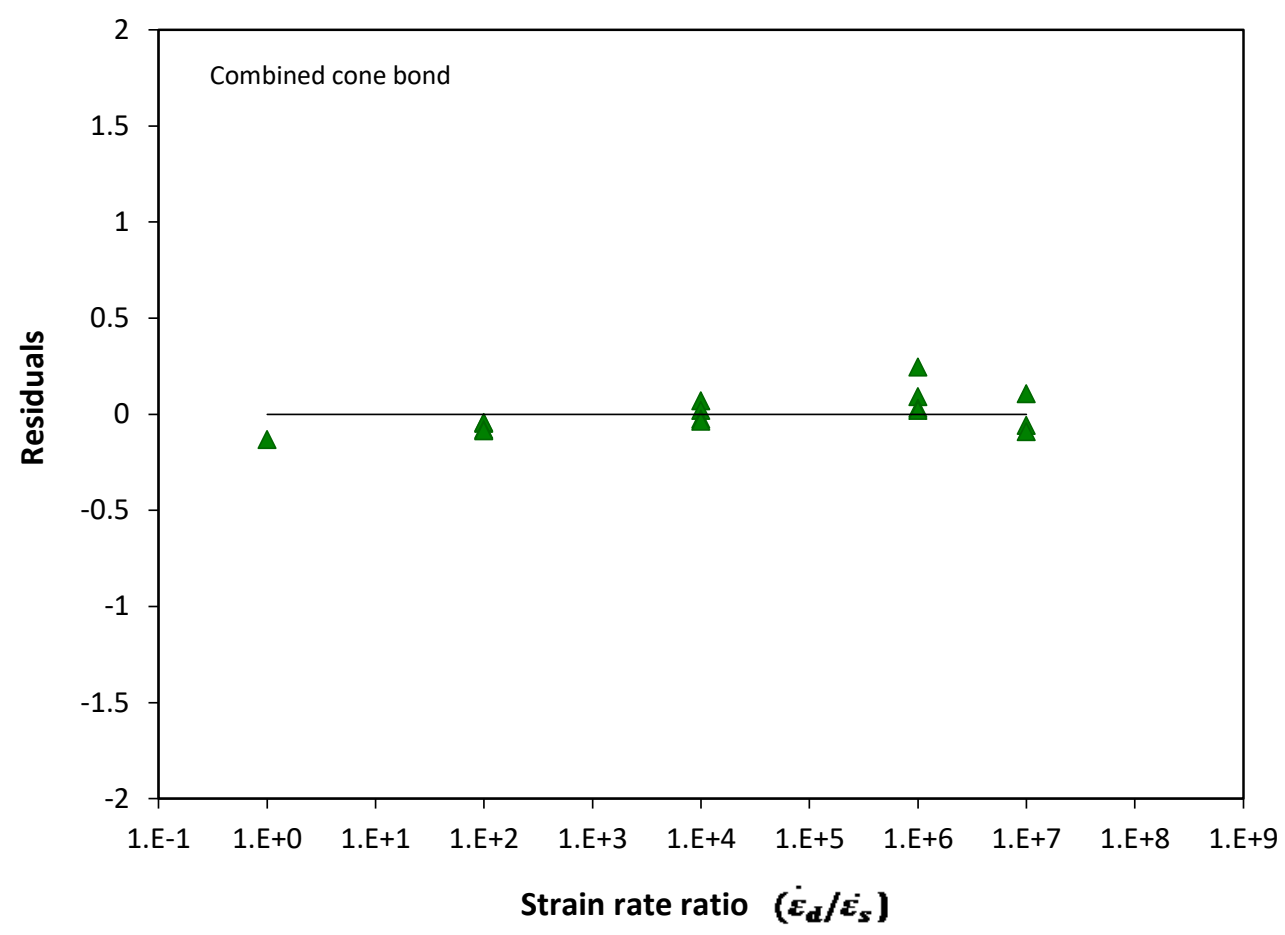

Figure 5-30: Residual versus strain rate ratio for the adhesive anchor exhibited combined cone bond failure 


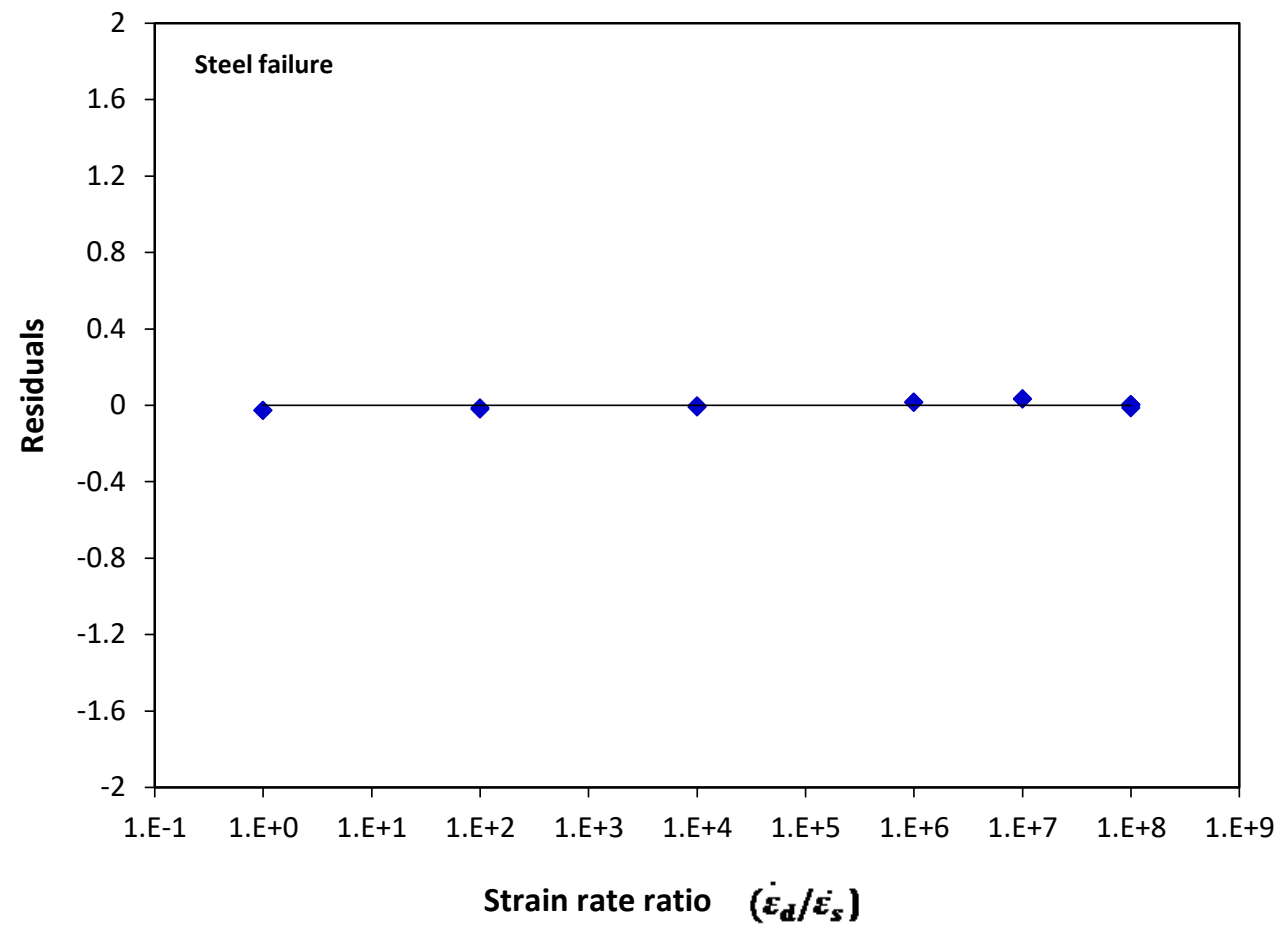

Figure 5-31: Residual versus strain rate ratio for the adhesive anchor exhibited steel failure

In addition, to evaluate the adequacy of the proposed equations, new adhesive anchor models with diameters of $9.5 \mathrm{~mm}, 12.7 \mathrm{~mm}, 15.9 \mathrm{~mm}$ and $19.1 \mathrm{~mm}$ and embedment depths of $89 \mathrm{~mm}, 114 \mathrm{~mm}$ and $140 \mathrm{~mm}$ were developed. The relation between the DIF obtained from the finite element analysis of the new developed models and the regression models (Equations (5.1) and (5.2)) are presented as shown in Figures 5-32 and 5-33 for the combined cone-bond failure and steel failure modes respectively. As shown in the figures, the DIF is distributed around the equality line. However, some divergence was observed for the higher values of the DIF for the combined cone bond failure mode where the residual increased at higher strain rates. 


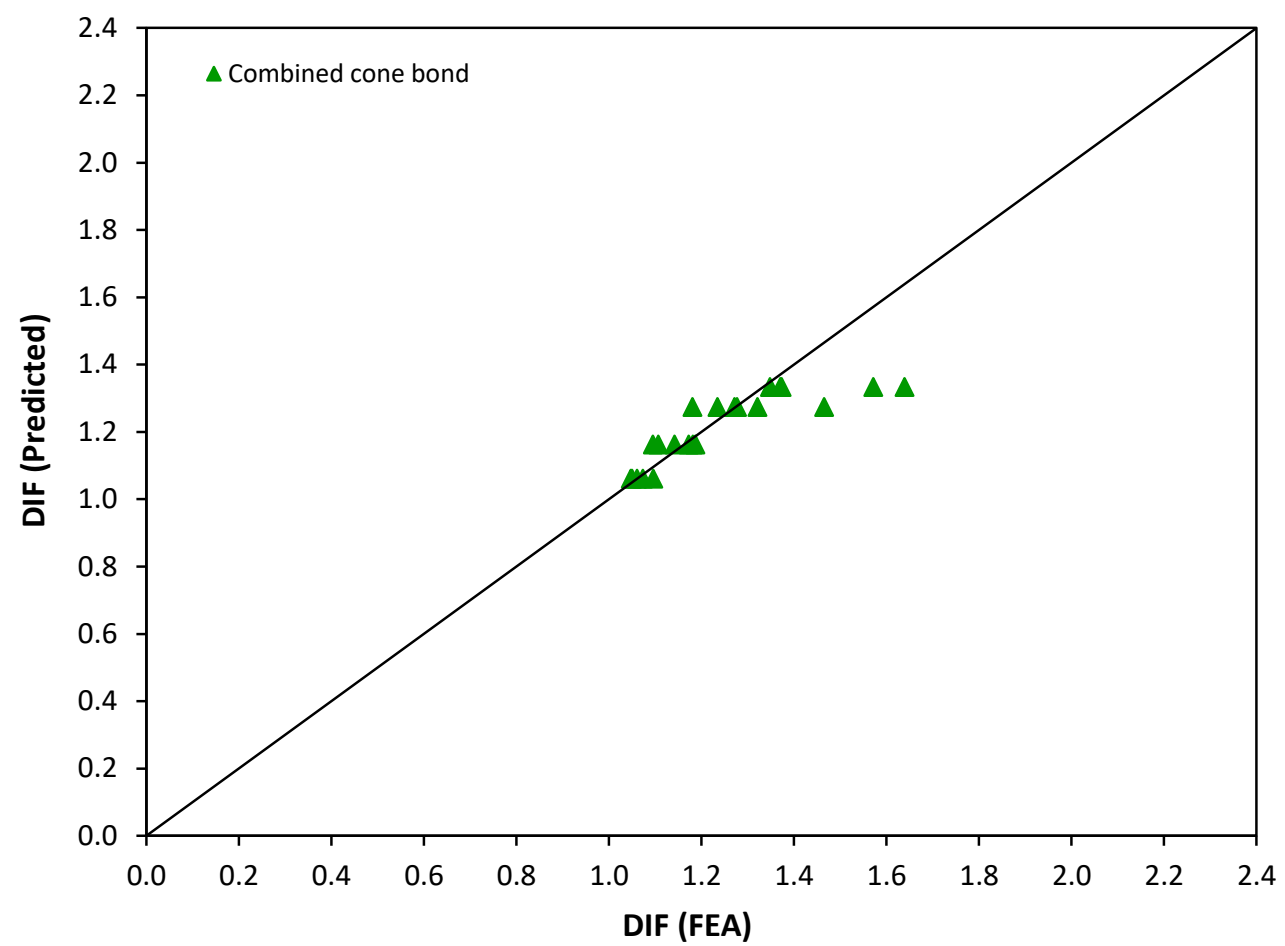

Figure 5-32: DIF obtained from the finite element analysis versus the predicted DIF for the adhesive anchors exhibited combined cone bond failure

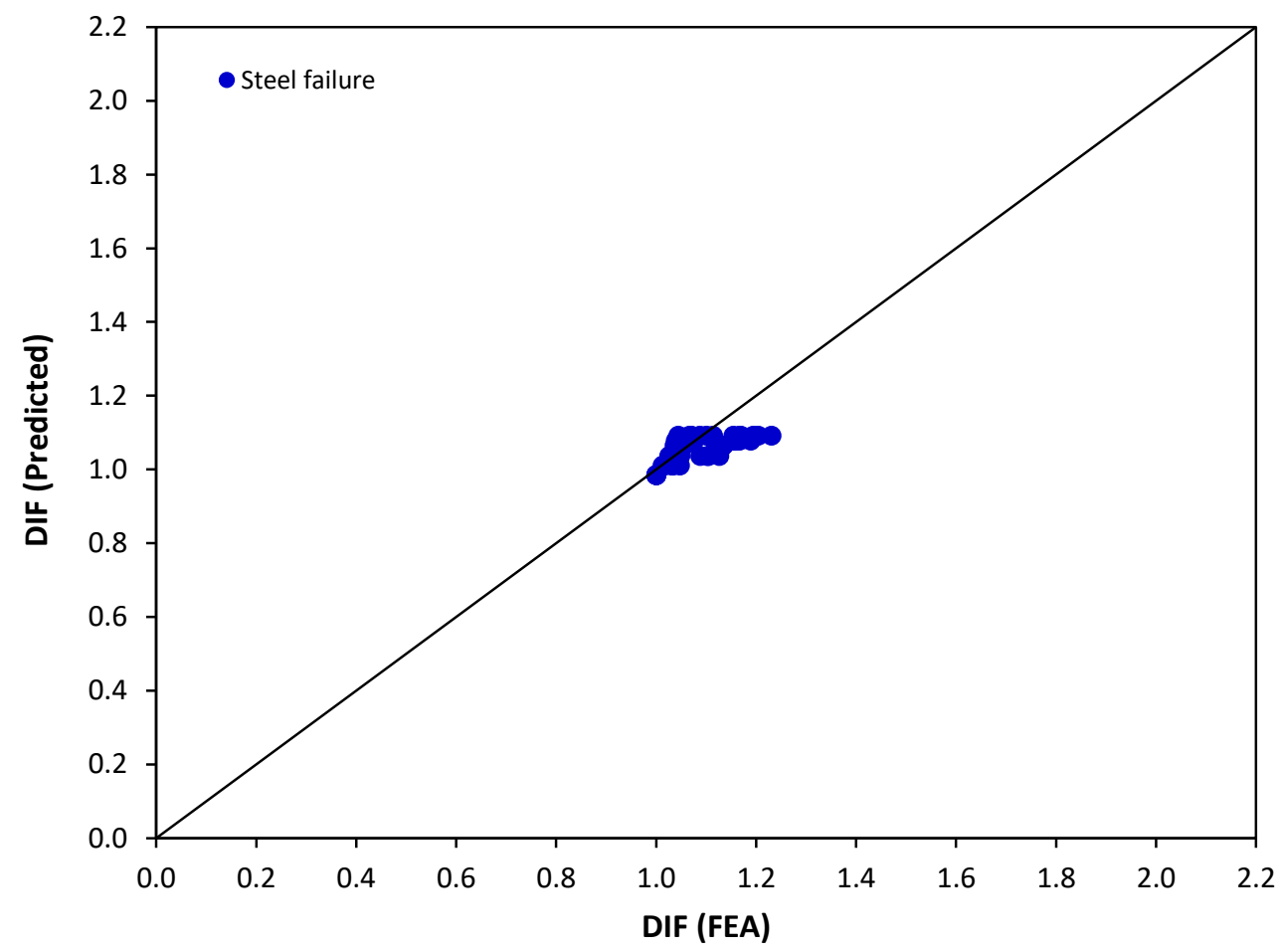

Figure 5-33: DIF obtained from the finite element analysis versus the predicted DIF for the adhesive anchors exhibited steel failure 
As mentioned in Chapter 4, Fujikake et al. (2003) proposed equation to determine the ultimate dynamic load of the anchorage system exhibited concrete cone breakout failure (Equation 4.10). Also, Fujikake et al. (2003) proposed equations to determine the ultimate dynamic load for the combined cone bond failure mode (Fujikake et al., 2003) as follows:

$F_{c c b}=F_{c d}+F_{b}$

$F_{b}=\pi \cdot d_{h} \cdot h_{b} \cdot \tau_{b s} \cdot\left(\frac{\dot{\varepsilon}_{d}}{\dot{\varepsilon}_{s}}\right)^{0.013}$

Where $F_{c c b}$ is the ultimate tensile load for the combined cone bond failure, $F_{c d}$ is the ultimate dynamic concrete cone breakout calculated by Equation (4.10), $F_{b}$ is the ultimate tensile load for the bond failure mode, $h_{b}$ is the bond failure depth $\left(h_{b}=h_{e f}-h_{c}\right), h_{c}$ is the failure cone depth in the combined cone bond failure mode $\left(h_{c}=35 \mathrm{~mm}\right), \alpha=3.48 \times 10^{-3}$, $\theta$ is the crack propagation angle $\left(\theta=60^{\circ}\right)$, where the concrete cone breakout angle is equal to $30^{\circ}, \tau_{b s}$ is the static bond strength $\left(\tau_{b s}=19 \mathrm{MPa}\right)$.

In order to verify the results obtained from the finite element analysis, a comparison has been made between the ultimate load obtained from the finite element analysis and the proposed equations by Fujikake et al. (Equations 4.10 and 5.5) for the concrete cone breakout failure and the combined cone-bond failure modes respectively (Fujikake et al., 2003). The embedment depth $\left(h_{e f}\right)$ substituted in Equation (5.5) is equal to the cone failure depth $\left(h_{c}\right)$ for the combined cone-bond failure mode. Tables 5-11 and 5-12 show a comparison of the ultimate load obtained from the finite element analysis for the concrete cone breakout failure and combined cone-bond failure modes and the proposed equations 
by Fujikake et al. (Fujikake et al., 2003). It can be seen from Tables 5-11 and 5-12 that the ultimate loads obtained from the finite element analysis agree well with the proposed equations by Fujikake et al. (2003).

Table 5-11: Comparison between ultimate load obtained from the FEA and the proposed equations by Fujikake et al. (2003) for concrete cone breakout failure mode

\begin{tabular}{|c|c|c|c|c|}
\hline $\begin{array}{c}\mathbf{d} \\
(\mathbf{m m})\end{array}$ & $\begin{array}{c}\mathbf{h}_{\text {ef }} \\
(\mathbf{m m})\end{array}$ & $\begin{array}{c}\text { Fus FEA } \\
(\mathbf{k N})\end{array}$ & $\begin{array}{c}\text { Fdd }_{\text {cd }} \text { Fujikake } \\
(\mathbf{k N})\end{array}$ & $\begin{array}{c}\text { Fus FEA/ } \\
\text { Fcd }_{\text { Fujikake }}\end{array}$ \\
\hline 12.7 & 76.2 & 57.12 & 53.10 & 1.08 \\
\hline 12.7 & 101.6 & 80.45 & 79.96 & 1.01 \\
\hline 15.9 & 76.2 & 65.69 & 54.27 & 1.21 \\
\hline 15.9 & 101.6 & 87.31 & 81.31 & 1.07 \\
\hline 19.1 & 76.2 & 69.62 & 55.45 & 1.26 \\
\hline 19.1 & 101.6 & 105.49 & 82.67 & 1.28 \\
\hline
\end{tabular}


Table 5-12: Comparison between ultimate dynamic load obtained from the FEA and the proposed equations by Fujikake et al. (2003) for combined cone bond failure mode

\begin{tabular}{|c|c|c|c|c|c|c|c|c|c|c|c|}
\hline \multirow{2}{*}{$\begin{array}{c}\mathrm{d} \\
(\mathrm{mm})\end{array}$} & \multirow{2}{*}{$\begin{array}{c}h_{\text {ef }} \\
(\mathbf{m m})\end{array}$} & \multirow{2}{*}{$\begin{array}{c}\dot{\varepsilon}_{s} \\
\left(\mathbf{s}^{-1}\right)\end{array}$} & \multirow{2}{*}{$\begin{array}{c}\dot{\varepsilon}_{d} \\
\left(\mathbf{s}^{-1}\right)\end{array}$} & \multirow{2}{*}{$\dot{\varepsilon}_{d} / \dot{\varepsilon}_{s}$} & \multicolumn{2}{|c|}{ FEA } & \multirow{2}{*}{$\begin{array}{c}\text { Regression } \\
\text { Fu }_{\mathbf{u}}(\mathbf{k N})\end{array}$} & \multicolumn{3}{|c|}{ Fujikake Equation (5.5) } & \multirow{2}{*}{$\begin{array}{c}\text { Fud FEA/ } \\
\text { Fecb Fujikake }\end{array}$} \\
\hline & & & & & $F_{\text {us }}(\mathbf{k N})$ & $F_{u d}(k N)$ & & $F_{c d}(k N)$ & $F_{b}(k N)$ & $F_{\text {ccb }}(k N)$ & \\
\hline 12.7 & 76.2 & $10^{-5}$ & $10^{-3}$ & $10^{2}$ & 57.12 & 63.33 & 60.63 & 18.48 & 38.38 & 56.86 & 1.11 \\
\hline 12.7 & 76.2 & $10^{-5}$ & $10^{-1}$ & $10^{4}$ & 57.12 & 67.39 & 66.42 & 20.88 & 40.75 & 61.63 & 1.09 \\
\hline 12.7 & 76.2 & $10^{-5}$ & 10 & $10^{6}$ & 57.12 & 71.14 & 72.76 & 31.01 & 43.26 & 74.28 & 0.96 \\
\hline 12.7 & 76.2 & $10^{-5}$ & $10^{2}$ & $10^{7}$ & 57.12 & 88.11 & 76.15 & 44.55 & 44.58 & 89.12 & 0.99 \\
\hline 12.7 & 101.6 & $10^{-5}$ & $10^{-3}$ & $10^{2}$ & 80.45 & 88.59 & 85.39 & 18.48 & 62.04 & 80.52 & 1.10 \\
\hline 12.7 & 101.6 & $10^{-5}$ & $10^{-1}$ & $10^{4}$ & 80.45 & 92.31 & 93.54 & 20.88 & 65.87 & 86.75 & 1.06 \\
\hline 12.7 & 101.6 & $10^{-5}$ & 10 & $10^{6}$ & 80.45 & 95.49 & 102.47 & 31.01 & 69.93 & 100.95 & 0.95 \\
\hline 12.7 & 101.6 & $10^{-5}$ & $10^{2}$ & $10^{7}$ & 80.45 & 98.60 & 107.25 & 44.55 & 72.06 & 116.61 & 0.85 \\
\hline 15.9 & 76.2 & $10^{-5}$ & $10^{-3}$ & $10^{2}$ & 65.69 & 69.70 & 69.72 & 19.28 & 47.00 & 66.28 & 1.05 \\
\hline 15.9 & 76.2 & $10^{-5}$ & $10^{-1}$ & $10^{4}$ & 65.69 & 74.94 & 76.38 & 21.79 & 49.90 & 71.69 & 1.05 \\
\hline 15.9 & 76.2 & $10^{-5}$ & 10 & $10^{6}$ & 65.69 & 87.36 & 83.67 & 32.37 & 52.97 & 85.34 & 1.02 \\
\hline 15.9 & 76.2 & $10^{-5}$ & $10^{2}$ & $10^{7}$ & 65.69 & 118.21 & 87.57 & 46.49 & 54.58 & 101.07 & 1.17 \\
\hline 15.9 & 101.6 & $10^{-5}$ & $10^{-3}$ & $10^{2}$ & 87.31 & 94.88 & 92.67 & 19.28 & 75.97 & 95.25 & 1.00 \\
\hline 15.9 & 101.6 & $10^{-5}$ & $10^{-1}$ & $10^{4}$ & 87.31 & 101.48 & 101.52 & 21.79 & 80.66 & 102.45 & 0.99 \\
\hline 15.9 & 101.6 & $10^{-5}$ & 10 & $10^{6}$ & 87.31 & 108.31 & 111.21 & 32.37 & 85.63 & 118.00 & 0.92 \\
\hline 15.9 & 101.6 & $10^{-5}$ & $10^{2}$ & $10^{7}$ & 87.31 & 124.28 & 116.40 & 46.49 & 88.23 & 134.73 & 0.92 \\
\hline 19.1 & 76.2 & $10^{-5}$ & $10^{-1}$ & $10^{4}$ & 69.62 & 89.32 & 80.95 & 22.70 & 60.98 & 83.68 & 1.07 \\
\hline 19.1 & 76.2 & $10^{-5}$ & 10 & $10^{6}$ & 69.62 & 114.01 & 88.68 & 33.72 & 64.75 & 98.47 & 1.16 \\
\hline
\end{tabular}




\begin{tabular}{|c|c|c|c|c|c|c|c|c|c|c|c|}
\hline 19.1 & 101.6 & $10^{-5}$ & $10^{-3}$ & $10^{2}$ & 105.49 & 113.94 & 111.97 & 20.09 & 92.85 & 112.94 & 1.01 \\
\hline 19.1 & 101.6 & $10^{-5}$ & $10^{-1}$ & $10^{4}$ & 105.49 & 120.78 & 122.66 & 22.70 & 98.58 & 121.28 & 1.00 \\
\hline 19.1 & 101.6 & $10^{-5}$ & 10 & $10^{6}$ & 105.49 & 139.14 & 134.37 & 33.72 & 104.66 & 138.38 & 1.01 \\
\hline 19.1 & 101.6 & $10^{-5}$ & $10^{2}$ & $10^{7}$ & 105.49 & 196.53 & 140.63 & 48.44 & 107.84 & 156.28 & 1.26 \\
\hline 19.1 & 127.0 & $10^{-5}$ & $10^{-3}$ & $10^{2}$ & 157.28 & 161.79 & 166.94 & 20.09 & 128.26 & 148.35 & 1.09 \\
\hline 19.1 & 127.0 & $10^{-5}$ & $10^{-1}$ & $10^{4}$ & 157.28 & 169.02 & 182.87 & 22.70 & 136.18 & 158.88 & 1.06 \\
\hline 19.1 & 127.0 & $10^{-5}$ & 10 & $10^{6}$ & 157.28 & 185.58 & 200.33 & 33.72 & 144.58 & 178.30 & 1.04 \\
\hline 19.1 & 127.0 & $10^{-5}$ & $10^{2}$ & $10^{7}$ & 157.28 & 217.96 & 209.68 & 48.44 & 148.97 & 197.41 & 1.10 \\
\hline 19.1 & 152.4 & $10^{-5}$ & $10^{-3}$ & $10^{2}$ & 179.40 & 187.48 & 190.42 & 20.09 & 163.67 & 183.76 & 1.02 \\
\hline 19.1 & 152.4 & $10^{-5}$ & $10^{-1}$ & $10^{4}$ & 179.40 & 196.47 & 208.59 & 22.70 & 173.77 & 196.47 & 1.00 \\
\hline 19.1 & 152.4 & $10^{-5}$ & 10 & $10^{6}$ & 179.40 & 211.45 & 228.51 & 33.72 & 184.49 & 218.22 & 0.97 \\
\hline 19.1 & 152.4 & $10^{-5}$ & $10^{2}$ & $10^{7}$ & 179.40 & 234.18 & 239.17 & 48.44 & 190.10 & 238.54 & 0.98 \\
\hline
\end{tabular}

${ }^{*} \mathrm{~F}_{\mathrm{us}}$ : ultimate static load obtained from FEA, $\mathrm{F}_{\mathrm{ud}}$ : ultimate dynamic load obtained from FEA 


\subsection{Adhesive anchors under shear load}

\subsubsection{Finite element modelling for adhesive anchors under shear load}

Shear behaviour of adhesive anchors was investigated using LS-DYNA finite element program. Adhesive anchor diameters of $12.7 \mathrm{~mm}, 15.9 \mathrm{~mm}$ and $19.1 \mathrm{~mm}$ with embedment depths of $76.2 \mathrm{~mm}, 101.6 \mathrm{~mm}$, and $152.4 \mathrm{~mm}$, respectively, were investigated. Figure 534 shows the geometric configuration and boundary conditions for the adhesive anchor model.

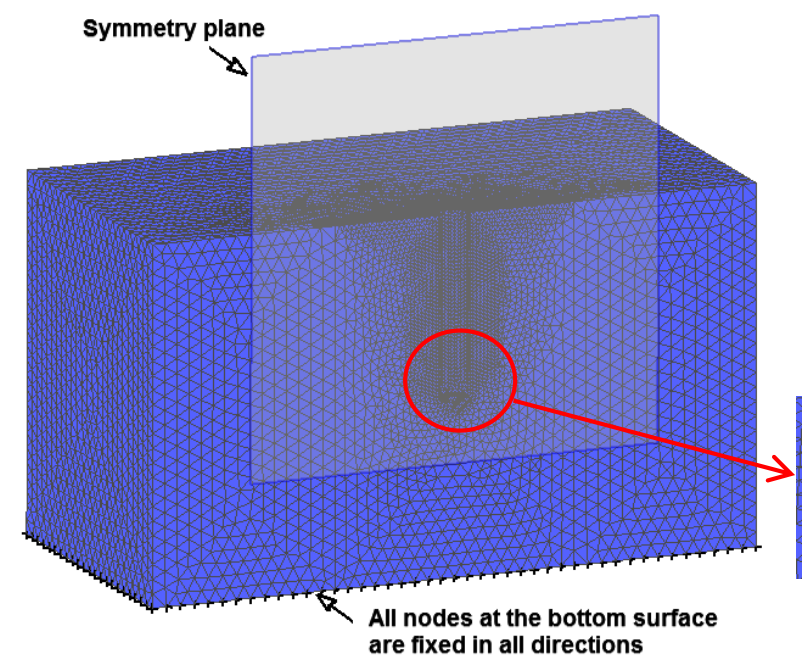

(a) Concrete mesh
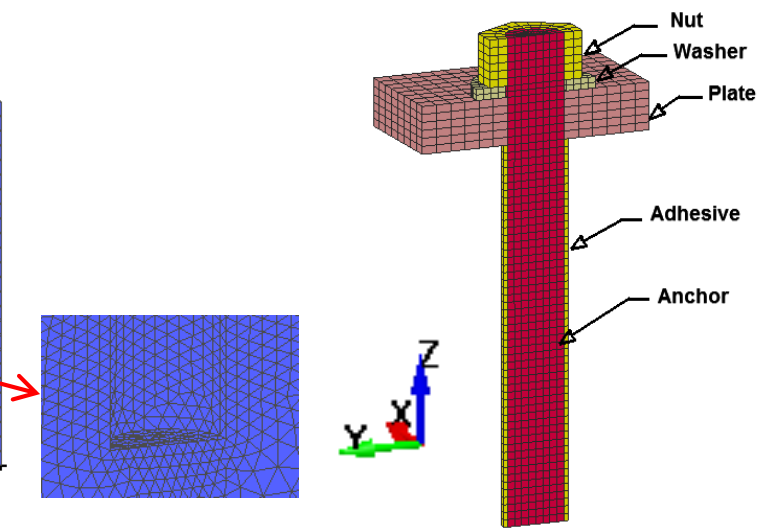

(b) Adhesive anchor mesh

Figure 5-34: Geometric configuration and boundary conditions of adhesive anchor model

\subsubsection{Validation of adhesive anchor model under shear load}

The adhesive anchor model was validated by comparing the finite element results with the experimental results obtained by Cattaneo and Muciaccia (Cattaneo \& Muciaccia, 2015). A finite element model, representing the test conducted by Cattaneo and Muciaccia, was 
developed using LS-DYNA. The adhesive anchor was placed at a distance of $40 \mathrm{~mm}$ from the concrete free edge. Epoxy adhesive of $1 \mathrm{~mm}$ thickness was used. The steel anchor used had yield strength of $400 \mathrm{MPa}$, diameter of $12-\mathrm{mm}$ and embedment depth of $110 \mathrm{~mm}$. The compressive strength of the concrete block used in the experimental test and numerical model was $90 \mathrm{MPa}$.

A mesh sensitivity analysis was carried out to obtain optimum mesh size that gives converged results to the experimental test results. BOUNDARY_PRESCRIBED_MOTION_SET in y direction was applied on the anchor plate to represent the shear load. Table 5-13 shows the results of the mesh sensitivity analysis for the adhesive anchor under shear load.

Table 5-13: Effect of mesh size refinement on the convergence of ultimate shear load for adhesive anchor

\begin{tabular}{|c|c|c|c|c|c|c|c|c|c|}
\hline \multirow[t]{3}{*}{$\begin{array}{l}\text { Model } \\
\text { No. }\end{array}$} & \multicolumn{4}{|c|}{$\begin{array}{c}\text { Mesh size } \\
(\mathrm{mm})\end{array}$} & \multicolumn{2}{|c|}{$\begin{array}{c}\text { Ultimate shear load } \\
(\mathrm{kN})\end{array}$} & \multicolumn{2}{|c|}{$\begin{array}{l}\text { Displacement } \\
(\mathrm{mm})\end{array}$} & \multirow[t]{3}{*}{$\begin{array}{l}\text { Failure } \\
\text { mode }\end{array}$} \\
\hline & \multirow[t]{2}{*}{ Anchor } & \multirow[t]{2}{*}{ Adhesive } & \multicolumn{2}{|c|}{ Concrete } & \multirow[t]{2}{*}{ FEA } & \multirow[t]{2}{*}{ EXP. } & \multirow[t]{2}{*}{ FEA } & \multirow[t]{2}{*}{ EXP. } & \\
\hline & & & Min. & Max. & & & & & \\
\hline 1 & 1 & 1 & 2 & 8 & 17.26 & \multirow{3}{*}{18.65} & 1.27 & \multirow{3}{*}{0.85} & $\begin{array}{l}\text { Concrete } \\
\text { cracking }\end{array}$ \\
\hline 2 & 1 & 1 & 1 & 8 & 17.96 & & 1.27 & & $\begin{array}{l}\text { Concrete } \\
\text { cracking }\end{array}$ \\
\hline 3 & 0.75 & 0.50 & 1 & 8 & 19.31 & & 1.24 & & $\begin{array}{l}\text { Concrete } \\
\text { cracking }\end{array}$ \\
\hline
\end{tabular}

Mesh sizes of $1 \times 1 \times 2 \mathrm{~mm}, 1 \times 1 \times 1 \mathrm{~mm}$ and $0.75 \times 0.5 \times 1 \mathrm{~mm}$ give percentage difference of $8.1 \%, 3.8 \%$ and $3.5 \%$ in the ultimate shear load respectively compared to the experimental work. Mesh size of model No. 3 yielded converged shear load-displacement behaviour to the experimental behaviour reported by Cattaneo and Muciaccia and selected for the analysis. A comparison of shear load-displacement response of the experimental tests and the finite element analysis is presented in Figure 5-35 and shows that the shear load 
increased with the displacement up to the ultimate load followed by a reduction in the shear load until failure. A good agreement was obtained between the finite element analysis and the experimental results. Concrete cracking failure mode was observed from the finite element results similar to that observed by Cattaneo and Muciaccia as shown in Figure 536.

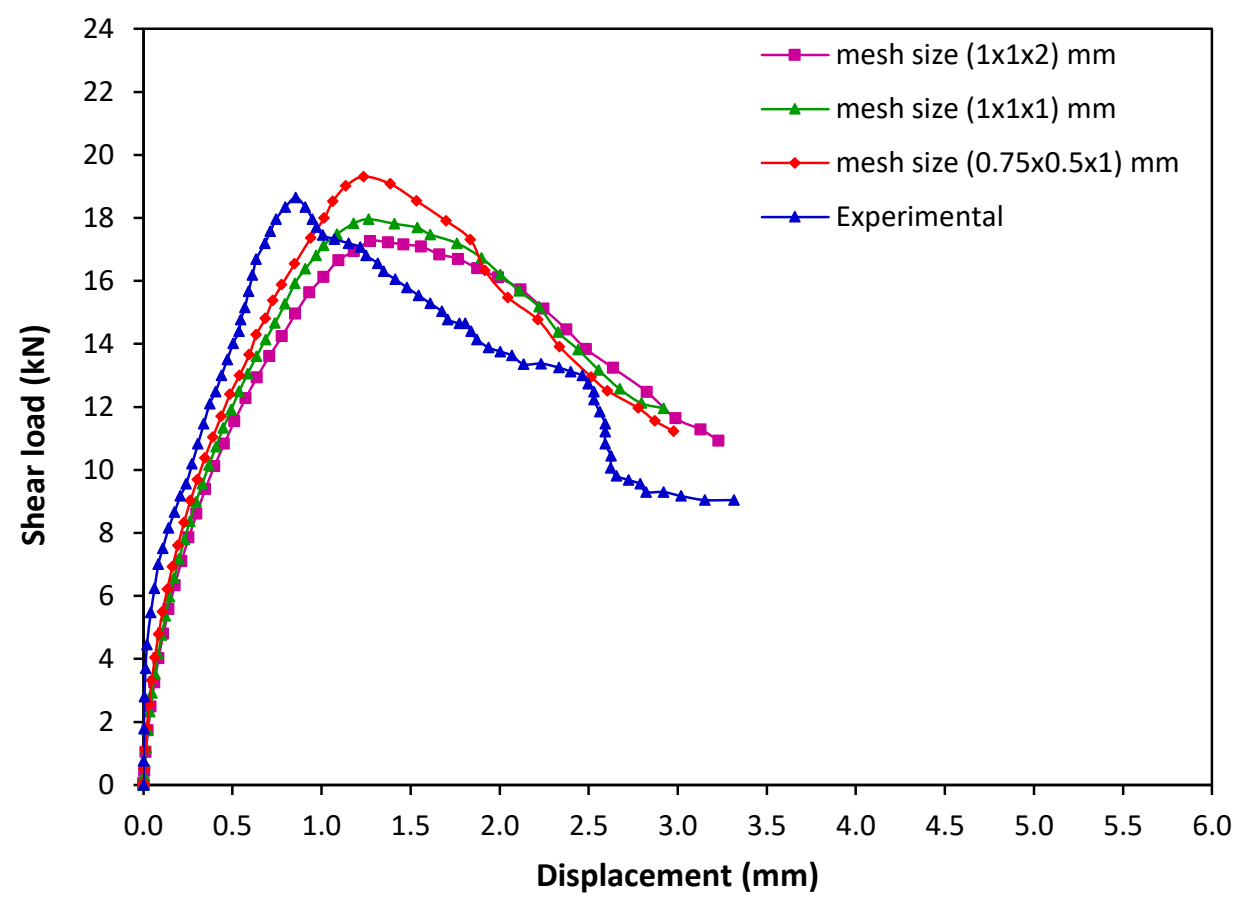

Figure 5-35: Comparison of shear load-displacement relation between FEA and experimental results obtained by Cattaneo et al. (Cattaneo \& Muciaccia, 2015)

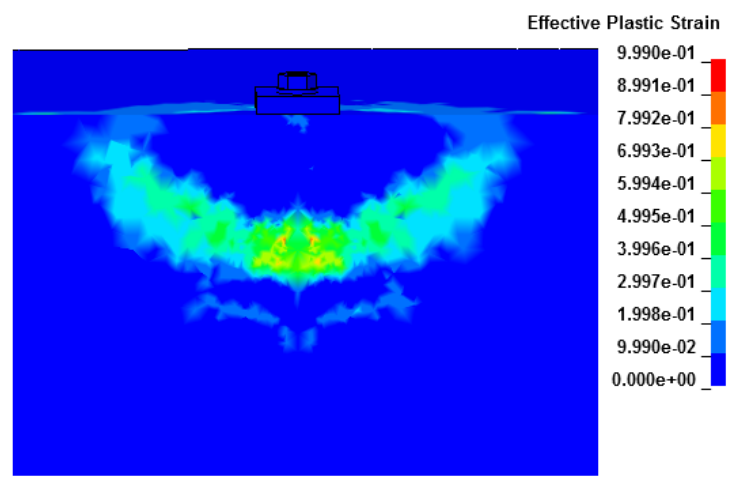

(a)

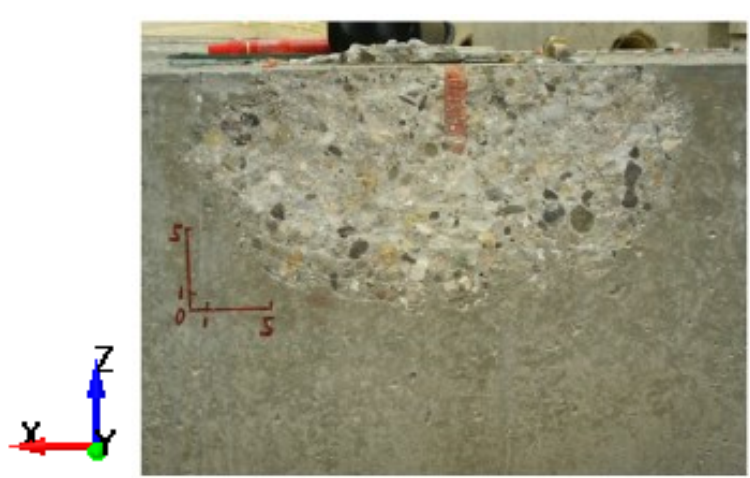

(b)

Figure 5-36: Failure mode obtained from:(a) finite element analysis and (b) experimental results obtained by Cattaneo et al. (Cattaneo \& Muciaccia, 2015) 


\subsubsection{Comparison of finite element results with design methods}

For the adhesive anchors placed far away from the concrete free edge, pryout failure mode or anchor failure is often the dominant failure modes. The shear failure load of anchors exhibiting pryout failure can be calculated according to ACI 318 (ACI Committee 318, 2011) Equation (4.14). The shear failure load of anchors that exhibit steel anchor failure can be calculated according to ACI 318 as in Equation (4.18) (ACI Committee 318, 2011). A comparison of the ultimate shear load obtained from the finite element analysis at strain rate of $10^{-5} \mathrm{~s}^{-1}$ and ACI 318 method (Equations 4.14 and 4.18) is presented in Table 5-14. It can be seen that the ultimate shear capacity increased with increase in the anchor diameter from $12.7 \mathrm{~mm}$ to $19.1 \mathrm{~mm}$, at the same embedment depth. Similar observation on the effect of anchor diameter on the shear capacity was reported by Jebara et al. (Jebara et al., 2016). A good agreement is obtained between the ultimate shear load obtained from the finite element analysis and the ACI 318 method.

Table 5-14: Comparison between FEA and ACI 318 method

\begin{tabular}{|c|c|c|c|c|c|c|}
\hline \multirow{2}{*}{$\begin{array}{c}\text { Model } \\
\text { No. }\end{array}$} & \multirow{2}{*}{$\begin{array}{c}\mathrm{d} \\
(\mathrm{mm})\end{array}$} & $\begin{array}{c}\mathrm{h}_{\mathrm{ef}} \\
(\mathrm{mm})\end{array}$ & \multicolumn{2}{|c|}{$\begin{array}{c}\text { Failure load } \\
(\mathrm{kN})\end{array}$} & FEA/ & *Failure mode \\
\cline { 4 - 5 } & & & $\begin{array}{c}\text { FEA static } \\
\left(\dot{\varepsilon}=10^{-5} \mathrm{~s}^{-1}\right)\end{array}$ & ACI 318 & & \\
\hline 1 & 12.7 & 76.2 & 79.93 & 78.59 & 1.02 & $\mathrm{~S}$ \\
\hline 2 & 12.7 & 101.6 & 81.64 & 78.59 & 1.04 & $\mathrm{~S}$ \\
\hline 3 & 12.7 & 152.4 & 81.85 & 78.59 & 1.04 & $\mathrm{~S}$ \\
\hline 4 & 15.9 & 76.2 & 94.31 & 109.24 & 0.86 & PR \\
\hline 5 & 15.9 & 101.6 & 103.73 & 123.18 & 0.84 & $\mathrm{~S}$ \\
\hline 6 & 15.9 & 152.4 & 106.5 & 123.18 & 0.86 & $\mathrm{~S}$ \\
\hline 7 & 19.1 & 76.2 & 116.26 & 109.24 & 1.06 & $\mathrm{PR}$ \\
\hline 8 & 19.1 & 101.6 & 146.45 & 168.19 & 0.87 & PR \\
\hline 9 & 19.1 & 152.4 & 164.33 & 177.76 & 0.92 & $\mathrm{~S}$ \\
\hline
\end{tabular}

*Failure mode: $\mathrm{S}=$ Steel anchor failure, $\mathrm{PR}=$ Pryout failure 


\subsubsection{Crack pattern for adhesive anchors under shear load}

Contours of effective plastic strain for adhesive anchors with $12.7 \mathrm{~mm}, 15.9 \mathrm{~mm}$ and 19.1 $\mathrm{mm}$ diameters and embedment depths of $76.2 \mathrm{~mm}, 101.6 \mathrm{~mm}$ and $152.4 \mathrm{~mm}$ at strain rate of $10^{-5} \mathrm{~s}^{-1}$ are shown in Figure 5-37. Two failure modes were observed for the adhesive anchors at low strain rate of $10^{-5} \mathrm{~s}^{-1}$ : pryout failure and steel anchor failure.

As shown in the figure, pryout failure was observed for the 15.9-mm and 19.1-mm diameter anchors with embedment depth of $76.2 \mathrm{~mm}$ whereas steel anchor failure was observed for the $12.7-\mathrm{mm}$ anchor diameter. For the embedment depth of $101.6 \mathrm{~mm}$ pryout failure was observed for the anchor diameter of $19.1 \mathrm{~mm}$. Steel anchor failure was observed for the 12.7- $\mathrm{mm}$ and 15.9-mm anchor diameters wherein the embedment depth to anchor diameter ratio was sufficient to prevent pryout failure. Also, steel anchor failure was observed for all the diameters at embedment depth of $152.4 \mathrm{~mm}$. In general, the failure mode of the adhesive anchor system depends on the anchor stiffness (diameter) and embedment depth. For the same anchor diameter, increase in the embedment depth can change the failure mode from concrete pryout failure to steel anchor fracture. Conversely at the same embedment depth, increase in anchor diameter can lead to concrete pryout failure. When the shear load is applied on the anchor plate, the anchor resists the shear load and transfers the load to the adhesive and then to the concrete resulting in compressive stresses in the concrete in front of the anchor. These stresses increase with the increase in the applied shear load, create concrete cracking under the anchor plate, result in displacement of the anchor plate in vertical direction, rotation of the anchor plate and generating a compression force on the concrete at the front end tip of the plate. A rotational moment will be generated due to the tensile force in the steel anchor and the compression force on the concrete that 
results in breaking half concrete cone at the back side on the anchor resulting in pryout failure. On the other hand, when the concrete resistance to the applied shear load is higher than the anchor strength, the anchor bends due to the applied shear load resulted in steel anchor failure. Cook et al. attributed the steel anchor failure of the anchorage to concrete system under shear load to the interaction of the shear, tensile and bending stresses (Cook et al., 2013).

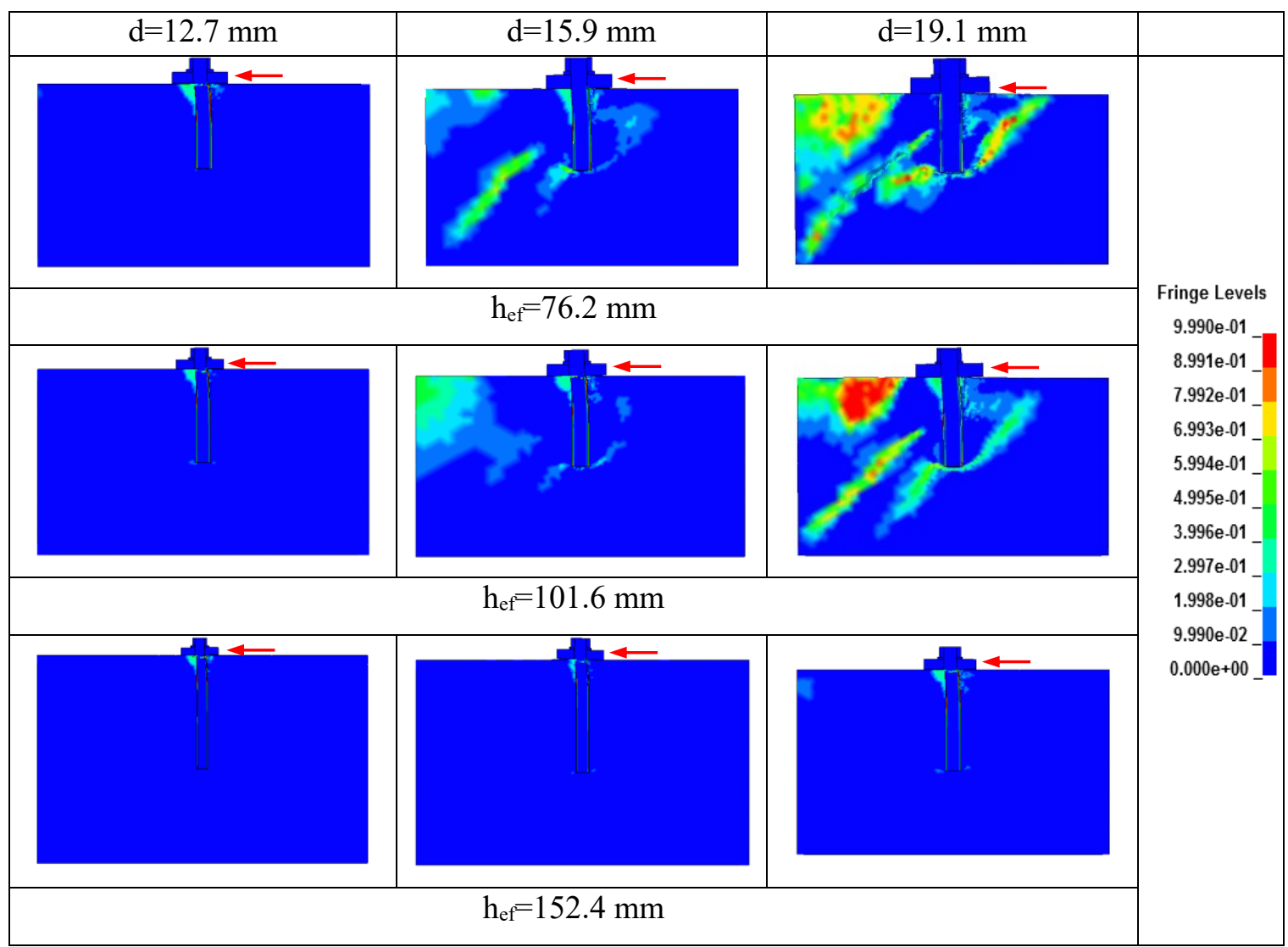

Figure 5-37: Plastic strain contours for adhesive anchor under shear load at strain rate of $10^{-5} \mathrm{~s}^{-1}$ 


\subsubsection{Effect of strain rate on the level of damage and failure mode}

Figures 5-38, 5-39 and 5-40 present failure mechanism for 12.7-mm, 15.9-mm and 19.1$\mathrm{mm}$ diameter adhesive anchors subjected to different shear strain rates of loading. The cracks initiated at the interface between concrete and adhesive on the top surface of the concrete in the direction of the applied load (ahead of the anchor) and propagated along the anchor embedment depth leading to failure. As shown in Figures 5-38 and 5-39, at strain rates ranging from $10^{-3} \mathrm{~s}^{-1}$ to $10^{3} \mathrm{~s}^{-1}$, concrete spalling followed by steel anchor failure was observed for the 12.7- $\mathrm{mm}$ and 15.9-mm diameter anchors. For anchor diameter of $19.1 \mathrm{~mm}$ (Figure 5-40) concrete spalling was observed at strain rates of $10^{-3} \mathrm{~s}^{-1}, 10^{-1} \mathrm{~s}^{-1}$ and $10 \mathrm{~s}^{-1}$ at embedment depth of $76.2 \mathrm{~mm}$, while steel anchor failure was observed at strain rates of $10^{2} \mathrm{~s}^{-1}$ and $10^{3} \mathrm{~s}^{-1}$. Also, steel anchor failure was observed for embedment depths of 101.6 $\mathrm{mm}$ and $152.4 \mathrm{~mm}$ at strain rates ranging from $10^{-3} \mathrm{~s}^{-1}$ to $10^{3} \mathrm{~s}^{-1}$. When concrete spalling occurs, the stresses are concentrated in the concrete in front of the anchor resulting in concrete cracking without breakout of a concrete cone behind the anchor.

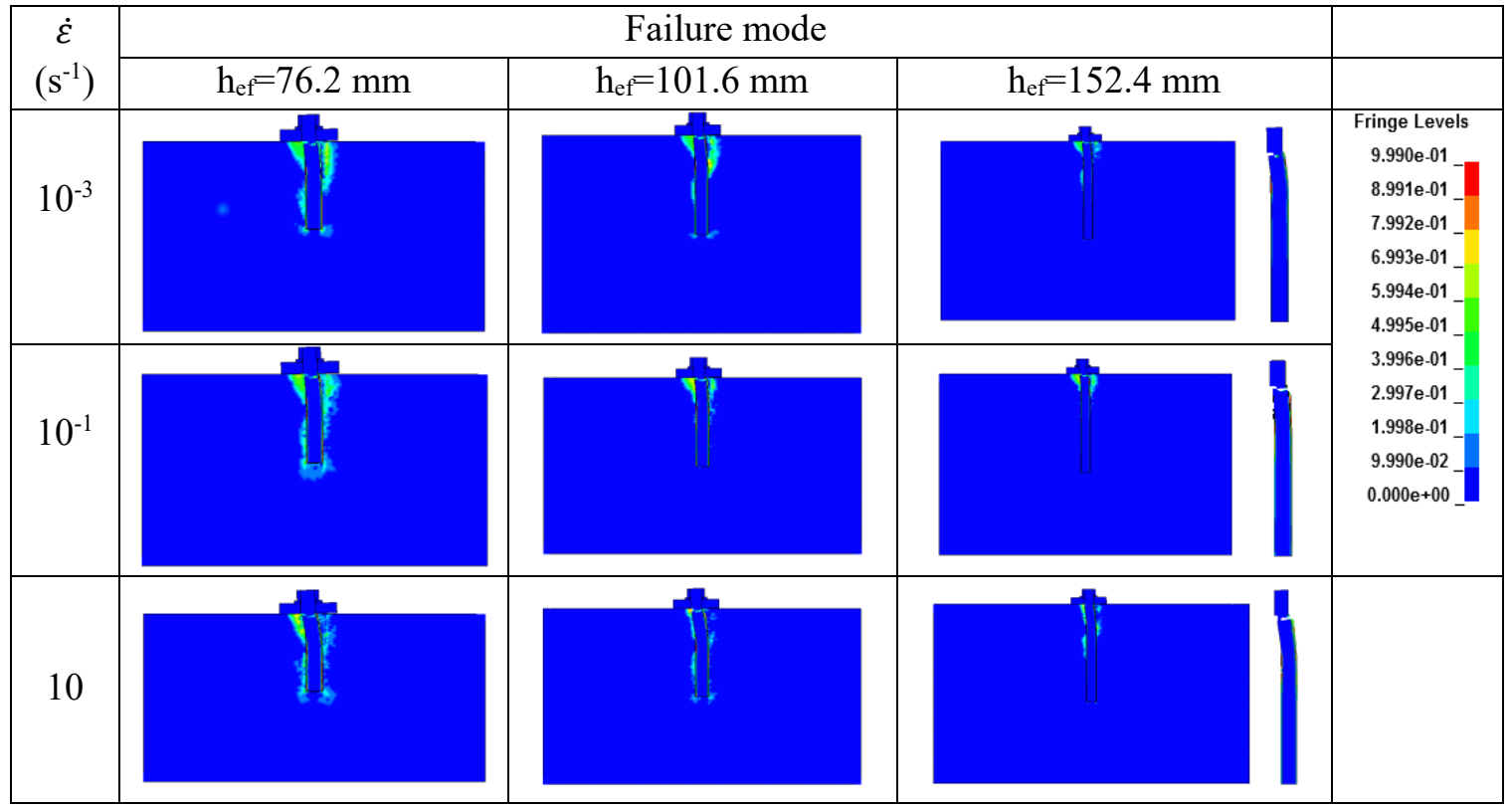




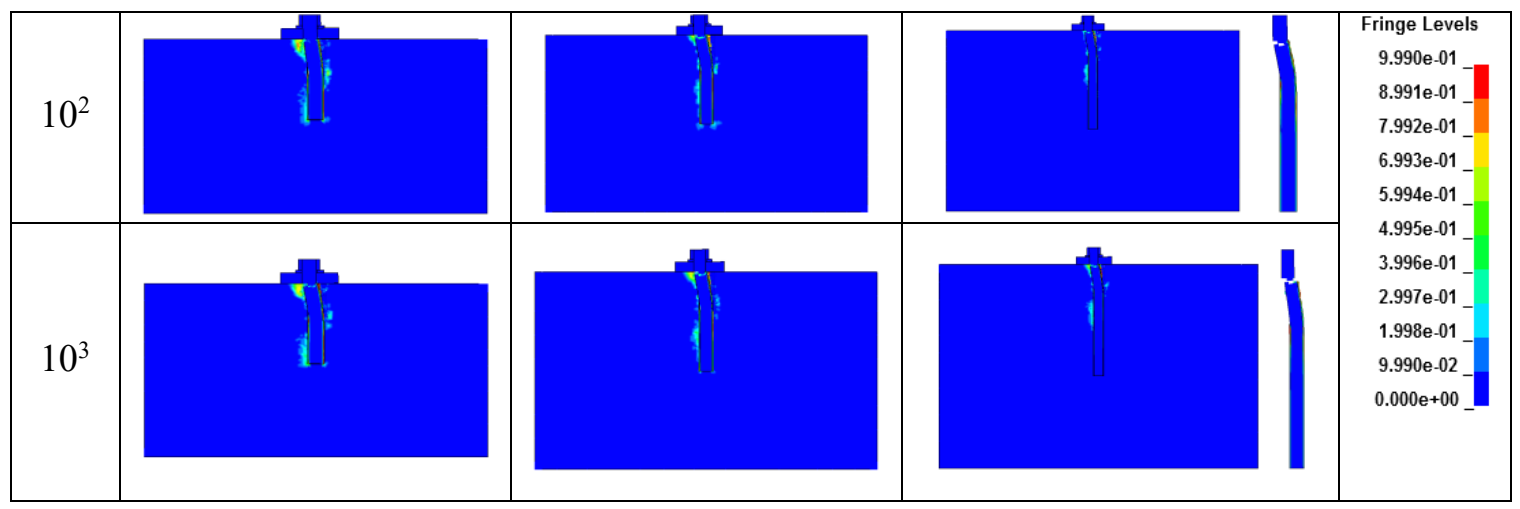

Figure 5-38: Failure mode for $12.7 \mathrm{~mm}$ diameter adhesive anchor at different strain rates

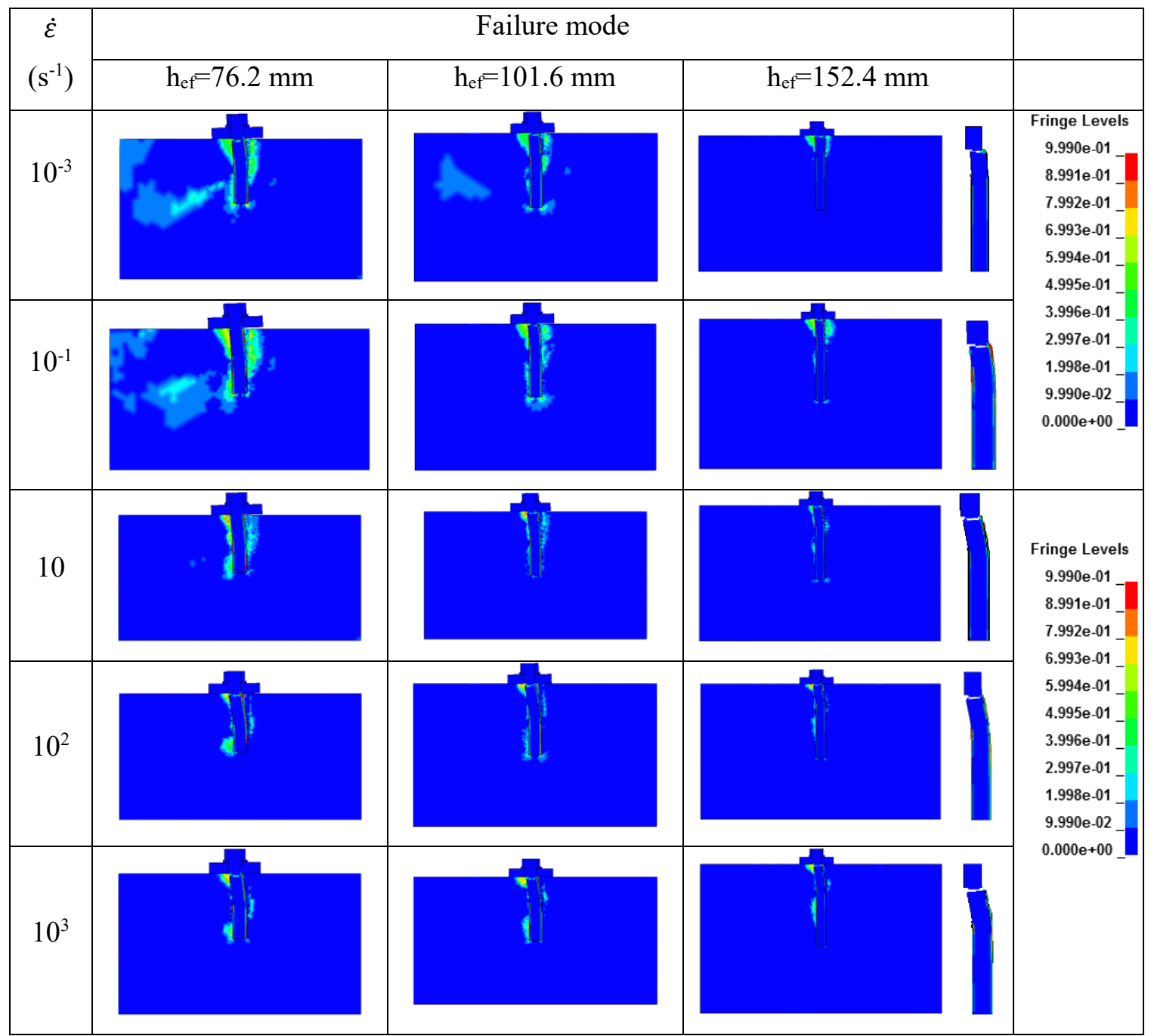

Figure 5-39: Failure mode for $15.9 \mathrm{~mm}$ diameter adhesive anchor at different strain rates 


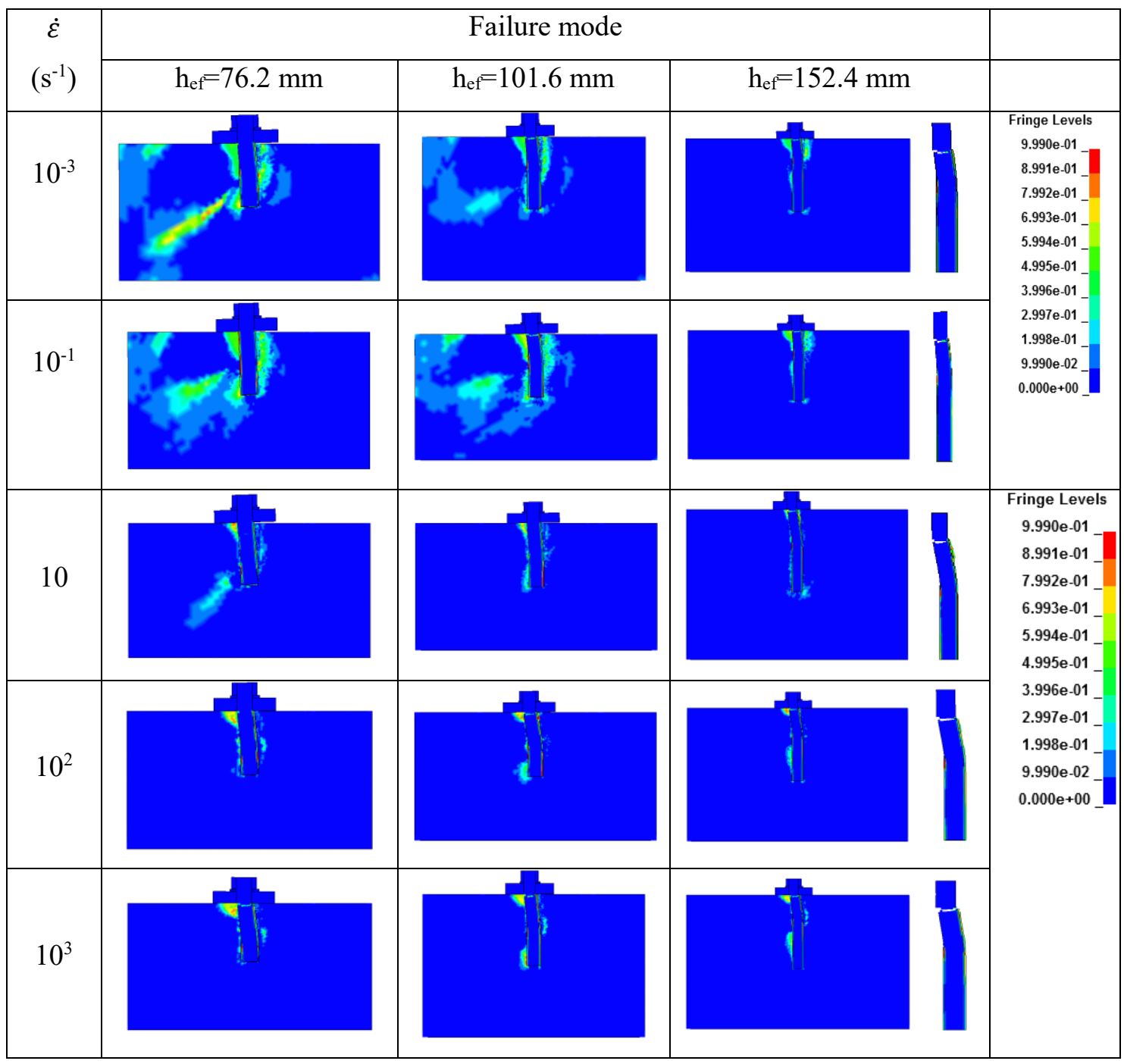

Figure 5-40: Failure mode for $19.1 \mathrm{~mm}$ diameter adhesive anchor at different strain rates

It can be seen from Figure 5-38, 5-39 and 5-40 that the strain rate has an influence on the failure mode. The figures show that, anchor diameter of $19.1 \mathrm{~mm}$ exhibited higher level of concrete damage compared to the smaller anchor diameter of $12.7-\mathrm{mm}$ for all the strain rates investigated.

Typical Von-Mises stress contours (MPa) in the steel anchor are presented in Figure 5-41 to show the mechanism of steel anchor failure of $19.1-\mathrm{mm}$ diameter adhesive anchor with $152.4 \mathrm{~mm}$ embedment depth at strain rate of $10 \mathrm{~s}^{-1}$. As shown in the figure, the Von-Mises 
stresses are concentrated around the top part of the anchor where the shear load is applied then propagated along the embedment depth of the anchor, followed by anchor bending, bond failure and steel anchor failure.

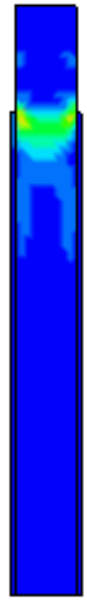

(a) $\mathrm{t}=0.05 \mathrm{~ms}$

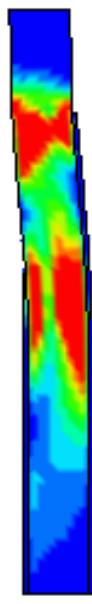

(b) $\mathrm{t}=0.28 \mathrm{~ms}$

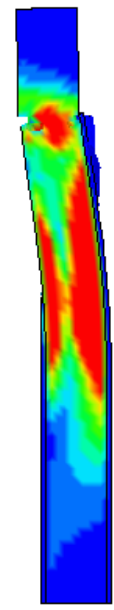

(c) $\mathrm{t}=0.40 \mathrm{~ms}$

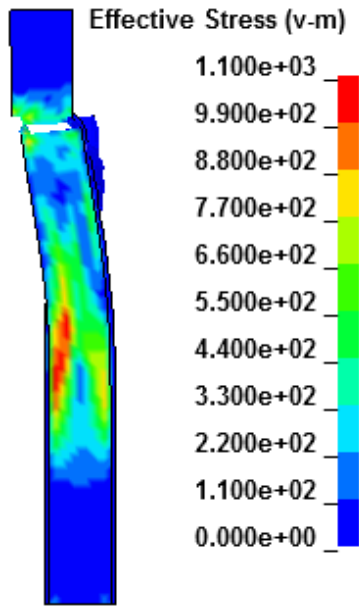

(d) $\mathrm{t}=0.5 \mathrm{~ms}$

Figure 5-41: Failure mechanism of $19.1 \mathrm{~mm}$ diameter adhesive anchor with $152.4 \mathrm{~mm}$ embedment depth; (a) stress concentration around the anchor, (b) anchor bending with bond failure, (c) initiation of anchor fracture, (d) complete anchor failure

Table 5-15 presents the failure modes for the adhesive anchors at diffent strain rates.

Table 5-15: Failure mode for adhesive anchors under shear load at different strain rates

\begin{tabular}{|c|c|c|c|c|c|c|c|c|}
\hline \multirow{3}{*}{$\begin{array}{c}\text { Model } \\
\text { No. }\end{array}$} & \multirow{3}{*}{$\begin{array}{c}d \\
(\mathrm{~mm})\end{array}$} & \multirow{3}{*}{$\begin{array}{c}\mathbf{h}_{\text {ef }} \\
(\mathbf{m m})\end{array}$} & \multicolumn{6}{|c|}{ "Failure mode } \\
\hline & & & \multicolumn{6}{|c|}{ Strain rate $\left(\mathrm{s}^{-1}\right)$} \\
\hline & & & $\dot{\varepsilon}=10^{-5}$ & $\dot{\varepsilon}=10^{-3}$ & $\dot{\varepsilon}=10^{-1}$ & $\dot{\varepsilon}=10$ & $\dot{\varepsilon}=10^{2}$ & $\dot{\varepsilon}=10^{3}$ \\
\hline 1 & 12.7 & 76.2 & $\mathrm{~S}$ & $\mathrm{~S}$ & $\mathrm{~S}$ & $\mathrm{~S}$ & $\mathrm{~S}$ & $\mathrm{~S}$ \\
\hline 2 & 12.7 & 101.6 & $\mathrm{~S}$ & $\mathrm{~S}$ & $\mathrm{~S}$ & $\mathrm{~S}$ & $\mathrm{~S}$ & $S$ \\
\hline 3 & 12.7 & 152.4 & $\mathrm{~S}$ & $\mathrm{~S}$ & $\mathrm{~S}$ & $\mathrm{~S}$ & $\mathrm{~S}$ & $\mathrm{~S}$ \\
\hline 4 & 15.9 & 76.2 & PR & $\bar{S}$ & $\mathrm{~S}$ & $\mathrm{~S}$ & $\mathrm{~S}$ & $\bar{S}$ \\
\hline 5 & 15.9 & 101.6 & $\mathrm{~S}$ & $\mathrm{~S}$ & $\mathrm{~S}$ & $\mathrm{~S}$ & $\mathrm{~S}$ & $\mathrm{~S}$ \\
\hline 6 & 15.9 & 152.4 & $S$ & $\mathrm{~S}$ & $\mathrm{~S}$ & $\mathrm{~S}$ & $S$ & $\mathrm{~S}$ \\
\hline 7 & 19.1 & 76.2 & PR & $\mathrm{CS}$ & $\mathrm{CS}$ & $\mathrm{CS}$ & $S$ & $S$ \\
\hline 8 & 19.1 & 101.6 & PR & $\mathrm{S}$ & $\mathrm{S}$ & $\mathrm{S}$ & $\mathrm{S}$ & $\mathrm{S}$ \\
\hline 9 & 19.1 & 152.4 & $\mathrm{~S}$ & $\mathrm{~S}$ & $\mathrm{~S}$ & $\mathrm{~S}$ & $\mathrm{~S}$ & $\mathrm{~S}$ \\
\hline
\end{tabular}

*Failure mode: $\mathrm{PR}=$ pryout failure, $\mathrm{CS}=$ concrete spalling, $\mathrm{S}=$ steel anchor failure 


\subsubsection{Effect of design parameters on failure mode and ultimate shear load}

Figures 5-42, 5-43 and 5-44 show the effect of strain rate on the failure mode and ultimate shear load for the adhesive anchors. As shown in Figure 5-42 and Figure 5-43, slight increase in the ultimate shear load with the increase in the strain rate is observed for the anchor diameters of $12.7 \mathrm{~mm}$ and $15.9 \mathrm{~mm}$. Also, it can be seen that increasing the embedment depth from $76.2 \mathrm{~mm}$ to $152.4 \mathrm{~mm}$ at the same strain rate has insignificant influence on the ultimate shear load. Where, steel anchor failure was observed for the 12.7$\mathrm{mm}$ and 15.9-mm diameter adhesive anchors at all the strain rates investigated except for the $15.9-\mathrm{mm}$ diameter adhesive anchor with $76.2 \mathrm{~mm}$ embedment depth which exhibited pryout failure. This is attributed to the concrete resistance to the applied load is higher than the capacity of the small anchor diameters of $12.7-\mathrm{mm}$ and $15.9-\mathrm{mm}$ leads to steel fracture. However, the increase in the shear load with increasing the embedment depth is remarkable for the 19.1-mm diameter adhesive anchor (Figure 5-44).

As shown in Figure 5-44 for the 19.1-mm diameter adhesive anchor with embedment depth of $76.2 \mathrm{~mm}$, the failure mode transitioned from pryout failure (PR) at strain rate of $10^{-5} \mathrm{~s}^{-1}$ to concrete spalling (CS) at strain rates of $10^{-3} \mathrm{~s}^{-1}, 10^{-1} \mathrm{~s}^{-1}$, and $10 \mathrm{~s}^{-1}$ and then to steel failure (S) at strain rates of $10^{2} \mathrm{~s}^{-1}$ and $10^{3} \mathrm{~s}^{-1}$. Concrete spalling failure mode occurs when the anchor placed far from the concrete free edge where the concrete resists the shear forces transferred from the anchor causing concrete spall. However, when the anchor placed near the concrete free edge, concrete breakout can occur. It can be seen from Figure 5-44, at strain rates of $10^{-3} \mathrm{~s}^{-1}, 10^{-1} \mathrm{~s}^{-1}$ and $10 \mathrm{~s}^{-1}$ transitions in the failure mode from concrete spalling to steel anchor failure is observed when the embedment depth increased from 76.2 $\mathrm{mm}$ to $152.4 \mathrm{~mm}$. At the $101.6 \mathrm{~mm}$ and $152.4 \mathrm{~mm}$ embedment depths, steel anchor failure 
was observed at all the strain rates investigated, while at low strain rate of $10^{-5} \mathrm{~s}^{-1}$ with embedment depth of $101.6 \mathrm{~mm}$, pryout failure was observed.

It can be seen from Figure 5-44 the ultimate shear load for the $76.2 \mathrm{~mm}$ embedment depth increased from $116.26 \mathrm{kN}$ at strain rate of $10^{-5} \mathrm{~s}^{-1}$ to $154.81 \mathrm{kN}$ at strain rate of $10 \mathrm{~s}^{-1}$. These values are $180.18 \mathrm{kN}$ and $184.17 \mathrm{kN}$ at strain rates of $10^{2} \mathrm{~s}^{-1}$ and $10^{3} \mathrm{~s}^{-1}$ respectively. The increase in the ultimate shear load is due to the increase in the concrete and steel strength with the increase in the strain rate from $10^{-5} \mathrm{~s}^{-1}$ to $10 \mathrm{~s}^{-1}$, where concrete spalling failure mode is observed. However, at high strain rates of $10^{2} \mathrm{~s}^{-1}$ and $10^{3} \mathrm{~s}^{-1}$, the increase in the ultimate shear load is due to the increase of steel strength at high strain rate where steel anchor failure is observed. For the embedment depth of $101.6 \mathrm{~mm}$, the increase in the ultimate load was from $146.45 \mathrm{kN}$ at strain rate of $10^{-5} \mathrm{~s}^{-1}$ to $170.49 \mathrm{kN}$ at strain rate of $10^{-}$ ${ }^{3} \mathrm{~s}^{-1}$. Further increase in the strain rate exhibited slight increase in the ultimate shear load. Similar behaviour was observed for the anchor embedment depth of $152.4 \mathrm{~mm}$ where steel anchor failure is the dominant failure mode at all the strain rates investigated.

As shown in Figure 5-44, the strain rate affects the failure mode for the anchor diameter of 19.1-mm where transition from pryout failure to concrete spalling and then to steel anchor failure is observed. Also, it can be seen from Figures 5-43 and 5-44 that the failure mode is influenced by the anchor diameter and embedment depth. Pryout failure (PR) is observed at low strain rate of $10^{-5} \mathrm{~s}^{-1}$ for anchor diameters of $15.9-\mathrm{mm}$ and $19.1 \mathrm{~mm}$ at embedment depth of $76.2 \mathrm{~mm}$. Also, pryout failure was observed for anchor diameter of 19.1-mm at embedment depth of $101.6 \mathrm{~mm}$. Concrete spalling (CS) was observed at strain rate of $10^{-}$ 
${ }^{3} \mathrm{~s}^{-1}$ to $10 \mathrm{~s}^{-1}$ for anchor diameter of $19.1-\mathrm{mm}$ at embedment depth of $76.2 \mathrm{~mm}$. Steel anchor failure (S) was observed for most of the adhesive anchors investigated. Also, it can be seen from the figures that the ultimate shear load increased with the increase in the strain rate and diameter for the adhesive anchors investigated. Anchor diameter of 19.1-mm gave the highest ultimate shear load at all the embedment depths investigated. This is attributed to the increase in the anchor stiffness and anchor resistance to the applied shear load and thus higher load is required for fracturing the anchor.

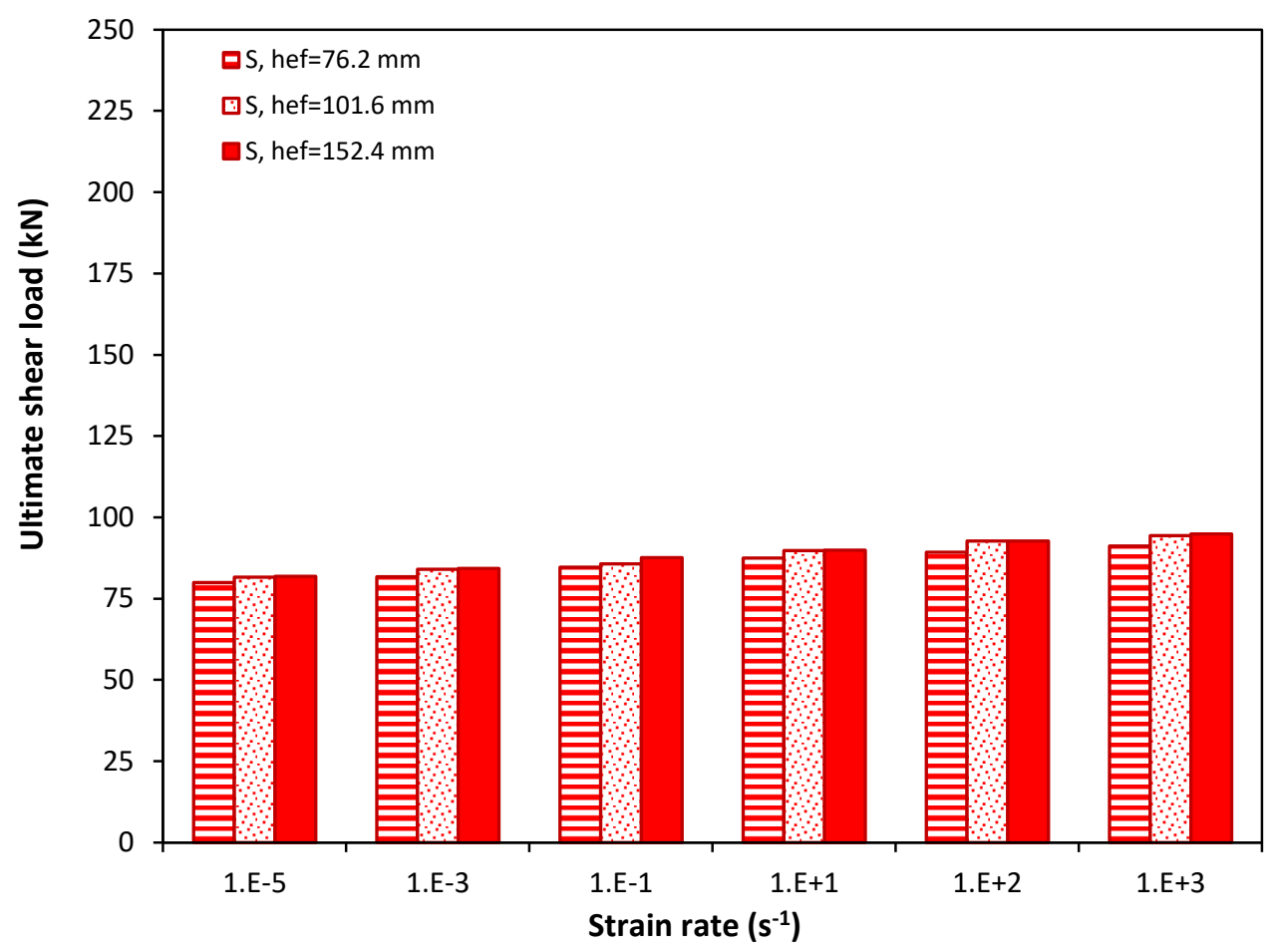

Figure 5-42: Effect of strain rate on the failure mode and ultimate shear load for the adhesive anchor diameter of $12.7 \mathrm{~mm}$ 


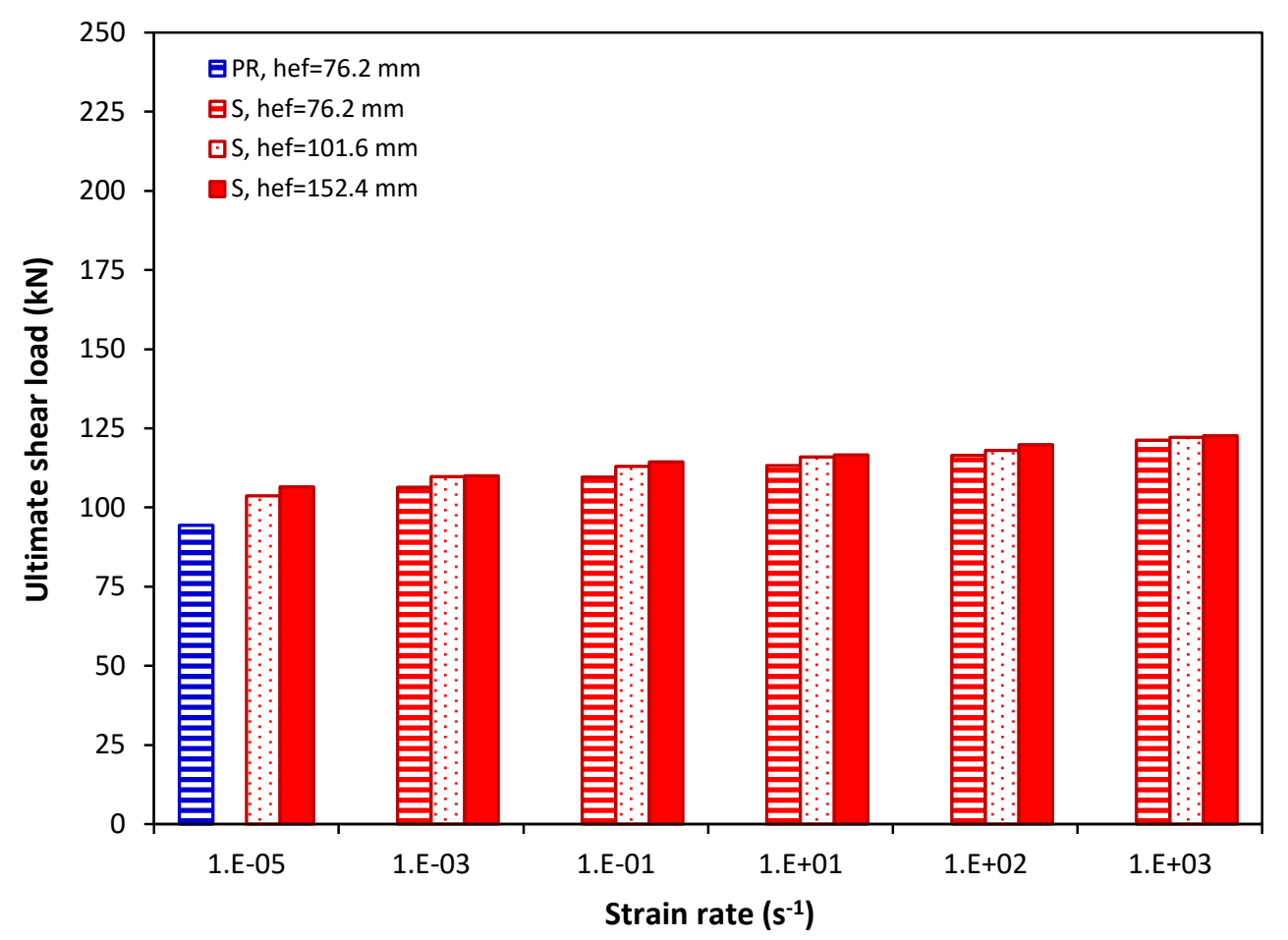

Figure 5-43: Effect of strain rate on the failure mode and ultimate shear load for the adhesive anchor diameter of $15.9 \mathrm{~mm}$

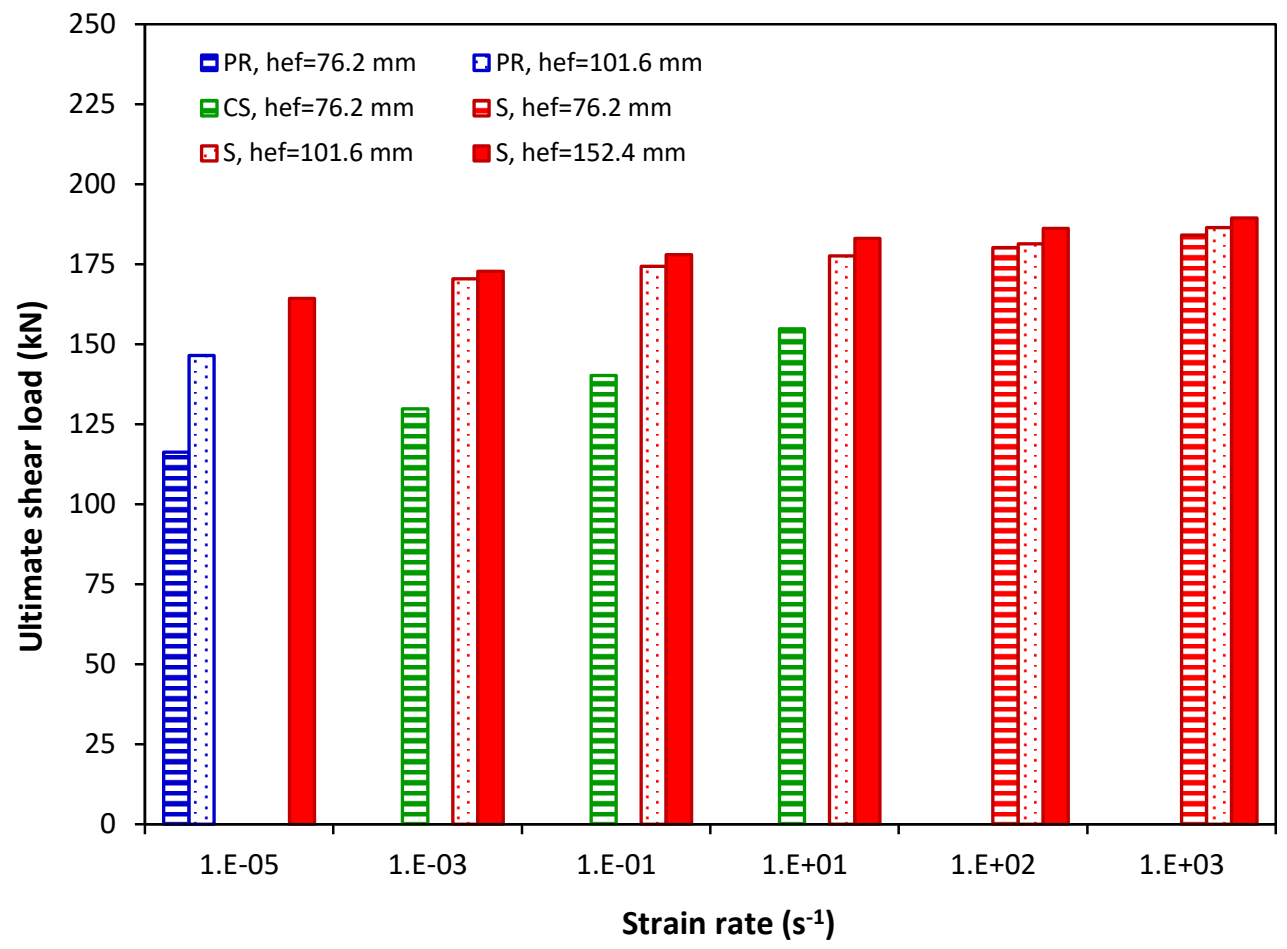

Figure 5-44: Effect of strain rate on the failure mode and ultimate shear load for the adhesive anchor diameter of $19.1 \mathrm{~mm}$ 


\subsubsection{Effect of strain rate on the shear behaviour of adhesive anchors}

The shear behaviour of the adhesive anchors embedded into concrete at strain rates in the range of $10^{-5} \mathrm{~s}^{-1}$ to $10^{3} \mathrm{~s}^{-1}$ was investigated. Load-displacement behaviour of the $12.7-\mathrm{mm}$ diameter adhesive anchor at strain rates of $10^{-5} \mathrm{~s}^{-1}, 10^{-3} \mathrm{~s}^{-1}, 10^{-1} \mathrm{~s}^{-1}, 10 \mathrm{~s}^{-1}, 10^{2} \mathrm{~s}^{-1}$ and $10^{3} \mathrm{~s}^{-}$ ${ }^{1}$ are shown in Figures 5-45, 5-46, 5-47, 5-48, 5-49 and 5-50 respectively at embedment depths of $76.2 \mathrm{~mm}, 101.6 \mathrm{~mm}$ and $152.4 \mathrm{~mm}$.

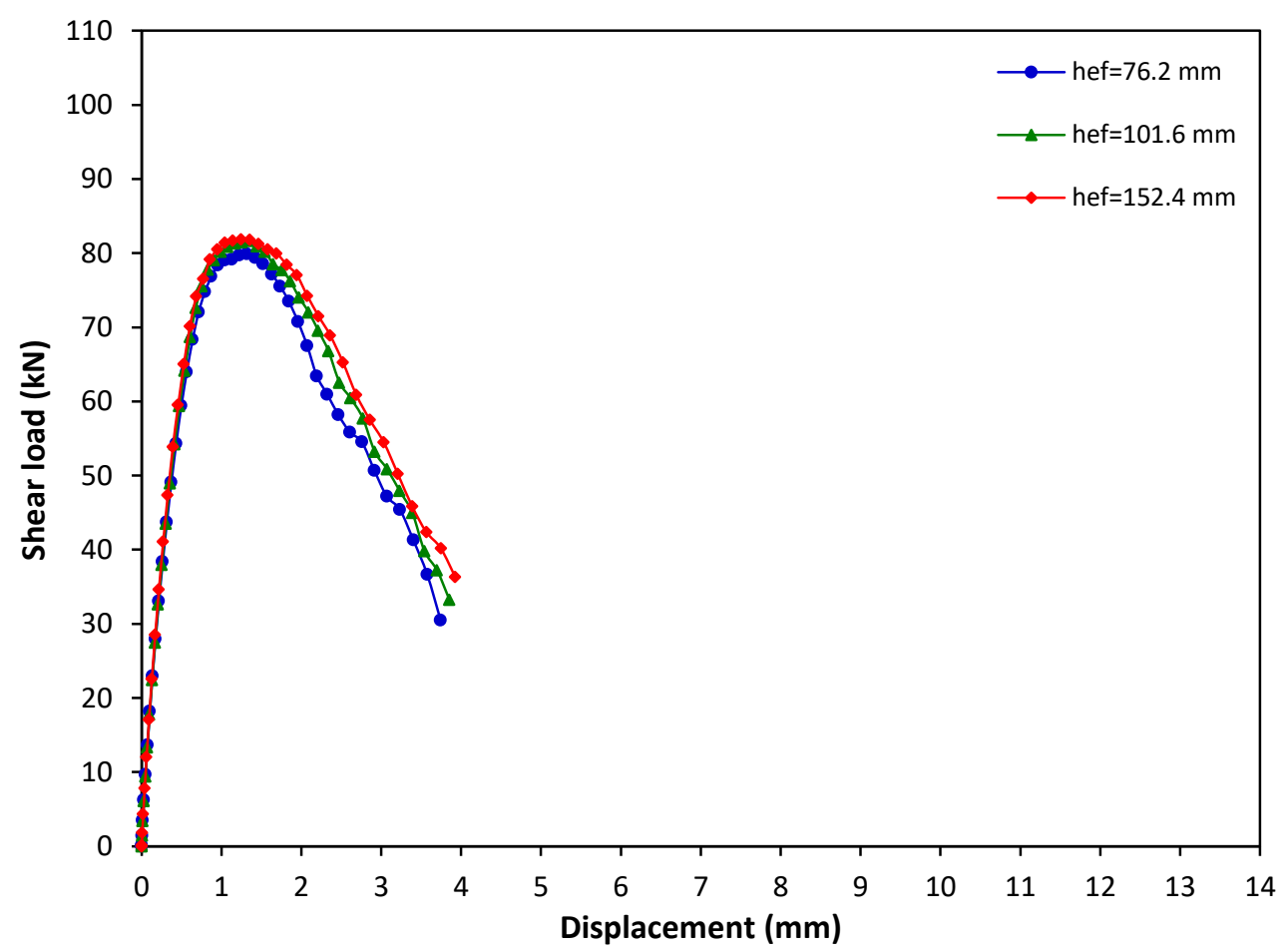

Figure 5-45: Shear load-displacement graph for 12.7-mm diameter adhesive anchor at strain rate of $10^{-5} \mathrm{~s}^{-1}$ 


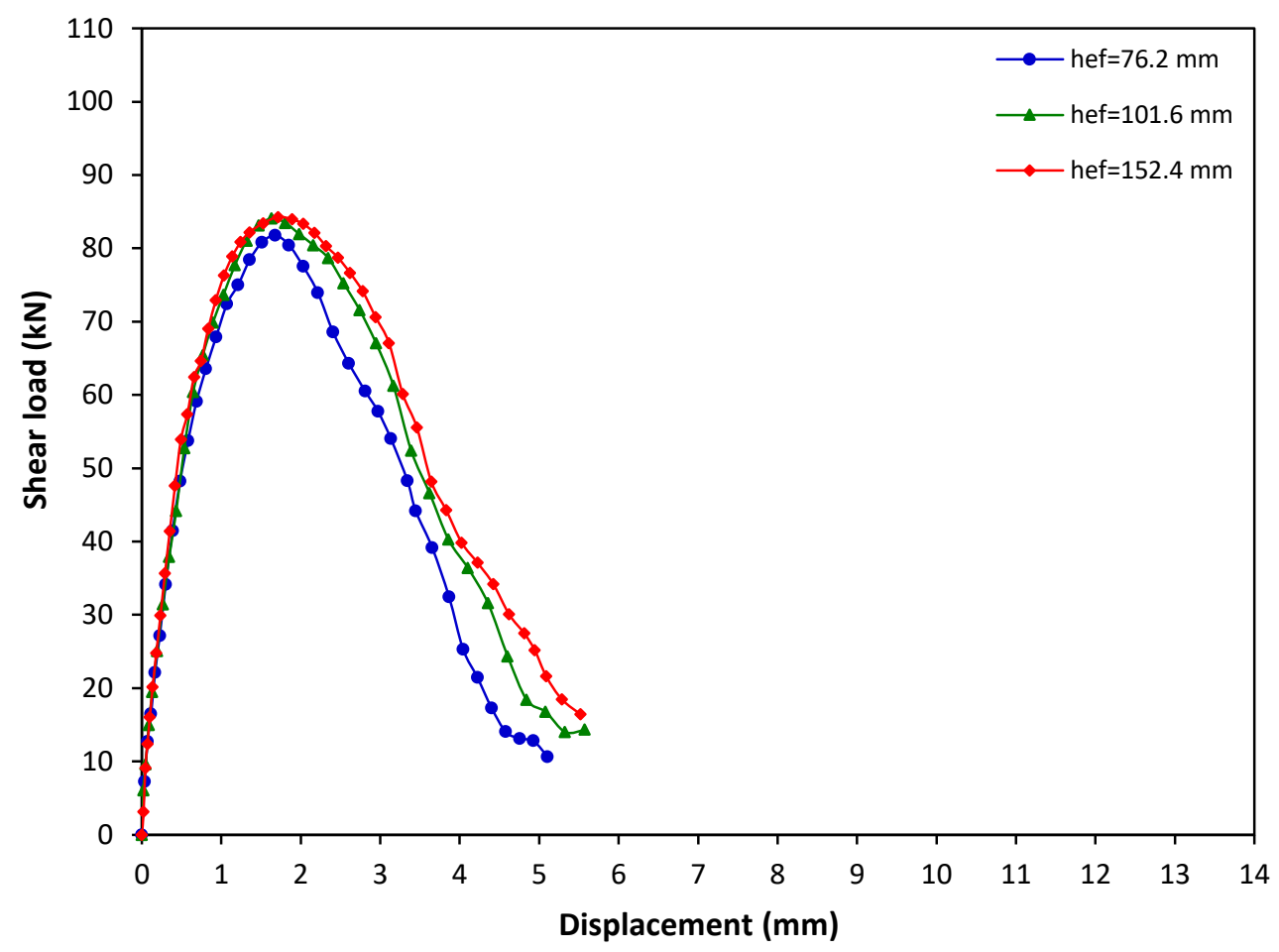

Figure 5-46: Shear load-displacement graph for 12.7-mm diameter adhesive anchor at

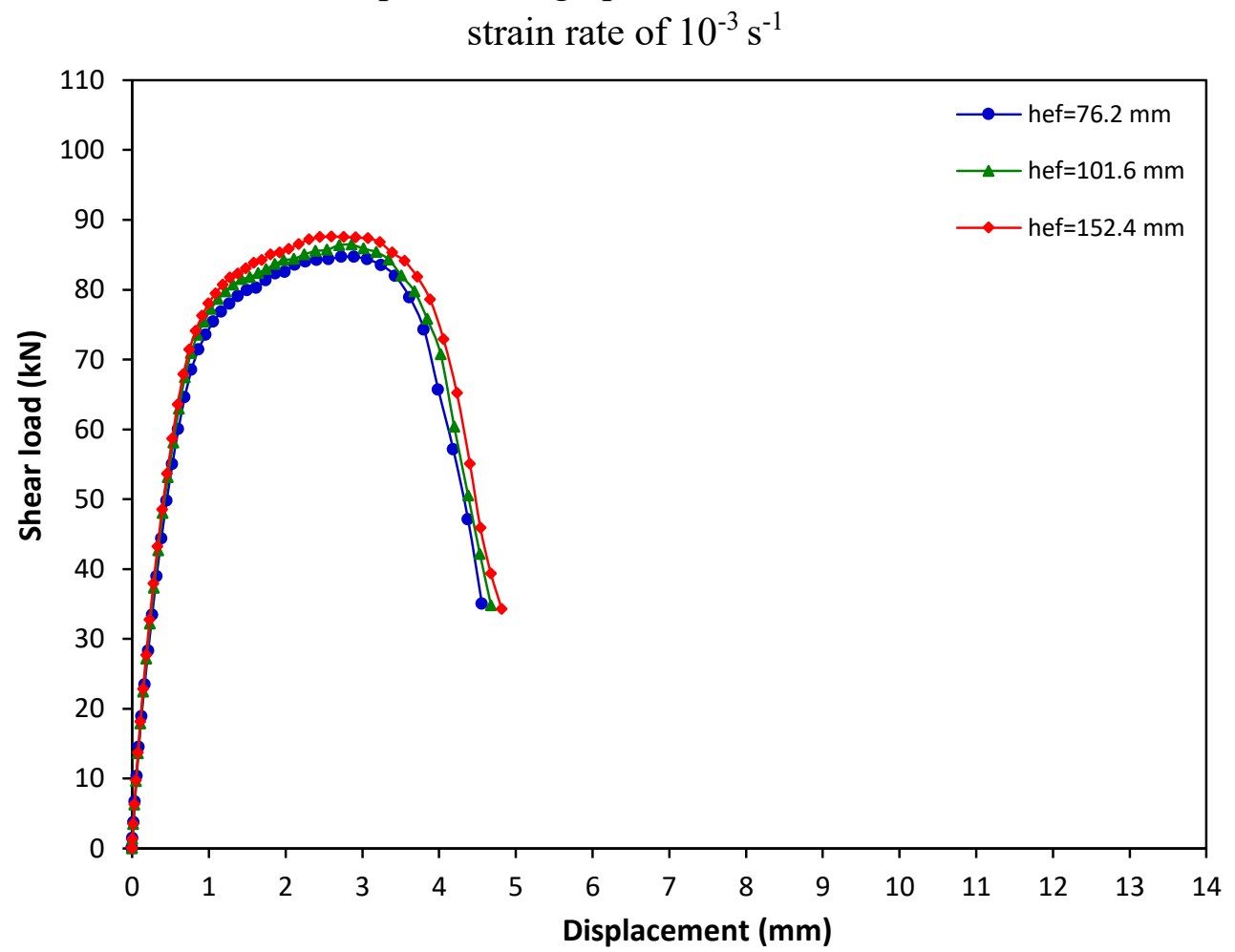

Figure 5-47: Shear load-displacement graph for 12.7-mm diameter adhesive anchor at strain rate of $10^{-1} \mathrm{~s}^{-1}$ 


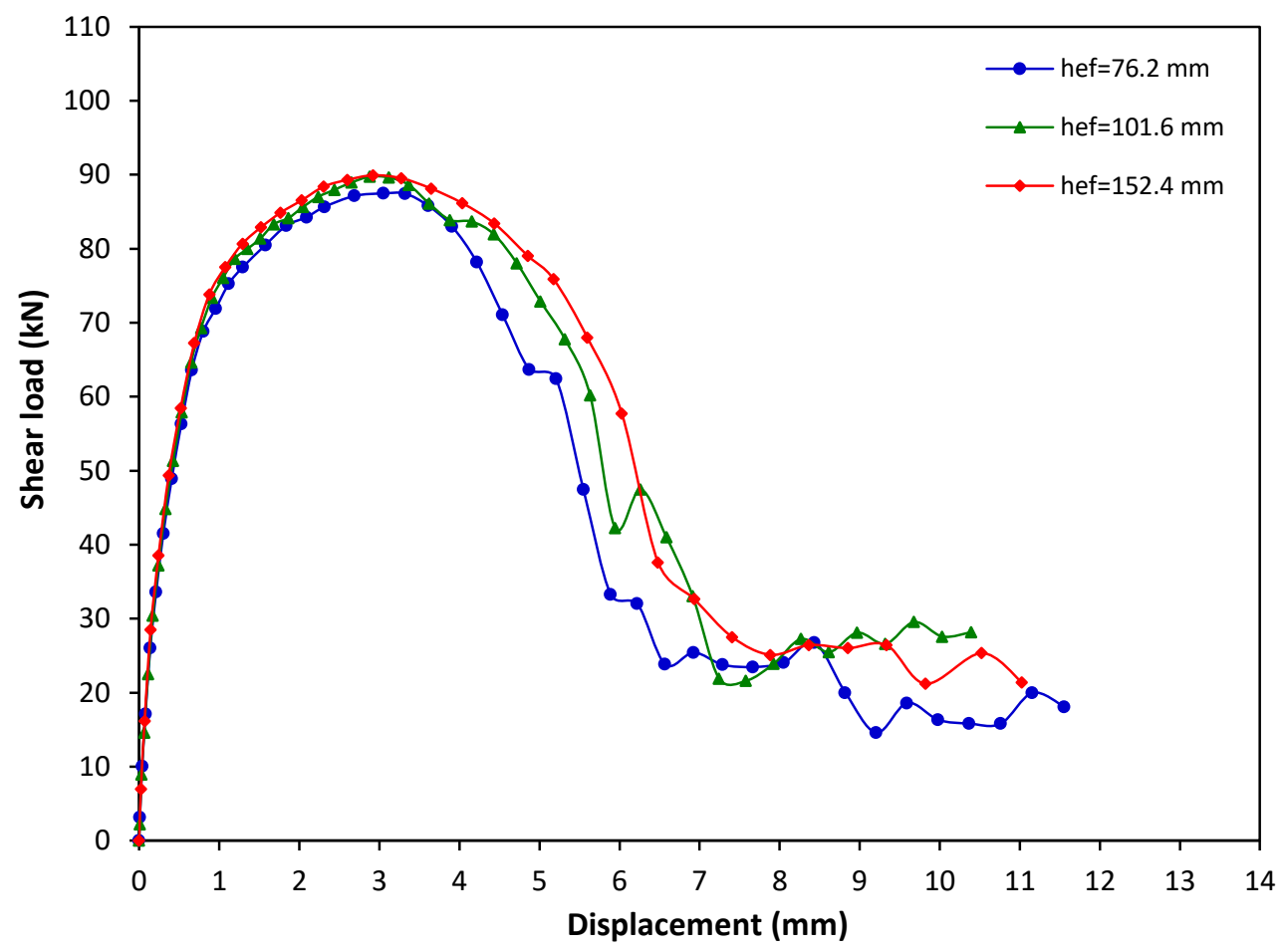

Figure 5-48: Shear load-displacement graph for 12.7-mm diameter adhesive anchor at

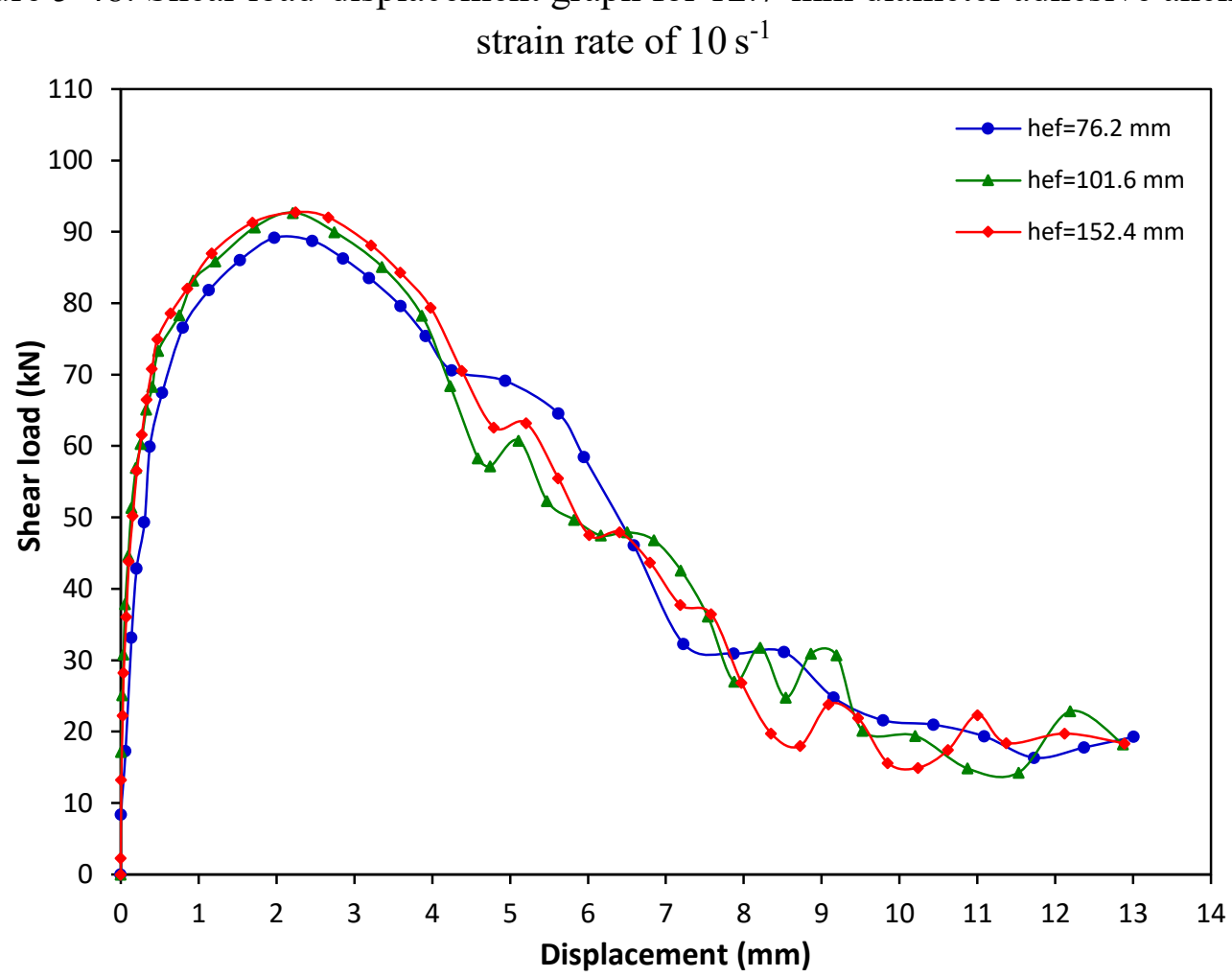

Figure 5-49: Shear load-displacement graph for 12.7-mm diameter adhesive anchor at strain rate of $10^{2} \mathrm{~s}^{-1}$ 


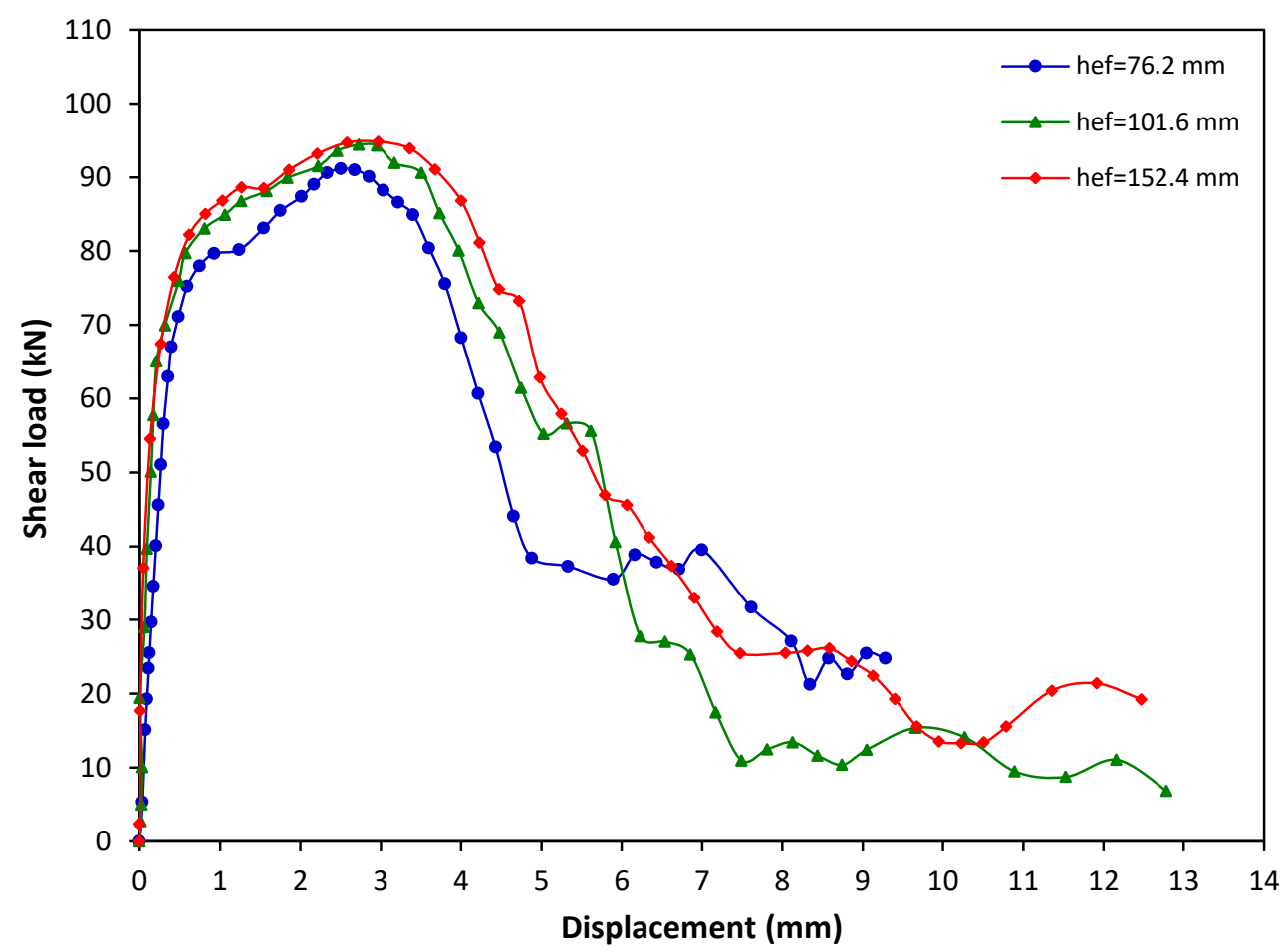

Figure 5-50: Shear load-displacement graph for 12.7-mm diameter adhesive anchor at strain rate of $10^{3} \mathrm{~s}^{-1}$

As shown from the Figures 5-45 to 5-50, the shear load increased with the displacement until the ultimate value. This is attributed to the concrete resistance to the applied load where the shear load is transferred from the anchor plate to the anchor and then to the concrete through the adhesive material. The post-peak response shows a reduction in the load with further increase in displacement until failure. It can be seen from Table 5-16 that the shear capacity of the adhesive anchor increased as the strain rate increased from $10^{-5} \mathrm{~s}^{-}$ ${ }^{1}$ to $10^{3} \mathrm{~s}^{-1}$. At strain rates of $10^{-5} \mathrm{~s}^{-1}, 10^{-3} \mathrm{~s}^{-1}$ and $10^{-1} \mathrm{~s}^{-1}$ (Figures 5-45, 5-46 and 5-47) the shear load increased with the displacement until reaching the ultimate value, and then decreased until complete failure. At strain rates of $10 \mathrm{~s}^{-1}, 10^{2} \mathrm{~s}^{-1}$ and $10^{3} \mathrm{~s}^{-1}$ (Figures 5-48, 5-49 and 5-50), the shear load increased with the displacement up to the ultimate shear load followed by a decrease in the shear load and fluctuation about the residual shear load. It 
can be seen from the figures that the ultimate shear loads of the anchors at strain rates ranging from $10^{-5} \mathrm{~s}^{-1}$ to $10^{3} \mathrm{~s}^{-1}$ were almost the same irrespective of the embedment depth as the predominant failure mode was steel anchor fracture.

The shear load-displacement behaviour of $15.9-\mathrm{mm}$ and $19.1-\mathrm{mm}$ diameter adhesive anchors with embedment depths of $76.2 \mathrm{~mm}, 101.6 \mathrm{~mm}$ and $152.4 \mathrm{~mm}$ at strain rates in the range between $10^{-5} \mathrm{~s}^{-1}$ to $10^{3} \mathrm{~s}^{-1}$ is showed similar response to the $12.7-\mathrm{mm}$ diameter adhesive anchor. The results for these adhesive anchors are presented in Appendix F.

Tables 5-16, 5-17 and 5-18 present the ultimate shear load-displacement results for the 12.7- $\mathrm{mm}, 15.9-\mathrm{mm}$ and $19.1-\mathrm{mm}$ diameter adhesive anchors respectively. Where $\delta$ is the displacement at the ultimate shear load. It can be seen from Tables 5-16, 5-17 and 5-18 that as the strain rate increased the ultimate shear capacity of the adhesive anchorage systems increased. The increased shear capacity is enhanced with increased anchor diameter from 12.7- $\mathrm{mm}$ to $19.1-\mathrm{mm}$ and for all the embedment depths investigated. It can be seen from Tables 5-16 and 5-17, steel failure is the dominant failure mode for adhesive anchor diameters of $12.7-\mathrm{mm}$ and $15.9-\mathrm{mm}$ for all embedment depths and strain rates investigated, except for the 15.9-mm diameter anchor with embedment depth of $76.2 \mathrm{~mm}$ at low strain rate of $10^{-5} \mathrm{~s}^{-1}$ that exhibited pryout failure, where the embedment depth to diameter ratio is sufficient to obtain pryout failure. For adhesive anchor diameter of 19.1-mm (Table 518), pryout failure was observed for the embedment depths of $76.2 \mathrm{~mm}$ and $101.6 \mathrm{~mm}$ while steel failure was observed at embedment depth of $152.4 \mathrm{~mm}$ at low strain rate of $10^{-}$ ${ }^{5} \mathrm{~s}^{-1}$. Concrete spalling was observed for the shallow embedment depth of $76.2 \mathrm{~mm}$ at strain 
rates of $10^{-3} \mathrm{~s}^{-1}, 10^{-1} \mathrm{~s}^{-1}$ and $10 \mathrm{~s}^{-1}$ while steel anchor failure was observed for the embedment depths of $101.6 \mathrm{~mm}$ and $152.4 \mathrm{~mm}$. Also, steel failure was observed at strain rates of $10^{2} \mathrm{~s}^{-1}$ and $10^{3} \mathrm{~s}^{-1}$ and for all embedment depths investigated. It can be stated that the strain rate has an effect on the shear load and failure mode.

Table 5-16: Ultimate shear load and displacement results for the 12.7-mm diameter adhesive anchor

\begin{tabular}{|c|c|c|c|c|c|c|}
\hline $\begin{array}{l}\text { Model } \\
\text { No. }\end{array}$ & $\begin{array}{c}\mathrm{d} \\
(\mathrm{mm})\end{array}$ & $\begin{array}{c}\mathrm{h}_{\mathrm{hf}} \\
(\mathrm{mm})\end{array}$ & $\begin{array}{c}\dot{\varepsilon} \\
\left(\mathrm{s}^{-1}\right)\end{array}$ & $\begin{array}{c}\mathrm{V}_{\mathrm{u}} \\
(\mathrm{kN})\end{array}$ & $\begin{array}{c}\delta \\
(\mathrm{mm}) \\
\end{array}$ & Failure mode \\
\hline 1 & \multirow{3}{*}{12.7} & 76.2 & \multirow{3}{*}{$10^{-5}$} & 79.93 & 1.32 & $\mathrm{~S}$ \\
\hline 2 & & 101.6 & & 81.64 & 1.35 & $\mathrm{~S}$ \\
\hline 3 & & 152.4 & & 81.85 & 1.25 & $\mathrm{~S}$ \\
\hline 4 & \multirow{3}{*}{12.7} & 76.2 & \multirow{3}{*}{$10^{-3}$} & 81.75 & 1.68 & $\mathrm{~S}$ \\
\hline 5 & & 101.6 & & 84.07 & 1.63 & $\mathrm{~S}$ \\
\hline 6 & & 152.4 & & 84.26 & 1.72 & $\mathrm{~S}$ \\
\hline 7 & \multirow{3}{*}{12.7} & 76.2 & \multirow{3}{*}{$10^{-1}$} & 84.69 & 2.89 & $\mathrm{~S}$ \\
\hline 8 & & 101.6 & & 85.72 & 2.54 & $\mathrm{~S}$ \\
\hline 9 & & 152.4 & & 87.63 & 2.56 & $\mathrm{~S}$ \\
\hline 10 & \multirow{3}{*}{12.7} & 76.2 & \multirow{3}{*}{$10^{1}$} & 87.47 & 3.00 & $\mathrm{~S}$ \\
\hline 11 & & 101.6 & & 89.74 & 2.89 & $\mathrm{~S}$ \\
\hline 12 & & 152.4 & & 89.91 & 2.92 & $\mathrm{~S}$ \\
\hline 13 & \multirow{3}{*}{12.7} & 76.2 & \multirow{3}{*}{$10^{2}$} & 89.19 & 1.98 & $\mathrm{~S}$ \\
\hline 14 & & 101.6 & & 92.68 & 2.21 & $\mathrm{~S}$ \\
\hline 15 & & 152.4 & & 92.75 & 2.25 & $\mathrm{~S}$ \\
\hline 16 & \multirow{3}{*}{12.7} & 76.2 & \multirow{3}{*}{$10^{3}$} & 91.14 & 2.50 & $\mathrm{~S}$ \\
\hline 17 & & 101.6 & & 94.39 & 2.73 & $\mathrm{~S}$ \\
\hline 18 & & 152.4 & & 94.82 & 2.97 & $\mathrm{~S}$ \\
\hline
\end{tabular}

Table 5-17: Ultimate shear load and displacement results for the 15.9-mm diameter

\begin{tabular}{|c|c|c|c|c|c|c|}
\hline \multicolumn{7}{|c|}{ adhesive anchor } \\
\hline $\begin{array}{l}\text { Model } \\
\text { No. }\end{array}$ & $\begin{array}{c}\mathrm{d} \\
(\mathrm{mm})\end{array}$ & $\begin{array}{c}\mathrm{h}_{\mathrm{ef}} \\
(\mathrm{mm})\end{array}$ & $\begin{array}{c}\dot{\varepsilon} \\
\left(\mathrm{s}^{-1}\right)\end{array}$ & $\begin{array}{c}\mathrm{V}_{\mathrm{u}} \\
(\mathrm{kN})\end{array}$ & $\begin{array}{c}\delta \\
(\mathrm{mm})\end{array}$ & Failure mode \\
\hline 1 & \multirow{3}{*}{15.9} & 76.2 & \multirow{3}{*}{$10^{-5}$} & 94.31 & 1.57 & PR \\
\hline 2 & & 101.6 & & 103.73 & 1.41 & $\mathrm{~S}$ \\
\hline 3 & & 152.4 & & 106.50 & 1.39 & $\mathrm{~S}$ \\
\hline 4 & \multirow{3}{*}{15.9} & 76.2 & \multirow{3}{*}{$10^{-3}$} & 106.38 & 3.07 & $\mathrm{~S}$ \\
\hline 5 & & 101.6 & & 109.69 & 2.81 & $\mathrm{~S}$ \\
\hline 6 & & 152.4 & & 109.98 & 2.57 & $\mathrm{~S}$ \\
\hline 7 & \multirow{3}{*}{15.9} & 76.2 & \multirow{3}{*}{$10^{-1}$} & 109.55 & 2.52 & $\mathrm{~S}$ \\
\hline 8 & & 101.6 & & 113.02 & 2.73 & $\mathrm{~S}$ \\
\hline 9 & & 152.4 & & 114.41 & 2.45 & $\mathrm{~S}$ \\
\hline 10 & 15.9 & 76.2 & 10 & 113.19 & 2.65 & $\mathrm{~S}$ \\
\hline
\end{tabular}




\begin{tabular}{|c|c|c|c|c|c|c|}
\hline 11 & & 101.6 & & 115.97 & 3.51 & $\mathrm{~S}$ \\
\hline 12 & & 152.4 & & 116.58 & 3.29 & $\mathrm{~S}$ \\
\hline 13 & \multirow{3}{*}{15.9} & 76.2 & \multirow{3}{*}{$10^{2}$} & 116.43 & 2.62 & $\mathrm{~S}$ \\
\hline 14 & & 101.6 & & 117.99 & 3.16 & $\mathrm{~S}$ \\
\hline 15 & & 152.4 & & 119.87 & 3.09 & S \\
\hline 16 & \multirow{3}{*}{15.9} & 76.2 & \multirow{3}{*}{$10^{3}$} & 121.21 & 2.59 & $\mathrm{~S}$ \\
\hline 17 & & 101.6 & & 122.16 & 3.17 & S \\
\hline 18 & & 152.4 & & 122.62 & 3.22 & $\mathrm{~S}$ \\
\hline
\end{tabular}

Table 5-18: Ultimate shear load and displacement results for the 19.1-mm diameter adhesive anchor

\begin{tabular}{|c|c|c|c|c|c|c|}
\hline $\begin{array}{c}\text { Model } \\
\text { No. }\end{array}$ & $\begin{array}{c}\mathrm{d} \\
(\mathrm{mm})\end{array}$ & $\begin{array}{c}\mathrm{h}_{\mathrm{ef}} \\
(\mathrm{mm})\end{array}$ & $\begin{array}{c}\dot{\varepsilon} \\
\left(\mathrm{s}^{-1}\right)\end{array}$ & $\begin{array}{c}\mathrm{V}_{\mathrm{u}} \\
(\mathrm{kN})\end{array}$ & $\begin{array}{c}\delta \\
(\mathrm{mm})\end{array}$ & Failure mode \\
\hline 1 & \multirow{3}{*}{19.1} & 76.2 & \multirow{3}{*}{$10^{-5}$} & 116.26 & 1.12 & PR \\
\hline 2 & & 101.6 & & 146.45 & 1.26 & PR \\
\hline 3 & & 152.4 & & 164.33 & 1.47 & $\mathrm{~S}$ \\
\hline 4 & \multirow{3}{*}{19.1} & 76.2 & \multirow{3}{*}{$10^{-3}$} & 129.89 & 2.08 & $\mathrm{CS}$ \\
\hline 5 & & 101.6 & & 170.49 & 1.93 & $\mathrm{~S}$ \\
\hline 6 & & 152.4 & & 172.78 & 1.91 & $\mathrm{~S}$ \\
\hline 7 & \multirow{3}{*}{19.1} & 76.2 & \multirow{3}{*}{$10^{-1}$} & 140.24 & 2.74 & $\mathrm{CS}$ \\
\hline 8 & & 101.6 & & 174.37 & 3.56 & $\mathrm{~S}$ \\
\hline 9 & & 152.4 & & 178.02 & 3.43 & $\mathrm{~S}$ \\
\hline 10 & \multirow{3}{*}{19.1} & 76.2 & \multirow{3}{*}{10} & 154.81 & 1.36 & $\mathrm{CS}$ \\
\hline 11 & & 101.6 & & 177.65 & 1.11 & $\mathrm{~S}$ \\
\hline 12 & & 152.4 & & 183.08 & 1.28 & $\mathrm{~S}$ \\
\hline 13 & \multirow{3}{*}{19.1} & 76.2 & \multirow{3}{*}{$10^{2}$} & 180.18 & 1.19 & $\mathrm{~S}$ \\
\hline 14 & & 101.6 & & 181.34 & 1.24 & $\mathrm{~S}$ \\
\hline 15 & & 152.4 & & 186.14 & 1.23 & $\mathrm{~S}$ \\
\hline 16 & \multirow{3}{*}{19.1} & 76.2 & \multirow{3}{*}{$10^{3}$} & 184.17 & 1.66 & $\mathrm{~S}$ \\
\hline 17 & & 101.6 & & 186.51 & 1.58 & $\mathrm{~S}$ \\
\hline 18 & & 152.4 & & 189.42 & 1.77 & $\mathrm{~S}$ \\
\hline
\end{tabular}

\subsubsection{Effect of strain rate on the ultimate shear load and DIF of adhesive anchors}

Figures 5-51, 5-52 and 5-53 present the effect of strain rate on the ultimate shear load and DIF for the 12.7-mm, 15.9-mm and 19.1-mm diameter adhesive anchors with different embedment depths. As shown in the figures, the ultimate shear load increased with the increase in the strain rate from $10^{-5} \mathrm{~s}^{-1}$ to $10^{3} \mathrm{~s}^{-1}$ for the adhesive anchors investigated. Similar observation on the cast-in-place and post-installed (undercut and expansion) 
anchors subjected to dynamic shear load was reported by Gross et al. (2001) (Gross et al., 2001). Gross et al. found that the ultimate shear loads for the investigated anchors under dynamic load is higher than that under static load.

The relationship between the ultimate shear load and strain rate is approximately linear for the 12.7- $\mathrm{mm}$ and 15.9-mm diameter adhesive anchors at all embedment depths. The linear relationship between the ultimate shear load and strain rate for the $12.7-\mathrm{mm}$ and $15.9-\mathrm{mm}$ diameter adhesive anchors is attributed to the slight increase in ultimate shear load with the increase in the strain rate from $10^{-5} \mathrm{~s}^{-1}$ to $10^{3} \mathrm{~s}^{-1}$ where steel anchor failure is the dominant failure mode at all the strain rates. The steel strength increased with the increase in the strain rate resulting in increased ultimate shear load. However, for the $19.1 \mathrm{~mm}$ diameter adhesive anchor with embedment depth of $76.2 \mathrm{~mm}$ an almost bilinear relation is obtained with change in slope at strain rate of $10 \mathrm{~s}^{-1}$. The change in slope is attributed to the transition from pryout failure mode to steel anchor failure. At embedment depth of $101.6 \mathrm{~mm}$, linear relation was observed for the 19.1-mm diameter anchor for the strain rates ranging from $10^{-3} \mathrm{~s}^{-1}$ to $10^{3} \mathrm{~s}^{-1}$. At strain rate of $10^{-5}$ pryout failure was observed, transition from pryout failure mode to steel anchor failure was observed at strain rate of $10^{-3} \mathrm{~s}^{-1}$. For pryout failure mode both steel and concrete materials contribute in increasing the shear load where their strength increased with increasing the strain rate. Increasing anchor embedment depth from $76.2 \mathrm{~mm}$ to $152.4 \mathrm{~mm}$ increased the ultimate shear load for the anchors exhibiting pryout or concrete spalling failure. Maximum ultimate shear loads of $94.82 \mathrm{kN}, 122.62 \mathrm{kN}$ and $189.42 \mathrm{kN}$ were obtained for the 12.7- $\mathrm{mm}, 15.9-\mathrm{mm}$ and 19.1-mm diameter adhesive anchors respectively with embedment depth of $152.4 \mathrm{~mm}$ at high strain rate of $10^{3} \mathrm{~s}^{-1}$. At 
low strain rate of $10^{-5} \mathrm{~s}^{-1}$ the shear loads were $81.85 \mathrm{kN}, 106.50 \mathrm{kN}$ and $164.33 \mathrm{kN}$ for the 12.7- $\mathrm{mm}, 15.9-\mathrm{mm}$ and 19.1-mm diameter adhesive anchors, respectively.

Comparing with the ultimate shear load at embedment depth of $76.2 \mathrm{~mm}$ (Figure 5-51), the increase in the ultimate shear load for the 12.7-mm, 15.9-mm and 19.1-mm diameter adhesive anchors over the static (strain rate of $10^{-5} \mathrm{~s}^{-1}$ ) was $14 \%, 28.5 \%$ and $58 \%$, respectively at high strain rate of $10^{3} \mathrm{~s}^{-1}$. While the increase in the ultimate shear load was $16 \%, 18 \%$ and $27 \%$ for the $12.7-\mathrm{mm}, 15.9-\mathrm{mm}$ and $19.1-\mathrm{mm}$ diameter adhesive anchors, respectively for the embedment depth of $101.6 \mathrm{~mm}$ at high strain rate of $10^{3} \mathrm{~s}^{-1}$ (Figure 552). The increase in the ultimate shear load was $16 \%, 15 \%$ and $15 \%$ for the $12.7-\mathrm{mm}, 15.9-$ $\mathrm{mm}$ and 19.1-mm diameter adhesive anchors, respectively for the embedment depth of $152.4 \mathrm{~mm}$ at high strain rate of $10^{3} \mathrm{~s}^{-1}$ where steel anchor failure is observed at all the strain rates investigated (Figure 5-53). At high strain rates of $10^{2} \mathrm{~s}^{-1}$ and $10^{3} \mathrm{~s}^{-1}$, the concrete strength increased and thus the concrete resistance to the applied shear load increased. The increase in the concrete strength at high strain rate is attributed to the internal cracking that is propagated severely in the concrete. The concrete is a brittle material when subjected to failure load there is no time for unloading and this resulted in confining stresses in the concrete and thus increase the dynamic strength (Bischoff \& Perry, 1991). Also, the strain rate has an influence on the mechanical properties of the steel, both the tensile and yield strength of the steel increased with the increase in the strain rate (Fu et al., 1991; Hopperstad et al., 2003; Yu et al., 2009). Furthermore, the strain rate has an influence on the flow stress-strain response and the microstructure of the steel (Lee et al., 2007). The increase in the strain rate increased the level of deformation of the steel where severe strain 
concentration is observed along narrow area called adiabatic shear band (Odeshi et al., 2005). The adiabatic shear band creates plastic instability that leads to fracture (Lee et al., 2007).

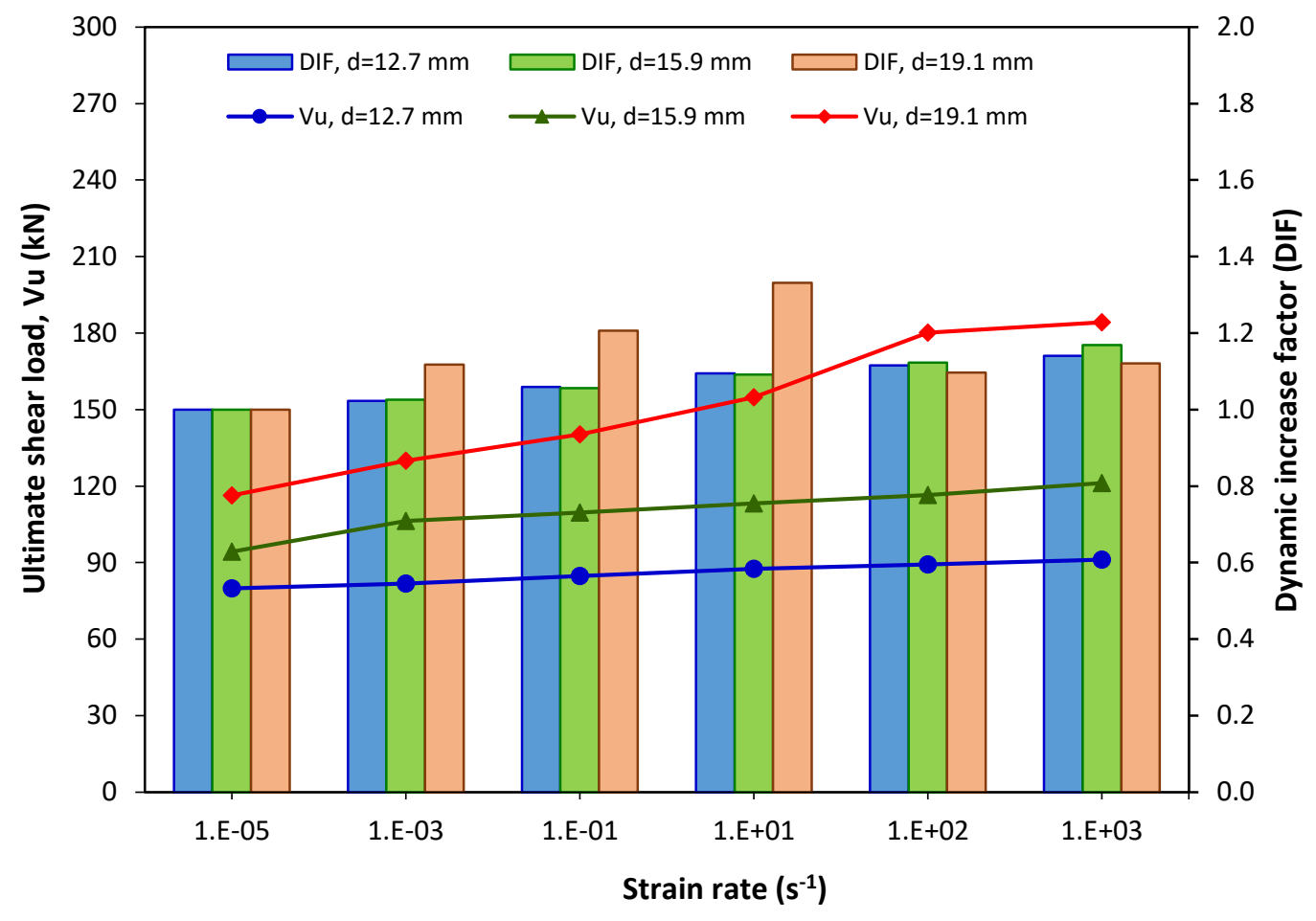

Figure 5-51: Ultimate shear load and DIF versus strain rate for adhesive anchors at 76.2 mm embedment depth 


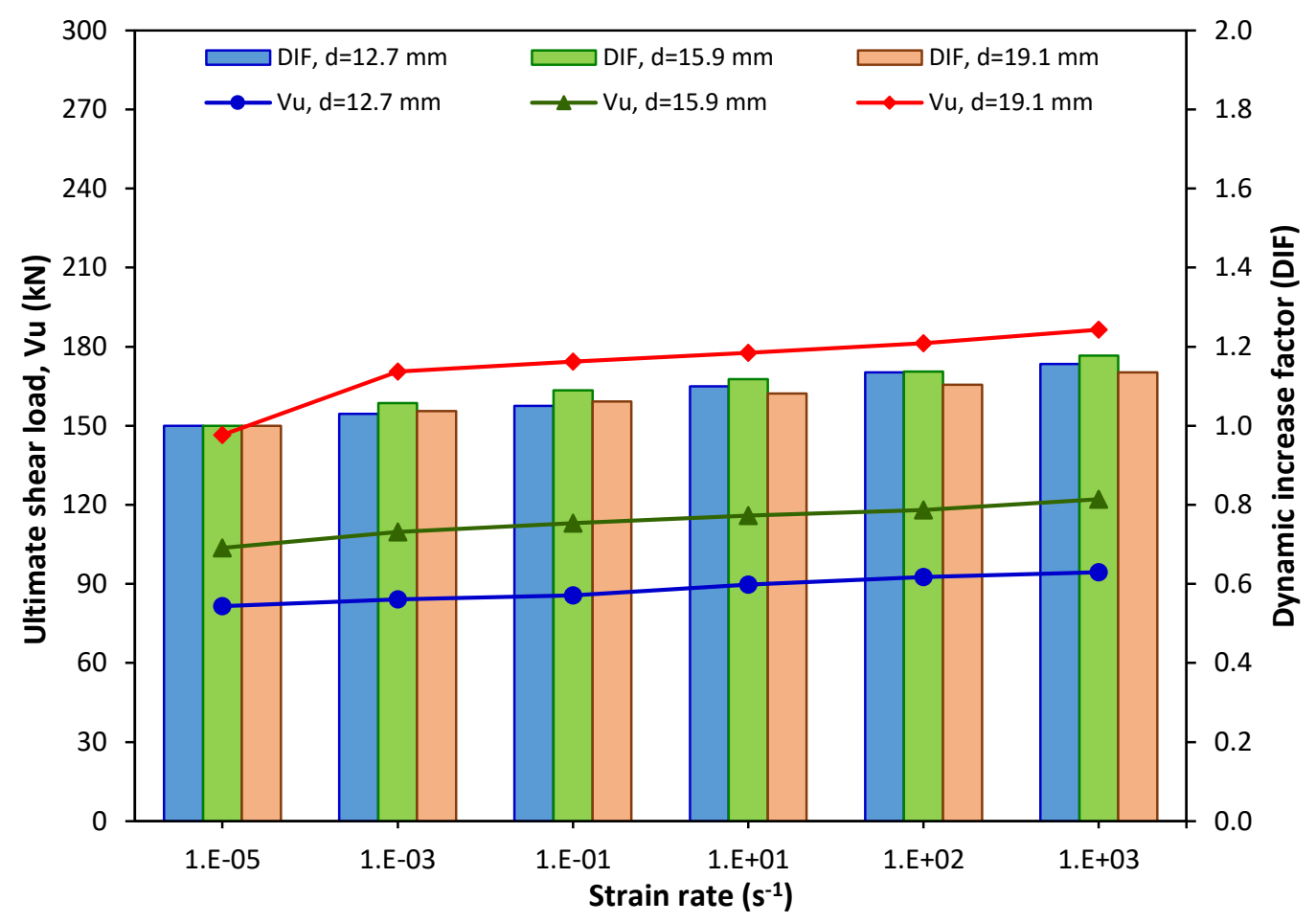

Figure 5-52: Ultimate shear load and DIF versus strain rate for adhesive anchors at 101.6 mm embedment depth

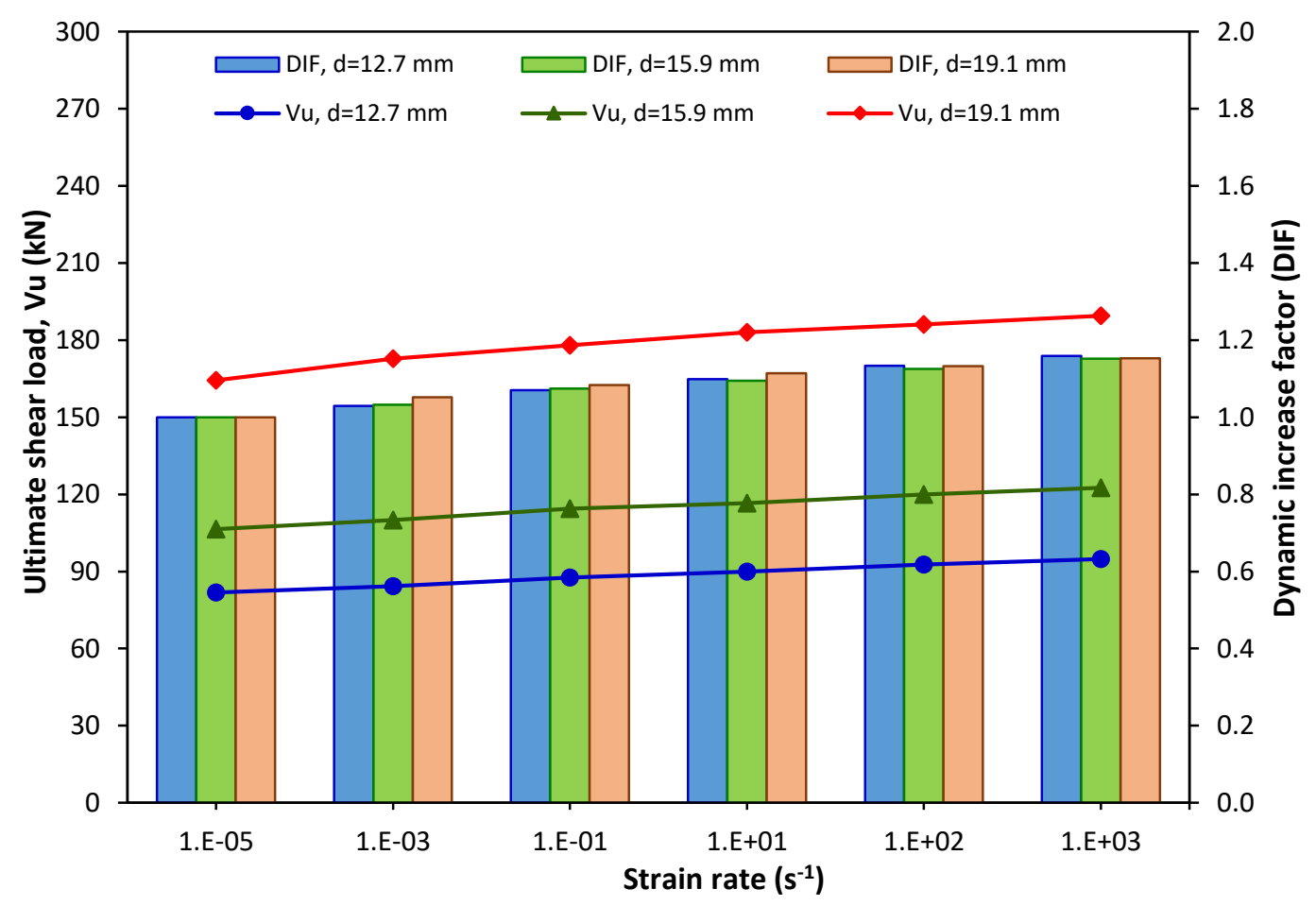

Figure 5-53: Ultimate shear load and DIF versus strain rate for adhesive anchors at 152.4 mm embedment depth 
The findings in the Figures 5-51, 5-52 and 5-53 demonstrate that the DIF increased with the increase in the strain rate from $10^{-5} \mathrm{~s}^{-1}$ to $10^{3} \mathrm{~s}^{-1}$. For the $19.1 \mathrm{~mm}$ diameter adhesive anchor with $76.2 \mathrm{~mm}$ embedment depth (Figure 5-51), the DIF increased with the increase in the strain rate up to $10 \mathrm{~s}^{-1}$ where transition in the failure mode from pryout failure to concrete spalling was observed. Steel failure was observed at high strain rates of $10^{2} \mathrm{~s}^{-1}$ and $10^{3} \mathrm{~s}^{-1}$ with a slight increase in the DIF to 1.12 . The increase in DIF is due to the increase in the strength of steel and concrete materials with the increase in strain rate. The increase in the concrete strength at high strain rate is higher than the increase in the steel strength. Maximum DIF of 1.33 was obtained for the $19.1 \mathrm{~mm}$ diameter adhesive anchor with embedment depth of $76.2 \mathrm{~mm}$ at strain rate of $10 \mathrm{~s}^{-1}$. It can be noticed from Figure 553 , that the influence of anchor diameter on the DIF at embedment depth of $152.4 \mathrm{~mm}$ is insignificant for the strain rates investigated where steel anchor failure is the dominant failure mode. Average DIF of 1.15 was obtained for adhesive anchors with embedment depth of $152.4 \mathrm{~mm}$ at high strain rate of $10^{3} \mathrm{~s}^{-1}$. This is comparable to the DIF of 1.2 reported by Braimah et al. for adhesive anchors subjected to impulse dynamic load and penetrated in normal substrate angle (Braimah et al., 2009). Cowell (1969) obtained DIF values of $1.25,1.33$ and 1.53 for A36 steel at strain rates of $0.03 \mathrm{~s}^{-1}, 0.1 \mathrm{~s}^{-1}$ and $1 \mathrm{~s}^{-1}$ respectively (Cowell, 1969). Wakabayashi et al. investigated the effect of strain rate on the yield strength of round and deformed steel. The authors obtained $1.07 \%$ and $1.08 \%$ increase in the yield strength at strain rate of $5 \times 10^{-3} \mathrm{~s}^{-1}$ for the deformed and round steel bars respectively, the increase was $1.18 \%$ and $1.16 \%$ at strain rate of $10^{-1} \mathrm{~s}^{-1}$ (Wakabayashi et al., 1980). 
Table 5-19 shows the maximum DIF for the adhesive anchors under shear load where pryout and steel anchor failure modes were observed. The value of DIF equal to one represents the DIF at the static strain rate of $10^{-5} \mathrm{~s}^{-1}$, where pryout failure mode is observed. Steel anchor failure was observed for most of the adhesive anchors at low strain rate of $10^{-}$ ${ }^{5} \mathrm{~s}^{-1}$ and higher strain rates up to $10^{3} \mathrm{~s}^{-1}$. Maximum DIF for the adhesive anchors exhibited steel failure was obtained at high strain rate of $10^{3} \mathrm{~s}^{-1}$ and presented in Table 5-19.

Table 5-19: Maximum dynamic increase factor for the adhesive anchors under shear load

\begin{tabular}{|c|c|c|c|c|c|c|}
\hline \multirow{2}{*}{$\begin{array}{c}\text { Model } \\
\text { No. }\end{array}$} & \multirow{2}{*}{$\begin{array}{c}\mathbf{d} \\
(\mathrm{mm})\end{array}$} & \multirow{2}{*}{$\begin{array}{c}\mathbf{h}_{\mathrm{ef}} \\
(\mathbf{m m})\end{array}$} & \multirow{2}{*}{$\begin{array}{c}\dot{\dot{\varepsilon}} \\
\left(\mathrm{s}^{-1}\right)\end{array}$} & DIF & \multirow{2}{*}{$\begin{array}{c}\dot{\dot{\varepsilon}} \\
\left(\mathrm{s}^{-1}\right)\end{array}$} & \multirow{2}{*}{$\frac{\text { DIF }}{\text { S }}$} \\
\hline & & & & PR & & \\
\hline 1 & 12.7 & 76.2 & - & - & $10^{3}$ & 1.14 \\
\hline 2 & 12.7 & 101.6 & - & - & $10^{3}$ & 1.16 \\
\hline 3 & 12.7 & 152.4 & - & - & $10^{3}$ & 1.16 \\
\hline 4 & 15.9 & 76.2 & $10^{-5}$ & 1.00 & $10^{3}$ & 1.17 \\
\hline 5 & 15.9 & 101.6 & - & - & $10^{3}$ & 1.18 \\
\hline 6 & 15.9 & 152.4 & - & - & $10^{3}$ & 1.15 \\
\hline 7 & 19.1 & 76.2 & $10^{-5}$ & 1.00 & $10^{3}$ & 1.12 \\
\hline 8 & 19.1 & 101.6 & $10^{-5}$ & 1.00 & $10^{3}$ & 1.13 \\
\hline 9 & 19.1 & 152.4 & - & - & $10^{3}$ & 1.15 \\
\hline
\end{tabular}

\subsubsection{Regression analysis for adhesive anchor under shear load}

Regression analysis was carried out to predict the relation between the DIF and the strain rate ratio for the adhesive anchors subjected to shear load. Steel anchor failure was observed as the dominant failure mode for the adhesive anchors subjected to shear load investigated in this research (Figures 5-38, 5-39 and 5-40). Hence regression analysis was conducted to this failure mode. Average value of the DIF for anchor diameters of $12.7 \mathrm{~mm}$, $15.9 \mathrm{~mm}$ and $19.1 \mathrm{~mm}$ was calculated to adjust the DIF for the effect of anchor diameter. Figure 5-54 presents the relation between the DIF and the strain rate ratio $\left(\dot{\varepsilon}_{d} / \dot{\varepsilon}_{s}\right)$ for the 
adhesive anchors exhibiting steel failure mode. The minimum recommended embedment depth for the adhesive anchor diameter of $19.1 \mathrm{~mm}$ is $89 \mathrm{~mm}$ (Hilti, 2014), hence anchor diameter of $19.1 \mathrm{~mm}$ with embedment depth of $76.2 \mathrm{~mm}$ was excluded from the regression analysis.

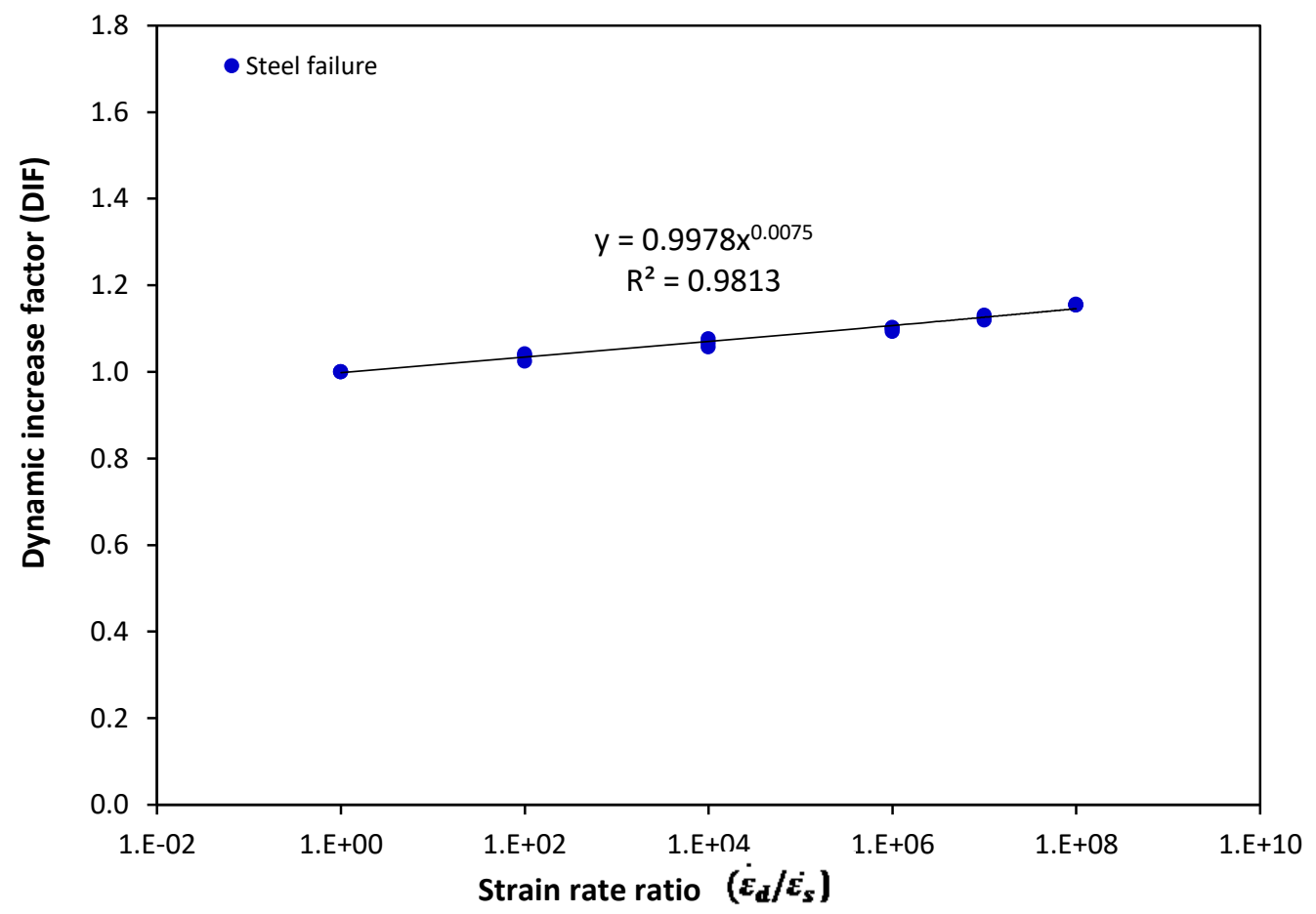

Figure 5-54: Effect of strain rate ratio on the DIF for adhesive anchor exhibiting steel failure under shear load

Various statistical models are used to predict the relation between the DIF and strain rate ratio as shown in Table 5-20. 
Table 5-20: Statistical models and coefficient of determination to predict the DIF for adhesive anchor exhibited steel failure under shear load

\begin{tabular}{|c|c|c|}
\hline Statistical models & Formulae & Coefficient of determination $\left(\mathrm{R}^{2}\right)$ \\
\hline Exponential & $D I F=1.0607 e^{9 E-10\left(\frac{\dot{\varepsilon}_{d}}{\dot{\varepsilon}_{s}}\right)}$ & 0.454 \\
\hline Linear & $D I F=1 E-9\left(\frac{\dot{\varepsilon}_{d}}{\dot{\varepsilon}_{s}}\right)+1.0616$ & 0.471 \\
\hline Logarithmic & $D I F=0.0081 \ln \left(\frac{\dot{\varepsilon}_{d}}{\dot{\varepsilon}_{s}}\right)+0.9963$ & 0.978 \\
\hline Power & $D I F=0.9978\left(\frac{\dot{\varepsilon}_{d}}{\dot{\varepsilon}_{s}}\right)^{0.0075}$ & 0.981 \\
\hline
\end{tabular}

From Table 5-20, it can be seen that power model exhibits the highest coefficient of determination $\left(\mathrm{R}^{2}\right)$ of $98 \%$ for the adhesive anchors exhibiting steel failure mode. The predicted formulae for the DIF of the adhesive anchor under shear load can be presented using the power model as in Equation (5.7).

$D I F=0.9978\left(\frac{\dot{\varepsilon}_{d}}{\dot{\varepsilon}_{s}}\right)^{0.0075}$

The proposed Equation (5.7) can be used to predict the ultimate dynamic shear load $\left(V_{u d}\right)$ as follows:

$V_{u d}=V_{u s} \times 0.9978\left(\frac{\dot{\varepsilon}_{d}}{\dot{\varepsilon}_{s}}\right)^{0.0075}$

Where the ultimate static load $\left(V_{u s}\right)$ can be determined from Equation (4.18) for steel anchor failure mode.

Residual analysis has been performed for the adhesive anchors to measure the difference between the results obtained from finite element analysis and results of DIF obtained from 
Equation (5.7). Figure 5-55 presents the residual plots for the adhesive anchors. As shown in Figure 5-55, the residual exhibits horizontal trend line with strain rate ratio.

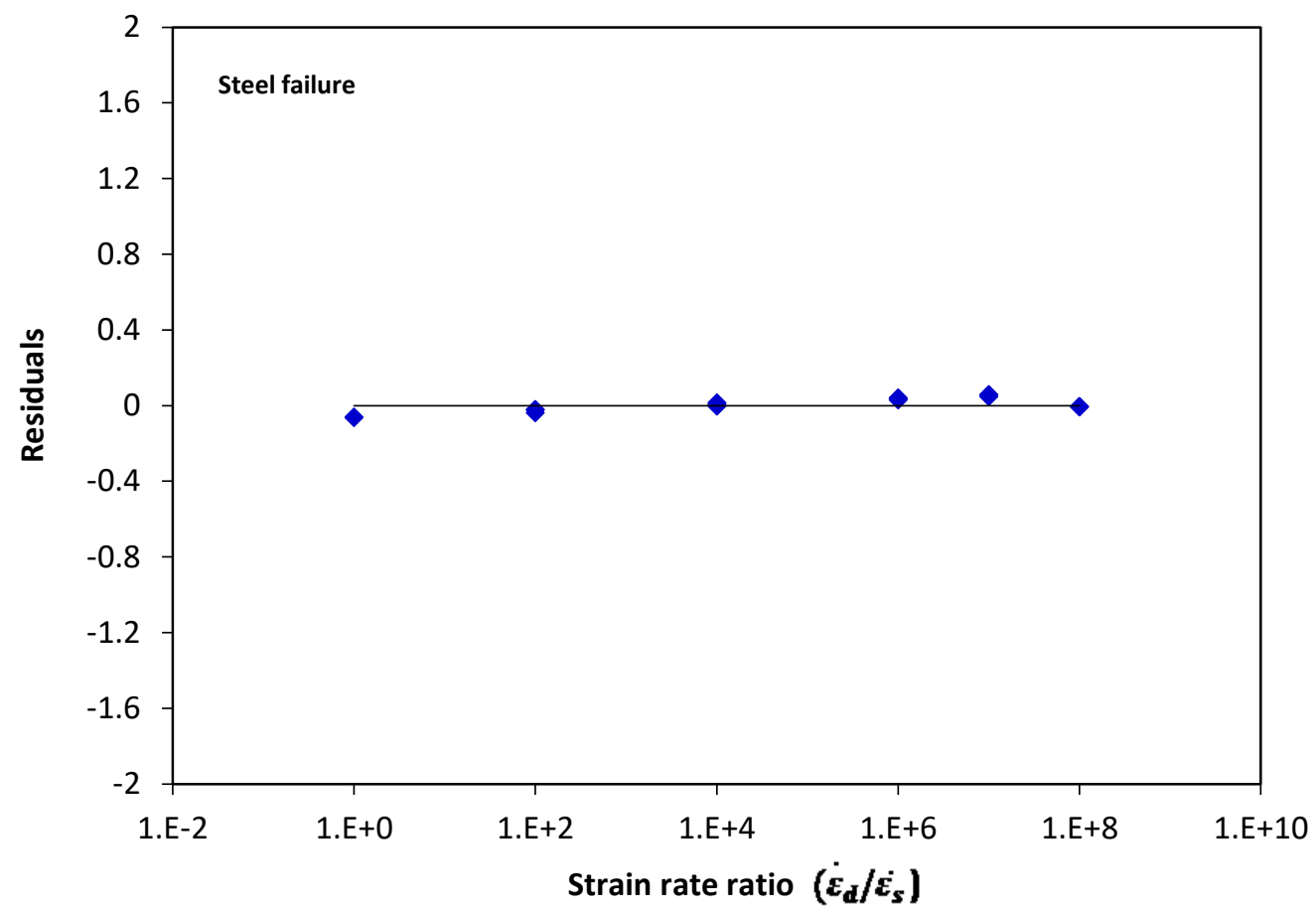

Figure 5-55: Residual versus strain rate ratio for the adhesive anchor subjected to shear load and exhibited steel failure

New adhesive anchor models with diameters of $9.5 \mathrm{~mm}, 12.7 \mathrm{~mm}, 15.9 \mathrm{~mm}$ and $19.1 \mathrm{~mm}$ and embedment depths of $89 \mathrm{~mm}, 114 \mathrm{~mm}$ and $140 \mathrm{~mm}$ were developed. The adequacy of the proposed equation was validated by comparing the results with the new adhesive anchor models with different design parameters. Figure 5-56 presents the relation between the DIF obtained from the finite element analysis of the newly developed models and the regression models (Equation (5.7)) for adhesive anchors exhibited steel failure. As shown in the figure, the DIF is observed distributed around the equality line. 


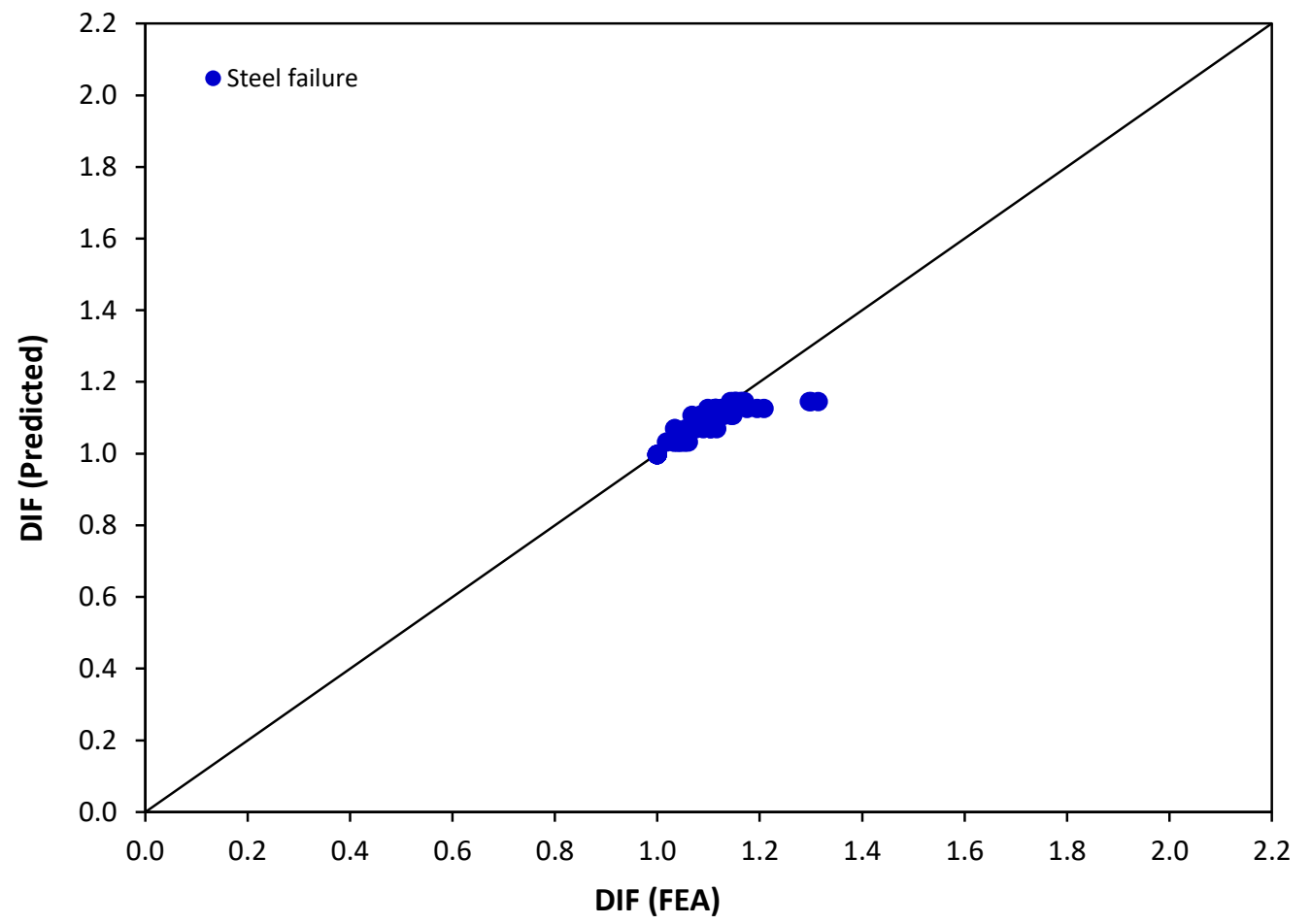

Figure 5-56: DIF obtained from the finite element analysis versus the predicted DIF for the adhesive anchor exhibited steel failure under shear load

\subsection{Summary}

In this chapter finite element analyses were performed to investigate the tensile and shear behaviour of the adhesive anchorage to concrete system models. The chapter begun with validation of the adhesive anchor models with the experimental results from the literature. Mesh sensitivity analyses were performed to obtain the optimum mesh size that can represent the experimental results accurately. The results of the finite element analysis have been compared with the ACI and CCD design methods. Also, the results of the finite element analysis were compared with the results obtained from the literature. Level of damage and failure mode for the adhesive anchors subjected to tensile and shear loads at strain rates ranging from $10^{-5} \mathrm{~s}^{-1}$ to $10^{3} \mathrm{~s}^{-1}$ were drawn and discussed. For the adhesive 
anchors subjected to tensile load, three types of failure modes were observed: concrete cone breakout, combined cone-bond failure and steel anchor failure. For the adhesive anchors subjected to shear loads three types of failure modes were observed: pryout, concrete spalling and steel anchor failure. Concrete cone depth and concrete cone breakout diameter for adhesive anchor under tensile load were determined. Load-displacement relations for adhesive anchor under tensile and shear loads were drawn and analyzed. The relation between ultimate load, failure mode and strain rate for the adhesive anchors subjected to tensile and shear loads were drawn and discussed. Dynamic increase factor (DIF) was determined for the adhesive anchors at all the strain rates investigated. Equations were developed to determine the relation between the DIF and strain rate for the adhesive anchors investigated. 


\section{Chapter 6 : Strain rate effect on the undercut anchors}

\subsection{Finite element modelling for undercut anchors under tensile load}

Finite element analyses were performed to investigate the tensile behaviour of the undercut anchors using LS-DYNA. Undercut anchor diameters of $12 \mathrm{~mm}, 16 \mathrm{~mm}$ and $20 \mathrm{~mm}$ with recommended effective embedment depths of $125 \mathrm{~mm}, 190 \mathrm{~mm}$ and $250 \mathrm{~mm}$, respectively (Hilti, 2011) were investigated. In addition, embedment depth of $100 \mathrm{~mm}$ was investigated. Figure 6-1 shows a schematic view of the undercut anchorage to concrete system. Figure 6-2 shows the geometric configuration and boundary conditions for the undercut anchor model under tensile load.

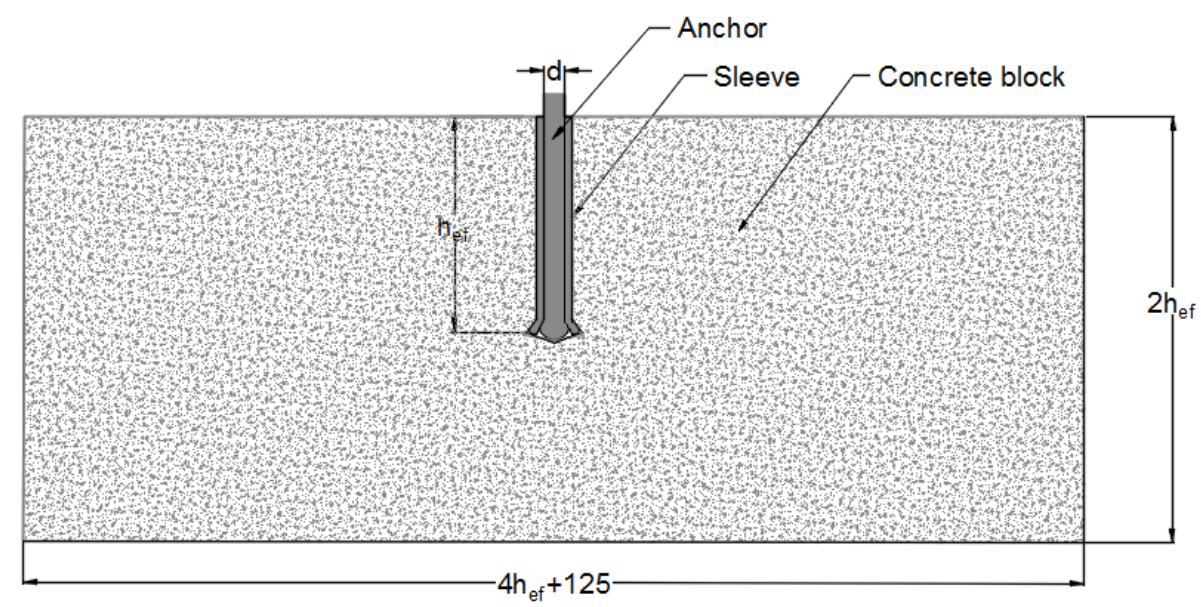

Figure 6-1: A schematic view for the undercut anchor model 


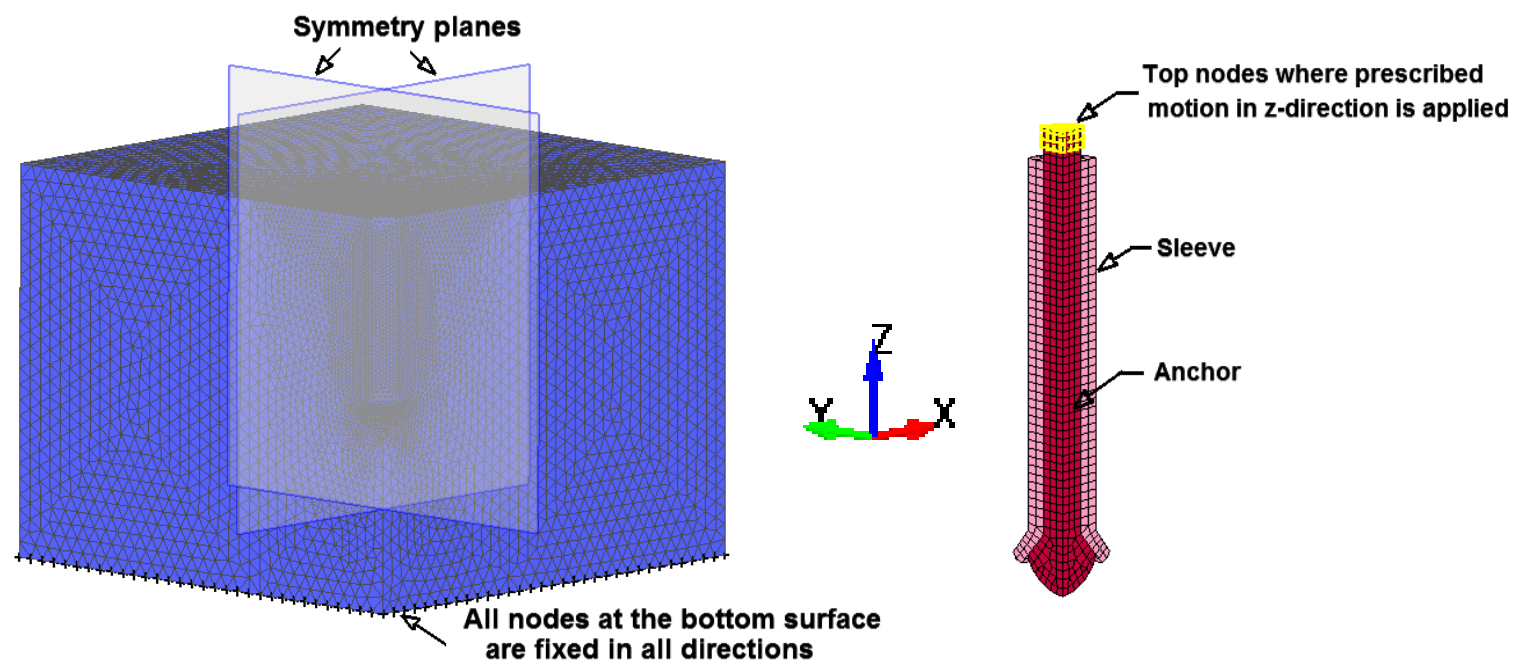

$\begin{array}{ll}\text { (a) concrete mesh } & \text { (b) undercut anchor mesh }\end{array}$

Figure 6-2: Geometric configuration and boundary conditions for the undercut anchor model

\subsubsection{Validation of undercut anchor model under tensile load}

To assess the ability of LS-DYNA model to predict the tensile behaviour of the undercut anchor, a finite element model representing the experimental test conducted by Mahadik et al. (Mahadik et al., 2016) was developed to validate the undercut anchor model. Concrete block size of $(700 \times 300 \times 400) \mathrm{mm}$ and cube compressive strength of $42.6 \mathrm{MPa}\left(f_{c}^{\prime}=33.5\right.$ $\mathrm{MPa}$ ) was used. Hilti undercut anchor, through-set type, of 12-mm diameter, $125 \mathrm{~mm}$ embedment depth and $640 \mathrm{MPa}$ yield strength was used for the analysis. Mesh sensitivity analyses were carried out to determine the optimum mesh size for both the steel undercut anchor and concrete block to maximize accuracy of the results while minimizing required computer resources. Table 6-1 shows various element mesh sizes for the steel anchor and concrete, the ultimate load, and failure mode for each mesh size. 
Table 6-1: Effect of mesh size refinement on the convergence of ultimate tensile load for undercut anchor

\begin{tabular}{|c|c|c|c|c|c|c|c|c|}
\hline \multirow[t]{3}{*}{$\begin{array}{c}\text { Model } \\
\text { No. }\end{array}$} & \multicolumn{3}{|c|}{$\begin{array}{l}\text { Mesh size } \\
(\mathrm{mm})\end{array}$} & \multicolumn{2}{|c|}{$\begin{array}{l}\text { Ultimate tensile load } \\
\qquad(\mathrm{kN})\end{array}$} & \multicolumn{2}{|c|}{$\begin{array}{l}\text { Displacement } \\
(\mathrm{mm})\end{array}$} & \multirow[t]{3}{*}{ Failure mode } \\
\hline & \multirow[t]{2}{*}{ Anchor } & \multicolumn{2}{|c|}{ Concrete } & \multirow[t]{2}{*}{ FEA } & \multirow[t]{2}{*}{ EXP. } & \multirow[t]{2}{*}{ FEA } & \multirow[t]{2}{*}{ EXP } & \\
\hline & & Min. & Max. & & & & & \\
\hline 1 & 2 & 4 & 12 & 66.5 & \multirow{3}{*}{72.8} & 5.13 & \multirow{3}{*}{4.67} & Steel failure \\
\hline 2 & 2 & 2 & 12 & 71.4 & & 4.74 & & Steel failure \\
\hline 3 & 1 & 1 & 12 & 74.2 & & 4.71 & & Steel failure \\
\hline
\end{tabular}

A percentage difference between the finite element analysis and the experimental results of $9.47 \%, 1.96 \%$ and $1.92 \%$ for the ultimate tensile load were obtained for the models No. 1,2 and 3 respectively. Mesh size of model No. 2 gave converged results to the experimental results conducted by Mahadik et al. with better computation time and was selected for the analysis. Figure 6-3 shows a comparison of tensile load-displacement response between the experimental results obtained by Mahadik et al. (Mahadik et al., 2016) and the finite element results. Ultimate tensile loads of $71.4 \mathrm{kN}$ and $72.8 \mathrm{kN}$ were obtained from the finite element analysis and the experimental test conducted by Mahadik et al. (Mahadik et al., 2016), respectively. The experimental results exhibited 1.96\% higher ultimate tensile load than the finite element analysis results. Both the finite element analysis and experimental test resulted in steel anchor failure mode as shown in Figure 64. 


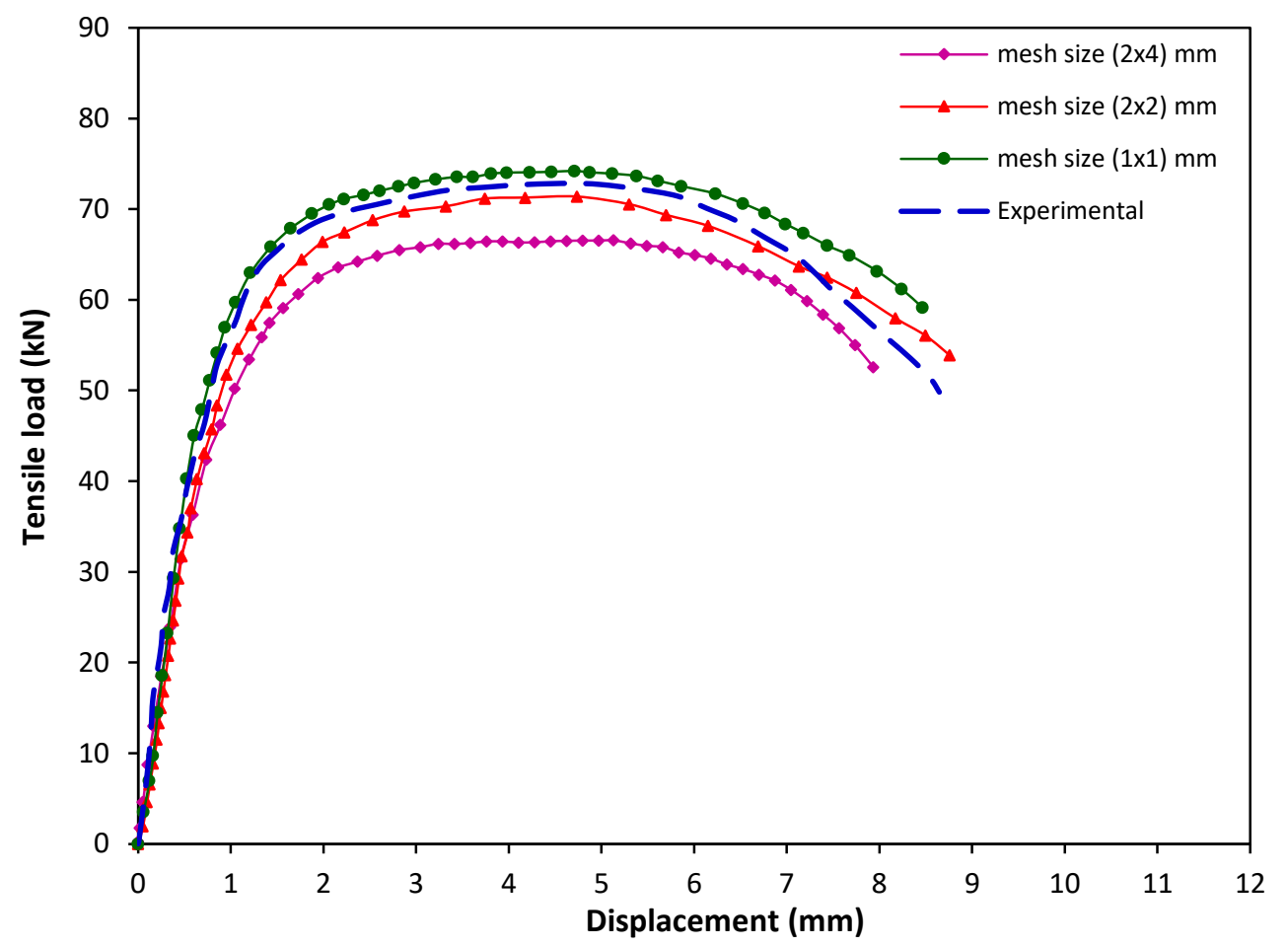

Figure 6-3: Tensile load-displacement response of the FEA and experimental results obtained by Mahadik et al. (Mahadik et al., 2016)

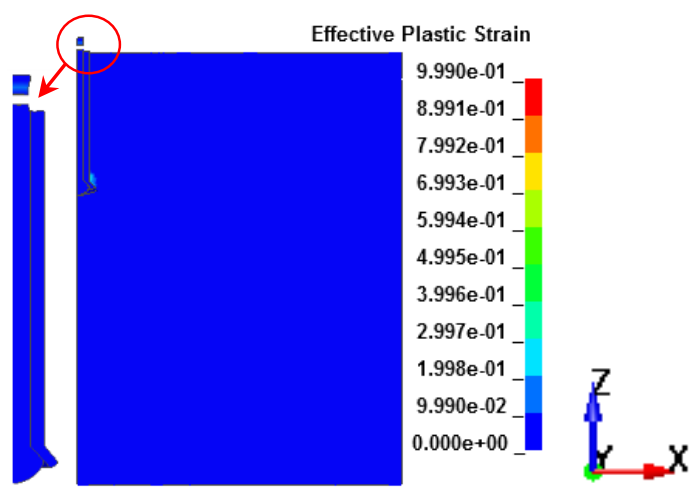

(a) Finite element analysis

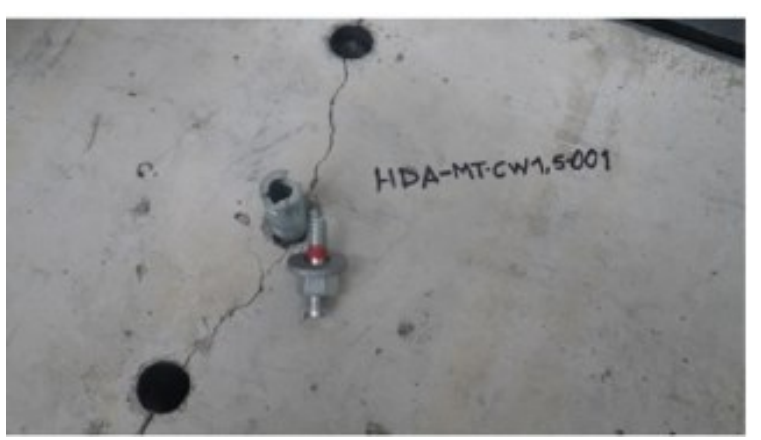

(b) Experimental (Mahadik et al., 2016)

Figure 6-4: Failure mode of the undercut anchors under tensile load observed from the finite element analysis and the experimental results by (Mahadik et al., 2016) 


\subsubsection{Comparison of FEA results with the ACI and CCD design methods}

Ultimate tensile loads obtained from the finite element analysis (FEA) at strain rate of $10^{-}$ ${ }^{5} \mathrm{~s}^{-1}$ were compared with anchor capacity obtained by use of the American Concrete Institute ACI 349-85 (Equations 4.1 for concrete cone breakout failure and 4.2 for steel anchor failure) and Concrete Capacity Design (CCD) (Equation 4.3) methods, as presented in Table 6-2. Fuchs et al. (1995) proposed an equation to predict the concrete cone breakout failure for the cast-in-place and post-installed anchors (Equation 4.3) which was subsequently adopted by ACI 318 code (Eligehausen, et al., 2006; Fuchs et al., 1995). The equivalent anchor head diameter $\left(d_{h}\right)$ (in Equation 4.1) of undercut anchors is $\geq 2.5 d$ (Hilti, 2011). For safe anchorage to concrete design, minimum ultimate load obtained from (Equations 4.1 and 4.2) is considered for the comparison of the FEA results with the ACI method. Also, minimum ultimate value obtained from (Equations 4.2 and 4.3 ) is considered for the comparison of the FEA results with the CCD method and included in Table 6-2.

It can be seen from Table 6-2 that the ACI and CCD methods underpredict the ultimate load for the anchor with a diameter of $12 \mathrm{~mm}$ and embedment depth of 100 and anchor diameters of $16 \mathrm{~mm}$ and $20 \mathrm{~mm}$ with embedment depths of $100 \mathrm{~mm}$ and $125 \mathrm{~mm}$. The ACI method underestimate the failure load for the shallow embedment depths (Fuchs et al., 1995). Also, the anchor diameter is not considered in the ACI (Equation 4.1) and CCD (Equation 4.3) methods. In general the finite element analysis results are in good agreement with the ACI and CCD methods. 
Table 6-2: Comparison of ultimate tensile loads obtained from FEA with ACI and CCD methods

\begin{tabular}{|c|c|c|c|c|c|c|c|c|}
\hline \multirow[t]{2}{*}{$\begin{array}{c}\mathrm{d} \\
(\mathrm{mm})\end{array}$} & \multirow[t]{2}{*}{$\begin{array}{c}\mathrm{h}_{\mathrm{ef}} \\
(\mathrm{mm})\end{array}$} & \multicolumn{3}{|c|}{$\begin{array}{l}\text { Ultimate tensile load } \\
\qquad(\mathrm{kN})\end{array}$} & \multirow[t]{2}{*}{$\begin{array}{c}\delta \\
(\mathrm{mm})\end{array}$} & \multirow[t]{2}{*}{ FEA/ACI } & \multirow[t]{2}{*}{ FEA/CCD } & \multirow{2}{*}{$\begin{array}{l}\text { *Failure } \\
\text { mode } \\
\text { (FEA) }\end{array}$} \\
\hline & & FEA & $\mathrm{ACI}$ & CCD & & & & \\
\hline \multirow{3}{*}{12} & 100 & 83.14 & 75.91 & 82.12 & 1.32 & 1.10 & 1.01 & $\mathrm{~S}$ \\
\hline & 125 & 83.22 & 90.48 & 90.48 & 1.34 & 0.92 & 0.92 & $\mathrm{~S}$ \\
\hline & 190 & 83.94 & 90.48 & 90.48 & 1.38 & 0.93 & 0.93 & $\mathrm{~S}$ \\
\hline \multirow{3}{*}{16} & 100 & 101.09 & 81.75 & 82.12 & 1.78 & 1.24 & 1.23 & $\mathrm{CC}$ \\
\hline & 125 & 126.62 & 120.44 & 114.76 & 1.88 & 1.05 & 1.10 & $\mathrm{~S}$ \\
\hline & 190 & 127.16 & 160.85 & 160.85 & 1.87 & 0.79 & 0.79 & $\mathrm{~S}$ \\
\hline \multirow{3}{*}{20} & 100 & 118.41 & 87.59 & 82.12 & 1.27 & 1.35 & 1.44 & $\mathrm{CC}$ \\
\hline & 125 & 168.66 & 127.74 & 114.76 & 1.81 & 1.32 & 1.47 & $\mathrm{CC}$ \\
\hline & 190 & 211.46 & 251.33 & 215.06 & 2.03 & 0.84 & 0.98 & $\mathrm{~S}$ \\
\hline
\end{tabular}

*Failure mode: $\mathrm{CC}=$ concrete cone breakout, $\mathrm{S}=$ steel anchor fracture

\subsubsection{Effect of strain rate on the level of damage and failure mode}

Figure 6-5 shows the level of damage for the undercut anchor diameters of $12 \mathrm{~mm}, 16 \mathrm{~mm}$ and $20 \mathrm{~mm}$ with different embedment depths at low strain rate of $10^{-5} \mathrm{~s}^{-1}$. As shown in the figure, two types of failure modes were observed at low strain rate of $10^{-5} \mathrm{~s}^{-1}:$ concrete cone breakout failure and steel failure. Concrete cone breakout failure was observed at embedment depth of $100 \mathrm{~mm}$ for anchor diameters of $16 \mathrm{~mm}$ and $20 \mathrm{~mm}$ whereas steel anchor failure was observed for the anchor diameter of $12 \mathrm{~mm}$. At the beginning of the loading, cracks started to appear at the lower ends of the sleeve, then the cracks grow and propagate diagonally along the embedment depth and transferred to a wider area on the concrete forming concrete cone breakout failure. Steel anchor failure was observed at embedment depths of $125 \mathrm{~mm}, 190 \mathrm{~mm}$ and $250 \mathrm{~mm}$ for all the undercut anchor diameters except anchor diameter of $20 \mathrm{~mm}$ with embedment depth of $125 \mathrm{~mm}$ where concrete cone breakout failure was observed. 


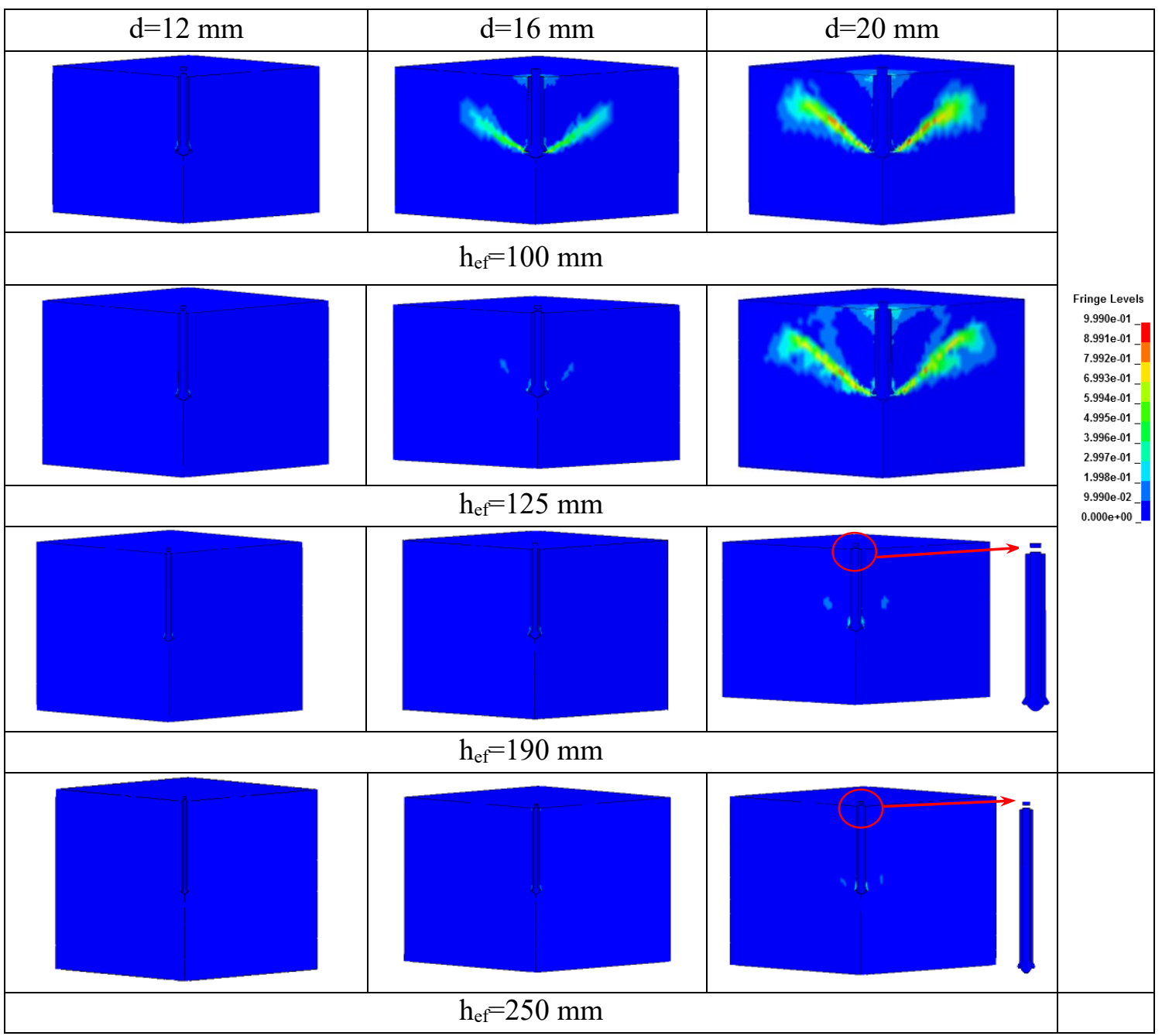

Figure 6-5: Plastic strain contours for the undercut anchors at strain rate of $10^{-5} \mathrm{~s}^{-1}$

Figures 6-6, 6-7 and 6-8 present the failure mode of $12 \mathrm{~mm}, 16 \mathrm{~mm}$ and $20 \mathrm{~mm}$ diameter undercut anchors with $100 \mathrm{~mm}, 125 \mathrm{~mm}, 190 \mathrm{~mm}$ and $250 \mathrm{~mm}$ embedment depths at different strain rates. As shown in the Figures 6-6 and 6-7, steel anchor failure was the dominant failure mode for the 12-mm and 16-mm diameter undercut anchors at strain rates ranging from $10^{-3} \mathrm{~s}^{-1}$ to $10^{3} \mathrm{~s}^{-1}$. 


\begin{tabular}{|c|c|c|c|c|c|}
\hline$\dot{\varepsilon}\left(\mathrm{s}^{-1}\right)$ & $\mathrm{h}_{\mathrm{ef}}=100 \mathrm{~mm}$ & $h_{\mathrm{ef}}=125 \mathrm{~mm}$ & $\mathrm{~h}_{\mathrm{ef}}=190 \mathrm{~mm}$ & $\mathrm{~h}_{\mathrm{ef}}=250 \mathrm{~mm}$ & \\
\hline $10^{-3}$ & w & & & & \\
\hline $10^{-1}$ & ". & & & & Fringe Levels \\
\hline 10 & , & i. & & & 5.994 e.01 \\
\hline $10^{2}$ & 14 & & & & $\begin{array}{c}9.990 \mathrm{e}-02 \\
0.000 \mathrm{e}+00\end{array}$ \\
\hline $10^{3}$ & & & & & \\
\hline
\end{tabular}

Figure 6-6: Failure mode of $12 \mathrm{~mm}$ diameter undercut anchor at different strain rates

\begin{tabular}{|c|c|c|c|c|c|}
\hline$\dot{\varepsilon}\left(\mathrm{s}^{-1}\right)$ & $\mathrm{h}_{\mathrm{ef}}=100 \mathrm{~mm}$ & $\mathrm{~h}_{\mathrm{ef}}=125 \mathrm{~mm}$ & $\mathrm{~h}_{\mathrm{ef}}=190 \mathrm{~mm}$ & $\mathrm{~h}_{\mathrm{ef}}=250 \mathrm{~mm}$ & \\
\hline $10^{-3}$ & & & & & \\
\hline $10^{-1}$ & & 1,1 & & & $\begin{array}{c}\text { Fringe Levels } \\
9.9900-01\end{array}$ \\
\hline 10 & 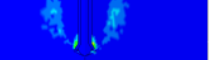 & 1,1 & " & & $\begin{array}{l}5.994 \mathrm{e}-01 \\
4.995 \mathrm{e}-01\end{array}$ \\
\hline $10^{2}$ & & & & & $0.000 \mathrm{e}+00$ \\
\hline $10^{3}$ & & & & & \\
\hline
\end{tabular}

Figure 6-7: Failure mode of $16 \mathrm{~mm}$ diameter undercut anchor at different strain rates 
As shown in Figure 6-8, concrete cone breakout failure was observed for the undercut anchor diameter of $20 \mathrm{~mm}$ with embedment depth of $100 \mathrm{~mm}$ at strain rates of $10^{-3} \mathrm{~s}^{-1}, 10^{-}$ ${ }^{1} \mathrm{~s}^{-1}$ and $10 \mathrm{~s}^{-1}$ while steel anchor failure was observed at strain rates of $10^{2} \mathrm{~s}^{-1}$ and $10^{3} \mathrm{~s}^{-1}$. At strain rates of $10^{-3}$ and $10^{-1} \mathrm{~s}^{-1}$ there was sufficient time to initiate and propagate the cracks in the concrete, formation of the conical shape resulting in concrete cone breakout failure. Whereas at high strain rates of $10^{2} \mathrm{~s}^{-1}$ and $10^{3} \mathrm{~s}^{-1}$, the steel anchor failure is attributed to the fast pullout of the anchors and increase in the concrete tensile strength at high strain rate. At embedment depth of $125 \mathrm{~mm}$, concrete cone breakout failure was observed at strain rates of $10^{-3} \mathrm{~s}^{-1}$ and $10^{-1} \mathrm{~s}^{-1}$ whereas steel anchor failure was observed at strain rates of $10 \mathrm{~s}^{-1}, 10^{2} \mathrm{~s}^{-1} 10^{3} \mathrm{~s}^{-1}$. Also, steel anchor failure was observed at embedment depths of $190 \mathrm{~mm}$ and $250 \mathrm{~mm}$ for all the strain rates investigated.

\begin{tabular}{|c|c|c|c|c|c|}
\hline$\dot{\varepsilon}\left(\mathrm{s}^{-1}\right)$ & $\mathrm{h}_{\mathrm{ef}}=100 \mathrm{~mm}$ & $\mathrm{~h}_{\mathrm{ef}}=125 \mathrm{~mm}$ & $\mathrm{~h}_{\mathrm{ef}}=190 \mathrm{~mm}$ & $\mathrm{~h}_{\mathrm{ef}}=250 \mathrm{~mm}$ & \\
\hline $10^{-3}$ & & & $(1,9$ & $\therefore$ & \\
\hline $10^{-1}$ & & & "I & w. & Fringe Levels \\
\hline 10 & & & $1 H^{4}$ & 11 & $\begin{array}{l}6.993 \mathrm{e}-01 \\
5.994 \mathrm{e}-01\end{array}$ \\
\hline $10^{2}$ & 2 & * & & A & $9.990 \mathrm{e}-02$ \\
\hline $10^{3}$ & & & & & \\
\hline
\end{tabular}

Figure 6-8: Failure mode of $20 \mathrm{~mm}$ diameter undercut anchor at different strain rates 


\subsubsection{Effect of design parameters on failure mode and ultimate tensile load}

Effect of strain rate on the ultimate tensile load and failure mode for the undercut anchors is shown in Figures 6-9, 6-10 and 6-11 for the $12 \mathrm{~mm}, 16 \mathrm{~mm}$ and $20 \mathrm{~mm}$ diameter undercut anchors respectively. It can be seen from the figures that the tensile load increased with the increase in the strain rate from $10^{-5} \mathrm{~s}^{-1}$ to $10^{3} \mathrm{~s}^{-1}$ where steel anchor failure is observed for most of the undercut anchors investigated. From Figures 6-9, 6-10 and 6-11, it can be seen that the strain rate has an influence on the failure mode of the undercut anchors.

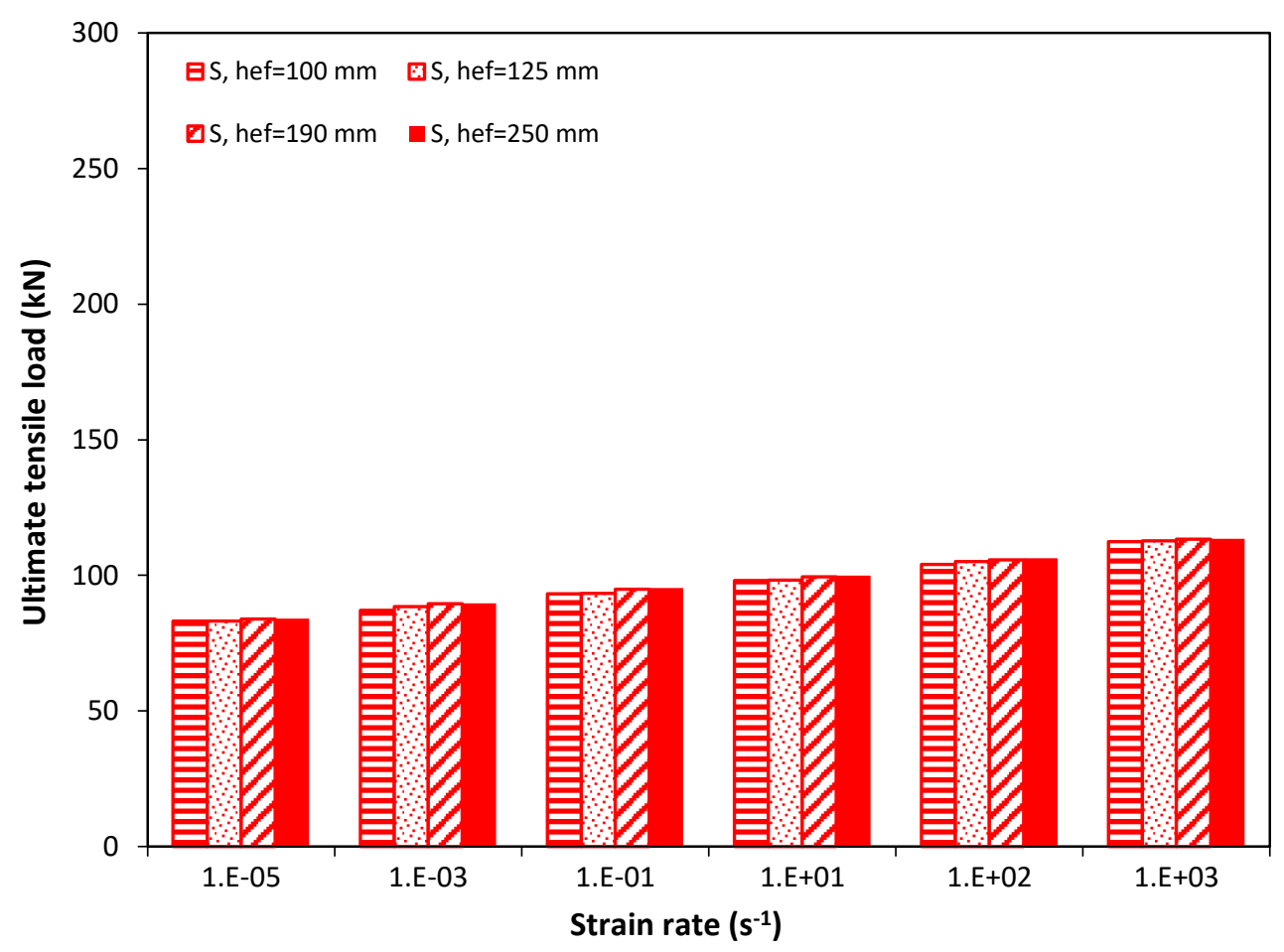

Figure 6-9: Strain rate effect on the failure mode and ultimate tensile load for undercut anchor diameter of $12 \mathrm{~mm}$ 


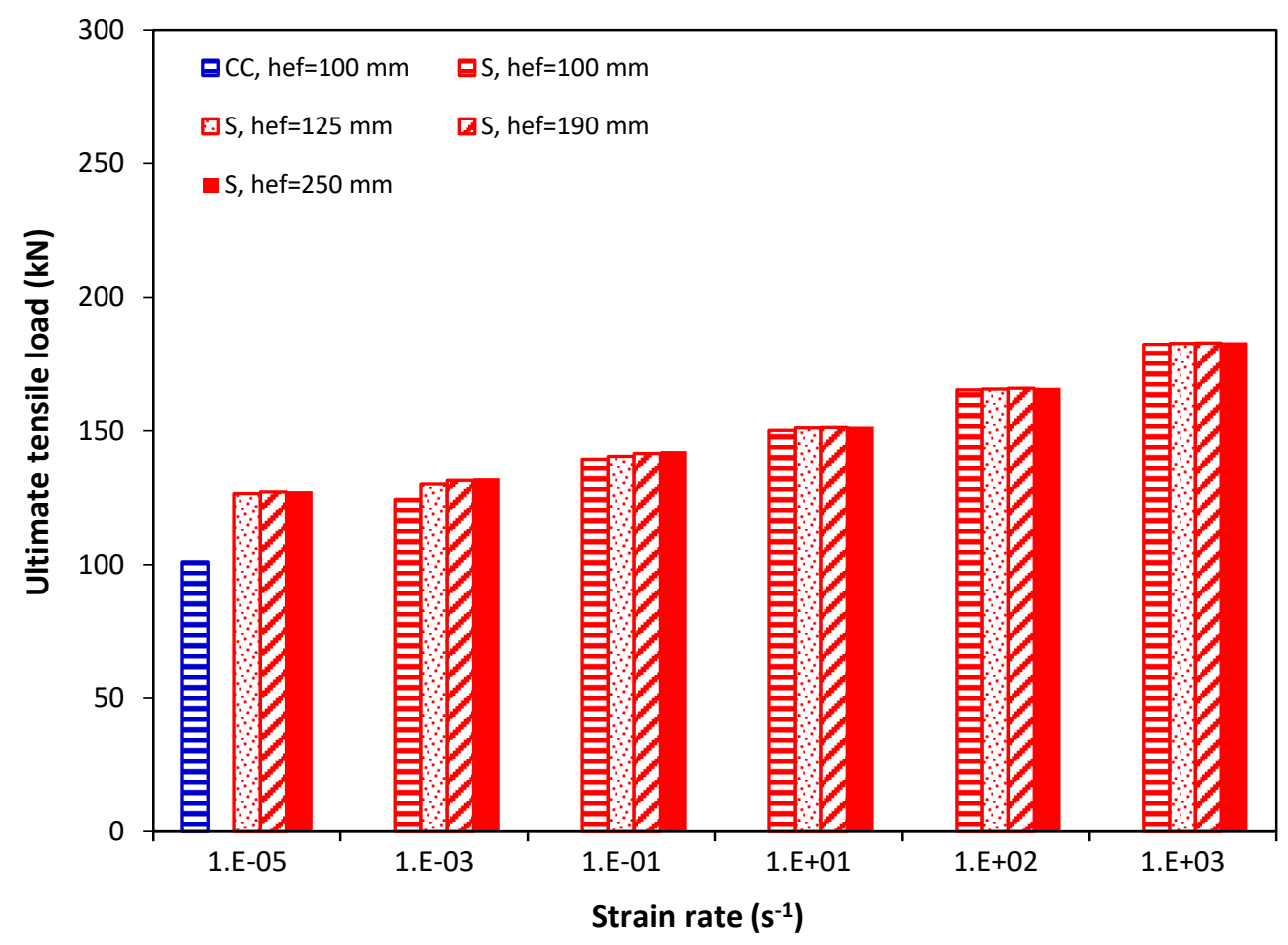

Figure 6-10: Strain rate effect on the failure mode and ultimate tensile load for undercut anchor diameter of $16 \mathrm{~mm}$

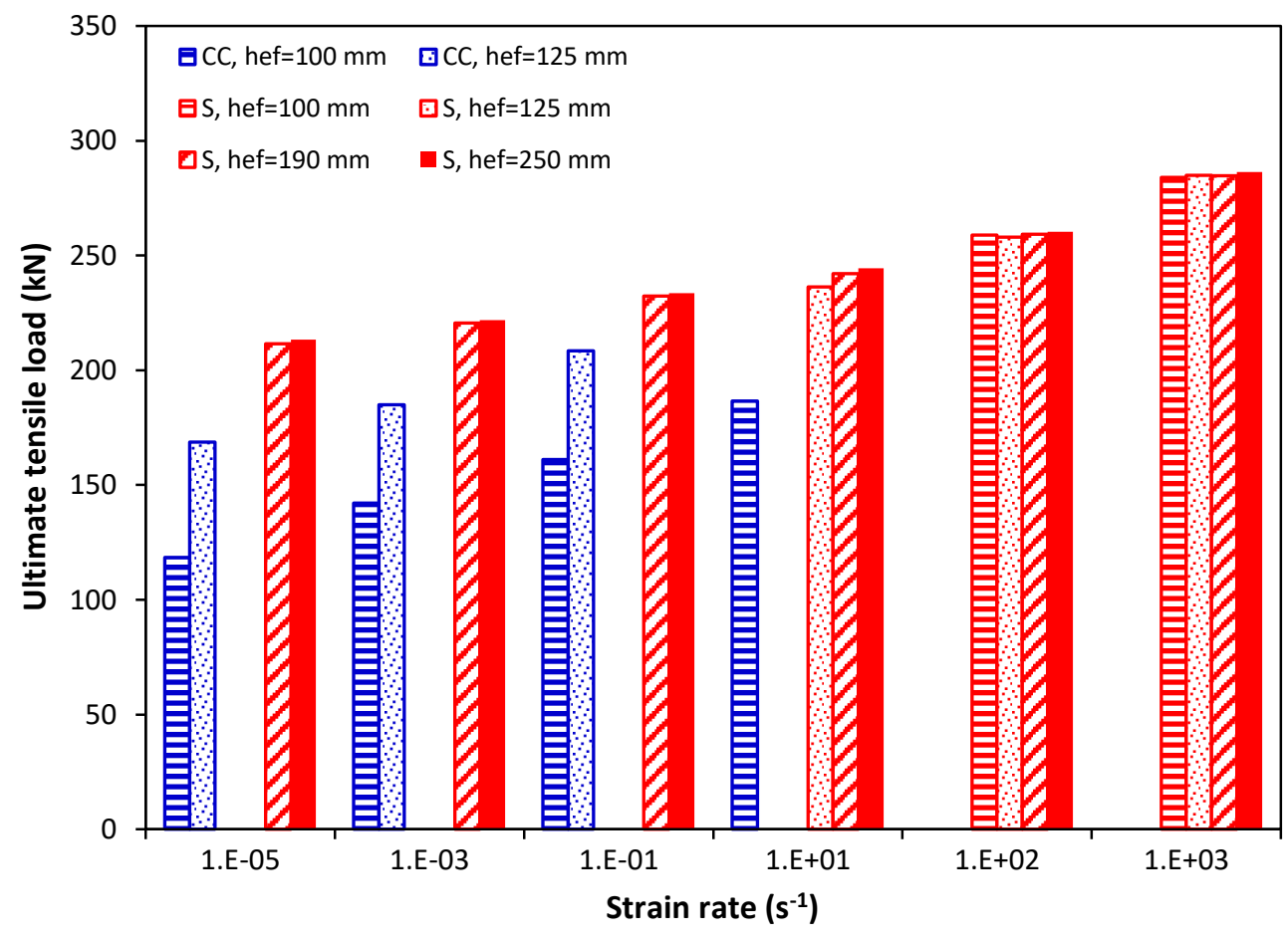

Figure 6-11: Strain rate effect on the failure mode and ultimate tensile load for undercut anchor diameter of $20 \mathrm{~mm}$ 
Figures 6-9, 6-10 and 6-11, show that the embedment depth and anchor diameter also have an effect on the failure mode. Increasing the embedment depth from $100 \mathrm{~mm}$ to $190 \mathrm{~mm}$ resulted in steel anchor failure for the anchor diameter of $20 \mathrm{~mm}$ at low strain rate of $10^{-5}$ $\mathrm{s}^{-1}$. Increasing the anchor diameter from $12 \mathrm{~mm}$ to $20 \mathrm{~mm}$ resulted in changing the failure mode from steel anchor failure to concrete cone breakout for the embedment depths of 100 $\mathrm{mm}$ and $125 \mathrm{~mm}$ at strain rates of $10^{-5} \mathrm{~s}^{-1}, 10^{-3} \mathrm{~s}^{-1}$ and $10^{-1} \mathrm{~s}^{-1}$. Also, the failure mode changed from steel failure to concrete cone breakout for embedment depth of $100 \mathrm{~mm}$ at strain rate of $10 \mathrm{~s}^{-1}$ with the increase in the anchor diameter from $12 \mathrm{~mm}$ to $20 \mathrm{~mm}$. Table 6-3 presents the failure mode for the undercut anchors under tensile load at different strain rates.

Table 6-3: Failure mode for undercut anchors under tensile load at different strain rates

\begin{tabular}{|c|c|c|c|c|c|c|c|c|}
\hline \multirow{3}{*}{$\begin{array}{c}\text { Model } \\
\text { No. }\end{array}$} & \multirow{3}{*}{$\begin{array}{c}\mathrm{d} \\
(\mathrm{mm})\end{array}$} & \multirow{3}{*}{$\begin{array}{c}h_{\text {ef }} \\
(\mathbf{m m})\end{array}$} & \multicolumn{6}{|c|}{ "Failure mode } \\
\hline & & & \multicolumn{6}{|c|}{ Strain rate $\left(\mathrm{s}^{-1}\right)$} \\
\hline & & & $\dot{\varepsilon}=10^{-5}$ & $\dot{\varepsilon}=10^{-3}$ & $\dot{\varepsilon}=10^{-1}$ & $\dot{\varepsilon}=10$ & $\dot{\varepsilon}=10^{2}$ & $\dot{\varepsilon}=10^{3}$ \\
\hline 1 & 12 & 100 & $\mathrm{~S}$ & $\mathrm{~S}$ & $\mathrm{~S}$ & $\mathrm{~S}$ & $\mathrm{~S}$ & $\mathrm{~S}$ \\
\hline 2 & 12 & 125 & $\mathrm{~S}$ & $\mathrm{~S}$ & $\mathrm{~S}$ & $\mathrm{~S}$ & $\bar{S}$ & $\bar{S}$ \\
\hline 3 & 12 & 190 & $\mathrm{~S}$ & $\mathrm{~S}$ & $\mathrm{~S}$ & $\mathrm{~S}$ & $\mathrm{~S}$ & $\bar{S}$ \\
\hline 4 & 12 & 250 & $\mathrm{~S}$ & $\mathrm{~S}$ & $\mathrm{~S}$ & $\mathrm{~S}$ & $\mathrm{~S}$ & $\mathrm{~S}$ \\
\hline 5 & 16 & 100 & $\mathrm{CC}$ & $\mathrm{S}$ & $\mathrm{S}$ & $\mathrm{S}$ & $\bar{S}$ & $\bar{S}$ \\
\hline 6 & 16 & 125 & $\mathrm{~S}$ & $\mathrm{~S}$ & $\mathrm{~S}$ & $\mathrm{~S}$ & $\bar{S}$ & $\bar{S}$ \\
\hline 7 & 16 & 190 & $\mathrm{~S}$ & $\mathrm{~S}$ & $\mathrm{~S}$ & $\mathrm{~S}$ & $\mathrm{~S}$ & $\mathrm{~S}$ \\
\hline 8 & 16 & 250 & $\mathrm{~S}$ & $\mathrm{~S}$ & $\mathrm{~S}$ & $\mathrm{~S}$ & $\mathrm{~S}$ & $\mathrm{~S}$ \\
\hline 9 & 20 & 100 & $\mathrm{CC}$ & $\mathrm{CC}$ & $\mathrm{CC}$ & $\mathrm{CC}$ & $S$ & $S$ \\
\hline 10 & 20 & 125 & $\mathrm{CC}$ & $\mathrm{CC}$ & $\mathrm{CC}$ & $\mathrm{S}$ & $S$ & $S$ \\
\hline 11 & 20 & 190 & 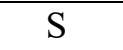 & $S$ & $\mathrm{~S}$ & $S$ & $S$ & $S$ \\
\hline 12 & 20 & 250 & $\mathrm{~S}$ & $\mathrm{~S}$ & $\mathrm{~S}$ & $\mathrm{~S}$ & $\mathrm{~S}$ & $\mathrm{~S}$ \\
\hline
\end{tabular}

*Failure mode: $\mathrm{CC}=$ concrete cone breakout, $\mathrm{S}=$ steel anchor failure 


\subsubsection{Effect of anchor embedment depth on the ultimate tensile load}

Figures 6-12, 6-13 and 6-14 show the ultimate tensile load and concrete cone depth for the 12-mm, 16-mm and 20-mm diameter undercut anchors for different embedment depths at low strain rate of $10^{-5} \mathrm{~s}^{-1}$. It can be seen that nearly same ultimate tensile load is obtained for the 12-mm anchor diameter at all the embedment depths investigated where steel anchor failure is observed as shown in Figure 6-12. For the anchor diameter of $16 \mathrm{~mm}$ (Figure 613) the ultimate tensile load increased from $101.09 \mathrm{kN}$ to $126.62 \mathrm{kN}$ as the embedment depth increased from $100 \mathrm{~mm}$ to $125 \mathrm{~mm}$. No increase in the ultimate tensile load was obtained for further increase in the embedment depth to $190 \mathrm{~mm}$. Concrete cone breakout failure was observed at embedment depth of $100 \mathrm{~mm}$ with concrete cone breakout diameter of $291 \mathrm{~mm}$. Steel anchor failure was observed at embedment depths of $125 \mathrm{~mm}$ and 190 $\mathrm{mm}$.

For the anchor diameter of $20 \mathrm{~mm}$ as shown in Figure 6-14, the ultimate tensile load increased with the increase in the embedment depth from $100 \mathrm{~mm}$ to $190 \mathrm{~mm}$. Concrete cone breakout failure mode was observed at embedment depths of $100 \mathrm{~mm}$ and $125 \mathrm{~mm}$. The concrete cone breakout diameter increased from $340 \mathrm{~mm}$ to $378 \mathrm{~mm}$ as the embedment depth increased from $100 \mathrm{~mm}$ to $125 \mathrm{~mm}$. Steel anchor failure was observed at embedment depth of $190 \mathrm{~mm}$. 


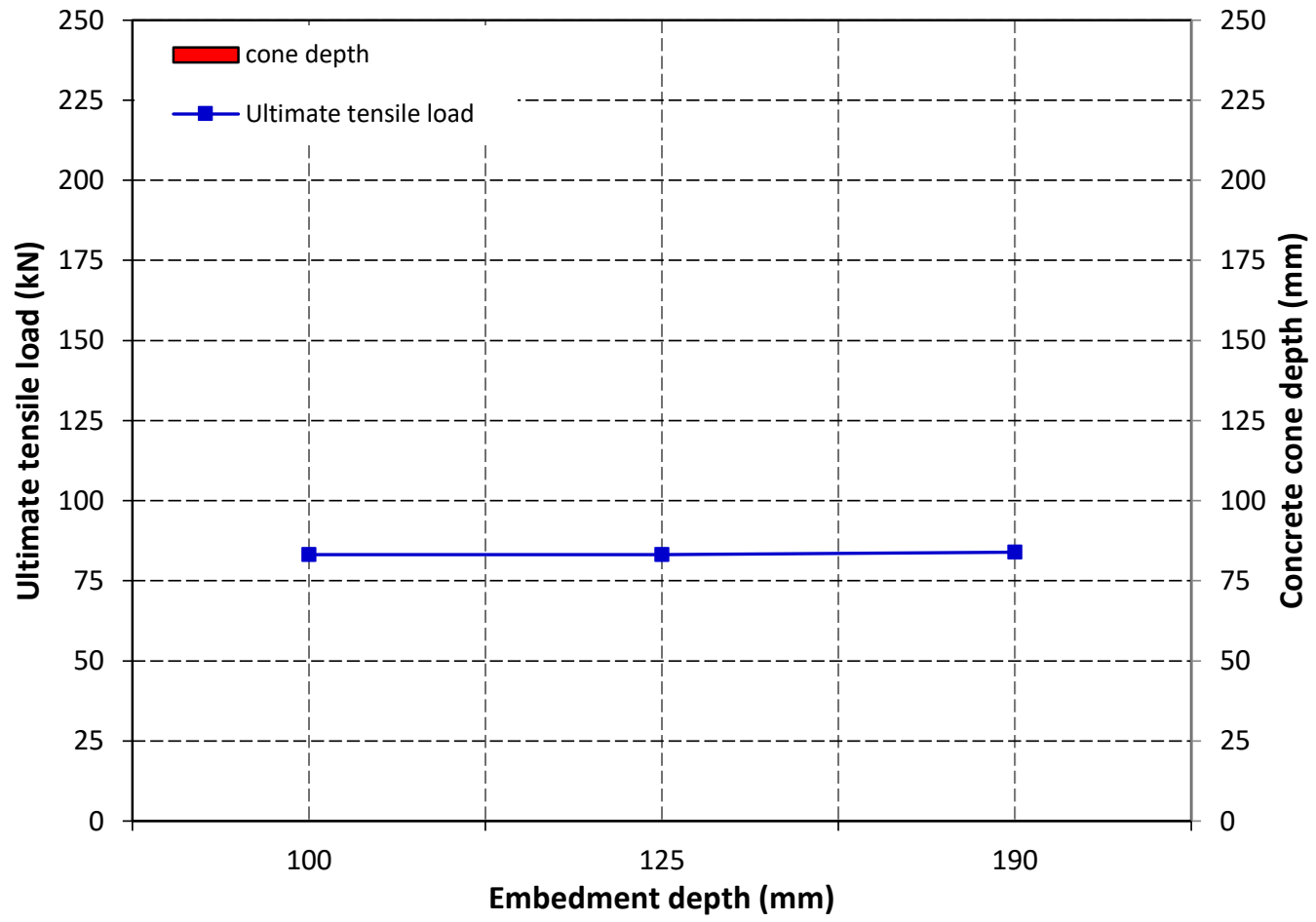

Figure 6-12: Effect of anchor embedment depth on the concrete cone depth and ultimate tensile load for $12 \mathrm{~mm}$ diameter undercut anchor

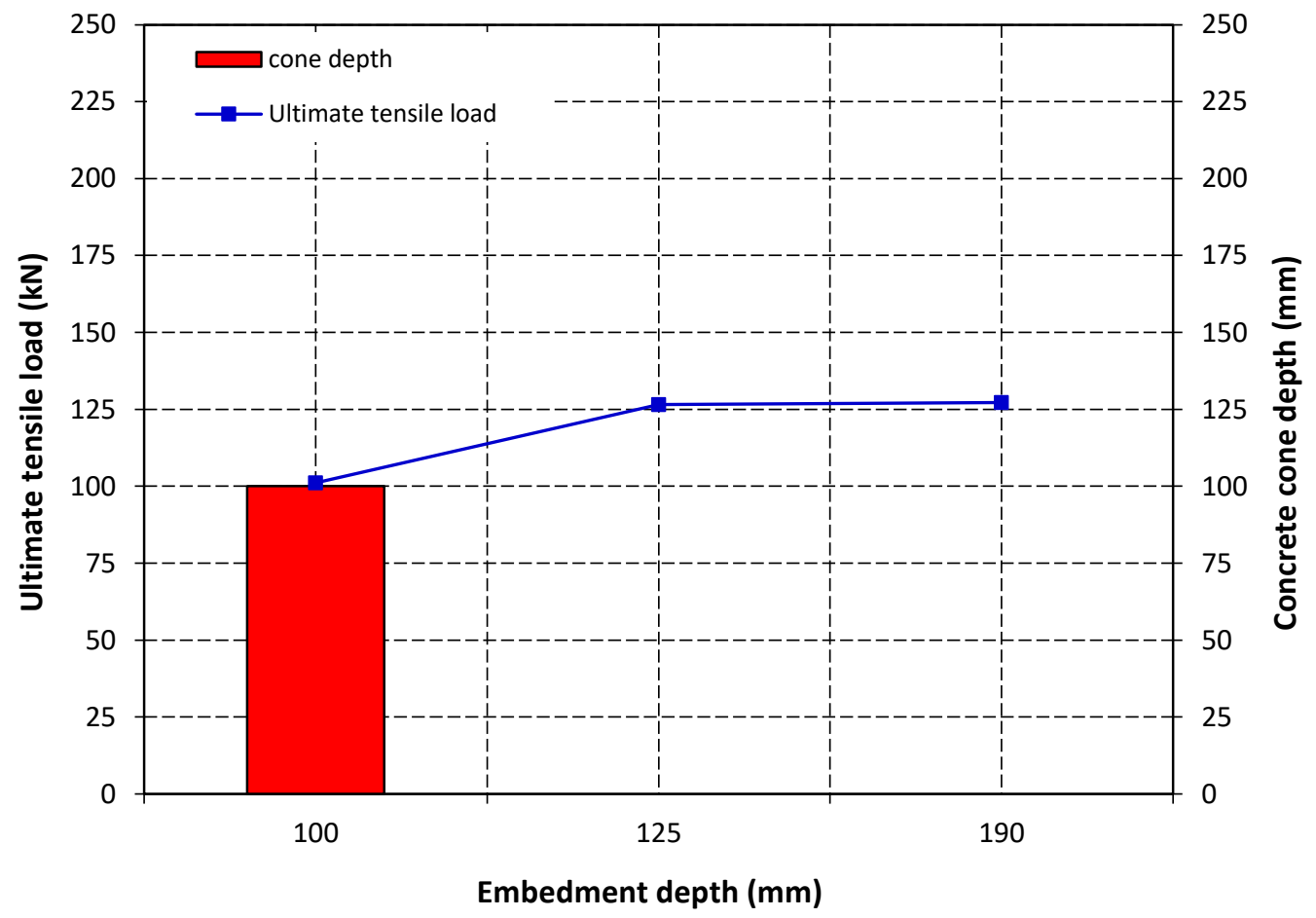

Figure 6-13: Effect of anchor embedment depth on the concrete cone depth and ultimate tensile load for $16 \mathrm{~mm}$ diameter undercut anchor 


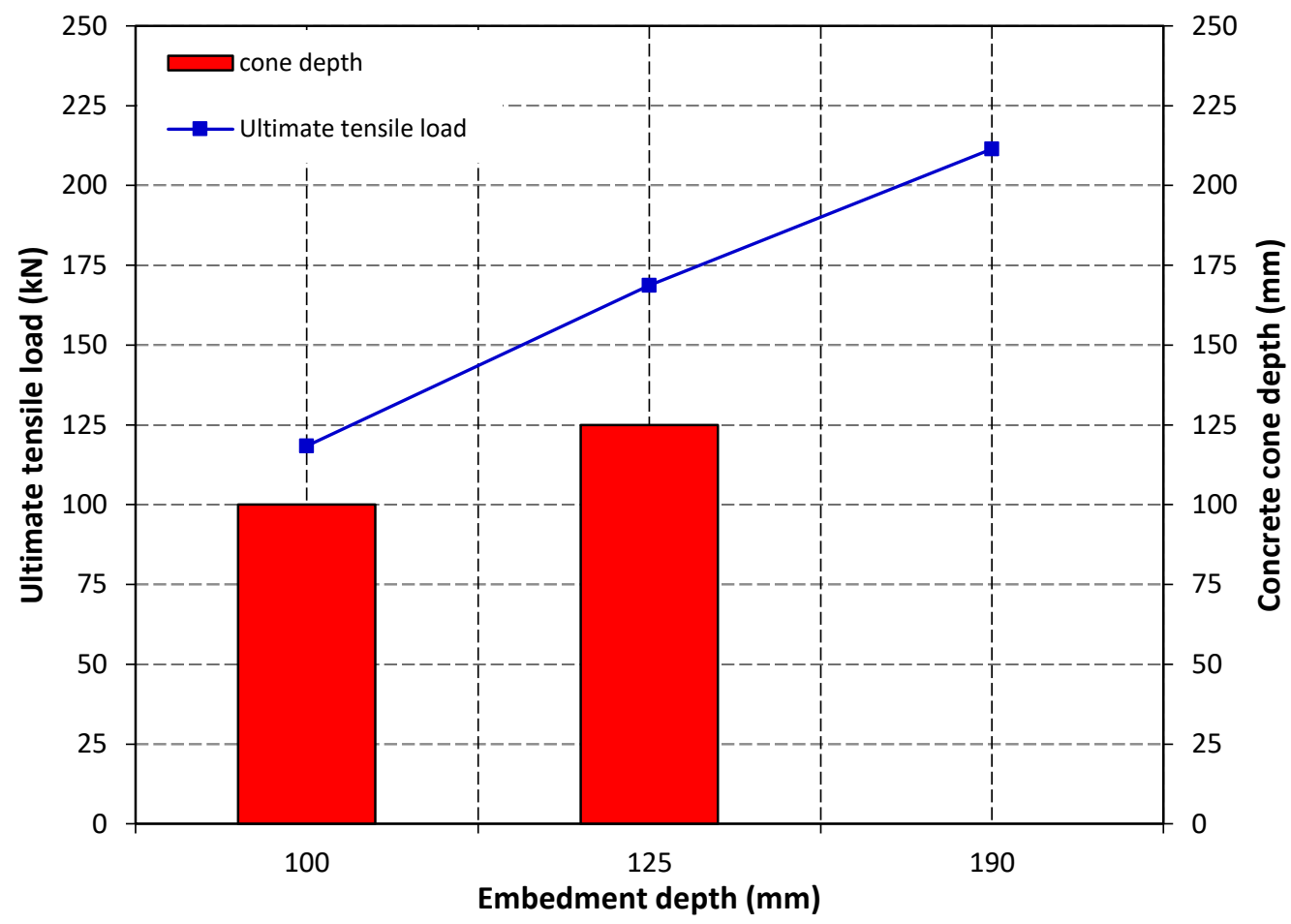

Figure 6-14: Effect of anchor embedment depth on the concrete cone depth and ultimate tensile load for $20 \mathrm{~mm}$ diameter undercut anchor

\subsubsection{Effect of strain rate on concrete cone breakout diameter and cone propagation angle}

Figure 6-15 shows the displacement contours of 20-mm diameter undercut anchor with embedemnt depth of $100 \mathrm{~mm}$ under tensile load at strain rates ranging from $10^{-5} \mathrm{~s}^{-1}$ to $10^{3}$ $\mathrm{s}^{-1}$. As shown in the figure, the increase in the strain rate from $10^{-5} \mathrm{~s}^{-1}$ to $10 \mathrm{~s}^{-1}$ decreased the concrete cone breakout diameter. At high strain rates of $10^{2} \mathrm{~s}^{-1}$ and $10^{3} \mathrm{~s}^{-1}$, steel anchor failure is observed. 


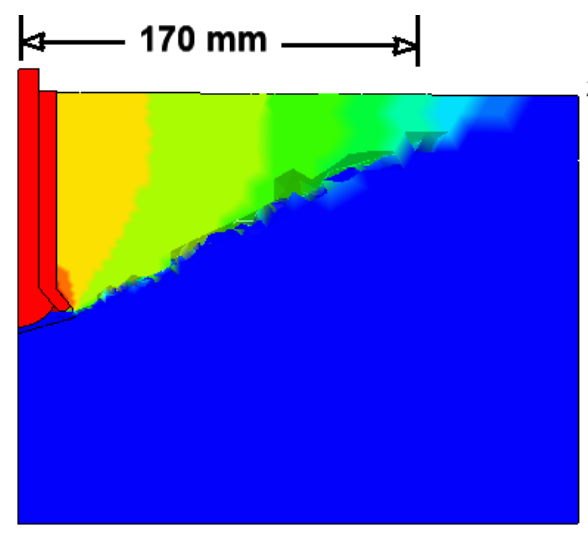

(a) $\dot{\varepsilon}=10^{-5} \mathrm{~s}^{-1}$

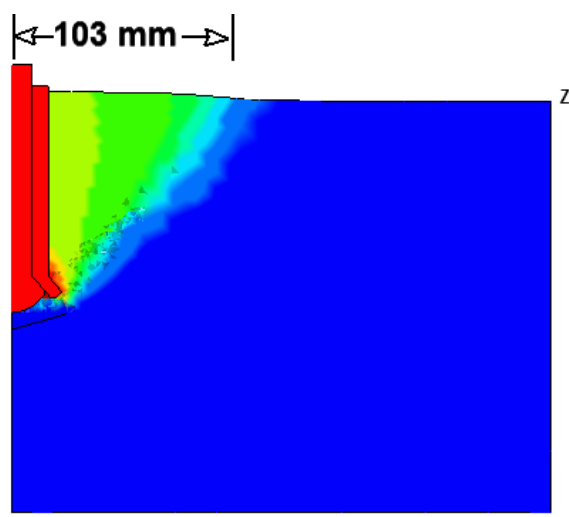

(c) $\dot{\varepsilon}=10^{-1} \mathrm{~s}^{-1}$

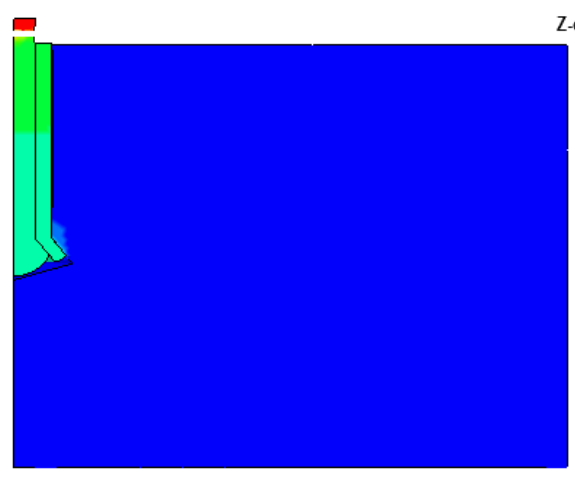

(e) $\dot{\varepsilon}=10^{2} \mathrm{~s}^{-1}$

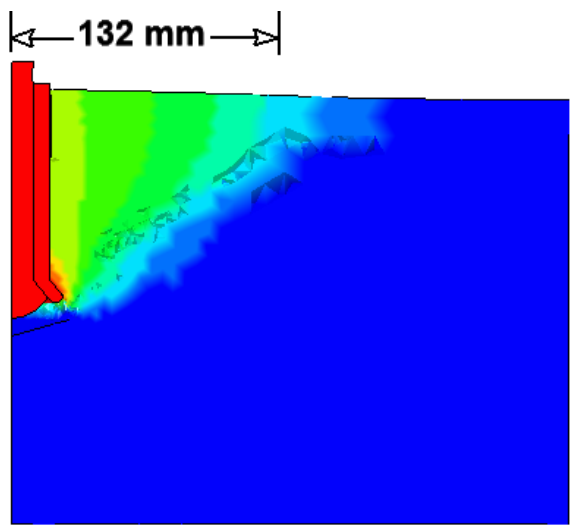

Z-displacement

$7.814 \mathrm{e}+00$

$7.033 \mathrm{e}+00$

$6.251 \mathrm{e}+00$

$5.470 \mathrm{e}+00$

$4.688 \mathrm{e}+00$

$3.907 \mathrm{e}+00$

$3.126 \mathrm{e}+00$

$2.344 \mathrm{e}+00$

$1.563 \mathrm{e}+00$

7.814e-01

$0.000 \mathrm{e}+00$

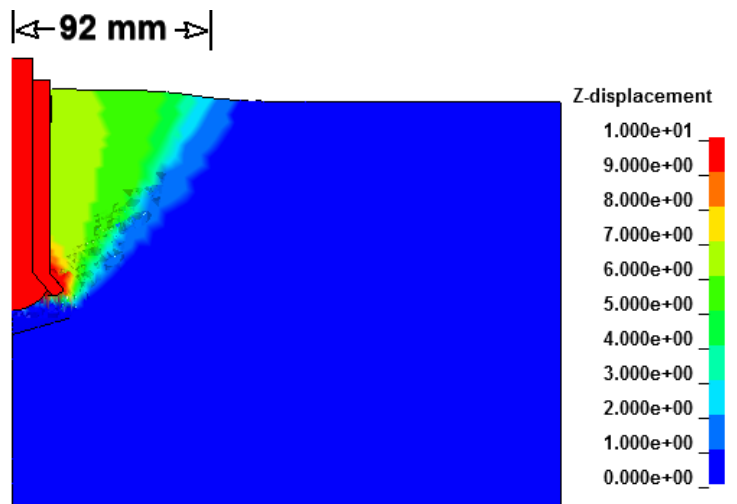

(d) $\dot{\varepsilon}=10 \mathrm{~s}^{-1}$

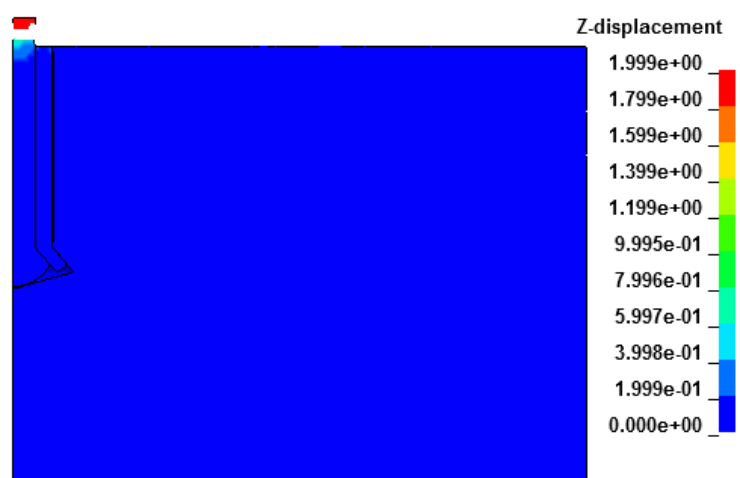

(f) $\dot{\varepsilon}=10^{3} \mathrm{~s}^{-1}$

Figure 6-15: Displacement contours for $20 \mathrm{~mm}$ undercut anchor diameter at strain rates ranging from $10^{-5} \mathrm{~s}^{-1}$ to $10^{3} \mathrm{~s}^{-1}$

The crack propagation angle was monitored and recorded at the ultimate capacity and

presented in Table 6-4. As shown in the table, the crack propagation angle decreases with 
the increase in the strain rate for the undercut anchors exhibiting concrete cone breakout failure. The crack propagation angle was measured from the direction of the applied load to the failure surface.

Table 6-4: Crack propagation angle for the undercut anchors at different strain rates

\begin{tabular}{|c|c|c|c|c|c|c|c|c|}
\hline \multirow{3}{*}{$\begin{array}{c}\text { Model } \\
\text { No. }\end{array}$} & \multirow{3}{*}{$\begin{array}{c}\mathrm{d} \\
(\mathrm{mm})\end{array}$} & \multirow{3}{*}{$\begin{array}{c}\mathbf{h}_{\text {ef }} \\
(\mathrm{mm})\end{array}$} & \multicolumn{6}{|c|}{ Crack propagation angle (deg) } \\
\hline & & & \multicolumn{6}{|c|}{ Strain rate $\left(\mathrm{s}^{-1}\right)$} \\
\hline & & & $\dot{\varepsilon}=10^{-5}$ & $\dot{\varepsilon}=10^{-3}$ & $\dot{\varepsilon}=10^{-1}$ & $\dot{\varepsilon}=10$ & $\dot{\varepsilon}=10^{2}$ & $\dot{\varepsilon}=10^{3}$ \\
\hline 1 & 12 & 100 & - & - & - & - & - & - \\
\hline 2 & 12 & 125 & - & - & - & - & - & - \\
\hline 3 & 12 & 190 & - & - & - & - & - & - \\
\hline 4 & 12 & 250 & - & - & - & - & - & - \\
\hline 5 & 16 & 100 & 63 & - & - & - & - & - \\
\hline 6 & 16 & 125 & - & - & - & - & - & - \\
\hline 7 & 16 & 190 & - & - & - & - & - & - \\
\hline 8 & 16 & 250 & - & - & - & - & - & - \\
\hline 9 & 20 & 100 & 62 & 50 & 46 & 42 & - & - \\
\hline 10 & 20 & 125 & 62 & 52 & 47 & - & - & - \\
\hline 11 & 20 & 190 & - & - & - & - & - & - \\
\hline 12 & 20 & 250 & - & - & - & - & - & - \\
\hline
\end{tabular}

\subsubsection{Effect of strain rate on the tensile behaviour of the undercut anchors}

Tensile behaviour of $12-\mathrm{mm}, 16-\mathrm{mm}$ and $20-\mathrm{mm}$ diameter undercut anchors with embedment depths of $100 \mathrm{~mm}, 125 \mathrm{~mm}, 190 \mathrm{~mm}$ and $250 \mathrm{~mm}$ were investigated. Figures 6-16, 6-17, 6-18, 6-19, 6-20 and 6-21 present the load-displacement relation for the 12-mm diameter undercut anchors subjected to tensile load. Strain rates from $10^{-5} \mathrm{~s}^{-1}$ to $10^{3} \mathrm{~s}^{-1}$ were investigated. 


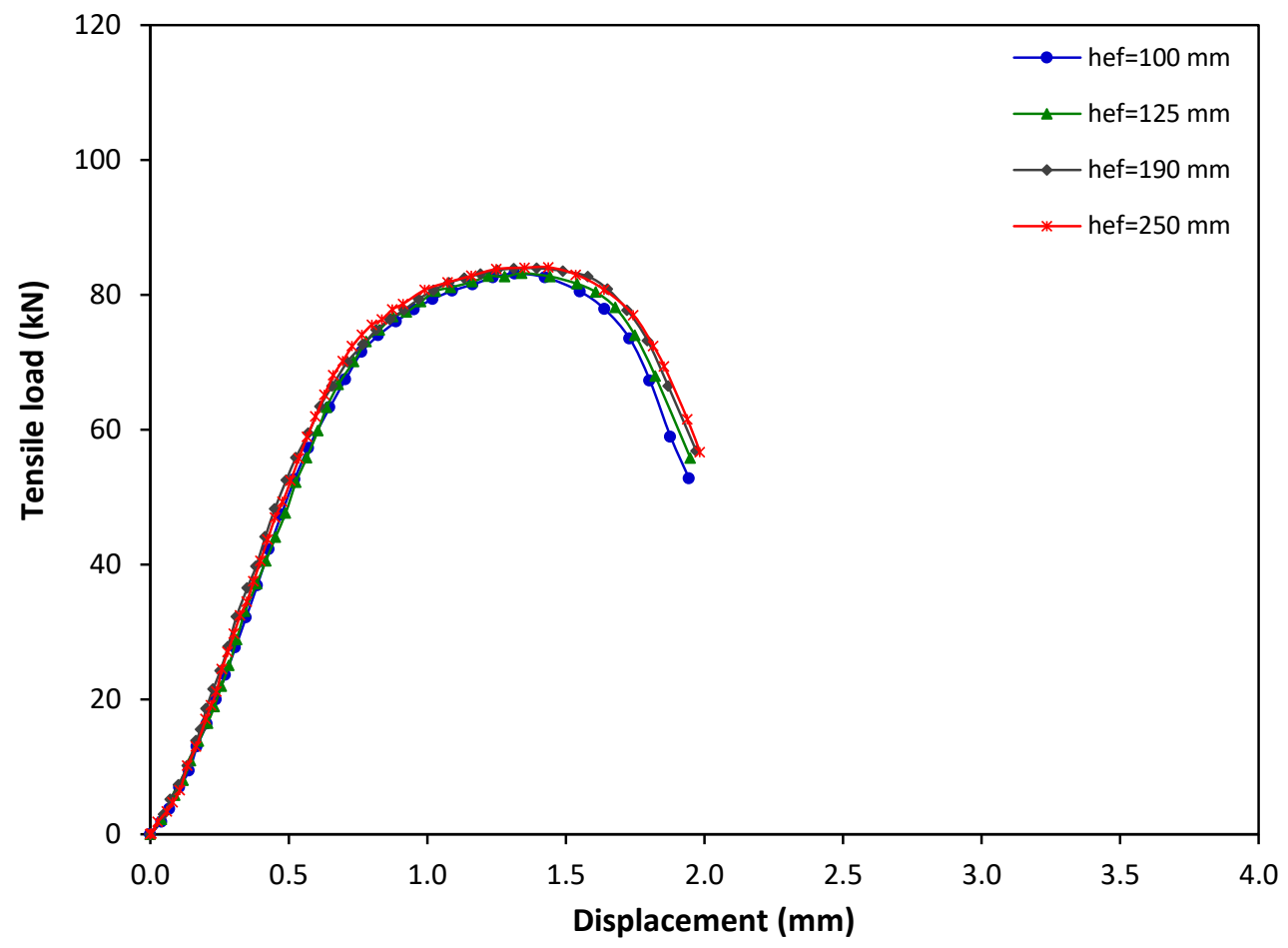

Figure 6-16: Tensile load-displacement graph for the 12-mm diameter undercut anchor at strain rate of $10^{-5} \mathrm{~s}^{-1}$

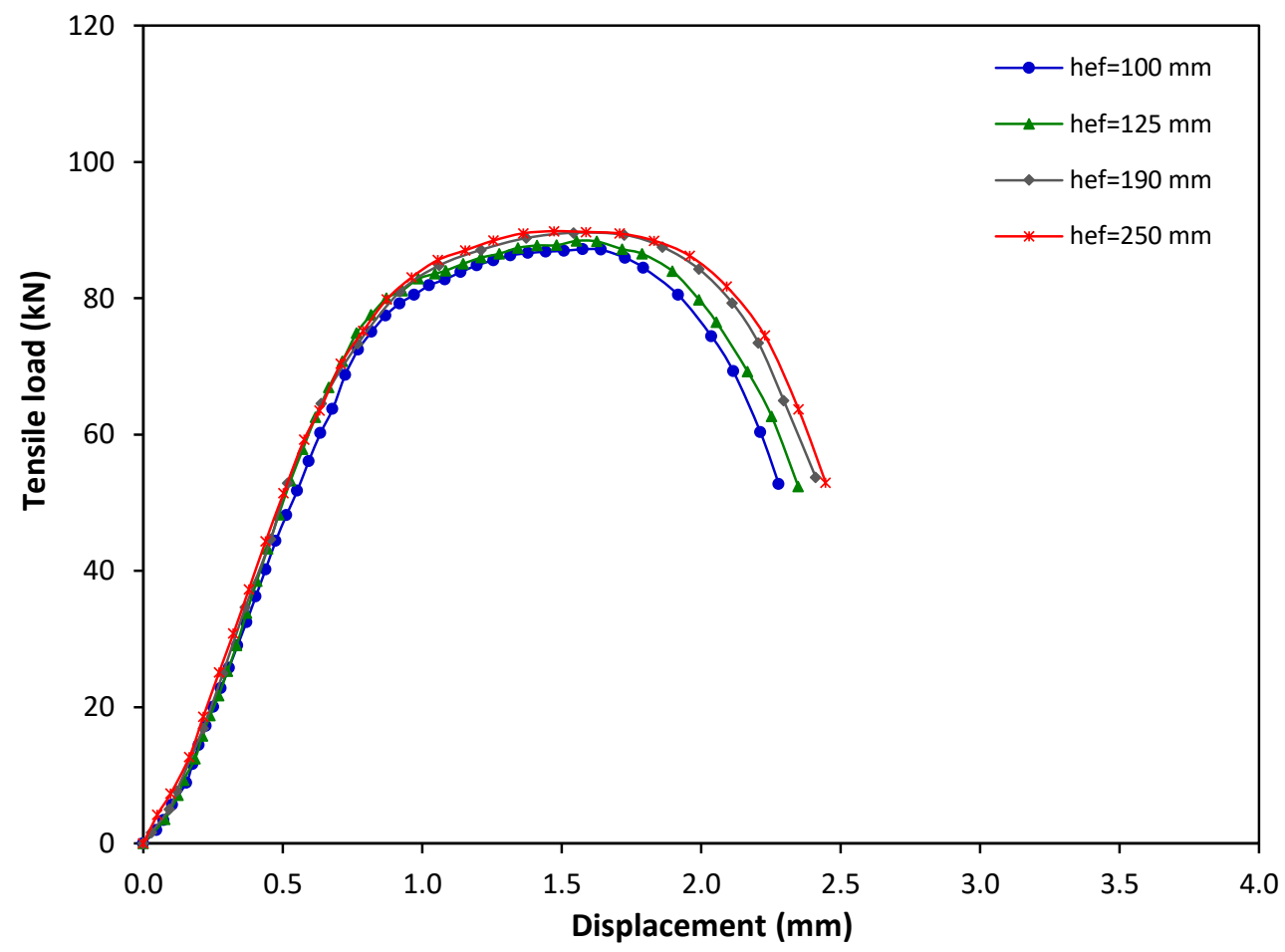

Figure 6-17: Tensile load-displacement graph for the 12-mm diameter undercut anchor at strain rate of $10^{-3} \mathrm{~s}^{-1}$ 


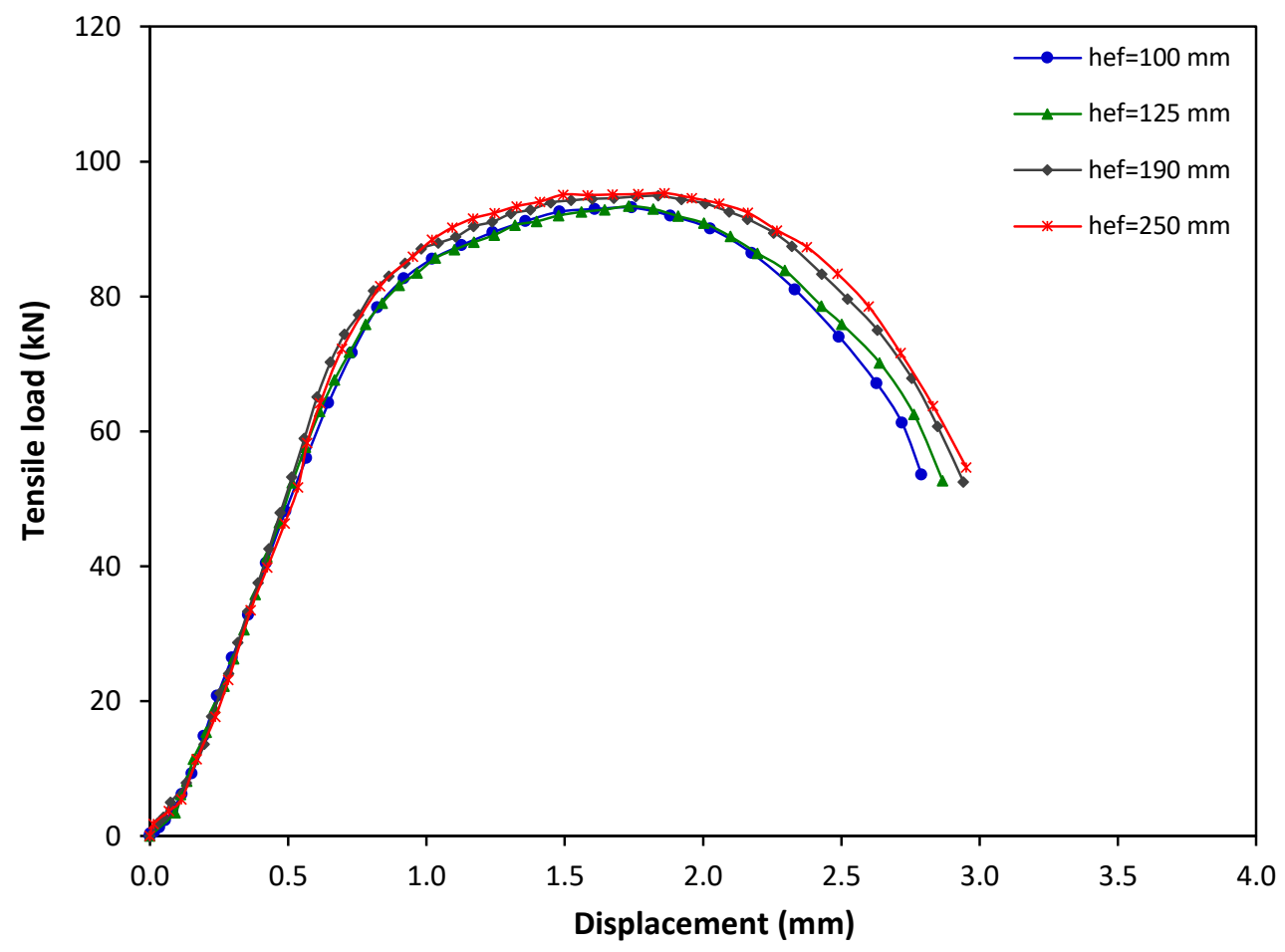

Figure 6-18: Tensile load-displacement graph for the 12-mm diameter undercut anchor at strain rate of $10^{-1} \mathrm{~s}^{-1}$

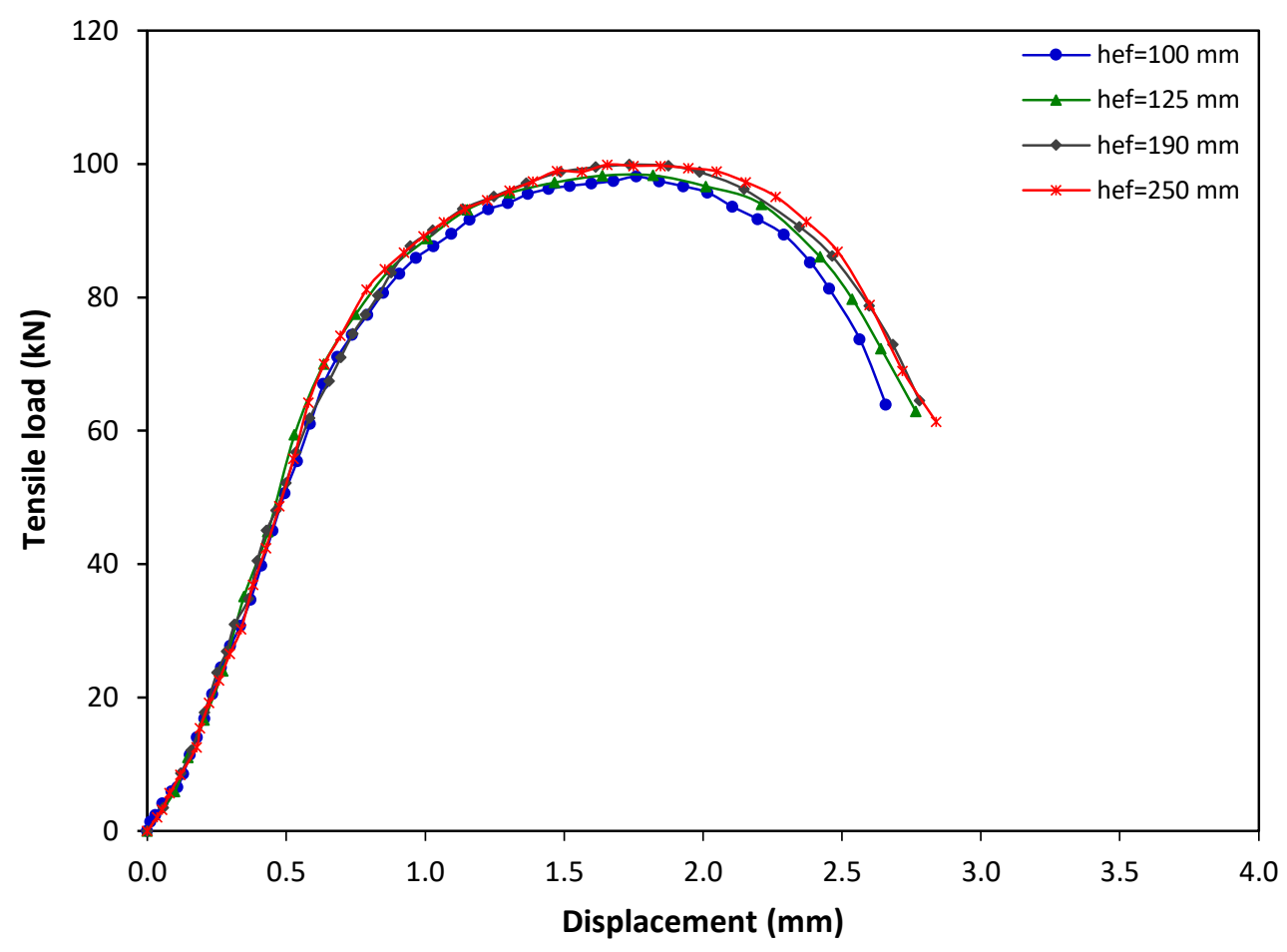

Figure 6-19: Tensile load-displacement graph for the 12-mm diameter undercut anchor at strain rate of $10 \mathrm{~s}^{-1}$ 


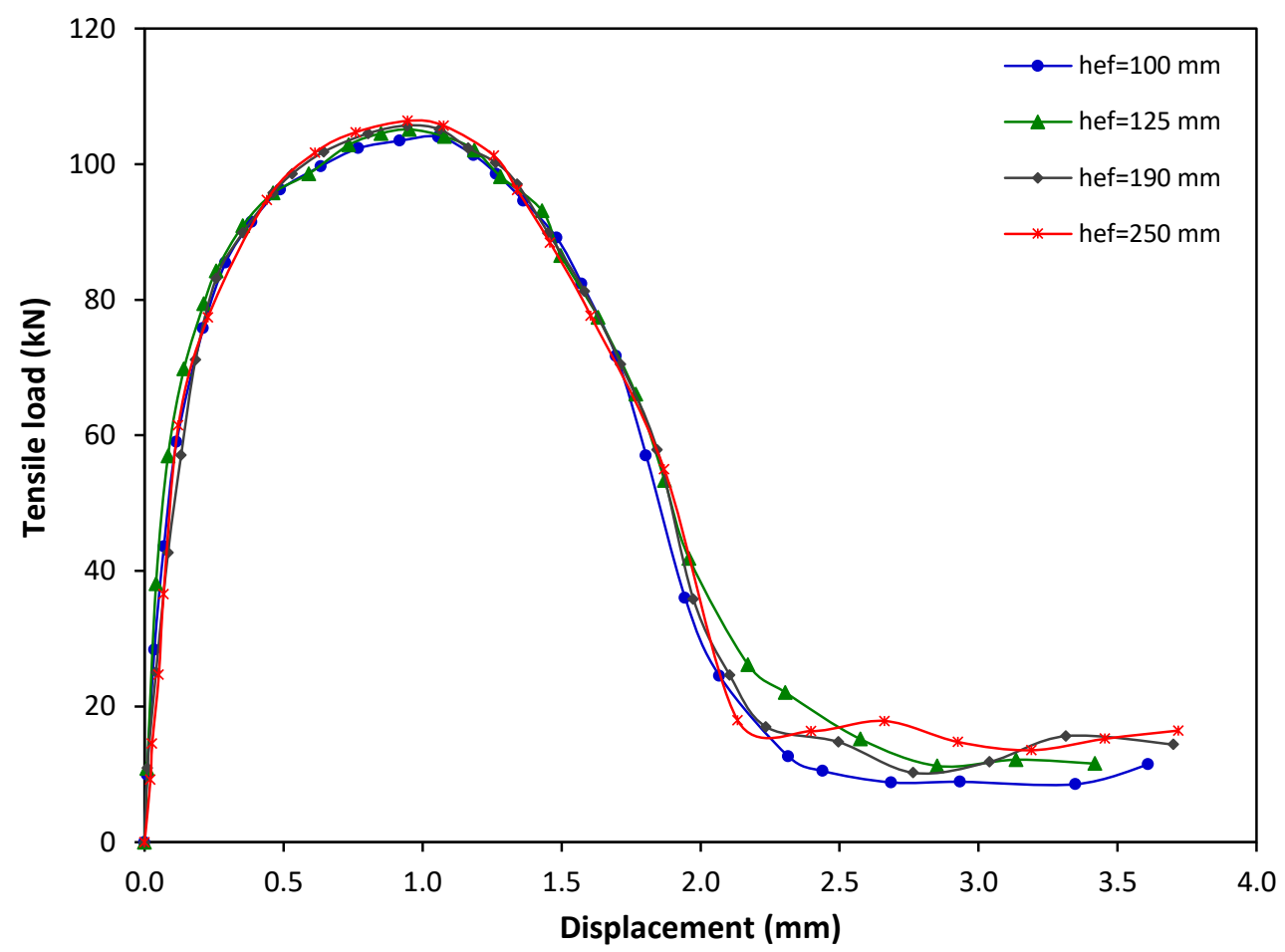

Figure 6-20: Tensile load-displacement graph for the 12-mm diameter undercut anchor at strain rate of $10^{2} \mathrm{~s}^{-1}$

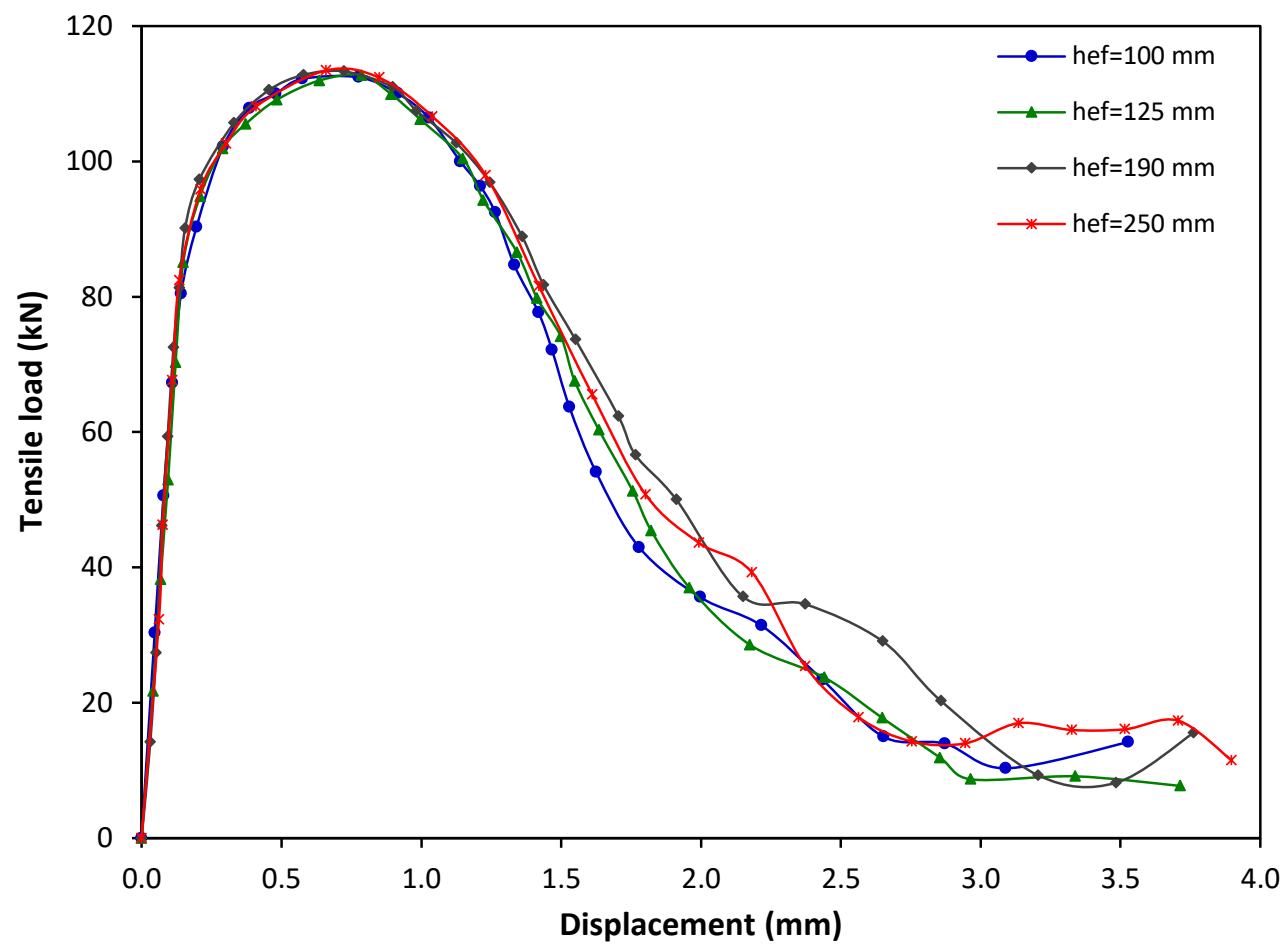

Figure 6-21: Tensile load-displacement graph for the 12-mm diameter undercut anchor at strain rate of $10^{3} \mathrm{~s}^{-1}$ 
As shown in the Figures 6-16 to 6-21, the load increased with displacement up to the ultimate capacity and then decreased until failure. Higher tensile loads were obtained at high strain rate of $10^{3} \mathrm{~s}^{-1}$ compared to that obtained at low strain rate of $10^{-5} \mathrm{~s}^{-1}$. Maximum tensile load for the $12-\mathrm{mm}$ diameter undercut anchor of $113.46 \mathrm{kN}$ was obtained at high strain rate of $10^{3} \mathrm{~s}^{-1}$. The increase in the embedment depth has no effect on the tensile capacity where steel anchor failure is observed.

The results of the load-displacement graphs for the 16-mm and 20-mm diameter undercut anchors with embedment depths of $100 \mathrm{~mm}, 125 \mathrm{~mm}, 190 \mathrm{~mm}$ and $250 \mathrm{~mm}$ at different strain rates are presented in Appendix G. Similar behaviour for the load-displacement relation of the 16-mm and 20-mm diameter undercut anchors were observed at the different strain rates.

Figures 6-22 and 6-23, show the load-displacement behaviour for undercut anchors with embedment depths of $100 \mathrm{~mm}$ and $190 \mathrm{~mm}$ respectively at the static strain rate of $10^{-5} \mathrm{~s}^{-1}$. 


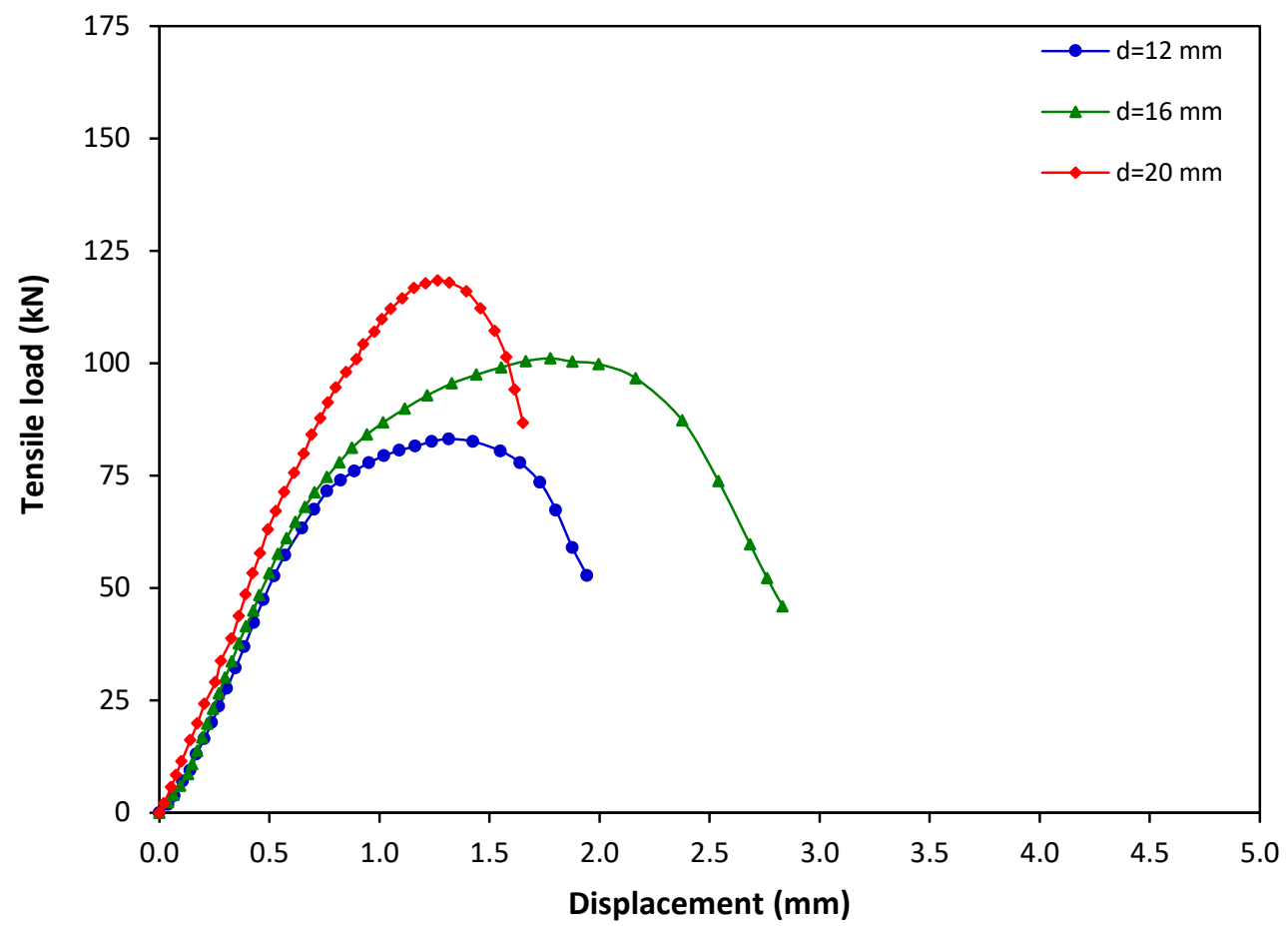

Figure 6-22: Tensile load-displacement response of $100 \mathrm{~mm}$ embedment depth undercut anchors at strain rate of $10^{-5} \mathrm{~s}^{-1}$

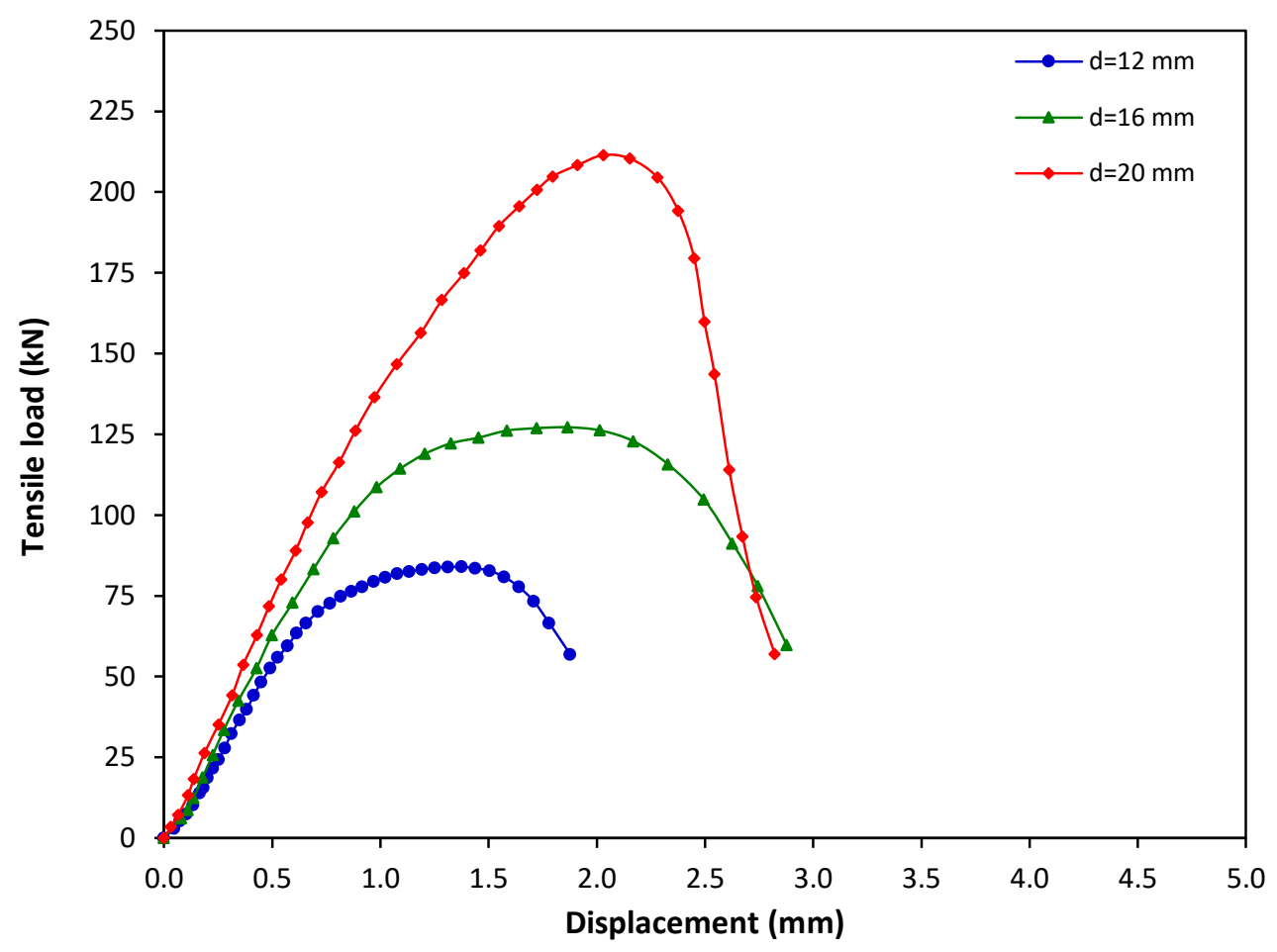

Figure 6-23: Tensile load-displacement response of $190 \mathrm{~mm}$ embedment depth undercut anchors at strain rate of $10^{-5} \mathrm{~s}^{-1}$ 
As shown in Figure 6-22 the increase in the anchor diameter from $12 \mathrm{~mm}$ to $20 \mathrm{~mm}$ resulted in an increase in tensile capacity from $83.1 \mathrm{kN}$ to $118.4 \mathrm{kN}$ and from $83.9 \mathrm{kN}$ to $211.5 \mathrm{kN}$ for embedment depths of $100 \mathrm{~mm}$ and $190 \mathrm{~mm}$ respectively. At embedment depth of 100 $\mathrm{mm}$, steel anchor failure was observed for anchor diameter of $12 \mathrm{~mm}$. Concrete cone breakout failure was observed for the anchor diameters of $16 \mathrm{~mm}$ and $20 \mathrm{~mm}$. At embedment depth of $190 \mathrm{~mm}$ steel anchor failure was observed for all the anchor diameters.

Tables 6-5, 6-6 and 6-7, show the ultimate tensile load and corresponding displacement $(\delta)$ for the $12-\mathrm{mm}, 16-\mathrm{mm}$ and $20-\mathrm{mm}$ diameter undercut anchors respectively at strain rates ranging from $10^{-5} \mathrm{~s}^{-1}$ to $10^{3} \mathrm{~s}^{-1}$. As shown in the tables, the increase in the strain rate resulted in increased ultimate tensile load for the undercut anchors investigated. It can be seen that the increase in the anchor diameter increased the ultimate tensile load for the undercut anchors. This is attributed to the increase in the cross-sectional area of the anchor and this requires higher load to cause steel failure. On the other hand, the increase in the anchor diameter increased the concrete cone breakout diameter that require higher load to breakout the concrete cone and hence increasing the tensile capacity.

Table 6-5: Ultimate tensile load and displacement for the $12 \mathrm{~mm}$ diameter undercut

\begin{tabular}{|c|c|c|c|c|c|c|}
\hline $\begin{array}{c}\text { Model } \\
\text { No. }\end{array}$ & $\begin{array}{c}\mathrm{d} \\
(\mathrm{mm})\end{array}$ & $\begin{array}{c}\mathbf{h}_{\mathrm{ef}} \\
(\mathbf{m m})\end{array}$ & $\begin{array}{c}\dot{\varepsilon} \\
\left(\mathrm{s}^{-1}\right)\end{array}$ & $\begin{array}{c}\mathrm{F}_{\mathrm{u}} \\
(\mathrm{kN})\end{array}$ & $\begin{array}{c}\delta \\
(\mathrm{mm})\end{array}$ & $\begin{array}{c}\text { Failure } \\
\text { mode }\end{array}$ \\
\hline 1 & \multirow{4}{*}{12} & 100 & \multirow{4}{*}{$10^{-5}$} & 83.14 & 1.32 & $\mathrm{~S}$ \\
\hline 2 & & 125 & & 83.22 & 1.34 & S \\
\hline 3 & & 190 & & 83.94 & 1.39 & $\mathrm{~S}$ \\
\hline 4 & & 250 & & 84.07 & 1.44 & $S$ \\
\hline 5 & \multirow{4}{*}{12} & 100 & \multirow{4}{*}{$10^{-3}$} & 87.19 & 1.58 & S \\
\hline 6 & & 125 & & 88.43 & 1.55 & $\mathrm{~S}$ \\
\hline 7 & & 190 & & 89.61 & 1.54 & $\mathrm{~S}$ \\
\hline 8 & & 250 & & 89.72 & 1.59 & $\mathrm{~S}$ \\
\hline 9 & \multirow{2}{*}{12} & 100 & \multirow{2}{*}{$10^{-1}$} & 93.18 & 1.74 & S \\
\hline 10 & & 125 & & 93.37 & 1.73 & $\mathrm{~S}$ \\
\hline
\end{tabular}




\begin{tabular}{|c|c|c|c|c|c|c|}
\hline 11 & & 190 & & 94.90 & 1.84 & $\mathrm{~S}$ \\
\hline 12 & & 250 & & 95.30 & 1.86 & $\mathrm{~S}$ \\
\hline 13 & \multirow{4}{*}{12} & 100 & \multirow{4}{*}{10} & 98.09 & 1.76 & $\mathrm{~S}$ \\
\hline 14 & & 125 & & 98.25 & 1.64 & $\mathrm{~S}$ \\
\hline 15 & & 190 & & 99.52 & 1.61 & $\mathrm{~S}$ \\
\hline 16 & & 250 & & 99.86 & 1.65 & $\mathrm{~S}$ \\
\hline 17 & \multirow{4}{*}{12} & 100 & \multirow{4}{*}{$10^{2}$} & 104.06 & 1.06 & $\mathrm{~S}$ \\
\hline 18 & & 125 & & 105.13 & 0.95 & $\mathrm{~S}$ \\
\hline 19 & & 190 & & 105.70 & 0.95 & $\mathrm{~S}$ \\
\hline 20 & & 250 & & 106.40 & 0.95 & $\mathrm{~S}$ \\
\hline 21 & \multirow{4}{*}{12} & 100 & \multirow{4}{*}{$10^{3}$} & 112.46 & 0.78 & $\mathrm{~S}$ \\
\hline 22 & & 125 & & 112.74 & 0.79 & $\mathrm{~S}$ \\
\hline 23 & & 190 & & 113.32 & 0.72 & $\mathrm{~S}$ \\
\hline 24 & & 250 & & 113.46 & 0.66 & $\mathrm{~S}$ \\
\hline
\end{tabular}

Table 6-6: Ultimate tensile load and displacement for the $16 \mathrm{~mm}$ diameter undercut anchor

\begin{tabular}{|c|c|c|c|c|c|c|}
\hline $\begin{array}{c}\text { Model } \\
\text { No. }\end{array}$ & $\underset{(\mathbf{m m})}{\mathbf{d}}$ & $\begin{array}{c}\mathbf{h}_{\text {eff }} \\
(\mathbf{m m})\end{array}$ & $\begin{array}{c}\dot{\varepsilon} \\
\left(\mathrm{s}^{-1}\right)\end{array}$ & $\begin{array}{c}\mathbf{F}_{\mathbf{u}} \\
(\mathbf{k N})\end{array}$ & $\begin{array}{c}\delta \\
(\mathbf{m m})\end{array}$ & $\begin{array}{c}\text { Failure } \\
\text { mode }\end{array}$ \\
\hline 1 & \multirow{4}{*}{16} & 100 & \multirow{4}{*}{$10^{-5}$} & 101.09 & 1.78 & $\mathrm{CC}$ \\
\hline 2 & & 125 & & 126.62 & 1.88 & $\mathrm{~S}$ \\
\hline 3 & & 190 & & 127.16 & 1.87 & $\mathrm{~S}$ \\
\hline 4 & & 250 & & 127.43 & 1.82 & $\mathrm{~S}$ \\
\hline 5 & \multirow{4}{*}{16} & 100 & \multirow{4}{*}{$10^{-3}$} & 124.46 & 2.29 & $\mathrm{~S}$ \\
\hline 6 & & 125 & & 130.07 & 2.26 & $\mathrm{~S}$ \\
\hline 7 & & 190 & & 131.56 & 2.19 & $\mathrm{~S}$ \\
\hline 8 & & 250 & & 132.25 & 2.31 & $\mathrm{~S}$ \\
\hline 9 & \multirow{4}{*}{16} & 100 & \multirow{4}{*}{$10^{-1}$} & 139.35 & 1.96 & $\mathrm{~S}$ \\
\hline 10 & & 125 & & 140.31 & 1.93 & $\mathrm{~S}$ \\
\hline 11 & & 190 & & 141.42 & 2.05 & $\mathrm{~S}$ \\
\hline 12 & & 250 & & 142.33 & 1.95 & $\mathrm{~S}$ \\
\hline 13 & \multirow{4}{*}{16} & 100 & \multirow{4}{*}{10} & 150.14 & 1.79 & $\mathrm{~S}$ \\
\hline 14 & & 125 & & 151.05 & 1.66 & $\mathrm{~S}$ \\
\hline 15 & & 190 & & 151.19 & 1.59 & $\mathrm{~S}$ \\
\hline 16 & & 250 & & 151.63 & 1.73 & $\mathrm{~S}$ \\
\hline 17 & \multirow{4}{*}{16} & 100 & \multirow{4}{*}{$10^{2}$} & 165.21 & 0.99 & $\mathrm{~S}$ \\
\hline 18 & & 125 & & 165.58 & 0.99 & S \\
\hline 19 & & 190 & & 165.84 & 0.99 & $\mathrm{~S}$ \\
\hline 20 & & 250 & & 165.94 & 1.02 & $\mathrm{~S}$ \\
\hline 21 & \multirow{4}{*}{16} & 100 & \multirow{4}{*}{$10^{3}$} & 182.46 & 0.87 & $\mathrm{~S}$ \\
\hline 22 & & 125 & & 182.76 & 0.88 & $\mathrm{~S}$ \\
\hline 23 & & 190 & & 182.88 & 0.79 & $\mathrm{~S}$ \\
\hline 24 & & 250 & & 183.16 & 0.79 & $\mathrm{~S}$ \\
\hline
\end{tabular}


Table 6-7: Ultimate tensile load and displacement for the $20 \mathrm{~mm}$ diameter undercut anchor

\begin{tabular}{|c|c|c|c|c|c|c|}
\hline $\begin{array}{c}\text { Model } \\
\text { No. }\end{array}$ & $\begin{array}{c}\mathbf{d} \\
(\mathbf{m m})\end{array}$ & $\begin{array}{c}\begin{array}{c}\mathbf{h}_{\mathrm{ef}} \\
(\mathbf{m m})\end{array} \\
\end{array}$ & $\begin{array}{c}\dot{\dot{\varepsilon}} \\
\left(\mathbf{s}^{-1}\right)\end{array}$ & $\begin{array}{c}\mathbf{F}_{\mathbf{u}} \\
(\mathbf{k N})\end{array}$ & $\begin{array}{c}\boldsymbol{\delta} \\
(\mathbf{m m})\end{array}$ & $\begin{array}{c}\text { Failure } \\
\text { mode }\end{array}$ \\
\hline 1 & \multirow{4}{*}{20} & 100 & \multirow{4}{*}{$10^{-5}$} & 118.41 & 1.27 & $\mathrm{CC}$ \\
\hline 2 & & 125 & & 168.66 & 1.81 & $\mathrm{CC}$ \\
\hline 3 & & 190 & & 211.46 & 2.10 & $\mathrm{~S}$ \\
\hline 4 & & 250 & & 213.29 & 2.14 & $\mathrm{~S}$ \\
\hline 5 & \multirow{4}{*}{20} & 100 & \multirow{4}{*}{$10^{-3}$} & 142.16 & 3.2 & $\mathrm{CC}$ \\
\hline 6 & & 125 & & 185.01 & 2.75 & $\mathrm{CC}$ \\
\hline 7 & & 190 & & 220.52 & 2.81 & $\mathrm{~S}$ \\
\hline 8 & & 250 & & 221.83 & 2.79 & $\mathrm{~S}$ \\
\hline 9 & \multirow{4}{*}{20} & 100 & \multirow{4}{*}{$10^{-1}$} & 161.18 & 3.19 & $\mathrm{CC}$ \\
\hline 10 & & 125 & & 208.53 & 3.31 & $\mathrm{CC}$ \\
\hline 11 & & 190 & & 232.39 & 2.74 & $\mathrm{~S}$ \\
\hline 12 & & 250 & & 233.64 & 2.84 & $\mathrm{~S}$ \\
\hline 13 & \multirow{4}{*}{20} & 100 & \multirow{4}{*}{10} & 186.60 & 3.49 & $\mathrm{CC}$ \\
\hline 14 & & 125 & & 236.30 & 3.59 & $\mathrm{~S}$ \\
\hline 15 & & 190 & & 242.02 & 4.02 & $\mathrm{~S}$ \\
\hline 16 & & 250 & & 244.50 & 4.10 & $\mathrm{~S}$ \\
\hline 17 & \multirow{4}{*}{20} & 100 & \multirow{4}{*}{$10^{2}$} & 258.89 & 0.99 & $\mathrm{~S}$ \\
\hline 18 & & 125 & & 258.05 & 0.95 & $\mathrm{~S}$ \\
\hline 19 & & 190 & & 259.19 & 1.09 & $\mathrm{~S}$ \\
\hline 20 & & 250 & & 260.39 & 1.07 & $\mathrm{~S}$ \\
\hline 21 & \multirow{4}{*}{20} & 100 & \multirow{4}{*}{$10^{3}$} & 283.96 & 0.83 & $\mathrm{~S}$ \\
\hline 22 & & 125 & & 284.84 & 0.65 & $\mathrm{~S}$ \\
\hline 23 & & 190 & & 284.66 & 0.74 & $\mathrm{~S}$ \\
\hline 24 & & 250 & & 286.36 & 0.78 & $\mathrm{~S}$ \\
\hline
\end{tabular}

\subsubsection{Effect of strain rate on the ultimate tensile load and DIF of undercut anchors}

Figures 6-24, 6-25, 6-26 and 6-27 present the relation between the ultimate tensile load, DIF and the strain rate, for undercut anchor diameters of $12 \mathrm{~mm}, 16 \mathrm{~mm}$ and $20 \mathrm{~mm}$, at embedment depths of $100 \mathrm{~mm}, 125 \mathrm{~mm}, 190 \mathrm{~mm}$ and $250 \mathrm{~mm}$ respectively. The ultimate tensile load increases with increasing anchor diameter from $12 \mathrm{~mm}$ to $20 \mathrm{~mm}$ for the same strain rate. The ultimate tensile load increased almost linearly with the strain rate for anchor diameters of $12 \mathrm{~mm}$ and $16 \mathrm{~mm}$ where steel anchor failure is the dominant failure mode at all the strain rates investigated (Figure 6-24, 6-25, 6-26 and 6-27). Hence the increase in 
the tensile load is attributed to the increase in steel strength at high strain rate. For $20-\mathrm{mm}$ diameter undercut anchor, as the strain rate increased from $10^{-5} \mathrm{~s}^{-1}$ to $10 \mathrm{~s}^{-1}$, the ultimate tensile load was observed to increase according to a nearly bilinear relationship with a change in slope at a strain rate of about $10 \mathrm{~s}^{-1}$ where concrete cone breakout failure was observed (Figures 6-24, 6-25, 6-26 and 6-27). The ultimate tensile load increased with further increase in the strain rate to $10^{2} \mathrm{~s}^{-1}$ where the failure mode transitioned to steel anchor failure. The increase in the ultimate load with the increase in the strain rate is attributed to the increase in the tensile strength of the concrete and steel materials with the increase in the strain rate. Maximum tensile loads of $113.5 \mathrm{kN}, 183.2 \mathrm{kN}$ and $286.4 \mathrm{kN}$ were obtained for the 12-mm, 16- $\mathrm{mm}$ and 20-mm diameter undercut anchors respectively at high strain rate of $10^{3} \mathrm{~s}^{-1}$.

Strain rate of $10^{-5} \mathrm{~s}^{-1}$ represents the static strain rate and is taken as the base line to determine the DIF at different strain rates for the anchors exhibited concrete cone failure. For the anchors exhibited steel failure, the DIF is taken as the ratio of the ultimate dynamic load at strain rates higher than $10^{-5} \mathrm{~s}^{-1}$ to the ultimate static steel failure load for the anchors. As shown in Figures 6-24 and 6-25, the DIF for anchor diameters of $12 \mathrm{~mm}$ and $16 \mathrm{~mm}$ increases with the increase in the strain rate from $10^{-3} \mathrm{~s}^{-1}$ to $10^{3} \mathrm{~s}^{-1}$ where steel failure was observed. The increase in the DIF is attributed to the increase in the steel strength with the increase in the strain rate. For anchor diameter of $20 \mathrm{~mm}$ with embedment depth of 100 $\mathrm{mm}$ (Figure 6-24), the DIF increased to 1.58 at strain rate of $10 \mathrm{~s}^{-1}$ where concrete cone failure was observed. At high strain rates of $10^{2} \mathrm{~s}^{-1}$ and $10^{3} \mathrm{~s}^{-1}$ steel failure was observed and the DIF was taken as the ratio of the dynamic ultimate load to the static steel failure 
load with DIFs of 1.22 and 1.34 respectively. For anchor diameter of $20 \mathrm{~mm}$ with embedment depth of $125 \mathrm{~mm}$ (Figure 6-25), the DIF increased to 1.24 at strain rate of $10^{-1}$ $\mathrm{s}^{-1}$ where concrete cone failure was observed. At higher strain rates of $10 \mathrm{~s}^{-1}$ up to $10^{3} \mathrm{~s}^{-1}$ steel failure was observed with maximum DIF of 1.35.

It can be seen from Figures 6-26 and 6-27, the DIF increased with the increase in the strain rate from $10^{-5} \mathrm{~s}^{-1}$ to $10^{3} \mathrm{~s}^{-1}$ for all anchor diameters investigated. Maximum DIF of 1.35 and 1.44 are obtained for the anchor diameters of $12 \mathrm{~mm}, 16 \mathrm{~mm}$ respectively at highest strain rate of $10^{3} \mathrm{~s}^{-1}$ where steel failure was the dominant failure mode at all the strain rates investigated. For the anchor diameter of $20 \mathrm{~mm}$ maximum DIF of 1.58 was obtained at strain rate of $10 \mathrm{~s}^{-1}$ with embedment depth of $100 \mathrm{~mm}$ where concrete cone breakout failure was observed. The failure mode transitioned from concrete cone breakout failure to steel failure at high strain rates of $10^{2} \mathrm{~s}^{-1}$ and $10^{3} \mathrm{~s}^{-1}$.

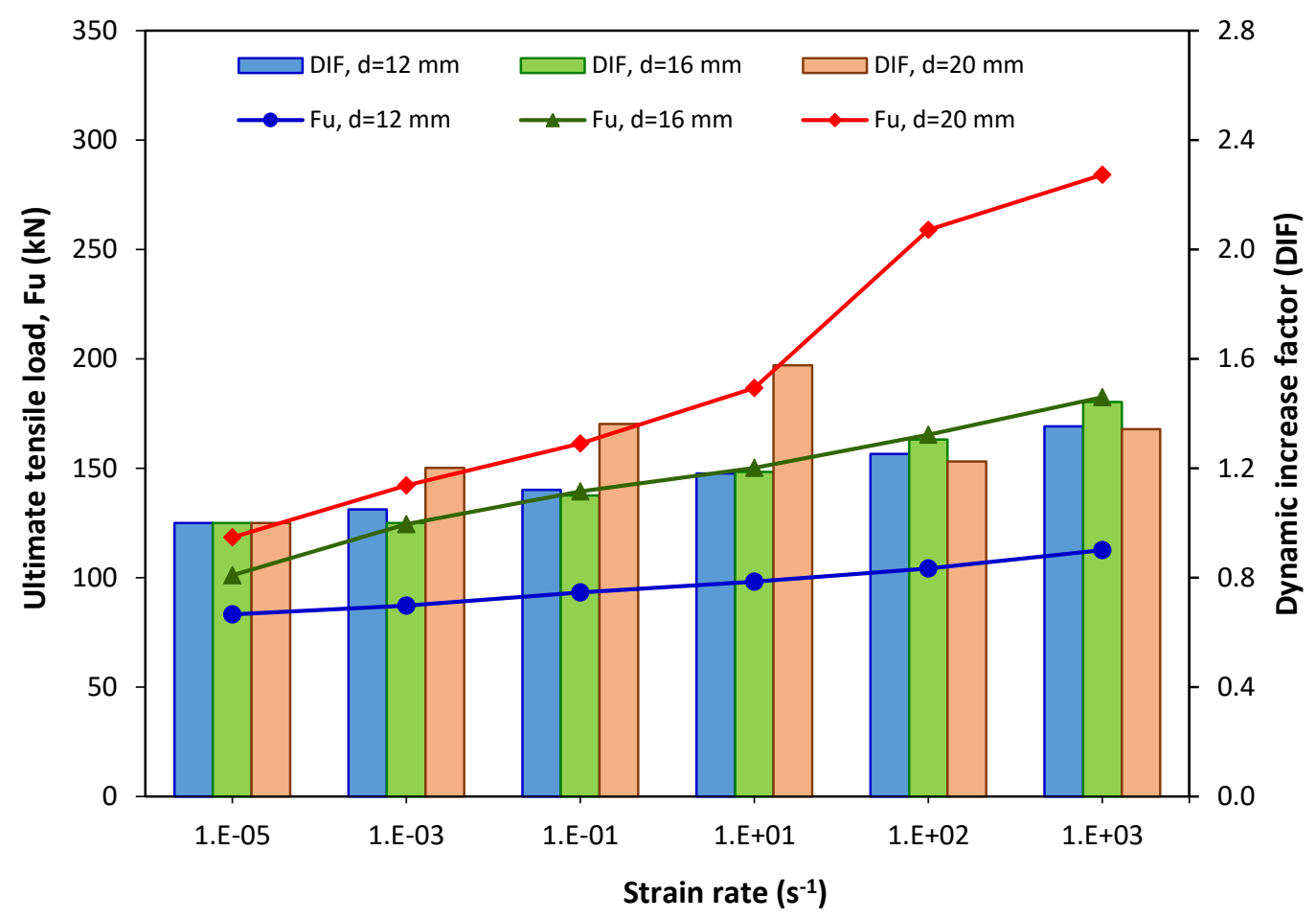

Figure 6-24: Ultimate tensile load and DIF versus strain rate for the undercut anchor at $100 \mathrm{~mm}$ embedment depth 


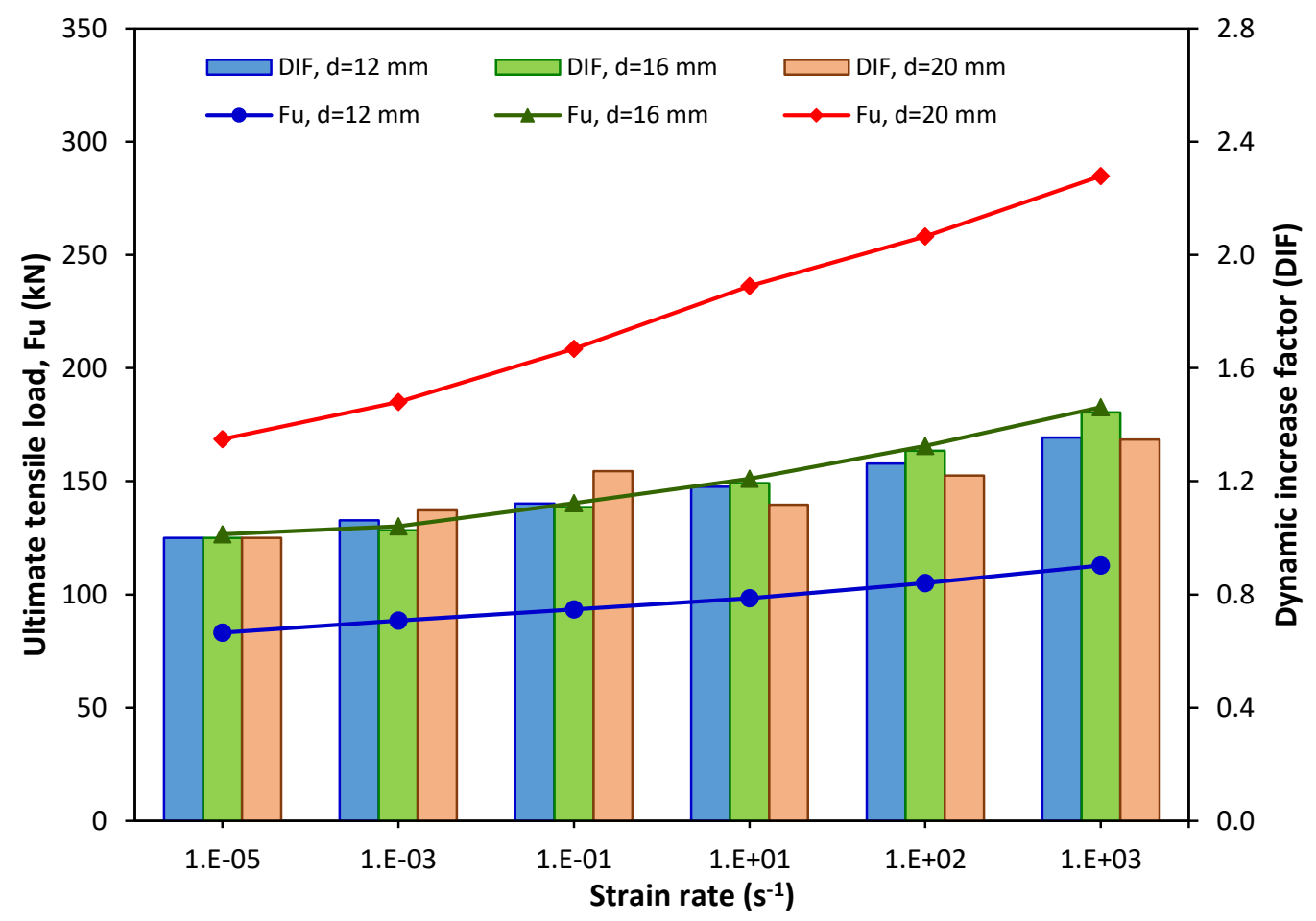

Figure 6-25: Ultimate tensile load and DIF versus strain rate for the undercut anchor at $125 \mathrm{~mm}$ embedment depth

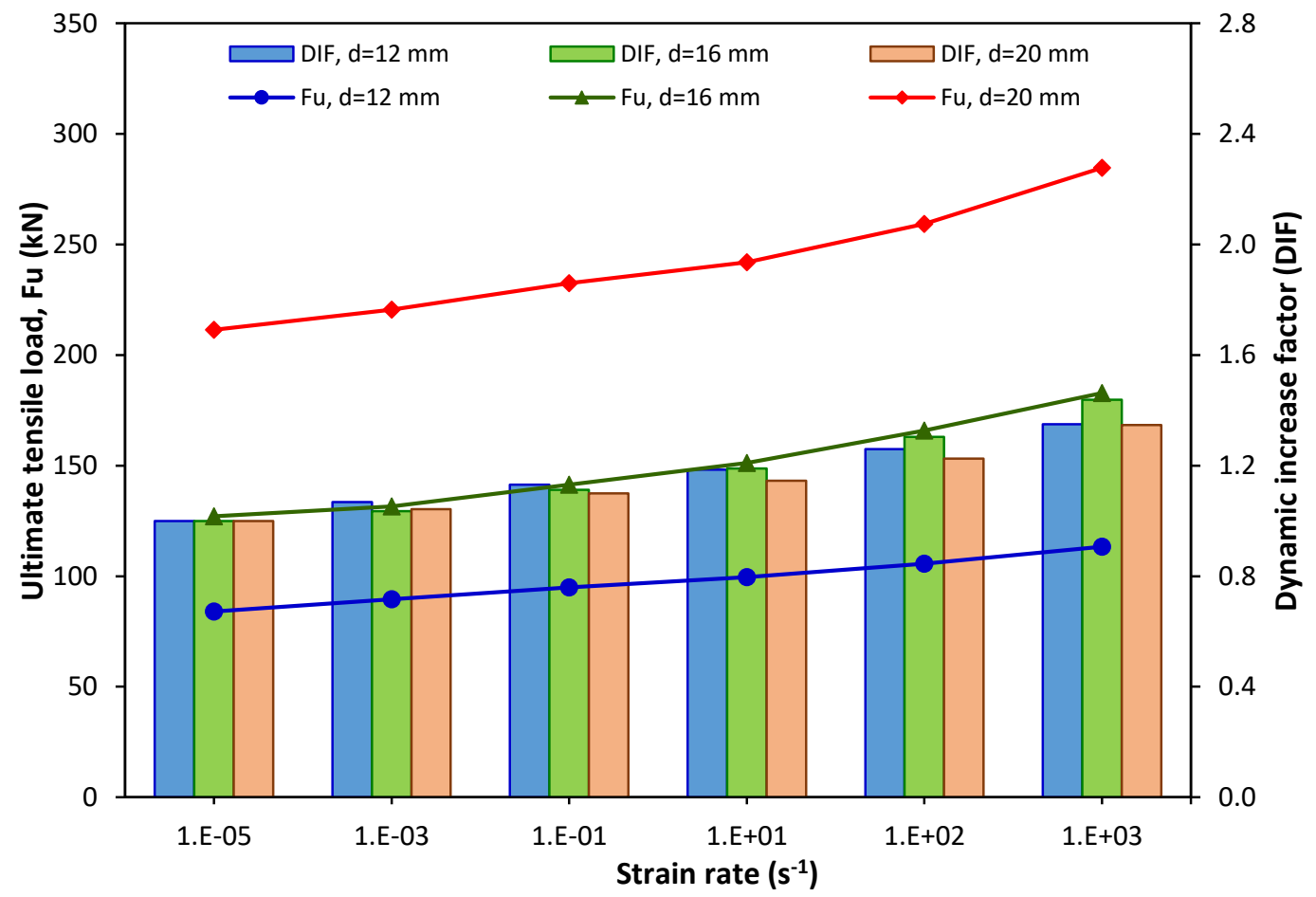

Figure 6-26: Ultimate tensile load and DIF versus strain rate for the undercut anchor at $190 \mathrm{~mm}$ embedment depth 


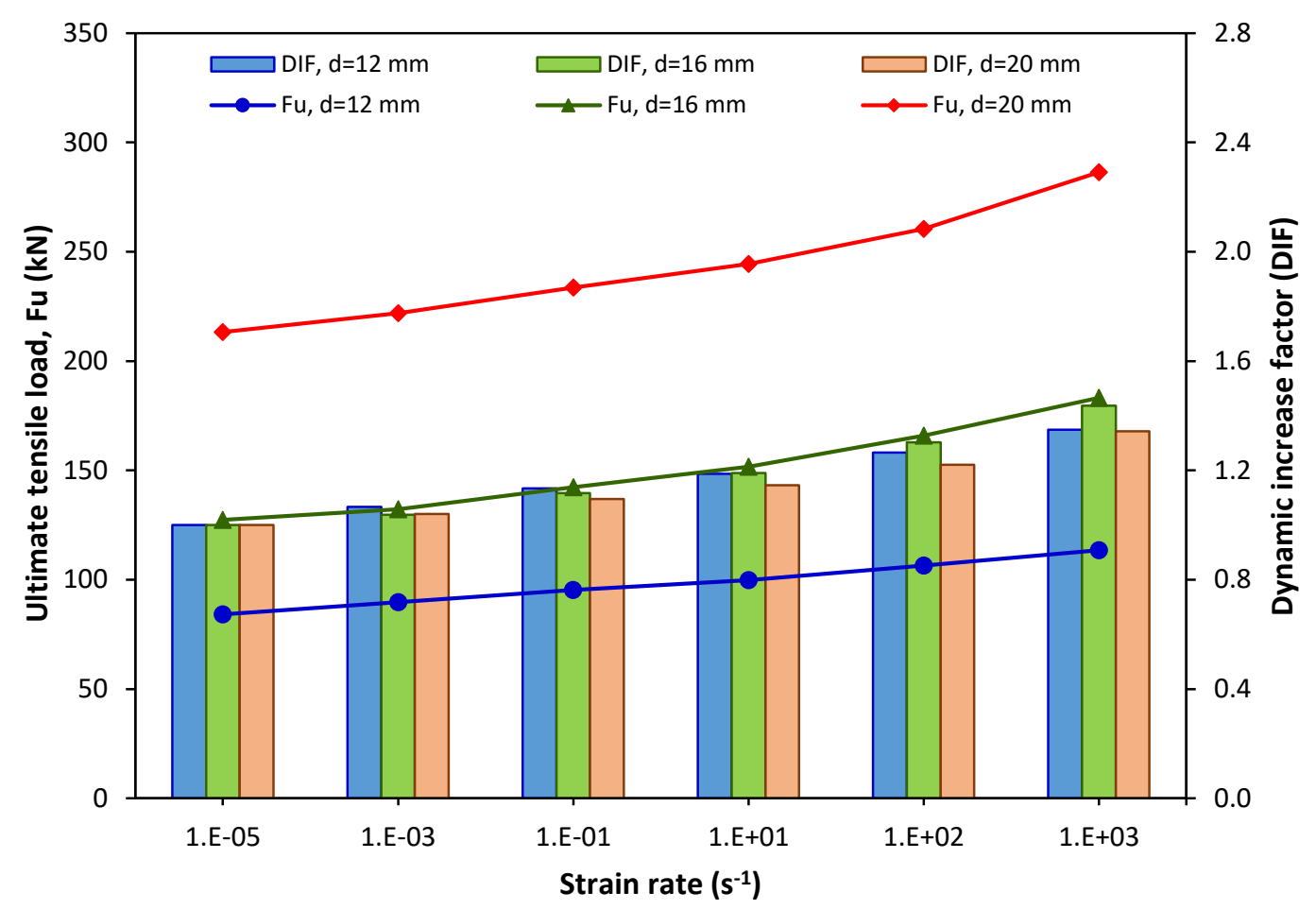

Figure 6-27: Ultimate tensile load and DIF versus strain rate for the undercut anchor at $250 \mathrm{~mm}$ embedment depth

Table 6-8 shows maximum DIF for the undercut anchors under tensile load where concrete cone breakout and steel anchor failure modes were observed. The value of DIF equal to one represents the DIF at the static strain rate of $10^{-5} \mathrm{~s}^{-1}$ where concrete cone breakout failure mode is observed for the $16 \mathrm{~mm}$ and $20 \mathrm{~mm}$ diameter undercut anchors with embedment depth of $100 \mathrm{~mm}$. Also, concrete cone breakout failure was observed for the $20 \mathrm{~mm}$ diameter with $125 \mathrm{~mm}$ embedment depth. Steel anchor failure was the dominant failure mode for most of the undercut anchors at the static strain rate of $10^{-5} \mathrm{~s}^{-1}$ and higher strain rates up to $10^{3} \mathrm{~s}^{-1}$. Maximum value of the DIF for the undercut anchors exhibited steel failure was obtained at high strain rate of $10^{3} \mathrm{~s}^{-1}$ and presented in Table 6-8. 
Table 6-8: Maximum Dynamic increase factor for the undercut anchors under tensile load

\begin{tabular}{|c|c|c|c|c|c|c|}
\hline \multirow{2}{*}{$\begin{array}{c}\text { Model } \\
\text { No. }\end{array}$} & \multirow{2}{*}{$\begin{array}{c}\mathbf{d} \\
(\mathbf{m m})\end{array}$} & \multirow{2}{*}{$\begin{array}{c}\mathbf{h}_{\text {ef }} \\
(\mathbf{m m})\end{array}$} & \multirow{2}{*}{$\begin{array}{c}\dot{\boldsymbol{\varepsilon}} \\
\left(\mathbf{s}^{-1}\right)\end{array}$} & \multirow{2}{*}{$\begin{array}{l}\text { DIF } \\
\text { CC }\end{array}$} & \multirow{2}{*}{$\begin{array}{c}\dot{\boldsymbol{\varepsilon}} \\
\left(\mathbf{s}^{-1}\right)\end{array}$} & \multirow{2}{*}{$\begin{array}{c}\text { DIF } \\
\mathrm{S}\end{array}$} \\
\hline & & & & & & \\
\hline 1 & 12 & 100 & - & - & $10^{3}$ & 1.35 \\
\hline 2 & 12 & 125 & - & - & $10^{3}$ & 1.35 \\
\hline 3 & 12 & 190 & - & - & $10^{3}$ & 1.35 \\
\hline 4 & 12 & 250 & - & - & $10^{3}$ & 1.35 \\
\hline 5 & 16 & 100 & $10^{-5}$ & 1.00 & $10^{3}$ & 1.44 \\
\hline 6 & 16 & 125 & - & - & $10^{3}$ & 1.44 \\
\hline 7 & 16 & 190 & - & - & $10^{3}$ & 1.44 \\
\hline 8 & 16 & 250 & - & - & $10^{3}$ & 1.44 \\
\hline 9 & 20 & 100 & 10 & 1.58 & $10^{3}$ & 1.34 \\
\hline 10 & 20 & 125 & $10^{-1}$ & 1.24 & $10^{3}$ & 1.35 \\
\hline 11 & 20 & 190 & - & - & $10^{3}$ & 1.35 \\
\hline 12 & 20 & 250 & - & - & $10^{3}$ & 1.34 \\
\hline
\end{tabular}

\subsubsection{Regression analysis for the undercut anchors under tensile load}

Regression analysis was performed for the undercut anchors under tensile load to develop an accurate predictive model based on the finite element results for determining the DIF of undercut anchors. Most of the undercut anchors exhibited steel anchor failure when subjected to strain rates in the range of $10^{-3} \mathrm{~s}^{-1}$ to $10^{3} \mathrm{~s}^{-1}$, hence regression analysis has been performed for the steel anchor failure mode. The relation between the DIF and the strain rate ratio $\left(\dot{\varepsilon}_{d} / \dot{\varepsilon}_{s}\right)$ for the undercut anchor diameters of $12 \mathrm{~mm}, 16 \mathrm{~mm}$ and $20 \mathrm{~mm}$ exhibited steel failure is shown in Figure 6-28. 


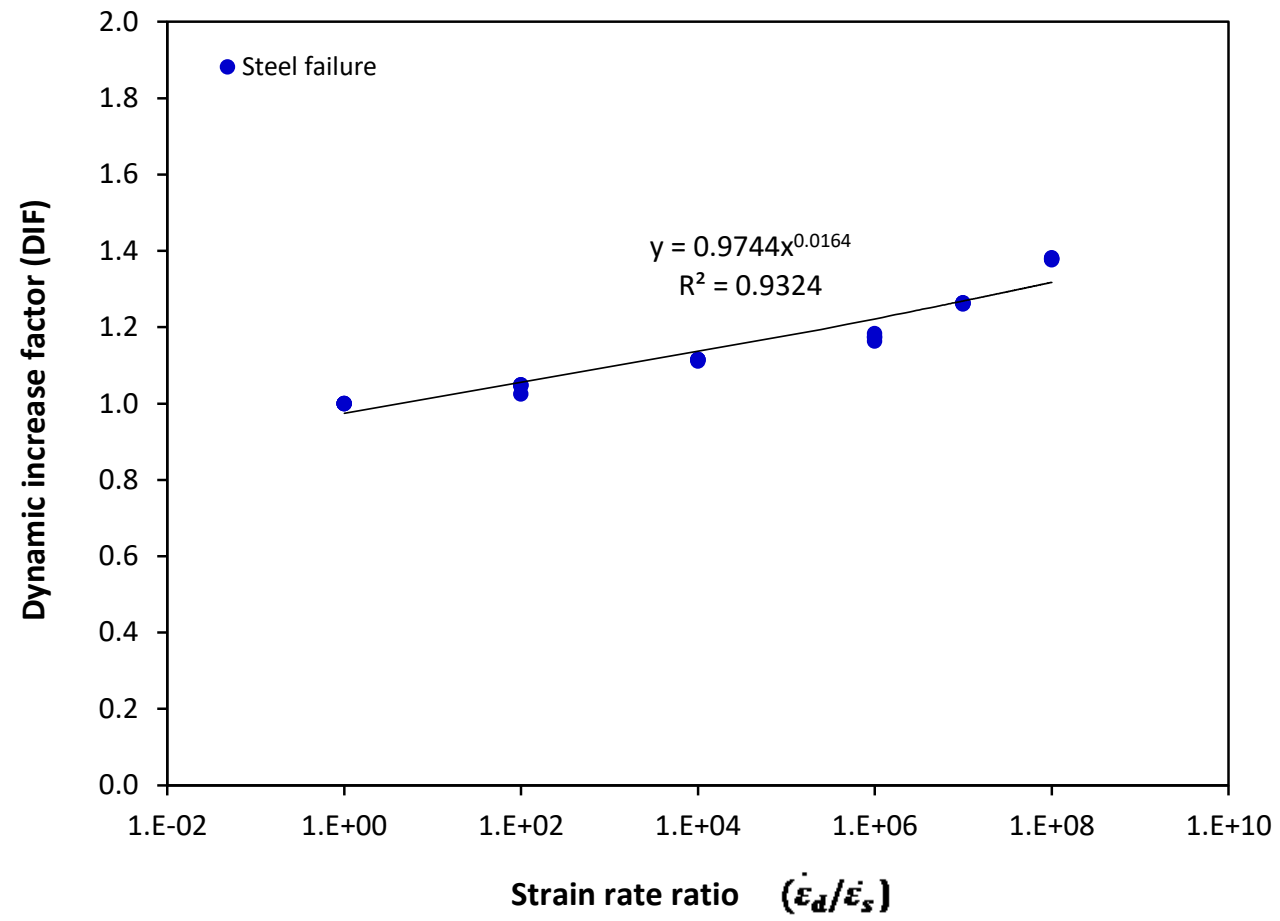

Figure 6-28: Effect of strain rate ratio on the DIF for the undercut anchors exhibited steel failure under tensile load

A formula relating DIF with the strain rate was developed using various regression models; namely, exponential, linear, logarithmic and power regression models. The predicted formulae obtained from various regression models is shown in Table 6-9.

Table 6-9: Statistical models and coefficient of determination to predict the DIF for the undercut anchors exhibited steel failure under tensile load

\begin{tabular}{|c|c|c|}
\hline Statistical models & Formulae & Coefficient of determination $\left(\mathrm{R}^{2}\right)$ \\
\hline Exponential & $D I F=1.1061 e^{2 E-09\left(\frac{\dot{\varepsilon}_{d}}{\dot{\varepsilon}_{s}}\right)}$ & 0.597 \\
\hline Linear & $D I F=3 E-09\left(\frac{\dot{\varepsilon}_{d}}{\dot{\varepsilon}_{s}}\right)+1.1094$ & 0.639 \\
\hline Logarithmic & $D I F=0.019 \ln \left(\frac{\dot{\varepsilon}_{d}}{\dot{\varepsilon}_{s}}\right)+0.9645$ & 0.908 \\
\hline Power & $D I F=0.9744\left(\frac{\dot{\varepsilon}_{d}}{\dot{\varepsilon}_{s}}\right)^{0.0164}$ & 0.932 \\
\hline
\end{tabular}


As shown in Table 6-9, the power regression model was observed to be best fit for the results with coefficients of determination $\left(R^{2}\right)$ of $93 \%$ for the undercut anchors exhibited steel failure. The DIF is represented by Equation (6.1) for the undercut anchors subjected to tensile load and exhibited steel failure.

$D I F=0.9744\left(\frac{\dot{\varepsilon}_{d}}{\dot{\varepsilon}_{s}}\right)^{0.0164}$

The ultimate dynamic load $\left(F_{u d}\right)$ for the undercut anchors exhibited steel anchor failure can be determined as in Equation (6.2) as follows:

$F_{u d}=F_{u s} \times 0.9744\left(\frac{\dot{\varepsilon}_{d}}{\dot{\varepsilon}_{s}}\right)^{0.0164}$

Where the ultimate static load $\left(F_{u s}\right)$ can be determined from Equation (4.2) for steel anchor failure mode.

Figure 6-29 presents the residual analysis for undercut anchor subjected to tensile load and exhibited steel anchor failure. Where the difference between the finite element analysis and the DIF results obtained from Equations (6.1) is presented. As shown in Figure 6-29, approximately horizontal trend line was observed for the residual with the increase in the strain rate ratio. 


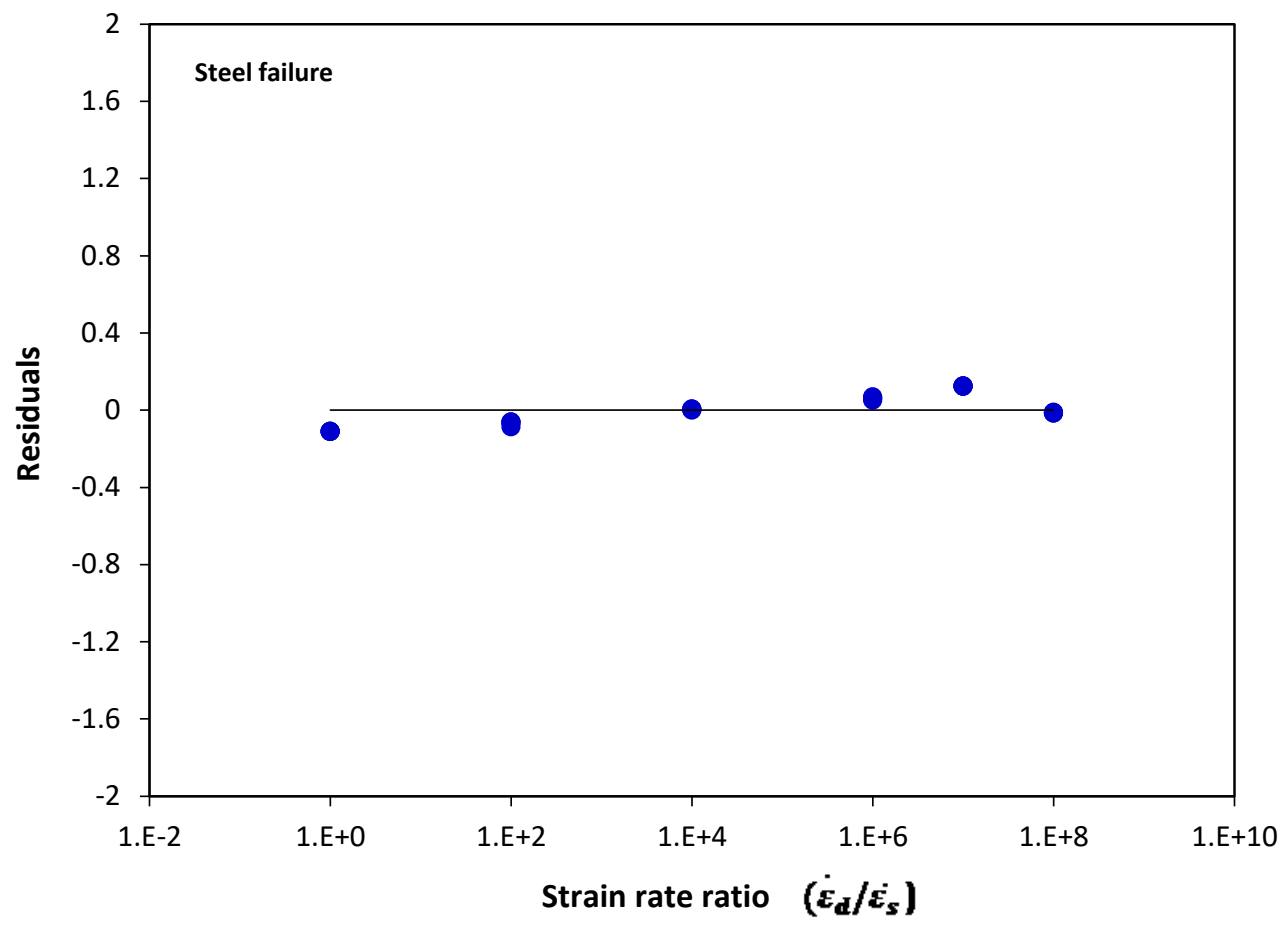

Figure 6-29: Residual versus strain rate ratio for the undercut anchors exhibited steel failure

In order to verify the accuracy of the proposed equation, new undercut anchors with diameters of $12 \mathrm{~mm}, 16 \mathrm{~mm}$ and $20 \mathrm{~mm}$ with embedment depths of $140 \mathrm{~mm}, 160 \mathrm{~mm}$ and $220 \mathrm{~mm}$ were developed. Figure 6-30 shows the relation between the DIF obtained from the newly developed numerical models and the predicted DIF obtained from Equation (6.1). As shown in the figure, the DIF values are uniformly distributed around the equality line. This means that power model presented in the Equation (6.1) can best represent the finite element results. 


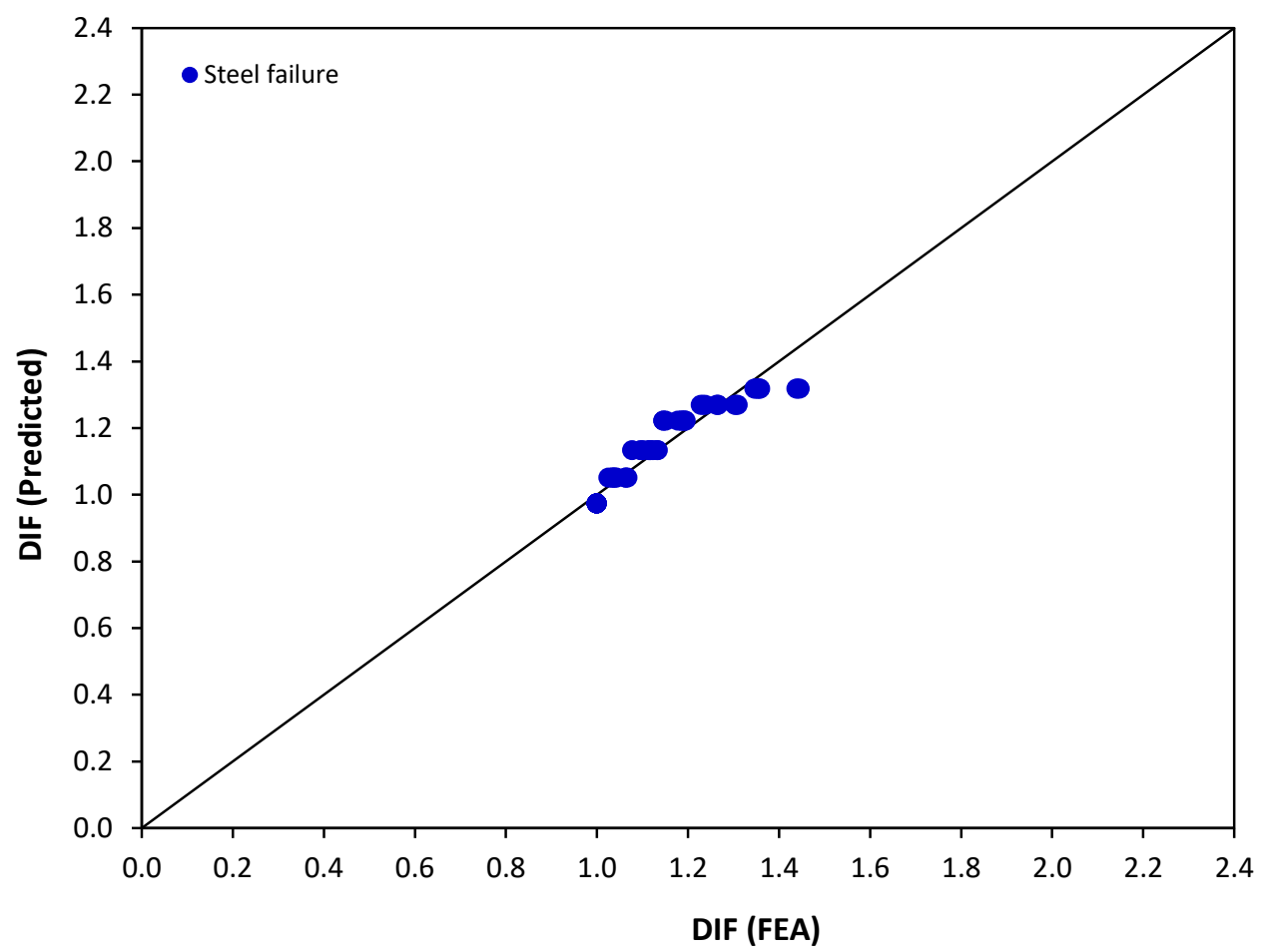

Figure 6-30: DIF obtained from the finite element analysis versus the predicted DIF for the undercut anchors exhibited steel failure

Also, in order to verify the results obtained from the finite element analysis, a comparison has been made between the ultimate dynamic load obtained from the finite element analysis for the undercut anchors exhibited concrete cone breakout and the proposed equation by Fujikake et al. (Fujikake et al., 2003) Equation (4.10) as shown in Table 6-10. As shown in the table the finite element results for the undercut anchors overpredict the dynamic load obtained from the proposed equation by Fujikake et al. 
Table 6-10: Comparison between ultimate dynamic load for the undercut anchor obtained from the FEA and proposed equation by Fujikake et al. (2003)

\begin{tabular}{|c|c|c|c|c|c|c|c|c|}
\hline $\begin{array}{c}\mathbf{d} \\
(\mathbf{m m})\end{array}$ & $\begin{array}{c}\mathbf{h}_{\mathrm{ef}} \\
(\mathbf{m m})\end{array}$ & $\begin{array}{c}\dot{\varepsilon}_{s} \\
\left(\mathrm{~s}^{-1}\right)\end{array}$ & $\begin{array}{c}\dot{\varepsilon}_{d} \\
\left(\mathrm{~s}^{-1}\right)\end{array}$ & $\dot{\varepsilon}_{d} / \dot{\varepsilon}_{s}$ & $\begin{array}{c}\text { Fus }_{\text {us }} \text { FEA } \\
(\mathbf{k N})\end{array}$ & $\begin{array}{c}\text { Fud }_{\text {ud }} \text { FEA } \\
(\mathbf{k N})\end{array}$ & $\begin{array}{c}\text { Fud }_{\text {udjikake }} \text { Fujo } \\
(k N)\end{array}$ & $\begin{array}{l}\text { Fud FEA/ } \\
\text { Fujikake }\end{array}$ \\
\hline 16 & 100 & $10^{-5}$ & $10^{-5}$ & 1 & 101.09 & 101.09 & 101.12 & 1.00 \\
\hline 20 & 100 & $10^{-5}$ & $10^{-5}$ & 1 & 118.41 & 118.41 & 103.02 & 1.15 \\
\hline 20 & 100 & $10^{-5}$ & $10^{-3}$ & $10^{2}$ & 118.41 & 142.16 & 104.38 & 1.36 \\
\hline 20 & 100 & $10^{-5}$ & $10^{-1}$ & $10^{4}$ & 118.41 & 161.18 & 117.94 & 1.37 \\
\hline 20 & 100 & $10^{-5}$ & 10 & $10^{6}$ & 118.41 & 186.6 & 175.20 & 1.07 \\
\hline 20 & 125 & $10^{-5}$ & $10^{-5}$ & 1 & 168.66 & 168.66 & 141.32 & 1.19 \\
\hline 20 & 125 & $10^{-5}$ & $10^{-3}$ & $10^{2}$ & 168.66 & 185.01 & 143.17 & 1.29 \\
\hline 20 & 125 & $10^{-5}$ & $10^{-1}$ & $10^{4}$ & 168.66 & 208.53 & 161.78 & 1.29 \\
\hline
\end{tabular}




\subsection{Finite element modeling for undercut anchors under shear load}

Finite element analyses were performed to investigate the shear behaviour of undercut anchors. Three anchor diameters of $12 \mathrm{~mm}, 16 \mathrm{~mm}$ and $20 \mathrm{~mm}$ with embedment depths of $100 \mathrm{~mm}, 125 \mathrm{~mm}, 190 \mathrm{~mm}$ and $250 \mathrm{~mm}$ were investigated. Figure 6-31 shows the geometric configuration and boundary conditions for the undercut anchor model under shear load.

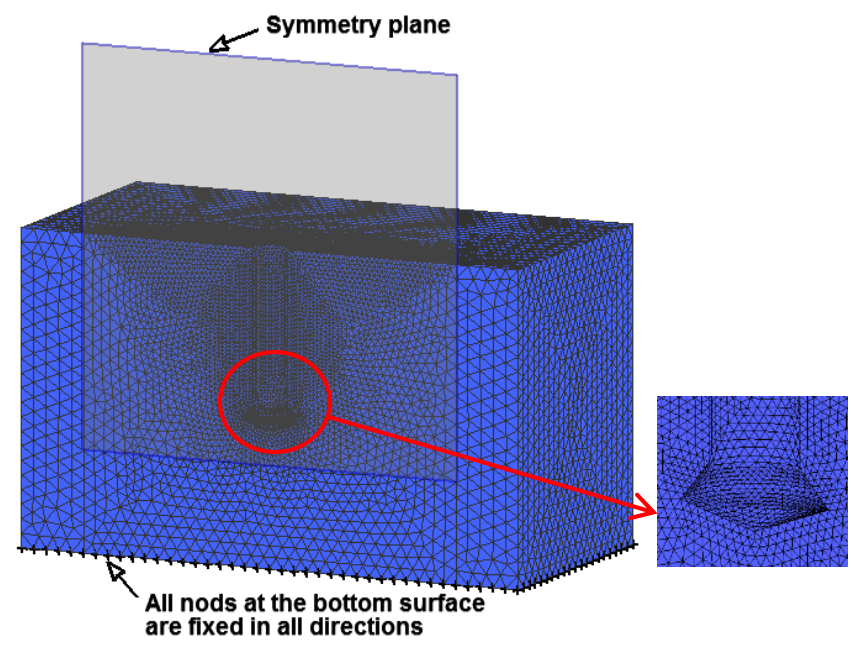

(a) Concrete mesh

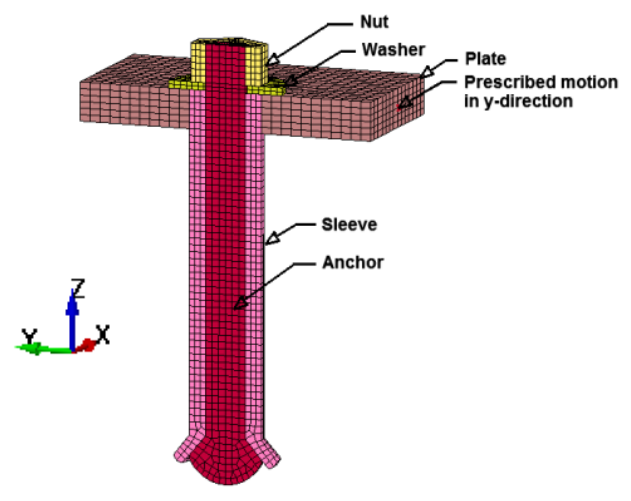

(b) Undercut anchor mesh

Figure 6-31: Geometric configuration and boundary conditions for the undercut anchor model

\subsubsection{Validation of undercut anchor model under shear load}

The numerical model of the undercut anchor under shear load was validated by comparing the finite element results with the experimental results obtained by Mahadik et al. (Mahadik et al., 2016). A finite element model, representing the test conducted by Mahadik et al., was developed using LS-DYNA software. Hilti undercut anchor through-set type was used. The undercut anchor used had yield strength of $640 \mathrm{MPa}$, tensile strength of $800 \mathrm{MPa}$, 
diameter of $12 \mathrm{~mm}$ and embedment depth of $125 \mathrm{~mm}$. The cube compressive strength of the concrete block used in the finite element analysis of $42.6 \mathrm{MPa}\left(f_{c}^{\prime}=33.5 \mathrm{MPa}\right)$ same as in the experimental test conducted by Mahadik et al. Mesh sensitivity analyses were carried out to obtain a mesh size that improve the accuracy and give converged results to the experimental results conducted by Mahadik et al. Table 6-11 shows the effect of mesh size refinement on the convergence of the ultimate shear load and failure mode.

Table 6-11: Effect of mesh size refinement on the convergence of ultimate shear load for undercut anchor

\begin{tabular}{|c|c|c|c|c|c|c|c|c|}
\hline \multirow[t]{3}{*}{$\begin{array}{c}\text { Model } \\
\text { No. }\end{array}$} & \multicolumn{3}{|c|}{$\begin{array}{l}\text { Mesh size } \\
(\mathrm{mm})\end{array}$} & \multicolumn{2}{|c|}{$\begin{array}{l}\text { Ultimate shear load } \\
\qquad(\mathrm{kN})\end{array}$} & \multicolumn{2}{|c|}{$\begin{array}{l}\text { Displacement } \\
(\mathrm{mm})\end{array}$} & \multirow[t]{3}{*}{ Failure mode } \\
\hline & \multirow[t]{2}{*}{ Anchor } & \multicolumn{2}{|c|}{ Concrete } & \multirow[t]{2}{*}{ FEA } & \multirow[t]{2}{*}{ EXP. } & \multirow[t]{2}{*}{ FEA } & \multirow[t]{2}{*}{ EXP. } & \\
\hline & & Min. & Max. & & & & & \\
\hline 1 & 2 & 4 & 12 & 126.21 & \multirow{3}{*}{131.9} & 19.10 & \multirow{3}{*}{18.71} & Steel failure \\
\hline 2 & 2 & 2 & 12 & 128.65 & & 18.89 & & Steel failure \\
\hline 3 & 1 & 1 & 12 & 134.73 & & 18.82 & & Steel failure \\
\hline
\end{tabular}

The finite element analysis exhibited a percentage difference of $4.5 \%, 2.5 \%$ and $2.1 \%$ for the ultimate shear load for the models No. 1, 2 and 3 respectively in comparison with the experimental results. Mesh size of model No. 2 gave load-displacement results agree well with the experimental results reported by Mahadik et al. with better computation time and selected for the analysis. A comparison of shear load-displacement response of the finite element analysis and experimental results is shown in Figure 6-32. As shown in the figure same shear load-displacement behaviour was observed for the all mesh sizes investigated. Finite element analysis results exhibited steel anchor failure mode similar to that observed by Mahadik et al. as shown in Figure 6-33. 


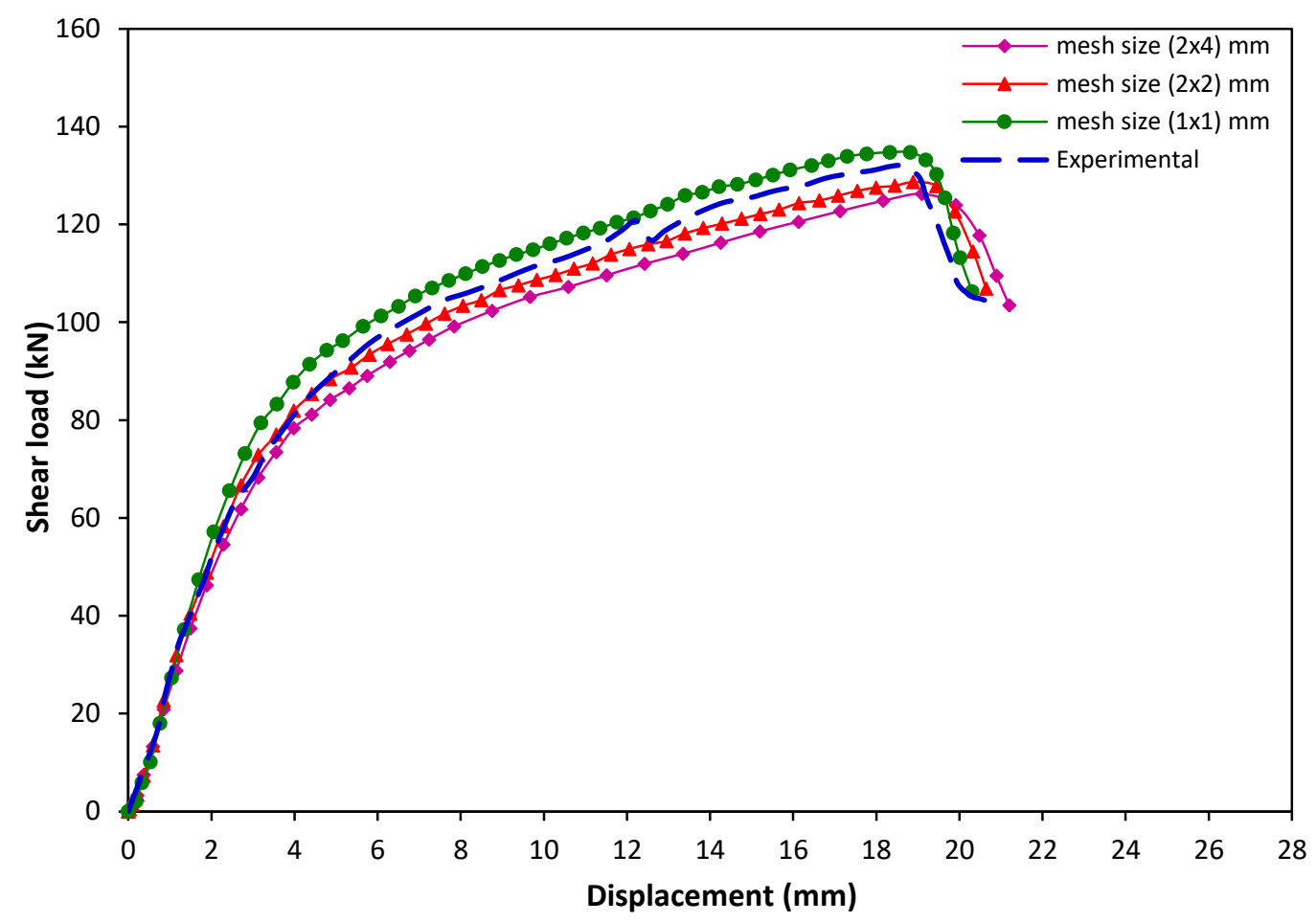

Figure 6-32: Comparison of shear load-displacement relation between FEA and experimental results obtained by Mahadik et al. (Mahadik et al., 2016)

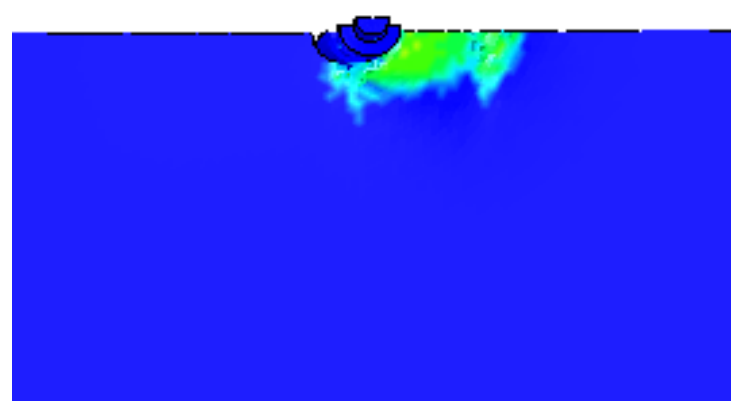

(a)

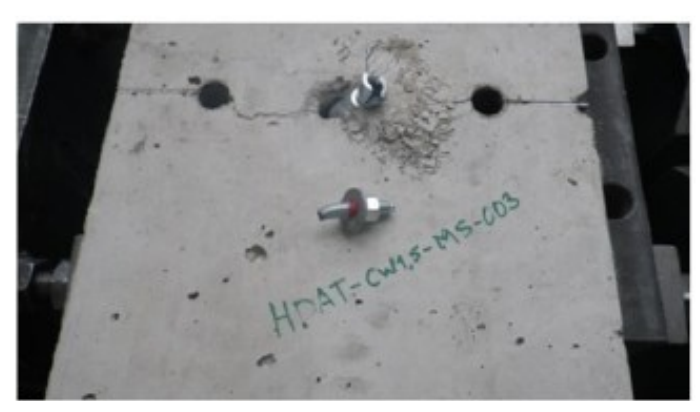

(b)

Figure 6-33: Failure mode obtained from:(a) finite element analysis and (b) experimental results obtained by Mahadik et al. (Mahadik et al., 2016)

\subsubsection{Comparison of finite element results with design methods}

For the anchors placed far away from the concrete free edge, pryout failure mode or anchor failure is often the dominant failure modes. The shear failure load of anchors exhibiting pryout failure can be calculated according to ACI 318 Equation (4.14) (ACI Committee 
$318,2011)$. The shear failure load of anchors that exhibit steel fracture failure can be calculated according to ACI 318 method as in Equation (4.18) (ACI Committee 318, 2011). The ultimate shear load obtained from the finite element analysis was compared with ACI 318 method (Table 6-12). It can be seen that for the anchor diameter of 12-mm with embedment depths of $125 \mathrm{~mm}$ and $190 \mathrm{~mm}$, a slight difference in the shear load where steel failure is observed. This can be attributed to the influence of embedment depth on the bending, tensile and shear stresses that are generated in the anchor due to the applied shear load. As shown in Table 6-12, the ACI 318 method overestimates the shear capacity for the undercut anchors investigated except for the anchor diameter of 20 -mm where pryout failure mode was observed. The ACI method takes into account the effect of embedment depth, however the influence of anchor diameter is not considered in the case of pryout failure mode. In general, the FEA results show good agreement with the ACI 318 method.

Table 6-12: Comparison of ultimate shear load obtained from FEA and ACI 318 method

\begin{tabular}{|c|c|c|c|c|c|}
\hline \multirow{2}{*}{$\begin{array}{c}\mathrm{d} \\
(\mathrm{mm})\end{array}$} & $\begin{array}{c}\mathrm{h}_{\mathrm{ef}} \\
(\mathrm{mm})\end{array}$ & \multicolumn{2}{|c|}{$\begin{array}{c}\text { Failure Load } \\
(\mathrm{kN})\end{array}$} & \multirow{2}{*}{$\begin{array}{c}\text { FEA } \\
\text { ACI } 318\end{array}$} & \\
\cline { 3 - 5 } & & $\begin{array}{c}\text { FEA static } \\
\left(\dot{\varepsilon}=10^{-5} \mathrm{~s}^{-1}\right)\end{array}$ & ACI 318 & & \\
\hline 12 & 100 & 132.27 & 164.23 & 0.81 & $\mathrm{PR}$ \\
\hline 12 & 125 & 148.09 & 166.25 & 0.89 & $\mathrm{~S}$ \\
\hline 12 & 190 & 153.57 & 166.25 & 0.92 & $\mathrm{~S}$ \\
\hline 16 & 100 & 152.26 & 164.23 & 0.93 & $\mathrm{PR}$ \\
\hline 16 & 125 & 199.42 & 229.52 & 0.87 & PR \\
\hline 16 & 190 & 270.05 & 317.05 & 0.85 & PR \\
\hline 16 & 250 & 304.6 & 317.05 & 0.96 & $\mathrm{~S}$ \\
\hline 20 & 100 & 175.37 & 164.23 & 1.07 & PR \\
\hline 20 & 125 & 227.49 & 229.52 & 0.99 & PR \\
\hline 20 & 190 & 344.63 & 430.13 & 0.80 & PR \\
\hline 20 & 250 & 420.84 & 488.58 & 0.86 & $\mathrm{~S}$ \\
\hline
\end{tabular}

*Failure mode: $\mathrm{PR}=$ pryout failure; $\mathrm{S}=$ steel anchor fracture 


\subsubsection{Crack pattern for the undercut anchors under shear load}

Figure 6-34 shows the crack patterns for the undercut anchors of different diameters and embedment depths at strain rate of $10^{-5} \mathrm{~s}^{-1}$. As shown in the figure, pryout failure mode was observed for the undercut anchors with embedment depth of $100 \mathrm{~mm}$. At embedment depths of $125 \mathrm{~mm}$ and $190 \mathrm{~mm}$, steel anchor failure was observed for the 12-mm diameter anchor whereas pryout failure was observed for the 16-mm and 20-mm diameter anchors. At embedment depth of $250 \mathrm{~mm}$, steel anchor failure was observed for all the anchor diameters investigated.

Level of damage and cracking in the concrete increased with the increase in the anchor diameter. It can be seen that, at the low strain rate of $10^{-5} \mathrm{~s}^{-1}$ the anchor diameter and embedment depth have an effect on the failure mode. The increase in the embedment depth from $100 \mathrm{~mm}$ to $125 \mathrm{~mm}$ resulted in concrete spalling followed by anchor fracture for the 12-mm diameter undercut anchor. The increase in the anchor diameter from $12 \mathrm{~mm}$ to 20 $\mathrm{mm}$ for the same embedment depth of $125 \mathrm{~mm}$ resulted in pryout failure of the anchor. It can be stated that, the increase in the undercut anchor diameter at low strain rate increases the probability of pryout failure as the anchor stiffness increases. 


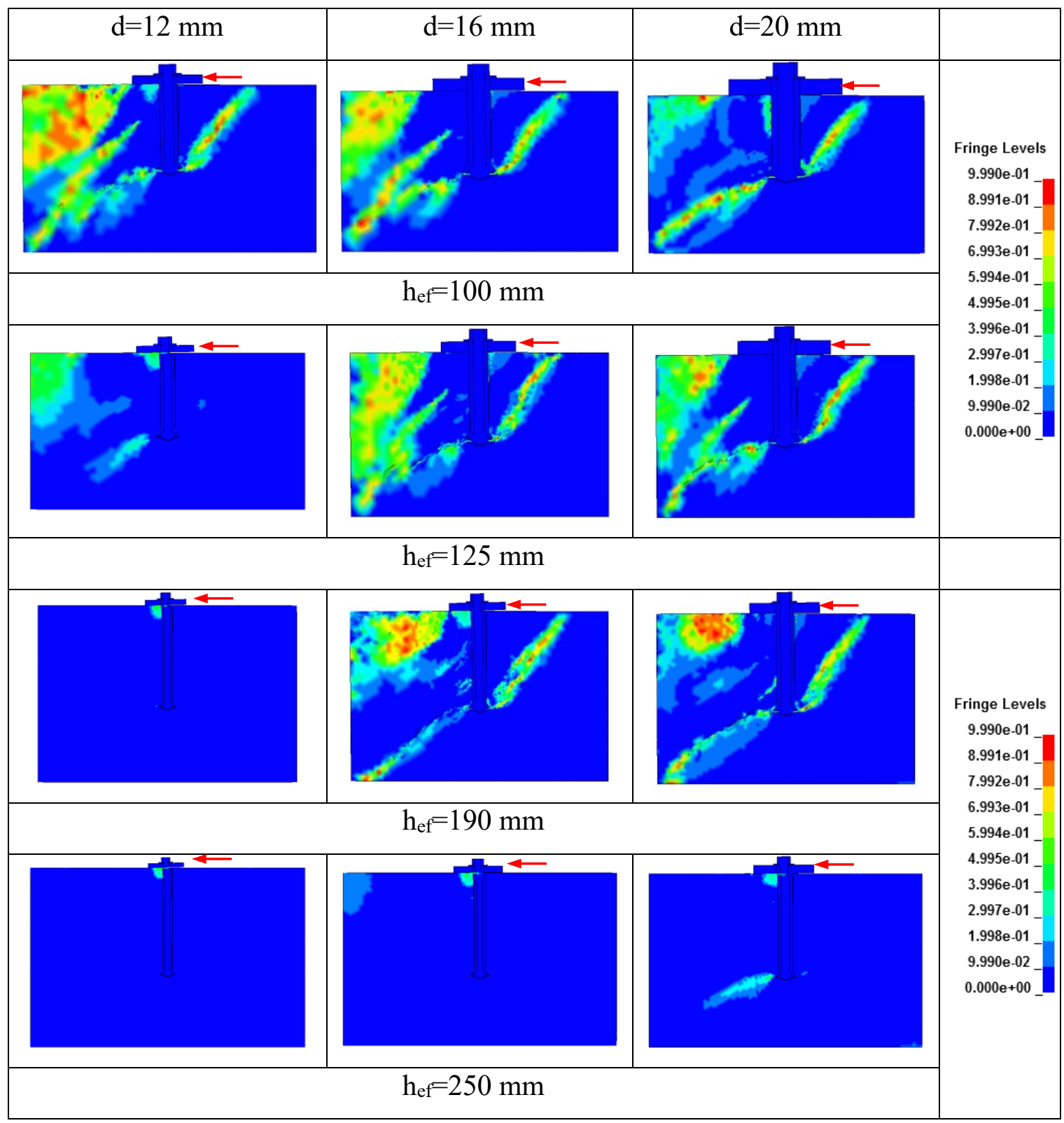

Figure 6-34: Plastic strain contours for undercut anchors under shear load at strain rate of $10^{-5} \mathrm{~s}^{-1}$

\subsubsection{Effect of strain rate on the level of damage and failure mode}

When the shear load is applied on the anchor plate, the load is transferred from the anchor plate to the undercut anchor. For the through-set type undercut anchor used in the analysis, both the sleeve and the anchor body share in resisting the shear load and transfer it to the 
concrete resulting in developing compressive stresses in the concrete in front of the anchor. These stresses increase with the increase in the applied shear load, create concrete cracking under the anchor plate and result in displacement of the anchor plate in vertical direction and rotation about the point of contact on the concrete surface. Three failure modes were observed; concrete pryout, concrete spalling and steel anchor failure modes. For the pryout failure mode, cracks initiate at the bottom of the sleeve and propagate diagonally towards the concrete surface resulting in breakout of a half concrete cone at the backside of the anchor, leading to pryout failure. For the concrete spalling, the stresses are concentrated in the concrete in front of the anchor resulting in concrete cracking without breakout of a half concrete cone behind the anchor. On the other hand, when the tensile, bending and shear stresses concentrated on the anchor, steel failure is observed. Failure mode for the 12-mm, 16-mm and $20-\mathrm{mm}$ diameter undercut anchors at different strain rates is presented in Figures 6-35, 6-36 and 6-37 respectively. 


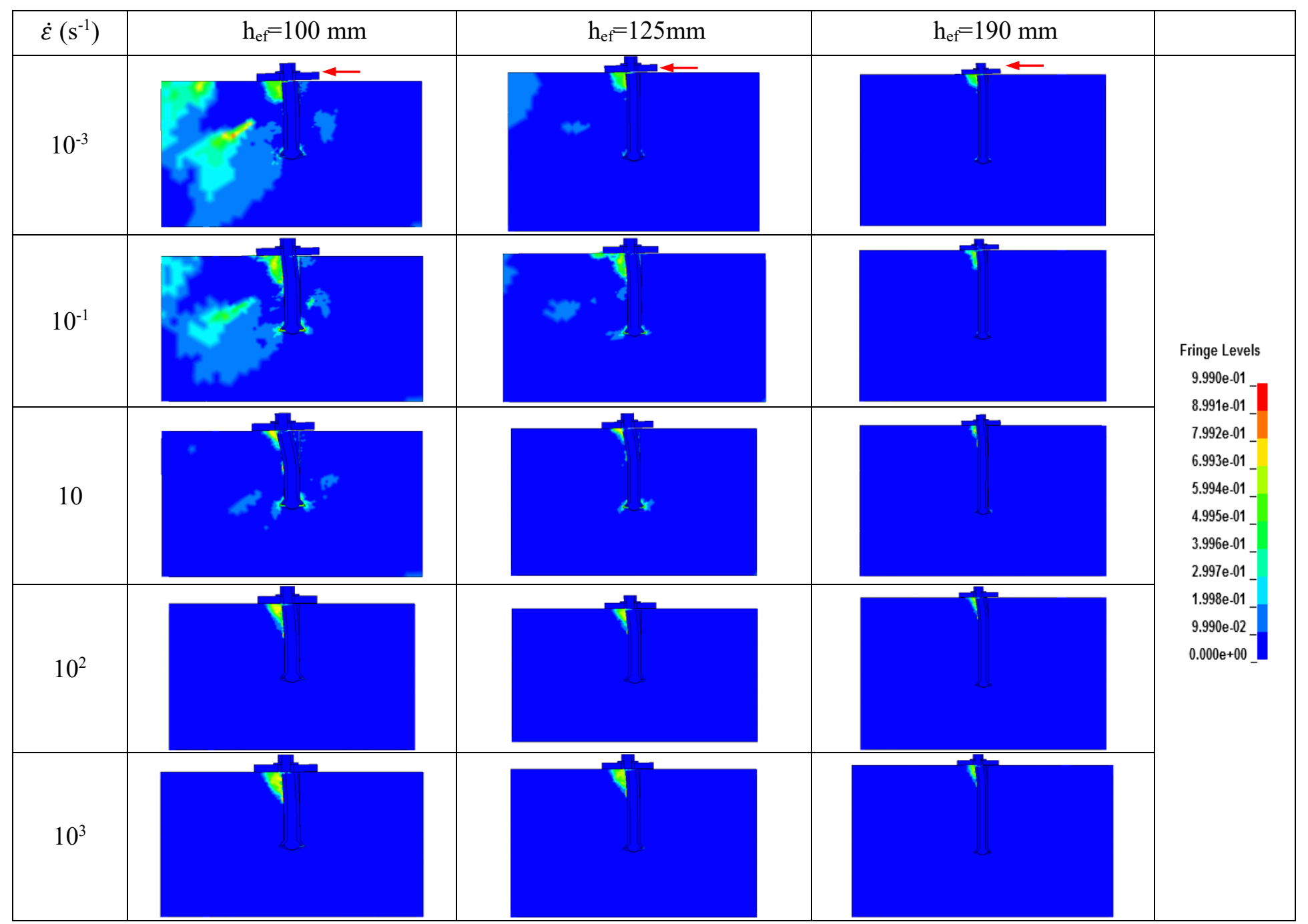

Figure 6-35: Failure mode of 12-mm diameter undercut anchor at different strain rates 


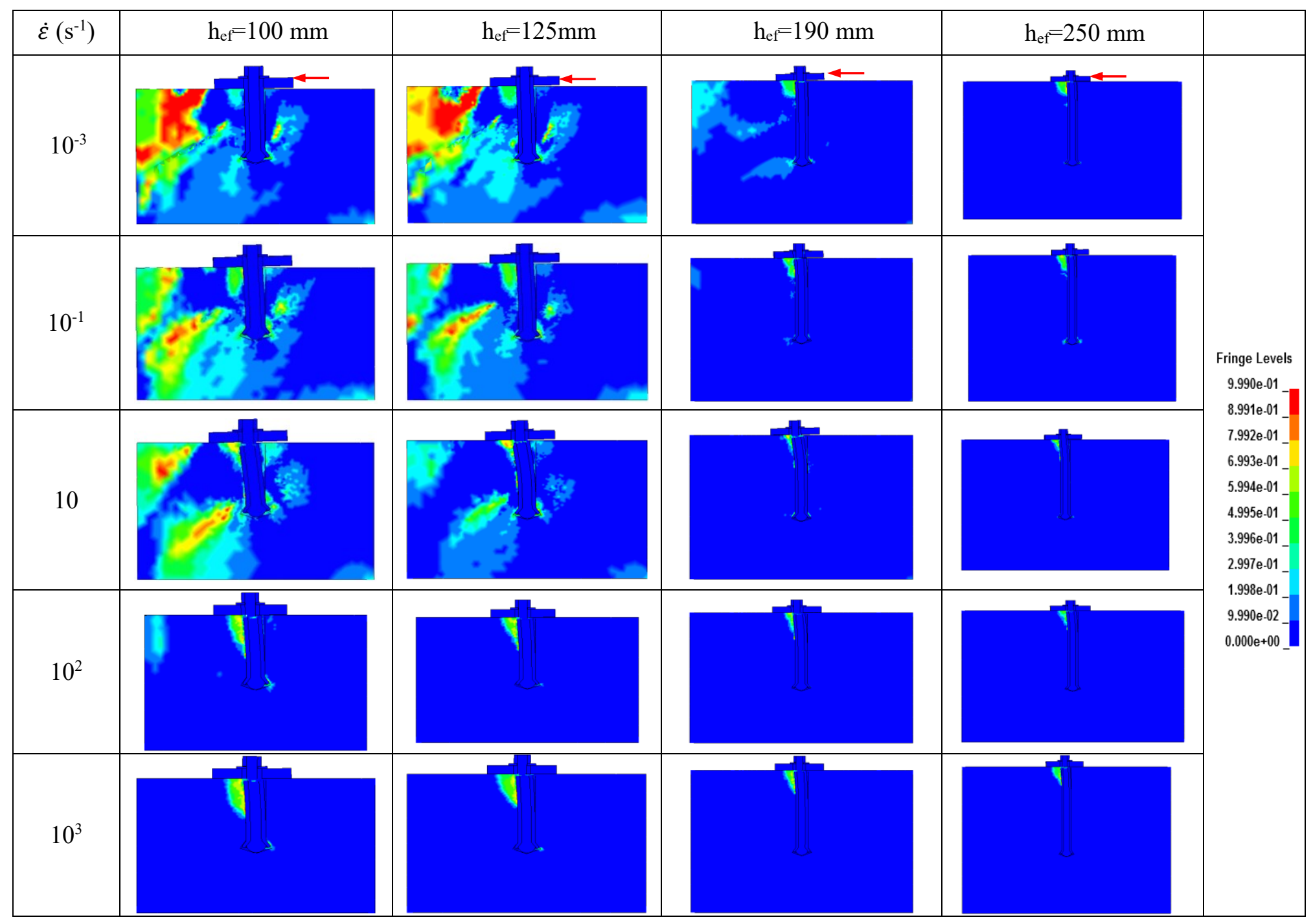

Figure 6-36: Failure mode of 16-mm diameter undercut anchor at different strain rates 


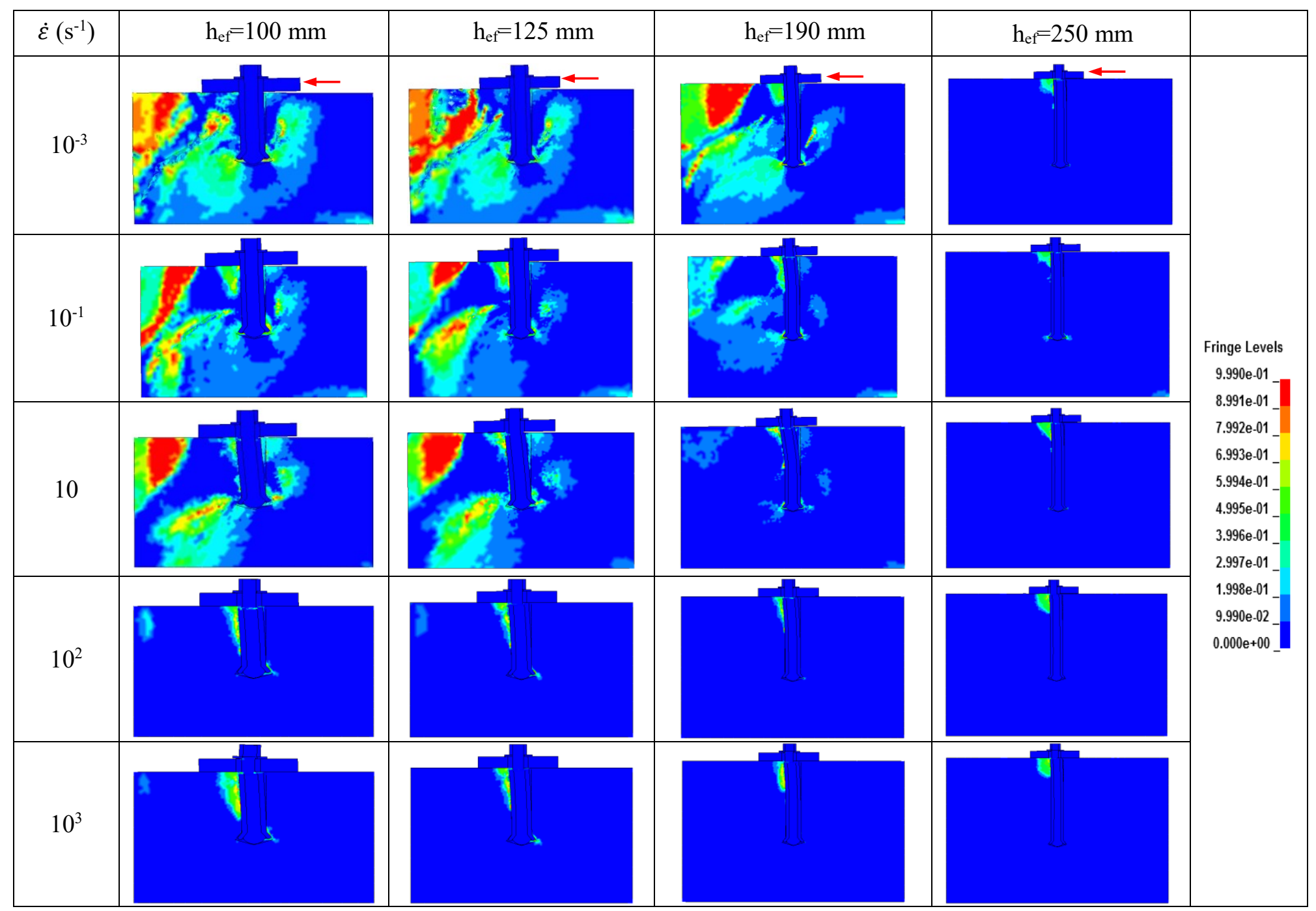

Figure 6-37: Failure mode of 20-mm diameter undercut anchor at different strain rates 
From Figures 6-35, 6-36 and 6-37, it can be seen that the behaviour and failure mode of the undercut anchor is affected by the strain rate. As shown in Figure 6-35, steel anchor failure is observed for anchor diameter of $12 \mathrm{~mm}$ at strain rates of $10^{-3} \mathrm{~s}^{-1}$ to $10^{3} \mathrm{~s}^{-1}$. As shown in Figure 6-36 for the anchor diameter of $16 \mathrm{~mm}$, at strain rates of $10^{-3} \mathrm{~s}^{-1}$ and $10^{-1}$ $\mathrm{s}^{-1}$, concrete spalling is observed for embedment depths of $100 \mathrm{~mm}$ and $125 \mathrm{~mm}$ while steel anchor failure is observed for embedment depths of $190 \mathrm{~mm}$ and $250 \mathrm{~mm}$. At strain rates of $10 \mathrm{~s}^{-1}, 10^{2} \mathrm{~s}^{-1}$ and $10^{3} \mathrm{~s}^{-1}$ concrete spalling followed by steel anchor failure is observed for all the embedment depths investigated. As shown in Figure 6-36 more concrete cracking and damage was observed for the embedment depths of $100 \mathrm{~mm}$ and $125 \mathrm{~mm}$ compared to the deeper embedment depths of $190 \mathrm{~mm}$ and $250 \mathrm{~mm}$. For the shallow embedment depths of $100 \mathrm{~mm}$ and $125 \mathrm{~mm}$ the cracks initiated in front of the anchor and at the lower end of the anchor resulted in concrete cracking in a wider area. However, for the deeper embedment depths concrete spalling followed by steel anchor failure was observed.

It can be seen from Figure 6-37 for the 20-mm diameter undercut anchor, at strain rate of $10^{-3} \mathrm{~s}^{-1}$, pryout failure is observed for embedment depths of $100 \mathrm{~mm}$ and $125 \mathrm{~mm}$, concrete spalling is observed for embedment depth of $190 \mathrm{~mm}$ whereas steel anchor failure is observed for embedment depth of $250 \mathrm{~mm}$. At strain rate of $10^{-1} \mathrm{~s}^{-1}$ concrete spalling is observed for embedment depths of $100 \mathrm{~mm}$ and $125 \mathrm{~mm}$ while steel anchor failure is observed for embedment depths of $190 \mathrm{~mm}$ and $250 \mathrm{~mm}$. Also, steel anchor failure preceded by concrete spalling was observed for all embedment depths at strain rates of 10 $\mathrm{s}^{-1}, 10^{2} \mathrm{~s}^{-1}$ and $10^{3} \mathrm{~s}^{-1}$. As shown in Figure 6-37 more concrete cracking is observed for the 
shallow embedment depths. It can be seen from Figures 6-35, 6-36 and 6-37 that concrete cracks and damage increased with the increase in anchor diameter from $12 \mathrm{~mm}$ to $20 \mathrm{~mm}$.

Figure 6-38 shows the failure progression of a 12-mm diameter undercut anchor embedded $190 \mathrm{~mm}$ in the concrete. Contours of Von Misses stresses (MPa) are shown in the figure. It can be seen that the stresses are concentrated around the sleeve and anchor at the top where the shear load is applied. Combination of the tensile, bending and shear stresses due to the applied load leads to initiation and propagation of cracks in the top part of the undercut anchor resulting in steel anchor failure.

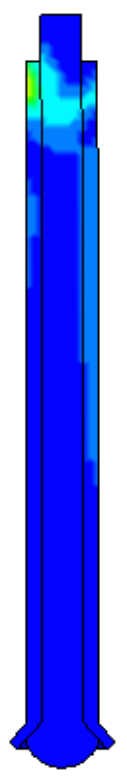

(a)

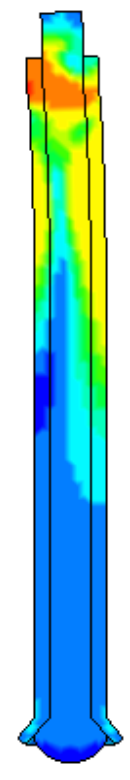

(b)

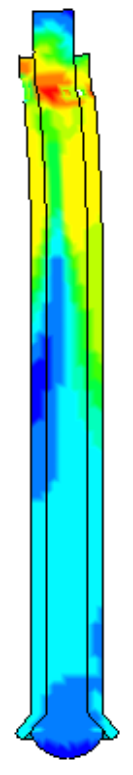

(c)

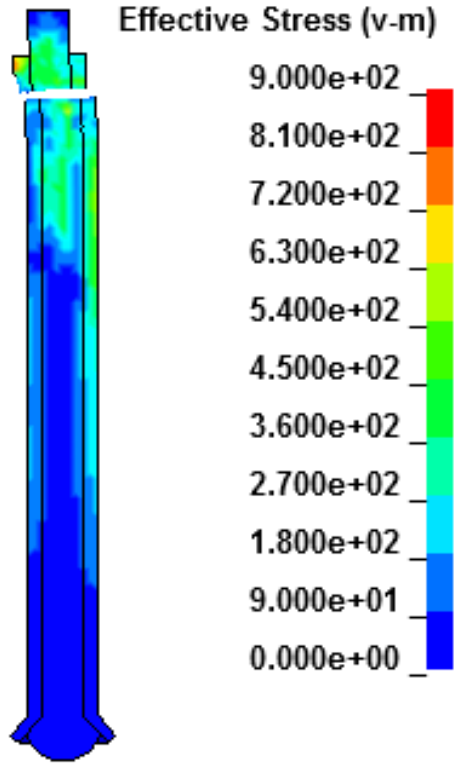

(d)

Figure 6-38: Failure process of 12-mm diameter undercut anchor with $190 \mathrm{~mm}$ embedment depth; (a) stress concentration around the undercut anchor, (b) anchor bending, (c) crack initiation and (d) anchor fracture 


\subsubsection{Effect of design parameters on failure mode and ultimate shear load}

Figures 6-39, 6-40 and 6-41 show the effect of strain rate on the failure mode and ultimate shear load for the 12-mm, 16-mm and 20-mm diameter undercut anchors respectively. As shown in the figures, the strain rate affects the failure mode for the 16-mm and $20-\mathrm{mm}$ diameter undercut anchors where transition from pryout failure to concrete spalling and then to steel anchor failure is observed. Also, the anchor diameter and embedment depth affect the failure mode. It can be seen from the figures that the ultimate shear load increased with the increase in the strain rate and anchor diameter for the undercut anchors.

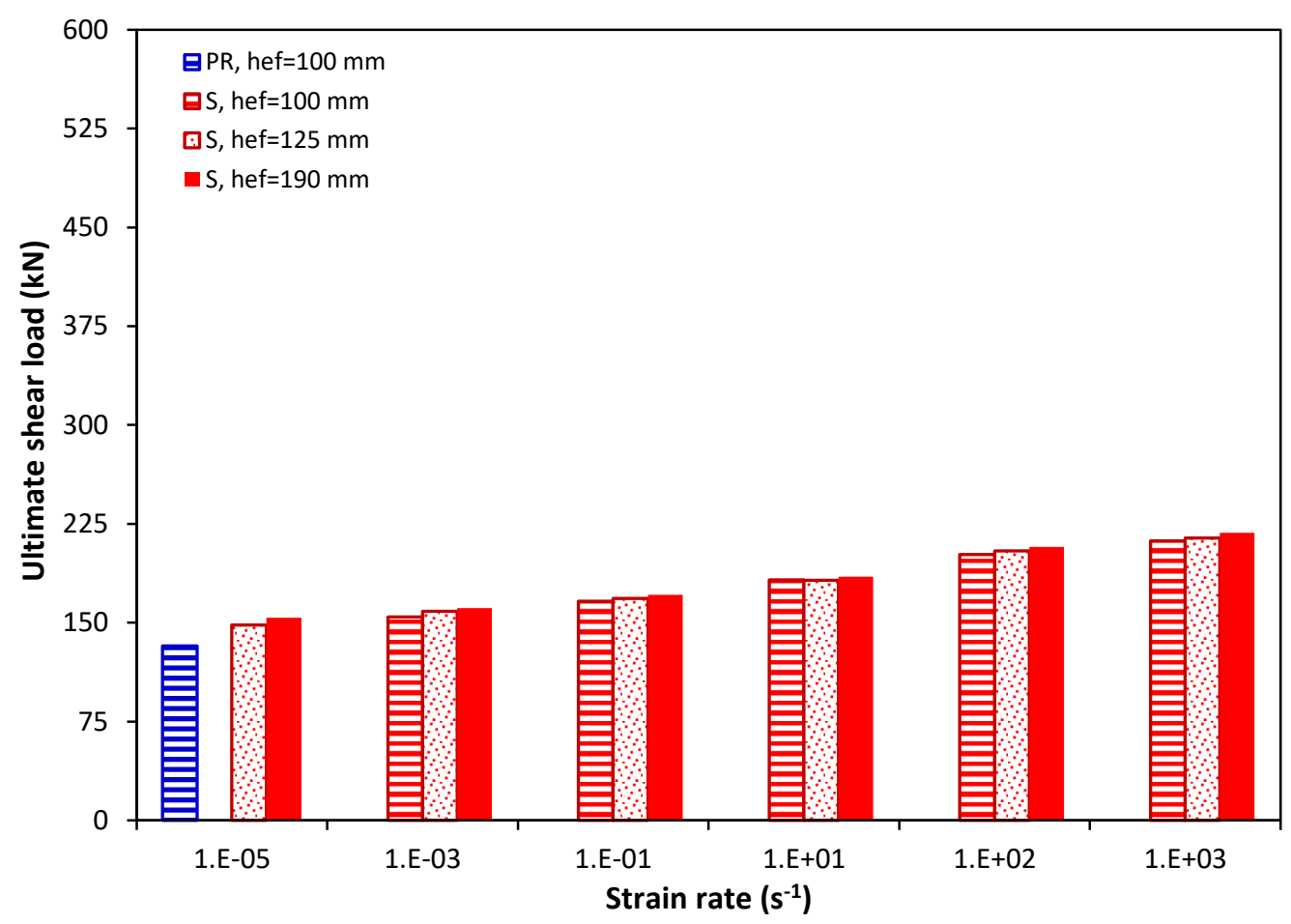

Figure 6-39: Effect of strain rate on the failure mode and ultimate shear load for the undercut anchor diameter of $12 \mathrm{~mm}$ 


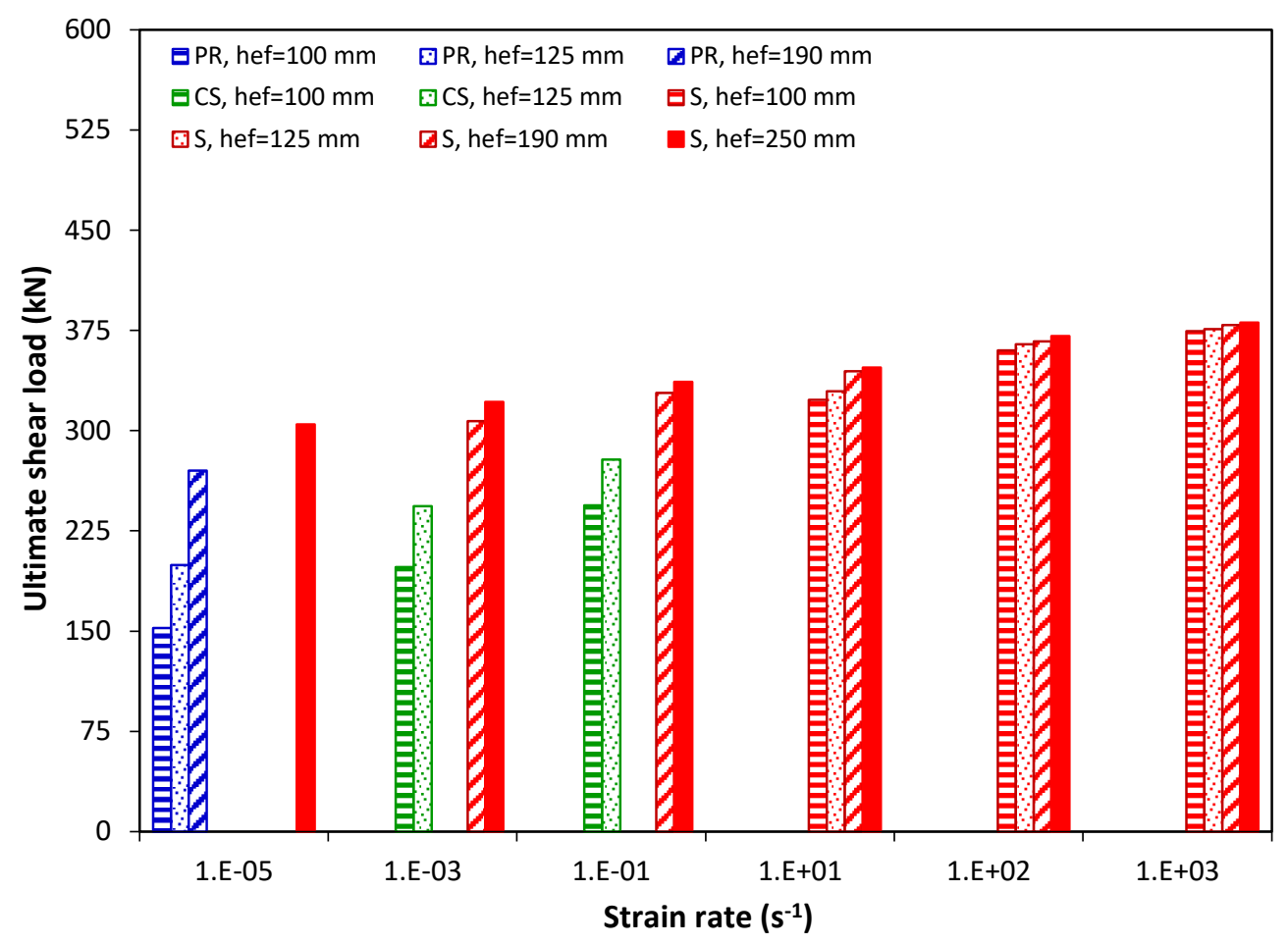

Figure 6-40: Effect of strain rate on the failure mode and ultimate shear load for the undercut anchor diameter of $16 \mathrm{~mm}$

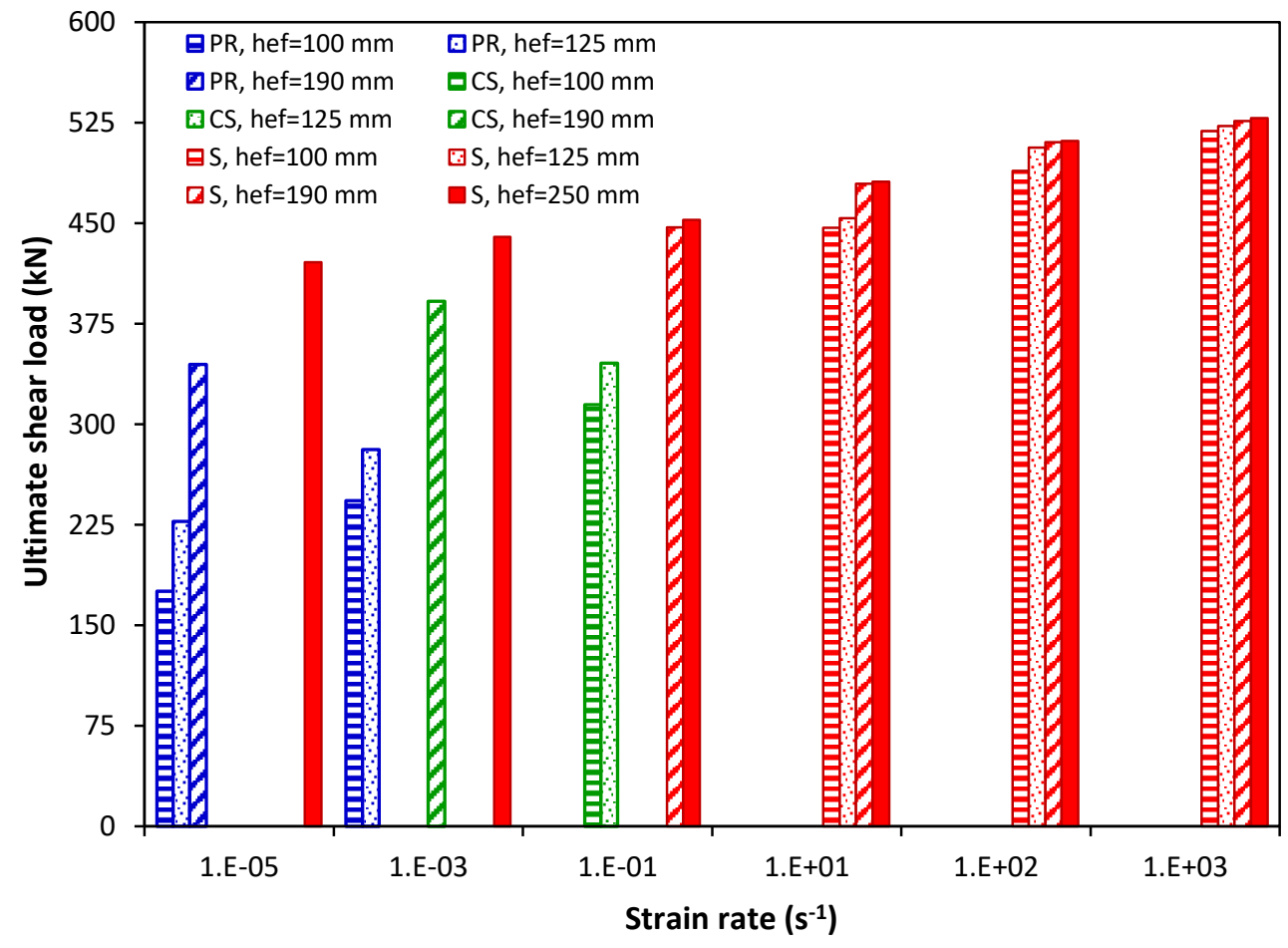

Figure 6-41: Effect of strain rate on the failure mode and ultimate shear load for the undercut anchor diameter of $20 \mathrm{~mm}$ 
Table 6-13 presents the failure modes for the undercut anchors subjected to shear loads at different strain rates.

Table 6-13: Failure mode for the undercut anchors under shear load at different strain rates

\begin{tabular}{|c|c|c|c|c|c|c|c|c|}
\hline \multirow{3}{*}{$\begin{array}{l}\text { Model } \\
\text { No. }\end{array}$} & \multirow{3}{*}{$\begin{array}{c}d \\
(\mathrm{~mm})\end{array}$} & \multirow{3}{*}{$\begin{array}{c}\mathbf{h}_{\text {ef }} \\
(\mathbf{m m})\end{array}$} & \multicolumn{6}{|c|}{ "Failure mode } \\
\hline & & & \multicolumn{6}{|c|}{ Strain rate $\left(\mathrm{s}^{-1}\right)$} \\
\hline & & & $\dot{\varepsilon}=10^{-5}$ & $\dot{\varepsilon}=10^{-3}$ & $\dot{\varepsilon}=10^{-1}$ & $\dot{\varepsilon}=10$ & $\dot{\varepsilon}=10^{2}$ & $\dot{\varepsilon}=10^{3}$ \\
\hline 1 & 12 & 100 & PR & $\mathrm{S}$ & $\mathrm{S}$ & $\mathrm{S}$ & $\mathrm{S}$ & $\mathrm{S}$ \\
\hline 2 & 12 & 125 & $\mathrm{~S}$ & $\mathrm{~S}$ & $\mathrm{~S}$ & $\mathrm{~S}$ & $\mathrm{~S}$ & $\mathrm{~S}$ \\
\hline 3 & 12 & 190 & $\mathrm{~S}$ & $\mathrm{~S}$ & $\mathrm{~S}$ & $\mathrm{~S}$ & $\mathrm{~S}$ & $\mathrm{~S}$ \\
\hline 4 & 16 & 100 & PR & $\mathrm{CS}$ & $\mathrm{CS}$ & $\mathrm{S}$ & $\mathrm{S}$ & $\mathrm{S}$ \\
\hline 5 & 16 & 125 & PR & $\mathrm{CS}$ & $\mathrm{CS}$ & $\mathrm{S}$ & $\mathrm{S}$ & $S$ \\
\hline 6 & 16 & 190 & PR & $\mathrm{S}$ & $\mathrm{S}$ & $\mathrm{S}$ & $\mathrm{S}$ & $\mathrm{S}$ \\
\hline 7 & 16 & 250 & $\mathrm{~S}$ & $S$ & $\mathrm{~S}$ & $\mathrm{~S}$ & $\mathrm{~S}$ & $\mathrm{~S}$ \\
\hline 8 & 20 & 100 & PR & PR & $\mathrm{CS}$ & $\mathrm{S}$ & $\mathrm{S}$ & $\mathrm{S}$ \\
\hline 9 & 20 & 125 & PR & PR & $\mathrm{CS}$ & $\mathrm{S}$ & $\mathrm{S}$ & $\mathrm{S}$ \\
\hline 10 & 20 & 190 & PR & $\mathrm{CS}$ & $\mathrm{S}$ & $\mathrm{S}$ & $\mathrm{S}$ & $\mathrm{S}$ \\
\hline 11 & 20 & 250 & $\mathrm{~S}$ & $\mathrm{~S}$ & $\mathrm{~S}$ & $\mathrm{~S}$ & $\mathrm{~S}$ & $\mathrm{~S}$ \\
\hline
\end{tabular}

*Failure mode: $\mathrm{PR}=$ pryout, $\mathrm{CS}=$ concrete spalling, $\mathrm{S}=$ steel anchor failure

\subsubsection{Effect of strain rate on the shear behaviour of undercut anchors}

Figures 6-42, 6-43, 6-44, 6-45, 6-46, and 6-47 present the shear load-displacement graphs for the 12-mm diameter undercut anchor at strain rates of $10^{-5} \mathrm{~s}^{-1}, 10^{-3} \mathrm{~s}^{-1}, 10^{-1} \mathrm{~s}^{-1}, 10 \mathrm{~s}^{-1}$, $10^{2} \mathrm{~s}^{-1}$ and $10^{3} \mathrm{~s}^{-1}$ respectively. 


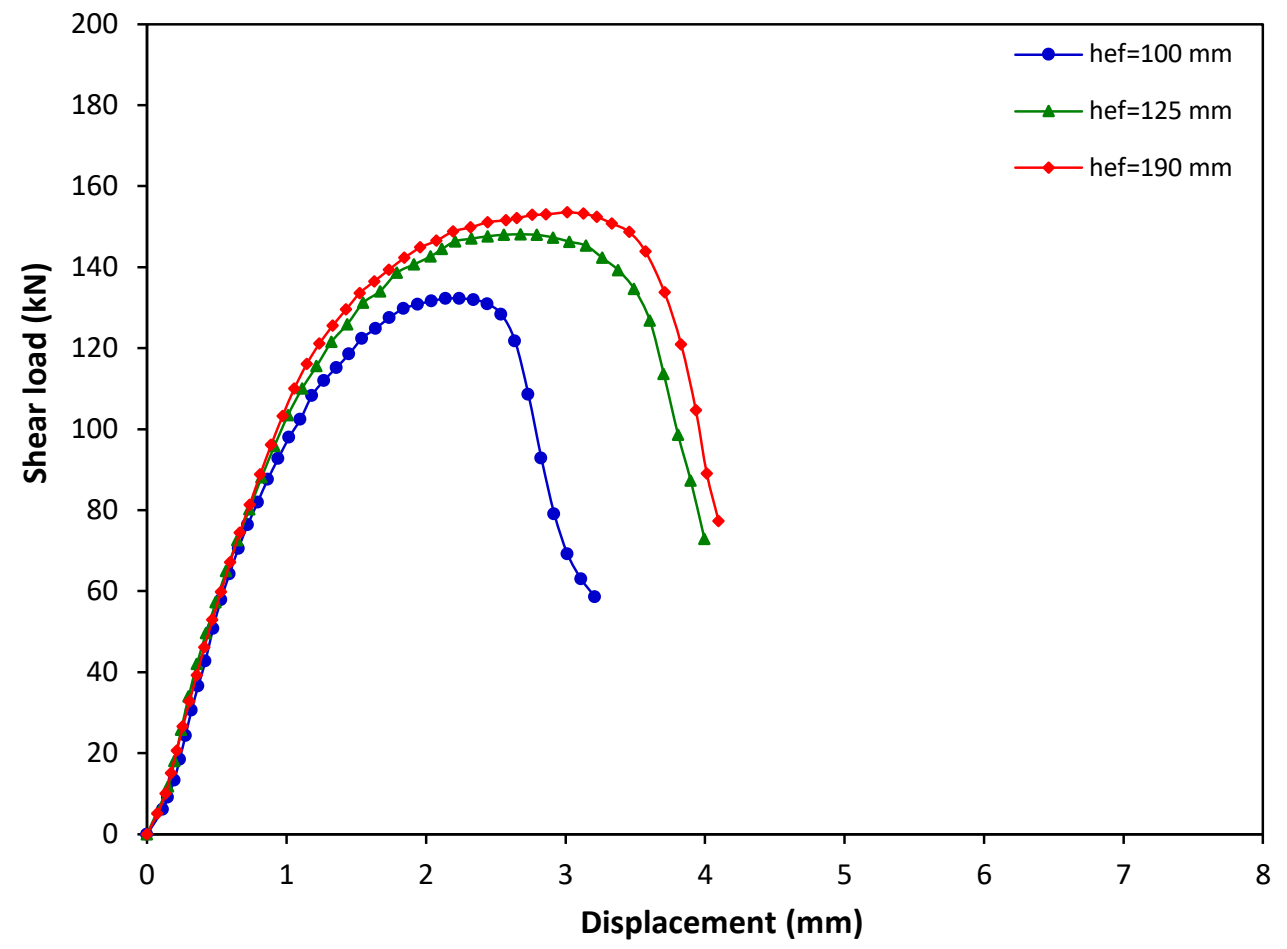

Figure 6-42: Shear load-displacement graph for 12-mm diameter undercut anchor at strain rate of $10^{-5} \mathrm{~s}^{-1}$

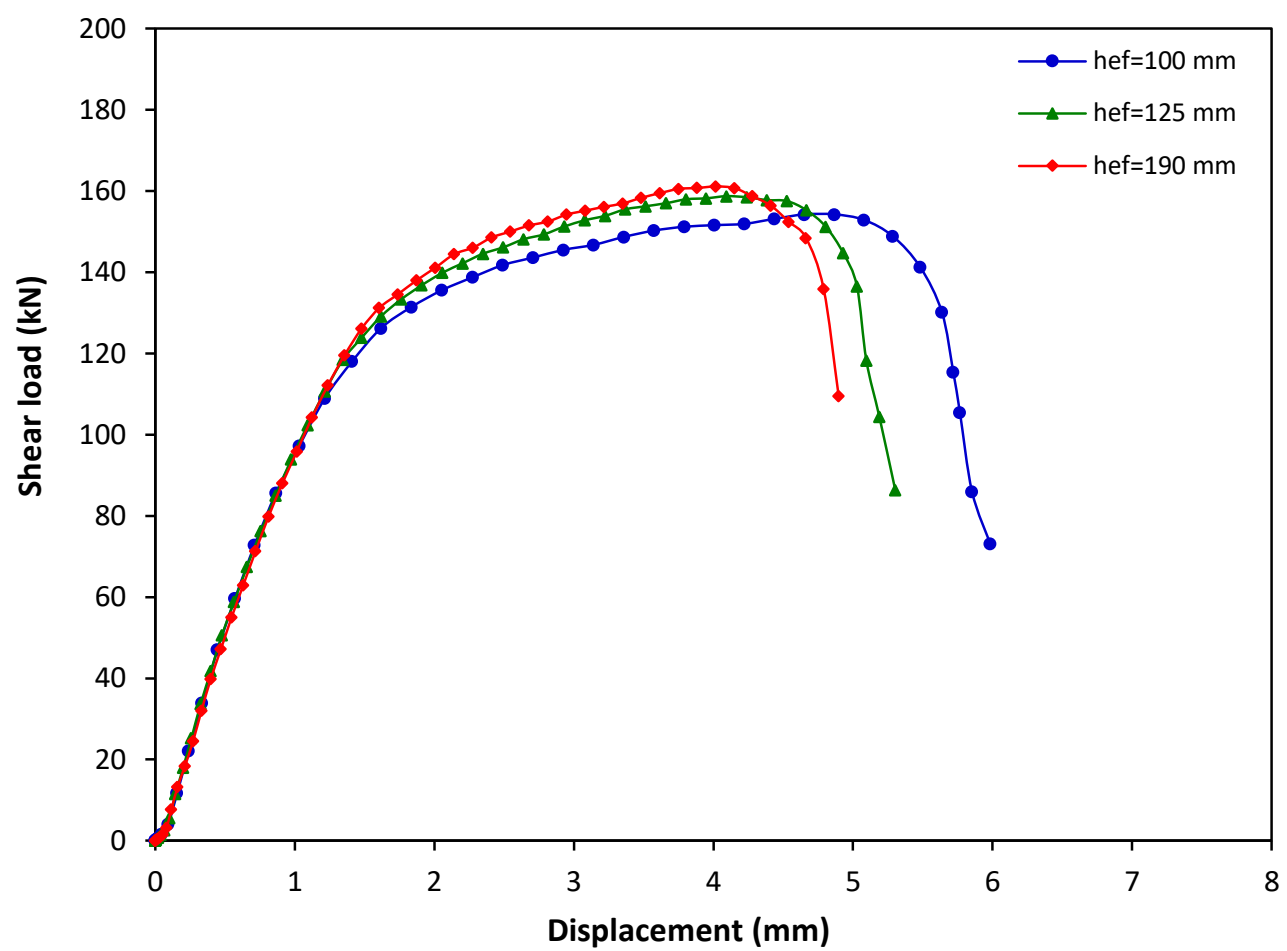

Figure 6-43: Shear load-displacement graph for 12-mm diameter undercut anchor at strain rate of $10^{-3} \mathrm{~s}^{-1}$ 


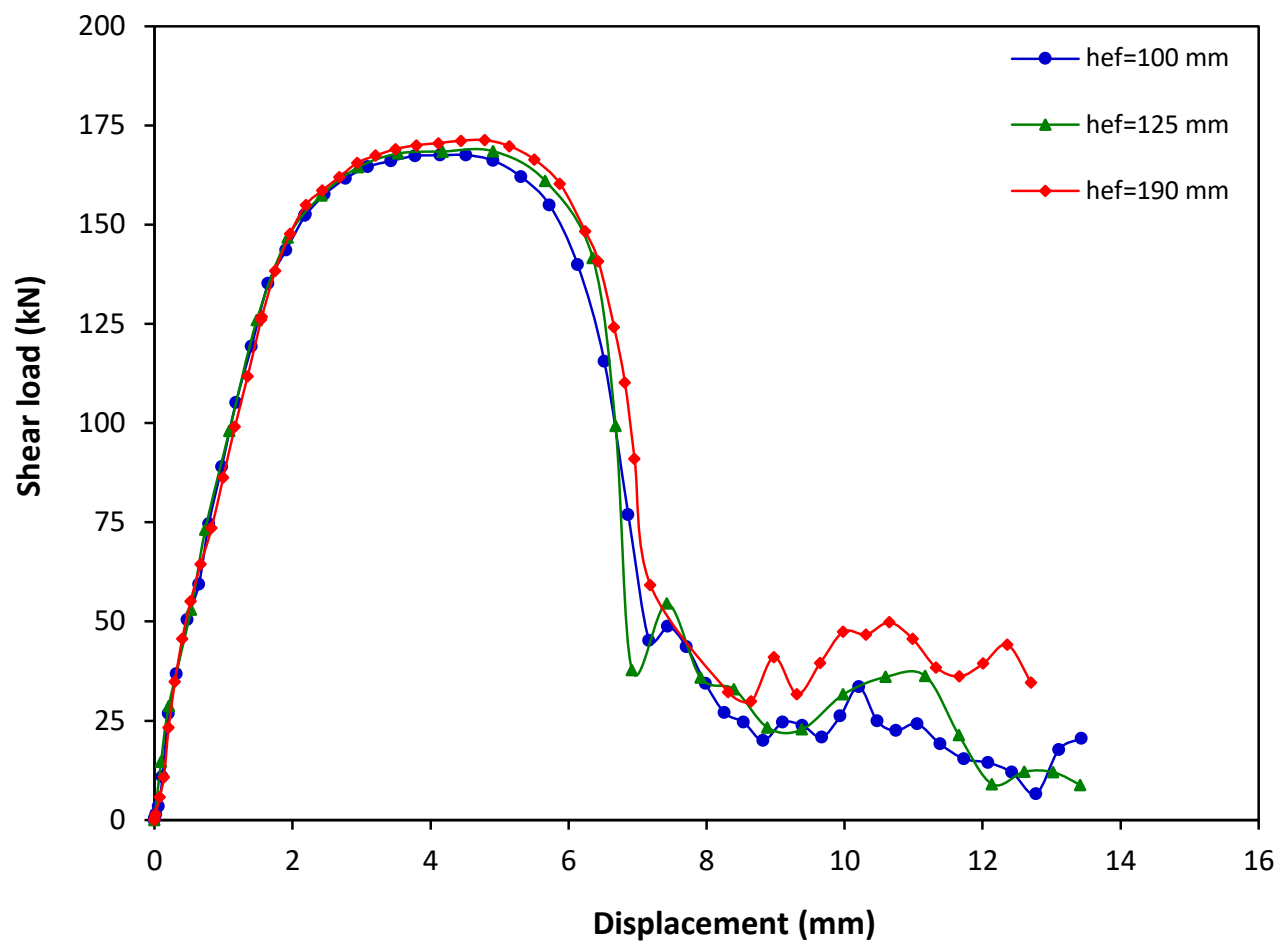

Figure 6-44: Shear load-displacement graph for 12-mm diameter undercut anchor at

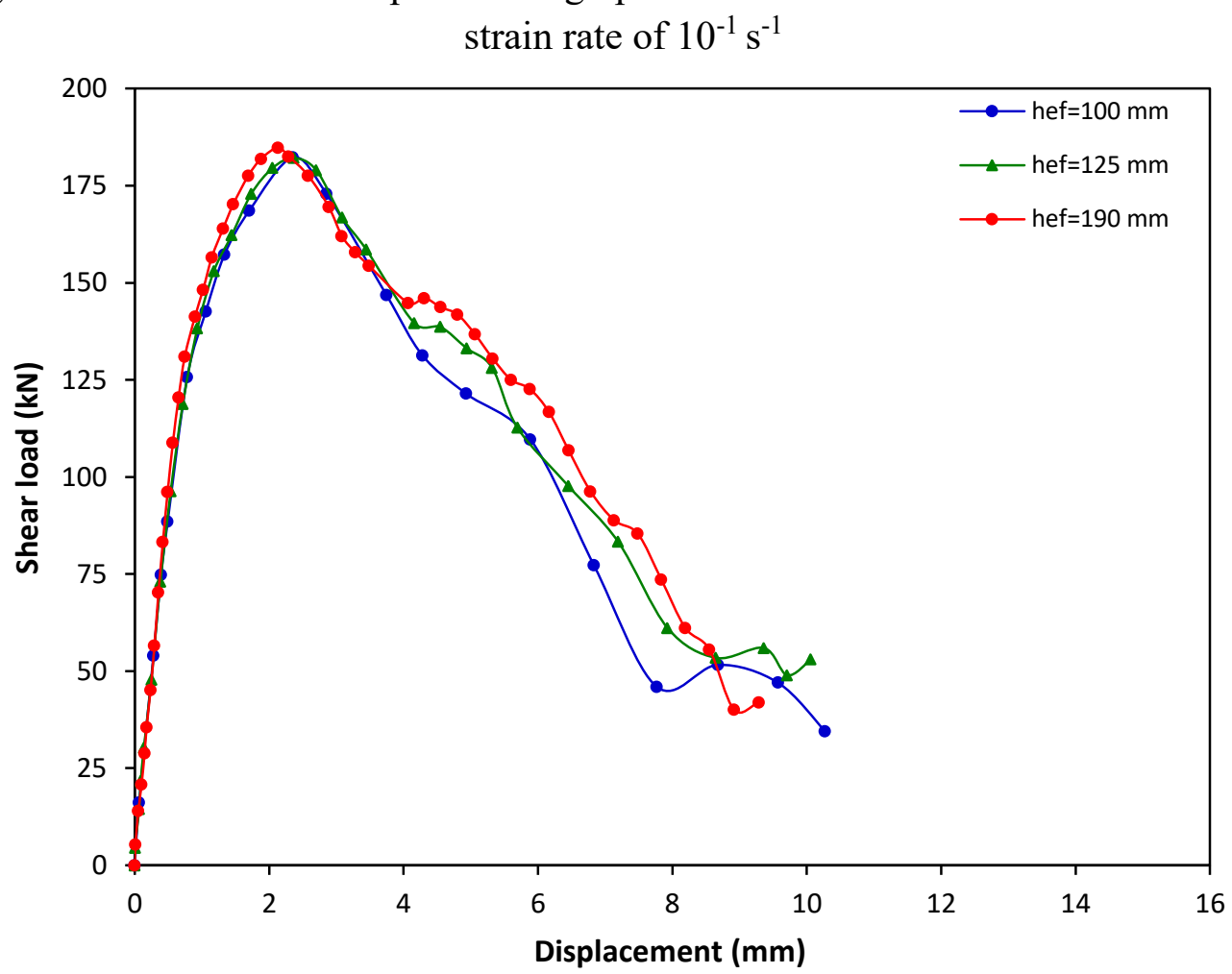

Figure 6-45: Shear load-displacement graph for 12-mm diameter undercut anchor at strain rate of $10 \mathrm{~s}^{-1}$ 


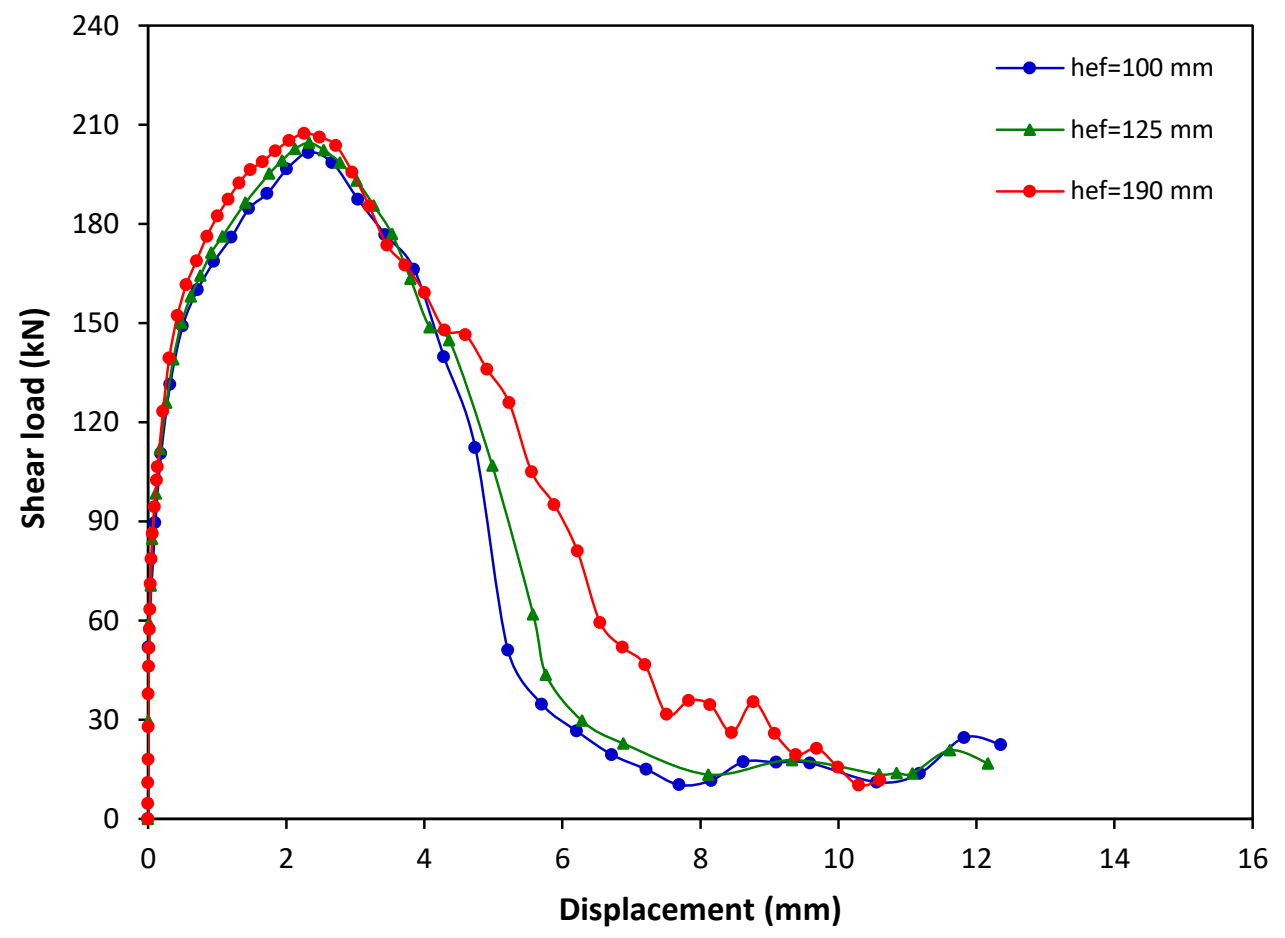

Figure 6-46: Shear load-displacement graph for 12-mm diameter undercut anchor at strain rate of $10^{2} \mathrm{~s}^{-1}$

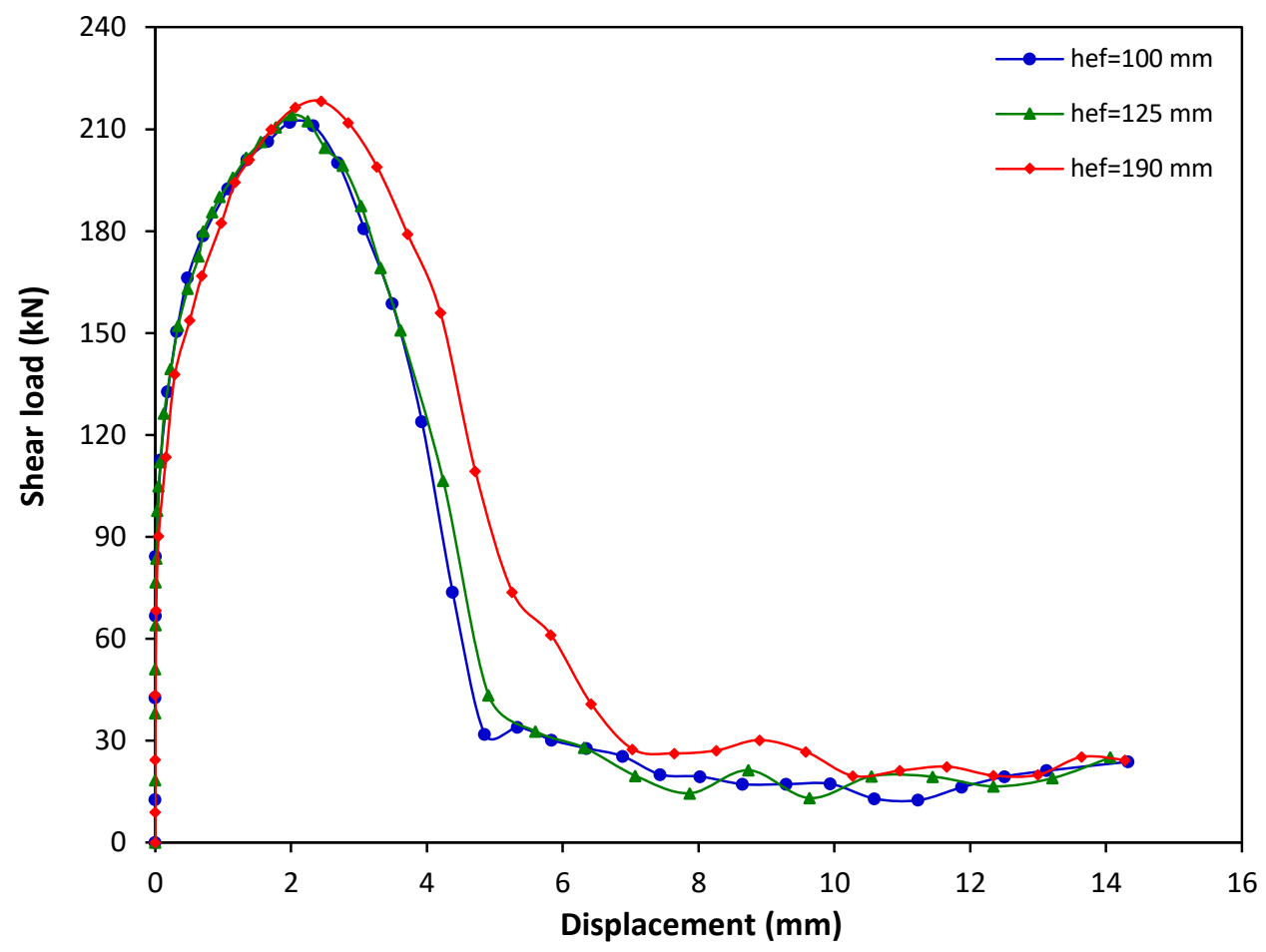

Figure 6-47: Shear load-displacement graph for 12-mm diameter undercut anchor at strain rate of $10^{3} \mathrm{~s}^{-1}$ 
As shown from Figures 6-42 to 6-47, Tables 6-14, 6-15 and 6-16 the shear load for the undercut anchors increased with the increase in the strain rate. At strain rates of $10^{-5} \mathrm{~s}^{-1}$ to $10^{3} \mathrm{~s}^{-1}$, the shear load increased with the displacement until maximum value then decreased with further increase in displacement until failure. At high strain rate, load fluctuations were observed in the post peak behaviour of the undercut anchors. This is attributed to the progressive propagation of the cracks in the concrete at high strain rate. Also, it can be seen that at low strain rate of $10^{-5} \mathrm{~s}^{-1}$ the increase in the embedment depth from $100 \mathrm{~mm}$ to 190 $\mathrm{mm}$ increased the ultimate shear capacity. However, at high strain rates the increase in the embedment depths increased the ultimate shear load slightly where steel anchor failure mode was observed.

Shear load-displacement graphs for 16- $\mathrm{mm}$ and 20-mm diameter undercut anchors with embedment depths of $100 \mathrm{~mm}, 125 \mathrm{~mm}, 190 \mathrm{~mm}$ and $250 \mathrm{~mm}$ at strain rates ranging from $10^{-5} \mathrm{~s}^{-1}$ to $10^{3} \mathrm{~s}^{-1}$ are presented in Appendix $\mathrm{H}$.

The ultimate shear load and displacement results for the $12-\mathrm{mm}, 16-\mathrm{mm}$ and $20-\mathrm{mm}$ diameter undercut anchors are presented in Tables 6-14, 6-15 and 6-16 respectively. Where $\delta$ is the displacement of the undercut anchor at the ultimate shear load. As shown in the tables, the ultimate shear loads increased with the increase in the strain rate from $10^{-5} \mathrm{~s}^{-1}$ to $10^{3} \mathrm{~s}^{-1}$ for all the undercut anchors investigated. Also, it can be seen that the ultimate shear load for the undercut anchors increased with the increase in the anchor diameter. 
Table 6-14: Ultimate shear load and displacement results for the 12-mm diameter undercut anchor

\begin{tabular}{|c|c|c|c|c|c|c|}
\hline $\begin{array}{c}\text { Model } \\
\text { No. }\end{array}$ & $\begin{array}{c}\mathbf{d} \\
(\mathrm{mm})\end{array}$ & $\begin{array}{c}\mathbf{h}_{\text {ef }} \\
(\mathbf{m m})\end{array}$ & $\begin{array}{c}\dot{\dot{\varepsilon}} \\
\left(\mathrm{s}^{-1}\right)\end{array}$ & $\begin{array}{c}V_{u} \\
(k N)\end{array}$ & $\begin{array}{c}\delta \\
(\mathbf{m m})\end{array}$ & $\begin{array}{c}\text { Failure } \\
\text { mode }\end{array}$ \\
\hline 1 & \multirow{3}{*}{12} & 100 & \multirow{3}{*}{$10^{-5}$} & 132.27 & 2.14 & PR \\
\hline 2 & & 125 & & 148.09 & 2.68 & $\mathrm{~S}$ \\
\hline 3 & & 190 & & 153.57 & 3.01 & $\mathrm{~S}$ \\
\hline 4 & \multirow{3}{*}{12} & 100 & \multirow{3}{*}{$10^{-3}$} & 154.21 & 4.65 & S \\
\hline 5 & & 125 & & 158.74 & 4.09 & $\mathrm{~S}$ \\
\hline 6 & & 190 & & 161.19 & 4.02 & $S$ \\
\hline 7 & \multirow{3}{*}{12} & 100 & \multirow{3}{*}{$10^{-1}$} & 166.17 & 4.91 & $\mathrm{~S}$ \\
\hline 8 & & 125 & & 168.49 & 4.91 & $\mathrm{~S}$ \\
\hline 9 & & 190 & & 171.25 & 4.79 & $\mathrm{~S}$ \\
\hline 10 & \multirow{3}{*}{12} & 100 & \multirow{3}{*}{10} & 182.34 & 2.35 & $\mathrm{~S}$ \\
\hline 11 & & 125 & & 182.16 & 2.36 & $\mathrm{~S}$ \\
\hline 12 & & 190 & & 184.79 & 2.13 & $\mathrm{~S}$ \\
\hline 13 & \multirow{3}{*}{12} & 100 & \multirow{3}{*}{$10^{2}$} & 201.66 & 2.32 & $\mathrm{~S}$ \\
\hline 14 & & 125 & & 204.59 & 2.33 & $\mathrm{~S}$ \\
\hline 15 & & 190 & & 207.46 & 2.26 & $\mathrm{~S}$ \\
\hline 16 & \multirow{3}{*}{12} & 100 & \multirow{3}{*}{$10^{3}$} & 211.98 & 1.98 & $\mathrm{~S}$ \\
\hline 17 & & 125 & & 214.21 & 2.01 & $\mathrm{~S}$ \\
\hline 18 & & 190 & & 218.27 & 2.44 & $\mathrm{~S}$ \\
\hline
\end{tabular}

Table 6-15: Ultimate shear load and displacement results for the 16-mm diameter undercut anchor

\begin{tabular}{|c|c|c|c|c|c|c|}
\hline $\begin{array}{c}\text { Model } \\
\text { No. }\end{array}$ & $\underset{(\mathrm{mm})}{\mathrm{d}}$ & $\begin{array}{c}\mathbf{h}_{\mathrm{ef}} \\
(\mathbf{m m})\end{array}$ & $\begin{array}{c}\dot{\varepsilon} \\
\left(\mathbf{s}^{-1}\right)\end{array}$ & $\begin{array}{c}\mathbf{V}_{\mathrm{u}} \\
(\mathrm{kN})\end{array}$ & $\begin{array}{c}\boldsymbol{\delta} \\
(\mathbf{m m})\end{array}$ & $\begin{array}{c}\text { Failure } \\
\text { mode }\end{array}$ \\
\hline 1 & \multirow{4}{*}{16} & 100 & \multirow{4}{*}{$10^{-5}$} & 152.26 & 1.13 & PR \\
\hline 2 & & 125 & & 199.42 & 1.65 & PR \\
\hline 3 & & 190 & & 270.05 & 3.73 & PR \\
\hline 4 & & 250 & & 304.60 & 3.76 & $\mathrm{~S}$ \\
\hline 5 & \multirow{4}{*}{16} & 100 & \multirow{4}{*}{$10^{-3}$} & 197.88 & 2.14 & CS \\
\hline 6 & & 125 & & 243.42 & 2.98 & $\mathrm{CS}$ \\
\hline 7 & & 190 & & 307.24 & 4.44 & $\mathrm{~S}$ \\
\hline 8 & & 250 & & 321.59 & 3.67 & $\mathrm{~S}$ \\
\hline 9 & \multirow{4}{*}{16} & 100 & \multirow{4}{*}{$10^{-1}$} & 244.19 & 3.26 & $\mathrm{CS}$ \\
\hline 10 & & 125 & & 278.38 & 3.94 & $\mathrm{CS}$ \\
\hline 11 & & 190 & & 328.13 & 3.51 & $\mathrm{~S}$ \\
\hline 12 & & 250 & & 336.46 & 3.61 & $\mathrm{~S}$ \\
\hline 13 & \multirow{4}{*}{16} & 100 & \multirow{4}{*}{10} & 322.91 & 2.67 & $S$ \\
\hline 14 & & 125 & & 329.50 & 2.90 & $\mathrm{~S}$ \\
\hline 15 & & 190 & & 344.53 & 2.97 & $\mathrm{~S}$ \\
\hline 16 & & 250 & & 347.23 & 2.71 & $\mathrm{~S}$ \\
\hline 17 & \multirow{4}{*}{16} & 100 & \multirow{4}{*}{$10^{2}$} & 360.02 & 2.55 & $\mathrm{~S}$ \\
\hline 18 & & 125 & & 364.76 & 2.92 & $\mathrm{~S}$ \\
\hline 19 & & 190 & & 366.70 & 2.34 & $\mathrm{~S}$ \\
\hline 20 & & 250 & & 370.66 & 2.28 & $\mathrm{~S}$ \\
\hline
\end{tabular}




\begin{tabular}{|c|c|c|c|c|c|c|}
\hline 21 & \multirow{4}{*}{16} & 100 & \multirow{4}{*}{$10^{3}$} & 374.52 & 1.95 & $\mathrm{~S}$ \\
\hline 22 & & 125 & & 376.11 & 1.84 & $\mathrm{~S}$ \\
\hline 23 & & 190 & & 379.07 & 1.89 & $\mathrm{~S}$ \\
\hline 24 & & 250 & & 380.84 & 2.10 & $\mathrm{~S}$ \\
\hline
\end{tabular}

Table 6-16: Ultimate shear load and displacement results for the 20-mm diameter

\begin{tabular}{|c|c|c|c|c|c|c|}
\hline $\begin{array}{l}\text { Model } \\
\text { No. }\end{array}$ & $\begin{array}{c}\mathbf{d} \\
(\mathbf{m m})\end{array}$ & $\begin{array}{c}\begin{array}{c}\mathbf{h}_{\text {ef }} \\
(\mathbf{m m})\end{array} \\
\end{array}$ & $\begin{array}{c}\dot{\varepsilon} \\
\left(\mathrm{s}^{-1}\right)\end{array}$ & $\begin{array}{c}V_{\mathbf{u}} \\
(\mathbf{k N})\end{array}$ & $\begin{array}{c}\delta \\
(\mathbf{m m})\end{array}$ & $\begin{array}{c}\begin{array}{c}\text { Failure } \\
\text { mode }\end{array} \\
\end{array}$ \\
\hline 1 & \multirow{4}{*}{20} & 100 & \multirow{4}{*}{$10^{-5}$} & 175.37 & 0.86 & PR \\
\hline 2 & & 125 & & 227.49 & 1.15 & PR \\
\hline 3 & & 190 & & 344.63 & 2.22 & PR \\
\hline 4 & & 250 & & 420.84 & 2.93 & $\mathrm{~S}$ \\
\hline 5 & \multirow{4}{*}{20} & 100 & \multirow{4}{*}{$10^{-3}$} & 242.86 & 1.90 & PR \\
\hline 6 & & 125 & & 280.99 & 2.22 & PR \\
\hline 7 & & 190 & & 391.77 & 4.04 & $\mathrm{CS}$ \\
\hline 8 & & 250 & & 439.63 & 3.79 & $\mathrm{~S}$ \\
\hline 9 & \multirow{4}{*}{20} & 100 & \multirow{4}{*}{$10^{-1}$} & 314.72 & 3.47 & $\mathrm{CS}$ \\
\hline 10 & & 125 & & 345.56 & 4.52 & $\mathrm{CS}$ \\
\hline 11 & & 190 & & 446.87 & 6.41 & $\mathrm{~S}$ \\
\hline 12 & & 250 & & 452.59 & 3.89 & $\mathrm{~S}$ \\
\hline 13 & \multirow{4}{*}{20} & 100 & \multirow{4}{*}{10} & 446.46 & 2.41 & S \\
\hline 14 & & 125 & & 453.73 & 2.33 & $\mathrm{~S}$ \\
\hline 15 & & 190 & & 479.50 & 2.19 & $\mathrm{~S}$ \\
\hline 16 & & 250 & & 480.93 & 2.79 & $\mathrm{~S}$ \\
\hline 17 & \multirow{4}{*}{20} & 100 & \multirow{4}{*}{$10^{2}$} & 489.11 & 2.42 & $\mathrm{~S}$ \\
\hline 18 & & 125 & & 506.24 & 2.44 & $\mathrm{~S}$ \\
\hline 19 & & 190 & & 510.41 & 2.81 & $S$ \\
\hline 20 & & 250 & & 511.22 & 2.17 & S \\
\hline 21 & \multirow{4}{*}{20} & 100 & \multirow{4}{*}{$10^{3}$} & 518.84 & 1.18 & $\mathrm{~S}$ \\
\hline 22 & & 125 & & 522.53 & 1.34 & S \\
\hline 23 & & 190 & & 526.32 & 1.59 & $\mathrm{~S}$ \\
\hline 24 & & 250 & & 528.37 & 1.61 & $\mathrm{~S}$ \\
\hline
\end{tabular}

\subsubsection{Effect of strain rate on the ultimate shear load and DIF of undercut anchors}

Figures 6-48, 6-49, 6-50 and 6-51 present the relation between the ultimate shear load, DIF and the strain rate for the $12-\mathrm{mm}, 16-\mathrm{mm}$ and $20-\mathrm{mm}$ diameter undercut anchors at embedment depths of $100 \mathrm{~mm}, 125 \mathrm{~mm}, 190 \mathrm{~mm}$ and $250 \mathrm{~mm}$ respectively. It can be seen that the increase in the strain rate from $10^{-5} \mathrm{~s}^{-1}$ to $10^{3} \mathrm{~s}^{-1}$ increased the ultimate shear load 
for the undercut anchors. In general, the ultimate shear load increased with the increase in the anchor diameter and embedment depth. As shown in Figure 6-48 the ultimate shear load increased for anchor diameters of $16 \mathrm{~mm}$ and $20 \mathrm{~mm}$ with the increase in the strain rate from $10^{-5} \mathrm{~s}^{-1}$ to $10 \mathrm{~s}^{-1}$ where the failure mode changed from pryout to concrete spalling and then to steel failure at strain rate of $10 \mathrm{~s}^{-1}$. A slight increase in the ultimate shear load of $4 \%$ and $6 \%$ is obtained with the increase in the strain rate from $10^{2} \mathrm{~s}^{-1}$ and $10^{3} \mathrm{~s}^{-1}$ for the 16- $\mathrm{mm}$ and 20-mm diameter undercut anchors respectively. The increase in the ultimate load is attributed to the increase in steel strength with the increase in strain rate. For the anchor diameter of $12 \mathrm{~mm}$ the ultimate shear load increased with the increase in the strain rate from $10^{-5} \mathrm{~s}^{-1}$ to $10^{3} \mathrm{~s}^{-1}$ where transition in the failure mode from pryout failure at strain rate of $10^{-5} \mathrm{~s}^{-1}$ to steel anchor failure is observed.

At embedment depth of $125 \mathrm{~mm}$ (Figure 6-49), a similar behaviour of the ultimate shear load with the strain rate was observed for the anchor diameters of $16 \mathrm{~mm}$ and $20 \mathrm{~mm}$. The ultimate shear load for the anchor diameter of $12 \mathrm{~mm}$ and embedment depth of $125 \mathrm{~mm}$ increased with the increase in the strain rate from $10^{-5} \mathrm{~s}^{-1}$ to $10^{3} \mathrm{~s}^{-1}$, where steel failure is the dominant failure mode at all the strain rates investigated.

As shown in Figure 6-50, for embedment depth of $190 \mathrm{~mm}$, the ultimate shear load increased with the increase in the strain rate from $10^{-5} \mathrm{~s}^{-1}$ to $10^{3} \mathrm{~s}^{-1}$ for anchor diameters of $12 \mathrm{~mm}, 16 \mathrm{~mm}$ and $20 \mathrm{~mm}$ respectively. At embedment depth of $250 \mathrm{~mm}$ (Figure 6-51) the ultimate shear load increased $25 \%$ and $25.6 \%$ for the $16-\mathrm{mm}$ and $20-\mathrm{mm}$ diameters undercut anchor with the increase in the strain rate from $10^{-5} \mathrm{~s}^{-1}$ to $10^{3} \mathrm{~s}^{-1}$ where the steel fracture is the dominant failure mode. The increase in the ultimate shear load is attributed 
to the fact that steel material is sensitive to the increase in the strain rate that increases the anchor capacity by increasing the yield and tensile strengths of the steel material.

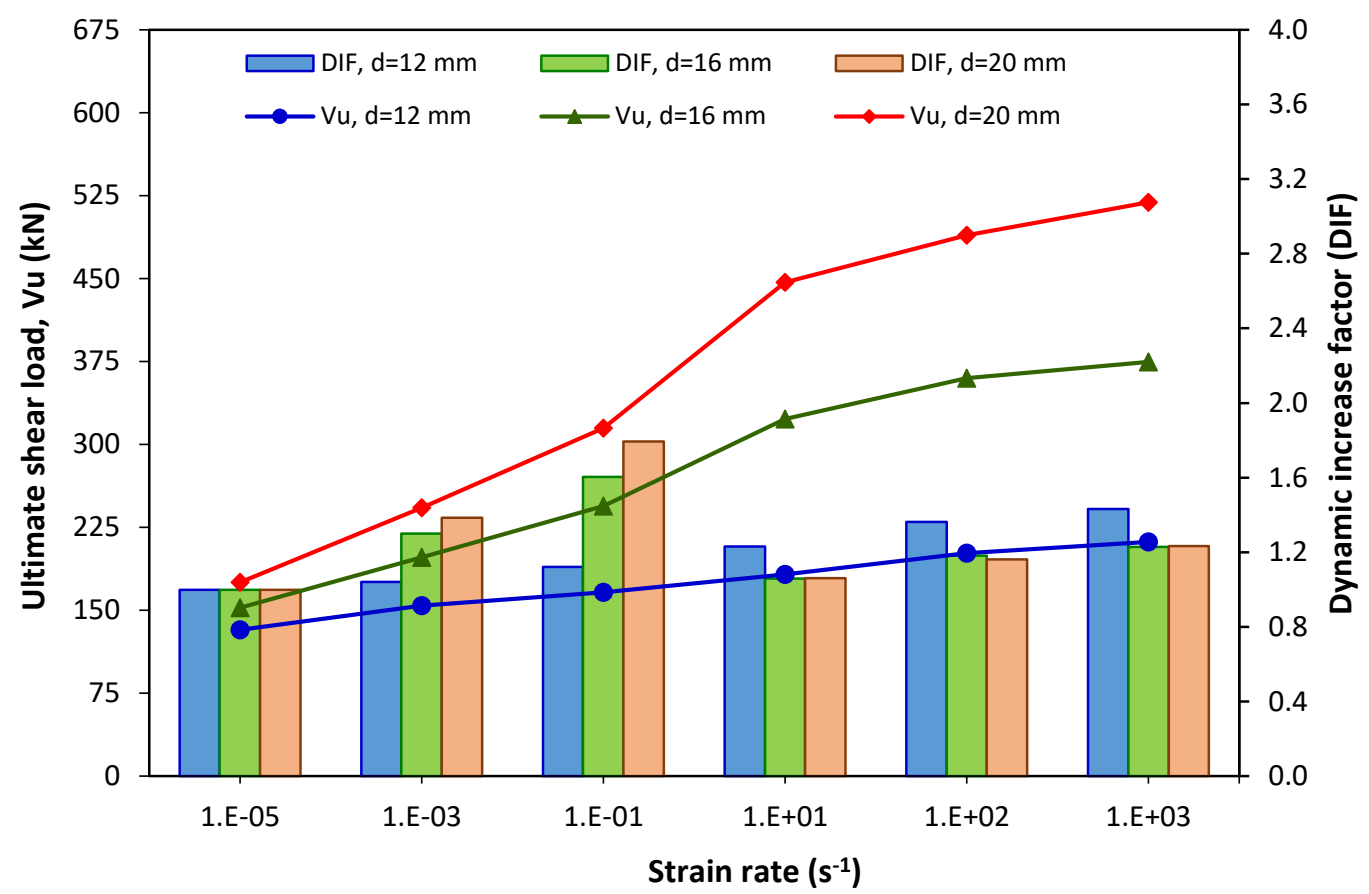

Figure 6-48: Ultimate shear load and DIF versus strain rate for undercut anchors at 100 mm embedment depth

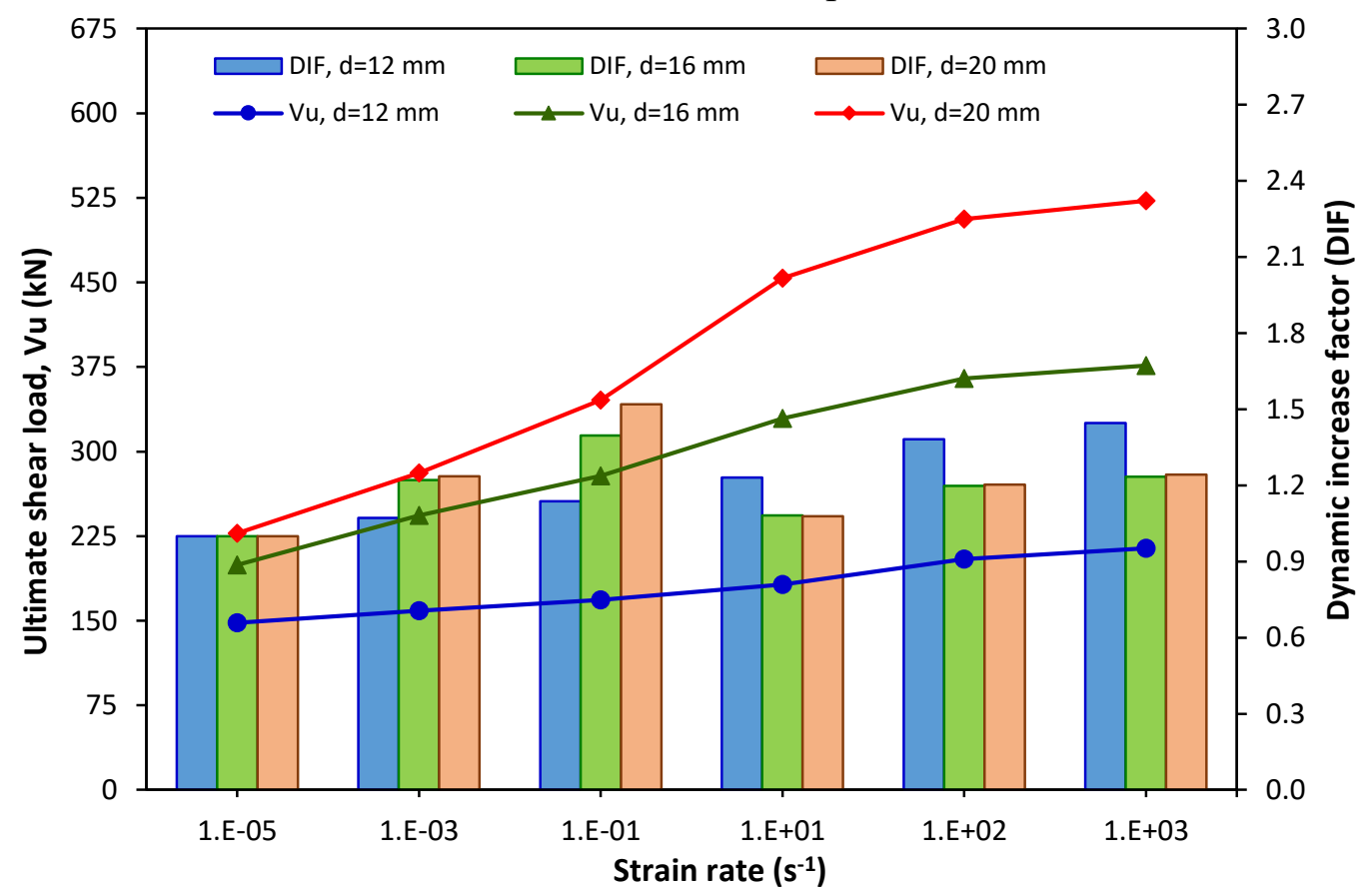

Figure 6-49: Ultimate shear load and DIF versus strain rate for undercut anchors at 125 mm embedment depth 


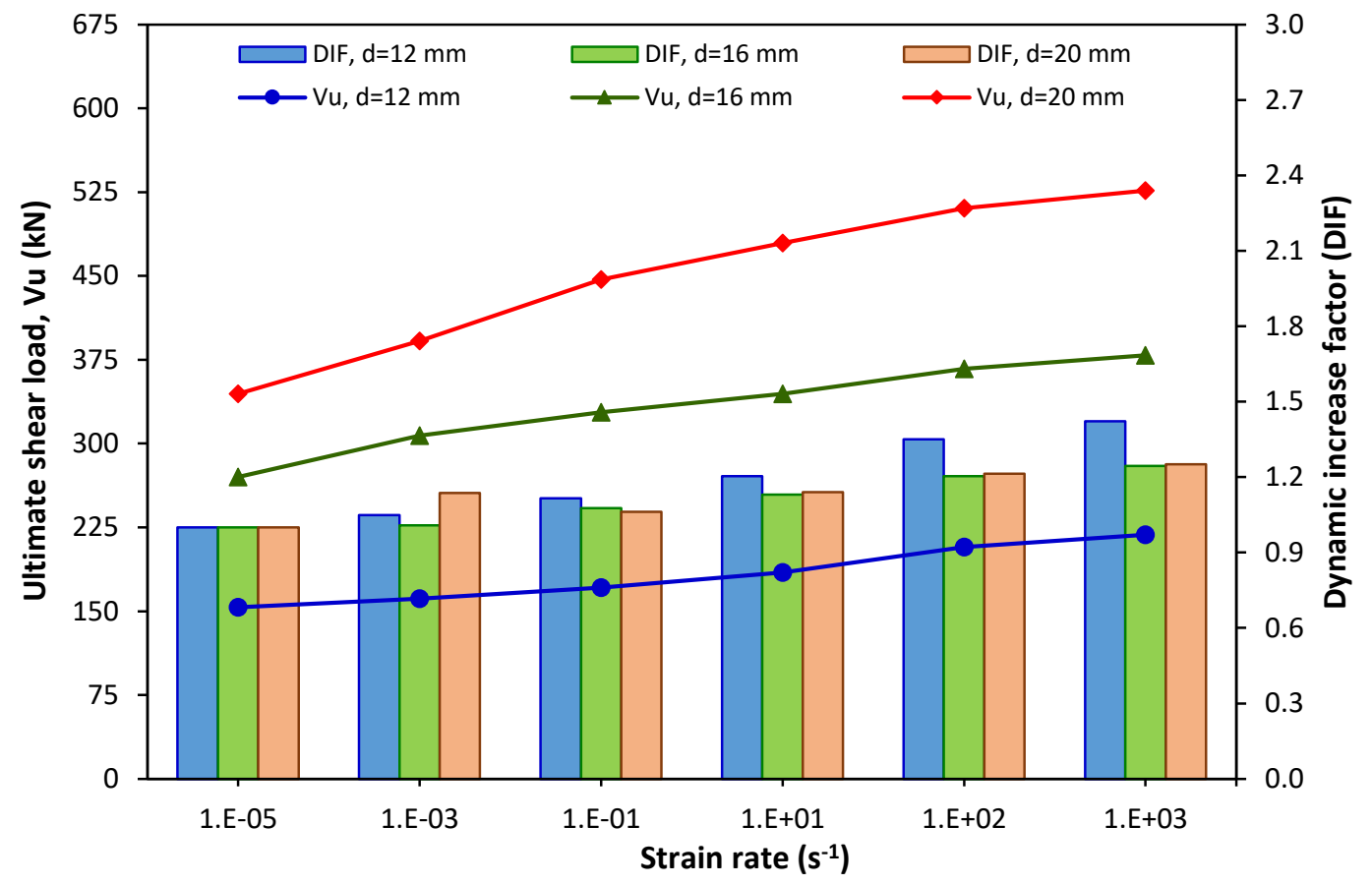

Figure 6-50: Ultimate shear load and DIF versus strain rate for undercut anchors at 190 mm embedment depth

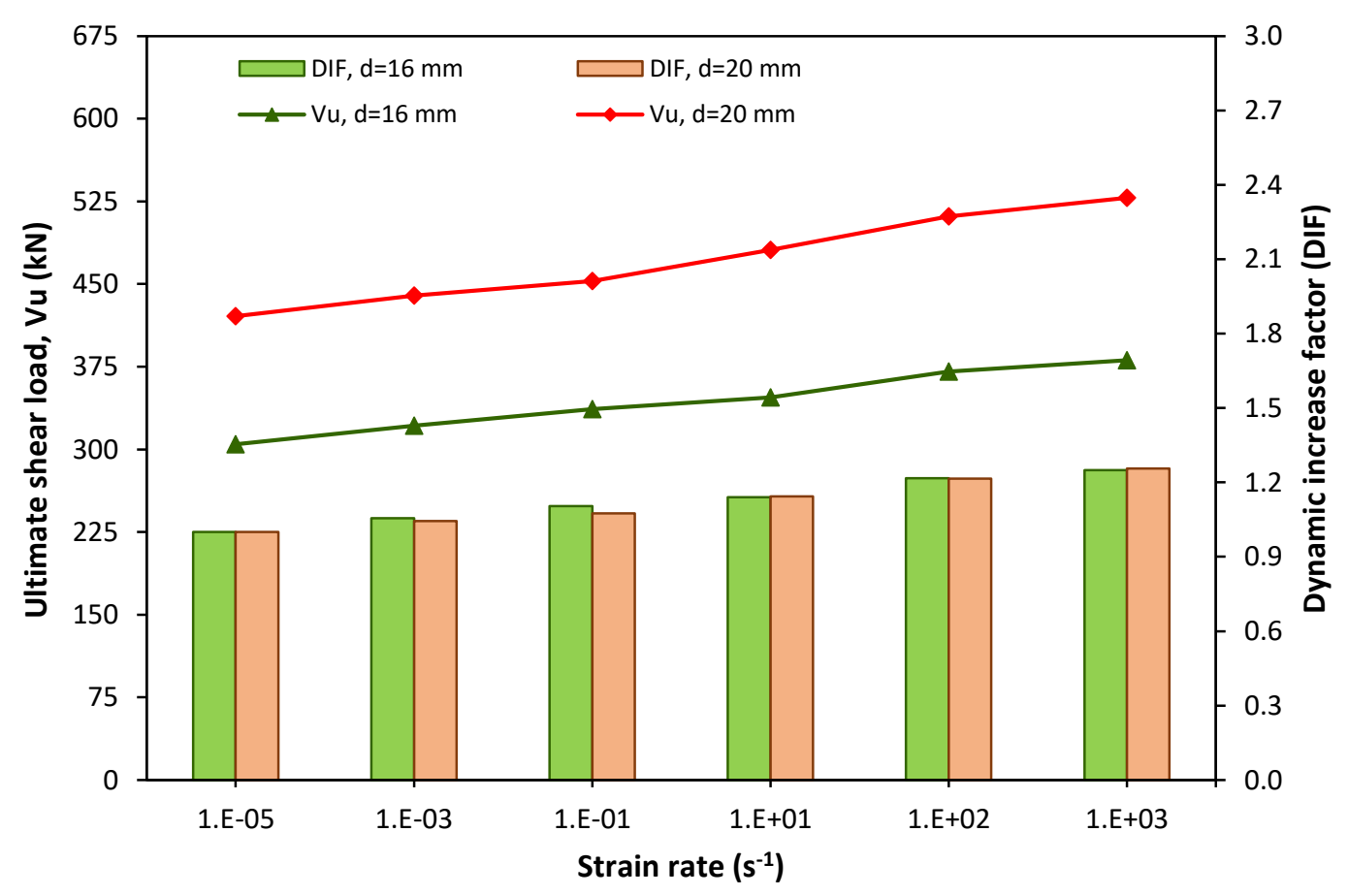

Figure 6-51: Ultimate shear load and DIF versus strain rate for undercut anchors at 250 mm embedment depth 
It can be seen from Figures 6-48 and 6-49 that the DIF increased with the increase in the strain rate from $10^{-3} \mathrm{~s}^{-1}$ to $10^{3} \mathrm{~s}^{-1}$ for anchor diameter of $12 \mathrm{~mm}$ where steel failure was observed. For anchor diameters of $16 \mathrm{~mm}$ and $20 \mathrm{~mm}$, DIF increased with the increase in the strain rate up to $10^{-1} \mathrm{~s}^{-1}$ where transition in the failure mode from pryout to concrete spalling was observed. Steel failure was observed at strain rates higher than $10^{-1} \mathrm{~s}^{-1}$. The increase in the strain rate from $10 \mathrm{~s}^{-1}$ to $10^{3} \mathrm{~s}^{-1}$ increased the DIF from 1.06 to 1.23 for the anchor diameters of $16 \mathrm{~mm}$ and $20 \mathrm{~mm}$ respectively with embedment depth of $100 \mathrm{~mm}$. The increase in the DIF was from 1.08 to 1.23 and from 1.08 to 1.24 for anchor diameters of $16 \mathrm{~mm}$ and $20 \mathrm{~mm}$ respectively with embedment depth of $125 \mathrm{~mm}$.

As shown in Figure 6-50, the DIF increased with the increase in the strain rate from $10^{-5} \mathrm{~s}^{-}$ ${ }^{1}$ to $10^{3} \mathrm{~s}^{-1}$ for the $12 \mathrm{~mm}$ and $16 \mathrm{~mm}$ diameter anchors. For anchor diameter of $20 \mathrm{~mm}$ the DIF increased to 1.14 at strain rate of $10^{-3} \mathrm{~s}^{-1}$ where transition from pryout failure to concrete spalling was observed. The increase in the strain rate from $10^{-1} \mathrm{~s}^{-1}$ to $10^{3} \mathrm{~s}^{-1}$ increased the DIF from 1.06 to 1.25 where steel failure was observed. As shown in Figure 6-51, average DIF of 1.25 was obtained for the undercut anchors with embedment depth of $250 \mathrm{~mm}$ at high strain rate of $10^{3} \mathrm{~s}^{-1}$.

It can be seen from Figures 6-48, 6-49, 6-50 and 6-51, maximum DIF of 1.6 and 1.79 were obtained at strain rate of $10^{-1} \mathrm{~s}^{-1}$ for the $16-\mathrm{mm}$ and $20-\mathrm{mm}$ diameter undercut anchors with $100 \mathrm{~mm}$ embedment depth where concrete spalling was observed. Maximum DIF of 1.45 was obtained for the $12 \mathrm{~mm}$ diameter anchor with embedment depth of $125 \mathrm{~mm}$ at high strain rate of $10^{3} \mathrm{~s}^{-1}$ where steel failure was observed. It can be seen from the figures that the influence of anchor diameter on the DIF decreased with the increase in the embedment 
depth from $100 \mathrm{~mm}$ to $250 \mathrm{~mm}$. At the deeper embedment depth of $250 \mathrm{~mm}$ the influence of anchor diameter is insignificant where steel anchor failure is observed for all the strain rates.

Table 6-17 shows the maximum DIF for the undercut anchors under shear load where pryout, concrete spalling and steel anchor failure modes were observed.

Table 6-17: Maximum dynamic increase factor for the undercut anchors under shear load

\begin{tabular}{|c|c|c|c|c|c|c|c|c|}
\hline \multirow{2}{*}{$\begin{array}{c}\text { Model } \\
\text { No. }\end{array}$} & \multirow{2}{*}{$\begin{array}{c}\mathrm{d} \\
(\mathrm{mm})\end{array}$} & \multirow{2}{*}{$\begin{array}{c}\mathbf{h}_{\text {eff }} \\
(\mathbf{m m})\end{array}$} & \multirow{2}{*}{$\begin{array}{c}\dot{\dot{\varepsilon}} \\
\left(\mathbf{s}^{-1}\right)\end{array}$} & \multirow{2}{*}{$\begin{array}{l}\text { DIF } \\
\text { PR }\end{array}$} & \multirow{2}{*}{$\begin{array}{c}\dot{\varepsilon} \\
\left(\mathbf{s}^{-1}\right)\end{array}$} & \multirow{2}{*}{$\begin{array}{l}\text { DIF } \\
\text { CS }\end{array}$} & \multirow{2}{*}{$\begin{array}{c}\dot{\varepsilon} \\
\left(\mathrm{s}^{-1}\right)\end{array}$} & \multirow{2}{*}{$\frac{\text { DIF }}{S}$} \\
\hline & & & & & & & & \\
\hline 1 & 12 & 100 & $10^{-5}$ & 1.00 & - & - & $10^{3}$ & 1.43 \\
\hline 2 & 12 & 125 & - & - & - & - & $10^{3}$ & 1.45 \\
\hline 3 & 12 & 190 & - & - & - & - & $10^{3}$ & 1.42 \\
\hline 5 & 16 & 100 & $10^{-5}$ & 1.00 & $10^{-1}$ & 1.60 & $10^{3}$ & 1.23 \\
\hline 6 & 16 & 125 & $10^{-5}$ & 1.00 & $10^{-1}$ & 1.40 & $10^{3}$ & 1.23 \\
\hline 7 & 16 & 190 & $10^{-5}$ & 1.00 & - & - & $10^{3}$ & 1.24 \\
\hline 8 & 16 & 250 & - & - & - & - & $10^{3}$ & 1.25 \\
\hline 9 & 20 & 100 & $10^{-3}$ & 1.38 & $10^{-1}$ & 1.79 & $10^{3}$ & 1.23 \\
\hline 10 & 20 & 125 & $10^{-3}$ & 1.24 & $10^{-1}$ & 1.52 & $10^{3}$ & 1.24 \\
\hline 11 & 20 & 190 & $10^{-5}$ & 1.00 & $10^{-3}$ & 1.14 & $10^{3}$ & 1.25 \\
\hline 12 & 20 & 250 & - & - & - & - & $10^{3}$ & 1.26 \\
\hline
\end{tabular}

\subsubsection{Regression analysis for undercut anchors under shear load}

Regression analysis was performed for the undercut anchors subjected to shear loading to predict the relation between the DIF and the strain rate. Steel anchor failure is observed as the dominant failure mode for most of the undercut anchors investigated. Average DIF for the anchor diameters of $12 \mathrm{~mm}, 16 \mathrm{~mm}$ and $20 \mathrm{~mm}$ were considered in the regression analysis for the undercut anchors to adjust DIF for the effect of anchor diameter. The relation between the DIF and the strain rate ratio $\left(\dot{\varepsilon}_{d} / \dot{\varepsilon}_{s}\right)$ is shown in Figure 6-52 for the undercut anchors exhibiting steel failure mode. 


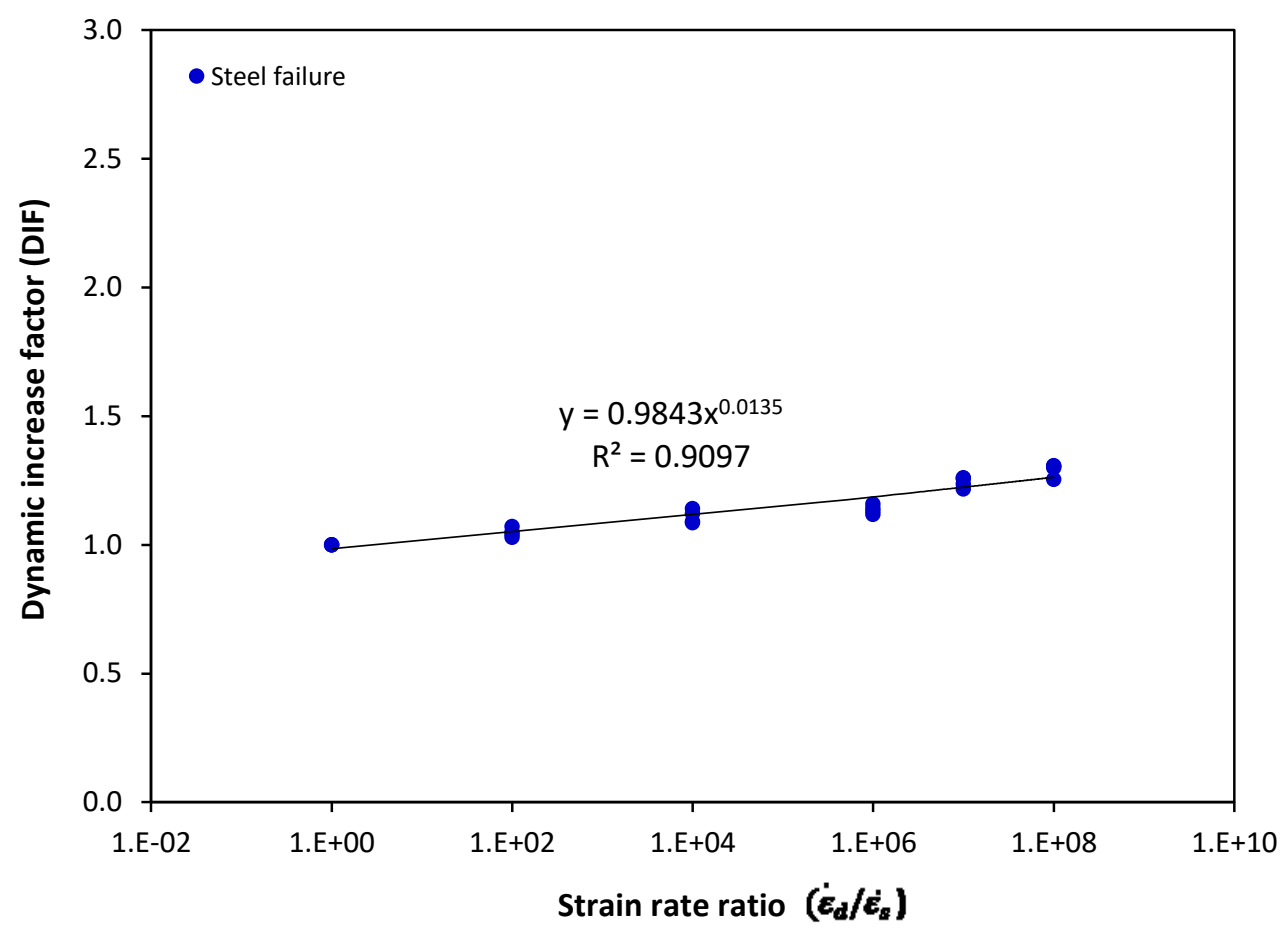

Figure 6-52: Effect of strain rate ratio on the DIF for undercut anchors exhibited steel failure under shear load

Various regression models were used to develop a formula that relates the DIF with the strain rate. Table 6-18 shows the statistical models used to predict the relation between the DIF with the strain rate for the undercut anchors subjected to shear load.

Table 6-18: Statistical models and coefficient of determination to predict the DIF for the undercut anchors exhibited steel failure under shear load

\begin{tabular}{|c|c|c|}
\hline Statistical models & Formulae & Coefficient of determination $\left(\mathrm{R}^{2}\right)$ \\
\hline Exponential & $D I F=1.1028 e^{2 E-9\left(\frac{\dot{\varepsilon}_{d}}{\dot{\varepsilon}_{s}}\right)}$ & 0.499 \\
\hline Linear & $D I F=2 E-9\left(\frac{\dot{\varepsilon}_{d}}{\dot{\varepsilon}_{s}}\right)+1.1055$ & 0.525 \\
\hline Logarithmic & $D I F=0.0154 \ln \left(\frac{\dot{\varepsilon}_{d}}{\dot{\varepsilon}_{s}}\right)+0.9774$ & 0.894 \\
\hline Power & $D I F=0.9843\left(\frac{\dot{\varepsilon}_{d}}{\dot{\varepsilon}_{s}}\right)^{0.0135}$ & 0.910 \\
\hline
\end{tabular}


As shown in the table, the power regression model gave the highest coefficient of determination of 0.91 and was used for prediction of DIF of undercut anchors exhibited steel failure as in Equation (6.3).

$D I F=0.9843\left(\frac{\dot{\varepsilon}_{d}}{\dot{\varepsilon}_{s}}\right)^{0.0135}$

The ultimate dynamic load $\left(V_{u d}\right)$ for the undercut anchors subjected to shear load exhibited steel anchor failure can be determined as in Equations (6.4) as follows:

$V_{u d}=V_{u s} \times 0.9843\left(\frac{\dot{\varepsilon}_{d}}{\dot{\varepsilon}_{s}}\right)^{0.0135}$

Where the ultimate static load $\left(V_{u s}\right)$ can be determined from Equation (4.18) for steel anchor failure mode.

Residual analysis is performed for the undercut anchors subjected to shear load to determine the difference between the DIF obtained from the finite element analysis and the predictive equation (Equation 6.3). The relation between the residual and the strain rate ratio is shown in Figure 6-53. As shown in the figure, the residual exhibits a horizontal trend line with the strain rate ratio. However, a slight divergence of the residual is observed. 


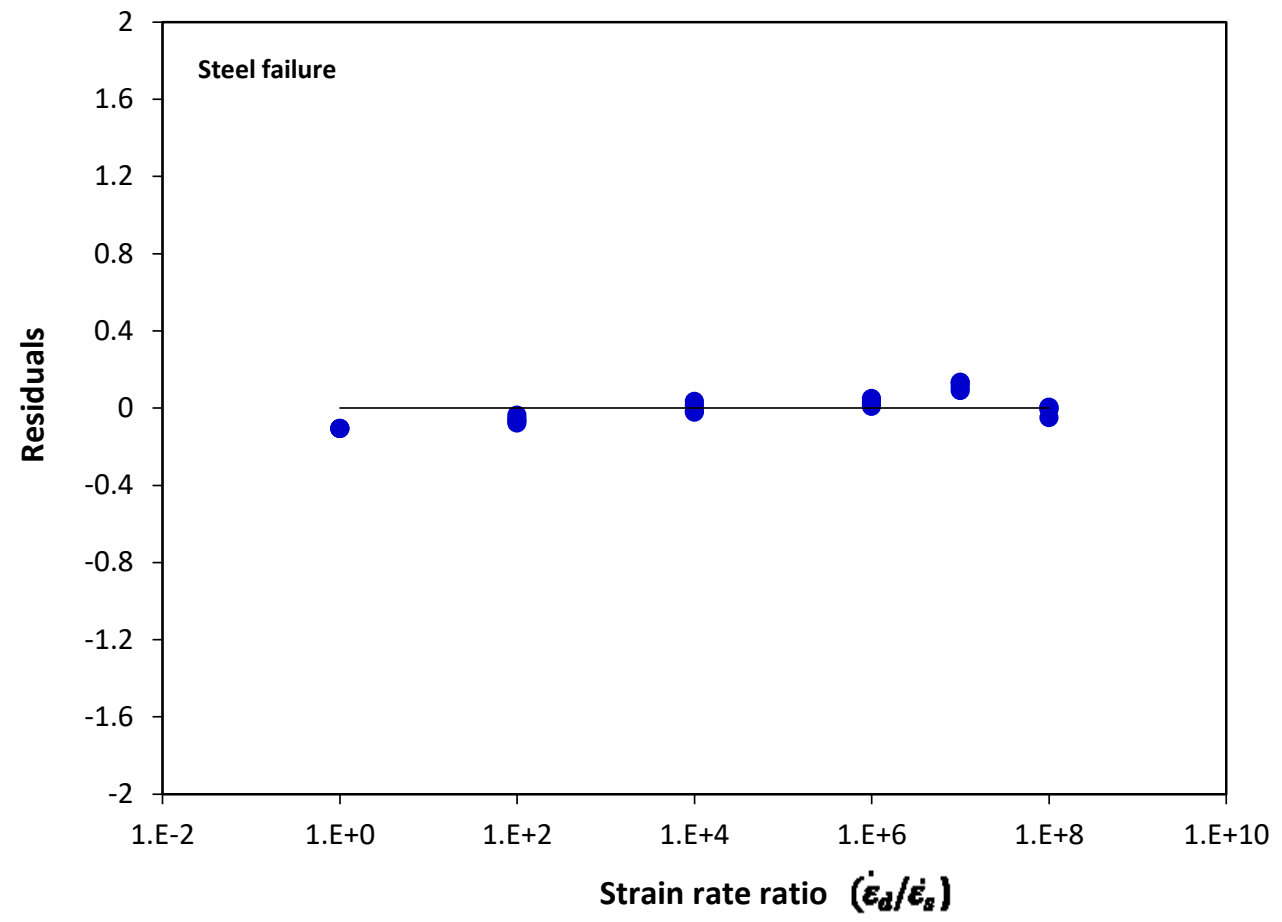

Figure 6-53: Residual versus strain rate ratio for the undercut anchors under shear load exhibited steel failure

New undercut anchorage to concrete models subjected to shear load were developed to verify the accuracy of the proposed equation (Equation 6.3). Undercut anchor diameters of $12 \mathrm{~mm}, 16 \mathrm{~mm}$ and $20 \mathrm{~mm}$ with embedment depths of $160 \mathrm{~mm}$ and $220 \mathrm{~mm}$ were investigated. The DIF results obtained from Equation (6.3) were compared with the DIF results of the newly developed numerical models. Figure 6-54 shows the relation between the DIF obtained from the finite element analysis and the DIF results obtained from the predictive equation. Normal distribution of the DIF values around the equality line is observed. This indicates that the finite element results well represented using the power model proposed in Equation (6.3). 


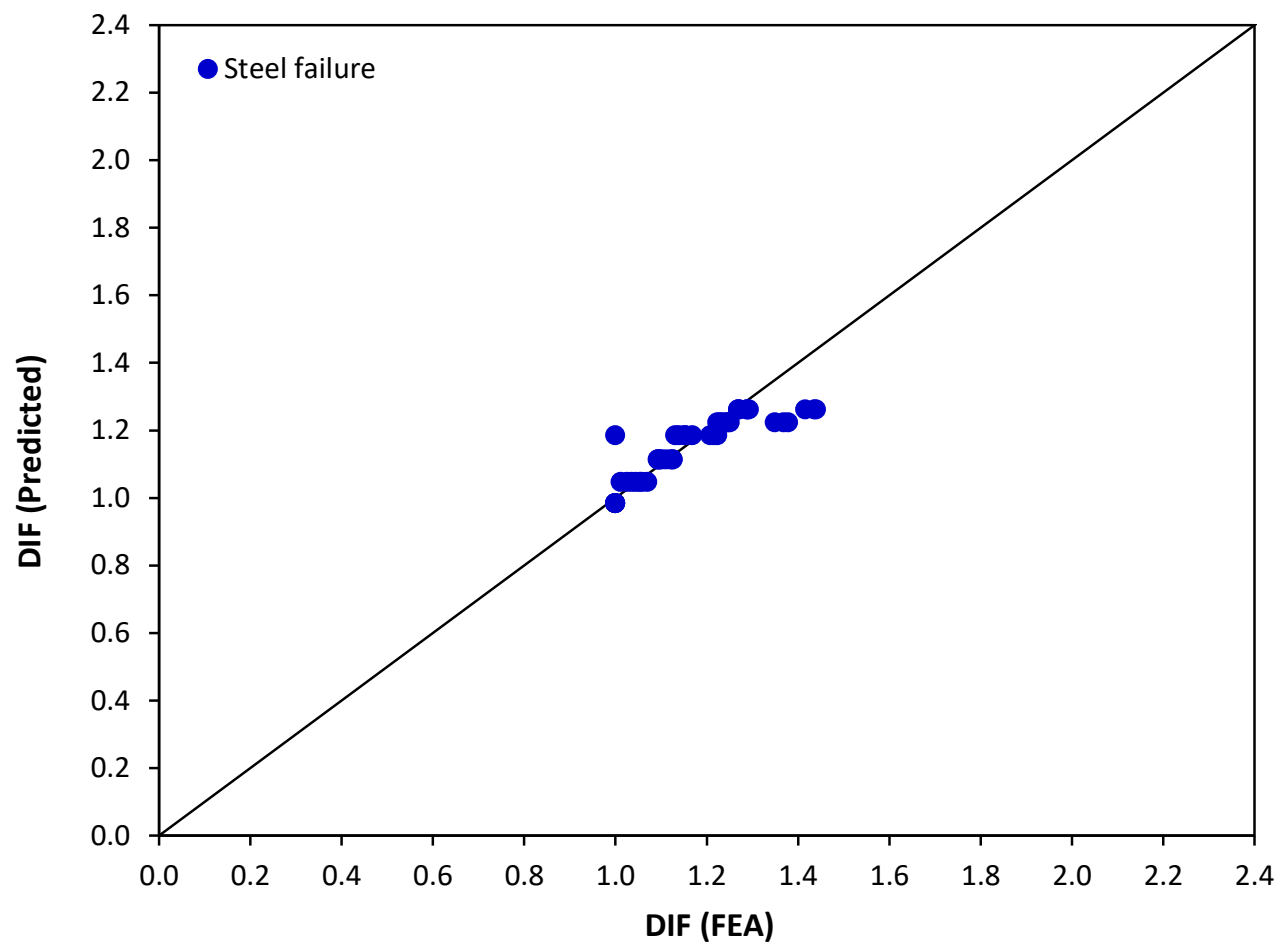

Figure 6-54: DIF obtained from the finite element analysis versus the predicted DIF for the undercut anchors exhibited steel failure under shear load

\subsection{Summary}

This chapter addressed the tensile and shear behaviour for the undercut anchorage to concrete systems subjected to strain rates of $10^{-5} \mathrm{~s}^{-1}, 10^{-3} \mathrm{~s}^{-1}, 10^{-1} \mathrm{~s}^{-1}, 10 \mathrm{~s}^{-1}, 10^{2} \mathrm{~s}^{-1}$ and $10^{3}$ $\mathrm{s}^{-1}$. The chapter begins with validation of the undercut anchor model with the experimental results in the literature. To improve the accuracy of the analysis, mesh sensitivity analyses were performed to determine the adequate mesh size that can best represent the experimental results in the literature. The results obtained from the finite element analysis were compared with the ACI and CCD prediction analytical methods. Level of damage and failure mode for the undercut anchorage to concrete systems for different design parameters and at different strain rates were presented and discussed. For the undercut 
anchors subjected to tensile load, two failure modes were observed: concrete cone breakout and steel anchor failure. On the other hand, for the undercut anchors subjected to shear load, three types of failure modes were observed: pryout, concrete spalling and steel anchor failure.

It is observed that the strain rate has influence on the failure mode of the undercut anchors, where the failure mode transitioned from concrete cone breakout failure or pryout failure to steel anchor failure with the increase in the strain rate from $10^{-5} \mathrm{~s}^{-1}$ to $10^{3} \mathrm{~s}^{-1}$. Concrete cone depth, concrete cone breakout diameter and cone propagation angle for the undercut anchors subjected to tensile load were determined. Ultimate load-displacement, failure mode and strain rate relations for the undercut anchorage to concrete systems subjected to tensile and shear loads were analyzed. Predicting the failure mode and the ultimate load for the undercut anchorage to concrete systems can improve the level of safety for the structures that contain undercut anchors.

DIFs for the undercut anchorage to concrete systems subjected to tensile and shear loads were determined at different strain rates. Equations were developed to determine the relation between the DIF and the strain rate for the undercut anchorage to concrete systems subjected to tensile and shear loads. 


\section{Chapter 7 :Conclusions and recommendations for future research}

\subsection{Introduction}

In this research, numerical models for cast-in-place, adhesive and undercut anchorage to concrete systems were developed to investigate the tensile and shear behaviour of the anchorage systems subjected to strain rates in the range from $10^{-5} \mathrm{~s}^{-1}$ to $10^{3} \mathrm{~s}^{-1}$ using LSDYNA- a multi-physics based finite element analysis program. Various design parameters (anchor diameter and embedment depths) of the anchorage systems were investigated. A procedure was considered for developing the numerical model for the anchorage to concrete systems, to best represent the tensile and shear behaviour of the anchorage to concrete systems, started with selecting the design parameters for the anchors and concrete block, contact formulation and boundary conditions. The commonly used material constitutive models in LS-DYNA for concrete, steel and adhesive materials were evaluated to select the most suitable material models that can best represent the behaviour of the anchorage to concrete systems under different strain rates. The selected material models have the required features to represent the strain rate effect, damage and failure criteria of the material. The numerical model results were validated with the experimental results and with the design prediction methods. The contributions of this thesis is in providing a comprehensive and validated database on the behaviour of anchorage to concrete systems subjected to different strain rates, that can assist designers to predict the performance of anchorage systems under different strain rate loading conditions and accordingly put the suitable measures to mitigate or prevent the failure. 


\subsection{General conclusions}

The main conclusions obtained from the finite element analyses on the cast-in-place, adhesive and undercut anchors can be summarized as follows:

- The developed cast-in-place, adhesive and undercut anchorage to concrete system models are capable to predict the tensile and shear behaviour, failure load and failure mode of the anchorage systems subjected to different strain rates.

- Increasing the strain rate from $10^{-5} \mathrm{~s}^{-1}$ to $10^{3} \mathrm{~s}^{-1}$ increases the ultimate tensile and shear capacity of cast-in-place, adhesive and undercut anchors. Irrespective of the anchor diameter or embedment depth.

- The ultimate tensile load increased with the increase in the embedment depth at the same strain rate when concrete cone breakout failure is dominant.

- The ultimate shear load capacity of anchors depends on embedment depth when the pryout failure mode is the dominant failure mode. The longer the embedment depth the higher the ultimate shear load.

- The ultimate shear load capacity of the anchors depends on the anchor diameter; the larger the diameter the higher the ultimate shear load. This is also observed for the tensile capacity of the anchors.

- The DIF for the cast-in-place, adhesive and undercut anchors under tensile and shear loads increases with the increase in the strain rate from $10^{-5} \mathrm{~s}^{-1}$ to $10^{3} \mathrm{~s}^{-1}$.

- Crack propagation is influenced by the anchor diameter and embedment depth. The increase in the anchor diameter from $12.7 \mathrm{~mm}$ to $19.1 \mathrm{~mm}$ increased the concrete cracking and level of damage sustained by the concrete substrate. 
- Crack propagation angle decreased with the increase in the strain rate from $10^{-5} \mathrm{~s}^{-1}$ to $10^{3} \mathrm{~s}^{-1}$ for the cast-in-place, adhesive and undercut anchors subjected to tensile load.

- The failure mode of the cast-in-place, adhesive and undercut anchors subjected to tensile and shear loads is influenced by the strain rate.

- The concrete cone breakout diameter increased with the increase in the anchor diameter. However, the concrete breakout diameter decreased with the increase in the strain rate.

- Regression analysis was performed to develop an accurate predictive formula to relate the DIF and the strain rate of the cast-in-place, adhesive and undercut anchors at different strain rates and proposed for use in design of anchors under high strain rate loading.

\subsection{Behaviour of cast-in-place anchorage system under different strain rates}

- For cast-in-place anchors subjected to tensile load, maximum DIF of 1.74 was obtained for the anchors exhibited concrete cone breakout failure at strain rate of $10 \mathrm{~s}^{-1}$. Maximum DIF of 1.17 was obtained for the cast-in-place anchors exhibited steel failure at high strain rate of $10^{3} \mathrm{~s}^{-1}$.

- For cast-in-place anchors subjected to shear load, maximum DIF of 1.24 was obtained for the anchors exhibited pryout failure at strain rate of $10^{-1} \mathrm{~s}^{-1}$. Maximum DIF of 1.15 was obtained for the anchors exhibited steel failure at high strain rate of $10^{3} \mathrm{~s}^{-1}$ 
- Average value of the crack propagation angle decreased from $58^{\circ}$ at strain rate of $10^{-5} \mathrm{~s}^{-1}$ to $48^{\circ}$ at strain rate of $10 \mathrm{~s}^{-1}$ for the cast-in-place anchors subjected to tensile load. Steel failure was observed at high strain rates of $10^{2} \mathrm{~s}^{-1}$ and $10^{3} \mathrm{~s}^{-1}$.

- The failure mode transitioned from concrete cone breakout failure at low strain rate of $10^{-5} \mathrm{~s}^{-1}$ to steel anchor failure at higher strain rates for the cast-in-place anchors subjected to tensile load. While the failure mode transitioned from pryout failure to steel anchor failure with the increase in the strain rate from $10^{-5} \mathrm{~s}^{-1}$ to $10^{3}$ $\mathrm{s}^{-1}$ for the cast-in-place anchors subjected to shear load.

- Concrete cone breakout diameter increased from $230 \mathrm{~mm}$ to $255 \mathrm{~mm}$ for the increase in anchor diameter from $12.7 \mathrm{~mm}$ to $19.1 \mathrm{~mm}$ at embedment depth of 76.2 $\mathrm{mm}$. The increase in the concrete cone breakout diameter at embedment depth of $101.6 \mathrm{~mm}$ was from $292 \mathrm{~mm}$ to $314 \mathrm{~mm}$ when the anchor diameter increased from $15.9 \mathrm{~mm}$ to $19.1 \mathrm{~mm}$. While steel anchor failure was observed for the $12.7 \mathrm{~mm}$ anchor diameter.

- The ultimate shear load increased with the increase in the concrete compressive strength when pryout failure is the dominant failure mode. Maximum increase in the ultimate shear load of $40.8 \%$ was obtained for the cast-in-place anchors when the concrete compressive strength increased from $20 \mathrm{MPa}$ to $40 \mathrm{MPa}$ at low strain rate of $10^{-5} \mathrm{~s}^{-1}$. While at high strain rate of $10^{3} \mathrm{~s}^{-1}$ increasing concrete compressive strength has no influence on the failure mode. 


\subsection{Behaviour of adhesive anchorage system under different strain rates}

- For adhesive anchors subjected to tensile load, maximum DIF of 1.13 was obtained for the adhesive anchors exhibited concrete cone breakout failure at strain rate of $10^{-3} \mathrm{~s}^{-1}$. Maximum DIF of 2.59 was obtained for the adhesive anchors exhibited combined cone bond failure at strain rate of $10^{2} \mathrm{~s}^{-1}$. Maximum DIF of 1.13 was obtained for the adhesive anchors exhibited steel failure at high strain rate of $10^{3} \mathrm{~s}^{-1}$.

- For adhesive anchors subjected to shear load, maximum DIF of 1.18 was obtained for the adhesive anchors exhibited steel failure at high strain rate of $10^{3} \mathrm{~s}^{-1}$.

- Concrete cone failure was observed at the static strain rate of $10^{-5} \mathrm{~s}^{-1}$, while combined cone-bond failure was observed at strain rates of $10^{-3} \mathrm{~s}^{-1}, 10^{-1} \mathrm{~s}^{-1}$ and 10 $\mathrm{s}^{-1}$ for most of the adhesive anchors subjected to tensile load. Steel anchor failure was observed at high strain rate of $10^{3} \mathrm{~s}^{-1}$.

- Average crack propagation angle of $56.5^{\circ}$ was observed at low strain rate of $10^{-5}$ $\mathrm{s}^{-1}$ for the adhesive anchors exhibited concrete cone breakout failure under tensile load. Increasing the strain rates higher than $10^{-5} \mathrm{~s}^{-1}$ exhibited combined cone bond failure and steel failure.

- Failure mode transition from pryout failure at low strain rate of $10^{-5} \mathrm{~s}^{-1}$ to steel anchor failure at high strain rate of $10^{3} \mathrm{~s}^{-1}$ was observed for most of the adhesive anchors subjected to shear load. However, failure mode transition from pryout failure at low strain rate of $10^{-5} \mathrm{~s}^{-1}$ to concrete spalling and steel anchor failure at high strain rate of $10^{3} \mathrm{~s}^{-1}$ was observed for $19.1 \mathrm{~mm}$ diameter adhesive anchor with embedment depth of $76.2 \mathrm{~mm}$. 
- The concrete cone breakout diameter increased from $225 \mathrm{~mm}$ to $236 \mathrm{~mm}$ with the increase in the anchor diameter from $12.7 \mathrm{~mm}$ to $15.9 \mathrm{~mm}$ at embedment depth of $76.2 \mathrm{~mm}$. The increase was from $268 \mathrm{~mm}$ to $291 \mathrm{~mm}$ at embedment depth of 101.6 $\mathrm{mm}$. At embedment depth of $127 \mathrm{~mm}$, steel anchor failure was observed for the $12.7 \mathrm{~mm}$ diameter and combined concrete cone bond failure for $15.9 \mathrm{~mm}$ diameter adhesive anchor. Steel anchor failure was observed at embedment depth of 152.4 $\mathrm{mm}$. The concrete cone breakout diameter increased from $244 \mathrm{~mm}$ to $421 \mathrm{~mm}$ for the $19.1 \mathrm{~mm}$ diameter adhesive anchor as the embedment depth increased from $76.2 \mathrm{~mm}$ to $152.4 \mathrm{~mm}$.

\subsection{Behaviour of undercut anchorage system under different strain rates}

- Maximum DIF of 1.58 was obtained for the undercut anchors subjected to tensile load at strain rate of $10 \mathrm{~s}^{-1}$, where concrete cone breakout failure is observed. Maximum DIF of 1.44 was obtained at strain rate of $10^{3} \mathrm{~s}^{-1}$, where steel failure is observed.

- For the undercut anchors subjected to shear load. Maximum DIF of 1.38 was obtained for the undercut anchors exhibited pryout failure at strain rate of $10^{-3} \mathrm{~s}^{-1}$. Maximum DIF of 1.79 was obtained for the undercut anchors exhibited concrete spalling at strain rate of $10^{-1} \mathrm{~s}^{-1}$. Maximum DIF of 1.45 was obtained for the undercut anchors exhibited steel failure at high strain rate of $10^{3} \mathrm{~s}^{-1}$.

- Steel anchor failure is the dominant failure mode for the $12-\mathrm{mm}$ and $16-\mathrm{mm}$ diameter undercut anchors subjected to tensile load at all the embedment depths and strain rates investigated. While concrete cone breakout failure is observed for 
the 20-mm diameter undercut anchor with $100 \mathrm{~mm}$ and $125 \mathrm{~mm}$ embedment depths at strain rates up to $10^{-1} \mathrm{~s}^{-1}$. Also, concrete cone breakout failure was observed for embedment depth of $100 \mathrm{~mm}$ at strain rate of $10 \mathrm{~s}^{-1}$. Steel anchor failure is observed at high strain rates of $10^{2} \mathrm{~s}^{-1}$ and $10^{3} \mathrm{~s}^{-1}$. Also, steel anchor failure was observed at embedment depths of $190 \mathrm{~mm}$ and $250 \mathrm{~mm}$ at all the strain rates investigated.

- Average crack propagation angle decreased from $62^{\circ}$ at strain rate of $10^{-5} \mathrm{~s}^{-1}$ to $42^{\circ}$ at strain rate of $10 \mathrm{~s}^{-1}$ for the undercut anchors exhibited concrete cone breakout failure.

- Failure mode transition from pryout failure at strain rate of $10^{-5} \mathrm{~s}^{-1}$ to concrete spalling and then to steel anchor failure at high strain rate of $10^{3} \mathrm{~s}^{-1}$ was observed for the undercut anchors subjected to shear load.

- The concrete cone breakout diameter increased from $291 \mathrm{~mm}$ to $340 \mathrm{~mm}$ with the increase in the anchor diameter from $16 \mathrm{~mm}$ to $20 \mathrm{~mm}$ at embedment depth of 100 $\mathrm{mm}$. While steel anchor failure was observed for anchor diameter of $12 \mathrm{~mm}$. Further increase in the anchor embedment depths for the $12 \mathrm{~mm}, 16 \mathrm{~mm}$ diameter undercut anchors resulted in steel failure. Concrete cone breakout diameter of 378 $\mathrm{mm}$ was observed for the $20 \mathrm{~mm}$ diameter undercut anchor at $125 \mathrm{~mm}$ embedment depth.

\subsection{Research limitations}

In this research plain concrete was used. Effect of reinforcement confinement was not considered for the analysis. Effect of edge distance was not considered where single anchor 
is placed at the center of the concrete block far from the concrete free edge. Effect of group anchors on the behaviour of anchorage to concrete system was not considered for the analysis. This research is limited to investigate the tensile and shear behaviour of anchorage to concrete system. Effect of combined tensile and shear loads on the behaviour of the anchorage to concrete system was not investigated.

\subsection{Future resreach}

The research performed in this thesis focused on the effect of strain rate on the tensile and shear capacity for the cast-in-place, adhesive and undercut anchors. The recommended future work can be summarized as follows:

- Effect of strain rate on the combination of the tensile and shear load of the anchorage systems.

- Influence of concrete confinement on the tensile and shear capacity of the anchorage system at different strain rates.

- Effect of hole shape (tapered shape) for the adhesive anchor on the tensile and shear capacity.

- Strain rate effect on the tensile and shear capacity for group of anchorage systems.

- Influence of temperature on the tensile and shear behaviour of anchorage to concrete systems at high strain rates.

- Strain rate effect on anchors near the concrete edge. 


\subsection{Contributions}

\subsubsection{Journal papers}

- Lenda T. Ahmed and Abass Braimah (2019). Tensile behaviour of adhesive anchors under different strain rates. Engineering Structures, (192), 113-125.

- Lenda T. Ahmed and Abass Braimah (2017). Behaviour of undercut anchors subjected to high strain rate loading. Procedia Engineering, (210), 326-333.

- Lenda T. Ahmed and Abass Braimah (2019). Shear behaviour of adhesive anchors under different strain rates. (Submitted to Engineering Structures).

\subsubsection{Conference papers}

- Lenda T. Ahmed and Abass Braimah (2018). Shear behaviour of cast-in-place anchors at low and high strain rates. CSCE 2018 Annual Conference, New Brunswick, Canada.

- Lenda T. Ahmed and Abass Braimah (2016). Strain Rate Effect on the behaviour of cast-in-place anchors under tensile load. $2^{\text {nd }}$ International Conference on Advances in Civil Architecture and Environmental Engineering (ICCAEE), KL, Malaysia.

- Lenda T. Ahmed and Abass Braimah (2016). Strain rate effect on the behaviour of cast-in-place concrete anchors under shear loading. $2^{\text {nd }}$ International Conference on Advances in Civil Architecture and Environmental Engineering (ICCAEE), KL, Malaysia. (Best paper presentation award). 


\section{References}

A Mitek' Company. (1997). DUC UNDERCUT ANCHORS.

Abebe, M. S., \& Qiu, H. S. (2016). Numerical modeling of geotextile reinforcement of soft subgrade ballasted railway under high speed train. Electronic Journal of Geotechnical Engineering, 21(12), 4327-4343.

ACI Committee (349). (1990). Code requirements for nuclear safety related structures (ACI) 349-85, Appendix B-steel embedment”. American concrete institute, Detroit.

ACI Committee 318. (2005). Building Code Requirements for Structural Concrete ( ACI 318-05) and Commentrary (ACI 318R-05).

ACI Committee 318. (2011). Building Code Requirements for Structural Concrete (ACI 318-11) and Commentary.

ACI Committee 355. (2000). Commentary on Evaluating the Performance of PostInstalled Mechanical Anchors in Concrete (ACI 355.2R-00).

Ansell, A. (2006). Dynamic testing of steel for a new type of energy absorbing rock bolt. Journal of Constructional Steel Research, 62(5), 501-512.

Ashour, A. F., \& Alqedra, M. A. (2005). Concrete breakout strength of single anchors in tension using neural networks. Advances in Engineering Software, 36, 87-97.

Bala, S. (2008). Tolerance used for Tying Slave Nodes in TIED contacts.

Barnat, J., Bajer, M., \& Vyhnankova, M. (2012). Bond strength of chemical anchor in highstrength concrete. Procedia Engineering, 40, 38-43.

Bathe, K.-J., Walczak, J., Guillermin, O., Bouzinov, P. A., \& Chen, H.-Y. (1999). Advances in crush analysis. Computers \& Structures, 72, 31-47.

Benmokrane, B., Tighiouart, B., \& Chaallal, O. (1996). Bond strength and load distribution 
of composite GFRP reinforcing bars in concrete. ACI Materials Journal, 93(3), 246252.

Bergan, P. G., Horrigmoe, G., Krakeland, B., \& Soreide, T. H. (1978). Solution techniques for non-linear finite element problems. International Journal for Numerical Methodds in Engineering, 12, 1677-1696.

Bermejo, M., Goicolea, J. M., Gabald'on, F., \& Santos, A. (2011). Impact And Explosive Loads On Concrete Buildings Using Shell And Beam Type Elements. 3rd ECCOMAS Thematic Conference on Computational Methods in Structural Dynamics and Earthquake Engineering, 1-14.

Bi, K., \& Hao, H. (2013). Numerical simulation of pounding damage to bridge structures under spatially varying ground motions. Engineering Structures, 46, 62-76.

Bickel, T. S., \& Shaikh, A. F. (2002). Shear Strength of Adhesive Anchors. PCI Journal, 47(5), 92-101.

Bischoff, P. H., \& Perry, S. H. (1991). Compressive behaviour of concrete at high strain rates. Materials and Structures, 24(6), 425-450.

Boh, J. W., Louca, L. A., \& Choo, Y. S. (2004). Strain rate effects on the response of stainless steel corrugated firewalls subjected to hydrocarbon explosions. Journal of Constructional Steel Research, 60(1), 1-29.

Børvik, T., Hopperstad, O. S., \& Berstad, T. (2003). On the influence of stress triaxiality and strain rate on the behaviour of a structural steel. Part II. Numerical study. European Journal of Mechanics, A/Solids, 22, 15-32.

Braimah, A., Contestabile, E., \& Guilbeault, R. (2009). Behaviour of adhesive steel anchors under impulse-type loading. Canadian Journal of Civil Engineering, 36(11), 
$1835-1847$.

Braimah, A., Guilbeault, R., \& Contestabile, E. (2004). High strain rate behaviour of chemically bonded steel anchors. Canadian explosives research laboratory (CERL), Report No. 20, Natural Resources Canada (Vol. 20).

Braimah, A., Guilbeault, R., \& Contestabile, E. (2014). Strain rate behaviour of adhesive anchors in masonry. Engineering Structures, 67, 96-108.

Bridge Design Aids. (2012). Anchorage to Concrete.

British Standards Institution Draft for development. (1992). Concrete performane, production, placing, and compliance criteria.

BS EN 206. (2013). BSI Standards Publication Concrete - Specification , performance, production and conformity. British Standard, (May), 1-93.

Cadoni, E., Labibes, K., Albertini, C., Berra, M., \& Giangrasso, M. (2001). Strain-rate effect on the tensile behaviour of concrete at different relative humidity levels. Materials and Structures, 34, 21-26.

Cai, M., Champaigne, D., \& Kaiser, P. K. (2010). Development of a fully debonded conebolt for rockburst support. In 5 th International Seminar on Deep and High Stress Mining (pp. 329-342).

Çalışkan, Ö., Yılmaz, S., Kaplan, H., \& Kıraç, N. (2013). Shear strength of epoxy anchors embedded into low strength concrete. Construction and Building Materials, 38, 723730.

Cattaneo, S., \& Muciaccia, G. (2015). Adhesive anchors in high performance concrete. Materials and Structures, 1-12.

Ceci, A. M., Casas, J. R., \& Ghosn, M. (2012). Statistical analysis of existing models for 
flexural strengthening of concrete bridge beams using FRP sheets. Construction and Building Materials, 27(1), 490-520.

Cement Association of Canada. (2010). Concrete design handbook, third edition.

Chan, Y., Chen, Y., \& Liu, Y. (2003). Development of bond strength of reinforcement steel in self-consolidating concrete. ACI Structural Journal, 100(4), 490-498.

Chapman, R. A., \& Shah, S. P. (1987). Early-Age bond strength in reinforced concrete. ACI Materials Journal, 501-510.

Chen, S., Zang, M., Wang, D., Yoshimura, S., \& Yamada, T. (2017). Numerical analysis of impact failure of automotive laminated glass: A review. Composites Part B, 122, $47-60$.

Chen, W., \& Hao, H. (2012). Numerical study of a new multi-arch double-layered blastresistance door panel. International Journal of Impact Engineering, 43, 16-28.

Choi, S., Joh, C., \& Chun, S.-C. (2015). Behavior and strengths of single cast-in anchors in Ultra-High-Performance Fiber-Reinforced Concrete (UHPFRC) subjected to a monotonic tension or shear. KSCE Journal of Civil Engineering, 19(4), 964-973.

Chou, C., Chen, P., Le, J., \& Tamini, N. (2004). A benchmark study of CAE sensor modeling using LS-DYNA. Eighth International LS-DYNA User Conference, (2), 322.

Committee Euro-International du Beton (CEB). (1994). Fastenings to concrete and masonry structures. State of the Art Report. Thomas Telford Ltd.

Cook, R. A. (1993). Behavior of chemically bonded anchors. Journal of Structural Engineering, 119(9), 2744-2762.

Cook, R. A., Collins, D. M., Klingner, R. E., \& Polyzois, D. (1992). Load-Deflection 
Behavior of Cast-in-Place and Retrofit Concrete Anchors. ACI Structural Journal, $89(6), 639-649$.

Cook, R. A., Doerr, G. T., \& Klingner, R. E. (1993). Bond stress model for design of adhesive anchors. ACI Structural Journal, 90(5), 514-524.

Cook, R. a., Eligehausen, R., \& Appl, J. J. (2007). Overview: Behavior of Adhesive Bonded Anchors. Beton- Und Stahlbetonbau, 102, 16-21.

Cook, R. A., Fagundo, F. E., Biller, M. H., \& Richardson, D. E. (1991). Tensile behavior and design of single adhesive anchors. Structures and Materials Res, (91-3).

Cook, R. A., \& Konz, R. C. (2001). Factors Influencing Bond Strength of Adhesive Anchors. ACI Structural Journal, 98(1), 76-86.

Cook, R. A., Kunz, J., Fuchs, W., \& Konz, R. C. (1998). Behavior and design of single adhesive anchors under tensile load in uncracked concrete. ACI Struct., 95(1), 9-26.

Cook, R. A., Prevatt, D. O., Mcbride, K. E., \& Potter, W. (2013). Steel shear strength of anchors with stand-off base plates.

Cowell, W. L. (1969). Dynamic tests on selected structural steels.

da Silva Lucas F. M., Öchsner, A., \& Adams, R. D. (2011). Handbook of Adhesion Technology.

Das, A., Ghosh, M., Tarafder, S., Sivaprasad, S., \& Chakrabarti, D. (2017). Micromechanisms of deformation in dual phase steels at high strain rates. Materials Science and Engineering A, 680, 249-258.

Davidson, J. S., Fisher, J. W., Hammons, M. I., Porter, J. R., \& Dinan, R. J. (2005). Failure Mechanisms of Polymer-Reinforced Concrete Masonry Walls Subjected to Blast. Journal of Structural Engineering, 131(August), 1194-1205. 
de Larrard, F., Schaller, I., \& Fuchs, J. (1993). Effect of bar diameter on the bond strength of passive reinforcement in high-performance concrete. ACI Materials Journal, 90(4), $333-339$.

Delhomme, F., Roure, T., Arrieta, B., \& Limam, A. (2015a). Static and cyclic pullout behavior of cast-in-place headed and bonded anchors with large embedment depths in cracked concrete. Nuclear Engineering and Design, 287, 139-150.

Delhomme, F., Roure, T., Arrieta, B., \& Limam, A. (2015b). Tensile behaviour of cast-inplace headed anchors with different embedment depths. European Journal of Environmental and Civil Engineering, 19(6), 703-716.

Dogan, F., Hadavinia, H., Donchev, T., \& Bhonge, P. S. (2012). Delamination of impacted composite structures by cohesive zone interface elements and tiebreak contact. Central European Journal of Engineering, 2(4), 612-626.

El-Hage, H., Mallick, P. K., \& Zamani, N. (2005). A numerical study on the quasi-static axial crush characteristics of square aluminum tubes with chamfering and other triggering mechanisms. International Journal of Crashworthiness, 10(2), 183-195.

Eligehausen, R. (1987). Anchorage to Concrete by Metallic Expansion Anchors. Anchorage to Concrete, SP-103, 181-201.

Eligehausen, R., Bouska, P., Cervenka, V., \& Pukl, R. (1992). Size effect of the concrete cone failure load of anchor bolts. In First international conference on fracture mechanics of concrete structures (FraMCoS1) (pp. 517-525).

Eligehausen, R., Cook, R. A., \& Appl, J. (2006). Behavior and Design of Adhesive Bonded Anchors. ACI Structural Journal, 103(6), 822-831.

Eligehausen, R., Fuchs, W., \& Sippel, T. M. (1998). Anchorage to concrete. Progress in 
Structural Engineering and Materials, 1(4), 392-403.

Eligehausen, R., Hofacker, I., \& Lettow, S. (2001). Fastening technique-current status and future trends. In International symposium on connections between steel and concrete (pp. 11-27).

Eligehausen, R., Mallee, R., \& Rehm. (1984). Fastenings with bonded anchors. Betonwerk+ Fertigteil -Technik, 10, 686-692.

Eligehausen, R., Mallée, R., \& Silva, J. F. (2006). Anchorage in concrete construction. Ernst \& Sohn, 1st edition.

Epackachi, S., Esmaili, O., Mirghaderi, S. R., \& Behbahani, A. A. T. (2015). Behavior of adhesive bonded anchors under tension and shear loads. Journal of Constructional Steel Research, 114, 269-280.

Fabbrocino, G., Verderame, G. M., \& Manfredi, G. (2005). Experimental behaviour of anchored smooth rebars in old type reinforced concrete buildings. Engineering Structures, 27(10), 1575-1585.

Fang, Q., \& Zhang, J. (2013). Three-dimensional modelling of steel fiber reinforced concrete material under intense dynamic loading. Construction and Building Materials, 44, 118-132.

Fu, B. H. C., Erki, M. A., \& Seckin, M. (1991). Review o f effects of loading rate on reinforced concrete. Journal of Structural Engineering, 117(12), 3660-3679.

Fu, X., \& Chung, D. D. L. (1999). Interface between steel rebar and concrete, studied by electromechanical pull out testing. Composite Interfaces, 6(2), 81-92.

Fuchs, W. (2001). Evolution of fastening design methods in Europe. In International Symposium on connections between steel and concrete (pp. 45-60). 
Fuchs, W., Eligehausen, R., \& Breen, J. E. (1995). Concrete capacity design (CCD) approach for fastening to concrete. ACI Structural Journal, 92(1), 73-94.

Fujikake, K., Nakayama, J., Sato, H., Mindess, S., \& Ishibashi, T. (2003). Chemically Bonded Anchors Subjected to Rapid Pullout Loading. ACI Materials Journal, 100(3), $246-252$.

Gebbeken, N., Greulich, S., \& Pietzsch, A. (2001). Performance of Concrete Based Building Materials Against Blast and Impact. In Proceedings of the fib-Symposium on Concrete and Environment, Berlin (pp. 1-11).

Gebbeken, N., \& Ruppert, M. (2000). A new material model for concrete in high-dynamic hydrocode simulations. Archive of Applied Mechanics, 70, 463-478.

Georgin, J. F., \& Reynouard, J. M. (2003). Modeling of structures subjected to impact: concrete behaviour under high strain rate. Cement and Concrete Composites, 25(1), $131-143$.

Gesoğlu, M., Güneyisi, E. M., Güneyisi, E., Yılmaz, M. E., \& Mermerdaş, K. (2014). Modeling and analysis of the shear capacity of adhesive anchors post-installed into uncracked concrete. Composites : Part B, 60, 716-724.

Gesoglu, M., Ozturan, T., Ozel, M., \& Guneyisi, E. (2005). Tensile Behavior of PostInstalled Anchors in Plain and Steel Fiber-Reinforced Normal- and High-Strength Concretes. Structural Journal, 102(2), 224-231.

Goncalves, M. C., \& Margarido, F. (2015). Materials for Construction and Civil Engineering.

González, F., Fernández, J., Agranati, G., \& Villanueva, P. (2018). Influence of construction conditions on strength of post installed bonded anchors. Construction 
and Building Materials, 165, 272-283.

Graf, T., Haufe, A., \& Andrade, F. (2014). Adhesives modeling with LS-DYNA : Recent developments and future work.

Gross, H. J., Klingner, R. E., \& Graves III, H. L. (2001). Dynamic Behavior of Single and Double Near-Edge Anchors Loaded in Shear. ACI Structural Journal, 98(5), 665676.

Hallquist, J. O. (2006). LS-DYNA theory manual. Livermore Software Technology Corporation.

Han, H., Taheri, F., Pegg, N., \& Lu, Y. (2007). A numerical study on the axial crushing response of hybrid pultruded and $\pm 45^{\circ}$ braided tubes. Composite Structures, $80(2)$, $253-264$

Hariyadi, Munemoto, S., \& Sonoda, Y. (2017). Experimental Analysis of Anchor Bolt in Concrete under the Pull-Out Loading. Procedia Engineering, 171, 926-933.

Hashimoto, J., \& Takiguchi, K. (2004). Experimental study on pullout strength of anchor bolt with an embedment depth of $30 \mathrm{~mm}$ in concrete under high temperature. Nuclear Engineering and Design, 229, 151-163.

Hawkins, N. (1987). Strength in shear and tension of cast-in-place anchor bolts. American Concrete Institute (ACI), 103(12), 233-255.

Hentz, S., Donzé, F. V., \& Daudeville, L. (2004). Discrete element modelling of concrete submitted to dynamic loading at high strain rates. Computers \& Structures, 82, 25092524.

Hilti. (2011). Anchor Fastening Technical Guide 2011.

Hilti. (2014). Adhesive anchoring systems. 
Hopperstad, O. S., Børvik, T., Langseth, M., Labibes, K., \& Albertini, C. (2003). On the influence of stress triaxiality and strain rate on the behaviour of a structural steel. Part I. Experiments. European Journal of Mechanics, A/Solids, 22, 1-13.

Jaime, M. C. (2011). Numerical modeling of rock cutting and its associated fragmentation process using the finite element method. PHD Thesis, Civil Engineering Department, University of Pittsburgh.

Jang, J. B., \& Suh, Y. P. (2006). The experimental investigation of a crack's influence on the concrete breakout strength of a cast-in-place anchor. Nuclear Engineering and Design, 236(9), 948-953.

Jebara, K., Ozbolt, J., \& Hofmann, J. (2016). Pryout failure capacity of single headed stud anchors. Materials and Structures, 49, 1775-1792.

Jensen, M. R., Hallquist, J., Grimes, R., Day, J., Lu, H., \& Tabiei, A. (2007). Introduction to LS-DYNA, 1-145.

Johnsonwindowfilms.com. (2018). http://www.johnsonwindowfilms.com/protective-films /anchoring-systems/.

Kashani, M. H., Alavijeh, H. S., Akbarshahi, H., \& Shakeri, M. (2013). Bitubular square tubes with different arrangements under quasi-static axial compression loading. Materials and Design, 51, 1095-1103.

Keller, T., \& Vallée, T. (2005). Adhesively bonded lap joints from pultruded GFRP profiles. Part I: Stress-strain analysis and failure modes. Composites Part B: Engineering, 36(4), 331-340.

Kim, J.-S., Jung, W.-Y., Kwon, M.-H., \& Ju, B.-S. (2013). Performance evaluation of the post-installed anchor for sign structure in South Korea. Construction and Building 
Materials, 44, 496-506.

Kinsho, T., Satake, M., \& Miyazaki, T. (2000). Epoxy curing agent and one component (type) epoxy resin composition.

Kulkarni, S. M., \& Shah, S. P. (1998). Response of Reinforced Concrete Beams at High Strain Rates. ACI Structural Journal, 95(6), 705-715.

Kwasniewski, L. (2010). Nonlinear dynamic simulations of progressive collapse for a multistory building. Engineering Structures, 32(5), 1223-1235.

Kyei, C., \& Braimah, A. (2013). Seismic Reinforcement Detailing Effects on Blast Resistance of Reinforced Concrete Columns. In 3rd Specialty Conference on Diasaster Prevention and Mitigation (pp. 1-9).

Lee, N. H., Park, K. R., \& Suh, Y. P. (2011). Shear behavior of headed anchors with large diameters and deep embedments in concrete. Nuclear Engineering and Design, 241(3), 608-616.

Lee, W.-S., \& Lam, H.-F. (1996). The deformation behaviour and microstructure evolution of high-strength alloy steel at high rate of strain. Journal of Materials Processing Technology, 57(3-4), 233-240.

Lee, W.-S., Lin, C.-F., \& Liu, T.-J. (2007). Impact and fracture response of sintered 316L stainless steel subjected to high strain rate loading. Materials Characterization, 58(4), $363-370$.

Li, Y., Eligehausen, R., Ozbolt, J., \& Lehr, B. (2002). Numerical Analysis of Quadruple Fastenings with Bonded Anchors. ACI Structural Journal, 99(2), 149-156.

Liu, Y. (2008). ANSYS and LS-DYNA used for structural analysis. International Journal of Computer Aided Engineering and Technology, 1(1), 31-44. 
Livermore software technology Corporation, L. (2012). LS-DYNA Keyword User' S Manual VOLUME II Material Models (Vol. II).

Livermore Software Technology Corporation, L. (2015). LS-DYNA Theory Manual.

Lou, K.-A., \& Perciballi, W. (2008). Finite Element Modeling of Preloaded Bolt Under Static Three-Point Bending Load. In 10th International LS-DYNA Users Conference (pp. 1-10).

LSTC, Livermore Software Technology Corporation. (2015). LS-DYNA Keyword User'S Manual Volume II material models.

LSTC, Livermore Software Technology Corporation. (2014a). LS-DYNA Keyword User's Manual Volume II Material Models. Livermore California (Vol. II).

LSTC, Livermore Software Technology Corporation. (2014b). Strain rate LS-DYNA support.

Lu, J. Z., Zhong, J. S., Luo, K. Y., Zhang, L., Qi, H., Luo, M., XU, X. J., Zhou, J. Z. (2013). Strain rate correspondence of fracture surface features and tensile properties in AISI304 stainless steel under different LSP impact time. Surface and Coatings Technology, 221, 88-93.

Mackay-Sim, R. (1990). Limit State Design: Fastening to Concrete (Vol. 355).

Madico Safety Shield Premier Partener. (2012). http://www.windowfilmuk.com/windowfilm-bomb-blast-protection/lifeline-anchoring-window-film.html.

Mahadik, V., Sharma, A., \& Hofmann, J. (2016). Inelastic seismic behavior of postinstalled anchors for nuclear safety related structures: Generation of experimental database. Nuclear Engineering and Design, 297, 231-250.

Mahrenholtz, C., \& Eligehausen, R. (2013). Dynamic performance of concrete undercut 
anchors for Nuclear Power Plants. Nuclear Engineering \& Design, 265, 1091-1100.

Mahrenholtz, P., \& Eligehausen, R. (2015). Post-installed concrete anchors in nuclear power plants: Performance and qualification. Nuclear Engineering and Design, 287, $48-56$.

Maker, B., \& Zhu, X. (2000). Input parameters for metal forming simulation using LSDYNA. Livermore Software Technology Corporation, 2, 1-10.

Mallonee, S., Shariat, S., Stennies, G., Waxweiler, R., Hogan, D., \& Jordan, F. (1996). Physical Injuries and Fatalities Resulting From the Oklahoma City Bombing. JAMA, 276(5), 382-387.

Malvar, L. J., \& Crawford, J. E. (1998). Dynamic Increase Factors for Concrete. In TwentyEight DDESB Seminar, Orlando, FL (pp. 1-17).

Malvar, L. J., \& Ross, C. A. (1998). Review of Strain Rate Effects for Concrete in Tension. ACI Materials Journal, 95(6), 735-739.

Marcon, M., Ninčević, K., Boumakis, I., Czernuschka, L.-M., \& Wan-Wendner, R. (2018). Aggregate effect on the concrete cone capacity of an undercut anchor under quasistatic tensile load. Materials, 11(5), 1-17.

Marzi, S., Hesebeck, O., Brede, M., \& Kleiner, F. (2009). A Rate-Dependent Cohesive Zone Model for Adhesively Bonded Joints Loaded in Mode I. Journal of Adhesion Science and Technology, 23(6), 881-898.

Marzi, S., Ramon-villalonga, L., Poklitar, M., \& Kleiner, F. (2008). Usage of Cohesive Elements in Crash Analysis of Large, Bonded Vehicle Structures Experimental Tests and Simulation. In LS DYNA FORUM, Bamberg.

May, M., Voß, H., \& Hiermaier, S. (2014). Predictive modeling of damage and failure in 
adhesively bonded metallic joints using cohesive interface elements. International Journal of Adhesion and Adhesives, 49, 7-17.

McMullin, P. W., Price, J. S., \& Persellin, E. H. (2016). Concrete design, Routledge Taylor and Francis Group, New York.

McVay, M., Cook, R. A., \& Krishnamurthy, K. (1996). Pullout Simulation Of Postinstalled Chemically Bonded Anchors. Structural Engineering, 122(9), 1016-1024.

Min, F., Yao, Z., \& Jiang, T. (2014). Experimental and numerical study on tensile strength of concrete under different strain rates. The Scientific World Journal, 1-11.

Montgomery, D. C. (2013). Design and Analysis of Experiments (8th Edition). John Wiley \& Sons, New York.

Mousavi, R., Champiri, M. D., \& Willam, K. J. (2016). Efficiency of damage-plasticity models in capturing compaction-expansion transition of concrete under different compression loading conditions. In VII European Congress on Computational Methods in Applied Sciences and Engineering (pp. 1-12).

Moutoussamy, L., Herve, G., \& Barbier, F. (2011). Qualification of * Constrained _ Lagrange_In_Solid command for steel / concrete interface modeling. In 8th European LS-DYNA Conference.

Muratli, H., Klingner, R. E., \& Graves III, H. L. (2001). Behavior of shear anchors in concrete: statistical analysis and design recommendations. In International Symposium on connections between steel and concrete (pp. 220-230).

Muratli, H., Klingner, R. E., \& Graves III, H. L. (2004). Breakout Capacity of Anchors in Concrete - Part 2 : Shear. ACI Structural Journal, 101(6), 821-829.

Murray, Y. (2007). Users Manual for LS-DYNA Concrete Material Model 159. 
Nammur Jr, G., \& Naaman, A. (1989). Bond stress model for fiber reinforced concrete based on bond stress-slip relationship. ACI Materials Journal, 45-57.

Nilforoush, R., Nilsson, M., \& Elfgren, L. (2017). Experimental evaluation of tensile behaviour of single cast-in-place anchor bolts in plain and steel fibre-reinforced normal- and high-strength concrete. Engineering Structures, 147, 195-206.

Nilforoush, R., Nilsson, M., \& Elfgren, L. (2018). Experimental evaluation of influence of member thickness, anchor-head size, and orthogonal surface reinforcement on the tensile capacity of headed anchors in uncracked concrete. Journal of Structural Engineering, 144(4), 1-14.

Norville, H. S., \& Conrath, E. J. (2001). Considerations for blast-resistant glazing design. Journal of Architectural Engineering, 7(3), 80-86.

Norville, H. S., \& Conrath, E. J. (2006). Blast-Resistant Glazing Design. Journal of Architectural Engineering, 12(3), 129-136.

Norville, H. S., Harvill, N., Conrath, E. J., Shariat, S., \& Mallonee, S. (1999). Glass-related injuries in Oklahoma City Bombing. Journal of Performance of Constructed Facilities, 13(2), 50-56.

Odeshi, A. G., Al-Ameeri, S., \& Bassim, M. N. (2005). Effect of high strain rate on plastic deformation of a low alloy steel subjected to ballistic impact. Journal of Materials Processing Technology, 162-163(SPEC. ISS.), 385-391.

Ozbolt, J., \& Eligehausen, R. (1990). Numerical analysis of headed studs embeded in large plain concrete blocks. Computer Aided Analysis and Design of Concrete Structures, $1,645-656$.

Ožbolt, J., Rah, K. K., \& Meštrović, D. (2006). Influence of loading rate on concrete cone 
failure. International Journal of Fracture, 139, 239-252.

Pallarés, L., \& Hajjar, J. F. (2009). Headed steel stud anchors in composite structures, Part II: Tension and interaction. Journal of Constructional Steel Research, 66, 1-38.

Park, S. W., Xia, Q., \& Zhou, M. (2001). Dynamic behavior of concrete at high strain rates and pressures : II . numerical simulation. International Journal of Impact Eng, 25, 887-910.

Paultre, P. (2010). Dynamics of Structures. ISTE Ltd, UK and John Wiley \& Sons, Inc, USA. John Wiley \& Sons, Inc, Hoboken, USA.

Petersen, D., Lin, Z., \& Zhao, J. (2013). Behavior and Design of Cast-in-Place Anchors under Simulated Seismic Loading (Vol. II).

precast-prestressed concrete Institute. (1999). PCI design handbook.

Primavera, E. J., Pinelli, J. P., \& Kalajian, E. H. (1997). Tensile behavior of cast-in-place and undercut anchors in high-strength concrete. ACI Structural Journal, 94(5), 583592.

Qian, S., \& Li, V. C. (2011). Headed Anchor/Engineered Cementitious Composites (ECC) Pullout Behavior. Journal of Advanced Concrete Technology, 9(3), 339-351.

Rao, G. A., \& Arora, J. (2013). Strength and Modes of Failure of Adhesive Anchors in Confined Concrete Under Direct Tensile Loading. In VIII International Conference on Fracture Mechanics of Concrete and Concrete Structures FraMCOS-8 (pp. 1-11).

Reinhardt, H., Rossi, P., \& van Mier, J. (1990). Joint investigation of concrete at high rates of loading. Materials and Structures, 23, 213-216.

Remani, C. (2013). Methods for Solving Systems of Nonlinear Equations.

Rodriguez, M., Zhang, Y., Lotez, D., Graves III, H. L., \& Klingner, R. E. (1997). Dynamic 
behaviour of anchors in cracked and uncracked concrete: a progress report 1. Nuclear Engineering and Design, 168, 23-34.

Ross, C. A., Jerome, D. M., Tedesco, J. W., \& Hughes, M. L. (1996). Moisture and Strain Rate Effects on Concrete Strength. ACI Materials Journal, 93(3), 293-298.

Ross, C. A., Tedesco, J. W., \& Kuennen, S. T. (1995). Effects of Strain Rate on Concrete Strength. ACI Materials Journal, 92(1), 37-45.

Ross, C. A., Thompson, P. Y., \& Tedesco, J. W. (1989). Split-Hopkinson Pressure-Bar Tests on Concrete and Mortar in Tension and Compression. ACI Materials Journal, $86(5), 475-481$.

Rossi, P., Mier, J. G. M. Van, Toutlemonde, F., Maou, fabrice le, \& Boulay, C. (1994). Effect of loading rate on the strength of concrete subjected to uniaxial tension. Materials and Structures, 27, 260-264.

Rust, W., \& Schweizerhof, K. (2003). Finite element limit load analysis of thin-walled structures by ANSYS (implicit), LS-DYNA (explicit) and in combination. ThinWalled Structures, 41(2-3), 227-244.

Sagals, G., Orbovic, N., \& Blahoianu, A. (2011). Sensitivity Studies of Reinforced Concrete Slabs Under Impact Loading. In Transactions, SMiRT 21 (pp. 1-8).

Sato, H., Fujikake, K., \& Mindess, S. (2004). Study on dynamic pullout strength of anchors based on failure modes. 13th World Conference on Earthquake Engineering, 1-7.

Shariat, S., Mallonee, S., \& Stidham, S. S. (1998). Summary of Reportable Injuries in Oklahoma: Oklahoma City Bombing Injuries.

Shima, H., Chou, L. L., \& Okamura, H. (1987). Micro and macro models for bond in reinforced concrete. Journal of the Faculty of Engineering, The Uinversity of Tokyo, 
XXXIX(2), 133-194.

Shkolnik, I. E. (2008). Influence of high strain rates on stress-strain relationship, strength and elastic modulus of concrete. Cement and Concrete Composites, 30(10), 10001012.

Solomos, G., \& Berra, M. (2006). Testing of anchorages in concrete under dynamic tensile loading. Materials and Structures, 39, 695-706.

Spyridis, P., \& Bergmeister, K. (2014). Effects of Assembly Tolerances on Bolted Anchorages in Concrete. Journal of Structural Engineering, 140(1), 1-12.

Subramanian, N. (2000). Recent developments in the design of anchor bolts. The Indian Concrete Journal, 407-414.

Sugiman, S., \& Ahmad, H. (2017). Comparison of cohesive zone and continuum damage approach in predicting the static failure of adhesively bonded single lap joints. Journal of Adhesion Science and Technology, 31(5), 552-570.

Tedesco, J. W., Hughes, M. L., \& Ross, C. a. (1994). Numerical simulation of high strain rate concrete compression tests. Computers \& Structures, 51(1), 65-77.

Toikka, L., Braimah, A., Razaqpur, G., \& Foo, S. (2015). Strain Rate Effect on Development Length of Steel Reinforcement. Journal of Structural Engineering, 141(11), 1-14.

Trautwein, B. (2017). Anchor US Patent, US9677586 B2.

Tserpes, K. I., \& Koumpias, A. S. (2012). Comparison between a Cohesive Zone Model and a Continuum Damage Model in Predicting Mode-I Fracture Behavior of Adhesively Bonded Joints. CMES, 83(2), 169-181.

Tu, Z., \& Lu, Y. (2009). Evaluation of typical concrete material models used in hydrocodes 
for high dynamic response simulations. International Journal of Impact Engineering, $36(1), 132-146$.

Ueda, T., Kitipornchai, S., \& Ling, K. (1990). Experimental Investigation of Anchor Bolts Under Shear. Structural Engineering, 116(4), 910-924.

Ueda, T., Stitmannaithum, B., \& Matupayont, S. (1991). Experimental Investigation on Shear Strength of Bolt Anchorage Group. ACI Structural Journal, 88(3), 292-300.

Upadhyaya, P., \& Kumar, S. (2015). Pull-out capacity of adhesive anchors: An analytical solution. International Journal of Adhesion and Adhesives, 60, 54-62.

Vuletic, R., \& Pearson, J. (2008). Use of Adhesive Anchors to Resist Long-Term Loads. Structural Engineering Magazine, (January), 1-6.

Wakabayashi, M., Nakamura, T., Yoshida, N., Iwai, S., \& Watanabe, Y. (1980). Dynamic loading effects on the structural performance of concrete and steel materials and beams. Proceedings of the Seventh World Conference on Earthquake Engineering, 6(3), 271-278.

Wang, D., Wu, D., He, S., Zhou, J., \& Ouyang, C. (2015). Behavior of post-installed largediameter anchors in concrete foundations. Construction and Building Materials, 95, $124-132$.

Wang, D., Wu, D., Ouyang, C., He, S., \& Sun, X. (2017). Simulation analysis of largediameter post-installed anchors in concrete. Construction and Building Materials, $143,558-565$.

Wu, Y., Crawford, J. E., \& Magallanes, J. M. (2012). Performance of LS-DYNA Concrete Constitutive Models. In 12 th International LS-DYNA Users Conference (pp. 1-13).

Xing, G., Zhou, C., Wu, T., \& Liu, B. (2015). Experimental study on bond behavior 
between plain reinforcing bars and concrete. Advances in Materials Science and Engineering, 1-9.

Xiong, R., Fu, R. Y., Su, Y., Li, Q., Wei, X.-C., \& Li, L. (2009). Tensile Properties of TWIP Steel at High Strain Rate. Journal of Iron and Steel Research International, $16(1), 81-86$.

Xu, S., Ruan, D., Beynon, J. H., \& Rong, Y. (2013). Dynamic tensile behaviour of TWIP steel under intermediate strain rate loading. Materials Science and Engineering A, $573,132-140$.

Yilmaz, S., Özen, M. A., \& Yardim, Y. (2013). Tensile behavior of post-installed chemical anchors embedded to low strength concrete. Construction and Building Materials, 47, $861-866$

Yoon, Y.-S., Kim, H.-S., \& Kim, S.-Y. (2001). Assessment of fracture behaviors for CIP anchors fastened to cracked and uncracked concretes. KCI Concrete Journal, 13(2), $33-41$.

Yu, H., Guo, Y., \& Lai, X. (2009). Rate-dependent behavior and constitutive model of DP600 steel at strain rate from $10^{-4}$ to $10^{3} \mathrm{~s}^{-1}$. Materials and Design, 30, 2501-2505.

Zamora, N. A., Cook, R. A., Konz, R. C., \& Consolazio, G. R. (2003). Behavior and Design of Single, Headed and Unheaded, Grouted Anchors under Tensile Load. ACI Structural Journal, 100(2), 222-230.

Zhao, C. F., Chen, J. Y., Wang, Y., \& Lu, S. J. (2012). Damage mechanism and response of reinforced concrete containment structure under internal blast loading. Theoretical and Applied Fracture Mechanics, 61, 12-20.

Zhao, G. (1994). Load-Carrying Behavior of Headed Stud Anchors in Concrete Breakout 
Away From an Edge. University Stuttgart, Germany.

Zhao, J. (2014). Seismic behavior of single anchors in plastic hinge zones of RC columns. In Tenth U.S. National Conference on Earthquake Engineering.

Zhao, T., Guo, W., Yin, Y., \& Tan, Y. (2015). Bolt Pull-Out Tests of Anchorage Body under Different Loading Rates. Shock and Vibration, 1-8.

Zhou, X. Q., Kuznetsov, V. A., Hao, H., \& Waschl, J. (2008). Numerical prediction of concrete slab response to blast loading. International Journal of Impact Engineering, $35,1186-1200$. 


\section{Appendix A: Design methods for cast-in-place anchors}

\section{A.1 Design methods for cast-in-place anchors under tensile load}

\section{A.1.1 American Concrete Institute (ACI)}

According to ACI 349-85 design method, the ultimate tensile failure load for cast-in-place headed anchors can be determined by Equation (4.1) (Fuchs et al., 1995; Subramanian, 2000; Fuchs, 2001). Equation (4.1) is derived assuming sufficient thickness of the concrete

member to ensure that there is no decrease in the failure load (Fuchs et al. 1995). For anchors with edge distance (c) less than $h_{e f}$, and/or the distance between anchors (spacing) (s) less than $2 \times h_{e f}$, the ultimate failure load can be determined by Equation (A.1) (Fuchs et al., 1995; Subramanian, 2000).

$N_{u}=\frac{A_{N}}{A_{N o}} \cdot N_{u o}$

$A_{N o}=\pi \cdot h_{e f}^{2}\left(1+\frac{d_{h}}{h_{e f}}\right)$

Where $A_{N}$ is the actual projected area of stress cone of a single anchor, $A_{N o}$ is the projected area of stress cone of a single anchor unlimited by edge effect or spacing between the anchors. According to Fuchs et al. (1995), the strength of anchor with shallow embedment depth is underestimated by using ACI 349 and is unconservative for anchors with large embedment depth. In addition, the ACI 349-85 method calculates the failure surface area using complex calculations (Subramanian, 2000). The complexity appears in determining 
the angle and the actual projected area $\left(\mathrm{A}_{\mathrm{N}}\right)$ for the case of multiple anchors and thin concrete member (member thickness less than edge distance) (Fuchs et al., 1995).

ACI 318-11 Appendix D is proposed to design structural anchors that transfer structural loads to the concrete by tension, shear or combination of tension and shear. These anchors are used either to connect the structural elements or in safety related attachments that are assembled externally to the structure such as sprinkler system, pipes and barrier rails. The ACI 318-11 code is concerned with the levels of safety for the structural anchors. The combination of the load factor and the capacity reduction factors ( $\varnothing$ factors) represent the levels of safety. These levels of safety are suitable for structural applications. The load factors are used to increase the load to consider the uncertainties in determining the dead and live loads. Capacity reduction factors are used to reduce the strength of the material to consider the uncertainties in material strengths and dimensions. The ACI 318-11 design provisions depend on the $\mathrm{CCD}$ method theory in predicting the concrete breakout capacity, where the failure surface forms a $35^{\circ}$ cone. The CCD method coefficients were calibrated based on extensive experimental tests, the theory is based on 5\% fractile failure, $90 \%$ confidence level, this means that for $95 \%$ of the performed tests the actual strength of the anchor is more than the nominal strength. Adhesive anchors were included in the design provisions of ACI 318-11 for the first time, as the previous versions of ACI code didn't include the adhesive anchors in the design (ACI Committee 318, 2011).

ACI 318 proposed equations to calculate the tensile load for each failure mode. Steel anchor failure (Figure 2-5a) is likely to happen in high strength concrete, the strength of 
the steel anchor can be determined by Equation (4.2). Where the ultimate tensile strength is calculated as the minimum value of $1.9 f_{y}$ and $125000 \mathrm{psi}$, to assure there is no steel anchor yielding during the service loads (Cement Association of Canada, 2010).

According to ACI 318 the nominal concrete breakout capacity (Fig. 2-5b) for a single anchor can be determined as in Equation (4.16). For group of anchors, the concrete breakout capacity can be determined as follows:

$$
\begin{aligned}
& N_{c b g}=\frac{A_{N}}{A_{N o}} \psi_{e c, N} \cdot \psi_{e d, N} \cdot \psi_{c, N} \cdot \psi_{c p, N} \cdot N_{b} \\
& \psi_{e c, N}=\frac{1}{1+2 e_{N}^{\prime} /\left(3 h_{e f}\right)} \leq 1 \\
& \psi_{e d, N}=1 \text { if } c \geq 1.5 h_{e f} \\
& \psi_{e d, N}=0.7+0.3 \frac{c}{1.5 h_{e f}} \text { if } c<1.5 h_{e f}
\end{aligned}
$$

For cracked concrete at service load:

$\psi_{c, N}=1$ for both cast in place and postinstalled anchors

For the uncracked concrete at service load:

$\psi_{c, N}=\left\{\begin{array}{l}1.25 \text { for cast in anchors } \\ 1.4 \text { for postinstalled anchors }\end{array}\right.$ 
$\psi_{c p, N}= \begin{cases}1 & \text { if } c_{\text {min }} \geq c_{c} \\ \frac{c_{\text {min }}}{c_{c}} \geq \frac{1.5 h_{e f}}{c_{c}} & \text { if } c_{\text {min }}<c_{c}\end{cases}$

Where $A_{N}$ is the actual projected area, $A_{N o}$ is the projected area for the failure surface of concrete where $A_{N} \leq n A_{N o}, \psi_{e c, N}$ is a modification factor account for eccentricity effect for group anchors, $\psi_{e d, N}$ is a modification factor account for edge effect, $\psi_{c, N}$ is a modification factor account for cracking, $\psi_{c p, N}$ is a modification factor account for post installed anchors embedded in uncracked concrete without reinforcement to control concrete splitting, $c_{c}$ is the critical distance, $N_{b}$ is the basic concrete breakout strength of a single anchor and can be determined using Equation (4.17), $e_{N}^{\prime}$ is the distance between the resultant tensile force of the group anchors and the centroid of the group anchors.

The geometrical calculations of the projected area $A_{N o}$ and the actual area $A_{N}$ are shown in Figure A-1.
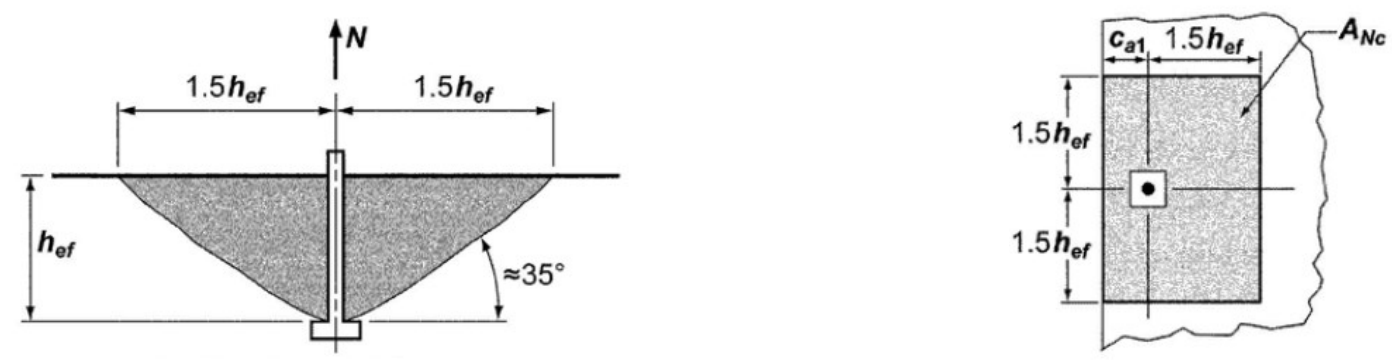

$$
A_{N}=\left(c_{a 1}+1.5 h_{e f}\right) \times\left(2 \times 1.5 h_{e f}\right) \text { For } c_{a 1}<1.5 h_{e f}
$$




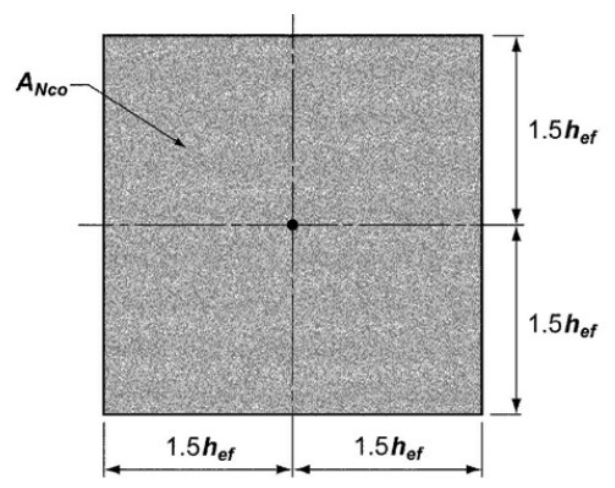

$A_{N o}=\left(2 \times 1.5 h_{e f}\right) \times\left(2 \times 1.5 h_{e f}\right)=9 h_{e f}^{2}$

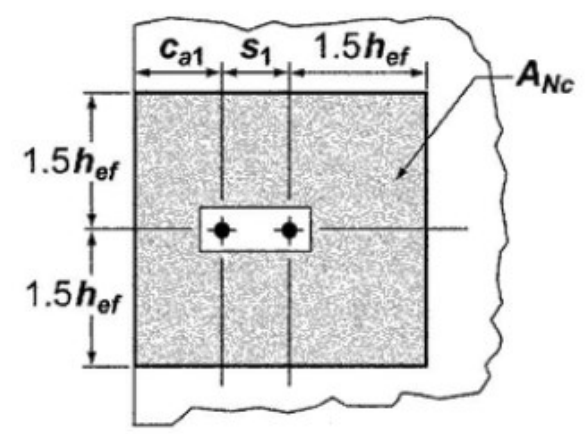

$$
A_{N}=\left(c_{a 1}+s_{1}+1.5 h_{e f}\right) \times\left(2 \times 1.5 h_{e f}\right)
$$

For $c_{a 1}<1.5 h_{e f}$ and $s_{1}<3 h_{e f}$

Figure A-1: Calculation of Projected area $A_{N o}$ and actual area $A_{N}$ for single anchor and double anchors (Cement Association of Canada 2010; ACI Committee 318 2011)

Side-face blowout failure (Figure 2-5c) occurs for single cast-in-place anchors with deep embedment depths and located near the concrete free edge (Cement Association of Canada, 2010; ACI Committee 318, 2011). The side face blowout strength can be determined as follows:

$N_{s b}=160 c_{1} \sqrt{A_{b r g}} \sqrt{f_{c}^{\prime}}$

Where $c_{1}<0.4 h_{e f}$

If $c_{2}<3 c_{1}$ Equation (A.7) shall be multiplied by $\left(1+\frac{c_{2}}{c_{1}}\right) / 4$ where the proportion of $\left(\frac{c_{2}}{c_{1}}\right)$ should be in the range of $1 \leq \frac{c_{2}}{c_{1}} \leq 3$

For group of anchors with deep embedment depths and $c_{1}<0.4 h_{e f}, s<6 c_{1}$, the strength of the anchors can be calculated as follows:

$$
N_{s b g}=\left(1+\frac{s}{6 c_{1}}\right) N_{s b}
$$


Where $A_{b r g}$ is the bearing area, $N_{s b}$ is the blowout strength for a single anchor, $N_{s b g}$ is the blowout strength for a group of anchors.

According to ACI 318 the pullout load of single anchor in tension can be determined as in Equation (A.9).

$N_{p n}=\psi_{c, p} \cdot N_{p}$

$\psi_{c, p}=\left\{\begin{array}{l}1 \quad \text { for craked concrete } \\ 1.4 \text { for uncracked concrete }\end{array}\right.$

$N_{p}=8 A_{b r g} \cdot f_{c}^{\prime}$

Where $N_{p n}$ is the pullout force of a single anchor, $N_{p}$ is the nominal pullout stress.

\section{A.1.2 Concrete Capacity Design (CCD)}

According to Concrete Capacity Design Method (CCD) the ultimate tensile load of the cast-in-place and post installed anchors in uncracked concrete can be determined as in Equation (4.3), where complete concrete cone is formed (Fuchs, 2001; Fuchs et al., 1995; Committee Euro-International du Beton (CEB), 1994; Subramanian, 2000; Ashour and Alqedra, 2005).

Large strain gradient in concrete for fastenings resulted in increasing the size effect and this behaviour is similar to linear elastic fracture mechanics (LEFM) mode. This means 
that, the nominal failure stress decreases with $\frac{1}{\sqrt{h_{e f}}}$ and the failure load increases with $h_{e f}^{1.5}$ (Fuchs et al., 1995).

When the anchor is placed near to the edge of the concrete, complete concrete cone will not be produced and resulted in a decrease in the ultimate tensile load. Also, when the anchors located near to each other, the ultimate tensile load will decrease due to the overlap in the concrete cone breakout. The CCD method takes into consideration the effect of edge distance, spacing, and eccentricity effect in determining the tensile load, and it uses simple geometric relations in calculating these effects (Fuchs et al., 1995).

For the edge distance effect, assuming that the tensile load is applied concentrically on the anchors, the ultimate tensile strength can be determined as in Equation (A.11).

$N_{u}=\frac{A_{N}}{A_{N O}} \cdot \psi_{2} \cdot N_{u o}$

Where $A_{N}$ is the actual projected area of stress cone of a single anchor. $A_{N o}$, is the projected area of single anchor, unlimited by edge effect or spacing between the anchors, adopting the approach of four-sided pyramids cone to predict the failure surface.

$$
A_{N o}=9 h_{e f}^{2}
$$

$\psi_{2}$ is a modification factor considering the disorder of the radial symmetric stress distribution due to the edge effect for the anchors far from the edge. Existence of free edge causes distortion in the stress distribution of the concrete around the anchor, this distortion 
is similar to that occurs in case of crack existence in the concrete around the anchor (Cement Association of Canada, 2010).

$\psi_{2}=1$ if $c_{1} \geq 1.5 h_{e f}, \psi_{2}=0.7+0.3 \frac{c_{1}}{1.5 h_{e f}}$ if $c_{1} \leq 1.5 h_{e f}$

where $c_{l}$ is the distance from center of anchor to edge of concrete in the direction of the applied load.

For the eccentricity effect and/or edge distance effect, the ultimate tensile strength can be determined as in Equation (A.14).

$N_{u}=\frac{A_{N}}{A_{N O}} \cdot \psi_{1} \cdot \psi_{2} \cdot N_{u o}$

$\psi_{1}$ is factor considering the eccentricity of the resultant tensile force on the anchors.

$\psi_{1}=\frac{1}{1+2 e_{N}^{\prime} /\left(3 h_{e f}\right)} \leq 1$

Where $e_{N}^{\prime}$ is the distance between the resultant tensile force of the set anchors and the centroid of these anchors. If the tensile load acts on one anchor of group anchors, the ultimate tensile load of the group of anchors is same as the ultimate tensile load of one anchor without spacing effects (Fuchs et al., 1995). 
When the largest edge distance $\left(\mathrm{c}_{\max }\right)$ is less than or equal to $1.5 h_{e f}$, embedment depth of $h_{e f}=\frac{c_{\max }}{1.5}$ is used in Equations (A.17) and (A.18) (Fuchs et al., 1995).

\section{A.2 Design methods for cast-in-place anchors under shear load}

\section{A.2.1 American concrete institute (ACI)}

ACI 349-85 design method assumes fully developed semi concrete cone with height equal to edge distance to predict the concrete failure surface. The concrete capacity for a single anchor is determined by assuming the angle between failure surface and the concrete surface $45^{\circ}$. ACI 349 is applied for the anchors used in safety related structures and nuclear power plant. The ultimate shear failure load for single anchor can be determined by Equation (A.16) (Fuchs et al., 1995; Subramanian, 2000; Fuchs, 2001; Zamora et al., 2003).

$V_{n o}=0.48 \sqrt{f_{c c}^{\prime}}\left(c_{1}\right)^{2}$

Where $V_{n o}$ is the ultimate shear load $(\mathrm{N}), f_{c c}^{\prime}$ is the cube compressive strength of concrete (MPa), and $c_{l}$ is the edge distance parallel to the direction of the applied load (mm).

For small concrete depth ( $h$ less than $c_{l}$ ) or anchor spacing, $s$, (less than $2 \times c_{l}$ ) or the edge distance perpendicular to the load direction, $c_{2}$, less than the edge distance parallel to the load, $c_{l}$, the ultimate shear failure load $\left(V_{n}\right)$ can be determined by the modified Equation (A.17) (Fuchs et al., 1995; Gross et al., 2001). 
$V_{n}=\frac{A_{v}}{A_{v o}} V_{n o}$

$A_{v o}=\frac{\pi}{2} c_{1}^{2}$

Where $A_{v}$ is the actual projected area considering edge effects and overlap with adjacent anchors and $A_{v o}$ is the projected area for one anchor unlimited by edge effects, concrete depth or cone overlapping.

According to ACI 318-11 the shear strength for the anchors can be calculated depending on the steel strength of the anchor, edge distance, concrete strength, and anchor spacing that in turn affects the failure mode.

Steel anchor failure (Figure 2-6a) occurs for anchors with deep embedment depths and far from the concrete edge, where the bending stresses in the anchor are higher than the tensile strength of the steel anchor. This failure characterized by bending followed by yielding and fracture the steel anchor. Concrete spall may be observed at the surface of the concrete in front of the anchor because of the high local pressure generated in front of the anchor (Cement Association of Canada, 2010). Steel strength of cast-in-place anchor under shear loading can be calculated as in Equation (4.18).

Concrete cone breakout failure (Figure 2-6c) occurs when the anchors located close to the concrete free edge and subjected to shear load towards the edge, where the tensile strength of the steel anchor is sufficient to prevent anchorage failure. According to ACI 318-11, 
assuming the failure surface makes $35^{\circ}$ cone with the surface of the concrete, the nominal concrete breakout strength for a single anchor subjected to shear load perpendicular to an edge can be determined as in the following Equation:

$V_{c b}=\frac{A_{v c}}{A_{v c o}} \cdot \psi_{e d, v} \cdot \psi_{c, v} \cdot V_{b}$

The concrete breakout strength for a group of anchors subjected to shear load can be determined as follows:

$V_{c b g}=\frac{A_{v c}}{A_{v c o}} \psi_{e c, v} \cdot \psi_{e d, v} \cdot \psi_{c, v} \cdot V_{b}$

$A_{v c o}=4.5 c_{1}^{2}$

$\psi_{e c, v}=\frac{1}{1+2 e_{v}^{\prime} /\left(3 c_{1}\right)} \leq 1$

$\psi_{e d, v}=1$ if $c_{2} \geq 1.5 c_{1}$

$\psi_{e d, V}=0.7+0.3 \frac{c_{2}}{1.5 c_{1}}$ if $c_{2}<1.5 c_{1}$

Where

$\psi_{c, V}=1.4$ in uncracked concrete 
The geometrical calculations of the projected area $A_{c o}$ are shown in Figure A-2.

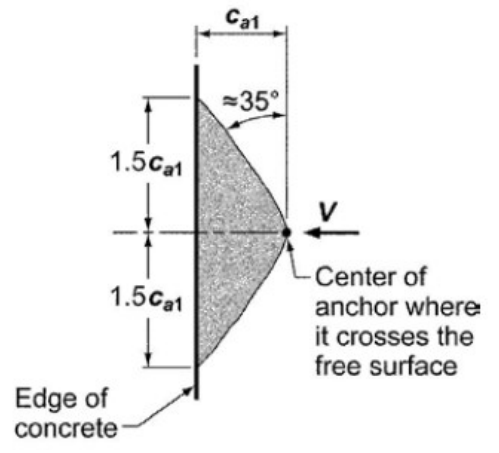

The critical edge distance is equal to $1.5 \mathrm{c}_{\mathrm{a} 1}$ for headed studs, headed bolts, expansion anchors, and undercut anchors

(a) Plan view

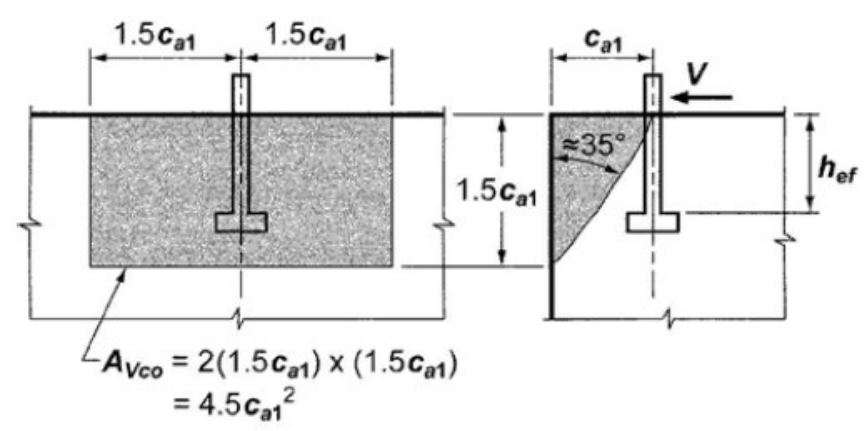

(b) Front view

(c) side view

Figure A-2: Calculation of projected area $A_{v c o}$ for single anchor (Cement Association of Canada, 2010; ACI Committee 318, 2011)

Where $A_{v c}$ is the actual projected area, $A_{v c o}$ is the projected area for the failure surface of concrete, $A_{v c}$ and $A_{v c o}$ are calculated assuming the failure surface as a half pyramid projected on the face of the concrete near the edge, where $A_{v c} \leq n A_{v c o}, \psi_{e c, v}$ is a modification factor accounts for eccentricity effect for group anchors subjected to eccentrically shear load, $\psi_{e d, v}$ is a modification factor accounts for edge effect, $\psi_{c, v}$ is a modification factor accounts for cracking, $V_{b}$ is the basic concrete breakout strength of a single anchor subjected to shear load in cracked concrete, $V_{c b}$ is the nominal shear strength 
acting perpendicular to the free edge of a single anchor, $V_{c b g}$ is the shear strength acting perpendicular to the free edge of a group anchors.

When the shear load acts parallel to the concrete free edge the shear load value obtained from Equations (A.19) and (A.20) will be doubled, with $\psi_{e d, v}=1$. For the anchors located far from the concrete free edge Equations (A.19 and A.20) will not be used where the concrete break out failure will not occur (ACI Committee 318, 2011).

Concrete pryout failure (Figure 2-6b) occurs when the anchors located far from the concrete free edge. The failure characterized by concrete cracking in the direction of the applied shear load accompanied by concrete spalling in the opposite direction. The concrete spalling initiated behind the anchor at its lower end and propagates forming a conical shape towards the surface of the concrete. This type of failure occurs for stiff anchors with shallow embedment depths (Cement Association of Canada, 2010). The shear load of the anchor that exhibits pryout failure can be calculated as in Equation (4.14) (ACI Committee $318,2011)$. The ACI 318 calculates the pryout capacity depending on the tensile concrete breakout capacity multiplied by a factor to consider the shear effect (ACI Committee 318, 2011). The ACI 318 method takes into account the effect of embedment depth and didn't consider the influence of anchor diameter in the calculation of the shear load for the case of the pryout failure.

Jebara et al. (Jebara et al., 2016) proposed an equation to determine the pryout capacity of cast-in-place concrete anchors embedded in concrete based on a regression analysis made as follows: 


$$
V_{c p}=6 \sqrt{d} \sqrt{f_{c c}}\left(h_{e f}\right)^{1.5}
$$

\section{A.2.2 Concrete Capacity Design (CCD)}

The ultimate shear load of single anchor in uncracked concrete can be determined in accordance with the CCD method as in Equation (A.26) (Fuchs, 2001; Fuchs et al., 1995; Committee Euro-International du Beton (CEB), 1994; Subramanian, 2000; Ashour and Alqedra 2005). The shear load is calculated based on the test results of a single anchor diameter of $d \leq 25$ and effective length of $h_{e f} \leq 8 d_{o}$ embedded in thick uncracked concrete (Fuchs et al., 1995).

$V_{n o}=\left(\frac{h_{e f}}{d}\right)^{0.2} \sqrt{d f_{c c}^{\prime}}\left(c_{1}\right)^{1.5}$

Where $h_{e f}$ is the effective length or embedment depth of cast-in-place anchors (mm), and $d$ is the diameter of the anchor (mm). As presented in Equation (A.26), the increment in

the ultimate shear load is proportional to $c_{1}^{1.5}$ due to the size effect. Also, the failure load is affected by the anchor stiffness and diameter (Fuchs et al., 1995; Subramanian, 2000). When the anchor or group of anchors is located near the edge of concrete specimen, the shear capacity for the concrete calculated in accordance with the CCD method is adjusted in accordance with Equation (A.27).

$V_{n}=\frac{A_{v}}{A_{v o}} \cdot \psi_{4} \cdot \psi_{5} \cdot V_{n o}$ 


$$
\psi_{4}=\frac{1}{1+2 e_{v}^{\prime} /\left(3 c_{1}\right)}
$$

$$
\psi_{5}= \begin{cases}1 & \text { for } c_{2} \geq 1.5 c_{1} \\ 0.7+0.3 \frac{c_{2}}{1.5 c_{1}} & \text { for } c_{2} \leq 1.5 c_{1}\end{cases}
$$

Where $A_{v}$ is the actual shear breakout area, $A_{v o}$ is the projected area for one anchor unlimited by edge effects, concrete depth or cone overlapping, adopting the approach of halfpyramids to predict the failure surface, $\psi_{4}$ is the eccentricity effect of shear load on anchor groups, $\psi_{5}$ is a modification factor takes into consideration disorder of symmetric stress distribution resulting from edge effects, $e_{v}^{\prime}$ is the distance between resultant shear force of the anchor group and the centroid of the anchors.

The CCD method assumes the fracture shear area as a half pyramid, the effect of edge distance and spacing is calculated based on rectangular projected area. This assumption makes the CCD method relatively simple compared to the ACI 349 method that assumes circular projected area in the calculation. In addition, the CCD method is concerned in the disorder of the stresses in the concrete due to the edge effect or eccentricity effect, while ACI 349 neglect this effect (Fuchs et al., 1995; Subramanian, 2000). 


\section{Appendix B: Design methods for adhesive anchors}

\section{B.1 Design methods for adhesive anchors under tensile load}

\section{B.1.1 Concrete cone breakout failure}

According to the Concrete Capacity Design Method (CCD), the pullout capacity for adhesive anchors under tensile load was introduced by Eligehausen et al.(1984) as in Equation (B.1)(Cook et al., 1998).

$N_{u}=0.92 h_{e f}^{2} \sqrt{f_{c}^{\prime}}$

Where $\mathrm{N}_{\mathrm{u}}$ is the pullout force $(\mathrm{N})$.

Cook et al. 1998 introduced another equation for determining the pullout capacity of adhesive anchors under tensile load and based on the model developed by Fuchs et al. 1995 for cast-in-place anchors (Cook et al.,1998).

$N_{u}=16.5 h_{e f}^{1.5} \sqrt{f_{c}^{\prime}}$

Anchor pullout failure occurs for the adhesive anchor when the friction between the anchor and the concrete is less than the applied tensile load. According to the American Concrete Institute method, the pullout capacity for adhesive anchors under tensile load is given as in the following equation ( Eligehausen, 1987; Fuchs et al., 1995; Gesoglu et al., 2005): 


$$
N_{u}=f_{c t} A_{N}
$$

$$
f_{c t}=0.33 \sqrt{f_{c}^{\prime}}
$$

$$
A_{N}=\pi h_{e f}^{2}\left(1+\frac{d_{h}}{h_{e f}}\right)
$$

Where

$f_{c t}$ is the concrete capacity, $\mathrm{A}_{\mathrm{N}}$ is the projected area of a single anchor, $d_{h}$ is the diameter of the anchor head, where anchor diameter (d) is used instead of $d_{h}$ for the adhesive anchors (Gesoglu et al., 2005).

Hence, the equation (B.3) can be expressed as follows (Eligehausen, 1987)

$$
N_{u}=1.043 \sqrt{f_{c}^{\prime}} h_{e f}^{2}\left(1+\frac{d_{h}}{h_{e f}}\right)
$$

\section{B.1.2 Anchor steel failure}

The pullout failure load for the steel anchor can be determined using Equation (4.2).

\section{B.1.3 Bond failure}

For the uniform bond stress model, where the bond failure occurs at the steel/adhesive interface, the pullout force can be predicted as follows (McVay et al., 1996; Cook et al., 1998; Cook, 1993; Eligehausen et al., 2007):

$$
N_{u}=\tau_{o} \pi d_{o} h_{e f}
$$


Where $\mathrm{N}_{\mathrm{u}}$ is the pullout force $(\mathrm{N}), \tau_{o}$ is the uniform bond stress in the adhesive layer (MPa), $d_{o}$ is the diameter of the hole $(\mathrm{mm}), h_{e f}$ is the effective embedment depth (mm).

According to Cook et al. (1991) and McVay et al.(1996), the uniform bond stress of the adhesive is in the range between (9-13 MPa). Equation (B.7) is suitable for $4 \leq \frac{h_{e f}}{d} \leq$ 20, $d \leq 50 \mathrm{~mm}$ and bond area of $\pi d h_{e f} \leq 58000 \mathrm{~mm}^{2}$ (Eligehausen et al., 2006).

On the other hand, for the uniform bond stress model, where the bond failure occurs at the adhesive/concrete interface, the pullout force can be predicted as follows (Cook et al., 1998).

$N_{u}=\tau_{o} \pi d_{o} h_{e f} \sqrt{\frac{f_{c}^{\prime}}{f_{c, \text { low }}^{\prime}}}$

Where $f_{c, \text { low }}^{\prime}$ is the low strength concrete.

For the elastic bond stress model, the bond strength can be predicted as in the following Equation (McVay et al., 1996; Cook et al., 1998; Cook et al., 1993; Cook, 1993):

$N_{u}=\tau_{\max } \pi d_{o}\left[\frac{\sqrt{d_{o}}}{\lambda^{\prime}} \tanh \frac{\lambda^{\prime} h_{e f}}{\sqrt{d_{o}}}\right]$

Where $\tau_{\max }$ is the maximum bond stress in the adhesive layer, $\lambda^{\prime}$ is the elastic constant that depends on the shear stiffness of the adhesive-concrete system and axial stiffness of the anchor, $\lambda^{\prime}$ is independent of the hole diameter. The maximum bond stress of the adhesive 
is in the range between (10-14.5 MPa) according to Cook et al. (Cook et al., 1991) and McVay et al. (McVay et al., 1996).

Similar results can be obtained from the uniform bond stress model and elastic bond stress model for embedment depths up to $40 \sqrt{d_{o}}$. Over prediction of the pullout force can be seen in the uniform bond stress model for the embedment depths higher than $40 \sqrt{d_{o}}$ (McVay et al., 1996; Cook et al., 1993).

\section{B.1.4 Combined cone-bond failure}

According to Cook (1993), the pullout failure load for the combined cone-bond model can be determined as in Equation (B.10).

$$
N_{u}=N_{\text {cone }}+N_{\text {bond }}
$$

So that, by combining concrete cone breakout failure with bond failure, combined conebond capacity equation can be written as follows for the uniform bond stress (McVay et al., 1996; Cook, 1993):

$N_{u}=0.92 h_{\text {cone }}^{2} \sqrt{f_{c}^{\prime}}+\tau_{o} \pi d_{o}\left(h_{e f}-h_{\text {cone }}\right)$

The concrete cone depth $\left(h_{\text {cone }}\right)$ at the minimum failure load can be determined by taking the derivative of the combined cone-bond capacity equation with respect to the cone depth as follows: 
$\frac{d N_{u}}{d h_{\text {cone }}}=0$

So that,

$$
h_{\text {cone }}=\frac{\tau_{o} \pi d_{o}}{1.84 \sqrt{f_{c}^{\prime}}}
$$

For the elastic bond stress, the combined cone-bond failure load can be determined as in the following Equation (McVay et al., 1996; Cook, 1993):

$$
N_{u}=0.92 h_{\text {cone }}^{2} \sqrt{f_{c}^{\prime}}+\tau_{\max } \pi d_{o}\left[\frac{d_{o}}{\lambda^{\prime}} \tan \frac{\lambda^{\prime}\left(h_{\left.e f-h_{\text {cone }}\right)}\right.}{\sqrt{d_{o}}}\right]
$$

By taking the derivative for Equation (B.14) with respect to $h_{\text {cone, }}$ resulted in the following Equation for the concrete cone depth:

$$
h_{\text {cone }}=\frac{\tau_{\max } \pi d_{o}}{1.84 \sqrt{f_{c}^{\prime}}}\left(\operatorname{sech}^{2} \frac{\lambda^{\prime}\left(h_{e f}-h_{\text {cone }}\right.}{\sqrt{d_{0}}}\right)
$$

The uniform shear stress $\left(\tau_{0}\right)$ and maximum shear stress $\left(\tau_{\max }\right)$ are identical for shallow embedment depths. While, the maximum shear stress $\left(\tau_{\max }\right)$ becomes higher than the uniform shear stress $\left(\tau_{0}\right)$ for the large embedment depths (Cook, 1993). 
For small concrete size, anchors located near to the edge of the concrete or small spacing distance between the anchors, concrete splitting would occur as shown in Figure 2-3d (Cement Association of Canada, 2010; Rao and Arora, 2013).

\section{B.2 Design methods for adhesive anchors under shear load}

The shear load of adhesive anchors can be determined according to Concrete Capacity Design method (CCD), American Concrete Institute (ACI) method and Precast/ Prestressed Concrete Institute (PCI) method.

The failure surface of the concrete become a semi-cone when the concrete thickness is less than edge distance parallel to the direction of the applied load $\left(\mathrm{c}_{1}\right)$, and or the spacing between two anchors (s) is less than $2 \mathrm{c}_{1}$, or $\left(\mathrm{c}_{2}\right)$ is less than $\mathrm{c}_{1}$ (Alqedra and Ashour, 2005; Fuchs et al., 1995).

The shear load of the adhesive anchor can be calculated based on (ACI 349-78) as follows (Ueda et al., 1990):

$$
V_{u}=0.522 c_{1}^{2} \sqrt{f_{c}^{\prime}}
$$

The anchor shear strength in Equation (B.16) is derived based on the tensile strength of the concrete acts on the projected area of half cone shaped (Ueda et al., 1990).

ACI 318 can be used to predict the ultimate load for cast-in-place and post installed expansion and undercut anchors (ACI Committee 318, 2005). According to ACI 318-05, the ultimate shear load can be predicted as follows: 
$V_{b}=0.6\left(\frac{h_{e f}}{d_{o}}\right)^{0.2} \sqrt{d_{o}} \sqrt{f_{c}^{\prime}}\left(c_{1}\right)^{1.5}$

ACI 318-11 deals with the structural anchors and can be used to predict the ultimate load for cast-in-place and post installed anchors including the adhesive anchors (ACI Committee 318,2011$)$. The failure surface of the concrete makes a $35^{\circ}$ angle with the contact concrete edge. Impact and blast loads on anchors are not included in the ACI 31811.

For single anchor, where the shear force perpendicular to the edge, the concrete breakout shear strength can be calculated as follows:

$V_{c b}=\frac{A_{v c}}{A_{v c o}} \psi_{e d, v} \cdot \psi_{c, v} \cdot \psi_{h, v} \cdot V_{b}$

$\psi_{c, v}=\left\{\begin{array}{cl}1 & \text { for anchors in cracked concrete without reinforcement } \\ 1.2 & \text { for anchors in cracked concrete with reinforcement } \\ 1.4 & \text { for anchors in uncracked concrete }\end{array}\right.$

$\psi_{h, v}=\sqrt{\frac{1.5 c_{1}}{h}}$

According to CCD method, the shear load of the adhesive anchor can be calculated as follows (Gesoğlu et al., 2014; Bickel and Shaikh, 2002):

$V_{u}=1.1\left(\frac{h_{e f}}{d_{0}}\right)^{0.2} \sqrt{d_{0}} \sqrt{f_{c c}^{\prime}} c_{1}^{1.5}$

Equation (B.20) can be used for calculating the shear force of a single anchor embedded into thick uncracked concrete, where the shear load is applied towards the free edge (Bickel and Shaikh 2002). 
Fuchs et al (1995) proposed equation (B.20) based on experimental shear tests for a single anchor with diameter $d_{o} \leq 25 \mathrm{~mm}$ and $h_{e f} \leq 8 d_{o}$. In order to include a wide range of anchor diameters and embedment depths Equation (B.20) is modified. The modification of the Equation (B.20) is based on experimental tests and numerical simulations. According to the modified CCD method the shear capacity of the adhesive anchor can be calculated as follows (Lee et al., 2011; Spyridis and Bergmeister, 2014):

$V_{u}=3 d_{o}^{a} h_{e f}^{b} \sqrt{f_{c c}^{\prime}} c_{1}^{1.5}$

Where

$a=0.1\left(\frac{h_{e f}}{c_{1}}\right)^{0.5}, b=0.1\left(\frac{d_{0}}{c_{1}}\right)^{0.2}$

Where $V_{u}$ is the shear capacity of the anchor near to edge and $h_{e f}$ is the effective length.

PCI 1978 can predict the failure of the concrete based on a conical failure surface. Since 2004 the PCI method relied on the ACI 318 which is in turn based on the CCD method by adopting the approach of four-sided pyramids cone to predict the failure surface (Pallarés and Hajjar, 2009). PCI method is more suitable for predicting the shear capacity for adhesive anchors than cast-in-place anchors (Bickel and Shaikh, 2002). According to Precast/ Prestressed Concrete Institute (PCI) Design Handbook (precast-prestressed concrete Institute, 1999), the shear capacity of single anchor in uncracked concrete can be calculated as follows:

$V_{u}=5.2 c_{1}^{1.5} \sqrt{f_{c}^{\prime}}$ 


\section{Appendix C:Tensile load-displacement relation for cast-in place anchors}

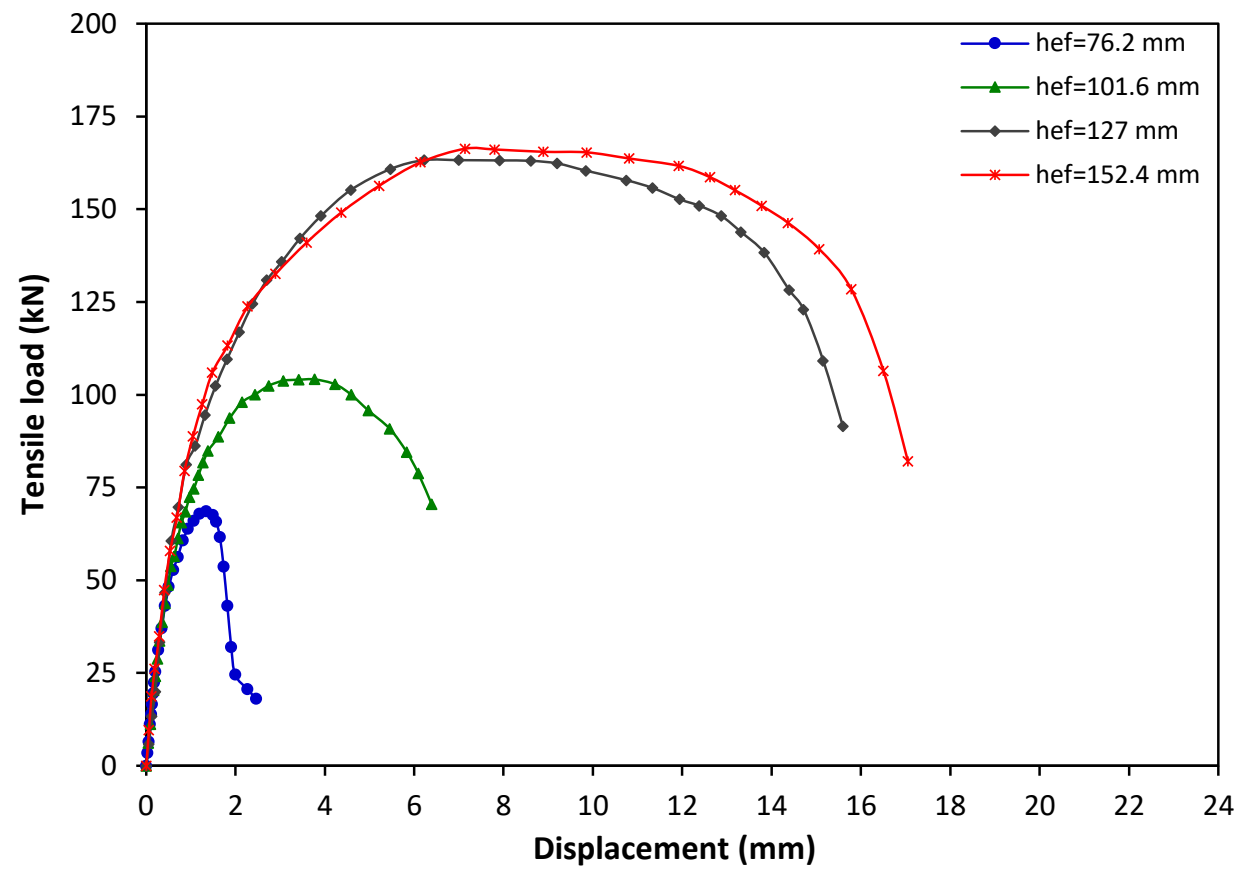

Figure C-1: Tensile load-displacement graph for $15.9 \mathrm{~mm}$ diameter cast-in-place anchor at strain rate of $10^{-5} \mathrm{~s}^{-1}$

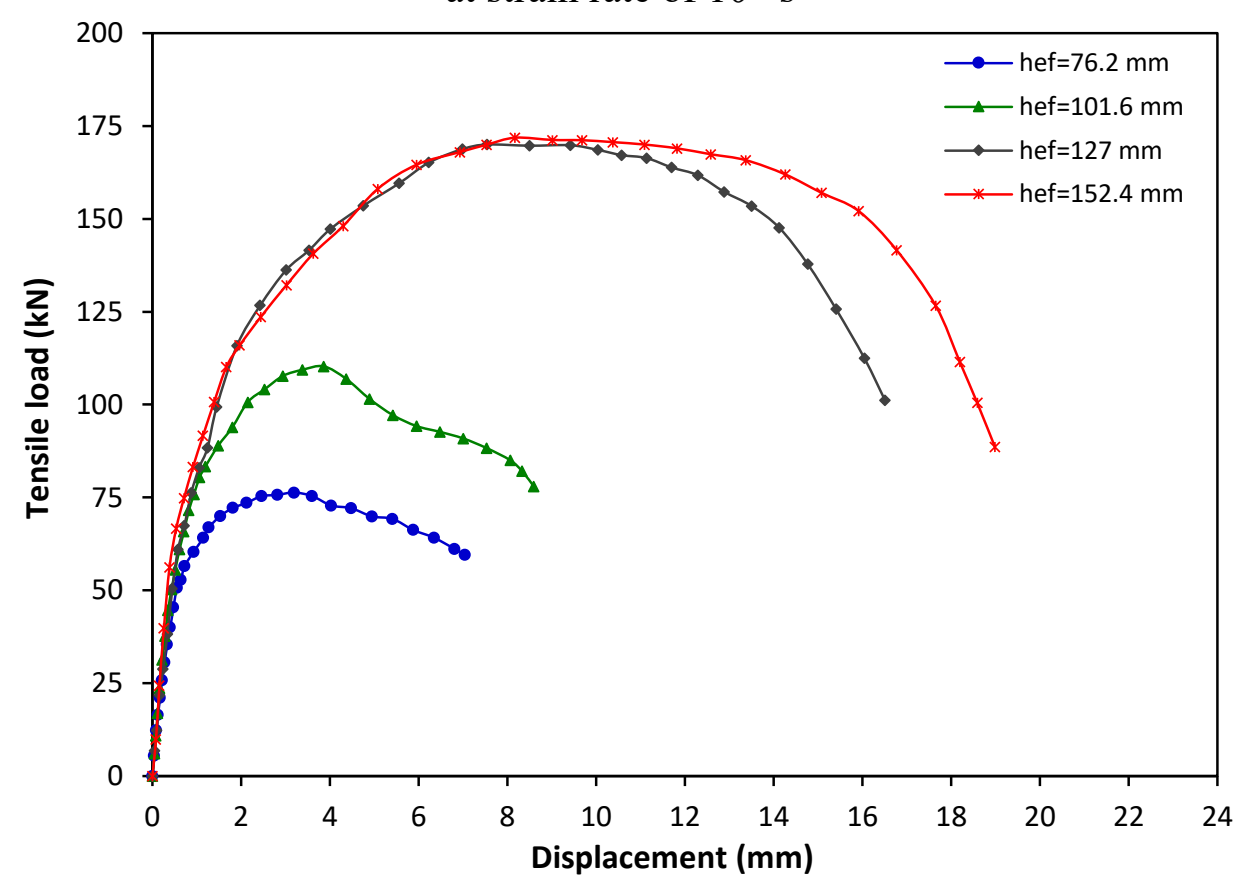

Figure C-2: Tensile load-displacement graph for $15.9 \mathrm{~mm}$ diameter cast-in-place anchor at strain rate of $10^{-3} \mathrm{~s}^{-1}$ 


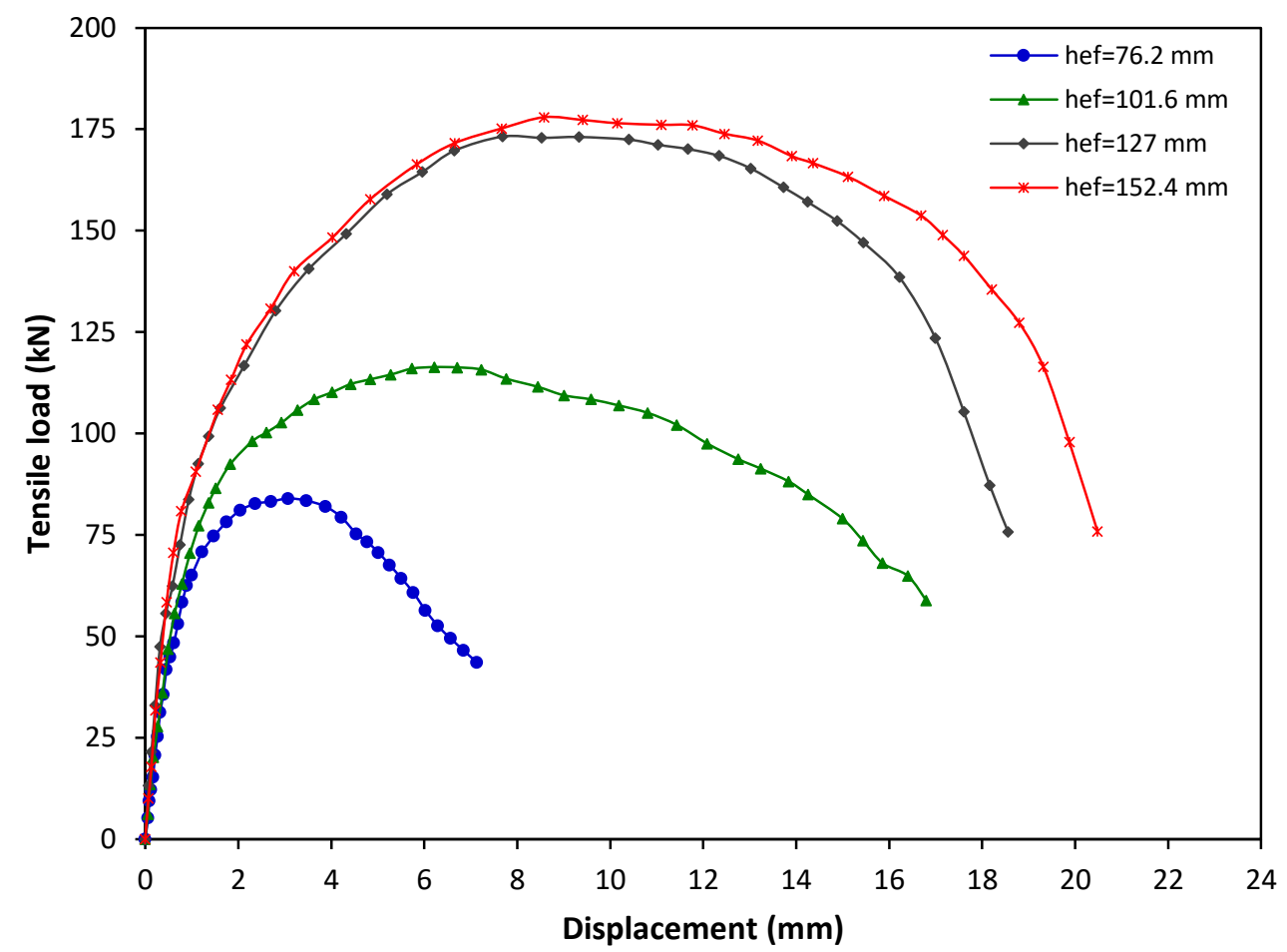

Figure C-3: Tensile load-displacement graph for $15.9 \mathrm{~mm}$ diameter cast-in-place anchor at strain rate of $10^{-1} \mathrm{~s}^{-1}$

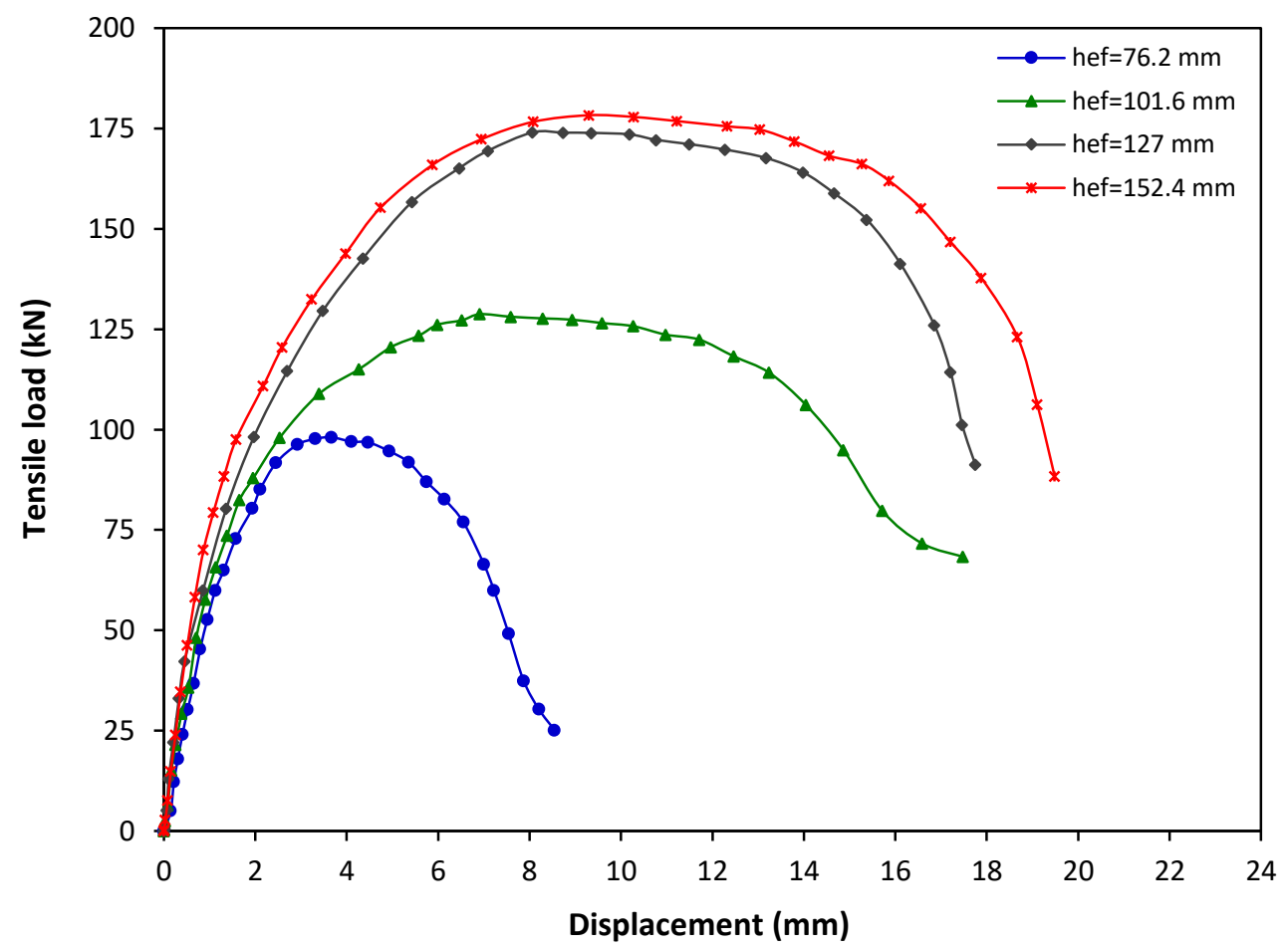

Figure C-4: Tensile load-displacement graph for $15.9 \mathrm{~mm}$ diameter cast-in-place anchor at strain rate of $10 \mathrm{~s}^{-1}$ 


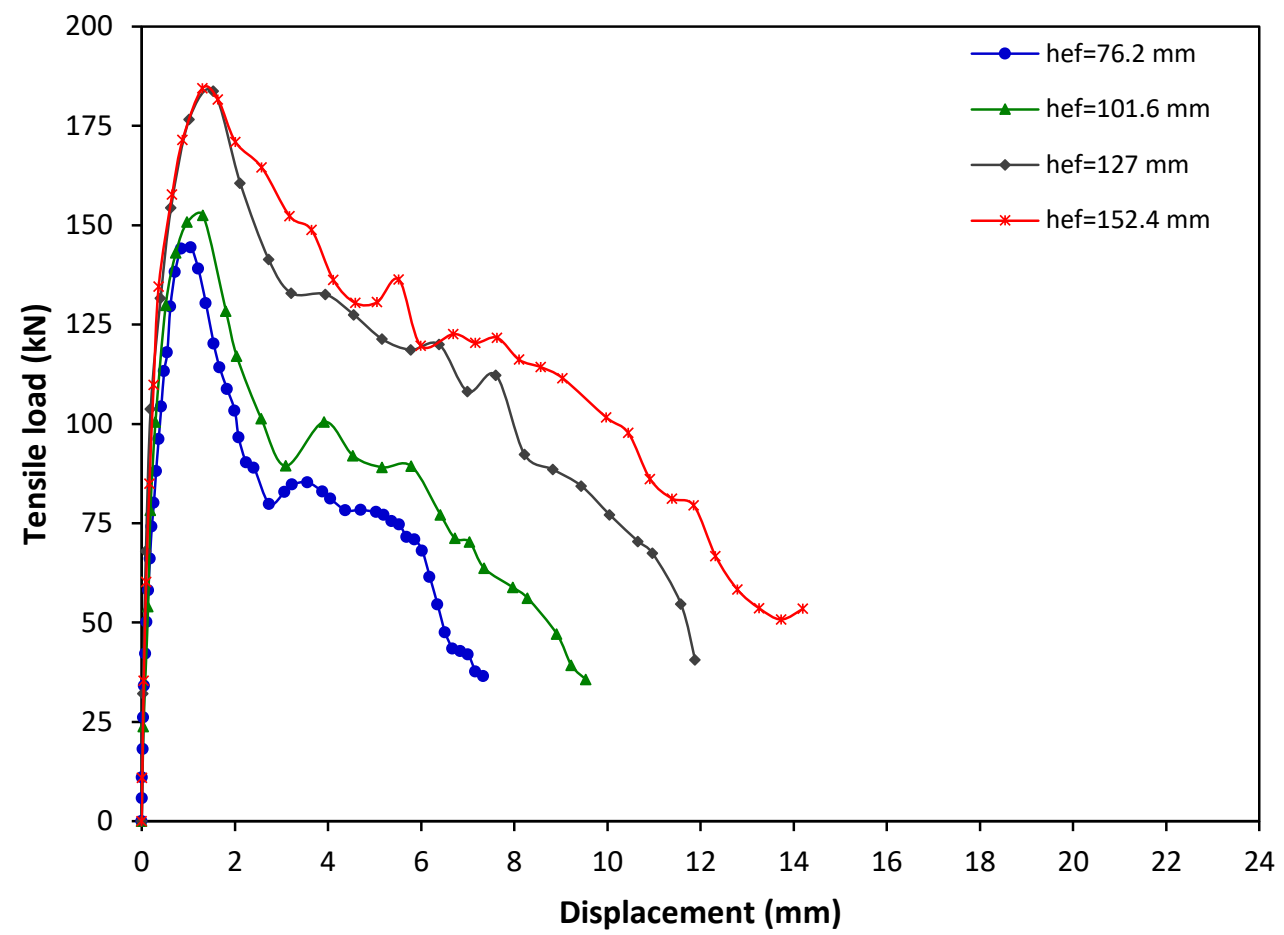

Figure C-5: Tensile load-displacement graph for $15.9 \mathrm{~mm}$ diameter cast-in-place anchor at strain rate of $10^{2} \mathrm{~s}^{-1}$

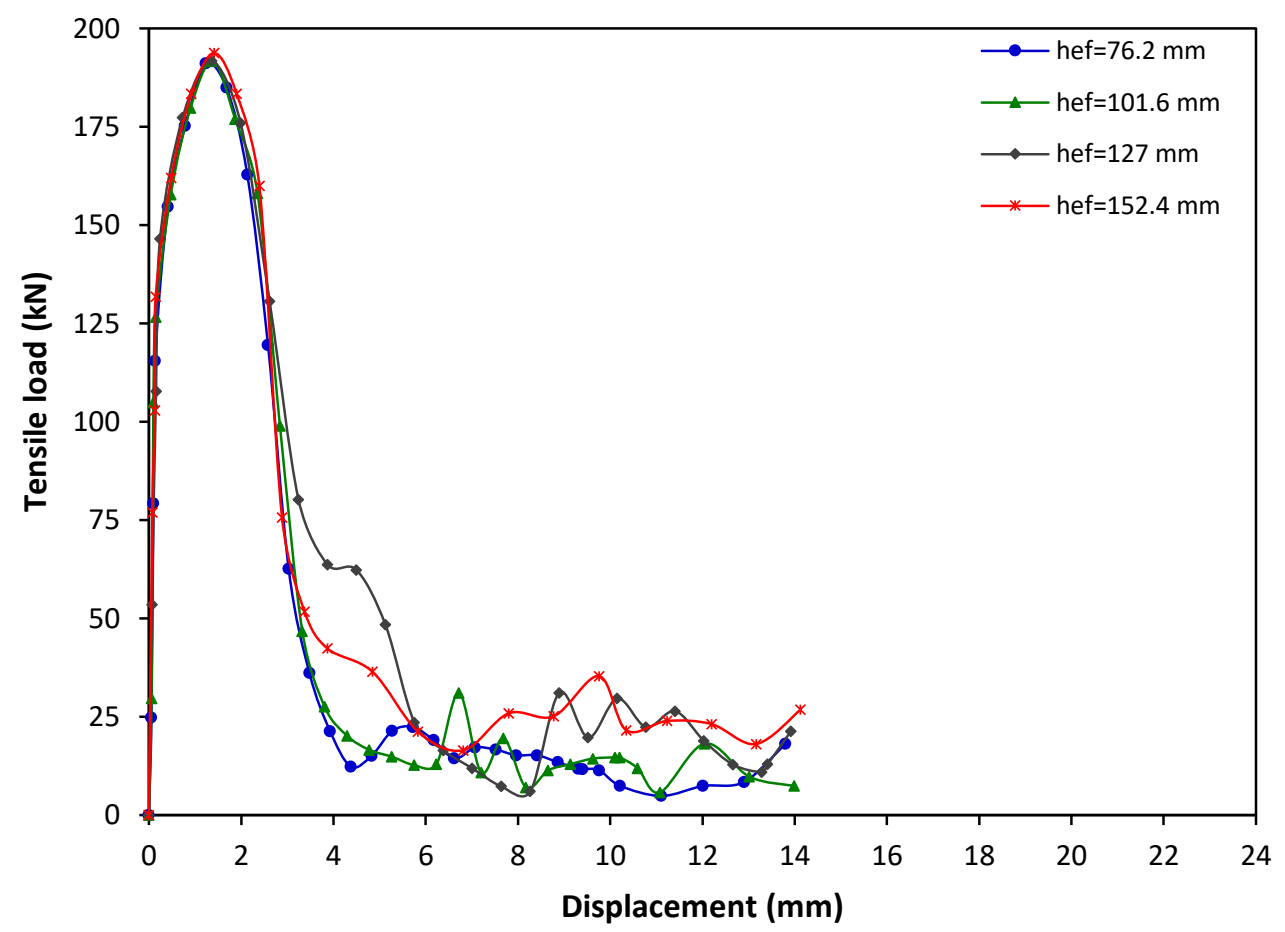

Figure C-6: Tensile load-displacement graph for $15.9 \mathrm{~mm}$ diameter cast-in-place anchor at strain rate of $10^{3} \mathrm{~s}^{-1}$ 


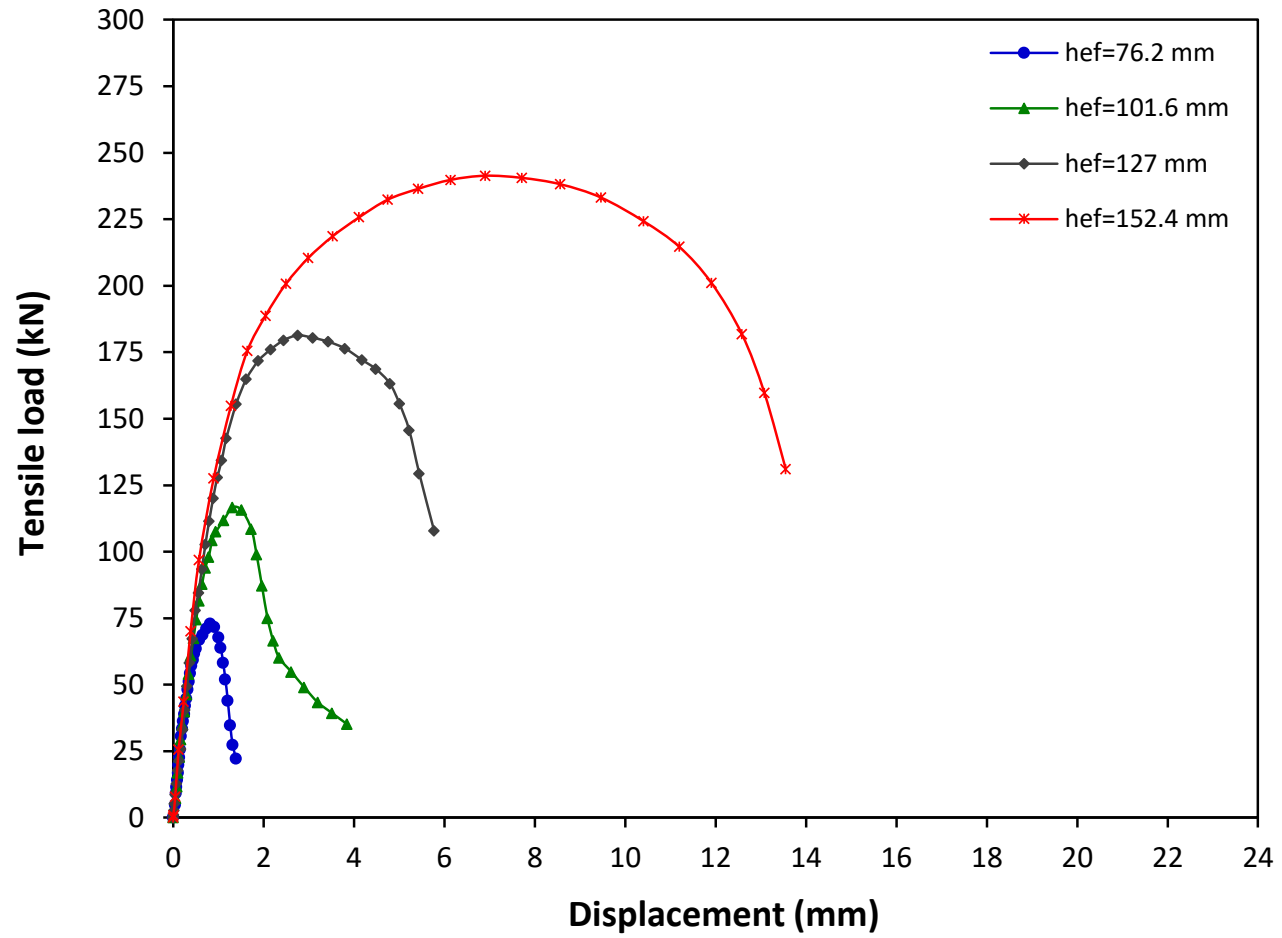

Figure C-7: Tensile load-displacement graph for $19.1 \mathrm{~mm}$ diameter cast-in-place anchor at strain rate of $10^{-5} \mathrm{~s}^{-1}$

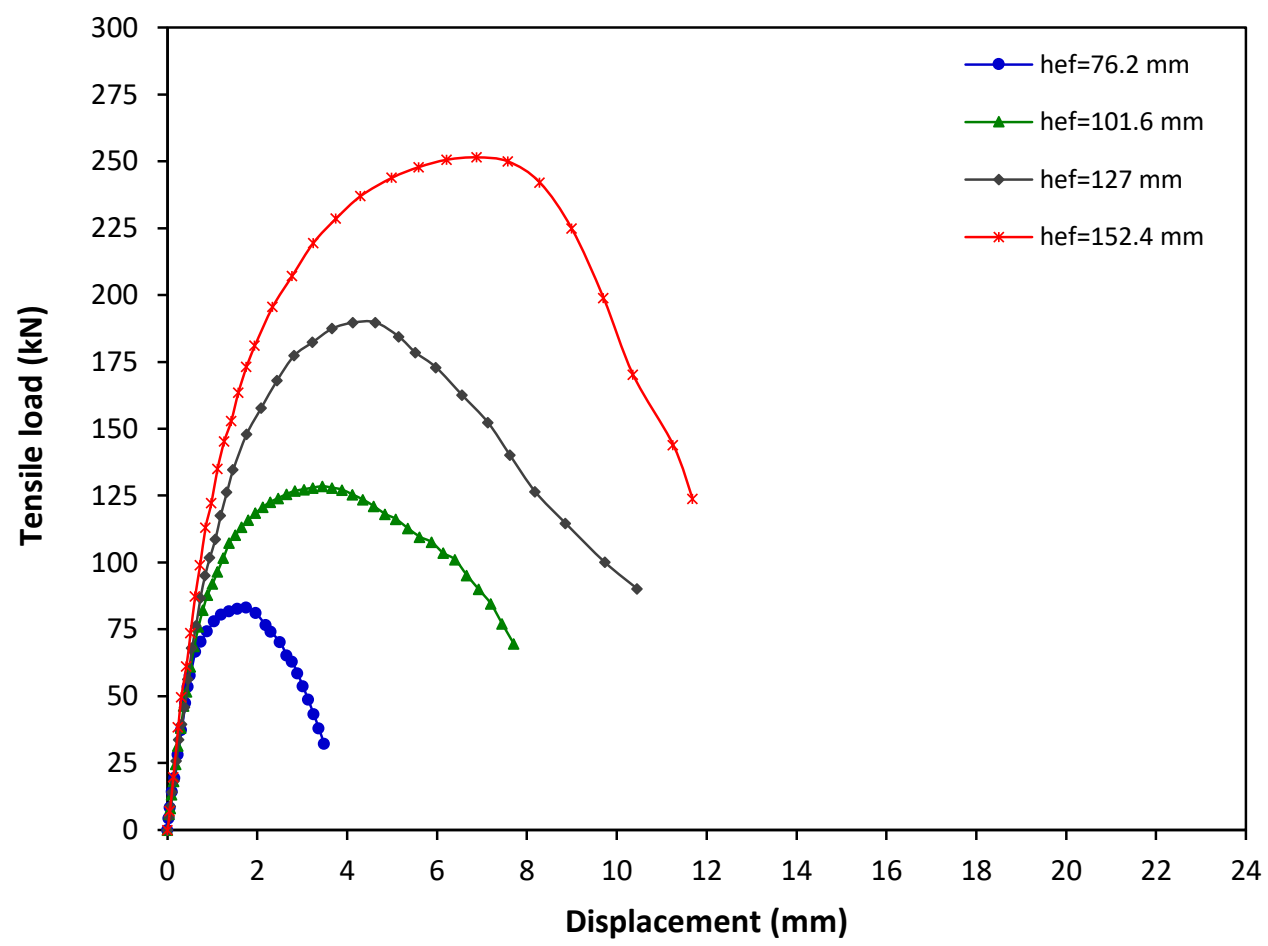

Figure C-8: Tensile load-displacement graph for $19.1 \mathrm{~mm}$ diameter cast-in-place anchor at strain rate of $10^{-3} \mathrm{~s}^{-1}$ 


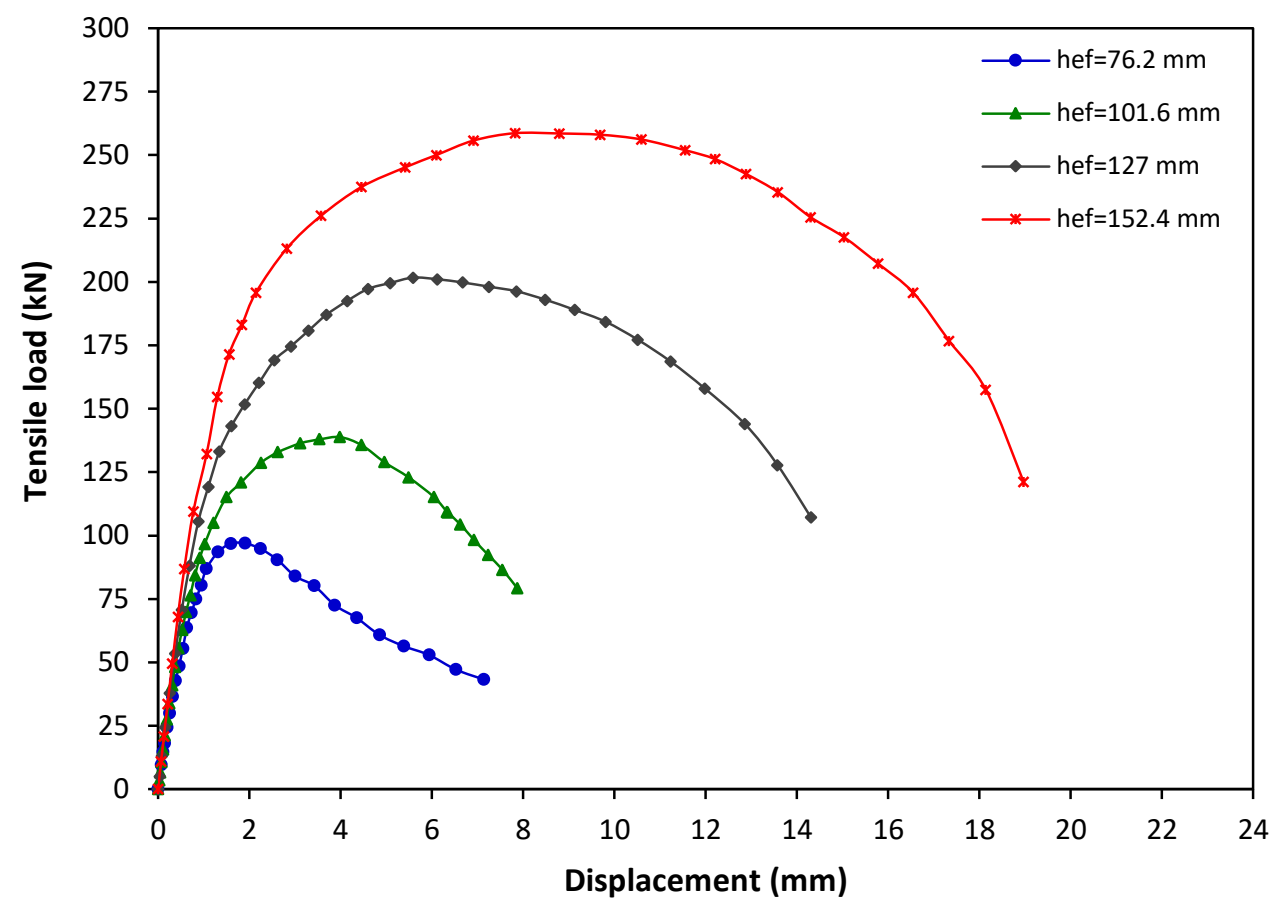

Figure C-9: Tensile load-displacement graph for $19.1 \mathrm{~mm}$ diameter cast-in-place anchor at strain rate of $10^{-1} \mathrm{~s}^{-1}$

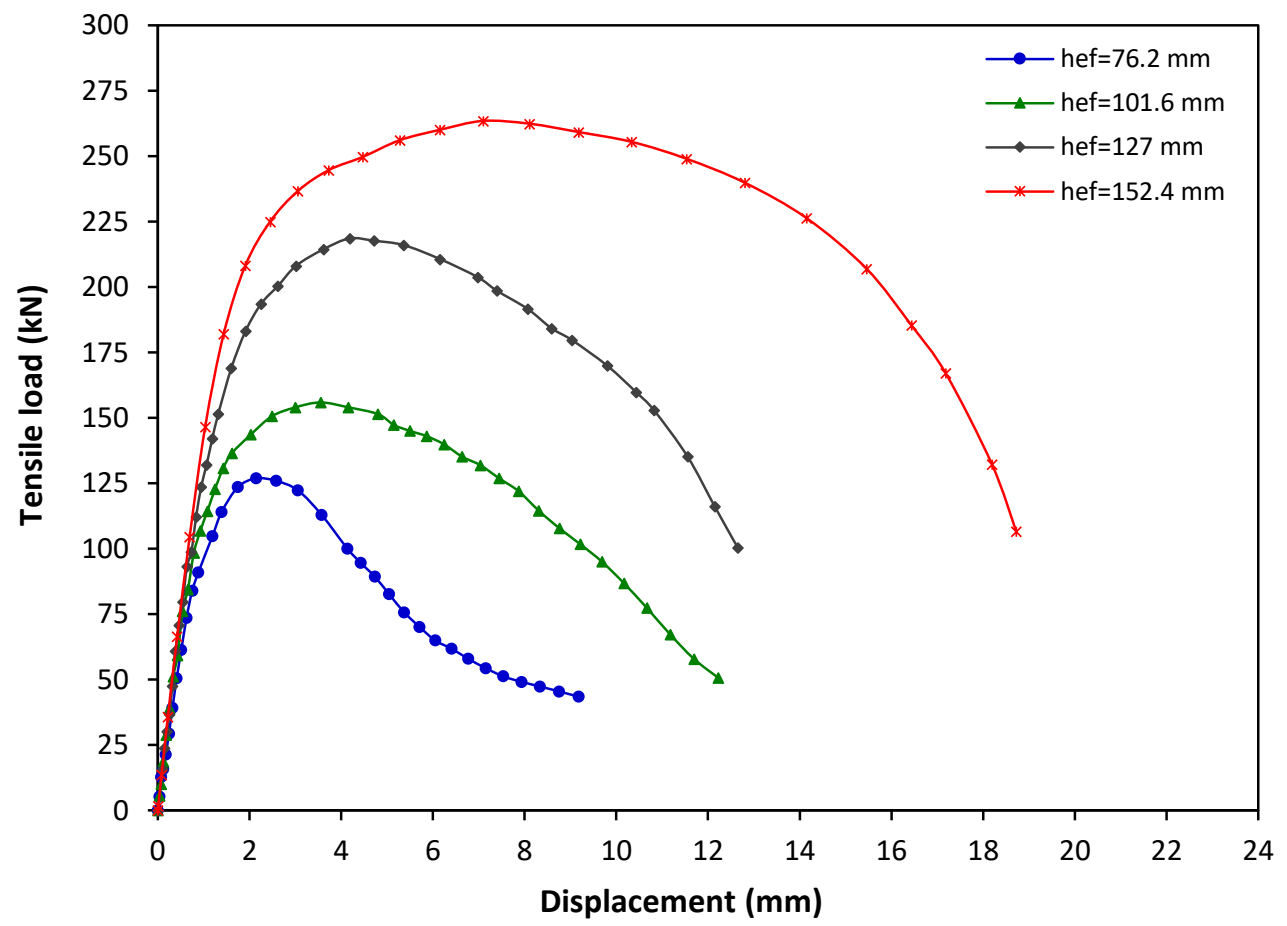

Figure C-10: Tensile load-displacement graph for $19.1 \mathrm{~mm}$ diameter cast-in-place anchor at strain rate of $10 \mathrm{~s}^{-1}$ 


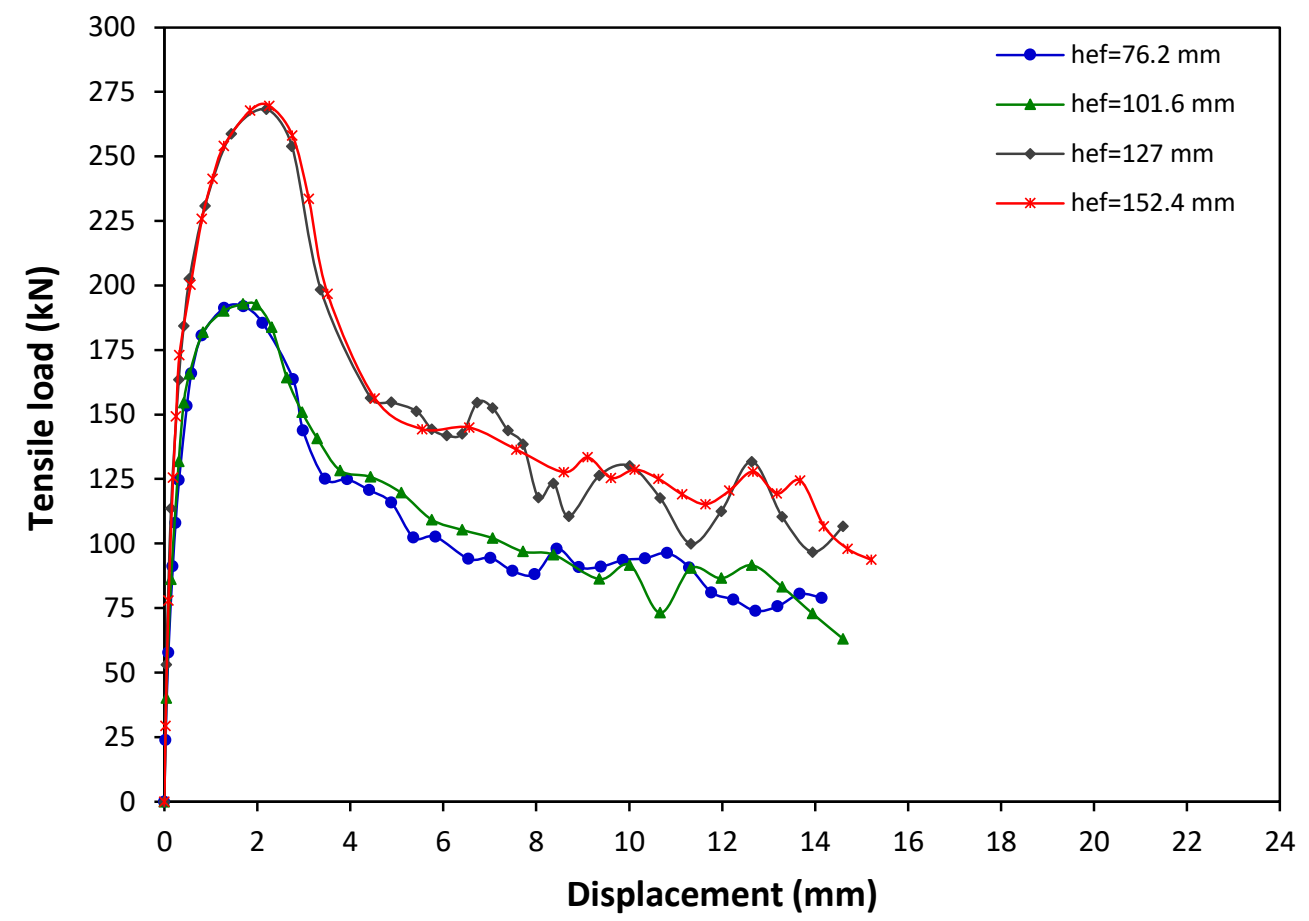

Figure C-11: Tensile load-displacement graph for $19.1 \mathrm{~mm}$ diameter cast-in-place anchor at strain rate of $10^{2} \mathrm{~s}^{-1}$

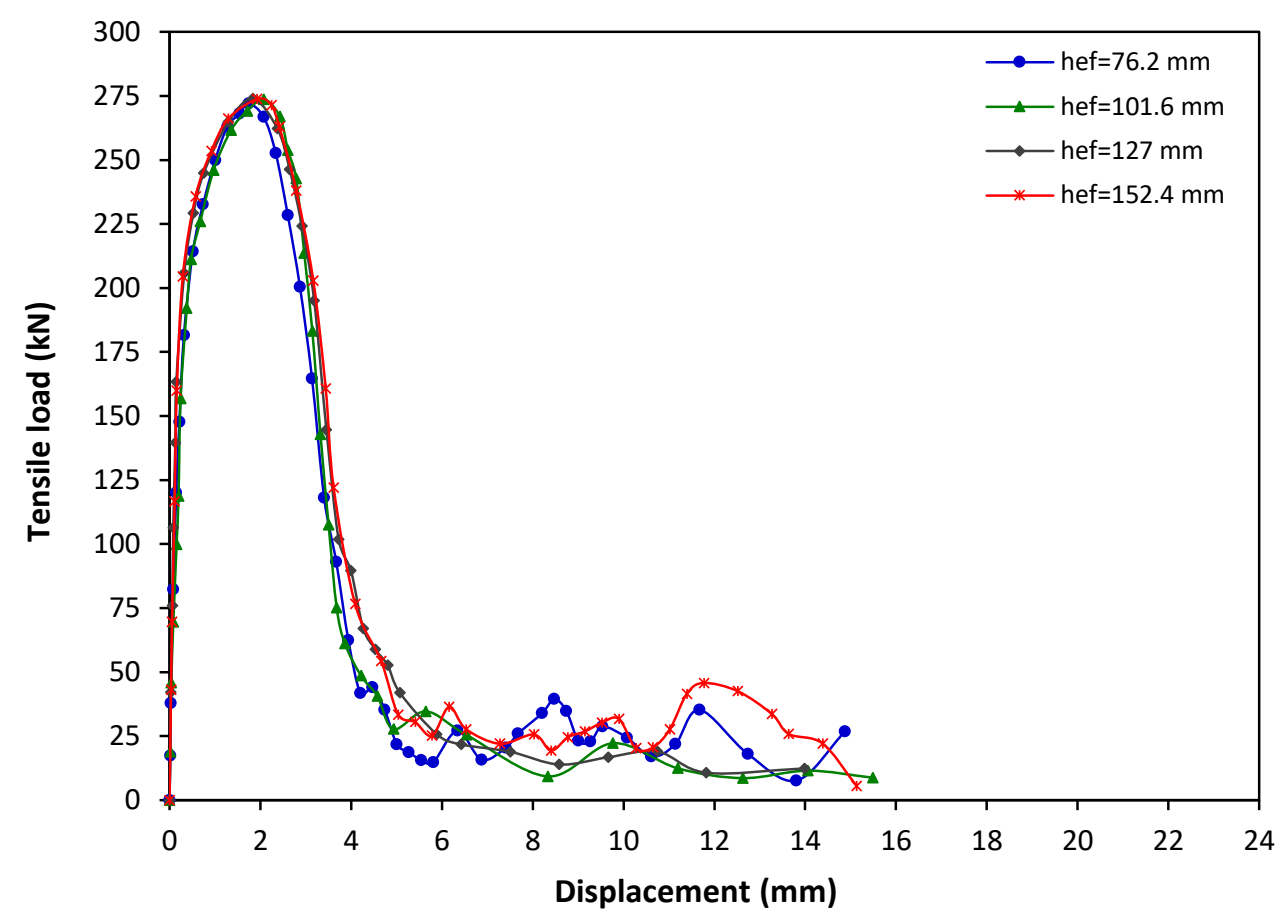

Figure C-12: Tensile load-displacement graph for $19.1 \mathrm{~mm}$ diameter cast-in-place anchor at strain rate of $10^{3} \mathrm{~s}^{-1}$ 


\section{Appendix D: Shear load-displacement relation for cast-in-place anchors}

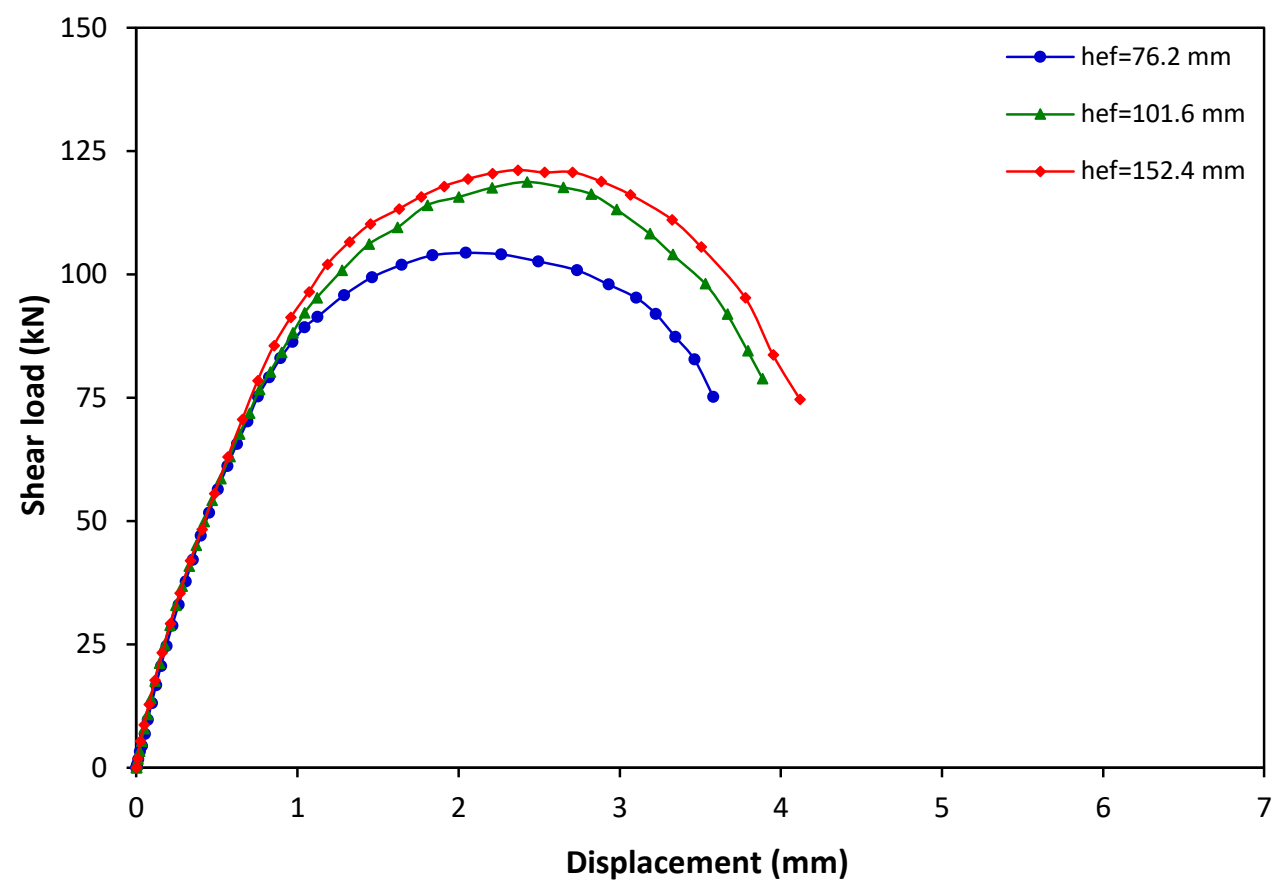

Figure D-1: Shear load-displacement graph for $15.9 \mathrm{~mm}$ diameter cast-in-place anchor at strain rate of $10^{-5} \mathrm{~s}^{-1}$

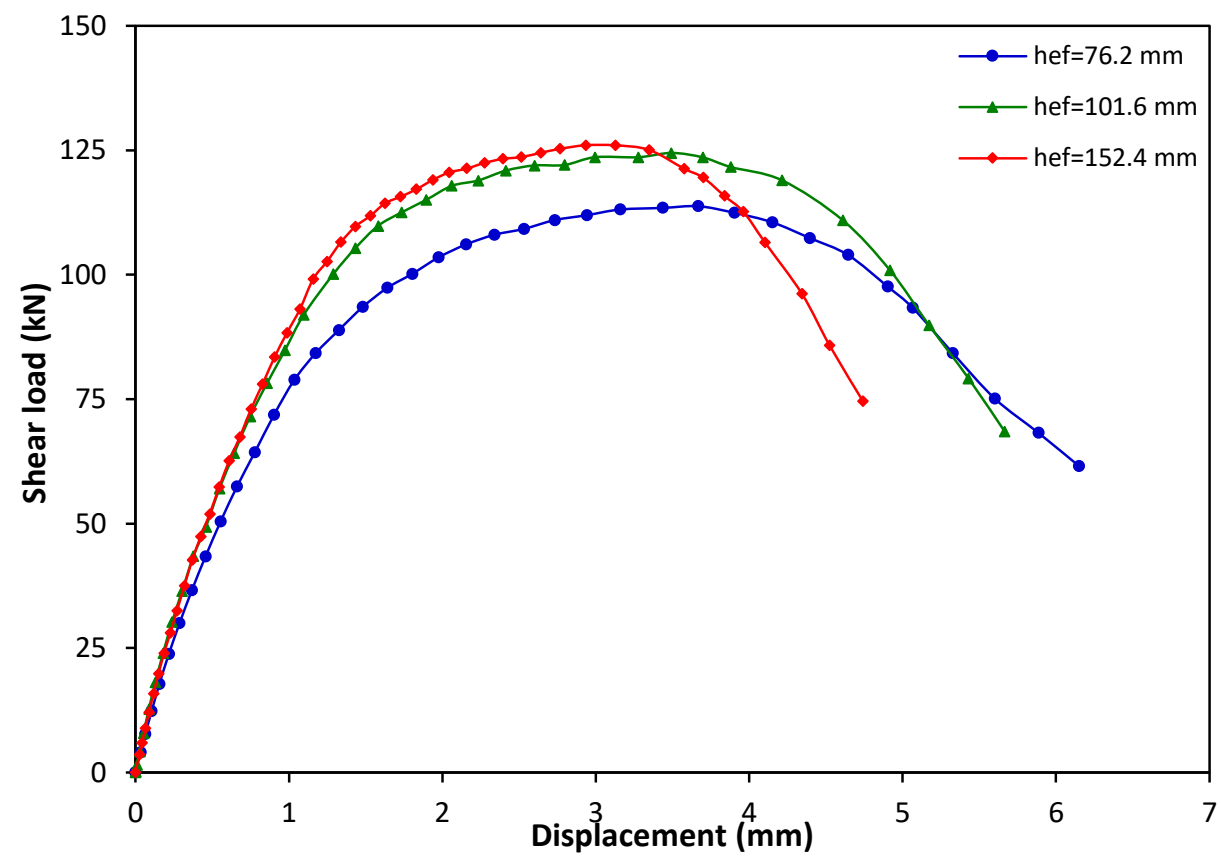

Figure D-2: Shear load-displacement graph for $15.9 \mathrm{~mm}$ diameter cast-in-place anchor at strain rate of $10^{-3} \mathrm{~s}^{-1}$ 


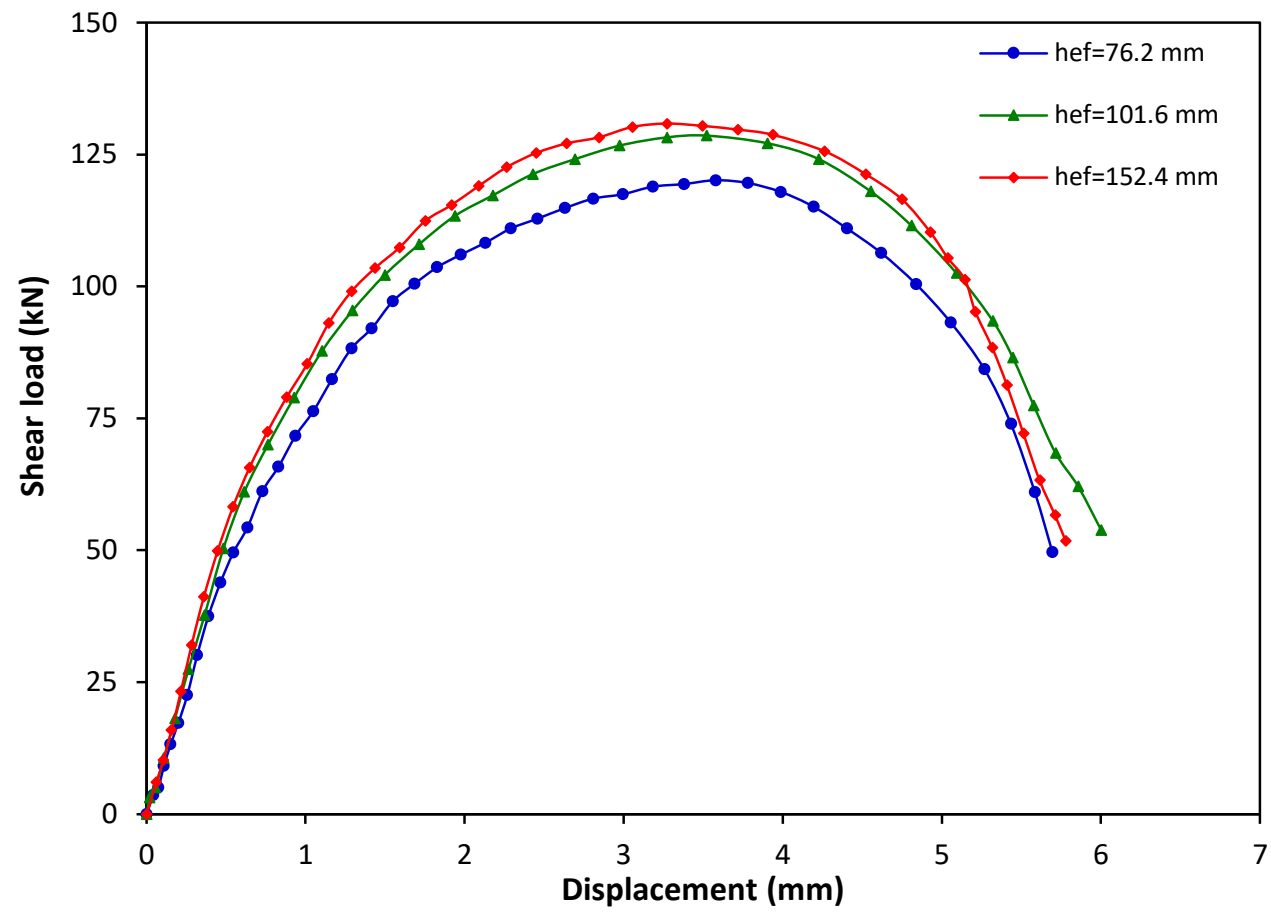

Figure D-3: Shear load-displacement graph for $15.9 \mathrm{~mm}$ diameter cast-in-place anchor at strain rate of $10^{-1} \mathrm{~s}^{-1}$

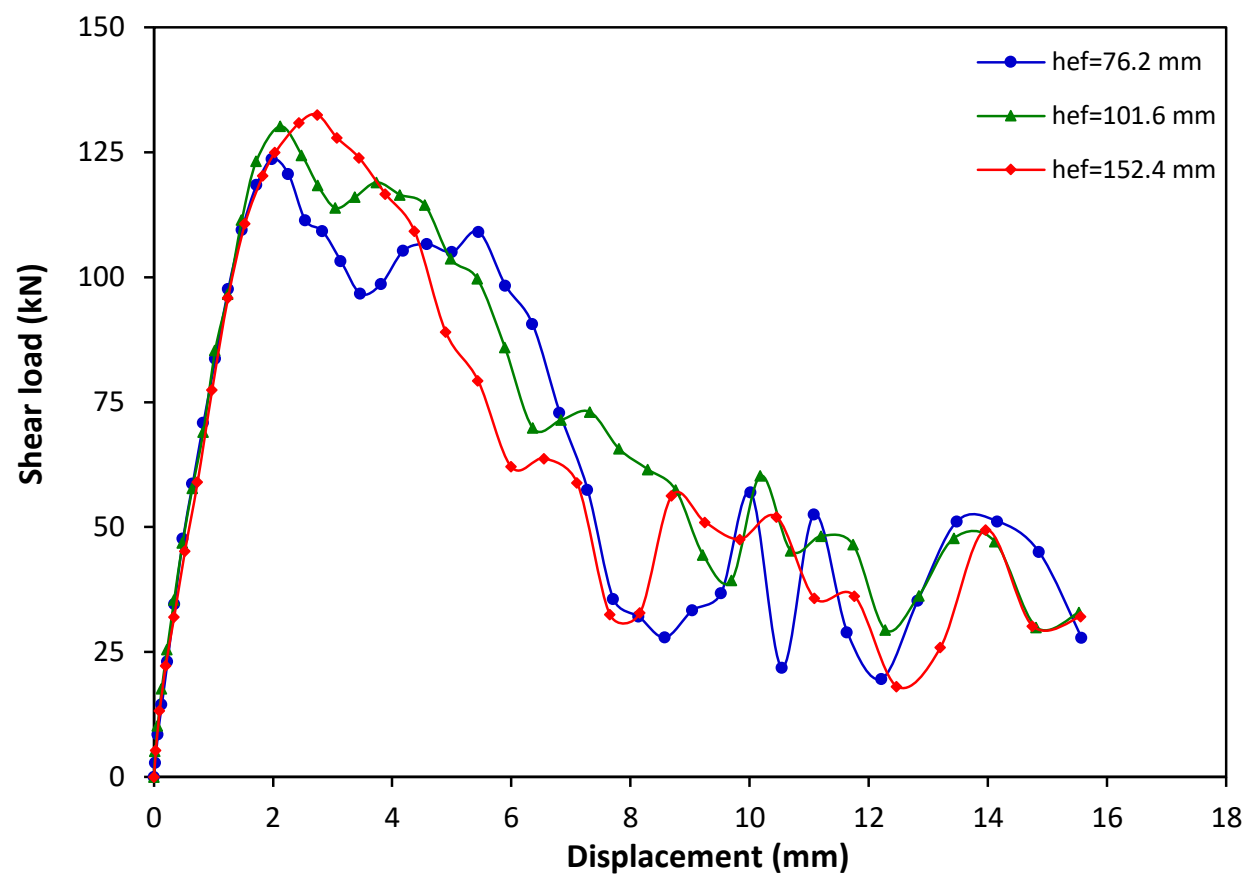

Figure D-4: Shear load-displacement graph for $15.9 \mathrm{~mm}$ diameter cast-in-place anchor at strain rate of $10 \mathrm{~s}^{-1}$ 


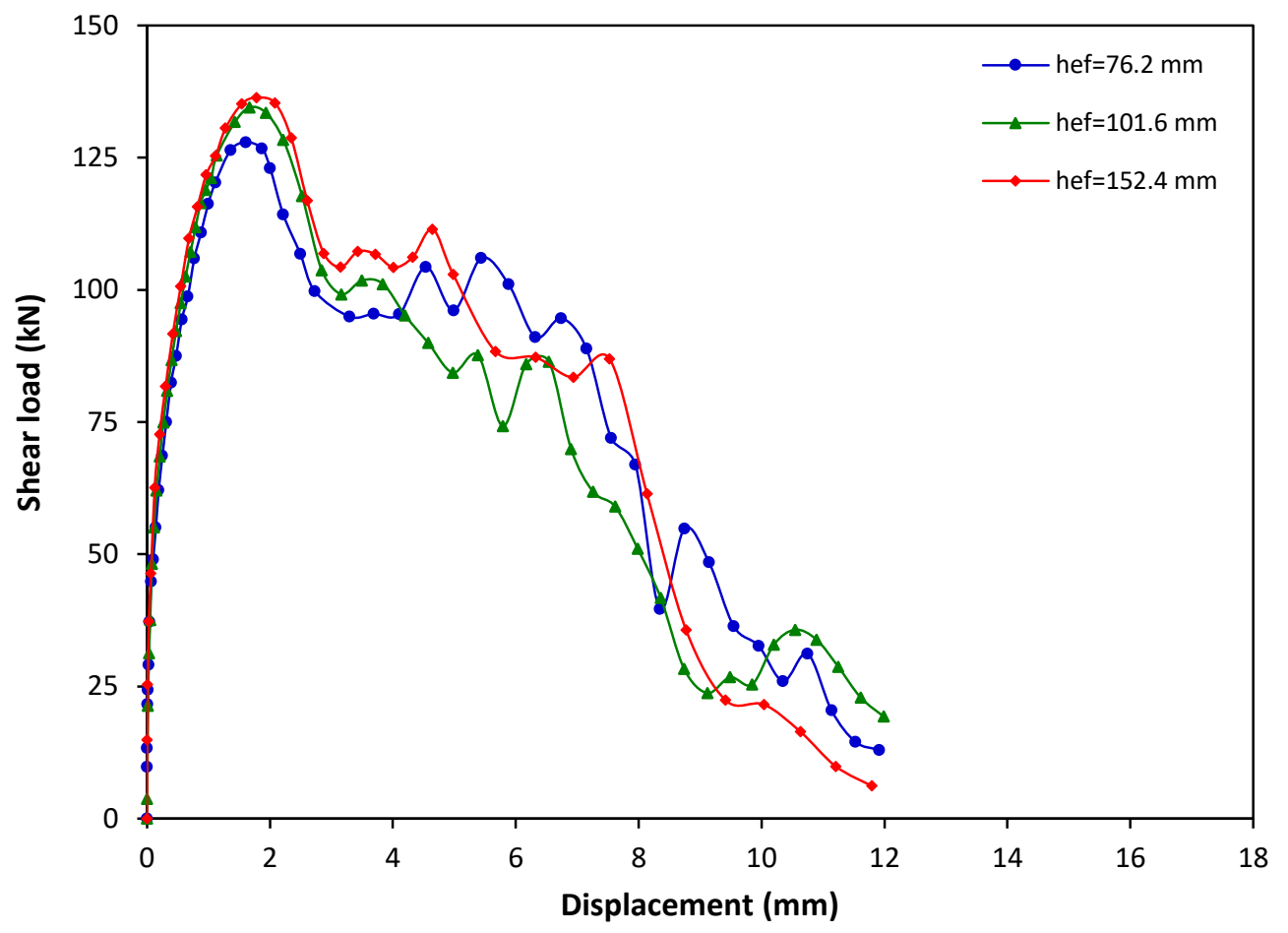

Figure D-5: Shear load-displacement graph for $15.9 \mathrm{~mm}$ diameter cast-in-place anchor at strain rate of $10^{2} \mathrm{~s}^{-1}$

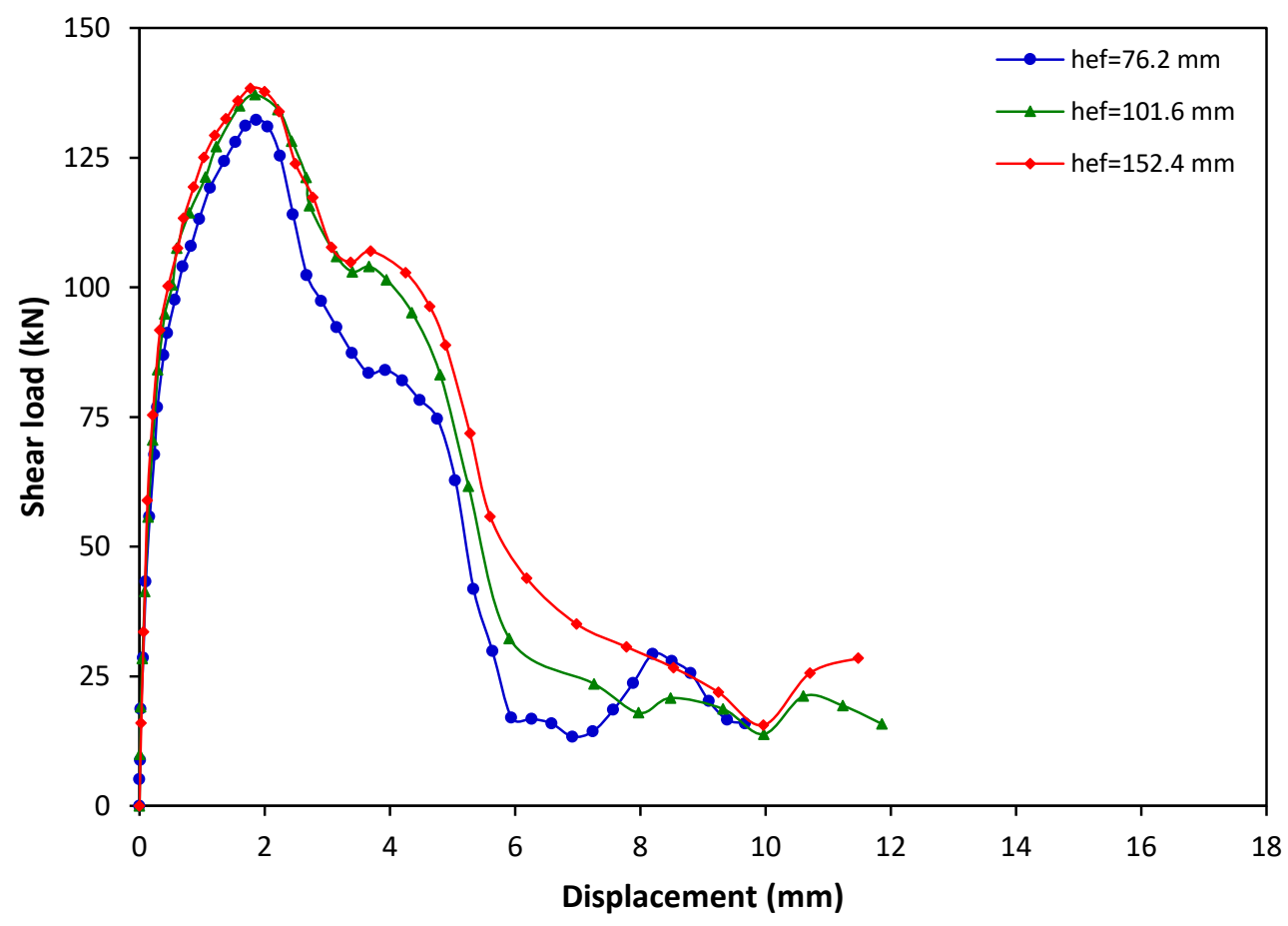

Figure D-6: Shear load-displacement graph for $15.9 \mathrm{~mm}$ diameter cast-in-place anchor at strain rate of $10^{3} \mathrm{~s}^{-1}$ 


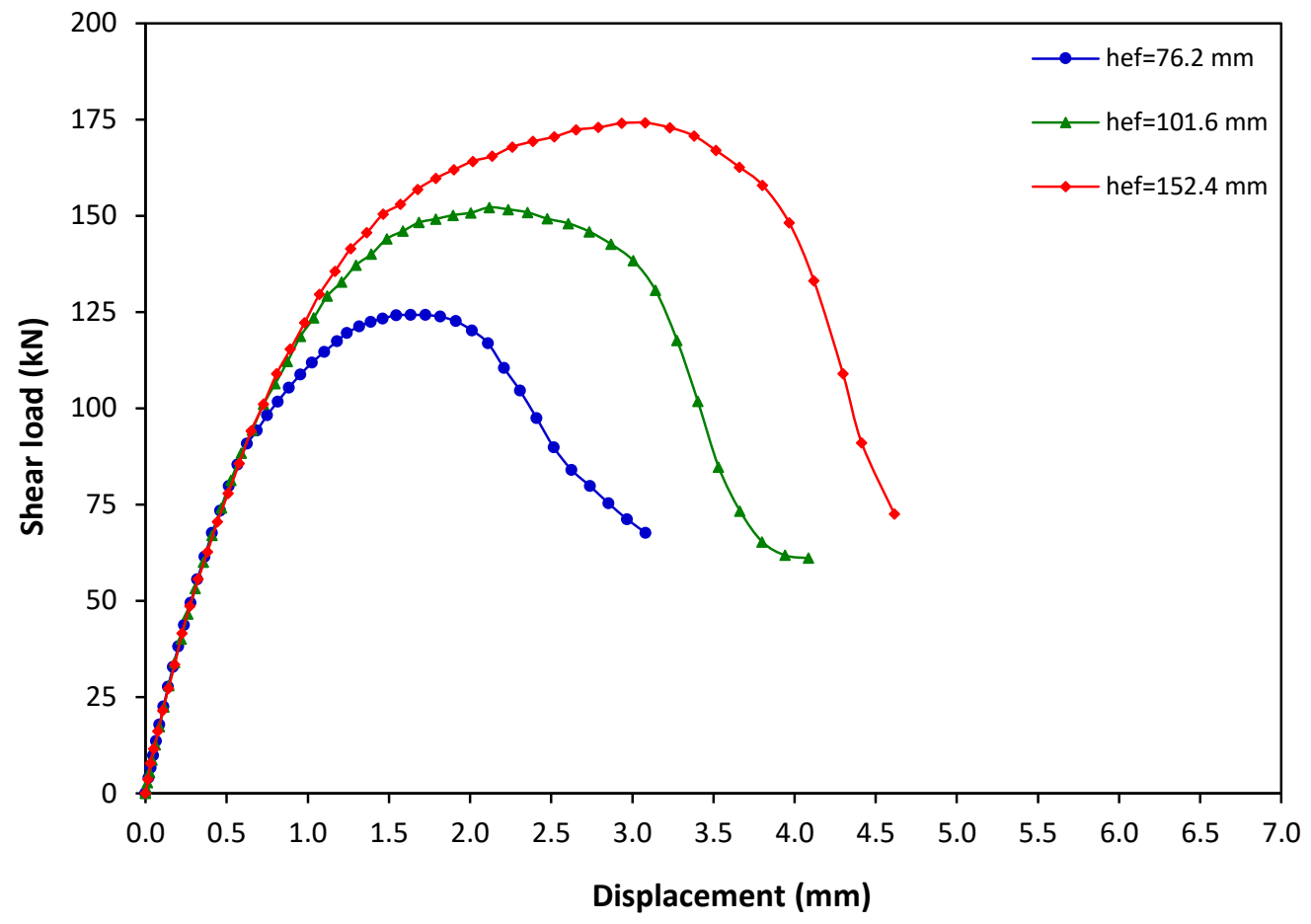

Figure D-7: Shear load-displacement graph for $19.1 \mathrm{~mm}$ diameter cast-in-place anchor at strain rate of $10^{-5} \mathrm{~s}^{-1}$

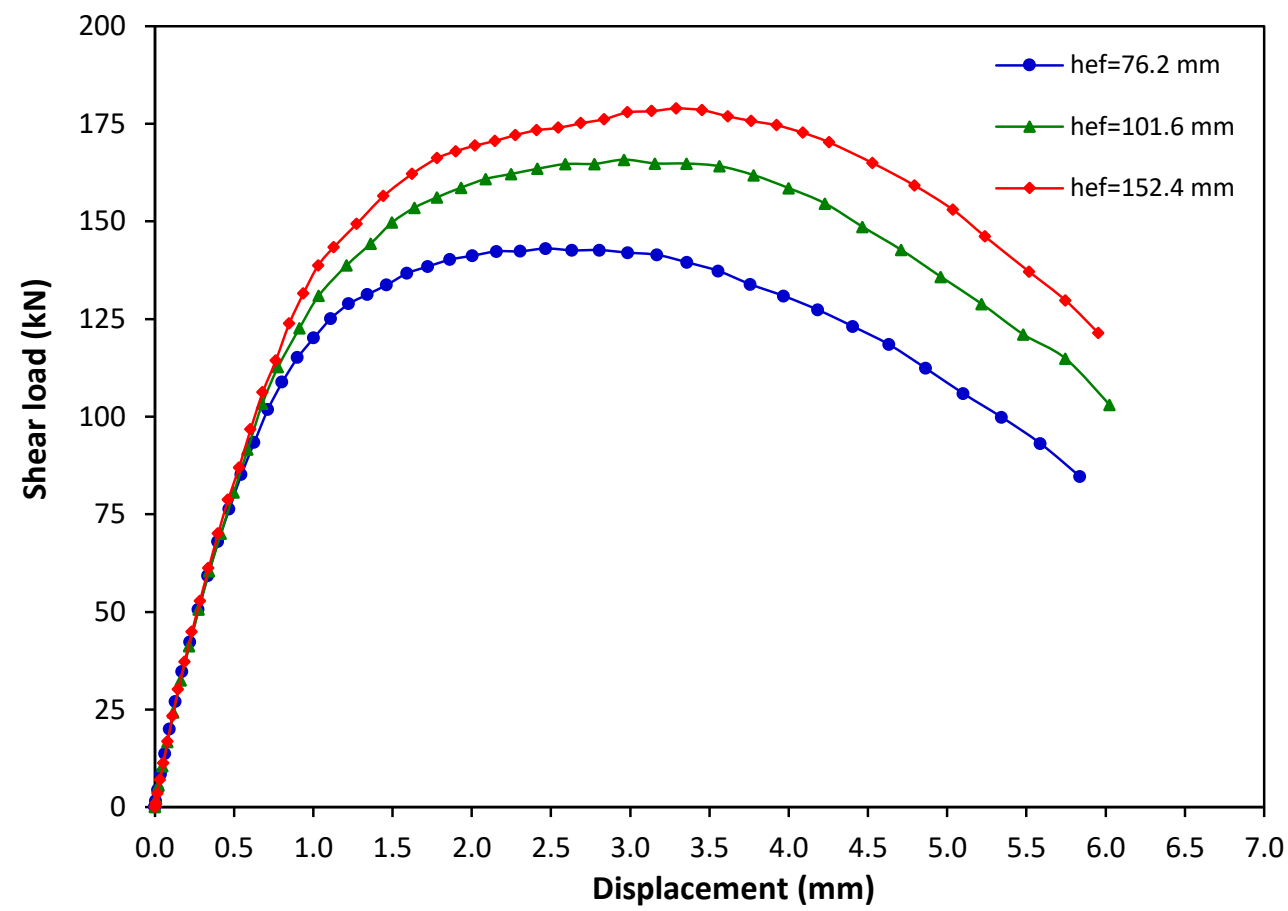

Figure D-8: Shear load-displacement graph for $19.1 \mathrm{~mm}$ diameter cast-in-place anchor at strain rate of $10^{-3} \mathrm{~s}^{-1}$ 


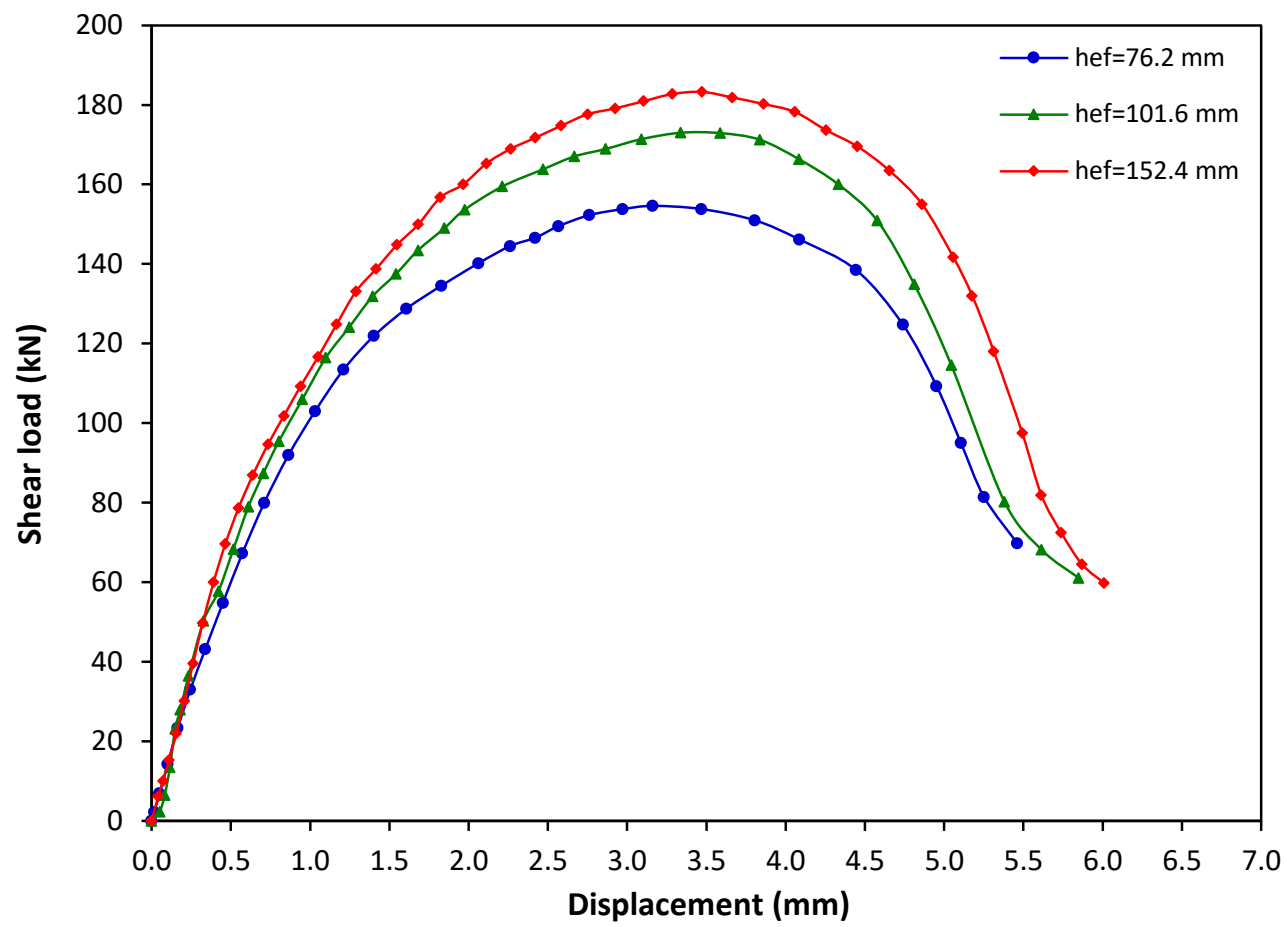

Figure D-9: Shear load-displacement graph for $19.1 \mathrm{~mm}$ diameter cast-in-place anchor $\mathrm{mm}$ at strain rate of $10^{-1} \mathrm{~s}^{-1}$

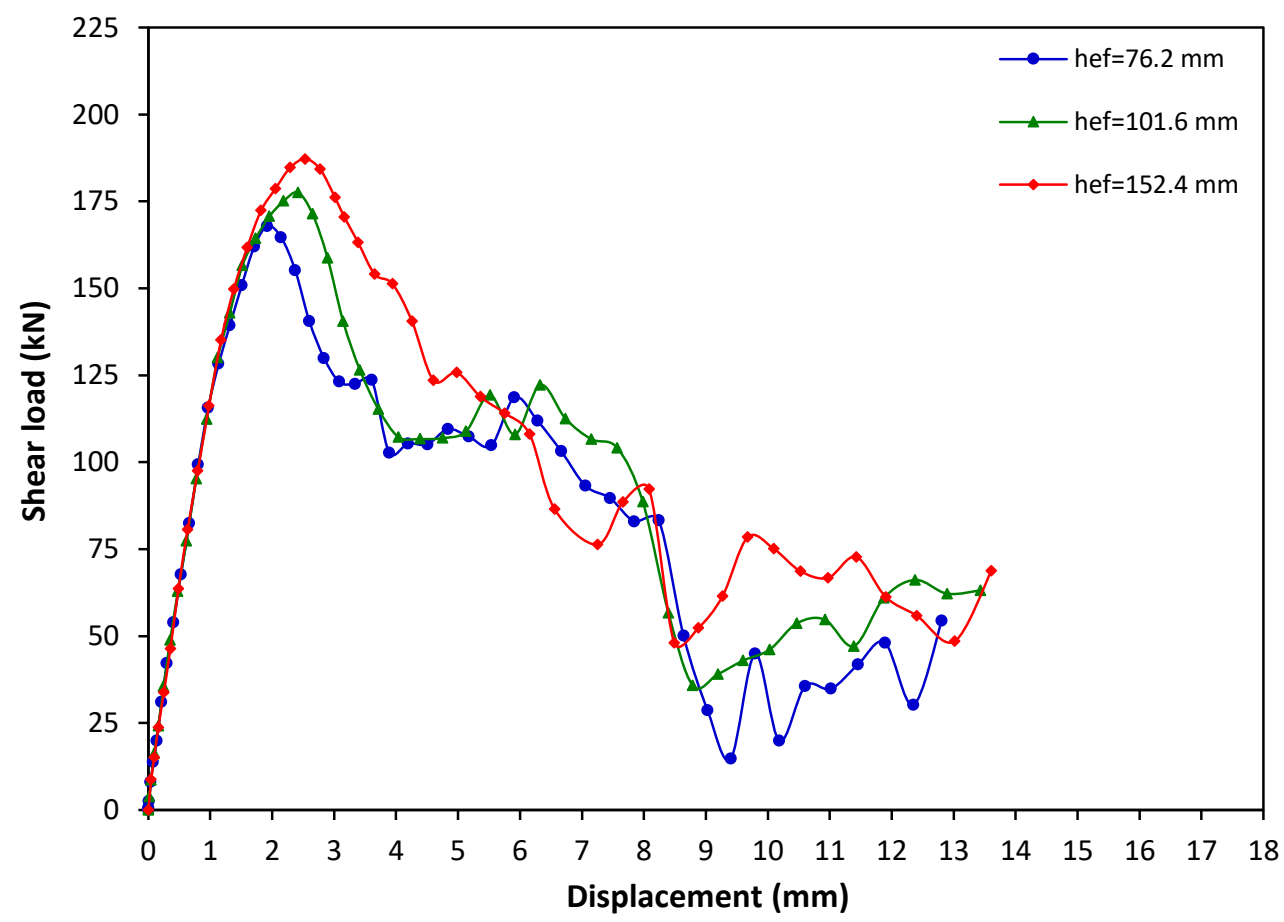

Figure D-10: Shear load-displacement graph for $19.1 \mathrm{~mm}$ diameter cast-in-place anchor at strain rate of $10 \mathrm{~s}^{-1}$ 


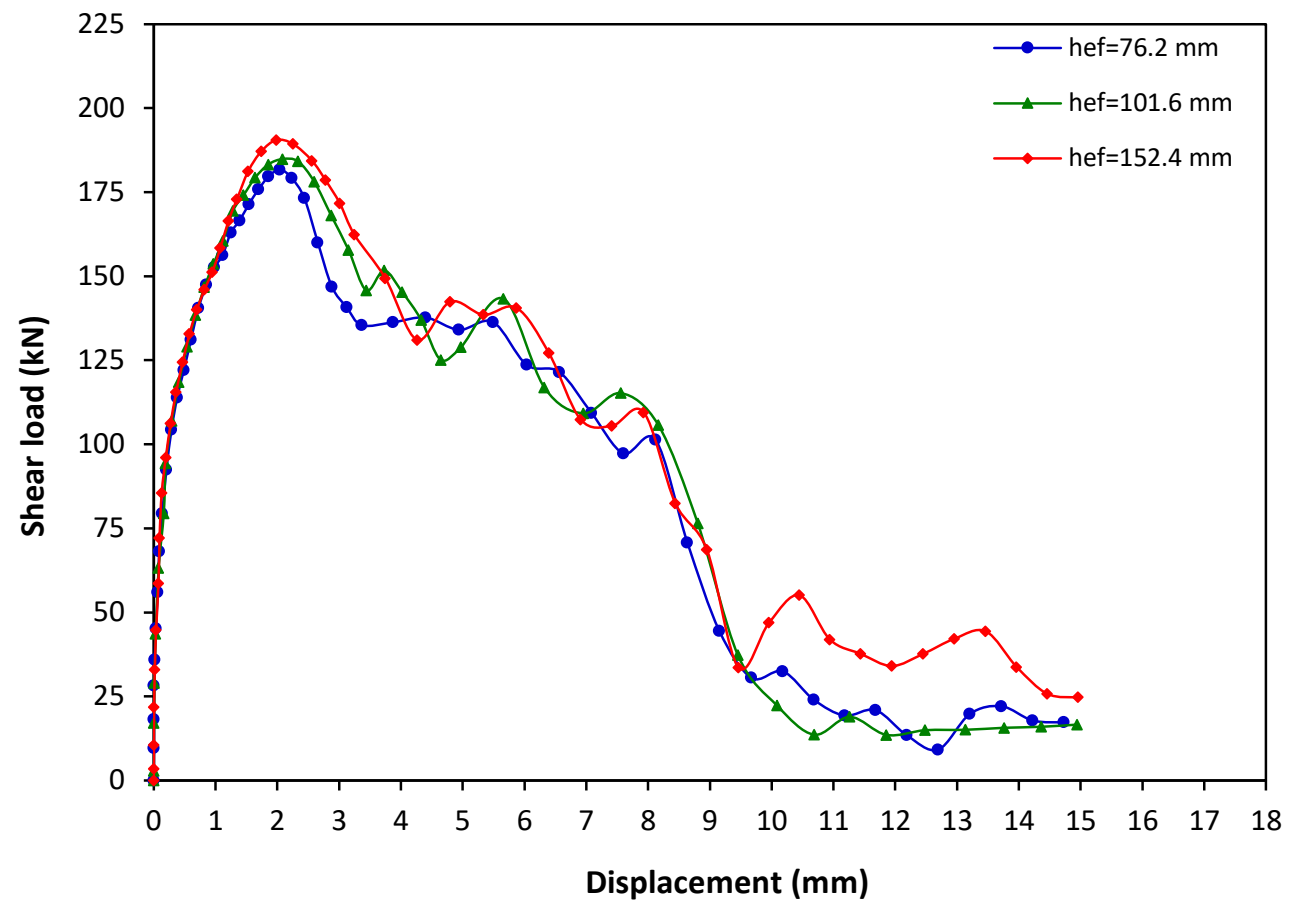

Figure D-11: Shear load-displacement graph for $19.1 \mathrm{~mm}$ diameter cast-in-place anchor at strain rate of $10^{2} \mathrm{~s}^{-1}$

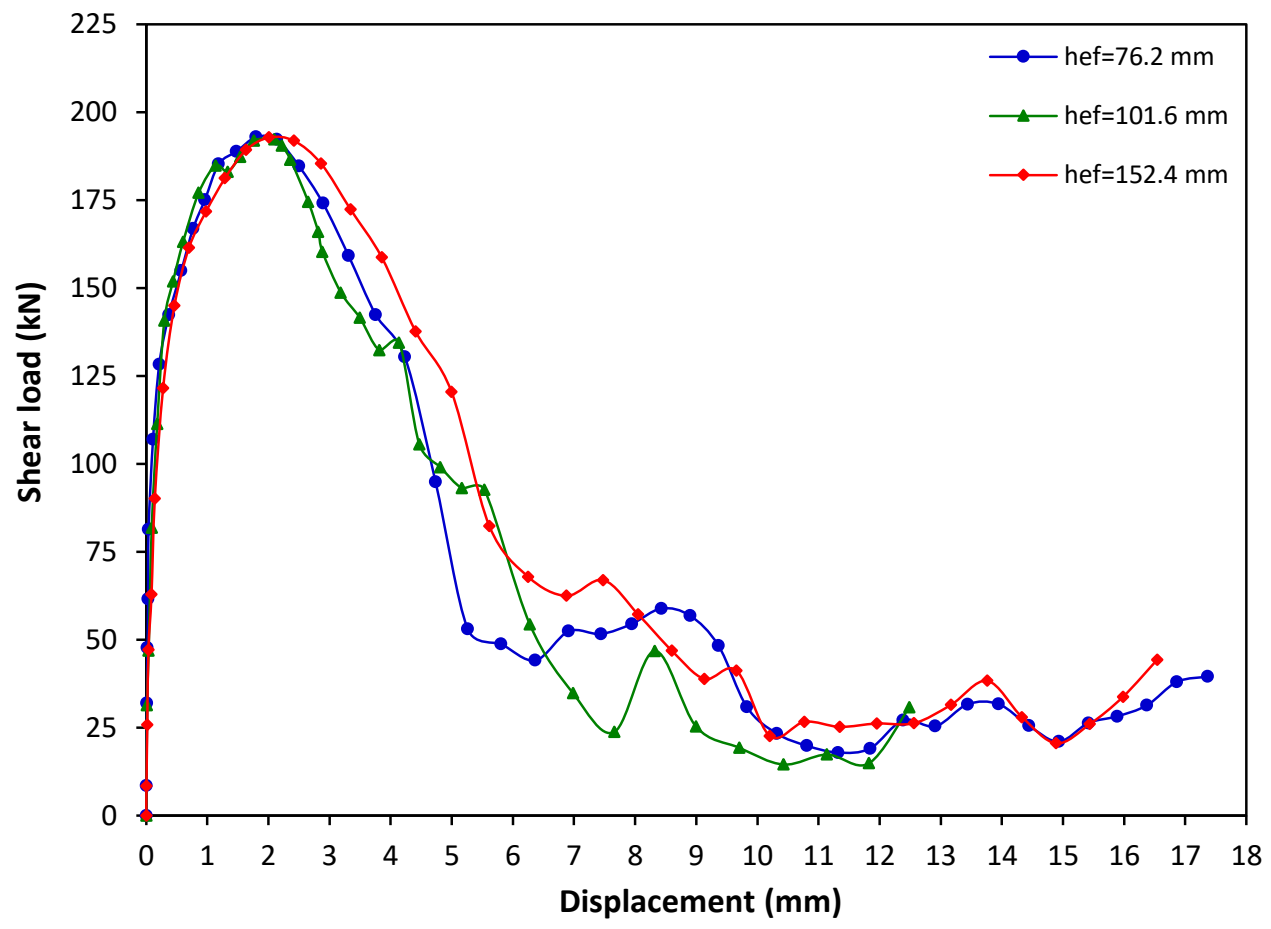

Figure D-12: Shear load-displacement graph for $19.1 \mathrm{~mm}$ diameter cast-in-place anchor at strain rate of $10^{3} \mathrm{~s}^{-1}$ 


\section{Appendix E: Tensile load-displacement relation for adhesive anchors}

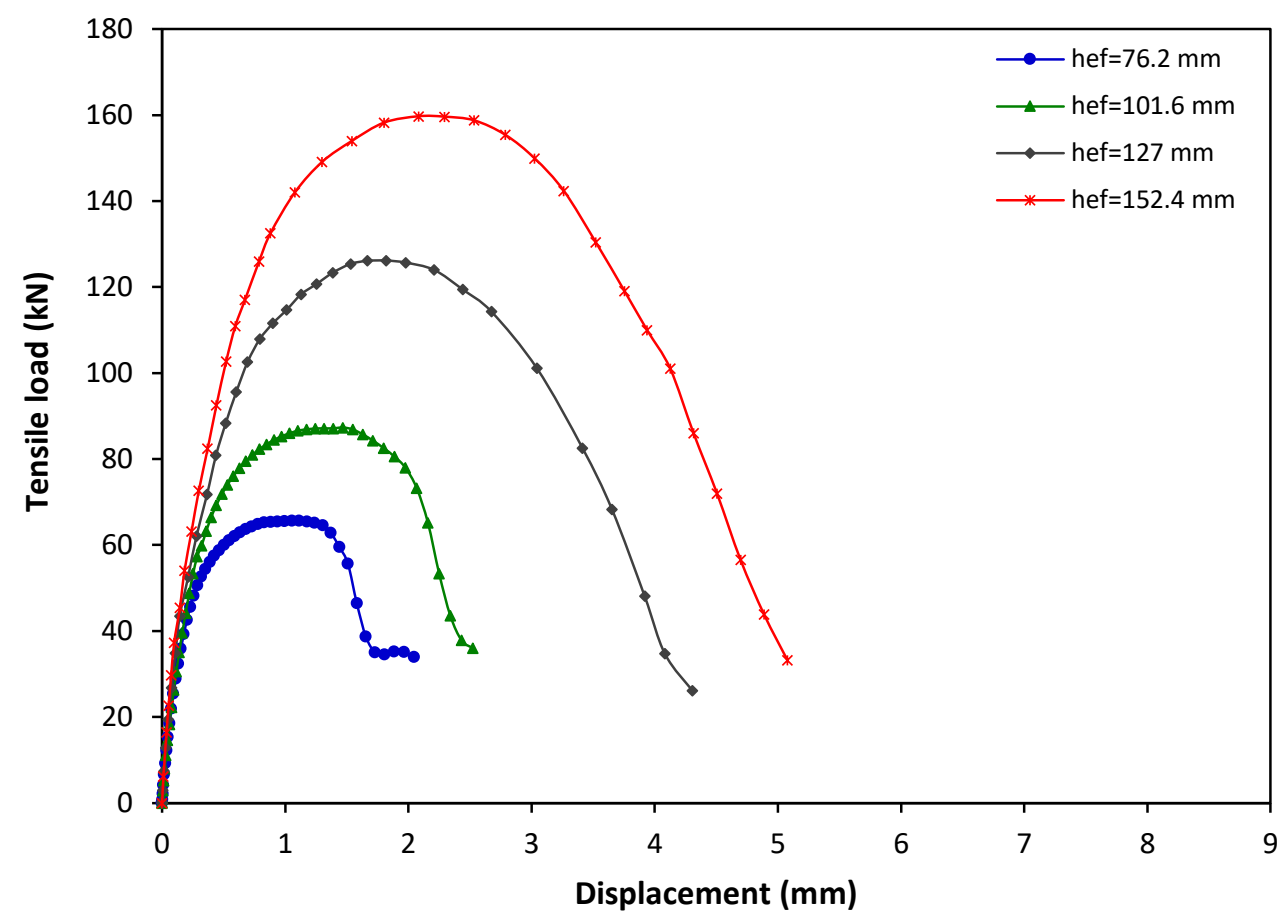

Figure E-1: Tensile load-displacement graph for $15.9 \mathrm{~mm}$ diameter adhesive anchor at strain rate of $10^{-5} \mathrm{~s}^{-1}$

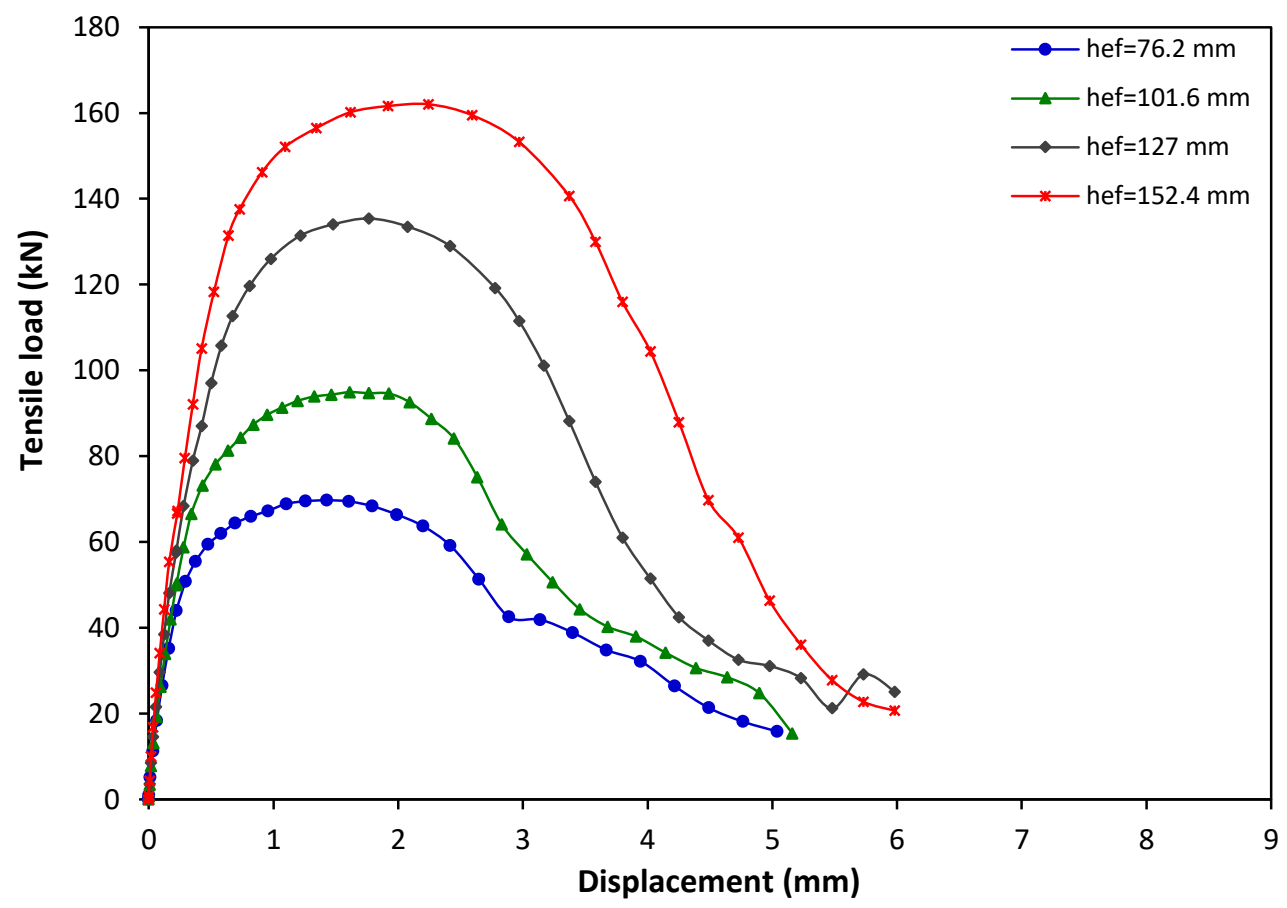

Figure E-2: Tensile load-displacement graph for $15.9 \mathrm{~mm}$ diameter adhesive anchor at strain rate of $10^{-3} \mathrm{~s}^{-1}$ 


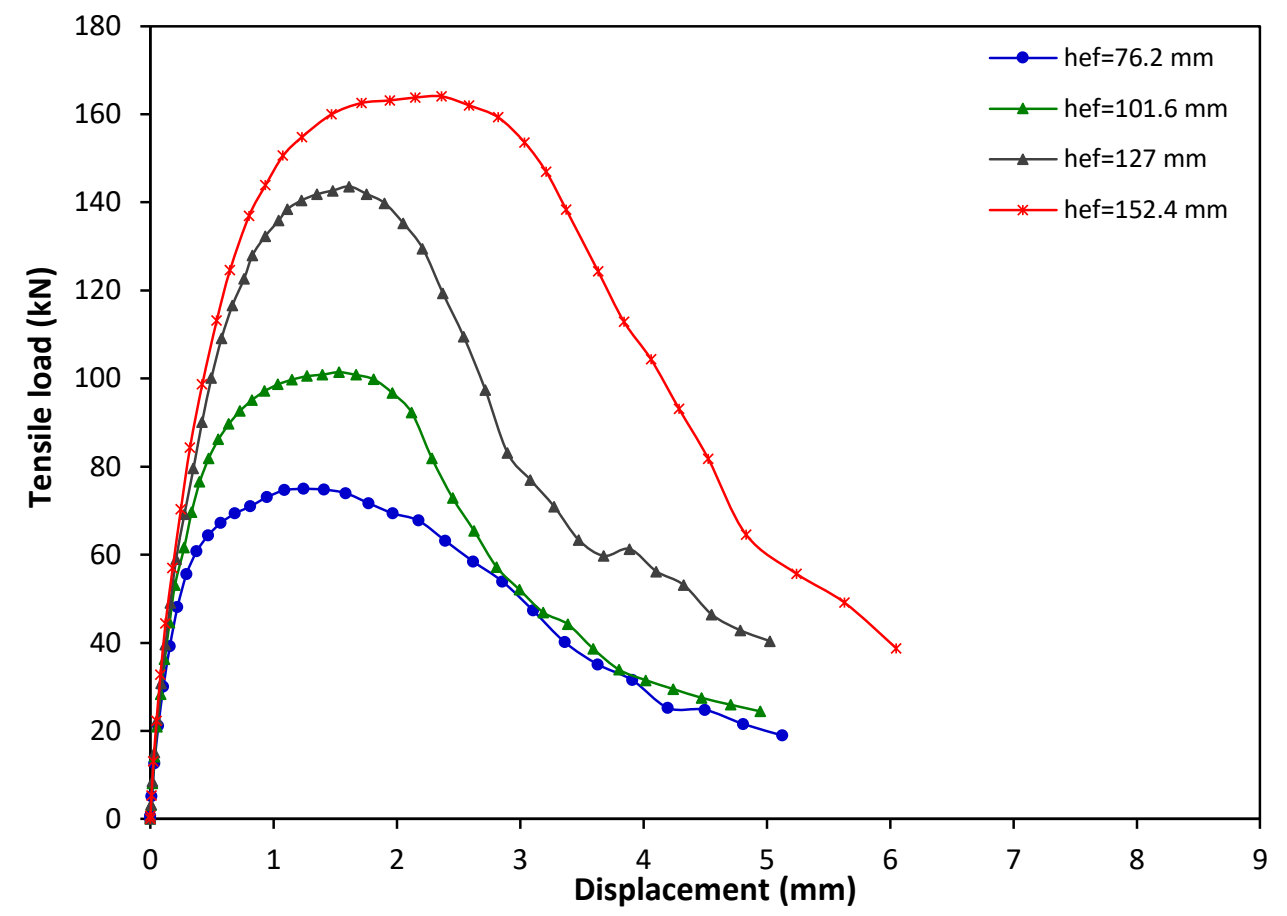

Figure E-3: Tensile load-displacement graph for $15.9 \mathrm{~mm}$ diameter adhesive anchor at strain rate of $10^{-1} \mathrm{~s}^{-1}$

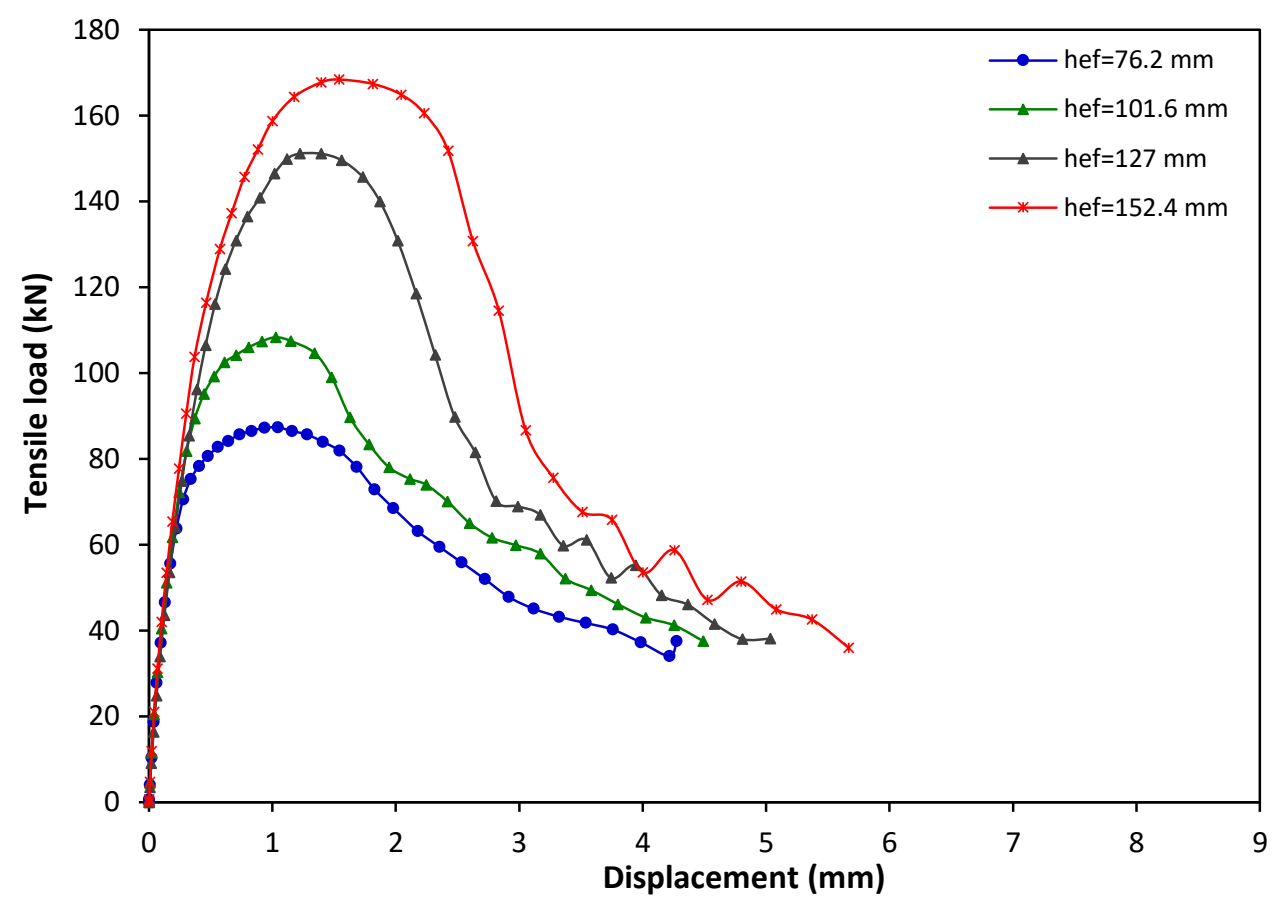

Figure E-4: Tensile load-displacement graph for $15.9 \mathrm{~mm}$ diameter adhesive anchor at strain rate of $10 \mathrm{~s}^{-1}$ 


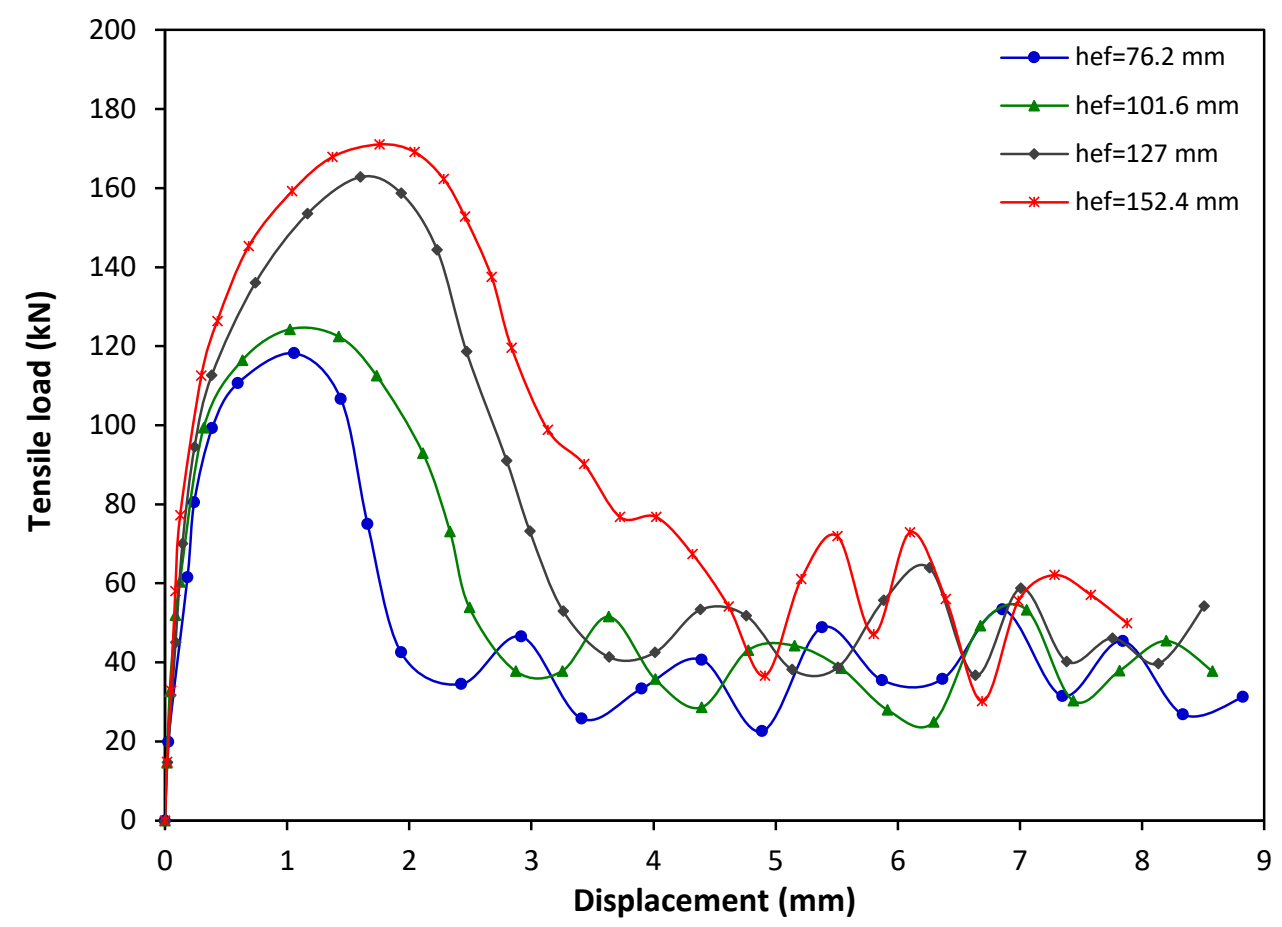

Figure E-5: Tensile load-displacement graph for $15.9 \mathrm{~mm}$ diameter adhesive anchor at strain rate of $10^{2} \mathrm{~s}^{-1}$

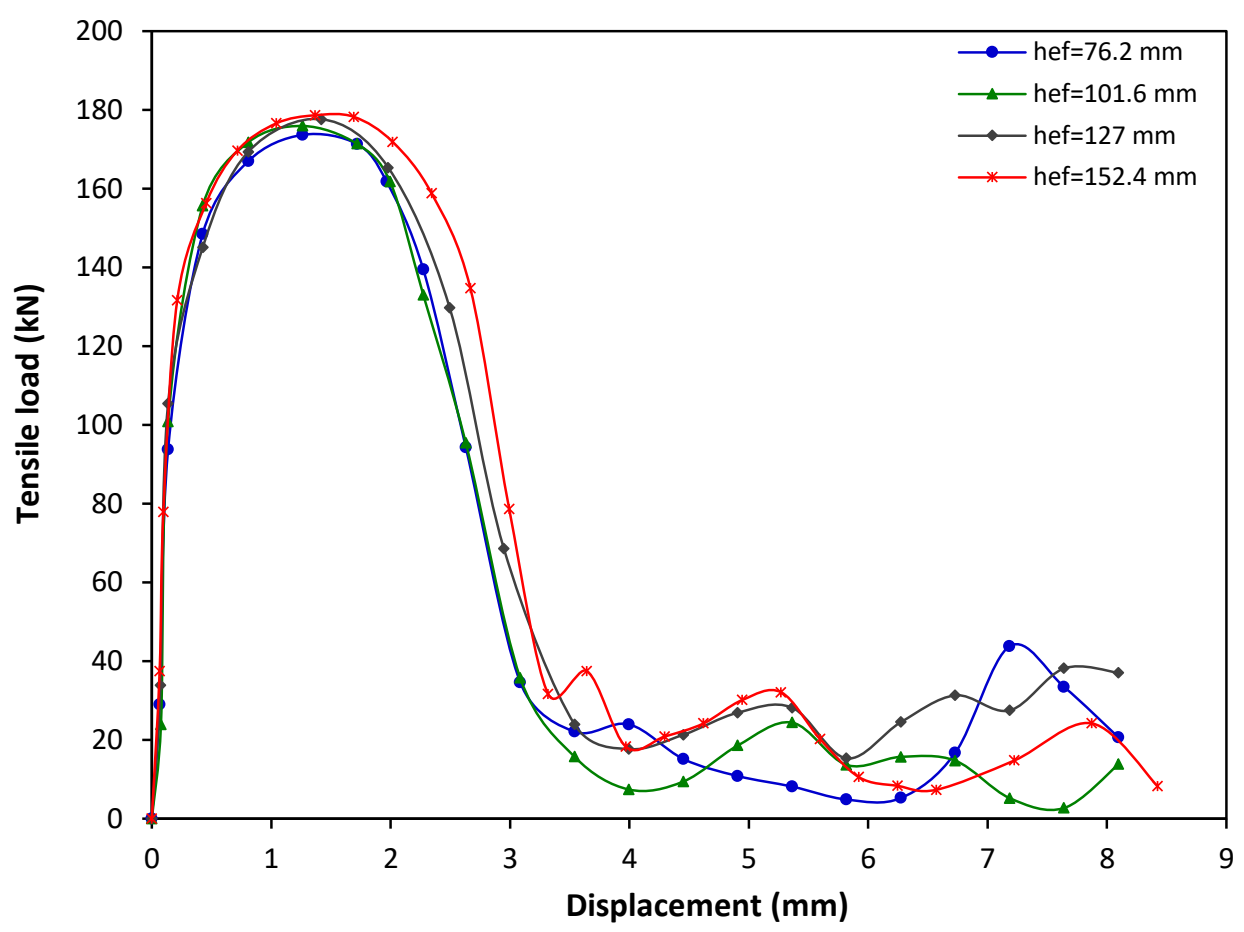

Figure E-6: Tensile load-displacement graph for $15.9 \mathrm{~mm}$ diameter adhesive anchor at strain rate of $10^{3} \mathrm{~s}^{-1}$ 


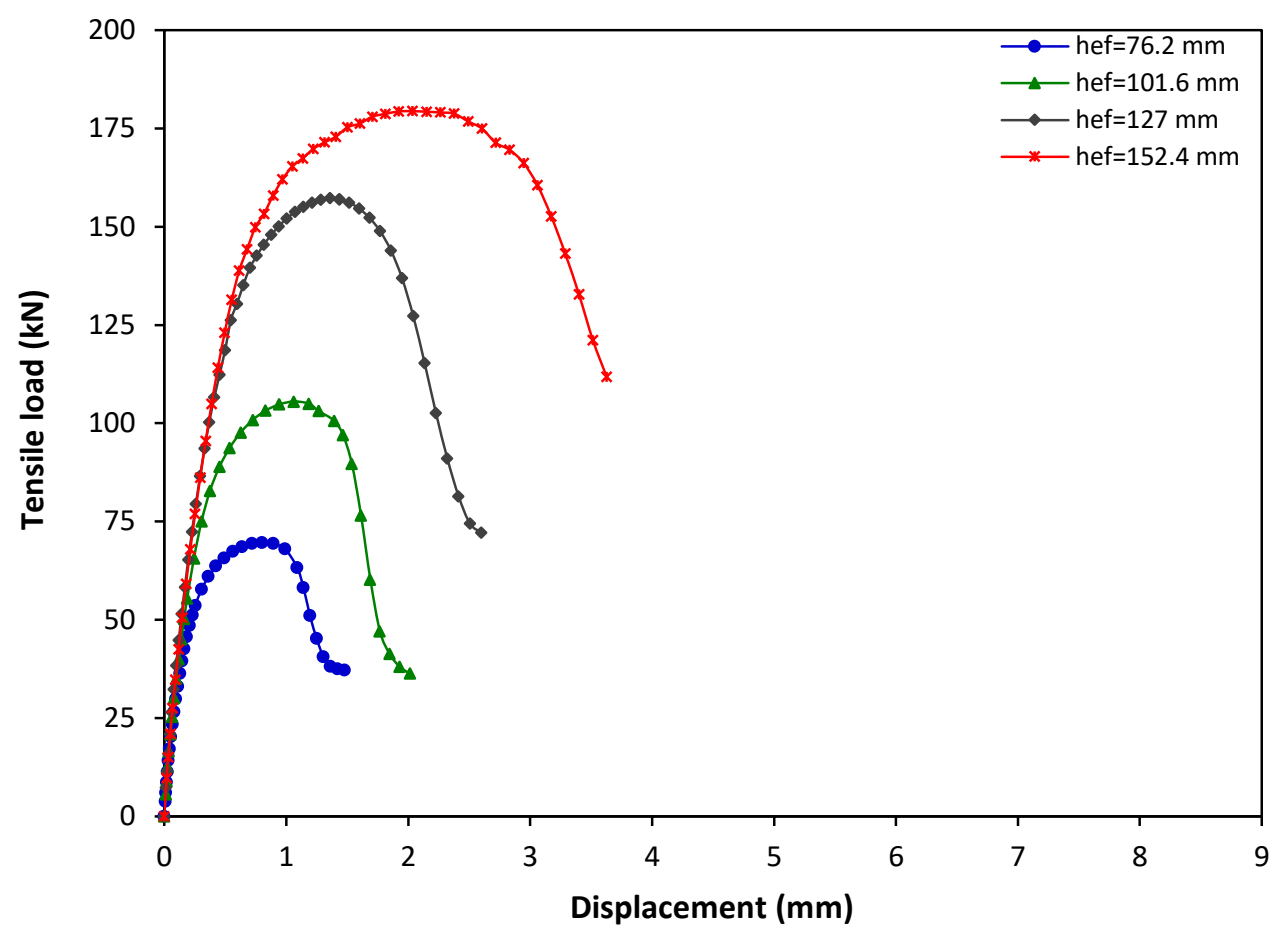

Figure E-7: Tensile load-displacement graph for $19.1 \mathrm{~mm}$ diameter adhesive anchor at strain rate of $10^{-5} \mathrm{~s}^{-1}$

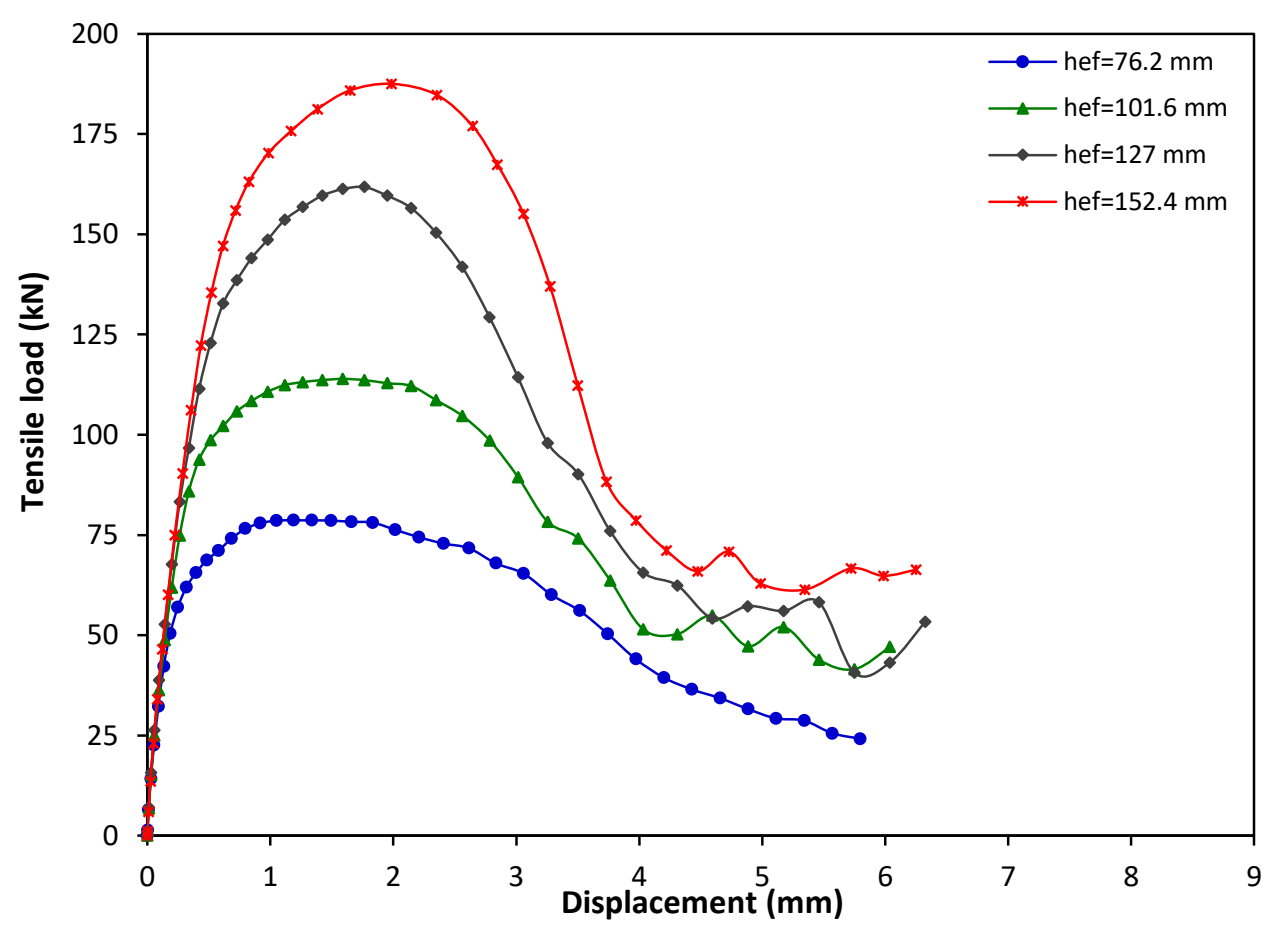

Figure E-8: Tensile load-displacement graph for $19.1 \mathrm{~mm}$ diameter adhesive anchor at strain rate of $10^{-3} \mathrm{~s}^{-1}$ 


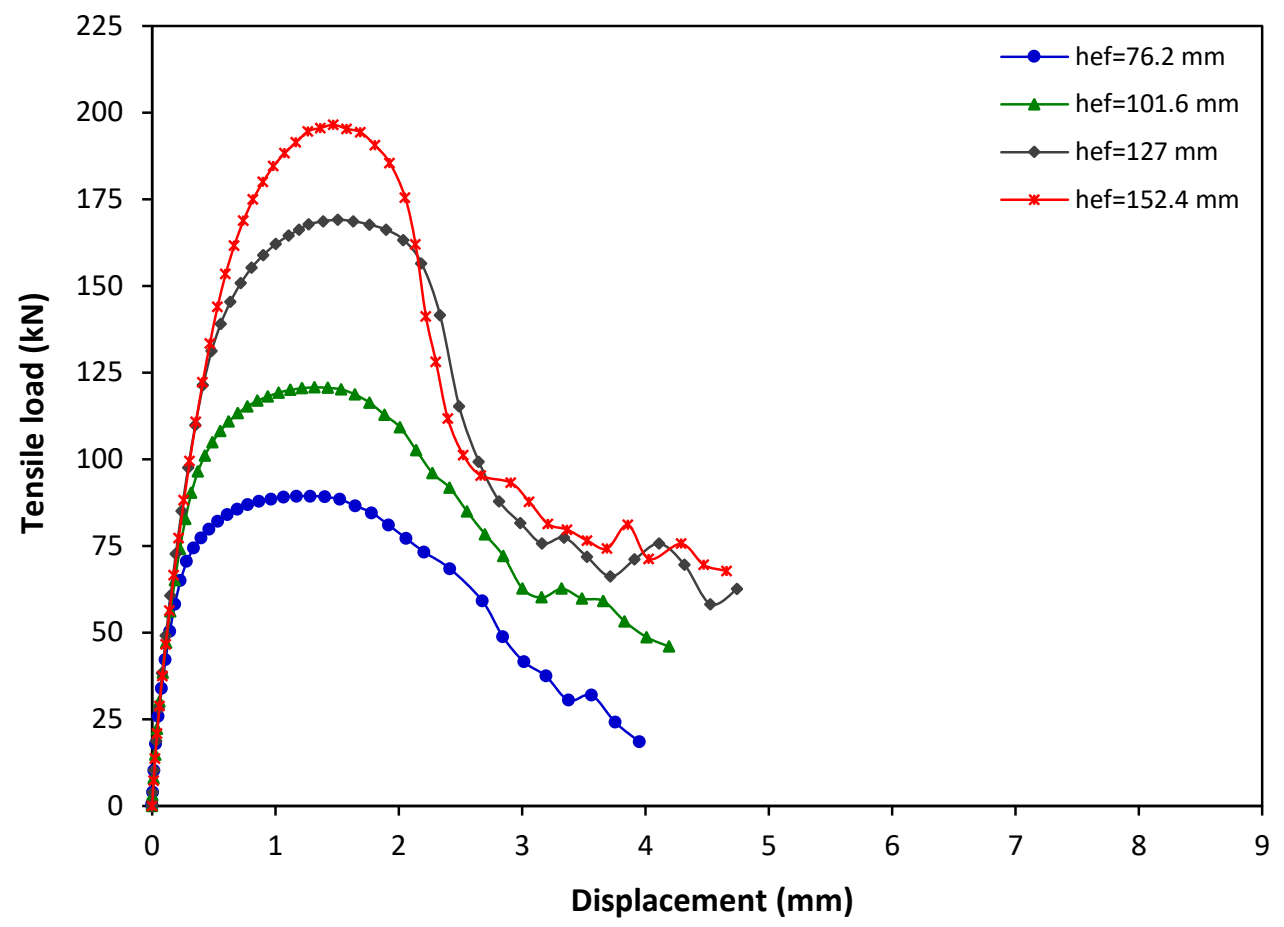

Figure E-9: Tensile load-displacement graph for $19.1 \mathrm{~mm}$ diameter adhesive anchor at strain rate of $10^{-1} \mathrm{~s}^{-1}$

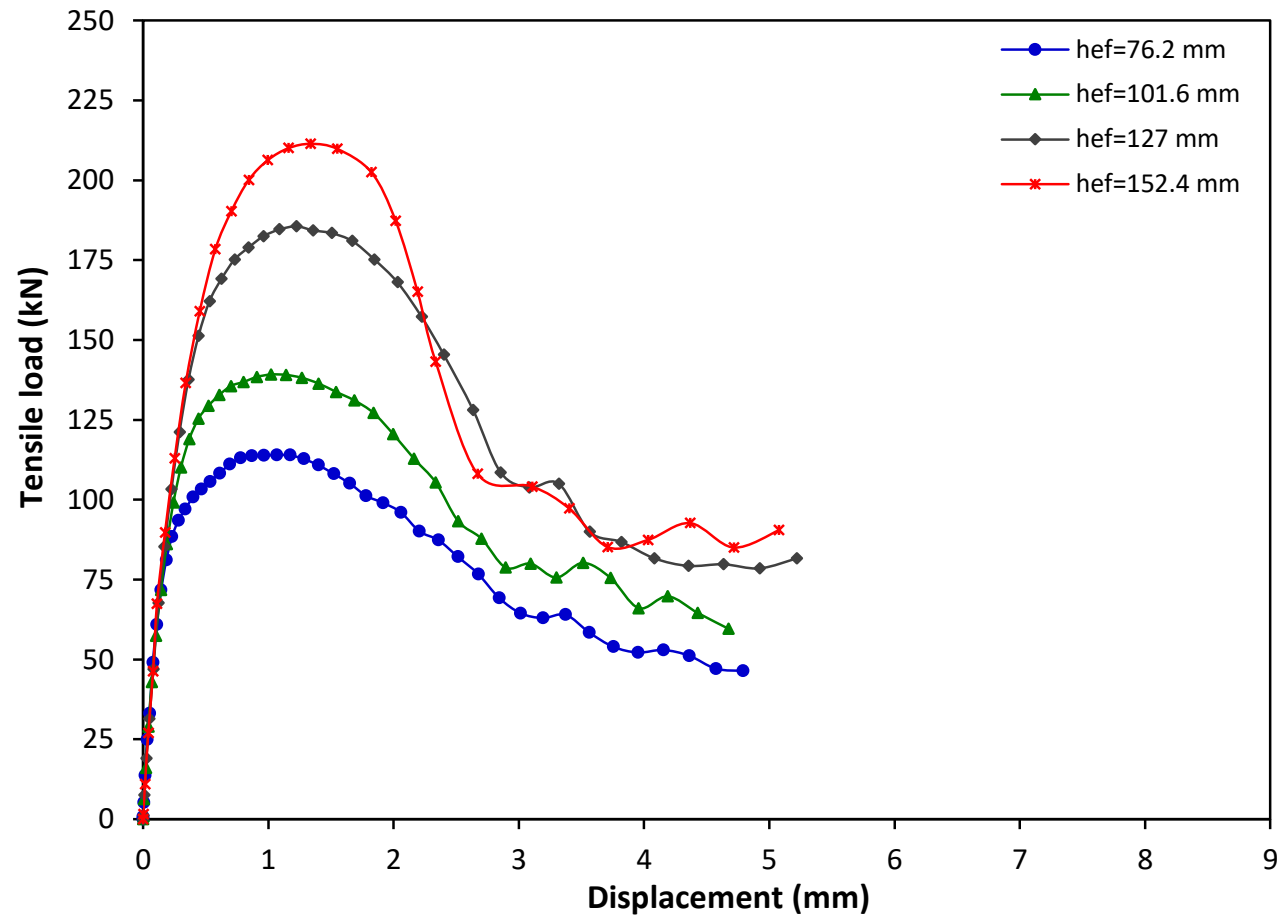

Figure E-10: Tensile load-displacement graph for $19.1 \mathrm{~mm}$ diameter adhesive anchor at strain rate of $10 \mathrm{~s}^{-1}$ 


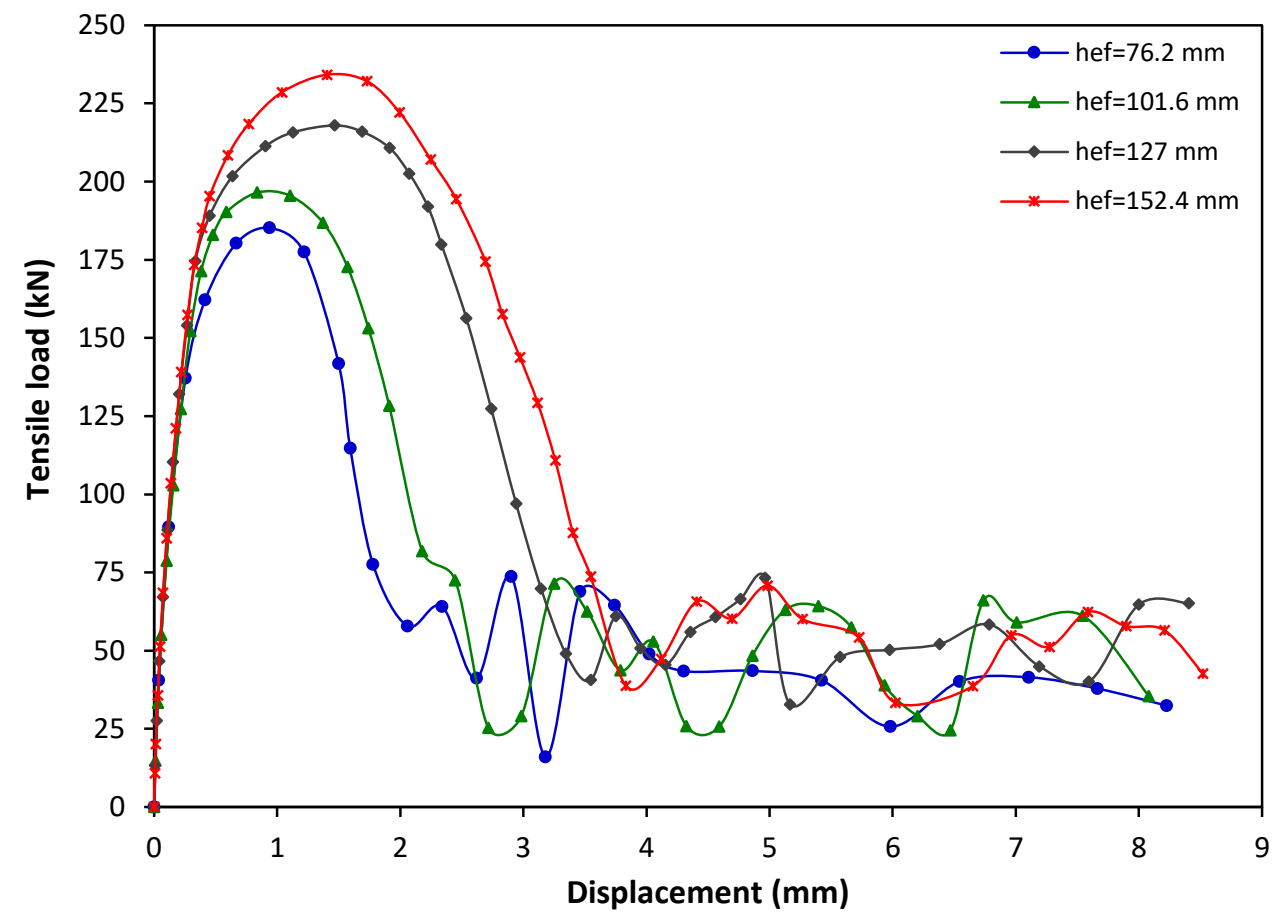

Figure E-11: Tensile load-displacement graph for $19.1 \mathrm{~mm}$ diameter adhesive anchor at strain rate of $10^{2} \mathrm{~s}^{-1}$

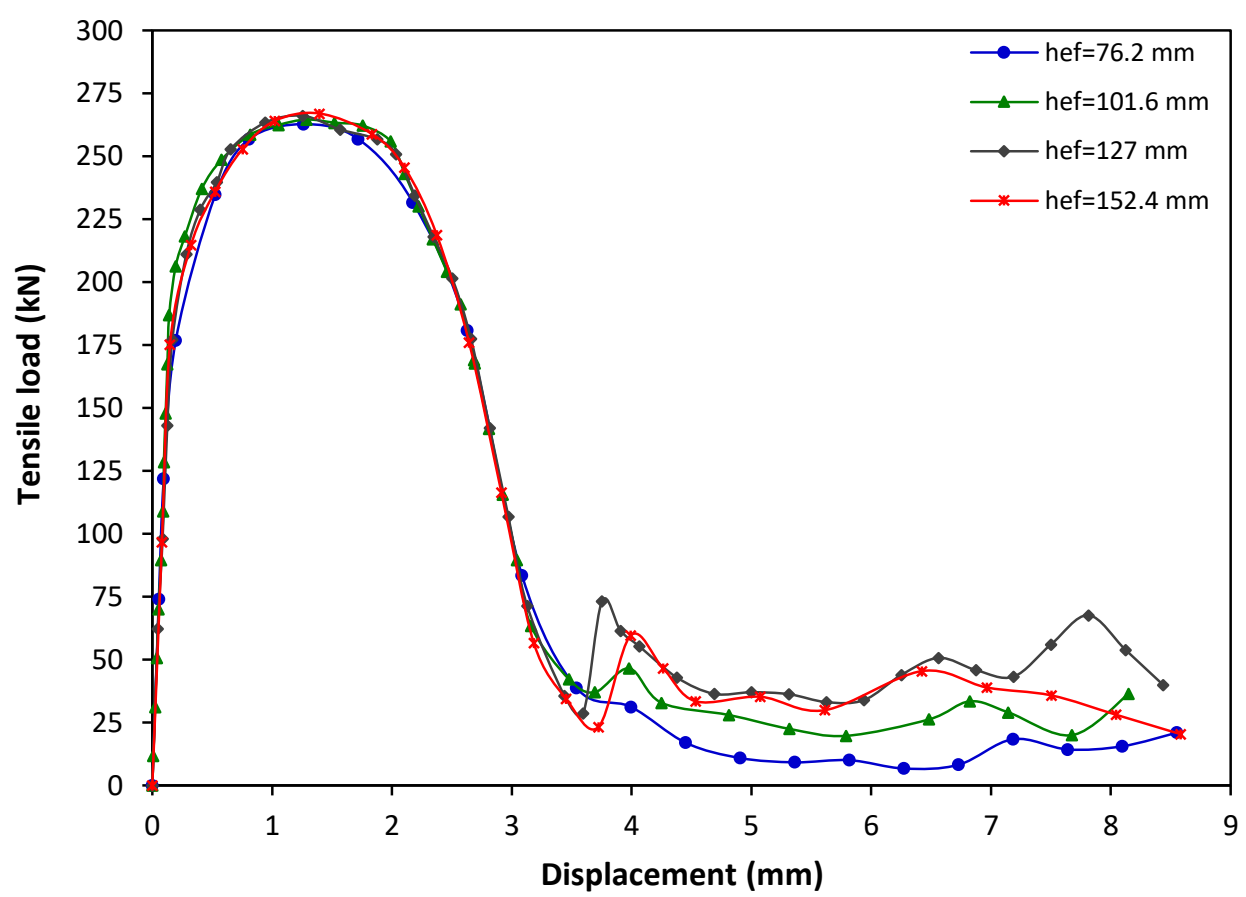

Figure E-12: Tensile load-displacement graph for $19.1 \mathrm{~mm}$ diameter adhesive anchor at strain rate of $10^{3} \mathrm{~s}^{-1}$ 


\section{Appendix F: Shear load-displacement relation for adhesive anchors}

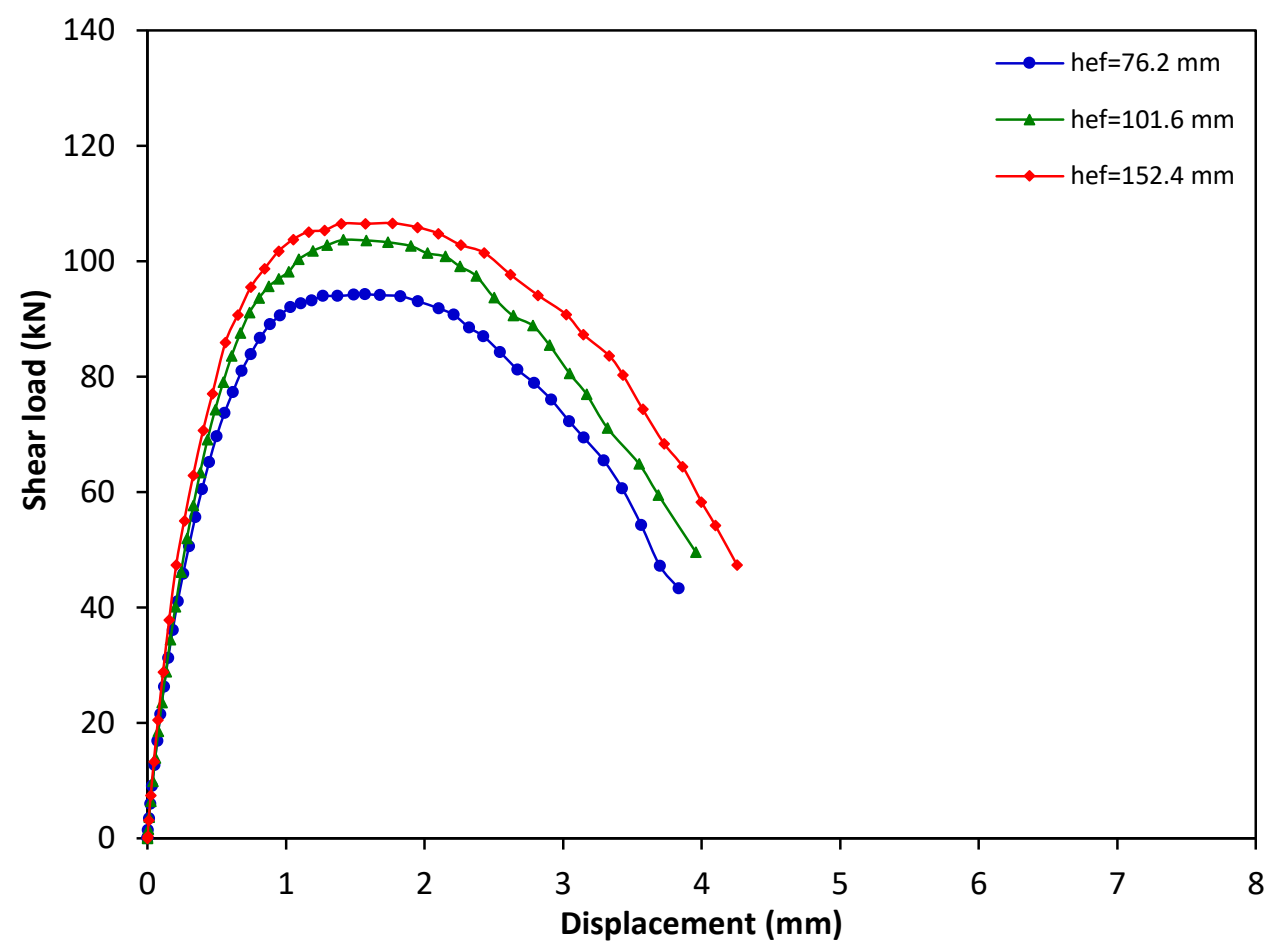

Figure F-1: Shear load-displacement graph for $15.9 \mathrm{~mm}$ diameter adhesive anchor at strain rate of $10^{-5} \mathrm{~s}^{-1}$

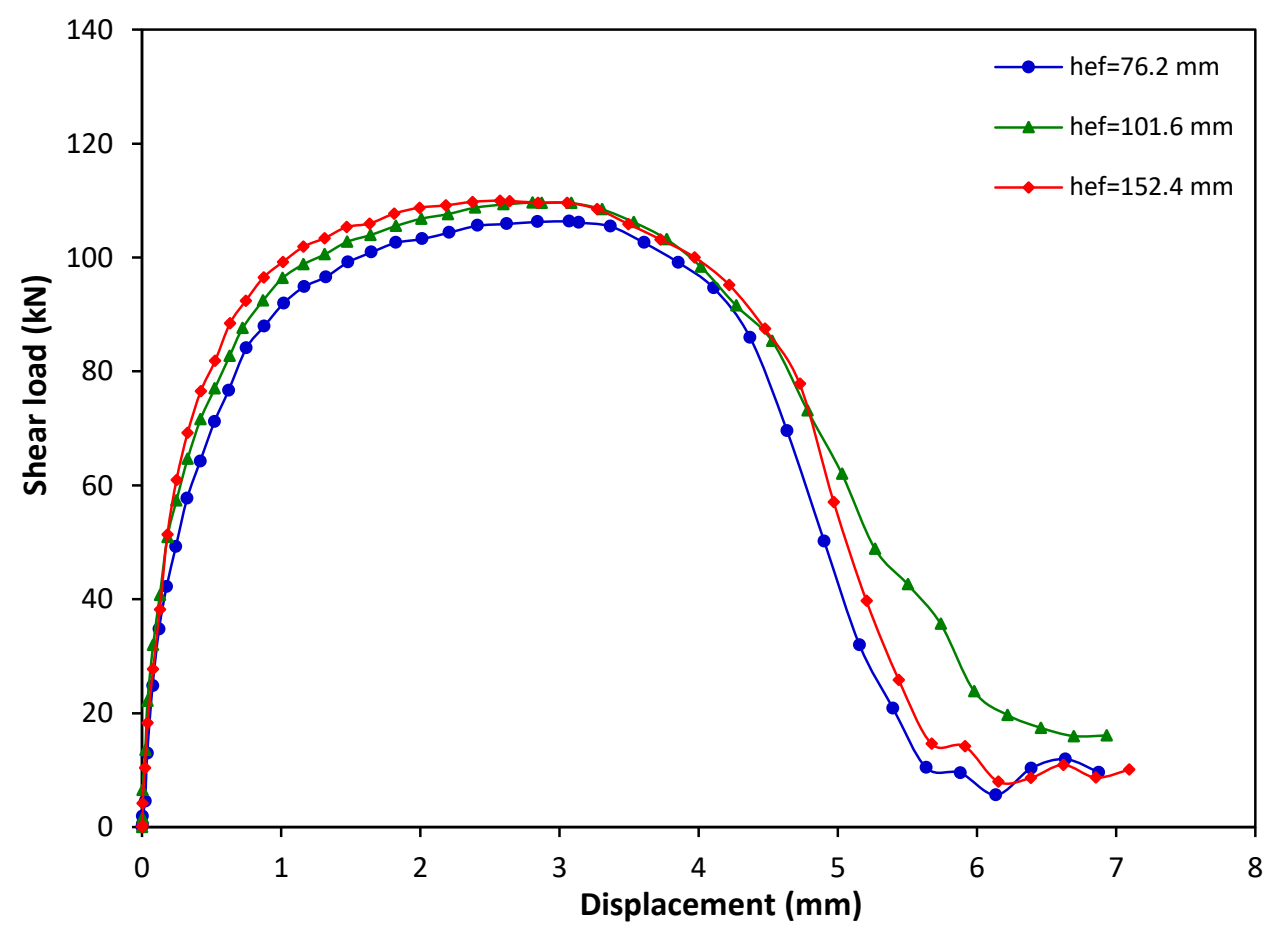

Figure F-2: Shear load-displacement graph for $15.9 \mathrm{~mm}$ diameter adhesive anchor at strain rate of $10^{-3} \mathrm{~s}^{-1}$ 


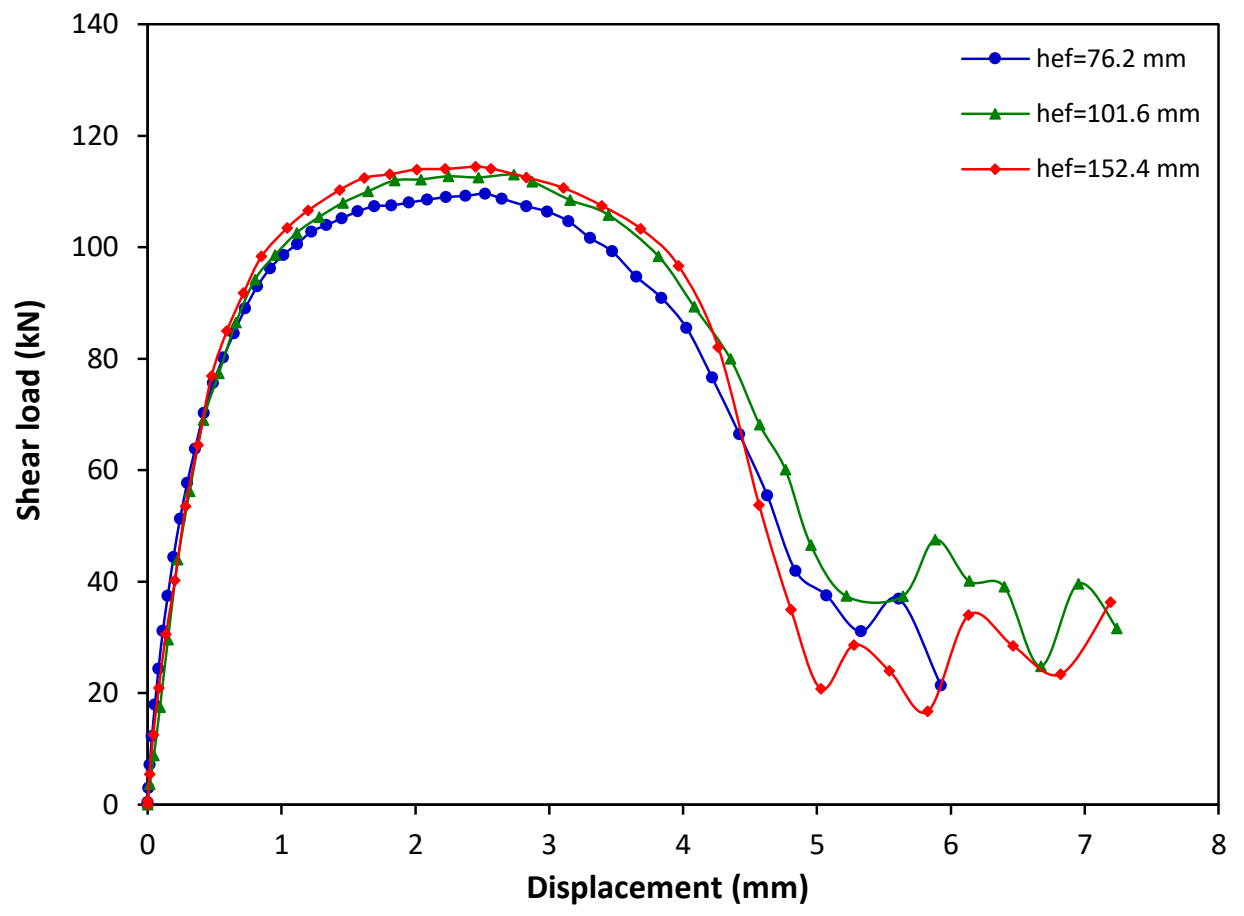

Figure F-3: Shear load-displacement graph for $15.9 \mathrm{~mm}$ diameter adhesive anchor at strain rate of $10^{-1} \mathrm{~s}^{-1}$

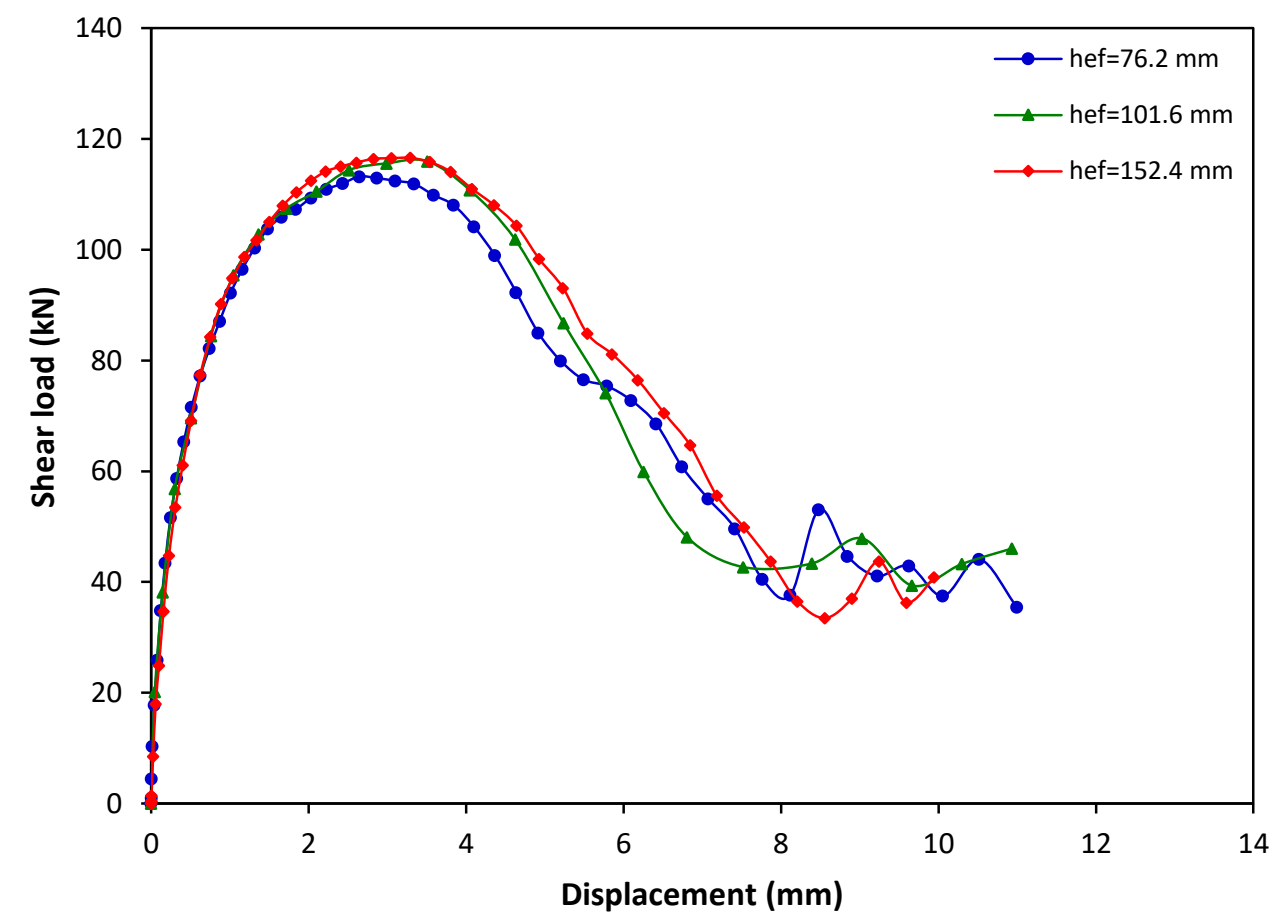

Figure F-4: Shear load-displacement graph for $15.9 \mathrm{~mm}$ diameter adhesive anchor at strain rate of $10 \mathrm{~s}^{-1}$ 


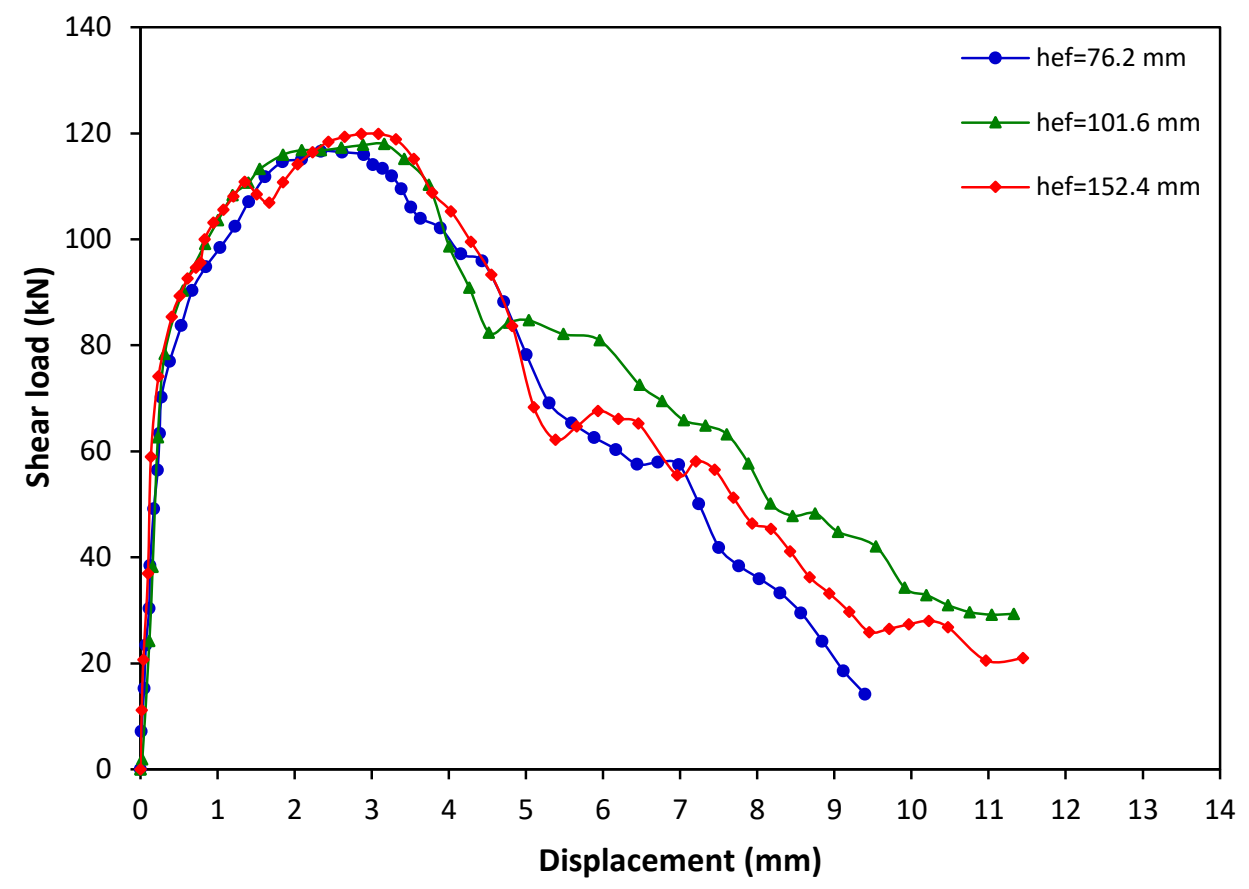

Figure F-5: Shear load-displacement graph for $15.9 \mathrm{~mm}$ diameter adhesive anchor at strain rate of $10^{2} \mathrm{~s}^{-1}$

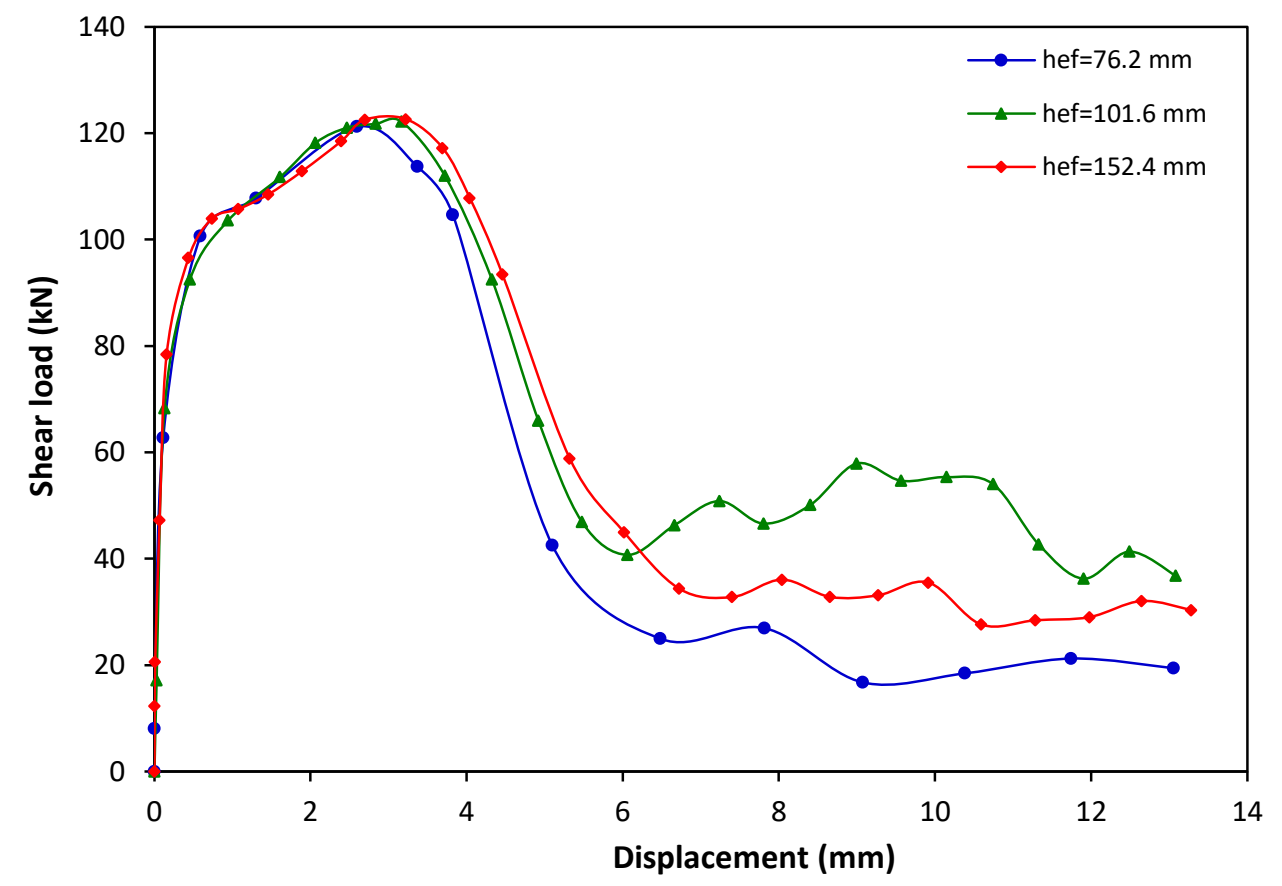

Figure F-6: Shear load-displacement graph for $15.9 \mathrm{~mm}$ diameter adhesive anchor at strain rate of $10^{3} \mathrm{~s}^{-1}$ 


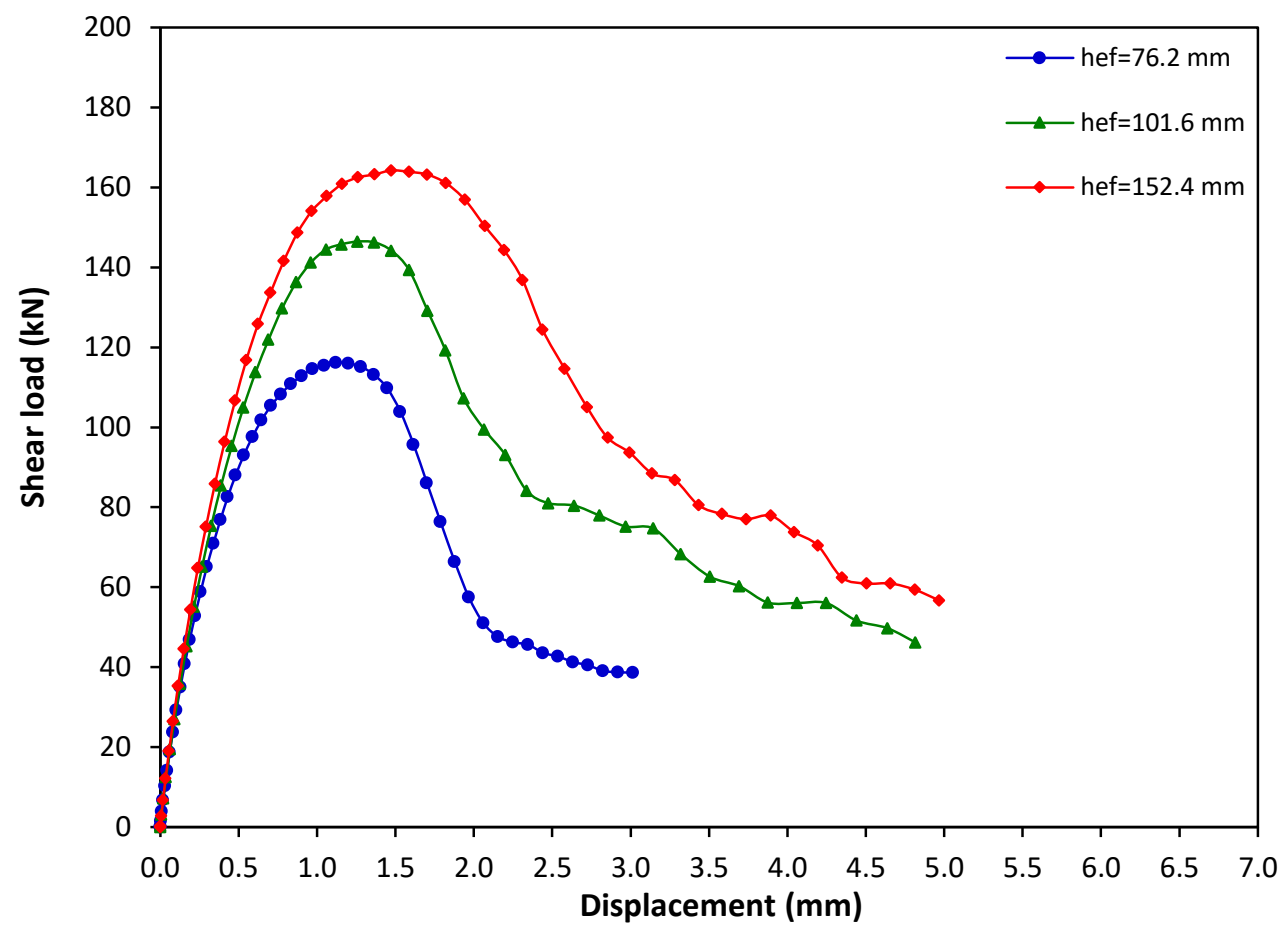

Figure F-7: Shear load-displacement graph for $19.1 \mathrm{~mm}$ diameter adhesive anchor at strain rate of $10^{-5} \mathrm{~s}^{-1}$

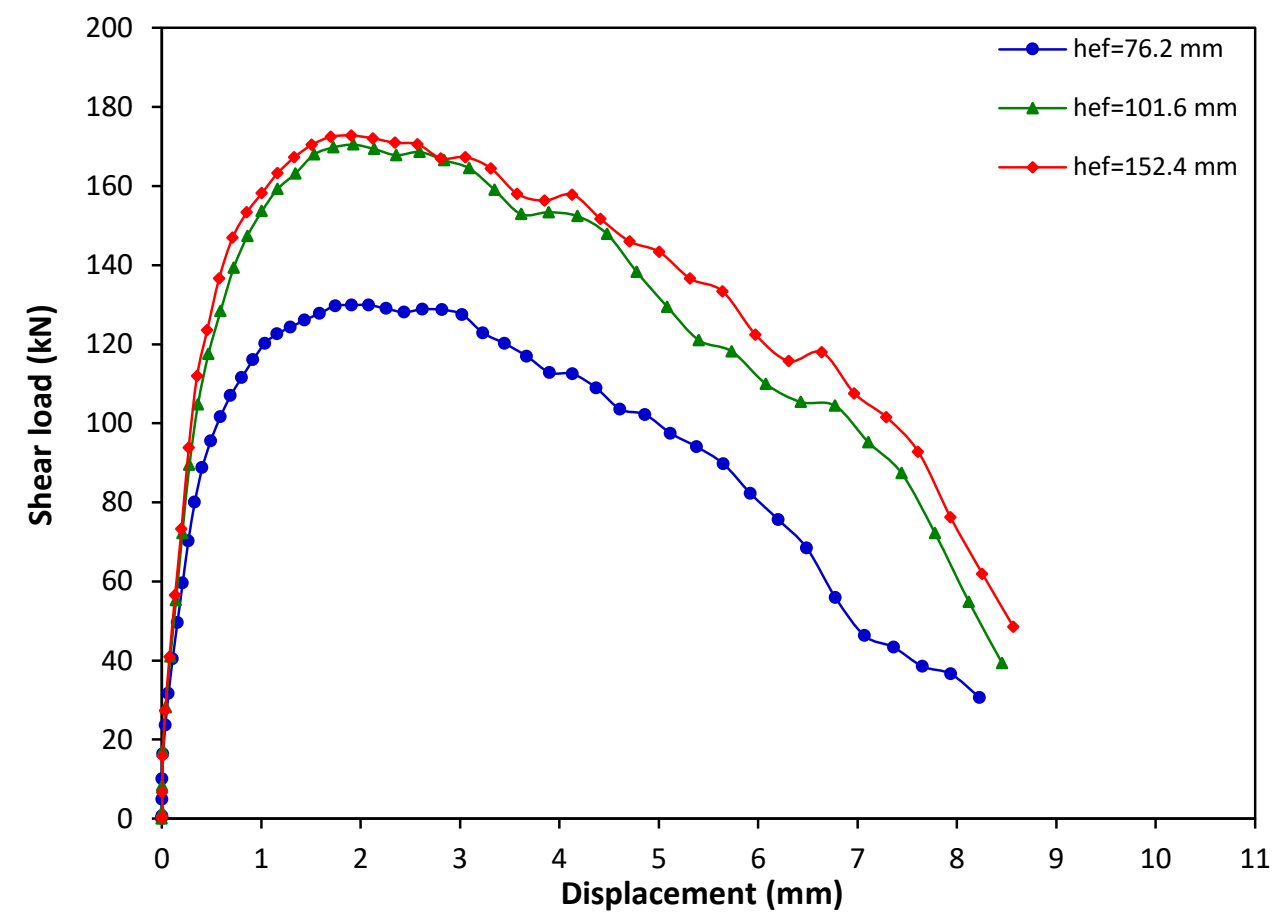

Figure F-8: Shear load-displacement graph for $19.1 \mathrm{~mm}$ diameter adhesive anchor at strain rate of $10^{-3} \mathrm{~s}^{-1}$ 


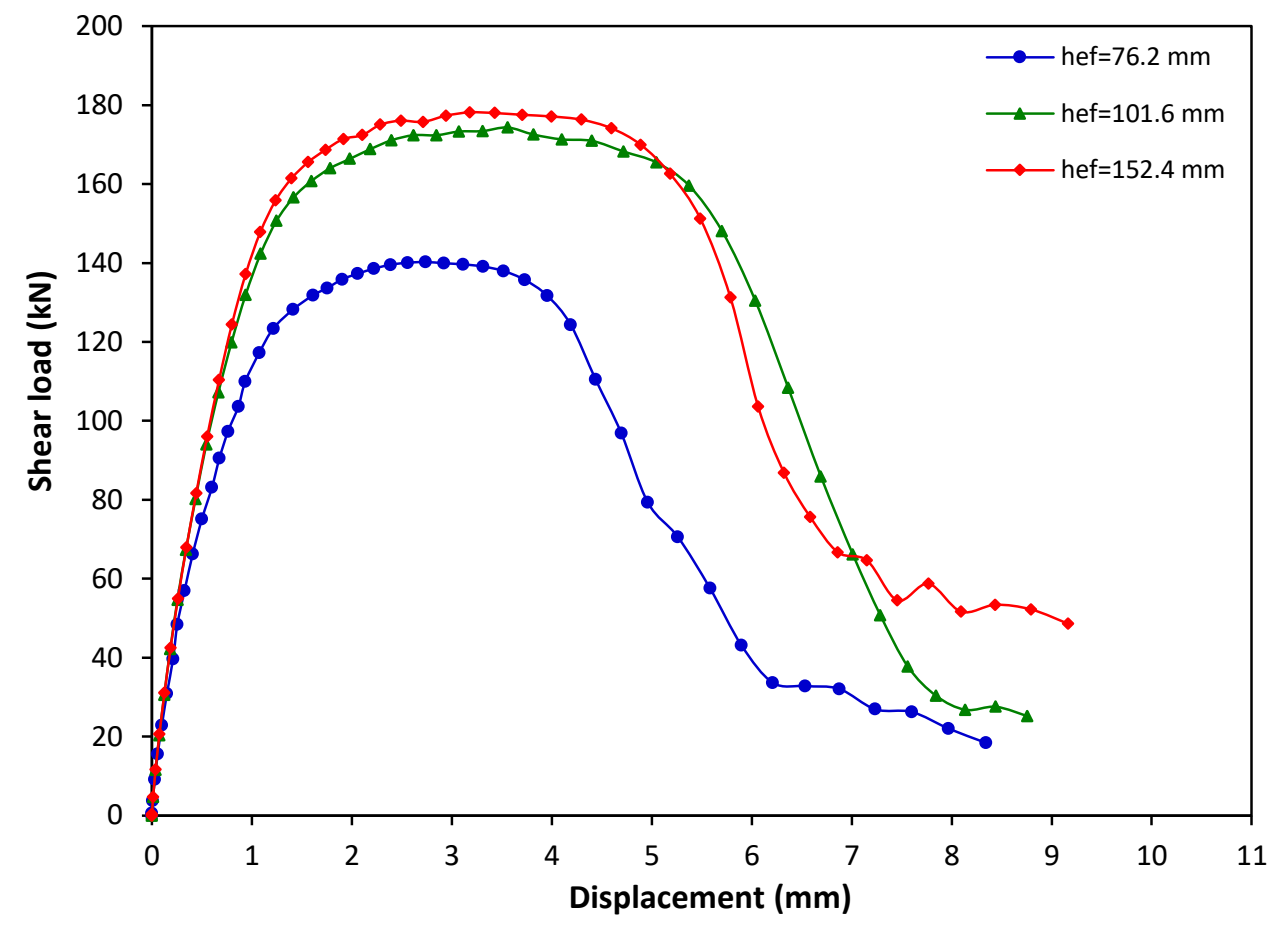

Figure F-9: Shear load-displacement graph for $19.1 \mathrm{~mm}$ diameter adhesive anchor at strain rate of $10^{-1} \mathrm{~s}^{-1}$

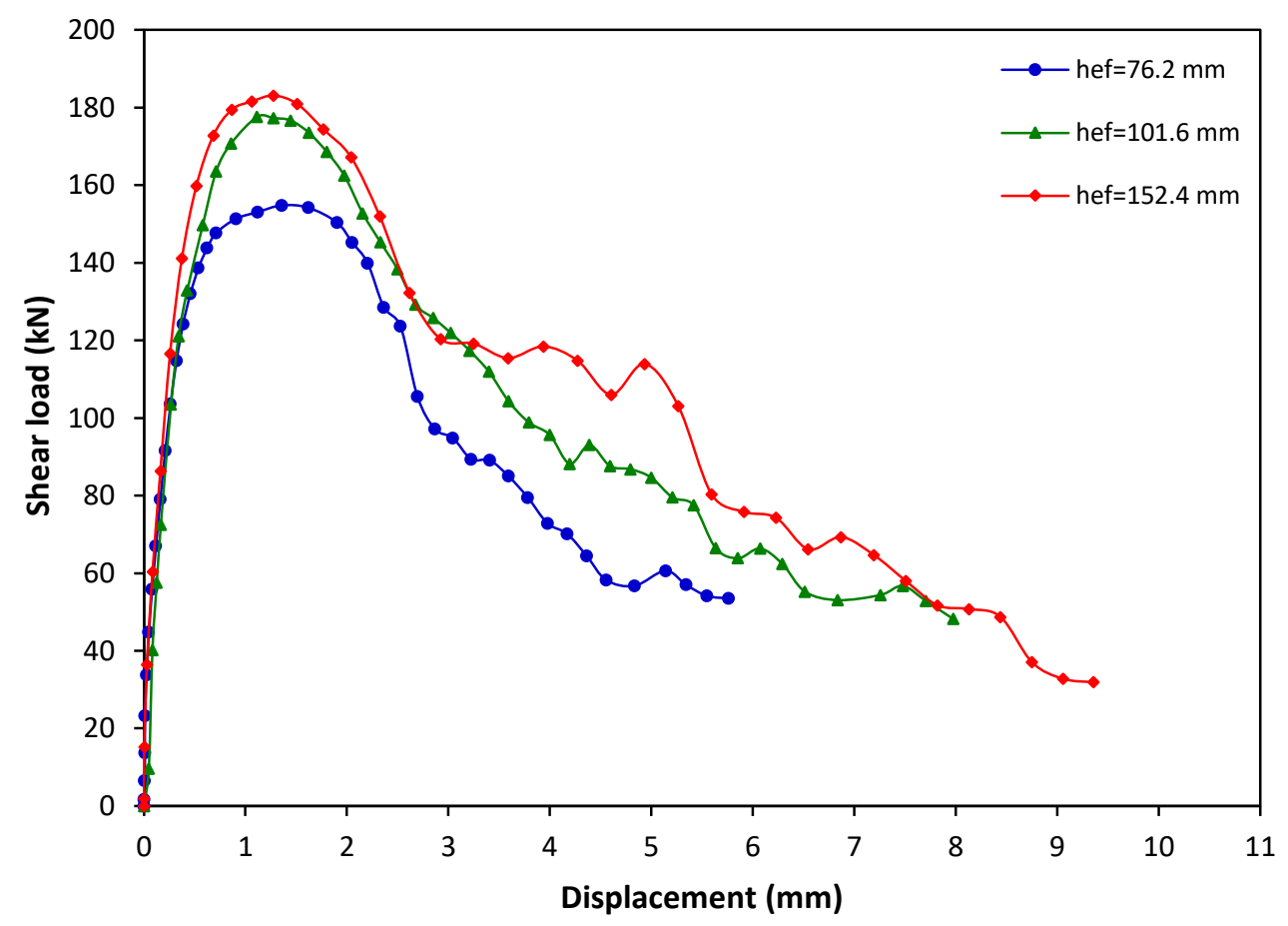

Figure F-10: Shear load-displacement graph for $19.1 \mathrm{~mm}$ diameter adhesive anchor at strain rate of $10 \mathrm{~s}^{-1}$ 


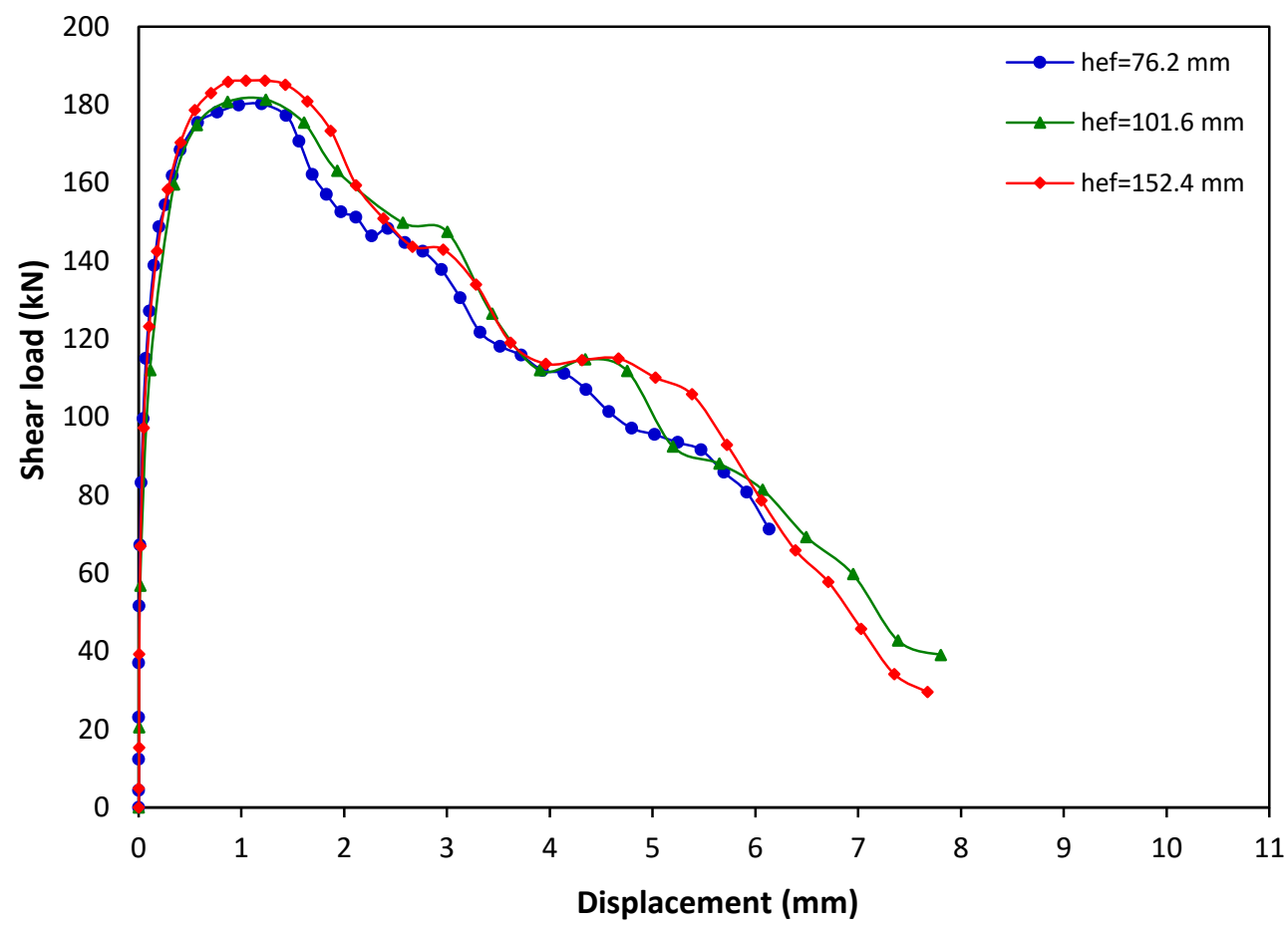

Figure F-11: Shear load-displacement graph for $19.1 \mathrm{~mm}$ diameter adhesive anchor at strain rate of $10^{2} \mathrm{~s}^{-1}$

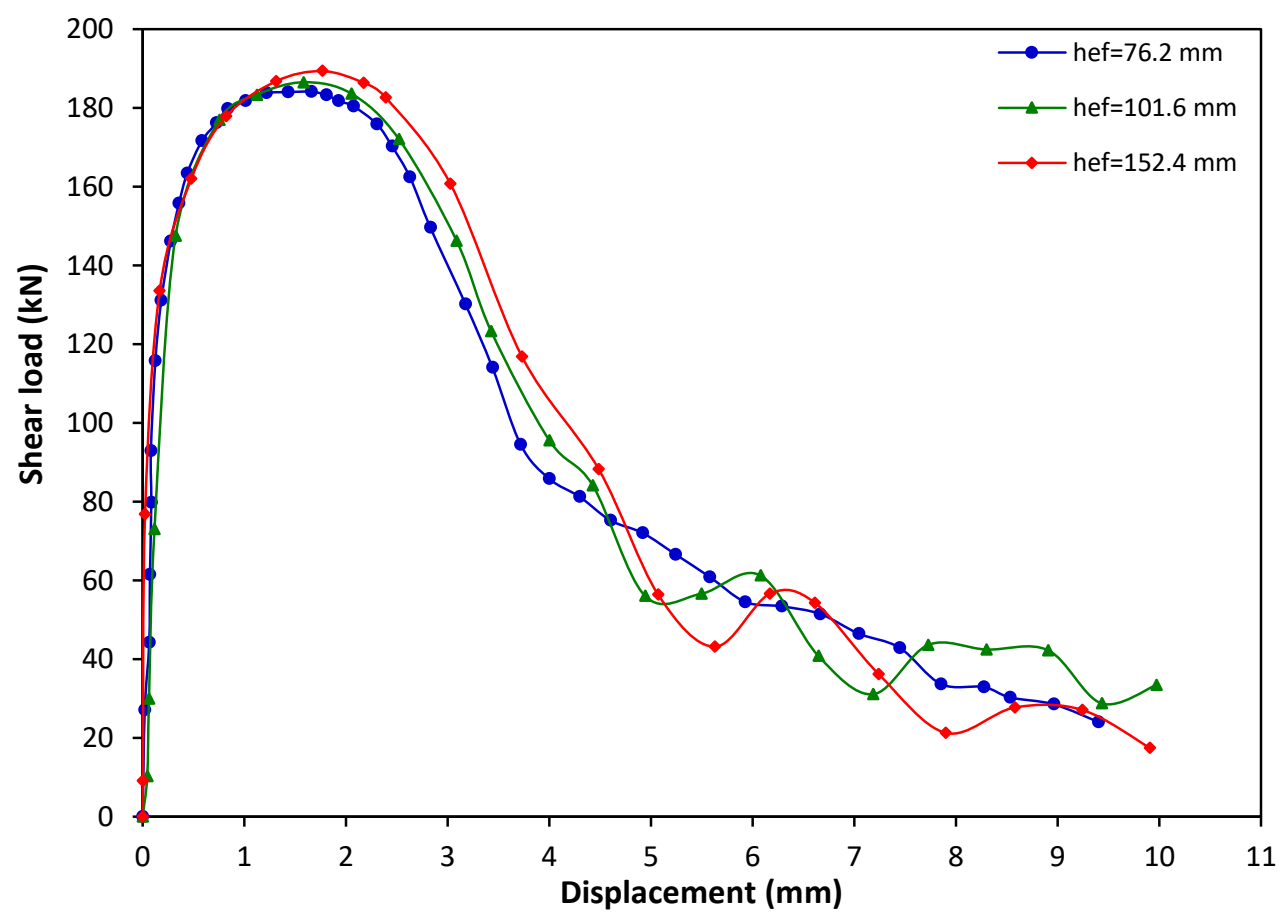

Figure F-12: Shear load-displacement graph for $19.1 \mathrm{~mm}$ diameter adhesive anchor at strain rate of $10^{3} \mathrm{~s}^{-1}$ 


\section{Appendix G: Tensile load-displacement relation for undercut anchors}

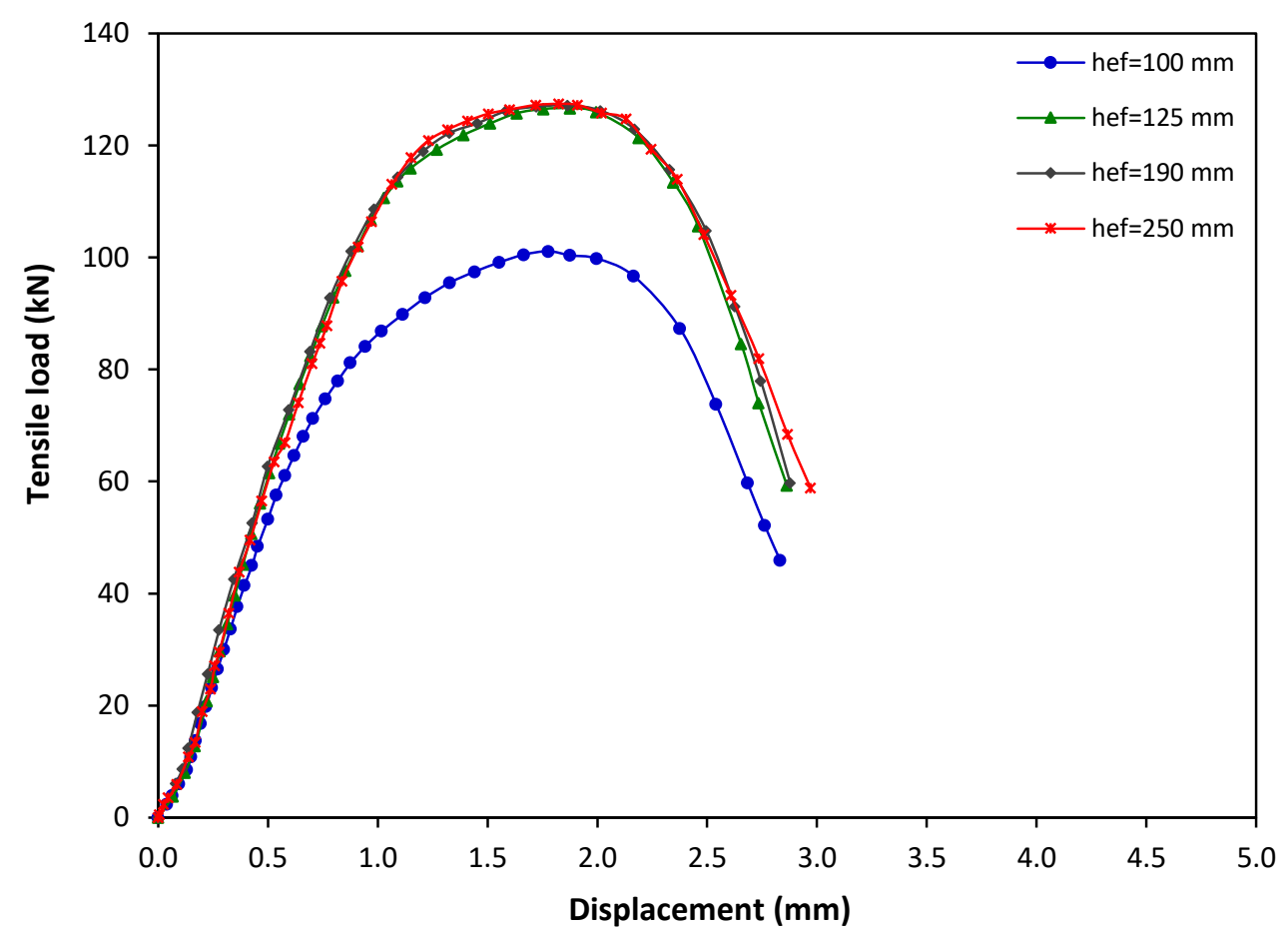

Figure G-1: Tensile load-displacement graph for the $16 \mathrm{~mm}$ diameter undercut anchor at

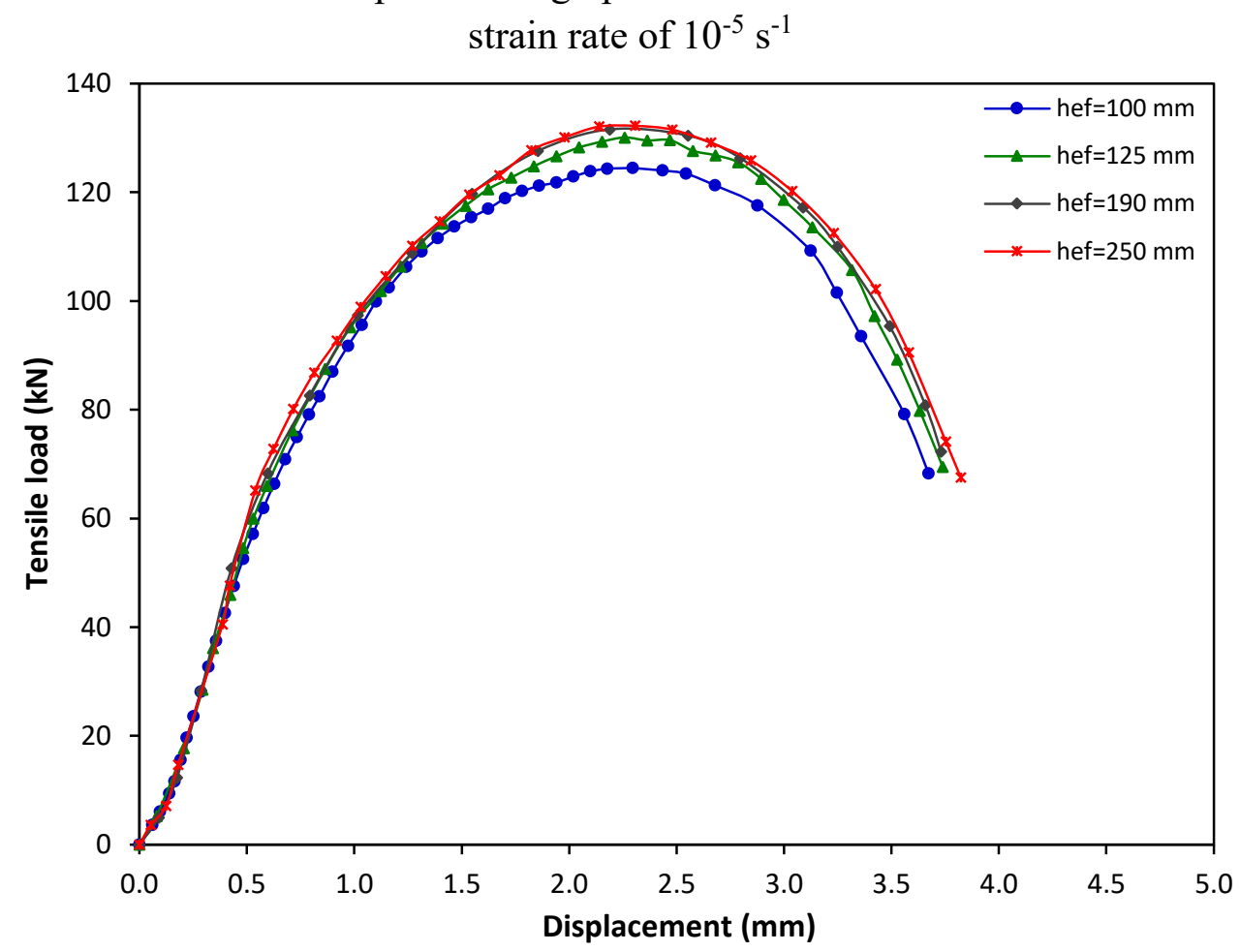

Figure G-2: Tensile load-displacement graph for the $16 \mathrm{~mm}$ diameter undercut anchor at strain rate of $10^{-3} \mathrm{~s}^{-1}$ 


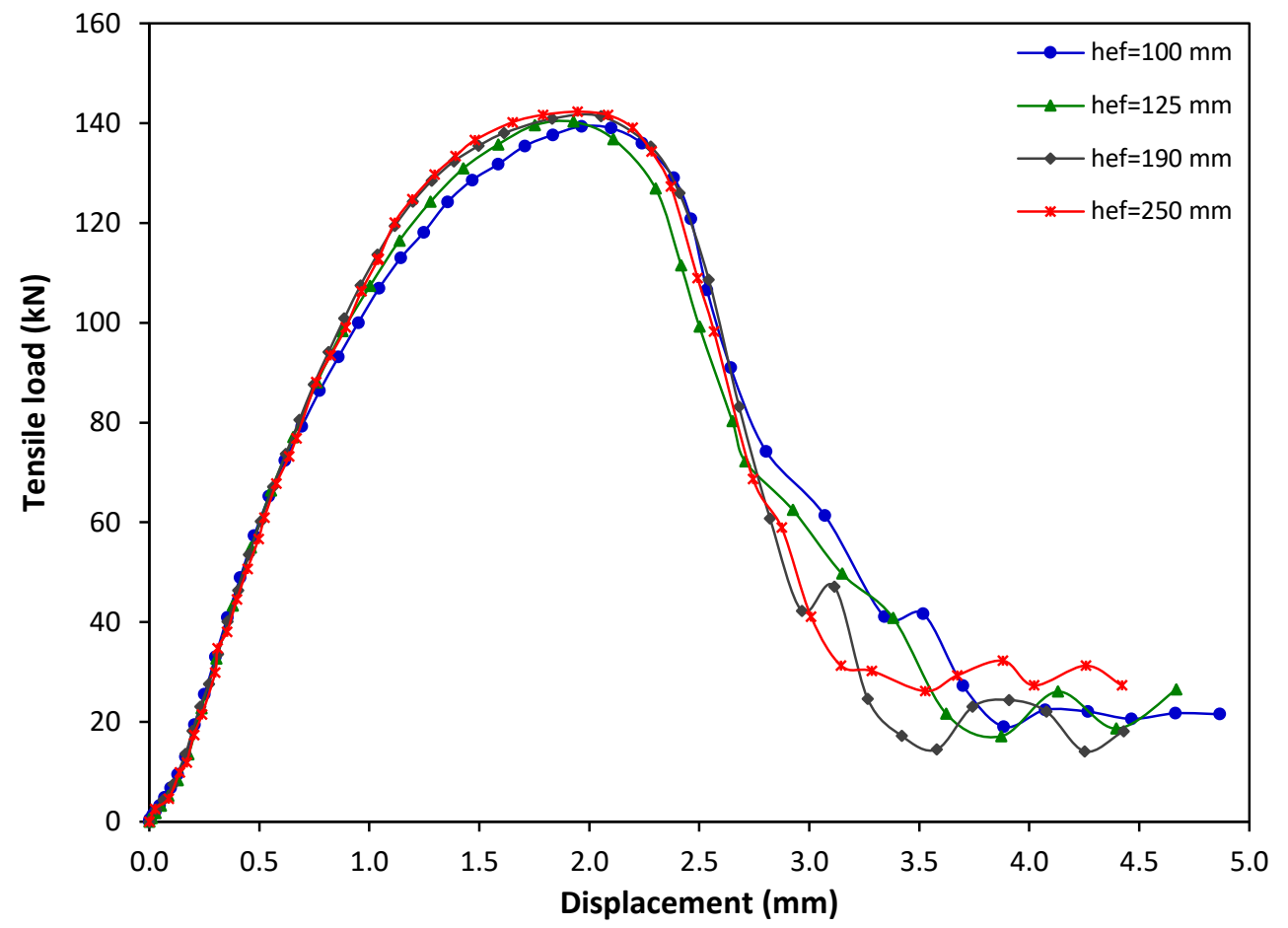

Figure G-3: Tensile load-displacement graph for the $16 \mathrm{~mm}$ diameter undercut anchor at strain rate of $10^{-1} \mathrm{~s}^{-1}$

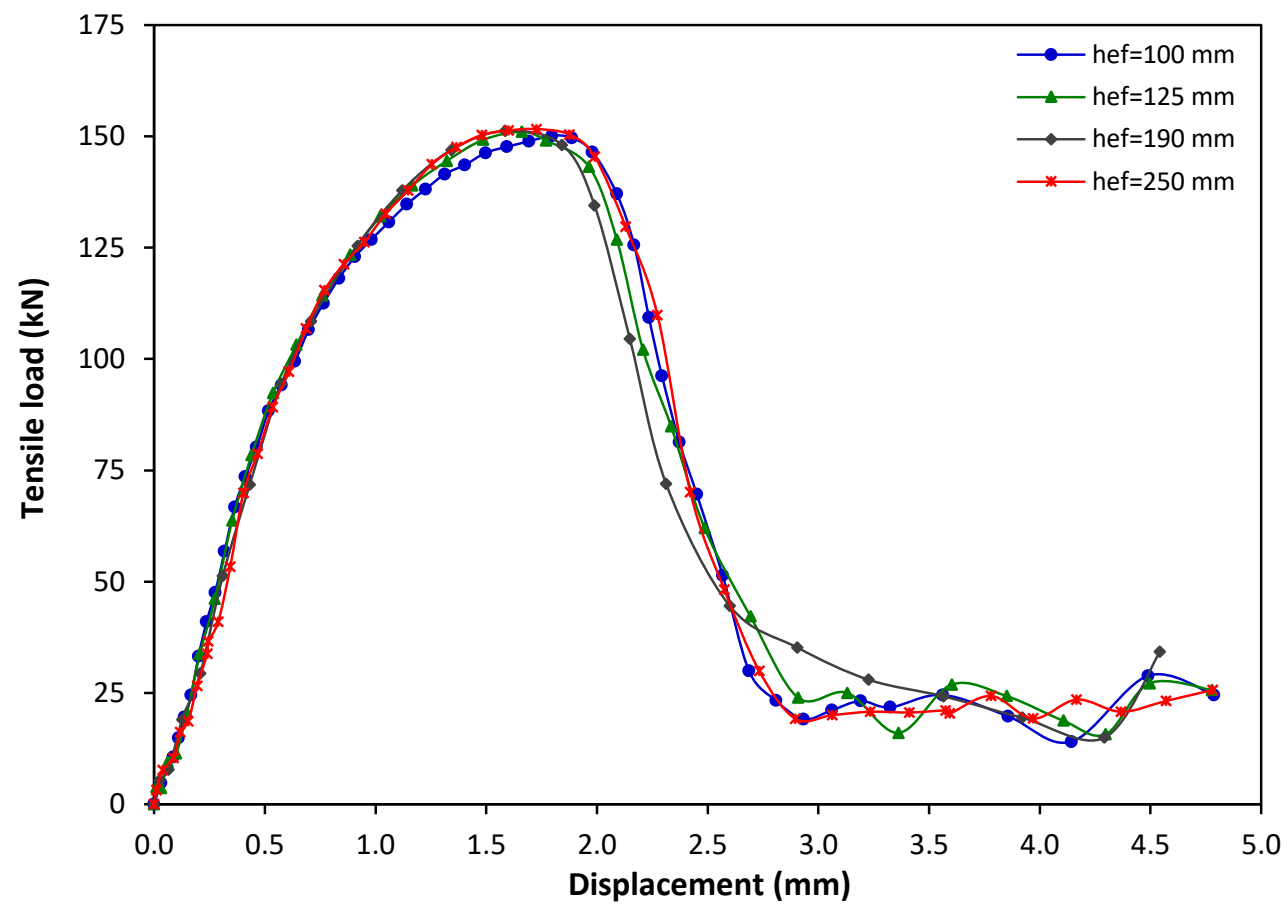

Figure G-4: Tensile load-displacement graph for the $16 \mathrm{~mm}$ diameter undercut anchor at strain rate of $10 \mathrm{~s}^{-1}$ 


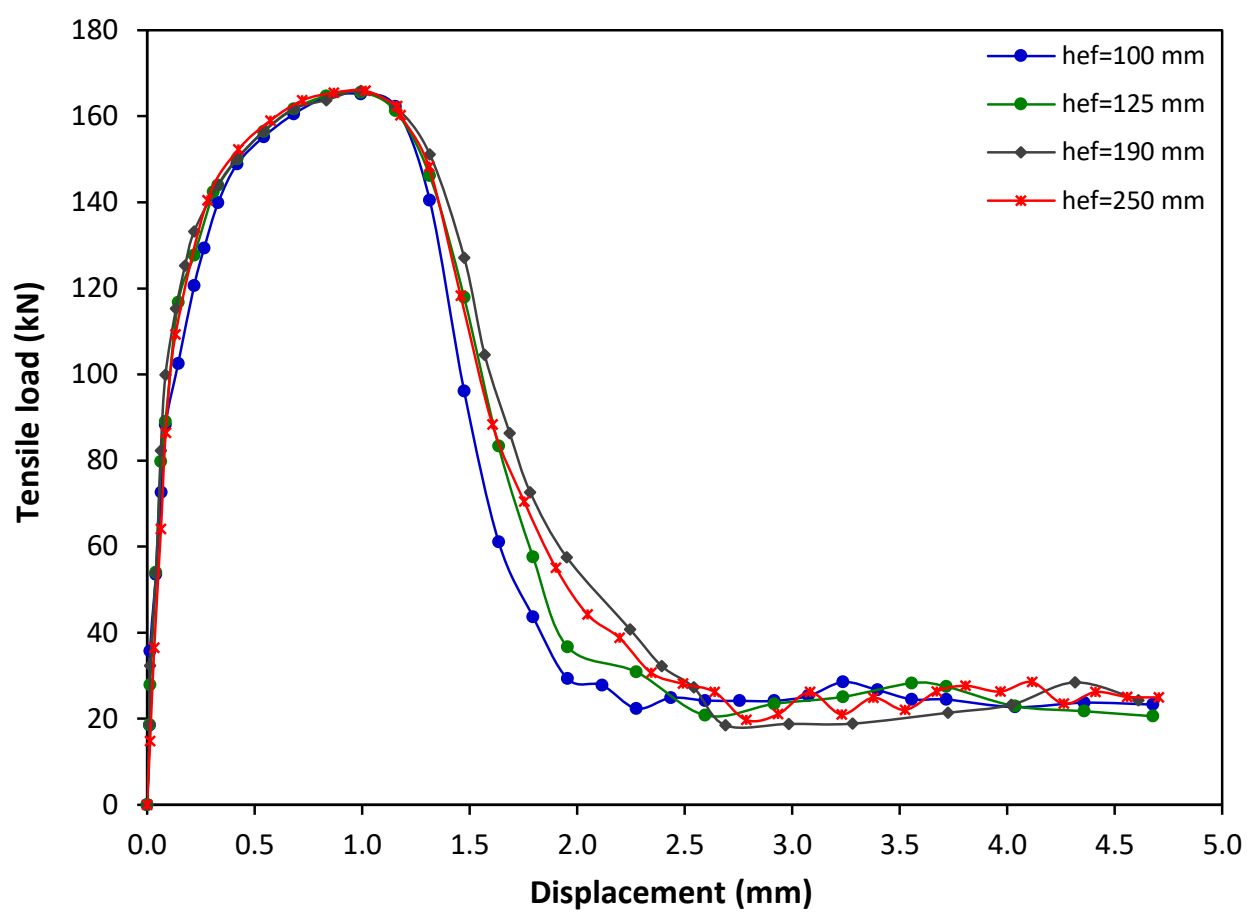

Figure G-5: Tensile load-displacement graph for the $16 \mathrm{~mm}$ diameter undercut anchor at strain rate of $10^{2} \mathrm{~s}^{-1}$

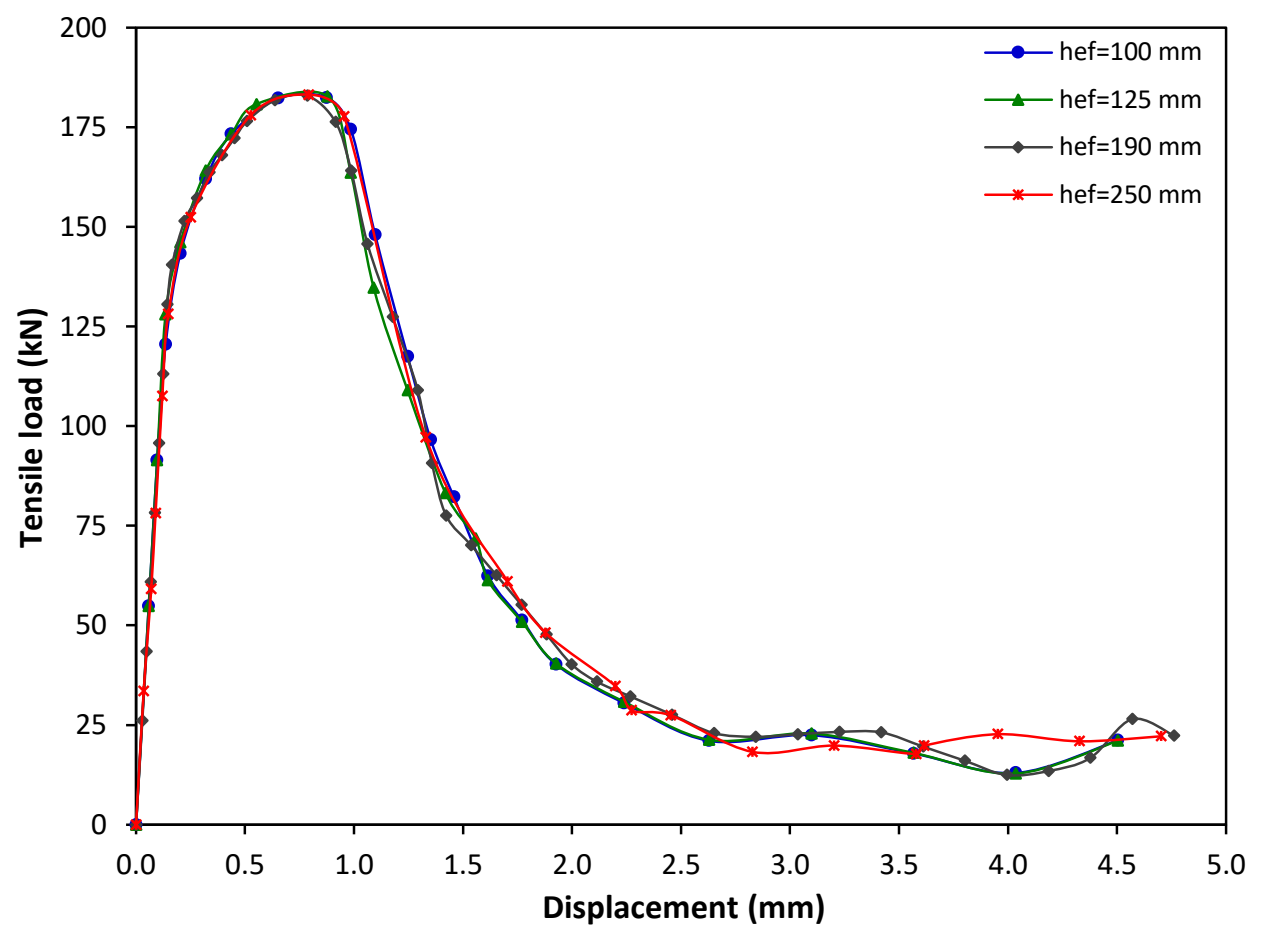

Figure G-6: Tensile load-displacement graph for the $16 \mathrm{~mm}$ diameter undercut anchor at strain rate of $10^{3} \mathrm{~s}^{-1}$ 


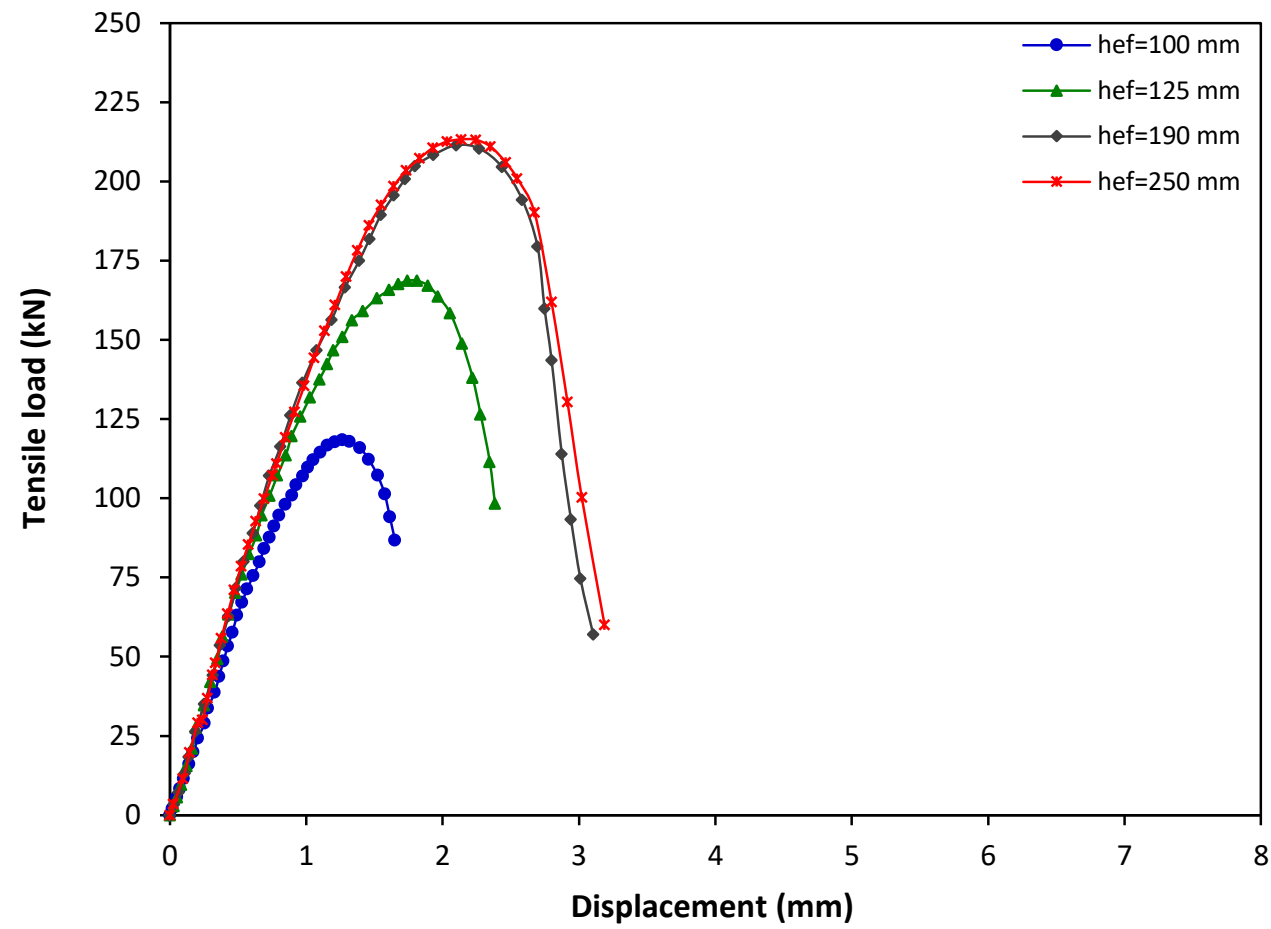

Figure G-7: Tensile load-displacement graph for the $20 \mathrm{~mm}$ diameter undercut anchor at strain rate of $10^{-5} \mathrm{~s}^{-1}$

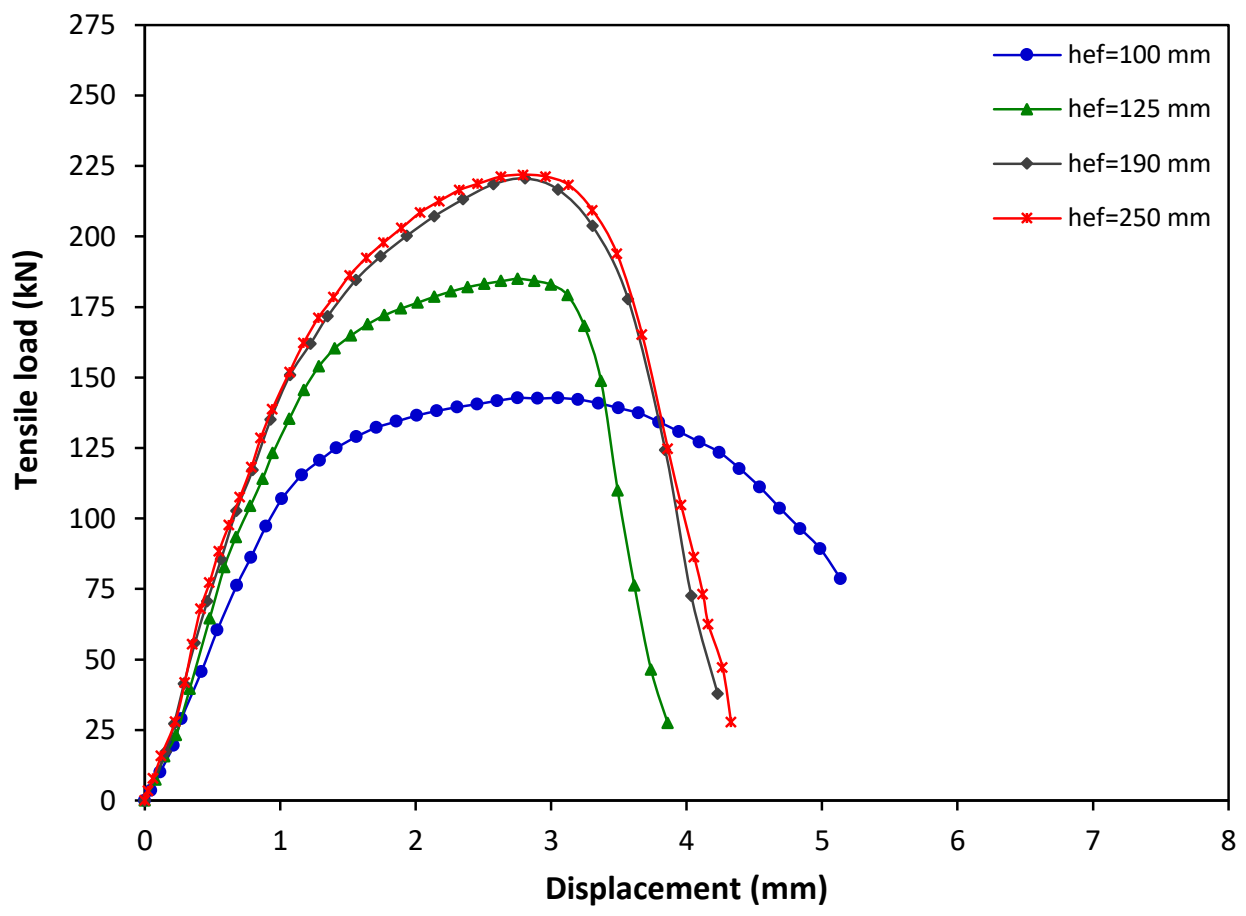

Figure G-8: Tensile load-displacement graph for the $20 \mathrm{~mm}$ diameter undercut anchor at strain rate of $10^{-3} \mathrm{~s}^{-1}$ 


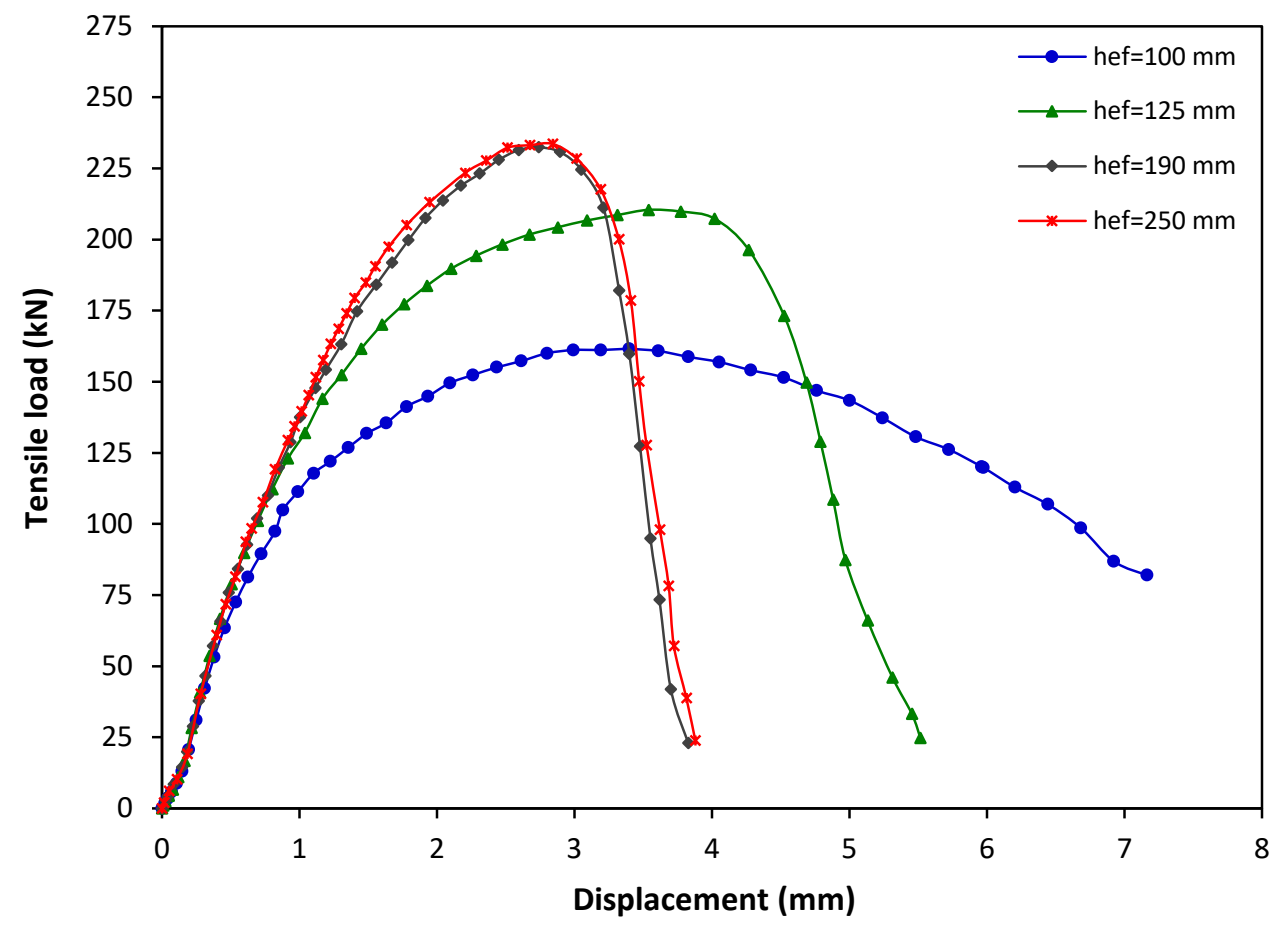

Figure G-9: Tensile load-displacement graph for the $20 \mathrm{~mm}$ diameter undercut anchor at strain rate of $10^{-1} \mathrm{~s}^{-1}$

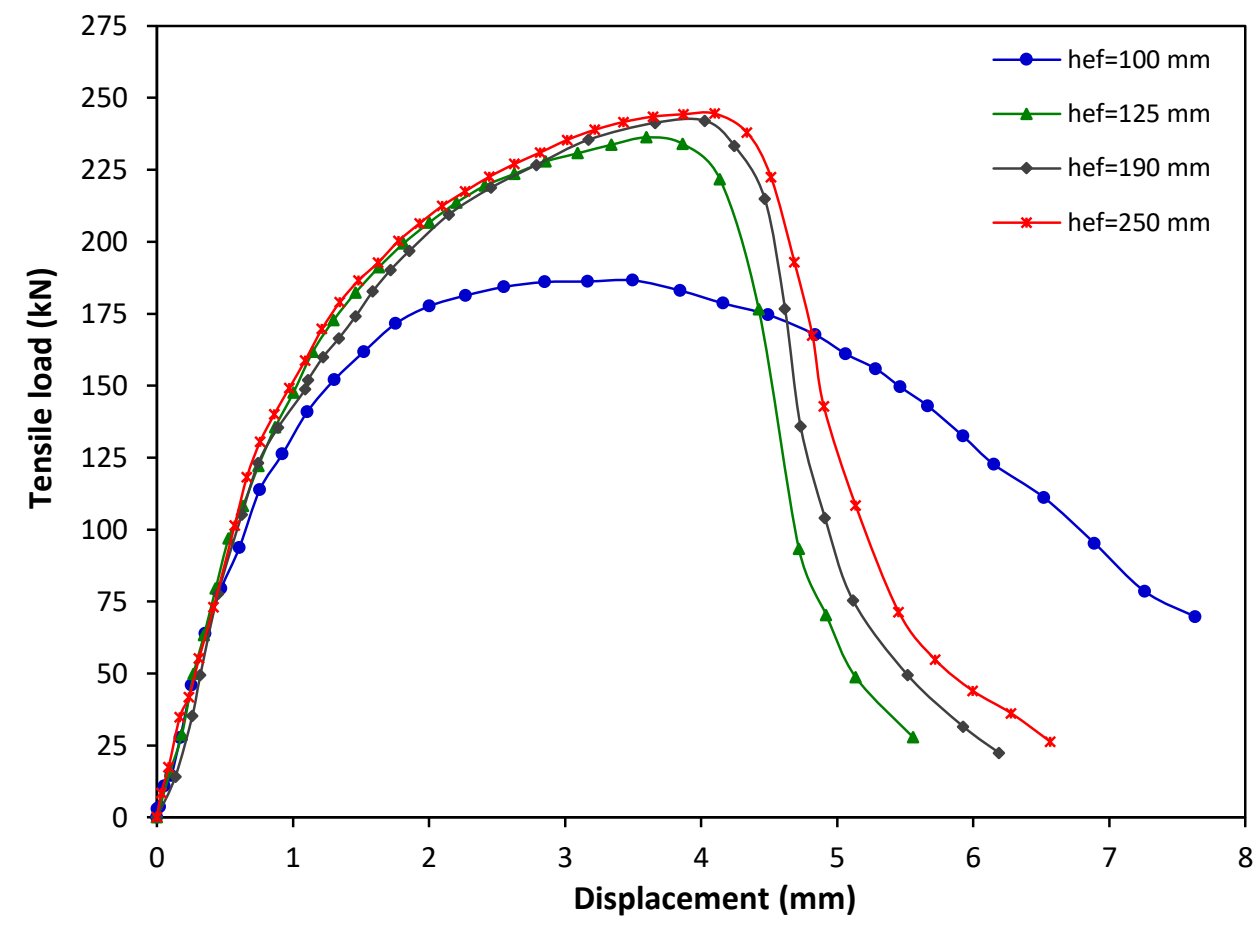

Figure G-10: Tensile load-displacement graph for the $20 \mathrm{~mm}$ diameter undercut anchor at strain rate of $10 \mathrm{~s}^{-1}$ 


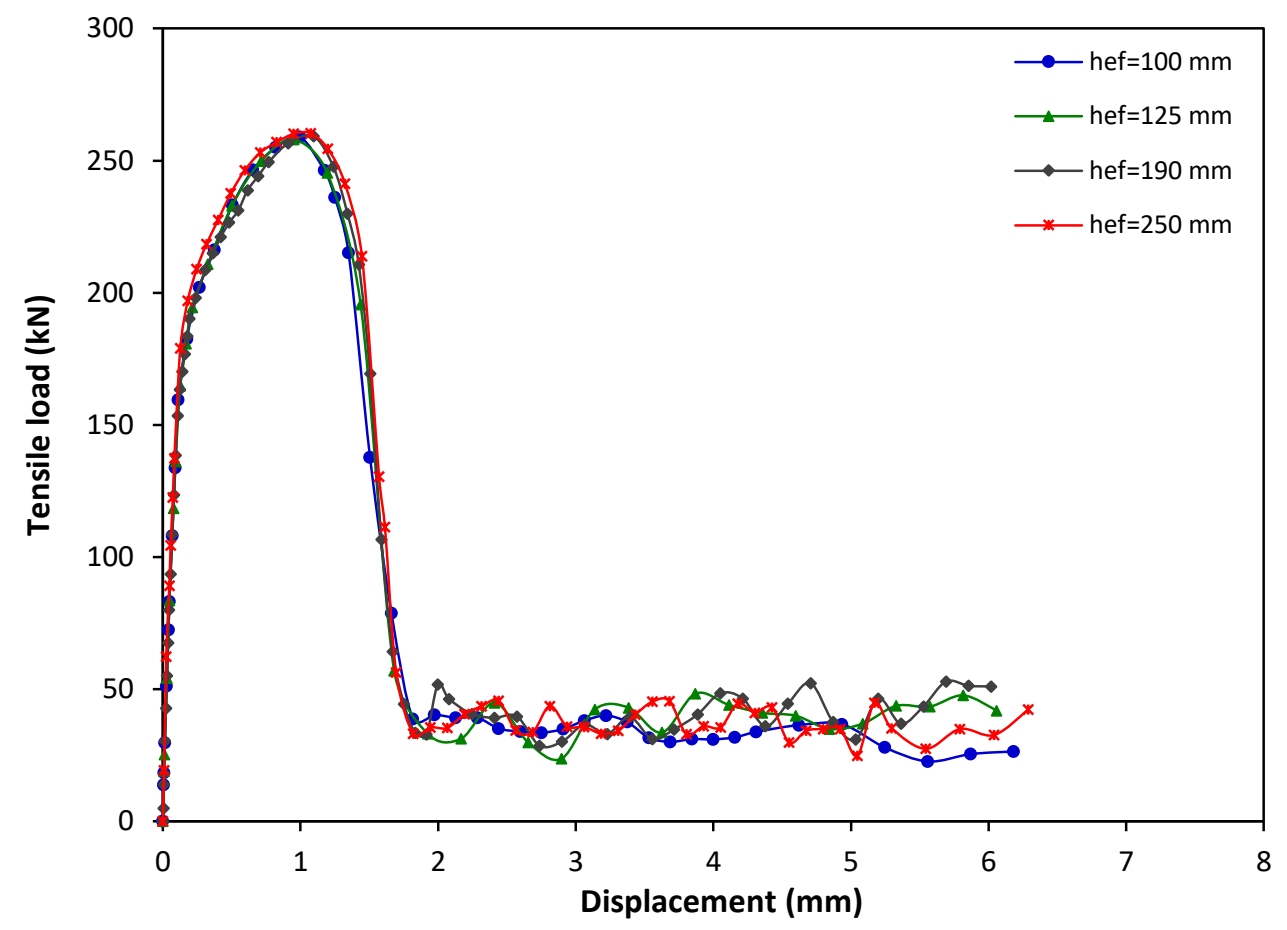

Figure G-11: Tensile load-displacement graph for the $20 \mathrm{~mm}$ diameter undercut anchor at strain rate of $10^{2} \mathrm{~s}^{-1}$

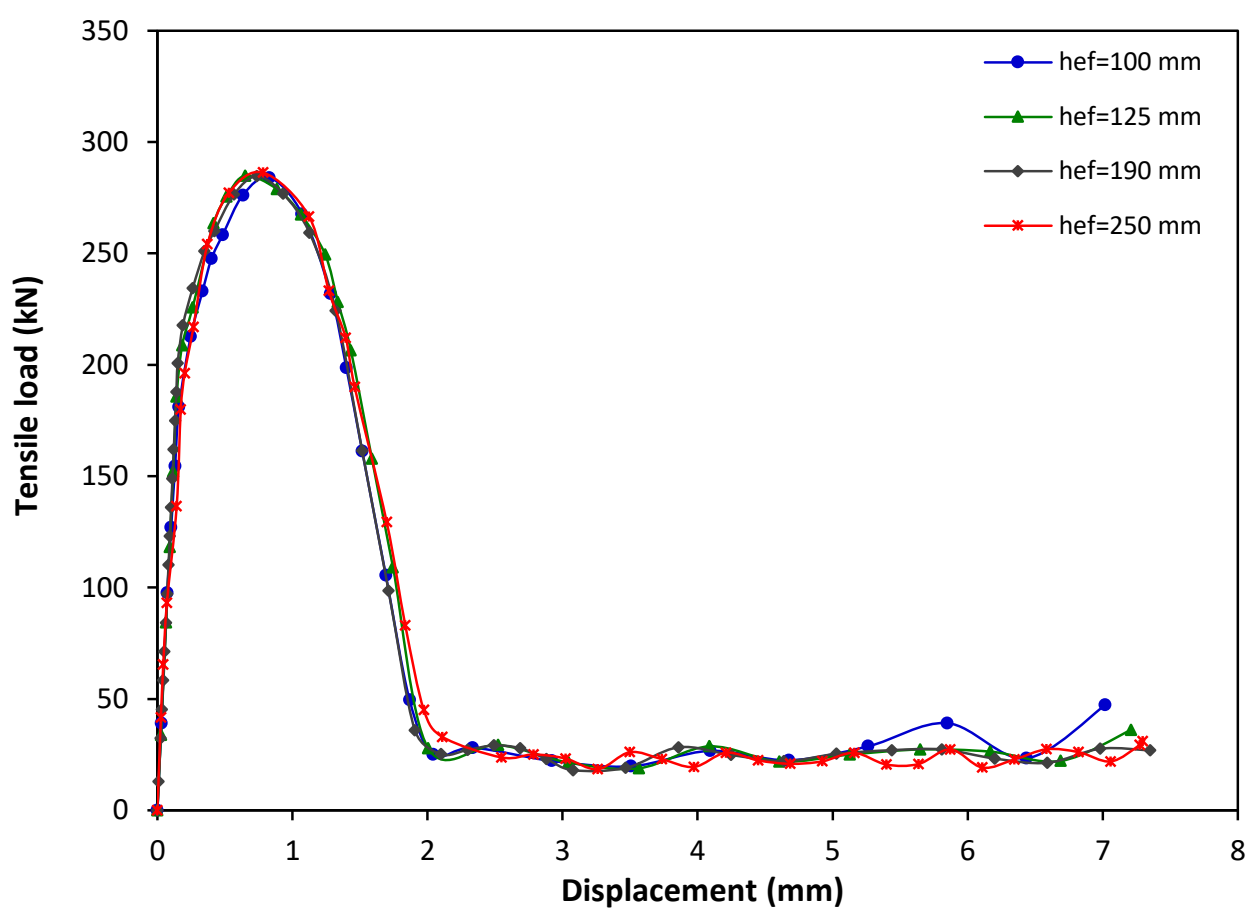

Figure G-12: Tensile load-displacement graph for the $20 \mathrm{~mm}$ diameter undercut anchor at strain rate of $10^{3} \mathrm{~s}^{-1}$ 


\section{Appendix H: Shear load-displacement relation for undercut anchors}

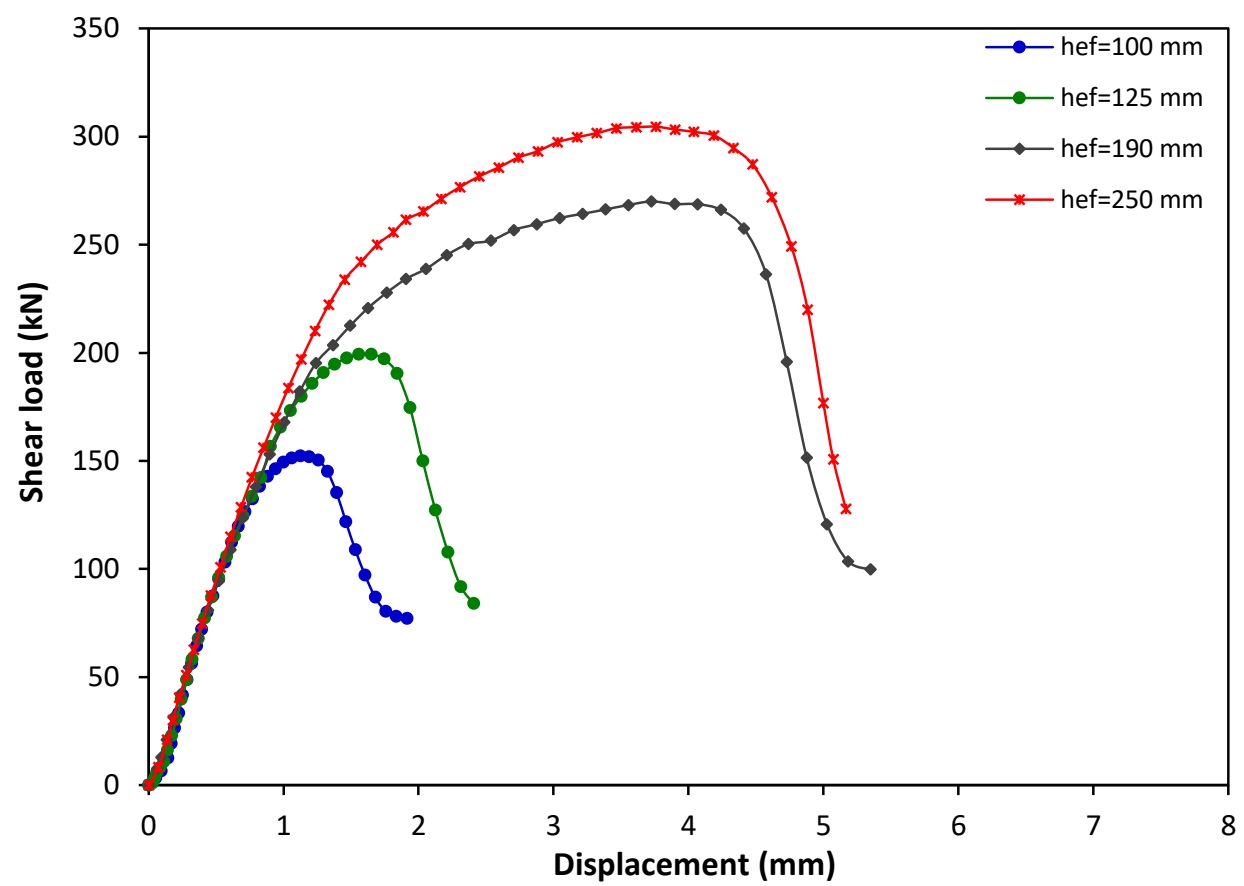

Figure H-1: Shear load-displacement graph for $16 \mathrm{~mm}$ diameter undercut anchor at strain rate of $10^{-5} \mathrm{~s}^{-1}$

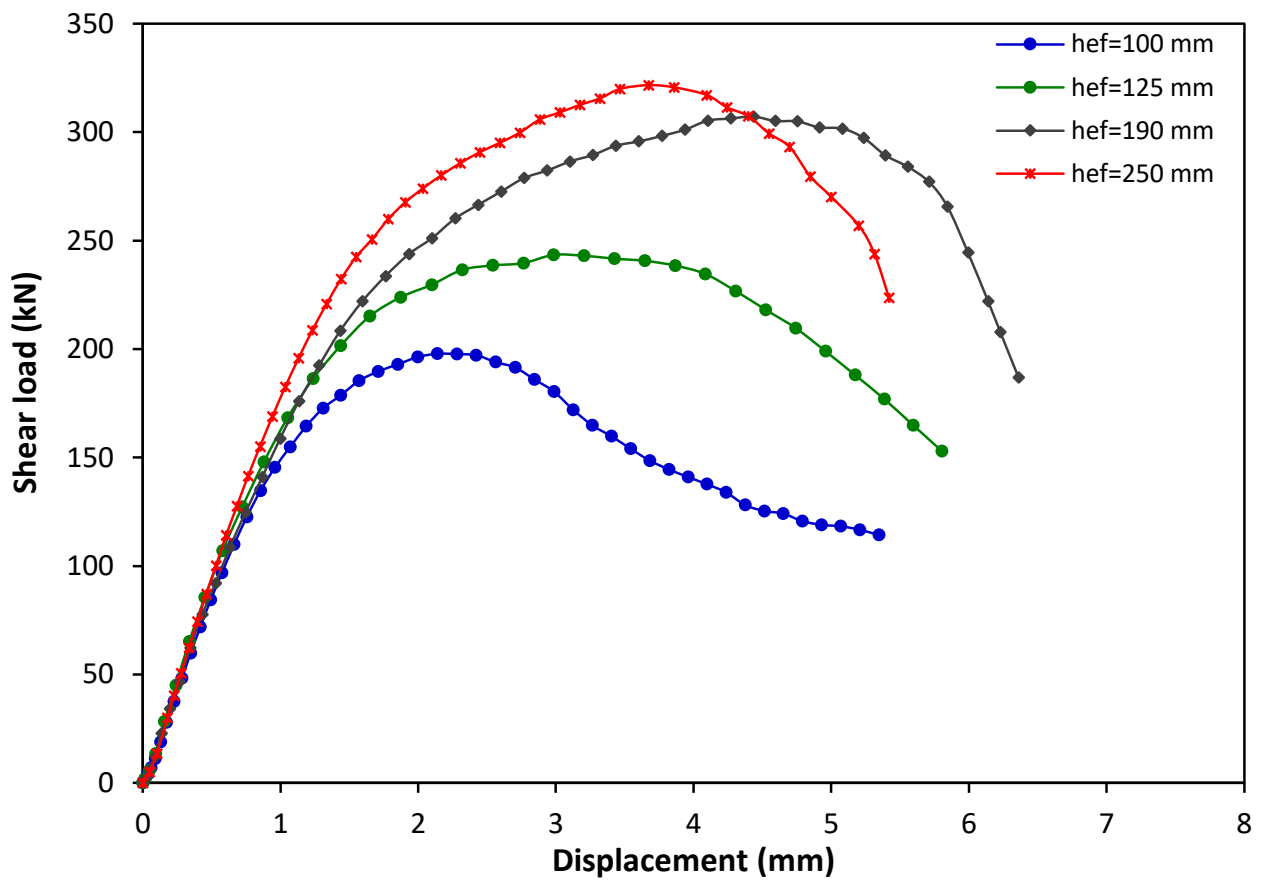

Figure H-2: Shear load-displacement graph for $16 \mathrm{~mm}$ diameter undercut anchor at strain rate of $10^{-3} \mathrm{~s}^{-1}$ 


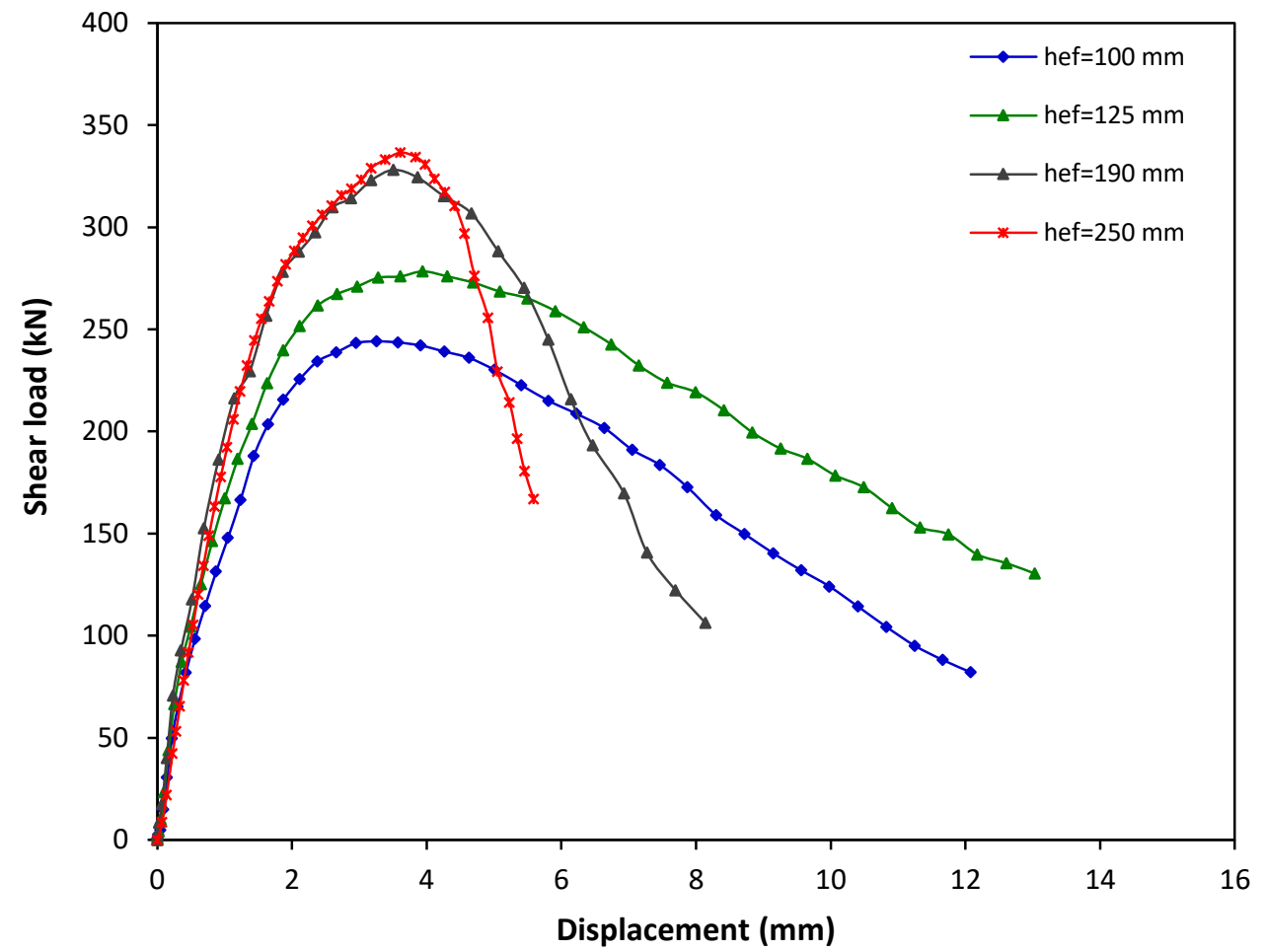

Figure H-3: Shear load-displacement graph for $16 \mathrm{~mm}$ diameter undercut anchor at strain rate of $10^{-1} \mathrm{~s}^{-1}$

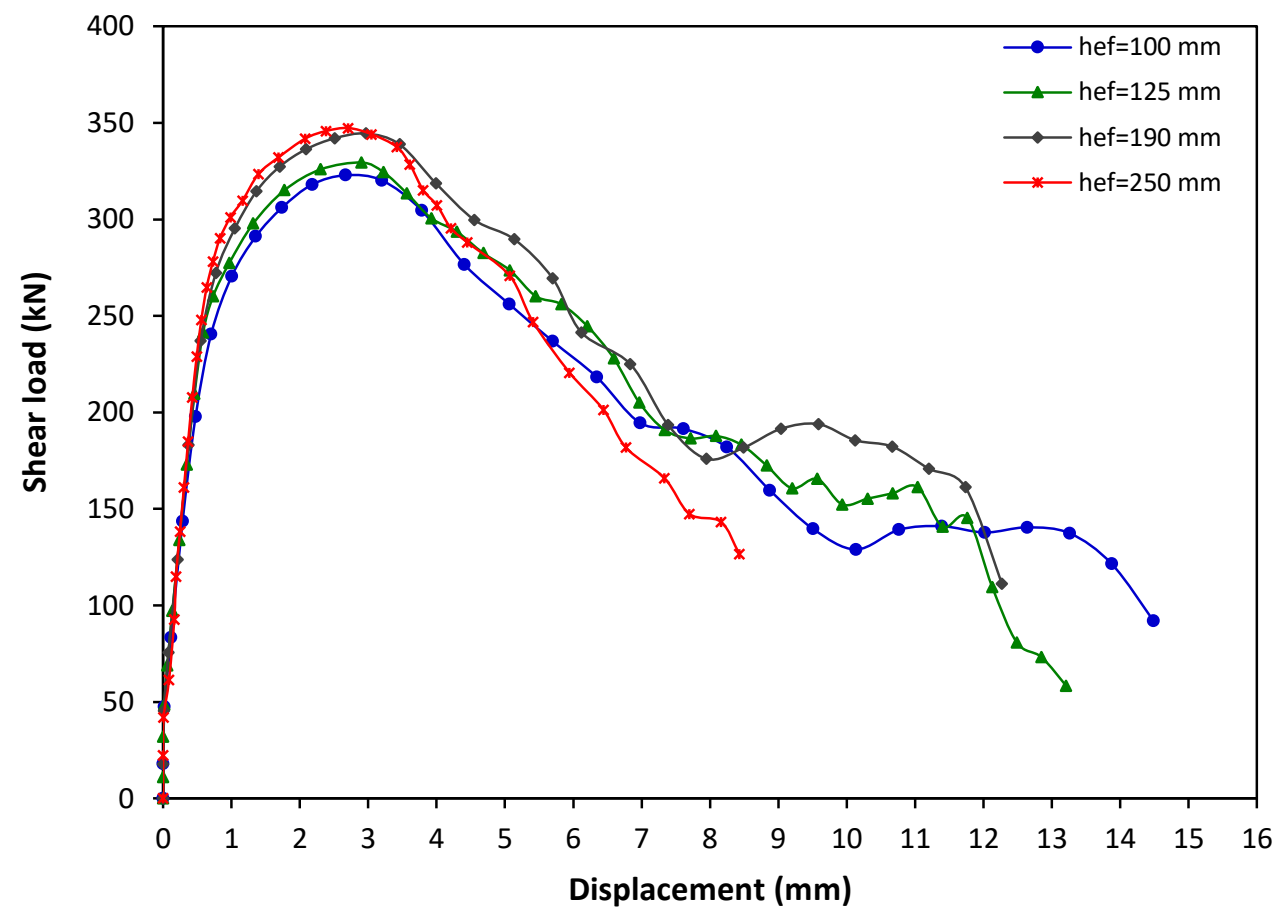

Figure H-4: Shear load-displacement graph for $16 \mathrm{~mm}$ diameter undercut anchor at strain rate of $10 \mathrm{~s}^{-1}$ 


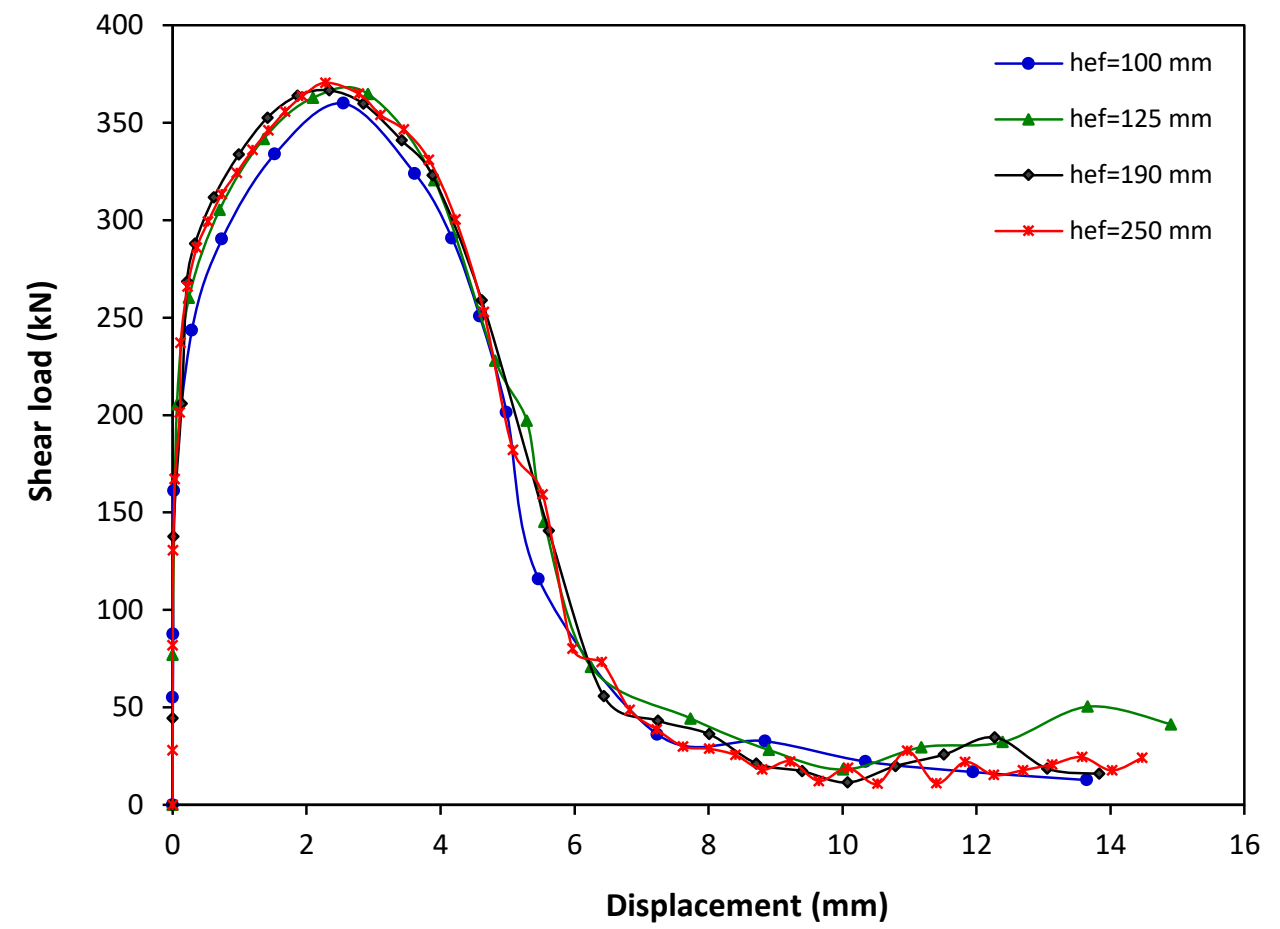

Figure H-5: Shear load-displacement graph for $16 \mathrm{~mm}$ diameter undercut anchor at strain rate of $10^{2} \mathrm{~s}^{-1}$

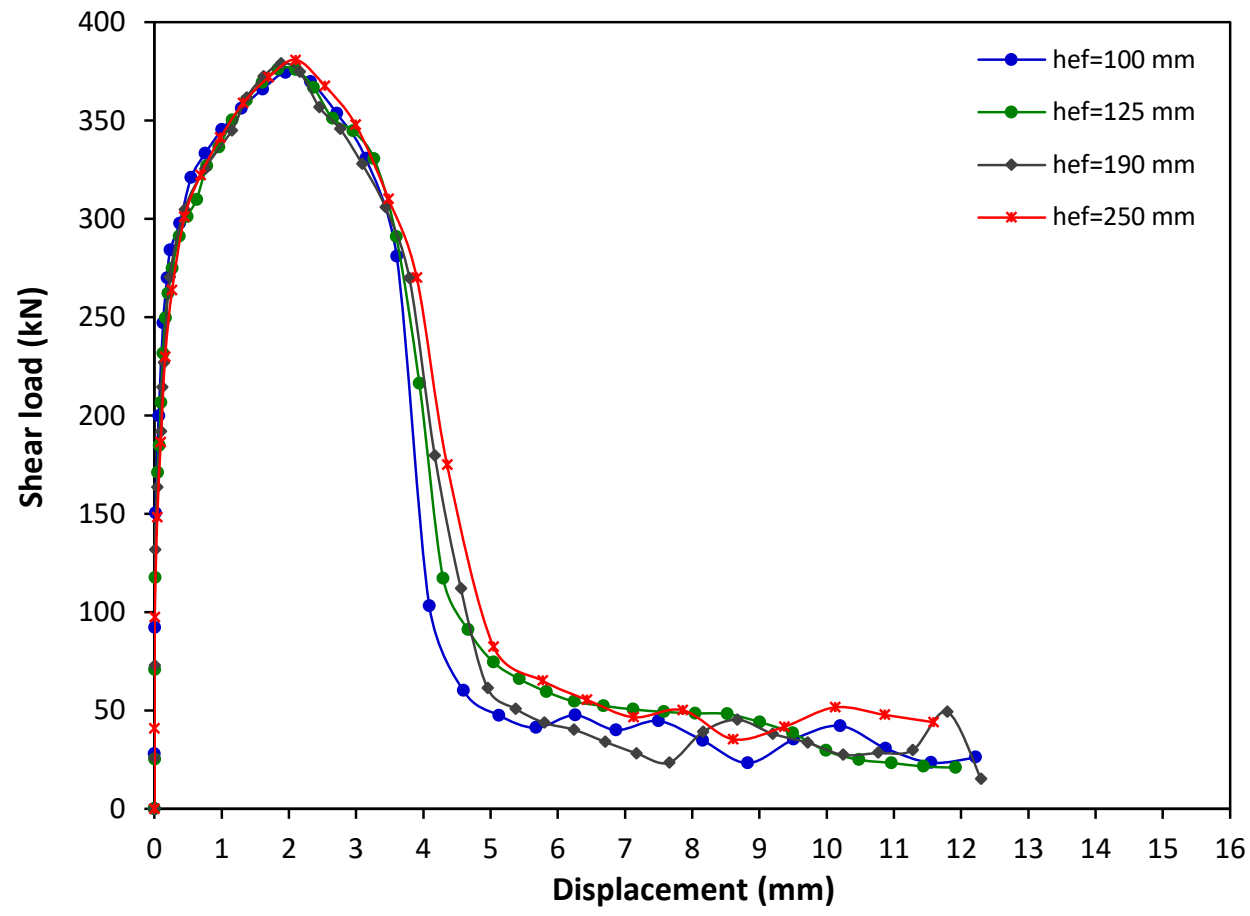

Figure H-6: Shear load-displacement graph for $16 \mathrm{~mm}$ diameter undercut anchor at strain rate of $10^{3} \mathrm{~s}^{-1}$ 


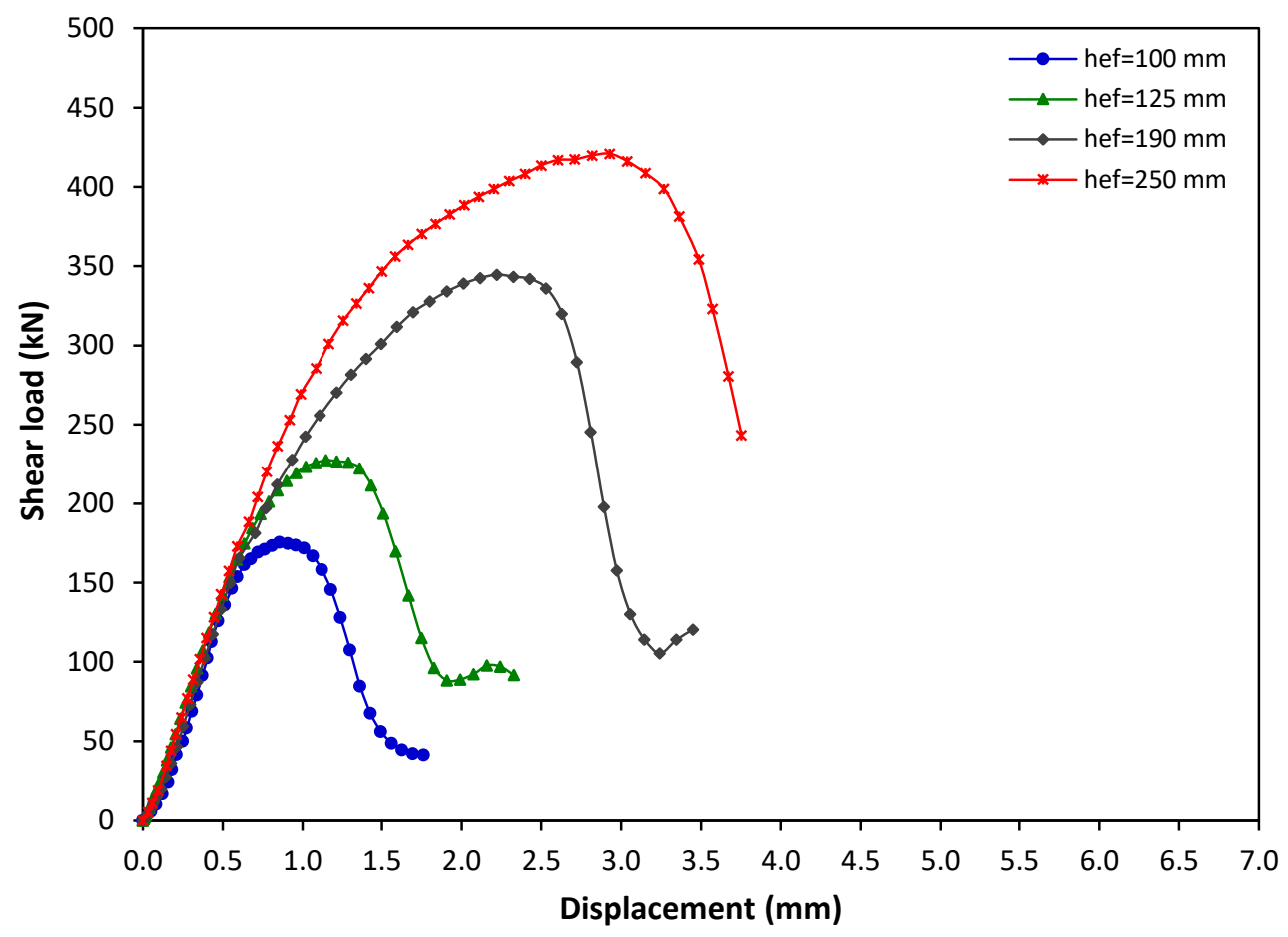

Figure H-7: Shear load-displacement graph for $20 \mathrm{~mm}$ diameter undercut anchor at strain rate of $10^{-5} \mathrm{~s}^{-1}$

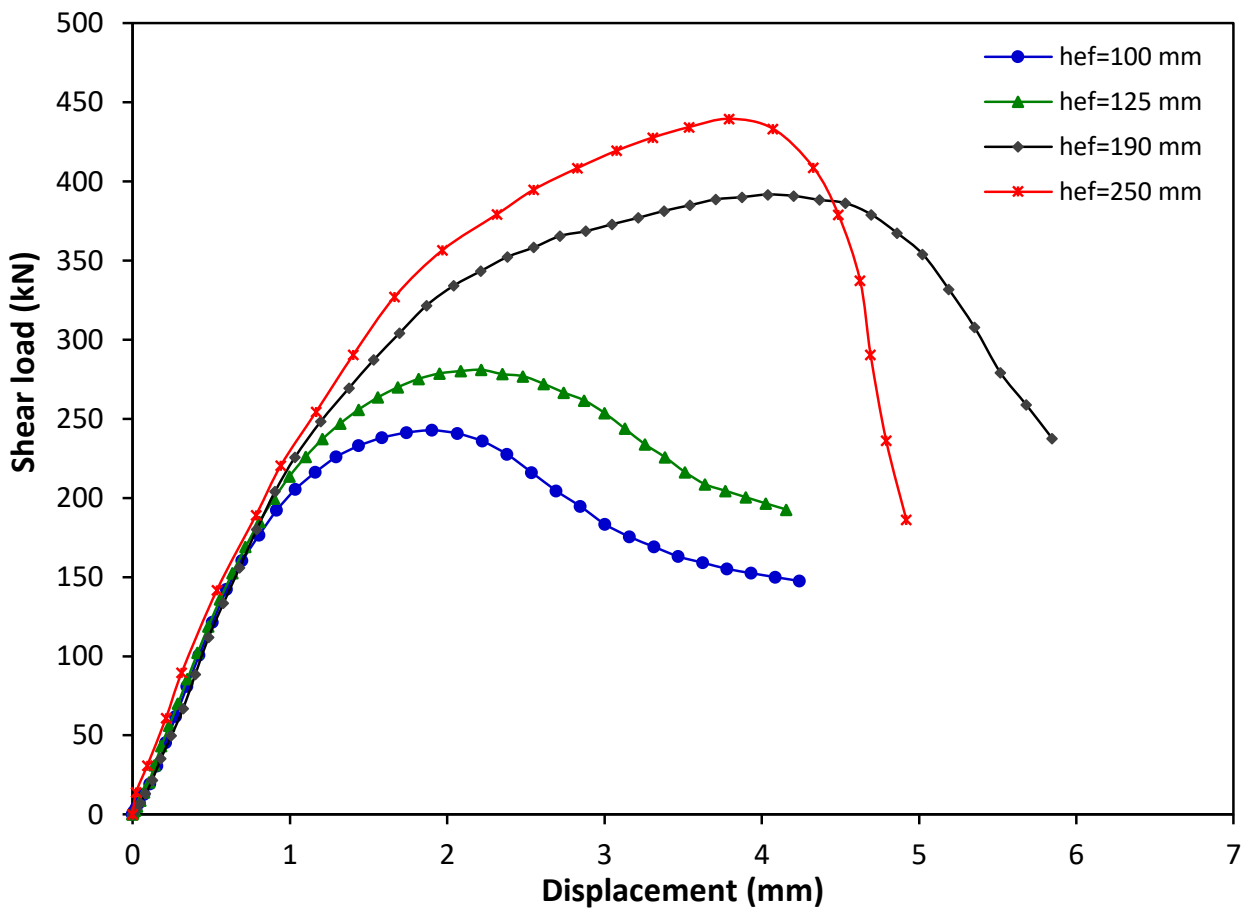

Figure H-8: Shear load-displacement graph for $20 \mathrm{~mm}$ diameter undercut anchor at strain rate of $10^{-3} \mathrm{~s}^{-1}$ 


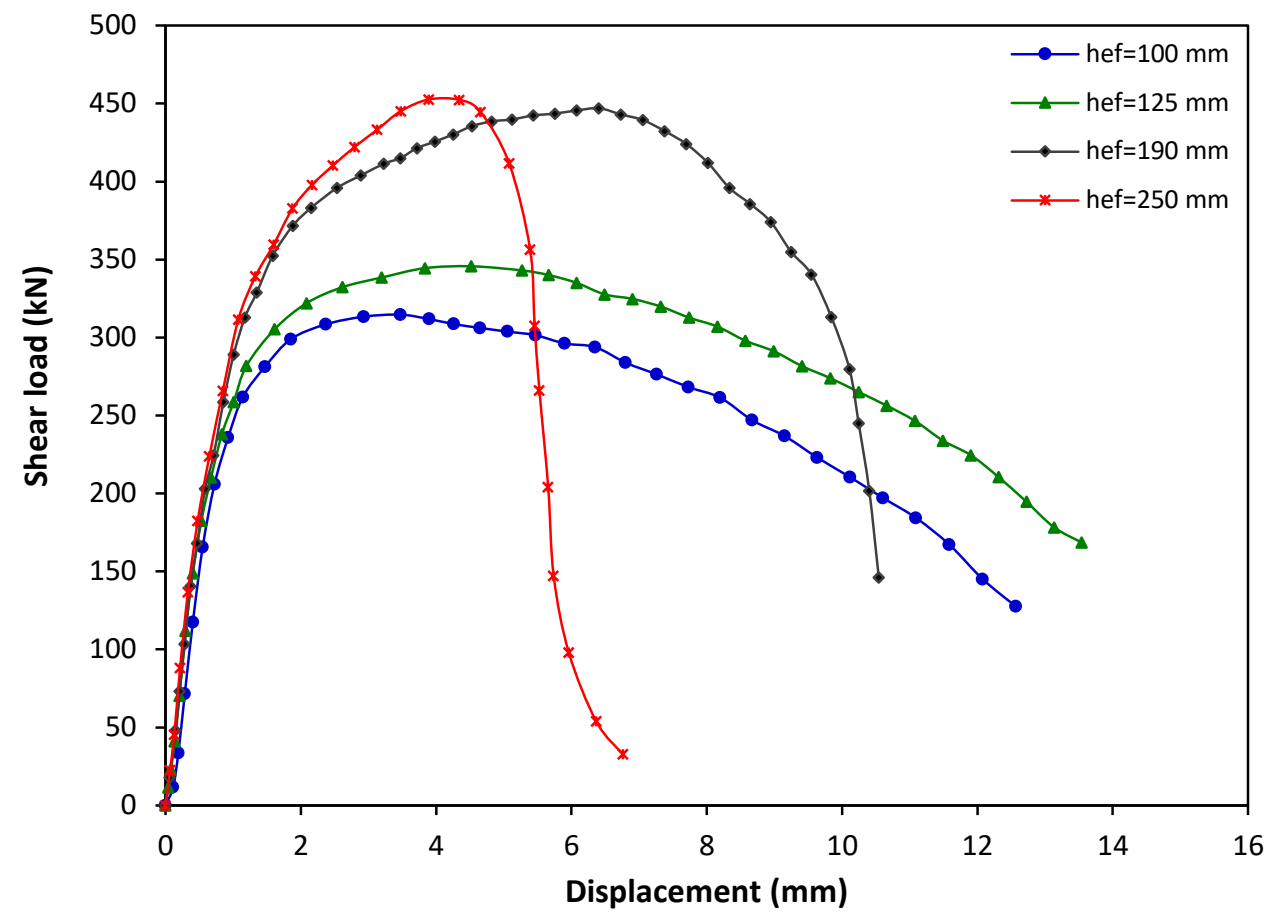

Figure H-9: Shear load-displacement graph for $20 \mathrm{~mm}$ diameter undercut anchor at strain rate of $10^{-1} \mathrm{~s}^{-1}$

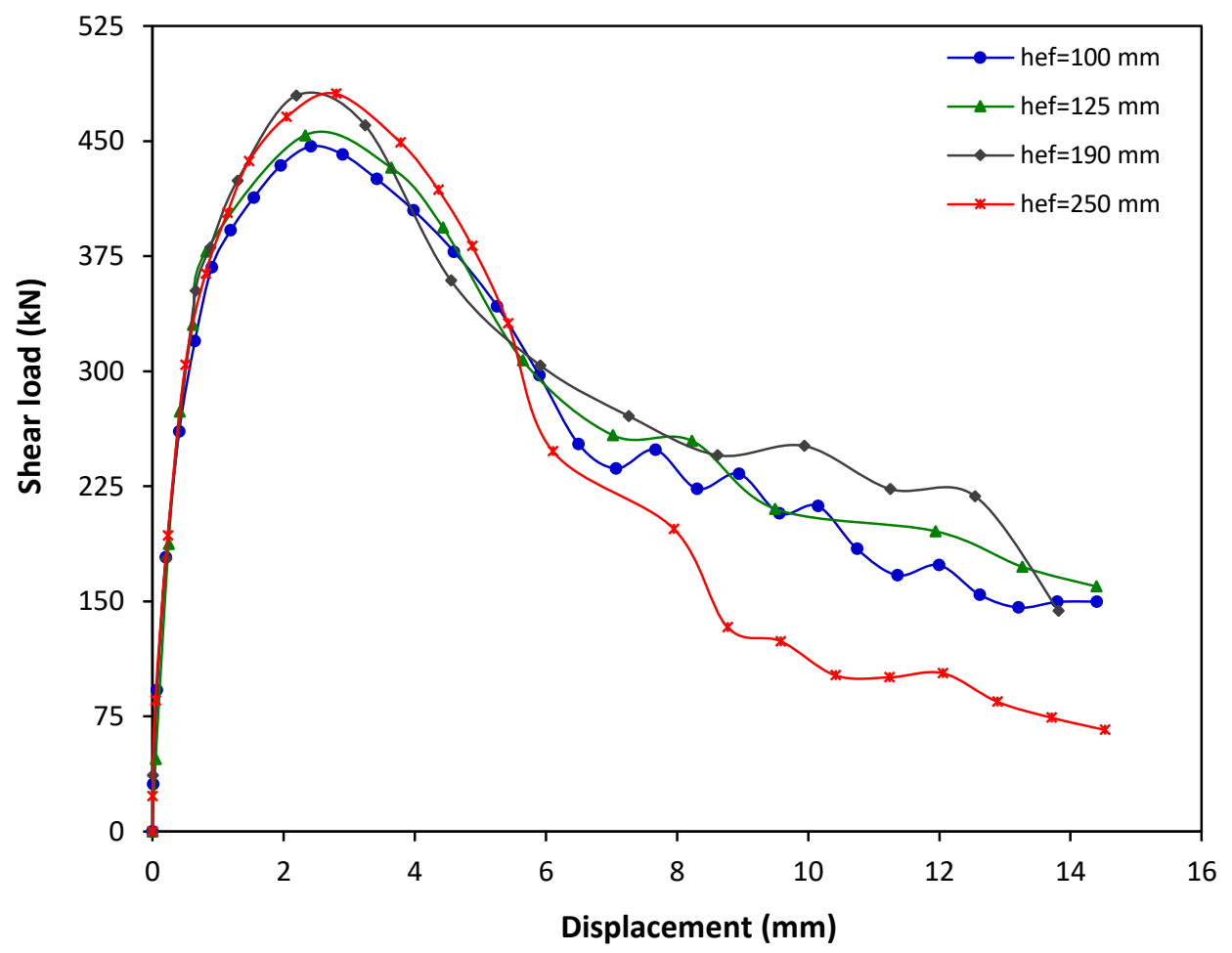

Figure H-10: Shear load-displacement graph for $20 \mathrm{~mm}$ diameter undercut anchor at strain rate of $10 \mathrm{~s}^{-1}$ 


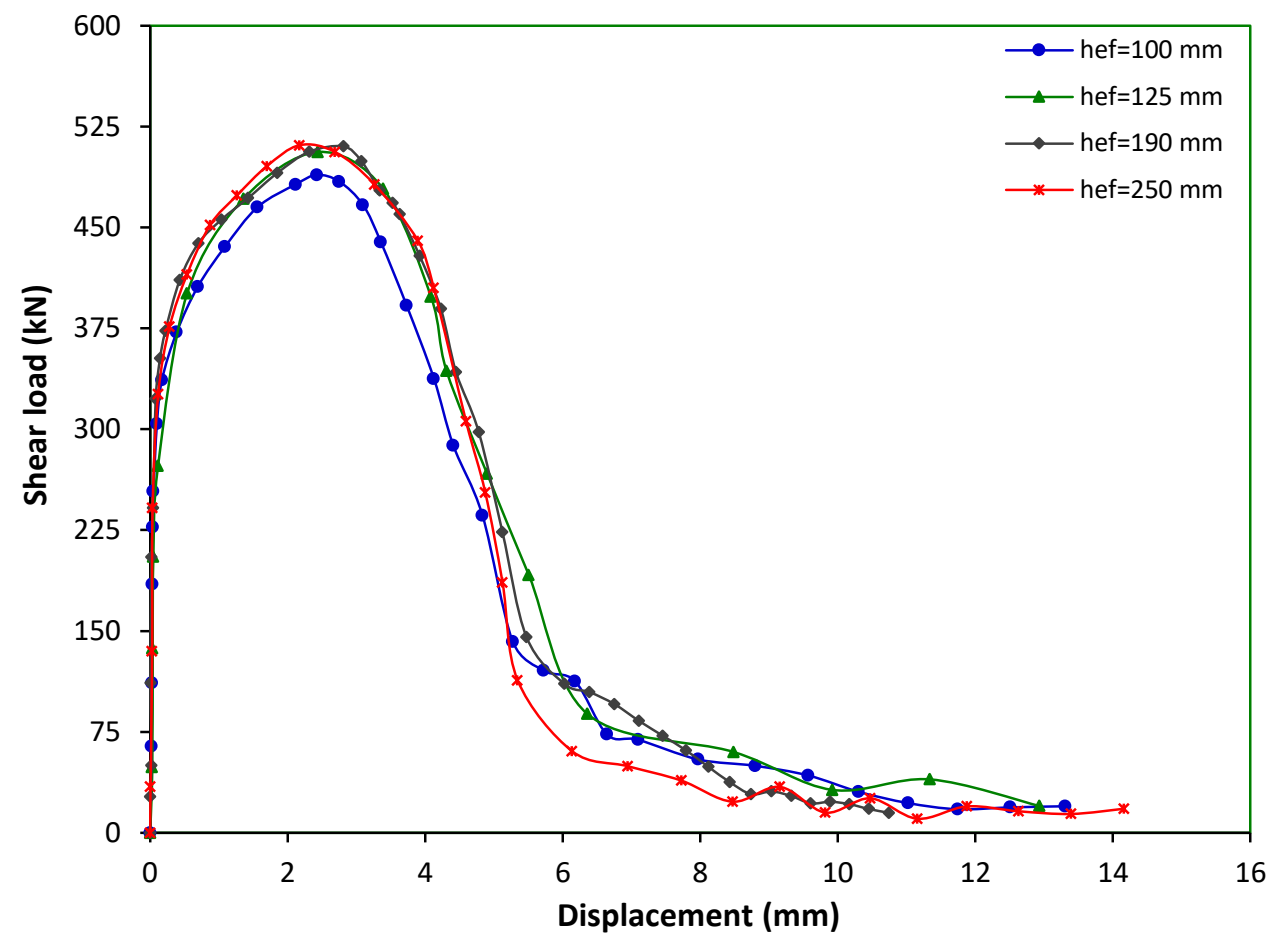

Figure H-11: Shear load-displacement graph for $20 \mathrm{~mm}$ diameter undercut anchor at strain rate of $10^{2} \mathrm{~s}^{-1}$

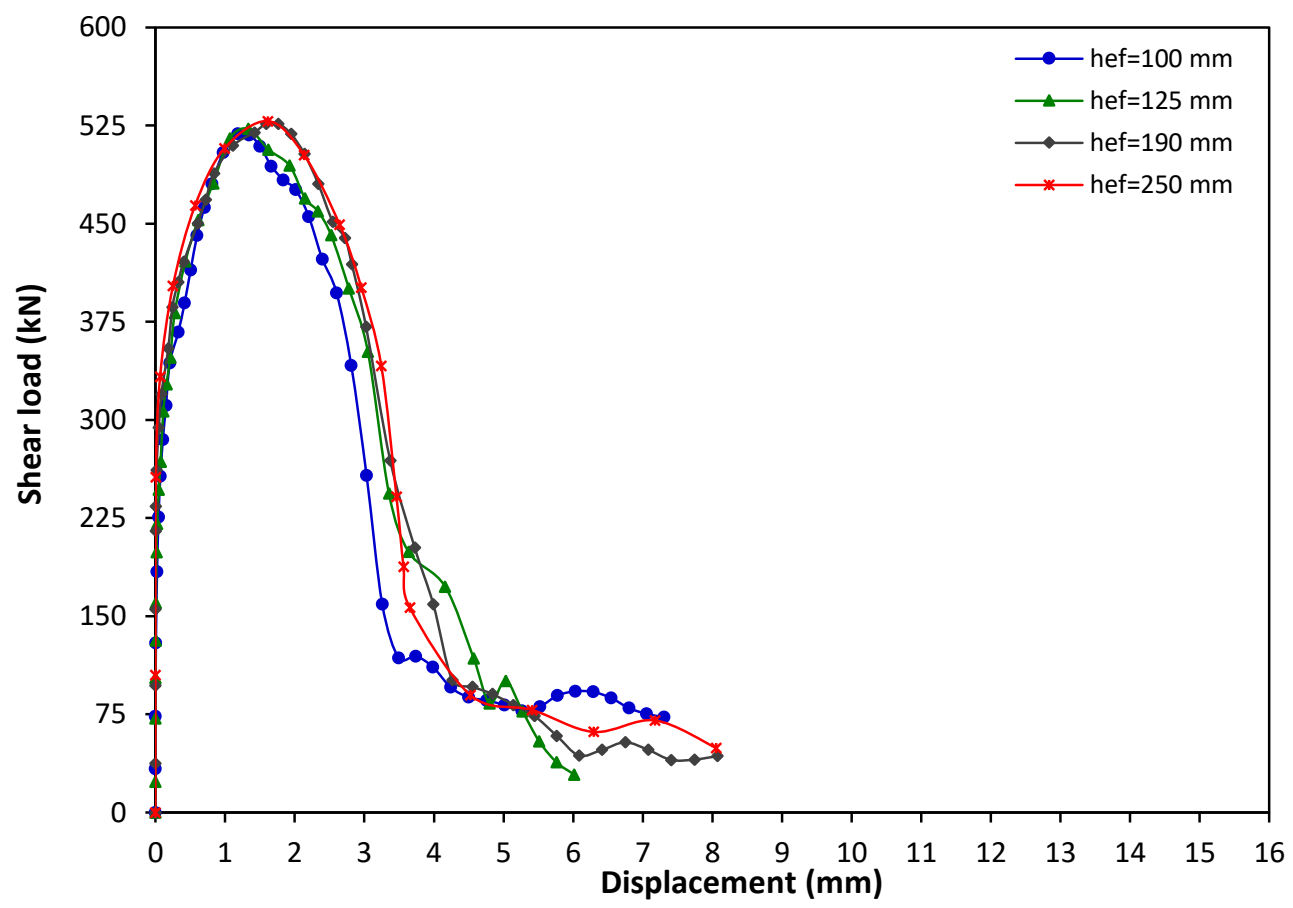

Figure H-12: Shear load-displacement graph for $20 \mathrm{~mm}$ diameter undercut anchor at strain rate of $10^{3}$ 


\section{Appendix I: LS-DYNA keyword files for anchorage to concrete systems}

\section{Cast-in-place anchor under tensile load}

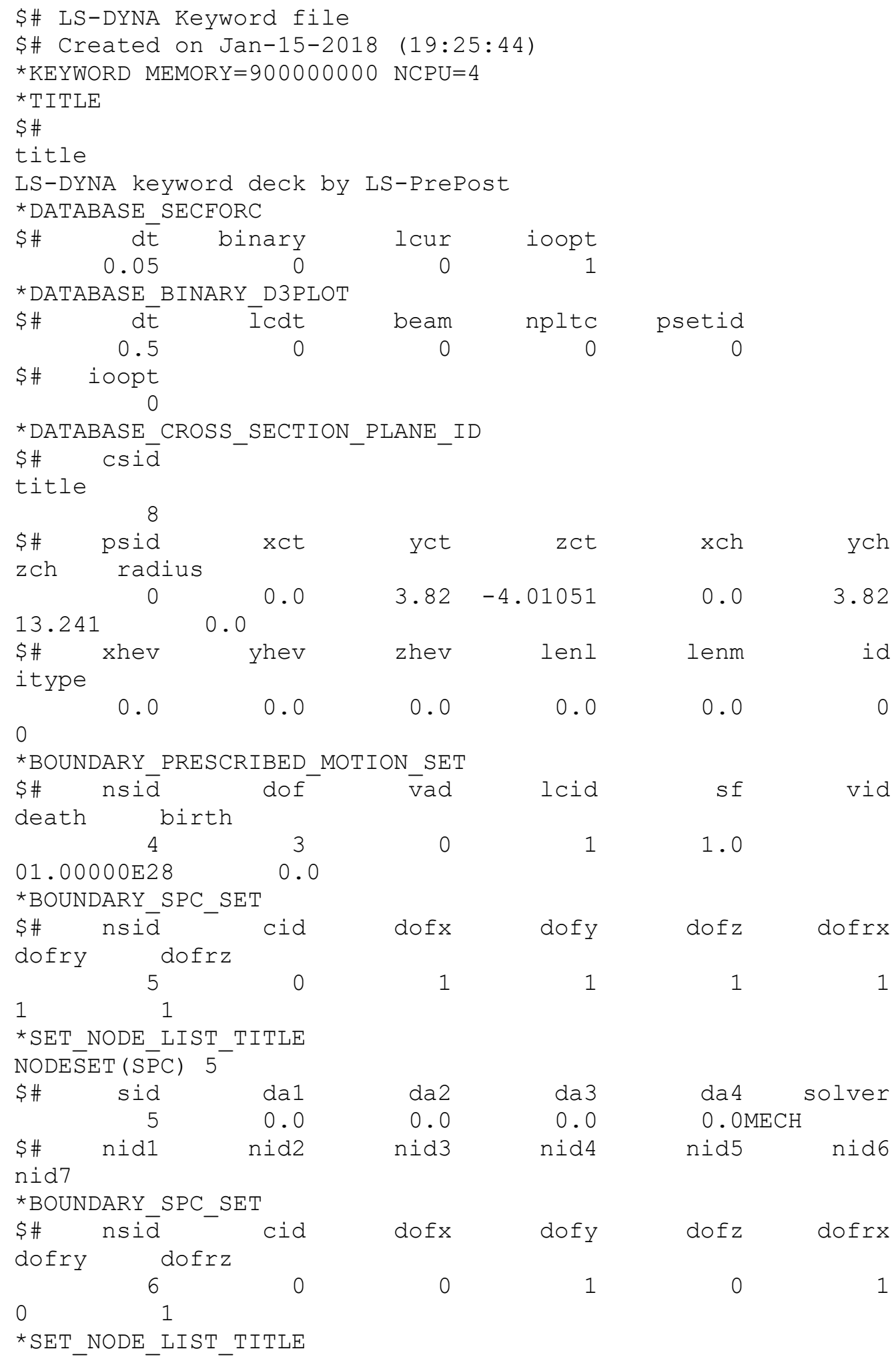




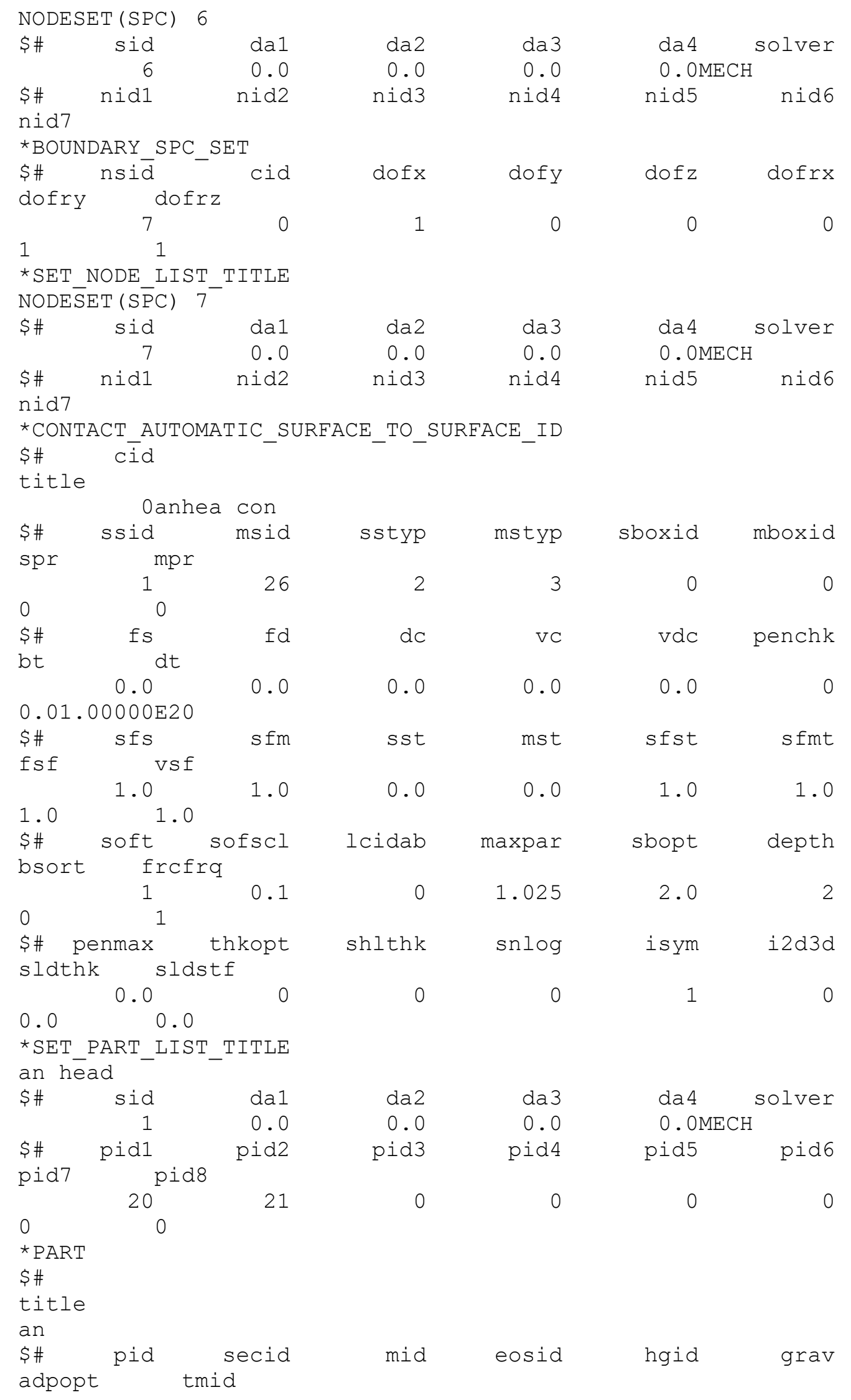




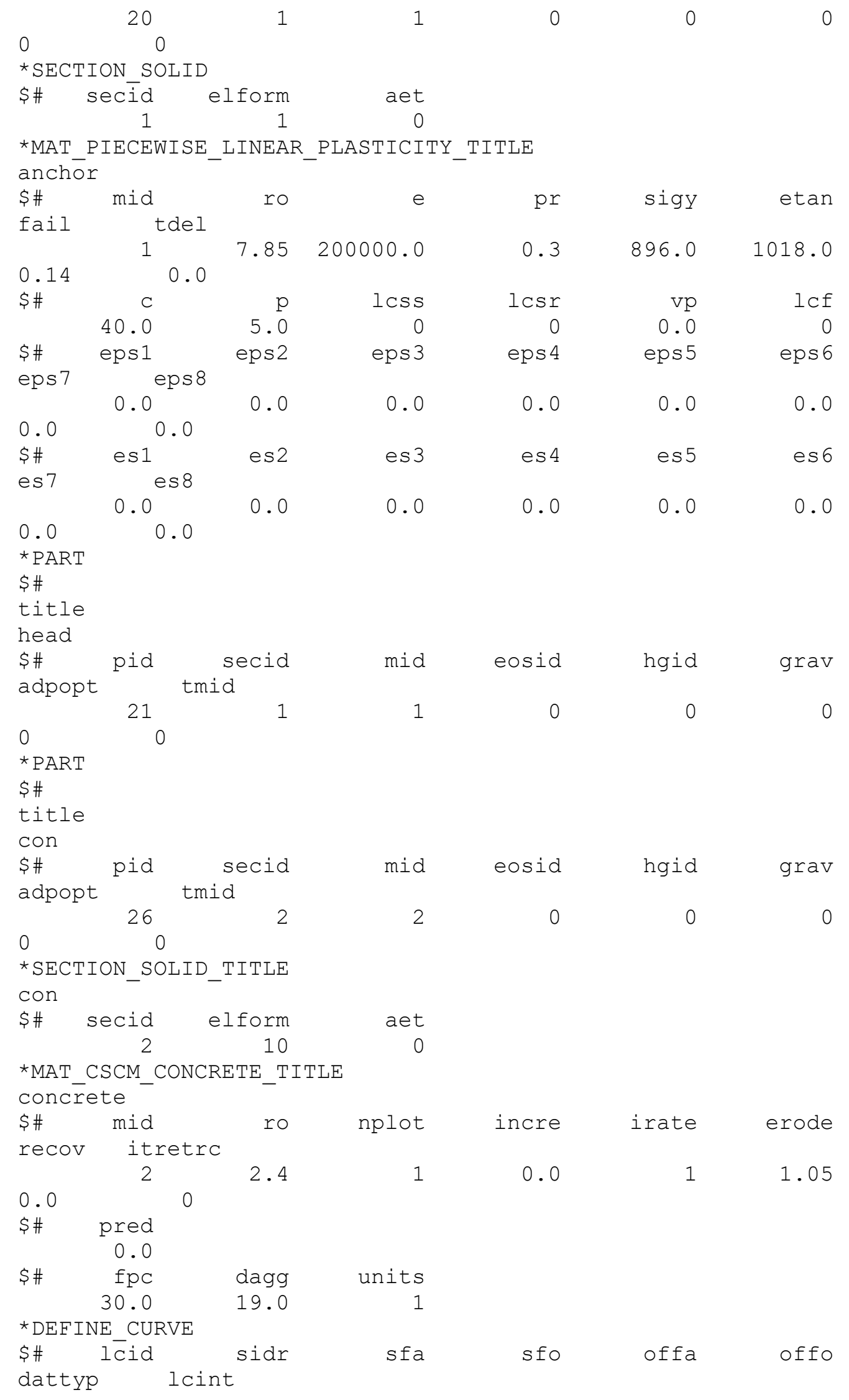




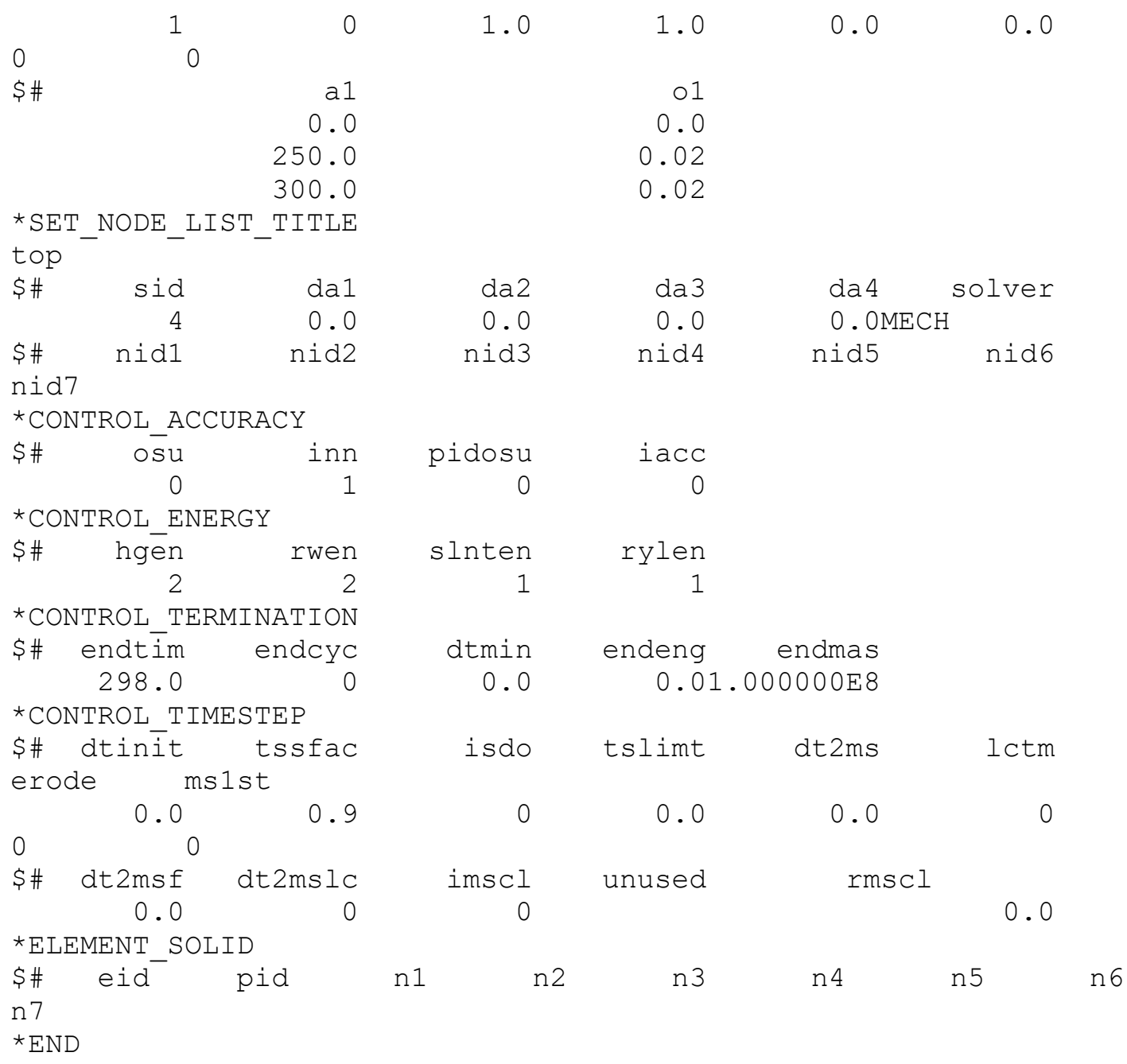

\section{Cast-in-place anchor under shear load}

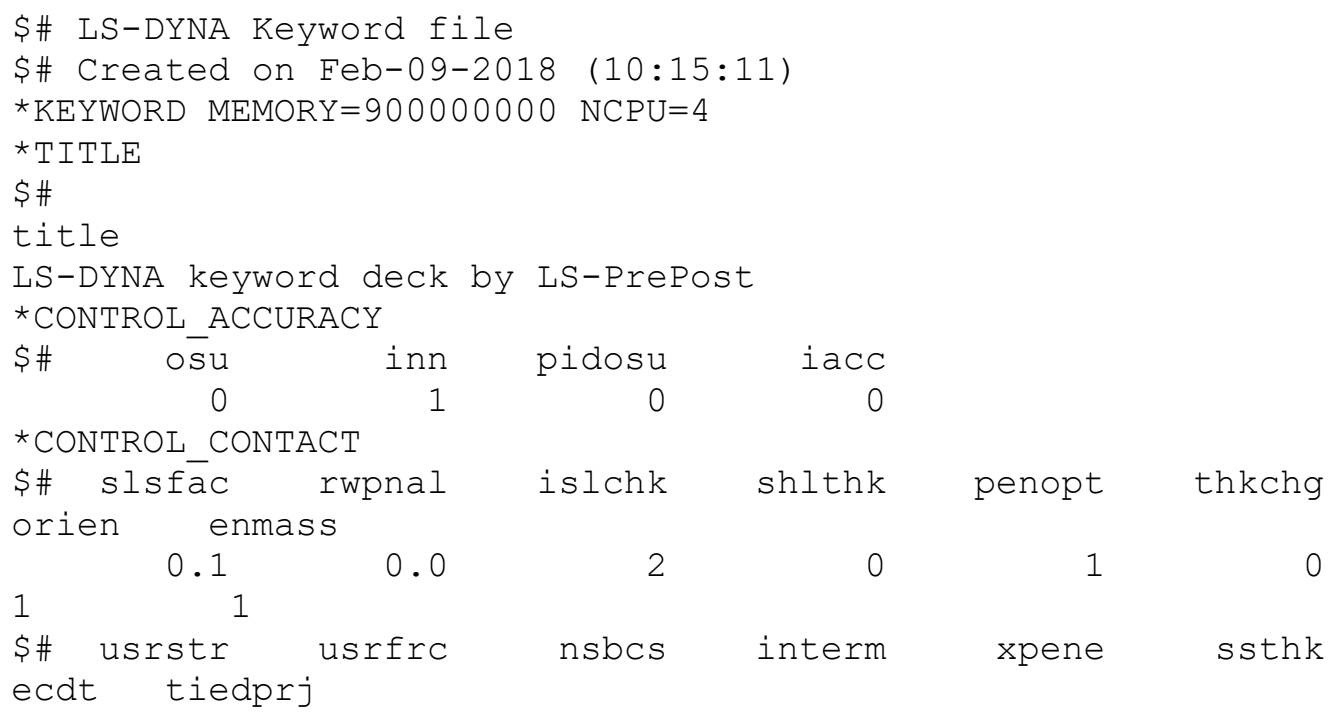




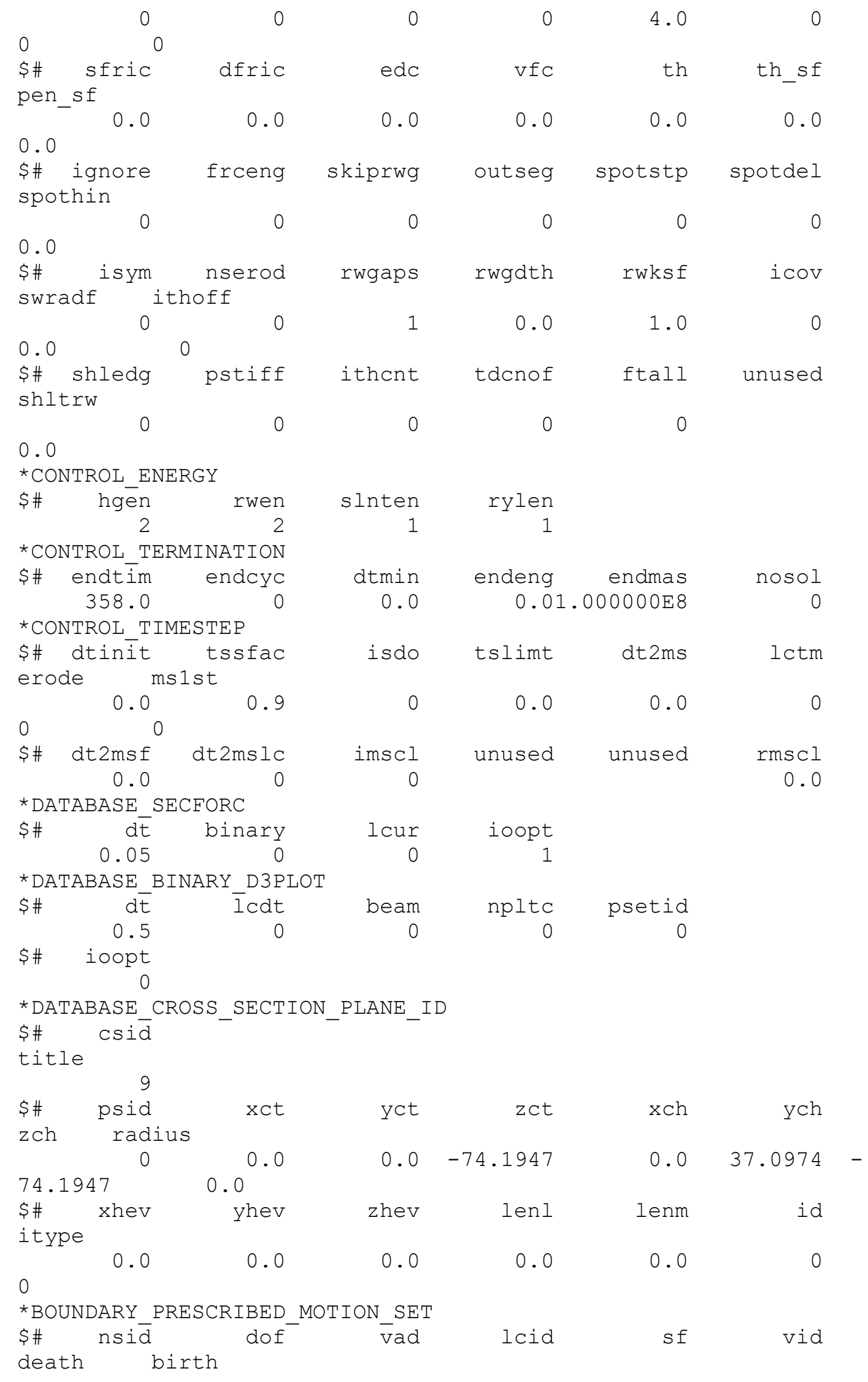




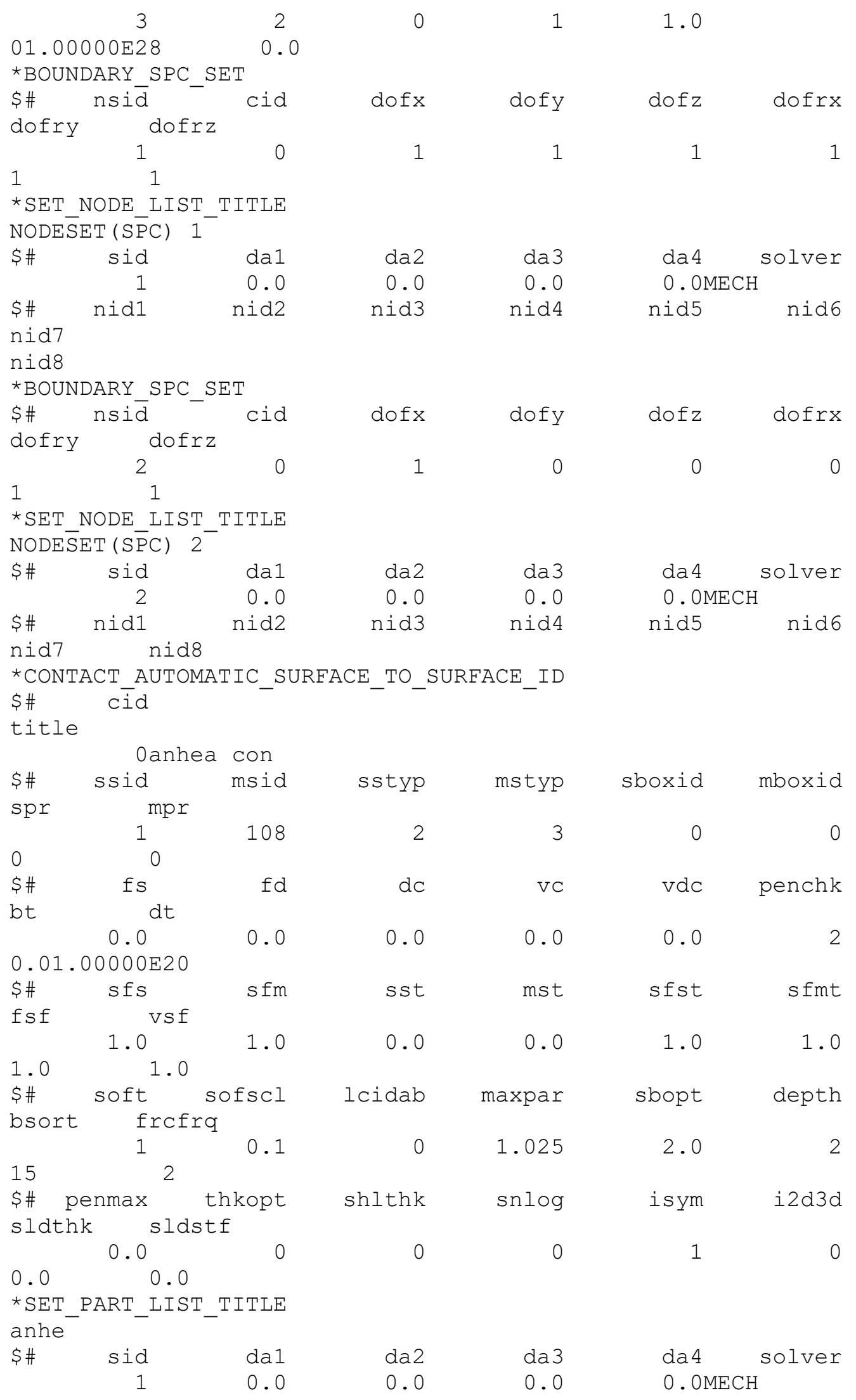




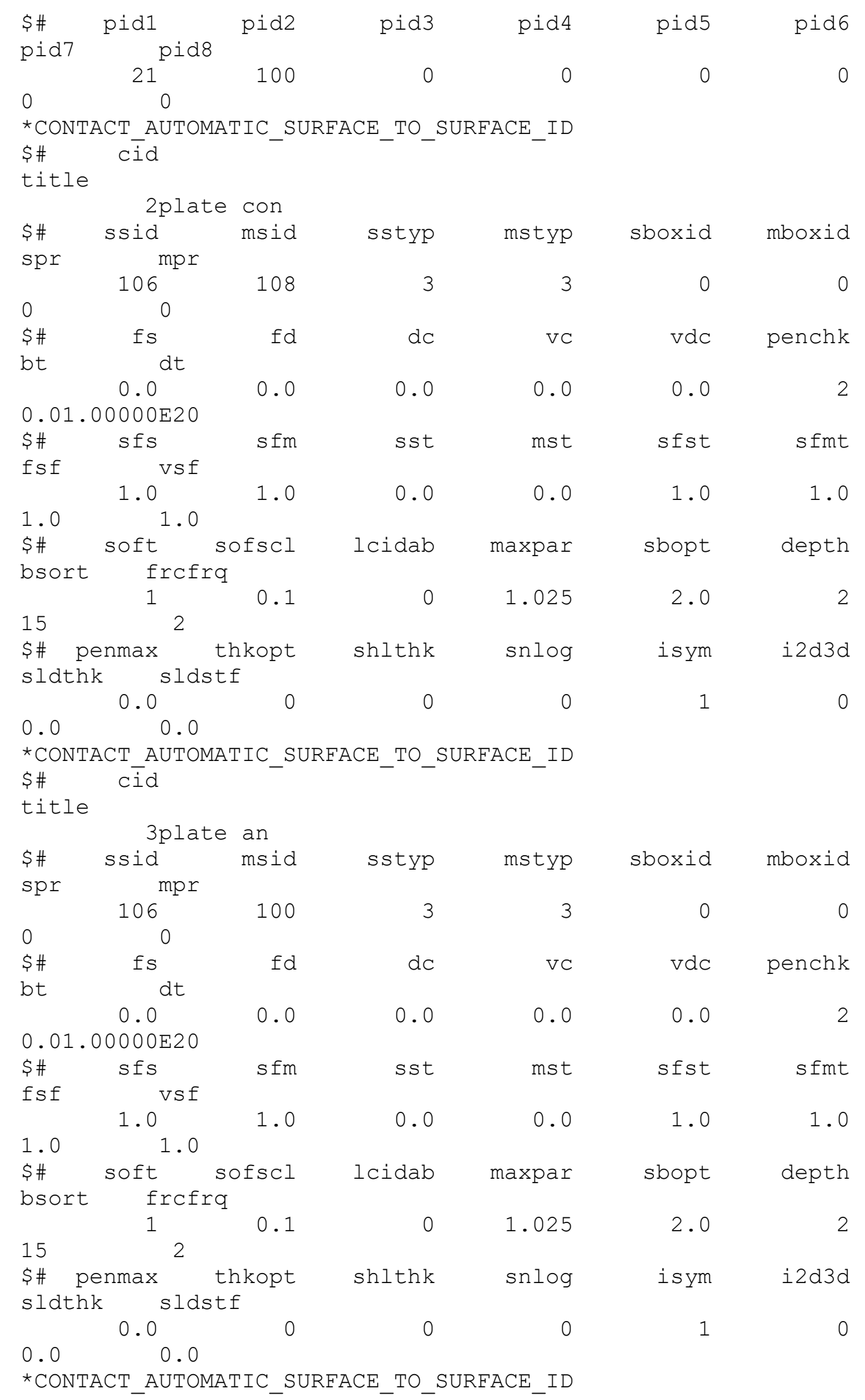




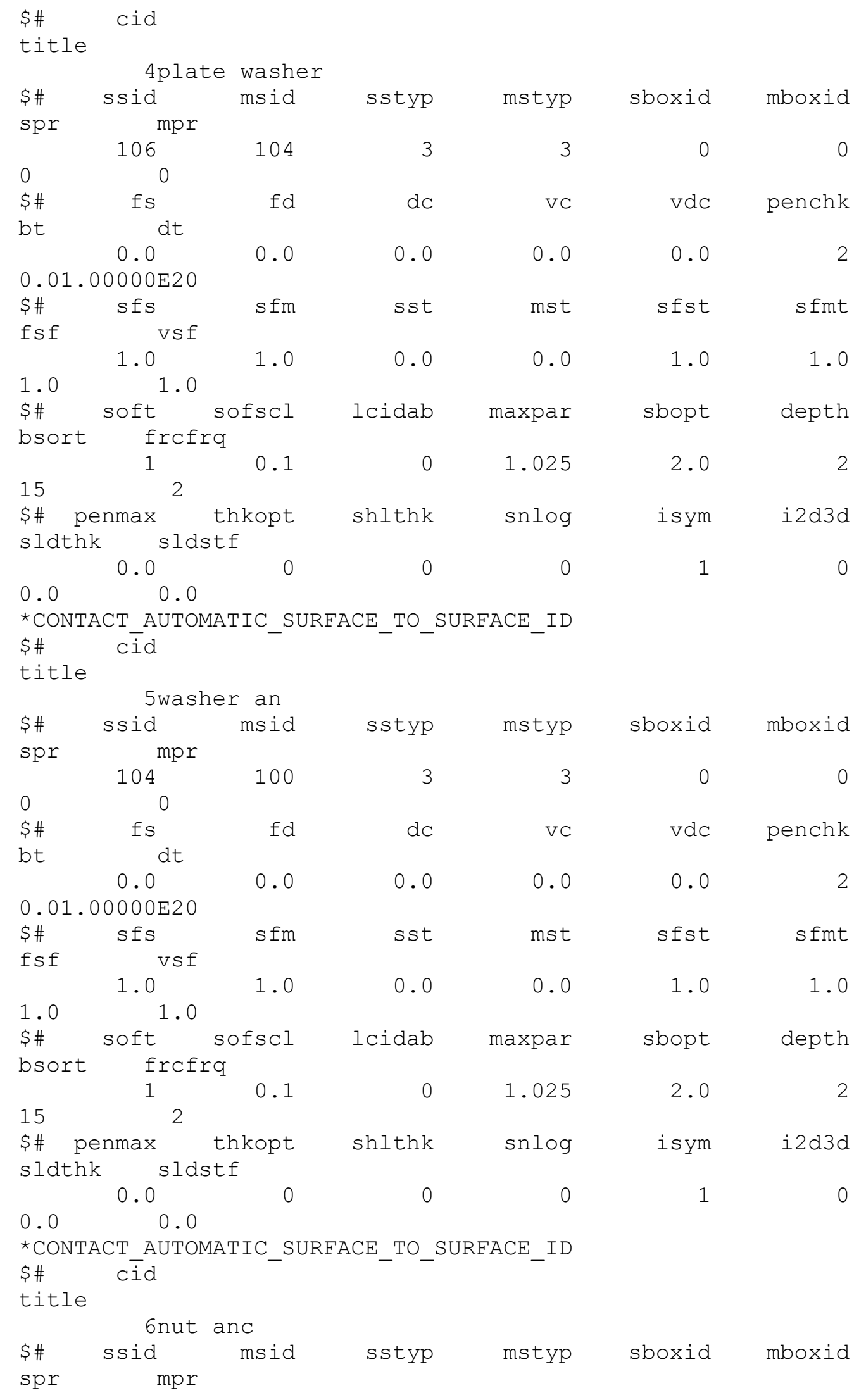




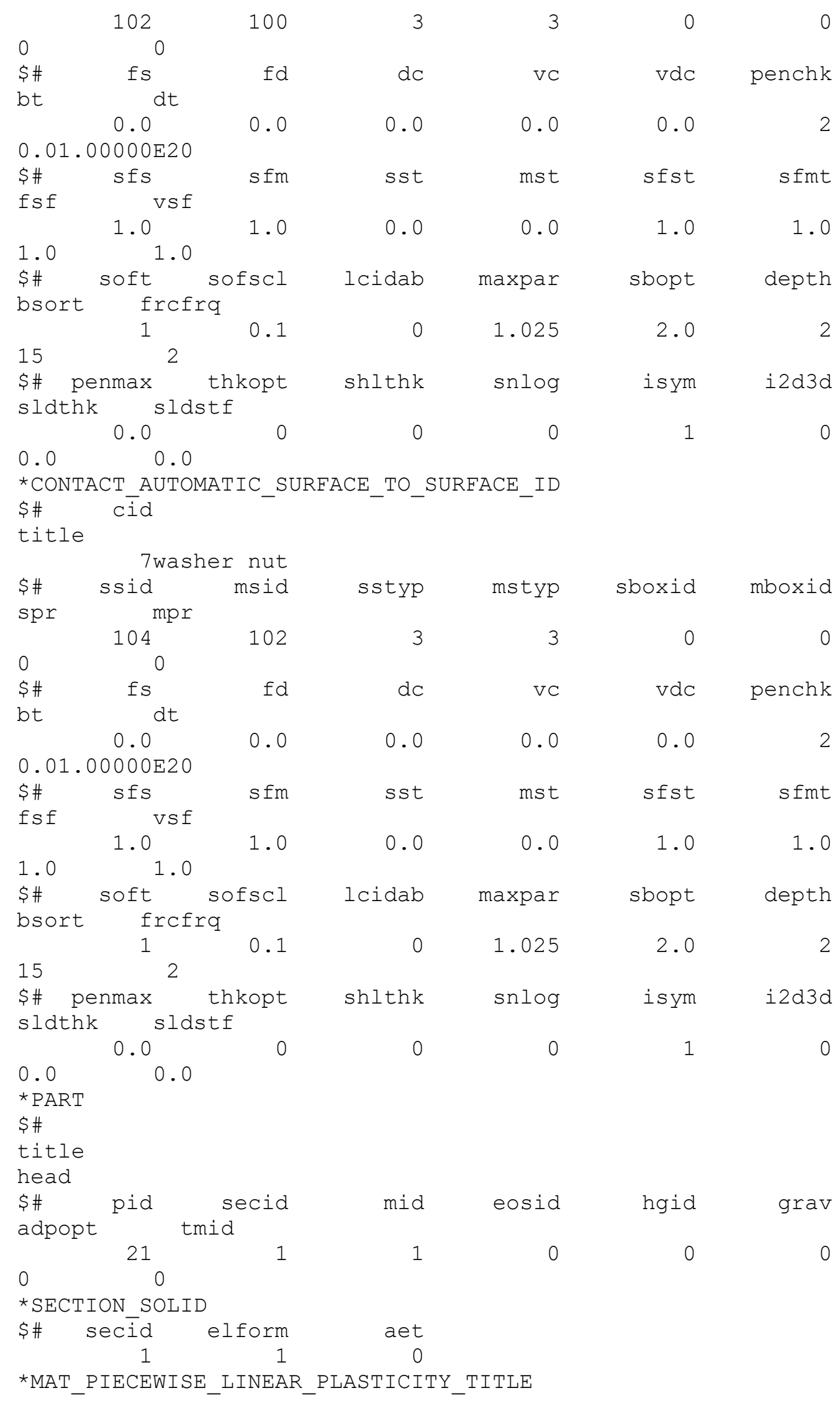




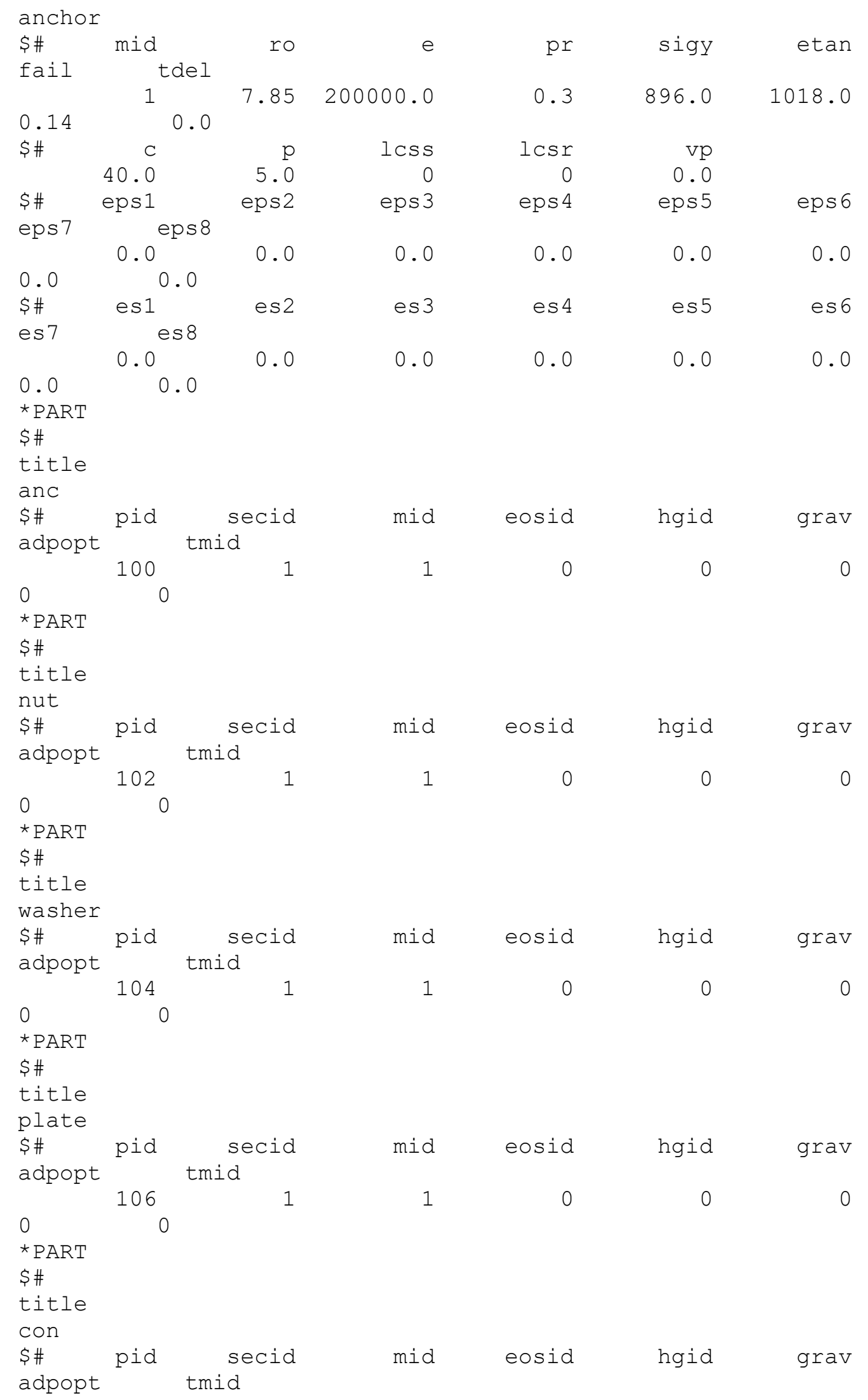




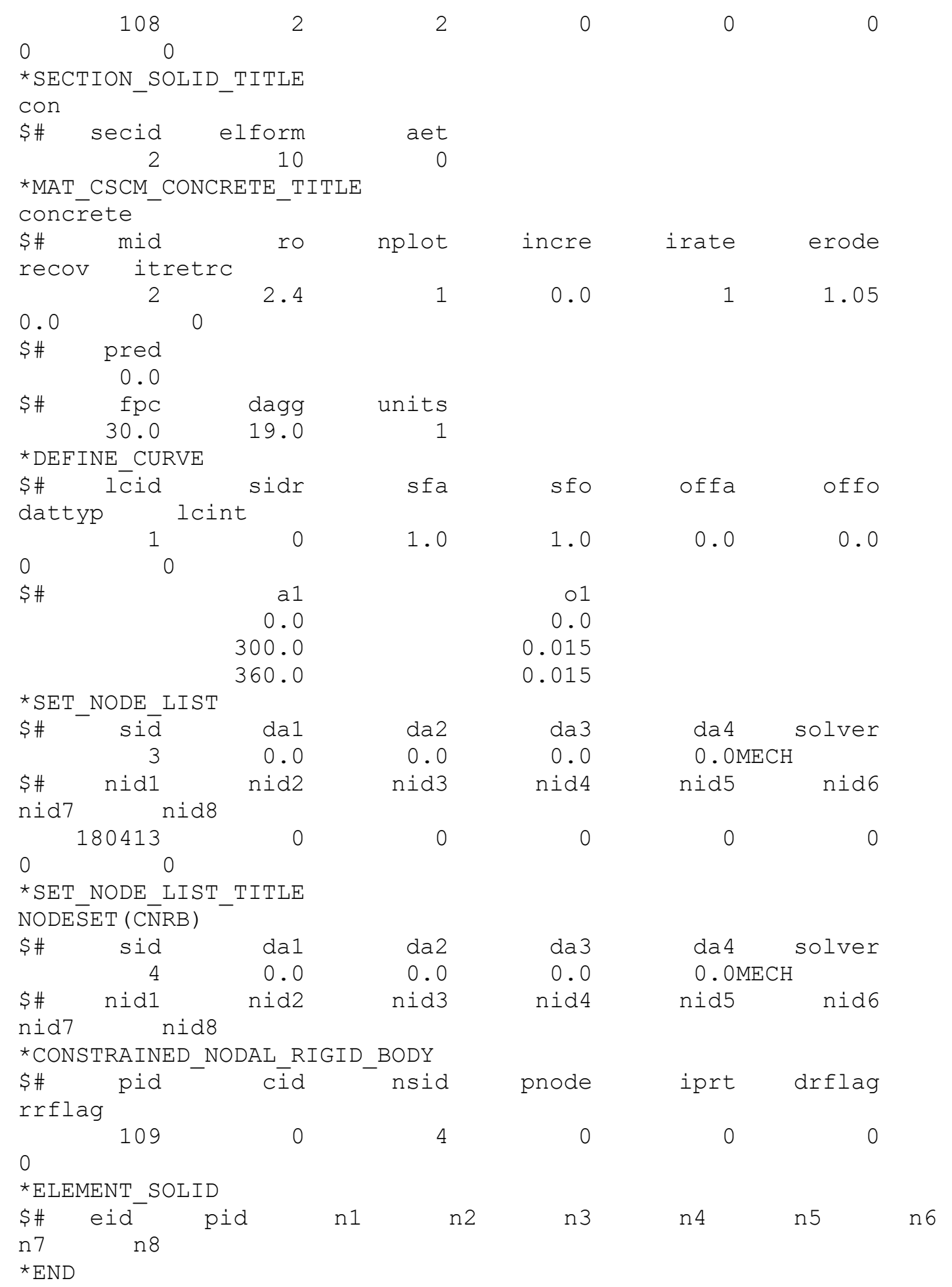




\section{Adhesive anchor under tensile load}

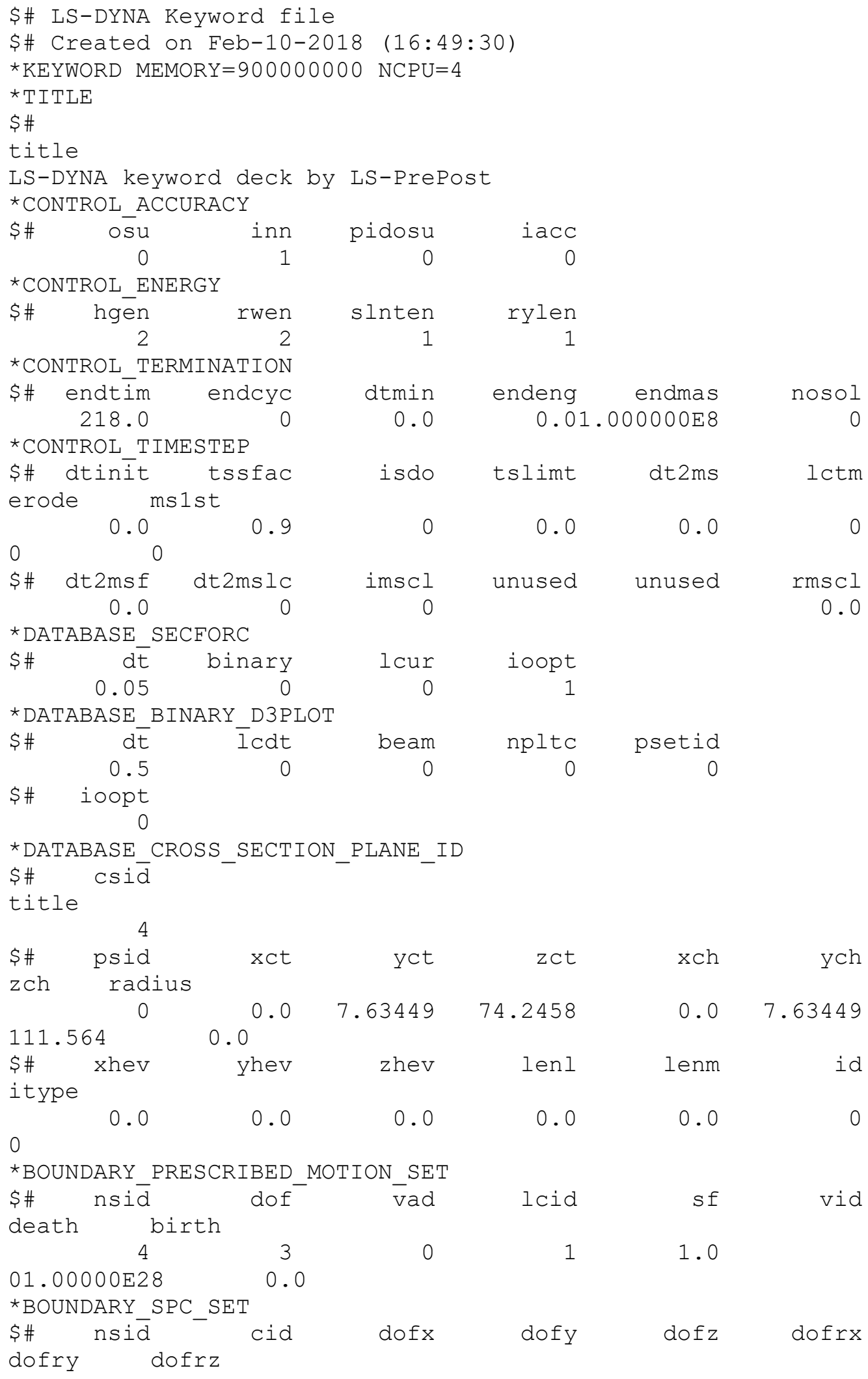




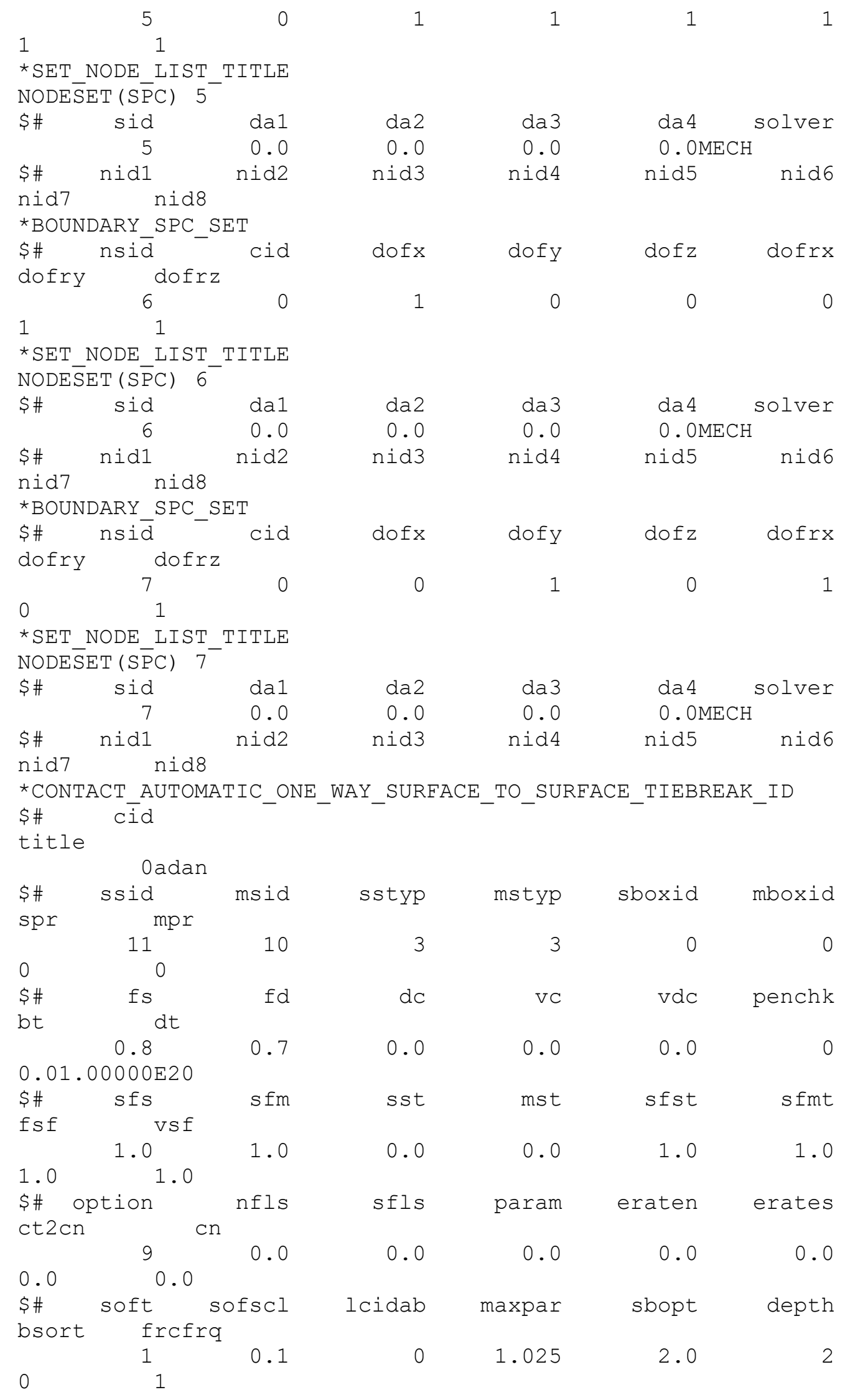




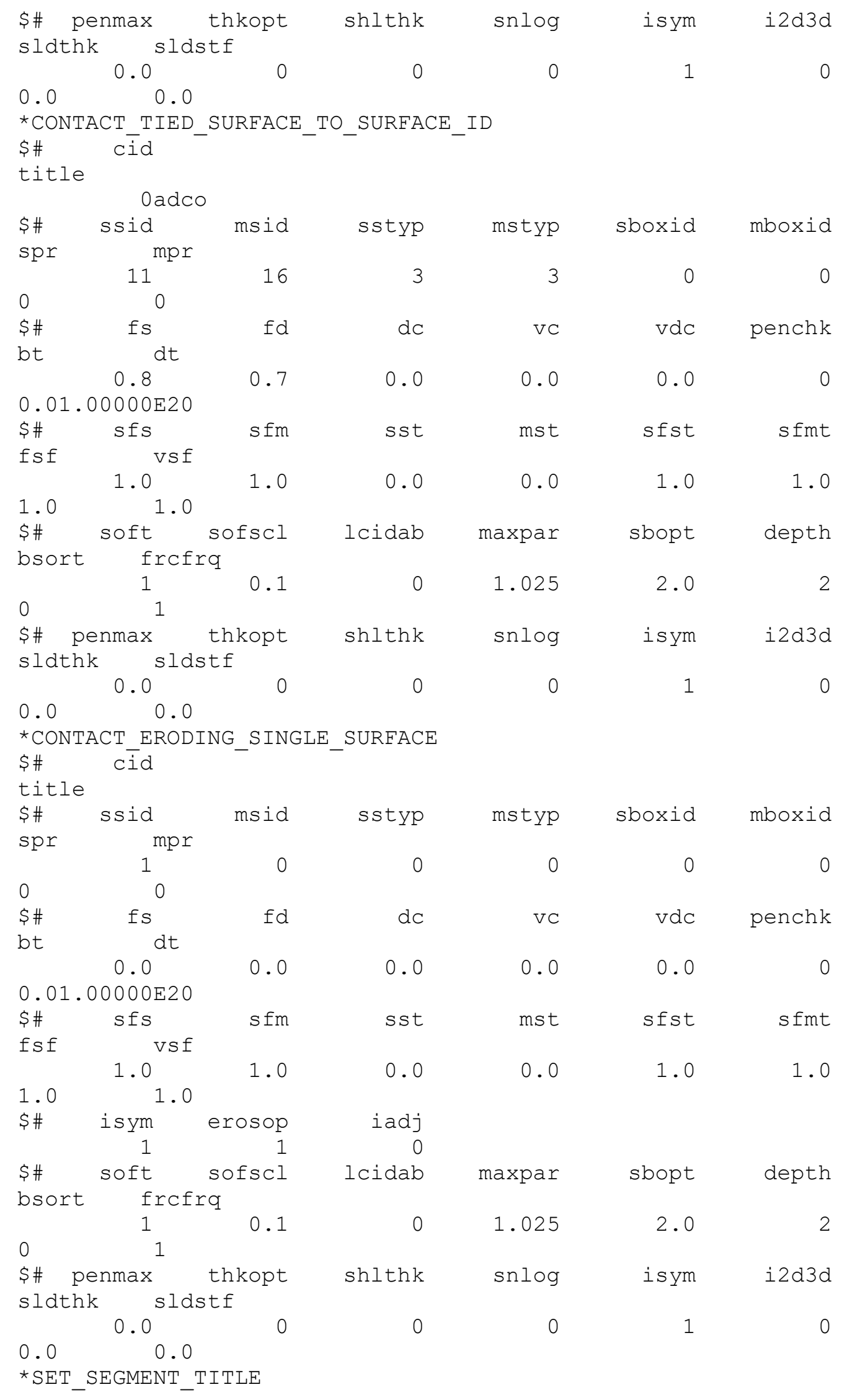




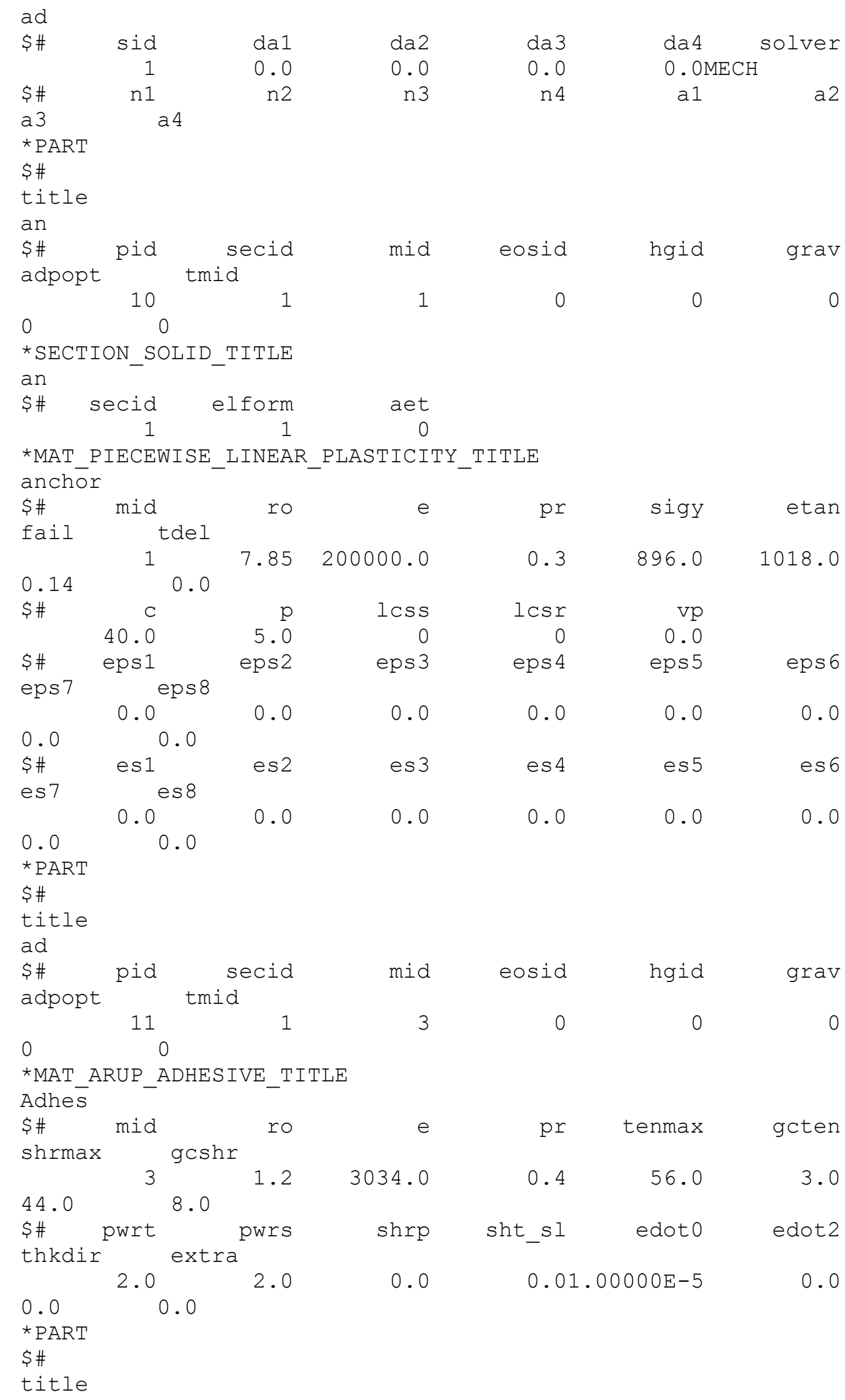




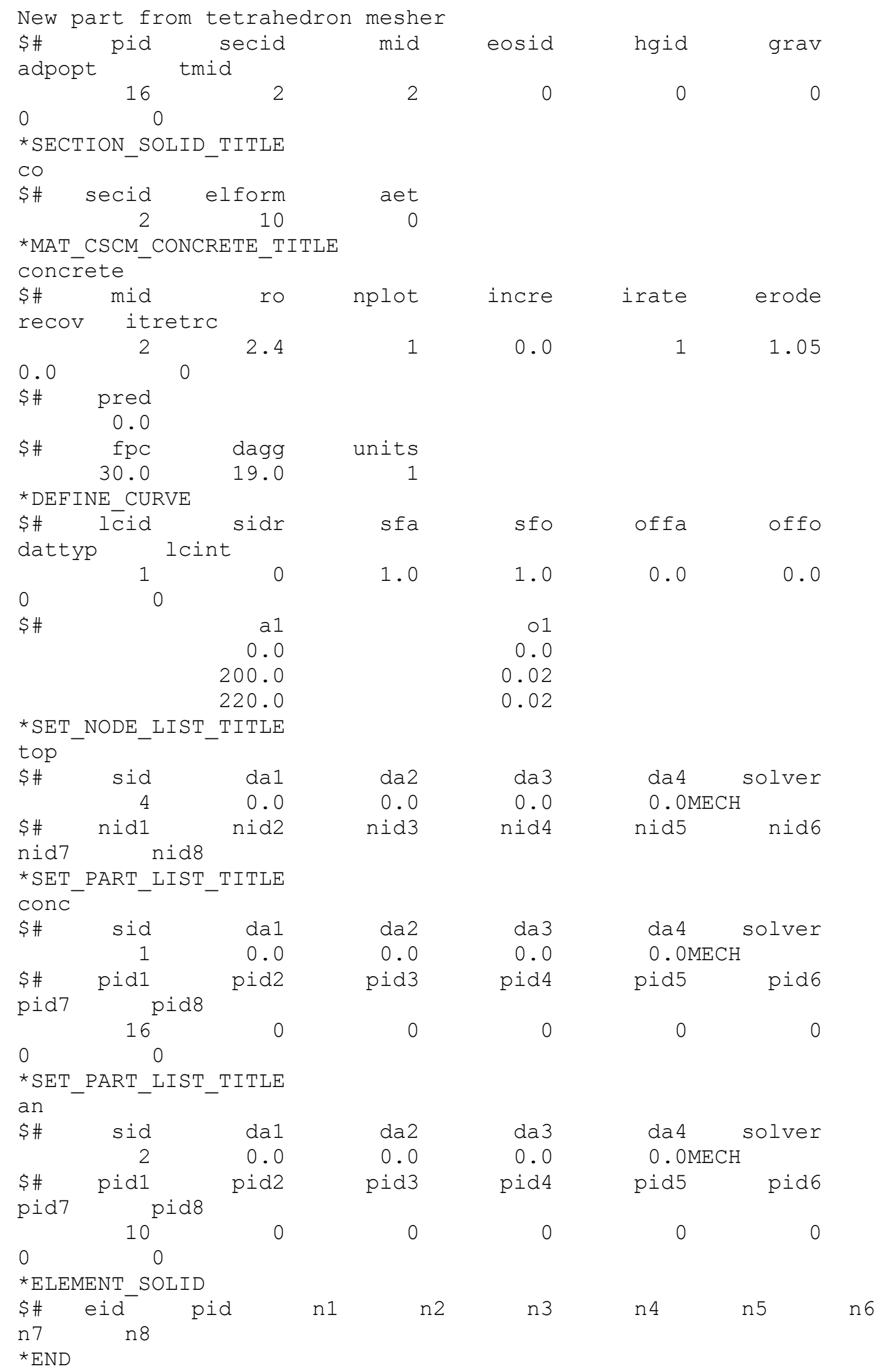




\section{Adhesive anchor under shear load}

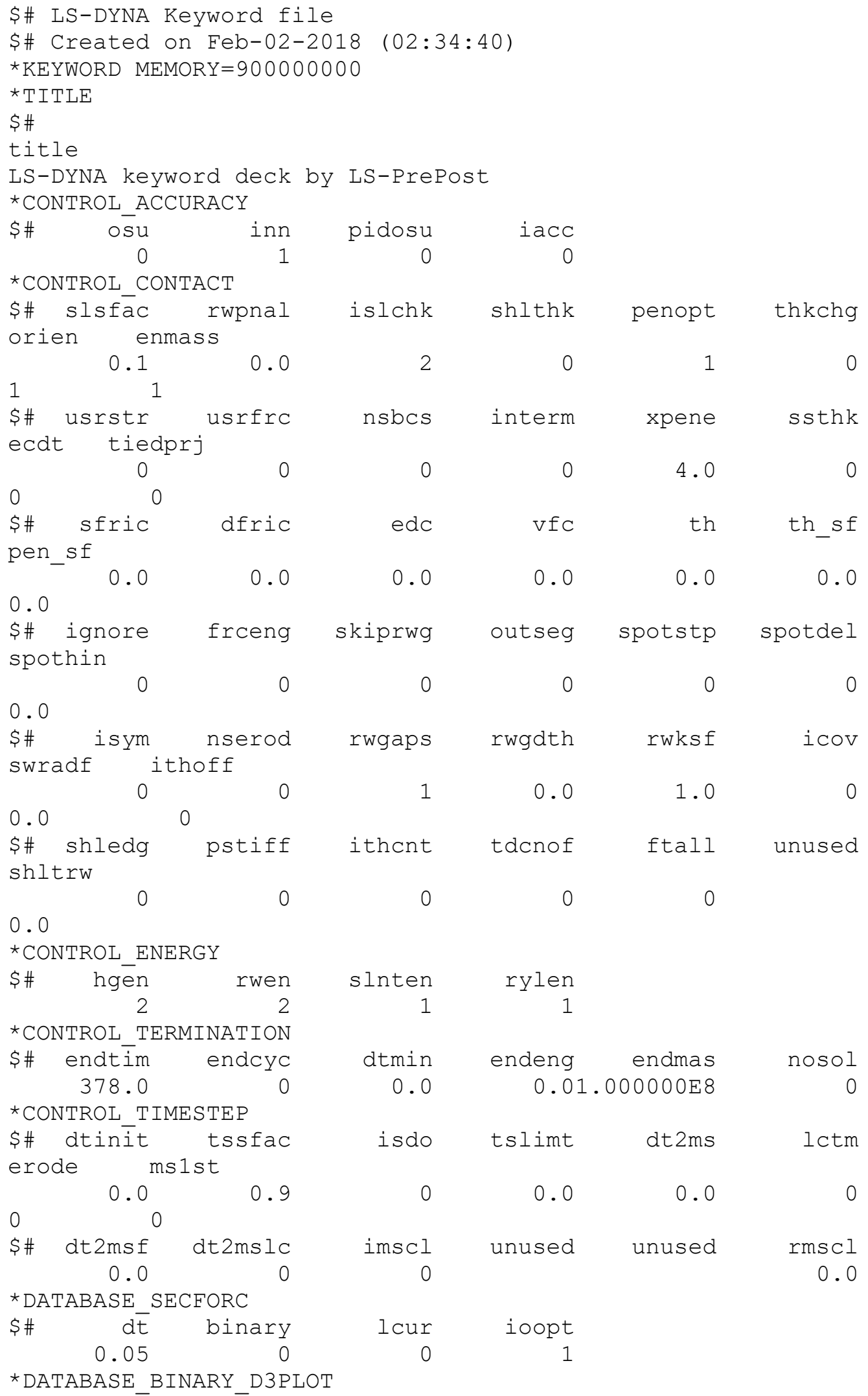




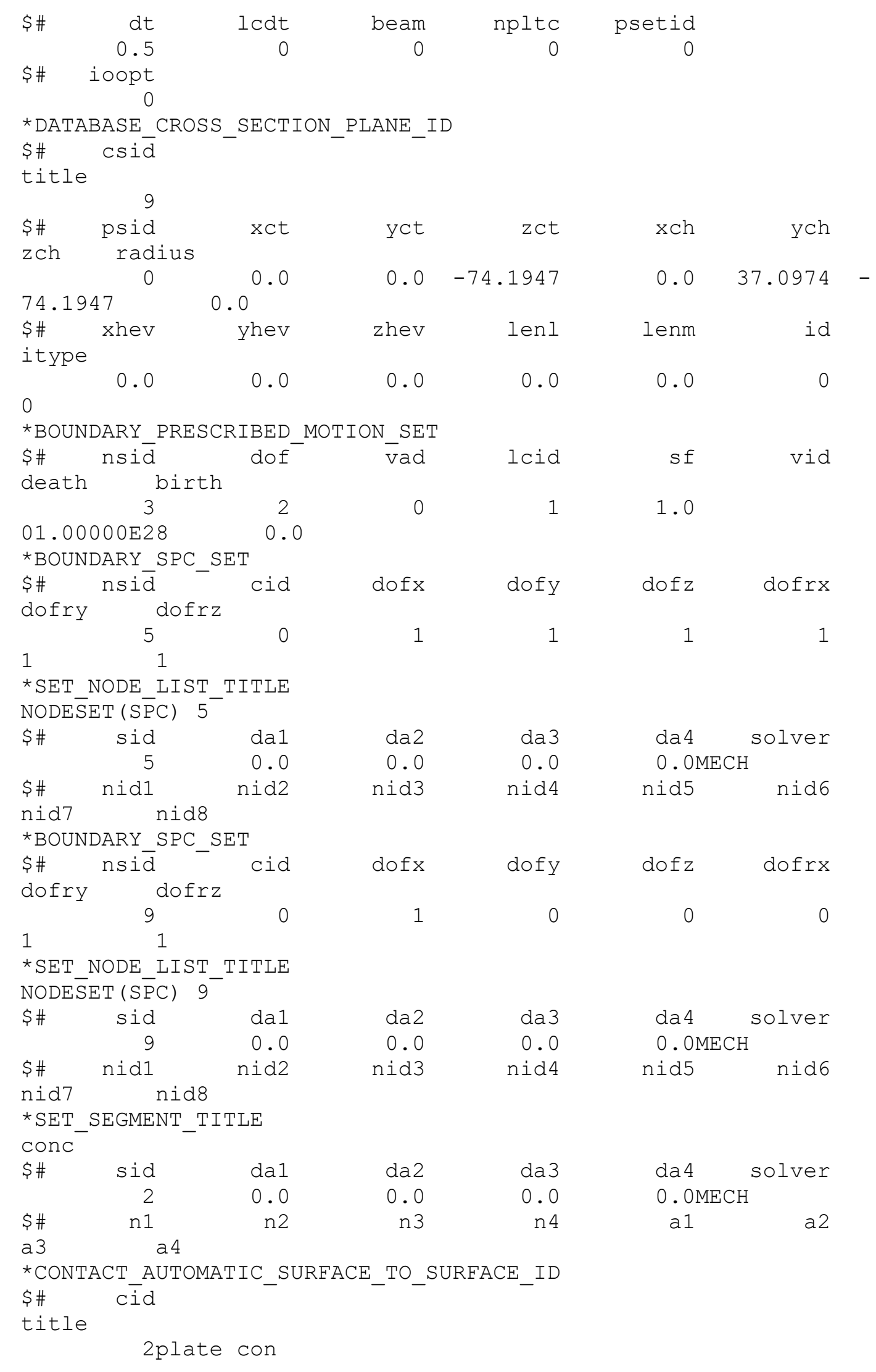




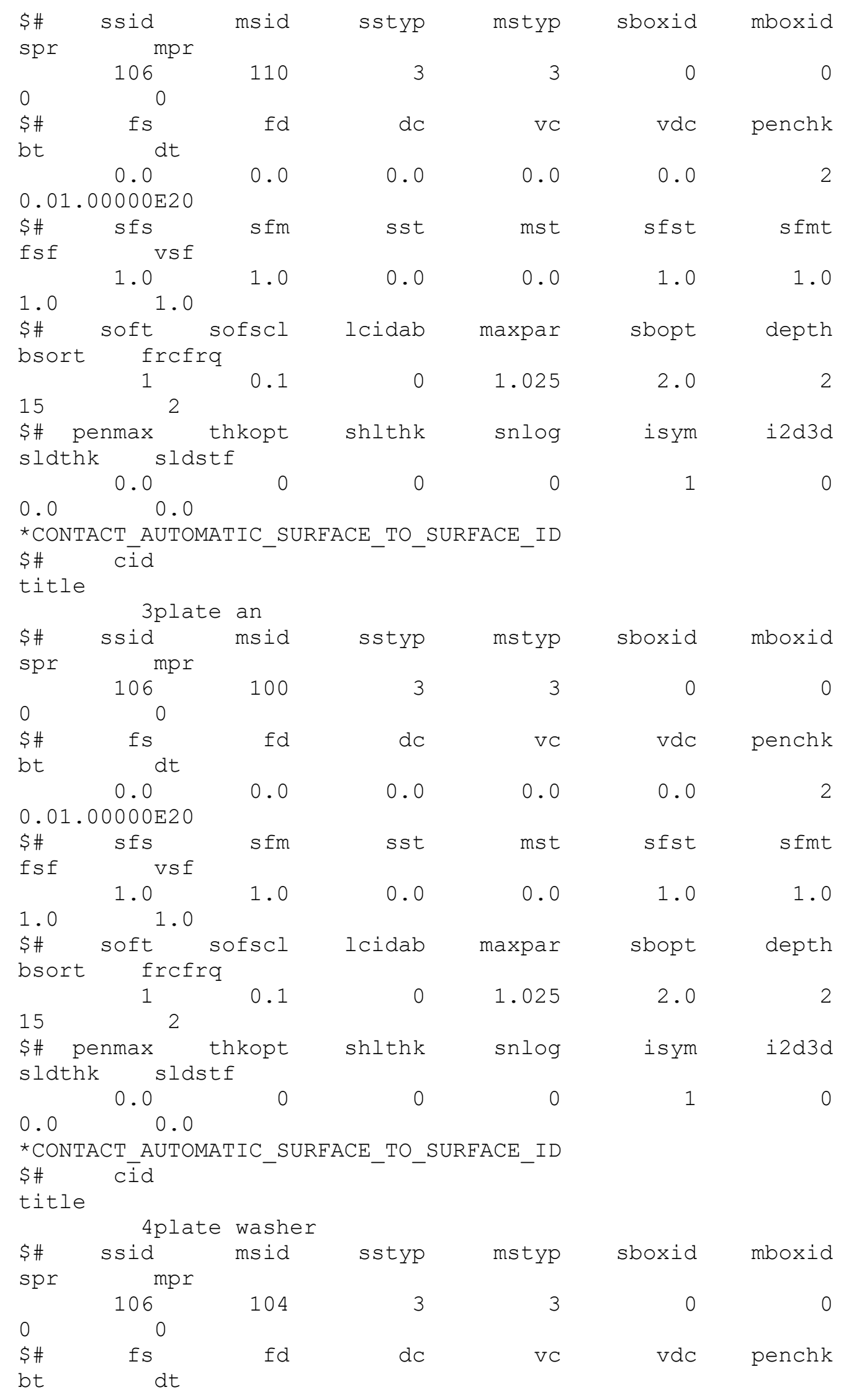




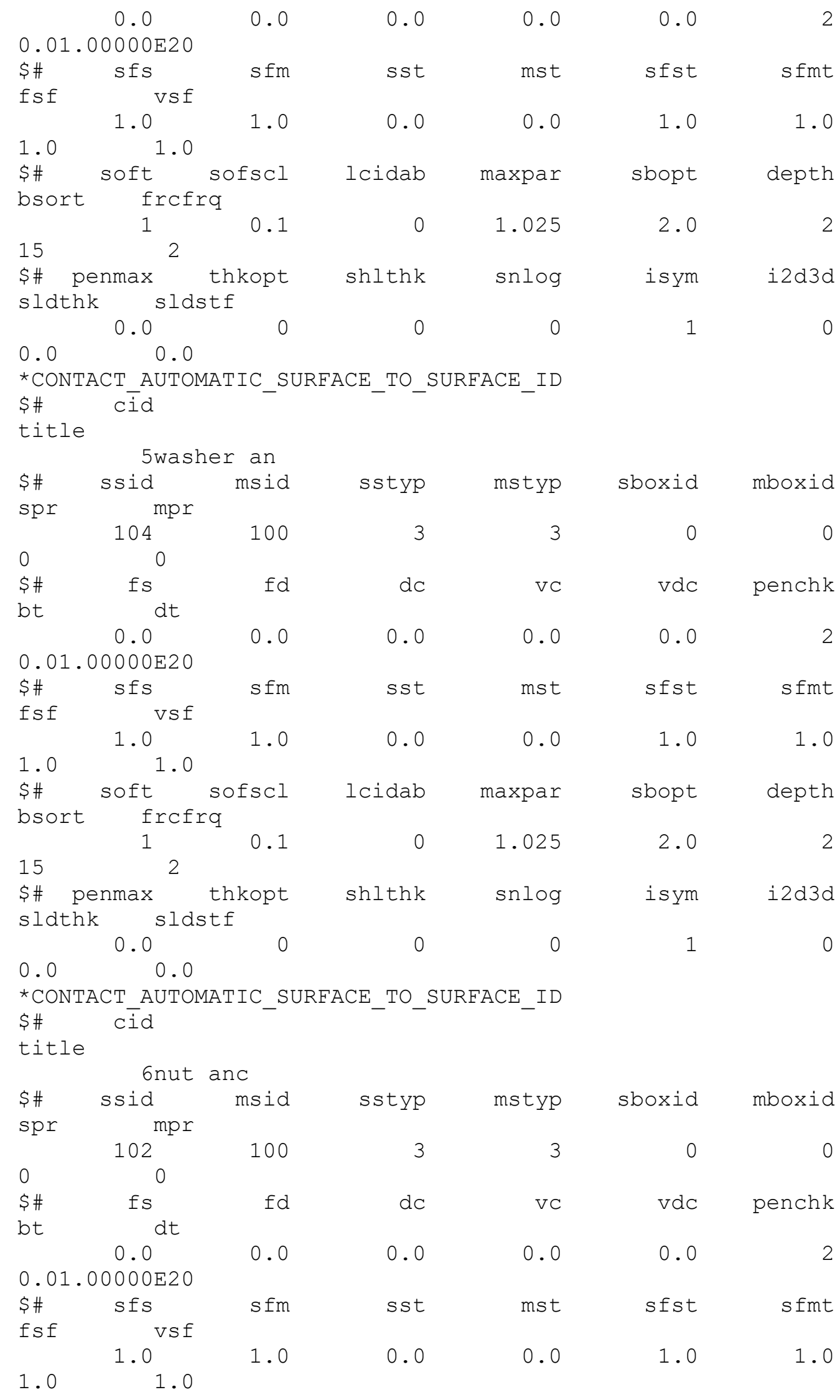




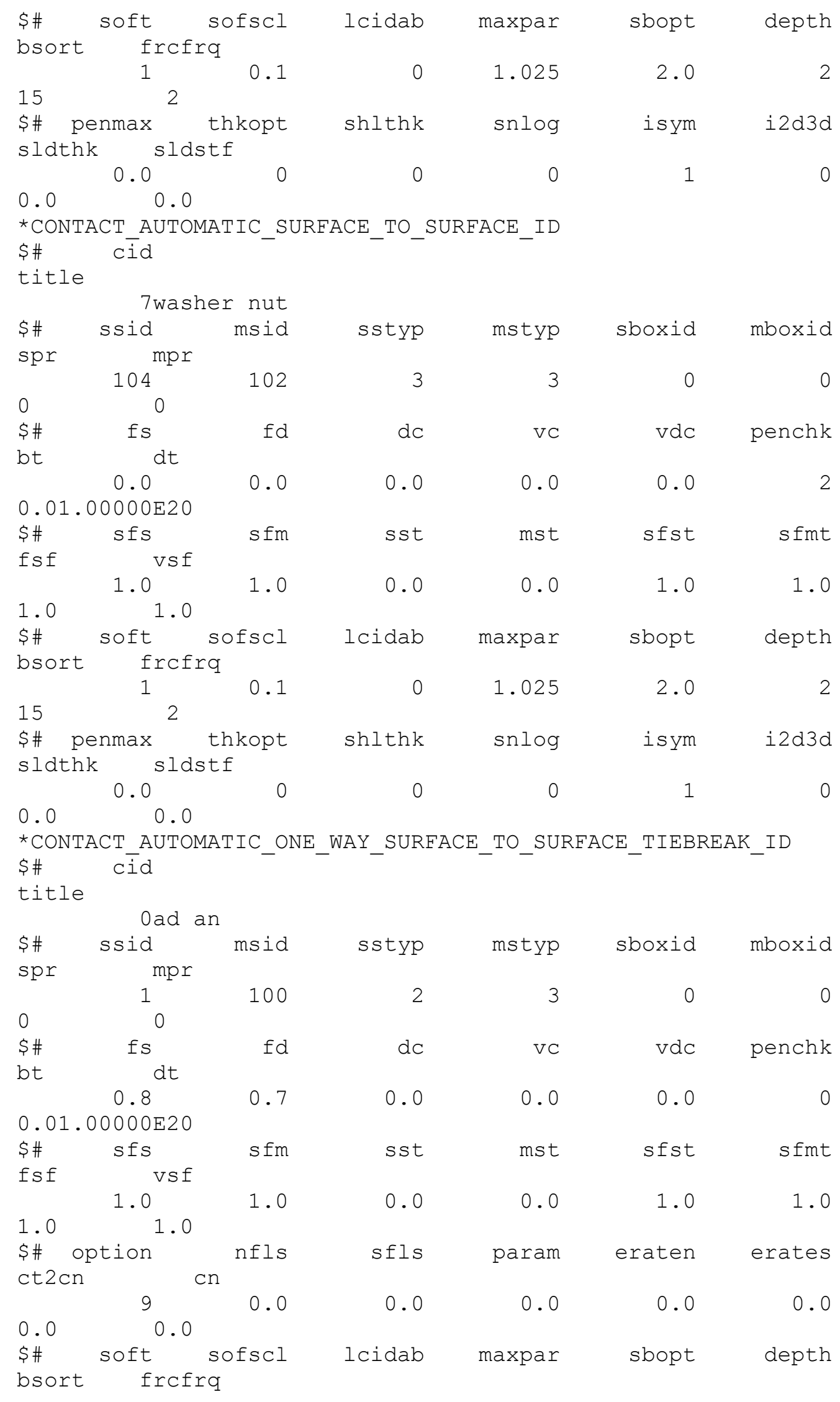




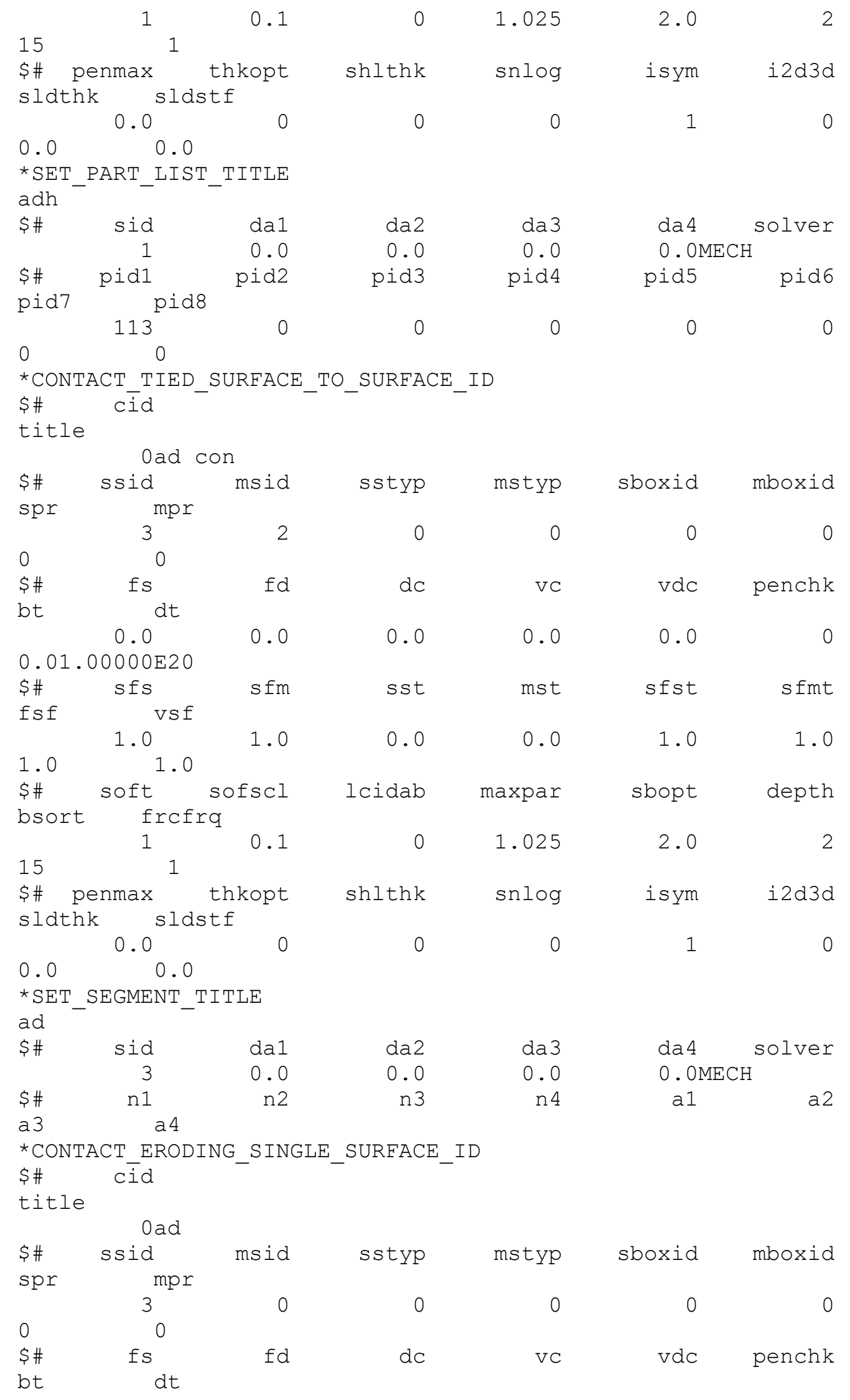




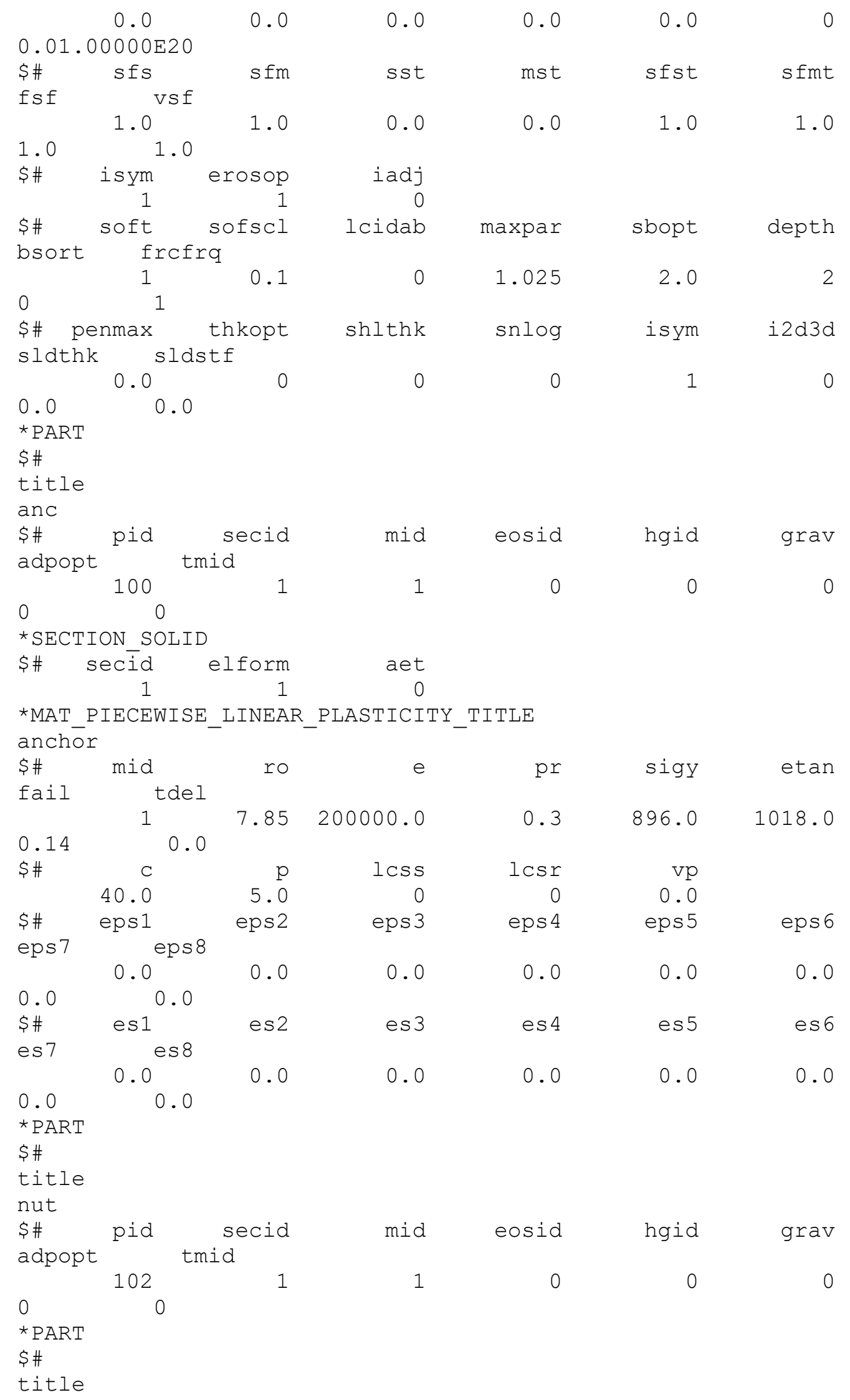




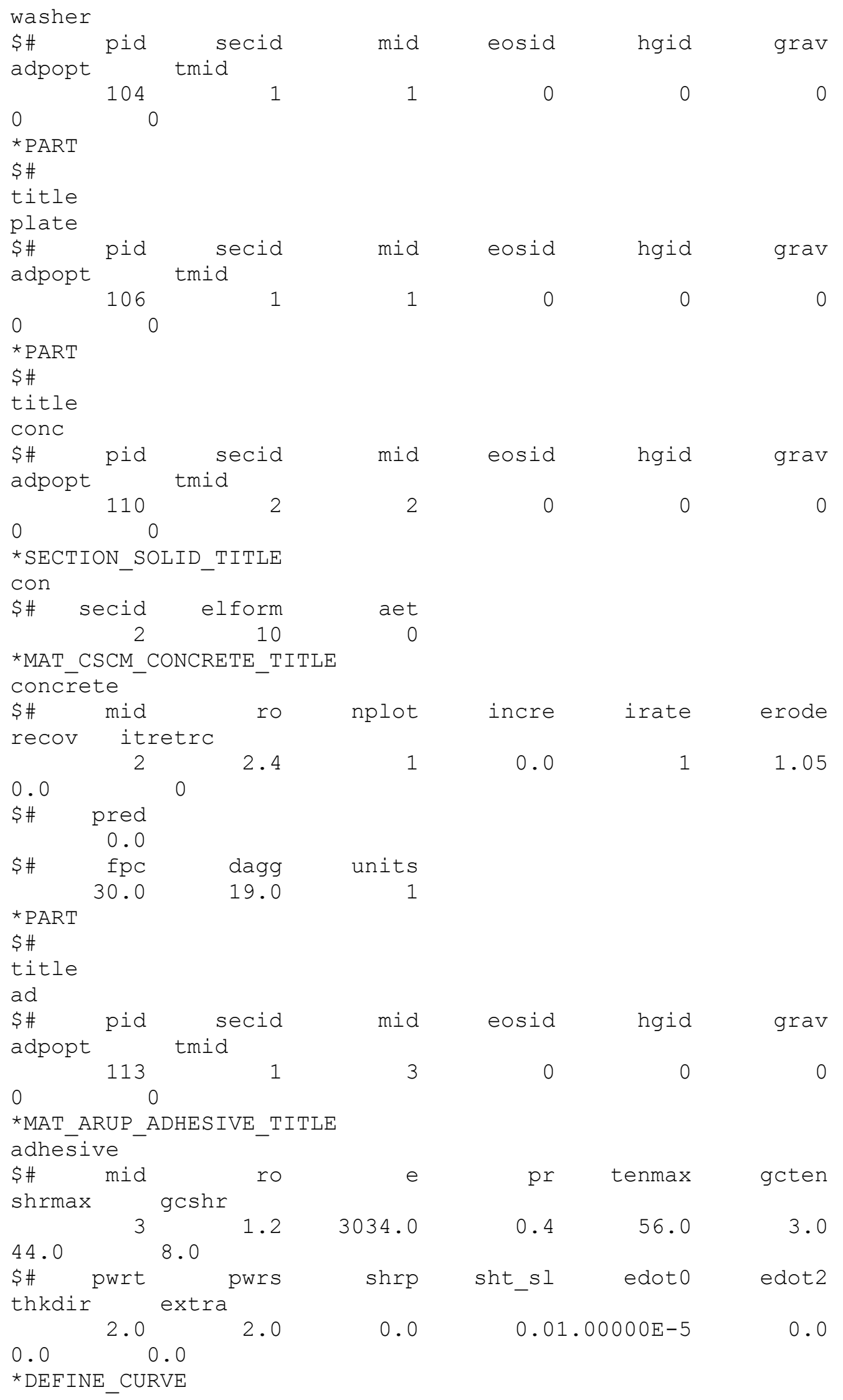




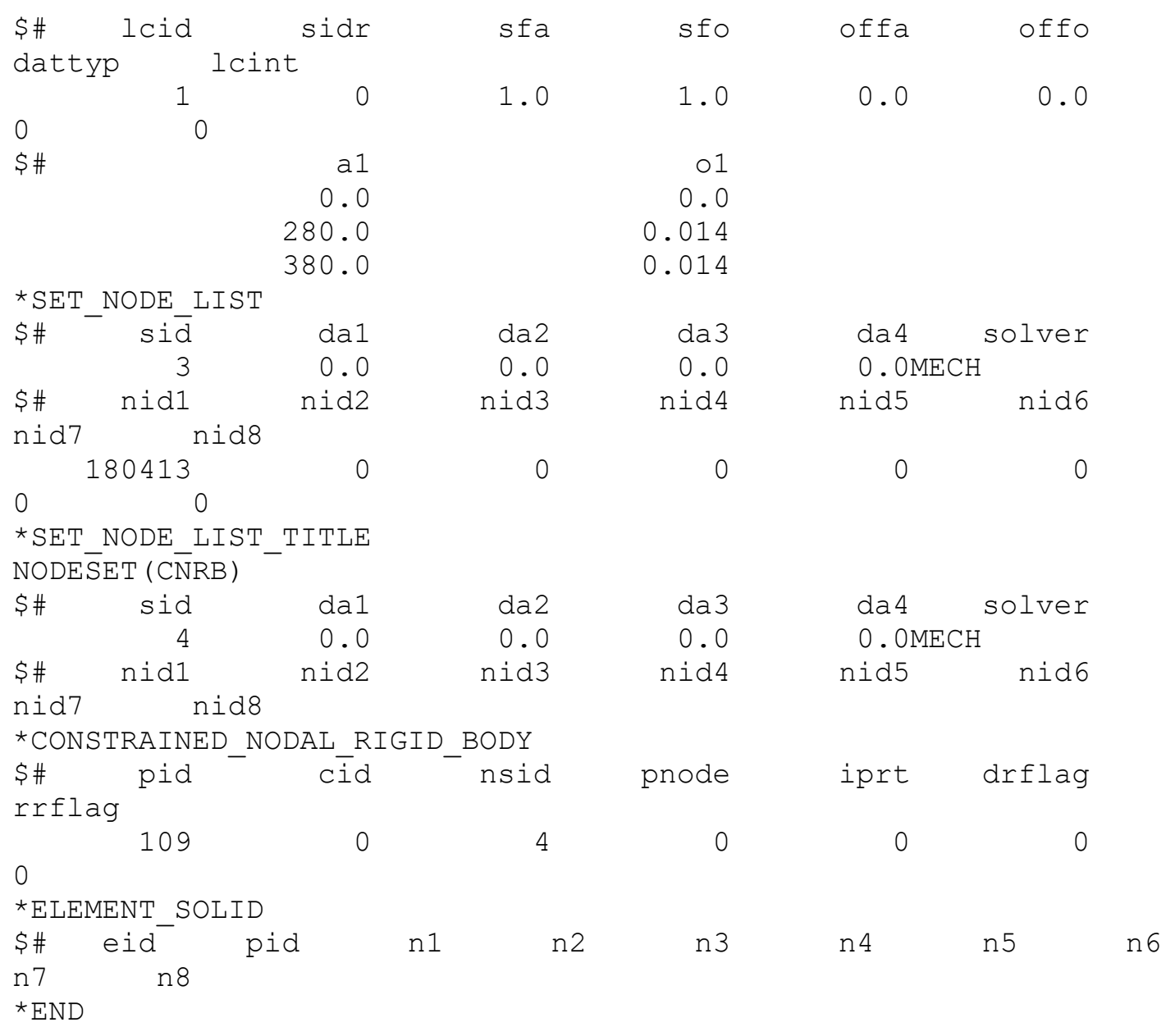

\section{Undercut anchor under tensile load}

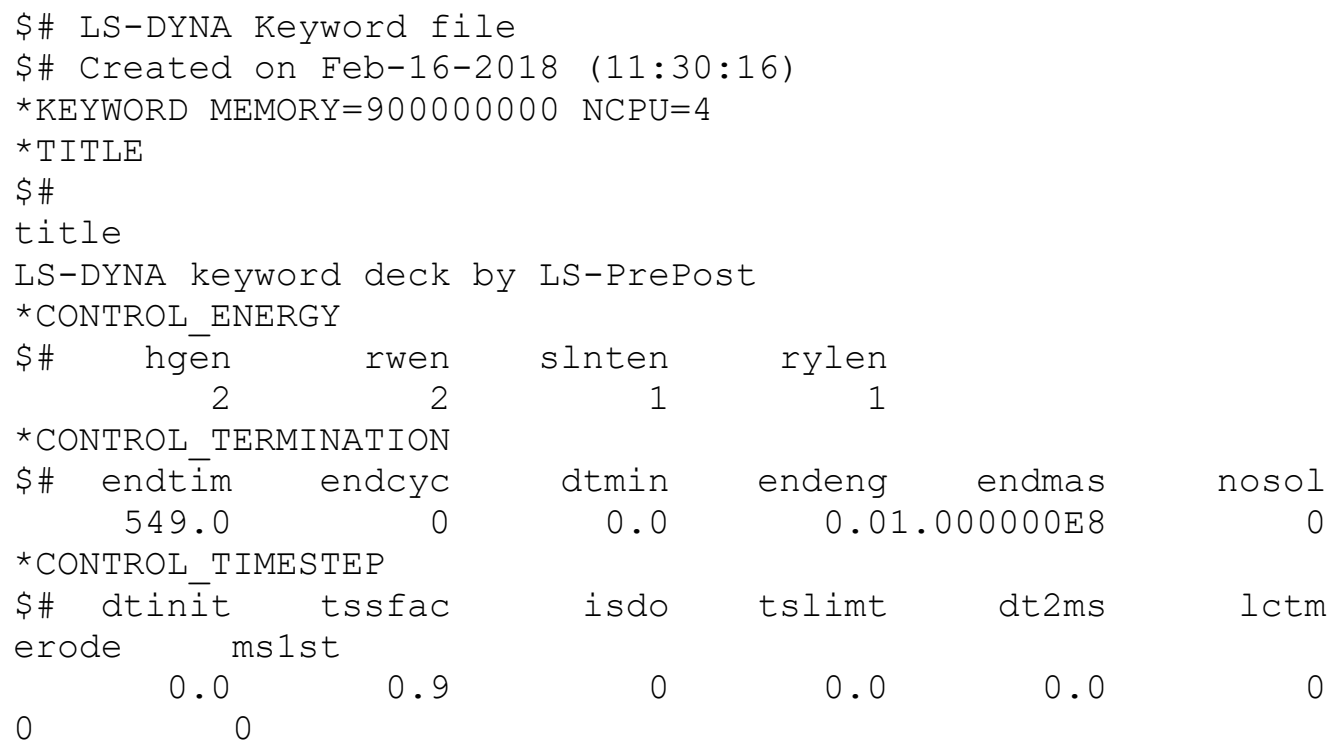




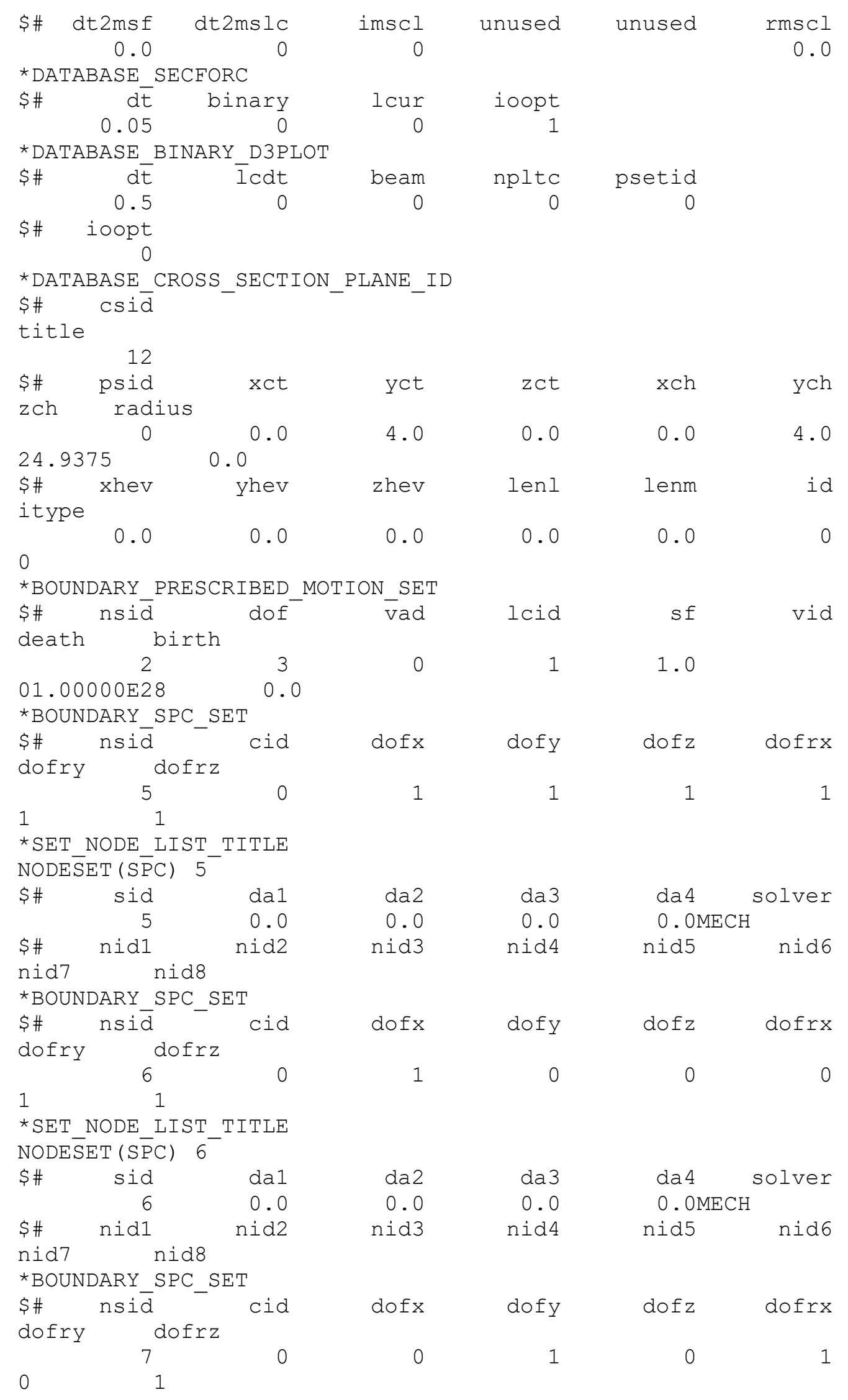




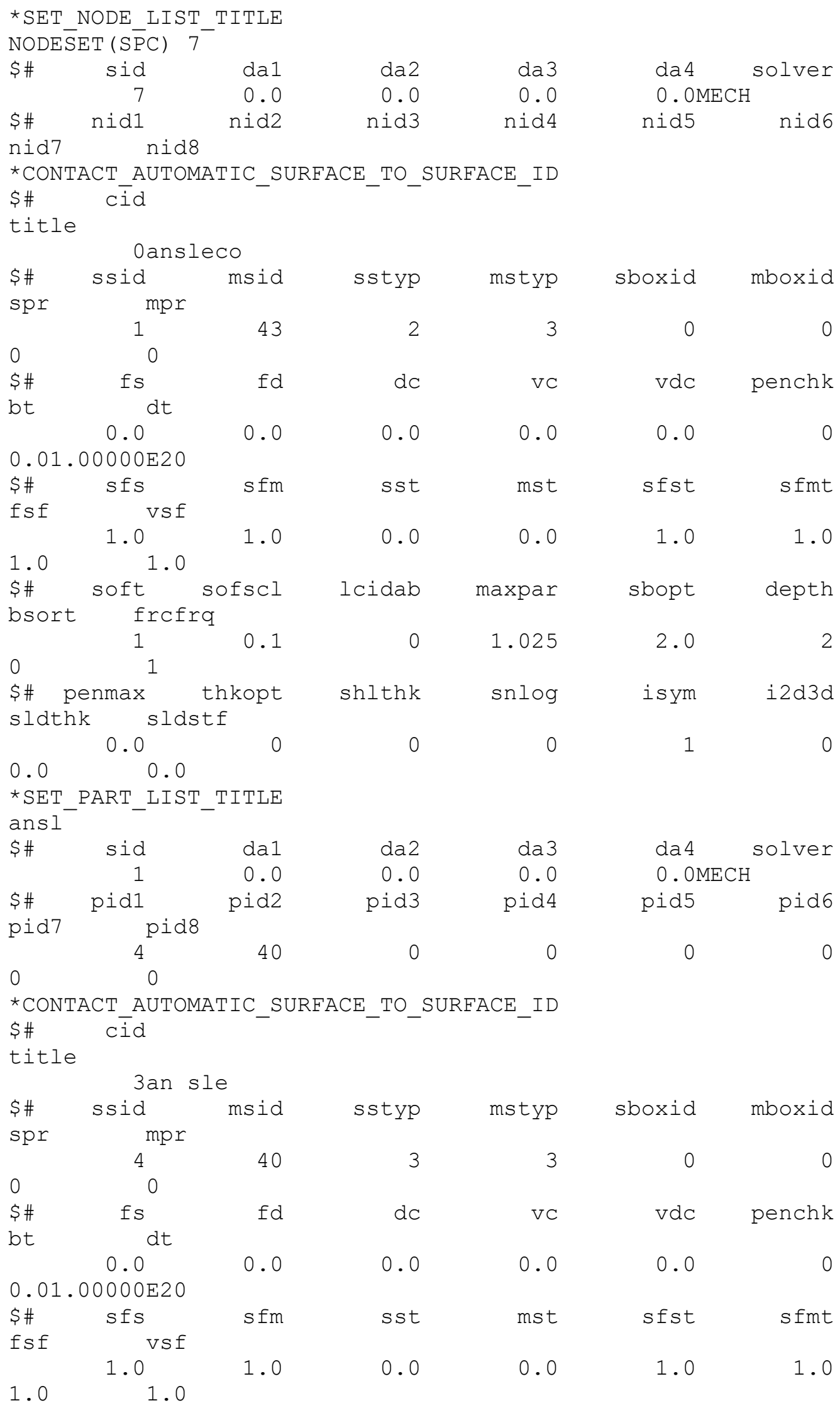




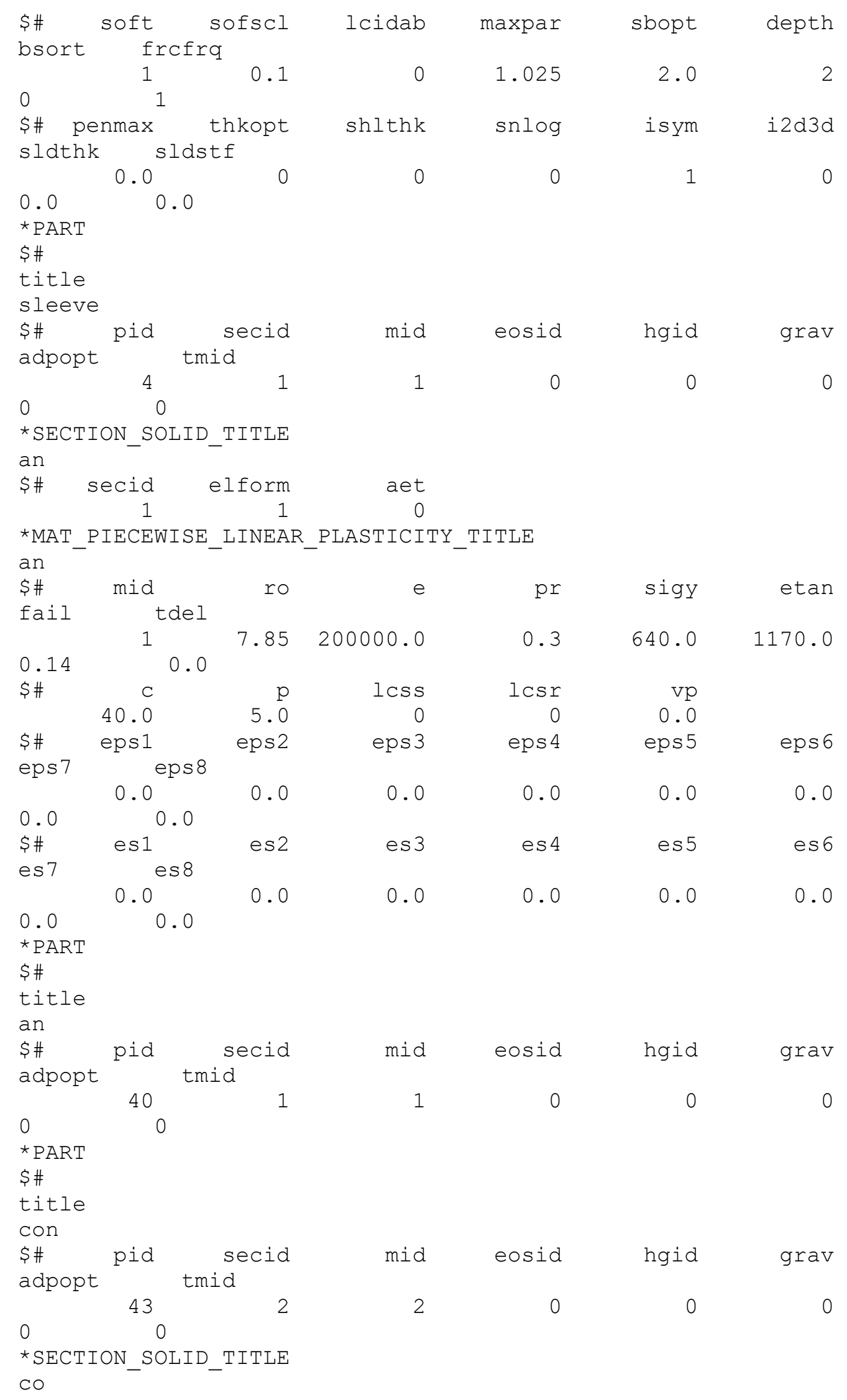




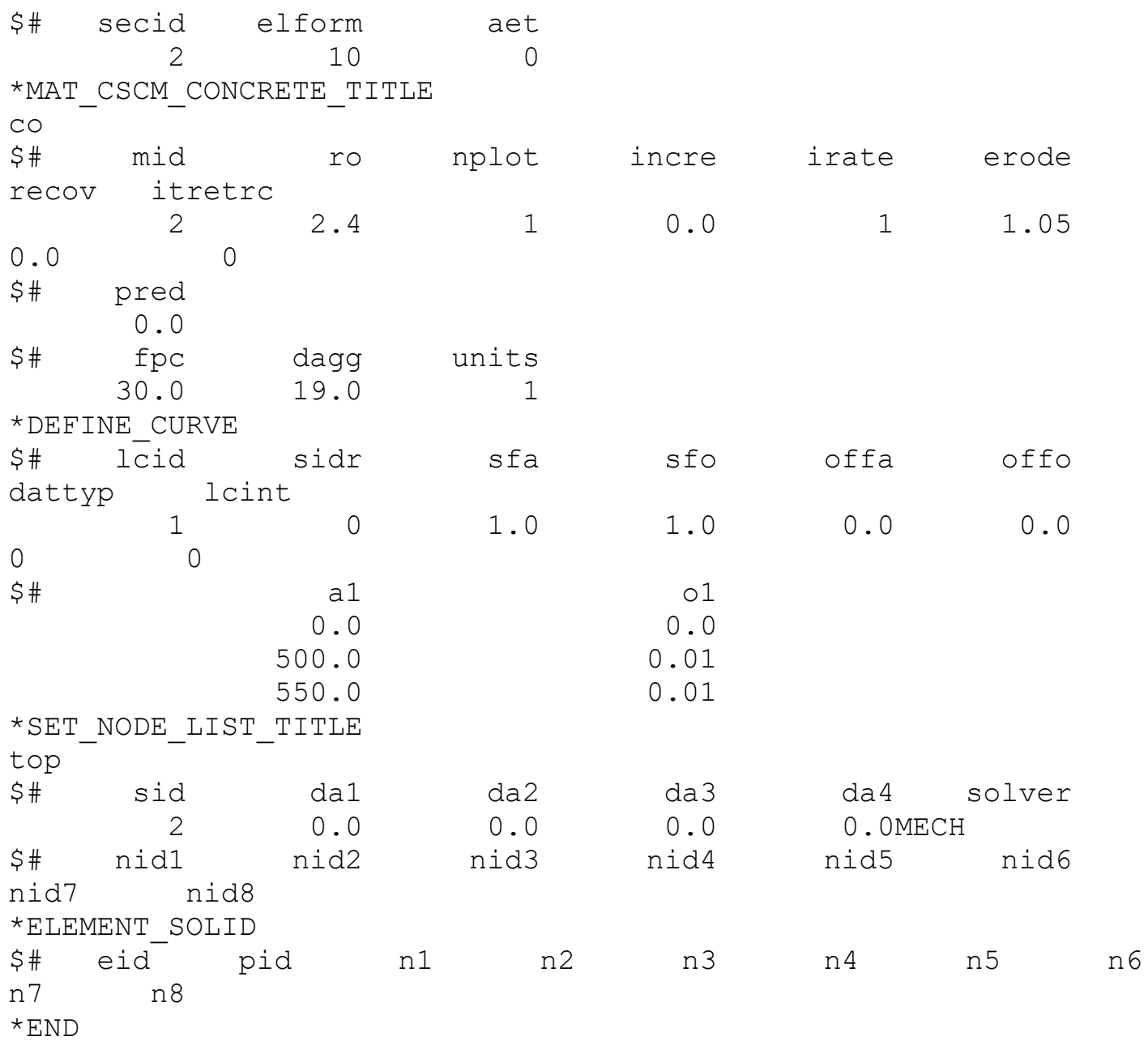

\section{Undercut anchor under shear load}

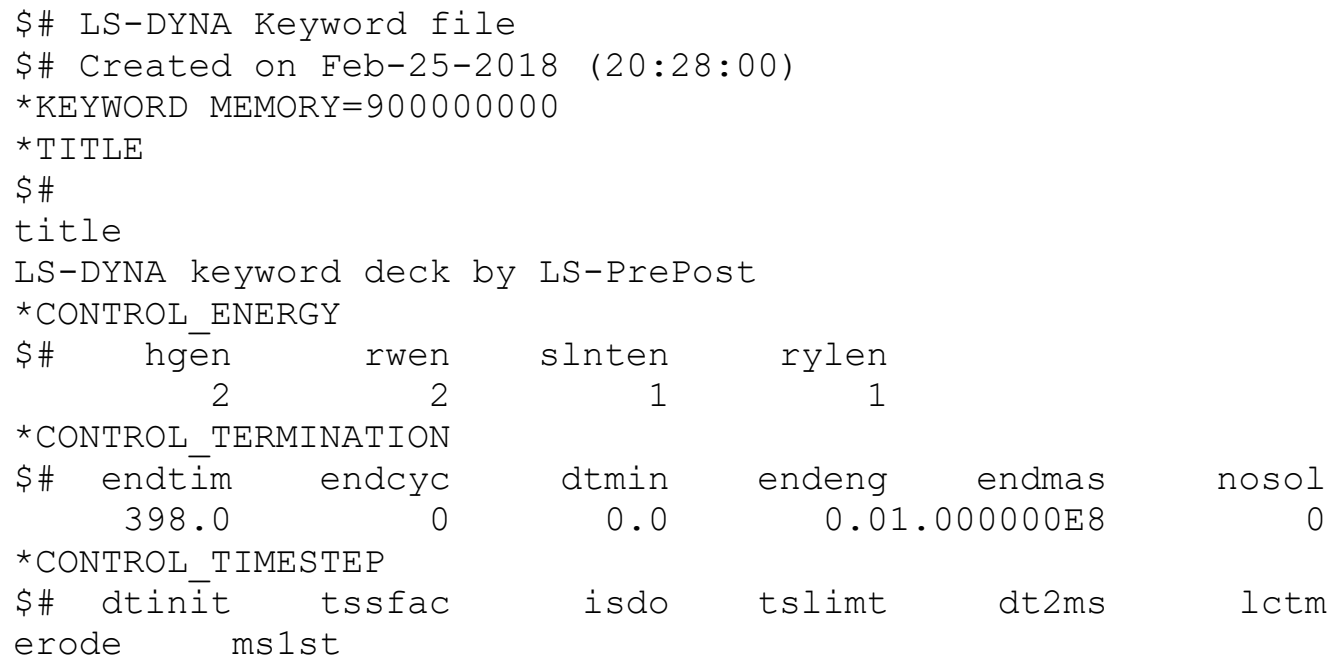




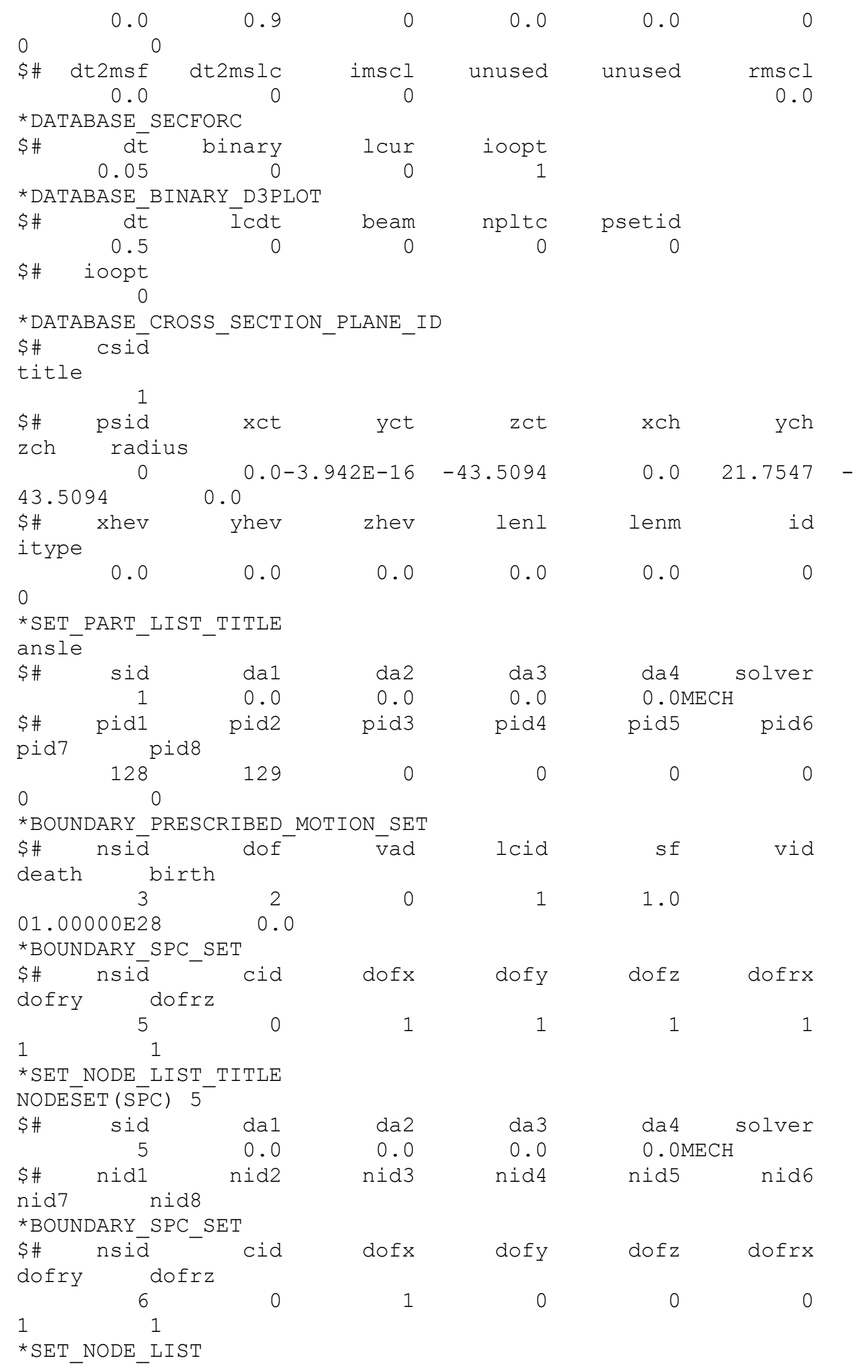




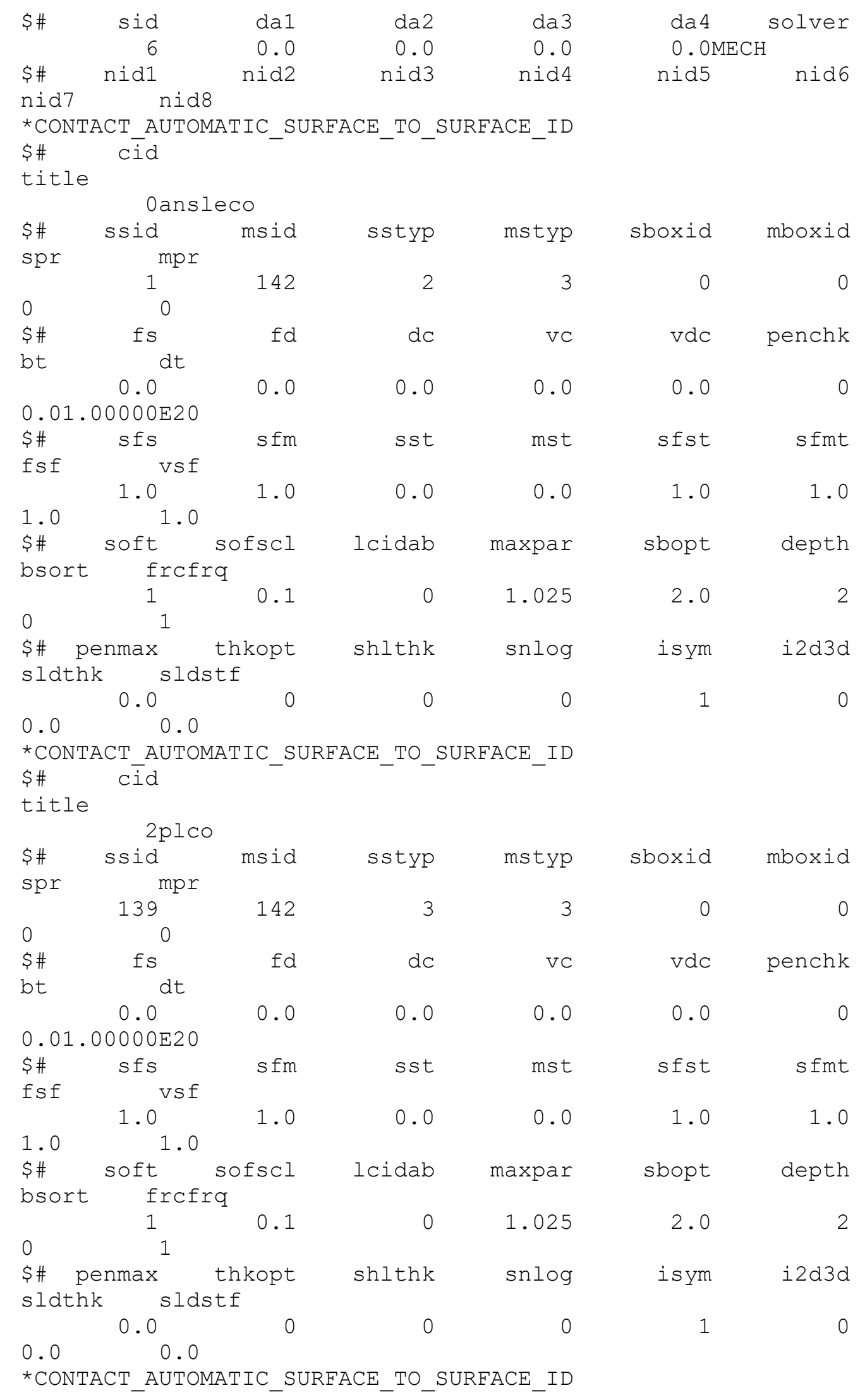




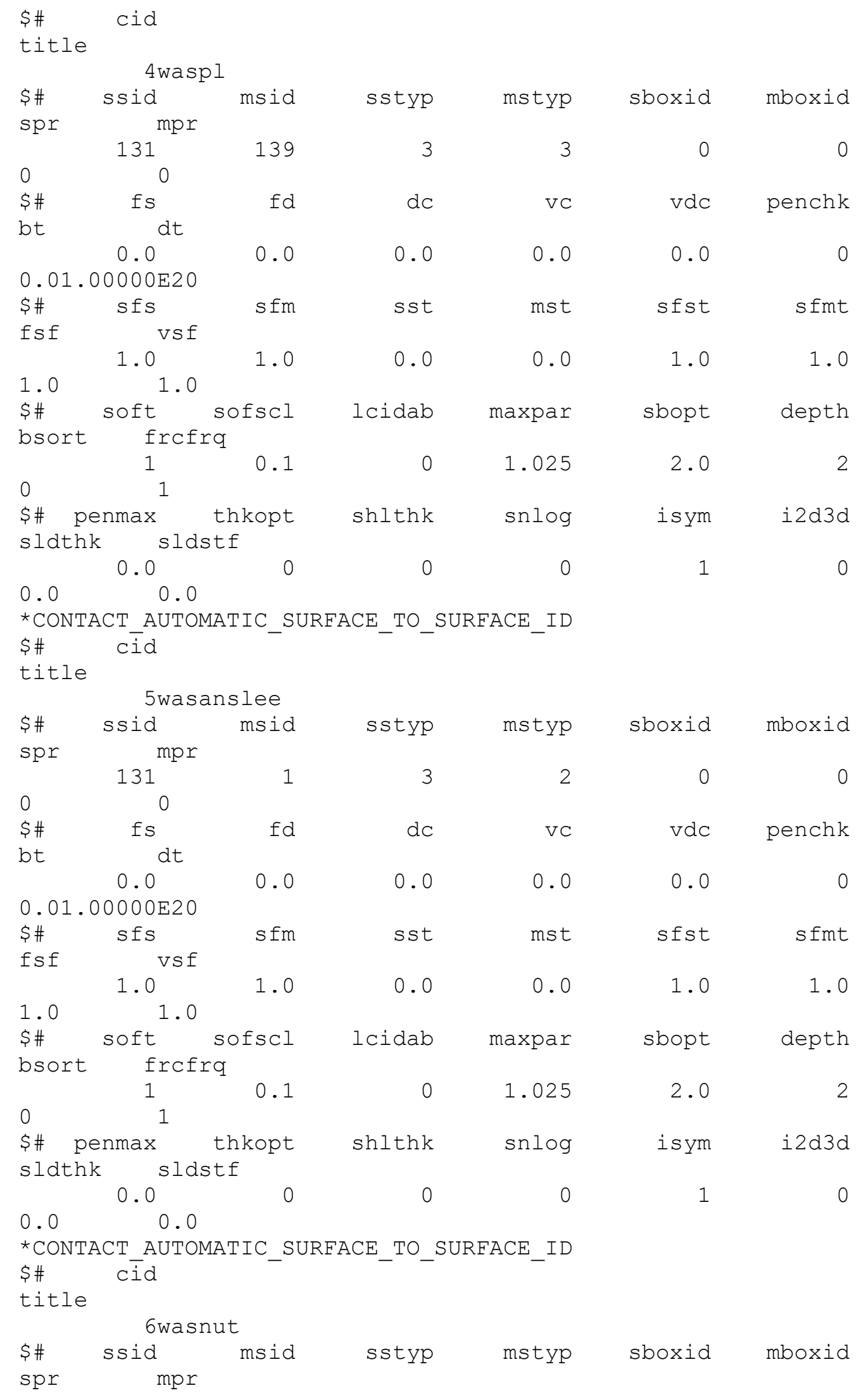




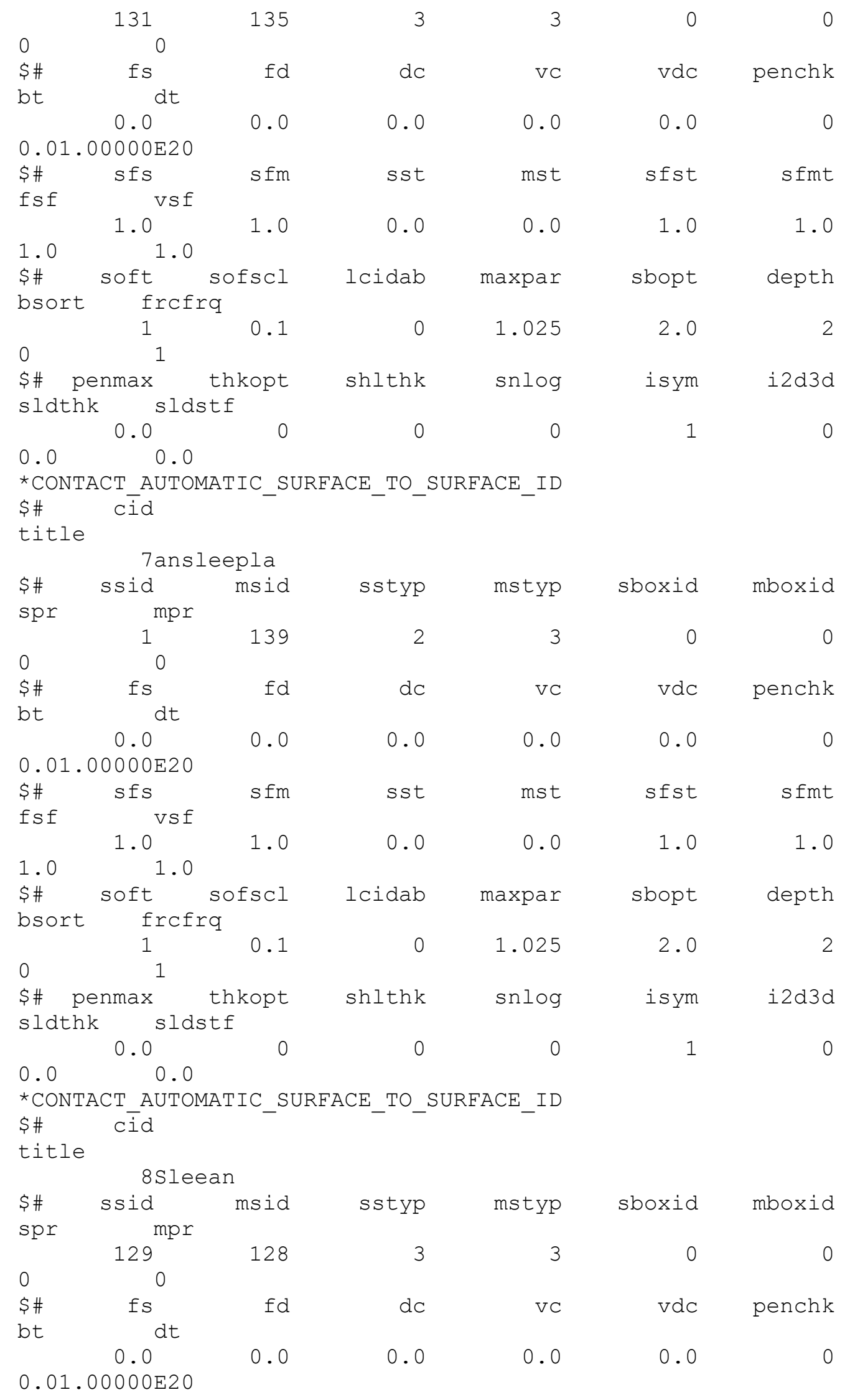




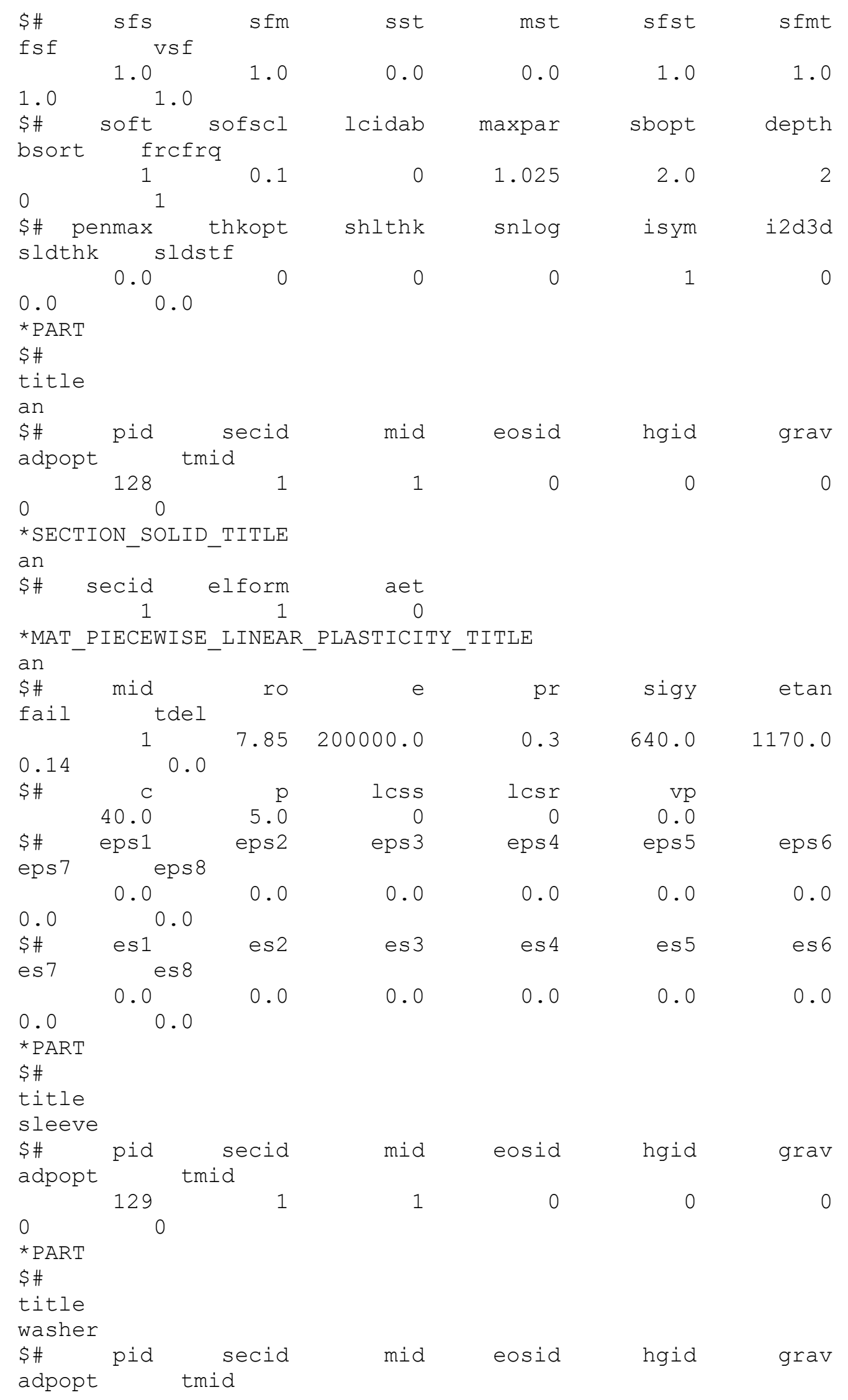




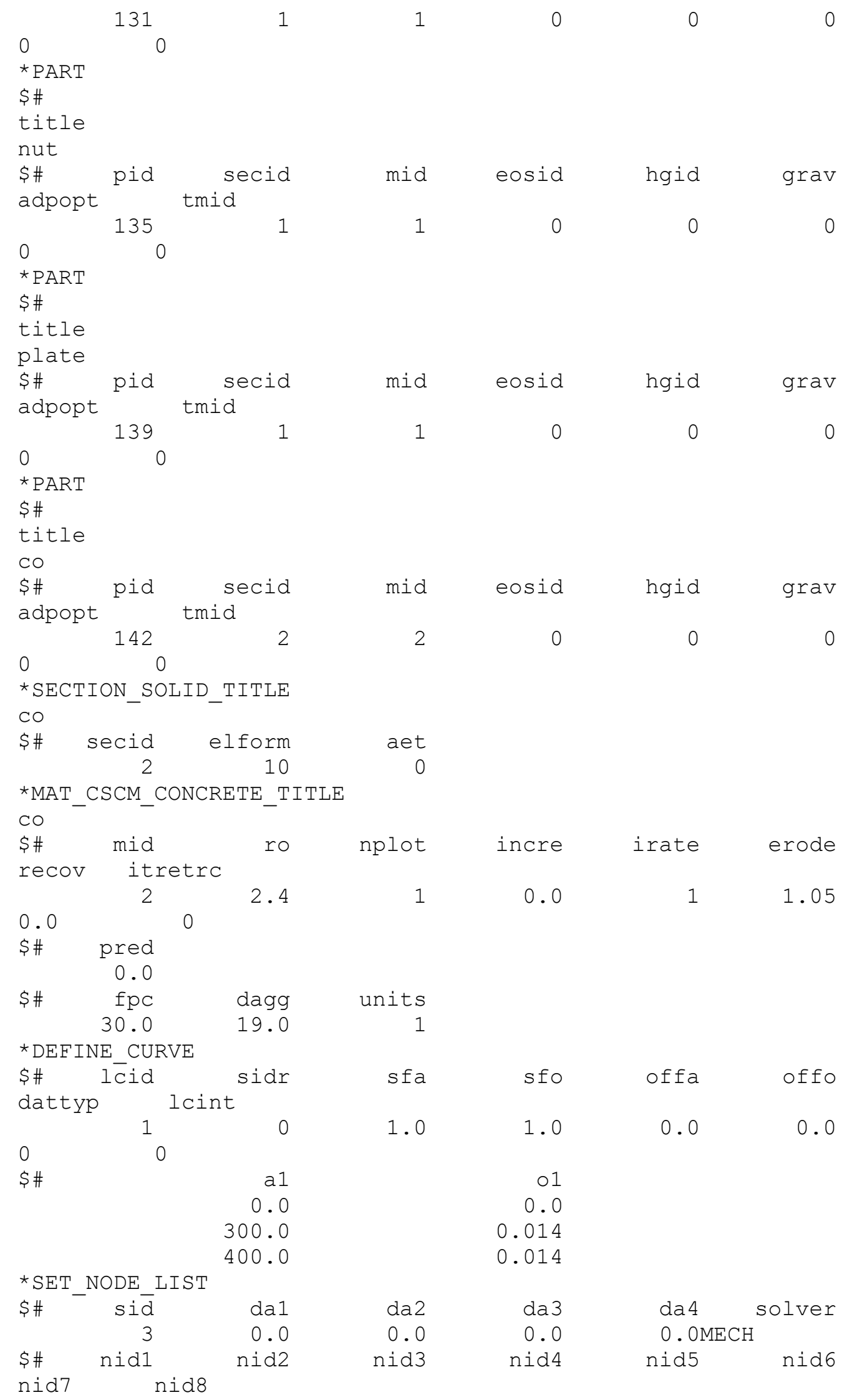




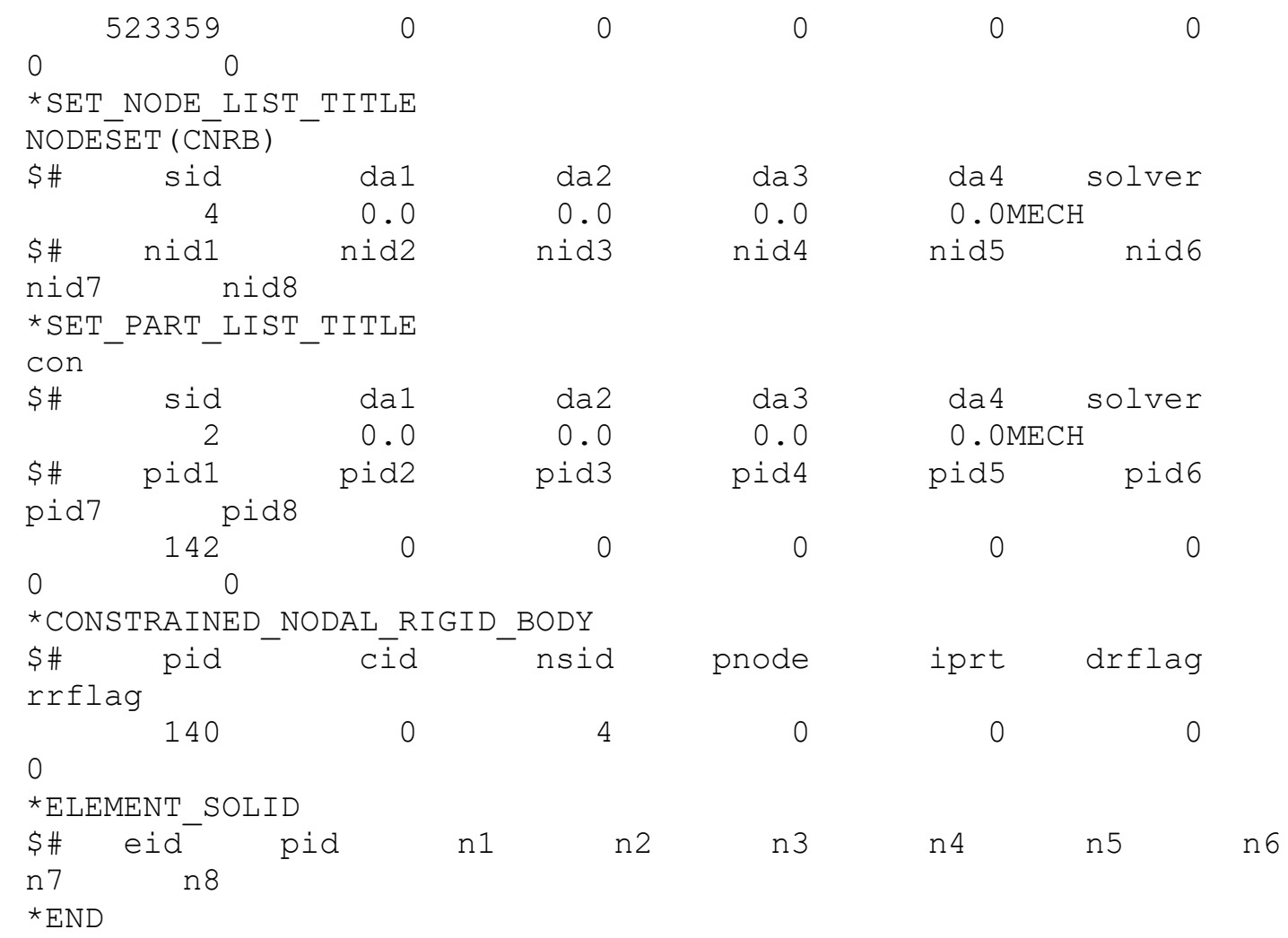

